\title{
AN ANALYSIS OF OWNERSHIP FORMS IN OFFSHORE HIGHER EDUCATION MARKETS: \\ A RESOURCE BASED PERSPECTIVE
}

By

Vikash A. Naidoo

\begin{abstract}
A thesis
submitted to the Victoria University of Wellington in fulfilment of the requirements for the degree of Doctor of Philosophy in International Business
\end{abstract}

Victoria University of Wellington 2010 
This $\mathrm{PhD}$ research seeks to consider overseas investment in a new and important context: education. Estimated at approximately US\$ 65 billion and representing roughly $3 \%$ of global services exports (Alderman, 2001), trade in education services is fast becoming a global business (Czinkota, 2006). In Australia, New Zealand and the United States, for example, educational service is estimated to be, respectively, the third, fourth and fifth largest service sector export (Vincent-Lancrin, 2004). The globalisation and internationalisation of higher education manifest themselves in various forms, of which transnational education or 'offshore' programmes - those taught outside of a host academic institution's country of origin - have been experiencing rapid increases over the past decade. Most of this growth, to date, has taken place through contractual arrangements such as licensing (e.g. twinning and articulation arrangements). However, there are also a substantial number of academic institutions that are currently delivering transnational education through equity modes of entry (e.g. branch campus operations). In this context, this $\mathrm{PhD}$ research, using universities as the unit of analysis, seeks to understand the dynamics of transnational education - how it is happening and why it is happening - grounded in the strategy and international business literatures. In particular, the research question being addressed in this study is: What resources are associated with entry mode choice for education providers entering overseas markets?

Using a multi-method research design consisting of both qualitative and quantitative analysis, seven different types of resources are specifically examined in this study: Geographical experience, Industry experience, Transfer experience, Organisational culture, Financial resources, Reputation and Learning intent. Using the resource-based view (RBV) as its theoretical underpinning, this study hypothesises that the more access to these resources an education service provider might have, the more they will favour a higher level of ownership in offshore education developments. This overall hypothesis builds on the basic assumption of the RBV that organisations in possession of resources which are potential sources of competitive advantage in a target market, would favour a 
mode of entry that facilitates control over and protection of the resources. This fundamental assumption of the RBV differs to that of the transaction cost approach, which typically views shared-control modes as the default mode of entry. The conceptual model developed in this study further postulates that the resource-entry mode relationship is moderated by institutional distance. The education sector in most countries is a regulated sector, where authorities monitor the quality of education. Therefore, when investing offshore, education service providers are likely to operate around some form of regulated institutional environments that are likely to affect their mode of entry decisions.

From the collected 308 instances of foreign market entry of universities in the United Kingdom (UK), United States (US), Canada (CA), Australia (AU), New Zealand (NZ) and Ireland (IR), analysis is conducted at both an aggregate and geographical grouping level (i.e. UK/IR, AU/NZ and US/CA). To assess the sensitivity of the obtained results, three estimation techniques are also analysed: Ordinary Least Squares (OLS), Tobit and Negative Binomial regressions. To further assess the sensitivity and robustness of the observed findings for the moderating role of institutional distance, three measures of distance are analysed: World Competitiveness Yearbook, Economic Freedom Index and Hofstede (1980) cultural indices.

From the different groupings and estimation techniques, the empirical findings show that support is obtained for Transfer experience, but only when using OLS estimation. Mixed support is obtained for Geographical experience, Industry experience and Financial resources. The hypotheses with respect to the other types of resources are not supported. These findings suggest that, contrary to the basic premise of the RBV, a higher level of ownership might not always be the preferred entry mode in the offshore education context. The observed findings also do not support the moderating hypothesis of institutional distance on the resource-entry mode relationship. This lack of support is consistent across all three measures of distance analysed. Several possible explanations for these observed findings are conjectured in Chapter 7. These explanations are not purely theoretical conjectures but are also enriched on the basis of the interviews 
conducted as part of the exploratory stage of this study. The greatest takeaway from this study is that the observed findings, which do not fully conform to mainstream international business and strategic management theories, can be attributed to context/industry specific conditions. Traditional international business and strategy research has largely focused on "for-profit" firms. Given that universities are "not-forprofit" organisations, it needs to be recognised that their international operations are different from those of regular multinational firms. These findings provide initial steps in improving our understanding of the internationalisation of the education services sector. 


\section{Acknowledgements}

"Nine tenths of education is encouragement" Anatole France (1844-1924)

As summed up in this quote by famous French novelist, Anatole France, being able to say that I have graduated from a $\mathrm{PhD}$ programme is a privilege that I cannot claim to have achieved all by myself. Indeed, this achievement is a tribute to the support that I have received from a number of people throughout my university career. Without them, my academic achievements to date would not have been possible and I wish to seize this opportunity to express my gratitude towards them.

First and foremost, I want to give special thanks to my parents, my sister and my partner. To enumerate all the support I have received from them and the sacrifices they have made for me is a task that cannot be confined to a few words. So, I would just like to say thank you for your constant encouragement. Dad, thanks for teaching me the importance of education right from a young age and for inculcating in me the work ethic that has been so useful in facing up to the challenges of the doctoral programme. Mum, thanks for always caring and for the emotional support you have given me. Although I might not always have shown it, I will forever be grateful for your love and support. To my sister Anna, thank you for putting up with my mood swings. To my partner, Sheonaigh, thank you for your constant encouragement through the countless hard times when I felt like giving up. The rigours and demands of writing a thesis can be stressful sometimes and I apologise if, at times, I have allowed this to come in the way of our relationship.

The contributions of my supervisors Dr Elizabeth Rose (Beth) in the School of Marketing and International Business at VUW and Professor Kiyohiko Ito of the University of Hawaii at Manoa, also permeate every aspect of this work. Thank you for being a constant source of motivation when I started doubting my own ability. Thank you also for allowing me the independence of framing my own research interest, a key 
success factor to my completion. I would like to particularly thank Beth for her unparalleled mentorship and guidance that she provided over the last three years. Despite her hectic schedule jet-setting around the world, her ability of guiding me through the $\mathrm{PhD}$ journey has been extraordinary. Thanks for everything Beth.

I also wish to thank the research committee of the School of Marketing and International Business at VUW for your understanding in allowing me to complete the $\mathrm{PhD}$ programme mostly by distance. Thank you for being sympathetic to my personal circumstances, which required me to be away from Wellington most of the time. I would especially like to thank Professor Peter Dowling of the research committee for his encouragement during the final stages of the $\mathrm{PhD}$ submission process.

Doing the PhD mostly via distance added some extra challenges in terms of finding a place to stay when I was in Wellington to meet my residency requirements. In this context, my thanks also go to my friend Dama Black for allowing me to stay at her place every time I needed to. Knowing that I always had a place to stay whenever I was in Wellington allowed me the luxury of having one less thing to worry about!

I am also deeply grateful to Dr Siah Hwee Ang, Senior Lecturer in the Department of Management and International Business at The University of Auckland and his colleague(s) at The National University of Singapore for providing access to the secondary data used in this study. Although you were not obligated to help, your willingness to help is extremely appreciated.

To Professor Doren Chadee, Head of the School of Management and Marketing at The University of Southern Queensland (previously of The University of Auckland), thank you for introducing the concept of education services as an area worthy of investigation. Not only did this lead to a Masters thesis but has now also been the subject of a $\mathrm{PhD}$ thesis as well as a number of referred publications. Many thanks also for your early encouragement to pursue the PhD academic journey. 
Last but not least, I want to thank all the participants who have contributed in one way or another with this particular project. While I cannot name you individually due to confidentiality reasons, I am sure you will recognise yourself.

On that note, I conclude my acknowledgements and wish to express my gratitude to anyone else whom I have failed to personally mention but who has contributed directly or indirectly in making this thesis possible. Thanks to you all and all the best for the future! 


\section{Table of Contents}

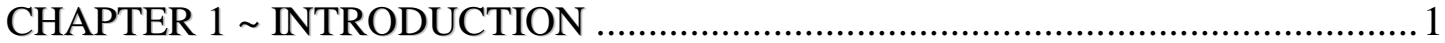

1.1 BACKGROUND AND RATIONALE OF STUDY ......................................... 1

1.2 DEFINITION OF TRANSNATIONAL EDUCATION …..............................4

1.3 STATEMENT OF THE PROBLEM AND RESEARCH OBJECTIVES ..........6

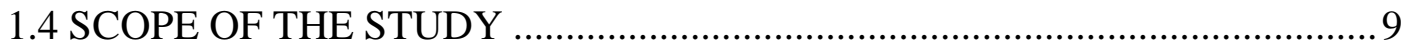

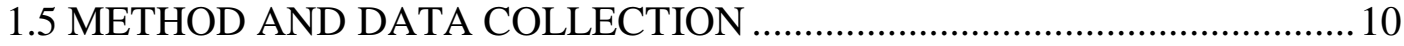

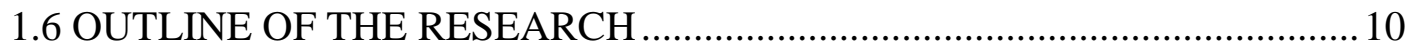

CHAPTER 2 DEVELOPMENTS IN TRANSNATIONAL HIGHER EDUCATION:

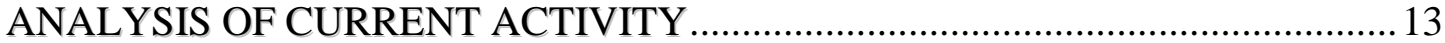

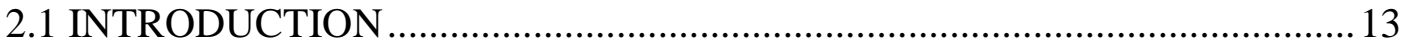

2.2 MODE 3 TRANSNATIONAL EDUCATION: A TYPOLOGY ......................19

2.3 MODE 3 TRANSNATIONAL EDUCATION: A CHANGING EDUCATIONAL MAP 23

2.3.1 Mode 3 Transnational Education via Wholly-Owned Subsidiaries and Joint

Ventures 25

2.3.2 Mode 3 Transnational Education via Non-Equity Arrangements ..............28

2.4 RATIONALES BEHIND THE GROWTH OF TRANSNATIONAL EDUCATION 37

2.5 TRANSNATIONAL EDUCATION AS A TOOL FOR BUILDING CAPACITY AND CAPABILITY 40

2.5.1 Risks Associated with Transnational Education for Capacity Building ....44 2.6 NATIONAL REGULATORY FRAMEWORK FOR TRANSNATIONAL

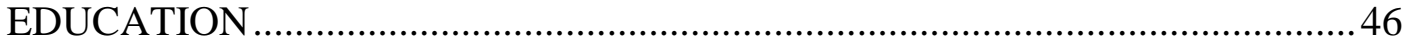

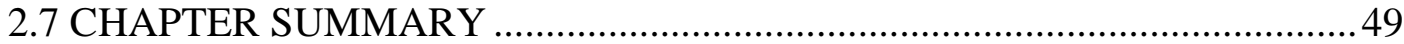

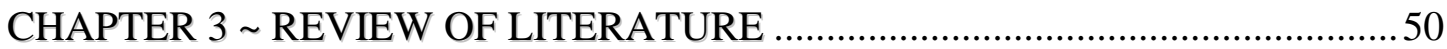

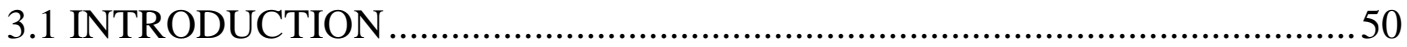

3.2 ENTRY MODE SELECTION: AN OVERVIEW OF THE LITERATURE ...51

3.2.1 Gradual, Incremental Involvement Models ..............................................57

3.2.2 Transaction Cost/Internalisation Theory …..............................................6 60

3.2.3 Competitive Strategy Models ..................................................................65

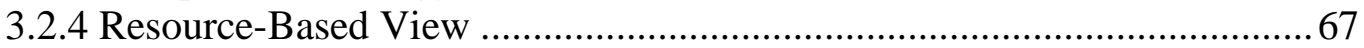

3.2.5 Organisational Capability Models ......................................................69

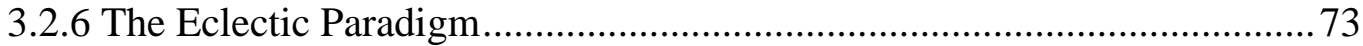

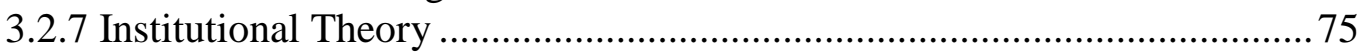

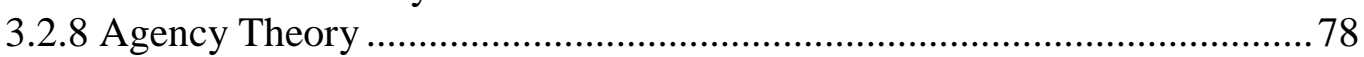

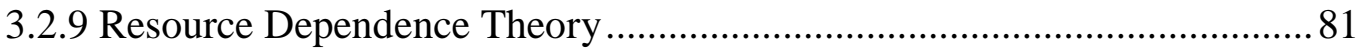

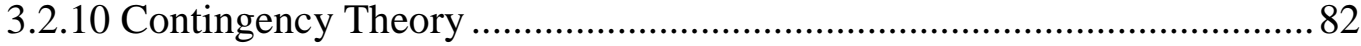

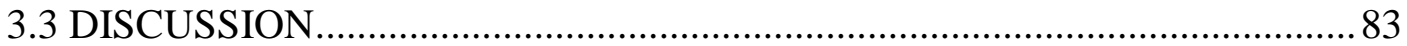

3.4 THE INTERNATIONALISATION PROCESS OF SERVICES......................85

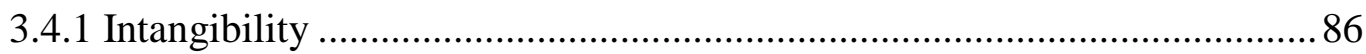

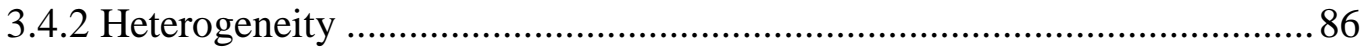




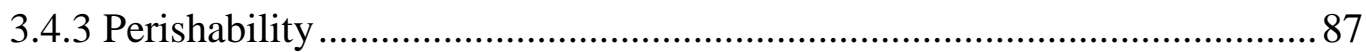

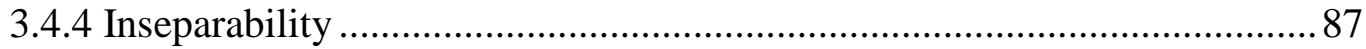

3.4.5 Implications of Service Characteristics in International Business ...............89

3.4.6 Implications of Service Characteristics in the Context of the

Internationalisation of Education Services ......................................................94

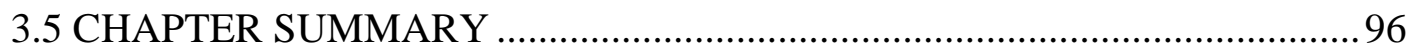

CHAPTER $4 \sim$ CONCEPTUAL FRAMEWORK \& HYPOTHESIS DEVELOPMENT

.

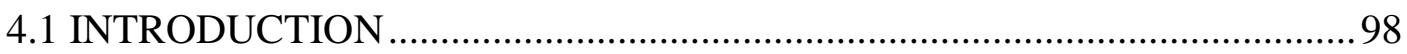

4.2 RESOURCES AND INTERNATIONALISATION: BUT WHAT TYPES OF

RESOURCES? ....................................................................................99

4.3 ENTRY MODE CHOICE IN EDUCATION SERVICES: A CONCEPTUAL

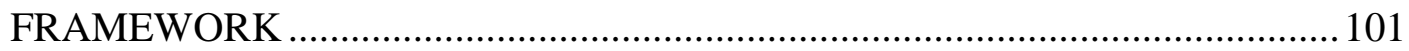

4.3.1 Elements of Organisation-Specific Resources ........................................... 104

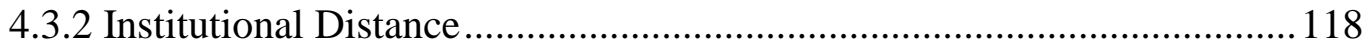

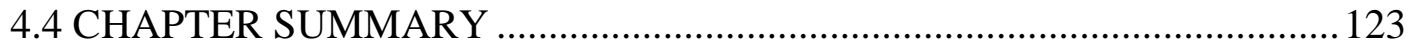

CHAPTER $5 \sim$ CONSTRUCTS, MEASURES \& RESEARCH METHODOLOGY125

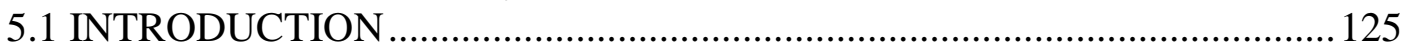

5.2 RESEARCH DESIGN, SAMPLE SELECTION AND DATA COLLECTION

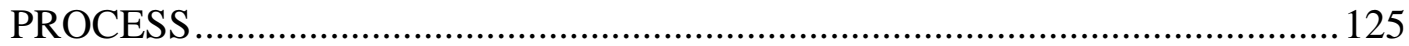

5.3 VARIABLES AND THEIR OPERATIONAL MEASURES ............................128

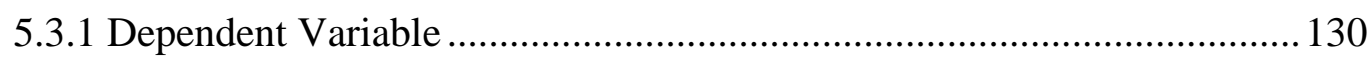

5.3.2 Independent Variables ............................................................................. 130

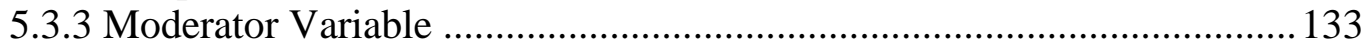

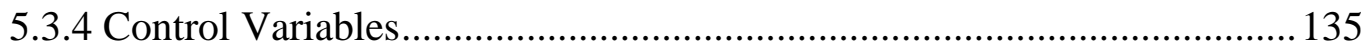

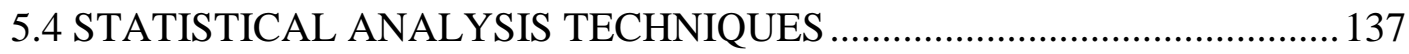

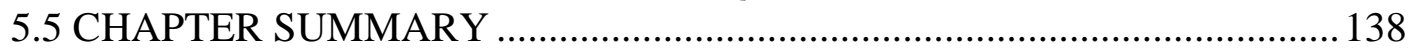

CHAPTER 6 FINDINGS FROM FIELD INTERVIEWS ...................................... 140

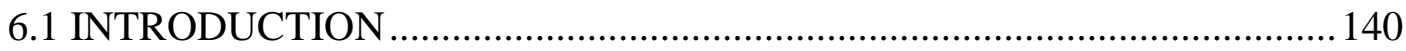

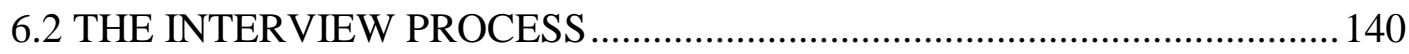

6.3 THE INTERVIEWS: INSIGHTS AND REFLECTIONS ………….................. 143

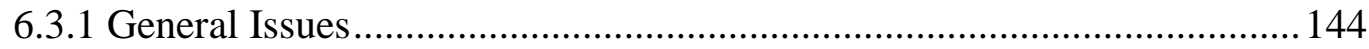

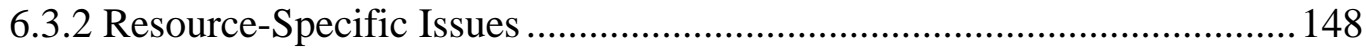

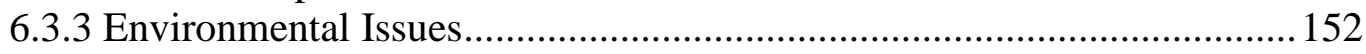

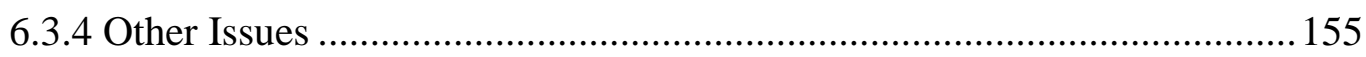

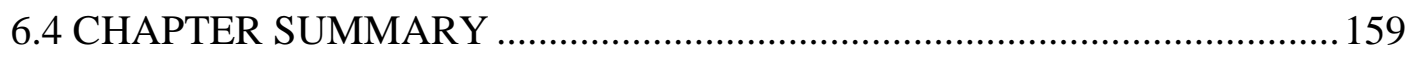

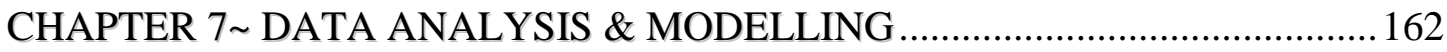

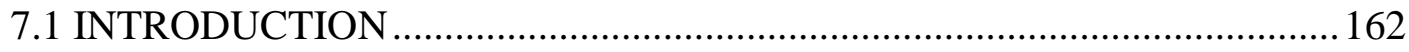

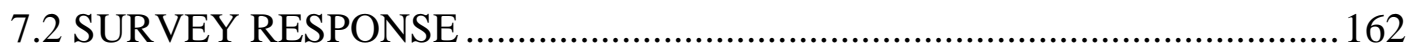

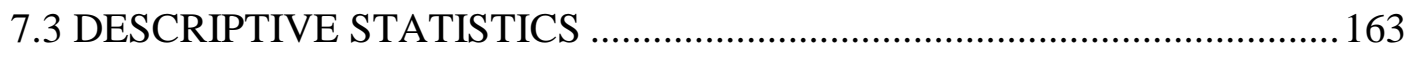

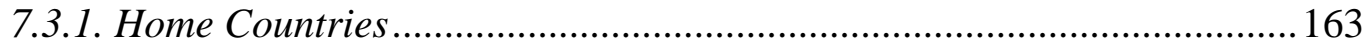

7.3.2. Host Countries......................................................................................... 163 


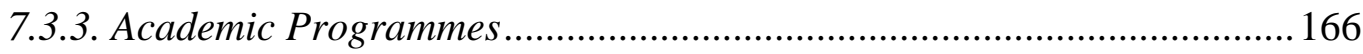

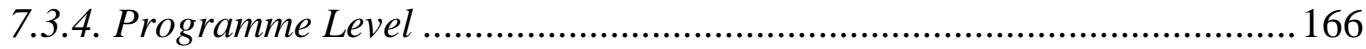

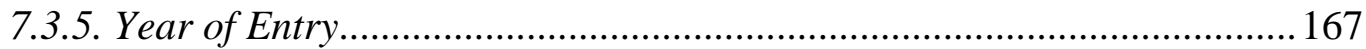

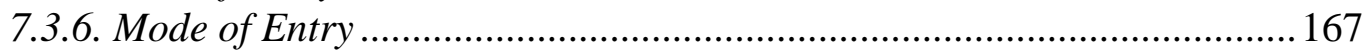

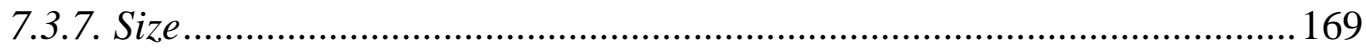

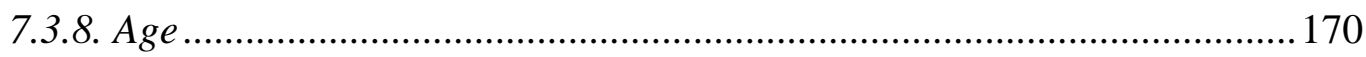

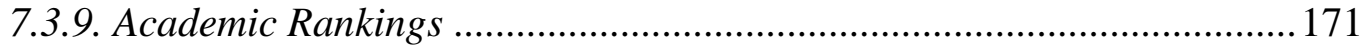

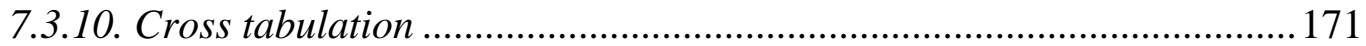

7.4 UNIVARIATE DESCRIPTIVE STATISTICS................................................ 172

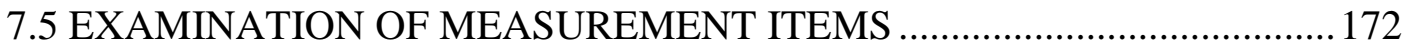

7.6 MODELLING AND HYPOTHESIS TESTING ............................................173

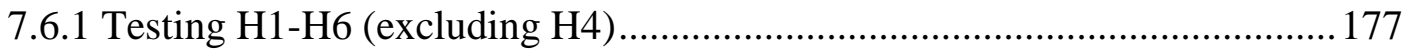

7.6.2 Testing for the moderating hypotheses of $\mathrm{H} 7$ and H8................................... 185

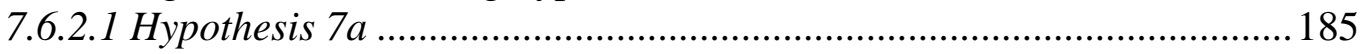

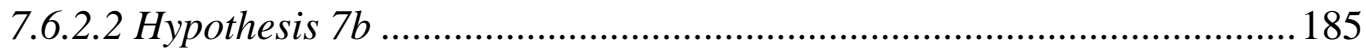

7.6.2.3 Hypothesis 7c................................................................................. 186

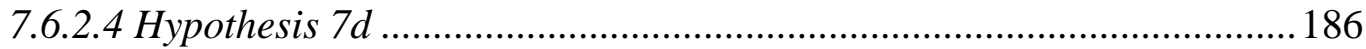

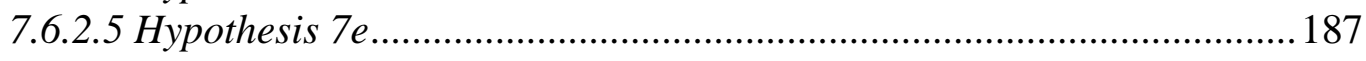

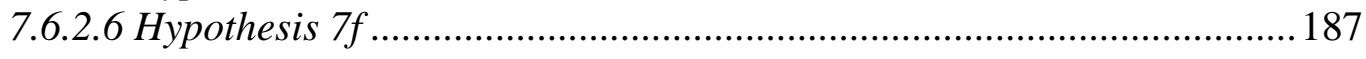

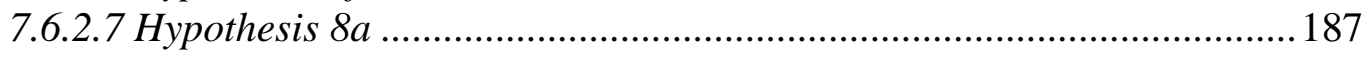

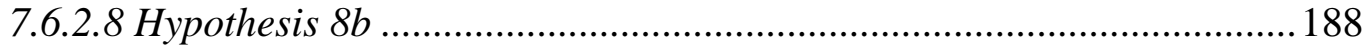

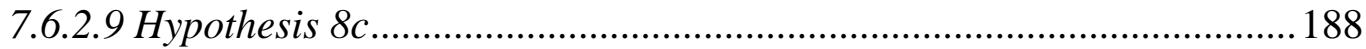

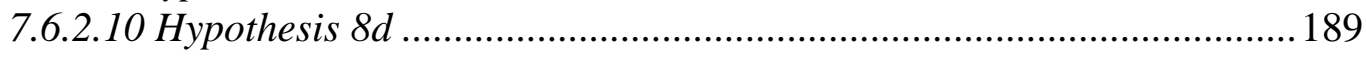

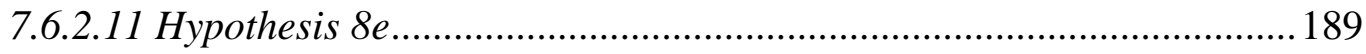

7.6.2.12 Hypothesis $8 f$.................................................................................. 189

7.7 TESTING THE ROBUSTNESS OF IMD NORMATIVE ID MEASURE USING HOFSTEDE'S CULTURAL DISTANCE MEASURE .......................................216

7.7.1 Direct hypotheses H1-6 (excluding H4) ...................................................... 216

7.7.2 Findings for moderating hypotheses of $\mathrm{H7}$................................................. 217

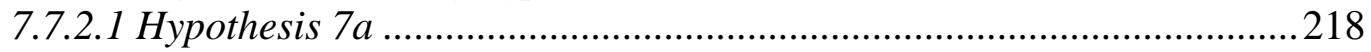

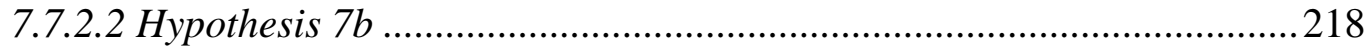

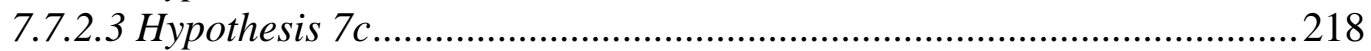

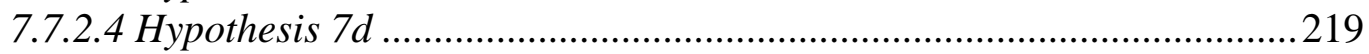

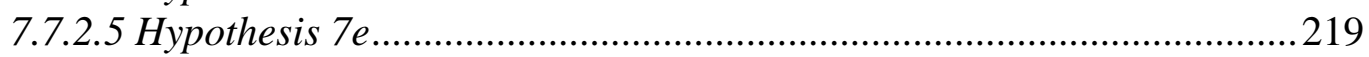

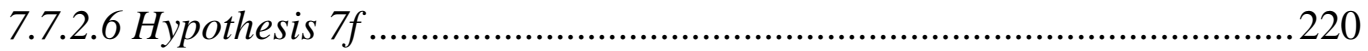

7.8 TESTING THE ROBUSTNESS OF IMD REGULATIVE ID MEASURE USING

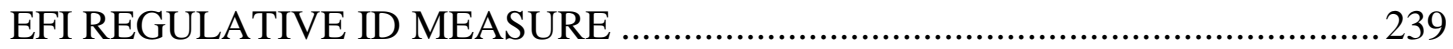

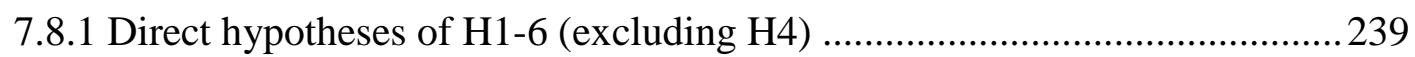

7.8.2 Findings for moderating hypotheses H8...................................................... 240

7.8.2.1 Hypothesis $8 a$..................................................................................... 241

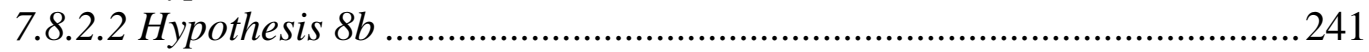

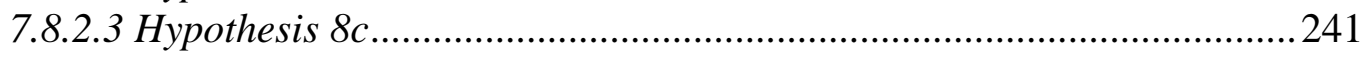

7.8.2.4 Hypothesis $8 d$..................................................................................... 242

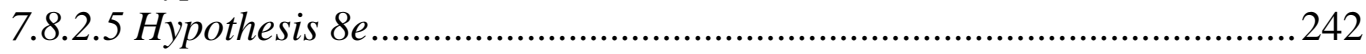

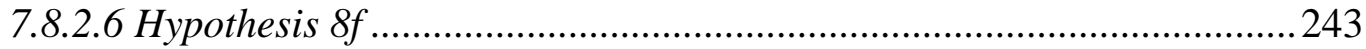

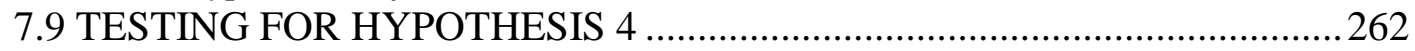




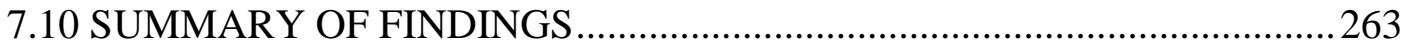

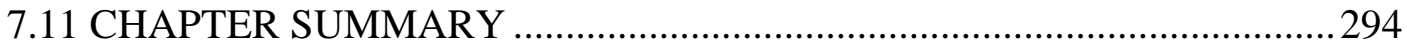

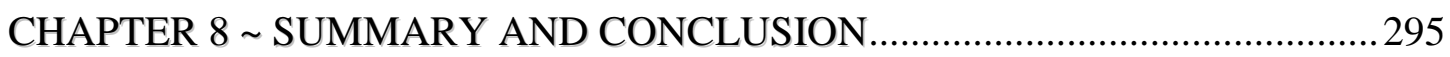

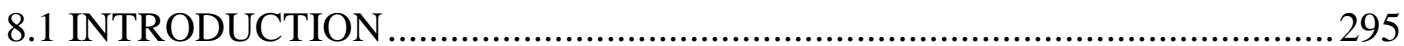

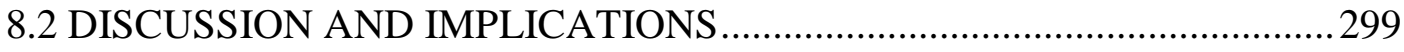

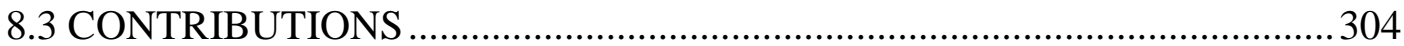

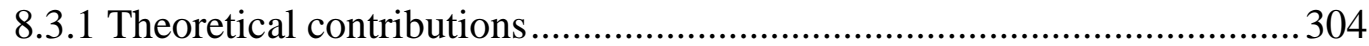

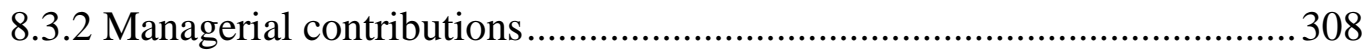

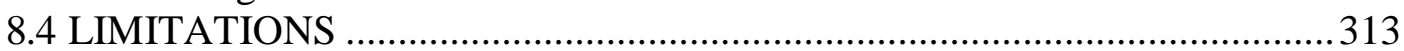

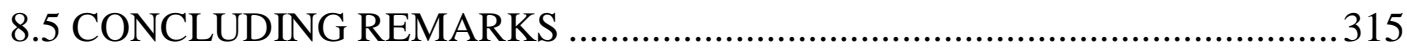

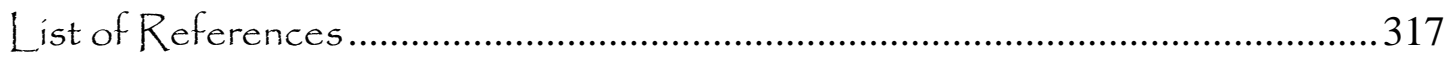

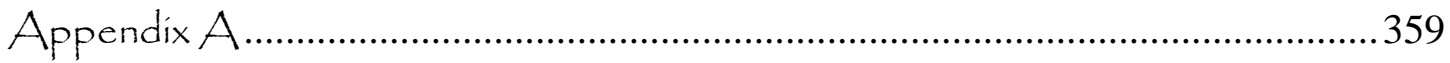

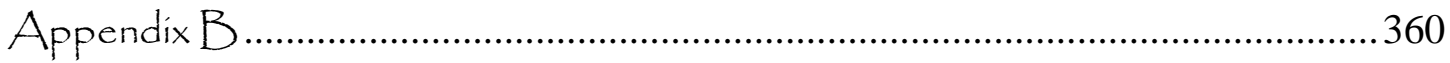

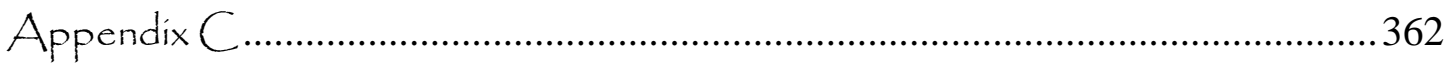

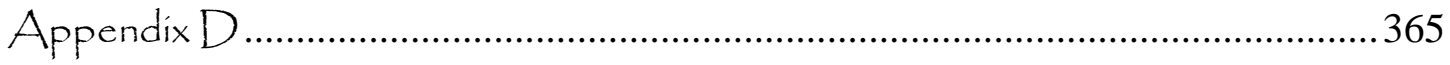

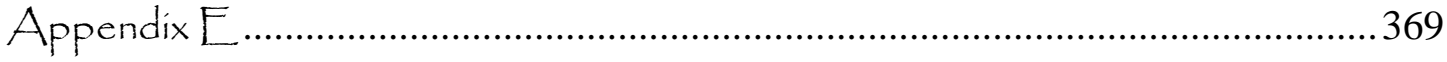

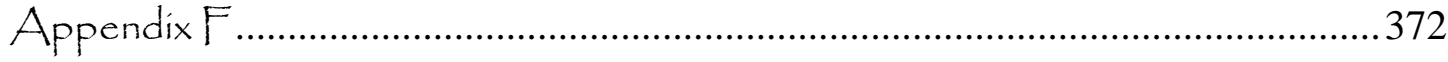

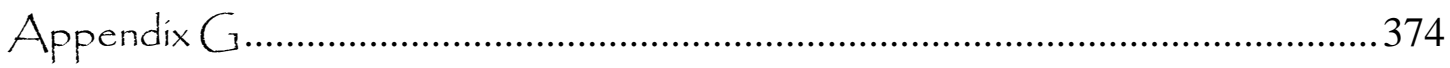

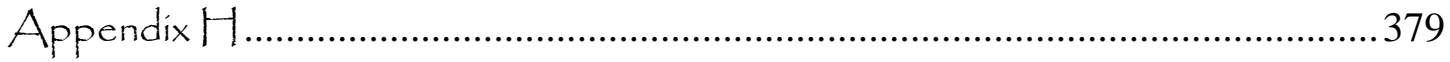

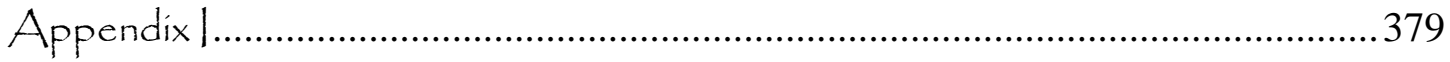

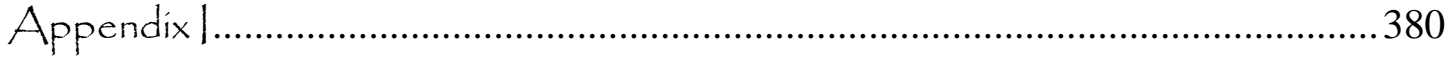

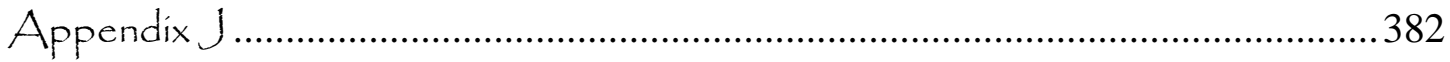

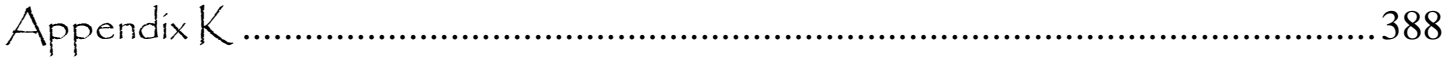

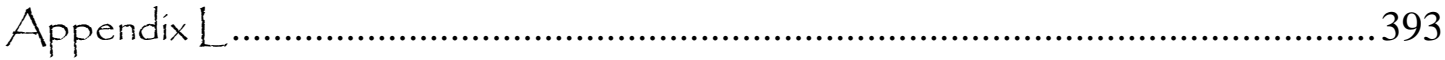

Appendix M

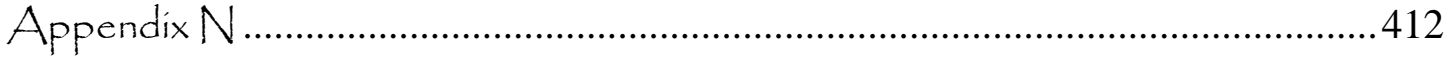

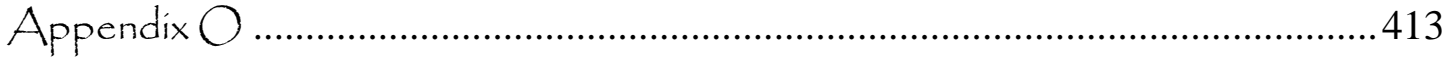

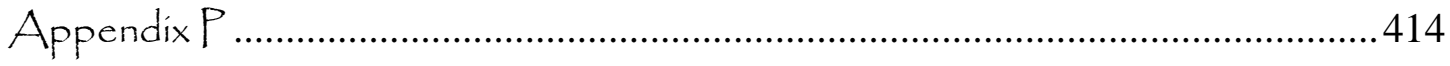

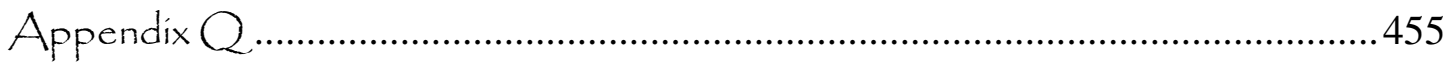

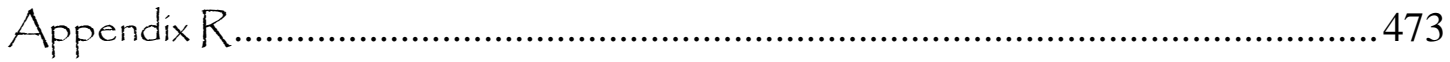

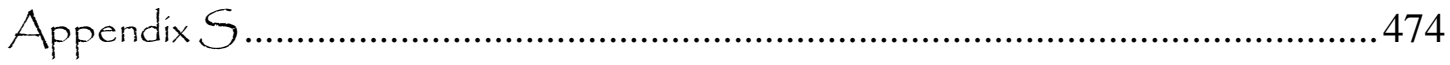

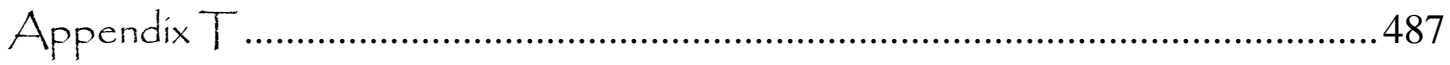

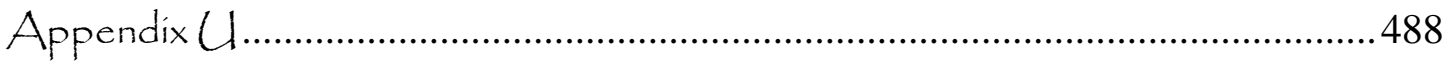




\section{List of Abbreviations}

AACSB Association to Advance Collegiate Schools of Business

AMBA Association of Master of Business Administration

APAIE Asia-Pacific Association for International Education

AUCC Association of Universities and Colleges of Canada

AUF Agence Universitaire de la Francophonie (translated to the Association of Francophone Universities)

AVCC Australian Vice-Chancellors’ Committee

CHEA Council on Higher Education Accreditation (US-based organisation)

CVCP Committee of Vice-Chancellors and Principals (UK-based organisation)

DAAD Deutscher Akademischer Austausch Dienst (the German Academic Exchange Service)

DEST Department of Education, Science and Training (Australian-based organisation)

EAIE European Association of International Education

EFI Economic Freedom Index

ENZ Education New Zealand (New Zealand's international education sector representative body)

EQUIS European Quality Improvement System

FDI Foreign Direct Investment

FTAs $\quad$ Free Trade Agreements

GATE Global Alliance for Transnational Education

GATS General Agreement on Trade in Services

HDI Human Development Index

ICT Information Communication Technologies 
IDP International Development Programme (Australian institution in charge of promoting Australian education service providers)

IMD IMD Business School, Switzerland

IIE Institute of International Education (US institution in charge of promoting education exchanges between the US and other countries)

IMF International Monetary Fund

LAN Lembaga Akreditasi Negara (Malaysian Accreditation Board)

MNEs Multinational Enterprises

NAFSA National Association of Foreign Student Advisers (US institution in charge of promoting the professional development of American college and university officials responsible for assisting and advising foreign students)

NIEPA National Institute of Educational Planning and Administration (Indian based organisation)

OBHE Observatory of Borderless Higher Education (UK-based institution)

OECD Organisation for Economic Co-operation and Development

OLS Ordinary Least Squares

RBV Resource-Based View

S-C-P Structure Conduct Performance

SME Small and Medium Enterprise

TCA Transaction Cost Analysis

UNCTAD United Nations Conference on Trade and Development

UNDP United Nations Development Programme

UNESCO United Nations Education, Scientific and Cultural Organisation

VIF Variance Inflation Factor

WEI World Education Indicators 


\section{Chapter One}

\section{INTRODUCTION}

\subsection{BACKGROUND AND RATIONALE OF STUDY}

This $\mathrm{PhD}$ research seeks to consider overseas investment in a new and important context: education. Estimated at approximately US\$65 billion and representing roughly $3 \%$ of global services export (Alderman 2001), trade in education services is fast becoming a global business (Czinkota, 2006) following the trend of other service sectors.

International expansion in the services sector is increasingly important (UNCTAD, 2004; Verikos and Zhang, 2004; Jensen, Rutherford and Tarr, 2007). Due largely to the non-tradeable nature of many services, the export of services has traditionally been low compared to the export of manufactured goods. The World Bank estimates that only one-tenth of the world's services output is internationally traded, compared to over half the production of merchandise goods (World Bank, 2003). This relatively low component of trade in services, largely due to their non-storability, requires that services are produced when and where they are required. Non-tradeability, therefore, results in international trade in services largely being undertaken through foreign direct investment (FDI) or non-equity arrangements (e.g. franchising/licensing). With the growth of e-commerce and new developments in information communication technology, this may change, but so far, trade in services over the computercommunication interface has not overtaken 'face-to-face' trade in services (UNCTAD, 2004).

The growth in services FDI has been more rapid than in the manufacturing sector. On average, UNCTAD (2004) estimates that services accounted for about two-thirds of 
total FDI inflows (and 70\% of outflows) in the early 2000s. The world's inward stock of services FDI quadrupled between 1990 and the early 2000s ${ }^{1}$ from an estimated US\$950 billion to over US\$4 trillion. One major reason for this surge in services FDI, is that until recently, many service firms have been limiting their investment offshore due to restrictive regulatory frameworks (UNCTAD, 2004). When the liberalisation of FDI policies began around the mid 1980s and gained momentum in the 1990s, services FDI surged (UNCTAD, 2004).

One of the services industries that is currently experiencing liberalisation of FDI policies is education, particularly higher education. In China, for example, authorities are actively encouraging FDI in the higher education sector as a means of developing domestic education capacity. The UK-based University of Nottingham took advantage of these policies and set up the first foreign-owned education institution in China in September 2004. Singapore is similarly pursuing such FDI liberalisation policies with respect to higher education. The Australia-based University of New South Wales (UNSW) launched a branch campus in Singapore in 2007, following other foreign universities such as University of Chicago and University of Nevada - Las Vegas, which also have a Singaporean presence ${ }^{2}$. The Middle East is another part of the world experiencing similar developments. The Education City in Qatar, on the outskirts of Doha, hosts branch campuses of some of the world's leading universities including USbased Texas A\&M University, Cornell Medical College and Georgetown University. Just a few miles away, the Knowledge Village in Dubai is host to Scotland-based Heriot Watt University, India-based Manipal Academy of Higher Education and Canada-based University of New Brunswick, among many other foreign providers. In Africa, Australia-based Monash University has set up a subsidiary ${ }^{3}$ campus in South Africa while, in Australia itself, US-based Carnegie Mellon University has established a campus in Adelaide. All of these examples illustrate the increasing pattern of FDI in

\footnotetext{
${ }^{1}$ This is the latest year of available statistics at the time of writing.

${ }^{2}$ Note that UNSW closed its operations in June 2007, five months after launching its first substantial offshore venture. The reasons for this sudden closure are unclear at the time of writing.

${ }^{3}$ In this study, I refer to offshore campuses as subsidiaries, consistent with the extant international business and strategy literatures.
} 
education services. In addition to FDI, growth in the trade of higher education services is also being materialised through non-equity ${ }^{4}$ contractual arrangements (e.g. twinning and articulation arrangements). Chapter 2 highlights these developments in more detail. This recent growth in overseas investment in education, termed as 'transnational' or 'offshore' education in the literature, departs from the traditional forms of international trade in education services, which have primarily been through student mobility (students from a source country ${ }^{5}$ travelling to a host country to access education services) and, to a much lesser extent, via distance and/or correspondence learning, where students access education services via a communication interface (e.g. the internet).

The growth in transnational ${ }^{6}$ or offshore education (used interchangeably in this thesis), consisting of programmes taught outside of an academic institution's country of origin, that provide students opportunities to earn a foreign qualification while remaining in their home country (see Section 1.2), calls for a more in-depth understanding of its dynamics. Currently, transnational education is an under-researched area and little theory has been developed about the nature of the forces affecting its development. While there are several excellent publications in this area, they tend to be grounded in the educational literature. With transnational education taking more of a market and trade approach in its expansion, there is a growing need to start addressing these developments from a business and managerial perspective, rather than purely through educational lenses. Suspicion of the commercial aspects of what is essentially a public

\footnotetext{
${ }^{4}$ Since the universities under consideration in this study do not use stocks to establish foreign operations, it is worth noting, for precision sake, that equity is used as a proxy for ownership in this dissertation.

${ }^{5}$ Source countries for the purpose of this research refer to the exporting countries of international education, i.e. where the education services originate. Conversely, host countries are the importing countries of education services, i.e. where the education services are consumed. In this study, the term 'source countries' is used interchangeably with exporting/sending/home countries. Similarly, the term 'host countries' is used interchangeably with importing/receiving countries.

${ }^{6}$ The term 'transnational' employed in this study is not to be confused with Bartlett and Ghoshal's use of the term, who define transnational organisations as being driven by simultaneous demands for global efficiency, national responsiveness and worldwide learning (Bartlett and Ghoshal, 1987). Section 1.2 provides a definition of 'transnational' as used in the education service context, the focus of this study. Similarly, the terms 'global' and 'international' as employed in this study, are not to be confused with Bartlett and Goshal's use of the term.
} 
good is suggested as a possible reason for this absence of research (Czinkota, 2006). With the commercialisation of education services growing at the phenomenal rate that it currently is, and all trends pointing to that continued growth, it is largely time that this research gap is addressed. This $\mathrm{PhD}$ research using universities as the unit of analysis (as opposed to other institutions of higher education; e.g. community colleges, polytechnics, etc. - see Section 1.4), seeks to understand the dynamics of transnational higher education, particularly how and why it is happening, through the lenses of strategy and international business literatures. The study is motivated by the growing importance of transnational education and the opportunity to combine different streams of research in a context of particular interest. By drawing from both the strategic management and international business literatures, and making both theoretical and practical contributions, the purpose of this study is to develop a more thorough and meaningful understanding of the choice of entry mode in transnational education developments (see Section 1.3).

\subsection{DEFINITION OF TRANSNATIONAL EDUCATION}

The term 'transnational education' first appeared in the Australian literature in the early 1990s when Australian education providers wanted to differentiate between international students recruited to Australian institutions and those who were enrolled in Australian degrees offshore (Knight, 2005a). Over the years, however, as will be highlighted in greater detail in Chapter 2, a great deal of terminological and conceptual confusion has arisen as to what constitutes transnational education. To preclude any such confusion, the definition provided by UNESCO's Council of Europe's (2000:2) Code of Good Practice in the Provision of Transnational Education is used for the purpose of this study, such that transnational or offshore education includes "all types of higher education study programmes or set of courses of study, or educational services....in which the learners are located in a country different from the one where the awarding institution is based". The Code specifies that transnational education programmes may belong to the educational system of a country different from the one in which they are offered, or they may be offered independently of any national system. Therefore, the prominent aspect of transnational education involves education 
programmes that are being delivered across the borders of national education systems, in which the notion of crossing borders is made explicit.

Transnational education can be delivered via several types of higher education programmes. The most common modalities include:

- Franchising: An education provider from source country A (the franchiser) grants another institution from host country $B$ the right to deliver the franchiser's educational programmes in country B or other countries. The qualification is then awarded by the franchiser in country A. Franchising agreements are usually for profit commercial arrangements and are often referred to as $3+0$ in the case of three year qualifications, $2+0$ in the case of two year qualifications, $4+0$ in the case of four year qualifications, and so forth, where the student undertakes the entire programme in the host or third country. For example, in the case of a 3+0 qualification franchised by a Malaysian institution from a British university, the student enrolled in Malaysia will obtain a British qualification without having to enroll at the institution in the UK.

- Twinning degrees: This is an arrangement where an education provider from source country A collaborates with another institution in host country B, allowing students studying at the latter institution to transfer their course credits to the institution in country A. One qualification is awarded by the education provider in country A. This may or may not be on a commercial basis, and is often referred to as $2+2,1+3$ (in the case of four year qualifications) or a similar combination for three year qualifications. For example, for the latter qualification, $1+2$ refers to the first year being delivered in the host country and the two remaining years in the source country.

- Programme articulations: In articulation arrangements, students undertake part of a source country qualification in a host country and then transfer to the source country institution with 'advanced standing' in terms of study credits and credit transfer to complete the qualification at the education institution in the source country. This sort of inter-institutional arrangement differs from twinning 
arrangements in that the students study the source country institution's curriculum in the host country. Under twinning arrangements, the student gets credits for the study they have undertaken in the host country irrespective of whether that study is based on the curriculum of the source country's institution.

- Branch campus: A subsidiary/satellite campus established by a source country education institution in a host country to deliver its own education programmes. Branch campuses can be established either through wholly-owned subsidiaries or via joint venture partnerships with local host country partners.

- Virtual/Distance learning: The education provider from a source country delivers the education service to students in a host country via a communication interface (usually via post and/or internet-based solutions) and the students self direct the learning process.

- Corporate programmes: Some major multinational corporations have their own higher education institutions or programmes of study offering qualifications that might not necessarily be affiliated with any national education system.

This doctoral thesis focuses solely on franchising, twinning arrangements, programme articulations and branch campus types of transnational education; distance learning and corporate programmes are excluded from consideration. The rationale behind this limited focus of transnational education is explained in Section 1.4 and in Chapter 2.

\subsection{STATEMENT OF THE PROBLEM AND RESEARCH OBJECTIVES}

The growth in transnational education gives impetus to three strands of enquiries:

- A strategic dimension that focuses on the decision making process behind the provision of transnational education (e.g. country selection factors, partner selection factors, mode of entry selection factors, etc.).

- An education dimension that focuses on the educational aspect of the provision of transnational education (e.g. quality assurance, approval and review mechanisms, curriculum development, etc.).

- A business/operational dimension that focuses on the delivery process of transnational education (e.g. market research, academic cost modelling, 
provision of physical facilities, operational plan, marketing plan, financial plan, etc.).

These three strands of enquiries are not necessarily mutually exclusive. For example, the need to adjust a curriculum to meet local demand (education dimension) can have an influence on the location that is selected for transnational delivery (strategic dimension). Similarly, the business/operational dimension of transnational education can also influence its strategic dimension, in terms of country selection factors. This thesis focuses on the strategic dimension of transnational higher education. Where relevant, this study also draws from the other two strands of enquiries. However, the education and business/operational dimensions of transnational education are not the primary focus of this study.

In particular, this study addresses the issue of entry modes for education service providers engaging in the transnational education market. As highlighted above, the growth in transnational education is taking place through both equity and non-equity arrangements. Drawing primarily from a resource-based perspective, the research question that thus poses itself is:

\section{What resources are associated with entry mode choice for education providers entering overseas markets?}

One of the most critical issues in international market entry strategy is the selection of an entry mode (Wind and Perlmuter, 1977; Terpstra and Sarathy, 2000). With ownership form being a relatively important determinant of performance (Sharma, 1993; Root, 1994; Li, 1995), it is crucial to gain an understanding of when equity channels are appropriate and when they are not, for transnational education provision. This study is motivated by the importance of the international entry mode phenomenon especially as it applies to the services sector. Erramilli and Rao (1990:136) note that "so little is known about how service firms enter foreign markets”. Clark, Rajaratnam and Smith (1996:9) further point out that "for international services, theory lags practice by 
a considerable degree and many important questions await answers”. Contractor, Kundu and Hsu (2003:9) more recently state that “...there is little research on the growth and internationalisation of service firms...” and further suggest that “...there are substantial differences among different types of services”. This $\mathrm{PhD}$ thesis addresses an important issue, in response to these calls for more understanding about internationalisation in the services sector. By developing a model of foreign market entry mode selection, this research seeks to understand entry decisions as they relate to education services. In particular, three modes of entry will be investigated as part of this research: whollyowned branch campuses, joint venture branch campuses and non-equity collaboration (i.e. twinning and articulation arrangements). In addressing the research question, the thesis will investigate the foreign market entry decisions of universities from three theoretical perspectives: resource-based view, organisational capability theory and institutional theory. It is postulated that these three perspectives play an important role in understanding the decision making process of universities entering foreign markets to deliver offshore education (see Chapter 3).

In addressing this research question, the overall objectives of this study are fourfold:

1. To gain an understanding of the emerging dynamics in transnational education through a comprehensive overview of patterns over the last few decades. The undertaking will assist in the development of a sampling frame for the current study.

2. To develop a conceptual model, drawing from a review of the relevant literature, explaining the foreign market entry decision of universities.

3. To empirically assess the extent to which the proposed model in (2) explains forms of ownership in education related overseas investment.

4. Based on the results from (1) - (3) above, make recommendations to allow universities to leverage their respective foreign market entry decision. 


\subsection{SCOPE OF THE STUDY}

Four main groups of providers of higher education are represented in transnational education developments: (1) traditional universities, colleges $^{7}$ and polytechnics, (2) professional associations (e.g. the Institute of Chartered Accountants), (3) virtual universities (i.e. e-learning - e.g. Open University in the UK, Indira Gandhi National Open University in India, the University of Phoenix in the US, etc.) and (4) corporate universities. Corporate universities not only include company specific training activity centres (e.g. McDonald's Hamburger University, Microsoft's Certified Technical Education Centres), but also include for-profit private companies such as Informatics (Singapore), Aptech (India), Apollo (US) and Laureate (US). These for-profit private companies are increasingly active in the transnational education market. Laureate, which is listed on the NASDAQ, is a case in point of the scale of transnational operations of these for-profit companies. It has acquired universities and business schools in Mexico, Spain, Chile, France, Switzerland and India, and enrolls more than 60,000 students worldwide (Vincent-Lancrin, 2004).

In spite of this growth in for-profit companies and the presence of professional associations and virtual universities in the transnational education landscape, the scope of this thesis will focus solely on existing/traditional universities and colleges. Other existing/traditional institutions of higher education such as community colleges and polytechnics are also excluded from the focus of this study. The analysis reported on in Chapter 2 reveals that traditional universities and colleges are the most active in the transnational education market (Larsen, Momii and Vincent-Lancrin, 2004). Virtual universities also lie outside of the scope of this study since, as highlighted in Chapter 2, they are not set up through offshore investment and as such do not fit well as part of the current study, which examines transnational education through 'FDI lenses'. Furthermore, corporate universities have operational dynamics that are very different to existing universities. For instance, companies like Laureate, being a publicly listed company, have to report to shareholders. Consequently, understanding the dynamics of corporate transnational education is different from understanding transnational

\footnotetext{
${ }^{7}$ College is used in its US sense representing a tertiary educational institution.
} 
education undertaken by universities. An investigation of the former would constitute a separate study in itself and as such lies outside the scope of this study. Similarly, professional associations deliver transnational education for objectives targeted to their membership, which differs from the objectives of traditional universities. They, therefore, also lie outside the scope of this study.

This study focuses primarily on traditional universities and colleges (used interchangeably hereafter) in the United States, the United Kingdom, Canada, Australia, New Zealand and Ireland. These six source countries are where the majority of transnational education developments are sourced from (see Chapter 2).

Last but not least, especially in light of some high profile failures of offshore education providers (e.g. UNSW in Singapore), it should be highlighted that the issue of performance of offshore education providers lies outside the scope of this study.

\subsection{METHOD AND DATA COLLECTION}

Using a multi-method design, this study develops and tests a model that explains the selection of ownership mode for new market entry in transnational education. First secondary analysis is conducted to learn about the state of play in the transnational education sector. Then, interviews, a blend of telephone and face-to-face, are developed to gain richer insights of the transnational education phenomenon and to inform the survey development process. The survey is developed to collect primary data that are used to test the proposed model. The target respondents for both the survey and the indepth interviews are appropriately knowledgeable senior managers at universities, responsible for foreign operations. In the university context, these are either Pro/Deputy Vice-Chancellor/President for International Affairs and/or the Directors of International Offices.

\subsection{OUTLINE OF THE RESEARCH}

This thesis consists of eight chapters, each discussing specific stages of the research process. The main content of each chapter is briefly outlined below: 


\section{Chapter One Introduction}

Starting off with a brief background to the issue under consideration, this chapter introduces the research problem and provides the study's justification. The objectives behind the study are outlined, along with the scope.

\section{Chapter Two Developments in Transnational Higher Education: Analysis of Current Activity}

Chapter 2 provides an overview of recent developments in transnational education. This is highlighted in the overall context of the growing trade of education. The analysis undertaken in Chapter 2 assists in the development of a sampling frame for the current study.

\section{Chapter Three $\sim$ Review of Literature}

Drawing from a review of the relevant literature, the key theoretical underpinnings of modes of entry are identified followed by a discussion as to which theoretical perspectives are most relevant to the study.

\section{Chapter Four Conceptual Framework and Hypothesis Development}

Drawing from the resource-based view, organisational capability theory and institutional theory, a model of entry mode is conceptualised by identifying key resources that explain ownership forms in the foreign market entry decision of universities. The relationships between these resources and ownership forms are hypothesised.

\section{Chapter Five - Constructs, Measures and Research Methodology}

This chapter discusses the methodology and data used in investigating the proposed conceptual model developed in Chapter 4. The research design is presented, the survey development process discussed, followed by a description of the techniques used to estimate the model. 


\section{Chapter Six Findings From Field Interviews}

The insights gained from interviews are presented, allowing for refinement, improvement and validation of the proposed conceptual model outlined in Chapter 4. The interviews also facilitate clarification and refining of the measures used in the questionnaire developed for this study.

\section{Chapter Seven Data Analysis and Modelling}

An analysis of the findings is presented and the results discussed.

\section{Chapter Eight Summary and Conclusion}

This chapter briefly summarises the study and draws conclusions from the main findings of the research. The implications of these findings are also discussed, as well as the limitations of the study and possible avenues for future research. 


\section{Chapter Two ${ }^{8}$ \\ DEVELOPMENTS IN TRANSNATIONAL HIGHER EDUCATION: ANALYSIS OF CURRENT ACTIVITY}

\subsection{INTRODUCTION}

Higher education is fast becoming a global business. While once traditionally thought of as a matter of national interest, the influence of 'globalisation' and 'internationalisation' has become increasingly widespread in the education industry (Enders, 2004). Unfortunately, the more widespread these two terms become in the literature, the more they seem to be used as catch-all phrases highlighting the international aspects of education (Enders, 2004). Yet, there are some important differences between these two terms that should not be overlooked. While 'globalisation' of education pertains to the external macro-socio-economic process that influences the way educational institutions operate, 'internationalisation' of education refers to the policy-based responses that educational institutions adopt, as a result of globalisation (Scott, 1998). These two terms, however, should not be viewed as distinct from each other. Rather, 'globalisation' and ‘internationalisation' of education should be seen as dynamically linked concepts. As stated by Knight (1999:14), “globalisation can be thought of as the catalyst while internationalisation is the response, albeit a response in a proactive way”.

Internationalisation is not a new phenomenon to many universities. Many already have international staff and students on campus, and have an international element to their curriculum. Globalisation, on the other hand, is potentially more challenging for higher

\footnotetext{
${ }^{8}$ A shortened version of this chapter has been published; see Naidoo, V. A Stock-take of Current Transnational Education Activity, Journal of Studies in International Education, Pre-published May 21, 2008; DOI: 10.1177/1028315308317938
} 
education, although it also offers considerable opportunities. One of these opportunities, as indicated in the opening chapter, is transnational education, which has emerged in a globalised higher education context that includes (Knight, 2005):

- An increased demand for tertiary education in societies and economies in which knowledge plays an increasingly important role.

- The introduction of a market and trade approach to international education.

- An increased prominence of education mobility.

- Advances in the use of information and communication technologies for delivery of education.

Transnational $^{9}$ education development (see Section 1.2 for definition) is not an entirely new international activity in the education services sector. From the mid 1950s, offshore education services were provided by US universities to both serve their students on study-abroad programmes and US military personnel (Verbik \& Merkley, 2006). However, the nature and scale of the global expansion of transnational education is currently in the throws of substantial change. Not only are 'traditional' public and private higher education institutions involved in this transnational education landscape, but an increasing number of 'new or alternate' providers, which include media companies such as Pearson (UK) and Thomson (Canada), multinational companies, such as Apollo (US) and Informatics (Singapore), corporate universities and professional associations, are also engaged in transnational education activities (Knight, 2005a). This increase in offshore education activity reflects, as highlighted in Chapter 1 , the increasing trend of FDI liberalisation in the education services sector. This liberalisation extends beyond individual country policy directives, surfacing to a multilateral level as part of the General Agreement on Trade in Services (GATS) and bilateral Free Trade Agreements (FTAs).

The liberalisation of the education services sector follows a boom in the international trade of education that has manifested itself over the last two decades. The OECD

\footnotetext{
${ }^{9}$ As discussed in Chapter 1, it is important to recall that the term 'transnational' employed in this study, is not to be confused with Bartlett and Goshal's use of the term.
} 
estimates that the international trade in education services accounts for roughly $3 \%$ of global services exports (Alderman, 2001; Johnson, 2002; Czinkota, 2006). This trade has largely developed through student mobility; see table 2.1.

Table 2.1: Global Trade in Education Services from 1980

\begin{tabular}{|c|c|c|c|c|c|c|}
\hline & 1980 & 1990 & 1995 & 2000 & 2004 & 2006 \\
\hline $\begin{array}{l}\text { Number of students } \\
\text { studying overseas } \\
\text { (million) } \\
\text { (avg. annual growth: \%) }\end{array}$ & 0.993 & $\begin{array}{l}1.2 \\
(1.8)\end{array}$ & $\begin{array}{l}1.3 \\
(1.4)\end{array}$ & $\begin{array}{l}1.8 \\
(6.4)\end{array}$ & $\begin{array}{l}2.3 \\
(5.6)\end{array}$ & $\begin{array}{l}2.7 \\
(5.8)\end{array}$ \\
\hline $\begin{array}{l}\text { Value of global education } \\
\text { market }^{1} \text { (US\$ bil.) }\end{array}$ & $\mathrm{n} / \mathrm{a}$ & 6 & 24 & 43 & 60 & 62 \\
\hline $\begin{array}{l}\text { Total value of global } \\
\text { services trade (US bil.) } \\
\text { (avg. annual growth: \%) }\end{array}$ & $\begin{array}{l}822 \\
\mathrm{n} / \mathrm{a}\end{array}$ & $\begin{array}{l}1691 \\
(10.6)\end{array}$ & $\begin{array}{l}2479 \\
(9.3)\end{array}$ & $\begin{array}{l}3045 \\
(4.6)\end{array}$ & $\begin{array}{l}4358 \\
(10.8)\end{array}$ & $\begin{array}{l}4736 \\
(4.3)\end{array}$ \\
\hline $\begin{array}{l}\text { Total value of } \\
\text { merchandise trade (US\$ } \\
\text { bil.) } \\
\text { (avg. annual growth: \%) }\end{array}$ & $\begin{array}{l}4108 \\
\mathrm{n} / \mathrm{a}\end{array}$ & $\begin{array}{l}7103 \\
(7.2)\end{array}$ & $\begin{array}{l}10393 \\
(9.3)\end{array}$ & $\begin{array}{l}13068 \\
(5.1)\end{array}$ & $\begin{array}{l}18219 \\
(9.9)\end{array}$ & $\begin{array}{l}21074 \\
(7.8)\end{array}$ \\
\hline $\begin{array}{l}\text { Higher Education as \% of } \\
\text { services trade (import }+ \\
\text { export) }\end{array}$ & $\mathrm{n} / \mathrm{a}$ & 0.3 & 1.0 & 1.5 & 1.4 & 1.3 \\
\hline
\end{tabular}

Source: UNESCO Statistical Yearbook, OECD Education Database; UNCTAD (Various)

${ }^{1}$ Note: Estimates based on Larsen et al's (2002) and assuming an average annual change in tuition fees based on the International Handbook of Universities, published by the International Association of Universities, in association with UNESCO.

Driven by the expectation of its ability to raise the status of the graduate, both economically and socially, demand for education, particularly higher education, has led to a large number of students, mostly from developing countries, travelling abroad for their studies. This global movement of students has led to a sort of 'academic trade', where knowledge and expertise are treated as traded services (McMahon, 1988). Figure 2.1 illustrates the trend in international student flows from a mere 149,590 in $1955^{10}$ to a peak of 2.7 million in $2006^{11}$. The growth in student flows has been particularly large in the late 2000s when mobility increased from roughly 1.9 million in 2002, representing a 42\% growth by 2006.

These data depict a shift in overseas study from an elitist experience to one involving mass movements. Indeed, no longer is overseas study limited to those earning

\footnotetext{
${ }^{10}$ This is the earliest year for which data are available.

${ }^{11}$ This is the latest year for which data are available at the time of writing.
} 
scholarships and fellowships. Rather, those who can afford it now have a chance for a cross-border education, reflecting a shift in overseas student policy from an 'aid' approach to a 'trade' rationale (Smart and Ang, 1993).

Figure 2.1: International Student Numbers at the Tertiary Level from 1955-2006

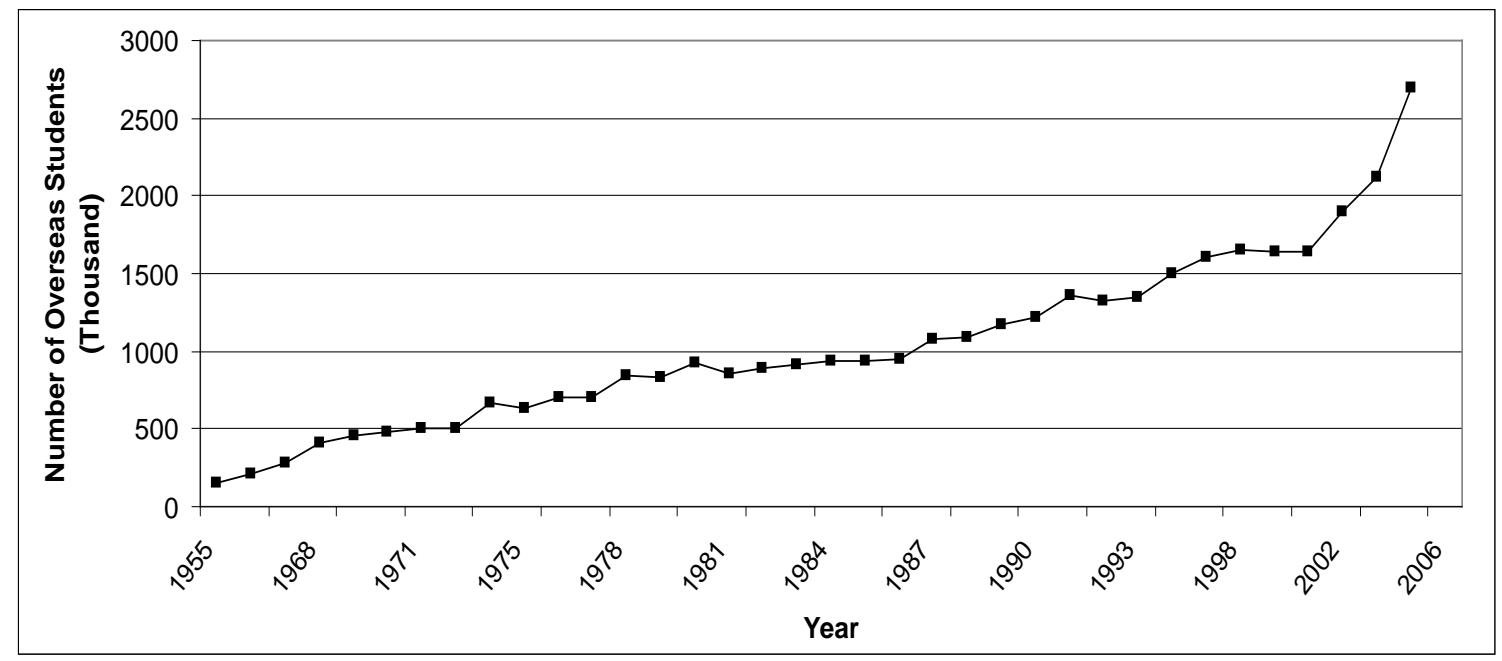

Source: UNESCO Statistical Yearbook; OECD Education Database

The shift to a 'trade' rationale has led to the emergence of a global education services industry (Mazzarol and Soutar, 2001, 2002; Mazzarol, Soutar, and Sim, M.Y-S, 2003). In Australia, New Zealand and the US, education services are estimated to be respectively the third, fourth and fifth largest service sector export (Vincent-Lancrin, 2004). To date, however, much of the trade in education services has focused quite narrowly on students travelling abroad for an education. A great deal of potential remains in the development of trade in education services via the other 'modes of supply’ through which education services can be traded; see Box 1.

In fact, growth in trade for higher education services might not necessarily be via student mobility. As indicated in Box 1 and based on the overall trend of services trade, growth in education services is likely to take place primarily through transnational education via Mode 3 and, to a lesser extent, Mode 1. With the promise of new satellite and Internet-based technologies, distance learning systems are expected to have great potential in the future (Wood, Tapsell, and Soutar, 2005). However, given that not all students are suited for distance learning and that not all subjects are readily taught via 
this form of learning (Palloff and Pratt, 2000), the uptake of distance learning may be slower than that of transnational education (Mode 3).

\section{Box 1: The different modes of services trade according to the GATS classification}

The GATS is the first set of multilateral rules covering international trade in services. It stipulates that a service can be traded in four ways.

Mode 1: Cross-border supply corresponds to the common form of trade in goods; only the service itself crosses the border. Cross-border supply of educational services is currently a small market but has the potential to grow rapidly in the future through the use of new information technologies for distance learning. A number of private companies and universities have launched recent initiatives in this area. In 2004, Mode 1 accounted for 35\% of the total world trade in services.

Mode 2: Consumption abroad refers to a situation in which a service consumer moves to another country to obtain the service (e.g. a student who travels abroad to study). International flows of students in higher education constitute at present by far the largest share of the global market for educational services. In 2004, Mode 2 accounted for $10-15 \%$ of total world trade in services.

Mode 3: Commercial presence of educational services refers to the commercial establishment of facilities abroad by education providers (e.g. 'local branch campuses' or partnerships with domestic education institutions). In 2004, Mode 3 accounted for $50 \%$ of the total world trade in services.

Mode 4: Presence of natural persons consists of a natural person (e.g. a professor, researcher, teacher, etc.) travelling to another country on a temporary basis to provide an educational service. In 2004, Mode 4 accounted for $1-2 \%$ of the total world trade in services.

Source: Adapted from Larsen et al (2002), Knight (2002a) \& World Trade Organisation (2004)

Mode 3 developments are expected to become more and more common with the liberalisation of FDI in education services. Already, the international education literature is full of reports of missed international recruitment targets via Mode 2 in a number of education exporting countries. Juxtaposing these missed targets with predictions of massive growth for transnational delivery (mostly from China), OBHE (2005a) argues that transnational education is beginning to 'cannibalise' trade in education services via Mode 2. Indeed, according to forecasts by the British Council (2004), transnational delivery is expected to outpace student mobility to the UK by 2010. At present, it already accounts for an estimated $50 \%$ of UK international 
enrolments (OBHE, 2006a). These forecasts build on work by IDP Education Australia, which has predicted a similar growth in transnational delivery for Australian higher education (Garrett and Verbik, 2004). Figure 2.2 shows the growth in transnational education enrolments between 1996 and 2005 accounting for roughly 29\% of all international students in the Australian tertiary education system in 2005 (DEST, 2006).

Figure 2.2: Distribution of International Students in Australian Universities by Mode of Study, 1996 to 2005

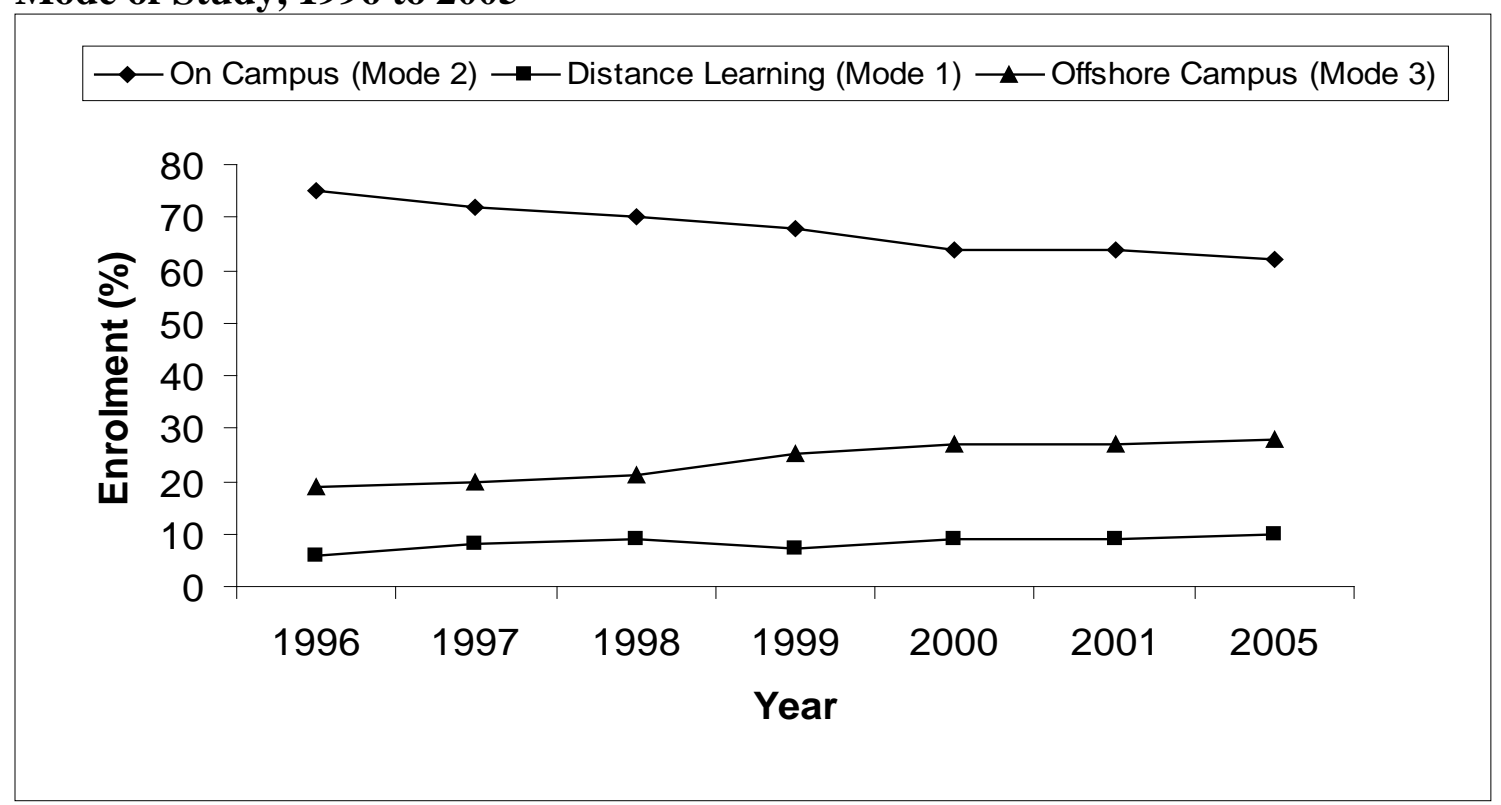

Source: DEST (2006)

In this chapter, a snapshot of the vast array of Mode 3 transnational developments in the trade of education services is provided. While transnational education can be delivered via Modes 1 and 3, only the latter developments are examined in this chapter, since transnational education is examined through 'FDI lenses' for the purpose of this research. Indeed, FDI in the education services context can be viewed as a movement of capital flows that provides ownership control through the management of assets and operations. This definition of FDI follows the IMF's Balance of Payments Manual (1993:86), which states:

Direct investment is the category of international investment that reflects the objective of obtaining a lasting interest by a resident entity in one economy in an enterprise resident in another economy...(it)...comprises 
not only the initial transaction...but also all subsequent transactions between ...the affiliated enterprises.

In Mode 1 transnational education, there is no flow of capital. Rather, distance learning providers invest domestically in communication and information technologies, to offer conventional education services via the internet or correspondence. No overseas investment of capital takes place via Mode 1 and the primary form of capital flow between a source and host country is the student in the latter, paying to obtain access to the education service being delivered 'virtually' from the former. This capital flow is a form of payment for services rendered, rather than investment on the part of the providers.

Mode 3 transnational education developments, on the other hand, clearly represent the transfer and investment of capital between source and host countries. Whether the flows are financial or non-financial (e.g. investment of human capital), Mode 3 transnational education developments clearly represent a form of FDI; consequently Mode 3 investments form the basis of this study.

This chapter opens with a section on the typology of Mode 3 transnational education, followed by an examination of current activities in transnational delivery. The latter addresses the first objective of this thesis, namely, "To gain an understanding of the emerging developments..." (see Chapter 1). The chapter acts as a prelude to the proposed research model for explaining modes of entry in Mode 3 transnational education developments. The goal of this exposition is to provide justification for better understanding the nature of this phenomenon, including developing a model that explains the choice of ownership forms.

\subsection{MODE 3 TRANSNATIONAL EDUCATION: A TYPOLOGY}

The existing conventions used in the educational literature to describe Mode 3 transnational education are multidimensional. Twinning programmes, articulation programmes, franchised (or licensed) programmes, joint award programmes are just some of the terms currently used in the literature, often inconsistently, to describe the 
complex range of activity; see Section 1.2. Davis, Olsen and Bohm (2000) reviewed the use of these conventions and found a lack of conceptual clarity. They reasoned that this lack of clarity stems from the current terminology of Mode 3 transnational education having been developed to suit a range of different purposes. For example, the differentiation between twinning and articulation programmes is rather murky, with the two groups sharing blurred boundaries. Similarly, franchised programmes represent business models, while twinning arrangements refer to the responsibility for educational delivery.

Knight (2003) further categorises transnational education into two different forms, programme and institutional mobility, adding to the myriad of terms that have emerged in the literature to describe transnational education. Programme mobility represents an educational programme that goes offshore while institution mobility pertains to an institution that goes overseas for education purposes. A common form of institution mobility is the opening of satellite branch campuses (i.e. a subsidiary campus) offshore. The downside of Knight's typology, however, is that it is oversimplified. Being generic in nature, Knight's nomenclature includes a wide spectrum of institutions and types of provision. For example, institution mobility can be undertaken via a wholly-owned satellite campus or via a joint venture operation. Distinguishing one form of institution mobility from the other is critical, as the issues faced under each of them have different implications. This categorisation therefore, needs to extend beyond the current terminology, in order to address the lack of conceptual clarity argument raised by Davis et al (2000).

In an attempt to bring structure to the debate of categorising and defining Mode 3 transnational education, this study approaches the task of developing a typology by building on international business literature. The internationalisation literature, in particular, has focused on the ownership decisions of multinational corporations investing offshore. In particular, three modes of entry have been examined in this 
literature: equity, joint ventures and non-equity arrangements that include franchising and licensing (e.g. Rugman, 1980; Davidson and McFetridge, 1985; Hennart, 1988) ${ }^{12}$.

Equity arrangements are those in which the multinational corporation invests offshore. This investment can represent any proportion of the equity of the overseas operation. Wholly-owned subsidiaries, at the top end of the scale, are equity arrangements where the multinational corporation owns roughly $100 \%$ of the operation. Wholly-owned subsidiaries can be established either by setting up a new operation offshore (greenfield) or by acquiring an established firm in the offshore market. At the other end of the spectrum, non-equity arrangements take place through licensing and franchising agreements. The former is an arrangement whereby a licensor grants the rights to an asset to a licensee for a specified time period and for a royalty fee. Franchising agreements are similar to licensing although they are for longer-term commitments ${ }^{13}$. Lastly, joint ventures can represent an ownership form that falls between equity and non-equity arrangements. An equity joint venture is a partnership between two or more otherwise independent firms to jointly own an organisation.

Applied to the education services sector, Mode 3 transnational education investment undertaken through equity channels therefore corresponds to equity-based investment in satellite and branch campuses offshore, such as Australia-based Monash University's campus in South Africa. Non-equity arrangement manifests itself through twinning, articulation and joint award programmes where students are awarded a qualification from the foreign institution ${ }^{14}$, while joint venture operations represent partnerships between two or more institutions to deliver an education programme offshore. For example, UK-based Middlesex University's operation in Dubai is established as a joint

\footnotetext{
12 As noted in Chapter 1, equity is used as a proxy for ownership in this study.

${ }^{13}$ In the education services context, franchising and licensing agreements are defined using the $i+j$ subscripts, where $i$ and $j$ respectively refer to the number of years of study in the host and source country (see Section 1.2).

${ }^{14}$ An example of a twinning programme is Victoria University of Wellington's partnership with The University of Economics in Ho Chi Minh City, where students study the first two years of an undergraduate degree in Vietnam before transferring to New Zealand.
} 
venture between the university and a private entity called Middlesex Associates in Dubai (Verbik and Merkley, 2006).

The definition of a joint venture, for the purpose of this study, should be clarified. This follows a lack of consistency in the literature, with some scholars using shared equity as the basis of their definition (e.g. Stopford and Wells, 1972; Harrigan, 1983, 1985; Gatignon and Anderson, 1988), while others focus on the establishment of a third joint venture entity between two or more independent agents as the definition (e.g. Shenkar and Zehra, 1987; Kogut, 1988). Pisano (1988) has referred to these as 'equity' and 'classic' joint ventures, respectively. This distinction becomes important when the focus of analysis is the managerial aspects of joint ventures, whereby the dyadic interactions between two partners will almost certainly differ from the dynamics between three or more partners (e.g. because of relationship, trust factors and behavioural patterns; see Pisano, 1988). However, with the focus of this present study investigating the decision prior to offshore investment, as opposed to post-entry management of the unit, the sharing of equity is assumed to be sufficient basis to classify an operation as a joint venture (Pisano, 1988).

Using these definitions, the analysis of current developments in Mode 3 transnational education, therefore excludes some operations. This includes the United States International University in Kenya, which, while having been established as a joint venture, has developed into an independent institution with no foreign control and with its own degree awarding powers and accreditations (Verbik and Merkley, 2006). Similarly, foreign-backed universities have also been excluded from this study. These institutions are typically new education providers set up in a host country with substantial foreign support, which tends to be more academic than financial. This academic support can be in the form of curriculum input, training opportunities for host country academics, and recognition in the source country for qualifications earned. Because foreign-backed institutions are mostly independent, foreign partners tend to have less ownership control, and these organisational forms do not fall into the definition of Mode 3 transnational education used for this study. Examples of foreignbacked institutions include the Swiss-German University of Indonesia, Westminster 
International University in Tashkent (Uzbekistan), the Kazakh-British Technical University in Kazakhstan, the British University in Dubai, the German University in Cairo, the British University in Egypt, Wadi Syrian-German University and the German-Jordanian University of Applied Sciences (Verbik and Merkeley, 2006). Another group of education institutions not included in this study are providers modelled on a foreign country's education system but with no affiliation to a mother institution there. These institutions are independently established. Examples of such institutions are the American Universities of Cairo, Beirut, Dubai (Qatar), Sharjah (United Arab Emirates), Bulgaria, Paris and the American International University in London.

\subsection{MODE 3 TRANSNATIONAL EDUCATION: A CHANGING EDUCATIONAL MAP}

Existing data on the growth and scope of transnational education is limited, because they tend to fall outside standard data gathering systems that focus primarily on domestic education programmes. It is, therefore, difficult to document precisely the extent of transnational education, and most of our understanding of this new phenomenon on the education landscape is based largely on anecdotal evidence (e.g. University of Nottingham's offshore campus in China). In this section, this study attempts to bridge this lack of data by synthesizing a range of intelligence scattered around books, academic journals, newspapers and institutional websites.

The following sources are accessed to develop an overview of the scope and scale of transnational education: Times Higher Education Supplement (UK-based publication), Observatory of Borderless Higher Education Reports (UK-based publication), Chronicles of Higher Education (US-based publication), The Australian higher education section (Australia-based publication), Campus Review (Australia-based publication), The Education Review (New Zealand-based publication) and Canadian Internationalist (Canada-based publication). Working papers, research reports and newsletters from the British Council, the Institute of International Education (US-based institution), the Australian Vice Chancellors' Committee, IDP Australia, the New Zealand Vice Chancellors’ Committee, Education New Zealand, Education Ireland and 
the Canadian Bureau for International Education are also consulted. Two academic publications specifically devoted to the field of international education, namely the Journal of Studies in International Education and the Journal of Research in International Education are also employed for compiling a sampling frame of transnational education developments. Data are also collected from international education-related conferences that I attended (IDP in Australia, NAFSA in North America, EAIE in Europe, APAIE in Asia and Going Global in the UK).

In undertaking the secondary data compilation, a literature search is conducted from 1970 onwards, to capture the few transnational education programmes that were set up prior to the late 1990s/early 2000s, when the scale of transnational education activity grew considerably. Transnational education programmes set up prior to 1970 are dissimilar to the contemporary ones, and were established either to serve study-abroad students or military personnel. Given that such transnational programmes are different in nature to the contemporary transnational education developments, which are established to serve fee-paying international students from an overseas location, they fall outside the scope of this study.

Furthermore, as a historical analysis, a number of transnational operations that are discussed in the literature, are not currently in operation. For example, UK-based De Montfort University and Australia-based Bond University both had transnational operations in South Africa. However, in 2004, these two operations were closed down, following a review of MBA programmes that resulted in their courses not being accredited. There are also cases in which the plans for transnational education development were captured by the media, but failed to materialise. This includes the Indian Institute of Management-Bangalore, which announced plans to open a campus in Singapore, but did not undertake the project. To ensure that the data compilation for this current study is as up-to-date and accurate as possible, any transnational education developments identified from the secondary sources accessed are cross-checked via the internet to ensure that they are still in operation. An active website is taken as an indication of operational existence, given that the internet tends to play a critical role in 
the recruitment of international students (for both cost effectiveness and marketing reach) (Gomes and Murphy, 2003). Where operational existence is in doubt, in spite of an internet presence, an attempt is made to contact the institution in question. If clarification is not obtained, then that operation is not included in the study. For example, some commentators have reported that New Zealand-based Victoria University of Wellington has a campus in Vietnam. However, clarification from Victoria University revealed that its Vietnamese operations are twinning arrangements, rather than a wholly-owned subsidiary or joint venture development ${ }^{15}$.

\subsubsection{Mode 3 Transnational Education via Wholly-Owned Subsidiaries and Joint Ventures}

The main conclusion that can be derived from the secondary analysis undertaken is that, while it is difficult to document, with precision, the scope and scale of transnational education, existing data shows that Mode 3 transnational education occurs mostly in the Asia-Pacific region, the Middle East, Eastern Europe and South America. It is most often undertaken by Australian, British and US institutions. Wholly-owned branch campuses or joint venture operations currently represent a very small share of the Mode 3 transnational education landscape while non-equity partnerships with local institutions remain the main vehicle for investment.

As highlighted in tables 2.2 and 2.3, the United States is clearly the leader in Mode 3 transnational education developments via wholly-owned subsidiaries and joint ventures; British institutions rank second to the US in absolute terms, while Australia leads in relative terms when the size of its post-secondary education system is taken into account.

Less than $1 \%$ of accredited not-for-profit universities and colleges in the US are active in wholly-owned subsidiaries and joint venture operations. However, Mode 3 transnational education operations via wholly-owned subsidiaries and joint ventures are becoming important parts of the operations of for-profit US educational institutions. Laureate Education Inc. (previously Sylvan International Universities), for example, has

\footnotetext{
${ }^{15}$ Correspondence with the International Office at Victoria University of Wellington.
} 
an enrolment of 209,000 students in 19 offshore operations across four continents (www.laureate-inc.com). Apollo International, an associated company of the Apollo Group (owner of US-based distance learning provider, the University of Phoenix) has five overseas campuses, in Canada, Brazil, Mexico, India and China (www.apollogrp.edu). DeVry Inc. has small operations in Canada and the Caribbean (www.devryinc.com) and Career Education Corporation has nine operations in France, one in the UK, two in Canada and one in the United Arab Emirates (www.careered.com). These for-profit operations are, however, outside the scope of this study as highlighted in Chapter 1, and are therefore, not included in tables 2.2 and 2.3.

Table 2.2: Mode 3 Transnational Education via Wholly-Owned subsidiary

\begin{tabular}{|c|c|c|c|c|c|}
\hline Location & USA & Australia & Ireland & Philippines & Total \\
\hline Austria & 1 & & & & $\overline{c 1}$ \\
\hline Belgium & 1 & & & & 1 \\
\hline Canada & 1 & 1 & & & 2 \\
\hline Czech Rep. & 1 & & & & 1 \\
\hline Fiji & & 1 & & & 1 \\
\hline France & 1 & & & & 1 \\
\hline Greece & 1 & & & & 1 \\
\hline Indonesia & & & & 1 & 1 \\
\hline Jamaica & 1 & & & & 1 \\
\hline Malaysia & & & 1 & & 1 \\
\hline Mexico & 2 & & & & 2 \\
\hline Netherlands & 1 & & & & 1 \\
\hline Singapore & 2 & & & & 2 \\
\hline Switzerland & 1 & & & & 1 \\
\hline S. Africa & & 1 & & & 1 \\
\hline Thailand & 1 & & & & 1 \\
\hline UK & 1 & & & & 1 \\
\hline Vietnam & & & & 1 & 1 \\
\hline Total & 15 & 3 & 1 & 2 & 21 \\
\hline
\end{tabular}


Table 2.3: Mode 3 Transnational Education Developments via Joint Ventures

\begin{tabular}{|c|c|c|c|c|c|c|c|c|c|c|c|c|}
\hline $\begin{array}{l}\text { Home } \\
\text { count }\end{array}$ & 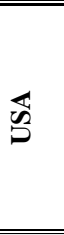 & 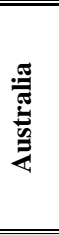 & 占 & $\stackrel{\widetilde{\Xi}}{\boldsymbol{\Xi}}$ & 胥 & $\underset{D}{.0}$ & ઇ త్ & 胥 & 矛 & 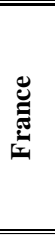 & 总 & 흄 \\
\hline "Australia & 1 & & & & & & & & & & & 1 \\
\hline China & 3 & & 1 & & & & & & & & & 4 \\
\hline Hungary & 1 & & & & & & & & & & & 1 \\
\hline Japan & 1 & & & & & & & & & & & 1 \\
\hline Jordan & 2 & & & & & & & & & & & 2 \\
\hline Kenya & & & & & & & & & 1 & & & 1 \\
\hline Malaysia & & 3 & 1 & & & & & & & & & 4 \\
\hline Panama & 1 & & & & & & & & & & & 1 \\
\hline Poland & 1 & & & & & & & & & & & 1 \\
\hline Qatar & 5 & & & & 1 & & & & & & & 6 \\
\hline Russia & & & & & & & & & & & 1 & 1 \\
\hline Singapore & & 2 & & & & & & & & 1 & & 3 \\
\hline UAE & 2 & 1 & 2 & 4 & 2 & 1 & 1 & 1 & 1 & & & 15 \\
\hline UK & 1 & & & & & & & & & & & 1 \\
\hline Vietnam & & 1 & & & & & & & & & & 1 \\
\hline Total & 18 & 7 & 4 & 4 & 3 & 1 & 1 & 1 & 2 & 1 & 1 & 43 \\
\hline
\end{tabular}

In addition to the institutions shown in tables 2.2 and 2.3, there are a number of other institutions whose ownership modes are unclear and are not easily categorised as either wholly-owned subsidiaries or joint ventures. These institutions include: Universidad Tecnica Federico Santa Maria de Chile (from Chile), based in Ecuador; SP Jain Centre of Management (from India), based in Singapore; Royal College of Surgeons (from Ireland), based in Bahrain; Griffith College, Dublin (from Ireland), based in Pakistan; University of Bologna (from Italy), based in Argentina; Christelijke Hogeschool NoordNederland (from the Netherlands), based in Qatar and South Africa; Seoul National University (from South Korea), based in Vietnam; John Hopkins University (from the US), based in China and Italy; Brookdale College (from the US), based in Ecuador; Troy University (from the US), based in Germany; and Clark University (from the US), based in Israel. Attempting to contact these institutions to clarify their ownership mode was not successful. These institutions are, therefore, omitted from the present study. Appendices A and B provide a detailed list of those institutions included in tables 2.2 and 2.3. 


\subsubsection{Mode 3 Transnational Education via Non-Equity Arrangements}

While wholly-owned subsidiary and joint venture Mode 3 transnational education is a small phenomenon, non-equity Mode 3 transnational education is much more established in the educational landscape. While it is similarly not easy to gauge the full extent of non-equity Mode 3 transnational education, some data are available to shed some light on its scope and scale.

Australia and New Zealand are particularly interesting cases to highlight, given that they are the only OECD countries currently collecting data on enrolment in non-equity transnational education programmes. In both countries, enrolments in non-equity offshore education have been increasing in the last few years. In 2006, this enrolment represented roughly 30\% of all international students enrolled in the Australian higher education system (DEST, 2007). This compares to 18\% in 1996 when the statistics on offshore education enrolments were first collected (DEST, 1997); see also figure 2.2. IDP, the company that markets Australian education internationally, forecasts that by 2025, 300,000 international students will be enrolled in Australian non-equity transnational education, representing 47\% of all international enrolments (Bohm, Davis, Meares \& Pearce, 2002). In 2003, 37 of the 38 Australian universities enrolled international students via non-equity offshore education programmes (AVCC, 2003). A survey conducted by the Australian Vice-Chancellors’ Committee (AVCC) shows that, as of May 2003, Australian Universities had 1569 non-equity offshore programmes with overseas education institutions. More than $70 \%$ of these non-equity offshore programmes were in Singapore, Malaysia and China (including Hong Kong). IDP further describes these non-equity offshore programmes as primarily postgraduate (56\%) and in business administration and economics (51\%) and with a mean enrolment of 40 students, 54\% of whom were enrolled in full-time study (Davis et al, 2000).

In New Zealand, a study commissioned by Education New Zealand (ENZ), the industry representative body of the international education sector, reveals that in 2006, 29 of the country’s 78 tertiary education providers surveyed (37\%) were offering non-equity transnational education programmes. Of these, 21 were state tertiary education 
providers (7 universities, 13 polytechnics and 1 college of education); the rest were private institutions. These 29 education providers represent a 53\% increase over the 19 providers that were involved in non-equity offshore education in 2003, when a similar study had been commissioned by the New Zealand Ministry of Education. 1,385 students were enrolled in New Zealand non-equity offshore education programmes. 137 courses were offered, of which 52 (38\%) were degree programmes, 49 (36\%) were diploma programmes and 30 (22\%) were at the certificate level and below. Six (4\%) programmes were not formal qualifications. Of the 52 degree programmes, 37 (71\%) were undergraduate programmes and 15 (29\%) were postgraduate programmes. Business administration and economics were by far the most popular types of courses offered offshore (46\%) followed by health (11\%), science and engineering (9\%), arts and social science (7\%), and tourism and hospitality (7\%). The offshore programmes surveyed were largely offered in China (including Hong Kong) (26\%), followed by the Pacific Islands (21\%) and Malaysia (12\%). The programmes in the Pacific were offered mainly in the Cook Islands and Tonga (9 programmes each).

In the United Kingdom, the overseas delivery of non-equity offshore education is a major and growing market and has been for some time (Committee of Vice-Chancellors and Principals, 2000). The British Council estimates that some 200,000 overseas-based students are involved in UK non-equity offshore education, and that this market is growing at $10 \%$ a year (www.britishcouncil.org/promotion/pmi.htm). The UK Prime Minister's Initiative, designed to market UK education to international students, targets offshore education as an area of growth (British Council, 2006).

In Canada, a survey conducted by the Association of Universities and Colleges of Canada (AUCC) in 2000, indicate that $42 \%$ of the responding institutions were delivering Mode 3 transnational education via non-equity modes (Knight, 2000). Of those $42 \%$, 29\% reported a low level of activity, $62 \%$ a medium level of activity and $8 \%$ described their level of activity as high. Those institutions that were not active in transnational education described their level of interest in delivering such programmes in the future as follows: low (50\%), medium (36\%) and high (11\%). These statistics, 
while fairly dated, reflect the observation that Canadian institutions have moderate to low interest in delivering transnational education programmes. This interest can be assumed to have probably risen over the last few years, but not hugely as highlighted in table 2.4.

In the United States, the Council on Higher Education Accreditation (CHEA), the national association for accredited degree-granting colleges and universities, indicates that, in 2006, 236 US-accredited programmes were operating outside the United States (CHEA, 2006). This figure does not include programmes that are accredited in the host country (or not accredited at all). It is estimated that, from the data presented in table 2.4, some 97 of the latter programmes existed in 2006. The data available from CHEA is only in aggregate form and unfortunately no further conclusions can be drawn. However, what can be mentioned from the aggregate data is that, while offshore education programmes are not overly common among traditional US universities and colleges, the for-profit education providers are quite active in this area. Indeed, not only are these providers active on the wholly-owned subsidiary and joint venture front of the transnational education market, but they are also very active via non-equity modes of entry, taking advantage of the low level of investment required. Although no comprehensive data is available to back this statement, figures from Sylvan Learning Systems show that college enrolments via non-equity arrangements are growing very rapidly, albeit from a small base (OBHE, 2003a). In fact, the same figures reveal that offshore education enrolments (delivered via all three modes of transnational education) are growing three times as fast as the US domestic level.

Evidence from the receiving host countries also makes it possible to assess the scale and scope of Mode 3 transnational education. With a few source countries suffering from a lack of comprehensive information, assessing the real extent of Mode 3 transnational education requires a triangulation methodology that examines source, host and third countries, in the case of franchised transnational qualifications. A number of host governments are increasingly requiring transnational education providers to go through a registration process, in order to maintain a degree of regulation on transnational 
education activity and avoid the emergence of 'degree mills' (i.e. rogue providers), all in the hope of guiding students toward 'legitimate' providers. This registration process provides lists of registered transnational education providers in China, Hong Kong, India, Jamaica, Malaysia, Pakistan, Singapore and Thailand. The online location of these lists is given in the reference section of this study.

While canvassing the available statistics from the host countries, efforts are made to compare them to those from the source countries whenever possible. Where the data do not match, they are cross-checked by contacting the source country institution in question. For cases in which this cross-reference is not obtained, due to a lack of response (a 78\% response rate is obtained), the more conservative estimate is then used so as not to run the risk of over-estimating the scale and scope of non-equity Mode 3 transnational education. In all cases, the conservative estimates are from the host countries; given that these are primarily from official government sources, they are assumed to be more accurate even in the presence of a large discrepancy. China is a particular case in point. While some commentators (e.g. Bjarnason, 2006) have suggested that there are currently more than 1000 non-equity Mode 3 transnational education programmes being delivered in China, data obtained from the Chinese Ministry of Education Website and the Australian Vice-Chancellors' Committee (AVCC) reveals that only about 400 such programmes are being delivered in China. This discrepancy may have arisen due to different data collection methods, or incomplete data, or could reflect the fact that unofficial provision exists in the delivery of non-equity Mode 3 transnational education, since the Chinese Ministry of Education and AVCC data account only for registered courses. Further analysis of the data would be needed to determine whether the discrepancy reflects additional programmes that are operating illegally. This analysis is, however, beyond the scope of the current study. Nevertheless, the discrepancy suggests that the true picture of transnational education activity currently remains outside of official information collection and the official Chinese and Australian data are used as they are more reliable. 
Table 2.4: Number of Joint Education Programmes from Selected Source Countries in Selected Host Countries as of 2006

\begin{tabular}{|c|c|c|c|c|c|c|c|}
\hline $\begin{array}{r}\text { Location of } \\
\text { Transnational } \\
\text { Source Country }\end{array}$ & 节 & 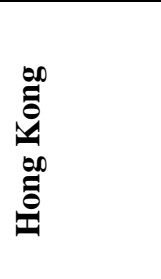 & 孚 & $\frac{\sqrt[\pi]{0}}{\stackrel{\vec{d}}{\tilde{J}}}$ & 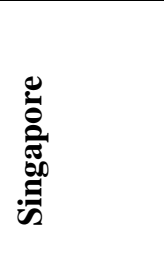 & ذّة & ฮีّ \\
\hline Australia & $199(23 *)$ & 291 (29) & 41 (9) & 315 (28) & 4991 (45) & 232 (37) & 1569 (37) \\
\hline Canada & $14(10)$ & $10(9)$ & 2(2) & $4(3)$ & $9(5)$ & 42 (n/a) & 81 (16) \\
\hline Ireland & $3(3)$ & $3(1)$ & $\mathrm{n} / \mathrm{a}$ & $1(1)$ & $15(2)$ & $\mathrm{n} / \mathrm{a}$ & $\mathrm{n} / \mathrm{a}$ \\
\hline New Zealand & $31(8)$ & $5(2)$ & $2(2)$ & $16(4)$ & $11(4)$ & 72 (18) & 137 (29) \\
\hline UK & $73(10)$ & 391 (51) & 105 (51) & $121(24)$ & 288 (55) & 27 (n/a) & 1002 (79) \\
\hline US & 50 (33) & $41(28)$ & 96 (64) & $23(8)$ & $86(45)$ & 37 (n/a) & 333 (n/a) \\
\hline Other & 40 (17) & $86(15)$ & $3(3)$ & $10(5)$ & $66(20)$ & $n / a$ & 205 (n/a) \\
\hline $\begin{array}{l}\text { Total programmes } \\
\text { identified }\end{array}$ & $410(104)$ & 827 (135) & 249 (131) & 490 (73) & $966(176)$ & n/a & 3327 (n/a) \\
\hline
\end{tabular}

Source: Australia: AVCC (2003); Canada: Knight (2000); China: China Ministry of Education (2006); Hong Kong: Education and Manpower Bureau (2006); India: National Institute of Educational Planning and Administration (NIEPA) (2006); Singapore: Ministry of Education (2006); Malaysia: LAN (2006); New Zealand: Education NZ (2006); UK: Education UK (2006); USA: CHEA (2006).

* Note: The number of higher education institutions from the selected source countries offering joint education programmes in the selected host countries is indicated in brackets.

Table 2.4 provides a snapshot of the number of non-equity Mode 3 transnational education programmes offered globally. The information reveals that Australia is by far the most active exporter of non-equity Mode 3 transnational education programmes. With 1569 education programmes and 37 institutions operating in the non-equity Mode 3 transnational education landscape, the overall 'intensity’ of Australia’s activity is 42.4 programmes per institution, compared to 12.7 programmes for UK-based institutions. In absolute terms, the US is the third most active exporter of non-equity Mode 3 transnational education programmes, followed by New Zealand and Canada. Ireland has some export activity, but not on the scale of market leaders. From the importers' side, the data presented in table 2.4 reinforce the point that the hotspots of non-equity Mode 3 transnational education are in Singapore, Hong Kong and Malaysia in descending order. China and India are emerging markets with each respectively having 410 and 249 nonequity transnational education programmes. It is to be highlighted that these numbers do 
not include distance learning transnational education programmes (i.e. Mode 1) since these lie outside the scope of this study. Appendices $C-G$ respectively provide a detailed overview of the institutions involved in the delivery of non-equity Mode 3 transnational education programmes in China, Hong Kong, India, Malaysia and Singapore.

In terms of the 'other' countries involved in the non-equity Mode 3 transnational education landscape, although it has not been possible to derive a comprehensive list of all institutions involved due to a lack of comprehensive secondary data, it is estimated that another 500-1000 education programmes are on offer worldwide with a similar number of institutions involved. This estimate is derived from information such as the following:

- In Argentina, there are currently 11 education programmes on offer via nonequity Mode 3; four from France and Spain respectively and three from Italy (García-Guadilla, Didou Aupetit and Marquis, 2002).

- In Brazil, there are currently five education programmes on offer, mostly from Portugal (Didou Aupetit, 2006).

- In the Dominican Republic, 45 foreign programmes are on offer; six from Latin America (mostly Cuba, Guadalupe and Puerto Rico), 23 from Spain, nine from the US and several from France, Canada and Belgium (OBHE, 2005b).

- In Eastern Europe, a report commissioned by the Swedish National Agency for Higher Education points to the emergence of Russia as a major exporter of transnational education. While constrained by a lack of data, the report does cite a number of examples of Russian transnational activity in former Soviet territories, serving Russian speaking minorities (e.g. Moscow University of Industry in Latvia and Moscow International Slavonic Institute in Bulgaria) (OBHE, 2003b).

- In France, some institutions are reported to be exporting transnational education programmes. These include Lille 2, Paris 4 and Grenoble 2 to Greece, and the Conservatoire National des Arts et Metiers, to Eastern Europe, Maghreb and sub-Saharan Africa (Adam, 2001). 
- In Francophone Africa, six undergraduate programmes are currently being offered by the Agence Universitaire de la Francophonie (AUF), a Montrealbased multilateral organisation comprising 525 francophone public and private universities. A graduate level training institute, located in Mauritius, is supported by AUF sponsored academics from French, Swiss, Belgian and Canadian universities (Jokivirta, 2005).

- In Germany, the DAAD (Deutscher Akademischer Austausch Dienst - the German Academic Exchange Service) estimates that 29 non-equity Mode 3 transnational educational programmes are currently being delivered by German institutions in 19 countries, 40\% of which are in Asia, about a third in Eastern Europe and the remaining in the Middle East, South America and Southern Africa (www.daad.org; Accessed February 2007).

- In Greece, some 600 transnational programmes are reported to be offered by European countries other than the UK (Adam, 2001).

- In Mexico, five education programmes are currently on offer, three from Spain and two from France (García-Guadilla, Didou Aupetit and Marquis, 2002).

- In Nigeria, Lagos Business School offers MBA programmes in partnership with Spanish based IESE Business School, which is part of the University of Navarra. (OBHE, 2005c).

- In Pakistan, seven foreign institutions are reported to be active: two from the UK, one each from Australia, Ireland, Malaysia, Singapore and the US (Higher Education Commission of Pakistan, 2006).

- In Portugal, Spanish education institutions are active in transnational education delivery. Portugal also exports transnational education with the Universidade Aberta, offering Portuguese qualifications to Portuguese emigrants in Africa and Western Europe (Adam, 2001).

- In Russia, the Indian Institute of Information Technology (IIT) is offering its masters and $\mathrm{PhD}$ programmes through a local teaching centre (Knight, 2005b).

- In South Africa, 14 programmes are offered by four institutions: two from Australia, one from the Netherlands and one from the UK (South African Qualifications Authority, 2006). 
- In Thailand, 17 programmes are offered by 14 institutions: nine from the UK, five from Australia, two from US and one from Canada (Thai Commission on Higher Education, 2006).

From the examples of these 'other' host countries, a broad observation can be made about Australia and the UK being the prominent source countries of transnational education. In terms of 'other' source countries, a handful of countries can be identified, including France, Germany, Italy, Portugal and Spain. Some major host countries such as Malaysia and Singapore, and even China and India, are also emerging as exporters of transnational education, although their level of activity is still extremely limited. Malaysia’s for-profit INTI International Group of Colleges, for example, has education programmes in Indonesia (OBHE, 2004). The National University of Singapore offers joint programmes with institutions based in the US, China, Sweden and India (OBHE, 2006b). China’s Jinan University is offering programmes in Thailand (Knight, 2005b) and Indian education institutions are targeting countries with an Indian Diaspora, offering Indian qualifications (e.g. the Indian NIIT education delivery network, which is spread over 30 countries in the Americas, Europe, Asia, Middle East, Africa; see www.niit.com).

Figures 2.3a and 2.3b below summarise these developments. Figure 2.3a highlights, on two axes, the extent of import and export of transnational education delivery, while figure $2.3 \mathrm{~b}$ provides a pictorial representation of that information.

What is not captured in figures 2.3a and 2.3b, however, is how national positions change over time. Vietnam, for example, is stepping up its import activities of transnational education; in time it can be expected that under present trends, Vietnam will move up the vertical axis in figure 2.3a. Conversely, some countries are moving to introduce regulations that are not welcoming to transnational delivery. Both China and India, for example, have recently introduced more stringent requirements for transnational education delivery, which are expected to consolidate their growth in transnational delivery experienced in recent years (Garrett and Verbik, 2003). 
Figure 2.3a: Mode 3 Transnational Education: Import and Export Activity in Selected Countries (Inclusive of All Three Modes of Ownership)

Major Importer

\begin{tabular}{|c|c|c|c|c|c|c|}
\hline \multirow{6}{*}{$\begin{array}{l}\text { Little or No } \\
\text { Export }\end{array}$} & & \multirow[t]{2}{*}{ Hong Kong } & \multirow{2}{*}{$\begin{array}{l}\begin{array}{l}\text { Malaysia } \\
\text { Singapore }\end{array} \\
\begin{array}{l}\text { China } \\
\text { India }\end{array} \\
\end{array}$} & 4 & & \\
\hline & & & & & & \\
\hline & $\begin{array}{l}\text { Mauritius } \\
\text { Qatar } \\
\text { UAE }\end{array}$ & & & & & \\
\hline & $\begin{array}{l}\text { Greece } \\
\text { Thailand } \\
\text { Vietnam }\end{array}$ & & Russia & & & \\
\hline & $\begin{array}{l}\text { Jamaica } \\
\text { Pakistan }\end{array}$ & $\begin{array}{l}\text { South } \\
\text { Africa }\end{array}$ & $\begin{array}{l}\text { Canada } \\
\text { Ireland }\end{array}$ & \begin{tabular}{|l|} 
France \\
Germany \\
New Zlnd
\end{tabular} & Spain & \\
\hline & & & & & USA & $\begin{array}{l}\text { Australia } \\
\text { UK }\end{array}$ \\
\hline
\end{tabular}

Major Exporter

Source: Adapted from Garrett and Verbik (2003)

Figure 2.3b: Mode 3 Transnational Education Markets: A Pictorial Representation of Import Activity (Inclusive of All Three Modes of Ownership)

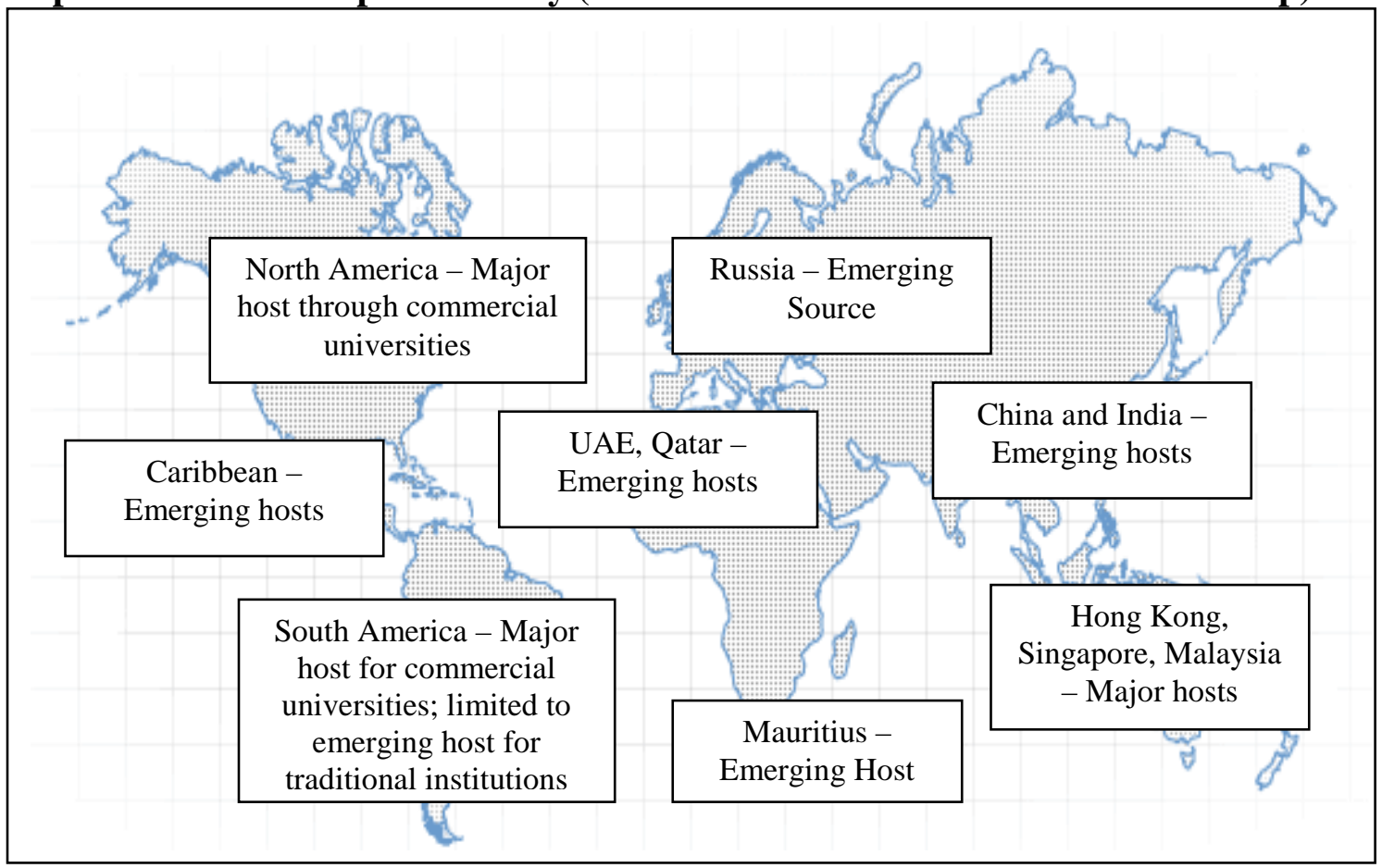

Source: Adapted from Bjarnason (2006) 
Overall, the secondary analysis conducted reveals that there were approximately 3327 non-equity Mode 3 transnational education programmes on offer worldwide as of 2006. This, however, is a very conservative estimate, and it is expected that the real scale of transnational activity is somewhere in the region of 3,800 - 4,300 programmes. As highlighted above, many transnational programmes delivered in the 'other' countries in table 2.4 are from non-English-speaking countries. Given that the methodology employed in collecting the data presented in table 2.4 is based on a canvassing of the English-medium literature, language barriers could have created an underestimation in the types and number of non-equity Mode 3 transnational programmes. Furthermore, the lack of most basic official data on transnational delivery might restrict press coverage in a lot of these 'other' countries, again causing an underestimation given the methodology employed. Lastly, the data examined in this chapter are those for registered and accredited transnational programmes; this excludes those programmes that are offered worldwide without being officially registered.

\subsection{RATIONALES BEHIND THE GROWTH OF TRANSNATIONAL EDUCATION}

Mode 3 transnational education is not a new phenomenon. The pace of its global expansion, however, is. The question that, therefore, arises is: why has this sudden growth occurred? This growth has taken place amidst liberalisation of FDI policies in the education sector (Altbach, 2007). However, FDI liberalisation is a facilitator of transnational education, not its catalyst. In the following discussion, four rationales are highlighted to help explain the growth in transnational education. These rationales, while distinct from each other, also share some overlapping elements and may be perceived as different ways of achieving the same goals. However, what distinguishes them lies in the presence of targeted policy tools, which are used under each of the rationales. These four rationales, derived from research undertaken by the OECD Centre for Educational Research and Innovation, are: (1) mutual understanding, (2) revenue generation, (3) skilled migration and (4) capacity building (OECD, 2004).

The mutual understanding rationale emphasises the academic, cultural, social and political grounds for the internationalisation of education, and does not consider 
education as part of an articulated economic policy. Under this rationale, the internationalisation policies for higher education are based on strengthening ties between countries through the creation of networks of political and business elites. For example, a number of Spanish institutions, especially the Catholic institutions, have apparently developed transnational programmes in order to increase the Spanish influence in the developing countries of Latin America (Adam, 2001).

Under the skilled migration rationale, internationalisation is meant to attract foreign students who are then encouraged to stay in the host country post graduation and contribute to its knowledge economy. Germany is an example where the skilled migration rationale is being employed (Garrett and Verbik, 2003).

The revenue-generation rationale highlights the market and trade approach of transnational education. It reflects the generation of income as an important rationale for recruiting international students. In the UK, for example, the recent Prime Minister's Initiative has highlighted offshore education as a means of diversifying the export of education services, which currently focus primarily on student mobility (i.e. via Mode 2) (British Council, 2006). Similarly, as highlighted in the preceding section, some traditional host countries have, in recent years, also shown an interest in developing their transnational delivery in order to take advantage of the export revenues provided by this approach to the internationalisation of education; Singapore is a key example of this strategy.

Lastly, the capacity development rationale views transnational education as a means of developing unmet demand for education from local constituents and building capacity and capability for quality education. This rationale for transnational education developments is especially important in countries such as Malaysia, where the higher education system does not meet domestic demand for higher education (VincentLancrin, 2005). 
These four different rationales for the growth of transnational education are reflected in figure 2.4. These four rationales are not mutually exclusive. For example, in encouraging transnational education, an exporting country might benefit from revenue generation, while the importing country benefits from capacity and capability building. The benefits of transnational education are not uni-dimensional; they are wide-ranging (from promoting mutual understanding to developing capacity) and accrue to both importing and exporting countries, depending on the perspective from which they are viewed.

\section{Figure 2.4 Policy Approaches to Transnational Education}

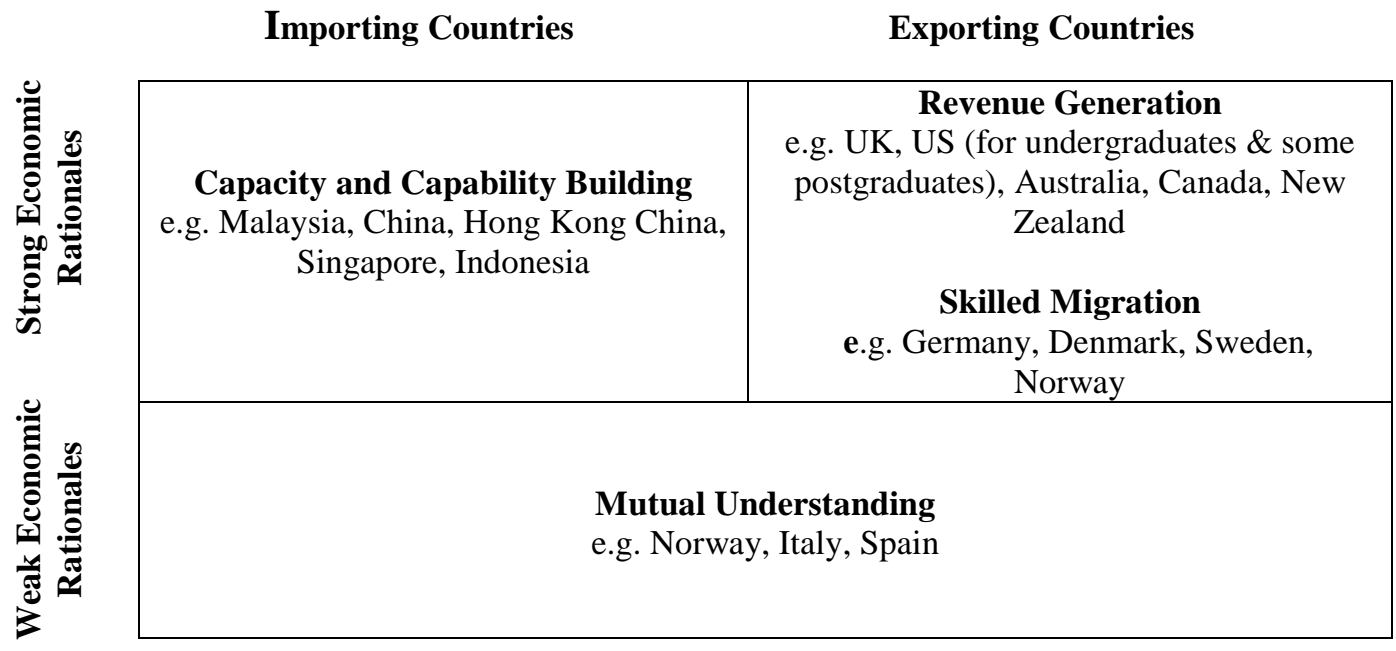

Source: Adapted from OECD (2004)

Of these four rationales, capacity building through transnational education developments has become an innovative strategy for economic development, and is increasing in popularity in a number of host countries (Vincent-Lancrin, 2005). While traditionally, transnational education has largely been a phenomenon developed out of source countries, a few host, importing countries are taking a proactive approach to encourage the delivery of transnational programmes in their domestic education system. Given this fairly new policy rationale to transnational education, the next section focuses more closely on the capacity building approach to transnational education. 


\subsection{TRANSNATIONAL EDUCATION AS A TOOL FOR BUILDING CAPACITY AND CAPABILITY}

Traditionally, many countries have relied on extensive scholarship programmes to promote student mobility as part of their capacity development strategies. Students educated abroad are expected to help build domestic capacity in higher education when they return home after their studies. However, given the substantial costs, the scale of any government sponsored scholarship programme is likely to be limited to a handful of students. Furthermore, the outcomes to be gained from such scholarship programmes depend on the students returning to the sending country, once their studies are completed. However, many sending countries suffer from 'brain drain' with nonreturning students representing a loss of investment. With receiving countries working hard to retain skilled foreign students to join the labour market post-graduation (i.e. the skilled migration approach), brain drain is becoming a substantial problem. In the United States, for instance, some 25\% of H1-B temporary visa holders have previously studied at US universities at either an undergraduate or postgraduate level (Cervantes and Guellec, 2002). The number is even higher for those with postgraduate qualifications, especially doctoral qualifications. Finn (2003) indicates that the percentage of foreign graduates with US doctoral qualifications in science or engineering, who are still in the US, four to five years after graduating, is above 95\% for Chinese and above 85\% for Indians. For these reasons, transnational education has become an important capacity building tool for many host countries. This is reflected in the policy statements of countries such as China where its law on Sino-Foreign Cooperation in the Running of Schools, has stated that it aims to "attract high-quality educational resources from overseas" and to "introduce globally advanced curriculum and teaching materials, which are in urgent need in China” (New China News Agency, 2003).

Three main reasons can be advanced as to why transnational education is gaining in

popularity as a capacity building tool: (1) expanding access to education, (2) increasing the variety/relevance of education and (3) enhancing the quality of domestic education (Vincent-Lancrin, 2005). 
Expanding access to education

Expanding access to education is an increasingly important element of economic development. An educated workforce is critical for sustained international competitiveness in a knowledge society (Romer, 1986). There is now wide recognition that an educated workforce is critical for sustained international competitiveness in the so-called knowledge economy. As a resource, knowledge not only contributes to the national wealth (Sobel, 1982), but also provides private advantages including incomeproducing benefits at an individual level (Becker, 1964). With the economic payback of education established in the literature, many countries are increasingly placing importance on higher education as part of their development strategies. Unfortunately, many are ill-equipped to cater to their development needs, suffering from a lack of supply of education. Indeed, whilst participation rates in tertiary education vary across OECD countries, it is expected that approximately $45 \%$ of the eligible age cohort in the OECD nations will enter higher education, compared to $26 \%$ in the 19 non-OECD countries that participated in the World Education Indicators (WEI) programme, which reports on this particular issue ${ }^{16}$ (Vincent-Lancrin, 2005). Although considerable progress has been made to improve access to education in developing countries, participation continues to be limited, relative to developed countries (Vincent-Lancrin, 2005). This is particularly the case in tertiary education, which is viewed as crucial for economic development.

Similarly, in emerging developing countries, rapid economic development has led to an increasing demand for tertiary education, especially among the middle class (VincentLancrin, 2005). However, many of these countries are not prepared to accommodate this increased demand for higher education. Larsen and Vincent-Lancrin (2002) reported that, in most developing countries, higher education institutions can only accommodate less than $5 \%$ of those who demand post-secondary education, adding that this shortage of higher education institutions in developing countries is likely to increase in the future, as the internationally-driven goal of providing basic education for

${ }^{16} 19$ countries participate in the OECD/UNESCO WEI programme: Argentina, Brazil, Chile, China, Egypt, India, Indonesia, Israel, Jamaica, Jordan, Malaysia, Paraguay, Peru, Philippines, Russia, Thailand, Tunisia, Uruguay and Zimbabwe. 
all is progressively achieved. With education, at least in the elementary and fundamental stages, being considered as a basic human right, and with numerous international governmental organisations (IGOs) supporting the educational development of several developing countries, the number of students who complete secondary education in those countries and want to continue on towards higher education is bound to increase in the coming years (Asian Development Bank, 2002). Unfortunately, unlike the primary and secondary education sectors, which have, to date, developed as a result of educational lending from IGOs, higher education in developing countries has not traditionally been the focus of educational assistance (World Bank, 2002). Due to a lack of resources, higher education institutions in most developing countries have been unable to keep pace with the associated demand that has occurred as more and more students have access to primary and secondary education.

Mode 3 transnational education can be a source for rapidly improving access to tertiary education. In order to provide education, a country needs to have a pool of human resources (academics at the tertiary level) at its disposal. Low levels of the necessary human resources preclude the production of new specialised human resources (VincentLancrin, 2005). For most developing countries, with few tertiary-trained citizens, the participation rate in tertiary education is not able to be raised as quickly as required, hampering efforts to catch up with their more developed counterparts. Even with adequate financial resources, developing countries with a low percentage of tertiary graduates may find it difficult to develop their domestic university education systems. This explains the case of the high-income Middle Eastern states, which still have less than OECD average rates of enrolments in their respective domestic tertiary education systems (Akkari, 2004). Having realised this, some countries, such as the United Arab Emirates and Qatar, have been encouraging foreign universities to establish Mode 3 transnational operations on their soil, to not only train their population for the labour market but also to boost the development of their domestic tertiary education systems. Malaysia is another example of a country employing such a strategy to boost the development of its own domestic tertiary education system. To date, there are five such campuses operating in Malaysia: Curtin University of Technology - Serawak Campus 
(Australia); De Montfort University (UK), Monash University (Australia), Swinburne University of Technology - Serawak Campus (Australia), the University of Nottingham (UK).

Increasing the variety and relevance of education

Transnational education can also offer students educational options that might not otherwise be available domestically (Vincent-Lancrin, 2005), especially where the tertiary education system is small and limited, in terms of range of disciplines and the level of study (i.e. undergraduate versus postgraduate). Often, this is the case in small countries, such as Luxembourg and Iceland, which have traditionally complemented their domestic education capacity with some form of transnational education (VincentLancrin, 2005).

Transnational education can also assist local education institutions in adjusting their course offerings to become more relevant in the global economy. In an era of economic integration, countries require a supply of cosmopolitans who carry what Kanter (1995) calls the three C's: concepts of the latest knowledge and ideas, competence in their ability to operate at the highest standards in any locations and connections that provide access to networked resources worldwide. By promoting knowledge circulation and inculcating students of the developing world with proficiency in the technologies and orientations of the industrialised world ${ }^{17}$, transnational education is an efficient way of developing a supply of cosmopolitans. Guoqing (2003), for instance, states that, for China, having a globally relevant education system is a must for its future workforce to be conversant with the practices of the advanced western countries. Similarly, Bennell and Pearce (2003) attribute the rapid growth in overseas student mobility from the socalled South East Asia tiger economies during the 1980s to the importance of 'learning from foreigners'. This learning can now take place domestically with the growth of transnational education.

${ }^{17}$ In general, developed countries export transnational education to developing countries, although mobility between developing countries also exists. 
Improving the quality of tertiary education

Some developing countries face a problem of quality, rather than quantity, of domestic tertiary education provision. It is, for instance, often commented in the literature that the domestic education systems of many developing countries suffer from a lack of reasonable facilities, absence of quality teachers, inadequacies in curricula and lack of flexible subject and course combinations (Avalos, 1992; World Bank, 2002). Transnational education, when delivered in partnership with local providers (i.e. through joint ventures and non-equity arrangements), can assist the latter to build capacity in teaching and curriculum design. Aside from generating such positive externalities through the employment of local staff, transnational education can also create constructive spillovers when domestic education institutions are faced with the need to consider and compare their offerings to the new transnational provision with which they compete when recruiting students (Vincent-Lancrin, 2005).

Furthermore, if transnational education provision involves research activities, in addition to purely teaching ones, it can add to the research capability development of the host country (Vincent-Lancrin, 2005). Depending on the subject matter, this can be the case in wholly-owned Mode 3 transnational operations, where the foreign education institutions invest into developing research programmes in the host country by establishing laboratories and acquiring expensive research equipment. These sorts of investments are important to foster an innovative research system. By establishing links with local industry, transnational education providers like their domestic counterparts in the host country, may also contribute to regional economic development (VincentLancrin, 2005).

\subsubsection{Risks Associated with Transnational Education for Capacity Building}

While transnational education does have a number of benefits for capacity building purposes, these benefits are not automatic outcomes of transnational delivery. For example, transnational delivery may not lead to quality enhancements or spillovers in the host country. The reasons for this can be manifold: transnational delivery not maintaining the same quality of education provided in the source country, partnerships with local education institutions not being appropriate mechanisms for delivery of the 
programmes, rogue providers leading to the emergence of 'degree mills', etc. (VincentLancrin, 2005). Spillovers may also be limited if source country education institutions do not partner with local institutions in the transnational delivery, through operating purely as wholly-owned subsidiaries.

Hence, while there are many reasons to suggest that transnational education can effectively assist in a capacity building strategy, the reality can be different. These risks, however, can be minimised via a policy framework that promotes an adequate national quality assurance framework, as has already been implemented in host countries such as Malaysia and South Africa (Vincent-Lancrin, 2005). It needs, though, to be acknowledged that some developing countries lack capacity in developing a quality assurance framework, and may be better advised to work at a supranational level, combining their resources and expertise (Lenn, 2003). Many such initiatives have emerged to address the need for improved quality assurance, accreditation and recognition of transnational education qualifications (e.g. joint UNESCO/OECD guidelines on quality provision in cross-border education, European Network for quality assurance in higher education, Global Alliance for Transnational Education (GATE)).

Ensuring that transnational education programmes meet national needs and objectives, goes beyond having an adequate quality assurance framework (Vincent-Lancrin, 2005). Rather, this needs to be addressed through regulatory mechanisms that cater to meeting national objectives. For example, countries wishing to build their capacity in research should promote branch campus Mode 3 transnational developments (preferably through a joint venture, to allow for knowledge spillover with local partner) as opposed to nonequity arrangements. Countries such as Hong Kong (China) are already making use of such regulatory frameworks. Since 1997, Hong Kong has implemented its 'Non-Local Higher and Professional Education Regulation', protecting local students against substandard transnational education programmes. In Singapore, partnerships with local institutions must also be government-approved, and partnerships with local universities can only be established if invited by the government (Vincent-Lancrin, 2005). While such mechanisms play an important role in ensuring that transnational education 
delivery is aligned to national needs and objectives, they should not, however, be overly restrictive, if they are to make the host country an attractive location for transnational delivery.

\subsection{NATIONAL REGULATORY FRAMEWORK FOR TRANSNATIONAL EDUCATION}

While the increasingly widespread phenomenon of transnational education is seen by many countries as adding value, there have also been growing concerns over how to regulate this type of activity and avoid the emergence of 'degree mills' or rogue education providers. While national regulations vary from country to country, Verbik and Jokivirta (2005) found six main regulatory models currently in existence: (1) no regulations, (2) liberal, (3) moderately liberal, (4) transitional - moving from liberal to more restrictive, (5) transitional - moving from very restrictive to more liberal and (6) very restrictive. These are briefly outlined in table 2.5, with examples drawn from both host and source countries. While one could assume that regulatory control to ensure the provision of quality transnational education is purely a host country issue, source countries also appear to be aware of the importance of adopting a sensitive approach to transnational delivery, in order to secure their brand name, as well as adopt pseudodevelopment based rhetoric to secure external support (and funding) from the wider public in both host and source countries (Verbik and Jokivirta, 2005).

Table 2.5: National Regulatory Frameworks for Transnational Higher Education

\begin{tabular}{lll}
\hline \hline Model & \multicolumn{1}{c}{ Regulations } & \multicolumn{1}{c}{$\begin{array}{c}\text { Examples of Source and Host } \\
\text { Countries }\end{array}$} \\
\hline \hline No regulations & $\begin{array}{l}\text { No special regulations or control } \\
\text { of foreign providers, which are } \\
\text { free to operate without seeking } \\
\text { permission from the host country. } \\
\text { The lack of regulations, however, } \\
\text { does not necessarily imply a }\end{array}$ & $\begin{array}{l}\text { Source: Austria, Czech Republic, } \\
\text { Denmark, France, Portugal, } \\
\text { 'laissez faire' approach but could }\end{array}$ \\
& $\begin{array}{l}\text { Hather represent a default } \\
\text { regulatory model where no }\end{array}$ & \\
& $\begin{array}{l}\text { attempt has been made to regulate } \\
\text { transnational delivery. }\end{array}$ & \\
& & \\
& Foreign providers must satisfy & Source: Canada, Finland, New \\
& certain minimum conditions prior & Zealand, Norway, United \\
& to commencing operations (e.g. & Kingdom, United States
\end{tabular}


official recognition in the home country).

Moderately liberal restrictive

Transitional: From restrictive to more liberal

The host country is actively involved in authorising and in some cases accrediting transnational providers. This model requires that foreign institutions gain accreditation or other formal permission by the host country (e.g. Ministry of Education) prior to commencing operations. This category is of academic criteria.

Requirements are generally straightforward and nonburdensome.

Transitional: From liberal to more diverse, ranging from compulsory registration to formal assessment

18 The case of Malaysia is ambiguous. For example, stricter regulations are being introduced for collaborative provision between domestic and foreign providers, but the requirements for foreign branch campus developments are becoming less demanding (Verbik and Jokivirta, 2005).
Host: Argentina, Peru

Source: Australia

Host: China, Hong Kong, Jamaica, Pakistan, Singapore, Vietnam

Host: India and Malaysia ${ }^{18}$

Host: Japan, South Korea
New legislation aimed at removing restrictions for foreign institutions wishing to operate in the country is being introduced. The new guidelines usually follow a period in which regulations have practically ruled out transnational provision. In some cases (e.g. South Korea), restrictions are only lifted in specified areas. In others (e.g. Japan), the changes in regulations apply to the entire country.

\begin{abstract}
introduced. Changes in legislation registration and/or accreditation order for foreign institutions to be allowed to operate and/or for their egrees to be recognised; presence in the country; criteria for collaboration between domestic and foreign institutions.
\end{abstract}


Very restrictive regulations:

a. concerning permission to operate b. concerning qualifications obtained through transnational provision
Strict requirements on foreign providers are imposed. Such institutions may be required to establish a physical presence in the country (i.e. non-equity arrangements are not allowed), only institutions/programmes accredited by the host country's agency are authorised, and/or foreign providers must change their curricula to be in line with domestic provision.

The government does not recognise foreign qualifications obtained through transnational provision. Foreign institutions wishing to grant recognised degrees must become a part of the national system (although that option may not be straightforward)
Host: Bulgaria, Cyprus, South Africa, United Arab Emirates ${ }^{19}$

Host: Belgium (Francophone), Greece $^{20}$

Source: Adapted from Verbik and Jokivirta (2005)

Juxtaposing the regulatory models presented above with the data on the scale of transnational education programmes highlighted in the previous sections, it becomes apparent that there might be a positive association between the regulatory environment and transnational education developments. China (mainland), Hong Kong (China) and Singapore, three of the most active host countries (territories) of transnational education, have moderately liberal regulatory frameworks. Malaysia is also one of the major sites for transnational delivery; although it has a transitional regulatory framework that is becoming more restrictive in some areas, current transnational provision would have taken place when the regulatory environment was more liberal. On the source country side, a regulatory framework that encourages the export of transnational education services appears to be important for transnational provision. The

\footnotetext{
${ }^{19}$ The UAE imposes very strict regulatory control on all foreign institutions with the exception of those operating in Dubai's Knowledge Village (where, accordingly, most of the transnational operations are located). The Knowledge Village, established in 2002, allows for $100 \%$ foreign ownership and is a taxfree zone.

${ }^{20}$ Private and foreign provision is not prohibited in Greece and foreign institutions offer qualifications in co-operation with local private colleges (as outlined in Section 2.3.2). However, these are not recognised by the Greek authorities. Interestingly, the demand for transnational education in Greece is high, in spite of its non-recognition.
} 
United Kingdom and Australia, two of the largest source country players in the transnational education market, respectively have a liberal and a moderately liberal regulatory environment. Therefore, it appears that transnational educational expansions do not occur in a vacuum, and that regulations in host and source countries can either impede or encourage these developments.

\subsection{CHAPTER SUMMARY}

This chapter presents an overview of the emerging patterns in the Mode 3 transnational education landscape. Through a review of both practitioner-oriented and academic literature pertaining to international education, the scale of transnational education development is outlined. In particular, wholly-owned operations, joint-venture arrangements and non-equity Mode 3 transnational education developments are discussed. It should be acknowledged that the transnational education landscape is constantly in a state of flux, subject to the changing patterns of demand and supply, as well as changes in regulation. Hence, the data provided in this chapter are descriptive and are current only at the time of writing. This information, nevertheless, provides a reasonable starting point for future mappings of the scope and scale of transnational education. Despite this caveat, some broad impressions may be drawn from this analysis. First, Mode 3 transnational education appears to be increasing very rapidly from a modest starting point. Second, it is developed countries that primarily export transnational education programmes to developing countries, although mobility between developing countries also exists. Lastly, transnational educational developments do not take place in an environmental vacuum. The institutional regulatory environment in both the source and host countries can encourage, as well as preclude, the development of transnational provision. It is the desire to develop an understanding of these conditions and how they are related to ownership forms in Mode 3 transnational delivery that leads us to the next objective of this study: developing a conceptual model to explain the foreign market entry decision of source-country universities. The theoretical considerations underpinning this research objective are outlined in Chapter 3. 


\section{Chapter Three}

\section{REVIEW OF LITERATURE}

\subsection{INTRODUCTION}

The conscious decision for international market expansion is almost always rooted in the prospect of profit maximisation (Root, 1994; Hill, 1997). When home markets stagnate, international expansion can be an effective means of expanding sales volume, reducing unit costs of overheads through economies of scale (and possibly also scope) and realising greater experience curve economics, offering the potential of strengthening competitiveness both at home and abroad (Vernon, 1966; Hamel and Prahalad, 1994; Hill, 1997). International expansion may also be undertaken to match the international market entry of a domestic competitor (Knickerbocker, 1973; Flowers, 1976; Yu and Ito, 1988), to counter foreign firms' penetrating domestic markets (Graham, 1978; Porter, 1980; Karnani and Wernerfelt, 1985; Wernerfelt and Karnani, 1987; Graham, 1998) or to take over weak competitors (Caves, 1996).

Whatever the firm's motivation to enter a foreign market, two major managerial decisions stand out when considering expanding business operations internationally: 1) the timing of market entry and 2) the mode of market entry (Day 1986). Karakaya and Stahl (1991) note that managers must additionally consider the type and magnitude of barriers to foreign entry, respond to these barriers through an appropriate strategy, and develop barriers to entry for competitors once established in the foreign market. Root (1987) adds that foreign market entry decisions can be categorised into five stages: 1) the choice of a target market; 2) the objectives and goals of operating in the target market; 3) the choice of a mode of entry for the target market; 4) the marketing plan, once in the target market; and 5) the implementation of a control system to monitor performance in the target market. Most of the literature that has been developed on international market entry seems to focus on four primary subjects: 1) entry barriers, 2) market selection, 3) entry timing and 4) entry mode selection (e.g. Anderson and 
Gatignon, 1986; Gatignon and Anderson, 1988; Agarwal and Ramaswami, 1992; Kumar and Subramaniam, 1997; Mani, Antia and Rindfleisch, 2007). This study deals with the latter issue. Hence, the focus of this chapter will be on the entry mode selection literature culminating with the intention of providing an integrated theoretical model of the foreign market entry process for education service providers that can be empirically tested.

Because this thesis focuses on education services, this chapter also reviews the relevant literature on the internationalisation of service firms, setting the stage for theoretical framework and hypothesis development in Chapter 4. Often, service firms internationalise their operations when their clients venture abroad (Weinstein, 1977; Vandermewe and Chadwick, 1989; Grönroos, 1999). For example, in the banking and the advertising industries, 'follow the client' strategy has been found to be a major driver for internationalisation (Nigh, Cho and Krishnan, 1986; Terpstra and Yu, 1988). However, the internationalisation of service firms has enjoyed explosive growth in recent decades and services trade play an increasingly important part in the global economy (Riddle, 1986; Erramilli, 1992; UNCTAD, 2004). This recognition has led to a growing literature on the internationalisation process of service firms, although this literature still remains very inconclusive in its findings (Anderson and Gatignon, 1986; Sarkar and Cavusgil, 1996). To understand the behaviour of internationalising service firms, it is, therefore, necessary to integrate the various existing strands of the literature.

\subsection{ENTRY MODE SELECTION: AN OVERVIEW OF THE LITERATURE}

Foreign market entry mode is a governance mechanism for organising and conducting international business transactions (Andersen, 1997). While there can be many forms of entry mode, the most common ones are exporting, licensing, strategic alliances, joint venture and wholly-owned subsidiaries (Agarwal and Ramaswami, 1992). Some scholars, such as Vandermerwe and Chadwick (1989), Erramilli (1991) and Hill, Hwang and Kim (1990), categorise these common modes of entry in terms of degree of equity involvement; full equity arrangements (wholly-owned subsidiary), partial equity arrangement (joint ventures in which the partner could be a majority, equal or minority partner) and non-equity arrangement (exporting, franchising and licensing). Others 
suggest that a natural hierarchy exists among these common modes of entry, such that managers tend to explore non-equity arrangements first before considering the more resource-intensive equity options (e.g. Johanson and Vahlne, 1977; Kumar and Subramaniam, 1997). Through a similar line of reasoning, it is also suggested in the literature that, within non-equity arrangements, direct exporting would likely be explored first, over the more costly indirect exporting, and licensing over the more resource intensive alliance option. Figure 3.1 depicts a hierarchical representation of modes of entry, developed by Pan and Tse (2000).

Figure 3.1: A Typology of Entry Modes

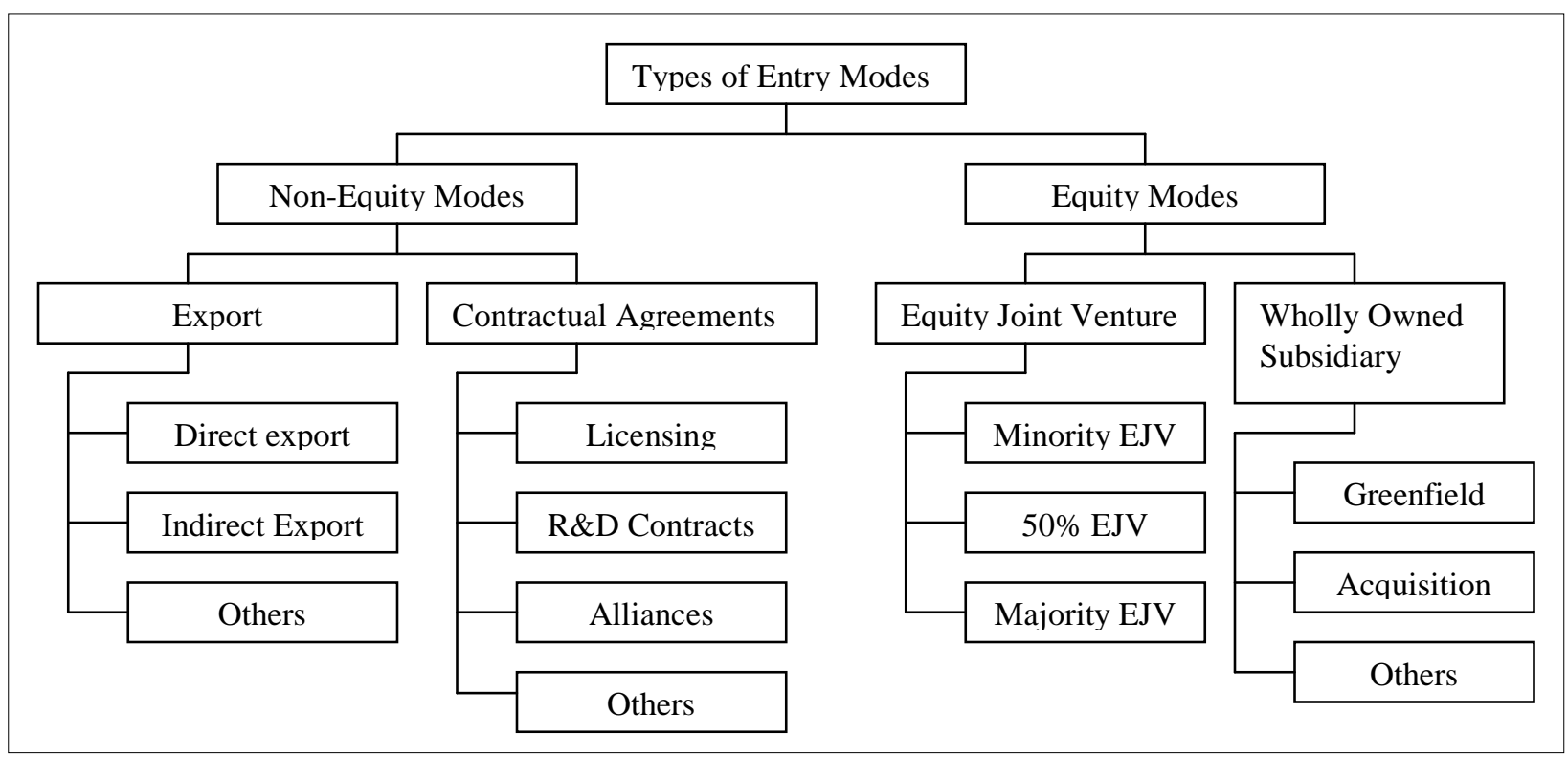

Source: Adapted from Pan and Tse (2000)

Entry modes are characterised by level of control and involvement (Anderson and Gatignon, 1986; Erramilli and Rao, 1990; Klein, Frazier and Roth, 1990; Kim and Hwang, 1992; Brown, Dev and Zhou, 2003). Control refers to the degree of authority over operational and strategic activities of the foreign subsidiary (Hill, Hwang and Kim, 1990), while involvement refers to the firm's level of participation in the target market (Erramilli and Rao, 1990). The entry mode literature focuses on control, because each mode is associated with a certain level of risk and resource commitment. The latter is, often the most important factor for determining investment risk and return (Anderson 
and Gatignon, 1986). High level of control and involvement is generally associated with wholly-owned subsidiaries, since they allow for the highest operational discretion (Anderson and Gatignon, 1986; Erramilli and Rao, 1993; Douglas and Craig, 1995). The downside of a wholly-owned subsidiary, however, is that it exposes the firm to the highest level of investment risk and is the most intensive resource commitment (Erramilli and Rao, 1990). If entry modes are to be placed on a continuum with respect to control, a wholly owned subsidiary will be at the top end of the scale, followed by a joint venture (subject to equity ownership), franchising, licensing and then exporting (Anderson and Gatignon, 1986; Erramilli and Rao, 1993; Zahra, Ireland and Hitt, 2000). The definition of these ownership forms, as used in the literature, is overviewed below.

Exporting is the most common entry point for international business transactions, enabling the firm to supply a target market from a domestic base or a third country (Douglas and Craig, 1995). Exporting can be either direct, indirect or involve some elements of collaboration (Douglas and Craig, 1995). Direct exporting occurs when the firm handles the export function internally, while indirect exporting involves the use of an intermediary such as an export agent or a trade office. Collaborative export involves arrangements between firms in delivering export related activities.

A joint venture involves some form of shared ownership of a foreign subsidiary that involves two or more independent organisations sharing their competitive strengths in the hopes of achieving a stronger market position (Keegan and Green, 1997). The level of control that each organisation exercises in the joint venture is proxied in the literature by the level of equity share each firm possesses in the venture. This level of equity contribution represents the extent of the partners' exposure to risks (Erramilli and Rao, 1990). In some environments, an organisation can be forced to undertake foreign entry through joint ventures because local laws prohibit 100\% ownership of local businesses by foreigners.

Licensing is a contractual arrangement whereby the owner of an asset (the licensor) grants another agent (the licensee) the right to use the asset in producing or distributing 
a good or service in return of some sort of consideration (Root, 1994). The licensed asset can be either tangible or intangible, in the form of trademarks, patents, trade secrets or production processes. While licensing can be an easy mode of entry into foreign markets, it can prove costly in the long term if the licensee uses the licensed asset, or ideas from the licensed asset, to develop its own know-how and capability. This know-how can then be used to compete directly with the licensor, not only in the foreign market but also in other national markets. A licensing contract can be used to minimise such contingencies but drawing up a contract, which is exhaustive of all contingencies, is an onerous and expensive task, not to mention that understanding such a contract would be a complex task (Arrow, 1974). This complexity adds to the intricacy of contractual enforcement, especially in countries where there is little respect for intellectual property rights.

Franchising, a form of licensing, is a mode of operation where a company that owns a protected trademark (the franchisor) grants another agent (the franchisee) the right to do business under the trademark, in exchange for some sort of consideration that often take the form of royalties, fees or profit sharing. A franchisee is basically a renter of a franchisor's brand name (Klein, Crawford and Alchian, 1978). Franchising differs from licensing in that it is more of a form of vertical integration than the standard licensing relationship (Klein et al, 1978). Largely due to the simultaneity of production and consumption, franchising is common in service industries (Palmer and Cole, 1995) as it allows the franchisor a great degree of control of the service delivery process (Bitner, Booms and Tetreault, 1990). Franchising shares many of the downsides of licensing including the difficulty of negotiating an agreement that effectively substitutes for vertical integration (Williamson, 1979), the difficulty in identifying and selecting a partner in the target foreign country (Geringer, 1991) and the complexity of drafting and enforcing a contract exhaustive of contingencies that would reduce the likelihood of opportunistic behaviour or shirking by the franchisee (Mathewson and Winter, 1985; Fladmoe-Lindquist and Jacque, 1995; Contractor and Kundu, 1998). 
Of the modes of entry defined above, this study, as outlined in Chapter 1, is concerned with wholly-owned subsidiaries, joint ventures and non-equity arrangements such as franchising. This follows the identification of these three modes of entry, in Chapter 2, as the key ownership forms being used by education service providers operating in the transnational education market.

Entry mode selection has been a topic of strong interest in the international business and strategic management literatures (e.g. Tse, Pan and Au, 1997; Brown et al, 2003). Scholars have long sought to identify and evaluate factors that determine an appropriate mode of entry in given situations where the firm's strengths and weaknesses, structural and strategic characteristics are aligned with the foreign environment (Brown et al, 2003). With different entry modes being somewhat consistent with different levels of control and dissemination of risk (Hill, Hwang and Kim, 1990), the choice of an appropriate entry mode, is therefore, a critical determinant of the success of the foreign operation (Davidson, 1982; Killing, 1982; Root, 1987).

Typically, entry mode selection is a two step process: (1) determining the location of production (export versus non-export) and (2) deciding on the level of control (i.e. fullcontrol versus shared control $^{21}$ ) or involvement (high versus low). Manufacturing businesses are concerned with both steps while services firms are concerned with the second step only, since for many type of services, production and consumption of services cannot be separated (see Section 3.4).

The firm's choice of a mode of entry has been linked to a large array of factors in the literature. For instance, it has been argued that it varies with product characteristics such as degree of differentiation (e.g. Stopford and Wells, 1972; Davidson, 1982; Goodnow, 1985; Gatignon and Anderson, 1988). It may further depend on firm characteristics such as size and resources (e.g. Davidson, 1982; Root, 1987). Entry mode choice can also be subject to external environmental factors, such as host country trade and investment

\footnotetext{
${ }^{21}$ Shared control includes joint venture, franchising, licensing, management contract or any other mode that entails joint ownership of the firm with one or more partners (Erramilli and Rao, 1993).
} 
restrictions, host market size, political stability, geographic and cultural distance, and exchange rate fluctuations (Aliber, 1970; Stopford and Wells, 1972; Goodnow and Hansz, 1972; Bauershmidt, Sullivan and Gillespie, 1985; Root, 1987; Gatignon and Anderson, 1988; Kogut and Singh, 1988). These products, firm and environmental determinants of entry modal choice are what Erramilli and Rao (1990) collectively classify as 'non-behavioural determinants'. Other studies have focused on behavioural aspects, identifying the decision making unit's knowledge of foreign markets, and the perceptions, opinions, beliefs and attitudes born out of this knowledge, as important determinants of entry model choice (Johanson and Vahlne, 1977).

Most studies on foreign mode of entry selection can be categorised into at least ten broad theoretical underpinnings, although these are not necessarily mutually exclusive from each other:

- Incremental involvement models whereby an organisation increases its resource commitments with respect to foreign market entry through stages, as more knowledge and experience in that foreign market is acquired (e.g. Johanson and Vahlne, 1977).

- Transaction cost models, which suggest that firms will opt for modes of entry that minimise the costs associated with the value chain by internalising those activities that they can perform at lower costs and externalising those activities that can be provided externally at a cost advantage (e.g. Anderson and Gatignon, 1986).

- Competitive strategy models, which are derived from the structure-conductperformance paradigm of industrial organisation theory and are concerned with the attainment of strategic competitiveness through competitive positioning. In the foreign market entry context, ownership mode is viewed as an important determinant of strategic positioning (e.g. McGahan and Porter, 1999).

- Resource-based view applied to the context of ownership forms, postulates that differential resource endowments are the underlying determinant of modes of entry (e.g. Barney, 1991). 
- Organisational capability models evaluate modes of entry from the perspective of enhancing the firm's performance and the demands they place on firms' capabilities (e.g. Root, 1994).

- Eclectic frameworks that propose that cross-border activities are influenced by location specific factors (e.g. investment risk), ownership specific factors (e.g. previous international experience) and internalisation specific factors (e.g. contractual risk) (e.g. Dunning, 1988).

- Institutional theory highlights the environment, both internal and external, as a major determinant of ownership forms (e.g. DiMaggio and Powell, 1983).

- Agency theory postulates that firms choose entry modes that maximise efficient principal-agent relationships and minimise monitoring costs (e.g. Jensen and Meckling, 1976).

- Resource dependence theory advances that firms will purposely structure exchange relationships through ownership forms that reduce environmental uncertainty and assist in the management of dependence (e.g. Pfeffer and Salancik, 1978).

- Contingency theory advances that there is no one best way of organising business operations and that any one way of organising is not equally effective under all conditions (e.g. Galbraith, 1973).

The literature on each of these theoretical approaches is briefly examined below.

\subsubsection{Gradual, Incremental Involvement Models}

Gradual, incremental involvement models of internationalisation are described through the 'Uppsala School' literature. Two theories of internationalisation stem from this literature: the Stages Model of Internationalisation and the Internationalisation Process Model.

The Stages Model of Internationalisation, first proposed by Johanson and WidersheimPaul (1975), is based on case studies of four Swedish firms. The model hypothesise a four-stage progressive/sequential internationalisation process spanning from no regular export activities, to export via independent representatives or agents, to a sales or 
marketing subsidiary, to production offshore. The basic assumption of the Stages Model is that an organisation develops its home market first, before it even contemplates offshore activities. The decision to internationalise eventuates when the firm faces factors such as economics, competition and growth (Johanson and Widersheim-Paul, 1975), and is related to the theory of the growth of the firm (Penrose, 1959). The incremental/stepwise internationalisation process of the Stages Model allows for the incremental assessment of challenges associated with offshore activities, which, in turn, allows for the incremental reduction of risks. Risk reduction is associated with the learning process that occurs over time, a process that allows for the psychic distance to be reduced between the home and host country. The greater the psychic distance between the two countries, the greater the time required to acquire the knowledge to reduce perceived risks. Indeed, as the firm learns more about a target host country, the perception of risks declines and the internationalisation process proceeds in that particular host country. The internationalisation process, according to the Stages Model, can be summarised as the firm's following the above outlined path, eventually culminating with it establishing a production unit offshore.

The Internationalisation Process Model, developed by Johanson and Vahlne (1977), builds on the Stages Model of Internationalisation. Its main proposition is that of successive and incremental levels of commitment, due to increasing levels of knowledge about the host country. The concepts of commitment and knowledge are expressed in greater depth than in the Stages Model, which focuses largely on the concept of psychic distance. In particular, the Internationalisation Process Model describes the sequential international involvement of an organisation from the initial export activities to the setting up of foreign production units, based on the firm's successively increasing experience and commitment to the foreign market. Based on behavioural theories, with the assumptions of lack of information and the importance of perceived risk and uncertainty (Cavusgil, 1980), the Internationalisation Process Model views internationalisation (and by implication, the use of selective modes of entry) not as a sequence of deliberately planned steps founded on rational analysis (Melin, 1992), but as an incremental process based on successive learning. This process of successive 
learning decreases psychic distance between the firm's country of origin and the target country of foreign entry, whereby more involved modes of entry will be used given lower degree of psychic distance and the associated perceived market uncertainty (Johanson and Vahlne, 1990).

The Internationalisation Process Model, therefore, postulates a direct relationship between market knowledge and market commitment, which can be explained through reduced uncertainty and perceived risks. Johanson and Vahlne (1977) go further in their explanation of the Internationalisation Process Model by distinguishing between objective and experiential knowledge. The former can be taught through some formal means, while the latter can only be acquired through operational experience. It is this experiential knowledge that is argued to be critical for facilitating resource commitments (Johanson and Vahlne, 1977). Cavusgil (1982) further adds that resource commitments in a foreign market are undertaken in small, incremental doses, based on each incremental increase in the firms' experiential knowledge.

While the simplicity of the Uppsala School of Internationalisation literature makes it attractive as a theoretical underpinning, it does, however, have some very serious limitations. Firstly, it has been suggested that the model is too deterministic and that it excludes other involved modes of entry at the early stages of internationalisation as important strategic options (e.g. Reid, 1983; Forsgren, 1990). Reid (1983) argues that strategic choice of an entry mode is a function of costs and, therefore, transaction cost models are better predictors of internationalisation. Johanson and Vahlne (1990) concede that neither the Stages Model nor the Internationalisation Process Model specifies strategic choice.

Young, Hamill, Wheeler and Davies (1989) also argue that distinct foreign markets may require different entry strategies, since they all have different market sizes, government policies, degree of risks and so forth. Consequently, it would be oversimplifying the case for entry modal choices to suggest, as the incremental models do, that firms follow a consistent path of internationalisation. 
The incremental model of internationalisation also appears to have been supported mainly in mature industries. Serious doubts are consistently raised when these models are applied to firms with small domestic markets, service firms, high-technology firms, entrepreneurial firms and international new ventures (Andersen, 1993). Bell and Young (1998) suggest that the incremental models of internationalisation merely identify the internationalisation patterns of certain firms, but fail to adequately explain those of others; this position is shared by other authors (e.g. Millington and Baylis, 1990; Oviatt and McDougall, 1997).

Furthermore, as the world becomes more and more homogeneous with the forces of globalisation, it has been suggested, although debatably, that the explanatory power of psychic distance is decreasing (Melin, 1992). While the extent of that decrease is contested in the scholarly literature, there is, however, wide acceptance that a decrease in psychic distance does lower the level of predictability of the model.

Additionally, it has been argued that this model pays no attention to environmental determinants of foreign market entry mode choice (Turnbull, 1987) and focuses purely on the concepts of psychic distance, commitment and knowledge. However, environmental determinants such as legal systems, host country governmental incentives, and geography can all have an impact on the internationalisation process (Gatignon \& Anderson, 1988; Gomes-Casseres, 1989, 1990; Agarwal 1994: Benito, 1996).

\subsubsection{Transaction Cost/Internalisation Theory}

While the Stages and Process Models of internationalisation are based largely on behavioural aspects, transaction cost theory highlights the economic rationale behind entry decisions. This approach views the firm as an efficiency-seeking entity that chooses modes of entry to minimise the costs of co-ordinating international business transactions (Contractor, 1990). This cost minimisation process is based on striking an appropriate balance between alternatives for ownership and control. Coase (1937) established the foundations for transaction cost theory. One of his initial propositions was that firms and markets are alternative governance structures that differ in their 
transaction costs and that, under certain conditions, the costs of organising an economic exchange within the boundaries of the firm may be lower than conducting that exchange in the market. Building on this proposition, Williamson (1975, 1979, 1981a) refined the concept of transaction cost analysis (TCA), to explore when an economic exchange might be performed more efficiently within an organisation (i.e. vertical integration) than through independent entities (i.e. outsourcing) in the market.

Transaction cost theory views the firm as a governance structure. Williamson and Ouchi (1981) broadly define governance as a mode of organising transactions that encompass the initiation, termination and ongoing relationship maintenance between parties (Palay, 1984; Moe 1984; Heide, 1994). Essentially, governance, therefore, includes elements of undertaking and structuring economic exchange transactions, as well as aspects of monitoring and enforcement.

Transaction costs, which are the costs of organising and running the economic system (Hobbs, 1996; Rindfleisch and Heide, 1997), include ex ante (e.g. negotiation costs to drafting a contract) and ex post costs (e.g. monitoring costs of enforcing the contact) (Jones and Hill, 1988; Hill, 1990; Rindfleisch and Heide, 1997). As suggested by Anderson (1985), transaction costs also include both actual and opportunity costs of transacting under various governance structures. If the total transaction costs (i.e. ex ante, ex post, actual and opportunity costs combined) of operating in the market outweigh the costs of managing an exchange within a hierarchy, then transaction cost theory postulates that it is more efficient to coordinate the exchange within the hierarchy (Williamson, 1985; Jones and Hill, 1988; Heide, 1994). In other words, the particular ownership structures, control modes or governance structures (the terminology used in transaction cost analysis) depend on comparative transaction costs, as opposed to simply production costs.

Transaction cost analysis begins with the approach that, under competitive market situations, market contracting arrangements (i.e. low involvement modes of entry) are favoured in the international context, given that the threat of competition forces 
suppliers to perform efficiently. On the other hand, in markets with low bargaining power vis-à-vis the target parent firm, wholly-owned operations (i.e. high involvement modes of entry) are preferred, as the firm can replace external suppliers with its own employees, whose behaviour can be more effectively monitored and controlled (Hennart, 1989). In other words, market failure is the most important antecedent to understand an organisation's decision to integrate and assume greater control (e.g. to use higher-involvement modes of entry in an international context). It should be noted that market failure does not refer to the fact that market mechanisms cannot be used. Rather, market failure, caused by the presence of transaction-specific assets (Williamson, 1986; Klein, Frazier and Roth, 1990) refers to the conditions in which integration is more efficient than market contracting (Anderson, 1985).

Williamson (1975) argues that the preference for integration over independent market agents is heightened when two sets of moderating factors co-exist in the market place, namely human and environmental factors. The former refers to bounded rationality that limits the ability to predict and prevent potential opportunistic behaviour by foreign agents (Beamish and Banks, 1987), while the latter refers to uncertainty and the level of competition among market agents.

Bounded rationality is based on the difficulty of gathering and analysing all the information necessary to make an informed choice. Because of this difficulty, the capacity to evaluate all strategic options is limited, in spite of the desire to make a rational decision (Simon, 1945). The second human factor, opportunism, has been defined by Williamson (1979:234) as "self-interest with guile", which refers to the incomplete or distorted disclosure of information. Transaction cost theory is based on the underlying assumption that opportunism is inherent in many transactions, since neither transacting party can be relied upon to voluntarily pass on complete information in an exchange process. This is not to say that all transactions necessarily involve opportunistic acts. Rather, transaction cost theory recognises that the risk of opportunism is often (if not always) present (Hobbs, 1996). Opportunism, therefore, plays a critical role in understanding the use of integration as a cost-minimising 
governance structure; without it, market contracting would be the norm to the exchange process (Williamson, 1985). Williamson (1985) further adds that, if asset specificity is high, the risk of opportunism is great enough to warrant opting for a hierarchical governance structure, since opportunism, as a strategic type of uncertainty (i.e. one that is intentional and incorporates ex ante behaviour) acts as a disturbance to the transaction.

Asset specificity, or what is often termed transaction-specific assets in the literature, refers to those assets, both financial and not, that are specialised and exclusive to the requirements of a particular exchange process and are, thus, non-redeployable. In other words, the higher the degree of re-deployability, the lower the asset specificity (Anderson, 1985; Anderson and Gatignon, 1986; Jones and Hill; 1988). The extent of asset specificity gives rise to safeguarding problems, in the sense that the risks of opportunistic exploitation need to be minimised (Klein, Crawford and Alchian, 1978; Williamson, 1985). Highly specific assets, such as specialised equipment, skilled workers or specialised investments in $R \& D$ and marketing, therefore, increase the costs of transactions since the market knowledge of the present and future value of these assets are likely to be limited and opportunism can easily take place in conditions of uncertainty (Simerly and Li, 2000). Thus, if the levels of asset specificity are low, it may be more efficient for firms to contract in the market place. If, on the other hand, asset specificity is high, it may be more efficient for firms to internalise their activities, from the market place to the hierarchy of the firm.

The greater the degree of uncertainty, the greater the level of difficulty in organising transactions, which may lead to a more formal type of integration, where control over the outcome of the transaction can be maintained (Hobbs, 1996). Two forms of uncertainty are outlined in the literature: environmental (Williamson, 1979) and internal (Williamson, 1981a). Environmental uncertainty is classified in the transaction cost literature as ex ante circumstances that surround an exchange, while internal uncertainty is classified as ex post circumstances. Environmental uncertainty, therefore, relates to the influence of the external environment on the transaction. The external environment 
causes an adaptation problem as part of the transaction, since contingencies are difficult to be specified ex ante in a contractual arrangement (Rubin, 1990). Therefore, under the transaction cost analysis framework, the likelihood of vertical integration is expected to increase with increasing uncertainty, given high asset specificity, since adaptation can be made without the need to revise contractual obligations between transacting agents (Williamson, 1979; Anderson and Schmittlein, 1984). In contrast, internal uncertainty relates to problems of behavioural opportunism that complicate the process of verifying compliance with established agreements, due to output measures of contractor performance that are often inadequate (Alchian and Demsetz, 1972; Rindfleisch and Heide, 1997). Hierarchical governance structures are assumed, a priori, to have better control and evaluation benefits (Anderson and Schhmittlein, 1984).

Another dimension of transaction cost analysis, is frequency, and involves the number of interactions involved in a particular transaction. It is contended that, as transactions become more infrequent, the incentive for opportunism increases, leading the costs of internalization to be more readily bearable than the losses from opportunistic behaviour (Anderson and Schmittlein, 1984). Hobbs (1996) contends that, when transactions are carried out frequently, the transacting parties are likely to value repeat business and are, thus, unlikely to act opportunistically at the risk of tarnishing their reputation. Frequent transactions also provide buyers and sellers with more information about one another, allowing frequent and repeated transactions to be carried out in the market.

One potential source of opportunistic behaviour is information asymmetry. Firstly, ex ante opportunism can take place when information is hidden prior to the transaction, leading to what Akerlof (1970) terms as 'adverse selection'. Secondly, ex post opportunism can take place, when actions of an agent are not observable by the other contractual parties, leading to a situation of moral hazard. The literature advances that, the greater the information asymmetry, the greater the likelihood of firms organising the transaction process through vertical integration, in order to mitigate the degree of opportunism (Williamson, 1979). 
The combining effects of bounded rationality, uncertainty, asset specificity, frequency and information asymmetry as sources of creating different levels of transaction costs, determine whether firms organise their business transactions through market exchange or hierarchy. This choice of market versus hierarchy, as identified in Williamson's original transaction cost analysis framework, has been expanded in recent years to cover a range of control mechanisms, rather than the polar extremes of integration or contractual arrangement (Williamson, 1985; Anderson and Gatignon, 1986). Osborn and Baughn (1990), for example, separated various forms of co-operation into quasimarket (i.e. market dominated) and quasi-hierarchy (i.e. hierarchy dominated) forms. Under this classification, non-equity agreements are defined as market-dominated, while joint venture operations are viewed as quasi-hierarchies.

Transaction cost theory, applied in the context of modes of entry has been enriched by the contributions of Anderson and Gatignon (1986) and Gatignon and Anderson (1988), who postulate that vertical integration is favoured when certain transaction-specific assets accumulate, when the external and internal uncertainties are high and when there are free-riding risks by agents and intermediaries. Anderson and Gatignon (1986) postulate that, in entry mode choice, trade-offs are made between control (the benefit of integration) and the costs of resource commitments (the cost of integration). In this context, the benefits of hierarchies must be compared with the costs of integration, since the latter entail considerable international organisation and bureaucratic costs.

\subsubsection{Competitive Strategy Models}

The competitive strategy perspective has its origins in Industrial Organisation (I/O) theory (Bain, 1956) and from the structure-conduct-performance (S-C-P) paradigm. It deals with the search for strategic competitiveness through the competitive positioning of the firm all with the goal of creating and claiming value (Ghosh and John, 1999). Under the assumptions of the S-C-P paradigm, an industry consists of homogeneous firms and the industry structure is assumed as a given. This scenario leaves no scope for strategic behaviour by firms, since structure determines conduct, which, in turn, determines performance. 
The competitive strategy model, though derived from the S-C-P paradigm, differs in terms of the level of analysis. While the S-C-P model focuses on the homogeneity of industry, the competitive strategy model perceives the industry as comprised of different groups, each with homogenous members, but relatively different from one another (Caves and Porter, 1977). This analytical focus on the industry sub-group level allows for the recognition of heterogeneity within an industry, which in turn, allows for an element of strategic behaviour since group composition can be partly attributed to the outcomes of strategic actions made by firms (McGee and Thomas, 1985). These strategic actions, which determine entry, exit and mobility barriers, become an important element of competitive strategy theory. In other words, under competitive strategy theory, these strategic actions are what define the boundaries of the firm. McGee and Thomas (1985) state that organisational boundary choices - the extent of vertical integration and the nature of contracts - are what influence strategic groupings. Porter (1980) highlights three kinds of vertical integration - full, tapered and quasiintegration (joint ownership) - to which Harrigan (1983) adds contracting as a fourth category. While these four categories of integration can be a source of competitive advantage, both Porter (1980) and Harrigan (1983) point to the fact that lesser levels of integration might also prove beneficial under certain conditions. These conditions, according to Harrigan (1983, 1985a), relate to the uncertainty of demand and the volatility of the competitive environment in which full integration might not prove to be the optimum strategy. Rather, greater flexibility is likely to be desirable under these conditions and can be achieved through lesser forms of integration that allow for the transfer of risks to others.

Applied to the context of modes of entry, the competitive strategy model postulates that firms choose entry modes that remove existing competition or minimise the possibility of new competition entering the market. Under this line of reasoning, a joint venture, as opposed to full integration, will be chosen only if it improves competitive positioning. This can be driven by a number of reasons. For example, joint ventures can be formed to split the costs of operations and the associated risks. Joint ventures also offer a way of accessing the resources of another firm by pooling complementary assets (Harrigan, 
1985a; Wille, 1988), along with lower exit barriers and greater flexibility than subsidiaries (Harrigan, 1985b, 1988). They may, therefore, be preferable in the early stages of an industry, when demand uncertainty is still fairly high. Wholly-owned subsidiaries can, however, be preferred when greater control over key value chain activities are required (Porter, 1980) and the risk of operations are lower.

Under the competitive strategy perspective, the rationale for non-equity modes of entry is similarly one based on the retention of flexibility, especially when the competitive environment is uncertain and volatile. Kobrin (1979) defines country risks in terms of the safety and profitability of business operations. This includes risks of expropriation or nationalisation; stability of the political, social and economic conditions of the host country; and the consistency of the host government's regulations towards foreign business activity. When country risk is high, an organisation is less likely to invest offshore through full integration, since this represents a higher degree of involvement and greater resource commitment (Kobrin, 1983; Vernon, 1983; Root, 1987).

An important argument, in the context of competitive strategy theory, is that the retention of flexibility is desirable unless strategic requirement necessitates full integration. Wholly-owned subsidiaries are only recommended when the costs of collaboration through joint ventures or trading through non-equity arrangements are too high. The treatment of the firm by competitive strategy theorists has, however, been criticised for its limited focus on the physical production configurations (e.g. value chains) at the expense of other flows, such as personnel, knowledge and information, which are equally important for efficient organisational performance (Ghoshal, 1987). This criticism leads us to the next theoretical approach that has been used to explain modes of entry determinants: the Resource-Based View.

\subsubsection{Resource-Based View}

Departing from traditional industrial organisation economics, which considers industry structure to affect strategy, which in turn, influences performance, the resource-based view (RBV) considers the firm, not the industry, as the source of competitive advantage (Penrose, 1959; Rubin, 1973; Wernerfelt, 1984; Barney, 1991; Capron and Hulland, 
1999). This competitive advantage, according to the RBV, is derived from the resources of the firm, which are assumed to be both heterogeneous and imperfectly mobile across firm borders (Barney, 1991; Peteraf, 1993; Hunt and Morgan, 1995). Conner (1991) advances that the core premise of the RBV remains the value-creating potential of the firm, rather than the avoidance of the negative impact of opportunism. This is not to deny the existence of opportunism. The RBV instead, provides an alternative explanation for the existence of the firm. While it accepts I/O-based views that the firm is efficiency-seeking in its operations, it goes further to explain that performance is not determined purely by the environment in which the firm operates, but also on the ability of the firm with respect to shaping that environment (Conner, 1991).

The RBV postulates that differential resource endowment is the ultimate determinant of strategic choices and actions that firms might take. Grant (1991) notes that resources underlie the direction of an organisation's strategy and are its primary source of profits. Wernerfelt (1989) contends that the focus of strategic analysis should, therefore, be on the firm rather than the industry. Focus on the firm allows for a dynamic view of strategy that the static equilibrium approach of I/O economics fails to address (Teece and Winter, 1984). This dynamic view of strategy views the firm as selecting a strategy based on its rent-generating assets and capabilities at any time (Grant, 1991).

The RBV views the firm as a unique bundle of resources, which are the sources of an organisation's competitive advantage as well as the main drivers of its performance (Rumelt, 1984, 1987; Wernerfelt, 1984, 1989). Grant (1991) adds that the amount, quality and type of resources place constraints on the firm's strategic actions. The type of resources, in particular, is an important qualifier in the RBV, in terms of the recognition that not all resources are sources of sustained competitive advantage. To be a source of sustained competitive advantage, a resource needs to be valuable, rare (Barney, 1991), inimitable (Amit and Shoemaker, 1993) and non-transferable (Rumelt, 1984, 1987). Non-transferable resources may take the form of proprietary technology, specialised assets, marketing skills, management style, location advantage, and 
organisational culture (Grant, 1991; Williams, 1992). Barney (1991) defines nontransferability in terms of the resource not having an equivalent strategic substitute.

Applied in the context of entry modes, the RBV, therefore, postulates that ownership forms in international business transactions are driven by the firm's resources. This argument goes further than competing entry mode selection theories by explaining not only the differences in entry mode choice observed across firms in an industry, but also why the firms in an industry do not all pursue strategies that are likely to offer the highest returns.

\subsubsection{Organisational Capability Models ${ }^{22}$}

Organisational capability theory, which is derived from organisation theory and evolutionary economics (Madhok, 1997), is based on the concepts of bounded rationality (Simon, 1945), incrementalism (Lindblom, 1959) and organisational routines (Cyert and March, 1963; Nelson and Winter, 1982). The organisational capability perspective views the firm as a set of input-output combinations, based on co-ordinated resources as the central factor for a productive organisational performance (Nelson and Winter, 1982). The focus of the organisational capability perspective is, in essence, based on co-ordinating the intangible resources of the firm, rather than on the traditional factors of capital and labour as determinants of firm output. This notion of organisational capability theory has gained in prominence under the resource-based view of strategy (Wernerfelt, 1984; Barney, 1991, 1996), in which resources, if valuable, rare, inimitable and non-transferable, are viewed as sources of competitive advantage (see previous section). Organisational capability theory and the resourcebased view of the firm are, however, distinct from each other. The distinction lies in the focus of analysis. While the resource-based view focuses on transferable input factors (i.e. resources) as the key source of competitive strategy, organisational capability

\footnotetext{
${ }^{22}$ There is debate in the literature as to whether Organisational Capability is a separate paradigm to the RBV. Some argue that it is not (e.g. Wernerfelt, 1984), while others suggest that it is, since capabilities refer to what a firm can do, whereas resources are the things it has (e.g. Amit and Shoemaker, 1993; Grant, 1996; Helfat and Peteraf, 2003). This study treats the two paradigms separately in order to explore their theoretical underpinnings individually. The study, however, acknowledges that there is a great deal of overlap between the two theoretical perspectives and views the capabilities approach as an extension of the RBV.
} 
theory views the firm's capability in managing resources as the actual value creator. Organisational capability theory, therefore, takes on a more dynamic focus, by analysing the firm-specific interactive processes that convert resources into valuecreating assets (Amit and Shoemaker, 1993). With this more dynamic focus, organisational capability theory goes beyond the resource-based view, and analyses the means by which firms amass and dissipate new capabilities and the forces that limit the rate and direction of this process. Key to these mechanisms are the information management attributes of the firm, which are based on developing and exchanging information through the firm's human capital (Amit and Shoemaker, 1993). This information management attribute of the firm is what makes human capital "repositories of embedded knowledge” (Badarocco, 1991: 129), where embeddedness limits transferability, adds to the inimitability of the knowledge and converts knowledge into rent-creating and sustaining resources.

Cohen and Levinthal (1990) have referred to the process of capability accumulation as absorptive capacity. Others, such as Ghoshal (1987) and Bartlett and Ghoshal (1987), have referred to this process as the firm's learning ability. Both of these notions refer to the firm's capability enhancement through acquiring, evaluating, diffusing, assimilating, deploying and exploiting knowledge (Duncan and Weiss, 1979). The capability of the firm to manage its knowledge acquisition and deployment strategy constitutes the focus of the organisational capability perspective.

The capability to acquire and deploy knowledge applies to both the stock and flow of knowledge (Dierickx and Cool, 1989). The former refers to an organisation's cumulative history, memory and experience, while knowledge flows refer to an organisation's current knowledge-related strategy. These two concepts of stock and flow are related, since it is the ability of the flow strategy to relate to the knowledge stock that creates value (Cool and Schendel, 1988). This is an important notion in the organisational capability literature, in that it establishes the argument that knowledge stocks are what embed the knowledge acquisition and deployment processes within the firm, thereby developing a source of inimitability and competitive advantage. Based on 
this argument, while an organisation's strategy can be imitated, it would be less than fully successful if it is incompatible with the imitating firm's knowledge stock. Dierickx and Cool (1989) refer the need for combining knowledge stock and flow in value creating activities as path-dependent processes, which are gradual and incremental, and based on an organisation's past experiences. The ability to acquire, evaluate, diffuse, assimilate, deploy and exploit knowledge, therefore, depends closely on the firm's existing stock of knowledge. Knowledge flow widens the firm's cognitive inventory of knowledge (i.e. knowledge stock) and enhances the firm's ability to acquire, develop and exploit new capabilities (Cohen and Levinthal, 1990).

In summary, the organisational capability perspective views the firm's absorptive capacity as essential to its innovative capabilities (Cohen and Levinthal, 1990). This absorptive capacity is based on both the stock and flow of knowledge, and managing the information flow within the path-dependent processes is what constitutes an organisation's invisible value creating resources, underlying the basis of durable competitive advantage (Itami, 1987). Accumulation of knowledge-based assets through a current strategy, therefore, not only has current value but also future benefits, in that these assets can be utilised as a basis for further accumulation.

Ownership forms are evaluated in terms of their contribution towards the enhancement of organisational capability and the demands they place on the firm's capabilities. This contrasts to the economic view of the firm as an entity capable of exploiting foreign market opportunities at will to one that chooses ownership forms that are within the limits of the firm's capabilities. Since capabilities are essentially embedded in firm and context-specific processes, they are not easily transferable to other contexts/firms without a consequent loss in value (Forsgren, 1990; Cantwell, 1991). Although these capabilities, being dynamic in nature, can be enhanced and applied to new environments, this is a gradual and incremental process (Dierickx and Cool, 1989). In the short term, an organisation's capabilities are, therefore, constrained to certain contexts and the more different the new context, the less transferable the capabilities are. Hence, the firm's existing knowledge stock both directs and limits its strategic 
feasibility of a particular market entry (Tallman, 1991). In this context, vertical integration might be preferable where the firm has a strong knowledge base, while more flexible ownership forms might be used in contexts where the firm lacks a strong knowledge base.

The nature of the environment is also critical in determining the firm's strategic evaluation of a particular market entry (Cool and Schendel, 1988). Given the pathdependent nature of the firm, resource accumulation is an incremental process (Dierickx and Cool, 1989). Under a stable environment, the gradual accumulation of knowledge is achievable, allowing knowledge flows to build on existing knowledge stock in selecting an appropriate mode of entry. In volatile environments, however, this gradual knowledge accumulation is not achievable with past experiences proving less directly applicable to rapidly changing dynamics, which require a considerable amount of new knowledge (March, 1991). Firm behaviour being constrained by past routines due to the stickiness of the knowledge base can lead to a 'competency trap' (Levitt and March, 1988), where the experiential learning that takes place is inadequate compared to the total set of knowledge needed in fast-changing environments. Therefore, in these dynamic uncertain environments where the firm's capabilities are limited to facilitate the accumulation of new know-how, the knowledge of other actors can be 'grafted' onto the target firm's resource base (Huber, 1991). This argument, applied in the context of foreign modes of entry, leads to the suggestion that in volatile environments, the use of ownership forms such as joint ventures may enhance organisational capabilities, compared to vertical integration, which may be viewed as too complex a process for the timely development of all necessary know-how (Pisano, 1988). Joint ventures may be useful when firm's organisational routines become less applicable in new foreign environments and additional routines are required to enhance organisational performance. These additional routines can be developed jointly with a partner, allowing for knowledge and skill transfer (Osborn and Baughn, 1990).

Franchising/licensing, as forms of non-equity ownership, may not involve adequate interaction for knowledge transfer exposure and, therefore, have lower utility as modes 
of entry under the organisational capability perspective. Although these non-equity ownership forms do provide scope for learning and capability enhancement, it is a matter of degree, which is relevant in this context.

\subsubsection{The Eclectic Paradigm ${ }^{23}$}

Dunning's $(1977,1988)$ model of internationalisation has been a work in progress, attempting to explain and predict the mode and pattern of the internationalisation process. His eclectic paradigm integrates many theories into a general framework that contends that three distinct advantages - ownership (O), location (L) and internalisation (I) - can be used to explain international investment.

Ownership-specific advantages are so specific to the firm that they become a key source of comparative advantage and make the multinational firm more competitive than the domestic/local firm, thereby offsetting the liability of foreignness. Location advantages occur when immobile factor endowments or "spatial market failure", such as trade barriers and high transportation costs, are present (Dunning, 1988:4). Put in other words, there must be some sort of location-specific advantages for a multinational firm to engage in foreign direct investment in a host country, rather than producing at home for export. Internalisation advantages occur when it is more effective for multinational firms to transfer their ownership-specific advantages across national borders through vertical integration than to depend on the international market-place, with its market imperfections.

The rise of alliance capitalism prompted Dunning (1995) to revise his initial framework, which largely viewed the firm as autonomous, to allow for greater use of collaborative transactional exchanges by multinational firms. This revision, allowing a sensitivity to the collaborative arrangements made for strategic imperatives, redefined ownershipspecific advantages to include not only those generated internally, but also the ability of the firm to seek out, harness and influence the income generating assets of other firms

\footnotetext{
${ }^{23}$ Although there are a number of eclectic models, which have been used in the mode of entry literature (e.g. Hill et al, 1990; Kim \& Hwang, 1990), the focus of this brief review is on Dunning's eclectic paradigm.
} 
with which they have ongoing co-operative relationships. Similarly, under alliance capitalism, location specific advantages will not only include location-bound resources and market imperfections affecting an organisation's costs of operation, but also the ability of the firm to acquire and exploit the ownership-specific assets of firms that are collaborative partners. Under these conditions, where ownership-specific and location advantages are co-ordinated through some sort of co-operative arrangement, the internalisation component of the eclectic paradigm, as initially applied in the context of the choice between markets and hierarchies, also needs to be widened to "embrace more 'voice'-oriented strategies of firms, which are directed to capturing the benefits of quasi-integration offered by transborder coalitions and cooperative relationships” (Dunning, 2001: 184). Another aspect of Dunning's (1995) revision is the dynamism introduced to the OLI framework. Alliance capitalism, by nature is a dynamic process, since the strategic forces in place at the beginning of an alliance could change, resulting in a new set of co-operative imperatives. Lastly, Dunning (2000) introduces a contextual element to the eclectic paradigm, suggesting that the importance of OLI advantages in explaining patterns of internationalisation is likely to be context-specific, varying across industries, geography and among firms.

The eclectic paradigm, applied in the context of entry modes, therefore, suggests that possession of an ownership-specific advantage can determine whether an organisation would engage in foreign operations or not (Dunning, 1980). If an organisation has such an advantage, the choice between foreign production and export depends upon the extent to which the firm's advantage could complement the location advantages of host markets. If factors align well with the host country, it might be more attractive to enter into foreign production rather than exporting (Dunning, 1980). The other central choice lies between non-equity ownership and integration/quasi-integration modes (i.e. whollyowned subsidiary/joint ventures). If the internalisation advantage is substantial, then the firm would be expected to favour integration/quasi-integration over non-equity modes.

The broadness and generality of the paradigm, allows for only general assertions and does not allow for more specific predictions about entry modes. Dunning (2001) notes 
that, the eclectic paradigm is a schema of generalised conditionals that provide a useful tool-kit for accommodating a variety of economic theories (Coasian, Williamsonian and Penrosian theories of the firm) explaining the patterns of internationalisation. It is not meant to offer a full explanation of all kinds of international production.

\subsubsection{Institutional Theory}

Institutional theory highlights the institutional environment as a major determinant of firm structure and behaviour (DiMaggio and Powell, 1983; DiMaggio and Powell, 1991; Scott, 1995, 2004). Seen as a complement to, rather than a substitute for, other theoretical perspectives (Eisenhardt, 1988; Oliver, 1997), institutional theory is a nonefficiency based perspective (DiMaggio and Powell, 1991; Selznick, 1996). Firms are perceived to conform to rules and belief systems prevailing in the environment of interest, in order to achieve legitimacy and confront isomorphic pressures (DiMaggio and Powell, 1983), rather than focusing purely on rationalism (Selznick, 1996). This is not to deny the existence of rationality as a theoretical insight, but reflects the argument that rationality itself is a social construct.

In an international business context, where multinational enterprises operate in multiple institutional environments across national boundaries, institutional theory underscores the argument that what is perceived as rational behaviour is dependent on a set of contextual, strategic and structural variables that vary from country to country (Rosenzweig and Singh, 1991).

Zucker (1983: 105) suggests that organisations gain a "common understanding of what is appropriate and fundamentally meaningful behaviour” as a result of pressures exerted by various types of institutions. Institutional theory aims to provide a theoretical basis to explain the range of influence these pressures exert on structural characteristics of organisations (Meyer, Scott and Strange, 1987), organisational change (Hinings and Greenwood, 1988) and strategic behaviours (Oliver, 1991). Institutional theory advances the notion that context can influence an organisation's course of actions (Roberts and Greenwood, 1997), focusing on three contextual pillars: regulative (i.e. 
rules and regulations), normative (i.e. cultural influences) and cognitive (i.e. behavioural influences) (Scott, 1995).

Institutional theorists have focused on the role of external institutions in affecting organisational behaviour (Davis, Desai and Francis, 2000), including regulatory structures, agencies, laws, courts, professions, interest groups and public opinion (Oliver, 1991). More recently, management scholars have included a focus on internal institutions' effects on organisational behaviour. Vladlamani (1996) and Kostova and Zaheer (1999), for example, define internal institutions as other business units within an organisational network, and suggest that business units can encounter institutional pressures from within the parent organisational network (e.g. from other business units) to become isomorphic (i.e. to conform) to the parent's organisational norms (Kostova and Zaheer, 1999).

In the context of entry modes, Rosenzweig and Singh (1991) suggest that the behaviour of the multinational firm might become institutionalised through isomorphic pressures, leading to the need to balance the pressures of (1) adaptation to the institutional context of the host country and (2) resembling other business units of the parent firm. Thus, understanding foreign entry modal choice from an institutional perspective requires an examination of the pressures that multinational enterprises encounter when managing international business transactions (Davis et al, 2000). This examination goes beyond a focus on external environmental factors (e.g. through SWOT analysis) to one that recognises internal isomorphic pressures. Management might be unconscious of, blind to or otherwise take for granted these isomorphic pressures since they have over time become institutionalised with the firm (Oliver, 1991). Similarly, the need for the parent firm to maintain strategic control over foreign subsidiaries adds to the internal isomorphic pressures to conform to intra-organisational norms (Davis et al, 2000). Conversely, when strategic control is vested at the subsidiary business unit level, pressures to maintain internal isomorphism are decreased (Davis et al, 2000). 
The degree of interdependency among subsidiaries, allowing for resource sharing, can also contribute to internal isomorphic pressures (Davis et al, 2000). Business units are indeed more likely to co-ordinate their modes of entry to facilitate the effective coordination of activities and strategies with sister units (Anderson and Gatignon, 1986), thereby reinforcing internal isomorphism. For example, business units sharing a common platform for research and development might all have to be operating offshore through high control modes of entry (e.g. wholly-owned subsidiaries) if they are to retain the rent creating assets that are generated through the research (e.g. intellectual property rights). A high-control mode of entry allows a parent firm the ability to centralise control, thereby allowing a higher level of convergence in institutionalised practices, which in turn, allow for higher levels of intra-organisational resource sharing and relatedness (Davis et al, 2000).

Organisational inertia is another explanation that is useful in explaining why parentcentred isomorphism can influence entry choice (Davis et al, 2000). As Porter (1990: 580-581) notes:

Firms would rather not change... Past approaches become institutionalised in procedures and management controls... Personnel are trained in one mode of behaviour. Self-selection attracts new employees who believe in the existing ways of doing things and are particularly suited to implementing them... Information that would challenge established wisdom is expelled or isolated.

This reinforcement of parent-centred isomorphism is based on collective and social beliefs (Vadlaman, 1996), which can possibly provide for common interpretive schemas across business units and facilitate strategic decision-making (Douglas, 1986). North (1990) argues that organisations operating offshore use these schemas and organisational routines to map out their investment and entry mode strategies.

Besides organisational schemas, conformity to the institutional norms and values of the host market might also be necessary to be perceived as legitimate entities (Davis et al, 
2000). While parent-business unit isomorphism might be institutionalised, it might not be applicable in certain host-country environments. In such contexts, the firm needs to be more closely aligned with the host country environment than with organisational schemas based on existing parent-business unit relationships (Davis et al, 2000). In the entry mode context, it would appear that some ownership forms facilitate alignment with the host-country contextual environment more than others (Davis et al, 2000). For example, low-control modes of entry, or what some scholars classify as adaptive modes of entry (i.e. partially-owned forms, such as joint ventures and franchising), are particularly suited in host regions characterised by high levels of uncertainty. Although these entry modes often imply a loss of internal control (Agarwal and Ramaswamy, 1992), the strategic flexibility gained in adapting to local market needs can more than offset this loss (Davis et al, 2000). Therefore, from the perspective of institutional theory, the firm seeking to enter foreign markets, must choose an appropriate mode of entry by balancing the conflicting demands of parental isomorphism and the need for local market adaptation.

\subsubsection{Agency Theory}

Agency theory, as developed by Berle and Means (1932) and later refined by Jensen and Meckling (1976), is associated with behavioural studies of employer-contractor or employer-employee interactions (Harris and Raviv, 1978). It posits that an agency relationship is present when a principal party, who is in charge of a set of assets/resources, depends on another party (the agent) to undertake some action on the principal's behalf (Ross, 1973). In this context, an agency problem arises when agents do not act in the best interest of the principal due to either an informational advantage over the principal or interests that differs to those of the principal (Hutchinson, 1999). Mechanisms such as investigating and selecting appropriate agents, monitoring them, and bonding payments by the agents can help to mitigate this agency problem (Jensen and Meckling, 1976). These mechanisms cannot, however, be implemented without extra agency costs, which add to the overall cost of operation. Agency costs, therefore, are a type of transaction cost reflecting the fact that without cost, it might be impossible to ensure that agents will act in the principal's interest. While reference is made to agency costs being transaction costs, agency theory should not be confused with 
transaction cost theory. While the latter focuses on macro external versus internal transactions (i.e. whether to internalise or use the market), agency theory has a purely internal focus, on the micro principal-agent relationship. The central issue in agency theory is how to resolve the conflict between principals and agents with respect to the control of corporate resources (Jensen, 1989). Efficient agency relationships are generally more complicated to achieve in international settings, with geographical distance and cross-cultural disparities compounding uncertainty, asymmetric information and monitoring (Bergen, Dutta and Walker, 1992; Alon and McKee, 1999). Efficient agency relationships depend on four key dimensions: information asymmetry, behavioural uncertainty, differing attitudes towards risks, and information as a commodity (Levintal, 1988; Eisenhardt, 1989). Each of these is reviewed below.

Information asymmetries arise when one party has access to information that the other desires but does not have. Agents, for example, in their day to day control of a company, develop detailed knowledge of its operations. The principals do not have access to that detailed knowledge, and agents might be unwilling to share that information with the principal, due to self-interest (Bergen et al, 1992).

In the context of agency theory, behavioural uncertainty can be categorised into two dimensions: moral hazard and adverse selection (Arrow, 1985). Moral hazard arises through shirking actions of the agent, with the principal being unable to monitor the agent's actions. Shane (1996) suggests that agents can engage in two types of moral hazard: sub-optimal effort and misdirected efforts. Adverse selection, on the other hand, refers to intentional misrepresentation of skills and abilities by the agent to the principal (Eisenhardt, 1988). This applies particularly in a hiring situation, where firms face uncertainty when hiring new employees (Coyte, 1984). Potential new employees have an incentive to exaggerate their skills, training and background, and firms incur costs in gathering information to determine who is most qualified for a job (Prescott and Visscher, 1980). This cost of overcoming adverse selection is even greater in international contexts, as the monitoring cost increases with business unit dispersion (Carney and Gegajlovic, 1991), potential language and institutional differences. 
Attitudes toward risk, the third dimension of efficient agency relationships, postulates that there is a disparity between the risk preferences of principal and agent, leading them toward disparate courses of action. Agency theory assumes that agents are more risk-averse than the principal (Harris and Raviv, 1978; Picard, 1987), on the basis that principals can decrease risk more easily through diversification of investments, while agents are less able to diversify their employment (Bergen et al, 1992).

Information, the fourth dimension, can be used by the principal to control the opportunistic behaviour of an agent (Eisenhardt, 1989). It is hypothesised that an organisation is more likely to succeed in minimising opportunistic behaviour by implementing adequate information systems in an internalised relationship, than by using a market based contract that focuses only on performance outcomes (FladmoeLindquist, 1991). The internalised relationship allows for monitoring problems to be overcome.

Applied in the context of entry modes, agency theory, therefore, postulates that firms choose entry modes that maximise efficient agency relationships and minimise the costs of monitoring. The intrinsic principal-agent problems and costs can be minimised through an organisational design that aligns the behaviour of the agent with the preferences of the principal. For example, agency theory proposes that, to mitigate agency costs such as moral hazard, contractual arrangements between agents and principals can be replaced with hybrid organisational arrangements like franchising (Jensen, 1983; Mathewson and Winter, 1985; Brickley and Dark, 1987; Norton, 1988a). Franchising can achieve goal alignment between agent and principal by placing the risk of failure from shirking or moral hazard on the agent (i.e. the franchisee) through tying business unit profits to the performance of the agent-principal relationship (Martin 1988). Non-performance penalties and franchise cancellation clauses help to align agent behaviour with principal preferences (Fladmoe-Lindquist, 1991). Norton (1988b) suggests that hybrid organisational arrangements deter moral hazard and adverse selection at lower monitoring costs than wholly-owned subsidiaries. Bergen et al (1992) add that, unless the principal is able to effectively monitor the behaviour of agents in 
foreign units, the increase in margin gained by ownership may not be sufficient to offset the greater efficiency of franchises.

\subsubsection{Resource Dependence Theory}

Resource dependence theory postulates that inter-firm governance is a strategic response to conditions of uncertainty and dependence (Pfeffer and Salancik, 1978), which are a reflection of the fact that few firms are completely self-sufficient with respect to their critical resources. This lack of self-sufficiency creates a potential dependence (Emerson, 1962) and adds uncertainty to the firm's decision making, to the extent that some resource flows are beyond the firm's control (Child 1972; Heide, 1994).

The resource dependence perspective assumes that firms will seek to reduce uncertainty and manage dependence by structuring their exchange relationships through formal or semi-formal links with other firms (Ulrich and Barney, 1984). Such structure is aimed to increase the extent of co-ordination with these firms and create a negotiated environment (Cyert and March, 1963); one that does not add to organisational complexity (Thompson 1967; Mintzberg, 1979), dynamism (Lawrence and Lorsch, 1967; Thompson, 1967) and hostility (Aldrich, 1979; Pfeffer and Salancik, 1978). In order to reduce the impact of environmental uncertainty, Kreiser and Marino (2002) highlight the importance of firms developing and sustaining effective relationships with their external environment. March and Simon (1958) add that the firm's ability to cope with these conditions by reducing its dependence on or increasing its control over its resources affects organisational effectiveness.

In the context of entry modes, resource dependence theory contends that environmental uncertainty influences organisational structure, as the firm aims to minimise or cope with this uncertainty. Resource dependence theory views governance as a response to environmental uncertainty and dependence (Pfeffer, 1972). In this context, environmental uncertainty becomes a critical determinant of entry mode choice (Erammilli, 1992). When environmental uncertainty is high, a large degree of ownership can decrease dependence and provide stronger control over the firm's 
operations. A large degree of ownership, however, also adds to switching costs, should undesirable outcomes occur in an uncertain environment. Williamson (1979) argues that firms should react to uncertainty by avoiding full ownership, since it commits them to a market that may not be appropriate when the operational environment changes. Harrigan (1986) adds that, in extreme market volatility, excessive integration can overexpose firms to demand uncertainty. At the other end of the spectrum, while complete reliance on the market will help manage the impact of uncertainty, it does not reduce the degree of dependence that the firm faces. Resource dependence theory, therefore, advances that the best way to manage environmental uncertainty and stabilise interorganisational relationships is through the use of hybrid ownership forms, such as joint ventures, franchising and licensing.

\subsubsection{Contingency Theory}

Lastly, contingency theory advances that there is no one best way of organising business operations, and that any one way of organising is not equally effective under all conditions (Galbraith, 1973; Ginsberg and Venkatraman, 1985). In the context of the international entry modes literature, the contingency relationships between firm-specific characteristics, environment and entry mode have been examined. Woodcock, Beamish and Makino (1994) argue for preferable entry modes being contingency driven. Stopford and Wells (1972) develop one of the first entry mode models that was contingent on the firm's international experience and product diversification. Since then, many other scholars have considered country, industry and firm-specific factors as contingent influences on entry mode decisions (Johanson and Vahlne, 1977; Davidson, 1980; Caves and Mehra, 1986; Gatigon and Anderson, 1988; Kogut and Singh, 1988).

While these studies did not specifically ground themselves in contingency theory, they do, by the nature of their analytical frameworks accept the premise of contingency theory, i.e. that the selected entry mode should conform to particular industry, firm and country factors that influence the entering firm (Woodcock et al, 1994). Therefore, contingency theory is viewed as a complementary theoretical perspective to other theories of mode of entry selection, rather than an independent theory in itself. 


\subsection{DISCUSSION}

Having reviewed these different theoretical approaches, the discussion that one needs to have is what theoretical approach to use in the context of this study. There is debate in the international business/strategic management literature as to which theory is most applicable in developing entry mode strategies in foreign markets (Dunning, 1977, 1980, 1988; Anderson and Gatignon, 1986; Hill, Hwang and Kim, 1990; Madhok, 1997). As outlined above, theories of entry modes range from industrial organisation economics to organisational behaviour (Buckley and Cason, 1976; Rugman, 1980; Williamson, 1975; Cyert and March, 1963). These theoretical perspectives overlap and complement each other and independently, none of these theories provide an exhaustive explanation of entry mode choices for multinational firms (Coviello and McAuley, 1999). There is, therefore, a need to choose the most useful set of perspectives for their applications. This study grounds itself in the resource based literature and the reasons for this undertaking are explained below.

Some scholars have lately been promoting the resource-based view as the richest framework for explaining entry modal choice (Madhok, 1997; Ekeledo and Sivakumar, 1998; Sharma and Erramilli, 2004) and more broadly strategy (Wernerfelt, 1989; Barney, 1991; Collis, 1991; Conner, 1991; Grant, 1991; Hall, 1992, Lado, Boyd and Wright, 1992, Mahoney and Pandian, 1992; Amit and Shoemaker, 1993; Bharadwaj, Varadarajan and Fahy, 1993; Madhok, 1997). The resource-based view acknowledges that an organisation's internal idiosyncratic characteristics also have an influence on strategy, including the choice of entry mode (Zou and Cavusgil, 1996). However, other scholars argue that a pure and simple focus on the internal resources of the firm fails to account for the external factors that have been linked to influence entry modal choice. These include the level of host government restrictions (Gatignon and Anderson, 1988; Gomes-Casseres, 1989, 1990), the level of competition in the host country (GomesCasseres, 1990), and cultural distance (Agarwal 1994; Benito, 1996), among others. Therefore, a broader framework that integrates the insights provided by the resourcebased view and addresses its short-comings is needed to provide a coherent perspective of internationalisation. It can be argued that the use of models combining various 
theoretical perspectives fulfils this requirement (Tallman, 1991). In this study, in an attempt to bring a more complete understanding and explanation of entry mode selection in the field of education services, a framework that draws from the resourcebased view, organisational capability perspective as an extension of the RBV and institutional theory is used.

The RBV and organisational capability perspectives are particularly applicable to the context of this study, with resources being particularly important in the education services sector. For example, without adequate resources (e.g. capital), education service providers are unlikely to contemplate offshore investment; unlike private firms, education service providers do not have ready access to alternate ways of raising capital (e.g. venture capital, initial public offerings ${ }^{24}$. Similarly, the service delivered by education providers is embedded in human resources (i.e. academics/faculty), without whom, offshore investment cannot take place.

The education services context is also a good fit to institutional theory, since the offshore environment in which the education provider operates may have a moderating role on the influence of resources on entry modes, by adding or reducing the transaction costs of the entry mode process. The education sector in most countries, is a regulated sector, where authorities monitor the delivery of quality education. Therefore, when investing offshore, education service providers are likely to operate around some form of regulated institutional environments that are likely to affect their mode of entry decisions. Education service providers must conform to the rules prevailing in the host environment (DiMaggio and Powell, 1983) and institutional isomorphism, both structural and procedural, to earn legitimacy (Deephouse, 1996; Daccin, 1997).

In developing the proposed analytical framework, another important issue to address is the applicability of theories and concepts developed to explain foreign entries of manufacturing firms to foreign entries of service firms (Aharoni, 1993). It has been

\footnotetext{
${ }^{24}$ As indicated in Chapter 1, the scope of this study is on the traditional education service providers as opposed to private corporate institutions, which are often listed on the stock exchange and have alternative means of raising capital.
} 
argued that concepts and theories developed for goods may not necessarily apply to services (Erramilli, 1990; Erramilli and Rao, 1990, 1993). This literature is overviewed in the next section. A discussion on the transferability of entry mode frameworks developed in manufacturing goods to the context of education services then follows.

\subsection{THE INTERNATIONALISATION PROCESS OF SERVICES}

In the entry mode literature, much attention has been devoted to the manufacturing sector, in spite of the growing phenomenon of services internationalisation (Stopford and Wells, 1972; Gomes-Casseres, 1985; Gatignon and Anderson, 1988; Erramilli, 1990; Erramilli and Rao, 1993; Sarkar and Cavusgil, 1996; Ekeledo and Sivakumar, 1998; Blomstermo, Sharma and Sallis, 2006). A growing debate has developed on whether service-specific characteristics (e.g. inseparability and intangibility) might affect foreign entry mode selection in ways that might not hold true in the manufacturing sector. Two schools of thought have emerged in the literature. The first is of the view that the research output on the choice of entry mode by manufacturing firms is also applicable to service firms (e.g. Weinstein, 1977; Boddewyn, Halbrich and Perry, 1986; Terpstra and Yu 1988; Agarwal and Ramaswami, 1992; Aharoni and Nachum, 2000). The other view-point argues that the factors influencing entry modal choice are not generalisable across the manufacturing and service sectors, given peculiar characteristics of service firms (e.g. Erramilli, 1990, 1991; Erramilli and Rao, 1990, 1993; O’Farrell, Wood and Zheng, 1996; Blomstermo et al, 2006). Still others have hypothesised that the concepts and theories that can be transferred from manufacturing to service contexts is contingent on the category of service involved i.e. hard or soft services (Ekeledo and Sivakumar, 1998).

In the manufacturing sector, research on entry mode choice is often grounded in a stages approach (Johanson and Wiedersheim-Paul, 1975; Johanson and Vahlne, 1977). In the services sector, however, firms may have to enter the foreign market all at once rather than in sequential stages (Grönroos, 1999; Zimmerman, 1999), especially when separating production and consumption is difficult (Prebble, 1992). Furthermore, in the services sector, industry-specific factors are more likely to impact on the mode of entry than in manufacturing firms, which are more likely to follow a sequential pattern 
favouring low-control modes of ownership when first engaging in international business and vice-versa (Buckley and Prescot, 1989). These service industry-specific factors include the degree of customisation, the degree of intangibility, the need for customer interaction, making international entry more difficult for service firms than for manufacturers (Knight, 1999b; Chase and Apte, 2007). Zeithaml, Parasuraman and Berry (1985) distinguish four key characteristics of services: intangibility (which leads to non-transportability, information asymmetry and ownership), heterogeneity, perishability and inseparability. It is to be noted, however, that few services display all of these four attributes, but most exhibit more than one. These four characteristics are central to the analysis of services and are outlined below (Aharoni and Nachum, 2000; Bouquet, Hebert and Delios, 2004).

\subsubsection{Intangibility}

Many have commented on the intangibility of services (Rathmell, 1966, Shostack, 1977; Chase, 1978; Berry, 1980; Lovelock, 1981) and how they cannot be touched, felt, seen, transported or stored (Kotler and Armstrong, 1991). Bateson (1979) argues that intangibility is the salient difference between services and goods, from which all other differences emerge. For example, this intangibility in turn gives rise to information asymmetry and lack of inventoriability between customer and service providers (Hölmstrom, 1985) resulting in service provision only being considered as an output at the instant it is sold and consumed. Until that moment, services are only potential outputs and can be considered as experiences that cannot be clearly assessed before consumption (Rathmell, 1966; Berry, 1980). In the case of education services, the act of imparting knowledge is highly intangible, although the use of physical symbols, such as text books and classroom design provides a physical reassurance of the presence of a good.

\subsubsection{Heterogeneity}

Services are highly heterogeneous, in the sense that their quality depends on who is delivering them and when, where and how they are provided (Kotler and Armstrong, 1991). Unlike products, service delivery is highly difficult to standardise and service performance is likely to differ from one consumption experience to another (Voss, 
Roth, Rosenzweig, Blackmon and Chase, 2004). This means that each service encounter is a unique interaction and often highly customised (Zeithaml et al, 1985). This heterogeneity can be problematic, in terms of consistency in service output, especially in labour-intensive and intellectual services (Knisely, 1979; Langeard, Bateson, Lovelock and Eiglier, 1981).

\subsubsection{Perishability}

The third attribute of services relates to its inability to be stored, meaning that services must be consumed at the time they are produced (Bessom and Jackson, 1975; Thomas, 1978; Lovelock, 1981; Holmström, 1985; Heskett, 1986, Mills, 1986). Some scholars, however, argue that perishability is a misleading characteristic of services, as there are many goods that are similarly perishable (e.g. bakery products), while there are also many services that may be as durable as goods (e.g. auto maintenance) (Riddle, 1986). Erramilli (1987) distinguishes between hard and soft services, and suggests that perishability is a key attribute of soft services, since the creation and delivery process of hard services (e.g. consulting services) can be separated. In spite of these different perspectives on perishability, it is largely accepted in the literature that most services are perishable in nature (Zimmerman, 1999). This perishability implies that direct delivery and short distribution channels are often essential in service industries (Chase, 1983). Managing service delivery, therefore, requires being situated close to the customer (Javalgi and White, 2002), which in an international context, is often achieved through foreign direct investment, joint ventures and/or licensing/franchising (Erramilli and Rao, 1993). It is to be acknowledged though, that the internationalisation of services through online communication interfaces (e.g. distance education) might slowly change the requirement of physical proximity in managing service delivery (Erramilli and Rao, 1993). Many services, however, still require real time delivery (Blomstermo et al, 2006).

\subsubsection{Inseparability}

Most services are also characterised by the simultaneous nature of their production and consumption (Zeithamal et al, 1985; Mills, 1986). Unlike physical goods, which are produced, stored and then sold, services are often sold first, then produced and 
consumed simultaneously (Regan, 1963; Grönroos, 1978). This inseparability attribute means that both producers and consumers must be present during the performance of the service (Erramilli, 1990) and consumers are forced into “...intimate contact with the production process” (Carmen and Langeard, 1980: 8). This provider-client interaction has led some scholars to suggest that inseparability is the most distinguishable feature in foreign entry behaviour of service firms and is at the centre of differences in entry behaviour between manufactured goods and services (Sampson and Snape, 1985; Erramilli and Rao, 1990, 1993). Indeed, entry modes that require a physical separation of production from consumption, such as exporting, might not always be appropriate in the internationalisation process of service firms (Carmen and Langeard, 1980; Root, 1987; Ekeledo and Sivakumar, 1998)

Inseparability also adds to the uncertainty of the service creation process, due to the diversity and unpredictability of customer demands, especially in culturally different foreign markets (Argote, 1982; Voss et al, 2004). McLaughlin and Fitzsimmons (1996) point out that, because of the intensity of customer involvement in services, substantial modification may be required to services in light of country specific circumstances, leading to a need for cultural adaptation. This cultural adaptation is potentially expensive, and may make internationalisation potentially difficult to achieve.

It is, however, also important to note that, while inseparability is an important attribute in services, it is not a universal phenomenon (Erramilli, 1990). Indeed, in some services, given their nature or owing to technological developments, the production and consumption stages of services can be separated (e.g. telecommunication allowing for long distance banking) (Riddle, 1986). Some services also have some elements of tangibility (e.g. disks by software services providers), and these services can be physically exported to a distant buyer. This selective, as opposed to universal, occurrence of inseparability is a reflection of the observed diversity within the service sector. 


\subsubsection{Implications of Service Characteristics in International Business}

While, in theory, services can be distinguished from pure goods based on the four key attributes highlighted above, in reality, the definition of services is complicated by the fact that there are few pure goods and services (Buckley, Pass and Prescott, 1992). Many goods can embody a service element as part of their production and distribution process, while many services can also incorporate some physical goods in their make up (Dunning, 1989). The distinction between goods and services, therefore, depends on the degree to which services are embodied in physical attributes and vice versa.

Several typologies have been advanced in the literature to define and classify the different types of services. Shostack (1977) developing Rathmell's (1966) concept of a good-service continuum, suggested that the greater the degree of intangibility in a market entity, the greater the divergence between product and services marketing, both in priorities and approach. Boddewyn, Halbrich and Perry (1986) further classify services into three categories according to their tradeability and based on the extent of inseparability in the provision of the service. These three categories are: (1) service commodities, which are exportable across national boundaries, since they are distinct from their production process; (2) location-bound where production cannot be separated from consumption; (3) an in-between category, where services comprise elements of commodities and location bound service elements. Sampson and Snape (1985) focus on inseparability and categorise services according to their tradeability. They propose that 'separated' services, i.e. those that do not require direct producer-consumer interaction, are only those services which can be exported.

Building on Lovelock (1983) and Schemenner (1986), Vandermerwe and Chandwick (1989) develop a two-axis configuration to explain the internationalisation of services, and come to the conclusion that the degree of service intangibility and inseparability of services are significant determinants of foreign market ownership in service firms.

Erramilli $(1987,1990)$ coined the terms hard and soft services. For soft services (e.g. health care, lodging services), it is argued that it is difficult, if not impossible, to 
separate production and consumption, but that such a separation is possible for hard services (e.g. architectural design, education). Applied in the context of foreign market entry, soft service firms, therefore, might not be able to export, while hard service firms can, and often do, export. Ekeledo and Sivakumar (1998) empirically tested Erramilli’s (1990) typology and found statistical differences between the foreign entry mode of hard and soft services.

Patterson and Cicic (1995) categorise services according to the degree of tangibility and the degree of face-to-face contact required with clients. They suggest that the more intangible a service and the greater the degree of producer-client interaction required, the less able service firms are to export.

Lovelock and Yip (1996) further classify services into three groups: (1) Peopleprocessing services that involve tangible action to consumers (e.g. restaurants, healthcare); (2) possession-processing services, which associate intangible elements to merchandise as a value added component to the consumer (e.g. transportation); (3) information-based services that provide some value for the consumer as a result of collection, analysis and manipulation of data (e.g. accounting). The second category does not involve the consumer in the production process, while the third category does only minimally.

Clark, Rajaratnam and Smith (1996) classify service firms engaged in international business into four types: (1) contact-based (2) vehicle-based (3) asset-based (4) objectbased. Contact-based service firms are those that cross national boundaries through the movement of people (producers or consumers) to engage in transactions (e.g. consultancy services). Vehicle-based services cross national boundaries through a communication interface (e.g. satellite transmissions). Asset-based services are those tied to international expansion through foreign direct investment (e.g. hotels). Lastly, object-based services cross national boundaries through physical goods, which are impregnated with services (e.g. computer software). Contact-based services are the 
purest form of services, exhibiting all the service attributes highlighted above, and are likely to engage in international business through non-export entry modes.

Domke-Damonte (2000) categorises services into three types of throughput technology: long-linked, mediating and intensive. Long-linked technologies (e.g. computer software) require the least producer-consumer interaction and can, therefore, be a foreign tradeable service (Boddewyn, Halbrich and Perry, 1986). In mediating throughput technologies (e.g. service restaurants) (Bowen, 1990), producer-consumer interaction can be partially decoupled, while in intensive throughput technologies (e.g. hotels) (Dunning and Kundu, 1995), the consumer is included, on a short-term basis, in the production process.

Of all the typologies sketched out above, Erramilli's (1990) is arguably the most widely quoted in the literature. This stems from the fact that the concept of hard and soft services is fairly easily understood and the classification schemas developed by other researchers can be easily applied and compressed to Errammilli's typology. For instance, from Patterson and Cicic's (1995) scheme, location-bound customised services and value-added customised services can also be classified as soft services, given their high producer-consumer interaction. Location-free professional services, on the other hand, are hard services, because they are characterised as low client-contact services (e.g. distance education courses). Similarly, in Clark et al's (1996) scheme, vehicle-based and object-based services are hard services, while contact-based and asset-based services are soft ones. In Lovelock and Yip's (1996) scheme, peopleprocessing and possession-processing services are soft services, while informationprocessing services (e.g. education) are hard services. The hard and soft service categorisation is particularly well embedded in the literature and applied to the context of entry modes, it provides useful insights that extend beyond individual classification schemes.

These insights can be summarised into the following points. First, the literature notes that firms whose outputs are more intangible tend to choose higher control entry modes 
in cross-border activities (Cloninger, 2004) as higher intangibility is associated with the need to produce service outputs physically near the client (Arvidson, 1997). With intangibility, performance ambiguity increases because the consumer has less evidence available to assess the service prior to the point of sale (Bowen and Jones, 1986; Roth and Menor, 2003). This ambiguity gives rise to the difficulty of evaluating exchanges between parties to a transaction (Bowen and Jones, 1986). Campbell and Verbeke (1994) suggest that this evaluation difficulty increases the importance of reputation, which are more easily managed through higher control modes (Kotabe, Murray and Javalgi, 1998; Enderwick, 1989). Campbell and Verbeke (1994) argue that service providers focus on providing a physical reassurance to consumers by creating, in their minds, tangible elements to an otherwise intangible experience. This tangible element tends to occur in marketing (e.g. through the development of branding/reputation) and is closely related to the service delivery process (Campbell and Verbeke, 1994).

Second, firms whose outputs are more heterogeneous are similarly expected to choose higher control entry modes than firms whose outputs are less heterogeneous (Cloninger, 2000). Since service delivery may be more difficult to standardise, with each service encounter being a unique and highly customised experience (Zeithaml et al, 1985), internationalisation may be driven through high control modes in order to maintain quality standards (Enderwick, 1989; Chase and Apte, 2007).

Third, since higher perishability increases the need to have production located close to the consumption point, service firms investing offshore may choose high control entry modes, since this allows them more control over the service delivery process (Cloninger, 2000) and facilitates service adaptation to the needs of foreign buyers (Voss et al, 2004; Blomstermo et al, 2006).

Fourth, it has been advanced that those service firms whose outputs are more inseparable, are more likely to choose higher control modes than firms whose outputs are more separable, because such modes allow them more control over the service delivery (Cloninger, 2000). Carman and Langeard (1980) suggest that firms providing 
inseparable service face additional risks, since they must begin operations in the host country through some form of physical presence without being able to export and learn first. Erramilli and Rao (1993) postulate that inseparability imposes considerable costs and risks on service firms, especially when faced with high cultural distance. Kogut and Singh (1988) argue that the extent of cultural distance might influence the entry mode and encourage the deployment of shared control modes (e.g. joint ventures).

Lastly, since soft services are more likely to be intangible, heterogeneous, perishable and inseparable, it has also been advanced in the literature that soft service firms are more likely to choose a high control entry mode than hard service firms (Blomstermo et al, 2006).

These insights, however, need to be treated with caution, since services, by their very nature, are diverse/heterogeneous; thus, generalisations about entry mode choices may represent over-simplification. Erramilli (1990) advances that a comparison of the entry mode choice patterns across various service industries reveals a picture of remarkable diversity. Richardson (1987) adds that, because of the diversity of services, no single theory of international services is likely to emerge as universally correct. Therefore, in any study of services, existing theories can only serve as a starting point in the development of context and service-industry specific analytical frameworks. With this point of departure, the next section highlights the application of the service characteristics literature in the context of the internationalisation of the education services industry. In particular, given the well-embedded nature of the soft/hard services classification scheme in the literature, the latter concepts are reviewed in the education services environment. It is questioned whether the hard and soft classification scheme can be applied to education services. From this analysis, it is then postulated whether the mode of entry theories highlighted above need to be adapted or not in their application to the education services context. 


\subsubsection{Implications of Service Characteristics in the Context of the Internationalisation of Education Services}

Education, like many service industries, exhibits elements of both hard and soft services. A hard service as indicated above, does not necessarily require a local presence by the exporter since it allows, to a major extent, the separation of production and consumption. Soft services, on the other hand, generally require more simultaneity of production and consumption; such services are more likely to require a major local presence by the service firm, or a representative acting on its behalf. The trade of education services through Mode 1 of supply (i.e. distance education - see Chapter 2) is, therefore, an example of a hard service, since the production and consumption process are separate from each other. Under distance education, the production process takes place at the home institution, while consumption takes place elsewhere, through an information communication technology (ICT) interface. This separation of production and consumption is feasible across national borders, when the student, enrols into an education programme being delivered outside his/her home country.

Education that is exported through Modes 2, 3 and 4 (international student mobility, offshore education and mobility of academics, respectively - see Chapter 2), are examples of soft services. With international student mobility, the consumer (i.e. the student) travels to the host institution to be provided with the education service. In offshore education, the service provider travels to the consumer to deliver the education service. With mobility of academics, an agent of the education service provider (i.e. an academic/teacher) travels to the consumer to deliver the education service. All three examples require physical proximity between the service provider and consumer, with consumer involvement during the service transfer, enabling production and consumption to take place simultaneously.

Offshore education, the focus of this study, can, therefore, be viewed as a soft service. In this context, offshore education service providers can be expected to prefer high control entry modes (Blomstermo et al, 2006). In reality, however, in spite of being soft services, offshore education service providers do not rely solely on high control modes of entry. In fact, few offshore education providers have, so far, embarked on 
international expansion through traditional high-control modes of entry (see Chapter 2). Instead, most offshore education service providers seem content to use non-equity modes of entry, such as franchising. Informal discussions with 40 senior university representatives involved in international education ${ }^{25}$, revealed that many education service providers are still at a stage where they are largely risk-averse with their offshore investment and, therefore, prefer low control entry modes over high control ones. This propensity to be risk averse stems from many universities being resourcepoor (e.g. capital, people) and preferring to undertake less risky investments than establish 'subsidiaries' ${ }^{26}$ overseas. It was further commented that while education services, to a large extent, demonstrates the characteristics of a soft service and that consequently, providers could benefit from high-control entry modes in their offshore investment, it is clear that education service providers are currently following an evolutionary approach to foreign market entry.

The university representatives talked to, mentioned how the internationalisation process of education service providers usually start on a small scale before commitment is deepened in the foreign market via joint ventures and offshore campus developments. This approach is depicted in figure 3.2, and is similar in nature to the sequential pattern of entry traditionally theorised in the manufacturing sector. The informal discussion with university representatives revealed that education service providers tend to approach their internationalisation process initially through an exporting process, whereby international students travel to the campus to study. This stage of internationalisation requires minimal resource investment, primarily in the form of an international recruitment/marketing and admissions team. The second stage of internationalisation tends to take place through non-equity arrangements such as franchising, twinning degrees and programme articulations (see Chapter 1). As indicated in Chapter 2, this is how most offshore education is currently taking place. Joint ventures and wholly-owned equity operations, the third and fourth respective

\footnotetext{
${ }^{25}$ Informal discussion held prior to my Ph.D. enrolment and which provided the foundation for this research topic - see Appendix H.

${ }^{26}$ As discussed in Chapter 1, in this study, I refer to offshore campuses as subsidiaries, consistent with the extant international business and strategy literatures.
} 
stages of the internationalisation process of education services, are not very common at this point, because of the resource commitments and the newness of the phenomenon on a large scale. It was further indicated that joint ventures and equity operations would be used only if the market dictated such activities or the market was to provide some sort of incentive for such operations (e.g. tax benefits, host government financial incentives, etc).

This evolutionary approach to the internationalisation of education services suggests that the theories of entry mode selection largely developed out of the manufacturing sector are applicable for understanding the education services context. This contention follows other studies, which also found the theories of manufacturing FDI relevant to services (e.g. Dunning and McQueen, 1982; Boddewyn et al, 1986; Agarwal and Ramaswami, 1992).

\section{Figure 3.2: Evolution of Education Services}

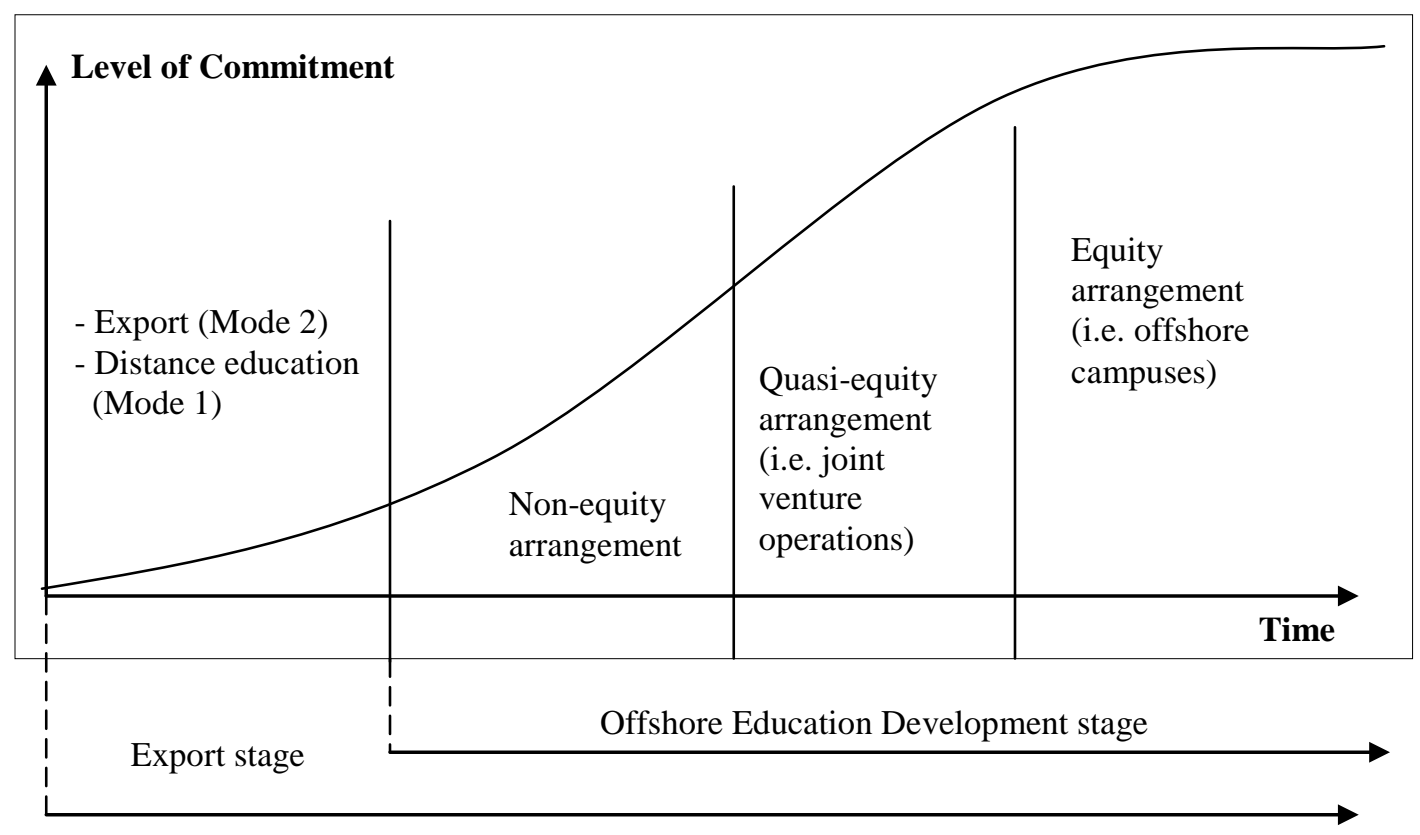

\subsection{CHAPTER SUMMARY}

This chapter has overviewed the literature on modes of entry and has highlighted the theoretical frameworks that have been used to explain modes of entry choices. In particular, ten broad theoretical approaches are reviewed: namely incremental 
involvement models, transaction cost theory, competitive strategy theory, resourcebased view theory, organisational capability theory, the eclectic paradigm, institutional theory, agency theory, resource dependence theory and contingency theory. These theories largely drawn from the manufacturing sector are then reviewed in terms of their applicability to service firms, given the context of this study focusing on education services. Two schools of thoughts have emerged in the literature on the internationalisation of services. The first is of the view that the research output on the choice of entry modes by manufacturing firms is applicable to service firms. The second argues that the factors influencing entry modal choice cannot be generalised across the manufacturing and service sectors given, peculiar characteristics of services: intangibility, heterogeneity, perishability and inseparability. Applying these service characteristics in the context of the internationalisation of education services, it is argued that the former school of thought is more appropriate to this study. This follows the identification of the internationalisation of education service providers as taking more of an evolutionary sequential approach, similar to manufacturing firms. 


\section{Chapter Four}

\section{CONCEPTUAL FRAMEWORK \& HYPOTHESIS DEVELOPMENT}

\subsection{INTRODUCTION}

As discussed in Section 3.3, the analytical framework developed for the purpose of this study is a function of internal organisation specific resources and institutional environments. While the central focus of the framework is on organisation specific resources, the external environment in which the organisation operates is viewed as an influential factor on entry choice. Furthermore, for the purpose of this study, resources encompass both rent-generating assets and capabilities, thus, building on both the RBV as well as organisational capability theory. As identified under Sections 3.2.4 and 3.2.5, while the RBV focuses on resources in terms of transferable input factors as the key source of competitive advantage, organisational capability theory views the organisation's capability in managing resources as the actual value creator. It is reasoned, for the purpose of the current study, that both views have their merits, and may be more effective when used in combination.

The key issue of the resource-based and organisational capability views of the organisation is the attainment of competitiveness through the development and exploitation of an organisation's assets and capabilities. Assets are things, both tangible and intangible that an organisation owns. Capabilities on the other hand, refer to what an organisation can do with its assets. According to the RBV and organisational capability perspectives, governance structures of organisations are, therefore, chosen based on the contributions towards and demands placed on those assets and capabilities. This logic is also structured around constraints that the organisation faces in terms of its assets and capabilities, which are mostly developed gradually and primarily through experiential knowledge. The know-how in managing these assets and capabilities is 
lodged in the organisation's routines and is largely organisation and context specific. Applying this know-how in a different offshore context can result in a reduction of its rent-generating potential due to its weakened applicability in the new context. This may lead to a less than effective transfer of knowledge, creating efficiency losses, especially in environments where the organisation is particularly not experienced. Therefore, the entry mode choice needs to be balanced against its benefits and the costs of efficiency losses and reduced effectiveness due to weak transferability. In other words, an organisation's decision regarding modal entry choice should be based on an examination of its existing resources, or a capability audit, and the requirements of the operational context (Tallman, 1991). The existing stock of an organisation's resources will, therefore, both direct and limit the strategic evaluation of a particular entry.

The types of resources examined in this study are organisation specific capabilities with respect to experience and tacit know-how, organisational culture, financial capital and reputation. This list of resources is by no means exhaustive. They are, rather, some of the resources most often identified in the literature as likely to drive an organisation's foreign entry strategy. These resources are discussed in greater depth in the next sections, along with the reasoning behind the consideration of these resources, as opposed to others.

\subsection{RESOURCES AND INTERNATIONALISATION: BUT WHAT TYPES OF RESOURCES?}

As discussed in the previous chapter, a resource becomes a source of sustainable competitive advantage when it meets four conditions: value, rareness, inimitability and non-substitutability. According to this reasoning, not all resources will be sources of advantage for an organisation and assist in the internationalisation process. The difficulty in ascertaining which resources might be such a source of competitive advantage stems from the multitude of resources that have benefited from empirical support in both the international business and strategic management literature. Newbert (2007), for example, in a review of empirical articles that have employed the RBV as a theoretical underpinning, finds a great deal of variation in how resources are operationalised (see Appendix I). Newbert finds that only two of the 26 resources 
examined (human capital and knowledge), one of the 32 capabilities (information technology) and none of the core competencies are included in more than $5 \%$ of the total articles reviewed. This variation in operationalising resources stems from a particular resource not necessarily having the same rent-generating capacity for one organisation compared to another. In other words, what constitutes a resource that provides a source of sustained competitive advantage would be organisation and/or industry/context specific.

To bring some sort of order to the discussion on resources as a source of competitive advantage and to minimise the variation in the way resources are operationalised, a number of scholars have sought to divide resources into different categories. Barney (1991), for example, categorises resources into physical capital, human capital and organisational capital (e.g. management systems). Grant (1991) classifies resources into six categories: financial, physical, human, technological, reputation and organisational. Miller and Shamsie (1996) classify resources into property-based (e.g. proprietary technology) and knowledge-based.

Of those three typologies, Grant (1991) has the added advantage of not being too narrow in the attempt to categorise the complex range of resources. While there is a real need to bring some structure to the debate, it is also acknowledged in this study that typologies might not be value added if they are too constrained in their categorisation. Therefore, building on Grant's (1991) typology of resources, this study focuses on financial, human, reputation and organisational resources as key rent-generating assets and capabilities. Physical and technological resources are de-emphasised in this study, since they are not of particular importance to education service providers in generating a source of sustainable competitive advantage offshore. Education service providers indeed do not generally have specialised physical resources, which influence their internationalisation strategies, nor do they generally have specialised technological resources. The only physical and technological resources that education service providers might have (e.g. specialised laboratory equipment such as a synchrotron), and which may be reputation-enhancing, tend to be location bound in the home country of 
the provider and do not currently assist greatly in offshore education developments. Rather, the majority of offshore education developments currently taking place tend to be in disciplines that do not require specialised physical and technological resources for teaching. These educational programmes tend to be in areas such as Business and Arts or those that would require a minimum investment in physical and technological resources (e.g. basic laboratory equipment, such as microscopes, for teaching the fundamentals of science) (OECD, 2004). The latter resources would not be sources of competitive advantage, as they would not meet the four conditions of value, rareness, inimitability and non-substitutability. Furthermore, educational programmes that are inclined to be developed around highly specialised physical and technological resources tend to be at the postgraduate level, especially in the scientific and engineering fields. These highly specialised programmes are currently not on offer offshore (OECD, 2004).

\subsection{ENTRY MODE CHOICE IN EDUCATION SERVICES: A CONCEPTUAL FRAMEWORK}

Figure 4.1 is a diagrammatic representation of the resource-based approach to entry mode decision-making developed for this study. The individual elements of the proposed framework in figure 4.1 are overviewed in the following sections. However, before doing so, it is important to address two important assumptions of the resourcebased approach to entry mode selection.

First, under the RBV, a higher level of ownership is presumed to be the preferred entry mode until proven otherwise, since it provides the organisation the most control in protecting its rent-generating assets and capabilities (Stopford and Wells, 1972; Ekeledo and Sivakumar, 2004). This assumption is consistent with empirical studies that have found that US firms tend to prefer sole ownership as a mode of entry (Anderson and Gatignon, 1986; Erramilli and Rao, 1993). There is also documented evidence in the literature that Japanese firms generally view alliance as a second-best alternative to sole ownership (Hamel, 1991). This fundamental assumption of the resource-based view differs to that of the transaction cost approach, which typically views shared-control modes as the default mode of entry (Anderson and Gatignon, 1986). Second, implicit in the first assumption, is the condition that the target foreign market has enough current 
or potential demand to make a higher level of ownership a viable entry mode. Otherwise, a higher level of ownership would not merit consideration, since it is more resource intensive (Anderson and Gatignon, 1986). These two assumptions provide the foundation for the analytical framework used in this study.

Building on these two assumptions, the basic premise of the conceptual framework presented in figure 4.1, therefore, assumes that an education service provider that possesses resources that are potential sources of competitive advantage in a target market would favour a mode of entry that allows control and protection over such resources. This premise, however, is moderated by aspects such as institutional distance between the home and host country, which as argued above, also has an important role to play in the entry mode decision process.

Figure 4.1: A Resource-based Framework for Entry Mode Choice in Education Services

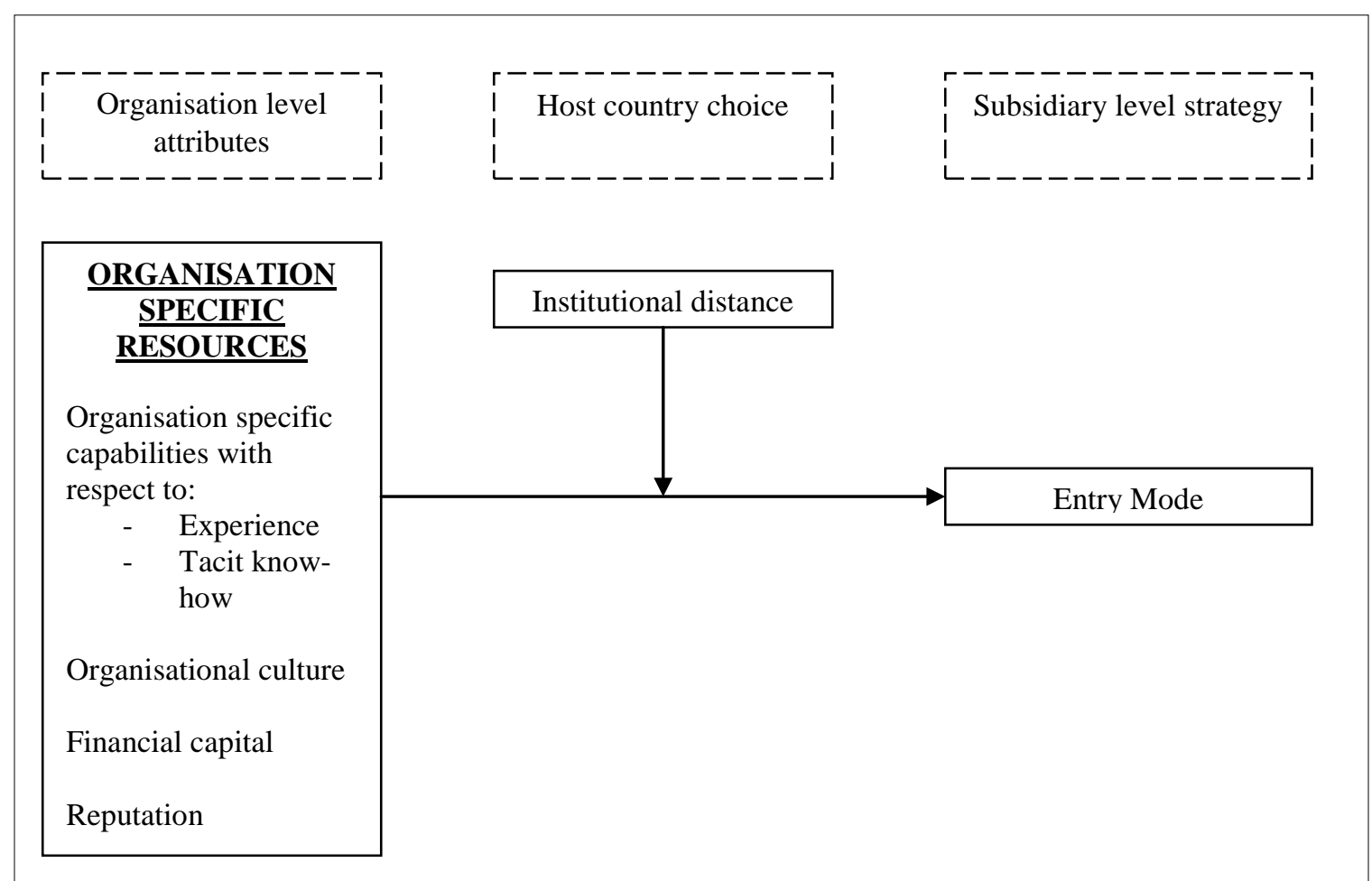

Note: As discussed in Section 5.3.1 of Chapter 5, the entry mode dependant variable in this study is measured as the focal university's equity stake for a single overseas investment. This approach is similar to previous studies (e.g. Rajan and Pangarkar, 2000; Rose and Ito, 2004). 
It should be noted that institutional distance can also be viewed as a mediating variable as opposed to a moderating variable. While the latter may reduce or enhance the direction of the relationship between a predictor variable and a dependent variable, or it may even change the direction of the relationship between the two variables from positive to negative or vice versa, a mediating variable represents an intervening variable through which an independent variable is able to influence a dependent variable. In the framework presented in figure 4.1, an argument for institutional distance as a mediating variable could run along those lines: first, offshore investment must be influenced by organisation-level attributes. The choice of host country, in terms of institutional distance, should be matched to organisation level attributes in such a way that allows the legitimacy of the foreign subsidiary to be ensured in the host country and the transfer of competitive advantage to be carried out successfully. Second, once a host country has been chosen, the choice of subsidiary level strategies (e.g. modes of entry choice) must be matched to the condition of that particular host country, in terms of institutional distance. This would help mitigate the impact caused by large institutional distance or enhance the competitive advantage of the organisation resulting from a small institutional distance.

In the education context however, it would appear that education service providers often do not have the luxury of selecting target markets with strong consideration of their institutional distance. A study conducted by the OECD (2004) reveals that most offshore investment undertaken by education providers is undertaken primarily for market seeking motives. This implies that education providers invest, first and foremost, in a target market if the demand conditions are right and then implement governance mechanisms to address the influence of institutional distance rather than basing their selection of target markets on institutional distance in the first place. As also discussed in Section 3.3, education service providers also often operate in regulated institutional environments, set in place by authorities in order to ensure the delivery of quality education. These institutional environments are, to a large extent, beyond the control of the education service provider and can be viewed as having a moderating role on the influence of resources on entry modes, by adding or reducing the transaction costs 
associated with the entry mode process. Institutional distance is, therefore, viewed as a moderating variable rather than a mediating one in this study.

\subsubsection{Elements of Organisation-Specific Resources}

Five elements of organisation-specific resources highlighted by previous studies are considered in the present study. These include organisation-specific capabilities with respect to experience and tacit know-how (e.g. organisation-specific capabilities related to human resources), organisational culture (as an element for organisational resources), financial capital and good reputation (Wernerfelt, 1984, 1989; Anderson and Gatignon, 1986; Aaker, 1989; Collis, 1991; Grant, 1991; Hall, 1992; Amit and Schoemaker, 1993). The focus on these specific resources is grounded in the literature and is supported, based on the informal discussions with 40 senior university representatives ${ }^{27}$. While it is acknowledged that these resources are by no means exhaustive, they are often identified as the basis of competitive advantage (Wernerfelt, 1984) and provide a reasonable basis for analysing entry mode choices from a resource-based approach in the context of education services. The literature behind the use of these specific resources and their influence on entry modes is highlighted below.

\section{A. Organisation-Specific Capabilities With Respect to Experience and Tacit Know- how}

Organisation-specific capabilities refer to the organisation's ability to use its resources for growth and profitability (Amit and Shoemaker, 1993). Capabilities can be thought of as the effectiveness with which a resource is used and converted to outputs (Dutta, Narasimham and Rajiv, 2005). Capabilities are, therefore, an 'intermediate transformation ability’ between resources (i.e. inputs) and outputs (i.e. an organisation’s objectives such as producing innovative technologies, introducing new products, reducing costs or, more specifically applied to this study, internationalisation). This intermediate transformative ability of capabilities has been operationalised in numerous ways (see Appendix I), but the one point of similarity across these operationalisations is that capabilities are based on developing, and exchanging information through the

\footnotetext{
${ }^{27}$ Informal discussion held prior to my Ph.D. enrolment and which provided the foundation for this research topic - see Appendix H
} 
organisation's human capital (Cavusgil, Seggie and Talay, 2007). This includes the cognitive processes by which tangible resources are translated into action (Foil, 1991). Human capital has a particularly large part to play in this conversion process, with organisation-specific capabilities often being embodied in employees' knowledge and experience (Day and Wensley, 1988; Hall, 1992).

In an international business setting, employees' international knowledge and experience is an invaluable source of competitive advantage. In the transnational ${ }^{28}$ education context for example, a major obstacle for an education service provider to be involved offshore is the lack of expertise needed to confidently undertake an international business development process (Education New Zealand, 2006). Many scholars have noted that organisations (and by implication, managers and employees) with little international exposure tend to be risk averse and underestimate potential returns (Johanson and Vahlne, 1977; Davidson, 1982; Caves and Mehra, 1986; Erramilli, 1991; Gatignon and Anderson, 1988; Agarwal and Ramaswami, 1992). In contrast, experienced organisations tend to be more aggressive in their market entry (Gatignon and Anderson, 1988; Gomes-Casseres, 1989; Erramilli, 1991). This is because for less experienced organisations with limited resources and capabilities, the administrative and coordination costs associated with internationalisation are high (Contractor, 1990) while with greater international experience, organisations develop the confidence and competence of managing the uncertainties and costs of operating offshore (Davidson, 1982).

Organisations with a greater stock of international knowledge and experience will pursue an extensive level of internationalisation for several reasons. First, prior experience can lead to the identification of opportunities (Shane, 2000; Shepherd and DeTienne, 2005) since managers are more alert to opportunities in those areas in which they have experience (Aldichvili, Cardozo and Ray, 2003). Second, managers with international experience may have international social networks that can compensate for

\footnotetext{
${ }^{28}$ As previously discussed in Chapter 1, the term 'transnational' employed in this study is not to be confused with Bartlett and Goshal's use of the term (see Section 1.2)
} 
an organisation's lack of established networks (McDougall, Shane and Oviatt, 1994; Oviatt and McDougall, 1994, 1995; Coviello and Munro, 1997) and, thereby, help the organisation to overcome international risks (Shrader, Oviatt and McDougall, 2000). Third, organisations with internationally experienced managers have been suggested to internationalise earlier in their life cycle (Reuber and Fischer, 1997). This has been attributed to an organisation's 'learning advantage of newness' in the case of international new ventures, which are argued to be able to better learn and adapt to changes in the environment than more mature organisations (Autio, Sapienza and Almeida, 2000). In addition, the organisational routines of a new venture with an internationally experienced management team will tend to have integrated internationalisation aspects from inception (Oviatt and McDougall, 1995), possibly resulting in faster international growth (Autio et al, 2000) and a higher level of achieved internationalisation (Reuber and Fischer, 1997). Therefore, when managers possess prior experience that is particularly relevant to foreign market expansion, it may allow organisations to accelerate their internationalisation, leading to greater entry mode commitments.

However, the relationship between experience and the level of control in terms of mode of entry is not always linear. Kogut and Singh (1988), for example find that experience does not explain why US organisations might prefer joint ventures over wholly-owned subsidiaries when investing offshore. Similarly, Erramilli (1991) finds that service organisations often favour sole ownership only at low and high levels of international experience and reason that this U-shaped phenomenon is more likely to apply to nonseparable services.

Gomes-Casseres (1989) suggests that two types of experience are relevant to the sole ownership issue; namely geographic and industry experience and that the contradictory finding about the relationship of experience on entry mode choice might be linked with the type of experience tested. Geographic experience refers to the organisation's familiarity with the host target country and/or region in which the target host country is located. Industry experience, on the other hand, is more a function of an organisation's 
age in the industry and the extent of its international business experience. GomesCasseres (1989) among others suggests that local organisations are likely to have more geographic experience than the multinational enterprises (MNEs) since they are likely to be more familiar with the local norms, values and customs of their home markets. Local organisations are also more likely to have access to economic elites and business groups that may provide them with a network of connections that MNEs would find hard to access (Gomes-Casseres, 1989). MNEs, on the other hand, may have an edge over local organisations in terms of industry experience. This advantage of the multinational organisation over local organisations can be linked to the ecological theory of organisations that views larger and older organisations as having more slack resources (often as a result of economies of scale, scope and learning) and well developed skills and routines that allow them to better withstand competitive pressures (Singh, House and Tucker, 1986). By this line of reasoning, it can be argued that MNEs poor in geographic experience are unlikely to favour a higher level of ownership to enter a market since they are likely to encounter stiff competition from the local organisations. It needs to be acknowledged, however, that MNEs might want to form joint ventures as opposed to using low-control non-equity arrangements in entering a host country in order to learn from the local partners and overcome their geographical inexperience (Hamel, 1991). However, it is reasoned that this geographical inexperience will limit the level of investment into these joint venture partnerships to the lower end of the scale of ownership. Conversely, MNEs rich in industry experience ${ }^{29}$ may choose higher levels of ownership. Put differently, the more an organisation's resources include both geographical and industrial experience, the more it is likely to favour a higher level of ownership as a mode of entry since this allows it to best exploit its experience in the foreign market (Tersptra and Sarathy, 1994; Douglas and Craig, 1995). Hence, these arguments lead to the first hypotheses of this study:

\section{Hypothesis 1a: The stronger the geographical experience of an education service provider, the more likely it will favour a higher level of ownership ${ }^{30}$.}

\footnotetext{
${ }^{29}$ In the context of this study, industry experience implies having previously been involved in exporting education and engaged in developing offshore education programmes.

${ }^{30}$ As discussed in Chapter 1, since universities do not use stocks to establish foreign operations, for precision sake, equity (the dependant variable) is used as a proxy for ownership in this study.
} 
Hypothesis 1b: The stronger the industrial experience of an education service provider, the more likely it will favour a higher level of ownership.

A related aspect of international experience, which is examined in this study, is transfer experience, defined as the prior successful experience an organisation has with a particular entry mode (Davidson and McFetridge, 1985). It is theorised that prior expertise with a particular governance form may encourage an organisation to use more resource intensive entry modes in subsequent investments because familiarity lowers the effective costs of further market entries. This follows the argument advanced by the Uppsala models of internationalisation (see Section 3.2.1), whereby an organisation increases its resource commitments with respect to foreign market entry as more knowledge and experience are acquired. While the focus of such knowledge and experience has traditionally been with respect to a particular foreign market, a similar argument can be extended to knowledge and experience with respect to a particular governance form. The internationalisation process is not strictly an economic decision but also a social, psychological and emotional phenomenon (Tallman and Shenkar, 1994). Tallman (1992) argues that managers, in developing entry strategies and structures, are subject to a variety of subjective concerns related to their inherent bounded rationality (Simon, 1945). This subjectivity leads to managers developing routine ways of doing things, such as choosing an entry mode (Nelson and Winter, 1982). Such routine responses, whether explicit or not, reduce the uncertainty under which managers operate, decreasing the perceived transaction costs associated with the activity (Beamish, 1988). Only after they are experienced in the use of a routinised entry mode might managers be comfortable in undertaking investments through higher resource commitments, in line with the incremental levels of commitment highlighted by the Uppsala School of internationalisation. However, routines can also result in a "locked-in" situation whereby managers might not adopt a more resource intensive entry mode, given that their accustomed level sets a benchmark of familiarity, effectively discouraging higher levels of resource commitments in subsequent foreign market entries (i.e. favouring investment of less or equal value to the routinised entry mode). Davidson and McFetridge (1985) find support for the mechanisms of past offshore investments increasing the probability of subsequent investments through 
similar mechanisms. In the education services context, this "locked-in" situation seems particularly plausible. The fact that the bulk of offshore investment takes place through non-equity arrangements (see Chapter 2) could be partly explained by the idea that education service providers might have developed routines for managing non-equity arrangements, and by default of those routines, choose subsequent foreign entries of, at most, the same type. Thus, it is hypothesised that:

\section{Hypothesis 1c: The more extensive the transfer experience of an education service provider with a particular mode of entry, the less likely it will favour a higher level of ownership.}

Experience also adds know-how to the organisation's capabilities as the more involved one is in a particular activity, the more knowledgeable one becomes about that activity. The critical role of knowledge in generating organisational advantage has increasingly gained prominence in the literature and the knowledge-based perspective, which views knowledge as the most strategically important of the organisation's resources (Grant, 1996) is essentially an outgrowth of the RBV. Tacit knowledge, in particular, has received attention in the entry mode literature (e.g. Kim \& Hwang, 1992; Madhok, 1998; Luo, 2001). Tacit know-how, the extent to which an organisation's capabilities are difficult to be articulated (Grant 1991), can be lodged not only in an organisation's human capital but also in its informal organisational routines (Nelson and Winter, 1982; Hill et al, 1990). Tacit know-how by definition is difficult to articulate, which adds to the difficulty of duplicating the organisation's routines and human capital in a foreign location (Teece 1981, 1988). Furthermore, since it is difficult to articulate tacit knowhow, it is by definition difficult to value tacit know-how. This is because to know what a know-how is worth, a prospective buyer would want the know-how disclosed (Anderson and Gatignon, 1986). Even if the know-how could be articulated, a problem arises in terms of the transferability of knowledge since a prospective buyer automatically acquires a know-how once it is disclosed before it is acquired. The difficulty of transferring tacit know-how can thus lead an organisation to use a high control mode of entry when investing offshore (Hill et al, 1990; Kogut and Zander, 1993). Kogut and Zander (1993), for example, found a positive relationship between 
tacit know-how and the adoption of sole ownership as a mode of operation. However, problems inherent in the transfer of tacit know-how can also be addressed through a low level mode of operation, such as exporting. Because the export of education services does not lie within the scope of this study, this argument is not addressed further in this section. Rather, it is hypothesised that:

Hypothesis 2: $\quad$ The less an education service provider is able to articulate its know-how specific to offshore expansion, the more likely it will favour a higher level of ownership.

\section{B. Organisational Culture}

Organisational culture consists of the values and beliefs that are common to members of an organisation. It provides them with norms of conduct (Davis, 1984; Kilman, Saxton and Serpa, 1986; Deshpande and Webster, 1989; Hofstede 1991). Culture embodies the beliefs, knowledge, attitudes and customs of an organisation and influences values and habits of its members. It can set members of an organisation apart from those of other organisations (Hall, 1992; Tallman and Shenkar, 1994). Barney (1986) adds that organisational culture can have a positive impact on organisational effectiveness and be a source of competitive advantage, if the culture is widely shared, is a positive attribute, and is deeply internalized by members of an organisation. Grant (1991) notes that organisational culture sustains an organisation's competitive advantage through the maintenance of organisation-specific capabilities. Organisational culture, especially the adaptive and entrepreneurial type, tends to encourage growth/change and influence an organisation's competitive environment to its own advantage (Bartol and Martin, 1998). In other words, an entrepreneurial culture pushes an organisation to innovate; successful organisations are often associated with a strongly entrepreneurial culture (Bartol and Martin, 1998).

An entrepreneurial culture has also been associated with a greater likelihood for internationalisation. In the education context, for example, offshore education is by and large an activity which depends on the entrepreneurial orientation of the leadership of the institution in question (Bannerman, Spiller, Yetton and Davis, 2005). Similarly, in 
the small and medium enterprise (SME) internationalisation literature, Anderson and Wictor (2003) suggest that entrepreneurial behaviour is arguably the most important internationalisation catalyst. McDougall et al (1994) indicate that entrepreneurs who have unique competencies in detecting international opportunities are more likely to internationalise. They equate these competencies to having an international entrepreneurial orientation. Similarly, Oviatt and McDougall (1995) suggest that a global vision is probably the most important characteristic of Born-Global entrepreneurs. An international entrepreneurial orientation reflects an organisation's overall innovativeness and proactiveness in pursuit of international markets (Miller and Friesen, 1984, Covin and Slevin, 1989; Lumpkin and Dess, 1996; Dess, Lumpkin and Covin, 1997). Knight and Cavusgil (2004) more recently also found that newly internationalised organisations tend to possess a distinctive entrepreneurial orientation that when combined with other resources and capabilities, allows them to see and exploit international business opportunities.

Numella’s (2004) 'global mindset' concept is closely associated with an international entrepreneurial orientation and includes both attitudinal and behavioural elements. The attitudinal element refers to the way managers make sense of the world; it refers to managers' openness to and awareness of the diversity of doing business internationally and the ability to handle it (Gupta and Govindarajan, 2002). This attitude may lead to the proactive managerial behaviour, in terms of taking risks in operating offshore. In this context, the 'global mindset' and 'international entrepreneurial orientation' concepts are quite similar.

In the education services context, involvement in offshore education can often arise from an opportunity presenting itself, with no proactive initiative on the part of the education institution. This is not to deny the existence of institutions with a formal policy to offshore education as part of a larger internationalisation strategy. For example, offshore developments may be sought by the international marketing manager whilst on recruitment trips, through extensions to already held articulation agreements or by seeking new business opportunities. However, new offshore activities can also 
often develop through personal contacts, such as academic staff members who have colleagues overseas with whom the new opportunity arises. It could also be the case for an institution to be approached by a provider offshore, seeking a business relationship (Education New Zealand, 2006).

Education service providers that have an interest in internationalising and are more entrepreneurial, may be more likely to take advantage of these opportunities as they arise. This might explain why offshore education tends to be more common among the so called 'new universities', as opposed to more established ones. New universities tend to be more entrepreneurial in their orientation than more established universities (Marginson and Considine, 2000; Prince, 2004; Harman, 2005). It can thus be argued that these education service providers with organisational cultures that are more entrepreneurial, are likely to be more innovative and accepting of risks in developing offshore education developments. Hence, it is hypothesised that:

\section{Hypothesis 3: The more entrepreneurial the organisational culture of an education service provider is, the more likely it will favour a higher level of ownership.}

With the current growth in offshore education (see Chapter 2), even the less entrepreneurial institutions are seeing the need to understand the dynamics of this phenomenon. Across a number of western English speaking countries, the pool of domestic students is either decreasing or increasing at a diminishing rate as populations age (OECD, 2006), so that a number of universities are starting to be concerned about their future funding streams with reliance solely on the domestic market. The offshore education market is, therefore, looking increasingly attractive. Slow domestic growth has been noted as an important factor in the internationalisation process of organisations (e.g. Coviello and Munro, 1995; McNaughton, 2003). Consequently, even the very established universities have recently announced plans to start looking into the offshore education market. The US Ivy League institutions for instance (e.g. Yale University in India, Cornell University in the Middle East), are examples of institutions that did not previously participate extensively in the offshore education phenomenon, but which are 
now increasingly aware of its importance (Selingo, 2007). These institutions, as latecomers into the offshore education market, may be at a competitive disadvantage with respect to operational experience, compared to institutions that have been involved in the offshore education market for a longer time. Therefore, these new-comers to the market, might decide to partner with local institutions in the host target market, rather than operate through non-equity arrangements (e.g. twinning arrangements) and/or wholly-owned subsidiaries, in order to learn the ropes of the offshore education business.

Learning is integral to the strategic management of an organisation. While, traditionally, strategy has been regarded as a match between an organisation and its external environment, it has more recently been acknowledged in the literature that the organisation-environment fit is an insufficient predictor of organisational success (Rumelt, Schendel and Teece, 1991; Madhok, 1997). Through the RBV, the influence of resources on competitive positioning has received renewed attention in the strategic management literature. In the RBV, the role of learning gains prominence, particularly if the resources of interest are knowledge and skills that cannot easily be purchased in the open market.

Several scholars have suggested that strategic alliances, particularly joint ventures, represent a potential mechanism for organisational learning (Westney, 1988; Kogut, 1988b; Powell, 1988; Hamel, 1991; Pucik, 1991). By internalising the skills of joint venture partners, organisations may gain access to the embedded knowledge of other organisations and, therefore, access to new skills, capabilities and resources. Huber (1991) refers to this process as 'grafting'.

Grafting, however, is not always successful as it depends on the organisation's learning capacity. This learning capacity in turn depends on several factors. First, the organisation's desire to learn is important. Second, is the perceived need to learn. Organisations in an industry faced with high competitive threats/intensity such as offshore education may have a greater need for learning, compared to organisations in 
more stable industries. A third factor is the organisation's attitude to learning. An organisation may take active steps to transfer learning but if the organisation's management does not appreciate the strategic value of learning, then skills may not be internalised (Inkpen, 1992).

These three factors have been captured in the literature in terms of the learning intent of the organisation. Hamel (1991: 89-90) defines learning intent as “an organisation's propensity to view collaboration as an opportunity to learn”. Thus, learning intent leads to collaboration being used as a vehicle to acquire knowledge where the requisite knowledge is lacking (Harrigan, 1985c; Hamel, 1991). Pucik (1988) adds that once an organisation decides to collaborate, the form of that collaboration would be determined by whether the organisation is interested in merely having access to resources or whether it wants to gain a greater understanding of the partners' routines, so that these can be internalised and leveraged for its own use. In the latter case, joint ventures have been argued to be a superior mode of entry, since they allow for a deeper exposure to the partner's expertise (Vernon and Wells, 1986). This is not to state that joint ventures cannot be formed to access resources, but that where an organisation has a conscious intention to learn, it may prefer joint ventures over other forms of collaboration (Pisano, 1988; Osbourn and Baughn, 1990). By this line of reasoning, it is hypothesised that education service providers that are intent on developing their capabilities in offshore education provision are likely to prefer joint ventures, particularly joint ventures with majority equity.

\section{Hypothesis 4: $\quad$ The stronger the learning intent of an education service provider, the more likely it will prefer joint ventures with majority equity.}

\section{Financial capital}

An organisation's financial capital/resources play a huge role in terms of what an organisation can and cannot do (Grant, 1991). An organisation with a large financial endowment usually has more resources at hand to pursue aggressive international expansion strategies (Buckley and Pearce, 1979; Kobrin, 1991). A small, less financially endowed organisation, on the other hand, may be restricted in its 
international expansion to low control modes of entry if the organisation cannot absorb the high costs or risks involved in high control modes of entry (Hennart, 1991; Agarwal and Ramaswami, 1992; Hennart and Park, 1993; Erramilli and Rao, 1993; Madhok, 1997). As a result, financial capital, often proxied by organisation size, is viewed as an important determinant of entry mode choice, and a positive relationship between organisation size and the likelihood of $100 \%$ ownership of foreign affiliates has been empirically supported in the literature (Buckley and Cason, 1976; Kimura, 1989; Chung and Enderwick, 2001).

In the education services context, it is similarly hypothesised that financial capital will be positively linked to the level of control of a mode of entry. Capital is a major obstacle for an education service provider looking to be involved offshore (Education New Zealand, 2006). Considerable costs are involved in offshore education developments including both opportunity costs and upfront expenditure. These include staff time, costs of travel and accommodation, due diligence costs, consultancy fees, course development costs, staff recruitment and others. For the education providers with financial constraints, the funds required to cover these costs might be unavailable or form a large proportion of the institution's cash reserves. Making a commitment to offshore education developments before having any guarantee of a return on investment is, therefore, argued to be more difficult for these not so financially rich institutions. This is even more difficult in high control modes of entry, which are resource intensive. Consequently, this leads us to the following hypothesis.

\section{Hypothesis 5: $\quad$ The larger the financial resources of an education service provider, the more likely it will favour a higher level of ownership.}

\section{Reputation}

From a resource-based view perspective, reputation, the "perceptual representation of a company's past actions and future prospects that describes the organisation's overall appeal to all of its key constituents when compared with other leading rivals" (Fombrun, 1996: 72), is a source of competitive advantage in that it acts as a sustainable basis for product differentiation (Grant, 1991; Hall, 1992; Dollinger, Golden and 
Saxton, 1997) and can, therefore, be a rent-generating asset (Barney, 1991). As a source of differentiation, reputation is often associated with product quality, management effectiveness or some other factor that appeals to the organisations' various constituencies (Tsui, 1984). This, in turn, allows an organisation to create competitive barriers (Deephouse, 2000).

Reputation is acknowledged as a source of competitive advantage because it is difficult to create, imitate or substitute (Barney, 1991). It is, for example, evident that reputation is difficult to create (i.e. rare) due to the variation of reputational capital among organisations (Fombrun and VanRiel, 2004). It is also difficult to imitate reputation, since it is a perceptual representation by key constituents and is often socially constructed (Barney, 1991).

Reputation is particularly important for service organisations because of the experience and the credence attributes that accompany service delivery (Aaker, 1989). The former relate to the inability of the consumer to accurately evaluate the quality of the service offering prior to the act of consumption, while the latter refers to situations where the consumer is unable to evaluate the service delivery even after it is consumed (Parasuraman et al, 1985; Bateson 1992). For example, the services of a doctor may not be evaluated immediately after the service offered. Consequently, prospective consumers often rely on reputation as an indicator of quality, especially in the case of non-separable services. This argument is reinforced by Weigelt and Camerer (1988) who suggest that reputation is especially important in situation of information asymmetry, where all players are not equally informed of the parameters involved. For example, when buying education services, a qualification from a prestigious university is deemed better than a qualification from a less reputable institution, even though both establishments could be using the same text books and the classes taught by professors with similar educational backgrounds. Because the students usually lack the information to assess the educational delivery of both institutions, they are likely to use prestige as a proxy for the quality of education service delivery. This is, in spite of the fact, that in real terms, both institutions might be delivering an equally good service. 
In an international business context, reputation can influence internationalisation in a number of ways. First, organisations can develop an international market base as a result of their reputation, whereby consumers in foreign countries demand their products and services (Goldberg and Hartwick, 1990). Aldrich and Fiol (1994) further suggest that reputation can help overcome legitimacy issues when entering a new market, making the organisation appear more credible to potential consumers in that market. Fombrun and Van Riel (2004) also argue that reputation influences the ability of the organisation to recruit and keep employees. This ability of attracting and maintaining local employees is critical to reduce the transaction costs associated with international business activities, since a loyal employee is less likely to act opportunistically (Rugman, 1981; Dollinger, Golden and Saxton, 1997; Brouthers, Brouthers and Werner, 2003). Reputation is also important in attracting investors (Frombrun and Van Riel, 2004). As indicated above, capital is a major obstacle to offshore education developments and those education service providers with good reputation might be in better positions to access enough capital to allow them to internationalise via resource intensive, high control modes of entry. Cornell University, for example, was invited by the United Arab Emirates authorities to open a campus in Qatar and was offered large financial incentives to offset their costs (Blumenstyk, 2006). Reputation, can also allow organisations the ability to charge a premium for their products and services, which in turn allows the organisation to more quickly offset its investments costs for going offshore (Tsui, 1984; Deephouse, 2000). Reputation also influences the ability of the organisation to develop exchange relationships, such as alliances and/or joint ventures (Larson, 1992) since the costs associated with assessing an organisation as a potential exchange partner are reduced when that organisation enjoys a good reputation. Through these exchange relationships, reputable organisations can gain access to resources that are crucial in their internationalisation process but which are beyond their reach (Fombrun and Van Riel, 2004). Access to these resources provides firms with a better leverage to achieve higher levels of growth and performance internationally (Kotah, Rindova and Rothaermel, 2001; Lu and Beamish, 2001). 
However, not all reputable organisations will seek to develop exchange relationships. Indeed, while the ability of developing these relationships is assisted by reputation, reputable organisations do not always seek, require or want to be associated with exchange partners (Fombrun, 1996). Reputable organisations may prefer to protect their reputation rather than having to share it with others. In the education services context, for example, an Ivy League university might not want to collaborate with a local partner when going offshore, unless that partner is of a similar reputation to it or at least has one of the best reputations in the target host country.

The need to protect the reputation of an organisation is likely to compel that organisation to adopt a mode of operation that allows the greatest control and protection of its public image. Modes of entry with a higher level of ownership fall into that category $^{31}$. It is therefore hypothesised in this study that:

\section{Hypothesis 6: The better an education service provider's reputation, the more likely it will favour a higher level of ownership.}

\subsubsection{Institutional Distance}

Kostova (1999) and Kostova and Zaheer (1999) highlight how psychic and cultural distance, two concepts that have been examined in the scholarly literature (e.g. Johanson and Vahlne, 1977; Hofstede, 1991), are too narrow to capture all the issues of concern in examining distance in an international business context (Shenkar, 2001). Kostova (1999) and Kostova and Zaheer (1999) propose a new measure of distance in terms of institutional distance. This key concept analyses the differences between the host and home countries of the multinational organisation in terms of Scott's (1995) three pillars of institutional environment: regulative, normative and cognitive domains. The regulative pillar refers to rule-setting, monitoring and sanctioning activities present

\footnotetext{
${ }^{31}$ Exporting also falls into that category but because the latter mode of entry falls outside the scope of this study, it will not be addressed further in this section. In addition, it also needs to be acknowledged that non-equity arrangements such as franchising are also often used among service organisations to safeguard reputation (Palmer and Cole, 1995; Lovelock 1996). However, this mode of operation is more applicable to service operations that can somewhat be standardised in terms of their product/service delivery (e.g. fast food service organisations) but is more difficult to achieve in an education services context where the consumer-supplier interaction plays a more important role in the service delivery.
} 
in the institutional environment. In other words, it refers to explicit regulatory processes under which the organisation has to operate. The normative pillar refers to norms and values that direct and govern behaviour in a particular environment (e.g. culture). The cognitive pillar refers to the cognitive rules that influence behaviour. For simplification purposes, the normative and cognitive aspects of institutions can be grouped into one concept, since they are quite similar to each other (Scott, 1995). Institutional distance is, therefore, the difference/similarity between the regulative and normative/cognitive (used interchangeably henceforth) aspects of institutions of any two countries (Kostova, 1999). These distinct constructs of institutional distance all exert different pressures and expectations on the organisation (D'Aunno, Sutton and Price, 1991; Oliver, 1991), given that they are based on different types of motivations; coercive, mimetic and normative (Scott, 1995). These different aspects of institutional distance also exert dissimilar pressures on the organisation because they have diverse levels of tacitness in their enforcement (Scott, 1995). Regulatory barriers, for example, may prohibit certain elements of organisational practices (e.g. bonus-based remuneration) and normative barriers may moderate the appreciation of standing out among colleagues by overperforming (Ionascu, Meyer and Erstin, 2004). It is, therefore, postulated that regulatory and normative aspects of institutional distance all have different bearings on the multinational organisation (Kostova, 1999).

In an institutional theory context, the 'liability of foreignness' (Zaheer, 1995) is augmented when the linkages between the parent organisation and the local subsidiary are inhibited by their embeddedness in different national contexts. Institutional distance, for instance, is assumed to moderate the application of the MNE's operational practices within the socially constructed systems of rules, norms and cognitive frames of the local environment of host country (Kostova and Zaheer, 1999). Institutional distance can also either facilitate or impede the transfer of organisational practices from the parent organisation to the foreign subsidiary (Kostova and Roth, 2002). Institutional theory postulates that, the larger the institutional distance, the more difficult it is for the multinational organisation to establish legitimacy and practise co-ordination and integration, notably the transfer of knowledge and practices (Kostova and Zaheer, 
1999). The issue of establishing legitimacy is particularly important in the case of service organisations, because the perceived quality of service delivery depends on who is delivering it, and when, where and how it is being provided (Lowendahl, 1997; Lowendahl and Revang, 1998). This is because services are largely consumed at the time it is produced and cannot be stored.

While operating in different institutional environments presents many challenges, it also presents opportunities for institutional arbitrage by exploiting location specific advantages (Delios and Beamish, 2001). It has, however, been established in the literature that the scope of this arbitrage narrows and its marginal benefits decline in increasingly different institutional environments (Delios and Beamish, 2001). In distant host countries, the MNE is more likely to be at a competitive disadvantage, with additional costs of doing business. These additional transaction costs are derived from unfamiliarity and relational hazards (Caves, 1971; Henisz and Williamson, 1999). The former arises from a lack of knowledge and experience in the host environment, while the latter occurs when relationships are managed at a distance and include problems of opportunistic behaviour that may arise as a result of a lack of trust in unknown partners. When institutional distance is low, these transaction costs of operating offshore are more likely to be marginal but they tend to increase when institutional distance rises (Xu and Shenkar, 2002). Linking this argument to an organisation's resources and capabilities, it is postulated in this study, that there is an inverted relationship between resources and levels of equity ownership. Specifically, when institutional distance is low, an organisation rich in resources should be able to outweigh the costs of doing business in a different institutional environment, such that they might even invest offshore via higher levels of equity ownership. However, as institutional distance and the associated level of unfamiliarity increases, the relationship between resources and the level of equity ownership becomes strongly negative since the transaction costs of operating in host countries with large institutional distance may eventually exceed the benefits. In such situations, MNEs may not sustain their operations, irrespective of their resource endowments. In some highly uncertain environments, organisations may even face the risks of appropriation by the host government (Delios and Henisz, 2003). 
Therefore, it is postulated that there will be a point at which the slope changes dramatically in the relationship between institutional distance and resources. Accordingly, it is expected that there will be an inverted relationship between regulative and normative distances and organisation specific resources, on the basis of Kostova (1999) who suggests that the regulatory and normative aspects of institutional distance are all expected to have different bearings on the multinational organisation. However, among those elements of resources examined in this study, transfer experience is expected to be unaffected by institutional distance. As indicated above, since previous transfer experiences are hypothesised to influence subsequent transfers, institutional distance is very unlikely to play a moderating influential role in the entry modal choice. Rather, as highlighted above, the decision making processes tend to be based on routines that are founded on previous transfer experiences (Tallman and Shenkar, 1994). Tallman (1992) argues that managers, in developing entry strategies and structures, are subject to a variety of subjective concerns related to their inherent bounded rationality (Simon, 1945). This subjectivity leads to managers developing routine ways of doing things, such as choosing an entry mode (Nelson and Winter, 1982). Building on this argument, it is suggested that managers will similarly adopt routine responses, irrespective of institutional distance. Therefore, only organisation specific capabilities with respect to geographic experience, industry experience, tacit know-how, organisational culture, financial resources and reputation are postulated to be moderated by institutional distance leading to the following hypotheses:

Hypothesis 7: The relationship between resources and the level of equity ownership of education service providers is not linear, such that the relationship between levels of equity ownership and resources is positive at low to medium levels of normative institutional distance but negative at higher levels.

Hypothesis 7a: The relationship between geographic experience as an element of organisation specific resources and the level of equity ownership of education service providers is not linear, such that the relationship between levels of equity ownership and geographic experience is positive at low to medium levels of normative institutional distance but negative at higher levels.

Hypothesis 7b: The relationship between industry experience as an element of organisation specific resources and the level of equity ownership of education service 
providers is not linear, such that the relationship between levels of equity ownership and industry experience is positive at low to medium levels of normative institutional distance but negative at higher levels.

Hypothesis 7c: The relationship between tacit know-how as an element of organisational specific resources and the level of equity ownership of education service providers is not linear, such that the relationship between levels of equity ownership and tacit know-how is positive at low to medium levels of normative institutional distance but negative at higher levels.

Hypothesis 7d: The relationship between an entrepreneurial culture as an element of organisation specific resources and the level of equity ownership of education service providers is not linear, such that the relationship between levels of equity ownership and entrepreneurial culture is positive at low to medium levels of normative institutional distance but negative at higher levels.

Hypothesis 7e: The relationship between financial resources as an element of organisation specific resources and the level of equity ownership of education service providers is not linear, such that the relationship between levels of equity ownership and financial resources is positive at low to medium levels of normative institutional distance but negative at higher levels.

Hypothesis 7f: The relationship between reputation as an element of organisation specific resources and the level of equity ownership of education service providers is not linear, such that the relationship between levels of equity ownership and reputation is positive at low to medium levels of normative institutional distance but negative at higher levels.

Hypothesis 8: The relationship between resources and the level of equity ownership of education service providers is not linear, such that the relationship between levels of equity ownership and resources is positive at low to medium levels of regulative institutional distance but negative at higher levels.

Hypothesis 8a: The relationship between geographic experience as an element of organisation specific resources and the level of equity ownership of education service providers is not linear, such that the relationship between levels of equity ownership and geographic experience is positive at low to medium levels of regulative institutional distance but negative at higher levels.

Hypothesis 8b: The relationship between industry experience as an element of organisation specific resources and the level of equity ownership of education service providers is not linear, such that the relationship between levels of equity ownership and industry experience is positive at low to medium levels of regulative institutional distance but negative at higher levels. 
Hypothesis 8c: The relationship between tacit know-how as an element of organisational specific resources and the level of equity ownership of education service providers is not linear, such that the relationship between levels of equity ownership and tacit know-how is positive at low to medium levels of regulative institutional distance but negative at higher levels.

Hypothesis 8d: The relationship between an entrepreneurial culture as an element of organisation specific resources and the level of equity ownership of education service providers is not linear, such that the relationship between levels of equity ownership and entrepreneurial culture is positive at low to medium levels of regulative institutional distance but negative at higher levels.

Hypothesis 8e: The relationship between financial resources as an element of organisation specific resources and the level of equity ownership of education service providers is not linear, such that the relationship between levels of equity ownership and financial resources is positive at low to medium levels of regulative institutional distance but negative at higher levels.

Hypothesis 8f: The relationship between reputation as an element of organisation specific resources and the level of equity ownership of education service providers is not linear, such that the relationship between levels of equity ownership and reputation is positive at low to medium levels of regulative institutional distance but negative at higher levels.

\subsection{CHAPTER SUMMARY}

This chapter describes the proposed research framework for this study, building on the RBV, organisational capability and institutional theories. The model components are discussed, leading to eight major hypotheses generated. In particular, five elements of resources and organisational capability are incorporated in the research model, namely, organisation specific capabilities with respect to experience and tacit know-how, organisational culture, financial capital and reputation. These resources are by no means exhaustive (see appendix I); rather they are some of the factors often identified in the literature as more likely to drive an organisation's foreign entry strategy. Besides a grounding in the literature, the focus on these specific elements of resources is also deductively derived based on informal discussions with senior university representatives (see Appendix $\mathrm{H}$ ). Table 4.1 provides a summary of the resources examined in this study and their hypothetical relationships with the levels of equity ownership in entry modal choice. The moderating influence of institutional distance on these hypothetical relationships is also highlighted. 
Table 4.1: Variables and their Expected Signs

Dependent variable: The level of equity ownership.
A. Direct relationships
H1a: Geographical experience
H1b: Industry experience
H1c: Transfer experience
H2: Tacit know-how
H3: Entrepreneurial culture
H4: Learning intent
H5: Financial resources
H6: Reputation

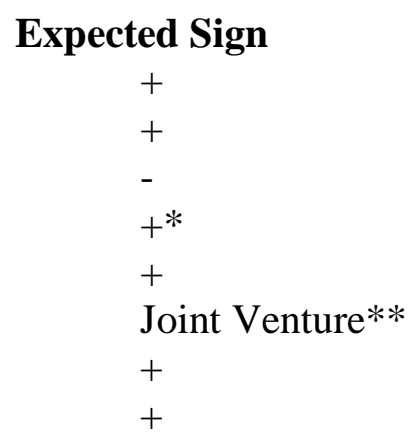

\section{B. Moderating relationships with respect to regulative institutional distance H7a: Normative institutional distance \& geographical experience Inverted H7b: Normative institutional distance \& industry experience Inverted H7c: Normative institutional distance \& tacit know-how Inverted H7d: Normative institutional distance \& entrepreneurial culture Inverted H7e: Normative institutional distance \& financial resources Inverted H7f: Normative institutional distance \& reputation Inverted}

\section{Moderating relationships with respect to normative institutional distance}

H8a: Regulative institutional distance \& geographical experience Inverted H8b: Regulative institutional distance \& industry experience Inverted H8c: Regulative institutional distance \& tacit know-how Inverted H8d: Regulative institutional distance \& entrepreneurial culture Inverted H8e: Regulative institutional distance \& financial resources $\quad$ Inverted H8f: Regulative institutional distance \& reputation Inverted

\footnotetext{
* A positive relationship is expected based on the way tacit know-how is measured in the survey instrument (see Chapter 5). Measured on a scale of 1-9, a higher number represents more difficulty/less ability to articulate know-how. Since the dependent variable is also measured with higher values representing more equity, a positive relationship is, therefore, expected.

** This relationship is only examined between JV majority ownership and non JV majority ownership (i.e. JV minority and JV 50/50 equity) only.
} 


\section{Chapter Five \\ CONSTRUCTS, MEASURES \& RESEARCH METHODOLOGY}

\subsection{INTRODUCTION}

This chapter highlights the research method used to examine the hypotheses advanced in the previous chapter. It sets the basis for outlining the proposed methods of validating, refining and testing the theoretical model advanced in this study. It describes the survey method and other procedures that are used to collect and analyse the data that constitute the basis of this study. In particular, the research design, sample selection procedure, data collection process, variables and their operational measures and statistical analysis techniques are described.

\subsection{RESEARCH DESIGN, SAMPLE SELECTION AND DATA COLLECTION PROCESS}

A multi-method design consisting of two stages was employed in this study. Using interviews with current universities operating offshore, the first stage was exploratory in nature, investigating education service providers' decision-making processes with respect to entering the offshore education market. The purpose of this stage of the research process was to become familiar with education service providers’ perspectives and to broadly explore issues pertinent to offshore market entry that they consider to be important. This allowed for validating and refining the research model proposed in the previous chapter (Churchill, 1979). The confidential interviews were also expected to provide depth in interpreting the results obtained in the second stage of the research, which is the administration of a survey (Brewer and Hunter, 1989). The interviews also informed the refinement of the survey instrument used in this study.

The interviews lasting about one hour each, adopted an in-depth and semi-structured approach through the use of open-ended questions, in the hope of gaining a better 
understanding of the subject matter at hand (Denzin \& Lincoln, 2000). A purposeful and maximum variation sampling approach was used to identify interviewees that were likely to contribute to the current study. This sampling strategy follows Eisenhardt (1989b) and Miles and Huberman (1994), who argue that random sampling in qualitative research is neither necessary nor generally desirable, since a purposeful sampling approach can help to focus the study and contribute more effectively to the research objectives. Similarly, a maximum variation sampling strategy was used to obtain access to a diverse set of opinions and perspectives about foreign market entry. In particular, the planned sample included education service providers involved across a wide range of offshore education entry modes. The interviews were conducted with the most senior knowledgeable managers responsible for foreign operations. These in the university context are Pro Vice-Chancellors/ Vice-Provosts (International)/ VicePresidents or Directors of International Offices. Appendix K presents a proposed interview protocol with a broad outline of the open-ended questions that were asked in the interview process. The outlined protocol is semi-structured and emerging questions not listed were also asked as judged appropriate by the interviewer.

To control the associated research costs of the study, telephone interviews were utilised, supplemented by field interviews wherever possible (e.g. in New Zealand). On the basis of the interviews, the research model was revised and refined as necessary. Once revised, the questionnaire was then pre-tested among a small group of potential respondents. Suggestions with respect to the content, quality and clarity of the questionnaire were sought. The returned questionnaires further assisted with refining the research instrument.

The second stage of the research was to administer the final research instrument. First, a comprehensive database of education service providers involved in offshore education market was developed. No secondary data currently exists for such a database. Therefore, the information compiled in Chapter 2 by pooling/synthesising a range of contextual intelligence on offshore education scattered around books, academic journals, newspapers and institutional websites was used to develop a database for the 
purpose of this study. This information is presented in Appendices A-G, which details education service providers active in offshore education as of late 2007. Because the scope of this study is on education service providers in English speaking countries operating offshore (see Chapter 1), a sampling frame of 258 institutions was developed to represent the population of interest listed in Appendices A-G. Of these 258 institutions, 15 were involved in wholly-owned operations, 27 in joint ventures and 216 in non-equity arrangements (see Appendix J).

Similar to the qualitative stage of the research, the most senior knowledgeable manager responsible for foreign operations, i.e. the Pro Vice-Chancellors/Vice-Presidents/Vice Pro-Vosts (International) or Directors of the International Offices, of each education institution listed in the sampling frame was initially contacted by telephone and asked to participate in the study; initial telephone contact has been shown to increase participation and response rate (Jobber and O'Reilly, 1998). The contact details of these target respondents were sourced via respective institutional websites. Once contact was made, the nature of the research was explained to motivate participation in the survey. A summary of the completed study was offered to respondents, as a tangible benefit of their participation.

During the initial phone contact, anonymity was also emphasised. The offshore education market is becoming extremely competitive and it was thought that the target respondents might be concerned about divulging confidential information due to sensitivity issues, especially when the current study was associated with Victoria University of Wellington (VUW), a competitor university involved in offshore education. In order to overcome the latter problem, the academic nature of the study was emphasised and a disconnection made with the international operations of VUW.

Third, once the initial phone contact was established, the questionnaire was posted (see Appendix M), accompanied by a covering letter that explained the purpose of the study (see Appendix L). A self-addressed, postage-paid return envelope was included to improve the response rate (Jobber and O’Reilly, 1998). A traditional mail survey 
administration was adopted over an email version, primarily for two reasons: (1) response rates appear to be lower for email questionnaires, since the email may be considered spam and ignored/discarded (Tse, 1998) and (2) respondent anonymity is more difficult to guarantee with an email delivery system (Jobber and O’Reilly, 1998). Three weeks after the initial mailing, follow up procedures were implemented to improve the response rate. These included a telephone contact, followed by a reminder letter two weeks later, and another questionnaire mail out a month after the original one (Dillman, 1978; Churchill and Iacobucci, 2002); see appendices N and O.

\subsection{VARIABLES AND THEIR OPERATIONAL MEASURES}

The questionnaire used in this study utilised existing scales and measures wherever possible. Table 5.1 outlines the various sources of the operational measures for each of the constructs. These measures, as appropriate, were modified to fit the context of this study, and the questionnaire pre-test was used to test these modifications and improvements of some of the measures. The survey items, where appropriate, used a nine-point Likert scale format, to create enough variation in the data, allowing for meaningful data analysis (Cox, 1980).

Table 5.1: Independent Variables and their Operational Measures as Used in the Literature

\begin{tabular}{|c|c|c|}
\hline Variable & Operational Measure & $\begin{array}{c}\text { Source of Measure/Related } \\
\text { Reference }\end{array}$ \\
\hline Geographic experience & $\begin{array}{l}\text { Knowledge of the foreign } \\
\text { market; similarity of culture } \\
\text { between the host and home } \\
\text { country; ratio of international } \\
\text { sales to total sales volume; } \\
\text { number of foreign countries on } \\
\text { the same continent as this foreign } \\
\text { market; ability to handle } \\
\text { international expansion in terms } \\
\text { of technological, managerial and } \\
\text { financial capabilities at the time } \\
\text { of entry; geographic knowledge } \\
\text { of the region where market is } \\
\text { located }\end{array}$ & $\begin{array}{l}\text { Davidson, 1980; Goodnow, } \\
\text { 1985; Gatignon and Anderson, } \\
\text { 1988; Gomes-Casseres, 1989; } \\
\text { Agarwal and Ramaswami, 1992; } \\
\text { Kim and Hwang, 1992; Aulakh } \\
\text { and Kotabe, 1997; Rajan and } \\
\text { Pangarkar, 2000; Ekeledo and } \\
\text { Sivakumar, } 2004\end{array}$ \\
\hline Industry experience & $\begin{array}{l}\text { Number of years in industry; age } \\
\text { of organisation }\end{array}$ & $\begin{array}{l}\text { Smith, Smith, Olian, Sims Jr., } \\
\text { O’Bannon and Scully, 1994; } \\
\text { Eisenhardt and Tabrizi, 1995; } \\
\text { Constant, Sproull and Kiesler, } \\
\text { 1996; Rajan and Pangarkar, } \\
\text { 2000; Ekeledo and Sivakumar, }\end{array}$ \\
\hline
\end{tabular}




\begin{tabular}{|c|c|c|}
\hline & & 2004 \\
\hline Transfer experience & $\begin{array}{l}\text { Aggregate number of prior } \\
\text { technology transfers by } \\
\text { transferor at the time of the } \\
\text { transfer }\end{array}$ & Davidson and McFetridge, 1985 \\
\hline Tacit know-how & $\begin{array}{l}\text { Codifiability of production } \\
\text { process; complexity of } \\
\text { production process; teachability } \\
\text { of production process; } \\
\text { transferability of marketing } \\
\text { know-how; difficulty in } \\
\text { assessing price of know-how }\end{array}$ & $\begin{array}{l}\text { Kim and Hwang, 1992; Kogut } \\
\text { and Zander, 1993; Ekeledo and } \\
\text { Sivakumar, } 2004\end{array}$ \\
\hline Organisational culture & $\begin{array}{l}\text { Product, process or marketing } \\
\text { innovations; encouragement of } \\
\text { open discussion; de-emphasis of } \\
\text { status distinction; encouragement } \\
\text { of experimentation; tolerance of } \\
\text { mistakes; customer service; } \\
\text { promotion from within }\end{array}$ & $\begin{array}{l}\text { Goodnow, 1985; Arogyaswamy } \\
\text { and Byles, 1987; Wernerfelt, } \\
\text { 1989; Hall, 1992; Tallman and } \\
\text { Shenkar, 1994: Ekeledo and } \\
\text { Sivakumar, 2004 }\end{array}$ \\
\hline Learning intent & $\begin{array}{l}\text { Access to skills and knowledge } \\
\text { an important consideration in } \\
\text { forming alliance; aggressiveness } \\
\text { in learning from partner; partner } \\
\text { efforts to gain information } \\
\text { outside of that agreed to } \\
\text { originally; objective to learn } \\
\text { about an unfamiliar market; } \\
\text { objective to learn about partner's } \\
\text { technology; objective to learn } \\
\text { about partner's management } \\
\text { techniques }\end{array}$ & $\begin{array}{l}\text { Kogut, 1988b; Westney, 1988; } \\
\text { Anderson, 1990; Hamel, 1991; } \\
\text { Inkpen, 1992; Norman, 1997; } \\
\text { Belderbos, } 2003\end{array}$ \\
\hline Financial capital & Total capital funding; size & $\begin{array}{l}\text { Harrigan, 1985c; Yu and Ito, } \\
\text { 1988; Rajan and Pangarkar, } \\
\text { 2000; Park, Chen and Gallagher, } \\
\text { 2002; Ekeledo and Sivakumar, } \\
2004\end{array}$ \\
\hline Reputation & $\begin{array}{l}\text { Reputation for superior } \\
\text { production process; reputation } \\
\text { for superior management; image } \\
\text { of your organisation held by } \\
\text { people; reputation for superior } \\
\text { quality product/service; } \\
\text { international recognition of } \\
\text { brand name }\end{array}$ & $\begin{array}{l}\text { Wernerfelt, 1989; Kim and } \\
\text { Hwang, 1992; Rajan and } \\
\text { Pangarkar, 2000;Ekeledo and } \\
\text { Sivakumar, 2004 }\end{array}$ \\
\hline
\end{tabular}

Wherever possible, multi-item measures were developed to help reduce measurement errors associated with single-item measures (Peter, 1979). Both exploratory factor and reliability analyses were conducted to identify and refine constructs to be used for data analysis and interpretation (Hair, Anderson, Rolph, Tatham and Black, 1998; Churchill 
and Iacobucci, 2002). In the next sections, I discuss the constructs and the choice of measures.

\subsubsection{Dependent Variable}

The dependent variable in this study is the focal university's equity ${ }^{32}$ stake for a single overseas investment. As previously discussed, equity is used as a proxy to represent ownership forms, consistent with previous international business studies such as Rajan and Pangarkar (2000) and Rose and Ito (2004). Although a categorical ownership variable is also captured in the survey (see question D in Appendix M), I opt to use the equity measure as the dependent variable (see question $\mathrm{C}$ in Appendix $\mathrm{M}$ ) over categorical ownership forms for two reasons. First, through the qualitative interviews (see Chapter 6), I identified that universities tend to proactively choose specific equity stakes (e.g. 20\% versus 25\%) when considering equity offshore investment. This warrants an equity-based dependent variable. Secondly, using only the categorical ownership variable would effectively be discarding useful information. For example, aggregating a majority joint venture of 95\% equity with a majority joint venture of $51 \%$ might mean that the analysis overlooks some important distinctions in these two types of investment. For these reasons and consistent with the international business literature, I use equity as a proxy for ownership, rather than the specific ownership forms.

\subsubsection{Independent Variables}

\section{Geographic, Industry and Transfer Experiences}

The measurements for these constructs are adapted from existing literature (see table 5.1). As highlighted previously, geographic experience refers to the organisation's familiarity with the host country and/or region in which the target country is located. Industry experience, on the other hand, is more a function of an organisation's age in the industry. Transfer experience refers to prior successful offshore entry through a particular institutional form. Geographic experience has been used widely in the literature while industry and transfer experience have been less frequently employed. Focusing on all three types of experience in this study should provide for a more

\footnotetext{
${ }^{32}$ As per the discussion in Chapter 1, equity is used as a proxy for ownership in this study.
} 
comprehensive understanding of the relationship of experience, as a resource, and mode of entry. Questions F, G and H of the questionnaire are, respectively, used to measure geographic, industry and transfer experience (see Appendix M).

\section{Tacit know-how}

The measure of tacit know-how has largely been grounded in the manufacturing literature and operationalised to a lesser extent in the services literature. Table 5.1 highlights some of the frequent measures of tacit know-how, which tend to focus on the codifiability and transferability of knowledge. To fit within the context of the current study, these measures are adapted and refined through the questionnaire pre-test. Question I of the questionnaire is used to develop this construct (see Appendix M).

\section{Organisational Culture}

In this study, the measures of organisational culture employed are largely adapted based on available literature (see table 5.1). Question J of the questionnaire is used to develop this construct (see Appendix M).

\section{Learning Intent}

Building on previous studies (see table 5.1), this construct is operationalised through the contents of question $\mathrm{D}$ (ii) of the questionnaire (see Appendix $\mathrm{M}$ ), based on the following indicators: (1) primacy of time and cost considerations in the formation of collaboration; (2) the length of perspective governing the collaboration (i.e. short-term focus versus long-term view); (3) the interest in developing a deeper understanding of desirable skills and (4) breadth of information in which the organisation is interested. It is reasoned that, if primacy of time and cost considerations in the formation of collaboration are high, then the learning intent is likely to be low. Similarly, if the length of perspective governing the collaboration is low, then the learning intent is also likely to be low. The last two indicators are self explanatory with positive relationships between them and learning intent, i.e. the higher the intent in developing a deeper understanding of desirable skills and the higher the breadth of information in which the organisation is interested, the higher the learning intent. 
Financial Capital

Table 5.1 highlights some of the measures that have been employed to operationalise financial capital. In this study, a one-item measure is used; namely the financial endowment of the education service provider. Question $\mathrm{K}$ of the questionnaire is used to capture this variable (see Appendix M).

\section{Reputation}

The measure of ranking used in this study is a subjective one. While there are a number of published academic rankings, such as the Times Higher Education and the Shanghai Jiao Tong University rankings that can be used as secondary sources, the fact that this study is anonymous in nature means that these sources are not appropriate for this study. Indeed, not being able to relate a survey item to a particular institution means that the use of secondary data measure for the ranking construct is unsuitable. Furthermore, the use of a subjective measure of ranking is deemed more useful given the politics of rankings. It is well documented in the higher education literature that different ranking systems have particular ways of recording and classifying institutions (Marginson and Van der Wende, 2007), so much so that no ranking systems can claim to cover all purposes of higher education from the point of view of all stakeholders (Usher and Savino, 2006). In other words, any ranking system will encompass the needs of some stakeholders better than others. This would explain why some institutions are better ranked on some ranking systems than on others. A subjective measure of ranking is, therefore, used in this study. As reputation is a function of the perceptions of key constituents (Fombrun, 1996), a subjective measure seeks to assess the respondent's beliefs of the institution's reputational image. Measurement items for this construct, grounded in the literature (see table 5.1), are adapted to fit the context of this study.

Question items $\mathrm{L}$ and $\mathrm{M}$ of the questionnaire are used to develop this construct (see Appendix M). 


\subsubsection{Moderator Variable}

\section{Institutional Distance}

Hypotheses 7 and 8 consider two measures of institutional distance: nominal and regulatory. The measures employed for these two constructs of institutional distance are discussed in this section.

Empirical studies that have examined the relationship between distance and entry mode choice have often opted for Kogut and Singh's (1998) index. However, the findings of these studies have often been inconclusive (Brouthers and Brouthers, 2001; Shenkar, 2001). Kogut and Singh (1998), for instance, find a negative relationship between the level of control and cultural distance while Pan (1996) observes the opposite relationship. This inconsistency, according to Ionascu et al (2004), is due to the imprecision of the measures used, since Kogut and Singh's index focuses largely on cultural distance (i.e. the normative influences of distance) and not regulatory distance. Ionascu et al (2004) call for a refined measurement that would complement Kogut and Singh's index with indicators of regulatory distance. Ionascu et al (2004) propose a measurement of regulatory institutional distance that is based on the 'Regulatory Factor' of the Economic Freedom Index (EFI) published by The Heritage Foundation as a proxy measure of regulatory distance. This index covers a broad range of aspects of regulations and is broken down in six sub-indices:

- Licensing requirements to operate a business

- Ease of obtaining a business license

- Corruption within the bureaucracy

- Labour regulations, such as established workweeks, paid vacations and parental leave, as well, as selected labour regulations

- Environmental, consumer safety and worker health regulations

- Regulations that impose a burden on business.

Following Ionascu et al (2004), I compute a similar measure of regulatory institutional distance. Using questions $\mathrm{A}$ and $\mathrm{E}$ of the questionnaire, I capture the necessary information about the home and host country in question to compute a measure of 
regulative institutional distance, based on data from the Economic Freedom Index (see Appendix M).

To compute a measure of nominal institutional distance, similar to Kogut and Singh (1988), I use the Hofstede’s (1980) indices of culture - namely power distance, individualism/collectivism, masculinity/femininity and uncertainty avoidance. Kogut and Singh (1998) use the following formula to calculate the normative aspect of institutional distance between two countries:

$$
C D_{j}=\sum_{i=1}^{4}\left\{\left(I_{i j}-I_{i k}\right)^{2} / V_{i}\right\} / 4
$$

where $\mathrm{I}_{\mathrm{ij}}$ stands for the index of the $\mathrm{i}^{\text {th }}$ cultural dimension and country $\mathrm{J}, \mathrm{I}_{\mathrm{ik}}$ stands for the index of the $\mathrm{i}^{\text {th }}$ cultural dimension and country $\mathrm{K}, \mathrm{V}_{\mathrm{i}}$ is the variance of the index of the $\mathrm{i}^{\text {th }}$ dimension, and $\mathrm{CD}_{\mathrm{j}}$ is the cultural distance of country $\mathrm{J}$ from country K. Using questions $\mathrm{A}$ and $\mathrm{E}$ of the questionnaire, I capture the necessary information about the home and host country in question in order to compute a measure of normative institutional distance based on Hofstede’s indices of culture (see Appendix M).

Following previous studies (e.g. Delios and Beamish, 1999; Gaur and Lu, 2007), I further supplement the measures of institutional distance using data from various editions of the World Competitiveness Yearbook. With Hofstede's data being a criticised measure of distance (e.g. Shenkar, 2001) and with the Economic Freedom Index being a measure that is rarely used in published scholarly work, it is deemed appropriate to conduct sensitivity assessment of these two measures of distance using a more established measure in the literature. The World Competitiveness Yearbook compiled by IMD business school has the benefit of capturing both the normative and regulative institutional distance concepts. Given that the yearbook, which employs a survey methodology, queries the same respondent with regards to both normative and regulative institutional distance, it provides the added advantage of consistency across both measures of institutional distance. In my computation of measures for institutional distance, I therefore, adopt three sources of relevant data - the Hofstede indices, the 
'regulatory factor' of the Economic Freedom Index and the World Competitiveness Yearbook.

With respect to the latter data source, using information from country-level indicators, a measure of normative and regulative distances is computed by means of a Euclidean distance calculation similar to that used in Kogut and Singh (1988):

$$
R D_{j k} / N D_{j k}=\sum_{i=1}^{n}\left\{\left(I_{j}-I_{k}\right)^{2} / V_{I}\right\} / n
$$

where $I_{j}$ refers to the institutional indicator (I) for country $J, I_{k}$ refers to the institutional indicator for country $K$, and $V_{I}$ is the variance of indicator $I$. $R D_{j k}$ and $N_{j k}$ are regulative and normative distances of country J from country K. Symbol $n$ refers to the number of indicators for a particular measure. Following Gaur and Lu (2007), table 5.2 outlines the specific indicators used as measures for normative and regulative distance. A time dimension is also added to equation 2 with the availability of time series data from the World Competitiveness Yearbook.

Table 5.2: Country-level indicators of normative and regulative distance

\begin{tabular}{|c|c|}
\hline Normative distance & Regulative distance \\
\hline $\begin{array}{l}\text { Adaptation of political system to today's economic } \\
\text { challenges }\end{array}$ & $\begin{array}{l}\text { Fiscal policy (govt. debt and total foreign debt as } \\
\% \text { of GDP) }\end{array}$ \\
\hline $\begin{array}{l}\text { Adaptation of government policies to new economic } \\
\text { realities }\end{array}$ & Antitrust regulation \\
\hline Transparency of government toward its citizens & Political transparency \\
\hline \multirow{4}{*}{$\begin{array}{l}\text { Degree to which bureaucracy hinders economic } \\
\text { development }\end{array}$} & Intellectual property protection \\
\hline & Judiciary system efficiency \\
\hline & Rarity of market dominance in key industries \\
\hline & Fiscal policy (inflation) \\
\hline
\end{tabular}

Source: Gaur and Lu (2007)

\subsubsection{Control Variables}

This study also includes controls for several variables that might be associated with the hypothesised relationships, including age, size, the existence of an offshore education policy, the influence of an internationalisation at home strategy and the host country's demand for higher education opportunities from overseas institutions. These variables are overviewed next. 
Age - Similar to other modes of entry studies, control variables are incorporated for the age of the education institution, since age might influence an institution's internationalisation processes. The business literature tends to argue that older organisations typically have more resources to rely on in their internationalisation process and, therefore, are more likely to favour higher control modes of entry (Burgel and Murray, 2000; Zahra, Ireland and Hitt, 2000). However, the education literature tends to highlight the opposing argument, with new universities often being much more entrepreneurial in their orientation than the more established universities (Marginson and Considine, 2000; Prince, 2004; Harman, 2005). This entrepreneurial orientation of the new universities is often manifested in the offshore education market. Age is operationalised through question $\mathrm{P}$ of the questionnaire (see Appendix M).

Size - The size of the educational institution is considered, due to larger organisations' generally having more resources that might influence the ability to internationalise (Bloodgood, Sapienza and Almeida, 1996; Zahra et al, 1997; Burgel and Murray, 2000). Size is operationalised through question $\mathrm{N}$ of the questionnaire (see Appendix M). Offshore education policy - The extent of planning of offshore education activities seems to vary considerably between institutions. Some have a formal offshore education policy as part of a larger internationalisation strategy, while others have a more ad hoc approach to initiating offshore projects. Institutions with a formal policy may seek specific offshore education opportunities that are more aligned to their resource endowment, as they are likely to have more developed planning procedures in place, compared to those institutions that approach offshore developments on an ad hoc basis. Consequently, the relationship between resources and modes of entry is likely to differ, depending on the presence of formal planning procedures. Question $\mathrm{O}$ in the questionnaire is intended to measure this variable (see Appendix M).

Internationalisation at home strategy - By the same logic outlined for using offshore education policy as a control variable, it is further reasoned that if an institutional offshore education policy could have an influence on mode of entry, then an institutional internationalisation at home strategy could also possibly influence mode of 
entry. Indeed, if the universities' strategic focus is on internationalising the home campus (e.g. through student exchanges, integrating international elements into the curricula, etc), could that lead to them not exhibiting an interest in going offshore? Could that also mean that instead of diverting limited resources through offshore activities, they would rather focus their commitments to internationalising at home? Question $\mathrm{T}$ in the questionnaire is intended to measure this variable (see Appendix M).

Host country's demand for overseas higher education opportunities - The size of the potential market in the host country could have an impact on the modes of entry that is used when entering particular foreign countries. For example, the bigger the market size, the more resource intensive the mode of entry could be given that the market potential could well outweigh the costs of market entry. Using question A of the questionnaire, I compute a measure of the host country's demand for overseas higher education based on the ratio of host country citizens who enrol for tertiary education overseas to those who enrol for tertiary education domestically. The relevant data are sourced from the World Bank Education Statistics database and various issues of the OECD's Education at a Glance publication. To deal with cases of missing data, relevant national education specific sources are accessed to complement the World Bank and OECD data. Given that the latter organisations source their data from the same national sources consulted, comparability of data across databases is not a problem.

\subsection{STATISTICAL ANALYSIS TECHNIQUES}

Ordinary least squares (OLS) regression is used to derive initial results. However, because the dependent variable of equity stake employed in this study is censored - it can only assume values between $0 \%$ and $100 \%$, respectively coded as 1 to 23 (see table 7.6a in Chapter 7) - and ordinary least squares with a censored dependent variable tends to yield coefficient estimators that are biased toward zero (Rose and Ito, 2004), Tobit analysis is also used to test the robustness of the OLS findings.

Subsequent to empirically testing the conceptual model presented in figure 4.1 and the associated hypotheses 1-6, another objective of this study is to assess how different 
types of distance might moderate the obtained results (hypotheses 7 and 8). Distance institutional (i.e. Scott's 1995 regulative pillar) and cultural (i.e. Scott's 1995 normative pillar) - is a key concept in the field of international business (see Section 4.3.2). Few studies have gone to the efforts of estimating models using both concepts of distance in order to compare and contrast their potential influences. A key contribution of this study to the discipline of international business is, therefore, the detailed consideration that I have given to analysing the different concepts of distance. That said, given that this study is exploratory, consideration needs to also be given for sensitivity assessment in order to comprehensively gauge the robustness of the obtained findings. Consequently, in addition to Tobit analysis, I also use negative binomial regression to test the hypotheses of interest (the dependent equity stake variable is measured using intervals, which effectively make it treatable as a count variable). Using multiple approaches to estimation is not common in international business literature, especially in the context of exploratory studies. The thoroughness of the estimation strategy adopted in this study to attain robust results, therefore, represents another contribution to the literature.

\subsection{CHAPTER SUMMARY}

This chapter outlines the research method proposed for this study. The research, employing a multi-method design, encompasses two stages. The first, building on interviews, is exploratory in nature and helps to validate, refine and improve the research model proposed in the previous chapter. The results from this stage also aid in the final questionnaire development, which utilises existing scales and measures as well as adapted measures developed specifically for this study.

Stage two of the research consists of a questionnaire-based survey, to test the research model proposed in the previous chapter. Questionnaires are distributed to 258 universities, which constitute the unit of analysis of this study. The most senior knowledgeable person responsible for foreign operations, i.e. the Pro ViceChancellor/Vice-President/Vice-Provosts (International) or Directors of the International Offices of each education institution listed in the sampling frame is targeted as respondents of this study. 
The obtained data from the interviews are then subjected to content analysis while the data from the administered survey are subjected to OLS, Tobit and negative binomial regression analysis. The use of the latter three regression approaches allows for sensitivity assessment of the results. The findings of both research stages (i.e. the interviews and the survey) are outlined next. 


\section{Chapter Six}

\section{FINDINGS FROM FIELD INTERVIEWS}

\subsection{INTRODUCTION}

As outlined in Chapter 5, Section 5.2, a multi-method research methodology is employed for this dissertation, consisting of in-depth interviews and a questionnaire. The qualitative phase of the research is aimed at developing the conceptualisation of the market entry decision model outlined in this study beyond the extant theory. It is also meant to validate and refine the proposed research model (see Chapter 4) and assist in the domain specification of the constructs used in this study. The confidential interviews also play an important part in informing and improving the questionnaire that is used in the quantitative phase of this study. In this chapter, the interviews and the resulting insights are discussed.

\subsection{THE INTERVIEW PROCESS}

As outlined in Section 5.2, because this is exploratory research, a semi-structured, openended interview format is adopted in this study, to allow key dimensions of entry mode selection process to emerge naturally through a conversational manner (Thompson, Locander and Polio, 1989). An initial interview guide is developed to elicit a discussion of pertinent issues (Patton, 1990). Appendix K highlights the interview protocol, with a broad outline of the open-ended questions that are asked in the interviews. The interview guide, however, is not static in nature, but rather developed as the series of interviews proceeded (Strauss and Corbin, 1998). The interviews took place over a three-month period between January and March 2008. Table 6.1 outlines the universities interviewed and provides some of their key demographic information. 
Table 6.1: Demographic Information as of 2008 of Universities Interviewed

\begin{tabular}{|c|c|c|c|c|c|c|c|c|c|c|}
\hline & $\begin{array}{c}\text { Canada } \\
\text { institution } \\
1 \text { (CA1) }\end{array}$ & $\begin{array}{c}\text { US } \\
\text { institution } \\
1 \text { (US1) }\end{array}$ & $\begin{array}{c}\text { US } \\
\text { institution } \\
2 \text { (US2) }\end{array}$ & $\begin{array}{c}\text { UK } \\
\text { institution } \\
1 \text { (UK1) }\end{array}$ & $\begin{array}{c}\text { Ireland } \\
\text { institution } \\
1 \text { (IR1) }\end{array}$ & $\begin{array}{c}\text { Australia } \\
\text { institution } \\
1 \text { (AU1) }\end{array}$ & $\begin{array}{c}\text { Australia } \\
\text { institution } \\
2 \text { (AU2) }\end{array}$ & $\begin{array}{c}\text { Australia } \\
\text { institution } \\
3 \text { (AU3) }\end{array}$ & $\begin{array}{c}\text { New } \\
\text { Zealand } \\
\text { institution } \\
1 \text { (NZ1) } \\
\end{array}$ & $\begin{array}{c}\text { New } \\
\text { Zealand } \\
\text { institution } \\
2 \text { (NZ2) } \\
\end{array}$ \\
\hline $\begin{array}{l}\text { Type of } \\
\text { offshore } \\
\text { education } \\
\text { activity }\end{array}$ & Non-equity & Non-equity & Non-equity & Non-equity & Non-equity & Non-equity & $\begin{array}{c}\text { Joint } \\
\text { venture }\end{array}$ & $\begin{array}{c}\text { Wholly- } \\
\text { owned } \\
\text { subsidiary }\end{array}$ & Non-equity & Non-equity \\
\hline Age* & $\begin{array}{l}50-100 \\
\text { yrs. }\end{array}$ & $\begin{array}{l}100-150 \\
\text { yrs. }\end{array}$ & $\begin{array}{l}0-50 \\
\text { yrs. }\end{array}$ & $\begin{array}{l}50-100 \\
\text { yrs. }\end{array}$ & $\begin{array}{l}150-200 \\
\text { yrs. }\end{array}$ & $\begin{array}{l}50-100 \\
\text { yrs. }\end{array}$ & $\begin{array}{l}150-200 \\
\text { yrs. }\end{array}$ & $\begin{array}{l}50-100 \\
\text { yrs. }\end{array}$ & $\begin{array}{l}100-150 \\
\text { yrs. }\end{array}$ & $\begin{array}{l}50-100 \\
\text { yrs. }\end{array}$ \\
\hline $\begin{array}{c}\text { Domestic } \\
\text { enrolment* }\end{array}$ & $\begin{array}{l}40,000- \\
45,000\end{array}$ & $\begin{array}{l}25,000- \\
30,000\end{array}$ & $\begin{array}{l}25,000- \\
30,000\end{array}$ & $\begin{array}{l}2,000- \\
5,000\end{array}$ & $\begin{array}{l}20,000- \\
25,000\end{array}$ & $\begin{array}{c}35,000- \\
40,000\end{array}$ & $\begin{array}{c}35,000- \\
40,000\end{array}$ & $\begin{array}{c}35,000- \\
40,000\end{array}$ & $\begin{array}{l}20,000- \\
25,000\end{array}$ & $\begin{array}{l}15,000- \\
20,000\end{array}$ \\
\hline $\begin{array}{l}\text { International } \\
\text { enrolment* }\end{array}$ & $\begin{array}{l}5,000- \\
10,000\end{array}$ & $\begin{array}{l}1,000- \\
5,000\end{array}$ & $\begin{array}{l}1,000- \\
5,000\end{array}$ & $\begin{array}{l}1,000- \\
5,000\end{array}$ & $\begin{array}{l}1,000- \\
5,000\end{array}$ & $\begin{array}{l}5,000- \\
10,000\end{array}$ & $\begin{array}{c}0- \\
5,000\end{array}$ & $\begin{array}{l}15,000- \\
20,000\end{array}$ & $\begin{array}{c}0- \\
5,000\end{array}$ & $\begin{array}{c}0- \\
5,000\end{array}$ \\
\hline $\begin{array}{c}\text { Research or } \\
\text { applied } \\
\text { university**** }\end{array}$ & Research & Research & Research & Research & Research & Applied & Applied & Research & Research & Research \\
\hline $\begin{array}{c}\text { Public/private } \\
\text { university }\end{array}$ & Public & Public & Public & Public & Public & Public & Public & Public & Public & Public \\
\hline $\begin{array}{l}\text { Academic } \\
\text { ranking** }\end{array}$ & Top 50 & $50-100$ & $50-100$ & Not ranked & $1-200 * * *$ & Not ranked & $150-200$ & Top 50 & $1-100 * * *$ & Not ranked \\
\hline Endowment* & $\begin{array}{l}\text { US\$1-1.5 } \\
\text { billion }\end{array}$ & $\begin{array}{l}\text { US\$ 500- } \\
600 \text { million }\end{array}$ & $\begin{array}{c}\text { US\$500- } \\
600 \text { million }\end{array}$ & $\begin{array}{l}\text { US\$45-50 } \\
\text { million }\end{array}$ & $\begin{array}{c}\text { US\$500- } \\
600 \text { million }\end{array}$ & $\begin{array}{l}\text { US\$500- } \\
600 \text { million }\end{array}$ & $\begin{array}{l}\text { US\$600- } \\
700 \text { million }\end{array}$ & $\begin{array}{l}\text { US\$1-1.5 } \\
\text { billion }\end{array}$ & $\begin{array}{c}\text { US\$800- } \\
900 \text { million }\end{array}$ & $\begin{array}{l}\text { US\$400- } \\
500 \text { million }\end{array}$ \\
\hline
\end{tabular}

* Note: Range values are reported for age, enrolments and endowment, as opposed to the actual values, in order to preserve the confidentialities of the universities interviewed.

**Note: The 2007 Times Higher Education ranking is used in this table. Although there are a number of ranking methodologies, the information provided in this table is indicative only and is not meant to be a scientific examination of these individual ranking systems. A categorical ranking is given, as opposed to the actual ranking in order to preserve the confidentialities of the universities interviewed. It is to be noted that this measure of ranking is not to be confused with the subjective measure of ranking used in the quantitative phase of this research (see Section 5.3.2 in Chapter 5 and Section 7.3.9 in Chapter 7). 
***Note: Ranking ranges of 50 are used for most of the universities. However, broader ranges are used for one Irish and one New Zealand university. These two institutions are the only universities in their respective countries in their ranges of 50, making them easy to identify. The use of the broader range for these two institutions ensures that confidentiality is maintained.

****Note: The categorisation of universities in terms of research intensive or applied institutions is derived from the institutions' own descriptions through their websites or strategic documents (e.g. annual reports).

The interviews, lasting about an hour each, are conducted with 10 senior university representatives responsible for international operations. The interviewees are either Pro Vice-Chancellors/Vice-Presidents (International), Directors of International Offices or equivalent/nominated persons.

Because the interviews are conducted on the basis of confidentiality, the names of the interviewees and their respective universities are not disclosed. Instead, the universities are categorised by their country of origin and the demographic information disclosed about them is categorised into ranges. The representatives of each of the universities targeted are also interviewed about one specific mode of entry. The latter is indicated by the "Type of offshore education activity" category in table 6.1. Questioning the representatives of the universities about one specific mode of entry does not imply that these universities are only involved in these types of offshore education activities. In fact, many of the universities targeted do have more than one type of mode of entry for their offshore education activities. However, for the interview purposes, the universities are canvassed regarding their involvement in one specific mode of entry. The universities targeted for the interviews and the specific mode of entry for which they are examined, are selected from the sampling frame shown in Appendix J. A conscious decision is made to target universities across all six English-speaking countries (i.e. Canada, United States, United Kingdom, Ireland, Australia, New Zealand) and all three modes of entry under investigation in this study: wholly-owned subsidiaries, joint ventures and non-equity arrangements. This purposeful and maximum variation sampling approach is used to obtain access to a diverse set of opinions and perspectives about foreign market entry in the educational sector. 
The breakdown of the 10 targeted universities, by the type of offshore education activity, reflects the respective proportion of each of the three entry modes in the sampling frame. In particular, one interview is respectively conducted with a university that is involved in a wholly-owned subsidiary and a joint venture operation. Each of these interviews correspondingly represents $1 \%$ of the targeted interviewee pool (the proportion for wholly-owned subsidiary in the sampling frame is $0.6 \%$ while the proportion of joint ventures in the sampling frame equals 1\%; see Appendix J). Eight interviews are conducted with universities involved in non-equity arrangements (sampling frame proportion for non-equity arrangements is $8 \%$ ). This sample size (10 interviews) is deemed adequate, as saturation of answers was perceived as the interviews progressed (Glaser, 1978). This sampling approach allows the emergent conceptual model to be adequately developed and provides it with precision (Strauss and Corbin, 1998).

\subsection{THE INTERVIEWS: INSIGHTS AND REFLECTIONS}

The qualitative data gathered from each individual interview session are transcribed verbatim. The data are analysed by coding the transcribed interviews using an inductive approach, allowing patterns and themes to emerge. These data are then subjected to content analysis using QSR*NUDIST, allowing for the exploration and explanation of the inter-relationships among the generated constructs. Two independent researchers (myself and a peer graduate student) conducted the data analysis and interpretation to allow for adequate triangulation and validation (Stöttinger, 2001).

Three broad themes emerge from the content analysis. The first is general issues relating to offshore education, providing an overview of the sector, from a practitioner perspective. The second theme deals primarily with issues related to resources, focusing on the link between resources and entry mode. The third theme is that of environmental pressures that impact on the relationship between resources and mode of entry. These themes are highlighted below and their associated excerpts are presented as close to the original form as possible. These are only altered to provide clarity and ensure confidentiality. Extraneous data that are not specifically relevant to this research are 
removed from analysis and given no further consideration, other than to be listed in Section 6.3.4 as avenues for future research.

\subsubsection{General Issues}

From a desk research perspective, offshore education is highlighted as an emerging phenomenon in Chapter 2. It is postulated that growth in the trade of education services has got most potential in mode 3 (i.e. offshore education; see Section 2.1) when contrasted with the growth patterns of other mainstream service industries (e.g. finance, consulting, etc). This view is shared by practitioners in the field. The general picture that emerged during the interviews is that the interviewees believed that there is a growing international trend for students to seek higher education in their home countries rather than travelling overseas. As the representative from AU2 put it:

A larger component of our intake of international students is taking place offshore. We anticipate that in five years' time, more than half of our international students will be based offshore. Currently, roughly $30 \%$ of the international cohort is enrolled through our offshore programmes.

The interviewee from NZ1 added:

Offshore education is here to stay whether you like it or not.... My office is almost inundated with requests seeking such collaborative partnerships.

In a similar vein, the interviewee from US1 said:

... senior management has realised that offshore education is a trend that is likely to continue in the future and that we, therefore, have no choice than to start actively addressing these offshore dynamics. The recent drive of some Ivy League universities to investigate offshore education activities is a reflection of the shift currently taking place in the US when it comes to the offshore education landscape.

These views are, however, not universal. Two of the 10 interviewees voiced concerns about engaging their universities offshore: 
The offshore education landscape is still very much in a state of flux. The collapse of UNSW [University of New South Wales] in Singapore is a case example. I am reluctant to advise my university that it should engage in offshore education even if the financial risks are small. The reputational risks more than outweigh the financial ones. (NZ2)

Even if the number of opportunities for offshore education engagement is growing, at the end of the day, I believe the uptake of these opportunities is going to be at the lower end of engagement for a number of reasons. First, universities have difficulties getting their academic staff members interested in teaching offshore. Second, offshore activities are costly, not only financially but also reputation-wise in the event of failure. Third, the regulatory landscape is still uncertain in a number of countries where offshore activities seem to be more in demand. For these reasons, I personally believe that a lot of universities will take $a$ 'wait and see' approach rather than seek to be too entrepreneurial. (UK1)

While the last two comments are somewhat of an exception to the norm, they do highlight the point that some universities appear to have little interest in engaging with offshore education. The traditional theories of internationalisation postulate that the decision to internationalise is always fraught with uncertainty. For example, the stages model advance that institutions internationalise in a slow and gradual manner with respect to geographical markets and market entry mode, due to uncertainty driven by a lack of knowledge and experience (see Chapter 3). The above concerns expressed by the representatives of NZ2 and UK1 could reflect this uncertainty in internationalising given the risky nature of offshore education and the risk averseness of many universities.

However, when asked whether they intend to increase their offshore involvement in the future, both of these interviewees commented that they would probably have no choice but to engage in the offshore arena.

... we can't afford to miss the boat. (UK1) 
... offshore is the only area for growth in the international student recruitment market. It is getting increasingly expensive to study abroad and this will have a negative impact on international student demand for onshore courses. (NZ2)

It is further postulated by these two interviewees that they are currently involved in offshore education, despite their reservations. In both cases, though, their universities' involvement is at the less resource-intensive end of the engagement spectrum.

In Section 3.4.6 of Chapter 3, it is advanced that the internationalisation of education service providers takes place in an evolutionary approach, similar in nature to the sequential pattern of entry traditionally theorised in the manufacturing sector. The above excerpts appear to complement to that perspective. The concerns expressed by the representatives of NZ2 and UK1, potentially reflect the fact that both these institutions have not reached the stage where they are confident to internationalise through more resource-intensive entry modes. Their current involvement in offshore education in spite of their concerns could further reinforce the point that both these institutions are increasing their offshore education activities in a slow but gradual fashion.

Other general comments from the interviews relate to the reasons for engaging with offshore education. While diverse responses are obtained, internationalisation and pathways to onshore provision are the most common. For example:

... we engage offshore primarily as a form of student recruitment onshore. We find that the pathway entry to our courses tends to be the most popular entrance option for our international students, especially those from South East Asia. (UK1)

We have a long tradition of providing advance standing for local courses delivered in Malaysia and Singapore. Increasingly, with the success of our recruitment strategy through such pathways, we have rolled out other articulation 
arrangements with other countries. More recently, we have even started exploring the Middle Eastern market. (AU2)

A number of countries that send us scholarship sponsored students require that we give credit to courses undertaken locally. In Saudi Arabia, for example, we have arrangements in place with local institutions to provide advance standing to their students. This provides us access to recruit students under the King Abdullah Scholarship Programme. (US1)

We engage in offshore activities primarily for internationalisation purposes. Dealing with local providers offshore is a way for our academics and, where possible, students to engage with their international counterparts. (IR1)

For us, international engagement with offshore providers is a way for the university to achieve its civic duties. A number of countries where we deliver offshore programmes are developing countries in dire need of capacity development. Assisting these countries in meeting their educational needs is a way for us to achieve our international mission of addressing issues of global reach. (US2)

In addition to internationalisation and pathways for onshore provision, other reasons mentioned for engaging with offshore education include financial and academic collaboration. The former pertains to the income generating potential of recruiting international students via offshore education, while the latter relates to the personal and professional interests of academic staff members in developing courses offshore in partnership with international colleagues.

With the interviews highlighting that offshore education activities are undertaken for various different objectives, it is important to introduce these different objectives as control variables. These are not previously thought of as controls (see Chapter 5) and, therefore, introduce a new analytical element to this study. 
Another point of interest is related to the presence of an offshore education policy. Only one of the 10 interviewed universities (AU3) had a formal offshore education policy. The other interviewed universities approach offshore education on an ad hoc basis. It is commented that these offshore opportunities often are developed based on some personal connections:

Often, these offshore engagements develop from somebody you know at the overseas institution. They are ad hoc approaches, really. (NZ1)

Interestingly, AU3, the only interviewed institution with a formal offshore policy, is also the one institution that operated offshore via full ownership. Therefore, the question arises as to whether there is a link between offshore policy and the level of offshore engagement. The question of why that particular institution had an offshore policy was put to its representative:

Our offshore engagement is so diverse and risky, ranging from twinning arrangements to satellite campuses, that we have to ensure that formal policies are in place in terms of our offshore engagement. We can ill afford to be complacent and ad hoc in our offshore engagement, given that our involvement is very resource intensive and that our financial and reputational risks are immense. We, therefore, have to be systematic in our offshore engagement to ensure that we are all on the same page. (AU3)

To examine the relationship between offshore policy and the level of offshore engagement, it is, therefore, appropriate to control for the presence of an offshore education policy when modelling the resource-entry mode relationship. This insight provides support for the use of offshore education policy as a control variable, as theorised in Chapter 5.

\subsubsection{Resource-Specific Issues}

The second theme that emerges from the content analysis is that the interviewed universities have different propensities towards the level of resource intensity associated with specific entry modes. It is clear from the interviews that this difference is largely 
due to the access to resources a particular university has. For example the following statements capture this point:

Offshore education is a very risky enterprise. How we engage with a particular offshore education opportunity largely depends on the resources we have access to invest. (UK1)

It simply requires too many academic and managerial resources from our home campus to currently operate offshore. We can ill afford this especially at a time when our student:staff ratios are increasing at home and where we have been criticised for this. (NZ2)

When asked about the specific types of resources which influence the resource-entry mode relationship, the representative from AU1 added that:

...cash is not the only thing important in determining our type of engagement offshore. Very often, host governments will provide substantial financial benefits for our university to set up campuses offshore. We, however, do not base our decision purely on cash, as there are other resources, such as reputation, which are equally, if not more important. The recent demise of UNSW [the University of New South Wales] in Singapore and the knock-on effect that its pull-out is having on the reputation of its Sydney-based campus is a case in point. (AU1)

The above excerpt captures not only the importance of financial resources (i.e. cash) but also highlights the importance of protecting reputational capital when engaging offshore. As highlighted in Chapter 4, reputation is particularly important for service organisations because of the experience and the credence attributes that accompany service delivery (Aaker, 1989). Weigelt and Camerer (1988) add that reputation is especially important in situation of information asymmetry, where all players are not equally informed of the parameters involved. For instance, when buying education services, a qualification from a prestigious university is deemed better than a qualification from a less reputable institution, even through both establishments could be using the same text books and the classes taught by professors with similar 
educational backgrounds. Because the students usually lack the information to assess the educational delivery of both institutions, they are likely to use prestige as a proxy for the quality of education service delivery.

The importance of organisational culture as a contributor to the resource-entry mode relationship is captured in the interview with US1:

The likelihood of engaging with offshore education also depends on the university's international strategy and the organisational support that is provided. With no support, we are, by default, restricted to modes of entry that are less resource intensive. However, if the university's senior management is willing to take greater risks offshore, then we are at least able to look into setting up branch campuses either through ownership or with a partner. Without the organisational support, we do not even address these opportunities when they arise, because we know they will be rejected higher up the hierarchy. We simply turn these opportunities down, as we know it is going to be an internal battle to get senior management interested in these more risky options. (US1)

As highlighted in Chapter 4, an entrepreneurial organisational culture has also been associated with a greater likelihood for internationalisation. In the education context, for example, offshore education is by and large an activity which depends on the entrepreneurial orientation of the leadership of the institution in question (Bannerman et al, 2005). The views expressed by the representative of US1 are complementary to the latter point.

The types of resources identified in the above comments are reinforcing of the research model conceptualised in Chapter 4 and developed from a theoretical perspective. Three of resource type included in the conceptual model, namely organisational culture, financial capital and reputation, are captured in the above statements. Further, it is advanced in Chapter 3 that the resource-based view perspective is particularly applicable to the context of this study, with resources being particularly important in the 
education services sector. The above findings from the interviews provide initial support for studying the phenomenon of offshore education using the RBV framework.

Additionally, the representative from CA1 and AU3 respectively stated:

We have to think about...our experience in managing the operational dynamics that come with a particular mode of entry.

Ultimately, I guess, it comes down to know-how and experience [for how we engage offshore].

The two latter comments capture the importance of experience and know-how, as key resources worth investigating. It is highlighted in Chapter 4 that in the offshore education context, a major obstacle for an education service provider to be involved offshore is the lack of expertise needed to confidently undertake an international business development process (Education New Zealand, 2006). Many scholars have noted that organisations (and by implication managers and employees) with little international exposure tend to be risk averse (e.g. Johanson and Vahlne, 1977). In contrast, experienced organisations tend to be more aggressive in their market entry (e.g. Gatignon and Anderson, 1988). This is because for less experienced organisations with limited resources and capabilities, the administrative and coordination costs associated with internationalisation are high (Contractor, 1990) while with greater international experience, organisations develop the confidence and competence of managing the uncertainties and costs of operating offshore (Davidson, 1982).

Experience also adds know-how to the organisation's capabilities as the more involved one is in a particular activity, the more knowledgeable one becomes about that activity. The critical role of knowledge in generating organisational advantage has increasingly gained prominence in the literature and the knowledge-based perspective, which views knowledge as the most strategically important of the organisation's resources (Grant, 1996) is essentially an outgrowth of the RBV. Tacit knowledge, in particular, has received attention in the entry mode literature (e.g. Kim \& Hwang, 1992; Madhok, 
1998; Luo, 2001) and as hypothesised in Chapter 4, a higher level of ownership is anticipated, the less able an education service provider can articulate its know-how specific to offshore expansion.

Another point related to experience is that three different types of experience are examined in this study: geographic, industry and transfer experience (see Chapter 4). While the influence of geographic and industry experience are well established in the literature, transfer experience, defined as the prior successful experience an organisation has with a particular entry mode, has been relatively less examined among current scholars. In fact, Davidson and McFetridge (1985) is the only published research that is identified as having examined the influence of transfer experience on mode of entry selection. Therefore, to inform the development of hypothesis 1C (see Chapter 4), the interviewees are specifically questioned about the potential influence of transfer experience. The representative of AU1 offers the following comment in support of H1C:

How we engage internationally is basically based on our previous international engagement. Because we know how to run twinning programmes, we always opt for that option whenever we get approached to deliver an offshore education programme. By default of that experience, we choose not to even investigate more resource-intensive engagement such as satellite campuses, should they come our way. We simply do not believe we can go in a type of offshore education engagement that we do not have the experience to engage with in the first place. It is a bit of a Catch 22, however, because you cannot gain that experience without trying it. The reality, though, is that offshore education is a risky undertaking and in the current tertiary education policy setting, we cannot be seen as being risk takers.

\subsubsection{Environmental Issues}

As conceptualised in Chapter 4, it is hypothesised in this study that institutional distance will act in a moderating capacity on the resource-entry mode relationship, such that the relationship between levels of equity ownership and resources is positive at low to medium levels of institutional distance but negative at higher levels. Consequently, 
questions are asked during the interviews on environmental influences on the resourceentry mode relationship. The findings from the interviews identify that environmental factors do indeed play a role in influencing the resource-mode of entry relationship. For example, here is what the representative from UK1 had to say:

No matter whether you are in India, China, Singapore or wherever else you might be, there are no quick answers to how resources influence foreign engagement. Basically, the operating environment in a country might be so risky that we choose to go with the least resource intensive engagement.

The representative from UK1 advocates that in risky environments, his/her institution might choose the least resource intensive entry mode, regardless of how resource rich his/her institution might be. As discussed in Chapter 4, as institutional distance and the associated level of unfamiliarity increase, the relationship between resources and the level of equity ownership becomes strongly negative since the transaction costs of operating in host countries with large institutional distance may eventually exceed the benefits. In such situations, MNEs may not sustain their operations irrespective of their resource endowments. In some highly uncertain environments, organisations may even face the risks of appropriation by the host government (Delios and Henisz, 2003).

Similar views are shared by other interviewees who commented that:

In some countries, we would not even dare to engage in offshore programmes because it would simply be too risky due to the political uncertainty. Take Iran for example. We have a large Iranian community here in ....[suppressed for confidentiality reasons]. We have a large number of Iranian students and staff members on campus. We are frequently approached through the relationships that these students and staff bring to campus, to establish offshore operations in Iran. We certainly have the resources to do so. We would, however, never even contemplate such an engagement because of the whole political climate in Iran. It is simply too risky. (US1) 
...take Indonesia for example. After the Bali bombings, we had a hard time getting our academics interested in going to teach in our programmes in Indonesia. And without them, we could not run our offshore education activities. Even though the university clearly had an articulated policy encouraging staff to teach offshore and even though our insurance policies covered staff teaching in hot spots like Indonesia, at the end of the day, our academic staff choose for themselves whether they want to engage in offshore education activities or not. We cannot force them to do so and yet we are completely dependent on them for running our offshore programmes. (AU3)

The above comments from US1 and AU3 highlight the importance of incorporating the external environmental influence as part of modelling the resource-entry mode relationship as well as provide support for looking into mode of entries as they apply to the context of offshore education using a resource-based perspective. The comment from AU3 in particular, is complementary to the latter point since it is highlighted that universities are completely dependent on academics for running offshore programmes. The same argument is put forward in Chapter 3 to position this study from a resourcebased perspective. In Section 3.3, it is stipulated that the service delivered by education providers is embedded in human resources (i.e. academics/faculty), without whom, offshore investment cannot take place.

When further questioned about environmental pressures, the representative from IR1 commented that host country regulation can influence the resource-mode of entry relationship.

It is very difficult to set up a 100\% owned operation in China. The two major foreign owned satellite campuses located in China, that of Nottingham University and The University of Liverpool, are not even wholly-owned. They are joint venture operations. To my knowledge, there are no fully owned operations in China. This is not due to a lack of interest in setting up full ownership operations in China. It's simply to do with the Chinese regulations that prohibit full 
ownership by foreign education providers. The same currently applies in the Indian higher education sector.

Interestingly, the representative from CA1 add that host country regulative distance might not be the only influencer on the resource-entry mode relationship, but that home country regulative distance could also play a role. The representative of CA1 notes that:

In Canada, our academic unions make it very hard for us to request our academics to teach overseas for an extended period of time. This means that if we cannot find suitable local academic hires to teach in our offshore education programmes, we are by default only able to operate through articulation arrangements where we do not have to worry about staffing issues.

The latter two comments from the representatives of IR1 and CA1 highlight the need to consider both the host and home country environments in modelling the impact of environmental influences on the resource-entry mode relationship. This finding provides support to our measure of institutional distance which computes a Euclidean distance of the difference between the host and home country.

\subsubsection{Other Issues}

In addition to the three broad themes discussed above, other issues not specifically relevant to this study emerge from the content analysis. These are outlined in this section as possible avenues for future research in the area of offshore education.

Quality assurance: All of the interviewees expressed general concerns surrounding quality assurance in education programmes delivered offshore. Key comments that arose are highlighted as the following possible research questions:

- How can quality provision of offshore education programmes be ensured?

- Who has the responsibility of ensuring quality, given that the delivery of offshore programmes often falls outside the jurisdiction of national accreditation systems? Is it the host country's or the home country's accreditation system that has this responsibility? What about the role of the universities themselves in ensuring quality? 
- Is there a role for supranational accreditation systems (e.g. UNESCO) and what might that role be?

- Is there a role for independent, discipline based accreditation systems (e.g. AACSB, EQUIS) and what might that role be?

- Who regulates these independent and supranational accreditation systems to ensure that they provide credible guarantees of quality assurance? Can the market self-regulate? What will be the role of consumer (i.e. universities and/or students) confidence in accreditation systems as part of a self-regulation system?

- What impact are commercial offshore education activities (e.g. Apollo and Laureate groups) likely to have on the quality of higher education?

- What are the key issues surrounding qualification recognition for courses undertaken through offshore provision?

These questions fall largely outside the scope of this study. Quality assurance is addressed indirectly, as a kind of brand protection when looking at reputation as a key resource. There are, however, many more avenues for research in the area of quality assurance, apart from brand protection, as alluded to in the above set of questions.

Quality of host country's students: One of the key concerns that emerged around delivering offshore programmes via wholly-owned ventures and joint ventures (as opposed to non-equity arrangements), relate to the quality of the local pool of students in the host country. For example, many of the research-intensive universities interviewed, questioned whether a satellite campus would be able to attract a sufficient number of students of the quality of students currently enrolled at the home campus. These institutions commented that the volume of students that would be required to make a branch campus academically and financially viable might be more than what the local talent supply would be able to provide. While institutions can be selective in terms of the entrance standards they set for students at the home campus, they might not have the same luxury at the satellite campus given pressure to recover investment costs and a lower volume of qualified students. Such concerns were primarily expressed with respect to the Middle East, an increasingly popular destination for offshore education. 
Several interviewees acknowledged the lack of students in the region with sufficient academic preparation. For example:

... a growing concern we have with our Middle Eastern offshore operations is whether the students there are suitably qualified to meet our strict university entrance criteria. Often, we find that we have to customise our programmes through the delivery of pathway programmes for these students to reach the same academic level of first year students back at our campus in Australia. (AU3)

A US-based study on offshore education developments in the Middle East highlights some reasons for the insufficient level of academic preparation in the region (Illuminate Consulting Group, 2008):

- The Middle East region has suffered tremendously from brain drain of its pool of talented and/or privileged students to study and work in North America and Europe

- Secondary education systems in the Middle East have largely been underperforming by western standards

- Cultural and social dynamics which have led to learning styles that are not aligned with western style curricula delivery.

This situation is not, however, restricted to the Middle East. In China for example, top students choose to study at a few very prestigious universities (e.g. Peking, Tsinghua) or to go overseas, as an overseas qualification is more highly valued than one from a less prestigious domestic institution (Xinhua News Agency, 2002). During the interviews, the question arose as to whether such top students would be as interested in the opportunity to enrol at a China-based satellite campus as they would have been in the overseas-based home campus. In other words, would a local satellite campus of a foreign university carry the same cache as its overseas campus? The same situation also appears to apply in other countries. In the context of Singapore for example, the representative from AU1 noted: 
UNSW [University of New South Wales] thought its Singapore campus would carry the same prestige as its Sydney based campus. Unfortunately, this did not prove true among students. The local student cohort seemed to still have a preference for the two top local, well established institutions: The National University of Singapore and Nanyang Technological University. Those students who wanted to go to UNSW seemed to still have a preference to enrol at the Sydney-based campus rather than the Singapore one. UNSW, therefore, had difficulty meeting its enrolment targets and was forced to close its operations.

Top students are also after more than just a qualification. They tend to be after an experience that not only includes having access to a premier academic environment, but to also feel connected to the ethos that such an environment can provide. Unfortunately, as discussed by the representative from US2, offshore campuses often, do not and cannot recreate this ethos. They might, therefore, be viewed by top students as only second best alternatives. The representative from US2 commented:

Top students want to learn from the best. That includes not only an institution's reputation, its teaching and research delivery model but also its organisational culture. While it might be possible to transfer the first two through an offshore education operation, it is much more difficult to transfer organisational culture offshore. This means that the totality of what constitutes a top quality institution as an organisational organism is not mobile. Therefore, an offshore operation will always prove to be a second best alternative to enrolling at the main campus. Talent, unfortunately, does not settle for second best.

Governance: For wholly-owned and joint venture operations, it is also highlighted that governance issues need to be addressed carefully, in terms of who ultimately controls the venture. To offset the high costs of such investments, institutions have entered into agreements with both local governments and private investors ${ }^{33}$. The interviews reveal that many institutions are still unsure about the implications of such partnerships, in

\footnotetext{
${ }^{33}$ In Dubai, for example, Michigan State University has forged a partnership with TECOM Investments to establish its Dubai campus (newsroom.msu.edu; accessed 17 May, 2008).
} 
terms of both academic and managerial governance. In the case of partnerships in the Middle East, for example, concerns are expressed around institutions' dealings with large financial conglomerates. These concerns are especially with regard to the lack of transparency, given the potential of unscrupulous investors laundering their money through the conglomerates. The representative from US1 commented:

...this lack of transparency is a real worry. Just imagine if 10 years down the road, we find out that the financial conglomerate we have been dealing with, have handled money of the next (worse if the current) Osama bin Laden. The implications of such an association would be catastrophic, not only on the offshore operation, but most importantly on our local domestic constituencies. America has not and will not forget the attacks of 9/11. Such an association would prove disastrous for our institution.

Such concerns highlight the need for proper due diligence systems to be factored into the offshore decision making process.

\subsection{CHAPTER SUMMARY}

This chapter highlights the findings from interviews conducted over a three-month period with 10 senior university representatives responsible for international operations. A purposeful and maximum variation approach is used to target the 10 universities across all the three modes of entry (wholly-owned subsidiaries, joint ventures and nonequity arrangements) and six countries (Canada, United States, United Kingdom, Ireland, Australia, New Zealand) under investigation in this study. The interviewees are either Pro Vice-Chancellors/Vice-Presidents/Vice-Provosts (International), Directors of International Offices or equivalent/nominated persons. Table 6.1 provides some key demographic information of the universities interviewed.

Based on content analysis of the interviews, three main themes emerge from the data. These are summarised in table 6.2. 
Table 6.2: Summary of Key Findings from Content Analysis

\section{General Issues}

(a) A larger component of the international student intake at the universities interviewed is taking place offshore, making it increasingly important to understand the dynamics of offshore education.

(b) Offshore education is risky and some universities have little interest in engaging with it. However, those institutions which expressed concerns still stated that they cannot afford to ignore offshore education developments.

(c) Internationalisation and pathways to onshore provision are highlighted as the most common reasons for engaging offshore. Financial gain and academic collaboration also emerge as key reasons for offshore engagement. Given the various objectives for offshore activities, it is advanced that offshore education objectives need to be introduced as a control variable in modelling the resource-entry mode relationship in the offshore education context.

(d) AU3, which operated offshore via a wholly-owned subsidiary, has a formal offshore policy. It is, therefore, questioned whether there is a link between offshore policy and the level of offshore engagement. Controlling for offshore policy in modelling the resource-entry mode relationship would help answer this question.

\section{Resource Specific Issues}

(a) The general findings from the content analysis provide support for looking at offshore involvement in the university sector from a resource-based perspective.

(b) Content analysis suggests that finance, reputation, entrepreneurial organisational culture, experience and know-how are important resources influencing offshore involvement in the education context. These findings provide support for the theoretical discussion presented previously in Chapter 4 and the associated hypotheses developed.

\section{Environmental Issues}

(a) The findings from the interviews identified that environmental factors do indeed play a role in moderating the resource-entry mode relationship.

(b) Content analysis revealed that it is important to consider both host and home country environments in modelling the impact of the environmental influences on the resource-entry mode relationship.

\section{Other Issues}

(a) Quality assurance, the lack of suitably qualified students in the host country and governance issues also emerge from the content analysis. Since these are not specifically relevant to the present study, they are presented as avenues for future research in the area of offshore education.

In general, many of the excerpts garnered from the interviews and presented in this chapter, are consistent with the theoretical discussion in Chapter 4. The numerous comments on the importance of resources reinforce the potential utility of the resourcebased view as the theoretical basis for considering offshore involvement in the university sector. The insights and reflections obtained from the interviews lay the 
foundation for the quantitative stage of this study. They provide a useful basis for refining the conceptual model and the questionnaire instrument. The results of the quantitative part of this research are outlined in the next chapter. 


\section{Chapter Seven}

\section{DATA ANALYSIS \& MODELLING}

\subsection{INTRODUCTION}

This chapter outlines the results of the data analysis conducted from the survey administered to university officials familiar with the foreign market entry of their respective institutions (see Chapter 5). The survey, developed based on extant literature, is revised and refined by means of pre-testing with three of the 10 university representatives interviewed during the qualitative phase of this study (see Chapter 6). The three pre-tests are conducted with universities that had employed the three modes of entry investigated in this study: wholly-owned subsidiary, joint venture and nonequity arrangement. Suggestions are solicited from these three interviewees on the content of the questionnaire, as well as the quality and clarity of the questions asked. The feedback obtained from the interviews does not reveal major problems with the survey development. The questionnaire is also shared among academic colleagues for comment, and minor changes are made to remove perceived ambiguities. The final version of the questionnaire, presented in Appendix $\mathrm{M}$, is sent out to the full sampling frame listed in Appendix J. The survey, which is international in scope, across institutions in six English-speaking nations, was conducted over a three month period between June and August 2008. The next section provides descriptive statistics on the responses collected. The following sections then present the results obtained from testing the hypotheses outlined in Chapter 4.

\subsection{SURVEY RESPONSE}

A total response rate of $63.0 \%$ is obtained from the surveys. Eleven of the received questionnaires are, however, unusable due to missing data, resulting in a usable response rate of 59.7\%. This represents 154 institutions from the 258 identified in the sampling frame outlined in Appendix J. Extensive follow-up telephone calls contributed to the high response rate. 
With each institution responding to the questionnaire with respect to two recent offshore education operations, a final data set of 308 instances of foreign market entry is obtained. Of these 308 observations, 13 represent the wholly-owned mode of entries, while 23 are joint ventures and 272 are non-equity arrangements. The next section highlights some relevant descriptive statistics of the compiled data set.

\subsection{DESCRIPTIVE STATISTICS}

\subsubsection{Home Countries}

Table 7.1 lists, in alphabetical order, the home countries of the universities represented in the sample. Confidence intervals for proportions are calculated between the sampled observations and the sampling frame. It can be noted that the distribution of the observations by home countries is broadly similar to the sampling frame highlighted in Appendix J.

Table 7.1: Home Countries in Sample

\begin{tabular}{l|l|l}
\hline \hline Home Country & Frequency & Percent \\
\hline \hline Australia & $70(76)$ & $22.7(14.7)^{*}$ \\
Canada & $30(36)$ & $9.7(7.0)$ \\
Ireland & $6(10)$ & $1.9(1.9)$ \\
New Zealand & $10(16)$ & $3.2(3.1)$ \\
United Kingdom & $94(144)$ & $30.5(27.9)$ \\
United States & $98(234)$ & $31.8(45.3)^{*}$ \\
\hline Total & $\mathbf{3 0 8 ( 5 1 6 )}$ & $\mathbf{1 0 0 . 0 ( 1 0 0 . 0 )}$ \\
\hline \hline
\end{tabular}

Note: Number in parentheses represents the equivalent frequency from the sampling frame outlined in Appendix $\mathrm{J} ;{ }^{*} \mathrm{p}<0.05$

\subsubsection{Host Countries}

Table 7.2 draws attention to the host countries represented in the collected sample. The bulk of the collected observations represent investments into Asian countries, particularly China, Singapore, India, Hong Kong and Malaysia in descending order. This distribution is broadly representative of the developments currently taking place in the offshore education landscape as overviewed in Chapter 2 (see figure 2.3b for a summary). 
Table 7.2: Host Countries Represented in Sample

\begin{tabular}{l|l|l}
\hline \hline Host Country & Frequency & Percent \\
\hline \hline Australia & 1 & 0.3 \\
Bahrain & 3 & 1.0 \\
Canada & 2 & 0.6 \\
China & 108 & 35.1 \\
Czech Rep & 1 & 0.3 \\
United Arab Emirates (Dubai) & 2 & 0.6 \\
Fiji & 1 & 0.3 \\
France & 1 & 0.3 \\
Greece & 1 & 0.3 \\
Hong Kong & 31 & 10.1 \\
Hungary & 1 & 0.3 \\
India & 39 & 12.7 \\
Indonesia & 8 & 2.6 \\
Malaysia & 29 & 9.4 \\
Mexico & 1 & 0.3 \\
Poland & 1 & 0.3 \\
Qatar & 3 & 1.0 \\
Singapore & 69 & 22.4 \\
South Africa & 3 & 1.0 \\
Sri Lanka & 2 & 0.6 \\
Thailand & 1 & 0.3 \\
\hline Total & $\mathbf{3 0 8}$ & $\mathbf{1 0 0 . 0}$ \\
\hline \hline
\end{tabular}

Of special note within the sample are Sri Lanka, Bahrain, UAE (Dubai) and Qatar. As highlighted in Chapter 5, one of the measures that I use in this study to compute the variable of institutional distance is the IMD World Competitiveness Yearbook, which does not include Sri Lanka, Bahrain, UAE (Dubai) and Qatar. To address this issue of missing data, I use available data as proxies: India for Sri Lanka and Jordan for Bahrain, UAE (Dubai) and Qatar. The extant cultural literature has used three major factors to cluster countries: (a) geographic proximity, (b) mass migrations and ethnic social capital and (c) religious and linguistic commonality (Gupta, Hanges and Dorfman, 2002). India and Sri Lanka share geographical proximity, historical and cultural affinities, along with complimentary regulatory policies founded on deep bilateral economic and political interactions (Mohan and Balendra, 2003). As such, it is reasonable to assume that India and Sri Lanka share a similar institutional environment. Similarly, Bahrain, UAE (Dubai) and Qatar are characterised by comparable institutional environments. Not only do they share similar political systems based on 
Islamic beliefs (Kshetri and Ajami, 2008), but they are also members of the Gulf Cooperation Council (GCC), a regional common market in the Middle East; effective common markets are based on members having complementary institutional environments. Although Jordan is not a member of the GCC, it is further extended that Jordan has a comparable institutional environment to the GCC states. Priess (1998:19) indicates that Jordan and the GCC states are "like-minded and western looking monarchies”. Priess further points out that there have been talks of extending the GCC membership to include Jordan (Priess, 1998, Bilaterals.Org, 2006). Since, for common markets to work, member states need to share a great deal of similarity in their economic, political and institutional regimes, it is reasonable to assume that Jordan is fairly similar to Bahrain, UAE (Dubai) and Qatar in terms of institutional environment.

This somewhat ad hoc approach to dealing with missing data, is deemed preferable to, losing these observations from the analysis $(n=10)$. Furthermore, sensitivity of the results is assessed to ensure the robustness of the missing data treatment, using the complimentary measures of institutional distance employed in this study, i.e. the measure of regulatory institutional distance from the Economic Freedom Index (EFI) and the measure of normative institutional distance using Hofstede's indices (see Chapter 5). With the latter measure, India is also used to proxy Sri Lanka, while an "Arab World" regional estimated value computed by Hofstede is used for Bahrain, UAE (Dubai) and Qatar. The Economic Freedom Index, covers all the host countries represented in this study, avoiding the problem of missing data for that measure.

The sample contains one instance of market entry into Fiji. Unfortunately, the World Competitiveness Yearbook does not provide a reasonable proxy, as there is no obvious cluster that would include Fiji. Similarly, Fiji is not included in the Hofstede data on cultural dimensions. The only relevant available data for Fiji pertains to regulatory institutional distance, from the Economic Freedom Index. Therefore, it is necessary to omit this one observation from consideration of the moderating role of institutional distance on the resource-mode of entry relationship. 


\subsubsection{Academic Programmes}

Table 7.3 highlights the academic programmes being delivered in the offshore education developments in the sample. 'Business and Management' is the most popular discipline being offered offshore by the sampled universities followed by 'Arts and Social Sciences' and 'Engineering'.

Table 7.3: Academic Programmes in Sample

\begin{tabular}{l|l|l}
\hline \hline Academic Programmes & Frequency & Percent \\
\hline \hline Arts and Social Sciences & 49 & 15.9 \\
Business and Management & 140 & 45.5 \\
Engineering & 48 & 15.6 \\
Sciences (including Life Sciences) & 28 & 9.1 \\
Law & 15 & 4.9 \\
Information Technology (including Computer Science) & 11 & 3.6 \\
Education & 10 & 3.2 \\
Creative Arts (e.g. Fine Arts, Music, Dance) & 5 & 1.6 \\
Architecture, Planning and Design & 2 & 0.6 \\
\hline Total & $\mathbf{3 0 8}$ & $\mathbf{1 0 0 . 0}$ \\
\hline \hline
\end{tabular}

The conceptual model developed in Chapter 4, grounded in Grant's (1991) typology of resources, omits a focus on physical and technological resources on the basis that the majority of extant offshore education developments tend to be in disciplines that do not require such specialised resources. It is further suggested in Chapter 4 that offshore education programmes tend to be in areas such as Business and Arts, or those that would require a minimal investment in physical and technological resources. The collected data presented in table 7.3 support these expectations.

\subsubsection{Programme Level}

Table 7.4 displays the programme levels being delivered in the sampled offshore education developments. Undergraduate programmes are the most common, accounting for nearly $71 \%$ of the observations, followed by Master's by coursework programmes (11.7\%), diplomas (7.8\%) and associate degrees (4.2\%). 
Table 7.4: Programme Level in Sample

\begin{tabular}{l|l|l}
\hline \hline Programme level & Frequency & Percent \\
\hline \hline Professional doctorates (e.g. DBA, EdD) & 5 & 1.6 \\
Master's by coursework only & 36 & 11.7 \\
Master's by both coursework and research & 8 & 2.6 \\
Undergraduate degree (three or four years in duration) & 218 & 70.8 \\
Diploma (two years in duration) & 24 & 7.8 \\
Associate degree (two years in duration) & 13 & 4.2 \\
$\begin{array}{l}\text { Certificate (at least one year in duration) } \\
\text { Executive/Professional short programme (less than one year in } \\
\text { duration) }\end{array}$ & 3 & 1.0 \\
\hline Total & 1 & 0.3 \\
\hline \hline
\end{tabular}

\subsubsection{Year of Entry}

Table 7.5 shows the year of entry of the offshore education developments represented in the sample.

Table 7.5: Entry Year of Offshore Education Developments in Sample

\begin{tabular}{l|l|l}
\hline \hline Year of Entry & Frequency & Percent \\
\hline \hline 1999 & 51 & 16.6 \\
2000 & 33 & 10.7 \\
2001 & 44 & 14.3 \\
2002 & 16 & 5.2 \\
2003 & 26 & 8.4 \\
2004 & 40 & 13.0 \\
2005 & 43 & 14.0 \\
2006 & 27 & 8.8 \\
2007 & 28 & 9.1 \\
\hline Total & $\mathbf{3 0 8}$ & $\mathbf{1 0 0}$ \\
\hline \hline
\end{tabular}

The distribution of the data is broadly reflective of the point made in Chapters 1 and 2, that offshore education, in its contemporary form, is a recent phenomenon in the education landscape.

\subsubsection{Mode of Entry}

Data obtained from the respondents on entry mode choice are summarized in tables 7.6a and 7.6b. Two measures of entry mode, the dependent variable used in this study, are captured from the survey: equity stake using ranges and a nominal measure reflecting the entry type. 
Table 7.6a: Equity Stake in Sample

\begin{tabular}{l|l|l}
\hline \hline Ownership Level & Frequency & Percent \\
\hline \hline $100 \%$ & 1 & 0.3 \\
$95-99 \%$ & 12 & 3.9 \\
$55-59 \%$ & 5 & 1.6 \\
$51-54 \%$ & 11 & 3.6 \\
$50 \%$ & 4 & 1.3 \\
$45-49 \%$ & 3 & 1.0 \\
$40-44 \%$ & 35 & 11.4 \\
$35-39 \%$ & 31 & 10.1 \\
$30-34 \%$ & 34 & 11.0 \\
$25-29 \%$ & 41 & 13.3 \\
$20-24 \%$ & 33 & 10.7 \\
$15-19 \%$ & 30 & 9.7 \\
$10-14 \%$ & 37 & 12.0 \\
$5-9 \%$ & 29 & 9.4 \\
$1-4 \%$ & 1 & 0.6 \\
$0 \%$ & 0 & 0 \\
\hline Total & 308 & $\mathbf{1 0 0 . 0}$ \\
\hline \hline
\end{tabular}

Table 7.6b: Entry Mode in Sample

\begin{tabular}{|c|c|c|}
\hline Ownership Type & Frequency & Percent \\
\hline $\begin{array}{l}\text { Non-equity (e.g. twinning programmes of the } 2+2 \text { type, } \\
\text { programme articulations, franchise programmes) (less than } \\
45 \% \text { equity stake) }\end{array}$ & 272 & 88.3 \\
\hline $\begin{array}{l}\text { Joint venture with minority equity share (less than } 50 \% \\
\text { equity share) }\end{array}$ & 3 & 1.0 \\
\hline Joint venture with $50 / 50$ equity share & 4 & 1.3 \\
\hline $\begin{array}{l}\text { Joint venture with majority equity share (between 51-95\% } \\
\text { equity share) }\end{array}$ & 16 & 5.2 \\
\hline $\begin{array}{l}\text { Sole ownership (i.e. greater than 95\% ownership of the } \\
\text { offshore education development) }\end{array}$ & 13 & 4.2 \\
\hline Total & 308 & 100.0 \\
\hline
\end{tabular}

Note: As discussed in Chapter 5, the entry mode dependant variable in this study is measured as the focal university's equity stake for a single overseas investment (see Section 5.3.1)

Of the 308 observations, 13 (4.2\%) represent wholly-owned entries, while 23 (7.5\%) are joint ventures. Most of the joint ventures are majority equity (5.2\% of the total sample), with $50 / 50$ equity share constituting $1.3 \%$ of the total and minority share $1.0 \%$. Nonequity arrangements account for $88.3 \%$ of the sample (see table $7.6 \mathrm{~b}$ ), with the majority being at the lower end of the scale in terms of equity stake; $89.2 \%$ of the sample represent equity shares below 50\% (see table 7.6a). Confidence intervals for proportions 
at the $95 \%$ level are calculated between the sampled observations and the sampling frame. No statistical difference is found, indicating that the overall distribution of the sample is representative of the data outlined in the sampling frame (see Appendix $\mathrm{J}$ where wholly-owned entries comprise $5.8 \%$ of the population, joint ventures $10.5 \%$ and non-equity arrangements $83.7 \%$ ).

Of particular interest is the interpretation of "non-equity" by respondents. While in table 7.6b, $88.3 \%$ of the respondents indicated that they conduct offshore education operations through non-equity arrangements, none of the respondents opted for $0 \%$ under the measure of equity stake. Further discussion with the interviewees (see Chapter 6) revealed that, although institutions are not investing in terms of capital in those non-equity arrangements, the respondents perceived that they are nevertheless investing in terms of academic input. For example, in non-equity twinning programmes, where students undertake a set number of years of study in their country of origin before transferring to institutions abroad with advanced credit (see Chapter 1), the institutions in question need to collaborate on curriculum design to ensure that courses are academically equivalent. It is, therefore, important to understand this interpretation of "non-equity" in the context of education services, as it differs from the more commercial interpretation of the term.

\subsubsection{Size}

The tables below illustrate the size of the universities represented in the sample (exclusive of offshore campus enrolments). Three measures of size are captured in the survey: numbers of undergraduate students, postgraduate students and academic staff. These are shown in tables 7.7a, 7.7b and 7.7c.

Table 7.7a: Size of Universities (Undergraduate Students) in Sample

\begin{tabular}{l|l|l}
\hline \hline Undergraduate Students & Frequency & Percent \\
\hline$<1000$ & 6 & 1.9 \\
$1001-5000$ & 21 & 6.8 \\
$5001-10000$ & 54 & 17.5 \\
$10001-15000$ & 63 & 20.5 \\
$15001-20000$ & 62 & 20.1 \\
$20001-25000$ & 42 & 13.6
\end{tabular}




\begin{tabular}{l|l|l}
\hline \hline $25001-30000$ & 32 & 10.4 \\
$30001-35000$ & 15 & 4.9 \\
$35001-40000$ & 11 & 3.6 \\
$40001-45000$ & 0 & 0.0 \\
$45001-50000$ & 2 & 0.6 \\
\hline Total & $\mathbf{3 0 8}$ & $\mathbf{1 0 0 . 0}$ \\
\hline \hline
\end{tabular}

Table 7.7b: Size of Universities (Postgraduate Students) in Sample

\begin{tabular}{l|l|l}
\hline \hline Postgraduate Students & Frequency & Percent \\
\hline \hline$<1000$ & 15 & 4.9 \\
$1001-5000$ & 180 & 58.4 \\
$5001-10000$ & 89 & 28.9 \\
$10001-15000$ & 22 & 7.1 \\
$150001-20000$ & 2 & 0.6 \\
\hline Total & $\mathbf{3 0 8}$ & $\mathbf{1 0 0 . 0}$ \\
\hline \hline
\end{tabular}

Table 7.7c: Size of Universities (Academic Staff) in Sample

\begin{tabular}{l|l|l}
\hline \hline Staff & Frequency & Percent \\
\hline \hline$<500$ & 34 & 11 \\
$501-1000$ & 62 & 20.1 \\
$1001-1500$ & 50 & 16.2 \\
$1501-2000$ & 46 & 14.9 \\
$2001-2500$ & 25 & 8.1 \\
$2501-3000$ & 43 & 14 \\
$3001-3500$ & 12 & 3.9 \\
$3501-4000$ & 20 & 6.5 \\
$4001-4500$ & 1 & 0.3 \\
$4501-5000$ & 9 & 2.9 \\
$5001-5500$ & 2 & 0.6 \\
$5501-6000$ & 2 & 0.6 \\
$7501-8000$ & 2 & 0.6 \\
\hline Total & $\mathbf{3 0 8}$ & $\mathbf{1 0 0 . 0}$ \\
\hline \hline
\end{tabular}

\subsubsection{Age}

In Chapter 4, based on studies such as Marginson and Considine (2000), Prince (2004) and Harman (2005), it is suggested that new universities tend to be more entrepreneurial in their approach to offshore education, compared to more established institutions. From table 7.8, the sample reflects this position with $35.7 \%$ of the respondent universities being 50 years or younger. 
Table 7.8: Age of Universities Represented in Sample

\begin{tabular}{l|l|l}
\hline \hline Years & Frequency & Percent \\
\hline \hline$<10$ & 8 & 2.6 \\
$11-50$ & 102 & 33.1 \\
$51-100$ & 58 & 18.8 \\
$101-150$ & 74 & 24 \\
$151-200$ & 56 & 18.2 \\
$201-250$ & 8 & 2.6 \\
$251-300$ & 2 & 0.6 \\
\hline Total & $\mathbf{3 0 8}$ & $\mathbf{1 0 0 . 0}$ \\
\hline \hline
\end{tabular}

\subsubsection{Academic Rankings}

Table 7.9 highlights the subjective measure of academic rankings - domestic and worldwide - of the respondent universities. Most of the universities report being ranked in the $40^{\text {th }}-60^{\text {th }}$ percentile range with respect to both worldwide and domestic rankings, consistent with the notion that fewer highly ranked institutions are active in the offshore education sector. The fact that few lower-ranked institutions, in terms of both worldwide and domestic rankings, are represented in the sample could reflect the perspective that reputation is a resource for engaging in offshore education.

Table 7.9: Worldwide Academic Ranking of Universities Represented in Sample

\begin{tabular}{l|l|l||l|l}
\hline \hline & \multicolumn{2}{|c|}{ World Ranking } & \multicolumn{2}{c}{ Domestic Ranking } \\
\cline { 2 - 5 } & Frequency & Percent & Frequency & Percent \\
\hline \hline Ranked in the $90^{\text {th }}$ percentile & 10 & 3.2 & 28 & 9.1 \\
Ranked in the $80^{\text {th }}$ percentile & 28 & 9.1 & 44 & 14.3 \\
Ranked in the $70^{\text {th }}$ percentile & 34 & 11 & 38 & 12.3 \\
Ranked in the $60^{\text {th }}$ percentile & 50 & 16.2 & 50 & 16.2 \\
Ranked in the $50^{\text {th }}$ percentile & 86 & 27.9 & 98 & 31.8 \\
Ranked in the $40^{\text {th }}$ percentile & 58 & 18.8 & 38 & 12.3 \\
Ranked in the $30^{\text {th }}$ percentile & 40 & 13 & 8 & 2.6 \\
Ranked in the $20^{\text {th }}$ percentile & 2 & 0.6 & 2 & 0.6 \\
Ranked in the $10^{\text {th }}$ percentile & 0 & 0.0 & 2 & 0.6 \\
\hline Total & $\mathbf{3 0 8}$ & $\mathbf{1 0 0 . 0}$ & $\mathbf{3 0 8}$ & $\mathbf{1 0 0 . 0}$ \\
\hline \hline
\end{tabular}

\subsubsection{Cross tabulation}

To further explore the sampled data, extensive cross-tabulations are also computed and outlined in Appendix P. The overall findings are consistent with what has been suggested in the previous chapters. 


\subsection{UNIVARIATE DESCRIPTIVE STATISTICS}

Univariate analysis examining the variables captured in the survey is conducted to gain a sense of the data and to assess the assumptions necessary for analysis. Appendix Q presents key descriptive statistics used in evaluating the assumptions of normality. Appendix R presents the findings from Levene's test for homogeneity of variance across the six different home countries considered in the study (i.e. United States, United Kingdom, Canada, Australia, New Zealand and Ireland). From the observed findings, variance is not consistent across the six countries. To address this constraint, the following approaches are adopted. First, the data set is split by home country and factor analysis is conducted on the two largest home country subsets: UK (n=98) and US ( $\mathrm{n}=94$ ) (see Section 7.5). The rationale is that, if similar results are obtained for these two home countries, then the analysis could logically be conducted using the whole data set, with the results assumed to be robust against the lack of homogeneity of variance. However, different factors resulted from analysing the UK and US subsamples. Therefore, the alternative approach adopted to deal with the heterogeneity of variance is to consider the data using three different sub-groups and to run each analysis separately for each group. The three home country sub-groups, developed on the basis of Gupta et al's (2002) cluster approach, are Australia/New Zealand, UK/Ireland and US/Canada. Rather than analysing each country separately, the clustering approach allows the consideration of two countries with small sample sizes (i.e. 10 for New Zealand and 6 for Ireland) $)^{34}$.

\subsection{EXAMINATION OF MEASUREMENT ITEMS}

Appendix $S$ highlights the factors created using exploratory factor analysis with principal component extraction and varimax rotation. Factors are identified based on eigenvalues (generally, eigenvalues of 1 are used, with some discretion around eigenvalues close to the cut-off of 1). Items pertaining to Know-how (question I in the survey) and Reputation (question $\mathrm{M}$ in the survey) all load onto one factor for the complete data set as well as the geographical groupings outlined in the previous section. Items representing Organisational culture (question $\mathrm{J}$ in the survey) load onto one

\footnotetext{
${ }^{34}$ Other conceptually logical groupings are analysed (e.g. AU/UK/US and NZ/IR/CA on the basis of scale of offshore activity), but do not substantially add to the reported models.
} 
factor for the complete data set and the Australia/New Zealand and US/Canada subsets. For the UK/Ireland grouping, only items 2-4 of question J load onto a single factor. For Industry experience, only items 3 and 4 of question $\mathrm{G}$ in the survey load onto a factor; this factor is common across the complete data set and the three geographical groupings. Lastly, no factors are created for Learning intent and Geographical experience. Contrary to the theorisation, no commonality is found across the items representing these two concepts. Thus, the individual items are used as explanatory variables in the analysis.

The distributions of the created factors are examined and do not display major departures from normality. Cronbach $\alpha$ values are computed for each of the factors, to assess reliability; all of the factors created across the complete data set, as well as the three home country geographical groupings, display reliability above the standard $\alpha=$ 0.6 threshold, except for the Organisational culture factor for the UK/IR grouping (see Appendix T). For the UK/IR subset, the individual items pertaining to Organisational culture are used as exploratory variables in the regression modelling.

Correlations between pairs of variables (factors and applicable individual items) are computed, in order to assess potential multicollinearity for the regression modelling. Appendix $U$ presents the correlation matrix, highlighting statistically significant bivariate correlations. In addition, multicollinearity is assessed via the Variance Inflation Factors (VIF) in the regression models.

\subsection{MODELLING AND HYPOTHESIS TESTING}

Three variables of interest display strong correlations with each other: transfer experience, know-how and organisational culture. These high correlations have the potential to result in multicollinearity if the three variables are all used as explanatory variables in regression model(s). Therefore, to prevent any distortion of the estimated coefficients, several model specifications are employed, including the variables of transfer experience, know-how and organisational culture into separate regressions. The three models, estimated for the full data set and the various geographical subsets, used to test the hypotheses $\mathrm{H} 1, \mathrm{H} 2, \mathrm{H} 3$, H5 and H6 are: 
Equation 1: Equity $=f$ (Geographic experience, Industry experience, $\underline{\text { Transfer }}$ experience, Financial resources, Reputation, IMD normative institutional distance, IMD regulative institutional distance, Control variables, Geographical grouping dummies ${ }^{35 \pm}$, random error)

Equation 2: Equity $=f$ (Geographic experience, Industry experience, Know-how, Financial resources, Reputation, IMD normative institutional distance, IMD regulative institutional distance, Control variables, Geographical grouping dummies, random error)

Equation 3: Equity $=f$ (Geographic experience, Industry experience, Organisational culture, Financial resources, Reputation, IMD normative institutional distance, IMD regulative institutional distance, Control variables, Geographical grouping dummies, random error)

Learning intent, used to test $\mathrm{H} 4$, is not included in these equations because it applies only to joint ventures (see Section 7.9). Rather, given our interest in investigating the relationship between learning intent and the propensity to have a majority stake in a joint venture, t-testing is conducted to compare mean levels of learning intent between majority and minority JVs (joint ventures with 50/50 ownership are classified as having minority ownership).

To test the moderating hypotheses of $\mathrm{H7}$ and $\mathrm{H} 8$, the data are split into two groups using the medians of the institutional distance measures as cut off points. Equations 4-9, as specified below, are then estimated separately for both groups. Given that H7 and H8 address two different types of institutional distance - normative and regulative equations 4-9 are estimated separately for both types of distances. As hypothesised in H7 and H8, a positive coefficient associated with the examined type of resource is expected for smaller values of institutional distance and a negative coefficient is expected for larger values.

Equation 4: Equity $=f$ (Geographic experience, Control variables, Geographical grouping dummies, random error)

\footnotetext{
${ }^{35 \pm}$ The geographical grouping dummies are included only for the complete data set.
} 
Equation 5: Equity $=f$ (Industry experience, Control variables, Geographical grouping dummies, random error)

Equation 6: Equity $=f$ (Know-how, Control variables, Geographical grouping dummies, random error)

Equation 7: Equity $=f$ (Organisational culture, Control variables, Geographical grouping dummies, random error)

Equation 8: Equity $=f$ (Financial resources, Control variables, Geographical grouping dummies, random error)

Equation 9: Equity $=f$ (Reputation, Control variables, Geographical grouping dummies, random error)

To further assess the robustness of the IMD normative and regulative institutional distance measure (see Section 5.3.3), equations 1-9 are re-estimated using the Hofstede cultural distance and EFI regulative institutional distance measures. Box 7.1 highlights the different models analysed and their associated hypotheses.

\section{Box 7.1: Regression equations and their associated hypotheses}

- Equations 1-3 are associated with the direct hypotheses of H1 - H6.

- Equations 4-9 are associated with the moderating hypotheses of H7 and H8.

- Equations 10-18 are equations 1-9 re-estimated using the Hofstede cultural distance measure to assess the robustness of the IMD normative institutional distance measure.

- Equations 19-27 are equations 1-9 re-estimated using the EFI regulative institutional distance measure to assess the robustness of the IMD regulative institutional distance measure.

Available data makes it possible to lag the institutional distance measures by up to three years $^{36}$, which is intuitively appealing, as the entry mode decision is likely to be made a few years prior to the time of entry. Using the aggregate data set, the equations are estimated using the three possible lags. A one-year lag provides the best fit to the data,

\footnotetext{
${ }^{36}$ Lagging is not conducted for the models using Hofstede data as this data is not collected across time.
} 
based on the adjusted $\mathrm{R}^{2}$ statistic. For consistency purposes, the lag 1 model specifications are then adopted across the three geographical sub-groupings (Australia/New Zealand, UK/Ireland and US/Canada).

A collection of control variables is included in the regression models. In addition to the basic five variables outlined in Chapter 5 (age, size, presence of an offshore education policy, internationalisation at home policy and host country's demand for overseas higher education opportunities; see Section 5.3.4), other control variables addressed in the survey include offshore engagement objectives (e.g. financial gain, investment opportunity, internationalisation, etc), types of offshore education policy (e.g. quality assurance policy, teaching and learning policy, business risks, etc), and the extent of anticipated future offshore engagement. Because the extra control variables do not add marginal explanatory power to the various models, only the five original control variables are retained in the models.

Three correlated measures of size are captured in the survey: numbers of undergraduate students, postgraduate students and academic staff (see question $\mathrm{N}$ in Appendix $\mathrm{M}$ ). The latter measure of size is retained in the models, as it provides the best fit to the data. Retaining the size measure pertaining to staff is intuitively appealing, as academics constitute a key resource in the provision of education services. Without them, offshore engagement cannot take place (see Chapter 6).

As indicated in section 5.4, OLS regression is used to estimate initial results. However, because the dependent equity stake variable employed in this study is censored - it can only assume values between 0 and 100 (see table 7.6a) - and OLS with a censored dependent variable tends to yield coefficient estimators that are biased toward zero (e.g. Rose and Ito, 2004), Tobit regression is also used, to assess the robustness of the OLS findings. Similarly, because the dependent equity stake variable is measured as a discrete, ordinal variable, which effectively represent a count variable, the models are also estimated using negative binomial regression. Using these three different estimation approaches allows for an assessment of the robustness of the results. 
A cut-off VIF score of 2, with some discretion for values of up to 3, is used in assessing multicollinearity. When a variable with a VIF of greater than 2 is retained in a model, robustness of the results is assessed to ensure that distortion of the observed significance levels did not take place. The highest VIF obtained in each of the models is presented in the tables below. Residual analysis is also conducted and the plots of standardised residuals against standardised predictions do not indicate any evidence of heteroscedasticity or lack of independence.

The section below presents the findings from the modelling. While the presented results are for the home country groupings, other possible stratifications of the data on the basis of the descriptive elements captured in the survey (e.g. academic rankings) are also examined. The results obtained are similar to these of the home country stratification. Therefore, because of space constraints, only the results for the home country data split are presented.

\subsubsection{Testing H1-H6 (excluding H4)}

As a result of multicollinearity among the variables of transfer experience, know-how and organisational culture, equations 1-3 (see tables 7.10-7.12) include each of these three variables separately. The $\mathrm{R}^{2}$ values for equations $1-3$ range between 0.42 and 0.69 , indicating reasonable explanatory power. The offshore policy control variable adds explanatory power (at least $p<0.10$ ) to the aggregate and US/CA grouping across equations 1-3 (OLS, Tobit, negative binomial in tables 7.10, 7.11 and 7.12). In the UK/IR grouping, the coefficient associated with offshore policy is significant (at least $p$ $<0.10$ ) across equations 1-3 for the OLS and Tobit models, but not the negative binomial. In the AU/NZ grouping, offshore policy contributes significant exploratory power $(p<0.05)$ only in equation 1 (Tobit in table 7.10). Age and host country demand for overseas education opportunities are the only other control variables which add explanatory power to equations $1-3$. Age contributes $(p<0.05)$ only in equation 1 , for the UK/IR OLS estimation. The coefficient associated with host country demand is significant in the full sample (Tobit in table 7.10), AU/NZ (Tobit in table 7.11; OLS and Tobit in table 7.12), and UK/IR (OLS in table 7.10). 
H1a, which pertains to geographic experience, receives no support across any of the full samples tested in equations 1-3. Some support $(p<0.10)$ is received for: AU/NZ (OLS in table 7.10), US/CA (negative binomial in table 7.10, OLS and Tobit in table 7.11, Tobit in table 7.12), UK/IR (OLS in tables 7.10 and 7.11). Support $(p<0.05$ ) is also received for: AU/NZ (OLS and Tobit in table 7.12), US/CA (Tobit in table 7.10, OLS and Tobit in table 7.12). Of the five aspects of geographic experience tested (i.e. previous knowledge of the education sector in the region of interest, academic/ managerial/financial capabilities for international expansion in the region of interest and previous international student recruitment experience in the region of interest), only previous knowledge of the education sector does not demonstrate a significant relationship with equity stake ${ }^{37}$.

H1b, with respect to industry experience, receives some support $(p<0.10)$ for: full sample (OLS and Tobit in table 7.10, Tobit in table 7.11), AU/NZ (Tobit in table 7.10), UK/IR (Tobit in table 7.12). H1b receives stronger support $(p<0.05)$ for the full sample (OLS in table 7.11) and US/CA (Tobit in table 7.10). Of the different aspects of industry experience examined, previous practice in offering offshore programmes at the undergraduate and graduate levels and previous experience in marketing offshore education programmes to graduate students do not demonstrate significant relationships with equity. Only industry experience with respect to recruitment of undergraduate and postgraduate students and previous experience in marketing offshore education programmes to undergraduate students provide support for H1b (at least $p<0.10$ ).

H1c, pertaining to transfer experience, is strongly supported $(p<0.01)$ in table 7.10, for the OLS models using the full sample and all three regional sub-samples.

Similarly, H2, regarding know-how, is not supported $(p<0.01)$ in all of the models except for the UK/IR negative binomial (see table 7.11).

\footnotetext{
${ }^{37}$ As discussed in Chapter 5 , the entry mode dependant variable in this study is measured as the local university's equity stake for a single overseas investment (see Section 5.3.1)
} 
Furthermore, the hypothesised relationship between organisational culture and equity stake (H3) is not supported, with very strong coefficients $(p<0.01)$ observed for a negative relationship obtained across the full sample, and the AU/NZ and US/CA subsamples. Similarly, at least some support $(p<0.10)$ for a negative relationship is found in the UK/IR group (see table 7.12).

$\mathrm{H} 5$, with respect to financial resources, is only partially supported $(p<0.10)$ for the full sample in equation 2 (OLS in table 7.11).

Finally, H6, dealing with reputation, is not supported. Strong coefficients $(p<0.01)$ are found for a negative relationship between reputation and equity stake in the full sample (Tobit and negative binomial in table 7.10). Support $(p<0.05)$ is also found for this negative relationship in the UK/IR subsample (Tobit in table 7.10). Similarly, partial support $(p<0.10)$ for a negative relationship between reputation and equity stake is found in the AU/NZ (Tobit in table 7.10) and US/CA sub-groups (Tobit in table 7.12).

In sum, based on the modelling results shown in tables 7.10-7.12, strong support is obtained for H1c (Transfer experience). Partial support is garnered only for H1a (Geographic experience) and H1b (Industry experience). While H2 (Know-how), H3 (Organisational culture), H5 (Financial resources) and H6 (Reputation) are not supported, some interesting results are obtained with respect to H2, H3 and H6, for which the highly significant results contradict the hypothesised relationships. 
Table 7.10: Regression estimates for equation 1

\begin{tabular}{|c|c|c|c|c|c|c|c|c|c|c|c|c|}
\hline & \multicolumn{3}{|c|}{ Complete data set } & \multicolumn{3}{|c|}{ AU/NZ } & \multicolumn{3}{|c|}{ US/CA } & \multicolumn{3}{|c|}{$\overline{\text { UK/IR }}$} \\
\hline & OLS & Tobit & $\begin{array}{l}\text { Negative } \\
\text { binomial }\end{array}$ & OLS & Tobit & $\begin{array}{l}\text { Negative } \\
\text { binomial } \\
\end{array}$ & OLS & Tobit & $\begin{array}{l}\text { Negative } \\
\text { binomial }\end{array}$ & OLS & Tobit & $\begin{array}{l}\text { Negative } \\
\text { binomial }\end{array}$ \\
\hline Intercept & $\begin{array}{l}12.770 * * \\
(3.579)\end{array}$ & $\begin{array}{l}19.127 * * \\
(3.473)\end{array}$ & $\begin{array}{l}2.924 * * \\
(0.359)\end{array}$ & $\begin{array}{l}4.426 \\
(6.882)\end{array}$ & $\begin{array}{l}16.214^{*} \\
(6.823)\end{array}$ & $\begin{array}{l}2.778 * * \\
(0.924)\end{array}$ & $\begin{array}{l}14.241 * \\
(6.109)\end{array}$ & $\begin{array}{l}22.294 * * \\
(6.419)\end{array}$ & $\begin{array}{l}\text { 3.114** } \\
(0.5715)\end{array}$ & $\begin{array}{l}\begin{array}{l}17.057 \dagger \\
(6.326)\end{array} \\
\end{array}$ & $\begin{array}{l}18.629 * * \\
(4.586)\end{array}$ & $\begin{array}{l}2.910 * * \\
(0.9756)\end{array}$ \\
\hline $\begin{array}{l}\text { Geo exp - knowledge } \\
\text { of edu sector }(H I a)\end{array}$ & $\begin{array}{l}0.010 \\
(0.137)\end{array}$ & $\begin{array}{l}-0.142 \\
(0.137)\end{array}$ & $\begin{array}{l}-0.010 \\
(0.013)\end{array}$ & $\begin{array}{l}0.487 \\
(0.303)\end{array}$ & $\begin{array}{l}0.376 \\
(0.306)\end{array}$ & $\begin{array}{l}0.020 \\
(0.042)\end{array}$ & $\begin{array}{l}0.084 \\
(0.221)\end{array}$ & $\begin{array}{l}-0.099 \\
(0.235)\end{array}$ & $\begin{array}{l}-0.006 \\
(0.020)\end{array}$ & $\begin{array}{l}-0.259 \\
(0.253)\end{array}$ & $\begin{array}{l}-0.289 \\
(0.182)\end{array}$ & $\begin{array}{l}-0.018 \\
(0.397)\end{array}$ \\
\hline $\begin{array}{l}\text { Geo exp - academic } \\
\text { capabilities }(H 1 a)\end{array}$ & $\begin{array}{l}0.064 \\
(0.131)\end{array}$ & $\begin{array}{l}0.090 \\
(0.134)\end{array}$ & $\begin{array}{l}0.006 \\
(0.012)\end{array}$ & $\begin{array}{l}0.522 \dagger \\
(0.297)\end{array}$ & $\begin{array}{l}0.079 \\
(0.303)\end{array}$ & $\begin{array}{l}0.005 \\
(0.037)\end{array}$ & $\begin{array}{l}-0.023 \\
(0.221)\end{array}$ & $\begin{array}{l}0.172 \\
(0.236)\end{array}$ & $\begin{array}{l}0.011 \\
(0.018)\end{array}$ & $\begin{array}{l}0.079 \\
(0.220)\end{array}$ & $\begin{array}{l}-0.017 \\
(0.169)\end{array}$ & $\begin{array}{l}-0.0003 \\
(0.032)\end{array}$ \\
\hline $\begin{array}{l}\text { Geo exp - managerial } \\
\text { capabilities (H1a) }\end{array}$ & $\begin{array}{l}0.122 \\
(0.158)\end{array}$ & $\begin{array}{l}0.051 \\
(0.164)\end{array}$ & $\begin{array}{l}0.003 \\
(0.017)\end{array}$ & $\begin{array}{l}0.005 \\
(0.348)\end{array}$ & $\begin{array}{l}0.137 \\
(0.356)\end{array}$ & $\begin{array}{l}0.009 \\
(0.054)\end{array}$ & $\begin{array}{l}0.324 \\
(0.256)\end{array}$ & $\begin{array}{l}0.102 \\
(0.276)\end{array}$ & $\begin{array}{l}0.005 \\
(0.024)\end{array}$ & $\begin{array}{l}-0.094 \\
(0.273)\end{array}$ & $\begin{array}{l}-0.090 \\
(0.211)\end{array}$ & $\begin{array}{l}-0.006 \\
(0.044)\end{array}$ \\
\hline $\begin{array}{l}\text { Geo exp - financial } \\
\text { capabilities }(H 1 a)\end{array}$ & $\begin{array}{l}0.000 \\
(0.146)\end{array}$ & $\begin{array}{l}0.034 \\
(0.151)\end{array}$ & $\begin{array}{l}0.002 \\
(0.015)\end{array}$ & $\begin{array}{l}-0.117 \\
(0.335)\end{array}$ & $\begin{array}{l}-0.289 \\
(0.319)\end{array}$ & $\begin{array}{l}-0.017 \\
(0.404)\end{array}$ & $\begin{array}{l}0.329 \\
(0.226)\end{array}$ & $\begin{array}{l}0.285 \\
(0.245)\end{array}$ & $\begin{array}{l}0.020 \\
(0.023)\end{array}$ & $\begin{array}{l}-0.474 \dagger \\
(0.265)\end{array}$ & $\begin{array}{l}-0.282 \\
(0.210)\end{array}$ & $\begin{array}{l}-0.017 \\
(0.039)\end{array}$ \\
\hline $\begin{array}{l}\text { Geo exp - intl } \\
\text { recruitment (H1a) }\end{array}$ & $\begin{array}{l}-0.056 \\
(0.163)\end{array}$ & $\begin{array}{l}-0.175 \\
(0.169)\end{array}$ & $\begin{array}{l}-0.011 \\
(0.015)\end{array}$ & $\begin{array}{l}0.492 \dagger \\
(0.289)\end{array}$ & $\begin{array}{l}0.226 \\
(0.320)\end{array}$ & $\begin{array}{l}0.013 \\
(0.039)\end{array}$ & $\begin{array}{l}-0.409 \\
(0.287)\end{array}$ & $\begin{array}{l}-0.692^{*} \\
(0.299)\end{array}$ & $\begin{array}{l}-0.047 \dagger \\
(0.025)\end{array}$ & $\begin{array}{l}-0.010 \\
(0.311)\end{array}$ & $\begin{array}{l}0.109 \\
(0.228)\end{array}$ & $\begin{array}{l}0.007 \\
(0.048)\end{array}$ \\
\hline $\begin{array}{l}\text { Ind exp - UG offshore } \\
\text { edu }(H 1 b)\end{array}$ & $\begin{array}{l}0.071 \\
(0.126)\end{array}$ & $\begin{array}{l}0.073 \\
(0.131)\end{array}$ & $\begin{array}{l}0.005 \\
(0.012)\end{array}$ & $\begin{array}{l}-0.268 \\
(0.256)\end{array}$ & $\begin{array}{l}-0.080 \\
(0.280)\end{array}$ & $\begin{array}{l}-0.005 \\
(0.033)\end{array}$ & $\begin{array}{l}0.095 \\
(0.228)\end{array}$ & $\begin{array}{l}0.112 \\
(0.244)\end{array}$ & $\begin{array}{l}0.010 \\
(0.023)\end{array}$ & $\begin{array}{l}0.017 \\
(0.208)\end{array}$ & $\begin{array}{l}-0.004 \\
(0.168)\end{array}$ & $\begin{array}{l}-0.0009 \\
(0.033)\end{array}$ \\
\hline $\begin{array}{l}\text { Ind exp - PG offshore } \\
\text { edu }(H 1 b)\end{array}$ & $\begin{array}{l}0.034 \\
(0.144)\end{array}$ & $\begin{array}{l}0.039 \\
(0.149)\end{array}$ & $\begin{array}{l}0.002 \\
(0.013)\end{array}$ & $\begin{array}{l}0.062 \\
(0.302)\end{array}$ & $\begin{array}{l}-0.042 \\
(0.316)\end{array}$ & $\begin{array}{l}-0.002 \\
(0.037)\end{array}$ & $\begin{array}{l}0.010 \\
(0.966)\end{array}$ & $\begin{array}{l}-0.032 \\
(0.246)\end{array}$ & $\begin{array}{l}-0.003 \\
(0.019)\end{array}$ & $\begin{array}{l}0.157 \\
(0.256)\end{array}$ & $\begin{array}{l}-0.031 \\
(0.212)\end{array}$ & $\begin{array}{l}-0.002 \\
(0.049)\end{array}$ \\
\hline $\begin{array}{l}\text { Ind exp - intl } \\
\text { recruitment (Factor) } \\
(\text { H1b) }\end{array}$ & $\begin{array}{l}0.297 \dagger \\
(0.166)\end{array}$ & $\begin{array}{l}0.408 \dagger \\
(0.163)\end{array}$ & $\begin{array}{l}0.025 \\
(0.017)\end{array}$ & $\begin{array}{l}0.246 \\
(0.346)\end{array}$ & $\begin{array}{l}0.471 \\
(0.343)\end{array}$ & $\begin{array}{l}0.027 \\
(0.042)\end{array}$ & $\begin{array}{l}0.425 \\
(0.287)\end{array}$ & $\begin{array}{l}0.648 * \\
(0.291)\end{array}$ & $\begin{array}{l}0.043 \\
(0.030)\end{array}$ & $\begin{array}{l}0.367 \\
(0.305)\end{array}$ & $\begin{array}{l}0.250 \\
(0.212)\end{array}$ & $\begin{array}{l}0.015 \\
(0.041)\end{array}$ \\
\hline $\begin{array}{l}\text { Ind exp - UG } \\
\text { marketing }(H 1 b)\end{array}$ & $\begin{array}{l}-0.063 \\
(0.130)\end{array}$ & $\begin{array}{l}-0.042 \\
(0.130)\end{array}$ & $\begin{array}{l}-0.003 \\
(0.014)\end{array}$ & $\begin{array}{l}-0.366 \\
(0.330)\end{array}$ & $\begin{array}{l}-0.632 \dagger \\
(0.335)\end{array}$ & $\begin{array}{l}-0.037 \\
(0.039)\end{array}$ & $\begin{array}{l}-0.179 \\
(0.199)\end{array}$ & $\begin{array}{l}0.096 \\
(0.210)\end{array}$ & $\begin{array}{l}0.007 \\
(0.018)\end{array}$ & $\begin{array}{l}0.096 \\
(0.227)\end{array}$ & $\begin{array}{l}0.126 \\
(0.163)\end{array}$ & $\begin{array}{l}0.007 \\
(0.039)\end{array}$ \\
\hline $\begin{array}{l}\text { Ind exp - PG } \\
\text { marketing }(H 1 b)\end{array}$ & $\begin{array}{l}0.074 \\
(0.124)\end{array}$ & $\begin{array}{l}0.028 \\
(0.126)\end{array}$ & $\begin{array}{l}0.002 \\
(0.012)\end{array}$ & $\begin{array}{l}0.335 \\
(0.244)\end{array}$ & $\begin{array}{l}0.101 \\
(0.265)\end{array}$ & $\begin{array}{l}0.005 \\
(0.029)\end{array}$ & $\begin{array}{l}-0.132 \\
(0.208)\end{array}$ & $\begin{array}{l}-0.142 \\
(0.208)\end{array}$ & $\begin{array}{l}-0.009 \\
(0.018)\end{array}$ & $\begin{array}{l}0.366 \\
(0.219)\end{array}$ & $\begin{array}{l}0.176 \\
(0.173)\end{array}$ & $\begin{array}{l}0.010 \\
(0.036)\end{array}$ \\
\hline $\begin{array}{l}\text { Transfer experience } \\
(\text { H1c) }\end{array}$ & $\begin{array}{l}-7.897 * * \\
(0.695)\end{array}$ & $\begin{array}{l}-0.0005 \\
(0.0005)\end{array}$ & $\begin{array}{l}-0.294 \mathrm{E}-4 \\
(0.70 \mathrm{E}-4)\end{array}$ & $\begin{array}{l}-10.827^{* *} \\
(1.544)\end{array}$ & $\begin{array}{l}-0.001 \\
(0.001)\end{array}$ & $\begin{array}{l}-0.720 \mathrm{E}-4 \\
(0.0002)\end{array}$ & $\begin{array}{l}-9.013^{* *} \\
(1.158)\end{array}$ & $\begin{array}{l}-0.001 \\
(0.001)\end{array}$ & $\begin{array}{l}-0.798 \mathrm{E}-4 \\
(0.0001)\end{array}$ & $\begin{array}{l}-4.576 * * \\
(1.385)\end{array}$ & $\begin{array}{l}0.945 \mathrm{E}-4 \\
(0.0007)\end{array}$ & $\begin{array}{l}0.551 \mathrm{E}-6 \\
(0.0001)\end{array}$ \\
\hline $\begin{array}{l}\text { Financial resources } \\
\text { (H5) }\end{array}$ & $\begin{array}{l}0.009 \\
(0.113)\end{array}$ & $\begin{array}{l}-0.085 \\
(0.110)\end{array}$ & $\begin{array}{l}-0.006 \\
(0.011)\end{array}$ & $\begin{array}{l}0.451 \\
(0.280)\end{array}$ & $\begin{array}{l}-0.088 \\
(0.259)\end{array}$ & $\begin{array}{l}-0.0046 \\
(0.030)\end{array}$ & $\begin{array}{l}0.104 \\
(0.176)\end{array}$ & $\begin{array}{l}-0.026 \\
(0.170)\end{array}$ & $\begin{array}{l}-0.004 \\
(0.015)\end{array}$ & $\begin{array}{l}-0.066 \\
(0.200)\end{array}$ & $\begin{array}{l}-0.059 \\
(0.152)\end{array}$ & $\begin{array}{l}-0.004 \\
(0.034)\end{array}$ \\
\hline $\begin{array}{l}\text { Reputation (Factor) - } \\
\text { (H6) }\end{array}$ & $\begin{array}{l}-0.309 \\
(0.235)\end{array}$ & $\begin{array}{l}-1.512^{* *} \\
(0.213)\end{array}$ & $\begin{array}{l}-0.094 * * \\
(0.021)\end{array}$ & $\begin{array}{l}0.184 \\
(0.504)\end{array}$ & $\begin{array}{l}-0.996 \dagger \\
(0.524)\end{array}$ & $\begin{array}{l}-0.061 \\
(0.067)\end{array}$ & $\begin{array}{l}-0.403 \\
(0.424)\end{array}$ & $\begin{array}{l}-2.364^{* *} \\
(0.352)\end{array}$ & $\begin{array}{l}-0.161 * * \\
(0.032)\end{array}$ & $\begin{array}{l}-0.479 \\
(0.391)\end{array}$ & $\begin{array}{l}-0.669 * \\
(0.289)\end{array}$ & $\begin{array}{l}-0.040 \\
(0.055)\end{array}$ \\
\hline $\begin{array}{l}\text { IMD Normative ID } \\
\text { (lag 1) }\end{array}$ & $\begin{array}{l}-0.213 \\
(0.202)\end{array}$ & $\begin{array}{l}-0.048 \\
(0.185)\end{array}$ & $\begin{array}{l}-0.002 \\
(0.018)\end{array}$ & $\begin{array}{l}-0.290 \\
(0.337)\end{array}$ & $\begin{array}{l}0.152 \\
(0.354)\end{array}$ & $\begin{array}{l}0.007 \\
(0.043)\end{array}$ & $\begin{array}{l}-0.580 \\
(0.351)\end{array}$ & $\begin{array}{l}-0.336 \\
(0.312)\end{array}$ & $\begin{array}{l}-0.020 \\
(0.027)\end{array}$ & $\begin{array}{l}-0.333 \\
(0.359)\end{array}$ & $\begin{array}{l}0.143 \\
(0.249)\end{array}$ & $\begin{array}{l}0.009 \\
(0.058)\end{array}$ \\
\hline $\begin{array}{l}\text { IMD Regulative ID } \\
\text { (lag 1) }\end{array}$ & $\begin{array}{l}0.154 \\
(0.235)\end{array}$ & $\begin{array}{l}0.044 \\
(0.185)\end{array}$ & $\begin{array}{l}0.001 \\
(0.020)\end{array}$ & - & - & - & $\begin{array}{l}0.254 \\
(0.398)\end{array}$ & $\begin{array}{l}0.331 \\
(0.312)\end{array}$ & $\begin{array}{l}0.019 \\
(0.031)\end{array}$ & $\begin{array}{l}-0.048 \\
(0.564)\end{array}$ & $\begin{array}{l}-0.380 \\
(0.353)\end{array}$ & $\begin{array}{l}-0.023 \\
(0.085)\end{array}$ \\
\hline
\end{tabular}




\begin{tabular}{|c|c|c|c|c|c|c|c|c|c|c|c|c|}
\hline Age & $\begin{array}{l}0.005 \\
(0.004)\end{array}$ & $\begin{array}{l}0.003 \\
(0.004)\end{array}$ & $\begin{array}{l}0.0002 \\
(0.0004)\end{array}$ & $\begin{array}{l}-0.005 \\
(0.013)\end{array}$ & $\begin{array}{l}-0.0001 \\
(0.012)\end{array}$ & $\begin{array}{l}-0.383 E-4 \\
(0.002)\end{array}$ & $\begin{array}{l}0.004 \\
(0.006)\end{array}$ & $\begin{array}{l}0.003 \\
(0.007)\end{array}$ & $\begin{array}{l}0.0003 \\
(0.0006)\end{array}$ & $\begin{array}{l}\text { 0.011* } \\
(0.006)\end{array}$ & $\begin{array}{l}0.004 \\
(0.004)\end{array}$ & $\begin{array}{l}0.0002 \\
(0.0008)\end{array}$ \\
\hline Size (Staff) & $\begin{array}{l}8.447 \mathrm{E}-5 \\
(0.000)\end{array}$ & $\begin{array}{l}0.0002 \\
(0.0002)\end{array}$ & $\begin{array}{l}0.119 E-4 \\
(0.168 E-4)\end{array}$ & - & - & - & $\begin{array}{l}0.000 \\
(0.000)\end{array}$ & $\begin{array}{l}0.876 \mathrm{E}-4 \\
(0.0003)\end{array}$ & $\begin{array}{l}0.710 \mathrm{E}-5 \\
(0.239 \mathrm{E}-4)\end{array}$ & $\begin{array}{l}9.917 \mathrm{E}-5 \\
(0.000)\end{array}$ & $\begin{array}{l}0.0001 \\
(0.0002)\end{array}$ & $\begin{array}{l}0.680 \mathrm{E}-5 \\
(0.485 \mathrm{E}-4)\end{array}$ \\
\hline Offshore policy & $\begin{array}{l}1.981 * * \\
(0.717)\end{array}$ & $\begin{array}{l}3.698 * * \\
(0.775)\end{array}$ & $\begin{array}{l}0.278 * * \\
(0.046)\end{array}$ & $\begin{array}{l}2.040 \\
(1.463)\end{array}$ & $\begin{array}{l}4.085^{*} \\
(1.627)\end{array}$ & $\begin{array}{l}0.269 \\
(0.198)\end{array}$ & $\begin{array}{l}1.908 \dagger \\
(1.130)\end{array}$ & $\begin{array}{l}4.391 * * \\
(1.220)\end{array}$ & $\begin{array}{l}0.380 * * \\
(0.071)\end{array}$ & $\begin{array}{l}2.583 \dagger \\
(1.435)\end{array}$ & $\begin{array}{l}3.490 * * \\
(1.205)\end{array}$ & $\begin{array}{l}0.231 \\
(0.189)\end{array}$ \\
\hline $\begin{array}{l}\text { Internationalisation at } \\
\text { home strategy }\end{array}$ & $\begin{array}{l}0.074 \\
(0.147)\end{array}$ & $\begin{array}{l}0.930 \mathrm{E}-4 \\
(0.001)\end{array}$ & $\begin{array}{l}0.117 \mathrm{E}-4 \\
(0.0001)\end{array}$ & $\begin{array}{l}-0.011 \\
(0.365)\end{array}$ & $\begin{array}{l}0.564 \mathrm{E}-4 \\
(0.002)\end{array}$ & $\begin{array}{l}0.143 \mathrm{E}-4 \\
(0.0003)\end{array}$ & $\begin{array}{l}-0.074 \\
(0.229)\end{array}$ & $\begin{array}{l}0.0002 \\
(0.003)\end{array}$ & $\begin{array}{l}0.249 \mathrm{E}-4 \\
(0.0002)\end{array}$ & $\begin{array}{l}0.086 \\
(0.271)\end{array}$ & $\begin{array}{l}0.0008 \\
(0.0009)\end{array}$ & $\begin{array}{l}0.493 \mathrm{E}-4 \\
(0.0002)\end{array}$ \\
\hline Host country demand & $\begin{array}{l}2.208 \\
(2.122)\end{array}$ & $\begin{array}{l}4.085 \dagger \\
(2.145)\end{array}$ & $\begin{array}{l}0.241 \\
(0.210)\end{array}$ & $\begin{array}{l}2.992 \\
(4.162)\end{array}$ & $\begin{array}{l}6.282 \\
(4.239)\end{array}$ & $\begin{array}{l}0.352 \\
(0.621)\end{array}$ & $\begin{array}{l}-1.384 \\
(-0.028)\end{array}$ & $\begin{array}{l}2.618 \\
(3.537)\end{array}$ & $\begin{array}{l}0.150 \\
(0.300)\end{array}$ & $\begin{array}{l}8.371 \dagger \\
(4.656)\end{array}$ & $\begin{array}{l}3.088 \\
(3.221)\end{array}$ & $\begin{array}{l}0.169 \\
(0.678)\end{array}$ \\
\hline AU/NZ dummy & $\begin{array}{l}0.240 \\
(0.612)\end{array}$ & $\begin{array}{l}0.602 \\
(0.618)\end{array}$ & $\begin{array}{l}0.039 \\
(0.059)\end{array}$ & - & - & - & - & - & - & - & - & - \\
\hline UK/IR dummy & $\begin{array}{l}0.287 \\
(0.552)\end{array}$ & $\begin{array}{l}0.849 \\
(0.562)\end{array}$ & $\begin{array}{l}0.052 \\
(0.056)\end{array}$ & - & - & - & - & - & - & - & - & - \\
\hline $\mathrm{R}^{2}$ & 0.564 & & & 0.634 & & & 0.695 & & & 0.422 & & \\
\hline $\operatorname{AdjR}^{2}$ & 0.522 & & & 0.496 & & & 0.623 & & & 0.227 & & \\
\hline Log likelihood & & -815.69 & -863.99 & & -211.69 & -224.98 & & -334.87 & -358.36 & & -241.36 & -265.09 \\
\hline $\mathrm{N}$ & 308 & 308 & 308 & 80 & 80 & 80 & 124 & 124 & 124 & 104 & 104 & 104 \\
\hline Max. VIF & 2.064 & & & 2.361 & & & 2.528 & & & 2.182 & & \\
\hline
\end{tabular}

Standard errors in parentheses, all $t$ tests are two-tailed, $\uparrow p<.10,{ }^{*} p<.05$, ${ }^{* *} p<.01$

Table 7.11: Regression estimates for equation 2

\begin{tabular}{|c|c|c|c|c|c|c|c|c|c|c|c|c|}
\hline & \multicolumn{3}{|c|}{ Complete data set } & \multicolumn{3}{|c|}{ AU/NZ } & \multicolumn{3}{|c|}{ US/CA } & \multicolumn{3}{|c|}{ UK/IR } \\
\hline & OLS & Tobit & $\begin{array}{l}\text { Negative } \\
\text { binomial }\end{array}$ & OLS & Tobit & $\begin{array}{l}\text { Negative } \\
\text { binomial }\end{array}$ & OLS & Tobit & $\begin{array}{l}\text { Negative } \\
\text { binomial }\end{array}$ & OLS & Tobit & $\begin{array}{l}\text { Negative } \\
\text { binomial }\end{array}$ \\
\hline Intercept & $\begin{array}{l}17.740 * * \\
(3.024)\end{array}$ & $\begin{array}{l}18.834 * * \\
(2.720)\end{array}$ & $\begin{array}{l}3.026 * * \\
(0.396)\end{array}$ & $\begin{array}{l}13.093^{*} \\
(6.238)\end{array}$ & $\begin{array}{l}16.666 * * \\
(5.153)\end{array}$ & $\begin{array}{l}2.896 * * \\
(0.877)\end{array}$ & $\begin{array}{l}18.096 * \\
(5.552)\end{array}$ & $\begin{array}{l}17.597 * * \\
(4.877)\end{array}$ & $\begin{array}{l}2.954 * * \\
(0.761)\end{array}$ & $\begin{array}{l}20.573 * * \\
(4.647)\end{array}$ & $\begin{array}{l}19.943 * * \\
(3.898)\end{array}$ & $\begin{array}{l}3.050 * * \\
(1.106)\end{array}$ \\
\hline $\begin{array}{l}\text { Geo exp - } \\
\text { knowledge of edu } \\
\text { sector (HIa) }\end{array}$ & $\begin{array}{l}-0.038 \\
(0.116)\end{array}$ & $\begin{array}{l}-0.047 \\
(0.108)\end{array}$ & $\begin{array}{l}-0.004 \\
(0.016)\end{array}$ & $\begin{array}{l}0.415 \\
(0.279)\end{array}$ & $\begin{array}{l}0.363 \\
(0.233)\end{array}$ & $\begin{array}{l}0.020 \\
(0.046)\end{array}$ & $\begin{array}{l}-0.057 \\
(0.198)\end{array}$ & $\begin{array}{l}-0.043 \\
(0.178)\end{array}$ & $\begin{array}{l}-0.004 \\
(0.027)\end{array}$ & $\begin{array}{l}-0.195 \\
(0.190)\end{array}$ & $\begin{array}{l}-0.168 \\
(0.156)\end{array}$ & $\begin{array}{l}-0.011 \\
(0.048)\end{array}$ \\
\hline $\begin{array}{l}\text { Geo exp - academic } \\
\text { capabilities (H1a) }\end{array}$ & $\begin{array}{l}0.011 \\
(0.113)\end{array}$ & $\begin{array}{l}-0.023 \\
(0.105)\end{array}$ & $\begin{array}{l}-0.003 \\
(0.014)\end{array}$ & $\begin{array}{l}0.228 \\
(0.263)\end{array}$ & $\begin{array}{l}0.189 \\
(0.231)\end{array}$ & $\begin{array}{l}0.006 \\
(0.042)\end{array}$ & $\begin{array}{l}0.041 \\
(0.196)\end{array}$ & $\begin{array}{l}0.022 \\
(0.177)\end{array}$ & $\begin{array}{l}0.002 \\
(0.025)\end{array}$ & $\begin{array}{l}-0.067 \\
(0.170)\end{array}$ & $\begin{array}{l}-0.081 \\
(0.144)\end{array}$ & $\begin{array}{l}-0.005 \\
(0.036)\end{array}$ \\
\hline $\begin{array}{l}\text { Geo exp - } \\
\text { managerial } \\
\text { capabilities }(H 1 a)\end{array}$ & $\begin{array}{l}0.069 \\
(0.135)\end{array}$ & $\begin{array}{l}0.078 \\
(0.128)\end{array}$ & $\begin{array}{l}0.005 \\
(0.018)\end{array}$ & $\begin{array}{l}-0.040 \\
(0.306)\end{array}$ & $\begin{array}{l}-0.016 \\
(0.269)\end{array}$ & $\begin{array}{l}0.0008 \\
(0.048)\end{array}$ & $\begin{array}{l}0.334 \\
(0.231)\end{array}$ & $\begin{array}{l}0.350 \dagger \\
(0.210)\end{array}$ & $\begin{array}{l}0.022 \\
(0.031)\end{array}$ & $\begin{array}{l}-0.068 \\
(0.203)\end{array}$ & $\begin{array}{l}-0.030 \\
(0.178)\end{array}$ & $\begin{array}{l}-0.003 \\
(0.045)\end{array}$ \\
\hline
\end{tabular}




\begin{tabular}{|c|c|c|c|c|c|c|c|c|c|c|c|c|}
\hline $\begin{array}{l}\text { Geo exp - financial } \\
\text { capabilities }(H 1 a)\end{array}$ & $\begin{array}{l}0.055 \\
(0.128)\end{array}$ & $\begin{array}{l}0.022 \\
(0.118)\end{array}$ & $\begin{array}{l}0.0009 \\
(0.016)\end{array}$ & $\begin{array}{l}-0.055 \\
(0.306)\end{array}$ & $\begin{array}{l}-0.207 \\
(0.243)\end{array}$ & $\begin{array}{l}-0.013 \\
(0.043)\end{array}$ & $\begin{array}{l}0.326 \\
(0.206)\end{array}$ & $\begin{array}{l}0.305 \dagger \\
(0.185)\end{array}$ & $\begin{array}{l}0.021 \\
(0.025)\end{array}$ & $\begin{array}{l}-0.378 \dagger \\
(0.210)\end{array}$ & $\begin{array}{l}-0.288 \\
(0.179)\end{array}$ & $\begin{array}{l}-0.020 \\
(0.048)\end{array}$ \\
\hline $\begin{array}{l}\text { Geo exp - intl } \\
\text { recruitment (H1a) }\end{array}$ & $\begin{array}{l}-0.074 \\
(0.142)\end{array}$ & $\begin{array}{l}-0.049 \\
(0.133)\end{array}$ & $\begin{array}{l}-0.002 \\
(0.018)\end{array}$ & $\begin{array}{l}-0.055 \\
(0.306)\end{array}$ & $\begin{array}{l}0.331 \\
(0.243)\end{array}$ & $\begin{array}{l}0.022 \\
(0.045)\end{array}$ & $\begin{array}{l}-0.449 \dagger \\
(0.254)\end{array}$ & $\begin{array}{l}-0.396 \dagger \\
(0.227)\end{array}$ & $\begin{array}{l}-0.029 \\
(0.034)\end{array}$ & $\begin{array}{l}0.043 \\
(0.226)\end{array}$ & $\begin{array}{l}0.095 \\
(0.194)\end{array}$ & $\begin{array}{l}0.008 \\
(0.056)\end{array}$ \\
\hline $\begin{array}{l}\text { Ind exp - UG } \\
\text { offshore edu (H1b) }\end{array}$ & $\begin{array}{l}0.073 \\
(0.109)\end{array}$ & $\begin{array}{l}0.063 \\
(0.103)\end{array}$ & $\begin{array}{l}0.005 \\
(0.014)\end{array}$ & $\begin{array}{l}0.315 \\
(0.276)\end{array}$ & $\begin{array}{l}-0.178 \\
(0.214)\end{array}$ & $\begin{array}{l}-0.010 \\
(0.032)\end{array}$ & $\begin{array}{l}0.033 \\
(0.204)\end{array}$ & $\begin{array}{l}0.033 \\
(0.184)\end{array}$ & $\begin{array}{l}0.005 \\
(0.027)\end{array}$ & $\begin{array}{l}0.023 \\
(0.165)\end{array}$ & $\begin{array}{l}0.076 \\
(0.144)\end{array}$ & $\begin{array}{l}0.004 \\
(0.036)\end{array}$ \\
\hline $\begin{array}{l}\text { Ind exp - PG } \\
\text { offshore edu (H1b) }\end{array}$ & $\begin{array}{l}-0.054 \\
(0.124)\end{array}$ & $\begin{array}{l}-0.050 \\
(0.116)\end{array}$ & $\begin{array}{l}-0.004 \\
(0.016)\end{array}$ & $\begin{array}{l}-0.104 \\
(0.247)\end{array}$ & $\begin{array}{l}0.199 \\
(0.243)\end{array}$ & $\begin{array}{l}0.012 \\
(0.040)\end{array}$ & $\begin{array}{l}-0.089 \\
(0.206)\end{array}$ & $\begin{array}{l}-0.078 \\
(0.186)\end{array}$ & $\begin{array}{l}-0.007 \\
(0.026)\end{array}$ & $\begin{array}{l}-0.184 \\
(0.208)\end{array}$ & $\begin{array}{l}-0.241 \\
(0.181)\end{array}$ & $\begin{array}{l}-0.015 \\
(0.051)\end{array}$ \\
\hline $\begin{array}{l}\text { Ind exp - intl } \\
\text { recruitment (Factor) } \\
\text { (H1b) }\end{array}$ & $\begin{array}{l}0.281^{*} \\
(0.142)\end{array}$ & $\begin{array}{l}0.230 \dagger \\
(0.129)\end{array}$ & $\begin{array}{l}0.015 \\
(0.019)\end{array}$ & $\begin{array}{l}0.369 \\
(0.317)\end{array}$ & $\begin{array}{l}0.188 \\
(0.264)\end{array}$ & $\begin{array}{l}0.014 \\
(0.046)\end{array}$ & $\begin{array}{l}0.253 \\
(0.252)\end{array}$ & $\begin{array}{l}0.191 \\
(0.224)\end{array}$ & $\begin{array}{l}0.012 \\
(0.034)\end{array}$ & $\begin{array}{l}0.357 \\
(0.228)\end{array}$ & $\begin{array}{l}0.235 \\
(0.181)\end{array}$ & $\begin{array}{l}0.015 \\
(0.043)\end{array}$ \\
\hline $\begin{array}{l}\text { Ind exp - UG } \\
\text { marketing (H1b) }\end{array}$ & $\begin{array}{l}0.015 \\
(0.109)\end{array}$ & $\begin{array}{l}0.009 \\
(0.101)\end{array}$ & $\begin{array}{l}-0.0002 \\
(0.016)\end{array}$ & $\begin{array}{l}-0.459 \\
(0.298)\end{array}$ & $\begin{array}{l}-0.370 \\
(0.258)\end{array}$ & $\begin{array}{l}-0.026 \\
(0.051)\end{array}$ & $\begin{array}{l}0.171 \\
(0.175)\end{array}$ & $\begin{array}{l}0.150 \\
(0.159)\end{array}$ & $\begin{array}{l}0.012 \\
(0.024)\end{array}$ & $\begin{array}{l}0.078 \\
(0.159)\end{array}$ & $\begin{array}{l}0.103 \\
(0.135)\end{array}$ & $\begin{array}{l}0.005 \\
(0.040)\end{array}$ \\
\hline $\begin{array}{l}\text { Ind exp - PG } \\
\text { marketing }(H 1 b)\end{array}$ & $\begin{array}{l}0.014 \\
(0.06)\end{array}$ & $\begin{array}{l}-0.035 \\
(0.099)\end{array}$ & $\begin{array}{l}-0.003 \\
(0.014)\end{array}$ & $\begin{array}{l}0.157 \\
(0.233)\end{array}$ & $\begin{array}{l}0.105 \\
(0.202)\end{array}$ & $\begin{array}{l}0.004 \\
(0.036)\end{array}$ & $\begin{array}{l}-0.078 \\
(0.174)\end{array}$ & $\begin{array}{l}-0.076 \\
(0.157)\end{array}$ & $\begin{array}{l}-0.006 \\
(0.024)\end{array}$ & $\begin{array}{l}0.137 \\
(0.178)\end{array}$ & $\begin{array}{l}0.026 \\
(0.147)\end{array}$ & $\begin{array}{l}0.001 \\
(0.042)\end{array}$ \\
\hline $\begin{array}{l}\text { Know-how (Factor) } \\
-(\mathrm{H} 2)\end{array}$ & $\begin{array}{l}-1.544 * * \\
(0.117)\end{array}$ & $\begin{array}{l}-1.538 * * \\
(0.110)\end{array}$ & $\begin{array}{l}-0.120^{* *} \\
(0.013)\end{array}$ & $\begin{array}{l}-1.765 * * \\
(0.255)\end{array}$ & $\begin{array}{l}-1.726 * * \\
(0.225)\end{array}$ & $\begin{array}{l}-0.130 * * \\
(0.034)\end{array}$ & $\begin{array}{l}-1.671 * * \\
(0.191)\end{array}$ & $\begin{array}{l}-1.651 * * \\
(0.169)\end{array}$ & $\begin{array}{l}-0.130 * * \\
(0.024)\end{array}$ & $\begin{array}{l}-1.215^{* *} \\
(0.228)\end{array}$ & $\begin{array}{l}-1.266 * * \\
(0.202)\end{array}$ & $\begin{array}{l}-0.092 \\
(0.060)\end{array}$ \\
\hline $\begin{array}{l}\text { Financial resources - } \\
\text { (H5) }\end{array}$ & $\begin{array}{l}0.006 \dagger \\
(0.095)\end{array}$ & $\begin{array}{l}0.029 \\
(0.087)\end{array}$ & $\begin{array}{l}0.458 E-4 \\
(0.012)\end{array}$ & $\begin{array}{l}0.354 \\
(0.259)\end{array}$ & $\begin{array}{l}0.287 \\
(0.204)\end{array}$ & $\begin{array}{l}0.018 \\
(0.040)\end{array}$ & $\begin{array}{l}0.069 \\
(0.146)\end{array}$ & $\begin{array}{l}0.108 \\
(0.129)\end{array}$ & $\begin{array}{l}0.004 \\
(0.020)\end{array}$ & $\begin{array}{l}-0.117 \\
(0.158)\end{array}$ & $\begin{array}{l}-0.081 \\
(0.129)\end{array}$ & $\begin{array}{l}-0.007 \\
(0.036)\end{array}$ \\
\hline $\begin{array}{l}\text { Reputation (Factor) } \\
-(\text { H6) }\end{array}$ & $\begin{array}{l}-0.233 \\
(0.207)\end{array}$ & $\begin{array}{l}-0.280 \\
(0.189)\end{array}$ & $\begin{array}{l}-0.022 \\
(0.028)\end{array}$ & $\begin{array}{l}0.044 \\
(0.481)\end{array}$ & $\begin{array}{l}-0.063 \\
(0.408)\end{array}$ & $\begin{array}{l}-0.006 \\
(0.080)\end{array}$ & $\begin{array}{l}-0.361 \\
(0.378)\end{array}$ & $\begin{array}{l}-0.391 \\
(0.335)\end{array}$ & $\begin{array}{l}-0.038 \\
(0.055)\end{array}$ & $\begin{array}{l}-0.190 \\
(0.320)\end{array}$ & $\begin{array}{l}-0.173 \\
(0.259)\end{array}$ & $\begin{array}{l}-0.011 \\
(0.069)\end{array}$ \\
\hline $\begin{array}{l}\text { IMD Normative ID } \\
\text { (lag 1) }\end{array}$ & $\begin{array}{l}-0.171 \\
(0.176)\end{array}$ & $\begin{array}{l}-0.131 \\
(0.144)\end{array}$ & $\begin{array}{l}-0.007 \\
(0.023)\end{array}$ & $\begin{array}{l}-0.198 \\
(0.318)\end{array}$ & $\begin{array}{l}-0.119 \\
(0.272)\end{array}$ & $\begin{array}{l}-0.007 \\
(0.053)\end{array}$ & $\begin{array}{l}-0.590 \dagger \\
(0.309)\end{array}$ & $\begin{array}{l}-0.468^{*} \\
(0.235)\end{array}$ & $\begin{array}{l}-0.029 \\
(0.038)\end{array}$ & $\begin{array}{l}0.100 \\
(0.281)\end{array}$ & $\begin{array}{l}0.189 \\
(0.212)\end{array}$ & $\begin{array}{l}0.012 \\
(0.066)\end{array}$ \\
\hline $\begin{array}{l}\text { IMD Regulative ID } \\
\text { (lag 1) }\end{array}$ & $\begin{array}{l}0.120 \\
(0.190)\end{array}$ & $\begin{array}{l}0.128 \\
(0.144)\end{array}$ & $\begin{array}{l}0.007 \\
(0.024)\end{array}$ & (0.010) & - & $(8000)$ & $\begin{array}{l}0.356 \\
(0.315)\end{array}$ & $\begin{array}{l}0.465 * \\
(0.235)\end{array}$ & $\begin{array}{l}0.029 \\
(0.033)\end{array}$ & $\begin{array}{l}-0.325 \\
(0.338)\end{array}$ & $\begin{array}{l}-0.300 \\
(0.293)\end{array}$ & $\begin{array}{l}-0.016 \\
(0.098)\end{array}$ \\
\hline Age & $\begin{array}{l}0.004 \\
(0.003)\end{array}$ & $\begin{array}{l}0.003 \\
(0.003)\end{array}$ & $\begin{array}{l}0.0001 \\
(0.0004)\end{array}$ & $\begin{array}{l}-0.006 \\
(0.012)\end{array}$ & $\begin{array}{l}-0.004 \\
(0.009)\end{array}$ & $\begin{array}{l}-0.0004 \\
(0.002)\end{array}$ & $\begin{array}{l}0.002 \\
(0.006)\end{array}$ & $\begin{array}{l}0.002 \\
(0.005)\end{array}$ & $\begin{array}{l}0.0001 \\
(0.0007)\end{array}$ & $\begin{array}{l}0.007 \\
(0.004)\end{array}$ & $\begin{array}{l}0.004 \\
(0.004)\end{array}$ & $\begin{array}{l}0.0002 \\
(0.0009)\end{array}$ \\
\hline Size (Staff) & $\begin{array}{l}0.000 \\
(0.000)\end{array}$ & $\begin{array}{l}0.984 \mathrm{E}-4 \\
(0.0001)\end{array}$ & $\begin{array}{l}0.760 \mathrm{E}-5 \\
(0.176 \mathrm{E}-4)\end{array}$ & - & - & - & $\begin{array}{l}0.000 \\
(0.000)\end{array}$ & $\begin{array}{l}-0.0001 \\
(0.0002)\end{array}$ & $\begin{array}{l}-0.826 \mathrm{E}-5 \\
(0.306 \mathrm{E}-4)\end{array}$ & $\begin{array}{l}0.000 \\
(0.000)\end{array}$ & $\begin{array}{l}0.0001 \\
(0.0001)\end{array}$ & $\begin{array}{l}0.873 \mathrm{E}-5 \\
(0.520 \mathrm{E}-4)\end{array}$ \\
\hline Offshore policy & $\begin{array}{l}1.328 * \\
(0.666)\end{array}$ & $\begin{array}{l}1.339^{*} \\
(0.629)\end{array}$ & $\begin{array}{l}0.111 \dagger \\
(0.063)\end{array}$ & $\begin{array}{l}0.865 \\
(1.487)\end{array}$ & $\begin{array}{l}0.847 \\
(1.313)\end{array}$ & $\begin{array}{l}0.077 \\
(0.200)\end{array}$ & $\begin{array}{l}1.985 \dagger \\
(1.071)\end{array}$ & $\begin{array}{l}2.101 * \\
(0.949)\end{array}$ & $\begin{array}{l}0.205^{*} \\
(0.094)\end{array}$ & $\begin{array}{l}2.451^{*} \\
(1.182)\end{array}$ & $\begin{array}{l}2.490^{*} \\
(1.038)\end{array}$ & $\begin{array}{l}0.169 \\
(0.296)\end{array}$ \\
\hline $\begin{array}{l}\text { Internationalisation } \\
\text { at home strategy }\end{array}$ & $\begin{array}{l}0.035 \\
(0.127)\end{array}$ & $\begin{array}{l}-0.0007 \\
(0.0007)\end{array}$ & $\begin{array}{l}-0.462 \mathrm{E}-4 \\
(0.0001)\end{array}$ & $\begin{array}{l}0.155 \\
(0.332)\end{array}$ & $\begin{array}{l}-0.001 \\
(0.001)\end{array}$ & $\begin{array}{l}-0.672 \mathrm{E}-4 \\
(0.0002)\end{array}$ & $\begin{array}{l}-0.030 \\
(0.205)\end{array}$ & $\begin{array}{l}-0.0007 \\
(0.002)\end{array}$ & $\begin{array}{l}-0.452 \\
(0.0003)\end{array}$ & $\begin{array}{l}-0.016 \\
(0.211)\end{array}$ & $\begin{array}{l}0.0005 \\
(0.0008)\end{array}$ & $\begin{array}{l}0.241 \mathrm{E}-4 \\
(0.0002)\end{array}$ \\
\hline $\begin{array}{l}\text { Host country } \\
\text { demand }\end{array}$ & $\begin{array}{l}2.258 \\
(1.826)\end{array}$ & $\begin{array}{l}2.399 \\
(1.680)\end{array}$ & $\begin{array}{l}0.154 \\
(0.228)\end{array}$ & $\begin{array}{l}6.356 \\
(3.879)\end{array}$ & $\begin{array}{l}\text { 8.143* } \\
(3.240)\end{array}$ & $\begin{array}{l}0.487 \\
(0.603)\end{array}$ & $\begin{array}{l}-2.597 \\
(3.034)\end{array}$ & $\begin{array}{l}-2.183 \\
(2.720)\end{array}$ & $\begin{array}{l}-0.148 \\
(0.415)\end{array}$ & $\begin{array}{l}3.538 \\
(3.450)\end{array}$ & $\begin{array}{l}1.949 \\
(2.722)\end{array}$ & $\begin{array}{l}0.114 \\
(0.685)\end{array}$ \\
\hline AU/NZ dummy & $\begin{array}{l}0.352 \\
(0.518)\end{array}$ & $\begin{array}{l}0.395 \\
(0.485)\end{array}$ & $\begin{array}{l}0.022 \\
(0.065)\end{array}$ & - & - & - & - & - & - & - & - & - \\
\hline UK/IR dummy & $\begin{array}{l}0.167 \\
(0.479)\end{array}$ & $\begin{array}{l}0.198 \\
(0.443)\end{array}$ & $\begin{array}{l}0.011 \\
(0.064)\end{array}$ & - & - & - & - & - & - & - & - & - \\
\hline
\end{tabular}




\begin{tabular}{|c|c|c|c|c|c|c|c|c|c|c|c|c|}
\hline $\mathrm{R}^{2}$ & 0.573 & & & 0.586 & & & 0.683 & & & 0.494 & & \\
\hline $\operatorname{AdjR}^{2}$ & 0.538 & & & 0.455 & & & 0.619 & & & 0.357 & & \\
\hline Log likelihood & & -740.47 & -809.38 & & -189.99 & -210.15 & & -300.20 & -329.54 & & -224.71 & -258.86 \\
\hline $\mathrm{N}$ & 308 & 308 & 308 & 80 & 80 & 80 & 124 & 124 & 124 & 104 & 104 & \\
\hline Max. VIF & 1.971 & & & 2.234 & & & 2.222 & & & 2.057 & & \\
\hline
\end{tabular}

Standard errors in parentheses, all $t$ tests are two-tailed, $\dagger p<.10,{ }^{*} p<.05,{ }^{* *} p<.01$

Table 7.12: Regression estimates for equation 3

\begin{tabular}{|c|c|c|c|c|c|c|c|c|c|c|c|c|}
\hline & \multicolumn{3}{|c|}{ Complete data set } & \multicolumn{3}{|c|}{ AU/NZ } & \multicolumn{3}{|c|}{$\overline{\text { US/CA }}$} & \multicolumn{3}{|c|}{$\overline{\mathrm{UK} / \mathrm{IR}}$} \\
\hline & OLS & Tobit & $\begin{array}{l}\text { Negative } \\
\text { binomial }\end{array}$ & OLS & Tobit & $\begin{array}{l}\text { Negative } \\
\text { binomial }\end{array}$ & OLS & Tobit & $\begin{array}{l}\text { Negative } \\
\text { binomial }\end{array}$ & OLS & Tobit & $\begin{array}{l}\text { Negative } \\
\text { binomial }\end{array}$ \\
\hline Intercept & $\begin{array}{l}\text { 21.204** } \\
(3.272)\end{array}$ & $\begin{array}{l}\text { 22.711** } \\
(2.959)\end{array}$ & $\begin{array}{l}3.265 * * \\
(0.373)\end{array}$ & $\begin{array}{l}21.758 * \\
(6.580)\end{array}$ & $\begin{array}{l}23.695 * * \\
(5.373)\end{array}$ & $\begin{array}{l}3.356 * * \\
(0.896)\end{array}$ & $\begin{array}{l}20.769 * * \\
(6.212)\end{array}$ & $\begin{array}{l}20.806 * * \\
(5.442)\end{array}$ & $\begin{array}{l}3.138 * * \\
(0.660)\end{array}$ & $\begin{array}{l}24.735 * * \\
(5.325)\end{array}$ & $\begin{array}{l}23.199 * * \\
(4.336)\end{array}$ & $\begin{array}{l}3.236 * * \\
(1.049)\end{array}$ \\
\hline $\begin{array}{l}\text { Geo exp - knowledge } \\
\text { of edu sector (HIa) }\end{array}$ & $\begin{array}{l}-0.041 \\
(0.126)\end{array}$ & $\begin{array}{l}-0.059 \\
(0.117)\end{array}$ & $\begin{array}{l}-0.005 \\
(0.015)\end{array}$ & $\begin{array}{l}0.436 \\
(0.287)\end{array}$ & $\begin{array}{l}0.362 \\
(0.238)\end{array}$ & $\begin{array}{l}0.020 \\
(0.045)\end{array}$ & $\begin{array}{l}-0.078 \\
(0.222)\end{array}$ & $\begin{array}{l}-0.060 \\
(0.199)\end{array}$ & $\begin{array}{l}-0.005 \\
(0.024)\end{array}$ & $\begin{array}{l}-0.231 \\
(0.213)\end{array}$ & $\begin{array}{l}-0.186 \\
(0.167)\end{array}$ & $\begin{array}{l}-0.012 \\
(0.045)\end{array}$ \\
\hline $\begin{array}{l}\text { Geo exp - academic } \\
\text { capabilities }(H 1 a)\end{array}$ & $\begin{array}{l}0.118 \\
(0.121)\end{array}$ & $\begin{array}{l}0.067 \\
(0.113)\end{array}$ & $\begin{array}{l}0.006 \\
(0.013)\end{array}$ & $\begin{array}{l}0.614^{*} \\
(0.283)\end{array}$ & $\begin{array}{l}0.559 * \\
(0.244)\end{array}$ & $\begin{array}{l}0.035 \\
(0.048)\end{array}$ & $\begin{array}{l}0.067 \\
(0.220)\end{array}$ & $\begin{array}{l}0.045 \\
(0.199)\end{array}$ & $\begin{array}{l}0.004 \\
(0.020)\end{array}$ & $\begin{array}{l}0.030 \\
(0.185)\end{array}$ & $\begin{array}{l}-0.046 \\
(0.153)\end{array}$ & $\begin{array}{l}-0.002 \\
(0.034)\end{array}$ \\
\hline $\begin{array}{l}\text { Geo exp - managerial } \\
\text { capabilities }(H 1 a)\end{array}$ & $\begin{array}{l}0.151 \\
(0.146)\end{array}$ & $\begin{array}{l}0.157 \\
(0.139)\end{array}$ & $\begin{array}{l}0.009 \\
(0.017)\end{array}$ & $\begin{array}{l}-0.028 \\
(0.316)\end{array}$ & $\begin{array}{l}0.010 \\
(0.273)\end{array}$ & $\begin{array}{l}0.002 \\
(0.048)\end{array}$ & $\begin{array}{l}0.410 \\
(0.262)\end{array}$ & $\begin{array}{l}0.421 \dagger \\
(0.237)\end{array}$ & $\begin{array}{l}0.026 \\
(0.028)\end{array}$ & $\begin{array}{l}-0.107 \\
(0.233)\end{array}$ & $\begin{array}{l}-0.029 \\
(0.199)\end{array}$ & $\begin{array}{l}-0.003 \\
(0.042)\end{array}$ \\
\hline $\begin{array}{l}\text { Geo exp - financial } \\
\text { capabilities }(H 1 a)\end{array}$ & $\begin{array}{l}-0.011 \\
(0.138)\end{array}$ & $\begin{array}{l}-0.049 \\
(0.128)\end{array}$ & $\begin{array}{l}-0.004 \\
(0.017)\end{array}$ & $\begin{array}{l}-0.361 \\
(0.315)\end{array}$ & $\begin{array}{l}-0.508 * \\
(0.250)\end{array}$ & $\begin{array}{l}-0.035 \\
(0.046)\end{array}$ & $\begin{array}{l}0.197 \\
(0.231)\end{array}$ & $\begin{array}{l}0.174 \\
(0.208)\end{array}$ & $\begin{array}{l}0.012 \\
(0.023)\end{array}$ & $\begin{array}{l}-0.384 \\
(0.236)\end{array}$ & $\begin{array}{l}-0.329 \\
(0.201)\end{array}$ & $\begin{array}{l}-0.021 \\
(0.050)\end{array}$ \\
\hline $\begin{array}{l}\text { Geo exp - intl } \\
\text { recruitment (H1a) }\end{array}$ & $\begin{array}{l}-0.185 \\
(0.152)\end{array}$ & $\begin{array}{l}-0.178 \\
(0.143)\end{array}$ & $\begin{array}{l}-0.011 \\
(0.017)\end{array}$ & $\begin{array}{l}0.267 \\
(0.284)\end{array}$ & $\begin{array}{l}0.293 \\
(0.248)\end{array}$ & $\begin{array}{l}0.019 \\
(0.041)\end{array}$ & $\begin{array}{l}-0.599 * \\
(0.284)\end{array}$ & $\begin{array}{l}-0.563^{*} \\
(0.253)\end{array}$ & $\begin{array}{l}-0.040 \\
(0.030)\end{array}$ & $\begin{array}{l}0.055 \\
(0.250)\end{array}$ & $\begin{array}{l}0.081 \\
(0.214)\end{array}$ & $\begin{array}{l}0.006 \\
(0.057)\end{array}$ \\
\hline $\begin{array}{l}\text { Ind exp - UG offshore } \\
\text { edu }(H 1 b)\end{array}$ & $\begin{array}{l}0.024 \\
(0.118)\end{array}$ & $\begin{array}{l}0.013 \\
(0.111)\end{array}$ & $\begin{array}{l}0.0008 \\
(0.013)\end{array}$ & $\begin{array}{l}-0.240 \\
(0.257)\end{array}$ & $\begin{array}{l}-0.283 \\
(0.219)\end{array}$ & $\begin{array}{l}-0.016 \\
(0.038)\end{array}$ & $\begin{array}{l}0.039 \\
(0.229)\end{array}$ & $\begin{array}{l}0.030 \\
(0.207)\end{array}$ & $\begin{array}{l}0.004 \\
(0.023)\end{array}$ & $\begin{array}{l}0.058 \\
(0.189)\end{array}$ & $\begin{array}{l}0.087 \\
(0.158)\end{array}$ & $\begin{array}{l}0.004 \\
(0.038)\end{array}$ \\
\hline $\begin{array}{l}\text { Ind exp - PG offshore } \\
\text { edu }(H 1 b)\end{array}$ & $\begin{array}{l}-0.043 \\
(0.133)\end{array}$ & $\begin{array}{l}-0.043 \\
(0.126)\end{array}$ & $\begin{array}{l}-0.003 \\
(0.015)\end{array}$ & $\begin{array}{l}-0.043 \\
(0.285)\end{array}$ & $\begin{array}{l}0.029 \\
(0.245)\end{array}$ & $\begin{array}{l}0.001 \\
(0.039)\end{array}$ & $\begin{array}{l}-0.030 \\
(0.231)\end{array}$ & $\begin{array}{l}-0.035 \\
(0.208)\end{array}$ & $\begin{array}{l}-0.003 \\
(0.023)\end{array}$ & $\begin{array}{l}-0.046 \\
(0.233)\end{array}$ & $\begin{array}{l}-0.056 \\
(0.198)\end{array}$ & $\begin{array}{l}-0.002 \\
(0.047)\end{array}$ \\
\hline $\begin{array}{l}\text { Ind exp - intl } \\
\text { recruitment (Factor) } \\
(H 1 b)\end{array}$ & $\begin{array}{l}0.238 \\
(0.154)\end{array}$ & $\begin{array}{l}0.207 \\
(0.140)\end{array}$ & $\begin{array}{l}0.013 \\
(0.019)\end{array}$ & $\begin{array}{l}-0.069 \\
(0.340)\end{array}$ & $\begin{array}{l}-0.185 \\
(0.280)\end{array}$ & $\begin{array}{l}-0.013 \\
(0.046)\end{array}$ & $\begin{array}{l}0.375 \\
(0.282)\end{array}$ & $\begin{array}{l}0.308 \\
(0.251)\end{array}$ & $\begin{array}{l}0.021 \\
(0.029)\end{array}$ & $\begin{array}{l}0.382 \\
(0.254)\end{array}$ & $\begin{array}{l}\mathbf{0 . 3 2 7} \dagger \\
\mathbf{( 0 . 1 9 8 )}\end{array}$ & $\begin{array}{l}0.020 \\
(0.047)\end{array}$ \\
\hline $\begin{array}{l}\text { Ind exp - UG } \\
\text { marketing }(H 1 b)\end{array}$ & $\begin{array}{l}0.046 \\
(0.117)\end{array}$ & $\begin{array}{l}0.040 \\
(0.110)\end{array}$ & $\begin{array}{l}0.002 \\
(0.015)\end{array}$ & $\begin{array}{l}-0.288 \\
(0.311)\end{array}$ & $\begin{array}{l}-0.210 \\
(0.267)\end{array}$ & $\begin{array}{l}-0.015 \\
(0.056)\end{array}$ & $\begin{array}{l}0.282 \\
(0.199)\end{array}$ & $\begin{array}{l}0.257 \\
(0.179)\end{array}$ & $\begin{array}{l}0.019 \\
(0.022)\end{array}$ & $\begin{array}{l}0.024 \\
(0.178)\end{array}$ & $\begin{array}{l}0.065 \\
(0.147)\end{array}$ & $\begin{array}{l}0.003 \\
(0.039)\end{array}$ \\
\hline $\begin{array}{l}\text { Ind exp - PG } \\
\text { marketing }(H 1 b)\end{array}$ & $\begin{array}{l}0.029 \\
(0.115)\end{array}$ & $\begin{array}{l}-0.032 \\
(0.107)\end{array}$ & $\begin{array}{l}-0.003 \\
(0.013)\end{array}$ & $\begin{array}{l}0.279 \\
(0.241)\end{array}$ & $\begin{array}{l}0.262 \\
(0.207)\end{array}$ & $\begin{array}{l}0.015 \\
(0.037)\end{array}$ & $\begin{array}{l}-0.168 \\
(0.195)\end{array}$ & $\begin{array}{l}-0.171 \\
(0.176)\end{array}$ & $\begin{array}{l}-0.013 \\
(0.019)\end{array}$ & $\begin{array}{l}0.184 \\
(0.197)\end{array}$ & $\begin{array}{l}0.062 \\
(0.164)\end{array}$ & $\begin{array}{l}0.003 \\
(0.042)\end{array}$ \\
\hline $\begin{array}{l}\text { Org culture (Factor) - } \\
\text { (H3) }\end{array}$ & $\begin{array}{l}-1.865^{* *} \\
(0.176)\end{array}$ & $\begin{array}{l}-1.801^{* *} \\
(0.163)\end{array}$ & $\begin{array}{l}-0.133 * * \\
(0.020)\end{array}$ & $\begin{array}{l}-2.229 * * \\
(0.345)\end{array}$ & $\begin{array}{l}-2.208^{* *} \\
(0.301)\end{array}$ & $\begin{array}{l}-0.158^{* *} \\
(0.042)\end{array}$ & $\begin{array}{l}-2.024 * * \\
(0.320)\end{array}$ & $\begin{array}{l}-1.939 * * \\
(0.273)\end{array}$ & $\begin{array}{l}-0.147^{* *} \\
(0.033)\end{array}$ & - & - & - \\
\hline
\end{tabular}




\begin{tabular}{|c|c|c|c|c|c|c|c|c|c|c|c|c|}
\hline $\begin{array}{l}\text { Org culture - open } \\
\text { discussion }(H 3)\end{array}$ & - & - & - & - & - & - & - & - & - & $\begin{array}{l}-0.429 \dagger \\
(0.242)\end{array}$ & $\begin{array}{l}-0.439 * \\
(0.204)\end{array}$ & $\begin{array}{l}-0.028 \\
(0.044)\end{array}$ \\
\hline $\begin{array}{l}\text { Org culture - no } \\
\text { status distinction (H3) }\end{array}$ & - & - & - & - & - & - & - & - & - & $\begin{array}{l}-0.450 \dagger \\
(0.252)\end{array}$ & $\begin{array}{l}-0.407 \dagger \\
(0.212)\end{array}$ & $\begin{array}{l}-0.027 \\
(0.047)\end{array}$ \\
\hline $\begin{array}{l}\text { Org culture - } \\
\text { experimentation (H3) }\end{array}$ & - & - & - & - & - & - & - & - & - & $\begin{array}{l}-0.051 \\
(0.225)\end{array}$ & $\begin{array}{l}-0.175 \\
(0.177)\end{array}$ & $\begin{array}{l}-0.012 \\
(0.043)\end{array}$ \\
\hline $\begin{array}{l}\text { Org culture - offshore } \\
\text { development (H3) }\end{array}$ & - & - & - & - & - & - & - & - & - & $\begin{array}{l}-0.503^{*} \\
(0.231)\end{array}$ & $\begin{array}{l}-0.396^{*} \\
(0.185)\end{array}$ & $\begin{array}{l}-0.025 \\
(0.051)\end{array}$ \\
\hline $\begin{array}{l}\text { Org culture - offshore } \\
\text { responsiveness }(H 3)\end{array}$ & - & - & - & - & - & - & - & - & - & $\begin{array}{l}-0.083 \\
(0.229)\end{array}$ & $\begin{array}{l}0.035 \\
(0.187)\end{array}$ & $\begin{array}{l}0.0003 \\
(0.043)\end{array}$ \\
\hline $\begin{array}{l}\text { Financial resources } \\
\text { (H5) }\end{array}$ & $\begin{array}{l}0.102 \\
(0.104)\end{array}$ & $\begin{array}{l}0.095 \\
(0.095)\end{array}$ & $\begin{array}{l}0.005 \\
(0.011)\end{array}$ & $\begin{array}{l}0.202 \\
(0.263)\end{array}$ & $\begin{array}{l}0.102 \\
(0.203)\end{array}$ & $\begin{array}{l}0.006 \\
(0.040)\end{array}$ & $\begin{array}{l}0.172 \\
(0.167)\end{array}$ & $\begin{array}{l}0.203 \\
(0.148)\end{array}$ & $\begin{array}{l}0.011 \\
(0.015)\end{array}$ & $\begin{array}{l}0.019 \\
(0.176)\end{array}$ & $\begin{array}{l}-0.025 \\
(0.140)\end{array}$ & $\begin{array}{l}-0.002 \\
(0.040)\end{array}$ \\
\hline $\begin{array}{l}\text { Reputation (Factor) } \\
\text { (H6) }\end{array}$ & $\begin{array}{l}-0.408 \dagger \\
(0.222)\end{array}$ & $\begin{array}{l}-0.510^{*} \\
(0.202)\end{array}$ & $\begin{array}{l}-0.035 \\
(0.026)\end{array}$ & $\begin{array}{l}-0.322 \\
(0.485)\end{array}$ & $\begin{array}{l}-0.333 \\
(0.408)\end{array}$ & $\begin{array}{l}-0.026 \\
(0.071)\end{array}$ & $\begin{array}{l}-0.554 \\
(0.443)\end{array}$ & $\begin{array}{l}-0.659 \dagger \\
(0.384)\end{array}$ & $\begin{array}{l}-0.050 \\
(0.050)\end{array}$ & $\begin{array}{l}-0.368 \\
(0.347)\end{array}$ & $\begin{array}{l}-0.421 \\
(0.274)\end{array}$ & $\begin{array}{l}-0.026 \\
(0.077)\end{array}$ \\
\hline $\begin{array}{l}\text { IMD Normative ID } \\
\text { (lag 1) }\end{array}$ & $\begin{array}{l}-0.113 \\
(0.190)\end{array}$ & $\begin{array}{l}-0.057 \\
(0.156)\end{array}$ & $\begin{array}{l}-0.002 \\
(0.020)\end{array}$ & $\begin{array}{l}-0.049 \\
(0.326)\end{array}$ & $\begin{array}{l}0.050 \\
(0.275)\end{array}$ & $\begin{array}{l}0.006 \\
(0.057)\end{array}$ & $\begin{array}{l}0.595 \dagger \\
(0.349)\end{array}$ & $\begin{array}{l}-0.392 \\
(0.263)\end{array}$ & $\begin{array}{l}-0.024 \\
(0.032)\end{array}$ & $\begin{array}{l}0.048 \\
(0.314)\end{array}$ & $\begin{array}{l}0.238 \\
(0.240)\end{array}$ & $\begin{array}{l}0.015 \\
(0.063)\end{array}$ \\
\hline $\begin{array}{l}\text { IMD Regulative ID } \\
\text { (lag 1) }\end{array}$ & $\begin{array}{l}0.052 \\
(0.205)\end{array}$ & $\begin{array}{l}0.055 \\
(0.156)\end{array}$ & $\begin{array}{l}0.002 \\
(0.023)\end{array}$ & - & - & - & $\begin{array}{l}0.204 \\
(0.358)\end{array}$ & $\begin{array}{l}0.390 \\
(0.264)\end{array}$ & $\begin{array}{l}0.024 \\
(0.031)\end{array}$ & $\begin{array}{l}-0.610 \\
(0.382)\end{array}$ & $\begin{array}{l}-0.535 \dagger \\
(0.323)\end{array}$ & $\begin{array}{l}-0.032 \\
(0.071)\end{array}$ \\
\hline Age & $\begin{array}{l}0.003 \\
(0.004)\end{array}$ & $\begin{array}{l}0.002 \\
(0.003)\end{array}$ & $\begin{array}{l}0.0001 \\
(0.0004)\end{array}$ & $\begin{array}{l}0.002 \\
(0.012)\end{array}$ & $\begin{array}{l}0.005 \\
(0.009)\end{array}$ & $\begin{array}{l}0.0002 \\
(0.001)\end{array}$ & $\begin{array}{l}0.003 \\
(0.006)\end{array}$ & $\begin{array}{l}0.002 \\
(0.006)\end{array}$ & $\begin{array}{l}0.0002 \\
(0.0006)\end{array}$ & $\begin{array}{l}0.002 \\
(0.005)\end{array}$ & $\begin{array}{l}0.001 \\
(0.004)\end{array}$ & $\begin{array}{l}0.866 \mathrm{E}-4 \\
(0.001)\end{array}$ \\
\hline Size (Staff) & $\begin{array}{l}2.612 \mathrm{E}-6 \\
(0.000)\end{array}$ & $\begin{array}{l}0.143 \mathrm{E}-4 \\
(0.0001)\end{array}$ & $\begin{array}{l}0.125 E-5 \\
(0.185 E-4)\end{array}$ & (1) & (10, & (1) & $\begin{array}{l}0.000 \\
(0.000)\end{array}$ & $\begin{array}{l}-0.0002 \\
(0.0002)\end{array}$ & $\begin{array}{l}-0.132 \mathrm{E}-4 \\
(0.271 \mathrm{E}-4)\end{array}$ & $\begin{array}{l}2.817 \mathrm{E}-5 \\
(0.000)\end{array}$ & $\begin{array}{l}0.342 \mathrm{E}-4 \\
(0.0001)\end{array}$ & $\begin{array}{l}0.252 \mathrm{E}-5 \\
(0.564 \mathrm{E}-4)\end{array}$ \\
\hline Offshore policy & $\begin{array}{l}1.669 * \\
(0.718)\end{array}$ & $\begin{array}{l}1.753^{* *} \\
(0.679)\end{array}$ & $\begin{array}{l}0.144 * \\
(0.059)\end{array}$ & $\begin{array}{l}1.376 \\
(1.514)\end{array}$ & $\begin{array}{l}1.275 \\
(1.323)\end{array}$ & $\begin{array}{l}0.110 \\
(0.229)\end{array}$ & $\begin{array}{l}2.515^{*} \\
(1.201)\end{array}$ & $\begin{array}{l}2.689 * \\
(1.058)\end{array}$ & $\begin{array}{l}0.244 * * \\
(0.083)\end{array}$ & $\begin{array}{l}2.242 \dagger \\
(1.318)\end{array}$ & $\begin{array}{l}2.512 * \\
(1.140)\end{array}$ & $\begin{array}{l}0.170 \\
(0.274)\end{array}$ \\
\hline $\begin{array}{l}\text { Internationalisation at } \\
\text { home strategy }\end{array}$ & $\begin{array}{l}0.014 \\
(0.137)\end{array}$ & $\begin{array}{l}0.0002 \\
(0.0008)\end{array}$ & $\begin{array}{l}0.217 \mathrm{E}-4 \\
(0.0001)\end{array}$ & $\begin{array}{l}-0.057 \\
(0.344)\end{array}$ & $\begin{array}{l}-0.0001 \\
(0.001)\end{array}$ & $\begin{array}{l}0.126 E-4 \\
(0.0003)\end{array}$ & $\begin{array}{l}0.053 \\
(0.231)\end{array}$ & $\begin{array}{l}-0.0005 \\
(0.002)\end{array}$ & $\begin{array}{l}-0.396 \mathrm{E}-4 \\
(0.0003)\end{array}$ & $\begin{array}{l}-0.167 \\
(0.241)\end{array}$ & $\begin{array}{l}0.001 \\
(0.0009)\end{array}$ & $\begin{array}{l}0.826 E-4 \\
(0.0001)\end{array}$ \\
\hline Host country demand & $\begin{array}{l}2.112 \\
(1.973)\end{array}$ & $\begin{array}{l}2.139 \\
(1.821)\end{array}$ & $\begin{array}{l}0.124 \\
(0.209)\end{array}$ & $\begin{array}{l}7.665 \dagger \\
(4.013)\end{array}$ & $\begin{array}{l}\text { 8.612** } \\
\text { (3.307) }\end{array}$ & $\begin{array}{l}0.520 \\
(0.539)\end{array}$ & $\begin{array}{l}-1.267 \\
(3.396)\end{array}$ & $\begin{array}{l}-0.676 \\
(3.033)\end{array}$ & $\begin{array}{l}-0.057 \\
(0.321)\end{array}$ & $\begin{array}{l}2.531 \\
(3.932)\end{array}$ & $\begin{array}{l}0.631 \\
(3.039)\end{array}$ & $\begin{array}{l}0.020 \\
(0.784)\end{array}$ \\
\hline AU/NZ dummy & $\begin{array}{l}0.691 \\
(0.558)\end{array}$ & $\begin{array}{l}0.674 \\
(0.524)\end{array}$ & $\begin{array}{l}0.045 \\
(0.065)\end{array}$ & - & - & - & - & - & - & - & - & - \\
\hline UK/IR dummy & $\begin{array}{l}0.460 \\
(0.515)\end{array}$ & $\begin{array}{l}0.414 \\
(0.478)\end{array}$ & $\begin{array}{l}0.027 \\
(0.060)\end{array}$ & - & - & - & - & - & - & - & - & - \\
\hline $\mathrm{R}^{2}$ & 0.504 & & & 0.560 & & & 0.600 & & & 0.440 & & \\
\hline $\operatorname{AdjR}^{2}$ & 0.463 & & & 0.421 & & & 0.520 & & & 0.248 & & \\
\hline Log likelihood & & -764.50 & -825.47 & & -191.46 & -211.38 & & -314.31 & -340.05 & & -231.12 & -261.31 \\
\hline $\mathrm{N}$ & 308 & 308 & 308 & 80 & 80 & 80 & 124 & 124 & 124 & 104 & 104 & 104 \\
\hline Max. VIF & 2.041 & & & 2.161 & & & 2.570 & & & 2.199 & & \\
\hline
\end{tabular}

Standard errors in parentheses, all $t$ tests are two-tailed, $\uparrow p<.10, * p<.05,{ }^{* *} p<.01$ 


\subsubsection{Testing for the moderating hypotheses of $\mathrm{H7}$ and $\mathrm{H} 8$}

In order to test for the moderating relationship hypothesised in $\mathrm{H7}$ and H8, the data are stratified using the median of the respective institutional distance measures. It is hypothesised that, for lower values of institutional distance, positive relationships between specific resources and equity stake will be observed, while the relationships will be negative for larger values of institutional distance.

\subsubsection{Hypothesis 7a}

The findings from tables 7.13a and 7.13b do not lend support to H7a, which posits a positive relationship between geographic experience and equity stake, given low institutional distance and a negative relationship at higher levels of distance. From table 7.13a, the estimated coefficients associated with geographic experience - knowledge of education sector and geographic experience - financial capabilities are significant (at least $p<0.10$ ), but negative (OLS, Tobit and negative binomial in the full sample, Tobit for US/CA, OLS for UK/IR). From table 7.13b, which shows the results of modelling the relationship between geographic experience and equity stake for higher normative institutional distance, the estimated coefficients associated with geographic experience - knowledge of education sector and geographic experience - managerial capabilities are significant (at least $p<0.10$ ), but positive (OLS and Tobit in the full sample, AU/NZ, UK/IR). With a negative relationship hypothesised at higher levels of institutional distance, H7a is, thus, not supported.

\subsubsection{Hypothesis $7 b$}

The results in table 7.14a lend support to H7b, with respect to the hypothesised positive relationship between industry experience - international recruitment and equity stake at low levels of normative institutional distance. Significantly positive results (at least at $p$ $<0.10$ ) are obtained for the aggregate and US/CA groupings with all three estimation approaches (OLS, Tobit and negative binomial), and similar findings (at least $p<0.05$ ) are obtained for the AU/NZ grouping when using both OLS and Tobit estimation. H7b, with respect to the hypothesised positive relationship between industry experience postgraduate marketing and equity stake is also supported in table 7.14a (OLS for UK/IR). However, the associated negative hypothesised relationships for both aspects 
of industry experience at higher levels of normative institutional distance are not supported in table 7.14b. Thus, the combined findings from table 7.14a and 7.14b, do not lend support to H7b across the range of institutional distance.

\subsubsection{Hypothesis 7c}

H7c, which pertains to the moderating role of normative institutional distance on the know-how and equity stake relationship, is not supported from the results in tables 7.15a and 7.15b. Although significant results are obtained in the full and sub-samples in table 7.15a (except for UK/IR negative binomial), the negative coefficients contradict the positive hypothesised relationship at low levels of institutional distance. At higher levels of institutional distance (see table 7.15b), significant coefficients are found in support of the negative hypothesised relationship (OLS, Tobit and negative binomial for the full sample, AU/NZ and US/CA). However, the combined findings from tables 7.15a and 7.15b do not support the inverted hypothesised relationship from low to higher levels of institutional distance. Rather, know-how appears to be negatively associated with equity stake regardless of the levels of normative institutional distance and after accounting for the other control variables.

\subsubsection{Hypothesis 7d}

H7d, which deals with the relationship of normative institutional distance on the hypothesised positive association between organisational culture and equity stake, is not supported from the findings in tables 7.16a and 7.16b. Significant findings are found in table 7.16a (except for UK/IR negative binomial), but the negative coefficients do not lend credence to the positive hypothesised relationship at low levels of normative institutional distance. At higher levels of normative institutional distance, a negative relationship is hypothesised. In table 7.16b, significant negative findings are found for the full sample, AU/NZ and US/CA subsamples. However, positive findings are also found in the UK/IR subgroup for organisational culture - open discussion and organisational culture - offshore responsiveness (OLS and Tobit in table 7.16b). 


\subsubsection{Hypothesis 7e}

H7e, which relates to how normative institutional distance moderates the hypothesised relationship between financial resources and equity stake, is not supported. In table 7.17a, a negative significant finding is found for the UK/IR sub-group (OLS). The finding does not support the positive hypothesised relationship at low levels of normative institutional distance. In table 7.17b, a negative significant finding is found for the full sample (Tobit). This is supportive of the hypothesised negative relationship between financial resources and equity stake at higher levels of normative institutional distance. The combined findings from tables 7.17a and 7.17b, do not, however, lend support for H7e.

\subsubsection{Hypothesis $7 f$}

H7f, which relates to the relationship of normative institutional distance on the hypothesised association between reputation and equity stake, is not supported. While a positive relationship is hypothesised at low levels of normative institutional distance, negative significant findings are reported in table 7.18a (OLS, Tobit and negative binomial for the full sample and US/CA, OLS for UK/IR). At higher levels of normative institutional distance, a negative relationship is hypothesised. While the findings from table 7.18b support this hypothesis (OLS, Tobit and negative binomial for full sample and US/CA, OLS and Tobit for AU/NZ), the combined findings from tables 7.18a and 7.18b, do not support an inverted relationship between reputation and equity stake.

\subsubsection{Hypothesis 8a}

H8a, which pertains to the moderating role of regulative institutional distance on the geographic experience and equity stake relationship, is not supported. A positive relationship is hypothesised at low levels of regulative institutional distance. Evidence towards this hypothesis is found for geographic experience - academic capabilities in the US/CA subgroup (OLS, Tobit and negative binomial in table 7.19a). However, the accompanying hypothesised negative relationship at higher levels of regulative institutional distance is not found in table 7.19b. Similarly, the significant negative coefficients associated with the various aspects of geographic experience in table 7.19b (Tobit for AU/NZ, OLS and Tobit for US/CA and UK/IR), do not present 
accompanying positive relationships at low levels of regulative institutional distance in table 7.19a. Therefore, the combined findings of tables 7.19a and 7.19b do not provide support towards H8a.

\subsubsection{Hypothesis $8 b$}

H8b, which relates to how regulative institutional distance moderates on the hypothesised relationship between industry experience and equity stake, is not supported. A positive relationship is hypothesised at low levels of regulative institutional distance. Evidence in support of this hypothesis is found for industry experience - international recruitment in the AU/NZ subsample (Tobit in table 7.20a). However, negative significant findings are also obtained for industry experience undergraduate marketing in the US/CA grouping (OLS and Tobit in table 7.20a). At higher levels of regulative institutional distance, a negative relationship between industry experience and equity stake is hypothesised. Such a hypothesis is not supported in table 7.20b, where significant positive findings are found for industry experience international recruitment in the US/CA grouping (OLS and Tobit). Thus, the combined findings from tables 7.20a and 7.20b, do not lend support to H8b.

\subsubsection{Hypothesis 8c}

H8c, which relates to the relationship of regulative institutional distance on the hypothesised association between know-how and equity stake, is not supported. While significant findings are obtained (at least $p<0.05$ ) across all the models analysed in table 7.21a, the observed negative coefficients do not support the positive hypothesised relationship at low levels of institutional distance. While the findings in table $7.21 \mathrm{~b}$ are more aligned with H8c, showing significant negative findings (at least $p<0.05$ ) between know-how and equity stake at higher levels of regulative institutional distance, the combined findings from tables 7.21a and 7.21b do not support H8c. In fact, the combined findings suggest that after accounting for the other control variables and regardless of the levels of regulative institutional distance, there is a negative relationship between know-how and equity stake rather than the hypothesised inverted relationship. 


\subsubsection{Hypothesis $8 d$}

H8d, which pertains to the weighting of regulative institutional distance on the hypothesised relationship between entrepreneurial organisation culture and equity stake, is not supported. At low levels of regulative institutional distance, a positive relationship is hypothesised. Negative significant findings are, however, obtained in table 7.22a (OLS, Tobit and negative binomial for the full sample, AU/NZ and US/CA). Similarly, when separately investigating the different aspects of organisation culture in the UK/IR grouping, the following negative coefficients are found: org. culture - open discussion (Tobit), org. culture - no status distinction and org. culture - offshore development (both OLS and Tobit). At higher levels of regulative institutional distance, where a negative relationship is hypothesised, negative coefficients associated with the organisation culture factor are found in the full sample and the US/CA grouping in table 7.22b. The individual aspects of organisation culture examined in the UK/IR subsample are not significant. Thus, the combined findings from tables 7.22a and 7.22b do not support H8d.

\subsubsection{Hypothesis $8 \mathrm{e}$}

H8e, which relates to the moderating role of regulative institutional distance on the hypothesised relationship between financial resources and equity stake, is not supported. At low levels of regulative institutional distance, a positive relationship is hypothesised between financial resources and equity stake. However, financial resources are not significant across any of the models examined in table 7.23a. At higher levels of regulative institutional distance, a negative relationship is hypothesised between financial resources and equity stake. Only in the full sample is this negative relationship found to be significant in table 7.23b (OLS and Tobit). Thus, the combined findings from tables 7.23a and 7.23b, do not lend support to H8e.

\subsubsection{Hypothesis $8 f$}

H8f, which pertains to the relationship of regulative institutional distance on the hypothesised association between reputation and equity stake, is not supported. A positive relationship is hypothesised at low levels of regulative institutional distance. The significant negative findings in table 7.24a (at least $p<0.05$ ) do not support this hypothesis. At higher levels of regulative institutional distance, a negative relationship 
is hypothesised. The significant negative findings in table 7.24b (at least $p<0.05$ ) supports this hypothesis. However, the combined findings outlined in tables 7.24a and 7.24b do not lend support to H8f. In fact, the combined findings suggest that in the case of the full sample and the US/CA grouping, that regardless of the levels of regulative institutional distance, reputation is negatively associated with equity stake after accounting for the other variables in the models. 
Table 7.13a: Regression estimates for equation 4a (low normative institutional distance)

\begin{tabular}{|c|c|c|c|c|c|c|c|c|c|c|c|c|}
\hline & \multicolumn{3}{|c|}{ Complete data set } & \multicolumn{3}{|c|}{ AU/NZ } & \multicolumn{3}{|c|}{$\overline{\text { US/CA }}$} & \multicolumn{3}{|c|}{ UK/IR } \\
\hline & OLS & Tobit & $\begin{array}{l}\text { Negative } \\
\text { binomial }\end{array}$ & OLS & Tobit & $\begin{array}{l}\text { Negative } \\
\text { binomial }\end{array}$ & OLS & Tobit & $\begin{array}{l}\text { Negative } \\
\text { binomial }\end{array}$ & OLS & Tobit & $\begin{array}{l}\text { Negative } \\
\text { binomial }\end{array}$ \\
\hline 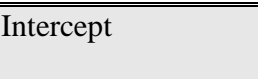 & $\begin{array}{l}17.000 * * \\
(4.663)\end{array}$ & $\begin{array}{l}18.830 * * \\
(4.055)\end{array}$ & $\begin{array}{l}2.936 * * \\
(0.373)\end{array}$ & $\begin{array}{l}13.326 \\
(14.442)\end{array}$ & $\begin{array}{l}14.141 \\
(9.896)\end{array}$ & $\begin{array}{l}2.590 \dagger \\
(1.373)\end{array}$ & $\begin{array}{l}21.840 * \\
(8.449)\end{array}$ & $\begin{array}{l}23.779 * * \\
(6.993)\end{array}$ & $\begin{array}{l}3.245 * * \\
(0.565)\end{array}$ & $\begin{array}{l}\text { 13.813* } \\
\text { (5.773) }\end{array}$ & $\begin{array}{l}14.804 * * \\
(4.984)\end{array}$ & $\begin{array}{l}2.657 * * \\
(0.694)\end{array}$ \\
\hline $\begin{array}{l}\text { Geo exp - } \\
\text { knowledge of edu } \\
\text { sector }(H 7 a)\end{array}$ & $\begin{array}{l}-0.480 \\
(0.228)\end{array}$ & $\begin{array}{l}-0.558 * * \\
(0.207)\end{array}$ & $\begin{array}{l}-0.035 \dagger \\
(0.020)\end{array}$ & $\begin{array}{l}-0.572 \\
(0.756)\end{array}$ & $\begin{array}{l}-0.583 \\
(0.580)\end{array}$ & $\begin{array}{l}-0.036 \\
(0.070)\end{array}$ & $\begin{array}{l}-0.622 \\
(0.400)\end{array}$ & $\begin{array}{l}-0.652 \dagger \\
(0.354)\end{array}$ & $\begin{array}{l}-0.043 \\
(0.028)\end{array}$ & $\begin{array}{l}-0.189 \\
(0.296)\end{array}$ & $\begin{array}{l}-0.315 \\
(0.251)\end{array}$ & $\begin{array}{l}-0.019 \\
(0.037)\end{array}$ \\
\hline $\begin{array}{l}\text { Geo exp - } \\
\text { academic } \\
\text { capabilities }(H 7 a)\end{array}$ & $\begin{array}{l}0.032 \\
(0.215)\end{array}$ & $\begin{array}{l}0.075 \\
(0.199)\end{array}$ & $\begin{array}{l}0.005 \\
(0.015)\end{array}$ & $\begin{array}{l}-0.262 \\
(0.731)\end{array}$ & $\begin{array}{l}-0.228 \\
(0.540)\end{array}$ & $\begin{array}{l}-0.015 \\
(0.063)\end{array}$ & $\begin{array}{l}0.191 \\
(0.414)\end{array}$ & $\begin{array}{l}0.169 \\
(0.366)\end{array}$ & $\begin{array}{l}0.011 \\
(0.024)\end{array}$ & $\begin{array}{l}-0.065 \\
(0.254)\end{array}$ & $\begin{array}{l}0.014 \\
(0.227)\end{array}$ & $\begin{array}{l}0.002 \\
(0.036)\end{array}$ \\
\hline $\begin{array}{l}\text { Geo exp - } \\
\text { managerial } \\
\text { capabilities }(H 7 a)\end{array}$ & $\begin{array}{l}0.174 \\
(0.273)\end{array}$ & $\begin{array}{l}0.103 \\
(0.252)\end{array}$ & $\begin{array}{l}0.008 \\
(0.023)\end{array}$ & $\begin{array}{l}0.909 \\
(1.177)\end{array}$ & $\begin{array}{l}0.836 \\
(0.836)\end{array}$ & $\begin{array}{l}0.054 \\
(0.097)\end{array}$ & $\begin{array}{l}0.411 \\
(0.492)\end{array}$ & $\begin{array}{l}0.408 \\
(0.435)\end{array}$ & $\begin{array}{l}0.028 \\
(0.030)\end{array}$ & $\begin{array}{l}-0.156 \\
(0.316)\end{array}$ & $\begin{array}{l}-0.227 \\
(0.281)\end{array}$ & $\begin{array}{l}-0.013 \\
(0.058)\end{array}$ \\
\hline $\begin{array}{l}\text { Geo exp - financial } \\
\text { capabilities }(H 7 a)\end{array}$ & $\begin{array}{l}-0.414 \dagger \\
(0.242)\end{array}$ & $\begin{array}{l}-0.342 \\
(0.221)\end{array}$ & $\begin{array}{l}-0.021 \\
(0.022)\end{array}$ & $\begin{array}{l}-0.535 \\
(0.797)\end{array}$ & $\begin{array}{l}-0.510 \\
(0.560)\end{array}$ & $\begin{array}{l}-0.032 \\
(0.072)\end{array}$ & $\begin{array}{l}-0.433 \\
(0.425)\end{array}$ & $\begin{array}{l}-0.426 \\
(0.376)\end{array}$ & $\begin{array}{l}-0.026 \\
(0.034)\end{array}$ & $\begin{array}{l}-0.567 \dagger \\
(0.315)\end{array}$ & $\begin{array}{l}-0.436 \\
(0.279)\end{array}$ & $\begin{array}{l}-0.027 \\
(0.034)\end{array}$ \\
\hline $\begin{array}{l}\text { Geo exp - intl } \\
\text { recruitment }(H 7 a)\end{array}$ & $\begin{array}{l}-0.154 \\
(0.273)\end{array}$ & $\begin{array}{l}-0.155 \\
(0.254)\end{array}$ & $\begin{array}{l}-0.010 \\
(0.020)\end{array}$ & $\begin{array}{l}0.079 \\
(0.749)\end{array}$ & $\begin{array}{l}0.072 \\
(0.575)\end{array}$ & $\begin{array}{l}0.006 \\
(0.047)\end{array}$ & $\begin{array}{l}-0.347 \\
(0.539)\end{array}$ & $\begin{array}{l}-0.346 \\
(0.468)\end{array}$ & $\begin{array}{l}-0.022 \\
(0.037)\end{array}$ & $\begin{array}{l}0.386 \\
(0.368)\end{array}$ & $\begin{array}{l}0.395 \\
(0.332)\end{array}$ & $\begin{array}{l}0.025 \\
(0.053)\end{array}$ \\
\hline Age & $\begin{array}{l}0.008 \\
(0.006)\end{array}$ & $\begin{array}{l}0.007 \\
(0.006)\end{array}$ & $\begin{array}{l}0.0004 \\
(0.0004)\end{array}$ & $\begin{array}{l}0.027 \\
(0.026)\end{array}$ & $\begin{array}{l}0.026 \\
(0.018)\end{array}$ & $\begin{array}{l}0.001 \\
(0.003)\end{array}$ & $\begin{array}{l}-0.004 \\
(0.012)\end{array}$ & $\begin{array}{l}-0.004 \\
(0.011)\end{array}$ & $\begin{array}{l}-0.0003 \\
(0.0008)\end{array}$ & $\begin{array}{l}0.009 \\
(0.007)\end{array}$ & $\begin{array}{l}0.007 \\
(0.006)\end{array}$ & $\begin{array}{l}0.0005 \\
(0.0007)\end{array}$ \\
\hline Size (Staff) & $\begin{array}{l}0.000 \\
(0.000)\end{array}$ & $\begin{array}{l}0.0002 \\
(0.0002)\end{array}$ & $\begin{array}{l}0.142 \mathrm{E}-4 \\
(0.220 \mathrm{E}- \\
4)\end{array}$ & $\begin{array}{l}-0.000 \\
(0.001)\end{array}$ & $\begin{array}{l}-0.181 \mathrm{E}-4 \\
(0.0005)\end{array}$ & $\begin{array}{l}-0.182 \mathrm{E}-5 \\
(0.486 \mathrm{E}-4)\end{array}$ & $\begin{array}{l}0.000 \\
(0.000)\end{array}$ & $\begin{array}{l}0.0003 \\
(0.0004)\end{array}$ & $\begin{array}{l}0.250 \mathrm{E}-4 \\
(0.369 \mathrm{E}-4)\end{array}$ & $\begin{array}{l}0.000 \\
(0.000)\end{array}$ & $\begin{array}{l}0.0004 \\
(0.0003)\end{array}$ & $\begin{array}{l}0.238 E-4 \\
(0.504 E-4)\end{array}$ \\
\hline Offshore policy & $\begin{array}{l}1.291 \\
(1.257)\end{array}$ & $\begin{array}{l}1.010 \\
(1.171)\end{array}$ & $\begin{array}{l}0.070 \\
(0.062)\end{array}$ & $\begin{array}{l}2.023 \\
(3.666)\end{array}$ & $\begin{array}{l}2.060 \\
(2.755)\end{array}$ & $\begin{array}{l}0.175 \\
(0.184)\end{array}$ & $\begin{array}{l}-2.881 \\
(2.571)\end{array}$ & $\begin{array}{l}-3.606 \dagger \\
(2.105)\end{array}$ & $\begin{array}{l}-0.224 \\
(0.158)\end{array}$ & $\begin{array}{l}4.365^{*} \\
(1.645)\end{array}$ & $\begin{array}{l}4.291 * * \\
(1.493)\end{array}$ & $\begin{array}{l}0.288^{*} \\
(0.139)\end{array}$ \\
\hline $\begin{array}{l}\text { Internationalisation } \\
\text { at home strategy }\end{array}$ & $\begin{array}{l}0.338 \\
(0.247)\end{array}$ & $\begin{array}{l}-0.002 \\
(0.001)\end{array}$ & $\begin{array}{l}-0.996 \mathrm{E}- \\
4 \\
(0.0001)\end{array}$ & $\begin{array}{l}0.178 \\
(1.026)\end{array}$ & $\begin{array}{l}-0.001 \\
(0.003)\end{array}$ & $\begin{array}{l}-0.785 E-4 \\
(0.002)\end{array}$ & $\begin{array}{l}0.317 \\
(0.471)\end{array}$ & $\begin{array}{l}-0.004 \\
(0.003)\end{array}$ & $\begin{array}{l}-0.0002 \\
(0.0006)\end{array}$ & $\begin{array}{l}0.232 \\
(0.301)\end{array}$ & $\begin{array}{l}-0.001 \\
(0.001)\end{array}$ & $\begin{array}{l}-0.710 \mathrm{E}-4 \\
(0.0002)\end{array}$ \\
\hline $\begin{array}{l}\text { Host country } \\
\text { demand }\end{array}$ & $\begin{array}{l}\text { 9.802* } \\
(3.824)\end{array}$ & $\begin{array}{l}9.294 * * \\
(3.414)\end{array}$ & $\begin{array}{l}0.559 \dagger \\
(0.324)\end{array}$ & $\begin{array}{l}7.806 \\
(9.823)\end{array}$ & $\begin{array}{l}7.824 \\
(6.993)\end{array}$ & $\begin{array}{l}0.468 \\
(0.798)\end{array}$ & $\begin{array}{l}11.372 \dagger \\
(6.633)\end{array}$ & $\begin{array}{l}\text { 12.468* } \\
(5.758)\end{array}$ & $\begin{array}{l}\mathbf{0 . 7 8 4} \dagger \\
(\mathbf{0 . 4 7 4 )}\end{array}$ & $\begin{array}{l}7.591 \\
(6.342)\end{array}$ & $\begin{array}{l}3.905 \\
(5.123)\end{array}$ & $\begin{array}{l}0.209 \\
(0.829)\end{array}$ \\
\hline AU/NZ dummy & $\begin{array}{l}0.404 \\
(1.008)\end{array}$ & $\begin{array}{l}0.177 \\
(0.932)\end{array}$ & $\begin{array}{l}0.011 \\
(0.077)\end{array}$ & - & - & - & - & - & - & - & - & - \\
\hline UK/IR dummy & $\begin{array}{l}1.371 \dagger \\
(\mathbf{0 . 7 5 8})\end{array}$ & $\begin{array}{l}1.249 \dagger \\
(0.700)\end{array}$ & $\begin{array}{l}0.078 \\
(0.058)\end{array}$ & - & - & - & - & - & - & - & - & - \\
\hline $\mathrm{R}^{2}$ & 0.155 & & & 0.224 & & & 0.221 & & & 0.267 & & \\
\hline $\operatorname{AdjR}^{2}$ & 0.08 & & & -0.184 & & & 0.052 & & & 0.123 & & \\
\hline
\end{tabular}




\begin{tabular}{|c|c|c|c|c|c|c|c|c|c|c|c|c|}
\hline Log likelihood & & -426.21 & -443.17 & & -89.48 & -95.44 & & -163.29 & -168.76 & & -159.07 & -171.15 \\
\hline $\mathrm{N}$ & 157 & 157 & 157 & 32 & 32 & 32 & 59 & 59 & 59 & 66 & 66 & 66 \\
\hline Max. VIF & 1.145 & & & 1.485 & & & 1.31 & & & 1.400 & & \\
\hline
\end{tabular}

Standard errors in parentheses, all $t$ tests are two-tailed, $\uparrow p<.10, * p<.05, * * p<.01$

Table 7.13b: Regression estimates for equation $4 \mathrm{~b}$ (high normative institutional distance)

\begin{tabular}{|c|c|c|c|c|c|c|c|c|c|c|c|c|}
\hline & \multicolumn{3}{|c|}{ Complete data set } & \multicolumn{3}{|c|}{ AU/NZ } & \multicolumn{3}{|c|}{ US/CA } & \multicolumn{3}{|c|}{ UK/IR } \\
\hline & OLS & Tobit & $\begin{array}{l}\text { Negative } \\
\text { binomial }\end{array}$ & OLS & Tobit & $\begin{array}{l}\text { Negative } \\
\text { binomial }\end{array}$ & OLS & Tobit & $\begin{array}{l}\text { Negative } \\
\text { binomial }\end{array}$ & OLS & Tobit & $\begin{array}{l}\text { Negative } \\
\text { binomial }\end{array}$ \\
\hline Intercept & $\begin{array}{l}2.736 \\
(3.871)\end{array}$ & $\begin{array}{l}3.283 \\
(3.573)\end{array}$ & $\begin{array}{l}1.852 * * \\
(0.348)\end{array}$ & $\begin{array}{l}3.982 \\
(6.432)\end{array}$ & $\begin{array}{l}4.15 \\
(5.588)\end{array}$ & $\begin{array}{l}\text { 2.051* } \\
(0.809)\end{array}$ & $\begin{array}{l}4.145 \\
(6.568)\end{array}$ & $\begin{array}{l}4.145 \\
(5.977)\end{array}$ & $\begin{array}{l}1.568 * \\
(0.613)\end{array}$ & $\begin{array}{l}11.344 * \\
(5.330)\end{array}$ & $\begin{array}{l}13.421 * * \\
(4.192)\end{array}$ & $\begin{array}{l}2.606 \\
(21.512)\end{array}$ \\
\hline $\begin{array}{l}\text { Geo exp - } \\
\text { knowledge of edu } \\
\text { sector }(H 7 a)\end{array}$ & $\begin{array}{l}0.401 \dagger \\
(0.218)\end{array}$ & $\begin{array}{l}\text { 0.401* } \\
(0.203)\end{array}$ & $\begin{array}{l}0.023 \\
(0.022)\end{array}$ & $\begin{array}{l}1.085^{*} \\
(0.423)\end{array}$ & $\begin{array}{l}1.138 * * \\
(0.366)\end{array}$ & $\begin{array}{l}0.067 \\
(0.051)\end{array}$ & $\begin{array}{l}0.172 \\
(0.340)\end{array}$ & $\begin{array}{l}0.172 \\
(0.309)\end{array}$ & $\begin{array}{l}0.013 \\
(0.037)\end{array}$ & $\begin{array}{l}0.041 \\
(0.327)\end{array}$ & $\begin{array}{l}0.077 \\
(0.232)\end{array}$ & $\begin{array}{l}0.004 \\
(0.078)\end{array}$ \\
\hline $\begin{array}{l}\text { Geo exp - academic } \\
\text { capabilities }(H 7 a)\end{array}$ & $\begin{array}{l}0.106 \\
(0.206)\end{array}$ & $\begin{array}{l}0.060 \\
(0.193)\end{array}$ & $\begin{array}{l}0.004 \\
(0.017)\end{array}$ & $\begin{array}{l}-0.049 \\
(0.347)\end{array}$ & $\begin{array}{l}-0.069 \\
(0.303)\end{array}$ & $\begin{array}{l}-0.004 \\
(0.045)\end{array}$ & $\begin{array}{l}0.314 \\
(0.371)\end{array}$ & $\begin{array}{l}0.314 \\
(0.338)\end{array}$ & $\begin{array}{l}0.023 \\
(0.033)\end{array}$ & $\begin{array}{l}-0.081 \\
(0.289)\end{array}$ & $\begin{array}{l}-0.205 \\
(0.210)\end{array}$ & $\begin{array}{l}-0.011 \\
(0.060)\end{array}$ \\
\hline $\begin{array}{l}\text { Geo exp - } \\
\text { managerial } \\
\text { capabilities }(H 7 a)\end{array}$ & $\begin{array}{l}0.221 \\
(0.233)\end{array}$ & $\begin{array}{l}0.196 \\
(0.220)\end{array}$ & $\begin{array}{l}0.012 \\
(0.023)\end{array}$ & $\begin{array}{l}0.038 \\
(0.395)\end{array}$ & $\begin{array}{l}0.053 \\
(0.344)\end{array}$ & $\begin{array}{l}0.004 \\
(0.053)\end{array}$ & $\begin{array}{l}0.224 \\
(0.382)\end{array}$ & $\begin{array}{l}0.223 \\
(0.348)\end{array}$ & $\begin{array}{l}0.013 \\
(0.036)\end{array}$ & $\begin{array}{l}0.695 \dagger \\
(0.393)\end{array}$ & $\begin{array}{l}0.566^{*} \\
(0.268)\end{array}$ & $\begin{array}{l}0.031 \\
(0.070)\end{array}$ \\
\hline $\begin{array}{l}\text { Geo exp - financial } \\
\text { capabilities }(H 7 a)\end{array}$ & $\begin{array}{l}0.178 \\
(0.240)\end{array}$ & $\begin{array}{l}0.096 \\
(0.221)\end{array}$ & $\begin{array}{l}0.006 \\
(0.026)\end{array}$ & $\begin{array}{l}-0.101 \\
(0.485)\end{array}$ & $\begin{array}{l}-0.162 \\
(0.389)\end{array}$ & $\begin{array}{l}-0.009 \\
(0.081)\end{array}$ & $\begin{array}{l}0.129 \\
(0.387)\end{array}$ & $\begin{array}{l}0.129 \\
(0.352)\end{array}$ & $\begin{array}{l}0.010 \\
(0.041)\end{array}$ & $\begin{array}{l}0.191 \\
(0.345)\end{array}$ & $\begin{array}{l}0.033 \\
(0.265)\end{array}$ & $\begin{array}{l}0.001 \\
(0.085)\end{array}$ \\
\hline $\begin{array}{l}\text { Geo exp - intl } \\
\text { recruitment }(H 7 a)\end{array}$ & $\begin{array}{l}0.014 \\
(0.256)\end{array}$ & $\begin{array}{l}-0.009 \\
(0.242)\end{array}$ & $\begin{array}{l}-0.0007 \\
(0.026)\end{array}$ & $\begin{array}{l}0.582 \\
(0.427)\end{array}$ & $\begin{array}{l}0.592 \\
(0.373)\end{array}$ & $\begin{array}{l}0.035 \\
(0.055)\end{array}$ & $\begin{array}{l}-0.393 \\
(0.436)\end{array}$ & $\begin{array}{l}-0.393 \\
(0.397)\end{array}$ & $\begin{array}{l}-0.025 \\
(0.049)\end{array}$ & $\begin{array}{l}-0.223 \\
(0.326)\end{array}$ & $\begin{array}{l}-0.205 \\
(0.266)\end{array}$ & $\begin{array}{l}-0.011 \\
(0.081)\end{array}$ \\
\hline Age & $\begin{array}{l}0.001 \\
(0.006)\end{array}$ & $\begin{array}{l}0.0006 \\
(0.005)\end{array}$ & $\begin{array}{l}0.507 \mathrm{E}-4 \\
(0.0004)\end{array}$ & $\begin{array}{l}0.000 \\
(0.013)\end{array}$ & $\begin{array}{l}0.0004 \\
(0.011)\end{array}$ & $\begin{array}{l}0.558 \mathrm{E}-4 \\
(0.002)\end{array}$ & $\begin{array}{l}0.002 \\
(0.009)\end{array}$ & $\begin{array}{l}0.002 \\
(0.008)\end{array}$ & $\begin{array}{l}0.891 \mathrm{E}-4 \\
(0.0007)\end{array}$ & $\begin{array}{l}0.000 \\
(0.007)\end{array}$ & $\begin{array}{l}-0.003 \\
(0.005)\end{array}$ & $\begin{array}{l}-0.0002 \\
(0.02)\end{array}$ \\
\hline Size (Staff) & $\begin{array}{l}-0.000 \\
(0.000)\end{array}$ & $\begin{array}{l}-0.0001 \\
(0.0002)\end{array}$ & $\begin{array}{l}-0.947 \mathrm{E}-5 \\
(0.279 \mathrm{E}-4)\end{array}$ & $\begin{array}{l}0.000 \\
(0.001)\end{array}$ & $\begin{array}{l}-0.0006 \\
(0.0004)\end{array}$ & $\begin{array}{l}-0.374 \mathrm{E}-4 \\
(0.737 \mathrm{E}-4)\end{array}$ & $\begin{array}{l}0.000 \\
(0.000)\end{array}$ & $\begin{array}{l}0.7211 \mathrm{E}-4 \\
(0.0003)\end{array}$ & $\begin{array}{l}0.070 \mathrm{E}-5 \\
(0.578 \mathrm{E}-4)\end{array}$ & $\begin{array}{l}-0.000 \\
(0.000)\end{array}$ & $\begin{array}{l}-0.0002 \\
(0.0002)\end{array}$ & $\begin{array}{l}-0.147 \\
(0 . .0001)\end{array}$ \\
\hline Offshore policy & $\begin{array}{l}\text { 8.060** } \\
(1.127)\end{array}$ & $\begin{array}{l}\text { 8.020** } \\
(1.072)\end{array}$ & $\begin{array}{l}0.631^{* *} \\
(0.076)\end{array}$ & $\begin{array}{l}2.854 \\
(2.128)\end{array}$ & $\begin{array}{l}2.972 \\
(1.840)\end{array}$ & $\begin{array}{l}0.181 \\
(0.277)\end{array}$ & $\begin{array}{l}\text { 11.595** } \\
(1.739)\end{array}$ & $\begin{array}{l}\text { 11.595** } \\
(1.582)\end{array}$ & $\begin{array}{l}\text { 1.161** } \\
(0.138)\end{array}$ & $\begin{array}{l}4.086^{*} \\
(1.759)\end{array}$ & $\begin{array}{l}3.685^{*} \\
(1.431)\end{array}$ & $\begin{array}{l}0.232 \\
(21.480)\end{array}$ \\
\hline $\begin{array}{l}\text { Internationalisation } \\
\text { at home strategy }\end{array}$ & $\begin{array}{l}-0.277 \\
(0.227)\end{array}$ & $\begin{array}{l}0.225 E-4 \\
(0.001)\end{array}$ & $\begin{array}{l}0.866 \mathrm{E}-6 \\
(0.0001)\end{array}$ & $\begin{array}{l}0.121 \\
(0.410)\end{array}$ & $\begin{array}{l}-0.003 \\
(0.002)\end{array}$ & $\begin{array}{l}-0.0002 \\
(0.0008)\end{array}$ & $\begin{array}{l}-0.614 \\
(0.369)\end{array}$ & $\begin{array}{l}-0.614 \dagger \\
(0.335)\end{array}$ & $\begin{array}{l}-0.418 \\
(0.037)\end{array}$ & $\begin{array}{l}-0.448 \\
(0.381)\end{array}$ & $\begin{array}{l}0.001 \\
(0.001)\end{array}$ & $\begin{array}{l}0.865 E-4 \\
(0.0003)\end{array}$ \\
\hline $\begin{array}{l}\text { Host country } \\
\text { demand }\end{array}$ & $\begin{array}{l}1.373 \\
(2.986)\end{array}$ & $\begin{array}{l}1.693 \\
(2.836)\end{array}$ & $\begin{array}{l}0.1010 \\
(0.306)\end{array}$ & $\begin{array}{l}5.691 \\
(5.769)\end{array}$ & $\begin{array}{l}6.8000 \\
(4.732)\end{array}$ & $\begin{array}{l}0.400 \\
(0.646)\end{array}$ & $\begin{array}{l}-2.486 \\
(4.862)\end{array}$ & $\begin{array}{l}-2.486 \\
(4.424)\end{array}$ & $\begin{array}{l}-0.204 \\
(0.527)\end{array}$ & $\begin{array}{l}-0.990 \\
(4.057)\end{array}$ & $\begin{array}{l}1.047 \\
(3.069)\end{array}$ & $\begin{array}{l}0.056 \\
(1.013)\end{array}$ \\
\hline AU/NZ dummy & $\begin{array}{l}0.891 \\
(0.785)\end{array}$ & $\begin{array}{l}1.135 \\
(0.733)\end{array}$ & $\begin{array}{l}0.071 \\
(0.083)\end{array}$ & - & - & - & - & - & - & - & - & - \\
\hline
\end{tabular}




\begin{tabular}{|c|c|c|c|c|c|c|c|c|c|c|c|c|}
\hline UK/IR dummy & $\begin{array}{l}1.642^{*} \\
(0.838)\end{array}$ & $\begin{array}{l}1.652 * \\
(0.779)\end{array}$ & $\begin{array}{l}0.098 \\
(0.093)\end{array}$ & - & - & - & - & - & - & - & - & - \\
\hline $\mathrm{R}^{2}$ & 0.346 & & & 0.274 & & & 0.531 & & & 0.359 & & \\
\hline $\operatorname{Adj}^{2}$ & 0.286 & & & 0.066 & & & 0.443 & & & 0.067 & & \\
\hline Log likelihood & & -397.15 & -424.33 & & -119.69 & -128.74 & & -172.06 & -184.36 & & -76.36 & -93.06 \\
\hline $\mathrm{N}$ & 150 & 150 & 150 & 48 & 48 & 48 & 64 & 64 & 64 & 38 & 38 & 38 \\
\hline Max. VIF & 1.496 & & & 1.348 & & & 1.298 & & & 1.885 & & \\
\hline
\end{tabular}

Standard errors in parentheses, all $t$ tests are two-tailed, $\uparrow p<.10,{ }^{*} p<.05,{ }^{* *} p<.01$

Table 7.14a: Regression estimates for equation 5a (low-median normative institutional distance)

\begin{tabular}{|c|c|c|c|c|c|c|c|c|c|c|c|c|}
\hline & \multicolumn{3}{|c|}{ Complete data set } & \multicolumn{3}{|c|}{ AU/NZ } & \multicolumn{3}{|c|}{ US/CA } & \multicolumn{3}{|c|}{ UK/IR } \\
\hline & OLS & Tobit & $\begin{array}{l}\text { Negative } \\
\text { binomial }\end{array}$ & OLS & Tobit & $\begin{array}{l}\text { Negative } \\
\text { binomial }\end{array}$ & OLS & Tobit & $\begin{array}{l}\text { Negative } \\
\text { binomial }\end{array}$ & OLS & Tobit & $\begin{array}{l}\text { Negative } \\
\text { binomial }\end{array}$ \\
\hline Intercept & $\begin{array}{l}5.184 \\
(3.215)\end{array}$ & $\begin{array}{l}7.610 * * \\
(2.909)\end{array}$ & $\begin{array}{l}2.248 * * \\
(0.230)\end{array}$ & $\begin{array}{l}4.357 \\
(9.882)\end{array}$ & $\begin{array}{l}5.580 \\
(6.306)\end{array}$ & $\begin{array}{l}2.046 * * \\
(0.679)\end{array}$ & $\begin{array}{l}5.621 \\
(6.859)\end{array}$ & $\begin{array}{l}8.668 \\
(5.560)\end{array}$ & $\begin{array}{l}2.272 * * \\
(0.412)\end{array}$ & $\begin{array}{l}7.288 \dagger \\
(3.671)\end{array}$ & $\begin{array}{l}\text { 8.868** } \\
(3.328)\end{array}$ & $\begin{array}{l}2.314 * * \\
(0.481)\end{array}$ \\
\hline $\begin{array}{l}\text { Ind exp - UG } \\
\text { offshore edu (H7b) }\end{array}$ & $\begin{array}{l}-0.131 \\
(0.199)\end{array}$ & $\begin{array}{l}-0.147 \\
(0.186)\end{array}$ & $\begin{array}{l}-0.009 \\
(0.014)\end{array}$ & $\begin{array}{l}-0.213 \\
(0.726)\end{array}$ & $\begin{array}{l}-0.321 \\
(0.497)\end{array}$ & $\begin{array}{l}-0.022 \\
(0.064)\end{array}$ & $\begin{array}{l}-0.314 \\
(0.383)\end{array}$ & $\begin{array}{l}-0.324 \\
(0.340)\end{array}$ & $\begin{array}{l}-0.021 \\
(0.037)\end{array}$ & $\begin{array}{l}0.052 \\
(0.240)\end{array}$ & $\begin{array}{l}0.029 \\
(0.219)\end{array}$ & $\begin{array}{l}0.001 \\
(0.039)\end{array}$ \\
\hline $\begin{array}{l}\text { Ind exp - PG } \\
\text { offshore edu }(H 7 b)\end{array}$ & $\begin{array}{l}0.034 \\
(0.244)\end{array}$ & $\begin{array}{l}0.058 \\
(0.227)\end{array}$ & $\begin{array}{l}0.003 \\
(0.016)\end{array}$ & $\begin{array}{l}0.167 \\
(0.634)\end{array}$ & $\begin{array}{l}0.199 \\
(0.471)\end{array}$ & $\begin{array}{l}0.011 \\
(0.064)\end{array}$ & $\begin{array}{l}-0.073 \\
(0.453)\end{array}$ & $\begin{array}{l}-0.050 \\
(0.403)\end{array}$ & $\begin{array}{l}-0.005 \\
(0.029)\end{array}$ & $\begin{array}{l}-0.160 \\
(0.329)\end{array}$ & $\begin{array}{l}-0.167 \\
(0.298)\end{array}$ & $\begin{array}{l}-0.009 \\
(0.049)\end{array}$ \\
\hline $\begin{array}{l}\text { Ind exp - Intl } \\
\text { recruitment (Factor) } \\
(H 7 b)\end{array}$ & $\begin{array}{l}0.807^{* *} \\
(\mathbf{0 . 2 8 2})\end{array}$ & $\begin{array}{l}0.695 * * \\
(0.252)\end{array}$ & $\begin{array}{l}0.043 \dagger \\
(0.023)\end{array}$ & $\begin{array}{l}2.006 * \\
(0.895)\end{array}$ & $\begin{array}{l}1.921 * * \\
(0.671)\end{array}$ & $\begin{array}{l}0.127 \\
(0.079)\end{array}$ & $\begin{array}{l}1.385^{*} \\
(0.538)\end{array}$ & $\begin{array}{l}1.270 * * \\
(0.472)\end{array}$ & $\begin{array}{l}0.085 \dagger \\
(0.046)\end{array}$ & $\begin{array}{l}0.034 \\
(0.338)\end{array}$ & $\begin{array}{l}-0.017 \\
(0.281)\end{array}$ & $\begin{array}{l}-0.001 \\
(0.057)\end{array}$ \\
\hline $\begin{array}{l}\text { Ind exp - UG } \\
\text { marketing }(H 7 b)\end{array}$ & $\begin{array}{l}0.025 \\
(0.197)\end{array}$ & $\begin{array}{l}0.026 \\
(0.181)\end{array}$ & $\begin{array}{l}0.001 \\
(0.018)\end{array}$ & $\begin{array}{l}-1.612 * \\
(0.754)\end{array}$ & $\begin{array}{l}-1.568 * * \\
(0.573)\end{array}$ & $\begin{array}{l}-0.100 \dagger \\
(0.054)\end{array}$ & $\begin{array}{l}0.175 \\
(0.347)\end{array}$ & $\begin{array}{l}0.142 \\
(0.308)\end{array}$ & $\begin{array}{l}0.008 \\
(0.035)\end{array}$ & $\begin{array}{l}0.176 \\
(0.234)\end{array}$ & $\begin{array}{l}0.209 \\
(0.211)\end{array}$ & $\begin{array}{l}0.012 \\
(0.040)\end{array}$ \\
\hline $\begin{array}{l}\text { Ind exp - PG } \\
\text { marketing }(H 7 b)\end{array}$ & $\begin{array}{l}0.300 \\
(0.215)\end{array}$ & $\begin{array}{l}0.234 \\
(0.198)\end{array}$ & $\begin{array}{l}0.014 \\
(0.016)\end{array}$ & $\begin{array}{l}0.261 \\
(0.549)\end{array}$ & $\begin{array}{l}0.213 \\
(0.411)\end{array}$ & $\begin{array}{l}0.011 \\
(0.042)\end{array}$ & $\begin{array}{l}0.365 \\
(0.386)\end{array}$ & $\begin{array}{l}0.325 \\
(0.344)\end{array}$ & $\begin{array}{l}0.021 \\
(0.029)\end{array}$ & $\begin{array}{l}\mathbf{0 . 5 5 5 \dagger} \\
\mathbf{( 0 . 2 9 9 )}\end{array}$ & $\begin{array}{l}0.371 \\
(0.258)\end{array}$ & $\begin{array}{l}0.022 \\
(0.040)\end{array}$ \\
\hline Age & $\begin{array}{l}0.007 \\
(0.006)\end{array}$ & $\begin{array}{l}0.005 \\
(0.005)\end{array}$ & $\begin{array}{l}0.0003 \\
(0.0004)\end{array}$ & $\begin{array}{l}0.009 \\
(0.023)\end{array}$ & $\begin{array}{l}0.011 \\
(0.015)\end{array}$ & $\begin{array}{l}0.0006 \\
(0.001)\end{array}$ & $\begin{array}{l}-0.006 \\
(0.012)\end{array}$ & $\begin{array}{l}-0.006 \\
(0.010)\end{array}$ & $\begin{array}{l}-0.0003 \\
(0.0008)\end{array}$ & $\begin{array}{l}0.009 \\
(0.007)\end{array}$ & $\begin{array}{l}0.007 \\
(0.005)\end{array}$ & $\begin{array}{l}0.0004 \\
(0.0008)\end{array}$ \\
\hline Size (Staff) & $\begin{array}{l}0.000 \\
(0.000)\end{array}$ & $\begin{array}{l}0.0002 \\
(0.0002)\end{array}$ & $\begin{array}{l}0.158 \mathrm{E}-4 \\
(0.229 \mathrm{E}-4)\end{array}$ & $\begin{array}{l}0.000 \\
(0.001)\end{array}$ & $\begin{array}{l}0.0001 \\
(0.0005)\end{array}$ & $\begin{array}{l}0.607 \mathrm{E}-5 \\
(0.640 \mathrm{E}-4)\end{array}$ & $\begin{array}{l}0.001 \\
(0.000)\end{array}$ & $\begin{array}{l}0.0005 \\
(0.0004)\end{array}$ & $\begin{array}{l}0.358 E-4 \\
(0.347 E-4)\end{array}$ & $\begin{array}{l}0.000 \\
(0.000)\end{array}$ & $\begin{array}{l}\mathbf{0 . 0 0 0 4} \dagger \\
(\mathbf{0 . 0 0 0 2})\end{array}$ & $\begin{array}{l}0.276 \mathrm{E}-4 \\
(0.517 \mathrm{E}-4)\end{array}$ \\
\hline Offshore policy & $\begin{array}{l}1.605 \\
(1.246)\end{array}$ & $\begin{array}{l}1.386 \\
(1.175)\end{array}$ & $\begin{array}{l}0.090 \\
(0.059)\end{array}$ & $\begin{array}{l}8.075 * \\
(3.562)\end{array}$ & $\begin{array}{l}8.062 * * \\
(2.753)\end{array}$ & $\begin{array}{l}0.566 \dagger \\
(0.329)\end{array}$ & $\begin{array}{l}-2.036 \\
(2.552)\end{array}$ & $\begin{array}{l}-2.966 \\
(2.087)\end{array}$ & $\begin{array}{l}-0.191 \\
(0.126)\end{array}$ & $\begin{array}{l}3.699 * \\
(1.670)\end{array}$ & $\begin{array}{l}3.953^{*} \\
(1.536)\end{array}$ & $\begin{array}{l}0.265 \dagger \\
(0.151)\end{array}$ \\
\hline $\begin{array}{l}\text { Internationalisation } \\
\text { at home strategy }\end{array}$ & $\begin{array}{l}0.321 \\
(0.239)\end{array}$ & $\begin{array}{l}-0.001 \\
(0.001)\end{array}$ & $\begin{array}{l}-0.665 \mathrm{E}-4 \\
(0.0001)\end{array}$ & $\begin{array}{l}0.116 \\
(0.978)\end{array}$ & $\begin{array}{l}0.002 \\
(0.002)\end{array}$ & $\begin{array}{l}0.0001 \\
(0.0009)\end{array}$ & $\begin{array}{l}0.362 \\
(0.468)\end{array}$ & $\begin{array}{l}-0.004 \\
(0.002)\end{array}$ & $\begin{array}{l}-0.0002 \\
(0.0002)\end{array}$ & $\begin{array}{l}0.219 \\
(0.283)\end{array}$ & $\begin{array}{l}-0.0002 \\
(0.001)\end{array}$ & $\begin{array}{l}-0.124 \mathrm{E}-4 \\
(0.0002)\end{array}$ \\
\hline
\end{tabular}




\begin{tabular}{|c|c|c|c|c|c|c|c|c|c|c|c|c|}
\hline $\begin{array}{l}\text { Host country } \\
\text { demand }\end{array}$ & $\begin{array}{l}\text { 9.358* } \\
\text { (3.810) }\end{array}$ & $\begin{array}{l}\text { 8.670* } \\
(3.430)\end{array}$ & $\begin{array}{l}0.519 \\
(0.342)\end{array}$ & $\begin{array}{l}4.133 \\
(8.749)\end{array}$ & $\begin{array}{l}5.474 \\
(6.215)\end{array}$ & $\begin{array}{l}0.336 \\
(0.806)\end{array}$ & $\begin{array}{l}10.562 \\
(6.522)\end{array}$ & $\begin{array}{l}\text { 11.800* } \\
\text { (5.694) }\end{array}$ & $\begin{array}{l}0.747 \\
(0.505)\end{array}$ & $\begin{array}{l}12.203 \\
(6.602)\end{array}$ & $\begin{array}{l}6.721 \\
(5.435)\end{array}$ & $\begin{array}{l}0.376 \\
(0.944)\end{array}$ \\
\hline AU/NZ dummy & $\begin{array}{l}0.525 \\
(1.013)\end{array}$ & $\begin{array}{l}0.301 \\
(0.945)\end{array}$ & $\begin{array}{l}0.019 \\
(0.078)\end{array}$ & - & - & - & - & - & - & - & - & - \\
\hline UK/IR dummy & $\begin{array}{l}1.203 \\
(0.751)\end{array}$ & $\begin{array}{l}1.112 \\
(0.698)\end{array}$ & $\begin{array}{l}0.069 \\
(0.058)\end{array}$ & - & - & - & - & - & - & - & - & - \\
\hline $\mathrm{R}^{2}$ & 0.158 & & & 0.401 & & & 0.239 & & & 0.258 & & \\
\hline $\operatorname{Adj}^{2}$ & 0.084 & & & 0.086 & & & 0.073 & & & 0.112 & & \\
\hline Log likelihood & & -427.27 & -444.09 & & -85.44 & -91.71 & & -163.20 & -168.54 & & -160.49 & -171.84 \\
\hline $\mathrm{N}$ & 157 & 157 & 157 & 32 & 32 & 32 & 59 & 59 & 59 & 66 & 66 & 66 \\
\hline Max. VIF & 1.656 & & & 2.194 & & & 1.214 & & & 1.222 & & \\
\hline
\end{tabular}

Standard errors in parentheses, all $t$ tests are two-tailed, $\dagger p<.10,{ }^{*} p<.05,{ }^{* *} p<.01$

Table 7.14b: Regression estimates for equation $5 b$ (median-high normative institutional distance)

\begin{tabular}{|c|c|c|c|c|c|c|c|c|c|c|c|c|}
\hline & \multicolumn{3}{|c|}{ Complete data set } & \multicolumn{3}{|c|}{ AU/NZ } & \multicolumn{3}{|c|}{ US/CA } & \multicolumn{3}{|c|}{ UK/IR } \\
\hline & OLS & Tobit & $\begin{array}{l}\text { Negative } \\
\text { binomial } \\
\end{array}$ & OLS & Tobit & $\begin{array}{l}\text { Negative } \\
\text { binomial }\end{array}$ & OLS & Tobit & $\begin{array}{l}\text { Negative } \\
\text { binomial } \\
\end{array}$ & OLS & Tobit & $\begin{array}{l}\text { Negative } \\
\text { binomial } \\
\end{array}$ \\
\hline Intercept & $\begin{array}{l}9.873 * * \\
(2.857)\end{array}$ & $\begin{array}{l}9.486 * * \\
(2.612)\end{array}$ & $\begin{array}{l}2.241^{* *} \\
(0.258)\end{array}$ & $\begin{array}{l}16.862 * \\
(5.396)\end{array}$ & $\begin{array}{l}18.517 * * \\
(4.595)\end{array}$ & $\begin{array}{l}2.902 * * \\
(0.739)\end{array}$ & $\begin{array}{l}7.969 \\
(5.285)\end{array}$ & $\begin{array}{l}7.968 \dagger \\
\mathbf{( 4 . 8 0 9 )}\end{array}$ & $\begin{array}{l}1.824 * * \\
(0.547)\end{array}$ & $\begin{array}{l}10.376 * \\
\text { (3.177) }\end{array}$ & $\begin{array}{l}10.945 * * \\
(2.628)\end{array}$ & $\begin{array}{l}2.470 \\
(2.276)\end{array}$ \\
\hline $\begin{array}{l}\text { Ind exp - UG } \\
\text { offshore edu (H7b) }\end{array}$ & $\begin{array}{l}0.111 \\
(0.197)\end{array}$ & $\begin{array}{l}0.076 \\
(0.185)\end{array}$ & $\begin{array}{l}0.004 \\
(0.022)\end{array}$ & $\begin{array}{l}-0.126 \\
(0.353)\end{array}$ & $\begin{array}{l}-0.135 \\
(0.310)\end{array}$ & $\begin{array}{l}-0.007 \\
(0.055)\end{array}$ & $\begin{array}{l}-0.009 \\
(0.368)\end{array}$ & $\begin{array}{l}-0.008 \\
(0.334)\end{array}$ & $\begin{array}{l}-0.116 \\
(0.046)\end{array}$ & $\begin{array}{l}0.243 \\
(0.258)\end{array}$ & $\begin{array}{l}0.298 \\
(0.203)\end{array}$ & $\begin{array}{l}0.016 \\
(0.068)\end{array}$ \\
\hline $\begin{array}{l}\text { Ind exp - PG } \\
\text { offshore edu }(H 7 b)\end{array}$ & $\begin{array}{l}-0.106 \\
(0.232)\end{array}$ & $\begin{array}{l}-0.122 \\
(0.219)\end{array}$ & $\begin{array}{l}-0.007 \\
(0.024)\end{array}$ & $\begin{array}{l}-0.384 \\
(0.424)\end{array}$ & $\begin{array}{l}-0.347 \\
(0.373)\end{array}$ & $\begin{array}{l}-0.019 \\
(0.058)\end{array}$ & $\begin{array}{l}-0.181 \\
(0.385)\end{array}$ & $\begin{array}{l}-0.181 \\
(0.350)\end{array}$ & $\begin{array}{l}-0.010 \\
(0.039)\end{array}$ & $\begin{array}{l}0.339 \\
(0.356)\end{array}$ & $\begin{array}{l}0.170 \\
(0.276)\end{array}$ & $\begin{array}{l}0.009 \\
(0.096)\end{array}$ \\
\hline $\begin{array}{l}\text { Ind exp }(H 7 b)-\text { Intl } \\
\text { recruitment (Factor) }\end{array}$ & $\begin{array}{l}0.122 \\
(0.244)\end{array}$ & $\begin{array}{l}0.101 \\
(0.229)\end{array}$ & $\begin{array}{l}0.005 \\
(0.026)\end{array}$ & $\begin{array}{l}0.151 \\
(0.447)\end{array}$ & $\begin{array}{l}-0.022 \\
(0.370)\end{array}$ & $\begin{array}{l}-0.001 \\
(0.055)\end{array}$ & $\begin{array}{l}0.174 \\
(0.444)\end{array}$ & $\begin{array}{l}0.173 \\
(0.404)\end{array}$ & $\begin{array}{l}0.010 \\
(0.046)\end{array}$ & $\begin{array}{l}0.383 \\
(0.354)\end{array}$ & $\begin{array}{l}0.421 \\
(0.289)\end{array}$ & $\begin{array}{l}0.023 \\
(0.096)\end{array}$ \\
\hline $\begin{array}{l}\text { Ind exp - UG } \\
\text { marketing }(H 7 b)\end{array}$ & $\begin{array}{l}-0.359 \\
(0.216)\end{array}$ & $\begin{array}{l}-0.320 \\
(0.201)\end{array}$ & $\begin{array}{l}-0.020 \\
(0.025)\end{array}$ & $\begin{array}{l}-0.174 \\
(0.443)\end{array}$ & $\begin{array}{l}-0.154 \\
(0.392)\end{array}$ & $\begin{array}{l}-0.008 \\
(0.064)\end{array}$ & $\begin{array}{l}-0.223 \\
(0.337)\end{array}$ & $\begin{array}{l}-0.222 \\
(0.306)\end{array}$ & $\begin{array}{l}-0.012 \\
(0.039)\end{array}$ & $\begin{array}{l}-0.173 \\
(0.378)\end{array}$ & $\begin{array}{l}-0.168 \\
(0.281)\end{array}$ & $\begin{array}{l}-0.009 \\
(0.101)\end{array}$ \\
\hline $\begin{array}{l}\text { Ind exp - PG } \\
\text { marketing }(H 7 b)\end{array}$ & $\begin{array}{l}0.169 \\
(0.184)\end{array}$ & $\begin{array}{l}0.122 \\
(0.173)\end{array}$ & $\begin{array}{l}0.007 \\
(0.016)\end{array}$ & $\begin{array}{l}0.418 \\
(0.363)\end{array}$ & $\begin{array}{l}0.393 \\
(0.319)\end{array}$ & $\begin{array}{l}0.022 \\
(0.038)\end{array}$ & $\begin{array}{l}-0.042 \\
(0.301)\end{array}$ & $\begin{array}{l}-0.041 \\
(0.274)\end{array}$ & $\begin{array}{l}-0.003 \\
(0.026)\end{array}$ & $\begin{array}{l}0.164 \\
(0.266)\end{array}$ & $\begin{array}{l}-0.0005 \\
(0.204)\end{array}$ & $\begin{array}{l}0.0002 \\
(0.095)\end{array}$ \\
\hline Age & $\begin{array}{l}-0.002 \\
(0.006)\end{array}$ & $\begin{array}{l}-0.002 \\
(0.005)\end{array}$ & $\begin{array}{l}-0.0001 \\
(0.0006)\end{array}$ & $\begin{array}{l}-0.017 \\
(0.014)\end{array}$ & $\begin{array}{l}-0.018 \\
(0.011)\end{array}$ & $\begin{array}{l}-0.001 \\
(0.001)\end{array}$ & $\begin{array}{l}0.001 \\
(0.010)\end{array}$ & $\begin{array}{l}0.001 \\
(0.008)\end{array}$ & $\begin{array}{l}0.957 \mathrm{E}-4 \\
(0.001)\end{array}$ & $\begin{array}{l}0.001 \\
(0.008)\end{array}$ & $\begin{array}{l}-0.002 \\
(0.005)\end{array}$ & $\begin{array}{l}-0.0001 \\
(0.002)\end{array}$ \\
\hline Size (Staff) & $\begin{array}{l}-0.000 \\
(0.000)\end{array}$ & $\begin{array}{l}-0.794 \mathrm{D}-4 \\
(0.0002)\end{array}$ & $\begin{array}{l}-0.446 \mathrm{E}-5 \\
(0.272 \mathrm{E}-4)\end{array}$ & $\begin{array}{l}0.000 \\
(0.001)\end{array}$ & $\begin{array}{l}-0.0004 \\
(0.0004)\end{array}$ & $\begin{array}{l}-0.257 \mathrm{E}-4 \\
(0.749 \mathrm{E}-4)\end{array}$ & $\begin{array}{l}0.000 \\
(0.000)\end{array}$ & $\begin{array}{l}0.0001 \\
(0.0003)\end{array}$ & $\begin{array}{l}0.843 \mathrm{E}-5 \\
(0.531 \mathrm{E}-4)\end{array}$ & $\begin{array}{l}0.000 \\
(0.000)\end{array}$ & $\begin{array}{l}-0.0004 \dagger \\
(0.0002)\end{array}$ & $\begin{array}{l}-0.242 \mathrm{E}-4 \\
(0.0001)\end{array}$ \\
\hline
\end{tabular}




\begin{tabular}{|c|c|c|c|c|c|c|c|c|c|c|c|c|}
\hline Offshore policy & $\begin{array}{l}\text { 8.385** } \\
(1.127)\end{array}$ & $\begin{array}{l}\text { 8.229** } \\
(1.071)\end{array}$ & $\begin{array}{l}0.649 * * \\
(0.070)\end{array}$ & $\begin{array}{l}1.595 \\
(2.291)\end{array}$ & $\begin{array}{l}1.949 \\
(2.000)\end{array}$ & $\begin{array}{l}0.124 \\
(0.292)\end{array}$ & $\begin{array}{l}\text { 11.775** } \\
(1.641)\end{array}$ & $\begin{array}{l}11.775 * * \\
(1.493)\end{array}$ & $\begin{array}{l}\text { 1.171** } \\
(0.124)\end{array}$ & $\begin{array}{l}4.759 \dagger \\
(2.422)\end{array}$ & $\begin{array}{l}4.083 * \\
(1.921)\end{array}$ & $\begin{array}{l}0.257 \\
(2.284)\end{array}$ \\
\hline $\begin{array}{l}\text { Internationalisation } \\
\text { at home strategy }\end{array}$ & $\begin{array}{l}-0.284 \\
(0.227)\end{array}$ & $\begin{array}{l}0.0006 \\
(0.001)\end{array}$ & $\begin{array}{l}0.432 \mathrm{E}-4 \\
(0.0001)\end{array}$ & $\begin{array}{l}0.378 \\
(0.444)\end{array}$ & $\begin{array}{l}-0.001 \\
(0.002)\end{array}$ & $\begin{array}{l}-0.695 \mathrm{E}-4 \\
(0.0004)\end{array}$ & $\begin{array}{l}-0.640 \dagger \\
(0.371)\end{array}$ & $\begin{array}{l}-0.640 \dagger \\
(0.337)\end{array}$ & $\begin{array}{l}-0.040 \\
(0.036)\end{array}$ & $\begin{array}{l}-0.253 \\
(0.360)\end{array}$ & $\begin{array}{l}0.001 \dagger \\
(0.001)\end{array}$ & $\begin{array}{l}0.965 E-4 \\
(0.0003)\end{array}$ \\
\hline $\begin{array}{l}\text { Host country } \\
\text { demand }\end{array}$ & $\begin{array}{l}0.388 \\
(3.015)\end{array}$ & $\begin{array}{l}0.719 \\
(2.864)\end{array}$ & $\begin{array}{l}0.041 \\
(0.297)\end{array}$ & $\begin{array}{l}2.511 \\
(5.932)\end{array}$ & $\begin{array}{l}5.084 \\
(4.871)\end{array}$ & $\begin{array}{l}0.288 \\
(0.741)\end{array}$ & $\begin{array}{l}-2.553 \\
(5.402)\end{array}$ & $\begin{array}{l}-2.552 \\
(4.916)\end{array}$ & $\begin{array}{l}-0.163 \\
(0.553)\end{array}$ & $\begin{array}{l}-0.852 \\
(4.241)\end{array}$ & $\begin{array}{l}-0.587 \\
(3.324)\end{array}$ & $\begin{array}{l}-0.035 \\
(1.090)\end{array}$ \\
\hline AU/NZ dummy & $\begin{array}{l}0.603 \\
(0.806)\end{array}$ & $\begin{array}{l}0.886 \\
(0.749)\end{array}$ & $\begin{array}{l}0.055 \\
(0.078)\end{array}$ & - & - & - & - & - & - & - & - & - \\
\hline UK/IR dummy & $\begin{array}{l}1.570 \\
(0.830)\end{array}$ & $\begin{array}{l}1.597^{*} \\
(0.771)\end{array}$ & $\begin{array}{l}0.098 \\
(0.095)\end{array}$ & - & - & - & - & - & - & - & - & - \\
\hline $\mathrm{R}^{2}$ & 0.343 & & & 0.184 & & & 0.522 & & & 0.347 & & \\
\hline $\operatorname{AdjR}^{2}$ & 0.282 & & & -0.049 & & & 0.432 & & & 0.050 & & \\
\hline Log likelihood & & -397.68 & -424.52 & & -123.08 & -130.60 & & -172.70 & -185.10 & & -76.95 & -93.17 \\
\hline $\mathrm{N}$ & 150 & 150 & 150 & 48 & 48 & 48 & 64 & 64 & 64 & 38 & 38 & 38 \\
\hline Max. VIF & 1.565 & & & 1.284 & & & 1.273 & & & 2.307 & & \\
\hline
\end{tabular}

Standard errors in parentheses, all $t$ tests are two-tailed, $\uparrow p<.10,{ }^{*} p<.05, * * p<.01$

Table 7.15a: Regression estimates for equation 6a (low normative institutional distance)

\begin{tabular}{|c|c|c|c|c|c|c|c|c|c|c|c|c|}
\hline & \multicolumn{3}{|c|}{ Complete data set } & \multicolumn{3}{|c|}{$\overline{\mathrm{AU} / \mathrm{NZ}}$} & \multicolumn{3}{|c|}{$\overline{\mathrm{US} / \mathrm{CA}}$} & \multicolumn{3}{|c|}{ 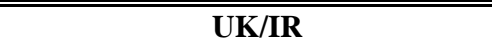 } \\
\hline & OLS & Tobit & $\begin{array}{l}\text { Negative } \\
\text { binomial }\end{array}$ & OLS & Tobit & $\begin{array}{l}\text { Negative } \\
\text { binomial }\end{array}$ & OLS & Tobit & $\begin{array}{l}\text { Negative } \\
\text { binomial }\end{array}$ & OLS & Tobit & $\begin{array}{l}\text { Negative } \\
\text { binomial }\end{array}$ \\
\hline Intercept & $\begin{array}{l}19.107 * * \\
(1.435)\end{array}$ & $\begin{array}{l}19.926 * * \\
(1.167)\end{array}$ & $\begin{array}{l}3.055^{* *} \\
(0.1402)\end{array}$ & $\begin{array}{l}20.825 * * \\
(3.600)\end{array}$ & $\begin{array}{l}20.056 * * \\
(2.536)\end{array}$ & $\begin{array}{l}3.092 * * \\
(0.234)\end{array}$ & $\begin{array}{l}21.315 * * \\
(2.480)\end{array}$ & $\begin{array}{l}22.757 * * \\
(1.770)\end{array}$ & $\begin{array}{l}3.225 * * \\
(0.306)\end{array}$ & $\begin{array}{l}16.513 * * \\
(1.859)\end{array}$ & $\begin{array}{l}16.472 * * \\
(1.641)\end{array}$ & $\begin{array}{l}2.810 * * \\
(0.436)\end{array}$ \\
\hline $\begin{array}{l}\text { Know-how (Factor) } \\
-(\mathrm{H} 7 \mathrm{c})\end{array}$ & $\begin{array}{l}-1.455^{* *} \\
(0.119)\end{array}$ & $\begin{array}{l}-1.470 * * \\
(0.113)\end{array}$ & $\begin{array}{l}-0.108 * * \\
(0.015)\end{array}$ & $\begin{array}{l}-1.935 * * \\
(0.358)\end{array}$ & $\begin{array}{l}-1.912 * * \\
(0.305)\end{array}$ & $\begin{array}{l}-0.147 * * \\
(0.026)\end{array}$ & $\begin{array}{l}-1.396 * * \\
(0.171)\end{array}$ & $\begin{array}{l}-1.395 * * \\
(0.159)\end{array}$ & $\begin{array}{l}-0.102 * * \\
(0.026)\end{array}$ & $\begin{array}{l}-1.299 * * \\
(0.219)\end{array}$ & $\begin{array}{l}-1.332 * * \\
(0.206)\end{array}$ & $\begin{array}{l}-0.097 \\
(0.063)\end{array}$ \\
\hline Age & $\begin{array}{l}0.006 \\
(0.004)\end{array}$ & $\begin{array}{l}0.005 \\
(0.003)\end{array}$ & $\begin{array}{l}0.0003 \\
(0.0005)\end{array}$ & $\begin{array}{l}-0.003 \\
(0.016)\end{array}$ & $\begin{array}{l}0.001 \\
(0.012)\end{array}$ & $\begin{array}{l}-0.672 \mathrm{E}-5 \\
(0.001)\end{array}$ & $\begin{array}{l}0.000 \\
(0.008)\end{array}$ & $\begin{array}{l}-0.0004 \\
(0.007)\end{array}$ & $\begin{array}{l}-0.412 \mathrm{E}-4 \\
(0.0009)\end{array}$ & $\begin{array}{l}0.010 \dagger \\
(0.005)\end{array}$ & $\begin{array}{l}0.008 \dagger \\
(0.004)\end{array}$ & $\begin{array}{l}0.0005 \\
(0.001)\end{array}$ \\
\hline Size (Staff) & $\begin{array}{l}0.000 \\
(0.000)\end{array}$ & $\begin{array}{l}0.0002 \dagger \\
(0.0001)\end{array}$ & $\begin{array}{l}0.160 \mathrm{E}-4 \\
(0.247 \mathrm{E}-4)\end{array}$ & $\begin{array}{l}0.001 \\
(0.000)\end{array}$ & $\begin{array}{l}0.0007 \dagger \\
(0.0004)\end{array}$ & $\begin{array}{l}0.490 \mathrm{E}-4 \\
(0.618 \mathrm{E}-4)\end{array}$ & $\begin{array}{l}0.000 \\
(0.000)\end{array}$ & $\begin{array}{l}0.0002 \\
(0.0002)\end{array}$ & $\begin{array}{l}0.137 \mathrm{E}-4 \\
(0.418 \mathrm{E}-4)\end{array}$ & $\begin{array}{l}0.000 \\
(0.000)\end{array}$ & $\begin{array}{l}0.0003 \\
(0.0002)\end{array}$ & $\begin{array}{l}0.209 \mathrm{E}-4 \\
(0.450 \mathrm{E}-4)\end{array}$ \\
\hline Offshore policy & $\begin{array}{l}0.136 \\
(0.891)\end{array}$ & $\begin{array}{l}-0.034 \\
(0.842)\end{array}$ & $\begin{array}{l}-0.002 \\
(0.088)\end{array}$ & $\begin{array}{l}0.105 \\
(2.250)\end{array}$ & $\begin{array}{l}-0.029 \\
(1.915)\end{array}$ & $\begin{array}{l}-0.004 \\
(0.158)\end{array}$ & $\begin{array}{l}-1.693 \\
(1.698)\end{array}$ & $\begin{array}{l}-2.350 \\
(1.448)\end{array}$ & $\begin{array}{l}-0.142 \\
(0.278)\end{array}$ & $\begin{array}{l}2.077 \\
(1.279)\end{array}$ & $\begin{array}{l}2.099 \dagger \\
(1.212)\end{array}$ & $\begin{array}{l}0.150 \\
(0.264)\end{array}$ \\
\hline $\begin{array}{l}\text { Internationalisation } \\
\text { at home strategy }\end{array}$ & $\begin{array}{l}0.183 \\
(0.168)\end{array}$ & $\begin{array}{l}-0.001 \\
(0.0009)\end{array}$ & $\begin{array}{l}-0.671 \mathrm{E}-4 \\
(0.0002)\end{array}$ & $\begin{array}{l}-0.137 \\
(0.596)\end{array}$ & $\begin{array}{l}0.0003 \\
(0.002)\end{array}$ & $\begin{array}{l}0.234 \mathrm{E}-4 \\
(0.0005)\end{array}$ & $\begin{array}{l}0.284 \\
(0.312)\end{array}$ & $\begin{array}{l}-0.001 \\
(0.002)\end{array}$ & $\begin{array}{l}-0.818 \\
(0.0004)\end{array}$ & $\begin{array}{l}0.162 \\
(0.216)\end{array}$ & $\begin{array}{l}-0.001 \\
(0.001)\end{array}$ & $\begin{array}{l}-0.699 \mathrm{E}-4 \\
(0.0002)\end{array}$ \\
\hline $\begin{array}{l}\text { Host country } \\
\text { demand }\end{array}$ & $\begin{array}{l}3.095 \\
(2.736)\end{array}$ & $\begin{array}{l}3.162 \\
(2.469)\end{array}$ & $\begin{array}{l}0.190 \\
(0.333)\end{array}$ & $\begin{array}{l}3.765 \\
(6.074)\end{array}$ & $\begin{array}{l}5.140 \\
(4.810)\end{array}$ & $\begin{array}{l}0.326 \\
(0.762)\end{array}$ & $\begin{array}{l}-0.301 \\
(4.471)\end{array}$ & $\begin{array}{l}0.620 \\
(4.102)\end{array}$ & $\begin{array}{l}0.033 \\
(0.523)\end{array}$ & $\begin{array}{l}9.267 \dagger \\
(4.922)\end{array}$ & $\begin{array}{l}5.611 \\
(4.100)\end{array}$ & $\begin{array}{l}0.331 \\
(0.841)\end{array}$ \\
\hline
\end{tabular}




\begin{tabular}{|c|c|c|c|c|c|c|c|c|c|c|c|c|}
\hline AU/NZ dummy & $\begin{array}{l}-0.051 \\
(0.712)\end{array}$ & $\begin{array}{l}-0.1817 \\
(0.668)\end{array}$ & $\begin{array}{l}-0.007 \\
(0.096)\end{array}$ & - & - & - & - & - & - & - & - & - \\
\hline UK/IR dummy & $\begin{array}{l}-0.586 \\
(0.550)\end{array}$ & $\begin{array}{l}-0.569 \\
(0.514)\end{array}$ & $\begin{array}{l}-0.033 \\
(0.074)\end{array}$ & - & - & - & - & - & - & - & - & - \\
\hline $\mathrm{R}^{2}$ & 0.562 & & & 0.623 & & & 0.619 & & & 0.508 & & \\
\hline $\operatorname{AdjR}^{2}$ & 0.537 & & & 0.524 & & & 0.573 & & & 0.454 & & \\
\hline Log likelihood & & -374.54 & -407.02 & & -78.27 & -85.94 & & -142.71 & -152.82 & & -146.03 & -165.51 \\
\hline $\mathrm{N}$ & 157 & 157 & 157 & 32 & 32 & 32 & 59 & 59 & 59 & 66 & 66 & 66 \\
\hline Max. VIF & 1.616 & & & 1.395 & & & 1.280 & & & 1.155 & & \\
\hline
\end{tabular}

Standard errors in parentheses, all $t$ tests are two-tailed, $\uparrow p<.10, * p<.05, * * p<.01$

Table 7.15b: Regression estimates for equation $6 \mathrm{~b}$ (high normative institutional distance)

\begin{tabular}{|c|c|c|c|c|c|c|c|c|c|c|c|c|}
\hline & \multicolumn{3}{|c|}{ Complete data set } & \multicolumn{3}{|c|}{ AU/NZ } & \multicolumn{3}{|c|}{ US/CA } & \multicolumn{3}{|c|}{ UK/IR } \\
\hline & OLS & Tobit & $\begin{array}{l}\text { Negative } \\
\text { binomial }\end{array}$ & OLS & Tobit & $\begin{array}{l}\text { Negative } \\
\text { binomial }\end{array}$ & OLS & Tobit & $\begin{array}{l}\text { Negative } \\
\text { binomial }\end{array}$ & OLS & Tobit & $\begin{array}{l}\text { Negative } \\
\text { binomial }\end{array}$ \\
\hline Intercept & $\begin{array}{l}19.861 * * \\
(1.455)\end{array}$ & $\begin{array}{l}20.005 * * \\
(1.391)\end{array}$ & $\begin{array}{l}3.038 * * \\
(0.176)\end{array}$ & $\begin{array}{l}20.850 * * \\
(2.215)\end{array}$ & $\begin{array}{l}21.435 * * \\
(2.050)\end{array}$ & $\begin{array}{l}3.144 * * \\
(0.616)\end{array}$ & $\begin{array}{l}23.120 * * \\
(2.516)\end{array}$ & $\begin{array}{l}23.120 * * \\
(2.373)\end{array}$ & $\begin{array}{l}3.263 * * \\
(0.357)\end{array}$ & $\begin{array}{l}17.046 * * \\
(2.890)\end{array}$ & $\begin{array}{l}17.050 * * \\
(2.517)\end{array}$ & $\begin{array}{l}2.808 \\
(2.363)\end{array}$ \\
\hline $\begin{array}{l}\text { Know-how (Factor) } \\
-(H 7 c)\end{array}$ & $\begin{array}{l}-1.961 * * \\
(0.187)\end{array}$ & $\begin{array}{l}-1.962^{* *} \\
(0.181)\end{array}$ & $\begin{array}{l}-0.165 * * \\
(0.017)\end{array}$ & $\begin{array}{l}-1.661 * * \\
(0.305)\end{array}$ & $\begin{array}{l}-1.676 * * \\
(0.292)\end{array}$ & $\begin{array}{l}-0.121 * * \\
(0.032)\end{array}$ & $\begin{array}{l}-2.367^{* *} \\
(0.300)\end{array}$ & $\begin{array}{l}-2.366^{* *} \\
(0.282)\end{array}$ & $\begin{array}{l}-0.227 * * \\
(0.038)\end{array}$ & $\begin{array}{l}-0.713 \\
(0.641)\end{array}$ & $\begin{array}{l}-0.680 \\
(0.566)\end{array}$ & $\begin{array}{l}-0.037 \\
(0.170)\end{array}$ \\
\hline Age & $\begin{array}{l}0.001 \\
(0.004)\end{array}$ & $\begin{array}{l}-0.0006 \\
(0.004)\end{array}$ & $\begin{array}{l}-0.910 \mathrm{E}-4 \\
(0.0005)\end{array}$ & $\begin{array}{l}-0.007 \\
(0.010)\end{array}$ & $\begin{array}{l}-0.008 \\
(0.009)\end{array}$ & $\begin{array}{l}-0.0005 \\
(0.001)\end{array}$ & $\begin{array}{l}0.001 \\
(0.006)\end{array}$ & $\begin{array}{l}0.0008 \\
(0.005)\end{array}$ & $\begin{array}{l}-0.701 \mathrm{E}-4 \\
(0.0008)\end{array}$ & $\begin{array}{l}0.000 \\
(0.006)\end{array}$ & $\begin{array}{l}-0.003 \\
(0.005)\end{array}$ & $\begin{array}{l}-0.0001 \\
(0.002)\end{array}$ \\
\hline Size (Staff) & $\begin{array}{l}-0.000 \\
(0.000)\end{array}$ & $\begin{array}{l}-0.918 \mathrm{E}-4 \\
(0.0001)\end{array}$ & $\begin{array}{l}-0.888 \mathrm{D}-6 \\
(0.268 \mathrm{D}-4)\end{array}$ & $\begin{array}{l}0.000 \\
(0.000)\end{array}$ & $\begin{array}{l}-0.0001 \\
(0.0003)\end{array}$ & $\begin{array}{l}-0.694 \mathrm{E}-5 \\
(0.632 \mathrm{E}-4)\end{array}$ & $\begin{array}{l}0.000 \\
(0.000)\end{array}$ & $\begin{array}{l}-0.0001 \\
(0.0002)\end{array}$ & $\begin{array}{l}-0.304 \mathrm{E}-5 \\
(0.444 \mathrm{E}-4)\end{array}$ & $\begin{array}{l}0.000 \\
(0.000)\end{array}$ & $\begin{array}{l}-0.0003 \\
(0.0002)\end{array}$ & $\begin{array}{l}-0.173 E-4 \\
(0.999 E-4)\end{array}$ \\
\hline Offshore policy & $\begin{array}{l}2.008^{*} \\
(1.002)\end{array}$ & $\begin{array}{l}2.014 * \\
(0.966)\end{array}$ & $\begin{array}{l}0.202 \dagger \\
(0.115)\end{array}$ & $\begin{array}{l}-0.254 \\
(1.714)\end{array}$ & $\begin{array}{l}-0.006 \\
(1.607)\end{array}$ & $\begin{array}{l}-0.0001 \\
(0.584)\end{array}$ & $\begin{array}{l}1.651 \\
(1.685)\end{array}$ & $\begin{array}{l}1.651 \\
(1.589)\end{array}$ & $\begin{array}{l}0.266 \\
(0.222)\end{array}$ & $\begin{array}{l}3.855^{*} \\
(1.708)\end{array}$ & $\begin{array}{l}3.740 * \\
(1.521)\end{array}$ & $\begin{array}{l}0.236 \\
(2.255)\end{array}$ \\
\hline $\begin{array}{l}\text { Internationalisation } \\
\text { at home strategy }\end{array}$ & $\begin{array}{l}0.012 \\
(0.170)\end{array}$ & $\begin{array}{l}-0.0003 \\
(0.001)\end{array}$ & $\begin{array}{l}-0.345 \mathrm{D}-4 \\
(0.0001)\end{array}$ & $\begin{array}{l}0.385 \\
(0.319)\end{array}$ & $\begin{array}{l}-0.002 \\
(0.001)\end{array}$ & $\begin{array}{l}-0.0001 \\
(0.0003)\end{array}$ & $\begin{array}{l}-0.294 \\
(0.248)\end{array}$ & $\begin{array}{l}-0.293 \\
(0.234)\end{array}$ & $\begin{array}{l}-0.023 \\
(0.038)\end{array}$ & $\begin{array}{l}-0.142 \\
(0.324)\end{array}$ & $\begin{array}{l}0.001 \\
(0.0009)\end{array}$ & $\begin{array}{l}0.577 \mathrm{E}-4 \\
(0.0002)\end{array}$ \\
\hline $\begin{array}{l}\text { Host country } \\
\text { demand }\end{array}$ & $\begin{array}{l}1.306 \\
(2.190)\end{array}$ & $\begin{array}{l}1.613 \\
(2.122)\end{array}$ & $\begin{array}{l}0.116 \\
(0.283)\end{array}$ & $\begin{array}{l}8.513 \dagger \\
(4.332)\end{array}$ & $\begin{array}{l}\text { 10.873** } \\
(3.915)\end{array}$ & $\begin{array}{l}0.681 \\
(0.569)\end{array}$ & $\begin{array}{l}-4.815 \\
(3.276)\end{array}$ & $\begin{array}{l}-4.815 \\
(3.091)\end{array}$ & $\begin{array}{l}-0.289 \\
(0.484)\end{array}$ & $\begin{array}{l}-0.414 \\
(3.882)\end{array}$ & $\begin{array}{l}-0.003 \\
(3.232)\end{array}$ & $\begin{array}{l}-0.001 \\
(0.962)\end{array}$ \\
\hline AU/NZ dummy & $\begin{array}{l}0.668 \\
(0.577)\end{array}$ & $\begin{array}{l}0.687 \\
(0.550)\end{array}$ & $\begin{array}{l}0.043 \\
(0.074)\end{array}$ & - & - & - & - & - & - & - & - & - \\
\hline UK/IR dummy & $\begin{array}{l}1.093 \dagger \\
(0.611)\end{array}$ & $\begin{array}{l}0.926 \\
(0.582)\end{array}$ & $\begin{array}{l}0.060 \\
(0.090)\end{array}$ & - & - & - & - & - & - & - & - & - \\
\hline
\end{tabular}




\begin{tabular}{|c|c|c|c|c|c|c|c|c|c|c|c|c|}
\hline $\mathrm{R}^{2}$ & 0.626 & & & 0.486 & & & 0.768 & & & 0.288 & & \\
\hline $\operatorname{AdjR}^{2}$ & 0.604 & & & 0.407 & & & 0.744 & & & 0.123 & & \\
\hline Log likelihood & & -355.92 & -394.21 & & -112.36 & -124.59 & & -149.56 & -166.03 & & -78.25 & -93.42 \\
\hline $\mathrm{N}$ & 150 & 150 & 150 & 48 & 48 & 48 & 64 & 64 & 64 & 38 & 38 & 38 \\
\hline Max. VIF & 1.548 & & & 1.212 & & & 2.547 & & & 1.423 & & \\
\hline
\end{tabular}

Standard errors in parentheses, all $t$ tests are two-tailed, $\uparrow p<.10,{ }^{*} p<.05,{ }^{* *} p<.01$

Table 7.16a: Regression estimates for equation 7a (low normative institutional distance)

\begin{tabular}{|c|c|c|c|c|c|c|c|c|c|c|c|c|}
\hline & \multicolumn{3}{|c|}{ Complete data set } & \multicolumn{3}{|c|}{ AU/NZ } & \multicolumn{3}{|c|}{$\overline{\mathrm{US} / \mathrm{CA}}$} & \multicolumn{3}{|c|}{ 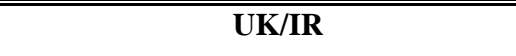 } \\
\hline & OLS & Tobit & $\begin{array}{l}\text { Negative } \\
\text { binomial }\end{array}$ & OLS & Tobit & $\begin{array}{l}\text { Negative } \\
\text { binomial }\end{array}$ & OLS & Tobit & $\begin{array}{l}\text { Negative } \\
\text { binomial }\end{array}$ & OLS & Tobit & $\begin{array}{l}\text { Negative } \\
\text { binomial }\end{array}$ \\
\hline Intercept & $\begin{array}{l}22.177^{* *} \\
(1.784)\end{array}$ & $\begin{array}{l}23.288 * * \\
(1.494)\end{array}$ & $\begin{array}{l}3.279 * * \\
(0.165)\end{array}$ & $\begin{array}{l}25.071 * * \\
(4.338)\end{array}$ & $\begin{array}{l}23.324 * * \\
(3.128)\end{array}$ & $\begin{array}{l}3.283 * * \\
(0.317)\end{array}$ & $\begin{array}{l}23.900 * * \\
(2.990)\end{array}$ & $\begin{array}{l}26.252^{* *} \\
(2.297)\end{array}$ & $\begin{array}{l}3.473^{* *} \\
(0.378)\end{array}$ & $\begin{array}{l}19.646 * * \\
(2.676)\end{array}$ & $\begin{array}{l}20.596 * * \\
(2.161)\end{array}$ & $\begin{array}{l}3.072 * * \\
(0.540)\end{array}$ \\
\hline $\begin{array}{l}\text { Org culture (Factor) } \\
-(H 7 d)\end{array}$ & $\begin{array}{l}-1.848^{* *} \\
(0.183)\end{array}$ & $\begin{array}{l}-1.876 * * \\
(0.173)\end{array}$ & $\begin{array}{l}-0.133^{* *} \\
(0.021)\end{array}$ & $\begin{array}{l}-2.444^{* *} \\
(0.496)\end{array}$ & $\begin{array}{l}-2.376 * * \\
(0.424)\end{array}$ & $\begin{array}{l}-0.175^{* *} \\
(0.041)\end{array}$ & $\begin{array}{l}-1.749 * * \\
(0.273)\end{array}$ & $\begin{array}{l}-1.730 * * \\
(0.255)\end{array}$ & $\begin{array}{l}-0.123 * * \\
(0.028)\end{array}$ & - & - & - \\
\hline $\begin{array}{l}\text { Org culture - open } \\
\text { discussion }(H 7 d)\end{array}$ & - & - & - & - & - & - & - & - & - & $\begin{array}{l}-0.570^{*} \\
(0.266)\end{array}$ & $\begin{array}{l}-0.623 * * \\
(0.229)\end{array}$ & $\begin{array}{l}-0.041 \\
(0.043)\end{array}$ \\
\hline $\begin{array}{l}\text { Org culture - no } \\
\text { status distinction } \\
(H 7 d)\end{array}$ & - & - & - & - & - & - & - & - & - & $\begin{array}{l}-0.393 \\
(0.275)\end{array}$ & $\begin{array}{l}-0.448^{*} \\
(0.236)\end{array}$ & $\begin{array}{l}-0.034 \\
(0.048)\end{array}$ \\
\hline $\begin{array}{l}\text { Org culture - } \\
\text { experimentation } \\
(H 7 d)\end{array}$ & - & - & - & - & - & - & - & - & - & $\begin{array}{l}-0.161 \\
(0.253)\end{array}$ & $\begin{array}{l}-0.168 \\
(0.212)\end{array}$ & $\begin{array}{l}-0.013 \\
(0.045)\end{array}$ \\
\hline $\begin{array}{l}\text { Org culture - } \\
\text { offshore } \\
\text { development }(H 7 d)\end{array}$ & - & - & - & - & - & - & - & - & - & $\begin{array}{l}-0.471 \\
(0.299)\end{array}$ & $\begin{array}{l}-0.537^{*} \\
(0.243)\end{array}$ & $\begin{array}{l}-0.032 \\
(0.057)\end{array}$ \\
\hline $\begin{array}{l}\text { Org culture - } \\
\text { offshore } \\
\text { responsiveness } \\
(H 7 d)\end{array}$ & - & - & - & - & - & - & - & - & - & $\begin{array}{l}-0.187 \\
(0.290)\end{array}$ & $\begin{array}{l}-0.092 \\
(0.231)\end{array}$ & $\begin{array}{l}-0.008 \\
(0.057)\end{array}$ \\
\hline Age & $\begin{array}{l}0.004 \\
(0.005)\end{array}$ & $\begin{array}{l}0.003 \\
(0.004)\end{array}$ & $\begin{array}{l}0.0001 \\
(0.0005)\end{array}$ & $\begin{array}{l}-0.006 \\
(0.017)\end{array}$ & $\begin{array}{l}0.0008 \\
(0.013)\end{array}$ & $\begin{array}{l}-0.614 \mathrm{E}-4 \\
(0.001)\end{array}$ & $\begin{array}{l}-0.003 \\
(0.009)\end{array}$ & $\begin{array}{l}-0.002 \\
(0.008)\end{array}$ & $\begin{array}{l}-0.0001 \\
(0.0009)\end{array}$ & $\begin{array}{l}0.005 \\
(0.006)\end{array}$ & $\begin{array}{l}0.004 \\
(0.005)\end{array}$ & $\begin{array}{l}0.0002 \\
(0.001)\end{array}$ \\
\hline Size (Staff) & $\begin{array}{l}0.000 \\
(0.000)\end{array}$ & $\begin{array}{l}0.0002 \dagger \\
(0.0001)\end{array}$ & $\begin{array}{l}0.180 \mathrm{E}-4 \\
(0.258 \mathrm{E}-4)\end{array}$ & $\begin{array}{l}0.001 \\
(0.000)\end{array}$ & $\begin{array}{l}0.0006 \\
(0.0004)\end{array}$ & $\begin{array}{l}0.395 E-4 \\
(0.690 E-4)\end{array}$ & $\begin{array}{l}0.000 \\
(0.000)\end{array}$ & $\begin{array}{l}0.0004 \\
(0.0003)\end{array}$ & $\begin{array}{l}0.276 \mathrm{E}-4 \\
(0.379 \mathrm{E}-4)\end{array}$ & $\begin{array}{l}0.000 \\
(0.000)\end{array}$ & $\begin{array}{l}0.0003 \\
(0.0002)\end{array}$ & $\begin{array}{l}0.222 \mathrm{E}-4 \\
(0.577 \mathrm{E}-4)\end{array}$ \\
\hline Offshore policy & 0.174 & -0.042 & 0.0003 & 0.872 & 0.770 & 0.083 & -2.258 & $-3.350 *$ & -0.217 & $2.411 \dagger$ & $2.403 \dagger$ & 0.1811 \\
\hline
\end{tabular}




\begin{tabular}{|c|c|c|c|c|c|c|c|c|c|c|c|c|}
\hline & $(0.976)$ & $(0.920)$ & $(0.083)$ & $(2.331)$ & (1.998) & $(0.172)$ & (1.919) & (1.641) & $(0.316)$ & (1.406) & (1.251) & $(0.238)$ \\
\hline $\begin{array}{l}\text { Internationalisation } \\
\text { at home strategy }\end{array}$ & $\begin{array}{l}0.237 \\
(0.183)\end{array}$ & $\begin{array}{l}-0.0002 \\
(0.001)\end{array}$ & $\begin{array}{l}-0.900 \mathrm{E}-5 \\
(0.0002)\end{array}$ & $\begin{array}{l}-0.344 \\
(0.629)\end{array}$ & $\begin{array}{l}0.001 \\
(0.002)\end{array}$ & $\begin{array}{l}0.785 E-4 \\
(0.0004)\end{array}$ & $\begin{array}{l}0.483 \\
(0.354)\end{array}$ & $\begin{array}{l}-0.001 \\
(0.002)\end{array}$ & $\begin{array}{l}-0.815 E-4 \\
(0.0008)\end{array}$ & $\begin{array}{l}0.110 \\
(0.247)\end{array}$ & $\begin{array}{l}0.829 \mathrm{E}-4 \\
(0.001)\end{array}$ & $\begin{array}{l}0.500 \mathrm{E}-5 \\
(0.0005)\end{array}$ \\
\hline $\begin{array}{l}\text { Host country } \\
\text { demand }\end{array}$ & $\begin{array}{l}2.895 \\
(3.006)\end{array}$ & $\begin{array}{l}2.945 \\
(2.706)\end{array}$ & $\begin{array}{l}0.170 \\
(0.309)\end{array}$ & $\begin{array}{l}4.259 \\
(6.381)\end{array}$ & $\begin{array}{l}5.731 \\
(5.094)\end{array}$ & $\begin{array}{l}0.376 \\
(0.644)\end{array}$ & $\begin{array}{l}0.106 \\
(5.107)\end{array}$ & $\begin{array}{l}1.624 \\
(4.688)\end{array}$ & $\begin{array}{l}0.093 \\
(0.441)\end{array}$ & $\begin{array}{l}5.789 \\
(5.755)\end{array}$ & $\begin{array}{l}3.205 \\
(4.360)\end{array}$ & $\begin{array}{l}0.153 \\
(0.967)\end{array}$ \\
\hline AU/NZ dummy & $\begin{array}{l}-0.258 \\
(0.781)\end{array}$ & $\begin{array}{l}-0.409 \\
(0.731)\end{array}$ & $\begin{array}{l}-0.026 \\
(0.091)\end{array}$ & - & - & - & - & - & - & - & - & - \\
\hline UK/IR dummy & $\begin{array}{l}-0.554 \\
(0.605)\end{array}$ & $\begin{array}{l}-0.572 \\
(0.565)\end{array}$ & $\begin{array}{l}-0.035 \\
(0.068)\end{array}$ & - & - & - & - & - & - & - & - & - \\
\hline $\mathrm{R}^{2}$ & 0.476 & & & 0.583 & & & 0.511 & & & 0.459 & & \\
\hline $\operatorname{Adj}^{2}$ & 0.446 & & & 0.474 & & & 0.453 & & & 0.353 & & \\
\hline Log likelihood & & -388.24 & -415.03 & & -80.12 & -87.26 & & -150.34 & -157.58 & & -147.48 & -166.19 \\
\hline $\mathrm{N}$ & 157 & 157 & 157 & 32 & 32 & 32 & 59 & 59 & 59 & 66 & 66 & 66 \\
\hline Max. VIF & 1.626 & & & 1.348 & & & 1.282 & & & 1.832 & & \\
\hline
\end{tabular}

Table 7.16b: Regression estimates for equation 7b (high normative institutional distance)

\begin{tabular}{|c|c|c|c|c|c|c|c|c|c|c|c|c|}
\hline & \multicolumn{3}{|c|}{ Complete data set } & \multicolumn{3}{|c|}{ AU/NZ } & \multicolumn{3}{|c|}{ US/CA } & \multicolumn{3}{|c|}{$\overline{\text { UK/IR }}$} \\
\hline & OLS & Tobit & $\begin{array}{l}\text { Negative } \\
\text { binomial }\end{array}$ & OLS & Tobit & $\begin{array}{l}\text { Negative } \\
\text { binomial }\end{array}$ & OLS & Tobit & $\begin{array}{l}\text { Negative } \\
\text { binomial }\end{array}$ & OLS & Tobit & $\begin{array}{l}\text { Negative } \\
\text { binomial }\end{array}$ \\
\hline Intercept & $\begin{array}{l}21.638 * * \\
(1.879)\end{array}$ & $\begin{array}{l}20 . .907 * * \\
(1.826)\end{array}$ & $\begin{array}{l}3.117 * * \\
(0.161)\end{array}$ & $\begin{array}{l}23.084 * * \\
(2.789)\end{array}$ & $\begin{array}{l}23.422 * * \\
(2.566)\end{array}$ & $\begin{array}{l}3.246 * * \\
(0.376)\end{array}$ & $\begin{array}{l}22.604 * * \\
(3.217)\end{array}$ & $\begin{array}{l}22.604 * * \\
(3.036)\end{array}$ & $\begin{array}{l}3.016 * * \\
(0.244)\end{array}$ & $\begin{array}{l}9.007 \dagger \\
(4.492)\end{array}$ & $\begin{array}{l}6.762 * \\
(3.254)\end{array}$ & $\begin{array}{l}2.232 \\
(64.275)\end{array}$ \\
\hline $\begin{array}{l}\text { Org culture (Factor) } \\
-(H 7 d)\end{array}$ & $\begin{array}{l}-2.204 * * \\
(0.266)\end{array}$ & $\begin{array}{l}-2.101 * * \\
(0.259)\end{array}$ & $\begin{array}{l}-0.155^{* *} \\
(0.020)\end{array}$ & $\begin{array}{l}-1.839 * * \\
(0.433)\end{array}$ & $\begin{array}{l}-1.844 * * \\
(0.409)\end{array}$ & $\begin{array}{l}-0.121 * * \\
(0.044)\end{array}$ & $\begin{array}{l}-2.475^{* *} \\
(0.433)\end{array}$ & $\begin{array}{l}-2.474 * * \\
(0.408)\end{array}$ & $\begin{array}{l}-0.203^{* *} \\
(0.029)\end{array}$ & $(-1.0-1$ & (-10-2) & (1) \\
\hline $\begin{array}{l}\text { Org culture - open } \\
\text { discussion }(H 7 d)\end{array}$ & (0.0) & ) & (1) & (- & (1) & (1) & (1) & (- & (1) & $\begin{array}{l}0.781 * \\
(0.346)\end{array}$ & $\begin{array}{l}\text { 0.7911** } \\
(0.273)\end{array}$ & $\begin{array}{l}0.043 \\
(0.109)\end{array}$ \\
\hline $\begin{array}{l}\text { Org culture - no } \\
\text { status distinction } \\
(H 7 d)\end{array}$ & - & - & - & - & - & - & - & - & - & $\begin{array}{l}-0.230 \\
(0.308)\end{array}$ & $\begin{array}{l}-0.001 \\
(0.231)\end{array}$ & $\begin{array}{l}0.0004 \\
(0.110)\end{array}$ \\
\hline $\begin{array}{l}\text { Org culture - } \\
\text { experimentation } \\
(H 7 d)\end{array}$ & - & - & - & - & - & - & - & - & - & $\begin{array}{l}0.109 \\
(0.335)\end{array}$ & $\begin{array}{l}0.062 \\
(0.232)\end{array}$ & $\begin{array}{l}0.003 \\
(0.138)\end{array}$ \\
\hline $\begin{array}{l}\text { Org culture - } \\
\text { offshore }\end{array}$ & - & - & - & - & - & - & - & - & - & $\begin{array}{l}0.041 \\
(0.363)\end{array}$ & $\begin{array}{l}0.248 \\
(0.256)\end{array}$ & $\begin{array}{l}0.013 \\
(0.143)\end{array}$ \\
\hline
\end{tabular}




\begin{tabular}{|c|c|c|c|c|c|c|c|c|c|c|c|c|}
\hline $\begin{array}{l}\text { Org culture - } \\
\text { offshore } \\
\text { responsiveness } \\
(H 7 d)\end{array}$ & - & - & - & - & - & - & - & - & - & $\begin{array}{l}0.315 \\
(0.313)\end{array}$ & $\begin{array}{l}0.435 \dagger \\
(0.242)\end{array}$ & $\begin{array}{l}0.024 \\
(0.106)\end{array}$ \\
\hline Age & $\begin{array}{l}0.002 \\
(0.005)\end{array}$ & $\begin{array}{l}0.001 \\
(0.004)\end{array}$ & $\begin{array}{l}0.883 E-4 \\
(0.0005)\end{array}$ & $\begin{array}{l}0.002 \\
(0.011)\end{array}$ & $\begin{array}{l}0.0007 \\
(0.010)\end{array}$ & $\begin{array}{l}0.936 \mathrm{E}-4 \\
(0.015)\end{array}$ & $\begin{array}{l}0.005 \\
(0.007)\end{array}$ & $\begin{array}{l}0.005 \\
(0.006)\end{array}$ & $\begin{array}{l}0.0004 \\
(0.0006)\end{array}$ & $\begin{array}{l}0.004 \\
(0.007)\end{array}$ & $\begin{array}{l}0.001 \\
(0.005)\end{array}$ & $\begin{array}{l}0.953 \mathrm{E}-4 \\
(0.002)\end{array}$ \\
\hline Size (Staff) & $\begin{array}{l}0.000 \\
(0.000)\end{array}$ & $\begin{array}{l}-0.0002 \\
(0.0001)\end{array}$ & $\begin{array}{l}-0.127 \mathrm{E}-4 \\
(0.265 \mathrm{E}-4)\end{array}$ & $\begin{array}{l}0.000 \\
(0.000)\end{array}$ & $\begin{array}{l}-0.0002 \\
(0.0003)\end{array}$ & $\begin{array}{l}-0.150 \mathrm{E}-4 \\
(0.534 \mathrm{E}-4)\end{array}$ & $\begin{array}{l}0.000 \\
(0.000)\end{array}$ & $\begin{array}{l}-0.0002 \\
(0.0002)\end{array}$ & $\begin{array}{l}-0.181 E-4 \\
(0.446 E-4)\end{array}$ & $\begin{array}{l}0.000 \\
(0.000)\end{array}$ & $\begin{array}{l}-0.0002 \\
(0.0002)\end{array}$ & $\begin{array}{l}-1.158 \mathrm{E}-4 \\
(0.0001)\end{array}$ \\
\hline Offshore policy & $\begin{array}{l}\text { 3.511** } \\
\text { (1.051) }\end{array}$ & $\begin{array}{l}\text { 3.684** } \\
(1.031)\end{array}$ & $\begin{array}{l}0.342 * * \\
(0.082)\end{array}$ & $\begin{array}{l}0.445 \\
(1.862)\end{array}$ & $\begin{array}{l}0.652 \\
(1.738)\end{array}$ & $\begin{array}{l}0.045 \\
(0.332)\end{array}$ & $\begin{array}{l}4.732 * * \\
(1.762)\end{array}$ & $\begin{array}{l}4.732 * * \\
(1.662)\end{array}$ & $\begin{array}{l}0.615^{* *} \\
(0.129)\end{array}$ & $\begin{array}{l}\text { 6.189** } \\
(1.807)\end{array}$ & $\begin{array}{l}6.595 * * \\
(1.445)\end{array}$ & $\begin{array}{l}0.393 \\
(64.265)\end{array}$ \\
\hline $\begin{array}{l}\text { Internationalisation } \\
\text { at home strategy }\end{array}$ & $\begin{array}{l}-0.100 \\
(0.185)\end{array}$ & $\begin{array}{l}0.0008 \\
(0.001)\end{array}$ & $\begin{array}{l}0.653 E-4 \\
(0.0001)\end{array}$ & $\begin{array}{l}0.284 \\
(0.349)\end{array}$ & $\begin{array}{l}-0.001 \\
(0.002)\end{array}$ & $\begin{array}{l}-0.944 \mathrm{E}-4 \\
(0.0002)\end{array}$ & $\begin{array}{l}-0.313 \\
(0.287)\end{array}$ & $\begin{array}{l}-0.313 \\
(0.270)\end{array}$ & $\begin{array}{l}-0.018 \\
(0.032)\end{array}$ & $\begin{array}{l}-0.057 \\
(0.347)\end{array}$ & $\begin{array}{l}0.0003 \\
(0.0009)\end{array}$ & $\begin{array}{l}0.178 \\
(0.0004)\end{array}$ \\
\hline $\begin{array}{l}\text { Host country } \\
\text { demand }\end{array}$ & $\begin{array}{l}1.420 \\
(2.401)\end{array}$ & $\begin{array}{l}1.740 \\
(2.367)\end{array}$ & $\begin{array}{l}0.106 \\
(0.267)\end{array}$ & $\begin{array}{l}4.230 \\
(4.628)\end{array}$ & $\begin{array}{l}6.394 \\
(4.119)\end{array}$ & $\begin{array}{l}0.361 \\
(0.575)\end{array}$ & $\begin{array}{l}-2.500 \\
(3.771)\end{array}$ & $\begin{array}{l}-2.499 \\
(3.558)\end{array}$ & $\begin{array}{l}-0.113 \\
(0.417)\end{array}$ & $\begin{array}{l}-1.101 \\
(3.942)\end{array}$ & $\begin{array}{l}-1.565 \\
(2.990)\end{array}$ & $\begin{array}{l}-0.087 \\
(1.487)\end{array}$ \\
\hline AU/NZ dummy & $\begin{array}{l}1.026 \\
(0.632)\end{array}$ & $\begin{array}{l}1.120 \dagger \\
(0.612)\end{array}$ & $\begin{array}{l}-0.076 \\
(0.073)\end{array}$ & - & - & - & - & - & - & - & - & - \\
\hline UK/IR dummy & $\begin{array}{l}1.052 \\
(0.671)\end{array}$ & $\begin{array}{l}0.980 \\
(0.650)\end{array}$ & $\begin{array}{l}-0.013 \\
(0.082)\end{array}$ & - & - & - & - & - & - & - & - & - \\
\hline $\mathrm{R}^{2}$ & 0.551 & & & 0.381 & & & 0.691 & & & 0.440 & & \\
\hline $\operatorname{AdjR}^{2}$ & 0.525 & & & 0.286 & & & 0.659 & & & 0.185 & & \\
\hline Log likelihood & & -372.24 & -406.12 & & -116.59 & -126.91 & & -158.67 & -173.38 & & -73.55 & -92.59 \\
\hline $\mathrm{N}$ & 150 & 150 & 150 & 48 & 48 & 48 & 64 & 64 & 64 & 38 & 38 & 38 \\
\hline Max. VIF & 1.454 & & & 1.192 & & & 2.123 & & & 1.763 & & \\
\hline
\end{tabular}

Standard errors in parentheses, all $t$ tests are two-tailed, $\dagger p<.10,{ }^{*} p<.05,{ }^{* *} p<.01$

Table 7.17a: Regression estimates for equation 8a (low normative institutional distance)

\begin{tabular}{|c|c|c|c|c|c|c|c|c|c|c|c|c|}
\hline & \multicolumn{3}{|c|}{ Complete data set } & \multicolumn{3}{|c|}{ AU/NZ } & \multicolumn{3}{|c|}{ 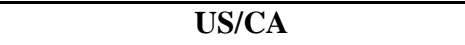 } & \multicolumn{3}{|c|}{ 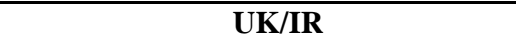 } \\
\hline & OLS & Tobit & $\begin{array}{l}\text { Negative } \\
\text { binomial }\end{array}$ & OLS & Tobit & $\begin{array}{l}\text { Negative } \\
\text { binomial }\end{array}$ & OLS & Tobit & $\begin{array}{l}\text { Negative } \\
\text { binomial }\end{array}$ & OLS & Tobit & $\begin{array}{l}\text { Negative } \\
\text { binomial }\end{array}$ \\
\hline Intercept & $\begin{array}{l}\text { 10.637** } \\
(1.947)\end{array}$ & $\begin{array}{l}11.954^{* *} \\
(1.696)\end{array}$ & $\begin{array}{l}\text { 2.517** } \\
(0.131)\end{array}$ & $\begin{array}{l}\text { 10.774* } \\
(4.110)\end{array}$ & $\begin{array}{l}\text { 10.416** } \\
(3.103)\end{array}$ & $\begin{array}{l}2.378 * * \\
(0.201)\end{array}$ & $\begin{array}{l}14.294 * * \\
(3.868)\end{array}$ & $\begin{array}{l}15.477^{* *} \\
(2.990)\end{array}$ & $\begin{array}{l}2.732 * * \\
(0.257)\end{array}$ & $\begin{array}{l}\text { 10.200** } \\
(1.976)\end{array}$ & $\begin{array}{l}11.342 * * \\
(1.783)\end{array}$ & $\begin{array}{l}\text { 2.463** } \\
(0.179)\end{array}$ \\
\hline
\end{tabular}




\begin{tabular}{|c|c|c|c|c|c|c|c|c|c|c|c|c|}
\hline $\begin{array}{l}\text { Financial resources } \\
(\mathrm{H} 7 \mathrm{e})\end{array}$ & $\begin{array}{l}0.027 \\
(0.182)\end{array}$ & $\begin{array}{l}0.102 \\
(0.166)\end{array}$ & $\begin{array}{l}0.006 \\
(0.015)\end{array}$ & $\begin{array}{l}0.178 \\
(0.624)\end{array}$ & $\begin{array}{l}0.094 \\
(0.471)\end{array}$ & $\begin{array}{l}0.006 \\
(0.052)\end{array}$ & $\begin{array}{l}0.273 \\
(0.301)\end{array}$ & $\begin{array}{l}0.312 \\
(0.262)\end{array}$ & $\begin{array}{l}0.019 \\
(0.028)\end{array}$ & $\begin{array}{l}-0.376 \dagger \\
(0.213)\end{array}$ & $\begin{array}{l}-0.282 \\
(0.198)\end{array}$ & $\begin{array}{l}-0.017 \\
(0.028)\end{array}$ \\
\hline Age & $\begin{array}{l}0.007 \\
(0.006)\end{array}$ & $\begin{array}{l}0.004 \\
(0.005)\end{array}$ & $\begin{array}{l}0.0002 \\
(0.0004)\end{array}$ & $\begin{array}{l}0.016 \\
(0.027)\end{array}$ & $\begin{array}{l}0.020 \\
(0.019)\end{array}$ & $\begin{array}{l}0.001 \\
(0.002)\end{array}$ & $\begin{array}{l}-0.006 \\
(0.012)\end{array}$ & $\begin{array}{l}-0.006 \\
(0.011)\end{array}$ & $\begin{array}{l}-0.0004 \\
(0.0008)\end{array}$ & $\begin{array}{l}0.011 \\
(0.006)\end{array}$ & $\begin{array}{l}0.008 \\
(0.005)\end{array}$ & $\begin{array}{l}0.0005 \\
(0.0006)\end{array}$ \\
\hline Size (Staff) & $\begin{array}{l}0.000 \\
(0.000)\end{array}$ & $\begin{array}{l}0.0002 \\
(0.0002)\end{array}$ & $\begin{array}{l}0.154 \mathrm{E}-4 \\
(0.213 \mathrm{E}-4)\end{array}$ & - & ( & r & $\begin{array}{l}0.000 \\
(0.000)\end{array}$ & $\begin{array}{l}0.0003 \\
(0.0004)\end{array}$ & $\begin{array}{l}0.208 \mathrm{E}-4 \\
(0.361 \mathrm{E}-4)\end{array}$ & $\begin{array}{l}\text { 0.001* } \\
(0.000)\end{array}$ & $\begin{array}{l}\text { 0.0006* } \\
(0.0002)\end{array}$ & $\begin{array}{l}0.398 \mathrm{E}-4 \\
(0.464 \mathrm{E}-4)\end{array}$ \\
\hline Offshore policy & $\begin{array}{l}1.641 \\
91.305)\end{array}$ & $\begin{array}{l}1.237 \\
(1.225)\end{array}$ & $\begin{array}{l}0.082 \\
(0.061)\end{array}$ & $\begin{array}{l}3.586 \\
(3.142)\end{array}$ & $\begin{array}{l}3.587 \\
(2.695)\end{array}$ & $\begin{array}{l}0.270 \dagger \\
(0.141)\end{array}$ & $\begin{array}{l}-2.905 \\
(2.583)\end{array}$ & $\begin{array}{l}-3.546 \\
(2.161)\end{array}$ & $\begin{array}{l}-0.222 \dagger \\
(0.122)\end{array}$ & $\begin{array}{l}4.665 * * \\
(1.618)\end{array}$ & $\begin{array}{l}4.609 * * \\
(1.549)\end{array}$ & $\begin{array}{l}0.308^{*} \\
(0.123)\end{array}$ \\
\hline $\begin{array}{l}\text { Internationalisation } \\
\text { at home strategy }\end{array}$ & $\begin{array}{l}0.390 \\
(0.247)\end{array}$ & $\begin{array}{l}-0.001 \\
(0.001)\end{array}$ & $\begin{array}{l}-0.811 \mathrm{E}-4 \\
(0.0001)\end{array}$ & $\begin{array}{l}-0.197 \\
(0.970)\end{array}$ & $\begin{array}{l}-0.001 \\
(0.003)\end{array}$ & $\begin{array}{l}-0.589 \mathrm{E}-4 \\
(0.001)\end{array}$ & $\begin{array}{l}0.280 \\
(0.483)\end{array}$ & $\begin{array}{l}-0.003 \\
(0.002)\end{array}$ & $\begin{array}{l}-0.0002 \\
(0.020)\end{array}$ & $\begin{array}{l}0.390 \\
(0.273)\end{array}$ & $\begin{array}{l}-0.001 \\
(0.001)\end{array}$ & $\begin{array}{l}-0.607 \mathrm{E}-4 \\
(0.0002)\end{array}$ \\
\hline $\begin{array}{l}\text { Host country } \\
\text { demand }\end{array}$ & $\begin{array}{l}\text { 9.232* } \\
(3.924)\end{array}$ & $\begin{array}{l}\text { 8.929* } \\
(3.550)\end{array}$ & $\begin{array}{l}0.534 \dagger \\
(0.3226)\end{array}$ & $\begin{array}{l}6.539 \\
(9.090)\end{array}$ & $\begin{array}{l}6.733 \\
(7.369)\end{array}$ & $\begin{array}{l}0.402 \\
(0.790)\end{array}$ & $\begin{array}{l}12.849 \dagger \\
(6.700)\end{array}$ & $\begin{array}{l}\text { 13.847* } \\
(5.966)\end{array}$ & $\begin{array}{l}0.861 \\
(0.559)\end{array}$ & $\begin{array}{l}10.030 \\
(6.132)\end{array}$ & $\begin{array}{l}5.252 \\
(5.155)\end{array}$ & $\begin{array}{l}0.282 \\
(0.793)\end{array}$ \\
\hline AU/NZ dummy & $\begin{array}{l}0.680 \\
(1.092)\end{array}$ & $\begin{array}{l}0.601 \\
(1.032)\end{array}$ & $\begin{array}{l}0.036 \\
(0.087)\end{array}$ & - & - & - & - & - & - & - & - & - \\
\hline UK/IR dummy & $\begin{array}{l}1.298 \\
(0.910)\end{array}$ & $\begin{array}{l}1.460 \dagger \\
(0.841)\end{array}$ & $\begin{array}{l}0.090 \\
(0.067)\end{array}$ & - & - & - & - & - & - & - & - & - \\
\hline $\mathrm{R}^{2}$ & 0.095 & & & 0.146 & & & 0.125 & & & 0.237 & & \\
\hline $\operatorname{AdjR}^{2}$ & 0.043 & & & -0.032 & & & 0.019 & & & 0.0154 & & \\
\hline Log likelihood & & -431.66 & -447.25 & & -91.04 & -96.96 & & -166.57 & -172.03 & & -161.14 & -172.11 \\
\hline $\mathrm{N}$ & 157 & 157 & 157 & 32 & 32 & 32 & 59 & 59 & 59 & 66 & 66 & 66 \\
\hline Max. VIF & 1.932 & & & 1.815 & & & 1.367 & & & 1.284 & & \\
\hline
\end{tabular}

Standard errors in parentheses, all $t$ tests are two-tailed, $\dagger p<.10,{ }^{*} p<.05,{ }^{* *} p<.01$

Table 7.17b: Regression estimates for equation $8 \mathrm{~b}$ (high normative institutional distance)

\begin{tabular}{|c|c|c|c|c|c|c|c|c|c|c|c|c|}
\hline & \multicolumn{3}{|c|}{ Complete data set } & \multicolumn{3}{|c|}{ AU/NZ } & \multicolumn{3}{|c|}{ US/CA } & \multicolumn{3}{|c|}{ UK/IR } \\
\hline & OLS & Tobit & $\begin{array}{l}\text { Negative } \\
\text { binomial }\end{array}$ & OLS & Tobit & $\begin{array}{l}\text { Negative } \\
\text { binomial }\end{array}$ & OLS & Tobit & $\begin{array}{l}\text { Negative } \\
\text { binomial }\end{array}$ & OLS & Tobit & $\begin{array}{l}\text { Negative } \\
\text { binomial }\end{array}$ \\
\hline$\overline{\text { Intercept }}$ & $\begin{array}{l}10.662 * * \\
(1.643)\end{array}$ & $\begin{array}{l}10.259 * * \\
(1.503)\end{array}$ & $\begin{array}{l}2.274 * * \\
(0.120)\end{array}$ & $\begin{array}{l}16.704^{* *} \\
(2.696)\end{array}$ & $\begin{array}{l}\text { 17.102** } \\
(2.439)\end{array}$ & $\begin{array}{l}2.826 * * \\
(0.275)\end{array}$ & $\begin{array}{l}8.617 * * \\
(2.488)\end{array}$ & $\begin{array}{l}8.616^{* *} \\
(2.348)\end{array}$ & $\begin{array}{l}1.878 * * \\
(0.225)\end{array}$ & $\begin{array}{l}13.259 * * \\
(1.973)\end{array}$ & $\begin{array}{l}14.308 * * \\
(1.748)\end{array}$ & $\begin{array}{l}2.655 \\
(1.625)\end{array}$ \\
\hline $\begin{array}{l}\text { Financial resources } \\
(\mathrm{H} 7 \mathrm{e})\end{array}$ & $\begin{array}{l}-0.277 \\
(0.173)\end{array}$ & $\begin{array}{l}-0.290 \dagger \\
(0.160)\end{array}$ & $\begin{array}{l}-0.017 \\
(0.016)\end{array}$ & $\begin{array}{l}-0.484 \\
(0.370)\end{array}$ & $\begin{array}{l}-0.450 \\
(0.341)\end{array}$ & $\begin{array}{l}-0.026 \\
(0.044)\end{array}$ & $\begin{array}{l}-0.320 \\
(0.253)\end{array}$ & $\begin{array}{l}-0.319 \\
(0.239)\end{array}$ & $\begin{array}{l}-0.020 \\
(0.025)\end{array}$ & $\begin{array}{l}0.406 \\
(0.320)\end{array}$ & $\begin{array}{l}0.073 \\
(0.209)\end{array}$ & $\begin{array}{l}0.004 \\
(0.061)\end{array}$ \\
\hline Age & $\begin{array}{l}0.004 \\
(0.006)\end{array}$ & $\begin{array}{l}0.003 \\
(0.005)\end{array}$ & $\begin{array}{l}0.0002 \\
(0.0004)\end{array}$ & $\begin{array}{l}0.000 \\
(0.015)\end{array}$ & $\begin{array}{l}-0.002 \\
(0.013)\end{array}$ & $\begin{array}{l}-0.0001 \\
(0.001)\end{array}$ & $\begin{array}{l}0.009 \\
(0.010)\end{array}$ & $\begin{array}{l}0.008 \\
(0.008)\end{array}$ & $\begin{array}{l}0.0005 \\
(0.0008)\end{array}$ & $\begin{array}{l}0.001 \\
(0.006)\end{array}$ & $\begin{array}{l}-0.003 \\
(0.005)\end{array}$ & $\begin{array}{l}-0.0001 \\
(0.001)\end{array}$ \\
\hline Size (Staff) & 0.000 & 0.0001 & $0.762 \mathrm{E}-5$ & 0.000 & -0.0001 & $-0.582 E-5$ & 0.000 & 0.0003 & $0.222 \mathrm{E}-4$ & 0.000 & -0.0004 & $-0.234 \mathrm{E}-4$ \\
\hline
\end{tabular}




\begin{tabular}{|c|c|c|c|c|c|c|c|c|c|c|c|c|}
\hline & $(0.000)$ & $(0.0002)$ & $(0.269 \mathrm{E}-4)$ & $(0.001)$ & $(0.0005)$ & $(0.776 \mathrm{E}-4)$ & $(0.000)$ & $(0.0004)$ & $(0.466 \mathrm{E}-4)$ & $(0.000)$ & $(0.0002)$ & $(0.0001)$ \\
\hline Offshore policy & $\begin{array}{l}\text { 7.988** } \\
(1.093)\end{array}$ & $\begin{array}{l}7.892 * * \\
(1.051)\end{array}$ & $\begin{array}{l}0.625^{* *} \\
(0.063)\end{array}$ & $\begin{array}{l}2.047 \\
(2.166)\end{array}$ & $\begin{array}{l}2.267 \\
(2.002)\end{array}$ & $\begin{array}{l}0.143 \\
(0.212)\end{array}$ & $\begin{array}{l}11.502 * * \\
(1.576)\end{array}$ & $\begin{array}{l}11.501 * * \\
(1.487)\end{array}$ & $\begin{array}{l}\text { 1.155** } \\
(0.117)\end{array}$ & $\begin{array}{l}4.417 * * \\
(1.587)\end{array}$ & $\begin{array}{l}4.34 * * \\
(1.449)\end{array}$ & $\begin{array}{l}0.270 \\
(1.604)\end{array}$ \\
\hline $\begin{array}{l}\text { Internationalisation } \\
\text { at home strategy }\end{array}$ & $\begin{array}{l}-0.214 \\
(0.228)\end{array}$ & $\begin{array}{l}0.0003 \\
(0.001)\end{array}$ & $\begin{array}{l}0.194 \mathrm{E}-4 \\
(0.0001)\end{array}$ & $\begin{array}{l}0.332 \\
(0.417)\end{array}$ & $\begin{array}{l}-0.001 \\
(0.002)\end{array}$ & $\begin{array}{l}-0.768 \mathrm{E}-4 \\
(0.0004)\end{array}$ & $\begin{array}{l}-0.585 \\
(0.350)\end{array}$ & $\begin{array}{l}-0.584 \dagger \\
(0.330)\end{array}$ & $\begin{array}{l}-0.038 \\
(0.035)\end{array}$ & $\begin{array}{l}-0.409 \\
(0.393)\end{array}$ & $\begin{array}{l}0.001 \\
(0.0009)\end{array}$ & $\begin{array}{l}0.707 \mathrm{E}-4 \\
(0.0002)\end{array}$ \\
\hline $\begin{array}{l}\text { Host country } \\
\text { demand }\end{array}$ & $\begin{array}{l}0.118 \\
(2.925)\end{array}$ & $\begin{array}{l}0.439 \\
(2.804)\end{array}$ & $\begin{array}{l}0.027 \\
(0.288)\end{array}$ & $\begin{array}{l}3.744 \\
(5.500)\end{array}$ & $\begin{array}{l}5.646 \\
(4.863)\end{array}$ & $\begin{array}{l}0.325 \\
(0.600)\end{array}$ & $\begin{array}{l}-3.624 \\
(4.682)\end{array}$ & $\begin{array}{l}-3.623 \\
(4.418)\end{array}$ & $\begin{array}{l}-0.249 \\
(0.526)\end{array}$ & $\begin{array}{l}0.972 \\
(3.760)\end{array}$ & $\begin{array}{l}1.136 \\
(3.304)\end{array}$ & $\begin{array}{l}0.060 \\
(0.852)\end{array}$ \\
\hline AU/NZ dummy & $\begin{array}{l}0.296 \\
(0.842)\end{array}$ & $\begin{array}{l}0.433 \\
(0.803)\end{array}$ & $\begin{array}{l}0.028 \\
(0.078)\end{array}$ & - & - & - & - & - & - & - & - & - \\
\hline UK/IR dummy & $\begin{array}{l}1.058 \\
(0.903)\end{array}$ & $\begin{array}{l}1.014 \\
(0.857)\end{array}$ & $\begin{array}{l}0.060 \\
(0.096)\end{array}$ & - & - & - & - & - & - & - & - & - \\
\hline $\mathrm{R}^{2}$ & 0.334 & & & 0.133 & & & 0.527 & & & 0.297 & & \\
\hline $\operatorname{AdjR}^{2}$ & 0.294 & & & 0.000 & & & 0.478 & & & 0.135 & & \\
\hline Log likelihood & & -397.96 & -424.82 & & -124.33 & -131.31 & & -172.33 & -184.69 & & -78.89 & -93.55 \\
\hline $\mathrm{N}$ & 150 & 150 & 150 & 48 & 48 & 48 & 64 & 64 & 64 & 38 & 38 & 38 \\
\hline Max. VIF & 2.003 & & & 2.066 & & & 1.607 & & & 2.411 & & \\
\hline
\end{tabular}

Standard errors in parentheses, all $t$ tests are two-tailed, $\dagger p<.10, * p<.05, * * p<.01$

Table 7.18a: Regression estimates for equation 9a (low normative institutional distance)

\begin{tabular}{|c|c|c|c|c|c|c|c|c|c|c|c|c|}
\hline & \multicolumn{3}{|c|}{ "Complete data set } & \multicolumn{3}{|c|}{ AU/NZ } & \multicolumn{3}{|c|}{ "US/CA } & \multicolumn{3}{|c|}{ 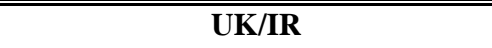 } \\
\hline & OLS & Tobit & $\begin{array}{l}\text { Negative } \\
\text { binomial }\end{array}$ & OLS & Tobit & $\begin{array}{l}\text { Negative } \\
\text { binomial }\end{array}$ & OLS & Tobit & $\begin{array}{l}\text { Negative } \\
\text { binomial }\end{array}$ & OLS & Tobit & $\begin{array}{l}\text { Negative } \\
\text { binomial }\end{array}$ \\
\hline Intercept & $\begin{array}{l}20.774 * * \\
(2.671)\end{array}$ & $\begin{array}{l}22.168 * * \\
(2.421)\end{array}$ & $\begin{array}{l}3.155^{* *} \\
(0.210)\end{array}$ & $\begin{array}{l}18.040 \dagger \\
(9.080)\end{array}$ & $\begin{array}{l}17.190 * \\
(6.897)\end{array}$ & $\begin{array}{l}2.801 * * \\
(0.644)\end{array}$ & $\begin{array}{l}\text { 28.052** } \\
(4.265)\end{array}$ & $\begin{array}{l}30.252 * * \\
(3.571)\end{array}$ & $\begin{array}{l}3.705^{* *} \\
(0.381)\end{array}$ & $\begin{array}{l}15.164 * * \\
(3.464)\end{array}$ & $\begin{array}{l}14.697 * * \\
(3.242)\end{array}$ & $\begin{array}{l}2.666^{* *} \\
(0.402)\end{array}$ \\
\hline $\begin{array}{l}\text { Reputation (Factor) } \\
\text { (H7f) }\end{array}$ & $\begin{array}{l}-1.465^{* *} \\
(0.310)\end{array}$ & $\begin{array}{l}-1.432 * * \\
(0.295)\end{array}$ & $\begin{array}{l}-0.089 * * \\
(0.028)\end{array}$ & $\begin{array}{l}-1.008 \\
(1.125)\end{array}$ & $\begin{array}{l}-0.976 \\
(0.936)\end{array}$ & $\begin{array}{l}-0.060 \\
(0.101)\end{array}$ & $\begin{array}{l}-2.163 * * \\
(0.506)\end{array}$ & $\begin{array}{l}-2.131 * * \\
(0.471)\end{array}$ & $\begin{array}{l}-0.142 * * \\
(0.053)\end{array}$ & $\begin{array}{l}-0.765 \dagger \\
(0.405)\end{array}$ & $\begin{array}{l}-0.522 \\
(0.367)\end{array}$ & $\begin{array}{l}-0.031 \\
(0.051)\end{array}$ \\
\hline Age & $\begin{array}{l}0.006 \\
(0.006)\end{array}$ & $\begin{array}{l}0.003 \\
(0.005)\end{array}$ & $\begin{array}{l}0.0002 \\
(0.0004)\end{array}$ & $\begin{array}{l}0.015 \\
(0.024)\end{array}$ & $\begin{array}{l}0.016 \\
(0.018)\end{array}$ & $\begin{array}{l}0.0009 \\
(0.001)\end{array}$ & $\begin{array}{l}-0.006 \\
(0.010)\end{array}$ & $\begin{array}{l}-0.005 \\
(0.009)\end{array}$ & $\begin{array}{l}-0.0003 \\
(0.0007)\end{array}$ & $\begin{array}{l}0.009 \\
(0.006)\end{array}$ & $\begin{array}{l}0.006 \\
(0.005)\end{array}$ & $\begin{array}{l}0.0004 \\
(0.0007)\end{array}$ \\
\hline Size (Staff) & $\begin{array}{l}0.000 \\
(0.000)\end{array}$ & $\begin{array}{l}0.0003 \\
(0.0002)\end{array}$ & $\begin{array}{l}0.197 \mathrm{E}-4 \\
(0.216 \mathrm{E}-4)\end{array}$ & $\begin{array}{l}0.000 \\
(0.024)\end{array}$ & $\begin{array}{l}0.0001 \\
(0.0005)\end{array}$ & $\begin{array}{l}0.107 \mathrm{E}-4 \\
(0.547 \mathrm{E}-4)\end{array}$ & $\begin{array}{l}0.001 \\
(0.000)\end{array}$ & $\begin{array}{l}0.0004 \\
(0.0003)\end{array}$ & $\begin{array}{l}0.301 \mathrm{E}-4 \\
(0.395 \mathrm{E}-4)\end{array}$ & $\begin{array}{l}0.000 \\
(0.000)\end{array}$ & $\begin{array}{l}0.0004 \dagger \\
(0.0002)\end{array}$ & $\begin{array}{l}0.290 \mathrm{E}-4 \\
(0.456 \mathrm{E}-4)\end{array}$ \\
\hline Offshore policy & $\begin{array}{l}1.413 \\
(1.175)\end{array}$ & $\begin{array}{l}1.142 \\
(1.123)\end{array}$ & $\begin{array}{l}0.072 \\
(0.059)\end{array}$ & $\begin{array}{l}3.614 \\
(3.184)\end{array}$ & $\begin{array}{l}3.637 \\
(2.670)\end{array}$ & $\begin{array}{l}0.275^{*} \\
(0.119)\end{array}$ & $\begin{array}{l}-0.638 \\
(2.264)\end{array}$ & $\begin{array}{l}-1.730 \\
(1.924)\end{array}$ & $\begin{array}{l}-0.111 \\
(0.160)\end{array}$ & $\begin{array}{l}\text { 3.045† } \\
\text { (1.599) }\end{array}$ & $\begin{array}{l}3.444^{*} \\
(1.523)\end{array}$ & $\begin{array}{l}0.232 * \\
(0.117)\end{array}$ \\
\hline
\end{tabular}




\begin{tabular}{|c|c|c|c|c|c|c|c|c|c|c|c|c|}
\hline $\begin{array}{l}\text { Internationalisation } \\
\text { at home strategy }\end{array}$ & $\begin{array}{l}0.396 \dagger \\
(0.222)\end{array}$ & $\begin{array}{l}-0.0001 \\
(0.001)\end{array}$ & $\begin{array}{l}-0.285 \mathrm{E}-5 \\
(0.0001)\end{array}$ & $\begin{array}{l}-0.192 \\
(0.893)\end{array}$ & $\begin{array}{l}-0.0001 \\
(0.003)\end{array}$ & $\begin{array}{l}0.388 \mathrm{E}-5 \\
(0.005)\end{array}$ & $\begin{array}{l}0.473 \\
(0.409)\end{array}$ & $\begin{array}{l}-0.0005 \\
(0.002)\end{array}$ & $\begin{array}{l}-0.391 \mathrm{E}-4 \\
(0.0009)\end{array}$ & $\begin{array}{l}0.375 \\
(0.270)\end{array}$ & $\begin{array}{l}-0.0007 \\
(0.001)\end{array}$ & $\begin{array}{l}-0.416 \mathrm{E}-4 \\
(0.0002)\end{array}$ \\
\hline $\begin{array}{l}\text { Host country } \\
\text { demand }\end{array}$ & $\begin{array}{l}\text { 8.518* } \\
(3.583)\end{array}$ & $\begin{array}{l}\text { 7.469* } \\
(3.278)\end{array}$ & $\begin{array}{l}0.448 \\
(0.295)\end{array}$ & $\begin{array}{l}6.332 \\
(8.987)\end{array}$ & $\begin{array}{l}6.213 \\
(7.052)\end{array}$ & $\begin{array}{l}0.357 \\
(0.789)\end{array}$ & $\begin{array}{l}6.500 \\
(5.657)\end{array}$ & $\begin{array}{l}7.939 \\
(5.161)\end{array}$ & $\begin{array}{l}0.529 \\
(0.448)\end{array}$ & $\begin{array}{l}\text { 11.001† } \\
(6.150)\end{array}$ & $\begin{array}{l}5.296 \\
(5.155)\end{array}$ & $\begin{array}{l}0.295 \\
(0.742)\end{array}$ \\
\hline AU/NZ dummy & $\begin{array}{l}0.800 \\
(0.945)\end{array}$ & $\begin{array}{l}0.507 \\
(0.896)\end{array}$ & $\begin{array}{l}0.032 \\
(0.076)\end{array}$ & - & - & - & - & - & - & - & - & - \\
\hline UK/IR dummy & $\begin{array}{l}1.029 \\
(0.706)\end{array}$ & $\begin{array}{l}0.987 \\
(0.667)\end{array}$ & $\begin{array}{l}0.061 \\
(0.059)\end{array}$ & - & - & - & - & - & - & - & - & - \\
\hline $\mathrm{R}^{2}$ & 0.224 & & & 0.172 & & & 0.348 & & & 0.243 & & \\
\hline $\operatorname{AdjR}^{2}$ & 0.179 & & & -0.044 & & & 0.270 & & & 0.160 & & \\
\hline Log likelihood & & -420.90 & -438.92 & & -90.53 & -96.46 & & -158.50 & -164.16 & & -161.15 & -172.15 \\
\hline $\mathrm{N}$ & 157 & 157 & 157 & 32 & 32 & 32 & 59 & 59 & 59 & 66 & 66 & 66 \\
\hline Max. VIF & 1.608 & & & 1.309 & & & 1.318 & & & 1.145 & & \\
\hline
\end{tabular}

Standard errors in parentheses, all $t$ tests are two-tailed, $\uparrow p<.10,{ }^{*} p<.05,{ }^{* *} p<.01$

Table 7.18b: Regression estimates for equation 9b (high normative institutional distance)

\begin{tabular}{|c|c|c|c|c|c|c|c|c|c|c|c|c|}
\hline & \multicolumn{3}{|c|}{ "Complete data set } & \multicolumn{3}{|c|}{ AU/NZ } & \multicolumn{3}{|c|}{$\overline{\text { US/CA }}$} & \multicolumn{3}{|c|}{ UK/IR } \\
\hline & OLS & Tobit & $\begin{array}{l}\text { Negative } \\
\text { binomial }\end{array}$ & OLS & Tobit & $\begin{array}{l}\text { Negative } \\
\text { binomial } \\
\end{array}$ & OLS & Tobit & $\begin{array}{l}\text { Negative } \\
\text { binomial }\end{array}$ & OLS & Tobit & $\begin{array}{l}\text { Negative } \\
\text { binomial }\end{array}$ \\
\hline Intercept & $\begin{array}{l}19.041 * * \\
(2.598)\end{array}$ & $\begin{array}{l}18.099 * * \\
(2.314)\end{array}$ & $\begin{array}{l}2.756 * * \\
(0.226)\end{array}$ & $\begin{array}{l}24.050 * * \\
(4.032)\end{array}$ & $\begin{array}{l}24.627 * * \\
(3.616)\end{array}$ & $\begin{array}{l}3.260 * * \\
(0.483)\end{array}$ & $\begin{array}{l}19.685 * * \\
(4.705)\end{array}$ & $\begin{array}{l}19.685 * * \\
(4.440)\end{array}$ & $\begin{array}{l}2.597 * * \\
(0.367)\end{array}$ & $\begin{array}{l}16.502^{* *} \\
(3.206)\end{array}$ & $\begin{array}{l}17.543^{* *} \\
(2.490)\end{array}$ & $\begin{array}{l}2.839 * \\
(1.469)\end{array}$ \\
\hline $\begin{array}{l}\text { Reputation (Factor) } \\
\text { (H7f) }\end{array}$ & $\begin{array}{l}-1.340 * * \\
(0.307)\end{array}$ & $\begin{array}{l}-1.342 * * \\
(0.285)\end{array}$ & $\begin{array}{l}-0.083^{* *} \\
(0.296)\end{array}$ & $\begin{array}{l}-1.366^{*} \\
(0.532)\end{array}$ & $\begin{array}{l}-1.429 * * \\
(0.488)\end{array}$ & $\begin{array}{l}-0.082 \\
(0.059)\end{array}$ & $\begin{array}{l}-1.584^{* *} \\
(0.529)\end{array}$ & $\begin{array}{l}-1.584^{* *} \\
(0.499)\end{array}$ & $\begin{array}{l}-0.102 * * \\
(0.045)\end{array}$ & $\begin{array}{l}-0.335 \\
(0.444)\end{array}$ & $\begin{array}{l}-0.515 \\
(0.352)\end{array}$ & $\begin{array}{l}-0.029 \\
(0.107)\end{array}$ \\
\hline Age & $\begin{array}{l}0.000 \\
(0.005)\end{array}$ & $\begin{array}{l}-0.0001 \\
(0.005)\end{array}$ & $\begin{array}{l}-0.166 \mathrm{E}-4 \\
(0.0004)\end{array}$ & $\begin{array}{l}-0.017 \\
(0.012)\end{array}$ & $\begin{array}{l}-0.018 \\
(0.011)\end{array}$ & $\begin{array}{l}-0.001 \\
(0.001)\end{array}$ & $\begin{array}{l}0.004 \\
(0.008)\end{array}$ & $\begin{array}{l}0.004 \\
(0.007)\end{array}$ & $\begin{array}{l}0.0002 \\
(0.0006)\end{array}$ & $\begin{array}{l}0.000 \\
(0.000)\end{array}$ & $\begin{array}{l}-0.002 \\
(0.005)\end{array}$ & $\begin{array}{l}-0.0001 \\
(0.001)\end{array}$ \\
\hline Size (Staff) & $\begin{array}{l}-0.000 \\
(0.000)\end{array}$ & $\begin{array}{l}-0.0001 \\
(0.0002)\end{array}$ & $\begin{array}{l}-0.462 E-5 \\
(0.248 E-4)\end{array}$ & $\begin{array}{l}0.000 \\
(0.000)\end{array}$ & $\begin{array}{l}-0.000 \\
(0.0004)\end{array}$ & $\begin{array}{l}-0.176 \mathrm{E}-4 \\
(0.630 \mathrm{E}-4)\end{array}$ & $\begin{array}{l}-0.000 \\
(0.000)\end{array}$ & $\begin{array}{l}-0.710 \mathrm{E}-4 \\
(0.0003)\end{array}$ & $\begin{array}{l}-0.298 \mathrm{E}-5 \\
(0.441 \mathrm{E}-4)\end{array}$ & $\begin{array}{l}0.000 \\
(0.000)\end{array}$ & $\begin{array}{l}-0.0003 \\
(0.0002)\end{array}$ & $\begin{array}{l}-0.181 \mathrm{E}-4 \\
(0.0001)\end{array}$ \\
\hline Offshore policy & $\begin{array}{l}\text { 6.878** } \\
(1.067)\end{array}$ & $\begin{array}{l}\text { 6.700** } \\
(1.026)\end{array}$ & $\begin{array}{l}0.552^{* *} \\
(0.065)\end{array}$ & $\begin{array}{l}2.037 \\
(2.046)\end{array}$ & $\begin{array}{l}2.134 \\
(1.872)\end{array}$ & $\begin{array}{l}0.132 \\
(0.303)\end{array}$ & $\begin{array}{l}9.540 * * \\
(1.649)\end{array}$ & $\begin{array}{l}9.539 * * \\
(1.556)\end{array}$ & $\begin{array}{l}1.026 * * \\
(0.120)\end{array}$ & $\begin{array}{l}4.561 * * \\
(1.615)\end{array}$ & $\begin{array}{l}4.360 * * \\
(1.402)\end{array}$ & $\begin{array}{l}0.272 \\
(1.343)\end{array}$ \\
\hline $\begin{array}{l}\text { Internationalisation } \\
\text { at home strategy }\end{array}$ & $\begin{array}{l}-0.364 \dagger \\
(0.213)\end{array}$ & $\begin{array}{l}0.0002 \\
(0.001)\end{array}$ & $\begin{array}{l}0.140 \mathrm{E}-4 \\
(0.0002)\end{array}$ & $\begin{array}{l}0.153 \\
(0393)\end{array}$ & $\begin{array}{l}0.0002 \\
(0.002)\end{array}$ & $\begin{array}{l}0.203 E-4 \\
(0.0007)\end{array}$ & $\begin{array}{l}-0.775^{*} \\
(0.334)\end{array}$ & $\begin{array}{l}-0.774 * \\
(0.315)\end{array}$ & $\begin{array}{l}-0.051 \\
(0.032)\end{array}$ & $\begin{array}{l}-0.181 \\
(0.336)\end{array}$ & $\begin{array}{l}0.0009 \\
(0.0009)\end{array}$ & $\begin{array}{l}0.555 E-4 \\
(0.0002)\end{array}$ \\
\hline $\begin{array}{l}\text { Host country } \\
\text { demand }\end{array}$ & $\begin{array}{l}1.045 \\
(2.765)\end{array}$ & $\begin{array}{l}1.520 \\
(2.650)\end{array}$ & $\begin{array}{l}0.094 \\
(0.298)\end{array}$ & $\begin{array}{l}6.687 \\
(5.365)\end{array}$ & $\begin{array}{l}7.941 \dagger \\
(4.639)\end{array}$ & $\begin{array}{l}0.441 \\
(0.844)\end{array}$ & $\begin{array}{l}-4.543 \\
(4.423)\end{array}$ & $\begin{array}{l}-4.543 \\
(4.173)\end{array}$ & $\begin{array}{l}-0.285 \\
(0.434)\end{array}$ & $\begin{array}{l}0.697 \\
(3.824)\end{array}$ & $\begin{array}{l}1.474 \\
(3.153)\end{array}$ & $\begin{array}{l}0.079 \\
(0.984)\end{array}$ \\
\hline
\end{tabular}




\begin{tabular}{|c|c|c|c|c|c|c|c|c|c|c|c|c|}
\hline AU/NZ dummy & $\begin{array}{l}0.599 \\
(0.729)\end{array}$ & $\begin{array}{l}0.812 \\
(0.686)\end{array}$ & $\begin{array}{l}0.053 \\
(0.075)\end{array}$ & - & - & - & - & - & - & - & - & - \\
\hline UK/IR dummy & $\begin{array}{l}1.289 \dagger \\
(0.773)\end{array}$ & $\begin{array}{l}1.471 * \\
(0.722)\end{array}$ & $\begin{array}{l}0.090 \\
(0.090)\end{array}$ & - & - & - & - & - & - & - & - & - \\
\hline $\mathrm{R}^{2}$ & 0.405 & & & 0.226 & & & 0.580 & & & 0.270 & & \\
\hline $\operatorname{AdjR}^{2}$ & 0.370 & & & 0.107 & & & 0.536 & & & 0.101 & & \\
\hline Log likelihood & & -389.27 & -418.74 & & -121.23 & -129.58 & & -168.54 & -181.75 & & -77.92 & -93.35 \\
\hline $\mathrm{N}$ & 150 & 150 & 150 & 48 & 48 & 48 & 64 & 64 & 64 & 38 & 38 & 38 \\
\hline Max. VIF & 1.461 & & & 1.218 & & & 1.388 & & & 1.344 & & \\
\hline
\end{tabular}

Standard errors in parentheses, all $t$ tests are two-tailed, $\uparrow p<.10,{ }^{*} p<.05,{ }^{* *} p<.01$

Table 7.19a: Regression estimates for equation 4c (low regulative institutional distance)

\begin{tabular}{|c|c|c|c|c|c|c|c|c|c|c|c|c|}
\hline & \multicolumn{3}{|c|}{ Complete data set } & \multicolumn{3}{|c|}{ AU/NZ } & \multicolumn{3}{|c|}{ US/CA } & \multicolumn{3}{|c|}{ UK/IR } \\
\hline & OLS & Tobit & $\begin{array}{l}\text { Negative } \\
\text { binomial } \\
\end{array}$ & OLS & Tobit & $\begin{array}{l}\text { Negative } \\
\text { binomial } \\
\end{array}$ & OLS & Tobit & $\begin{array}{l}\text { Negative } \\
\text { binomial } \\
\end{array}$ & OLS & Tobit & $\begin{array}{l}\text { Negative } \\
\text { binomial }\end{array}$ \\
\hline Intercept & $\begin{array}{l}7.530 \\
(5.301)\end{array}$ & $\begin{array}{l}9.766^{*} \\
(4.676)\end{array}$ & $\begin{array}{l}2.367 * * \\
(0.368)\end{array}$ & $\begin{array}{l}1.377 \\
(12.782)\end{array}$ & $\begin{array}{l}0.714 \\
(10.381)\end{array}$ & $\begin{array}{l}1.628 \dagger \\
(0.908)\end{array}$ & $\begin{array}{l}2.344 \\
(0.859)\end{array}$ & $\begin{array}{l}5.330 \\
(10.255)\end{array}$ & $\begin{array}{l}1.987 \\
(1.306)\end{array}$ & $\begin{array}{l}14.259 * * \\
(4.979)\end{array}$ & $\begin{array}{l}15.272^{* *} \\
(4.216)\end{array}$ & $\begin{array}{l}2.684 * * \\
(0.653)\end{array}$ \\
\hline $\begin{array}{l}\text { Geo exp - } \\
\text { knowledge of edu } \\
\text { sector }(H 8 a)\end{array}$ & $\begin{array}{l}-0.012 \\
(0.269)\end{array}$ & $\begin{array}{l}-0.071 \\
(0.239)\end{array}$ & $\begin{array}{l}-0.005 \\
(0.017)\end{array}$ & $\begin{array}{l}0.388 \\
(0.785)\end{array}$ & $\begin{array}{l}0.447 \\
(0.607)\end{array}$ & $\begin{array}{l}0.025 \\
(0.057)\end{array}$ & $\begin{array}{l}0.651 \\
(0.661)\end{array}$ & $\begin{array}{l}0.573 \\
(0.557)\end{array}$ & $\begin{array}{l}0.040 \\
(0.052)\end{array}$ & $\begin{array}{l}-0.257 \\
(0.266)\end{array}$ & $\begin{array}{l}-0.306 \\
(0.221)\end{array}$ & $\begin{array}{l}-0.017 \\
(0.381)\end{array}$ \\
\hline $\begin{array}{l}\text { Geo exp - academic } \\
\text { capabilities }(H 8 a)\end{array}$ & $\begin{array}{l}0.132 \\
(0.237)\end{array}$ & $\begin{array}{l}0.123 \\
(0.219)\end{array}$ & $\begin{array}{l}0.007 \\
(0.014)\end{array}$ & $\begin{array}{l}-0.463 \\
(0.575)\end{array}$ & $\begin{array}{l}-0.463 \\
(0.465)\end{array}$ & $\begin{array}{l}-0.031 \\
(0.045)\end{array}$ & $\begin{array}{l}1.2522 * \\
(0.509)\end{array}$ & $\begin{array}{l}1.247 * * \\
(0.439)\end{array}$ & $\begin{array}{l}0.087 * \\
(0.035)\end{array}$ & $\begin{array}{l}-0.030 \\
(0.257)\end{array}$ & $\begin{array}{l}-0.039 \\
(0.225)\end{array}$ & $\begin{array}{l}-0.001 \\
(0.037)\end{array}$ \\
\hline $\begin{array}{l}\text { Geo exp - } \\
\text { managerial } \\
\text { capabilities }(H 8 a)\end{array}$ & $\begin{array}{l}0.183 \\
(0.292)\end{array}$ & $\begin{array}{l}0.175 \\
(0.273)\end{array}$ & $\begin{array}{l}0.011 \\
(0.020)\end{array}$ & $\begin{array}{l}-0.342 \\
(1.005)\end{array}$ & $\begin{array}{l}-0.263 \\
(0.799)\end{array}$ & $\begin{array}{l}-0.019 \\
(0.080)\end{array}$ & $\begin{array}{l}-0.012 \\
(0.614)\end{array}$ & $\begin{array}{l}-0.061 \\
(0.524)\end{array}$ & $\begin{array}{l}-0.002 \\
(0.037)\end{array}$ & $\begin{array}{l}0.114 \\
(0.290)\end{array}$ & $\begin{array}{l}0.100 \\
(0.261)\end{array}$ & $\begin{array}{l}0.005 \\
(0.053)\end{array}$ \\
\hline $\begin{array}{l}\text { Geo exp - financial } \\
\text { capabilities }(H 8 a)\end{array}$ & $\begin{array}{l}-0.122 \\
(0.268)\end{array}$ & $\begin{array}{l}-0.064 \\
(0.243)\end{array}$ & $\begin{array}{l}-0.004 \\
(0.022)\end{array}$ & $\begin{array}{l}0.362 \\
(0.761)\end{array}$ & $\begin{array}{l}0.376 \\
(0.571)\end{array}$ & $\begin{array}{l}0.027 \\
(0.073)\end{array}$ & $\begin{array}{l}0.508 \\
(0.537)\end{array}$ & $\begin{array}{l}0.526 \\
(0.462)\end{array}$ & $\begin{array}{l}0.033 \\
(0.594)\end{array}$ & $\begin{array}{l}-0.513 \dagger \\
(0.294)\end{array}$ & $\begin{array}{l}-0.365 \\
(0.253)\end{array}$ & $\begin{array}{l}-0.021 \\
(0.034)\end{array}$ \\
\hline $\begin{array}{l}\text { Geo exp - intl } \\
\text { recruitment (H8a) }\end{array}$ & $\begin{array}{l}0.005 \\
(0.319)\end{array}$ & $\begin{array}{l}-0.038 \\
(0.292)\end{array}$ & $\begin{array}{l}-0.003 \\
(0.019)\end{array}$ & $\begin{array}{l}0.130 \\
(0.972)\end{array}$ & $\begin{array}{l}0.229 \\
(0.790)\end{array}$ & $\begin{array}{l}0.012 \\
(0.059)\end{array}$ & $\begin{array}{l}-0.636 \\
(0.666)\end{array}$ & $\begin{array}{l}-0.710 \\
(0.562)\end{array}$ & $\begin{array}{l}-0.044 \\
(0.083)\end{array}$ & $\begin{array}{l}0.124 \\
(0.336)\end{array}$ & $\begin{array}{l}0.122 \\
(0.289)\end{array}$ & $\begin{array}{l}0.007 \\
(0.064)\end{array}$ \\
\hline Age & $\begin{array}{l}0.005 \\
(0.007)\end{array}$ & $\begin{array}{l}0.004 \\
(0.006)\end{array}$ & $\begin{array}{l}0.0002 \\
(0.0004)\end{array}$ & $\begin{array}{l}-0.008 \\
(0.026)\end{array}$ & $\begin{array}{l}-0.006 \\
(0.006)\end{array}$ & $\begin{array}{l}-0.0004 \\
(0.002)\end{array}$ & $\begin{array}{l}0.009 \\
(0.017)\end{array}$ & $\begin{array}{l}0.008 \\
(0.014)\end{array}$ & $\begin{array}{l}0.0004 \\
(0.001)\end{array}$ & $\begin{array}{l}0.002 \\
(0.006)\end{array}$ & $\begin{array}{l}-0.279 \mathrm{E}-4 \\
(0.005)\end{array}$ & $\begin{array}{l}0.116 \mathrm{E}-4 \\
(0.0007)\end{array}$ \\
\hline Size (Staff) & $\begin{array}{l}0.000 \\
(0.000)\end{array}$ & $\begin{array}{l}0.0002 \\
(0.0002)\end{array}$ & $\begin{array}{l}1.588 \mathrm{E}-4 \\
(1.989 \mathrm{E}-4)\end{array}$ & $\begin{array}{l}0.001 \\
(0.001)\end{array}$ & $\begin{array}{l}0.0005 \\
(0.0006)\end{array}$ & $\begin{array}{l}0.365 \mathrm{E}-4 \\
(0.580 \mathrm{E}-4)\end{array}$ & $\begin{array}{l}0.001 \\
(0.001)\end{array}$ & $\begin{array}{l}\text { 0.001* } \\
(0.0004)\end{array}$ & $\begin{array}{l}0.705 E-4 \\
(0.629 E-4)\end{array}$ & $\begin{array}{l}0.000 \\
(0.000)\end{array}$ & $\begin{array}{l}0.856 E-4 \\
(0.0002)\end{array}$ & $\begin{array}{l}0.537 \mathrm{E}-5 \\
(0.485 \mathrm{E}-4)\end{array}$ \\
\hline Offshore policy & $2.848^{*}$ & $2.703 \dagger$ & $0.191 * *$ & 8.824* & $8.632 * *$ & $0.743 * *$ & $-5.395 \dagger$ & $-5.757^{*}$ & -0.395 & $5.117 * *$ & $5.055 * *$ & $0.336 * *$ \\
\hline
\end{tabular}




\begin{tabular}{|c|c|c|c|c|c|c|c|c|c|c|c|c|}
\hline & (1.497) & (1.413) & $(0.063)$ & (3.767) & (3.055) & $(0.254)$ & (3.159) & (2.666) & $(0.290)$ & (1.816) & (1.649) & $(0.122)$ \\
\hline $\begin{array}{l}\text { Internationalisation } \\
\text { at home strategy }\end{array}$ & $\begin{array}{l}0.372 \\
(0.260)\end{array}$ & $\begin{array}{l}-0.0002 \\
(0.001)\end{array}$ & $\begin{array}{l}-0.153 \mathrm{E}-4 \\
(0.0001)\end{array}$ & $\begin{array}{l}0.317 \\
(0.854)\end{array}$ & $\begin{array}{l}-0.001 \\
(0.003)\end{array}$ & $\begin{array}{l}-0.799 \\
(0.0007)\end{array}$ & $\begin{array}{l}0.284 \\
(0.535)\end{array}$ & $\begin{array}{l}-0.007 \dagger \\
(0.004)\end{array}$ & $\begin{array}{l}-0.0005 \\
(0.034)\end{array}$ & $\begin{array}{l}0.352 \\
(0.294)\end{array}$ & $\begin{array}{l}0.0009 \\
(0.001)\end{array}$ & $\begin{array}{l}0.539 E-4 \\
(0.0001)\end{array}$ \\
\hline $\begin{array}{l}\text { Host country } \\
\text { demand }\end{array}$ & $\begin{array}{l}12.133 \\
(3.595)\end{array}$ & $\begin{array}{l}10.359 * * \\
(3.235)\end{array}$ & $\begin{array}{l}0.624^{*} \\
(0.264)\end{array}$ & $\begin{array}{l}\text { 20.889* } \\
(9.321)\end{array}$ & $\begin{array}{l}21.580 * * \\
(7.218)\end{array}$ & $\begin{array}{l}1.388 \\
(0.875)\end{array}$ & $\begin{array}{l}\text { 13.690* } \\
(6.754)\end{array}$ & $\begin{array}{l}\text { 13.698* } \\
(5.831)\end{array}$ & $\begin{array}{l}0.943 \\
(0.616)\end{array}$ & $\begin{array}{l}7.503 \dagger \\
(4.330)\end{array}$ & $\begin{array}{l}3.809 \\
(3.386)\end{array}$ & $\begin{array}{l}0.216 \\
(0.641)\end{array}$ \\
\hline AU/NZ dummy & $\begin{array}{l}-0.059 \\
(1.101)\end{array}$ & $\begin{array}{l}-0.2887 \\
(1.012)\end{array}$ & $\begin{array}{l}-0.015 \\
(0.689)\end{array}$ & - & - & - & - & - & - & - & - & - \\
\hline UK/IR dummy & $\begin{array}{l}2.330 \\
(0.885)\end{array}$ & $\begin{array}{l}1.877^{*} \\
(0.812)\end{array}$ & $\begin{array}{l}0.115 * \\
(0.056)\end{array}$ & - & - & - & - & - & - & - & - & - \\
\hline $\mathrm{R}^{2}$ & 0.177 & & & 0.392 & & & 0.338 & & & 0.222 & & \\
\hline $\operatorname{AdjR}^{2}$ & 0.100 & & & 0.102 & & & 0.144 & & & 0.076 & & \\
\hline Log likelihood & & -426.19 & -452.24 & & -95.11 & -103.35 & & -130.52 & -140.80 & & -172.58 & -186.94 \\
\hline $\mathrm{N}$ & 152 & 152 & 152 & 34 & 34 & 34 & 46 & 46 & 46 & 72 & 72 & 72 \\
\hline Max. VIF & 1.672 & & & 1.564 & & & 1.339 & & & 1.508 & & \\
\hline
\end{tabular}

Standard errors in parentheses, all $t$ tests are two-tailed, $\uparrow p<.10,{ }^{*} p<.05,{ }^{* *} p<.01$

Table 7.19b: Regression estimates for equation 4d (high regulative institutional distance)

\begin{tabular}{|c|c|c|c|c|c|c|c|c|c|c|c|c|}
\hline & \multicolumn{3}{|c|}{ Complete data set } & \multicolumn{3}{|c|}{ AU/NZ } & \multicolumn{3}{|c|}{$\overline{\text { US/CA }}$} & \multicolumn{3}{|c|}{ UK/IR } \\
\hline & OLS & Tobit & $\begin{array}{l}\text { Negative } \\
\text { binomial }\end{array}$ & OLS & Tobit & $\begin{array}{l}\text { Negative } \\
\text { binomial }\end{array}$ & OLS & Tobit & $\begin{array}{l}\text { Negative } \\
\text { binomial }\end{array}$ & OLS & Tobit & $\begin{array}{l}\text { Negative } \\
\text { binomial }\end{array}$ \\
\hline Intercept & $\begin{array}{l}8.923 * \\
(3.674)\end{array}$ & $\begin{array}{l}9.175^{* *} \\
(3.368)\end{array}$ & $\begin{array}{l}2.273^{* *} \\
(0.328)\end{array}$ & $\begin{array}{l}16.412 * * \\
(4.581)\end{array}$ & $\begin{array}{l}16.984 * * \\
(3.786)\end{array}$ & $\begin{array}{l}\text { 2.830* } \\
(1.200)\end{array}$ & $\begin{array}{l}7.262 \\
(5.716)\end{array}$ & $\begin{array}{l}7.868 \\
(5.127)\end{array}$ & $\begin{array}{l}1.981 * * \\
(0.536)\end{array}$ & $\begin{array}{l}15.946 \dagger \\
(9.066)\end{array}$ & $\begin{array}{l}14.682 * \\
(7.070)\end{array}$ & $\begin{array}{l}0.065 \\
(0.063)\end{array}$ \\
\hline $\begin{array}{l}\text { Geo exp - } \\
\text { knowledge of edu } \\
\text { sector (H8a) }\end{array}$ & $\begin{array}{l}-0.000 \\
(0.191)\end{array}$ & $\begin{array}{l}-0.006 \\
(0.178)\end{array}$ & $\begin{array}{l}-0.0003 \\
(0.020)\end{array}$ & $\begin{array}{l}0.474 \dagger \\
(0.269)\end{array}$ & $\begin{array}{l}\text { 0.488* } \\
(0.227)\end{array}$ & $\begin{array}{l}0.027 \\
(0.074)\end{array}$ & $\begin{array}{l}0.010 \\
(0.277)\end{array}$ & $\begin{array}{l}0.005 \\
(0.254)\end{array}$ & $\begin{array}{l}0.0004 \\
(0.029)\end{array}$ & $\begin{array}{l}-0.368 \\
(0.444)\end{array}$ & $\begin{array}{l}-0.244 \\
(0.320)\end{array}$ & $\begin{array}{l}0.050 \\
(0.056)\end{array}$ \\
\hline $\begin{array}{l}\text { Geo exp - academic } \\
\text { capabilities }(H 8 a)\end{array}$ & $\begin{array}{l}0.141 \\
(0.196)\end{array}$ & $\begin{array}{l}0.112 \\
(0.182)\end{array}$ & $\begin{array}{l}0.007 \\
(0.017)\end{array}$ & $\begin{array}{l}0.229 \\
(0.282)\end{array}$ & $\begin{array}{l}0.226 \\
(0.232)\end{array}$ & $\begin{array}{l}0.012 \\
(0.069)\end{array}$ & $\begin{array}{l}0.080 \\
(0.296)\end{array}$ & $\begin{array}{l}0.082 \\
(0.272)\end{array}$ & $\begin{array}{l}0.005 \\
(0.026)\end{array}$ & $\begin{array}{l}0.231 \\
(0.337)\end{array}$ & $\begin{array}{l}0.209 \\
(0.267)\end{array}$ & $\begin{array}{l}0.032 \\
(0.062)\end{array}$ \\
\hline $\begin{array}{l}\text { Geo exp - } \\
\text { managerial } \\
\text { capabilities (H8a) }\end{array}$ & $\begin{array}{l}0.040 \\
(0.227)\end{array}$ & $\begin{array}{l}0.046 \\
(0.213)\end{array}$ & $\begin{array}{l}0.002 \\
(0.022)\end{array}$ & $\begin{array}{l}-0.256 \\
(0.300)\end{array}$ & $\begin{array}{l}-0.269 \\
(0.250)\end{array}$ & $\begin{array}{l}-0.015 \\
(0.081)\end{array}$ & $\begin{array}{l}0.536 \\
(0.346)\end{array}$ & $\begin{array}{l}0.545 \dagger \\
(0.318)\end{array}$ & $\begin{array}{l}0.034 \\
(0.037)\end{array}$ & $\begin{array}{l}-0.729 \dagger \\
(0.419)\end{array}$ & $\begin{array}{l}-0.731^{*} \\
(0.334)\end{array}$ & $\begin{array}{l}0.069 \\
(0.069)\end{array}$ \\
\hline $\begin{array}{l}\text { Geo exp - financial } \\
\text { capabilities (H8a) }\end{array}$ & $\begin{array}{l}-0.042 \\
(0.223)\end{array}$ & $\begin{array}{l}-0.073 \\
(0.204)\end{array}$ & $\begin{array}{l}-0.004 \\
(0.023)\end{array}$ & $\begin{array}{l}-0.519 \\
(0.344)\end{array}$ & $\begin{array}{l}-0.561 * \\
(0.267)\end{array}$ & $\begin{array}{l}-0.032 \\
(0.085)\end{array}$ & $\begin{array}{l}0.044 \\
(0.307)\end{array}$ & $\begin{array}{l}0.033 \\
(0.281)\end{array}$ & $\begin{array}{l}0.001 \\
(0.030)\end{array}$ & $\begin{array}{l}0.101 \\
(0.454)\end{array}$ & $\begin{array}{l}0.097 \\
(0.362)\end{array}$ & $\begin{array}{l}0.088 \\
(0.059)\end{array}$ \\
\hline $\begin{array}{l}\text { Geo exp - intl } \\
\text { recruitment (H8a) }\end{array}$ & $\begin{array}{l}0.022 \\
(0.233)\end{array}$ & $\begin{array}{l}0.032 \\
(0.220)\end{array}$ & $\begin{array}{l}0.002 \\
(0.020)\end{array}$ & $\begin{array}{l}0.383 \\
(0.296)\end{array}$ & $\begin{array}{l}0.381 \\
(0.245)\end{array}$ & $\begin{array}{l}0.021 \\
(0.084)\end{array}$ & $\begin{array}{l}-0.724 \dagger \\
(0.391)\end{array}$ & $\begin{array}{l}-0.729 * \\
(0.360)\end{array}$ & $\begin{array}{l}-0.046 \\
(0.030)\end{array}$ & $\begin{array}{l}0.126 \\
(0.458)\end{array}$ & $\begin{array}{l}0.145 \\
(0.364)\end{array}$ & $\begin{array}{l}0.001 \\
(0.002)\end{array}$ \\
\hline
\end{tabular}




\begin{tabular}{|c|c|c|c|c|c|c|c|c|c|c|c|c|}
\hline Age & $\begin{array}{l}0.001 \\
(0.006)\end{array}$ & $\begin{array}{l}0.001 \\
(0.005)\end{array}$ & $\begin{array}{l}0.842 \mathrm{E}-4 \\
(0.0005)\end{array}$ & $\begin{array}{l}0.001 \\
(0.010)\end{array}$ & $\begin{array}{l}-0.467 \mathrm{E}-4 \\
(0.007)\end{array}$ & $\begin{array}{l}0.776 \mathrm{E}-5 \\
(0.002)\end{array}$ & $\begin{array}{l}0.000 \\
(0.000)\end{array}$ & $\begin{array}{l}-0.001 \\
(0.007)\end{array}$ & $\begin{array}{l}-0.637 \mathrm{E}-4 \\
(0.0008)\end{array}$ & $\begin{array}{l}0.022 * \\
(0.011)\end{array}$ & $\begin{array}{l}0.021 * * \\
(0.008)\end{array}$ & $\begin{array}{l}0.762 \mathrm{E}-4 \\
(0.848 \mathrm{E}-4)\end{array}$ \\
\hline Size (Staff) & $\begin{array}{l}0.000 \\
(0.000)\end{array}$ & $\begin{array}{l}0.659 \mathrm{E}-4 \\
(0.0002)\end{array}$ & $\begin{array}{l}0.450 \mathrm{E}-5 \\
(0.247 \mathrm{E}-4)\end{array}$ & $\begin{array}{l}0.000 \\
(0.000)\end{array}$ & $\begin{array}{l}-0.0004 \\
(0.0002)\end{array}$ & $\begin{array}{l}-0.246 \mathrm{E}-4 \\
(0.760 \mathrm{E}-4)\end{array}$ & $\begin{array}{l}0.000 \\
(0.000)\end{array}$ & $\begin{array}{l}-0.0003 \\
(0.0003)\end{array}$ & $\begin{array}{l}-0.202 E-4 \\
(0.373 E-4)\end{array}$ & $\begin{array}{l}0.000 \\
(0.001)\end{array}$ & $\begin{array}{l}-0.979 \mathrm{E}-4 \\
(0.0004)\end{array}$ & $\begin{array}{l}0.328 \\
(0.862)\end{array}$ \\
\hline Offshore policy & $\begin{array}{l}5.984 * * \\
(0.992)\end{array}$ & $\begin{array}{l}5.987 * * \\
(0.937)\end{array}$ & $\begin{array}{l}0.439 * * \\
(0.062)\end{array}$ & $\begin{array}{l}-0.225 \\
(1.506)\end{array}$ & $\begin{array}{l}-0.178 \\
(1.275)\end{array}$ & $\begin{array}{l}-0.010 \\
(0.300)\end{array}$ & $\begin{array}{l}10.582 * * \\
(1.444)\end{array}$ & $\begin{array}{l}10.539 * * \\
(1.326)\end{array}$ & $\begin{array}{l}0.941^{* *} \\
(0.098)\end{array}$ & $\begin{array}{l}4.555^{*} \\
(1.796)\end{array}$ & $\begin{array}{l}4.070 * * \\
(1.302)\end{array}$ & $\begin{array}{l}-0.0001 \\
(0.069)\end{array}$ \\
\hline $\begin{array}{l}\text { Internationalisation } \\
\text { at home strategy }\end{array}$ & $\begin{array}{l}-0.010 \\
(0.227)\end{array}$ & $\begin{array}{l}-0.001 \\
(0.001)\end{array}$ & $\begin{array}{l}-0.857 \mathrm{E}-4 \\
(0.0003)\end{array}$ & $\begin{array}{l}0.097 \\
(0.311)\end{array}$ & $\begin{array}{l}-0.001 \\
(0.001)\end{array}$ & $\begin{array}{l}-0.968 \\
(0.001)\end{array}$ & $\begin{array}{l}0.164 \\
(0.342)\end{array}$ & $\begin{array}{l}0.001 \\
(0.003)\end{array}$ & $\begin{array}{l}0.0001 \\
(0.028)\end{array}$ & $\begin{array}{l}-0.288 \\
(0.439)\end{array}$ & $\begin{array}{l}-0.004 * \\
(0.002)\end{array}$ & $\begin{array}{l}0.705 \\
(0.979)\end{array}$ \\
\hline $\begin{array}{l}\text { Host country } \\
\text { demand }\end{array}$ & $\begin{array}{l}0.797 \\
(3.281)\end{array}$ & $\begin{array}{l}1.254 \\
(3.058)\end{array}$ & $\begin{array}{l}0.077 \\
(0.327)\end{array}$ & $\begin{array}{l}1.927 \\
(4.860)\end{array}$ & $\begin{array}{l}2.530 \\
(4.014)\end{array}$ & $\begin{array}{l}0.137 \\
(1.420)\end{array}$ & $\begin{array}{l}-2.554 \\
(4.711)\end{array}$ & $\begin{array}{l}-2.740 \\
(4.320)\end{array}$ & $\begin{array}{l}-0.170 \\
(0.492)\end{array}$ & $\begin{array}{l}4.027 \\
(5.611)\end{array}$ & $\begin{array}{l}5.184 \\
(4.246)\end{array}$ & $\begin{array}{l}0.310 \\
(1.455)\end{array}$ \\
\hline AU/NZ dummy & $\begin{array}{l}1.686^{*} \\
(0.803)\end{array}$ & $\begin{array}{l}1.716^{*} \\
(0.738)\end{array}$ & $\begin{array}{l}0.103 \\
(0.089)\end{array}$ & - & - & - & - & - & - & - & - & - \\
\hline UK/IR dummy & $\begin{array}{l}0.843 \\
(0.795)\end{array}$ & $\begin{array}{l}0.922 \\
(0.744)\end{array}$ & $\begin{array}{l}0.058 \\
(0.083)\end{array}$ & - & - & - & - & - & - & - & - & - \\
\hline $\mathrm{R}^{2}$ & 0.257 & & & 0.191 & & & 0.484 & & & 0.410 & & \\
\hline $\operatorname{Adj}^{2}$ & 0.192 & & & -0.054 & & & 0.404 & & & 0.115 & & \\
\hline Log likelihood & & -402.19 & -423.62 & & -96.05 & -113.36 & & -202.13 & -213.10 & & -67.37 & -82.61 \\
\hline $\mathrm{N}$ & 155 & 155 & 155 & 46 & 46 & 46 & 77 & 77 & 77 & 32 & 32 & 32 \\
\hline Max. VIF & 1.716 & & & 1.598 & & & 1.317 & & & 1.860 & & \\
\hline
\end{tabular}

Standard errors in parentheses, all $t$ tests are two-tailed, $\dagger p<.10,{ }^{*} p<.05, * * p<.01$

Table 7.20a: Regression estimates for equation 5c (low regulative institutional distance)

\begin{tabular}{|c|c|c|c|c|c|c|c|c|c|c|c|c|}
\hline & \multicolumn{3}{|c|}{ Complete data set } & \multicolumn{3}{|c|}{ AU/NZ } & \multicolumn{3}{|c|}{$\overline{\mathrm{US} / \mathrm{CA}}$} & \multicolumn{3}{|c|}{ 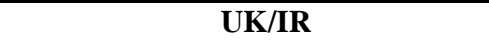 } \\
\hline & OLS & Tobit & $\begin{array}{l}\text { Negative } \\
\text { binomial } \\
\end{array}$ & OLS & Tobit & $\begin{array}{l}\text { Negative } \\
\text { binomial }\end{array}$ & OLS & Tobit & $\begin{array}{l}\text { Negative } \\
\text { binomial }\end{array}$ & OLS & Tobit & $\begin{array}{l}\text { Negative } \\
\text { binomial }\end{array}$ \\
\hline Intercept & $\begin{array}{l}\text { 8.482* } \\
(3.447)\end{array}$ & $\begin{array}{l}10.493 * * \\
(3.168)\end{array}$ & $\begin{array}{l}2.410 * * \\
(0.202)\end{array}$ & $\begin{array}{l}-1.992 \\
(8.282)\end{array}$ & $\begin{array}{l}-1.969 \\
(6.637)\end{array}$ & $\begin{array}{l}1.452 * \\
(0.707)\end{array}$ & $\begin{array}{l}13.819 \\
(8.254)\end{array}$ & $\begin{array}{l}17.020 * \\
(6.851)\end{array}$ & $\begin{array}{l}2.840 * * \\
(0.521)\end{array}$ & $\begin{array}{l}10.830 * \\
(3.612)\end{array}$ & $\begin{array}{l}12.208 * * \\
(3.218)\end{array}$ & $\begin{array}{l}2.510 * * \\
(0.514)\end{array}$ \\
\hline $\begin{array}{l}\text { Ind exp - UG } \\
\text { offshore edu (H8b) }\end{array}$ & $\begin{array}{l}-0.079 \\
(0.224)\end{array}$ & $\begin{array}{l}-0.103 \\
(0.208)\end{array}$ & $\begin{array}{l}-0.006 \\
(0.014)\end{array}$ & $\begin{array}{l}-0.244 \\
(0.601)\end{array}$ & $\begin{array}{l}-0.271 \\
(0.473)\end{array}$ & $\begin{array}{l}-0.016 \\
(0.045)\end{array}$ & $\begin{array}{l}0.047 \\
(0.554)\end{array}$ & $\begin{array}{l}-0.069 \\
(0.478)\end{array}$ & $\begin{array}{l}-0.001 \\
(0.057)\end{array}$ & $\begin{array}{l}-0.115 \\
(0.231)\end{array}$ & $\begin{array}{l}-0.077 \\
(0.203)\end{array}$ & $\begin{array}{l}-0.004 \\
(0.043)\end{array}$ \\
\hline $\begin{array}{l}\text { Ind exp - PG } \\
\text { offshore edu (H8b) }\end{array}$ & $\begin{array}{l}-0.212 \\
(0.267)\end{array}$ & $\begin{array}{l}-0.217 \\
(0.247)\end{array}$ & $\begin{array}{l}-0.013 \\
(0.016)\end{array}$ & $\begin{array}{l}0.133 \\
(0.651)\end{array}$ & $\begin{array}{l}0.130 \\
(0.506)\end{array}$ & $\begin{array}{l}0.005 \\
(0.052)\end{array}$ & $\begin{array}{l}-0.256 \\
(0.615)\end{array}$ & $\begin{array}{l}-0.101 \\
(0.527)\end{array}$ & $\begin{array}{l}-0.011 \\
(0.038)\end{array}$ & $\begin{array}{l}-0.312 \\
(0.302)\end{array}$ & $\begin{array}{l}-0.374 \\
(0.260)\end{array}$ & $\begin{array}{l}-0.021 \\
(0.050)\end{array}$ \\
\hline $\begin{array}{l}\text { Ind exp - Intl } \\
\text { recruitment (Factor) } \\
(\mathrm{H} 8 b)\end{array}$ & $\begin{array}{l}0.506 \\
(0.310)\end{array}$ & $\begin{array}{l}0.439 \\
(0.276)\end{array}$ & $\begin{array}{l}0.026 \\
(0.024)\end{array}$ & $\begin{array}{l}1.346 \\
(1.000)\end{array}$ & $\begin{array}{l}1.367 \dagger \\
(0.803)\end{array}$ & $\begin{array}{l}0.090 \\
(0.091)\end{array}$ & $\begin{array}{l}0.824 \\
(0.704)\end{array}$ & $\begin{array}{l}0.758 \\
(0.616)\end{array}$ & $\begin{array}{l}0.051 \\
(0.059)\end{array}$ & $\begin{array}{l}0.152 \\
(0.323)\end{array}$ & $\begin{array}{l}0.075 \\
(0.261)\end{array}$ & $\begin{array}{l}0.004 \\
(0.053)\end{array}$ \\
\hline
\end{tabular}




\begin{tabular}{|c|c|c|c|c|c|c|c|c|c|c|c|c|}
\hline $\begin{array}{l}\text { Ind exp - UG } \\
\text { marketing (H8b) }\end{array}$ & $\begin{array}{l}-0.296 \\
(0.236)\end{array}$ & $\begin{array}{l}-0.265 \\
(0.212)\end{array}$ & $\begin{array}{l}-0.016 \\
(0.019)\end{array}$ & $\begin{array}{l}-0.687 \\
(0.651)\end{array}$ & $\begin{array}{l}-0.680 \\
(0.518)\end{array}$ & $\begin{array}{l}-0.044 \\
(0.071)\end{array}$ & $\begin{array}{l}-1.048 \dagger \\
(0.546)\end{array}$ & $\begin{array}{l}-0.862 \dagger \\
(0.459)\end{array}$ & $\begin{array}{l}-0.061 \\
(0.057)\end{array}$ & $\begin{array}{l}0.130 \\
(0.249)\end{array}$ & $\begin{array}{l}0.163 \\
(0.206)\end{array}$ & $\begin{array}{l}0.009 \\
(0.045)\end{array}$ \\
\hline $\begin{array}{l}\text { Ind exp - PG } \\
\text { marketing (H8b) }\end{array}$ & $\begin{array}{l}0.239 \\
(0.233)\end{array}$ & $\begin{array}{l}0.171 \\
(0.212)\end{array}$ & $\begin{array}{l}0.009 \\
(0.016)\end{array}$ & $\begin{array}{l}0.804 \\
(0.540)\end{array}$ & $\begin{array}{l}0.807 \dagger \\
(0.423)\end{array}$ & $\begin{array}{l}0.047 \\
(0.047)\end{array}$ & $\begin{array}{l}-0.517 \\
(0.607)\end{array}$ & $\begin{array}{l}-0.472 \\
(0.531)\end{array}$ & $\begin{array}{l}-0.034 \\
(0.051)\end{array}$ & $\begin{array}{l}0.351 \\
(0.242)\end{array}$ & $\begin{array}{l}0.213 \\
(0.204)\end{array}$ & $\begin{array}{l}0.012 \\
(0.040)\end{array}$ \\
\hline Age & $\begin{array}{l}0.002 \\
(0.007)\end{array}$ & $\begin{array}{l}0.001 \\
(0.006)\end{array}$ & $\begin{array}{l}0.0001 \\
(0.0004)\end{array}$ & $\begin{array}{l}0.000 \\
(0.023)\end{array}$ & $\begin{array}{l}0.001 \\
(0.181)\end{array}$ & $\begin{array}{l}0.0001 \\
(0.002)\end{array}$ & $\begin{array}{l}0.002 \\
(0.017)\end{array}$ & $\begin{array}{l}0.001 \\
(0.015)\end{array}$ & $\begin{array}{l}0.789 \mathrm{E}-4 \\
(0.001)\end{array}$ & $\begin{array}{l}0.000 \\
(0.006)\end{array}$ & $\begin{array}{l}-0.0019 \\
(0.005)\end{array}$ & $\begin{array}{l}-0.0001 \\
(0.0008)\end{array}$ \\
\hline Size (Staff) & $\begin{array}{l}0.000 \\
(0.000)\end{array}$ & $\begin{array}{l}0.0002 \\
(0.0002)\end{array}$ & $\begin{array}{l}0.136 \mathrm{E}-4 \\
(0.201 \mathrm{E}-4)\end{array}$ & $\begin{array}{l}0.000 \\
(0.001)\end{array}$ & $\begin{array}{l}0.0001 \\
(0.0005)\end{array}$ & $\begin{array}{l}0.131 \mathrm{E}-4 \\
(0.510 \mathrm{E}-4)\end{array}$ & $\begin{array}{l}0.002^{*} \\
(0.001)\end{array}$ & $\begin{array}{l}0.0012 * \\
(0.0005)\end{array}$ & $\begin{array}{l}0.884 \mathrm{E}-4 \\
(0.683 \mathrm{E}-4)\end{array}$ & $\begin{array}{l}-0.000 \\
(0.000)\end{array}$ & $\begin{array}{l}0.121 \mathrm{E}-4 \\
(0.0002)\end{array}$ & $\begin{array}{l}0.698 E-6 \\
(0.535 E-4)\end{array}$ \\
\hline Offshore policy & $\begin{array}{l}2.809 \dagger \\
(1.464)\end{array}$ & $\begin{array}{l}2.694 \dagger \\
(1.385)\end{array}$ & $\begin{array}{l}\text { 0.189* } \\
(0.074)\end{array}$ & $\begin{array}{l}8.872 * \\
(3.260)\end{array}$ & $\begin{array}{l}\text { 8.810** } \\
(2.637)\end{array}$ & $\begin{array}{l}0.723 * * \\
(0.272)\end{array}$ & $\begin{array}{l}-3.383 \\
(3.305)\end{array}$ & $\begin{array}{l}-4.254 \\
(2.828)\end{array}$ & $\begin{array}{l}-0.292 \\
(0.220)\end{array}$ & $\begin{array}{l}4.196 * \\
(1.853)\end{array}$ & $\begin{array}{l}4.416 * * \\
(1.672)\end{array}$ & $\begin{array}{l}0.299 \dagger \\
(0.165)\end{array}$ \\
\hline $\begin{array}{l}\text { Internationalisation } \\
\text { at home strategy }\end{array}$ & $\begin{array}{l}0.304 \\
(0.254)\end{array}$ & $\begin{array}{l}0.802 \mathrm{E}-4 \\
(0.001)\end{array}$ & $\begin{array}{l}0.639 E-5 \\
(0.0001)\end{array}$ & $\begin{array}{l}0.085 \\
(0.825)\end{array}$ & $\begin{array}{l}0.0006 \\
(0.003)\end{array}$ & $\begin{array}{l}0.494 \mathrm{E}-4 \\
(0.001)\end{array}$ & $\begin{array}{l}0.666 \\
(0.562)\end{array}$ & $\begin{array}{l}-0.005 \\
(0.004)\end{array}$ & $\begin{array}{l}-0.000 \\
(0.023)\end{array}$ & $\begin{array}{l}0.237 \\
(0.295)\end{array}$ & $\begin{array}{l}0.0009 \\
(0.001)\end{array}$ & $\begin{array}{l}0.551 \mathrm{E}-4 \\
(0.0001)\end{array}$ \\
\hline $\begin{array}{l}\text { Host country } \\
\text { demand }\end{array}$ & $\begin{array}{l}11.847^{* *} \\
(3.516)\end{array}$ & $\begin{array}{l}10.297^{* *} \\
(3.171)\end{array}$ & $\begin{array}{l}0.616 * \\
(0.266)\end{array}$ & $\begin{array}{l}\text { 17.299† } \\
(9.149)\end{array}$ & $\begin{array}{l}18.424 * * \\
(7.148)\end{array}$ & $\begin{array}{l}1.120 \\
(0.961)\end{array}$ & $\begin{array}{l}17.135^{*} \\
(6.880)\end{array}$ & $\begin{array}{l}17.110 * * \\
(6.038)\end{array}$ & $\begin{array}{l}\text { 1.177* } \\
(0.579)\end{array}$ & $\begin{array}{l}5.333 \\
(4.445)\end{array}$ & $\begin{array}{l}2.681 \\
(3.376)\end{array}$ & $\begin{array}{l}0.151 \\
(0.744)\end{array}$ \\
\hline AU/NZ dummy & $\begin{array}{l}-0.337 \\
(1.053)\end{array}$ & $\begin{array}{l}-0.441 \\
(0.976)\end{array}$ & $\begin{array}{l}-0.024 \\
(0.071)\end{array}$ & - & - & - & - & - & - & - & - & - \\
\hline UK/IR dummy & $\begin{array}{l}\text { 2.159* } \\
(0.844)\end{array}$ & $\begin{array}{l}1.799 * * \\
(0.779)\end{array}$ & $\begin{array}{l}0.110 \dagger \\
(0.060)\end{array}$ & - & - & - & - & - & - & - & - & - \\
\hline $\mathrm{R}^{2}$ & 0.207 & & & 0.482 & & & 0.301 & & & 0.222 & & \\
\hline $\operatorname{AdjR}^{2}$ & 0.133 & & & 0.235 & & & 0.095 & & & 0.075 & & \\
\hline Log likelihood & & -423.85 & -449.56 & & -92.47 & -101.04 & & -132.55 & -142.56 & & -172.56 & -186.77 \\
\hline $\mathrm{N}$ & 152 & 152 & 152 & 34 & 34 & 34 & 46 & 46 & 46 & 72 & 72 & 72 \\
\hline Max. VIF & 1.585 & & & 1.607 & & & 1.480 & & & 1.521 & & \\
\hline
\end{tabular}

Standard errors in parentheses, all $t$ tests are two-tailed, $\dagger p<.10, * p<.05,{ }^{* *} p<.01$

Table 7.20b: Regression estimates for equation 5d (high regulative institutional distance)

\begin{tabular}{|c|c|c|c|c|c|c|c|c|c|c|c|c|}
\hline & \multicolumn{3}{|c|}{ Complete data set } & \multicolumn{3}{|c|}{ AU/NZ } & \multicolumn{3}{|c|}{$\overline{\text { US/CA }}$} & \multicolumn{3}{|c|}{ 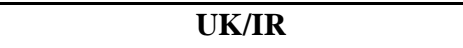 } \\
\hline & OLS & Tobit & $\begin{array}{l}\text { Negative } \\
\text { binomial } \\
\end{array}$ & OLS & Tobit & $\begin{array}{l}\text { Negative } \\
\text { binomial }\end{array}$ & OLS & Tobit & $\begin{array}{l}\text { Negative } \\
\text { binomial } \\
\end{array}$ & OLS & Tobit & $\begin{array}{l}\text { Negative } \\
\text { binomial } \\
\end{array}$ \\
\hline Intercept & $\begin{array}{l}5.525^{*} \\
(2.726)\end{array}$ & $\begin{array}{l}5.857 * \\
(2.408)\end{array}$ & $\begin{array}{l}2.083^{* *} \\
(0.257)\end{array}$ & $\begin{array}{l}20.463 * * \\
(4.237)\end{array}$ & $\begin{array}{l}20.819 * * \\
(3.024)\end{array}$ & $\begin{array}{l}3.046 * * \\
(0.825)\end{array}$ & $\begin{array}{l}-3.034 \\
(4.472)\end{array}$ & $\begin{array}{l}-2.013 \\
(3.932)\end{array}$ & $\begin{array}{l}1.357 * * \\
(0.428)\end{array}$ & $\begin{array}{l}7.593 \dagger \\
(3.851)\end{array}$ & $\begin{array}{l}\text { 7.499* } \\
\text { (3.088) }\end{array}$ & $\begin{array}{l}2.256^{* *} \\
(1.096)\end{array}$ \\
\hline $\begin{array}{l}\text { Ind exp - UG } \\
\text { offshore edu (H8b) }\end{array}$ & $\begin{array}{l}0.099 \\
(0.186)\end{array}$ & $\begin{array}{l}0.085 \\
(0.175)\end{array}$ & $\begin{array}{l}0.004 \\
(0.019)\end{array}$ & $\begin{array}{l}-0.186 \\
(0.270)\end{array}$ & $\begin{array}{l}-0.204 \\
(0.222)\end{array}$ & $\begin{array}{l}-0.011 \\
(0.070)\end{array}$ & $\begin{array}{l}0.097 \\
(0.298)\end{array}$ & $\begin{array}{l}0.098 \\
(0.275)\end{array}$ & $\begin{array}{l}0.005 \\
(0.033)\end{array}$ & $\begin{array}{l}0.132 \\
(0.315)\end{array}$ & $\begin{array}{l}0.167 \\
(0.250)\end{array}$ & $\begin{array}{l}0.010 \\
(0.062)\end{array}$ \\
\hline
\end{tabular}




\begin{tabular}{|c|c|c|c|c|c|c|c|c|c|c|c|c|}
\hline $\begin{array}{l}\text { Ind exp - PG } \\
\text { offshore edu (H8b) }\end{array}$ & $\begin{array}{l}0.209 \\
(0.207)\end{array}$ & $\begin{array}{l}0.213 \\
(0.195)\end{array}$ & $\begin{array}{l}0.012 \\
(0.020)\end{array}$ & $\begin{array}{l}0.234 \\
(0.289)\end{array}$ & $\begin{array}{l}0.248 \\
(0.244)\end{array}$ & $\begin{array}{l}0.013 \\
(0.061)\end{array}$ & $\begin{array}{l}-0.034 \\
(0.319)\end{array}$ & $\begin{array}{l}-0.037 \\
(0.294)\end{array}$ & $\begin{array}{l}-0.0009 \\
(0.029)\end{array}$ & $\begin{array}{l}0.392 \\
(0.431)\end{array}$ & $\begin{array}{l}0.399 \\
(0.345)\end{array}$ & $\begin{array}{l}0.023 \\
(0.139)\end{array}$ \\
\hline $\begin{array}{l}\text { Ind exp - Intl } \\
\text { recruitment (Factor) } \\
(\text { H8b) }\end{array}$ & $\begin{array}{l}0.351 \\
(0.228)\end{array}$ & $\begin{array}{l}0.318 \\
(0.212)\end{array}$ & $\begin{array}{l}0.019 \\
(0.022)\end{array}$ & $\begin{array}{l}0.012 \\
(0.324)\end{array}$ & $\begin{array}{l}-0.031 \\
(0.251)\end{array}$ & $\begin{array}{l}-0.001 \\
(0.068)\end{array}$ & $\begin{array}{l}0.781 * \\
(0.379)\end{array}$ & $\begin{array}{l}0.786^{*} \\
(0.350)\end{array}$ & $\begin{array}{l}0.049 \\
(0.043)\end{array}$ & $\begin{array}{l}0.082 \\
(0.475)\end{array}$ & $\begin{array}{l}0.096 \\
(0.380)\end{array}$ & $\begin{array}{l}0.005 \\
(0.098)\end{array}$ \\
\hline $\begin{array}{l}\text { Ind exp - UG } \\
\text { marketing }(H 8 b)\end{array}$ & $\begin{array}{l}0.040 \\
(0.178)\end{array}$ & $\begin{array}{l}0.050 \\
(0.168)\end{array}$ & $\begin{array}{l}0.003 \\
(0.019)\end{array}$ & $\begin{array}{l}-0.090 \\
(0.418)\end{array}$ & $\begin{array}{l}-0.083 \\
(0.355)\end{array}$ & $\begin{array}{l}-0.004 \\
(0.101)\end{array}$ & $\begin{array}{l}0.356 \\
(0.245)\end{array}$ & $\begin{array}{l}0.328 \\
(0.223)\end{array}$ & $\begin{array}{l}0.020 \\
(0.028)\end{array}$ & $\begin{array}{l}0.145 \\
(0.321)\end{array}$ & $\begin{array}{l}0.097 \\
(0.253)\end{array}$ & $\begin{array}{l}0.005 \\
(0.106)\end{array}$ \\
\hline $\begin{array}{l}\text { Ind exp - PG } \\
\text { marketing (H8b) }\end{array}$ & $\begin{array}{l}0.133 \\
(0.175)\end{array}$ & $\begin{array}{l}0.121 \\
(0.164)\end{array}$ & $\begin{array}{l}0.006 \\
(0.017)\end{array}$ & $\begin{array}{l}-0.254 \\
(0.276)\end{array}$ & $\begin{array}{l}-0.274 \\
(0.216)\end{array}$ & $\begin{array}{l}-0.015 \\
(0.054)\end{array}$ & $\begin{array}{l}0.319 \\
(0.247)\end{array}$ & $\begin{array}{l}0.323 \\
(0.227)\end{array}$ & $\begin{array}{l}0.020 \\
(0.027)\end{array}$ & $\begin{array}{l}0.360 \\
(0.419)\end{array}$ & $\begin{array}{l}0.271 \\
(0.323)\end{array}$ & $\begin{array}{l}0.014 \\
(0.132)\end{array}$ \\
\hline Age & $\begin{array}{l}0.002 \\
(0.006)\end{array}$ & $\begin{array}{l}0.001 \\
(0.005)\end{array}$ & $\begin{array}{l}0.0001 \\
(0.0005)\end{array}$ & $\begin{array}{l}-0.004 \\
(0.010)\end{array}$ & $\begin{array}{l}-0.003 \\
(0.007)\end{array}$ & $\begin{array}{l}-0.0001 \\
(0.002)\end{array}$ & $\begin{array}{l}0.000 \\
(0.008)\end{array}$ & $\begin{array}{l}-0.001 \\
(0.007)\end{array}$ & $\begin{array}{l}-0.0001 \\
(0.0007)\end{array}$ & $\begin{array}{l}0.019 \\
(0.011)\end{array}$ & $\begin{array}{l}\text { 0.018* } \\
(0.009)\end{array}$ & $\begin{array}{l}0.001 \\
(0.002)\end{array}$ \\
\hline Size (Staff) & $\begin{array}{l}0.000 \\
(0.000)\end{array}$ & $\begin{array}{l}0.736 \mathrm{E}-4 \\
(0.0002)\end{array}$ & $\begin{array}{l}0.498 \mathrm{E}-5 \\
(0.246 \mathrm{E}-4)\end{array}$ & $\begin{array}{l}0.000 \\
(0.000)\end{array}$ & $\begin{array}{l}-0.000 \\
(0.000)\end{array}$ & $\begin{array}{l}-0.118 \mathrm{E}-4 \\
(0.829 \mathrm{E}-4)\end{array}$ & $\begin{array}{l}0.000 \\
(0.000)\end{array}$ & $\begin{array}{l}-0.0001 \\
(0.0003)\end{array}$ & $\begin{array}{l}-0.114 \mathrm{E}-4 \\
(0.367 \mathrm{E}-4)\end{array}$ & $\begin{array}{l}-0.000 \\
(0.000)\end{array}$ & $\begin{array}{l}-0.234 \\
(0.0003)\end{array}$ & $\begin{array}{l}-0.407 \mathrm{E}-6 \\
(0.0001)\end{array}$ \\
\hline Offshore policy & $\begin{array}{l}5.804 * * \\
(0.968)\end{array}$ & $\begin{array}{l}5.811^{* *} \\
(0.914)\end{array}$ & $\begin{array}{l}0.428 * * \\
(0.060)\end{array}$ & $\begin{array}{l}-0.382 \\
(1.686)\end{array}$ & $\begin{array}{l}-0.399 \\
(1.417)\end{array}$ & $\begin{array}{l}-0.021 \\
(0.394)\end{array}$ & $\begin{array}{l}\text { 10.109** } \\
(1.384)\end{array}$ & $\begin{array}{l}10.022 * * \\
(1.272)\end{array}$ & $\begin{array}{l}0.909 * * \\
(0.091)\end{array}$ & $\begin{array}{l}4.209 \dagger \\
(2.147)\end{array}$ & $\begin{array}{l}3.585^{*} \\
(1.599)\end{array}$ & $\begin{array}{l}0.225 \\
(0.780)\end{array}$ \\
\hline $\begin{array}{l}\text { Internationalisation } \\
\text { at home strategy }\end{array}$ & $\begin{array}{l}0.037 \\
(0.224)\end{array}$ & $\begin{array}{l}-0.001 \\
(0.001)\end{array}$ & $\begin{array}{l}-0.820 \mathrm{E}-4 \\
(0.0003)\end{array}$ & $\begin{array}{l}-0.004 \\
(0.354)\end{array}$ & $\begin{array}{l}-0.001 \\
(0.001)\end{array}$ & $\begin{array}{l}-0.615 \mathrm{E}-4 \\
(0.0008)\end{array}$ & $\begin{array}{l}0.257 \\
(0.339)\end{array}$ & $\begin{array}{l}0.001 \\
(0.003)\end{array}$ & $\begin{array}{l}0.745 \mathrm{E}-4 \\
(0.025)\end{array}$ & $\begin{array}{l}-0.338 \\
(0.429)\end{array}$ & $\begin{array}{l}-0.003 \\
(0.002)\end{array}$ & $\begin{array}{l}-0.0001 \\
(0.071)\end{array}$ \\
\hline $\begin{array}{l}\text { Host country } \\
\text { demand }\end{array}$ & $\begin{array}{l}0.402 \\
(3.245)\end{array}$ & $\begin{array}{l}0.914 \\
(3.024)\end{array}$ & $\begin{array}{l}0.048 \\
(0.337)\end{array}$ & $\begin{array}{l}2.453 \\
(5.100)\end{array}$ & $\begin{array}{l}3.235 \\
(4.147)\end{array}$ & $\begin{array}{l}1.179 \\
(1.278)\end{array}$ & $\begin{array}{l}-3.131 \\
(4.733)\end{array}$ & $\begin{array}{l}-3.481 \\
(4.346)\end{array}$ & $\begin{array}{l}-0.234 \\
(0.578)\end{array}$ & $\begin{array}{l}2.709 \\
(5.844)\end{array}$ & $\begin{array}{l}3.604 \\
(4.598)\end{array}$ & $\begin{array}{l}0.206 \\
(1.155)\end{array}$ \\
\hline AU/NZ dummy & $\begin{array}{l}1.820 * \\
(0.781)\end{array}$ & $\begin{array}{l}\text { 1.826* } \\
(0.721)\end{array}$ & $\begin{array}{l}0.109 \\
(0.080)\end{array}$ & - & - & - & - & - & - & - & - & - \\
\hline UK/IR dummy & $\begin{array}{l}0.954 \\
(0.779)\end{array}$ & $\begin{array}{l}0.981 \\
(0.731)\end{array}$ & $\begin{array}{l}0.059 \\
(0.083)\end{array}$ & - & - & - & - & - & - & - & - & - \\
\hline $\mathrm{R}^{2}$ & 0.276 & & & 0.102 & & & 0.498 & & & 0.368 & & \\
\hline $\operatorname{AdjR}^{2}$ & 0.213 & & & -0.170 & & & 0.421 & & & 0.052 & & \\
\hline Log likelihood & & -400.32 & -422.47 & & -98.47 & -113.93 & & -201.20 & -212.47 & & -68.63 & -78.54 \\
\hline $\mathrm{N}$ & 155 & 155 & 155 & 46 & 46 & 46 & 77 & 77 & 77 & 32 & 32 & 32 \\
\hline Max. VIF & 1.663 & & & 1.631 & & & 1.262 & & & 1.883 & & \\
\hline
\end{tabular}


Table 7.21a: Regression estimates for equation 6c (low regulative institutional distance)

\begin{tabular}{|c|c|c|c|c|c|c|c|c|c|c|c|c|}
\hline & \multicolumn{3}{|c|}{ Complete data set } & \multicolumn{3}{|c|}{ AU/NZ } & \multicolumn{3}{|c|}{ US/CA } & \multicolumn{3}{|c|}{ UK/IR } \\
\hline & OLS & Tobit & $\begin{array}{l}\text { Negative } \\
\text { binomial }\end{array}$ & OLS & Tobit & $\begin{array}{l}\text { Negative } \\
\text { binomial } \\
\end{array}$ & OLS & Tobit & $\begin{array}{l}\text { Negative } \\
\text { binomial } \\
\end{array}$ & OLS & Tobit & $\begin{array}{l}\text { Negative } \\
\text { binomial }\end{array}$ \\
\hline Intercept & $\begin{array}{l}19.574 * * \\
(1.668)\end{array}$ & $\begin{array}{l}20.440 * * \\
(1.389)\end{array}$ & $\begin{array}{l}3.121 * * \\
(0.143)\end{array}$ & $\begin{array}{l}18.787 * * \\
(4.382)\end{array}$ & $\begin{array}{l}19.652 * * \\
(3.593)\end{array}$ & $\begin{array}{l}3.136 * * \\
(0.368)\end{array}$ & $\begin{array}{l}20.874 * * \\
(3.300)\end{array}$ & $\begin{array}{l}22.137 * * \\
(2.456)\end{array}$ & $\begin{array}{l}3.247 * * \\
(0.290)\end{array}$ & $\begin{array}{l}17.906 * * \\
(2.063)\end{array}$ & $\begin{array}{l}18.920 * * \\
(1.764)\end{array}$ & $\begin{array}{l}2.954 * * \\
(0.364)\end{array}$ \\
\hline $\begin{array}{l}\text { Know-how (Factor) } \\
\text { (H8c) }\end{array}$ & $\begin{array}{l}-1.720^{* *} \\
(0.144)\end{array}$ & $\begin{array}{l}-1.753 * * \\
(0.136)\end{array}$ & $\begin{array}{l}-0.139 * * \\
(0.013)\end{array}$ & $\begin{array}{l}-1.829 * * \\
(0.363)\end{array}$ & $\begin{array}{l}-1.882^{* *} \\
(0.323)\end{array}$ & $\begin{array}{l}-0.158^{* *} \\
(0.028)\end{array}$ & $\begin{array}{l}-1.811^{* *} \\
(0.259)\end{array}$ & $\begin{array}{l}-1.813^{* *} \\
(0.236)\end{array}$ & $\begin{array}{l}-0.149 * * \\
(0.024)\end{array}$ & $\begin{array}{l}-1.415^{* *} \\
(0.252)\end{array}$ & $\begin{array}{l}-1.441^{* *} \\
(0.233)\end{array}$ & $\begin{array}{l}-0.102^{*} \\
(0.051)\end{array}$ \\
\hline Age & $\begin{array}{l}0.005 \\
(0.005)\end{array}$ & $\begin{array}{l}0.004 \\
(0.004)\end{array}$ & $\begin{array}{l}0.0002 \\
(0.0005)\end{array}$ & $\begin{array}{l}0.004 \\
(0.015)\end{array}$ & $\begin{array}{l}0.005 \\
(0.012)\end{array}$ & $\begin{array}{l}0.0004 \\
(0.001)\end{array}$ & $\begin{array}{l}0.005 \\
(0.011)\end{array}$ & $\begin{array}{l}0.004 \\
(0.009)\end{array}$ & $\begin{array}{l}0.0003 \\
(0.001)\end{array}$ & $\begin{array}{l}0.003 \\
(0.005)\end{array}$ & $\begin{array}{l}0.0005 \\
(0.004)\end{array}$ & $\begin{array}{l}0.336 \mathrm{E}-4 \\
(0.0009)\end{array}$ \\
\hline Size (Staff) & $\begin{array}{l}0.000 \\
(0.000)\end{array}$ & $\begin{array}{l}0.0001 \\
(0.0001)\end{array}$ & $\begin{array}{l}0.810 \mathrm{E}-5 \\
(0.248 \mathrm{E}-4)\end{array}$ & $\begin{array}{l}0.000 \\
(0.001)\end{array}$ & $\begin{array}{l}0.0003 \\
(0.0004)\end{array}$ & $\begin{array}{l}0.135 \mathrm{E}-4 \\
(0.612 \mathrm{E}-4)\end{array}$ & $\begin{array}{l}0.001 \\
(0.000)\end{array}$ & $\begin{array}{l}0.0004 \\
(0.0003)\end{array}$ & $\begin{array}{l}0.290 \mathrm{E}-4 \\
(0.481 \mathrm{E}-4)\end{array}$ & $\begin{array}{l}-0.000 \\
(0.000)\end{array}$ & $\begin{array}{l}-0.344 \mathrm{E}-4 \\
(0.0001)\end{array}$ & $\begin{array}{l}-0.659 \mathrm{E}-6 \\
(0.415 \mathrm{E}-4)\end{array}$ \\
\hline Offshore policy & $\begin{array}{l}0.237 \\
(1.045)\end{array}$ & $\begin{array}{l}0.114 \\
(0.996)\end{array}$ & $\begin{array}{l}0.411 \mathrm{E}-4 \\
(0.097)\end{array}$ & $\begin{array}{l}0.009 \\
(2.637)\end{array}$ & $\begin{array}{l}-0.308 \\
(2.344)\end{array}$ & $\begin{array}{l}-0.045 \\
(0.194)\end{array}$ & $\begin{array}{l}-0.975 \\
(2.171)\end{array}$ & $\begin{array}{l}-1.315 \\
(1.927)\end{array}$ & $\begin{array}{l}-0.093 \\
(0.219)\end{array}$ & $\begin{array}{l}2.021 \\
(1.489)\end{array}$ & $\begin{array}{l}1.955 \\
(1.394)\end{array}$ & $\begin{array}{l}0.140 \\
(0.224)\end{array}$ \\
\hline $\begin{array}{l}\text { Internationalisation } \\
\text { at home strategy }\end{array}$ & $\begin{array}{l}0.122 \\
(0.173)\end{array}$ & $\begin{array}{l}-0.0004 \\
(0.0008)\end{array}$ & $\begin{array}{l}-0.416 \mathrm{E}-4 \\
(0.0001)\end{array}$ & $\begin{array}{l}0.199 \\
(0.557)\end{array}$ & $\begin{array}{l}-0.0001 \\
(0.002)\end{array}$ & $\begin{array}{l}-0.129 \mathrm{E}-5 \\
(0.022)\end{array}$ & $\begin{array}{l}0.229 \\
(0.351)\end{array}$ & $\begin{array}{l}-0.003 \\
(0.003)\end{array}$ & $\begin{array}{l}-0.0002 \\
(0.025)\end{array}$ & $\begin{array}{l}0.173 \\
(0.228)\end{array}$ & $\begin{array}{l}0.0002 \\
(0.0008)\end{array}$ & $\begin{array}{l}0.713 \mathrm{E}-5 \\
(0.0001)\end{array}$ \\
\hline $\begin{array}{l}\text { Host country } \\
\text { demand }\end{array}$ & $\begin{array}{l}3.934 \\
(2.530)\end{array}$ & $\begin{array}{l}3.252 \\
(2.289)\end{array}$ & $\begin{array}{l}0.207 \\
(0.297)\end{array}$ & $\begin{array}{l}11.436 \dagger \\
(6.332)\end{array}$ & $\begin{array}{l}12.303 \dagger \\
(5.338)\end{array}$ & $\begin{array}{l}0.768 \\
(0.857)\end{array}$ & $\begin{array}{l}-2.914 \\
(5.149)\end{array}$ & $\begin{array}{l}-2.788 \\
(4.702)\end{array}$ & $\begin{array}{l}-0.191 \\
(0.561)\end{array}$ & $\begin{array}{l}5.275 \\
(3.454)\end{array}$ & $\begin{array}{l}3.021 \\
(2.769)\end{array}$ & $\begin{array}{l}0.188 \\
(0.565)\end{array}$ \\
\hline AU/NZ dummy & $\begin{array}{l}0.392 \\
(0.726)\end{array}$ & $\begin{array}{l}0.270 \\
(0.682)\end{array}$ & $\begin{array}{l}0.027 \\
(0.086)\end{array}$ & - & - & - & - & - & - & - & - & - \\
\hline UK/IR dummy & $\begin{array}{l}0.445 \\
(0.604)\end{array}$ & $\begin{array}{l}0.233 \\
(0.559)\end{array}$ & $\begin{array}{l}0.016 \\
(0.072)\end{array}$ & - & - & - & - & - & - & - & - & - \\
\hline $\mathrm{R}^{2}$ & 0.601 & & & 0.674 & & & 0.640 & & & 0.455 & & \\
\hline $\operatorname{Adj}^{2}$ & 0.577 & & & 0.596 & & & 0.584 & & & 0.398 & & \\
\hline Log likelihood & & -370.82 & -404.89 & & -84.52 & -93.24 & & -116.57 & -126.14 & & -159.49 & -181.60 \\
\hline $\mathrm{N}$ & 152 & 152 & 152 & 34 & 34 & 34 & 46 & 46 & 46 & 72 & 72 & 72 \\
\hline Max. VIF & 1.547 & & & 1.640 & & & 1.336 & & & 1.400 & & \\
\hline
\end{tabular}

Standard errors in parentheses, all $t$ tests are two-tailed, $\uparrow p<.10, * p<.05, * * p<.01$ 
Table 7.21b: Regression estimates for equation 6d (high regulative institutional distance)

\begin{tabular}{|c|c|c|c|c|c|c|c|c|c|c|c|c|}
\hline & \multicolumn{3}{|c|}{ Complete data set } & \multicolumn{3}{|c|}{ AU/NZ } & \multicolumn{3}{|c|}{ US/CA } & \multicolumn{3}{|c|}{ UK/IR } \\
\hline & OLS & Tobit & $\begin{array}{l}\text { Negative } \\
\text { binomial }\end{array}$ & OLS & Tobit & $\begin{array}{l}\text { Negative } \\
\text { binomial }\end{array}$ & OLS & Tobit & $\begin{array}{l}\text { Negative } \\
\text { binomial }\end{array}$ & OLS & Tobit & $\begin{array}{l}\text { Negative } \\
\text { binomial }\end{array}$ \\
\hline Intercept & $\begin{array}{l}17.790 * * \\
(1.327)\end{array}$ & $\begin{array}{l}17.490 * * \\
(1.160)\end{array}$ & $\begin{array}{l}2.851 * * \\
(0.148)\end{array}$ & $\begin{array}{l}19.423 * * \\
(1.844)\end{array}$ & $\begin{array}{l}19.595 * * \\
(1.535)\end{array}$ & $\begin{array}{l}2.982 * * \\
(0.304)\end{array}$ & $\begin{array}{l}17.151^{* *} \\
(2.004)\end{array}$ & $\begin{array}{l}16.934 * * \\
(1.615)\end{array}$ & $\begin{array}{l}2.651 * * \\
(0.283)\end{array}$ & $\begin{array}{l}14.771 * * \\
(2.287)\end{array}$ & $\begin{array}{l}14.363 * * \\
(1.940)\end{array}$ & $\begin{array}{l}2.677 * * \\
(0.583)\end{array}$ \\
\hline $\begin{array}{l}\text { Know-how (Factor) } \\
\text { (H8c) }\end{array}$ & $\begin{array}{l}-1.374 * * \\
(0.146)\end{array}$ & $\begin{array}{l}-1.355^{* *} \\
(0.140)\end{array}$ & $\begin{array}{l}-0.102 * * \\
(0.020)\end{array}$ & $\begin{array}{l}-0.435 \\
(0.458)\end{array}$ & $\begin{array}{l}-0.386 \\
(0.414)\end{array}$ & $\begin{array}{l}-0.023 \\
(0.091)\end{array}$ & $\begin{array}{l}-1.418 * * \\
(0.189)\end{array}$ & $\begin{array}{l}-1.412 * * \\
(0.177)\end{array}$ & $\begin{array}{l}-0.107 * * \\
(0.026)\end{array}$ & $\begin{array}{l}-0.855^{*} \\
(0.384)\end{array}$ & $\begin{array}{l}-0.834^{*} \\
(0.335)\end{array}$ & $\begin{array}{l}-0.0567 \\
(0.080)\end{array}$ \\
\hline Age & $\begin{array}{l}0.001 \\
(0.004)\end{array}$ & $\begin{array}{l}0.001 \\
(0.004)\end{array}$ & $\begin{array}{l}0.531 \mathrm{E}-4 \\
(0.0006)\end{array}$ & $\begin{array}{l}-0.004 \\
(0.009)\end{array}$ & $\begin{array}{l}-0.005 \\
(0.008)\end{array}$ & $\begin{array}{l}-0.0002 \\
(0.001)\end{array}$ & $\begin{array}{l}0.002 \\
(0.006)\end{array}$ & $\begin{array}{l}0.001 \\
(0.005)\end{array}$ & $\begin{array}{l}0.913 E-4 \\
(0.0008)\end{array}$ & $\begin{array}{l}\mathbf{0 . 0 1 7} \dagger \\
(0.009)\end{array}$ & $\begin{array}{l}0.017 * \\
(0.008)\end{array}$ & $\begin{array}{l}0.001 \\
(0.001)\end{array}$ \\
\hline Size (Staff) & $\begin{array}{l}0.000 \\
(0.000)\end{array}$ & $\begin{array}{l}0.0001 \\
(0.0001)\end{array}$ & $\begin{array}{l}0.953 \mathrm{E}-5 \\
(0.263 \mathrm{E}-4)\end{array}$ & $\begin{array}{l}-0.000 \\
(0.000)\end{array}$ & $\begin{array}{l}-0.773 \mathrm{E}-4 \\
(0.0003)\end{array}$ & $\begin{array}{l}-0.448 \mathrm{E}-5 \\
(0.752 \mathrm{E}-4)\end{array}$ & $\begin{array}{l}0.000 \\
(0.000)\end{array}$ & $\begin{array}{l}-0.0002 \\
(0.0002)\end{array}$ & $\begin{array}{l}-0.138 \mathrm{E}-4 \\
(0.394 \mathrm{E}-4)\end{array}$ & $\begin{array}{l}0.000 \\
(0.000)\end{array}$ & $\begin{array}{l}0.859 \mathrm{E}-4 \\
(0.0003)\end{array}$ & $\begin{array}{l}0.618 E-5 \\
(0.985 E-4)\end{array}$ \\
\hline Offshore policy & $\begin{array}{l}2.817 * * \\
(0.821)\end{array}$ & $\begin{array}{l}2.828 * * \\
(0.792)\end{array}$ & $\begin{array}{l}0.227 * \\
(0.093)\end{array}$ & $\begin{array}{l}-0.890 \\
(1.487)\end{array}$ & $\begin{array}{l}-0.885 \\
(1.345)\end{array}$ & $\begin{array}{l}-0.048 \\
(0.244)\end{array}$ & $\begin{array}{l}4.693 * * \\
(1.284)\end{array}$ & $\begin{array}{l}4.729 * * \\
(1.204)\end{array}$ & $\begin{array}{l}0.519 * * \\
(0.145)\end{array}$ & $\begin{array}{l}3.285^{*} \\
(1.592)\end{array}$ & $\begin{array}{l}2.879 * \\
(1.303)\end{array}$ & $\begin{array}{l}0.181 \\
(0.474)\end{array}$ \\
\hline $\begin{array}{l}\text { Internationalisation } \\
\text { at home strategy }\end{array}$ & $\begin{array}{l}-0.044 \\
(0.175)\end{array}$ & $\begin{array}{l}-0.001 \\
(0.001)\end{array}$ & $\begin{array}{l}-0.0001 \\
(0.0002)\end{array}$ & $\begin{array}{l}0.131 \\
(0.298)\end{array}$ & $\begin{array}{l}-0.001 \\
(0.001)\end{array}$ & $\begin{array}{l}-0.886 \mathrm{E}-4 \\
(0.0005)\end{array}$ & $\begin{array}{l}-0.051 \\
(0.258)\end{array}$ & $\begin{array}{l}0.001 \\
(0.0026)\end{array}$ & $\begin{array}{l}0.0001 \\
(0.038)\end{array}$ & $\begin{array}{l}-0.259 \\
(0.354)\end{array}$ & $\begin{array}{l}-0.003 \\
(0.002)\end{array}$ & $\begin{array}{l}-0.0001 \\
(0.046)\end{array}$ \\
\hline $\begin{array}{l}\text { Host country } \\
\text { demand }\end{array}$ & $\begin{array}{l}-0.852 \\
(2.535)\end{array}$ & $\begin{array}{l}-0.055 \\
(2.408)\end{array}$ & $\begin{array}{l}0.012 \\
(0.329)\end{array}$ & $\begin{array}{l}2.744 \\
(4.764)\end{array}$ & $\begin{array}{l}4.027 \\
(4.178)\end{array}$ & $\begin{array}{l}0.226 \\
(1.005)\end{array}$ & $\begin{array}{l}-5.882 \\
(3.532)\end{array}$ & $\begin{array}{l}-5.804 \dagger \\
(3.325)\end{array}$ & $\begin{array}{l}-0.358 \\
(0.502)\end{array}$ & $\begin{array}{l}4.125 \\
(4.795)\end{array}$ & $\begin{array}{l}4.929 \\
(4.082)\end{array}$ & $\begin{array}{l}0.305 \\
(0.986)\end{array}$ \\
\hline AU/NZ dummy & $\begin{array}{l}0.432 \\
(0.615)\end{array}$ & $\begin{array}{l}0.520 \\
(0.578)\end{array}$ & $\begin{array}{l}0.031 \\
(0.084)\end{array}$ & - & - & - & - & - & - & - & - & - \\
\hline UK/IR dummy & $\begin{array}{l}-0.112 \\
(0.613)\end{array}$ & $\begin{array}{l}-0.384 \\
(0.587)\end{array}$ & $\begin{array}{l}0.003 \\
(0.091)\end{array}$ & - & - & - & - & - & - & - & - & - \\
\hline $\mathrm{R}^{2}$ & 0.541 & & & 0.073 & & & 0.691 & & & 0.403 & & \\
\hline $\operatorname{Adj}^{2}$ & 0.515 & & & -0.078 & & & 0.665 & & & 0.254 & & \\
\hline Log likelihood & & -366.11 & -401.41 & & -99.64 & -114.20 & & -182.19 & -200.24 & & -67.57 & -78.19 \\
\hline $\mathrm{N}$ & 155 & 155 & 155 & 46 & 46 & 46 & 77 & 77 & 77 & 32 & 32 & 32 \\
\hline Max. VIF & 1.674 & & & 1.490 & & & 1.591 & & & 1.419 & & \\
\hline
\end{tabular}

Standard errors in parentheses, all $t$ tests are two-tailed, $\uparrow p<.10, * p<.05, * * p<.01$ 
Table 7.22a: Regression estimates for equation 7c (low regulative institutional distance)

\begin{tabular}{|c|c|c|c|c|c|c|c|c|c|c|c|c|}
\hline & \multicolumn{3}{|c|}{ Complete data set } & \multicolumn{3}{|c|}{ AU/NZ } & \multicolumn{3}{|c|}{ US/CA } & \multicolumn{3}{|c|}{ UK/IR } \\
\hline & OLS & Tobit & $\begin{array}{l}\text { Negative } \\
\text { binomial }\end{array}$ & OLS & Tobit & $\begin{array}{l}\text { Negative } \\
\text { binomial }\end{array}$ & OLS & Tobit & $\begin{array}{l}\text { Negative } \\
\text { binomial }\end{array}$ & OLS & Tobit & $\begin{array}{l}\text { Negative } \\
\text { binomia }\end{array}$ \\
\hline Intercept & $\begin{array}{l}22.673 * * \\
(2.000)\end{array}$ & $\begin{array}{l}23.654 * * \\
(1.726)\end{array}$ & $\begin{array}{l}3.339 * * \\
(0.173)\end{array}$ & $\begin{array}{l}\text { 20.181** } \\
(5.118)\end{array}$ & $\begin{array}{l}20.795 * * \\
(4.166)\end{array}$ & $\begin{array}{l}\text { 3.084** } \\
(0.557)\end{array}$ & $\begin{array}{l}23.742 * * \\
(6.328)\end{array}$ & $\begin{array}{l}25.384 * * \\
(2.898)\end{array}$ & $\begin{array}{l}3.488 * * \\
(0.291)\end{array}$ & $\begin{array}{l}21.739 * * \\
(3.018)\end{array}$ & $\begin{array}{l}22.096 * * \\
(2.444)\end{array}$ & $\begin{array}{l}3.144 * * \\
(0.628)\end{array}$ \\
\hline $\begin{array}{l}\text { Org culture (Factor) } \\
-(H 8 d)\end{array}$ & $\begin{array}{l}-2.165 * * \\
(0.208)\end{array}$ & $\begin{array}{l}-2.186 * * \\
(0.199)\end{array}$ & $\begin{array}{l}-0.164 * * \\
(0.020)\end{array}$ & $\begin{array}{l}-2.119 * * \\
(0.486)\end{array}$ & $\begin{array}{l}-2.187 * * \\
(0.430)\end{array}$ & $\begin{array}{l}-0.167^{* *} \\
(0.050)\end{array}$ & $\begin{array}{l}-2.280 * * \\
(0.374)\end{array}$ & $\begin{array}{l}-2.275^{* *} \\
(0.343)\end{array}$ & $\begin{array}{l}-0.179 * * \\
(0.033)\end{array}$ & - & - & - \\
\hline $\begin{array}{l}\text { Org culture - open } \\
\text { discussion }(H 8 d)\end{array}$ & ( & ( & a & ( & ( & a & ( & a & ( & $\begin{array}{l}-0.377 \\
(0.250)\end{array}$ & $\begin{array}{l}-0.392 \dagger \\
(0.222)\end{array}$ & $\begin{array}{l}-0.026 \\
(0.039)\end{array}$ \\
\hline $\begin{array}{l}\text { Org culture }- \text { no } \\
\text { status distinction } \\
(\text { H8d) }\end{array}$ & - & - & - & - & - & - & - & - & - & $\begin{array}{l}-0.550^{*} \\
(0.256)\end{array}$ & $\begin{array}{l}-0.500 * \\
(0.220)\end{array}$ & $\begin{array}{l}-0.032 \\
(0.041)\end{array}$ \\
\hline $\begin{array}{l}\text { Org culture - } \\
\text { experimentation } \\
(\mathrm{H} 8 \mathrm{~d})\end{array}$ & - & - & - & - & - & - & - & - & - & $\begin{array}{l}-0.160 \\
(0.262)\end{array}$ & $\begin{array}{l}-0.164 \\
(0.212)\end{array}$ & $\begin{array}{l}-0.012 \\
(0.042)\end{array}$ \\
\hline $\begin{array}{l}\text { Org culture - } \\
\text { offshore } \\
\text { development (H8d) }\end{array}$ & - & - & - & - & - & - & - & - & - & $\begin{array}{l}-0.518 \dagger \\
(0.286)\end{array}$ & $\begin{array}{l}-0.558 * \\
(0.240)\end{array}$ & $\begin{array}{l}-0.035 \\
(0.071)\end{array}$ \\
\hline $\begin{array}{l}\text { Org culture - } \\
\text { offshore } \\
\text { responsiveness } \\
(\text { H8d) }\end{array}$ & - & - & - & - & - & - & - & - & - & $\begin{array}{l}-0.289 \\
(0.265)\end{array}$ & $\begin{array}{l}-0.197 \\
(0.218)\end{array}$ & $\begin{array}{l}-0.014 \\
(0.045)\end{array}$ \\
\hline Age & $\begin{array}{l}0.004 \\
(0.005)\end{array}$ & $\begin{array}{l}0.002 \\
(0.004)\end{array}$ & $\begin{array}{l}0.0001 \\
(0.0005)\end{array}$ & $\begin{array}{l}0.005 \\
(0.016)\end{array}$ & $\begin{array}{l}0.006 \\
(0.013)\end{array}$ & $\begin{array}{l}0.0005 \\
(0.001)\end{array}$ & $\begin{array}{l}0.008 \\
(0.012)\end{array}$ & $\begin{array}{l}0.007 \\
(0.010)\end{array}$ & $\begin{array}{l}0.0005 \\
(0.0009)\end{array}$ & $\begin{array}{l}-0.001 \\
(0.005)\end{array}$ & $\begin{array}{l}-0.003 \\
(0.004)\end{array}$ & $\begin{array}{l}-0.0002 \\
(0.001)\end{array}$ \\
\hline Size (Staff) & $\begin{array}{l}0.000 \\
(0.000)\end{array}$ & $\begin{array}{l}0.743 \mathrm{E}-4 \\
(0.0001)\end{array}$ & $\begin{array}{l}0.289 \mathrm{E}-5 \\
(0.243 \mathrm{E}-4)\end{array}$ & $\begin{array}{l}0.000 \\
(0.001)\end{array}$ & $\begin{array}{l}0.0002 \\
(0.0004)\end{array}$ & $\begin{array}{l}0.140 \mathrm{E}-4 \\
(0.581 \mathrm{E}-4)\end{array}$ & $\begin{array}{l}0.001 \\
(0.000)\end{array}$ & $\begin{array}{l}0.0004 \\
(0.003)\end{array}$ & $\begin{array}{l}0.226 E-4 \\
(0.499 E-4)\end{array}$ & $\begin{array}{l}0.000 \\
(0.000)\end{array}$ & $\begin{array}{l}-0.982 \\
(0.0001)\end{array}$ & $\begin{array}{l}-0.492 \mathrm{E}-5 \\
(0.461 \mathrm{E}-4)\end{array}$ \\
\hline Offshore policy & $\begin{array}{l}0.614 \\
(1.113)\end{array}$ & $\begin{array}{l}0.520 \\
(1.072)\end{array}$ & $\begin{array}{l}0.039 \\
(0.091)\end{array}$ & $\begin{array}{l}1.418 \\
(2.714)\end{array}$ & $\begin{array}{l}1.123 \\
(2.405)\end{array}$ & $\begin{array}{l}0.146 \\
(0.220)\end{array}$ & $\begin{array}{l}-1.136 \\
(2.338)\end{array}$ & $\begin{array}{l}-1.599 \\
(2.078)\end{array}$ & $\begin{array}{l}-0.105 \\
(0.209)\end{array}$ & $\begin{array}{l}2.483 \\
(1.630)\end{array}$ & $\begin{array}{l}2.549 \dagger \\
(1.481)\end{array}$ & $\begin{array}{l}0.186 \\
(0.264)\end{array}$ \\
\hline $\begin{array}{l}\text { Internationalisation } \\
\text { at home strategy }\end{array}$ & $\begin{array}{l}0.157 \\
(0.185)\end{array}$ & $\begin{array}{l}0.001 \\
(0.0009)\end{array}$ & $\begin{array}{l}0.800 \mathrm{E}-4 \\
(0.0001)\end{array}$ & $\begin{array}{l}0.063 \\
(0.598)\end{array}$ & $\begin{array}{l}0.0004 \\
(0.002)\end{array}$ & $\begin{array}{l}0.466 \mathrm{E}-4 \\
(0.003)\end{array}$ & $\begin{array}{l}0.305 \\
(0.378)\end{array}$ & $\begin{array}{l}-0.001 \\
(0.003)\end{array}$ & $\begin{array}{l}-0.0001 \\
(0.024)\end{array}$ & $\begin{array}{l}0.107 \\
(0.268)\end{array}$ & $\begin{array}{l}\text { 0.002* } \\
(0.001)\end{array}$ & $\begin{array}{l}0.0001 \\
(0.0002)\end{array}$ \\
\hline $\begin{array}{l}\text { Host country } \\
\text { demand }\end{array}$ & $\begin{array}{l}5.875^{*} \\
(2.670)\end{array}$ & $\begin{array}{l}\text { 4.921* } \\
(2.450)\end{array}$ & $\begin{array}{l}0.325 \\
(0.289)\end{array}$ & $\begin{array}{l}\text { 13.850* } \\
(6.681)\end{array}$ & $\begin{array}{l}\text { 14.498* } \\
(5.626)\end{array}$ & $\begin{array}{l}0.981 \\
(0.914)\end{array}$ & $\begin{array}{l}0.671 \\
(5.359)\end{array}$ & $\begin{array}{l}0.901 \\
(4.905)\end{array}$ & $\begin{array}{l}0.069 \\
(0.526)\end{array}$ & $\begin{array}{l}5.674 \\
(3.964)\end{array}$ & $\begin{array}{l}3.480 \\
(3.076)\end{array}$ & $\begin{array}{l}0.205 \\
(0.664)\end{array}$ \\
\hline AU/NZ dummy & $\begin{array}{l}0.027 \\
(0.774)\end{array}$ & $\begin{array}{l}-0.067 \\
(0.736)\end{array}$ & $\begin{array}{l}-0.002 \\
(0.080)\end{array}$ & (1000 & (1000 & $(2.01)$ & $(20000)$ & $(1.000)$ & $(8.06)$ & $(20004)$ & $(0.00)$ & - \\
\hline UK/IR dummy & $\begin{array}{l}0.463 \\
(0.648)\end{array}$ & $\begin{array}{l}0.202 \\
(0.608)\end{array}$ & $\begin{array}{l}0.013 \\
(0.070)\end{array}$ & - & - & - & - & - & - & - & - & - \\
\hline
\end{tabular}




\begin{tabular}{|c|c|c|c|c|c|c|c|c|c|c|c|c|}
\hline $\mathrm{R}^{2}$ & 0.545 & & & 0.627 & & & 0.583 & & & 0.404 & & \\
\hline $\operatorname{AdjR}^{2}$ & 0.517 & & & 0.538 & & & 0.518 & & & 0.292 & & \\
\hline Log likelihood & & -382.43 & -413.61 & & -86.69 & -95.25 & & -120.08 & -129.60 & & -163.57 & -183.21 \\
\hline $\mathrm{N}$ & 152 & 152 & 152 & 34 & 34 & 34 & 46 & 46 & 46 & 72 & 72 & 72 \\
\hline Max. VIF & 1.541 & & & 1.504 & & & 1.257 & & & 1.639 & & \\
\hline
\end{tabular}

Standard errors in parentheses, all $t$ tests are two-tailed, $\uparrow p<.10,{ }^{*} p<.05,{ }^{* *} p<.01$

Table 7.22b: Regression estimates for equation $7 \mathrm{~d}$ (high regulative institutional distance)

\begin{tabular}{|c|c|c|c|c|c|c|c|c|c|c|c|c|}
\hline & \multicolumn{3}{|c|}{ Complete data set } & \multicolumn{3}{|c|}{ AU/NZ } & \multicolumn{3}{|c|}{ US/CA } & \multicolumn{3}{|c|}{$\overline{\text { UK/IR }}$} \\
\hline & OLS & Tobit & $\begin{array}{l}\text { Negative } \\
\text { binomial } \\
\end{array}$ & OLS & Tobit & $\begin{array}{l}\text { Negative } \\
\text { binomial } \\
\end{array}$ & OLS & Tobit & $\begin{array}{l}\text { Negative } \\
\text { binomial } \\
\end{array}$ & OLS & Tobit & $\begin{array}{l}\text { Negative } \\
\text { binomial }\end{array}$ \\
\hline Intercept & $\begin{array}{l}19.431 * * \\
(1.725)\end{array}$ & $\begin{array}{l}19.002^{* *} \\
(1.546)\end{array}$ & $\begin{array}{l}2.939 * * \\
(0.179)\end{array}$ & $\begin{array}{l}20.339 * * \\
(2.253)\end{array}$ & $\begin{array}{l}20.239 * * \\
(1.895)\end{array}$ & $\begin{array}{l}3.020 * * \\
(0.369)\end{array}$ & $\begin{array}{l}16.791 * * \\
(2.759)\end{array}$ & $\begin{array}{l}17.188 * * \\
(2.360)\end{array}$ & $\begin{array}{l}2.619 * * \\
(0.246)\end{array}$ & $\begin{array}{l}\text { 16.001** } \\
(3.950)\end{array}$ & $\begin{array}{l}\text { 15.541** } \\
(3.106)\end{array}$ & $\begin{array}{l}2.742 * * \\
(0.975)\end{array}$ \\
\hline $\begin{array}{l}\text { Org culture (Factor) } \\
-(\text { H8d) }\end{array}$ & $\begin{array}{l}-1.587 * * \\
(0.219)\end{array}$ & $\begin{array}{l}-1.546^{* *} \\
(0.210)\end{array}$ & $\begin{array}{l}-0.109 * * \\
(0.027)\end{array}$ & $\begin{array}{l}-0.536 \\
(0.529)\end{array}$ & $\begin{array}{l}-0.430 \\
(0.471)\end{array}$ & $\begin{array}{l}-0.025 \\
(0.118)\end{array}$ & $\begin{array}{l}-1.460^{* *} \\
(0.315)\end{array}$ & $\begin{array}{l}-1.467 * * \\
(0.297)\end{array}$ & $\begin{array}{l}-0.101 * * \\
(0.032)\end{array}$ & (-) & (1) & (- \\
\hline $\begin{array}{l}\text { Org culture - open } \\
\text { discussion (H8d) }\end{array}$ & - & - & - & - & - & - & - & - & - & $\begin{array}{l}0.122 \\
(0.567)\end{array}$ & $\begin{array}{l}0.085 \\
(0.451)\end{array}$ & $\begin{array}{l}0.005 \\
(0.118)\end{array}$ \\
\hline $\begin{array}{l}\text { Org culture - no } \\
\text { status distinction } \\
(H 8 d)\end{array}$ & - & - & - & - & - & - & - & - & - & $\begin{array}{l}-0.262 \\
(0.514)\end{array}$ & $\begin{array}{l}-0.317 \\
(0.405)\end{array}$ & $\begin{array}{l}-0.020 \\
(0.095)\end{array}$ \\
\hline $\begin{array}{l}\text { Org culture - } \\
\text { experimentation } \\
\text { (H8d) }\end{array}$ & - & - & - & - & - & - & - & - & - & $\begin{array}{l}-0.119 \\
(0.364)\end{array}$ & $\begin{array}{l}-0.119 \\
(0.291)\end{array}$ & $\begin{array}{l}-0.007 \\
(0.071)\end{array}$ \\
\hline $\begin{array}{l}\text { Org culture - } \\
\text { offshore } \\
\text { development (H8d) }\end{array}$ & - & - & - & - & - & - & - & - & - & $\begin{array}{l}-0.453 \\
(0.373)\end{array}$ & $\begin{array}{l}-0.441 \\
(0.297)\end{array}$ & $\begin{array}{l}-0.026 \\
(0.097)\end{array}$ \\
\hline $\begin{array}{l}\text { Org culture - } \\
\text { offshore } \\
\text { responsiveness } \\
\text { (H8d) }\end{array}$ & - & - & - & - & - & - & - & - & - & $\begin{array}{l}-0.151 \\
(0.445)\end{array}$ & $\begin{array}{l}-0.042 \\
(0.330)\end{array}$ & $\begin{array}{l}-0.003 \\
(0.085)\end{array}$ \\
\hline Age & $\begin{array}{l}0.002 \\
(0.005)\end{array}$ & $\begin{array}{l}0.002 \\
(0.004)\end{array}$ & $\begin{array}{l}0.0001 \\
(0.0005)\end{array}$ & $\begin{array}{l}-0.002 \\
(0.009)\end{array}$ & $\begin{array}{l}-0.003 \\
(0.007)\end{array}$ & $\begin{array}{l}-0.0001 \\
(0.001)\end{array}$ & $\begin{array}{l}0.002 \\
(0.007)\end{array}$ & $\begin{array}{l}0.014 \\
(0.006)\end{array}$ & $\begin{array}{l}0.0001 \\
(0.0007)\end{array}$ & $\begin{array}{l}0.016 \\
(0.011)\end{array}$ & $\begin{array}{l}0.016 \dagger \\
(0.009)\end{array}$ & $\begin{array}{l}0.0009 \\
(0.002)\end{array}$ \\
\hline Size (Staff) & $\begin{array}{l}0.000 \\
(0.000)\end{array}$ & $\begin{array}{l}0.765 E-4 \\
(0.0001)\end{array}$ & $\begin{array}{l}0.674 \mathrm{E}-5 \\
(0.264 \mathrm{E}-4)\end{array}$ & $\begin{array}{l}0.000 \\
(0.000)\end{array}$ & $\begin{array}{l}-0.0001 \\
(0.0003)\end{array}$ & $\begin{array}{l}-0.781 \mathrm{E}-5 \\
(0.753 \mathrm{E}-4)\end{array}$ & $\begin{array}{l}0.000 \\
(0.000)\end{array}$ & $\begin{array}{l}-0.0001 \\
(0.0002)\end{array}$ & $\begin{array}{l}-0.107 \mathrm{E}-4 \\
(0.367 \mathrm{E}-4)\end{array}$ & $\begin{array}{l}0.000 \\
(0.000)\end{array}$ & $\begin{array}{l}0.917 \mathrm{E}-4 \\
(0.0003)\end{array}$ & $\begin{array}{l}0.703 E-5 \\
(0.0001)\end{array}$ \\
\hline Offshore policy & $\begin{array}{l}3.359 * * \\
(0.892)\end{array}$ & $\begin{array}{l}3.395 * * \\
(0.861)\end{array}$ & $\begin{array}{l}0.270 * * \\
(0.082)\end{array}$ & $\begin{array}{l}-0.781 \\
(1.504)\end{array}$ & $\begin{array}{l}-0.831 \\
(1.359)\end{array}$ & $\begin{array}{l}-0.046 \\
(0.263)\end{array}$ & $\begin{array}{l}\text { 6.076** } \\
(1.526)\end{array}$ & $\begin{array}{l}6.024^{* *} \\
(1.438)\end{array}$ & $\begin{array}{l}0.631 * * \\
(0.129)\end{array}$ & $\begin{array}{l}3.270 \dagger \\
\mathbf{( 1 . 8 9 0 )}\end{array}$ & $\begin{array}{l}2.837 * \\
(1.416)\end{array}$ & $\begin{array}{l}0.178 \\
(0.693)\end{array}$ \\
\hline
\end{tabular}




\begin{tabular}{|c|c|c|c|c|c|c|c|c|c|c|c|c|}
\hline $\begin{array}{l}\text { Internationalisation } \\
\text { at home strategy }\end{array}$ & $\begin{array}{l}-0.040 \\
(0.191)\end{array}$ & $\begin{array}{l}-0.001 \\
(0.001)\end{array}$ & $\begin{array}{l}-0.0001 \\
(0.0001)\end{array}$ & $\begin{array}{l}0.113 \\
(0.298)\end{array}$ & $\begin{array}{l}-0.001 \\
(0.001)\end{array}$ & $\begin{array}{l}-0.814 \mathrm{E}-4 \\
(0.0004)\end{array}$ & $\begin{array}{l}0.102 \\
(0.301)\end{array}$ & $\begin{array}{l}0.001 \\
(0.003)\end{array}$ & $\begin{array}{l}0.776 \\
(0.026)\end{array}$ & $\begin{array}{l}-0.289 \\
(0.431)\end{array}$ & $\begin{array}{l}-0.004 \dagger \\
(0.002)\end{array}$ & $\begin{array}{l}-0.000 \\
(0.058)\end{array}$ \\
\hline $\begin{array}{l}\text { Host country } \\
\text { demand }\end{array}$ & $\begin{array}{l}-2.486 \\
(2.790)\end{array}$ & $\begin{array}{l}-1.571 \\
(2.646)\end{array}$ & $\begin{array}{l}-0.110 \\
(0.298)\end{array}$ & $\begin{array}{l}1.579 \\
(4.727)\end{array}$ & $\begin{array}{l}3.123 \\
(4.113)\end{array}$ & $\begin{array}{l}0.175 \\
(1.013)\end{array}$ & $\begin{array}{l}-5.797 \\
(4.178)\end{array}$ & $\begin{array}{l}-5.940 \\
(3.937)\end{array}$ & $\begin{array}{l}-0.385 \\
(0.440)\end{array}$ & $\begin{array}{l}1.734 \\
(6.019)\end{array}$ & $\begin{array}{l}2.166 \\
(4.780)\end{array}$ & $\begin{array}{l}0.125 \\
(1.063)\end{array}$ \\
\hline AU/NZ dummy & $\begin{array}{l}0.885 \\
(0.663)\end{array}$ & $\begin{array}{l}0.942 \\
(0.624)\end{array}$ & $\begin{array}{l}0.061 \\
(0.082)\end{array}$ & - & - & - & - & - & - & - & - & - \\
\hline UK/IR dummy & $\begin{array}{l}0.097 \\
(0.667)\end{array}$ & $\begin{array}{l}0.198 \\
(0.638)\end{array}$ & $\begin{array}{l}0.018 \\
(0.087)\end{array}$ & - & - & - & - & - & - & - & - & - \\
\hline $\mathrm{R}^{2}$ & 0.455 & & & 0.076 & & & 0.574 & & & 0.371 & & \\
\hline $\operatorname{Adj}^{2}$ & 0.424 & & & -0.074 & & & 0.537 & & & 0.057 & & \\
\hline Log likelihood & & -379.43 & -409.02 & & -99.66 & -114.21 & & -194.65 & -207.86 & & -68.40 & -78.45 \\
\hline $\mathrm{N}$ & 155 & 155 & 155 & 46 & 46 & 46 & 77 & 77 & 77 & 32 & 32 & 32 \\
\hline Max. VIF & 1.640 & & & 1.305 & & & 1.628 & & & 2.140 & & \\
\hline
\end{tabular}

Standard errors in parentheses, all $t$ tests are two-tailed, $\uparrow p<.10,{ }^{*} p<.05,{ }^{* *} p<.01$

Table 7.23a: Regression estimates for equation 8c (low regulative institutional distance)

\begin{tabular}{|c|c|c|c|c|c|c|c|c|c|c|c|c|}
\hline & \multicolumn{3}{|c|}{ Complete data set } & \multicolumn{3}{|c|}{ AU/NZ } & \multicolumn{3}{|c|}{ US/CA } & \multicolumn{3}{|c|}{ 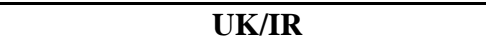 } \\
\hline & OLS & Tobit & $\begin{array}{l}\text { Negative } \\
\text { binomial } \\
\end{array}$ & OLS & Tobit & $\begin{array}{l}\text { Negative } \\
\text { binomial } \\
\end{array}$ & OLS & Tobit & $\begin{array}{l}\text { Negative } \\
\text { binomial } \\
\end{array}$ & OLS & Tobit & $\begin{array}{l}\text { Negative } \\
\text { binomial } \\
\end{array}$ \\
\hline Intercept & $\begin{array}{l}9.256 * * \\
(2.162)\end{array}$ & $\begin{array}{l}10.538 * * \\
(1.936)\end{array}$ & $\begin{array}{l}2.409 * * \\
(0.112)\end{array}$ & $\begin{array}{l}5.204 \\
(3.865)\end{array}$ & $\begin{array}{l}4.745 \\
(3.589)\end{array}$ & $\begin{array}{l}1.846 * * \\
(0.290)\end{array}$ & $\begin{array}{l}\text { 13.092* } \\
(5.003)\end{array}$ & $\begin{array}{l}14.155^{* *} \\
(3.929)\end{array}$ & $\begin{array}{l}\text { 2.642** } \\
(0.421)\end{array}$ & $\begin{array}{l}\text { 11.621** } \\
(2.117)\end{array}$ & $\begin{array}{l}\text { 12.990** } \\
(1.851)\end{array}$ & $\begin{array}{l}2.557 * * \\
(0.169)\end{array}$ \\
\hline $\begin{array}{l}\text { Financial resources } \\
(\mathrm{H} 8 \mathrm{e})\end{array}$ & $\begin{array}{l}-0.066 \\
(0.204)\end{array}$ & $\begin{array}{l}0.034 \\
(0.181)\end{array}$ & $\begin{array}{l}0.002 \\
(0.013)\end{array}$ & $\begin{array}{l}-0.279 \\
(0.608)\end{array}$ & $\begin{array}{l}-0.283 \\
(0.496)\end{array}$ & $\begin{array}{l}-0.013 \\
(0.050)\end{array}$ & $\begin{array}{l}0.224 \\
(0.437)\end{array}$ & $\begin{array}{l}0.256 \\
(0.391)\end{array}$ & $\begin{array}{l}0.016 \\
(0.046)\end{array}$ & $\begin{array}{l}-0.244 \\
(0.216)\end{array}$ & $\begin{array}{l}-0.096 \\
(0.179)\end{array}$ & $\begin{array}{l}-0.005 \\
(0.027)\end{array}$ \\
\hline Age & $\begin{array}{l}0.005 \\
(0.007)\end{array}$ & $\begin{array}{l}0.002 \\
(0.006)\end{array}$ & $\begin{array}{l}0.0001 \\
(0.0004)\end{array}$ & $\begin{array}{l}0.008 \\
(0.027)\end{array}$ & $\begin{array}{l}0.005 \\
(0.021)\end{array}$ & $\begin{array}{l}0.0003 \\
(0.002)\end{array}$ & $\begin{array}{l}0.001 \\
(0.018)\end{array}$ & $\begin{array}{l}0.0006 \\
(0.016)\end{array}$ & $\begin{array}{l}0.466 \mathrm{E}-4 \\
(0.001)\end{array}$ & $\begin{array}{l}0.000 \\
(0.006)\end{array}$ & $\begin{array}{l}-0.002 \\
(0.005)\end{array}$ & $\begin{array}{l}-0.0001 \\
(0.0007)\end{array}$ \\
\hline Size (Staff) & $\begin{array}{l}0.000 \\
(0.000)\end{array}$ & $\begin{array}{l}0.0002 \\
(0.0002)\end{array}$ & $\begin{array}{l}0.142 E-4 \\
(0.196 E-4)\end{array}$ & - & $\begin{array}{l}0.0005 \\
(0.0006)\end{array}$ & $\begin{array}{l}0.330 \mathrm{E}-4 \\
(0.673 \mathrm{E}-4)\end{array}$ & $\begin{array}{l}0.001 \\
(0.001)\end{array}$ & $\begin{array}{l}0.0007 \\
(0.0005)\end{array}$ & $\begin{array}{l}0.528 \mathrm{E}-4 \\
(0.680 \mathrm{E}-4)\end{array}$ & $\begin{array}{l}1.777 \mathrm{E}-5 \\
(0.000)\end{array}$ & $\begin{array}{l}0.880 \mathrm{E}-4 \\
(0.0002)\end{array}$ & $\begin{array}{l}0.505 E-5 \\
(0.381 E-4)\end{array}$ \\
\hline Offshore policy & $\begin{array}{l}\text { 3.006* } \\
(1.510)\end{array}$ & $\begin{array}{l}2.687 \dagger \\
(1.431)\end{array}$ & $\begin{array}{l}0.189 * * \\
(0.060)\end{array}$ & $\begin{array}{l}7.576^{*} \\
(2.929)\end{array}$ & $\begin{array}{l}8.067 * * \\
(2.735)\end{array}$ & $\begin{array}{l}0.697 * * \\
(0.215)\end{array}$ & $\begin{array}{l}-4.130 \\
(3.262)\end{array}$ & $\begin{array}{l}-4.498 \\
(2.862)\end{array}$ & $\begin{array}{l}-0.297 \\
(0.299)\end{array}$ & $\begin{array}{l}5.034 \dagger \\
(1.815)\end{array}$ & $\begin{array}{l}4.663 * * \\
(1.683)\end{array}$ & $\begin{array}{l}0.313^{* *} \\
(\mathbf{0 . 1 1 1})\end{array}$ \\
\hline $\begin{array}{l}\text { Internationalisation } \\
\text { at home strategy }\end{array}$ & $\begin{array}{l}0.371 \\
(0.259)\end{array}$ & $\begin{array}{l}-0.0003 \\
(0.001)\end{array}$ & $\begin{array}{l}-0.174 \mathrm{E}-4 \\
(0.0001)\end{array}$ & $\begin{array}{l}0.501 \\
(0.871)\end{array}$ & $\begin{array}{l}-0.001 \\
(0.003)\end{array}$ & $\begin{array}{l}-0.544 \mathrm{E}-4 \\
(0.0006)\end{array}$ & $\begin{array}{l}0.221 \\
(0.538)\end{array}$ & $\begin{array}{l}-0.005 \\
(0.004)\end{array}$ & $\begin{array}{l}-0.0003 \\
(0.037)\end{array}$ & $\begin{array}{l}0.447 \\
(0.303)\end{array}$ & $\begin{array}{l}0.0006 \\
(0.001)\end{array}$ & $\begin{array}{l}0.374 \mathrm{E}-4 \\
(0.0001)\end{array}$ \\
\hline $\begin{array}{l}\text { Host country } \\
\text { demand }\end{array}$ & $\begin{array}{l}\text { 12.145** } \\
\text { (3.510) }\end{array}$ & $\begin{array}{l}\text { 10.438** } \\
(3.216)\end{array}$ & $\begin{array}{l}0.629 * \\
(0.257)\end{array}$ & $\begin{array}{l}\text { 17.559* } \\
(8.616)\end{array}$ & $\begin{array}{l}19.625 * * \\
(7.415)\end{array}$ & $\begin{array}{l}1.265 \\
(0.837)\end{array}$ & $\begin{array}{l}\text { 14.515* } \\
(6.891)\end{array}$ & $\begin{array}{l}14.722 * \\
(6.261)\end{array}$ & $\begin{array}{l}0.986 \\
(0.689)\end{array}$ & $\begin{array}{l}7.230 \dagger \\
(4.295)\end{array}$ & $\begin{array}{l}3.286 \\
(3.417)\end{array}$ & $\begin{array}{l}0.187 \\
(0.571)\end{array}$ \\
\hline
\end{tabular}




\begin{tabular}{|c|c|c|c|c|c|c|c|c|c|c|c|c|}
\hline AU/NZ dummy & $\begin{array}{l}-0.329 \\
(1.125)\end{array}$ & $\begin{array}{l}-0.276 \\
(1.073)\end{array}$ & $\begin{array}{l}-0.013 \\
(0.070)\end{array}$ & - & - & - & - & - & - & - & - & - \\
\hline UK/IR dummy & $\begin{array}{l}\text { 2.126* } \\
\text { (1.003) }\end{array}$ & $\begin{array}{l}\text { 1.993* } \\
(0.946)\end{array}$ & $\begin{array}{l}0.123 \dagger \\
(0.064)\end{array}$ & - & - & - & - & - & - & - & - & - \\
\hline $\mathrm{R}^{2}$ & 0.172 & & & 0.340 & & & 0.183 & & & 0.172 & & \\
\hline $\operatorname{Adj}^{2}$ & 0.122 & & & 0.213 & & & 0.054 & & & 0.084 & & \\
\hline Log likelihood & & -426.66 & -452.79 & & -96.31 & -104.47 & & -135.29 & -145.04 & & -174.65 & -187.82 \\
\hline $\mathrm{N}$ & 152 & 152 & 152 & 34 & 34 & 34 & 46 & 46 & 46 & 72 & 72 & 72 \\
\hline Max. VIF & 2.006 & & & 2.265 & & & 1.439 & & & 1.435 & & \\
\hline
\end{tabular}

Standard errors in parentheses, all $t$ tests are two-tailed, $\uparrow p<.10, * p<.05, * * p<.01$

Table 7.23b: Regression estimates for equation 8d (high regulative institutional distance)

\begin{tabular}{|c|c|c|c|c|c|c|c|c|c|c|c|c|}
\hline & \multicolumn{3}{|c|}{ Complete data set } & \multicolumn{3}{|c|}{ AU/NZ } & \multicolumn{3}{|c|}{ US/CA } & \multicolumn{3}{|c|}{ U UK/IR } \\
\hline & OLS & Tobit & $\begin{array}{l}\text { Negative } \\
\text { binomial } \\
\end{array}$ & OLS & Tobit & $\begin{array}{l}\text { Negative } \\
\text { binomial } \\
\end{array}$ & OLS & Tobit & $\begin{array}{l}\text { Negative } \\
\text { binomial } \\
\end{array}$ & OLS & Tobit & $\begin{array}{l}\text { Negative } \\
\text { binomial } \\
\end{array}$ \\
\hline$\overline{\text { Intercept }}$ & $\begin{array}{l}11.512^{* *} \\
(1.571)\end{array}$ & $\begin{array}{l}11.408 * * \\
(1.370)\end{array}$ & $\begin{array}{l}2.417 * * \\
(0.117)\end{array}$ & $\begin{array}{l}19.079 * * \\
(1.864)\end{array}$ & $\begin{array}{l}19.248 * * \\
(1.554)\end{array}$ & $\begin{array}{l}2.961 * * \\
(0.296)\end{array}$ & $\begin{array}{l}7.140 * * \\
(2.350)\end{array}$ & $\begin{array}{l}7.847 * * \\
(1.928)\end{array}$ & $\begin{array}{l}1.979 * * \\
(0.180)\end{array}$ & $\begin{array}{l}12.533^{* *} \\
(2.168)\end{array}$ & $\begin{array}{l}12.366^{* *} \\
(1.852)\end{array}$ & $\begin{array}{l}2.535^{* *} \\
(0.587)\end{array}$ \\
\hline $\begin{array}{l}\text { Financial resources } \\
(\text { H8e) }\end{array}$ & $\begin{array}{l}-0.271 \dagger \\
(0.157)\end{array}$ & $\begin{array}{l}-0.251 \dagger \\
(0.149)\end{array}$ & $\begin{array}{l}-0.016 \\
(0.015)\end{array}$ & $\begin{array}{l}-0.082 \\
(0.273)\end{array}$ & $\begin{array}{l}-0.057 \\
(0.247)\end{array}$ & $\begin{array}{l}-0.003 \\
(0.077)\end{array}$ & $\begin{array}{l}-0.081 \\
(0.213)\end{array}$ & $\begin{array}{l}-0.082 \\
(0.201)\end{array}$ & $\begin{array}{l}-0.005 \\
(0.020)\end{array}$ & $\begin{array}{l}-0.333 \\
(0.350)\end{array}$ & $\begin{array}{l}-0.347 \\
(0.302)\end{array}$ & $\begin{array}{l}-0.024 \\
(0.089)\end{array}$ \\
\hline Age & $\begin{array}{l}0.005 \\
(0.006)\end{array}$ & $\begin{array}{l}0.004 \\
(0.005)\end{array}$ & $\begin{array}{l}0.0002 \\
(0.0004)\end{array}$ & $\begin{array}{l}0.000 \\
(0.010)\end{array}$ & $\begin{array}{l}-0.002 \\
(0.008)\end{array}$ & $\begin{array}{l}-0.0001 \\
(0.002)\end{array}$ & $\begin{array}{l}0.000 \\
(0.008)\end{array}$ & $\begin{array}{l}-0.001 \\
(0.007)\end{array}$ & $\begin{array}{l}-0.0001 \\
(0.0006)\end{array}$ & $\begin{array}{l}\text { 0.021* } \\
(0.010)\end{array}$ & $\begin{array}{l}\text { 0.020* } \\
(0.009)\end{array}$ & $\begin{array}{l}0.001 \\
(0.001)\end{array}$ \\
\hline Size (Staff) & $\begin{array}{l}0.000 \\
(0.000)\end{array}$ & $\begin{array}{l}0.0002 \\
(0.0002)\end{array}$ & $\begin{array}{l}0.146 E-4 \\
(0.241 E-4)\end{array}$ & $\begin{array}{l}0.000 \\
(0.000)\end{array}$ & $\begin{array}{l}-0.0002 \\
(0.0003)\end{array}$ & $\begin{array}{l}-0.116 \mathrm{E}-4 \\
(0.870 \mathrm{E}-4)\end{array}$ & $\begin{array}{l}0.000 \\
(0.000)\end{array}$ & $\begin{array}{l}-0.0001 \\
(0.0003)\end{array}$ & $\begin{array}{l}-0.587 E-5 \\
(0.377 E-4)\end{array}$ & $\begin{array}{l}0.000 \\
(0.000)\end{array}$ & $\begin{array}{l}0.0002 \\
(0.0003)\end{array}$ & $\begin{array}{l}0.182 \mathrm{E}-4 \\
(0.0001)\end{array}$ \\
\hline Offshore policy & $\begin{array}{l}5.960 * * \\
(0.947)\end{array}$ & $\begin{array}{l}5.949 * * \\
(0.907)\end{array}$ & $\begin{array}{l}0.437 * * \\
(0.056)\end{array}$ & $\begin{array}{l}-1.121 \\
(1.480)\end{array}$ & $\begin{array}{l}-1.099 \\
(1.336)\end{array}$ & $\begin{array}{l}-0.061 \\
(0.256)\end{array}$ & $\begin{array}{l}\text { 10.221** } \\
(1.416)\end{array}$ & $\begin{array}{l}10.160 * * \\
(1.340)\end{array}$ & $\begin{array}{l}0.918 * * \\
(0.089)\end{array}$ & $\begin{array}{l}4.247 * \\
(1.755)\end{array}$ & $\begin{array}{l}4.002 * * \\
(1.439)\end{array}$ & $\begin{array}{l}0.267 \\
(0.347)\end{array}$ \\
\hline $\begin{array}{l}\text { Internationalisation } \\
\text { at home strategy }\end{array}$ & $\begin{array}{l}0.019 \\
(0.221)\end{array}$ & $\begin{array}{l}-0.001 \\
(0.001)\end{array}$ & $\begin{array}{l}-0.659 \\
(0.0002)\end{array}$ & $\begin{array}{l}0.125 \\
(0.302)\end{array}$ & $\begin{array}{l}-0.001 \\
(0.001)\end{array}$ & $\begin{array}{l}-0.754 \mathrm{E}-4 \\
(0.0005)\end{array}$ & $\begin{array}{l}0.207 \\
(0.343)\end{array}$ & $\begin{array}{l}0.001 \\
(0.003)\end{array}$ & $\begin{array}{l}0.869 \mathrm{E}-4 \\
(0.024)\end{array}$ & $\begin{array}{l}-0.164 \\
(0.383)\end{array}$ & $\begin{array}{l}-0.002 \\
(0.002)\end{array}$ & $\begin{array}{l}-0.0001 \\
(0.065)\end{array}$ \\
\hline $\begin{array}{l}\text { Host country } \\
\text { demand }\end{array}$ & $\begin{array}{l}-0.628 \\
(3.275)\end{array}$ & $\begin{array}{l}0.006 \\
(3.082)\end{array}$ & $\begin{array}{l}-0.003 \\
(0.317)\end{array}$ & $\begin{array}{l}2.075 \\
(4.762)\end{array}$ & $\begin{array}{l}3.341 \\
(4.145)\end{array}$ & $\begin{array}{l}0.187 \\
(1.107)\end{array}$ & $\begin{array}{l}-2.926 \\
(4.858)\end{array}$ & $\begin{array}{l}-3.188 \\
(4.592)\end{array}$ & $\begin{array}{l}-0.203 \\
(0.464)\end{array}$ & $\begin{array}{l}2.900 \\
(5.507)\end{array}$ & $\begin{array}{l}3.316 \\
(4.709)\end{array}$ & $\begin{array}{l}0.186 \\
(0.997)\end{array}$ \\
\hline AU/NZ dummy & $\begin{array}{l}1.144 \\
(0.834)\end{array}$ & $\begin{array}{l}1.204 \\
(0.780)\end{array}$ & $\begin{array}{l}0.071 \\
(0.085)\end{array}$ & - & - & - & - & - & - & - & - & - \\
\hline UK/IR dummy & $\begin{array}{l}0.198 \\
(0.849)\end{array}$ & $\begin{array}{l}0.291 \\
(0.808)\end{array}$ & $\begin{array}{l}0.018 \\
(0.094)\end{array}$ & - & - & - & - & - & - & - & - & - \\
\hline
\end{tabular}




\begin{tabular}{|c|c|c|c|c|c|c|c|c|c|c|c|c|}
\hline $\mathrm{R}^{2}$ & 0.269 & & & 0.052 & & & 0.44 & & & 0.306 & & \\
\hline $\operatorname{AdjR}^{2}$ & 0.227 & & & -0.10 & & & 0.39 & & & 0.133 & & \\
\hline Log likelihood & & -401.13 & -422.87 & & -100.04 & -114.30 & & -205.17 & -215.32 & & -69.76 & -78.77 \\
\hline $\mathrm{N}$ & 155 & 155 & 155 & 46 & 46 & 46 & 77 & 77 & 77 & 32 & 32 & 32 \\
\hline Max. VIF & 2.068 & & & 1.980 & & & 1.55 & & & 1.623 & & \\
\hline
\end{tabular}

Standard errors in parentheses, all $t$ tests are two-tailed, $\uparrow p<.10, * p<.05, * * p<.01$

Table 7.24a: Regression estimates for equation 9c (low regulative institutional distance)

\begin{tabular}{|c|c|c|c|c|c|c|c|c|c|c|c|c|}
\hline & \multicolumn{3}{|c|}{ Complete data set } & \multicolumn{3}{|c|}{ AU/NZ } & \multicolumn{3}{|c|}{ US/CA } & \multicolumn{3}{|c|}{$\overline{\text { UK/IR }}$} \\
\hline & OLS & Tobit & $\begin{array}{l}\text { Negative } \\
\text { binomial }\end{array}$ & OLS & Tobit & $\begin{array}{l}\text { Negative } \\
\text { binomial }\end{array}$ & OLS & Tobit & $\begin{array}{l}\text { Negative } \\
\text { binomial }\end{array}$ & OLS & Tobit & $\begin{array}{l}\text { Negative } \\
\text { binomial }\end{array}$ \\
\hline Intercept & $\begin{array}{l}21.498 * * \\
(3.027)\end{array}$ & $\begin{array}{l}22.947 * * \\
(2.652)\end{array}$ & $\begin{array}{l}3.182 * * \\
(0.228)\end{array}$ & $\begin{array}{l}18.862 * \\
(7.207)\end{array}$ & $\begin{array}{l}18.806 * * \\
(5.817)\end{array}$ & $\begin{array}{l}2.733^{* *} \\
(0.697)\end{array}$ & $\begin{array}{l}32.904 * * \\
(5.738)\end{array}$ & $\begin{array}{l}33.952 * * \\
(4.688)\end{array}$ & $\begin{array}{l}4.067 * * \\
(0.357)\end{array}$ & $\begin{array}{l}15.078 * * \\
(3.492)\end{array}$ & $\begin{array}{l}16.418 * * \\
(3.010)\end{array}$ & $\begin{array}{l}2.755^{* *} \\
(0.408)\end{array}$ \\
\hline $\begin{array}{l}\text { Reputation (Factor) } \\
\text { (H8f) }\end{array}$ & $\begin{array}{l}-1.761 * * \\
(0.337)\end{array}$ & $\begin{array}{l}-1.753 * * \\
(0.310)\end{array}$ & $\begin{array}{l}-0.108 * * \\
(0.029)\end{array}$ & $\begin{array}{l}-2.116 * \\
(0.818)\end{array}$ & $\begin{array}{l}-2.081 * * \\
(0.701)\end{array}$ & $\begin{array}{l}-0.126 \\
(0.092)\end{array}$ & $\begin{array}{l}-3.075 * * \\
(\mathbf{0 . 6 8 8})\end{array}$ & $\begin{array}{l}-3.089 * * \\
(0.626)\end{array}$ & $\begin{array}{l}-0.227 * * \\
(0.049)\end{array}$ & $\begin{array}{l}-0.505 \\
(0.385)\end{array}$ & $\begin{array}{l}-0.501 \\
(0.332)\end{array}$ & $\begin{array}{l}-0.028 \\
(0.053)\end{array}$ \\
\hline Age & $\begin{array}{l}0.003 \\
(0.006)\end{array}$ & $\begin{array}{l}0.009 \\
(0.005)\end{array}$ & $\begin{array}{l}0.345 \mathrm{E}-4 \\
(0.0004)\end{array}$ & $\begin{array}{l}-0.006 \\
(0.019)\end{array}$ & $\begin{array}{l}-0.008 \\
(0.016)\end{array}$ & $\begin{array}{l}-0.0004 \\
(0.0016)\end{array}$ & $\begin{array}{l}0.005 \\
(0.013)\end{array}$ & $\begin{array}{l}0.004 \\
(0.012)\end{array}$ & $\begin{array}{l}0.0003 \\
(0.0008)\end{array}$ & $\begin{array}{l}0.000 \\
(0.006)\end{array}$ & $\begin{array}{l}-0.002 \\
(0.005)\end{array}$ & $\begin{array}{l}-0.0001 \\
(0.0007)\end{array}$ \\
\hline Size (Staff) & $\begin{array}{l}0.000 \\
(0.000)\end{array}$ & $\begin{array}{l}0.002 \\
(0.002)\end{array}$ & $\begin{array}{l}0.176 E-4 \\
(0.193 E-4)\end{array}$ & $\begin{array}{l}0.001 \\
(0.001)\end{array}$ & $\begin{array}{l}0.0007 \\
(0.0005)\end{array}$ & $\begin{array}{l}0.421 \mathrm{E}-4 \\
(0.487 \mathrm{E}-4)\end{array}$ & $\begin{array}{l}0.001 \\
(0.001)\end{array}$ & $\begin{array}{l}0.0006 \\
(0.0004)\end{array}$ & $\begin{array}{l}0.445 E-4 \\
(0.378 E-4)\end{array}$ & $\begin{array}{l}-0.000 \\
(0.000)\end{array}$ & $\begin{array}{l}0.478 \mathrm{E}-4 \\
(0.0002)\end{array}$ & $\begin{array}{l}0.265 \mathrm{E}-5 \\
(0.371 \mathrm{E}-4)\end{array}$ \\
\hline Offshore policy & $\begin{array}{l}1.947 \\
(1.353)\end{array}$ & $\begin{array}{l}1.801 \\
(1.291)\end{array}$ & $\begin{array}{l}0.129 * \\
(0.064)\end{array}$ & $\begin{array}{l}5.823^{*} \\
(2.798)\end{array}$ & $\begin{array}{l}5.810 * \\
(2.474)\end{array}$ & $\begin{array}{l}0.5488 * * \\
(0.173)\end{array}$ & $\begin{array}{l}-1.929 \\
(2.646)\end{array}$ & $\begin{array}{l}-2.185 \\
(2.343)\end{array}$ & $\begin{array}{l}-0.145 \\
(0.172)\end{array}$ & $\begin{array}{l}3.989 * \\
(1.791)\end{array}$ & $\begin{array}{l}3.946^{*} \\
(1.660)\end{array}$ & $\begin{array}{l}0.271^{*} \\
(0.113)\end{array}$ \\
\hline $\begin{array}{l}\text { Internationalisation } \\
\text { at home strategy }\end{array}$ & $\begin{array}{l}0.280 \\
(0.226)\end{array}$ & $\begin{array}{l}0.188 \mathrm{E}-4 \\
(0.001)\end{array}$ & $\begin{array}{l}0.358 \mathrm{E}-5 \\
(0.0001)\end{array}$ & $\begin{array}{l}-0.066 \\
(0.717)\end{array}$ & $\begin{array}{l}0.0004 \\
(0.002)\end{array}$ & $\begin{array}{l}0.514 \mathrm{E}-4 \\
(0.0003)\end{array}$ & $\begin{array}{l}0.176 \\
(0.431)\end{array}$ & $\begin{array}{l}-0.001 \\
(0.003)\end{array}$ & $\begin{array}{l}-0.837 \mathrm{E}-4 \\
(0.023)\end{array}$ & $\begin{array}{l}0.329 \\
(0.279)\end{array}$ & $\begin{array}{l}0.0005 \\
(0.001)\end{array}$ & $\begin{array}{l}0.346 \mathrm{E}-4 \\
(0.0001)\end{array}$ \\
\hline $\begin{array}{l}\text { Host country } \\
\text { demand }\end{array}$ & $\begin{array}{l}\text { 11.418** } \\
(3.200)\end{array}$ & $\begin{array}{l}9.771 * * \\
(2.921)\end{array}$ & $\begin{array}{l}0.595 * \\
(0.256)\end{array}$ & $\begin{array}{l}21.940 \dagger \\
(7.778)\end{array}$ & $\begin{array}{l}21.179 * * \\
(6.547)\end{array}$ & $\begin{array}{l}1.315 * \\
(0.603)\end{array}$ & $\begin{array}{l}10.730 \dagger \\
(5.609)\end{array}$ & $\begin{array}{l}10.832 * \\
(5.104)\end{array}$ & $\begin{array}{l}0.771 \dagger \\
(0.437)\end{array}$ & $\begin{array}{l}6.576 \\
(4.235)\end{array}$ & $\begin{array}{l}3.220 \\
(3.372)\end{array}$ & $\begin{array}{l}0.185 \\
(0.584)\end{array}$ \\
\hline AU/NZ dummy & $\begin{array}{l}-0.044 \\
(0.952)\end{array}$ & $\begin{array}{l}-0.267 \\
(0.895)\end{array}$ & $\begin{array}{l}-0.016 \\
(0.071)\end{array}$ & - & - & - & - & - & - & - & - & - \\
\hline UK/IR dummy & $\begin{array}{l}2.099 \dagger \\
(0.768)\end{array}$ & $\begin{array}{l}\text { 1.791* } \\
(0.716)\end{array}$ & $\begin{array}{l}0.109 \dagger \\
(0.057)\end{array}$ & - & - & - & - & - & - & - & - & - \\
\hline $\mathrm{R}^{2}$ & 0.313 & & & 0.482 & & & 0.461 & & & 0.178 & & \\
\hline $\operatorname{Adj}^{2}$ & 0.271 & & & 0.358 & & & 0.375 & & & 0.091 & & \\
\hline Log likelihood & & -412.21 & -439.64 & & -92.54 & -101.19 & & -125.73 & -135.43 & & -173.68 & -187.40 \\
\hline
\end{tabular}




\begin{tabular}{|c|c|c|c|c|c|c|c|c|c|}
\hline $\mathrm{N}$ & 152 & $152 \quad 152$ & 34 & 34 & 46 & 46 & 72 & 72 & 72 \\
\hline Max. VIF & 1.541 & & 1.217 & & 1.189 & & 1.384 & & \\
\hline
\end{tabular}

Standard errors in parentheses, all $t$ tests are two-tailed, $\dagger p<.10,{ }^{*} p<.05,{ }^{* *} p<.01$

Table 7.24b: Regression estimates for equation 9d (high regulative institutional distance)

\begin{tabular}{|c|c|c|c|c|c|c|c|c|c|c|c|c|}
\hline & \multicolumn{3}{|c|}{ Complete data set } & \multicolumn{3}{|c|}{ AU/NZ } & \multicolumn{3}{|c|}{$\overline{\mathrm{US} / \mathrm{CA}}$} & \multicolumn{3}{|c|}{ UK/IR } \\
\hline & OLS & Tobit & $\begin{array}{l}\text { Negative } \\
\text { binomial } \\
\end{array}$ & OLS & Tobit & $\begin{array}{l}\text { Negative } \\
\text { binomial } \\
\end{array}$ & OLS & Tobit & $\begin{array}{l}\text { Negative } \\
\text { binomial }\end{array}$ & OLS & Tobit & $\begin{array}{l}\text { Negative } \\
\text { binomial }\end{array}$ \\
\hline Intercept & $\begin{array}{l}18.163 * * \\
(2.292)\end{array}$ & $\begin{array}{l}\text { 17.918** } \\
\text { (2.071) }\end{array}$ & $\begin{array}{l}2.823 * * \\
(0.201)\end{array}$ & $\begin{array}{l}17.430 * * \\
(3.011)\end{array}$ & $\begin{array}{l}17.885 * * \\
(2.593)\end{array}$ & $\begin{array}{l}2.882 * * \\
(0.568)\end{array}$ & $\begin{array}{l}18.309 * * \\
(3.637)\end{array}$ & $\begin{array}{l}18.150 * * \\
(3.049)\end{array}$ & $\begin{array}{l}2.642^{* *} \\
(0.357)\end{array}$ & $\begin{array}{l}19.361 * * \\
(3.155)\end{array}$ & $\begin{array}{l}19.234 * * \\
(2.731)\end{array}$ & $\begin{array}{l}2.958 * * \\
(0.860)\end{array}$ \\
\hline $\begin{array}{l}\text { Reputation (Factor) } \\
\text { (H8f) }\end{array}$ & $\begin{array}{l}-1.141 * * \\
(0.269)\end{array}$ & $\begin{array}{l}-1.137 * * \\
(0.257)\end{array}$ & $\begin{array}{l}-0.071 * * \\
(0.026)\end{array}$ & $\begin{array}{l}0.267 \\
(0.436)\end{array}$ & $\begin{array}{l}0.228 \\
(0.394)\end{array}$ & $\begin{array}{l}0.013 \\
(0.092)\end{array}$ & $\begin{array}{l}-1.447^{* *} \\
(0.394)\end{array}$ & $\begin{array}{l}-1.440 * * \\
(0.365)\end{array}$ & $\begin{array}{l}-0.093^{*} \\
(0.041)\end{array}$ & $\begin{array}{l}-1.320^{* *} \\
(0.453)\end{array}$ & $\begin{array}{l}-1.330^{* *} \\
(0.393)\end{array}$ & $\begin{array}{l}-0.080 \\
(0.099)\end{array}$ \\
\hline Age & $\begin{array}{l}0.002 \\
(0.005)\end{array}$ & $\begin{array}{l}0.001 \\
(0.004)\end{array}$ & $\begin{array}{l}0.0001 \\
(0.0004)\end{array}$ & $\begin{array}{l}0.000 \\
(0.009)\end{array}$ & $\begin{array}{l}-0.001 \\
(0.008)\end{array}$ & $\begin{array}{l}-0.991 \mathrm{E}-4 \\
(0.001)\end{array}$ & $\begin{array}{l}-0.002 \\
(0.007)\end{array}$ & $\begin{array}{l}-0.001 \\
(0.006)\end{array}$ & $\begin{array}{l}-0.0001 \\
(0.0006)\end{array}$ & $\begin{array}{l}0.027 * * \\
(0.009)\end{array}$ & $\begin{array}{l}0.026 * * \\
(0.007)\end{array}$ & $\begin{array}{l}0.001 \\
(0.002)\end{array}$ \\
\hline Size (Staff) & $\begin{array}{l}1.428 \mathrm{E}-5 \\
(0.000)\end{array}$ & $\begin{array}{l}0.290 \mathrm{E}-4 \\
(0.0002)\end{array}$ & $\begin{array}{l}0.294 \mathrm{E}-5 \\
(0.242 \mathrm{E}-4)\end{array}$ & $\begin{array}{l}0.000 \\
(0.000)\end{array}$ & $\begin{array}{l}-0.0002 \\
(0.0002)\end{array}$ & $\begin{array}{l}-0.164 \mathrm{E}-4 \\
(0.603 \mathrm{E}-4)\end{array}$ & $\begin{array}{l}0.000 \\
(0.000)\end{array}$ & $\begin{array}{l}-0.0002 \\
(0.0002)\end{array}$ & $\begin{array}{l}-0.150 \mathrm{E}-4 \\
(0.372 \mathrm{E}-4)\end{array}$ & $\begin{array}{l}-0.000 \\
(0.000)\end{array}$ & $\begin{array}{l}-0.815 \mathrm{E}-4 \\
(0.0003)\end{array}$ & $\begin{array}{l}-0.415 \mathrm{E}-5 \\
(0.0001)\end{array}$ \\
\hline Offshore policy & $\begin{array}{l}5.288 * * \\
(0.915)\end{array}$ & $\begin{array}{l}5.263 * * \\
(0.876)\end{array}$ & $\begin{array}{l}0.395 * * \\
(0.057)\end{array}$ & $\begin{array}{l}-1.345 \\
(1.502)\end{array}$ & $\begin{array}{l}-1.280 \\
(1.354)\end{array}$ & $\begin{array}{l}-0.071 \\
(0.254)\end{array}$ & $\begin{array}{l}8.647 * * \\
(1.361)\end{array}$ & $\begin{array}{l}8.664^{* *} \\
(1.276)\end{array}$ & $\begin{array}{l}0.000^{* *} \\
(0.030)\end{array}$ & $\begin{array}{l}3.788^{*} \\
(1.486)\end{array}$ & $\begin{array}{l}3.550 * * \\
(1.199)\end{array}$ & $\begin{array}{l}0.220 \\
(0.547)\end{array}$ \\
\hline $\begin{array}{l}\text { Internationalisation } \\
\text { at home strategy }\end{array}$ & $\begin{array}{l}-0.057 \\
(0.211)\end{array}$ & $\begin{array}{l}-0.0002 \\
(0.001)\end{array}$ & $\begin{array}{l}-0.736 \mathrm{E}-5 \\
(0.0003)\end{array}$ & $\begin{array}{l}0.135 \\
(0.301)\end{array}$ & $\begin{array}{l}-0.001 \\
(0.001)\end{array}$ & $\begin{array}{l}-0.914 \mathrm{E}-4 \\
(0.0005)\end{array}$ & $\begin{array}{l}-0.027 \\
(0.321)\end{array}$ & $\begin{array}{l}0.002 \\
(0.003)\end{array}$ & $\begin{array}{l}0.000 \\
(0.030)\end{array}$ & $\begin{array}{l}-0.147 \\
(0.334)\end{array}$ & $\begin{array}{l}-0.002 \\
(0.002)\end{array}$ & $\begin{array}{l}-0.0001 \\
(0.046)\end{array}$ \\
\hline $\begin{array}{l}\text { Host country } \\
\text { demand }\end{array}$ & $\begin{array}{l}0.077 \\
(3.042)\end{array}$ & $\begin{array}{l}0.593 \\
(2.869)\end{array}$ & $\begin{array}{l}0.037 \\
(0.312)\end{array}$ & $\begin{array}{l}1.576 \\
(4.811)\end{array}$ & $\begin{array}{l}2.946 \\
(4.178)\end{array}$ & $\begin{array}{l}0.164 \\
(1.029)\end{array}$ & $\begin{array}{l}-5.390 \\
(4.385)\end{array}$ & $\begin{array}{l}-5.339 \\
(4.119)\end{array}$ & $\begin{array}{l}-0.351 \\
(0.416)\end{array}$ & $\begin{array}{l}5.130 \\
(4.521)\end{array}$ & $\begin{array}{l}5.581 \\
(3.824)\end{array}$ & $\begin{array}{l}0.357 \\
(0.966)\end{array}$ \\
\hline AU/NZ dummy & $\begin{array}{l}1.497 * \\
(0.722)\end{array}$ & $\begin{array}{l}1.540 * \\
(0.674)\end{array}$ & $\begin{array}{l}0.094 \\
(0.074)\end{array}$ & - & - & - & - & - & - & - & - & - \\
\hline UK/IR dummy & $\begin{array}{l}0.629 \\
(0.728)\end{array}$ & $\begin{array}{l}0.691 \\
(0.691)\end{array}$ & $\begin{array}{l}0.044 \\
(0.083)\end{array}$ & - & - & - & - & - & - & - & - & - \\
\hline $\mathrm{R}^{2}$ & 0.337 & & & 0.060 & & & 0.532 & & & 0.468 & & \\
\hline $\operatorname{AdjR}^{2}$ & 0.300 & & & -0.093 & & & 0.492 & & & 0.335 & & \\
\hline Log likelihood & & -393.37 & -418.06 & & -99.90 & -114.27 & & -198.19 & -210.31 & & -65.50 & -77.81 \\
\hline $\mathrm{N}$ & 155 & 155 & 155 & 46 & 46 & 46 & 77 & 77 & 77 & 32 & 32 & 32 \\
\hline Max. VIF & 1.598 & & & 1.309 & & & 1.209 & & & 1.506 & & \\
\hline
\end{tabular}

Standard errors in parentheses, all $t$ tests are two-tailed, $\dagger p<.10, * p<.05, * * p<.01$ 


\subsection{TESTING THE ROBUSTNESS OF IMD NORMATIVE ID MEASURE USING HOFSTEDE'S CULTURAL DISTANCE MEASURE}

To test the robustness of the normative institutional distance measure, equations 1-9 (see tables 7.10-7.24) are re-estimated replacing the IMD normative institutional distance measure with Hofstede's cultural indices (see Chapter 5). Equations 10-12 (see tables 7.25-7.27) are used to test the direct hypotheses of H1-6 (excluding H4). With $\mathrm{R}^{2}$ values ranging between 0.43 and 0.68 , equations $10-12$ have reasonable explanatory power.

\subsubsection{Direct hypotheses H1-6 (excluding H4)}

H1a, which pertains to the hypothesised relationship between geographic experience and equity stake, receives no support across any of the full samples tested in equations 10-12. Some support $(p<0.10)$ is found for geographic experience - managerial capabilities in the US/CA subsample (Tobit in table 7.27). Support $(p<0.05$ ) is also found for geographic experience - academic capabilities in the AU/NZ grouping (OLS in table 7.25, OLS and Tobit in table 7.27) Significant findings (at least $p<0.10$ ) are further noted for geographic experience - financial capabilities (OLS and Tobit for UK/IR in tables 7.25, 7.26 and 7.27) and geographic experience - international recruitment (Tobit and negative binomial for US/CA in table 7.25, OLS and Tobit for US/CA in tables 7.26, 7.27). The coefficients with the latter variables are, however, negative and do not support the positive hypothesised relationship.

H1b, which deals with industry experience, receives support for the hypothesised positive relationship in the full sample (OLS and Tobit in tables 7.25, 7.26 and OLS in 7.27) and US/CA grouping (Tobit in table 7.25). Of the different aspects of industry experience examined, these significant positive findings relate to industry experience international recruitment. Industry experience - undergraduate marketing and industry experience - undergraduate offshore education are also found to be significant (Tobit for AU/NZ in table 7.25 and 7.27 respectively). However, their associated negative coefficients do not demonstrate support for the hypothesised relationship. 
H1c, pertaining to transfer experience, is supported $(p<0.10)$ in table 7.25 for the OLS models using the full sample and all three geographical groupings.

H2, with respect to know-how, is not supported in table 7.26. Although significant findings are obtained across all models examined, the negative coefficients differ from the hypothesised positive relationship between tacit know-how and equity stake.

The results in table 7.27 also challenge H3, which relates to organisational culture. Although highly significant findings are obtained $(p<0.01)$ across the full sample, AU/NZ and US/CA using all three estimation techniques (i.e. OLS, Tobit and negative binomial), the negative coefficients do not support the hypothesised positive relationship. Of the different aspects of organisational culture analysed in the UK/IR grouping in table 7.27, significant findings are found for org. culture - open discussion (OLS and Tobit), org. culture - no status distinction (OLS) and org. culture - offshore development (OLS and Tobit). The associated coefficients of the latter three variables are, however, also negative, thereby lending no support to H3.

H5, which deals with financial resources, is not supported by equations 10-12 (see tables 7.25-7.27).

H6, which pertains to reputation, is also not supported in equations 10-12 (see tables 7.25-7.27). Significant findings (at least $p<0.10$ ) are obtained for reputation in tables 7.25 and 7.27. However, the negative coefficients contradict the hypothesised positive relationship.

\subsubsection{Findings for moderating hypotheses of $\mathrm{H7}$}

Similar to equations 4-9 (see tables 7.13-7.18), to test for the moderating relationship hypothesised in $\mathrm{H7}$, the data are stratified using the median of the normative institutional distance measure. It is hypothesised that, for lower values of institutional distance, a positive relationship between specific resources and equity stake will be observed, while the relationship will be negative for larger values of institutional distance. 


\subsubsection{Hypothesis 7a}

H7a, which pertains to the moderating role of normative institutional distance on the hypothesised relationship between geographic experience and equity stake, is not supported from the findings in tables 7.28a and 7.28b. While at low levels of institutional distance (see table 7.28a), significant positive findings are found, as hypothesised, for geographic experience - managerial capabilities (Tobit for US/CA) and geographic experience - international recruitment (OLS and Tobit for AU/NZ), the associated negative hypothesis is not supported at higher levels of institutional distance (see table 7.28b). Similarly, while geographic experience - financial capabilities is negatively significant, as hypothesised, at higher levels of institutional distance (Tobit for UK/IR in table 7.28b), the associated positive relationship at low levels of institutional distance is not supported (see table 7.28a).

\subsubsection{Hypothesis $7 b$}

H7b, which deals with the relationship of normative institutional distance on the hypothesised association between industry experience and equity stake, is not supported from the findings in tables 7.29a and 7.29b. While at low levels of institutional distance (see table 7.29a), significant positive findings are found, as hypothesised, for industry experience - international recruitment (OLS and Tobit for the full sample and US/CA), industry experience - undergraduate marketing (OLS and Tobit for UK/IR) and industry experience - postgraduate marketing (Tobit for AU/NZ), the associated negative hypothesis is not supported at higher levels of institutional distance (see table 7.29b). Similarly, while negative significant findings, as hypothesised, are found at higher levels of institutional distance (see table 7.29b) for industry experience - undergraduate offshore education (OLS and Tobit in the full sample, Tobit for AU/NZ), industry experience - postgraduate offshore education (OLS and Tobit for US/CA) and industry experience - undergraduate marketing (OLS and Tobit for the full sample, Tobit for US/CA), the associated positive relationship at low levels of institutional distance is not supported.

\subsubsection{Hypothesis 7c}

H7c, which pertains to the role of normative institutional distance on the know-how and equity relationship, is not supported. While significant findings are obtained across all 
the models examined in table 7.30a (except for UK/IR negative binomial), the observed negative coefficients do not support the positive relationship that is hypothesised at low levels of normative institutional distance. At higher levels of normative institutional distance, the findings in table 7.30b suggest that, know-how is negatively associated with equity stake $(p<0.01)$. While these findings are consistent with H7c at higher levels of normative institutional distance, the combined findings from tables 7.30a and 7.30b do not lend support to H7c. Rather than an inverted relationship, tables 7.30a and 7.30b suggest that, after accounting for the other variables, and regardless of the level of normative institutional distance, know-how is negatively associated with equity stake.

\subsubsection{Hypothesis 7d}

H7d, which deals with the role of normative institutional distance on the hypothesised relationship between organisation culture and equity stake, is not supported. While a positive relationship is hypothesised at low levels of institutional distance, the observed negative significant findings in table 7.31a (for the full sample, AU/NZ and US/CA) do not support this hypothesis. Similarly, for the UK/IR grouping, the negative significant coefficients associated with org. culture - offshore development challenge H7d. At higher levels of institutional distance, a negative relationship is hypothesised. Support towards this hypothesis is obtained for the full sample, AU/NZ and US/CA in table 7.31b. However, when combined with the findings from table 7.31a, the aggregate findings do not support H7d. Rather than the hypothesised inverted relationship, the findings from tables 7.31a and 7.31b suggest that organisation culture is negatively associated with equity stake regardless of the levels of institutional distance and after accounting for the other variables.

\subsubsection{Hypothesis 7e}

H7e, which pertains to the role of normative institutional distance on the hypothesised relationship between financial resources and equity stake is not supported. While a positive relationship is hypothesised at low levels of institutional distance, the insignificant findings do not support this hypothesis. At higher levels of institutional distance, a negative relationship is hypothesised. Some support towards this hypothesis is found in table 7.32b (OLS in the full sample, OLS and Tobit for US/CA). However, 
when combined with the findings from table 7.32a, the overall results do not lend support to H7e.

\subsubsection{Hypothesis 7f}

H7f, which deals with the moderating role of normative institutional distance on the hypothesised relationship between reputation and equity stake is not supported. A positive relationship is hypothesised at low levels of normative institutional distance. The findings from table 7.33a (across all models examined except the UK/IR negative binomial) suggest a significant negative association between reputation and equity stake (at least $p<0.10$ ). These findings do not lend credence to H7f at low levels of normative institutional distance. At higher levels of normative institutional distance, a negative relationship is hypothesised between reputation and equity stake. The findings in table 7.33b support this hypothesis in the full model, AU/NZ and US/CA (at least $p<$ 0.05). However, taken together, the findings of tables 7.33a and 7.33b do not indicate an inverted relationship between reputation and equity stake as hypothesised. Rather, the combined results suggest that regardless of the levels of normative institutional distance and after accounting for the other variables, reputation is negatively associated with equity stake. 
Table 7.25: Regression estimates for equation 10

\begin{tabular}{|c|c|c|c|c|c|c|c|c|c|c|c|c|}
\hline & \multicolumn{3}{|c|}{ Complete data set } & \multicolumn{3}{|c|}{$\mathrm{AU} / \mathrm{NZ}$} & \multicolumn{3}{|c|}{ US/CA } & \multicolumn{3}{|c|}{ UK/IR } \\
\hline & OLS & Tobit & $\begin{array}{l}\text { Negative } \\
\text { binomial } \\
\end{array}$ & OLS & Tobit & $\begin{array}{l}\text { Negative } \\
\text { binomial } \\
\end{array}$ & OLS & Tobit & $\begin{array}{l}\text { Negative } \\
\text { binomial } \\
\end{array}$ & OLS & Tobit & $\begin{array}{l}\text { Negative } \\
\text { binomial }\end{array}$ \\
\hline Intercept & $\begin{array}{l}12.090 * * \\
(3.653)\end{array}$ & $\begin{array}{l}\text { 19.161** } \\
\text { (3.472) }\end{array}$ & $\begin{array}{l}2.925 * * \\
(0.361)\end{array}$ & $\begin{array}{l}-1.246 \\
(6.890)\end{array}$ & $\begin{array}{l}14.405 * \\
(6.825)\end{array}$ & $\begin{array}{l}2.675 * * \\
(0.933)\end{array}$ & $\begin{array}{l}14.415^{*} \\
(6.241)\end{array}$ & $\begin{array}{l}22.775^{* *} \\
(6.432)\end{array}$ & $\begin{array}{l}3.137 * * \\
(0.573)\end{array}$ & $\begin{array}{l}\text { 18.514* } \\
\text { (6.415) }\end{array}$ & $\begin{array}{l}22.370 * * \\
(4.806)\end{array}$ & $\begin{array}{l}3.134 * * \\
(1.036)\end{array}$ \\
\hline $\begin{array}{l}\text { Geo exp - } \\
\text { knowledge of edu } \\
\text { sector (HIa) }\end{array}$ & $\begin{array}{l}0.000 \\
(0.137)\end{array}$ & $\begin{array}{l}-0.137 \\
(0.137)\end{array}$ & $\begin{array}{l}-0.009 \\
(0.013)\end{array}$ & $\begin{array}{l}0.422 \\
(0.292)\end{array}$ & $\begin{array}{l}0.380 \\
(0.317)\end{array}$ & $\begin{array}{l}0.021 \\
(0.050)\end{array}$ & $\begin{array}{l}0.070 \\
(0.224)\end{array}$ & $\begin{array}{l}-0.084 \\
(0.237)\end{array}$ & $\begin{array}{l}-0.005 \\
(0.020)\end{array}$ & $\begin{array}{l}-0.166 \\
(0.259)\end{array}$ & $\begin{array}{l}-0.223 \\
(0.181)\end{array}$ & $\begin{array}{l}-0.013 \\
(0.041)\end{array}$ \\
\hline $\begin{array}{l}\text { Geo exp - academic } \\
\text { capabilities (H1a) }\end{array}$ & $\begin{array}{l}0.059 \\
(0.131)\end{array}$ & $\begin{array}{l}0.084 \\
(0.132)\end{array}$ & $\begin{array}{l}0.005 \\
(0.012)\end{array}$ & $\begin{array}{l}0.600 * \\
(0.281)\end{array}$ & $\begin{array}{l}0.146 \\
(0.299)\end{array}$ & $\begin{array}{l}0.008 \\
(0.038)\end{array}$ & $\begin{array}{l}-0.007 \\
(0.225)\end{array}$ & $\begin{array}{l}0.165 \\
(0.236)\end{array}$ & $\begin{array}{l}0.019 \\
(0.018)\end{array}$ & $\begin{array}{l}-0.060 \\
(0.229)\end{array}$ & $\begin{array}{l}-0.083 \\
(0.170)\end{array}$ & $\begin{array}{l}-0.004 \\
(0.032)\end{array}$ \\
\hline $\begin{array}{l}\text { Geo exp - } \\
\text { managerial } \\
\text { capabilities }(\mathrm{H} 1 \mathrm{a})\end{array}$ & $\begin{array}{l}0.143 \\
(0.158)\end{array}$ & $\begin{array}{l}0.050 \\
(0.163)\end{array}$ & $\begin{array}{l}0.002 \\
(0.016)\end{array}$ & $\begin{array}{l}0.194 \\
(0.341)\end{array}$ & $\begin{array}{l}0.203 \\
(0.363)\end{array}$ & $\begin{array}{l}0.012 \\
(0.054)\end{array}$ & $\begin{array}{l}0.348 \\
(0.260)\end{array}$ & $\begin{array}{l}0.118 \\
(0.276)\end{array}$ & $\begin{array}{l}0.006 \\
(0.024)\end{array}$ & $\begin{array}{l}-0.047 \\
(0.260)\end{array}$ & $\begin{array}{l}-0.148 \\
(0.206)\end{array}$ & $\begin{array}{l}-0.009 \\
(0.044)\end{array}$ \\
\hline $\begin{array}{l}\text { Geo exp - financial } \\
\text { capabilities }(H 1 a)\end{array}$ & $\begin{array}{l}-0.004 \\
(0.147)\end{array}$ & $\begin{array}{l}0.026 \\
(0.150)\end{array}$ & $\begin{array}{l}0.001 \\
(0.014)\end{array}$ & $\begin{array}{l}-0.015 \\
(0.323)\end{array}$ & $\begin{array}{l}-0.282 \\
(0.334)\end{array}$ & $\begin{array}{l}-0.017 \\
(0.049)\end{array}$ & $\begin{array}{l}0.279 \\
(0.228)\end{array}$ & $\begin{array}{l}0.238 \\
(0.241)\end{array}$ & $\begin{array}{l}0.017 \\
(0.023)\end{array}$ & $\begin{array}{l}-0.513 \dagger \\
(0.264)\end{array}$ & $\begin{array}{l}-0.373 \dagger \\
(0.211)\end{array}$ & $\begin{array}{l}-0.022 \\
(0.042)\end{array}$ \\
\hline $\begin{array}{l}\text { Geo exp - intl } \\
\text { recruitment }(H 1 a)\end{array}$ & $\begin{array}{l}-0.060 \\
(0.164)\end{array}$ & $\begin{array}{l}-0.172 \\
(0.169)\end{array}$ & $\begin{array}{l}-0.010 \\
(0.015)\end{array}$ & $\begin{array}{l}0.373 \\
(0.285)\end{array}$ & $\begin{array}{l}0.160 \\
(0.344)\end{array}$ & $\begin{array}{l}0.010 \\
(0.052)\end{array}$ & $\begin{array}{l}-0.444 \\
(0.291)\end{array}$ & $\begin{array}{l}-0.687^{*} \\
(0.299)\end{array}$ & $\begin{array}{l}-0.047 \dagger \\
(0.024)\end{array}$ & $\begin{array}{l}0.047 \\
(0.305)\end{array}$ & $\begin{array}{l}0.099 \\
(0.224)\end{array}$ & $\begin{array}{l}0.006 \\
(0.045)\end{array}$ \\
\hline $\begin{array}{l}\text { Ind exp - UG } \\
\text { offshore edu (H1b) }\end{array}$ & $\begin{array}{l}0.072 \\
(0.127)\end{array}$ & $\begin{array}{l}0.073 \\
(0.130)\end{array}$ & $\begin{array}{l}0.004 \\
(0.011)\end{array}$ & $\begin{array}{l}-0.349 \\
(0.248)\end{array}$ & $\begin{array}{l}-0.183 \\
(0.289)\end{array}$ & $\begin{array}{l}-0.010 \\
(0.034)\end{array}$ & $\begin{array}{l}0.091 \\
(0.232)\end{array}$ & $\begin{array}{l}0.091 \\
(0.245)\end{array}$ & $\begin{array}{l}0.008 \\
(0.023)\end{array}$ & $\begin{array}{l}-0.065 \\
(0.207)\end{array}$ & $\begin{array}{l}-0.047 \\
(0.167)\end{array}$ & $\begin{array}{l}-0.003 \\
(0.032)\end{array}$ \\
\hline $\begin{array}{l}\text { Ind exp - PG } \\
\text { offshore edu (H1b) }\end{array}$ & $\begin{array}{l}0.042 \\
(0.144)\end{array}$ & $\begin{array}{l}0.035 \\
(0.148)\end{array}$ & $\begin{array}{l}0.002 \\
(0.012)\end{array}$ & $\begin{array}{l}0.089 \\
(0.293)\end{array}$ & $\begin{array}{l}-0.001 \\
(0.321)\end{array}$ & $\begin{array}{l}0.0005 \\
(0.038)\end{array}$ & $\begin{array}{l}0.019 \\
(0.238)\end{array}$ & $\begin{array}{l}-0.042 \\
(0.247)\end{array}$ & $\begin{array}{l}-0.003 \\
(0.018)\end{array}$ & $\begin{array}{l}0.135 \\
(0.254)\end{array}$ & $\begin{array}{l}-0.047 \\
(0.208)\end{array}$ & $\begin{array}{l}-0.002 \\
(0.048)\end{array}$ \\
\hline $\begin{array}{l}\text { Ind exp - intl } \\
\text { recruitment (Factor) } \\
\text { (H1b) }\end{array}$ & $\begin{array}{l}0.308 \dagger \\
(0.167)\end{array}$ & $\begin{array}{l}0.394^{*} \\
(0.166)\end{array}$ & $\begin{array}{l}0.024 \\
(0.016)\end{array}$ & $\begin{array}{l}0.309 \\
(0.334)\end{array}$ & $\begin{array}{l}0.453 \\
(0.342)\end{array}$ & $\begin{array}{l}0.026 \\
(0.044)\end{array}$ & $\begin{array}{l}0.347 \\
(0.288)\end{array}$ & $\begin{array}{l}0.589 * \\
(0.298)\end{array}$ & $\begin{array}{l}0.040 \\
(0.028)\end{array}$ & $\begin{array}{l}0.314 \\
(0.306)\end{array}$ & $\begin{array}{l}0.172 \\
(0.212)\end{array}$ & $\begin{array}{l}0.010 \\
(0.041)\end{array}$ \\
\hline $\begin{array}{l}\text { Ind exp - UG } \\
\text { marketing }(H 1 b)\end{array}$ & $\begin{array}{l}-0.077 \\
(0.130)\end{array}$ & $\begin{array}{l}-0.036 \\
(0.130)\end{array}$ & $\begin{array}{l}-0.002 \\
(0.014)\end{array}$ & $\begin{array}{l}-0.354 \\
(0.326)\end{array}$ & $\begin{array}{l}-0.636 \dagger \\
(0.344)\end{array}$ & $\begin{array}{l}-0.038 \\
(0.044)\end{array}$ & $\begin{array}{l}-0.186 \\
(0.202)\end{array}$ & $\begin{array}{l}0.099 \\
(0.210)\end{array}$ & $\begin{array}{l}0.007 \\
(0.017)\end{array}$ & $\begin{array}{l}0.174 \\
(0.229)\end{array}$ & $\begin{array}{l}0.154 \\
(0.160)\end{array}$ & $\begin{array}{l}0.008 \\
(0.037)\end{array}$ \\
\hline $\begin{array}{l}\text { Ind exp - PG } \\
\text { marketing }(H 1 b)\end{array}$ & $\begin{array}{l}0.066 \\
(0.124)\end{array}$ & $\begin{array}{l}0.020 \\
(0.126)\end{array}$ & $\begin{array}{l}0.001 \\
(0.011)\end{array}$ & $\begin{array}{l}0.349 \\
(0.234)\end{array}$ & $\begin{array}{l}0.140 \\
(0.275)\end{array}$ & $\begin{array}{l}0.008 \\
(0.033)\end{array}$ & $\begin{array}{l}-0.143 \\
(0.212)\end{array}$ & $\begin{array}{l}-0.152 \\
(0.208)\end{array}$ & $\begin{array}{l}-0.009 \\
(0.018)\end{array}$ & $\begin{array}{l}0.259 \\
(0.207)\end{array}$ & $\begin{array}{l}0.159 \\
(0.165)\end{array}$ & $\begin{array}{l}0.009 \\
(0.035)\end{array}$ \\
\hline $\begin{array}{l}\text { Transfer experience } \\
\text { (H1c) }\end{array}$ & $\begin{array}{l}-7.849 * * \\
(0.695)\end{array}$ & $\begin{array}{l}-0.0004 \\
(0.0005)\end{array}$ & $\begin{array}{l}-0.289 \mathrm{E}-4 \\
(0.698 \mathrm{E}-4)\end{array}$ & $\begin{array}{l}-10.884^{* *} \\
(1.464)\end{array}$ & $\begin{array}{l}-0.001 \\
(0.001)\end{array}$ & $\begin{array}{l}-0.765 \mathrm{E}-4 \\
(0.0001)\end{array}$ & $\begin{array}{l}-8.652 * * \\
(1.156)\end{array}$ & $\begin{array}{l}-0.001 \\
(0.001)\end{array}$ & $\begin{array}{l}-0.801 \mathrm{E}-4 \\
(0.0001)\end{array}$ & $\begin{array}{l}-4.126 * * \\
(1.361)\end{array}$ & $\begin{array}{l}0.879 \mathrm{E}-4 \\
(0.0007)\end{array}$ & $\begin{array}{l}0.634 \mathrm{E}-5 \\
(0.0001)\end{array}$ \\
\hline $\begin{array}{l}\text { Financial resources } \\
\text { (H5) }\end{array}$ & $\begin{array}{l}0.004 \\
(0.113)\end{array}$ & $\begin{array}{l}-0.081 \\
(0.110)\end{array}$ & $\begin{array}{l}-0.005 \\
(0.010)\end{array}$ & $\begin{array}{l}0.350 \\
(0.266)\end{array}$ & $\begin{array}{l}-0.071 \\
(0.298)\end{array}$ & $\begin{array}{l}-0.003 \\
(0.038)\end{array}$ & $\begin{array}{l}0.126 \\
(0.178)\end{array}$ & $\begin{array}{l}-0.017 \\
(0.170)\end{array}$ & $\begin{array}{l}-0.003 \\
(0.015)\end{array}$ & $\begin{array}{l}-0.077 \\
(0.197)\end{array}$ & $\begin{array}{l}-0.017 \\
(0.150)\end{array}$ & $\begin{array}{l}-0.001 \\
(0.035)\end{array}$ \\
\hline $\begin{array}{l}\text { Reputation (Factor) } \\
\text { (H6) }\end{array}$ & $\begin{array}{l}-0.281 \\
(0.235)\end{array}$ & $\begin{array}{l}-1.497^{* *} \\
(0.212)\end{array}$ & $\begin{array}{l}-0.093^{* *} \\
(0.021)\end{array}$ & $\begin{array}{l}0.422 \\
(0.483)\end{array}$ & $\begin{array}{l}-0.888 \dagger \\
(0.525)\end{array}$ & $\begin{array}{l}-0.054 \\
(0.064)\end{array}$ & $\begin{array}{l}-0.440 \\
(0.431)\end{array}$ & $\begin{array}{l}-2.335 * * \\
(0.349)\end{array}$ & $\begin{array}{l}-0.158^{* *} \\
(0.033)\end{array}$ & $\begin{array}{l}-0.376 \\
(0.377)\end{array}$ & $\begin{array}{l}-0.644^{*} \\
(0.280)\end{array}$ & $\begin{array}{l}-0.038 \\
(0.050)\end{array}$ \\
\hline $\begin{array}{l}\text { Hofstede cultural } \\
\text { distance }\end{array}$ & $\begin{array}{l}0.148 \\
(0.240)\end{array}$ & $\begin{array}{l}-0.076 \\
(0.152)\end{array}$ & $\begin{array}{l}-0.003 \\
(0.016)\end{array}$ & $\begin{array}{l}1.082 * \\
(0.451)\end{array}$ & $\begin{array}{l}0.664 \\
(0.498)\end{array}$ & $\begin{array}{l}0.039 \\
(0.047)\end{array}$ & $\begin{array}{l}0.077 \\
(0.418)\end{array}$ & $\begin{array}{l}-0.272 \\
(0.276)\end{array}$ & $\begin{array}{l}-0.014 \\
(0.029)\end{array}$ & $\begin{array}{l}-0.647 \\
(0.458)\end{array}$ & $\begin{array}{l}-0.679 \dagger \\
(0.354)\end{array}$ & $\begin{array}{l}-0.041 \\
(0.070)\end{array}$ \\
\hline
\end{tabular}




\begin{tabular}{|c|c|c|c|c|c|c|c|c|c|c|c|c|}
\hline $\begin{array}{l}\text { IMD Regulative ID } \\
\text { (lag 1) }\end{array}$ & $\begin{array}{l}0.029 \\
(0.199)\end{array}$ & $\begin{array}{l}0.071 \\
(0.153)\end{array}$ & $\begin{array}{l}0.003 \\
(0.018)\end{array}$ & $\begin{array}{l}-0.047 \\
(0.306)\end{array}$ & $\begin{array}{l}0.176 \\
(0.352)\end{array}$ & $\begin{array}{l}0.006 \\
(0.041)\end{array}$ & $\begin{array}{l}0.005 \\
(0.374)\end{array}$ & $\begin{array}{l}0.266 \\
(0.276)\end{array}$ & $\begin{array}{l}0.014 \\
(0.031)\end{array}$ & $\begin{array}{l}-0.319 \\
(0.546)\end{array}$ & $\begin{array}{l}-0.332 \\
(0.335)\end{array}$ & $\begin{array}{l}-0.019 \\
(0.086)\end{array}$ \\
\hline Age & $\begin{array}{l}0.006 \\
(0.004)\end{array}$ & $\begin{array}{l}0.003 \\
(0.003)\end{array}$ & $\begin{array}{l}0.0002 \\
(0.0003)\end{array}$ & $\begin{array}{l}-0.003 \\
(0.013)\end{array}$ & $\begin{array}{l}0.0003 \\
(0.012)\end{array}$ & $\begin{array}{l}0.366 \mathrm{E}-4 \\
(0.001)\end{array}$ & $\begin{array}{l}0.004 \\
(0.006)\end{array}$ & $\begin{array}{l}0.003 \\
(0.006)\end{array}$ & $\begin{array}{l}0.0002 \\
(0.0005)\end{array}$ & $\begin{array}{l}0.009 \\
(0.006)\end{array}$ & $\begin{array}{l}0.001 \\
(0.004)\end{array}$ & $\begin{array}{l}0.0001 \\
(0.0008)\end{array}$ \\
\hline Size (Staff) & $\begin{array}{l}9.985 E-5 \\
(0.000)\end{array}$ & $\begin{array}{l}0.0001 \\
(0.0001)\end{array}$ & $\begin{array}{l}0.121 \mathrm{E}-4 \\
(0.169 \mathrm{E}-4)\end{array}$ & - & - & - & $\begin{array}{l}0.000 \\
(0.000)\end{array}$ & $\begin{array}{l}0.0001 \\
(0.0002)\end{array}$ & $\begin{array}{l}0.943 E-5 \\
(0.232 E-5)\end{array}$ & $\begin{array}{l}6.904 \mathrm{E}-5 \\
(0.000)\end{array}$ & $\begin{array}{l}0.483 E-4 \\
(0.0002)\end{array}$ & $\begin{array}{l}0.288 \mathrm{E}-5 \\
(0.491 \mathrm{E}-4)\end{array}$ \\
\hline Offshore policy & $\begin{array}{l}2.024^{*} \\
(0.717)\end{array}$ & $\begin{array}{l}3.745 * * \\
(0.775)\end{array}$ & $\begin{array}{l}0.279 * * \\
(0.045)\end{array}$ & $\begin{array}{l}1.351 \\
(1.435)\end{array}$ & $\begin{array}{l}3.591^{*} \\
(1.655)\end{array}$ & $\begin{array}{l}0.238 \\
(0.198)\end{array}$ & $\begin{array}{l}2.175 \dagger \\
(1.142)\end{array}$ & $\begin{array}{l}4.493^{* *} \\
(1.219)\end{array}$ & $\begin{array}{l}0.384^{* *} \\
(0.072)\end{array}$ & $\begin{array}{l}3.154^{*} \\
(1.386)\end{array}$ & $\begin{array}{l}3.481 * * \\
(0.0009)\end{array}$ & $\begin{array}{l}0.232 \\
(0.189)\end{array}$ \\
\hline $\begin{array}{l}\text { Internationalisation } \\
\text { at home strategy }\end{array}$ & $\begin{array}{l}0.081 \\
(0.147)\end{array}$ & $\begin{array}{l}0.725 \mathrm{E}-4 \\
(0.0009)\end{array}$ & $\begin{array}{l}0.982 \mathrm{E}-5 \\
(0.0001)\end{array}$ & $\begin{array}{l}0.073 \\
(0.347)\end{array}$ & $\begin{array}{l}0.0001 \\
(0.001)\end{array}$ & $\begin{array}{l}0.177 \mathrm{E}-4 \\
(0.0002)\end{array}$ & $\begin{array}{l}-0.099 \\
(0.232)\end{array}$ & $\begin{array}{l}-0.757 \mathrm{E}-4 \\
(0.002)\end{array}$ & $\begin{array}{l}0.441 \mathrm{E}-5 \\
(0.0002)\end{array}$ & $\begin{array}{l}0.121 \\
(0.269)\end{array}$ & $\begin{array}{l}0.0006 \\
(0.0009)\end{array}$ & $\begin{array}{l}0.381 \mathrm{E}-4 \\
(0.0001)\end{array}$ \\
\hline $\begin{array}{l}\text { Host country } \\
\text { demand }\end{array}$ & $\begin{array}{l}1.415 \\
(2.093)\end{array}$ & $\begin{array}{l}\text { 4.181* } \\
(2.084)\end{array}$ & $\begin{array}{l}0.249 \\
(0.212)\end{array}$ & $\begin{array}{l}2.200 \\
(4.102)\end{array}$ & $\begin{array}{l}6.626 \\
(4.340)\end{array}$ & $\begin{array}{l}0.372 \\
(0.621)\end{array}$ & $\begin{array}{l}-2.255 \\
(3.455)\end{array}$ & $\begin{array}{l}2.712 \\
(3.599)\end{array}$ & $\begin{array}{l}0.157 \\
(0.323)\end{array}$ & $\begin{array}{l}6.868 \dagger \\
(3.755)\end{array}$ & $\begin{array}{l}4.941 \dagger \\
(2.632)\end{array}$ & $\begin{array}{l}0.282 \\
(0.583)\end{array}$ \\
\hline AU/NZ dummy & $\begin{array}{l}0.168 \\
(0.629)\end{array}$ & $\begin{array}{l}0.630 \\
(0.618)\end{array}$ & $\begin{array}{l}0.040 \\
(0.059)\end{array}$ & - & - & - & - & - & - & - & - & - \\
\hline UK/IR dummy & $\begin{array}{l}0.239 \\
(0.564)\end{array}$ & $\begin{array}{l}0.910 \\
(0.578)\end{array}$ & $\begin{array}{l}0.055 \\
(0.056)\end{array}$ & - & - & - & - & - & - & - & - & - \\
\hline $\mathrm{R}^{2}$ & 0.562 & & & 0.671 & & & 0.685 & & & 0.433 & & \\
\hline $\operatorname{AdjR}^{2}$ & 0.520 & & & 0.537 & & & 0.611 & & & 0.241 & & \\
\hline Log likelihood & & -815.61 & -863.94 & & -210.75 & -224.35 & & -334.96 & -358.46 & & -239.72 & -264.47 \\
\hline $\mathrm{N}$ & 308 & 308 & 308 & 80 & 80 & 80 & 124 & 124 & 124 & 104 & 104 & 104 \\
\hline Max. VIF & 2.151 & & & 2.315 & & & 2.444 & & & 1.721 & & \\
\hline
\end{tabular}

Standard errors in parentheses, all $t$ tests are two-tailed, $\dagger p<.10, * p<.05, * * p<.01$

Table 7.26: Regression estimates for equation 11

\begin{tabular}{|c|c|c|c|c|c|c|c|c|c|c|c|c|}
\hline & \multicolumn{3}{|c|}{ Complete data set } & \multicolumn{3}{|c|}{ AU/NZ } & \multicolumn{3}{|c|}{ US/CA } & \multicolumn{3}{|c|}{$\overline{\text { UK/IR }}$} \\
\hline & OLS & Tobit & $\begin{array}{l}\text { Negative } \\
\text { binomial }\end{array}$ & OLS & Tobit & $\begin{array}{l}\text { Negative } \\
\text { binomial }\end{array}$ & OLS & Tobit & $\begin{array}{l}\text { Negative } \\
\text { binomial }\end{array}$ & OLS & Tobit & $\begin{array}{l}\text { Negative } \\
\text { binomial }\end{array}$ \\
\hline Intercept & $\begin{array}{l}17.028 * * \\
(3.081)\end{array}$ & $\begin{array}{l}18.826 * * \\
(2.724)\end{array}$ & $\begin{array}{l}3.026 * * \\
(0.398)\end{array}$ & $\begin{array}{l}9.112 \\
(6.03)\end{array}$ & $\begin{array}{l}13.608 * * \\
(5.054)\end{array}$ & $\begin{array}{l}2.709 * * \\
(0.732)\end{array}$ & $\begin{array}{l}17.932 * * \\
(5.704)\end{array}$ & $\begin{array}{l}17.827 * * \\
(4.965)\end{array}$ & $\begin{array}{l}2.960 * * \\
(0.758)\end{array}$ & $\begin{array}{l}23.597 * * \\
(4.818)\end{array}$ & $\begin{array}{l}23.121 * * \\
(4.106)\end{array}$ & $\begin{array}{l}3.249 * * \\
(1.259)\end{array}$ \\
\hline $\begin{array}{l}\text { Geo exp - knowledge of edu } \\
\text { sector (HIa) }\end{array}$ & $\begin{array}{l}-0.052 \\
(0.117)\end{array}$ & $\begin{array}{l}-0.052 \\
(0.108)\end{array}$ & $\begin{array}{l}-0.004 \\
(0.015)\end{array}$ & $\begin{array}{l}0.326 \\
(0.272)\end{array}$ & $\begin{array}{l}0.340 \\
(0.237)\end{array}$ & $\begin{array}{l}0.019 \\
(0.044)\end{array}$ & $\begin{array}{l}-0.069 \\
(0.202)\end{array}$ & $\begin{array}{l}-0.071 \\
(0.181)\end{array}$ & $\begin{array}{l}-0.006 \\
(0.026)\end{array}$ & $\begin{array}{l}-0.129 \\
(0.191)\end{array}$ & $\begin{array}{l}-0.113 \\
(0.155)\end{array}$ & $\begin{array}{l}-0.006 \\
(0.052)\end{array}$ \\
\hline $\begin{array}{l}\text { Geo exp - academic } \\
\text { capabilities }(H 1 a)\end{array}$ & $\begin{array}{l}0.007 \\
(0.112)\end{array}$ & $\begin{array}{l}-0.032 \\
(0.104)\end{array}$ & $\begin{array}{l}-0.003 \\
(0.014)\end{array}$ & $\begin{array}{l}0.278 \\
(0.253)\end{array}$ & $\begin{array}{l}0.235 \\
(0.222)\end{array}$ & $\begin{array}{l}0.008 \\
(0.043)\end{array}$ & $\begin{array}{l}0.015 \\
(0.200)\end{array}$ & $\begin{array}{l}0.015 \\
(0.180)\end{array}$ & $\begin{array}{l}-0.0004 \\
(0.025)\end{array}$ & $\begin{array}{l}-0.125 \\
(0.170)\end{array}$ & $\begin{array}{l}-0.128 \\
(0.145)\end{array}$ & $\begin{array}{l}-0.007 \\
(0.034)\end{array}$ \\
\hline
\end{tabular}




\begin{tabular}{|c|c|c|c|c|c|c|c|c|c|c|c|c|}
\hline $\begin{array}{l}\text { Geo exp - managerial } \\
\text { capabilities (H1a) }\end{array}$ & $\begin{array}{l}0.083 \\
(0.135)\end{array}$ & $\begin{array}{l}0.087 \\
(0.128)\end{array}$ & $\begin{array}{l}0.005 \\
(0.018)\end{array}$ & $\begin{array}{l}0.064 \\
(0.302)\end{array}$ & $\begin{array}{l}0.078 \\
(0.269)\end{array}$ & $\begin{array}{l}0.005 \\
(0.048)\end{array}$ & $\begin{array}{l}0.334 \\
(0.235)\end{array}$ & $\begin{array}{l}0.343 \\
(0.213)\end{array}$ & $\begin{array}{l}0.021 \\
(0.029)\end{array}$ & $\begin{array}{l}-0.114 \\
(0.195)\end{array}$ & $\begin{array}{l}-0.088 \\
(0.174)\end{array}$ & $\begin{array}{l}-0.006 \\
(0.045)\end{array}$ \\
\hline $\begin{array}{l}\text { Geo exp - financial capabilities } \\
\text { (H1a) }\end{array}$ & $\begin{array}{l}0.053 \\
(0.129)\end{array}$ & $\begin{array}{l}0.016 \\
(0.117)\end{array}$ & $\begin{array}{l}0.0005 \\
(0.015)\end{array}$ & $\begin{array}{l}0.051 \\
(0.300)\end{array}$ & $\begin{array}{l}-0.184 \\
(0.250)\end{array}$ & $\begin{array}{l}-0.012 \\
(0.042)\end{array}$ & $\begin{array}{l}0.243 \\
(0.204)\end{array}$ & $\begin{array}{l}0.240 \\
(0.184)\end{array}$ & $\begin{array}{l}0.017 \\
(0.024)\end{array}$ & $\begin{array}{l}-0.454^{*} \\
(0.213)\end{array}$ & $\begin{array}{l}-0.362 * \\
(0.181)\end{array}$ & $\begin{array}{l}-0.024 \\
(0.047)\end{array}$ \\
\hline $\begin{array}{l}\text { Geo exp - intl recruitment } \\
\text { (H1a) }\end{array}$ & $\begin{array}{l}-0.076 \\
(0.142)\end{array}$ & $\begin{array}{l}-0.049 \\
(0.132)\end{array}$ & $\begin{array}{l}-0.002 \\
(0.018)\end{array}$ & $\begin{array}{l}0.168 \\
(0.278)\end{array}$ & $\begin{array}{l}0.236 \\
(0.256)\end{array}$ & $\begin{array}{l}0.015 \\
(0.048)\end{array}$ & $\begin{array}{l}-0.469 \dagger \\
(0.259)\end{array}$ & $\begin{array}{l}-0.426 \dagger \\
(0.231)\end{array}$ & $\begin{array}{l}-0.031 \\
(0.032)\end{array}$ & $\begin{array}{l}0.033 \\
(0.223)\end{array}$ & $\begin{array}{l}0.087 \\
(0.192)\end{array}$ & $\begin{array}{l}0.007 \\
(0.051)\end{array}$ \\
\hline $\begin{array}{l}\text { Ind exp - UG offshore edu } \\
\text { (H1b) }\end{array}$ & $\begin{array}{l}0.075 \\
(0.110)\end{array}$ & $\begin{array}{l}0.053 \\
(0.102)\end{array}$ & $\begin{array}{l}0.004 \\
(0.013)\end{array}$ & $\begin{array}{l}-0.164 \\
(0.241)\end{array}$ & $\begin{array}{l}-0.271 \\
(0.215)\end{array}$ & $\begin{array}{l}-0.013 \\
(0.037)\end{array}$ & $\begin{array}{l}0.037 \\
(0.208)\end{array}$ & $\begin{array}{l}0.042 \\
(0.188)\end{array}$ & $\begin{array}{l}0.005 \\
(0.027)\end{array}$ & $\begin{array}{l}-0.018 \\
(0.165)\end{array}$ & $\begin{array}{l}0.043 \\
(0.143)\end{array}$ & $\begin{array}{l}0.001 \\
(0.035)\end{array}$ \\
\hline $\begin{array}{l}\text { Ind exp - PG offshore edu } \\
(H 1 b)\end{array}$ & $\begin{array}{l}-0.048 \\
(0.124)\end{array}$ & $\begin{array}{l}-0.043 \\
(0.116)\end{array}$ & $\begin{array}{l}-0.003 \\
(0.015)\end{array}$ & $\begin{array}{l}0.144 \\
(0.241)\end{array}$ & $\begin{array}{l}0.230 \\
(0.242)\end{array}$ & $\begin{array}{l}0.013 \\
(0.039)\end{array}$ & $\begin{array}{l}-0.062 \\
(0.210)\end{array}$ & $\begin{array}{l}-0.043 \\
(0.189)\end{array}$ & $\begin{array}{l}-0.004 \\
(0.025)\end{array}$ & $\begin{array}{l}-0.192 \\
(0.205)\end{array}$ & $\begin{array}{l}-0.248 \\
(0.178)\end{array}$ & $\begin{array}{l}-0.015 \\
(0.049)\end{array}$ \\
\hline $\begin{array}{l}\text { Ind exp - intl recruitment } \\
\text { (Factor) (H1b) }\end{array}$ & $\begin{array}{l}0.304 * \\
(0.143)\end{array}$ & $\begin{array}{l}0.245 \dagger \\
(0.130)\end{array}$ & $\begin{array}{l}0.016 \\
(0.018)\end{array}$ & $\begin{array}{l}0.406 \\
(0.308)\end{array}$ & $\begin{array}{l}0.199 \\
(0.257)\end{array}$ & $\begin{array}{l}0.013 \\
(0.045)\end{array}$ & $\begin{array}{l}0.234 \\
(0.257)\end{array}$ & $\begin{array}{l}0.199 \\
(0.231)\end{array}$ & $\begin{array}{l}0.014 \\
(0.032)\end{array}$ & $\begin{array}{l}0.280 \\
(0.228)\end{array}$ & $\begin{array}{l}0.170 \\
(0.181)\end{array}$ & $\begin{array}{l}0.010 \\
(0.041)\end{array}$ \\
\hline Ind exp - UG marketing (H1b) & $\begin{array}{l}0.000 \\
(0.109)\end{array}$ & $\begin{array}{l}0.001 \\
(0.101)\end{array}$ & $\begin{array}{l}-0.0007 \\
(0.015)\end{array}$ & $\begin{array}{l}-0.447 \\
(0.297)\end{array}$ & $\begin{array}{l}-0.375 \\
(0.259)\end{array}$ & $\begin{array}{l}-0.026 \\
(0.050)\end{array}$ & $\begin{array}{l}0.147 \\
(0.179)\end{array}$ & $\begin{array}{l}0.140 \\
(0.161)\end{array}$ & $\begin{array}{l}0.011 \\
(0.024)\end{array}$ & $\begin{array}{l}0.111 \\
(0.158)\end{array}$ & $\begin{array}{l}0.124 \\
(0.133)\end{array}$ & $\begin{array}{l}0.006 \\
(0.039)\end{array}$ \\
\hline Ind exp - PG marketing (H1b) & $\begin{array}{l}0.013 \\
(0.107)\end{array}$ & $\begin{array}{l}-0.040 \\
(0.099)\end{array}$ & $\begin{array}{l}-0.003 \\
(0.013)\end{array}$ & $\begin{array}{l}0.176 \\
(0.225)\end{array}$ & $\begin{array}{l}0.130 \\
(0.205)\end{array}$ & $\begin{array}{l}0.006 \\
(0.037)\end{array}$ & $\begin{array}{l}-0.078 \\
(0.177)\end{array}$ & $\begin{array}{l}-0.072 \\
(0.160)\end{array}$ & $\begin{array}{l}-0.004 \\
(0.024)\end{array}$ & $\begin{array}{l}0.117 \\
(0.169)\end{array}$ & $\begin{array}{l}0.028 \\
(0.142)\end{array}$ & $\begin{array}{l}0.001 \\
(0.040)\end{array}$ \\
\hline Know-how (Factor) (H2) & $\begin{array}{l}-1.540 * * \\
(0.117)\end{array}$ & $\begin{array}{l}-1.536 * * \\
(0.110)\end{array}$ & $\begin{array}{l}-0.119 * * \\
(0.013)\end{array}$ & $\begin{array}{l}-1.803 * * \\
(0.246)\end{array}$ & $\begin{array}{l}-1.755^{* *} \\
(0.218)\end{array}$ & $\begin{array}{l}-0.131 * * \\
(\mathbf{0 . 0 3 0})\end{array}$ & $\begin{array}{l}-1.619 * * \\
(0.192)\end{array}$ & $\begin{array}{l}-1.635^{* *} \\
(0.173)\end{array}$ & $\begin{array}{l}-0.129 * * \\
(0.024)\end{array}$ & $\begin{array}{l}-1.176^{* *} \\
(0.225)\end{array}$ & $\begin{array}{l}-1.235 * * \\
(0.200)\end{array}$ & $\begin{array}{l}-0.090 \\
(0.059)\end{array}$ \\
\hline Financial resources (H5) & $\begin{array}{l}-0.003 \\
(0.095)\end{array}$ & $\begin{array}{l}0.025 \\
(0.086)\end{array}$ & $\begin{array}{l}-0.0001 \\
(0.012)\end{array}$ & $\begin{array}{l}0.286 \\
(0.250)\end{array}$ & $\begin{array}{l}0.252 \\
(0.227)\end{array}$ & $\begin{array}{l}0.015 \\
(0.044)\end{array}$ & $\begin{array}{l}0.073 \\
(0.148)\end{array}$ & $\begin{array}{l}0.100 \\
(0.131)\end{array}$ & $\begin{array}{l}0.003 \\
(0.019)\end{array}$ & $\begin{array}{l}-0.082 \\
(0.155)\end{array}$ & $\begin{array}{l}-0.043 \\
(0.128)\end{array}$ & $\begin{array}{l}-0.004 \\
(0.035)\end{array}$ \\
\hline Reputation (Factor) (H6) & $\begin{array}{l}-0.203 \\
(0.205)\end{array}$ & $\begin{array}{l}-0.270 \\
(0.188)\end{array}$ & $\begin{array}{l}-0.021 \\
(0.028)\end{array}$ & $\begin{array}{l}0.261 \\
(0.461)\end{array}$ & $\begin{array}{l}0.139 \\
(0.404)\end{array}$ & $\begin{array}{l}0.005 \\
(0.078)\end{array}$ & $\begin{array}{l}-0.374 \\
(0.384)\end{array}$ & $\begin{array}{l}-0.327 \\
(0.343)\end{array}$ & $\begin{array}{l}-0.033 \\
(0.057)\end{array}$ & $\begin{array}{l}-0.200 \\
(0.304)\end{array}$ & $\begin{array}{l}-0.183 \\
(0.250)\end{array}$ & $\begin{array}{l}-0.010 \\
(0.064)\end{array}$ \\
\hline Hofstede cultural distance & $\begin{array}{l}0.162 \\
(0.213)\end{array}$ & $\begin{array}{l}0.021 \\
(0.119)\end{array}$ & $\begin{array}{l}0.0008 \\
(0.022)\end{array}$ & $\begin{array}{l}0.995 * \\
(0.434)\end{array}$ & $\begin{array}{l}0.868 * \\
(0.375)\end{array}$ & $\begin{array}{l}0.057 \\
(0.050)\end{array}$ & $\begin{array}{l}0.191 \\
(0.381)\end{array}$ & $\begin{array}{l}0.003 \\
(0.214)\end{array}$ & $\begin{array}{l}0.002 \\
(0.038)\end{array}$ & $\begin{array}{l}-0.563 \\
(0.351)\end{array}$ & $\begin{array}{l}-0.551 \dagger \\
(0.303)\end{array}$ & $\begin{array}{l}-0.036 \\
(0.085)\end{array}$ \\
\hline IMD Regulative ID (lag 1) & $\begin{array}{l}0.026 \\
(0.163)\end{array}$ & $\begin{array}{l}-0.023 \\
(0.120)\end{array}$ & $\begin{array}{l}-0.0009 \\
(0.021)\end{array}$ & $\begin{array}{l}0.064 \\
(0.299)\end{array}$ & $\begin{array}{l}0.071 \\
(0.263)\end{array}$ & $\begin{array}{l}0.005 \\
(0.055)\end{array}$ & $\begin{array}{l}0.112 \\
(0.294)\end{array}$ & $\begin{array}{l}-0.006 \\
(0.214)\end{array}$ & $\begin{array}{l}-0.002 \\
(0.029)\end{array}$ & $\begin{array}{l}-0.292 \\
(0.322)\end{array}$ & $\begin{array}{l}-0.238 \\
(0.280)\end{array}$ & $\begin{array}{l}-0.011 \\
(0.110)\end{array}$ \\
\hline Age & $\begin{array}{l}0.004 \\
(0.003)\end{array}$ & $\begin{array}{l}0.002 \\
(0.003)\end{array}$ & $\begin{array}{l}0.0001 \\
(0.0004)\end{array}$ & $\begin{array}{l}-0.006 \\
(0.011)\end{array}$ & $\begin{array}{l}-0.003 \\
(0.008)\end{array}$ & $\begin{array}{l}-0.0002 \\
(0.001)\end{array}$ & $\begin{array}{l}0.002 \\
(0.006)\end{array}$ & $\begin{array}{l}0.002 \\
(0.005)\end{array}$ & $\begin{array}{l}0.0002 \\
(0.0007)\end{array}$ & $\begin{array}{l}0.005 \\
(0.004)\end{array}$ & $\begin{array}{l}0.002 \\
(0.003)\end{array}$ & $\begin{array}{l}0.0001 \\
(0.0009)\end{array}$ \\
\hline Size (Staff) & $\begin{array}{l}0.000 \\
(0.000)\end{array}$ & $\begin{array}{l}0.0001 \\
(0.0001)\end{array}$ & $\begin{array}{l}0.814 \mathrm{E}-5 \\
(0.173 \mathrm{E}-4)\end{array}$ & - & $\begin{array}{l}-0.0001 \\
(0.0003)\end{array}$ & $\begin{array}{l}-0.840 \mathrm{E}-5 \\
(0.636 \mathrm{E}-4)\end{array}$ & $\begin{array}{l}0.000 \\
(0.000)\end{array}$ & $\begin{array}{l}-0.757 \mathrm{E}-4 \\
(0.0002)\end{array}$ & $\begin{array}{l}-0.499 \mathrm{E}-5 \\
(0.278 \mathrm{E}-4)\end{array}$ & $\begin{array}{l}3.589 \mathrm{E}-5 \\
(0.000)\end{array}$ & $\begin{array}{l}0.495 E-4 \\
(0.0001)\end{array}$ & $\begin{array}{l}0.508 E-5 \\
(0.523 E-4)\end{array}$ \\
\hline Offshore policy & $\begin{array}{l}1.375^{*} \\
(0.662)\end{array}$ & $\begin{array}{l}1.380 * \\
(0.630)\end{array}$ & $\begin{array}{l}0.113 \dagger \\
(0.062)\end{array}$ & $\begin{array}{l}0.149 \\
(1.475)\end{array}$ & $\begin{array}{l}0.201 \\
(1.313)\end{array}$ & $\begin{array}{l}0.026 \\
(0.225)\end{array}$ & $\begin{array}{l}2.307 * \\
(1.079)\end{array}$ & $\begin{array}{l}2.238 * \\
(0.962)\end{array}$ & $\begin{array}{l}0.218^{*} \\
(0.092)\end{array}$ & $\begin{array}{l}\text { 2.462* } \\
(1.133)\end{array}$ & $\begin{array}{l}\text { 2.439* } \\
(1.009)\end{array}$ & $\begin{array}{l}0.168 \\
(0.317)\end{array}$ \\
\hline $\begin{array}{l}\text { Internationalisation at home } \\
\text { strategy }\end{array}$ & $\begin{array}{l}0.041 \\
(0.127)\end{array}$ & $\begin{array}{l}-0.0006 \\
(0.0007)\end{array}$ & $\begin{array}{l}-0.409 \mathrm{E}-4 \\
(0.0001)\end{array}$ & $\begin{array}{l}0.209 \\
(0.321)\end{array}$ & $\begin{array}{l}-0.001 \\
(0.001)\end{array}$ & $\begin{array}{l}-0.686 \mathrm{E}-4 \\
(0.0002)\end{array}$ & $\begin{array}{l}-0.047 \\
(0.208)\end{array}$ & $\begin{array}{l}-0.0007 \\
(0.002)\end{array}$ & $\begin{array}{l}-0.472 \mathrm{E}-4 \\
(0.0002)\end{array}$ & $\begin{array}{l}0.022 \\
(0.209)\end{array}$ & $\begin{array}{l}0.0003 \\
(0.0008)\end{array}$ & $\begin{array}{l}0.117 \mathrm{E}-4 \\
(0.0001)\end{array}$ \\
\hline Host country demand & $\begin{array}{l}1.537 \\
(1.790)\end{array}$ & $\begin{array}{l}1.770 \\
(1.641)\end{array}$ & $\begin{array}{l}0.123 \\
(0.226)\end{array}$ & $\begin{array}{l}6.327 \\
(3.858)\end{array}$ & $\begin{array}{l}8.652 * * \\
(3.255)\end{array}$ & $\begin{array}{l}0.546 \\
(0.625)\end{array}$ & $\begin{array}{l}-3.825 \\
(3.143)\end{array}$ & $\begin{array}{l}-3.672 \\
(2.846)\end{array}$ & $\begin{array}{l}-0.240 \\
(0.418)\end{array}$ & $\begin{array}{l}5.213 \dagger \\
(2.835)\end{array}$ & $\begin{array}{l}4.004 \dagger \\
(2.245)\end{array}$ & $\begin{array}{l}0.241 \\
(0.578)\end{array}$ \\
\hline AU/NZ dummy & $\begin{array}{l}0.252 \\
(0.535)\end{array}$ & $\begin{array}{l}0.416 \\
(0.485)\end{array}$ & $\begin{array}{l}0.024 \\
(0.065)\end{array}$ & - & - & s. & - & - & - & - & - & - \\
\hline UK/IR dummy & $\begin{array}{l}0.102 \\
(0.492)\end{array}$ & $\begin{array}{l}0.158 \\
(0.456)\end{array}$ & $\begin{array}{l}0.008 \\
(0.064)\end{array}$ & - & - & - & - & - & - & - & - & - \\
\hline
\end{tabular}




\begin{tabular}{|c|c|c|c|c|c|c|c|c|c|c|c|c|}
\hline $\mathrm{R}^{2}$ & 0.573 & & & 0.619 & & & 0.672 & & & 0.510 & & \\
\hline $\operatorname{AdjR}^{2}$ & 0.538 & & & 0.490 & & & 0.606 & & & 0.378 & & \\
\hline Log likelihood & & -740.86 & -809.52 & & -187.43 & -209.03 & & -302.14 & -330.40 & & -223.48 & -258.47 \\
\hline $\mathrm{N}$ & 308 & 308 & 308 & 80 & 80 & 80 & 124 & 124 & 124 & 104 & 104 & 104 \\
\hline Max. VIF & 2.033 & & & 2.213 & & & 2.172 & & & 1.671 & & \\
\hline
\end{tabular}

Standard errors in parentheses, all $t$ tests are two-tailed, $\dagger p<.10, * p<.05, * * p<.01$

Table 7.27: Regression estimates for equation 12

\begin{tabular}{|c|c|c|c|c|c|c|c|c|c|c|c|c|}
\hline & \multicolumn{3}{|c|}{ "Complete data set } & \multicolumn{3}{|c|}{$\overline{A U} / \mathbf{N Z}$} & \multicolumn{3}{|c|}{ 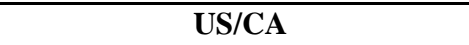 } & \multicolumn{3}{|c|}{ 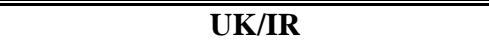 } \\
\hline & OLS & Tobit & $\begin{array}{l}\text { Negative } \\
\text { binomial }\end{array}$ & OLS & Tobit & $\begin{array}{l}\text { Negative } \\
\text { binomial }\end{array}$ & OLS & Tobit & $\begin{array}{l}\text { Negative } \\
\text { binomial }\end{array}$ & OLS & Tobit & $\begin{array}{l}\text { Negative } \\
\text { binomial }\end{array}$ \\
\hline Intercept & $\begin{array}{l}20.327 * * \\
(3.319)\end{array}$ & $\begin{array}{l}22.700 * * \\
(2.958)\end{array}$ & $\begin{array}{l}3.264 * * \\
(0.367)\end{array}$ & $\begin{array}{l}18.648 * \\
(6.411)\end{array}$ & $\begin{array}{l}21.147 * * \\
(5.244)\end{array}$ & $\begin{array}{l}3.203 * * \\
(0.825)\end{array}$ & $\begin{array}{l}20.078 * * \\
(6.343)\end{array}$ & $\begin{array}{l}20.687 * * \\
(5.489)\end{array}$ & $\begin{array}{l}3.122 * * \\
(0.661)\end{array}$ & $\begin{array}{l}26.836 * * \\
(5.565)\end{array}$ & $\begin{array}{l}25.862 * * \\
(4.614)\end{array}$ & $\begin{array}{l}3.401 * * \\
\text { (1.123) }\end{array}$ \\
\hline $\begin{array}{l}\text { Geo exp - } \\
\text { knowledge of edu } \\
\text { sector (HIa) }\end{array}$ & $\begin{array}{l}-0.060 \\
(0.126)\end{array}$ & $\begin{array}{l}-0.064 \\
(0.116)\end{array}$ & $\begin{array}{l}-0.005 \\
(0.015)\end{array}$ & $\begin{array}{l}0.358 \\
(0.282)\end{array}$ & $\begin{array}{l}0.354 \\
(0.241)\end{array}$ & $\begin{array}{l}0.020 \\
(0.041)\end{array}$ & $\begin{array}{l}-0.096 \\
(0.225)\end{array}$ & $\begin{array}{l}-0.106 \\
(0.202)\end{array}$ & $\begin{array}{l}-0.009 \\
(0.237)\end{array}$ & $\begin{array}{l}-0.195 \\
(0.214)\end{array}$ & $\begin{array}{l}-0.149 \\
(0.167)\end{array}$ & $\begin{array}{l}-0.009 \\
(0.045)\end{array}$ \\
\hline $\begin{array}{l}\text { Geo exp - academic } \\
\text { capabilities }(H 1 a)\end{array}$ & $\begin{array}{l}0.124 \\
(0.121)\end{array}$ & $\begin{array}{l}0.064 \\
(0.112)\end{array}$ & $\begin{array}{l}0.005 \\
(0.012)\end{array}$ & $\begin{array}{l}0.686^{*} \\
(0.274)\end{array}$ & $\begin{array}{l}0.634 * * \\
(0.236)\end{array}$ & $\begin{array}{l}0.039 \\
(0.048)\end{array}$ & $\begin{array}{l}0.034 \\
(0.223)\end{array}$ & $\begin{array}{l}0.037 \\
(0.200)\end{array}$ & $\begin{array}{l}0.002 \\
(0.020)\end{array}$ & $\begin{array}{l}-0.018 \\
(0.188)\end{array}$ & $\begin{array}{l}-0.079 \\
(0.155)\end{array}$ & $\begin{array}{l}-0.003 \\
(0.033)\end{array}$ \\
\hline $\begin{array}{l}\text { Geo exp - } \\
\text { managerial } \\
\text { capabilities }(\mathrm{H} 1 \mathrm{a})\end{array}$ & $\begin{array}{l}0.164 \\
(0.145)\end{array}$ & $\begin{array}{l}0.164 \\
(0.138)\end{array}$ & $\begin{array}{l}0.009 \\
(0.017)\end{array}$ & $\begin{array}{l}0.054 \\
(0.314)\end{array}$ & $\begin{array}{l}0.092 \\
(0.274)\end{array}$ & $\begin{array}{l}0.006 \\
(0.045)\end{array}$ & $\begin{array}{l}0.403 \\
(0.265)\end{array}$ & $\begin{array}{l}0.413 \dagger \\
(0.238)\end{array}$ & $\begin{array}{l}0.026 \\
(0.028)\end{array}$ & $\begin{array}{l}-0.143 \\
(0.231)\end{array}$ & $\begin{array}{l}-0.085 \\
(0.199)\end{array}$ & $\begin{array}{l}-0.006 \\
(0.044)\end{array}$ \\
\hline $\begin{array}{l}\text { Geo exp - financial } \\
\text { capabilities (H1a) }\end{array}$ & $\begin{array}{l}-0.003 \\
(0.138)\end{array}$ & $\begin{array}{l}-0.048 \\
(0.127)\end{array}$ & $\begin{array}{l}-0.003 \\
(0.016)\end{array}$ & $\begin{array}{l}-0.256 \\
(0.311)\end{array}$ & $\begin{array}{l}-0.497 \dagger \\
(0.256)\end{array}$ & $\begin{array}{l}-0.035 \\
(0.041)\end{array}$ & $\begin{array}{l}0.116 \\
(0.229)\end{array}$ & $\begin{array}{l}0.117 \\
(0.206)\end{array}$ & $\begin{array}{l}0.008 \\
(0.023)\end{array}$ & $\begin{array}{l}-0.441 \dagger \\
(0.241)\end{array}$ & $\begin{array}{l}-0.376 \dagger \\
(0.204)\end{array}$ & $\begin{array}{l}-0.024 \\
(0.047)\end{array}$ \\
\hline $\begin{array}{l}\text { Geo exp - intl } \\
\text { recruitment }(\mathrm{H} 1 \mathrm{a})\end{array}$ & $\begin{array}{l}-0.192 \\
(0.152)\end{array}$ & $\begin{array}{l}-0.180 \\
(0.143)\end{array}$ & $\begin{array}{l}-0.011 \\
(0.017)\end{array}$ & $\begin{array}{l}0.115 \\
(0.288)\end{array}$ & $\begin{array}{l}0.202 \\
(0.261)\end{array}$ & $\begin{array}{l}0.013 \\
(0.043)\end{array}$ & $\begin{array}{l}-0.618^{*} \\
(0.287)\end{array}$ & $\begin{array}{l}-0.598^{*} \\
(0.255)\end{array}$ & $\begin{array}{l}-0.043 \\
(0.030)\end{array}$ & $\begin{array}{l}0.055 \\
(0.248)\end{array}$ & $\begin{array}{l}0.075 \\
(0.212)\end{array}$ & $\begin{array}{l}0.005 \\
(0.054)\end{array}$ \\
\hline $\begin{array}{l}\text { Ind exp - UG } \\
\text { offshore edu (H1b) }\end{array}$ & $\begin{array}{l}0.030 \\
(0.118)\end{array}$ & $\begin{array}{l}0.006 \\
(0.110)\end{array}$ & $\begin{array}{l}0.0005 \\
(0.012)\end{array}$ & $\begin{array}{l}-0.304 \\
(0.252)\end{array}$ & $\begin{array}{l}-0.394 \dagger \\
(0.221)\end{array}$ & $\begin{array}{l}-0.020 \\
(0.045)\end{array}$ & $\begin{array}{l}0.053 \\
(0.232)\end{array}$ & $\begin{array}{l}0.052 \\
(0.209)\end{array}$ & $\begin{array}{l}0.005 \\
(0.023)\end{array}$ & $\begin{array}{l}0.025 \\
(0.189)\end{array}$ & $\begin{array}{l}0.076 \\
(0.157)\end{array}$ & $\begin{array}{l}0.003 \\
(0.037)\end{array}$ \\
\hline $\begin{array}{l}\text { Ind exp - PG } \\
\text { offshore edu (H1b) }\end{array}$ & $\begin{array}{l}-0.037 \\
(0.133)\end{array}$ & $\begin{array}{l}-0.035 \\
(0.125)\end{array}$ & $\begin{array}{l}-0.002 \\
(0.014)\end{array}$ & $\begin{array}{l}-0.042 \\
(0.279)\end{array}$ & $\begin{array}{l}0.065 \\
(0.244)\end{array}$ & $\begin{array}{l}0.002 \\
(0.039)\end{array}$ & $\begin{array}{l}0.007 \\
(0.235)\end{array}$ & $\begin{array}{l}0.018 \\
(0.210)\end{array}$ & $\begin{array}{l}0.001 \\
(0.023)\end{array}$ & $\begin{array}{l}-0.056 \\
(0.232)\end{array}$ & $\begin{array}{l}-0.073 \\
(0.197)\end{array}$ & $\begin{array}{l}-0.003 \\
(0.047)\end{array}$ \\
\hline $\begin{array}{l}\text { Ind exp - intl } \\
\text { recruitment (Factor) } \\
\text { (H1b) }\end{array}$ & $\begin{array}{l}0.264 \dagger \\
(0.154)\end{array}$ & $\begin{array}{l}0.225 \\
(0.141)\end{array}$ & $\begin{array}{l}0.014 \\
(0.018)\end{array}$ & $\begin{array}{l}-0.059 \\
(0.332)\end{array}$ & $\begin{array}{l}-0.207 \\
(0.273)\end{array}$ & $\begin{array}{l}-0.015 \\
(0.044)\end{array}$ & $\begin{array}{l}0.368 \\
(0.285)\end{array}$ & $\begin{array}{l}0.344 \\
(0.255)\end{array}$ & $\begin{array}{l}0.025 \\
(0.028)\end{array}$ & $\begin{array}{l}0.336 \\
(0.255)\end{array}$ & $\begin{array}{l}0.286 \\
(0.199)\end{array}$ & $\begin{array}{l}0.017 \\
(0.045)\end{array}$ \\
\hline $\begin{array}{l}\text { Ind exp - UG } \\
\text { marketing }(H 1 b)\end{array}$ & $\begin{array}{l}0.028 \\
(0.118)\end{array}$ & $\begin{array}{l}0.031 \\
(0.110)\end{array}$ & $\begin{array}{l}0.0011 \\
(0.015)\end{array}$ & $\begin{array}{l}-0.264 \\
(0.312)\end{array}$ & $\begin{array}{l}-0.200 \\
(0.268)\end{array}$ & $\begin{array}{l}-0.013 \\
(0.049)\end{array}$ & $\begin{array}{l}0.245 \\
(0.201)\end{array}$ & $\begin{array}{l}0.247 \\
(0.180)\end{array}$ & $\begin{array}{l}0.017 \\
(0.022)\end{array}$ & $\begin{array}{l}0.046 \\
(0.178)\end{array}$ & $\begin{array}{l}0.074 \\
(0.146)\end{array}$ & $\begin{array}{l}0.003 \\
(0.038)\end{array}$ \\
\hline
\end{tabular}




\begin{tabular}{|c|c|c|c|c|c|c|c|c|c|c|c|c|}
\hline $\begin{array}{l}\text { Ind exp - PG } \\
\text { marketing }(H 1 b)\end{array}$ & $\begin{array}{l}0.034 \\
(0.115)\end{array}$ & $\begin{array}{l}-0.030 \\
(0.107)\end{array}$ & $\begin{array}{l}-0.002 \\
(0.012)\end{array}$ & $\begin{array}{l}0.296 \\
(0.235)\end{array}$ & $\begin{array}{l}0.302 \\
(0.211)\end{array}$ & $\begin{array}{l}0.019 \\
(0.036)\end{array}$ & $\begin{array}{l}-0.158 \\
(0.197)\end{array}$ & $\begin{array}{l}-0.159 \\
(0.177)\end{array}$ & $\begin{array}{l}-0.011 \\
(0.019)\end{array}$ & $\begin{array}{l}0.168 \\
(0.187)\end{array}$ & $\begin{array}{l}0.090 \\
(0.156)\end{array}$ & $\begin{array}{l}0.005 \\
(0.042)\end{array}$ \\
\hline $\begin{array}{l}\text { Org culture (Factor) } \\
-(\mathrm{H} 3)\end{array}$ & $\begin{array}{l}-1.877 * * \\
(0.176)\end{array}$ & $\begin{array}{l}-1.810^{* *} \\
(0.163)\end{array}$ & $\begin{array}{l}-0.132 * * \\
(0.019)\end{array}$ & $\begin{array}{l}-2.278 * * \\
(0.336)\end{array}$ & $\begin{array}{l}-2.260 * * \\
(0.293)\end{array}$ & $\begin{array}{l}-0.163^{* *} \\
(0.040)\end{array}$ & $\begin{array}{l}-1.958 * * \\
(0.320)\end{array}$ & $\begin{array}{l}-1.992^{* *} \\
(0.286)\end{array}$ & $\begin{array}{l}-0.151 * * \\
(0.033)\end{array}$ & (1) & (1) & $(0,0.6$ \\
\hline $\begin{array}{l}\text { Org culture - open } \\
\text { discussion }(H 3)\end{array}$ & - & - & - & - & - & - & - & - & - & $\begin{array}{l}-0.439 \dagger \\
(0.239)\end{array}$ & $\begin{array}{l}-0.499 * \\
(0.200)\end{array}$ & $\begin{array}{l}-0.032 \\
(0.046)\end{array}$ \\
\hline $\begin{array}{l}\text { Org culture - no } \\
\text { status distinction } \\
\text { (H3) }\end{array}$ & - & - & - & - & - & - & - & - & - & $\begin{array}{l}-0.420 \dagger \\
(0.244)\end{array}$ & $\begin{array}{l}-0.322 \\
(0.201)\end{array}$ & $\begin{array}{l}-0.022 \\
(0.048)\end{array}$ \\
\hline $\begin{array}{l}\text { Org culture - } \\
\text { experimentation } \\
\text { (H3) }\end{array}$ & - & - & - & - & - & - & - & - & - & $\begin{array}{l}-0.009 \\
(0.227)\end{array}$ & $\begin{array}{l}-0.136 \\
(0.179)\end{array}$ & $\begin{array}{l}-0.009 \\
(0.046)\end{array}$ \\
\hline $\begin{array}{l}\text { Org culture - } \\
\text { offshore } \\
\text { development (H3) }\end{array}$ & - & - & - & - & - & - & - & - & - & $\begin{array}{l}-0.466 * \\
(0.232)\end{array}$ & $\begin{array}{l}-0.362 \dagger \\
(0.185)\end{array}$ & $\begin{array}{l}-0.022 \\
(0.047)\end{array}$ \\
\hline $\begin{array}{l}\text { Org culture - } \\
\text { offshore } \\
\text { responsiveness }(H 3)\end{array}$ & - & - & - & - & - & - & - & - & - & $\begin{array}{l}-0.091 \\
(0.226)\end{array}$ & $\begin{array}{l}0.021 \\
(0.186)\end{array}$ & $\begin{array}{l}-0.0002 \\
(0.042)\end{array}$ \\
\hline $\begin{array}{l}\text { Financial resources } \\
\text { (H5) }\end{array}$ & $\begin{array}{l}0.092 \\
(0.104)\end{array}$ & $\begin{array}{l}0.090 \\
(0.094)\end{array}$ & $\begin{array}{l}0.005 \\
(0.011)\end{array}$ & $\begin{array}{l}0.145 \\
(0.255)\end{array}$ & $\begin{array}{l}0.098 \\
(0.228)\end{array}$ & $\begin{array}{l}0.004 \\
(0.041)\end{array}$ & $\begin{array}{l}0.171 \\
(0.169)\end{array}$ & $\begin{array}{l}0.196 \\
(0.148)\end{array}$ & $\begin{array}{l}0.009 \\
(0.015)\end{array}$ & $\begin{array}{l}0.034 \\
(0.173)\end{array}$ & $\begin{array}{l}0.003 \\
(0.140)\end{array}$ & $\begin{array}{l}-0.0001 \\
(0.039)\end{array}$ \\
\hline $\begin{array}{l}\text { Reputation (Factor) } \\
\text { (H6) }\end{array}$ & $\begin{array}{l}-0.377 \dagger \\
(0.220)\end{array}$ & $\begin{array}{l}-0.506^{*} \\
(0.200)\end{array}$ & $\begin{array}{l}-0.035 \\
(0.026)\end{array}$ & $\begin{array}{l}-0.162 \\
(0.464)\end{array}$ & $\begin{array}{l}-0.148 \\
(0.403)\end{array}$ & $\begin{array}{l}-0.014 \\
(0.073)\end{array}$ & $\begin{array}{l}-0.564 \\
(0.448)\end{array}$ & $\begin{array}{l}-0.522 \\
(0.396)\end{array}$ & $\begin{array}{l}-0.040 \\
(0.055)\end{array}$ & $\begin{array}{l}-0.390 \\
(0.334)\end{array}$ & $\begin{array}{l}-0.470 \dagger \\
(0.266)\end{array}$ & $\begin{array}{l}-0.028 \\
(0.072)\end{array}$ \\
\hline $\begin{array}{l}\text { Hofstede cultural } \\
\text { distance }\end{array}$ & $\begin{array}{l}0.253 \\
(0.230)\end{array}$ & $\begin{array}{l}0.068 \\
(0.129)\end{array}$ & $\begin{array}{l}0.004 \\
(0.019)\end{array}$ & $\begin{array}{l}0.918^{*} \\
(0.449)\end{array}$ & $\begin{array}{l}0.850 * \\
(0.382)\end{array}$ & $\begin{array}{l}0.058 \\
(0.052)\end{array}$ & $\begin{array}{l}0.401 \\
(0.428)\end{array}$ & $\begin{array}{l}0.185 \\
(0.245)\end{array}$ & $\begin{array}{l}0.016 \\
(0.038)\end{array}$ & $\begin{array}{l}-0.421 \\
(0.401)\end{array}$ & $\begin{array}{l}-0.476 \\
(0.339)\end{array}$ & $\begin{array}{l}-0.031 \\
(0.088)\end{array}$ \\
\hline $\begin{array}{l}\text { IMD Regulative ID } \\
\text { (lag 1) }\end{array}$ & $\begin{array}{l}-0.010 \\
(0.175)\end{array}$ & $\begin{array}{l}-0.070 \\
(0.130)\end{array}$ & $\begin{array}{l}-0.004 \\
(0.019)\end{array}$ & $\begin{array}{l}0.152 \\
(0.309)\end{array}$ & $\begin{array}{l}0.181 \\
(0.268)\end{array}$ & $\begin{array}{l}0.014 \\
(0.051)\end{array}$ & $\begin{array}{l}-0.041 \\
(0.336)\end{array}$ & $\begin{array}{l}-0.187 \\
(0.245)\end{array}$ & $\begin{array}{l}-0.016 \\
(0.035)\end{array}$ & $\begin{array}{l}-0.604 \\
(0.368)\end{array}$ & $\begin{array}{l}-0.476 \\
(0.311)\end{array}$ & $\begin{array}{l}-0.028 \\
(0.068)\end{array}$ \\
\hline Age & $\begin{array}{l}0.003 \\
(0.004)\end{array}$ & $\begin{array}{l}0.002 \\
(0.003)\end{array}$ & $\begin{array}{l}0.001 \\
(0.0004)\end{array}$ & $\begin{array}{l}0.001 \\
(0.012)\end{array}$ & $\begin{array}{l}0.005 \\
(0.008)\end{array}$ & $\begin{array}{l}0.0004 \\
(0.001)\end{array}$ & $\begin{array}{l}0.003 \\
(0.007)\end{array}$ & $\begin{array}{l}0.002 \\
(0.005)\end{array}$ & $\begin{array}{l}0.0003 \\
(0.0006)\end{array}$ & $\begin{array}{l}0.001 \\
(0.005)\end{array}$ & $\begin{array}{l}-0.0004 \\
(0.004)\end{array}$ & $\begin{array}{l}-0.330 \mathrm{E}-4 \\
(0.001)\end{array}$ \\
\hline Size (Staff) & $\begin{array}{l}1.116 \mathrm{E}-5 \\
(0.000)\end{array}$ & $\begin{array}{l}0.168 \mathrm{E}-4 \\
(0.0001)\end{array}$ & $\begin{array}{l}0.129 E-5 \\
(0.185 E-4)\end{array}$ & - & $(2000)$ & - & $\begin{array}{l}0.000 \\
(0.000)\end{array}$ & $\begin{array}{l}-0.0001 \\
(0.0002)\end{array}$ & $\begin{array}{l}-0.111 E-4 \\
(0.263 E-4)\end{array}$ & $\begin{array}{l}-0.000 \\
(0.000)\end{array}$ & $\begin{array}{l}-0.308 \mathrm{E}-4 \\
(0.0001)\end{array}$ & $\begin{array}{l}-0.150 \mathrm{E}-5 \\
(0.589 \mathrm{E}-4)\end{array}$ \\
\hline Offshore policy & $\begin{array}{l}\text { 1.651* } \\
(0.714)\end{array}$ & $\begin{array}{l}\text { 1.735* } \\
(0.680)\end{array}$ & $\begin{array}{l}0.143^{*} \\
(0.058)\end{array}$ & $\begin{array}{l}0.725 \\
(1.509)\end{array}$ & $\begin{array}{l}0.586 \\
(1.325)\end{array}$ & $\begin{array}{l}0.053 \\
(0.277)\end{array}$ & $\begin{array}{l}\text { 2.859* } \\
\text { (1.199) }\end{array}$ & $\begin{array}{l}2.719 * \\
(1.067)\end{array}$ & $\begin{array}{l}0.251 * * \\
(0.083)\end{array}$ & $\begin{array}{l}2.320 \dagger \\
(1.274)\end{array}$ & $\begin{array}{l}\text { 2.425* } \\
(1.115)\end{array}$ & $\begin{array}{l}0.167 \\
(0.287)\end{array}$ \\
\hline $\begin{array}{l}\text { Internationalisation } \\
\text { at home strategy }\end{array}$ & $\begin{array}{l}0.019 \\
(0.137)\end{array}$ & $\begin{array}{l}0.0002 \\
(0.0008)\end{array}$ & $\begin{array}{l}0.256 \mathrm{E}-4 \\
(0.0001)\end{array}$ & $\begin{array}{l}-0.022 \\
(0.334)\end{array}$ & $\begin{array}{l}-0.0001 \\
(0.001)\end{array}$ & $\begin{array}{l}0.130 \mathrm{E}-4 \\
(0.0002)\end{array}$ & $\begin{array}{l}0.037 \\
(0.233)\end{array}$ & $\begin{array}{l}-0.0004 \\
(0.002)\end{array}$ & $\begin{array}{l}-0.258 \\
(0.0003)\end{array}$ & $\begin{array}{l}-0.138 \\
(0.241)\end{array}$ & $\begin{array}{l}0.001 \\
(0.0009)\end{array}$ & $\begin{array}{l}0.703 E-4 \\
(0.0001)\end{array}$ \\
\hline $\begin{array}{l}\text { Host country } \\
\text { demand }\end{array}$ & $\begin{array}{l}1.401 \\
(1.932)\end{array}$ & $\begin{array}{l}1.621 \\
(1.778)\end{array}$ & $\begin{array}{l}0.099 \\
(0.210)\end{array}$ & $\begin{array}{l}7.774 \dagger \\
\mathbf{( 4 . 0 1 4 )}\end{array}$ & $\begin{array}{l}9.236 * * \\
(3.323)\end{array}$ & $\begin{array}{l}0.587 \\
(0.586)\end{array}$ & $\begin{array}{l}-2.942 \\
(3.517)\end{array}$ & $\begin{array}{l}-2.860 \\
(3.172)\end{array}$ & $\begin{array}{l}-0.202 \\
(0.341)\end{array}$ & $\begin{array}{l}3.754 \\
(3.314)\end{array}$ & $\begin{array}{l}3.010 \\
(2.538)\end{array}$ & $\begin{array}{l}0.167 \\
(0.612)\end{array}$ \\
\hline AU/NZ dummy & $\begin{array}{l}0.535 \\
(0.575)\end{array}$ & $\begin{array}{l}0.669 \\
(0.523)\end{array}$ & $\begin{array}{l}0.044 \\
(0.064)\end{array}$ & - & (- & - & (2) & - & - & (20) & - & - \\
\hline UK/IR dummy & $\begin{array}{l}0.341 \\
(0.527)\end{array}$ & $\begin{array}{l}0.340 \\
(0.492)\end{array}$ & $\begin{array}{l}0.0227 \\
(0.062)\end{array}$ & - & 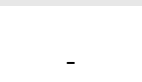 & - & - & - & - & - & - & - \\
\hline
\end{tabular}




\begin{tabular}{|c|c|c|c|c|c|c|c|c|c|c|c|c|}
\hline $\mathrm{R}^{2}$ & 0.505 & & & 0.590 & & & 0.592 & & & 0.449 & & \\
\hline $\operatorname{Adj}^{2}$ & 0.465 & & & 0.452 & & & 0.510 & & & 0.260 & & \\
\hline Log likelihood & & -764.42 & -825.42 & & -188.85 & -210.12 & & -315.12 & -340.29 & & -230.64 & -261.14 \\
\hline $\mathrm{N}$ & 308 & 308 & 308 & 80 & 80 & 80 & 124 & 124 & 124 & 104 & 104 & 104 \\
\hline Max. VIF & 2.048 & & & 2.153 & & & 2.518 & & & 1.827 & & \\
\hline
\end{tabular}

Standard errors in parentheses, all $t$ tests are two-tailed, $\dagger p<.10, * p<.05, * * p<.01$

Table 7.28a: Regression estimates for equation 13a (low normative institutional distance)

\begin{tabular}{|c|c|c|c|c|c|c|c|c|c|c|c|c|}
\hline & \multicolumn{3}{|c|}{ Complete data set } & \multicolumn{3}{|c|}{ AU/NZ } & \multicolumn{3}{|c|}{ US/CA } & \multicolumn{3}{|c|}{ UK/IR } \\
\hline & OLS & Tobit & $\begin{array}{l}\text { Negative } \\
\text { binomial }\end{array}$ & OLS & Tobit & $\begin{array}{l}\text { Negative } \\
\text { binomial }\end{array}$ & OLS & Tobit & $\begin{array}{l}\text { Negative } \\
\text { binomial }\end{array}$ & OLS & Tobit & $\begin{array}{l}\text { Negative } \\
\text { binomial }\end{array}$ \\
\hline Intercept & $\begin{array}{l}14.590 * * \\
(4.191)\end{array}$ & $\begin{array}{l}16.027 * * \\
(3.884)\end{array}$ & $\begin{array}{l}2.761 * * \\
(0.310)\end{array}$ & $\begin{array}{l}11.207 \\
(18.020)\end{array}$ & $\begin{array}{l}9.674 \\
(13.486)\end{array}$ & $\begin{array}{l}2.518 * \\
(1.132)\end{array}$ & $\begin{array}{l}14.615 * \\
(6.294)\end{array}$ & $\begin{array}{l}17.046 * * \\
(5.712)\end{array}$ & $\begin{array}{l}2.830 * * \\
(0.405)\end{array}$ & $\begin{array}{l}20.829 * * \\
(4.797)\end{array}$ & $\begin{array}{l}21.087 * * \\
(4.154)\end{array}$ & $\begin{array}{l}3.052 * * \\
(0.841)\end{array}$ \\
\hline $\begin{array}{l}\text { Geo exp - } \\
\text { knowledge of edu } \\
\text { sector }(H 7 a)\end{array}$ & $\begin{array}{l}-0.220 \\
(0.196)\end{array}$ & $\begin{array}{l}-0.273 \\
(0.185)\end{array}$ & $\begin{array}{l}-0.016 \\
(0.015)\end{array}$ & $\begin{array}{l}0.992 \\
(0.876)\end{array}$ & $\begin{array}{l}1.003 \\
(0.649)\end{array}$ & $\begin{array}{l}0.057 \\
(0.063)\end{array}$ & $\begin{array}{l}-0.346 \\
(0.292)\end{array}$ & $\begin{array}{l}-0.349 \\
(0.275)\end{array}$ & $\begin{array}{l}-0.022 \\
(0.022)\end{array}$ & $\begin{array}{l}-0.344 \\
(0.262)\end{array}$ & $\begin{array}{c}-0.443^{*} \\
(0.210)\end{array}$ & $\begin{array}{l}-0.025 \\
(0.052)\end{array}$ \\
\hline $\begin{array}{l}\text { Geo exp - academic } \\
\text { capabilities }(H 7 a)\end{array}$ & $\begin{array}{l}0.085 \\
(0.206)\end{array}$ & $\begin{array}{l}0.119 \\
(0.195)\end{array}$ & $\begin{array}{l}0.007 \\
(0.014)\end{array}$ & $\begin{array}{l}-0.224 \\
(1.004)\end{array}$ & $\begin{array}{l}-0.245 \\
(0.719)\end{array}$ & $\begin{array}{l}-0.017 \\
(0.071)\end{array}$ & $\begin{array}{l}0.250 \\
(0.310)\end{array}$ & $\begin{array}{l}0.236 \\
(0.292)\end{array}$ & $\begin{array}{l}0.014 \\
(0.020)\end{array}$ & $\begin{array}{l}0.075 \\
(0.230)\end{array}$ & $\begin{array}{l}0.173 \\
(0.201)\end{array}$ & $\begin{array}{l}0.010 \\
(0.046)\end{array}$ \\
\hline $\begin{array}{l}\text { Geo exp - } \\
\text { managerial } \\
\text { capabilities }(H 7 a)\end{array}$ & $\begin{array}{l}0.145 \\
(0.242)\end{array}$ & $\begin{array}{l}0.120 \\
(0.232)\end{array}$ & $\begin{array}{l}0.007 \\
(0.021)\end{array}$ & $\begin{array}{l}-0.380 \\
(1.024)\end{array}$ & $\begin{array}{l}-0.192 \\
(0.860)\end{array}$ & $\begin{array}{l}-0.023 \\
(0.086)\end{array}$ & $\begin{array}{l}0.535 \\
(0.369)\end{array}$ & $\begin{array}{l}0.579 \dagger \\
(0.346)\end{array}$ & $\begin{array}{l}0.036 \\
(0.026)\end{array}$ & $\begin{array}{l}-0.344 \\
(0.277)\end{array}$ & $\begin{array}{l}-0.386 \\
(0.247)\end{array}$ & $\begin{array}{l}-0.022 \\
(0.064)\end{array}$ \\
\hline $\begin{array}{l}\text { Geo exp - financial } \\
\text { capabilities }(H 7 a)\end{array}$ & $\begin{array}{l}-0.388 \\
(0.242)\end{array}$ & $\begin{array}{l}-0.314 \\
(0.226)\end{array}$ & $\begin{array}{l}-0.018 \\
(0.021)\end{array}$ & $\begin{array}{l}-1.879 \\
(1.457)\end{array}$ & $\begin{array}{l}-1.895 \dagger \\
(1.055)\end{array}$ & $\begin{array}{l}-0.127 \\
(0.107)\end{array}$ & $\begin{array}{l}-0.249 \\
(0.347)\end{array}$ & $\begin{array}{l}-0.213 \\
(0.326)\end{array}$ & $\begin{array}{l}-0.013 \\
(0.031)\end{array}$ & $\begin{array}{l}-0.433 \\
(0.280)\end{array}$ & $\begin{array}{l}-0.299 \\
(0.242)\end{array}$ & $\begin{array}{l}-0.017 \\
(0.047)\end{array}$ \\
\hline $\begin{array}{l}\text { Geo exp - intl } \\
\text { recruitment }(H 7 a)\end{array}$ & $\begin{array}{l}0.047 \\
(0.266)\end{array}$ & $\begin{array}{l}-0.005 \\
(0.252)\end{array}$ & $\begin{array}{l}-0.0005 \\
(0.020)\end{array}$ & $\begin{array}{l}1.910 \dagger \\
(1.022)\end{array}$ & $\begin{array}{l}2.003^{* *} \\
(0.759)\end{array}$ & $\begin{array}{l}0.124 \\
(0.109)\end{array}$ & $\begin{array}{l}-0.518 \\
(0.420)\end{array}$ & $\begin{array}{l}-0.635 \\
(0.388)\end{array}$ & $\begin{array}{l}-0.039 \\
(0.032)\end{array}$ & $\begin{array}{l}0.170 \\
(0.326)\end{array}$ & $\begin{array}{l}0.111 \\
(0.288)\end{array}$ & $\begin{array}{l}0.007 \\
(0.052)\end{array}$ \\
\hline Age & $\begin{array}{l}0.003 \\
(0.006)\end{array}$ & $\begin{array}{l}0.002 \\
(0.005)\end{array}$ & $\begin{array}{l}0.0001 \\
(0.0004)\end{array}$ & - & - & - & $\begin{array}{l}-0.004 \\
(0.009)\end{array}$ & $\begin{array}{l}-0.004 \\
(0.008)\end{array}$ & $\begin{array}{l}-0.0002 \\
(0.0005)\end{array}$ & $\begin{array}{l}0.007 \\
(0.006)\end{array}$ & $\begin{array}{l}0.005 \\
(0.005)\end{array}$ & $\begin{array}{l}0.0003 \\
(0.0009)\end{array}$ \\
\hline Size (Staff) & $\begin{array}{l}-0.000 \\
(0.000)\end{array}$ & $\begin{array}{l}-0.328 \mathrm{E}-4 \\
(0.0002)\end{array}$ & $\begin{array}{l}-0.173 E-5 \\
(0.228 E-4)\end{array}$ & $\begin{array}{l}-0.001 \\
(0.001)\end{array}$ & $\begin{array}{l}-0.001 \dagger \\
(0.0008)\end{array}$ & $\begin{array}{l}-0.813 \mathrm{E}-4 \\
(0.901 \mathrm{E}-4)\end{array}$ & $\begin{array}{l}0.000 \\
(0.000)\end{array}$ & $\begin{array}{l}0.0001 \\
(0.0003)\end{array}$ & $\begin{array}{l}0.838 \mathrm{E}-5 \\
(0.313 \mathrm{E}-4)\end{array}$ & $\begin{array}{l}0.000 \\
(0.000)\end{array}$ & $\begin{array}{l}-0.0001 \\
(0.0002)\end{array}$ & $\begin{array}{l}-0.683 E-5 \\
(0.613 E-4)\end{array}$ \\
\hline Offshore policy & $\begin{array}{l}1.805 \\
(1.141)\end{array}$ & $\begin{array}{l}1.690 \\
(1.096)\end{array}$ & $\begin{array}{l}0.114 \dagger \\
(0.061)\end{array}$ & $\begin{array}{l}4.976 \\
(3.920)\end{array}$ & $\begin{array}{l}4.167 \\
(3.065)\end{array}$ & $\begin{array}{l}0.352 \\
(0.216)\end{array}$ & $\begin{array}{l}2.051 \\
(1.890)\end{array}$ & $\begin{array}{l}1.669 \\
(1.764)\end{array}$ & $\begin{array}{l}0.111 \\
(0.094)\end{array}$ & $\begin{array}{l}1.514 \\
(1.456)\end{array}$ & $\begin{array}{l}1.546 \\
(1.273)\end{array}$ & $\begin{array}{l}0.092 \\
(0.375)\end{array}$ \\
\hline $\begin{array}{l}\text { Internationalisation } \\
\text { at home strategy }\end{array}$ & $\begin{array}{l}0.363 \\
(0.224)\end{array}$ & $\begin{array}{l}-0.0007 \\
(0.0015)\end{array}$ & $\begin{array}{l}-0.419 \mathrm{E}-4 \\
(0.0001)\end{array}$ & $\begin{array}{l}0.068 \\
(0.890)\end{array}$ & $\begin{array}{l}0.001 \\
(0.005)\end{array}$ & $\begin{array}{l}0.549 \mathrm{E}-4 \\
(0.111)\end{array}$ & $\begin{array}{l}0.506 \\
(0.358)\end{array}$ & $\begin{array}{l}-0.0008 \\
(0.004)\end{array}$ & $\begin{array}{l}-0.568 \mathrm{E}-4 \\
(0.012)\end{array}$ & $\begin{array}{l}0.125 \\
(0.292)\end{array}$ & $\begin{array}{l}-0.0004 \\
(0.001)\end{array}$ & $\begin{array}{l}-0.260 \mathrm{E}-4 \\
(0.0002)\end{array}$ \\
\hline
\end{tabular}




\begin{tabular}{|c|c|c|c|c|c|c|c|c|c|c|c|c|}
\hline $\begin{array}{l}\text { Host country } \\
\text { demand }\end{array}$ & $\begin{array}{l}\text { 8.259* } \\
(3.247)\end{array}$ & $\begin{array}{l}7.949 * * \\
(3.020)\end{array}$ & $\begin{array}{l}0.461 \\
(0.317)\end{array}$ & $\begin{array}{l}6.891 \\
(11.279)\end{array}$ & $\begin{array}{l}8.173 \\
(7.990)\end{array}$ & $\begin{array}{l}0.389 \\
(1.123)\end{array}$ & $\begin{array}{l}5.324 \\
(5.059)\end{array}$ & $\begin{array}{l}5.796 \\
(4.762)\end{array}$ & $\begin{array}{l}0.352 \\
(0.489)\end{array}$ & $\begin{array}{l}8.636 * \\
(4.266)\end{array}$ & $\begin{array}{l}\text { 6.191† } \\
(3.354)\end{array}$ & $\begin{array}{l}0.341 \\
(0.763)\end{array}$ \\
\hline AU/NZ dummy & $\begin{array}{l}0.251 \\
(1.080)\end{array}$ & $\begin{array}{l}-0.073 \\
(1.002)\end{array}$ & $\begin{array}{l}-0.001 \\
(0.090)\end{array}$ & - & - & - & - & - & - & - & - & - \\
\hline UK/IR dummy & $\begin{array}{l}1.569 * \\
(0.685)\end{array}$ & $\begin{array}{l}1.432^{*} \\
(0.650)\end{array}$ & $\begin{array}{l}0.086 \\
(0.069)\end{array}$ & - & - & - & - & - & - & - & - & - \\
\hline $\mathrm{R}^{2}$ & 0.124 & & & 0.503 & & & 0.143 & & & 0.220 & & \\
\hline $\operatorname{Adj}^{2}$ & 0.056 & & & 0.055 & & & 0.034 & & & 0.057 & & \\
\hline Log likelihood & & -476.68 & -498.75 & & -56.05 & -63.76 & & -255.26 & -267.14 & & -140.55 & -157.11 \\
\hline $\mathrm{N}$ & 175 & 175 & 175 & 21 & 21 & 21 & 91 & 91 & 91 & 63 & 63 & 63 \\
\hline Max. VIF & 1.390 & & & 1.637 & & & 1.126 & & & 1.754 & & \\
\hline
\end{tabular}

Standard errors in parentheses, all $t$ tests are two-tailed, $\dagger p<.10,{ }^{*} p<.05,{ }^{* *} p<.01$

Table 7.28b: Regression estimates for equation 13b (high normative institutional distance)

\begin{tabular}{|c|c|c|c|c|c|c|c|c|c|c|c|c|}
\hline & \multicolumn{3}{|c|}{ Complete data set } & \multicolumn{3}{|c|}{ AU/NZ } & \multicolumn{3}{|c|}{ US/CA } & \multicolumn{3}{|c|}{$\overline{\text { UK/IR }}$} \\
\hline & OLS & Tobit & $\begin{array}{l}\text { Negative } \\
\text { binomial }\end{array}$ & OLS & Tobit & $\begin{array}{l}\text { Negative } \\
\text { binomial }\end{array}$ & OLS & Tobit & $\begin{array}{l}\text { Negative } \\
\text { binomial }\end{array}$ & OLS & Tobit & $\begin{array}{l}\text { Negative } \\
\text { binomial }\end{array}$ \\
\hline$\overline{\text { Intercept }}$ & $\begin{array}{l}1.961 \\
(4.439)\end{array}$ & $\begin{array}{l}2.535 \\
(3.974)\end{array}$ & $\begin{array}{l}1.754 * * \\
(0.429)\end{array}$ & $\begin{array}{l}9.210 \\
(6.143)\end{array}$ & $\begin{array}{l}10.727 * \\
(5.316)\end{array}$ & $\begin{array}{l}2.450 * * \\
(0.717)\end{array}$ & $\begin{array}{l}3.651 \\
(12.003)\end{array}$ & $\begin{array}{l}-1.363 \\
(9.337)\end{array}$ & $\begin{array}{l}1.182 \\
(1.039)\end{array}$ & $\begin{array}{l}7.959 \\
(7.014)\end{array}$ & $\begin{array}{l}9.548 \dagger \\
(5.453)\end{array}$ & $\begin{array}{l}2.179 \\
(1.494)\end{array}$ \\
\hline $\begin{array}{l}\text { Geo exp - } \\
\text { knowledge of edu } \\
\text { sector }(H 7 a)\end{array}$ & $\begin{array}{l}0.272 \\
(0.272)\end{array}$ & $\begin{array}{l}0.310 \\
(0.242)\end{array}$ & $\begin{array}{l}0.019 \\
(0.028)\end{array}$ & $\begin{array}{l}0.243 \\
(0.414)\end{array}$ & $\begin{array}{l}0.242 \\
(0.360)\end{array}$ & $\begin{array}{l}0.014 \\
(0.059)\end{array}$ & $\begin{array}{l}-0.442 \\
(0.799)\end{array}$ & $\begin{array}{l}0.021 \\
(0.585)\end{array}$ & $\begin{array}{l}-0.001 \\
(0.067)\end{array}$ & $\begin{array}{l}0.288 \\
(0.393)\end{array}$ & $\begin{array}{l}0.278 \\
(0.290)\end{array}$ & $\begin{array}{l}0.015 \\
(0.081)\end{array}$ \\
\hline $\begin{array}{l}\text { Geo exp - academic } \\
\text { capabilities }(H 7 a)\end{array}$ & $\begin{array}{l}0.214 \\
(0.225)\end{array}$ & $\begin{array}{l}0.118 \\
(0.202)\end{array}$ & $\begin{array}{l}0.007 \\
(0.019)\end{array}$ & $\begin{array}{l}0.116 \\
(0.331)\end{array}$ & $\begin{array}{l}0.039 \\
(0.286)\end{array}$ & $\begin{array}{l}0.002 \\
(0.031)\end{array}$ & $\begin{array}{l}0.522 \\
(0.798)\end{array}$ & $\begin{array}{l}0.465 \\
(0.655)\end{array}$ & $\begin{array}{l}0.026 \\
(0.063)\end{array}$ & $\begin{array}{l}-0.144 \\
(0.307)\end{array}$ & $\begin{array}{l}-0.278 \\
(0.238)\end{array}$ & $\begin{array}{l}-0.015 \\
(0.058)\end{array}$ \\
\hline $\begin{array}{l}\text { Geo exp - } \\
\text { managerial } \\
\text { capabilities (H7a) }\end{array}$ & $\begin{array}{l}0.188 \\
(0.268)\end{array}$ & $\begin{array}{l}0.189 \\
(0.247)\end{array}$ & $\begin{array}{l}0.011 \\
(0.025)\end{array}$ & $\begin{array}{l}0.480 \\
(0.449)\end{array}$ & $\begin{array}{l}0.503 \\
(0.394)\end{array}$ & $\begin{array}{l}0.030 \\
(0.047)\end{array}$ & $\begin{array}{l}-0.721 \\
(0.715)\end{array}$ & $\begin{array}{l}-0.689 \\
(0.587)\end{array}$ & $\begin{array}{l}-0.045 \\
(0.048)\end{array}$ & $\begin{array}{l}0.194 \\
(0.392)\end{array}$ & $\begin{array}{l}0.218 \\
(0.312)\end{array}$ & $\begin{array}{l}0.011 \\
(0.077)\end{array}$ \\
\hline $\begin{array}{l}\text { Geo exp - financial } \\
\text { capabilities }(H 7 a)\end{array}$ & $\begin{array}{l}0.187 \\
(0.242)\end{array}$ & $\begin{array}{l}0.101 \\
(0.216)\end{array}$ & $\begin{array}{l}0.006 \\
(0.024)\end{array}$ & $\begin{array}{l}0.150 \\
(0.397)\end{array}$ & $\begin{array}{l}-0.008 \\
(0.325)\end{array}$ & $\begin{array}{l}-0.0005 \\
(0.041)\end{array}$ & $\begin{array}{l}0.833 \\
(0.670)\end{array}$ & $\begin{array}{l}0.899 \\
(0.549)\end{array}$ & $\begin{array}{l}0.056 \\
(0.059)\end{array}$ & $\begin{array}{l}-0.590 \\
(0.401)\end{array}$ & $\begin{array}{l}-0.593 \dagger \\
(0.311)\end{array}$ & $\begin{array}{l}-0.037 \\
(0.086)\end{array}$ \\
\hline $\begin{array}{l}\text { Geo exp - intl } \\
\text { recruitment }(H 7 a)\end{array}$ & $\begin{array}{l}-0.179 \\
(0.268)\end{array}$ & $\begin{array}{l}-0.194 \\
(0.246)\end{array}$ & $\begin{array}{l}-0.012 \\
(0.023)\end{array}$ & $\begin{array}{l}-0.227 \\
(0.401)\end{array}$ & $\begin{array}{l}-0.181 \\
(0.352)\end{array}$ & $\begin{array}{l}-0.010 \\
(0.040)\end{array}$ & $\begin{array}{l}0.056 \\
(0.797)\end{array}$ & $\begin{array}{l}-0.141 \\
(0.643)\end{array}$ & $\begin{array}{l}-0.006 \\
(0.055)\end{array}$ & $\begin{array}{l}-0.189 \\
(0.410)\end{array}$ & $\begin{array}{l}-0.199 \\
(0.325)\end{array}$ & $\begin{array}{l}-0.011 \\
(0.083)\end{array}$ \\
\hline Age & $\begin{array}{l}0.002 \\
(0.007)\end{array}$ & $\begin{array}{l}0.002 \\
(0.006)\end{array}$ & $\begin{array}{l}0.0001 \\
(0.0006)\end{array}$ & $\begin{array}{l}-0.002 \\
(0.012)\end{array}$ & $\begin{array}{l}-0.0003 \\
(0.010)\end{array}$ & $\begin{array}{l}-0.239 \mathrm{E}-4 \\
(0.001)\end{array}$ & $\begin{array}{l}0.002 \\
(0.018)\end{array}$ & $\begin{array}{l}0.003 \\
(0.014)\end{array}$ & $\begin{array}{l}0.0001 \\
(0.001)\end{array}$ & $\begin{array}{l}0.000 \\
(0.009)\end{array}$ & $\begin{array}{l}-0.002 \\
(0.006)\end{array}$ & $\begin{array}{l}-0.0001 \\
(0.002)\end{array}$ \\
\hline Size (Staff) & $\begin{array}{c}0.000 \\
(0.000)\end{array}$ & $\begin{array}{l}0.0001 \\
(0.0002)\end{array}$ & $\begin{array}{l}0.123 E-4 \\
(0.243 E-4)\end{array}$ & $\begin{array}{l}-0.000 \\
(0.000)\end{array}$ & $\begin{array}{l}-0.0001 \\
(0.0003)\end{array}$ & $\begin{array}{l}-0.741 \mathrm{E}-5 \\
(0.403 \mathrm{E}-4)\end{array}$ & $\begin{array}{l}0.001 \\
(0.001)\end{array}$ & $\begin{array}{l}0.001 \\
(0.0006)\end{array}$ & $\begin{array}{l}0.702 E-4 \\
(0.786 E-4)\end{array}$ & $\begin{array}{l}0.000 \\
(0.000)\end{array}$ & $\begin{array}{l}0.0002 \\
(0.0002)\end{array}$ & $\begin{array}{l}0.168 \mathrm{E}-4 \\
(0.541 \mathrm{E}-4)\end{array}$ \\
\hline
\end{tabular}




\begin{tabular}{|c|c|c|c|c|c|c|c|c|c|c|c|c|}
\hline Offshore policy & $\begin{array}{l}7.823 * * \\
(1.278)\end{array}$ & $\begin{array}{l}\text { 8.010** } \\
(1.191)\end{array}$ & $\begin{array}{l}0.673 * * \\
(0.084)\end{array}$ & $\begin{array}{l}2.226 \\
(2.279)\end{array}$ & $\begin{array}{l}2.119 \\
(2.008)\end{array}$ & $\begin{array}{l}0.134 \\
(0.136)\end{array}$ & $\begin{array}{l}6.718 \dagger \\
(3.434)\end{array}$ & $\begin{array}{l}\text { 6.615* } \\
(2.824)\end{array}$ & $\begin{array}{l}0.938 * * \\
(0.247)\end{array}$ & $\begin{array}{l}9.459 * * \\
(2.066)\end{array}$ & $\begin{array}{l}9.450 * * \\
(1.692)\end{array}$ & $\begin{array}{l}0.779 * * \\
(0.187)\end{array}$ \\
\hline $\begin{array}{l}\text { Internationalisation } \\
\text { at home strategy }\end{array}$ & $\begin{array}{l}-0.229 \\
(0.251)\end{array}$ & $\begin{array}{l}-0.0008 \\
(0.0012)\end{array}$ & $\begin{array}{l}-0.535 \mathrm{E}-4 \\
(0.0001)\end{array}$ & $\begin{array}{l}0.073 \\
(0.457)\end{array}$ & $\begin{array}{l}-0.001 \\
(0.002)\end{array}$ & $\begin{array}{l}-0.570 \mathrm{E}-4 \\
(0.0002)\end{array}$ & $\begin{array}{l}-0.785 \\
(0.604)\end{array}$ & $\begin{array}{l}-0.008 \dagger \\
(0.004)\end{array}$ & $\begin{array}{l}-0.0005 \\
(0.021)\end{array}$ & $\begin{array}{l}0.167 \\
(0.365)\end{array}$ & $\begin{array}{l}0.0007 \\
(0.001)\end{array}$ & $\begin{array}{l}0.444 \mathrm{E}-4 \\
(0.0003)\end{array}$ \\
\hline $\begin{array}{l}\text { Host country } \\
\text { demand }\end{array}$ & $\begin{array}{l}6.734 \dagger \\
(3.878)\end{array}$ & $\begin{array}{l}6.325 \dagger \\
(3.552)\end{array}$ & $\begin{array}{l}0.406 \\
(0.390)\end{array}$ & $\begin{array}{l}1.032 \\
(5.901)\end{array}$ & $\begin{array}{l}2.006 \\
(4.904)\end{array}$ & $\begin{array}{l}0.120 \\
(0.538)\end{array}$ & $\begin{array}{l}18.615 \\
(13.717)\end{array}$ & $\begin{array}{l}22.579 * \\
(10.994)\end{array}$ & $\begin{array}{l}1.443 \\
(1.082)\end{array}$ & $\begin{array}{l}\text { 13.632* } \\
(6.143)\end{array}$ & $\begin{array}{l}13.097 * * \\
(4.827)\end{array}$ & $\begin{array}{l}0.788 \\
(1.323)\end{array}$ \\
\hline AU/NZ dummy & $\begin{array}{l}2.091^{*} \\
(1.006)\end{array}$ & $\begin{array}{l}\text { 1.963* } \\
(0.923)\end{array}$ & $\begin{array}{l}\mathbf{0 . 1 3 0} \dagger \\
\mathbf{( 0 . 0 7 5 )}\end{array}$ & - & - & - & - & - & - & - & - & - \\
\hline UK/IR dummy & $\begin{array}{l}1.525 \\
(0.978)\end{array}$ & $\begin{array}{l}1.295 \\
(0.885)\end{array}$ & $\begin{array}{l}0.086 \\
(0.079)\end{array}$ & - & - & - & - & - & - & - & - & - \\
\hline $\mathrm{R}^{2}$ & 0.356 & & & 0.078 & & & 0.631 & & & 0.544 & & \\
\hline $\operatorname{AdjR}^{2}$ & 0.286 & & & -0.127 & & & 0.447 & & & 0.362 & & \\
\hline Log likelihood & & -351.47 & -371.72 & & -153.84 & -159.84 & & -87.91 & -94.27 & & -91.62 & -103.98 \\
\hline $\mathrm{N}$ & 132 & 132 & 132 & 59 & 59 & 59 & 32 & 32 & 32 & 41 & 41 & 41 \\
\hline Max. VIF & 2.252 & & & 1.266 & & & 2.349 & & & 1.363 & & \\
\hline
\end{tabular}

Table 7.29a: Regression estimates for equation 14a (low normative institutional distance)

\begin{tabular}{|c|c|c|c|c|c|c|c|c|c|c|c|c|}
\hline & \multicolumn{3}{|c|}{ Complete data set } & \multicolumn{3}{|c|}{ AU/NZ } & \multicolumn{3}{|c|}{ 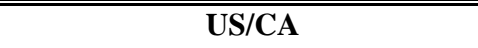 } & \multicolumn{3}{|c|}{ 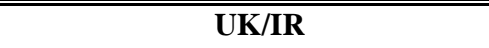 } \\
\hline & OLS & Tobit & $\begin{array}{l}\text { Negative } \\
\text { binomial }\end{array}$ & OLS & Tobit & $\begin{array}{l}\text { Negative } \\
\text { binomial }\end{array}$ & OLS & Tobit & $\begin{array}{l}\text { Negative } \\
\text { binomial }\end{array}$ & OLS & Tobit & $\begin{array}{l}\text { Negative } \\
\text { binomial }\end{array}$ \\
\hline Intercept & $\begin{array}{l}6.313^{*} \\
(2.894)\end{array}$ & $\begin{array}{l}7.929 * * \\
(2.694)\end{array}$ & $\begin{array}{l}2.268 * * \\
(0.219)\end{array}$ & $\begin{array}{l}0.965 \\
(11.988)\end{array}$ & $\begin{array}{l}5.204 \\
(7.959)\end{array}$ & $\begin{array}{l}2.055 \dagger \\
(1.166)\end{array}$ & $\begin{array}{l}4.461 \\
(5.185)\end{array}$ & $\begin{array}{l}7.582 \\
(4.622)\end{array}$ & $\begin{array}{l}2.234 * * \\
(0.295)\end{array}$ & $\begin{array}{l}10.806 * * \\
(3.104)\end{array}$ & $\begin{array}{l}\text { 12.011** } \\
(2.777)\end{array}$ & $\begin{array}{l}2.542 * * \\
(0.618)\end{array}$ \\
\hline $\begin{array}{l}\text { Ind exp - UG } \\
\text { offshore edu }(H 7 b)\end{array}$ & $\begin{array}{l}0.191 \\
(0.192)\end{array}$ & $\begin{array}{l}0.165 \\
(0.182)\end{array}$ & $\begin{array}{l}0.010 \\
(0.016)\end{array}$ & $\begin{array}{l}1.174 \\
(0.915)\end{array}$ & $\begin{array}{l}1.064 \\
(0.700)\end{array}$ & $\begin{array}{l}0.074 \\
(0.092)\end{array}$ & $\begin{array}{l}-0.043 \\
(0.322)\end{array}$ & $\begin{array}{l}-0.098 \\
(0.303)\end{array}$ & $\begin{array}{l}-0.006 \\
(0.028)\end{array}$ & $\begin{array}{l}0.239 \\
(0.217)\end{array}$ & $\begin{array}{l}0.245 \\
(0.191)\end{array}$ & $\begin{array}{l}0.014 \\
(0.038)\end{array}$ \\
\hline $\begin{array}{l}\text { Ind exp - PG } \\
\text { offshore edu }(H 7 b)\end{array}$ & $\begin{array}{l}0.081 \\
(0.223)\end{array}$ & $\begin{array}{l}0.113 \\
(0.211)\end{array}$ & $\begin{array}{l}0.007 \\
(0.014)\end{array}$ & $\begin{array}{l}-0.507 \\
(0.986)\end{array}$ & $\begin{array}{l}-0.343 \\
(0.738)\end{array}$ & $\begin{array}{l}-0.023 \\
(0.098)\end{array}$ & $\begin{array}{l}0.105 \\
(0.350)\end{array}$ & $\begin{array}{l}0.124 \\
(0.332)\end{array}$ & $\begin{array}{l}0.007 \\
(0.019)\end{array}$ & $\begin{array}{l}-0.170 \\
(0.281)\end{array}$ & $\begin{array}{l}-0.179 \\
(0.253)\end{array}$ & $\begin{array}{l}-0.010 \\
(0.057)\end{array}$ \\
\hline $\begin{array}{l}\text { Ind exp - intl } \\
\text { recruitment (Factor) } \\
(H 7 b)\end{array}$ & $\begin{array}{l}0.543^{*} \\
(0.260)\end{array}$ & $\begin{array}{l}0.486^{*} \\
(0.239)\end{array}$ & $\begin{array}{l}0.029 \\
(0.024)\end{array}$ & $\begin{array}{l}0.585 \\
(1.541)\end{array}$ & $\begin{array}{l}0.364 \\
(1.147)\end{array}$ & $\begin{array}{l}0.013 \\
(0.118)\end{array}$ & $\begin{array}{l}0.820^{*} \\
(0.428)\end{array}$ & $\begin{array}{l}0.792 \dagger \\
(0.406)\end{array}$ & $\begin{array}{l}0.050 \\
(0.037)\end{array}$ & $\begin{array}{l}0.116 \\
(0.306)\end{array}$ & $\begin{array}{l}0.053 \\
(0.247)\end{array}$ & $\begin{array}{l}0.002 \\
(0.053)\end{array}$ \\
\hline $\begin{array}{l}\text { Ind exp - UG } \\
\text { marketing }(H 7 b)\end{array}$ & $\begin{array}{l}0.070 \\
(0.183)\end{array}$ & $\begin{array}{l}0.070 \\
(0.174)\end{array}$ & $\begin{array}{l}0.004 \\
(0.017)\end{array}$ & $\begin{array}{l}-1.003 \\
(1.255)\end{array}$ & $\begin{array}{l}-0.957 \\
(0.964)\end{array}$ & $\begin{array}{l}-0.054 \\
(0.108)\end{array}$ & $\begin{array}{l}0.116 \\
(0.280)\end{array}$ & $\begin{array}{l}0.098 \\
(0.266)\end{array}$ & $\begin{array}{l}0.006 \\
(0.025)\end{array}$ & $\begin{array}{l}0.355 \dagger \\
(0.206)\end{array}$ & $\begin{array}{l}0.359 \dagger \\
(0.185)\end{array}$ & $\begin{array}{l}0.020 \\
(0.042)\end{array}$ \\
\hline $\begin{array}{l}\text { Ind exp - PG } \\
\text { marketing }(H 7 b)\end{array}$ & $\begin{array}{l}0.116 \\
(0.196)\end{array}$ & $\begin{array}{l}0.101 \\
(0.184)\end{array}$ & $\begin{array}{l}0.005 \\
(0.014)\end{array}$ & $\begin{array}{l}1.377 \\
(0.873)\end{array}$ & $\begin{array}{l}\mathbf{1 . 1 7 9} \dagger \\
(\mathbf{0 . 6 2 0})\end{array}$ & $\begin{array}{l}0.068 \\
(0.110)\end{array}$ & $\begin{array}{l}-0.056 \\
(0.296)\end{array}$ & $\begin{array}{l}0.028 \\
(0.277)\end{array}$ & $\begin{array}{l}0.002 \\
(0.019)\end{array}$ & $\begin{array}{l}0.174 \\
(0.247)\end{array}$ & $\begin{array}{l}0.087 \\
(0.211)\end{array}$ & $\begin{array}{l}0.004 \\
(0.044)\end{array}$ \\
\hline Age & $\begin{array}{l}0.003 \\
(0.006)\end{array}$ & $\begin{array}{l}0.002 \\
(0.005)\end{array}$ & $\begin{array}{l}0.0001 \\
(0.0004)\end{array}$ & - & - & - & $\begin{array}{l}0.000 \\
(0.009)\end{array}$ & $\begin{array}{l}-0.0006 \\
(0.008)\end{array}$ & $\begin{array}{l}-0.412 \mathrm{E}-4 \\
(0.0006)\end{array}$ & $\begin{array}{l}0.003 \\
(0.006)\end{array}$ & $\begin{array}{l}0.001 \\
(0.005)\end{array}$ & $\begin{array}{l}0.966 \mathrm{E}-4 \\
(0.0009)\end{array}$ \\
\hline
\end{tabular}




\begin{tabular}{|c|c|c|c|c|c|c|c|c|c|c|c|c|}
\hline Size (Staff) & $\begin{array}{l}0.000 \\
(0.000)\end{array}$ & $\begin{array}{l}-0.493 \mathrm{E}-4 \\
(0.0002)\end{array}$ & $\begin{array}{l}-0.291 \mathrm{E}-5 \\
(0.234 \mathrm{E}-4)\end{array}$ & - & - & - & $\begin{array}{l}0.000 \\
(0.000)\end{array}$ & $\begin{array}{l}0.0001 \\
(0.0003)\end{array}$ & $\begin{array}{l}0.104 \mathrm{E}-4 \\
(0.326 \mathrm{E}-4)\end{array}$ & $\begin{array}{l}0.000 \\
(0.000)\end{array}$ & $\begin{array}{l}-0.0001 \\
(0.0002)\end{array}$ & $\begin{array}{l}-0.648 \mathrm{E}-5 \\
(0.657 \mathrm{E}-4)\end{array}$ \\
\hline Offshore policy & $\begin{array}{l}1.771 \\
(1.139)\end{array}$ & $\begin{array}{l}1.704 \\
(1.098)\end{array}$ & $\begin{array}{l}0.114 \dagger \\
(0.065)\end{array}$ & $\begin{array}{l}4.718 \\
(3.982)\end{array}$ & $\begin{array}{l}4.159 \\
(3.032)\end{array}$ & $\begin{array}{l}0.305 \\
(0.275)\end{array}$ & $\begin{array}{l}2.288 \\
(1.945)\end{array}$ & $\begin{array}{l}1.609 \\
(1.809)\end{array}$ & $\begin{array}{l}0.105 \\
(0.101)\end{array}$ & $\begin{array}{l}0.665 \\
(1.385)\end{array}$ & $\begin{array}{l}0.860 \\
(1.248)\end{array}$ & $\begin{array}{l}0.052 \\
(0.314)\end{array}$ \\
\hline $\begin{array}{l}\text { Internationalisation } \\
\text { at home strategy }\end{array}$ & $\begin{array}{l}0.330 \\
(0.218)\end{array}$ & $\begin{array}{l}-0.0004 \\
(0.001)\end{array}$ & $\begin{array}{l}-0.021 \mathrm{E}-4 \\
(0.0001)\end{array}$ & $\begin{array}{l}0.770 \\
(1.093)\end{array}$ & $\begin{array}{l}-0.003 \\
(0.005)\end{array}$ & $\begin{array}{l}-0.0002 \\
(0.061)\end{array}$ & $\begin{array}{l}0.632 \dagger \\
(0.362)\end{array}$ & $\begin{array}{l}-0.744 \mathrm{E}-4 \\
(0.004)\end{array}$ & $\begin{array}{l}-0.667 \mathrm{E}-5 \\
(0.010)\end{array}$ & $\begin{array}{l}0.201 \\
(0.263)\end{array}$ & $\begin{array}{l}0.0005 \\
(0.001)\end{array}$ & $\begin{array}{l}0.319 \mathrm{E}-4 \\
(0.0002)\end{array}$ \\
\hline $\begin{array}{l}\text { Host country } \\
\text { demand }\end{array}$ & $\begin{array}{l}\text { 8.211* } \\
\text { (3.259) }\end{array}$ & $\begin{array}{l}\text { 7.972** } \\
(3.021)\end{array}$ & $\begin{array}{l}0.463 \\
(0.324)\end{array}$ & $\begin{array}{l}4.992 \\
(12.110)\end{array}$ & $\begin{array}{l}7.965 \\
(9.060)\end{array}$ & $\begin{array}{l}0.376 \\
(1.366)\end{array}$ & $\begin{array}{l}6.742 \\
(5.116)\end{array}$ & $\begin{array}{l}7.409 \\
(4.843)\end{array}$ & $\begin{array}{l}0.450 \\
(0.519)\end{array}$ & $\begin{array}{l}9.203^{*} \\
(4.314)\end{array}$ & $\begin{array}{l}6.403 \dagger \\
(3.464)\end{array}$ & $\begin{array}{l}0.354 \\
(0.938)\end{array}$ \\
\hline AU/NZ dummy & $\begin{array}{l}0.103 \\
(1.060)\end{array}$ & $\begin{array}{l}-0.097 \\
(0.991)\end{array}$ & $\begin{array}{l}-0.003 \\
(0.080)\end{array}$ & - & - & - & - & - & - & - & - & - \\
\hline UK/IR dummy & $\begin{array}{l}\text { 1.478* } \\
(0.672)\end{array}$ & $\begin{array}{l}1.408^{*} \\
(0.640)\end{array}$ & $\begin{array}{l}0.084 \\
(0.068)\end{array}$ & - & - & - & - & - & - & - & - & - \\
\hline $\mathrm{R}^{2}$ & 0.131 & & & 0.412 & & & 0.116 & & & 0.211 & & \\
\hline $\operatorname{AdjR}^{2}$ & 0.064 & & & -0.016 & & & 0.004 & & & 0.047 & & \\
\hline Log likelihood & & -476.41 & -498.53 & & -58.34 & -65.81 & & -257.24 & -269.11 & & -141.87 & -157.51 \\
\hline $\mathrm{N}$ & 175 & 175 & 175 & 21 & 21 & 21 & 91 & 91 & 91 & 63 & 63 & 63 \\
\hline Max. VIF & 1.204 & & & 1.872 & & & 1.158 & & & 1.415 & & \\
\hline
\end{tabular}

Standard errors in parentheses, all $t$ tests are two-tailed, $\uparrow p<.10, * p<.05, * * p<.01$

Table 7.29b: Regression estimates for equation 14b (high normative institutional distance)

\begin{tabular}{|c|c|c|c|c|c|c|c|c|c|c|c|c|}
\hline & \multicolumn{3}{|c|}{ Complete data set } & \multicolumn{3}{|c|}{ AU/NZ } & \multicolumn{3}{|c|}{ US/CA } & \multicolumn{3}{|c|}{ UK/IR } \\
\hline & OLS & Tobit & $\begin{array}{l}\text { Negative } \\
\text { binomial }\end{array}$ & OLS & Tobit & $\begin{array}{l}\text { Negative } \\
\text { binomial }\end{array}$ & OLS & Tobit & $\begin{array}{l}\text { Negative } \\
\text { binomial }\end{array}$ & OLS & Tobit & $\begin{array}{l}\text { Negative } \\
\text { binomial }\end{array}$ \\
\hline Intercept & $\begin{array}{l}9.618 * * \\
(3.171)\end{array}$ & $\begin{array}{l}9.480 * * \\
(2.735)\end{array}$ & $\begin{array}{l}2.236 * * \\
(0.278)\end{array}$ & $\begin{array}{l}16.531 * * \\
(4.903)\end{array}$ & $\begin{array}{l}18.043 * * \\
(3.977)\end{array}$ & $\begin{array}{l}2.870 * * \\
(0.470)\end{array}$ & $\begin{array}{l}17.785 * \\
(7.960)\end{array}$ & $\begin{array}{l}12.621 * \\
(6.458)\end{array}$ & $\begin{array}{l}2.142^{*} \\
(1.054)\end{array}$ & $\begin{array}{l}10.812 * \\
(3.954)\end{array}$ & $\begin{array}{l}11.607 * * \\
(3.393)\end{array}$ & $\begin{array}{l}2.306 * * \\
(0.591)\end{array}$ \\
\hline $\begin{array}{l}\text { Ind exp - UG } \\
\text { offshore edu (H7b) }\end{array}$ & $\begin{array}{l}-0.384 \dagger \\
(0.215)\end{array}$ & $\begin{array}{l}-0.383^{*} \\
(0.193)\end{array}$ & $\begin{array}{l}-0.016 \\
(0.018)\end{array}$ & $\begin{array}{l}-0.530 \\
(0.320)\end{array}$ & $\begin{array}{l}-0.636^{*} \\
(0.273)\end{array}$ & $\begin{array}{l}-0.036 \\
(0.040)\end{array}$ & $\begin{array}{l}-0.792 \\
(0.545)\end{array}$ & $\begin{array}{l}-0.623 \\
(0.463)\end{array}$ & $\begin{array}{l}-0.042 \\
(0.081)\end{array}$ & $\begin{array}{l}-0.346 \\
(0.307)\end{array}$ & $\begin{array}{l}-0.318 \\
(0.257)\end{array}$ & $\begin{array}{l}-0.020 \\
(0.048)\end{array}$ \\
\hline $\begin{array}{l}\text { Ind exp - PG } \\
\text { offshore edu }(H 7 b)\end{array}$ & $\begin{array}{l}-0.096 \\
(0.244)\end{array}$ & $\begin{array}{l}-0.040 \\
(0.221)\end{array}$ & $\begin{array}{l}-0.006 \\
(0.022)\end{array}$ & $\begin{array}{l}-0.101 \\
(0.366)\end{array}$ & $\begin{array}{l}-0.031 \\
(0.308)\end{array}$ & $\begin{array}{l}-0.001 \\
(0.039)\end{array}$ & $\begin{array}{l}-1.090 \dagger \\
(0.622)\end{array}$ & $\begin{array}{l}-1.072 * \\
(0.535)\end{array}$ & $\begin{array}{l}-0.090 \\
(0.073)\end{array}$ & $\begin{array}{l}0.135 \\
(0.386)\end{array}$ & $\begin{array}{l}-0.060 \\
(0.313)\end{array}$ & $\begin{array}{l}-0.001 \\
(0.080)\end{array}$ \\
\hline $\begin{array}{l}\text { Ind exp - intl } \\
\text { recruitment (Factor) } \\
(H 7 b)\end{array}$ & $\begin{array}{l}0.385 \\
(0.260)\end{array}$ & $\begin{array}{l}0.330 \\
(0.235)\end{array}$ & $\begin{array}{l}0.020 \\
(0.023)\end{array}$ & $\begin{array}{l}0.752 \dagger \\
(0.378)\end{array}$ & $\begin{array}{l}0.578 \dagger \\
(0.315)\end{array}$ & $\begin{array}{l}0.034 \\
(0.040)\end{array}$ & $\begin{array}{l}0.518 \\
(0.683)\end{array}$ & $\begin{array}{l}0.275 \\
(0.578)\end{array}$ & $\begin{array}{l}0.020 \\
(0.108)\end{array}$ & $\begin{array}{l}-0.471 \\
(0.375)\end{array}$ & $\begin{array}{l}-0.329 \\
(0.321)\end{array}$ & $\begin{array}{l}-0.021 \\
(0.076)\end{array}$ \\
\hline
\end{tabular}




\begin{tabular}{|c|c|c|c|c|c|c|c|c|c|c|c|c|}
\hline $\begin{array}{l}\text { Ind exp - UG } \\
\text { marketing }(H 7 b)\end{array}$ & $\begin{array}{l}-0.442 \dagger \\
(0.249)\end{array}$ & $\begin{array}{l}-0.484^{*} \\
(0.222)\end{array}$ & $\begin{array}{l}-0.030 \\
(0.021)\end{array}$ & $\begin{array}{l}-0.513 \\
(0.395)\end{array}$ & $\begin{array}{l}-0.442 \\
(0.346)\end{array}$ & $\begin{array}{l}-0.026 \\
(0.044)\end{array}$ & $\begin{array}{l}-1.027 \\
(0.599)\end{array}$ & $\begin{array}{l}-0.879 \dagger \\
(0.511)\end{array}$ & $\begin{array}{l}-0.082 \\
(0.078)\end{array}$ & $\begin{array}{l}-0.262 \\
(0.355)\end{array}$ & $\begin{array}{l}-0.271 \\
(0.265)\end{array}$ & $\begin{array}{l}-0.021 \\
(0.054)\end{array}$ \\
\hline $\begin{array}{l}\text { Ind exp - PG } \\
\text { marketing }(H 7 b)\end{array}$ & $\begin{array}{l}0.141 \\
(0.203)\end{array}$ & $\begin{array}{l}0.093 \\
(0.184)\end{array}$ & $\begin{array}{l}0.007 \\
(0.018)\end{array}$ & $\begin{array}{l}-0.040 \\
(0.310)\end{array}$ & $\begin{array}{l}-0.092 \\
(0.273)\end{array}$ & $\begin{array}{l}-0.005 \\
(0.032)\end{array}$ & $\begin{array}{l}-0.338 \\
(0.534)\end{array}$ & $\begin{array}{l}0.100 \\
(0.416)\end{array}$ & $\begin{array}{l}0.002 \\
(0.052)\end{array}$ & $\begin{array}{l}0.542 \\
(0.311)\end{array}$ & $\begin{array}{l}0.408 \\
(0.248)\end{array}$ & $\begin{array}{l}0.025 \\
(0.055)\end{array}$ \\
\hline Age & $\begin{array}{l}0.001 \\
(0.007)\end{array}$ & $\begin{array}{l}0.001 \\
(0.006)\end{array}$ & $\begin{array}{l}-0.0002 \\
(0.0005)\end{array}$ & $\begin{array}{l}0.000 \\
(0.011)\end{array}$ & $\begin{array}{l}0.0002 \\
(0.009)\end{array}$ & $\begin{array}{l}0.145 E-4 \\
(0.001)\end{array}$ & $\begin{array}{l}0.006 \\
(0.015)\end{array}$ & $\begin{array}{l}0.010 \\
(0.013)\end{array}$ & $\begin{array}{l}0.001 \\
(0.001)\end{array}$ & $\begin{array}{l}-0.003 \\
(0.009)\end{array}$ & $\begin{array}{l}-0.007 \\
(0.007)\end{array}$ & $\begin{array}{l}-0.0004 \\
(0.001)\end{array}$ \\
\hline Size (Staff) & $\begin{array}{l}0.000 \\
(0.000)\end{array}$ & $\begin{array}{l}0.467 \mathrm{E}-4 \\
(0.0002)\end{array}$ & $\begin{array}{l}0.880 \mathrm{E}-5 \\
(0.222 \mathrm{E}-4)\end{array}$ & $\begin{array}{l}-0.000 \\
(0.000)\end{array}$ & $\begin{array}{l}-0.0001 \\
(0.0003)\end{array}$ & $\begin{array}{l}-0.741 \mathrm{E}-5 \\
(0.397 \mathrm{E}-4)\end{array}$ & $\begin{array}{l}8.581 \mathrm{E}-5 \\
(0.001)\end{array}$ & $\begin{array}{l}0.0002 \\
(0.0005)\end{array}$ & $\begin{array}{l}0.771 E-5 \\
(0.798 E-4)\end{array}$ & $\begin{array}{l}0.000 \\
(0.000)\end{array}$ & $\begin{array}{l}-0.0001 \\
(0.0002)\end{array}$ & $\begin{array}{l}-0.954 \mathrm{E}-5 \\
(0.831 \mathrm{E}-4)\end{array}$ \\
\hline Offshore policy & $\begin{array}{l}\text { 8.146** } \\
(1.267)\end{array}$ & $\begin{array}{l}\text { 8.325** } \\
(1.177)\end{array}$ & $\begin{array}{l}\text { 0.721** } \\
(0.075)\end{array}$ & $\begin{array}{l}2.837 \\
(2.286)\end{array}$ & $\begin{array}{l}2.996 \\
(2.030)\end{array}$ & $\begin{array}{l}0.194 \\
(0.250)\end{array}$ & $\begin{array}{l}12.555^{* *} \\
(2.415)\end{array}$ & $\begin{array}{l}12.094 * * \\
(2.069)\end{array}$ & $\begin{array}{l}1.318^{* *} \\
(0.255)\end{array}$ & $\begin{array}{l}9.969 * * \\
(2.286)\end{array}$ & $\begin{array}{l}9.789 * * \\
(1.938)\end{array}$ & $\begin{array}{l}0.824 * * \\
(0.289)\end{array}$ \\
\hline $\begin{array}{l}\text { Internationalisation } \\
\text { at home strategy }\end{array}$ & $\begin{array}{l}-0.288 \\
(0.248)\end{array}$ & $\begin{array}{l}-0.0003 \\
(0.001)\end{array}$ & $\begin{array}{l}-0.121 \mathrm{E}-4 \\
(0.0001)\end{array}$ & $\begin{array}{l}0.110 \\
(0.442)\end{array}$ & $\begin{array}{l}-0.001 \\
(0.001)\end{array}$ & $\begin{array}{l}-0.749 \mathrm{E}-4 \\
(0.0002)\end{array}$ & $\begin{array}{l}-1.011 \dagger \\
(0.521)\end{array}$ & $\begin{array}{l}-0.002 \\
(0.004)\end{array}$ & $\begin{array}{l}-0.0001 \\
(0.022)\end{array}$ & $\begin{array}{l}0.190 \\
(0.358)\end{array}$ & $\begin{array}{l}0.001 \\
(0.001)\end{array}$ & $\begin{array}{l}0.877 \mathrm{E}-4 \\
(0.0002)\end{array}$ \\
\hline $\begin{array}{l}\text { Host country } \\
\text { demand }\end{array}$ & $\begin{array}{l}6.908 \dagger \\
(3.761)\end{array}$ & $\begin{array}{l}6.565 \dagger \\
(3.430)\end{array}$ & $\begin{array}{l}0.250 \\
(0.357)\end{array}$ & $\begin{array}{l}-1.978 \\
(5.309)\end{array}$ & $\begin{array}{l}-0.154 \\
(4.407)\end{array}$ & $\begin{array}{l}-0.020 \\
(0.695)\end{array}$ & $\begin{array}{l}4.237 \\
(10.096)\end{array}$ & $\begin{array}{l}5.030 \\
(8.688)\end{array}$ & $\begin{array}{l}0.703 \\
(1.525)\end{array}$ & $\begin{array}{l}13.816 \dagger \\
(7.311)\end{array}$ & $\begin{array}{l}14.041 * \\
(5.664)\end{array}$ & $\begin{array}{l}0.900 \\
(1.150)\end{array}$ \\
\hline AU/NZ dummy & $\begin{array}{l}2.152 * \\
(0.993)\end{array}$ & $\begin{array}{l}2.146^{*} \\
(0.900)\end{array}$ & $\begin{array}{l}0.139 \dagger \\
(0.084)\end{array}$ & - & - & - & - & - & - & - & - & - \\
\hline UK/IR dummy & $\begin{array}{l}1.410 \\
(0.947)\end{array}$ & $\begin{array}{l}1.211 \\
(0.850)\end{array}$ & $\begin{array}{l}0.078 \\
(0.078)\end{array}$ & - & - & - & - & - & - & - & - & - \\
\hline $\mathrm{R}^{2}$ & 0.397 & & & 0.208 & & & 0.715 & & & 0.591 & & \\
\hline $\operatorname{Adj}^{2}$ & 0.331 & & & 0.032 & & & 0.572 & & & 0.428 & & \\
\hline Log likelihood & & -347.18 & -370.80 & & -149.56 & -157.31 & & -85.27 & -90.23 & & -91.89 & -103.90 \\
\hline $\mathrm{N}$ & 132 & 132 & 132 & 59 & 59 & 59 & 32 & 32 & 32 & 41 & 41 & 41 \\
\hline Max. VIF & 2.340 & & & 1.334 & & & 1.754 & & & 1.905 & & \\
\hline
\end{tabular}

Standard errors in parentheses, all $t$ tests are two-tailed, $\uparrow p<.10, * p<.05, * * p<.01$

Table 7.30a: Regression estimates for equation 15a (low normative institutional distance)

\begin{tabular}{|c|c|c|c|c|c|c|c|c|c|c|c|c|}
\hline & \multicolumn{3}{|c|}{ Complete data set } & \multicolumn{3}{|c|}{ AU/NZ } & \multicolumn{3}{|c|}{ US/CA } & \multicolumn{3}{|c|}{ UK/IR } \\
\hline & OLS & Tobit & $\begin{array}{l}\text { Negative } \\
\text { binomial }\end{array}$ & OLS & Tobit & $\begin{array}{l}\text { Negative } \\
\text { binomial }\end{array}$ & OLS & Tobit & $\begin{array}{l}\text { Negative } \\
\text { binomial }\end{array}$ & OLS & Tobit & $\begin{array}{l}\text { Negative } \\
\text { binomial }\end{array}$ \\
\hline Intercept & $\begin{array}{l}19.895^{* *} \\
(1.280)\end{array}$ & $\begin{array}{l}\text { 20.528** } \\
(1.094)\end{array}$ & $\begin{array}{l}3.101 * * \\
(0.128)\end{array}$ & $\begin{array}{l}21.999 * * \\
(3.136)\end{array}$ & $\begin{array}{l}23.289 * * \\
(2.440)\end{array}$ & $\begin{array}{l}3.369 * * \\
(0.352)\end{array}$ & $\begin{array}{l}20.643 * * \\
(1.928)\end{array}$ & $\begin{array}{l}21.960 * * \\
(1.520)\end{array}$ & $\begin{array}{l}\text { 3.194** } \\
\text { (0.171) }\end{array}$ & $\begin{array}{l}18.135^{* *} \\
(1.769)\end{array}$ & $\begin{array}{l}18.758 * * \\
(1.587)\end{array}$ & $\begin{array}{l}2.957 * * \\
(0.438)\end{array}$ \\
\hline $\begin{array}{l}\text { Know-how (Factor) } \\
(H 7 c)\end{array}$ & $\begin{array}{l}-1.520^{* *} \\
(0.126)\end{array}$ & $\begin{array}{l}-1.542^{* *} \\
(0.121)\end{array}$ & $\begin{array}{l}-0.116^{* *} \\
(0.014)\end{array}$ & $\begin{array}{l}-2.618^{* *} \\
(0.425)\end{array}$ & $\begin{array}{l}-2.743^{* *} \\
(0.370)\end{array}$ & $\begin{array}{l}-0.264^{* *} \\
(0.057)\end{array}$ & $\begin{array}{l}-1.554^{* *} \\
(0.154)\end{array}$ & $\begin{array}{l}-1.578^{* *} \\
(0.147)\end{array}$ & $\begin{array}{l}-0.118 * * \\
(0.017)\end{array}$ & $\begin{array}{l}-0.886 * * \\
(0.282)\end{array}$ & $\begin{array}{l}-0.955^{* *} \\
(0.259)\end{array}$ & $\begin{array}{l}-0.062 \\
(0.057)\end{array}$ \\
\hline Age & 0.003 & 0.002 & 0.0001 & -0.026 & $-0.031 \dagger$ & -0.002 & 0.002 & 0.001 & 0.0001 & 0.004 & 0.002 & 0.0001 \\
\hline
\end{tabular}




\begin{tabular}{|c|c|c|c|c|c|c|c|c|c|c|c|c|}
\hline & $(0.004)$ & $(0.003)$ & $(0.0005)$ & $(0.025)$ & $(0.017)$ & $(0.004)$ & $(0.006)$ & $(0.005)$ & $(0.0007)$ & $(0.005)$ & $(0.004)$ & $(0.001)$ \\
\hline Size (Staff) & $\begin{array}{l}-0.000 \\
(0.000)\end{array}$ & $\begin{array}{l}-0.266 \mathrm{E}-4 \\
(0.0001)\end{array}$ & $\begin{array}{l}-0.183 \mathrm{E}-5 \\
(0.240 \mathrm{E}-4)\end{array}$ & $\begin{array}{l}9.170 \mathrm{E}-5 \\
(0.001)\end{array}$ & $\begin{array}{l}0.894 \mathrm{E}-4 \\
(0.0005)\end{array}$ & $\begin{array}{l}0.107 \mathrm{E}-4 \\
(0.0001)\end{array}$ & $\begin{array}{l}5.841 \mathrm{E}-5 \\
(0.000)\end{array}$ & $\begin{array}{l}0.393 E-4 \\
(0.0002)\end{array}$ & $\begin{array}{l}0.155 \mathrm{E}-5 \\
(0.314 \mathrm{E}-4)\end{array}$ & $\begin{array}{l}-0.000 \\
(0.000)\end{array}$ & $\begin{array}{l}0.500 \mathrm{E}-5 \\
(0.0002)\end{array}$ & $\begin{array}{l}0.324 \mathrm{E}-6 \\
(0.559 \mathrm{E}-4)\end{array}$ \\
\hline Offshore policy & $\begin{array}{l}0.272 \\
(0.835)\end{array}$ & $\begin{array}{l}0.200 \\
(0.810)\end{array}$ & $\begin{array}{l}0.012 \\
(0.093)\end{array}$ & $\begin{array}{l}1.772 \\
(2.253)\end{array}$ & $\begin{array}{l}1.502 \\
(1.874)\end{array}$ & $\begin{array}{l}0.188 \\
(0.255)\end{array}$ & $\begin{array}{l}-0.468 \\
(1.294)\end{array}$ & $\begin{array}{l}-0.786 \\
(1.219)\end{array}$ & $\begin{array}{l}-0.051 \\
(0.130)\end{array}$ & $\begin{array}{l}0.289 \\
(1.271)\end{array}$ & $\begin{array}{l}0.411 \\
(1.173)\end{array}$ & $\begin{array}{l}0.018 \\
(0.361)\end{array}$ \\
\hline $\begin{array}{l}\text { Internationalisation } \\
\text { at home strategy }\end{array}$ & $\begin{array}{l}0.172 \\
(0.159)\end{array}$ & $\begin{array}{l}-0.0007 \\
(0.001)\end{array}$ & $\begin{array}{l}-0.518 \mathrm{E}-4 \\
(0.0002)\end{array}$ & $\begin{array}{l}0.234 \\
(0.568)\end{array}$ & $\begin{array}{l}-0.004 \\
(0.002)\end{array}$ & $\begin{array}{l}-0.0002 \\
(0.064)\end{array}$ & $\begin{array}{l}0.288 \\
(0.241)\end{array}$ & $\begin{array}{l}0.001 \\
(0.002)\end{array}$ & $\begin{array}{l}0.520 \mathrm{E}-4 \\
(0.014)\end{array}$ & $\begin{array}{l}0.135 \\
(0.242)\end{array}$ & $\begin{array}{l}-0.0003 \\
(0.001)\end{array}$ & $\begin{array}{l}-0.216 \mathrm{E}-4 \\
(0.0002)\end{array}$ \\
\hline $\begin{array}{l}\text { Host country } \\
\text { demand }\end{array}$ & $\begin{array}{l}3.488 \\
(2.382)\end{array}$ & $\begin{array}{l}3.525 \\
(2.229)\end{array}$ & $\begin{array}{l}0.216 \\
(0.299)\end{array}$ & $\begin{array}{l}7.240 \\
(5.910)\end{array}$ & $\begin{array}{l}\text { 8.233† } \\
(4.859)\end{array}$ & $\begin{array}{l}0.546 \\
(0.927)\end{array}$ & $\begin{array}{l}-2.343 \\
(3.470)\end{array}$ & $\begin{array}{l}-2.100 \\
(3.336)\end{array}$ & $\begin{array}{l}-0.123 \\
(0.418)\end{array}$ & $\begin{array}{l}\text { 8.971* } \\
\text { (3.889) }\end{array}$ & $\begin{array}{l}\text { 6.625* } \\
(3.237)\end{array}$ & $\begin{array}{l}0.382 \\
(0.642)\end{array}$ \\
\hline AU/NZ dummy & $\begin{array}{l}-0.499 \\
(0.770)\end{array}$ & $\begin{array}{l}-0.572 \\
(0.727)\end{array}$ & $\begin{array}{l}-0.035 \\
(0.092)\end{array}$ & - & - & - & (2) & - & 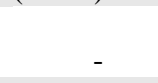 & (- & - & 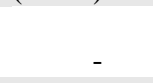 \\
\hline UK/IR dummy & $\begin{array}{l}0.174 \\
(0.497)\end{array}$ & $\begin{array}{l}0.130 \\
(0.477)\end{array}$ & $\begin{array}{l}0.008 \\
(0.070)\end{array}$ & - & - & - & - & - & - & - & - & - \\
\hline $\mathrm{R}^{2}$ & 0.527 & & & 0.798 & & & 0.578 & & & 0.259 & & \\
\hline $\operatorname{Adj}^{2}$ & 0.503 & & & 0.705 & & & 0.548 & & & 0.174 & & \\
\hline Log likelihood & & -422.33 & -459.22 & & -46.52 & -54.78 & & -222.61 & -240.06 & & -138.82 & -156.44 \\
\hline $\mathrm{N}$ & 175 & 175 & 175 & 21 & 21 & 21 & 91 & 91 & 91 & 63 & 63 & 63 \\
\hline Max. VIF & 1.344 & & & 1.667 & & & 1.125 & & & 1.378 & & \\
\hline
\end{tabular}

Standard errors in parentheses, all $t$ tests are two-tailed, $\uparrow p<.10,{ }^{*} p<.05,{ }^{* *} p<.01$

Table 7.30b: Regression estimates for equation 15b (high normative institutional distance)

\begin{tabular}{|c|c|c|c|c|c|c|c|c|c|c|c|c|}
\hline & \multicolumn{3}{|c|}{ Complete data set } & \multicolumn{3}{|c|}{ AU/NZ } & \multicolumn{3}{|c|}{ US/CA } & \multicolumn{3}{|c|}{ UK/IR } \\
\hline & OLS & Tobit & $\begin{array}{l}\text { Negative } \\
\text { binomial }\end{array}$ & OLS & Tobit & $\begin{array}{l}\text { Negative } \\
\text { binomial } \\
\end{array}$ & OLS & Tobit & $\begin{array}{l}\text { Negative } \\
\text { binomial }\end{array}$ & OLS & Tobit & $\begin{array}{l}\text { Negative } \\
\text { binomial }\end{array}$ \\
\hline Intercept & $\begin{array}{l}17.202 * * \\
(1.611)\end{array}$ & $\begin{array}{l}16.934 * * \\
(1.462)\end{array}$ & $\begin{array}{l}2.761 * * \\
(0.153)\end{array}$ & $\begin{array}{l}22.148 * * \\
(2.310)\end{array}$ & $\begin{array}{l}22.396 * * \\
(2.048)\end{array}$ & $\begin{array}{l}3.213 * * \\
(0.442)\end{array}$ & $\begin{array}{l}22.775 * * \\
(3.251)\end{array}$ & $\begin{array}{l}20.631 * * \\
(2.860)\end{array}$ & $\begin{array}{l}2.867 * * \\
(0.553)\end{array}$ & $\begin{array}{l}13.953 * * \\
(2.571)\end{array}$ & $\begin{array}{l}14.587 * * \\
(2.280)\end{array}$ & $\begin{array}{l}2.545 * * \\
(0.550)\end{array}$ \\
\hline $\begin{array}{l}\text { Know-how (Factor) } \\
(\mathrm{H} 7 \mathrm{c})\end{array}$ & $\begin{array}{l}-1.641 * * \\
(0.162)\end{array}$ & $\begin{array}{l}-1.635 * * \\
(0.155)\end{array}$ & $\begin{array}{l}-0.130 * * \\
(0.016)\end{array}$ & $\begin{array}{l}-1.578 * * \\
(0.245)\end{array}$ & $\begin{array}{l}-1.547^{* *} \\
(0.229)\end{array}$ & $\begin{array}{l}-0.109 * * \\
(0.026)\end{array}$ & $\begin{array}{l}-2.148 * * \\
(0.302)\end{array}$ & $\begin{array}{l}-2.166 * * \\
(0.281)\end{array}$ & $\begin{array}{l}-0.195 * * \\
(0.051)\end{array}$ & $\begin{array}{l}-1.476 * * \\
(0.314)\end{array}$ & $\begin{array}{l}-1.420^{* *} \\
(0.292)\end{array}$ & $\begin{array}{l}-0.109 \\
(0.075)\end{array}$ \\
\hline Age & $\begin{array}{l}0.003 \\
(0.005)\end{array}$ & $\begin{array}{l}0.002 \\
(0.004)\end{array}$ & $\begin{array}{l}0.0001 \\
(0.0006)\end{array}$ & $\begin{array}{l}-0.003 \\
(0.008)\end{array}$ & $\begin{array}{l}-0.002 \\
(0.007)\end{array}$ & $\begin{array}{l}-0.0001 \\
(0.001)\end{array}$ & $\begin{array}{l}-0.004 \\
(0.010)\end{array}$ & $\begin{array}{l}-0.001 \\
(0.008)\end{array}$ & $\begin{array}{l}-0.0002 \\
(0.002)\end{array}$ & $\begin{array}{l}0.008 \\
(0.006)\end{array}$ & $\begin{array}{l}0.003 \\
(0.005)\end{array}$ & $\begin{array}{l}0.0003 \\
(0.001)\end{array}$ \\
\hline Size (Staff) & $\begin{array}{l}0.000 \dagger \\
(0.000)\end{array}$ & $\begin{array}{l}0.0002 \dagger \\
(0.0001)\end{array}$ & $\begin{array}{l}0.201 \mathrm{E}-4 \\
(0.243 \mathrm{E}-4)\end{array}$ & $\begin{array}{l}0.000 \\
(0.000)\end{array}$ & $\begin{array}{l}0.0003 \\
(0.0002)\end{array}$ & $\begin{array}{l}0.222 \mathrm{E}-4 \\
(0.551 \mathrm{E}-4)\end{array}$ & $\begin{array}{l}0.000 \\
(0.000)\end{array}$ & $\begin{array}{l}-0.0001 \\
(0.0003)\end{array}$ & $\begin{array}{l}-0.974 \mathrm{E}-5 \\
(0.784 \mathrm{E}-4)\end{array}$ & $\begin{array}{l}1.115 \mathrm{E}-5 \\
(0.000)\end{array}$ & $\begin{array}{l}0.285 \mathrm{E}-4 \\
(0.0002)\end{array}$ & $\begin{array}{l}0.477 \mathrm{E}-5 \\
(0.581 \mathrm{E}-4)\end{array}$ \\
\hline Offshore policy & $\begin{array}{l}2.512 * \\
(1.050)\end{array}$ & $\begin{array}{l}2.593^{*} \\
(1.013)\end{array}$ & $\begin{array}{l}0.268 * * \\
(0.091)\end{array}$ & $\begin{array}{l}-2.194 \\
(1.749)\end{array}$ & $\begin{array}{l}-2.049 \\
(1.641)\end{array}$ & $\begin{array}{l}-0.144 \\
(0.409)\end{array}$ & $\begin{array}{l}5.116 * * \\
(1.615)\end{array}$ & $\begin{array}{l}4.983 * * \\
(1.506)\end{array}$ & $\begin{array}{l}0.662^{* *} \\
(0.186)\end{array}$ & $\begin{array}{l}5.040 * * \\
(1.761)\end{array}$ & $\begin{array}{l}5.148 * * \\
(1.659)\end{array}$ & $\begin{array}{l}0.485 \\
(0.312)\end{array}$ \\
\hline
\end{tabular}




\begin{tabular}{|c|c|c|c|c|c|c|c|c|c|c|c|c|}
\hline $\begin{array}{l}\text { Internationalisation } \\
\text { at home strategy }\end{array}$ & $\begin{array}{l}-0.142 \\
(0.180)\end{array}$ & $\begin{array}{l}-0.0009 \\
(0.0009)\end{array}$ & $\begin{array}{l}-0.715 \mathrm{E}-4 \\
(0.0001)\end{array}$ & $\begin{array}{l}0.168 \\
(0.326)\end{array}$ & $\begin{array}{l}-0.001 \\
(0.001)\end{array}$ & $\begin{array}{l}-0.837 \mathrm{E}-4 \\
(0.0002)\end{array}$ & $\begin{array}{l}-0.578 \dagger \\
(0.292)\end{array}$ & $\begin{array}{l}-0.001 \\
(0.002)\end{array}$ & $\begin{array}{l}-0.0001 \\
(0.018)\end{array}$ & $\begin{array}{l}0.170 \\
(0.265)\end{array}$ & $\begin{array}{l}0.0001 \\
(0.0009)\end{array}$ & $\begin{array}{l}-0.260 \mathrm{E}-5 \\
(0.0002)\end{array}$ \\
\hline $\begin{array}{l}\text { Host country } \\
\text { demand }\end{array}$ & $\begin{array}{l}3.920 \\
(2.768)\end{array}$ & $\begin{array}{l}3.819 \\
(2.609)\end{array}$ & $\begin{array}{l}0.305 \\
(0.370)\end{array}$ & $\begin{array}{l}3.393 \\
(4.094)\end{array}$ & $\begin{array}{l}4.906 \\
(3.641)\end{array}$ & $\begin{array}{l}0.333 \\
(0.553)\end{array}$ & $\begin{array}{l}-12.553 \dagger \\
(6.839)\end{array}$ & $\begin{array}{l}-11.317 \dagger \\
(6.357)\end{array}$ & $\begin{array}{l}-0.695 \\
(1.282)\end{array}$ & $\begin{array}{l}6.552 \\
(4.677)\end{array}$ & $\begin{array}{l}5.949 \\
(4.215)\end{array}$ & $\begin{array}{l}0.389 \\
(1.277)\end{array}$ \\
\hline AU/NZ dummy & $\begin{array}{l}1.400 * \\
(0.708)\end{array}$ & $\begin{array}{l}\text { 1.315* } \\
(0.669)\end{array}$ & $\begin{array}{l}0.099 \\
(0.087)\end{array}$ & - & - & - & (- & ( & 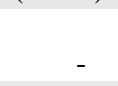 & (1 & ( & (2) \\
\hline UK/IR dummy & $\begin{array}{l}0586 \\
(0.701)\end{array}$ & $\begin{array}{l}0.412 \\
(0.655)\end{array}$ & $\begin{array}{l}0.034 \\
(0.098)\end{array}$ & - & - & - & - & - & - & - & - & - \\
\hline $\mathrm{R}^{2}$ & 0.651 & & & 0.474 & & & 0.862 & & & 0.701 & & \\
\hline $\operatorname{AdjR}^{2}$ & 0.626 & & & 0.409 & & & 0.828 & & & 0.639 & & \\
\hline Log likelihood & & -312.93 & -345.06 & & -138.28 & -151.01 & & -73.28 & -81.79 & & -85.78 & -101.67 \\
\hline $\mathrm{N}$ & 132 & 132 & 132 & 59 & 59 & 59 & 32 & 32 & 32 & 41 & 41 & 41 \\
\hline Max. VIF & 2.130 & & & 1.341 & & & 2.068 & & & 1.503 & & \\
\hline
\end{tabular}

Standard errors in parentheses, all $t$ tests are two-tailed, $\dagger p<.10,{ }^{*} p<.05,{ }^{* *} p<.01$

Table 7.31a: Regression estimates for equation 16a (low normative institutional distance)

\begin{tabular}{|c|c|c|c|c|c|c|c|c|c|c|c|c|}
\hline & \multicolumn{3}{|c|}{ Complete data set } & \multicolumn{3}{|c|}{ AU/NZ } & \multicolumn{3}{|c|}{ 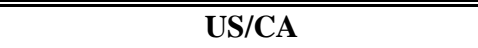 } & \multicolumn{3}{|c|}{ 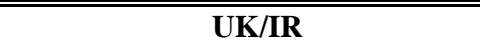 } \\
\hline & OLS & Tobit & $\begin{array}{l}\text { Negative } \\
\text { binomial }\end{array}$ & OLS & Tobit & $\begin{array}{l}\text { Negative } \\
\text { binomial }\end{array}$ & OLS & Tobit & $\begin{array}{l}\text { Negative } \\
\text { binomial }\end{array}$ & OLS & Tobit & $\begin{array}{l}\text { Negative } \\
\text { binomial }\end{array}$ \\
\hline Intercept & $\begin{array}{l}22.533^{* *} \\
(1.635)\end{array}$ & $\begin{array}{l}23.459 * * \\
(1.437)\end{array}$ & $\begin{array}{l}3.286 * * \\
(0.137)\end{array}$ & $\begin{array}{l}27.158 * * \\
(4.951)\end{array}$ & $\begin{array}{l}\text { 28.145** } \\
(3.882)\end{array}$ & $\begin{array}{l}3.714 * * \\
(0.404)\end{array}$ & $\begin{array}{l}22.389 * * \\
(2.517)\end{array}$ & $\begin{array}{l}24.399 * * \\
(2.091)\end{array}$ & $\begin{array}{l}3.358 * * \\
(0.169)\end{array}$ & $\begin{array}{l}21.903 * * \\
(2.443)\end{array}$ & $\begin{array}{l}22.879 * * \\
(2.060)\end{array}$ & $\begin{array}{l}3.216 * * \\
(0.653)\end{array}$ \\
\hline $\begin{array}{l}\text { Org culture (Factor) } \\
-(H 7 d)\end{array}$ & $\begin{array}{l}-1.852 * * \\
(0.194)\end{array}$ & $\begin{array}{l}-1.891 * * \\
(0.188)\end{array}$ & $\begin{array}{l}-0.134 * * \\
(0.019)\end{array}$ & $\begin{array}{l}-2.835 * * \\
(0.699)\end{array}$ & $\begin{array}{l}-3.000 * * \\
(0.594)\end{array}$ & $\begin{array}{l}-0.257 * * \\
(0.053)\end{array}$ & $\begin{array}{l}-1.816 * * \\
(0.256)\end{array}$ & $\begin{array}{l}-1.849 * * \\
(0.246)\end{array}$ & $\begin{array}{l}-0.131 * * \\
(0.023)\end{array}$ & $(-0.10)$ & (-.000) & (20000) \\
\hline $\begin{array}{l}\text { Org culture - open } \\
\text { discussion }(H 7 d)\end{array}$ & - & - & - & - & - & - & - & - & - & $\begin{array}{l}-0.200 \\
(0.290)\end{array}$ & $\begin{array}{l}-0.308 \\
(0.248)\end{array}$ & $\begin{array}{l}-0.019 \\
(0.059)\end{array}$ \\
\hline $\begin{array}{l}\text { Org culture - no } \\
\text { status distinction } \\
(H 7 d)\end{array}$ & - & - & - & - & - & - & - & - & - & $\begin{array}{l}-0.272 \\
(0.283)\end{array}$ & $\begin{array}{l}-0.376 \\
(0.234)\end{array}$ & $\begin{array}{l}-0.023 \\
(0.057)\end{array}$ \\
\hline $\begin{array}{l}\text { Org culture - } \\
\text { experimentation } \\
(H 7 d)\end{array}$ & - & - & - & - & - & - & - & - & - & $\begin{array}{l}-0.301 \\
(0.252)\end{array}$ & $\begin{array}{l}-0.330 \\
(0.206)\end{array}$ & $\begin{array}{l}-0.020 \\
(0.056)\end{array}$ \\
\hline $\begin{array}{l}\text { Org culture - } \\
\text { offshore } \\
\text { development }(H 7 d)\end{array}$ & - & - & - & - & - & - & - & - & - & $\begin{array}{l}-0.582 * \\
(0.249)\end{array}$ & $\begin{array}{l}-0.643^{* *} \\
(0.212)\end{array}$ & $\begin{array}{l}-0.040 \\
(0.062)\end{array}$ \\
\hline
\end{tabular}




\begin{tabular}{|c|c|c|c|c|c|c|c|c|c|c|c|c|}
\hline $\begin{array}{l}\text { Org culture - } \\
\text { offshore } \\
\text { responsiveness } \\
(H 7 d)\end{array}$ & - & - & - & - & - & - & - & - & - & $\begin{array}{l}-0.153 \\
(0.304)\end{array}$ & $\begin{array}{l}-0.021 \\
(0.237)\end{array}$ & $\begin{array}{l}-0.003 \\
(0.058)\end{array}$ \\
\hline Age & $\begin{array}{l}0.003 \\
(0.004)\end{array}$ & $\begin{array}{l}0.002 \\
(0.004)\end{array}$ & $\begin{array}{l}0.0001 \\
(0.0004)\end{array}$ & $\begin{array}{l}-0.014 \\
(0.032)\end{array}$ & $\begin{array}{l}-0.012 \\
(0.022)\end{array}$ & $\begin{array}{l}-0.0008 \\
(0.002)\end{array}$ & $\begin{array}{l}0.002 \\
(0.007)\end{array}$ & $\begin{array}{l}0.001 \\
(0.006)\end{array}$ & $\begin{array}{l}0.0001 \\
(0.0006)\end{array}$ & $\begin{array}{l}0.002 \\
(0.005)\end{array}$ & $\begin{array}{l}0.0006 \\
(0.004)\end{array}$ & $\begin{array}{l}0.191 \mathrm{E}-4 \\
(0.0012)\end{array}$ \\
\hline Size (Staff) & $\begin{array}{l}0.000 \\
(0.000)\end{array}$ & $\begin{array}{l}-0.522 \mathrm{E}-4 \\
(0.0001)\end{array}$ & $\begin{array}{l}-0.329 E-5 \\
(0.225 E-4)\end{array}$ & $\begin{array}{l}0.000 \\
(0.001)\end{array}$ & $\begin{array}{l}-0.0004 \\
(0.0006)\end{array}$ & $\begin{array}{l}-0.321 \mathrm{E}-4 \\
(0.587 \mathrm{E}-4)\end{array}$ & $\begin{array}{l}0.000 \\
(0.000)\end{array}$ & $\begin{array}{l}0.0001 \\
(0.0002)\end{array}$ & $\begin{array}{l}0.886 E-5 \\
(0.285 E-4)\end{array}$ & $\begin{array}{l}-0.000 \\
(0.000)\end{array}$ & $\begin{array}{l}0.388 \mathrm{E}-4 \\
(0.0002)\end{array}$ & $\begin{array}{l}0.186 \mathrm{E}-5 \\
(0.575 \mathrm{E}-4)\end{array}$ \\
\hline Offshore policy & $\begin{array}{l}0.295 \\
(0.926)\end{array}$ & $\begin{array}{l}0.208 \\
(0.897)\end{array}$ & $\begin{array}{l}0.017 \\
(0.081)\end{array}$ & $\begin{array}{l}1.459 \\
(2.965)\end{array}$ & $\begin{array}{l}1.001 \\
(2.449)\end{array}$ & $\begin{array}{l}0.139 \\
(0.239)\end{array}$ & $\begin{array}{l}-0.408 \\
(1.533)\end{array}$ & $\begin{array}{l}-0.882 \\
(1.455)\end{array}$ & $\begin{array}{l}-0.066 \\
(0.113)\end{array}$ & $\begin{array}{l}0.010 \\
(1.265)\end{array}$ & $\begin{array}{l}0.203 \\
(1.1116)\end{array}$ & $\begin{array}{l}0.006 \\
(0.390)\end{array}$ \\
\hline $\begin{array}{l}\text { Internationalisation } \\
\text { at home strategy }\end{array}$ & $\begin{array}{l}0.221 \\
(0.175)\end{array}$ & $\begin{array}{l}0.132 \mathrm{E}-4 \\
(0.001)\end{array}$ & $\begin{array}{l}0.765 E-5 \\
(0.0002)\end{array}$ & $\begin{array}{l}-0.040 \\
(0.745)\end{array}$ & $\begin{array}{l}-0.002 \\
(0.003)\end{array}$ & $\begin{array}{l}-0.0001 \\
(0.049)\end{array}$ & $\begin{array}{l}0.438 \\
(0.282)\end{array}$ & $\begin{array}{l}0.0003 \\
(0.003)\end{array}$ & $\begin{array}{l}0.921 \mathrm{E}-5 \\
(0.012)\end{array}$ & $\begin{array}{l}0.113 \\
(0.244)\end{array}$ & $\begin{array}{l}0.0009 \\
(0.001)\end{array}$ & $\begin{array}{l}0.603 E-4 \\
(0.0003)\end{array}$ \\
\hline $\begin{array}{l}\text { Host country } \\
\text { demand }\end{array}$ & $\begin{array}{l}3.859 \\
(2.635)\end{array}$ & $\begin{array}{l}3.698 \\
(2.465)\end{array}$ & $\begin{array}{l}0.219 \\
(0.276)\end{array}$ & $\begin{array}{l}7.076 \\
(7.799)\end{array}$ & $\begin{array}{l}7.674 \\
(6.380)\end{array}$ & $\begin{array}{l}0.489 \\
(0.549)\end{array}$ & $\begin{array}{l}-1.084 \\
(4.082)\end{array}$ & $\begin{array}{l}-0.646 \\
(3.945)\end{array}$ & $\begin{array}{l}-0.033 \\
(0.374)\end{array}$ & $\begin{array}{l}\text { 8.264† } \\
\mathbf{( 4 . 3 9 2 )}\end{array}$ & $\begin{array}{l}5.603 \dagger \\
(3.348)\end{array}$ & $\begin{array}{l}0.327 \\
(0.873)\end{array}$ \\
\hline AU/NZ dummy & $\begin{array}{l}-0.138 \\
(0.849)\end{array}$ & $\begin{array}{l}-0.259 \\
(0.801)\end{array}$ & $\begin{array}{l}-0.011 \\
(0.082)\end{array}$ & - & - & - & - & - & - & - & - & - \\
\hline UK/IR dummy & $\begin{array}{l}0.324 \\
(0.550)\end{array}$ & $\begin{array}{l}0.235 \\
(0.528)\end{array}$ & $\begin{array}{l}0.015 \\
(0.071)\end{array}$ & - & - & - & - & - & - & - & - & - \\
\hline $\mathrm{R}^{2}$ & 0.423 & & & 0.651 & & & 0.416 & & & 0.347 & & \\
\hline $\operatorname{Adj}^{2}$ & 0.394 & & & 0.490 & & & 0.374 & & & 0.211 & & \\
\hline Log likelihood & & -439.46 & -470.21 & & -52.11 & -58.82 & & -237.92 & -250.89 & & -133.99 & -155.15 \\
\hline $\mathrm{N}$ & 175 & 175 & 175 & 21 & 21 & 21 & 91 & 91 & 91 & 63 & 63 & 63 \\
\hline Max. VIF & 1.339 & & & 1.631 & & & 1.131 & & & 2.079 & & \\
\hline
\end{tabular}

Standard errors in parentheses, all $t$ tests are two-tailed, $\dagger p<.10, * p<.05,{ }^{* *} p<.01$

Table 7.31b: Regression estimates for equation 16b (high normative institutional distance)

\begin{tabular}{|c|c|c|c|c|c|c|c|c|c|c|c|c|}
\hline & \multicolumn{3}{|c|}{ Complete data set } & \multicolumn{3}{|c|}{ AU/NZ } & \multicolumn{3}{|c|}{ US/CA } & \multicolumn{3}{|c|}{ 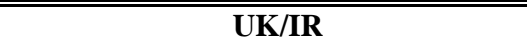 } \\
\hline & OLS & Tobit & $\begin{array}{l}\text { Negative } \\
\text { binomial }\end{array}$ & OLS & Tobit & $\begin{array}{l}\text { Negative } \\
\text { binomial }\end{array}$ & OLS & Tobit & $\begin{array}{l}\text { Negative } \\
\text { binomial }\end{array}$ & OLS & Tobit & $\begin{array}{l}\text { Negative } \\
\text { binomial }\end{array}$ \\
\hline Intercept & $\begin{array}{l}19.520 * * \\
(1.936)\end{array}$ & $\begin{array}{l}18.529 * * \\
(1.809)\end{array}$ & $\begin{array}{l}2.844 * * \\
(0.191)\end{array}$ & $\begin{array}{l}23.892 * * \\
(2.654)\end{array}$ & $\begin{array}{l}23.804 * * \\
(2.359)\end{array}$ & $\begin{array}{l}3.275 * * \\
(0.365)\end{array}$ & $\begin{array}{l}25.075^{* *} \\
(3.366)\end{array}$ & $\begin{array}{l}23.782 * * \\
(2.952)\end{array}$ & $\begin{array}{l}3.180 * * \\
(0.643)\end{array}$ & $\begin{array}{l}11.963 * \\
(4.853)\end{array}$ & $\begin{array}{l}10.642 * * \\
(3.791)\end{array}$ & $\begin{array}{l}2.269 * * \\
(0.610)\end{array}$ \\
\hline $\begin{array}{l}\text { Org culture (Factor) } \\
-(H 7 d)\end{array}$ & $\begin{array}{l}-1.986 * * \\
(0.222)\end{array}$ & $\begin{array}{l}-1.885^{* *} \\
(0.216)\end{array}$ & $\begin{array}{l}-0.139 * * \\
(0.024)\end{array}$ & $\begin{array}{l}-1.873^{* *} \\
(0.329)\end{array}$ & $\begin{array}{l}-1.810^{* *} \\
(0.308)\end{array}$ & $\begin{array}{l}-0.120 * * \\
(0.041)\end{array}$ & $\begin{array}{l}-2.734 * * \\
(0.367)\end{array}$ & $\begin{array}{l}-2.789 * * \\
(0.330)\end{array}$ & $\begin{array}{l}-0.252^{* *} \\
(0.066)\end{array}$ & - & - & - \\
\hline
\end{tabular}




\begin{tabular}{|c|c|c|c|c|c|c|c|c|c|c|c|c|}
\hline $\begin{array}{l}\text { Org culture - open } \\
\text { discussion }(H 7 d)\end{array}$ & - & - & - & - & - & - & - & - & - & $\begin{array}{l}-0.158 \\
(0.397)\end{array}$ & $\begin{array}{l}-0.237 \\
(0.334)\end{array}$ & $\begin{array}{l}-0.014 \\
(0.065)\end{array}$ \\
\hline $\begin{array}{l}\text { Org culture }- \text { no } \\
\text { status distinction } \\
(H 7 d)\end{array}$ & - & - & - & - & - & - & - & - & - & $\begin{array}{l}-0.344 \\
(0.437)\end{array}$ & $\begin{array}{l}-0.080 \\
(0.344)\end{array}$ & $\begin{array}{l}-0.006 \\
(0.071)\end{array}$ \\
\hline $\begin{array}{l}\text { Org culture - } \\
\text { experimentation } \\
(H 7 d)\end{array}$ & - & - & - & - & - & - & - & - & - & $\begin{array}{l}0.052 \\
(0.339)\end{array}$ & $\begin{array}{l}-0.152 \\
(0.259)\end{array}$ & $\begin{array}{l}-0.010 \\
(0.045)\end{array}$ \\
\hline $\begin{array}{l}\text { Org culture - } \\
\text { offshore } \\
\text { development }(H 7 d)\end{array}$ & - & - & - & - & - & - & - & - & - & $\begin{array}{l}-0.222 \\
(0.433)\end{array}$ & $\begin{array}{l}0.027 \\
(0.337)\end{array}$ & $\begin{array}{l}0.675 E-4 \\
(0.076)\end{array}$ \\
\hline $\begin{array}{l}\text { Org culture - } \\
\text { offshore } \\
\text { responsiveness } \\
(H 7 d)\end{array}$ & - & - & - & - & - & - & - & - & - & $\begin{array}{l}-0.283 \\
(0.378)\end{array}$ & $\begin{array}{l}-0.197 \\
(0.316)\end{array}$ & $\begin{array}{l}-0.012 \\
(0.068)\end{array}$ \\
\hline Age & $\begin{array}{l}0.002 \\
(0.005)\end{array}$ & $\begin{array}{l}0.001 \\
(0.004)\end{array}$ & $\begin{array}{l}0.0001 \\
(0.0007)\end{array}$ & $\begin{array}{l}0.003 \\
(0.009)\end{array}$ & $\begin{array}{l}0.003 \\
(0.008)\end{array}$ & $\begin{array}{l}0.0002 \\
(0.001)\end{array}$ & $\begin{array}{l}0.003 \\
(0.009)\end{array}$ & $\begin{array}{l}0.005 \\
(0.008)\end{array}$ & $\begin{array}{l}0.0003 \\
(0.001)\end{array}$ & $\begin{array}{l}0.001 \\
(0.008)\end{array}$ & $\begin{array}{l}-0.003 \\
(0.006)\end{array}$ & $\begin{array}{l}-0.0001 \\
(0.001)\end{array}$ \\
\hline Size (Staff) & $\begin{array}{l}0.000 \\
(0.000)\end{array}$ & $\begin{array}{l}0.0001 \\
(0.0001)\end{array}$ & $\begin{array}{l}0.127 \mathrm{E}-4 \\
(0.276 \mathrm{E}-4)\end{array}$ & $\begin{array}{l}0.000 \\
(0.000)\end{array}$ & $\begin{array}{l}0.0002 \\
(0.0002)\end{array}$ & $\begin{array}{l}0.122 E-4 \\
(0.462 E-4)\end{array}$ & $\begin{array}{l}0.000 \\
(0.000)\end{array}$ & $\begin{array}{l}-0.0005 \\
(0.0003)\end{array}$ & $\begin{array}{l}-0.484 \mathrm{E}-4 \\
(0.936 \mathrm{E}-4)\end{array}$ & $\begin{array}{l}0.000 \\
(0.000)\end{array}$ & $\begin{array}{l}0.562 \mathrm{E}-4 \\
(0.0002)\end{array}$ & $\begin{array}{l}0.396 \mathrm{E}-5 \\
(0.646 \mathrm{E}-4)\end{array}$ \\
\hline Offshore policy & $\begin{array}{l}3.792 * * \\
(1.064)\end{array}$ & $\begin{array}{l}4.069 * * \\
(1.046)\end{array}$ & $\begin{array}{l}0.398 * * \\
(0.085)\end{array}$ & $\begin{array}{l}-0.771 \\
(1.781)\end{array}$ & $\begin{array}{l}-0.626 \\
(1.673)\end{array}$ & $\begin{array}{l}-0.036 \\
(0.293)\end{array}$ & $\begin{array}{l}6.466 * * \\
(1.486)\end{array}$ & $\begin{array}{l}6.322 * * \\
(1.344)\end{array}$ & $\begin{array}{l}0.799 * * \\
(0.218)\end{array}$ & $\begin{array}{l}\text { 7.056** } \\
(2.505)\end{array}$ & $\begin{array}{l}7.430 * * \\
(2.140)\end{array}$ & $\begin{array}{l}0.648^{*} \\
(0.272)\end{array}$ \\
\hline $\begin{array}{l}\text { Internationalisation } \\
\text { at home strategy }\end{array}$ & $\begin{array}{l}-0.192 \\
(0.191)\end{array}$ & $\begin{array}{l}0.0001 \\
(0.0009)\end{array}$ & $\begin{array}{l}0.154 \mathrm{E}-4 \\
(0.994 \mathrm{E}-4)\end{array}$ & $\begin{array}{l}0.107 \\
(0.344)\end{array}$ & $\begin{array}{l}-0.0009 \\
(0.001)\end{array}$ & $\begin{array}{l}-0.520 \mathrm{E}-4 \\
(0.0002)\end{array}$ & $\begin{array}{l}-0.431 \\
(0.284)\end{array}$ & $\begin{array}{l}0.0005 \\
(0.002)\end{array}$ & $\begin{array}{l}0.744 \mathrm{E}-4 \\
(0.037)\end{array}$ & $\begin{array}{l}0.026 \\
(0.369)\end{array}$ & $\begin{array}{l}0.001 \\
(0.001)\end{array}$ & $\begin{array}{l}0.634 \mathrm{E}-4 \\
(0.0002)\end{array}$ \\
\hline $\begin{array}{l}\text { Host country } \\
\text { demand }\end{array}$ & $\begin{array}{l}3.162 \\
(2.939)\end{array}$ & $\begin{array}{l}3.158 \\
(2.826)\end{array}$ & $\begin{array}{l}0.222 \\
(0.347)\end{array}$ & $\begin{array}{l}1.159 \\
(4.267)\end{array}$ & $\begin{array}{l}2.661 \\
(3.794)\end{array}$ & $\begin{array}{l}0.165 \\
(0.523)\end{array}$ & $\begin{array}{l}-11.105 \\
(6.523)\end{array}$ & $\begin{array}{l}-10.449 \dagger \\
(5.900)\end{array}$ & $\begin{array}{l}-0.760 \\
(1.589)\end{array}$ & $\begin{array}{l}10.579 \\
(6.914)\end{array}$ & $\begin{array}{l}10.488 \dagger \\
(5.651)\end{array}$ & $\begin{array}{l}0.620 \\
(1.208)\end{array}$ \\
\hline AU/NZ dummy & $\begin{array}{l}1.269 \dagger \\
(0.751)\end{array}$ & $\begin{array}{l}1.246 \dagger \\
(0.724)\end{array}$ & $\begin{array}{l}0.089 \\
(0.088)\end{array}$ & - & - & - & - & - & - & - & - & - \\
\hline UK/IR dummy & $\begin{array}{l}0.480 \\
(0.744)\end{array}$ & $\begin{array}{l}0.346 \\
(0.710)\end{array}$ & $\begin{array}{l}0.027 \\
(0.091)\end{array}$ & - & - & - & - & - & - & - & - & - \\
\hline $\mathrm{R}^{2}$ & 0.609 & & & 0.414 & & & 0.871 & & & 0.536 & & \\
\hline $\operatorname{AdjR}^{2}$ & 0.581 & & & 0.342 & & & 0.839 & & & 0.350 & & \\
\hline Log likelihood & & -323.21 & -351.84 & & -141.55 & -152.82 & & -71.30 & -80.98 & & -94.08 & -104.73 \\
\hline $\mathrm{N}$ & 132 & 132 & 132 & 59 & 59 & 59 & 32 & 32 & 32 & 41 & 41 & 41 \\
\hline Max. VIF & 2.138 & & & 1.183 & & & 1.817 & & & 1.880 & & \\
\hline
\end{tabular}

Standard errors in parentheses, all $t$ tests are two-tailed, $\dagger p<.10,{ }^{*} p<.05,{ }^{* *} p<.01$ 
Table 7.32a: Regression estimates for equation 17a (low normative institutional distance)

\begin{tabular}{|c|c|c|c|c|c|c|c|c|c|c|c|c|}
\hline & \multicolumn{3}{|c|}{ Complete data set } & \multicolumn{3}{|c|}{ AU/NZ } & \multicolumn{3}{|c|}{ US/CA } & \multicolumn{3}{|c|}{ UK/IR } \\
\hline & OLS & Tobit & $\begin{array}{l}\text { Negative } \\
\text { binomial }\end{array}$ & OLS & Tobit & $\begin{array}{l}\text { Negative } \\
\text { binomial }\end{array}$ & OLS & Tobit & $\begin{array}{l}\text { Negative } \\
\text { binomial }\end{array}$ & OLS & Tobit & $\begin{array}{l}\text { Negative } \\
\text { binomial }\end{array}$ \\
\hline Intercept & $\begin{array}{l}12.502 * * \\
(1.698)\end{array}$ & $\begin{array}{l}13.508 * * \\
(1.527)\end{array}$ & $\begin{array}{l}2.610 * * \\
(0.099)\end{array}$ & $\begin{array}{l}15.093^{*} \\
(6.387)\end{array}$ & $\begin{array}{l}15.540 * * \\
(4.589)\end{array}$ & $\begin{array}{l}2.669 * * \\
(0.437)\end{array}$ & $\begin{array}{l}11.213 * * \\
(2.723)\end{array}$ & $\begin{array}{l}13.193 * * \\
(2.343)\end{array}$ & $\begin{array}{l}2.590 * * \\
(0.144)\end{array}$ & $\begin{array}{l}15.657 * * \\
(1.631)\end{array}$ & $\begin{array}{l}16.224 * * \\
(1.539)\end{array}$ & $\begin{array}{l}2.786 * * \\
(0.411)\end{array}$ \\
\hline $\begin{array}{l}\text { Financial resources } \\
(\mathrm{H} 7 \mathrm{e})\end{array}$ & $\begin{array}{l}-0.044 \\
(0.163)\end{array}$ & $\begin{array}{l}0.0008 \\
(0.155)\end{array}$ & $\begin{array}{l}-0.215 \mathrm{E}-4 \\
(0.014)\end{array}$ & $\begin{array}{l}-0.334 \\
(1.012)\end{array}$ & $\begin{array}{l}-0.400 \\
(0.825)\end{array}$ & $\begin{array}{l}-0.023 \\
(0.117)\end{array}$ & $\begin{array}{l}0.096 \\
(0.238)\end{array}$ & $\begin{array}{l}0.173 \\
(0.226)\end{array}$ & $\begin{array}{l}0.010 \\
(0.020)\end{array}$ & $\begin{array}{l}-0.308 \\
(0.201)\end{array}$ & $\begin{array}{l}-0.210 \\
(0.187)\end{array}$ & $\begin{array}{l}-0.012 \\
(0.040)\end{array}$ \\
\hline Age & $\begin{array}{l}0.003 \\
(0.006)\end{array}$ & $\begin{array}{l}0.001 \\
(0.005)\end{array}$ & $\begin{array}{l}0.0001 \\
(0.0004)\end{array}$ & $\begin{array}{c}0.005 \\
(0.049)\end{array}$ & $\begin{array}{l}0.006 \\
(0.034)\end{array}$ & $\begin{array}{l}0.0005 \\
(0.007)\end{array}$ & $\begin{array}{l}-0.003 \\
(0.009)\end{array}$ & $\begin{array}{l}-0.004 \\
(0.008)\end{array}$ & $\begin{array}{l}-0.0002 \\
(0.0005)\end{array}$ & $\begin{array}{l}0.006 \\
(0.006)\end{array}$ & $\begin{array}{l}0.003 \\
(0.005)\end{array}$ & $\begin{array}{l}0.0002 \\
(0.0008)\end{array}$ \\
\hline Size (Staff) & $\begin{array}{l}-0.000 \\
(0.000)\end{array}$ & $\begin{array}{l}-0.987 \mathrm{E}-5 \\
(0.0002)\end{array}$ & $\begin{array}{l}-0.192 \mathrm{E}-6 \\
(0.232 \mathrm{E}-4)\end{array}$ & $\begin{array}{l}0.000 \\
(0.002)\end{array}$ & $\begin{array}{l}-0.0006 \\
(0.001)\end{array}$ & $\begin{array}{l}-0.392 \mathrm{E}-4 \\
(0.0001)\end{array}$ & $\begin{array}{l}0.000 \\
(0.000)\end{array}$ & $\begin{array}{l}0.343 \mathrm{E}-4 \\
(0.0003)\end{array}$ & $\begin{array}{l}0.221 \mathrm{E}-5 \\
(0.346 \mathrm{E}-4)\end{array}$ & $\begin{array}{l}-0.000 \\
(0.000)\end{array}$ & $\begin{array}{l}0.848 E-4 \\
(0.0002)\end{array}$ & $\begin{array}{l}0.480 \mathrm{E}-5 \\
(0.487 \mathrm{E}-4)\end{array}$ \\
\hline Offshore policy & $\begin{array}{l}1.924 \dagger \\
(1.153)\end{array}$ & $\begin{array}{l}1.796 \\
(1.120)\end{array}$ & $\begin{array}{l}0.120 * \\
(0.060)\end{array}$ & $\begin{array}{l}2.707 \\
(4.713)\end{array}$ & $\begin{array}{l}2.434 \\
(3.834)\end{array}$ & $\begin{array}{l}0.200 \\
(0.437)\end{array}$ & $\begin{array}{l}1.996 \\
(1.895)\end{array}$ & $\begin{array}{l}1.424 \\
(1.802)\end{array}$ & $\begin{array}{l}0.095 \\
(0.083)\end{array}$ & $\begin{array}{l}1.126 \\
(1.414)\end{array}$ & $\begin{array}{l}1.304 \\
(1.347)\end{array}$ & $\begin{array}{l}0.078 \\
(0.387)\end{array}$ \\
\hline $\begin{array}{l}\text { Internationalisation } \\
\text { at home strategy }\end{array}$ & $\begin{array}{l}0.359 \\
(0.221)\end{array}$ & $\begin{array}{l}-0.0006 \\
(0.001)\end{array}$ & $\begin{array}{l}-0.353 E-4 \\
(0.0001)\end{array}$ & $\begin{array}{l}-0.012 \\
(1.131)\end{array}$ & $\begin{array}{l}-0.003 \\
(0.005)\end{array}$ & $\begin{array}{l}-0.0001 \\
(0.003)\end{array}$ & $\begin{array}{l}0.574 \\
(0.364)\end{array}$ & $\begin{array}{l}-0.001 \\
(0.004)\end{array}$ & $\begin{array}{l}-0.635 \mathrm{E}-4 \\
(0.012)\end{array}$ & $\begin{array}{l}0.360 \\
(0.260)\end{array}$ & $\begin{array}{l}-0.0002 \\
(0.001)\end{array}$ & $\begin{array}{l}-0.113 E-4 \\
(0.0002)\end{array}$ \\
\hline $\begin{array}{l}\text { Host country } \\
\text { demand }\end{array}$ & $\begin{array}{l}\text { 7.931* } \\
(3.280)\end{array}$ & $\begin{array}{l}\text { 7.688* } \\
(3.083)\end{array}$ & $\begin{array}{l}0.447 \\
(0.319)\end{array}$ & $\begin{array}{l}11.442 \\
(11.816)\end{array}$ & $\begin{array}{l}12.138 \\
(9.719)\end{array}$ & $\begin{array}{l}0.695 \\
(1.633)\end{array}$ & $\begin{array}{l}5.308 \\
(5.155)\end{array}$ & $\begin{array}{l}6.321 \\
(4.958)\end{array}$ & $\begin{array}{l}0.383 \\
(0.496)\end{array}$ & $\begin{array}{l}10.266^{*} \\
(4.139)\end{array}$ & $\begin{array}{l}6.591 \dagger \\
(3.535)\end{array}$ & $\begin{array}{l}0.366 \\
(0.601)\end{array}$ \\
\hline AU/NZ dummy & $\begin{array}{l}0.136 \\
(1.125)\end{array}$ & $\begin{array}{l}-0.014 \\
(1.076)\end{array}$ & $\begin{array}{l}0.001 \\
(0.083)\end{array}$ & $(1+0 \times)$ & - & - & - & - & - & $(70.00)$ & - & ) \\
\hline UK/IR dummy & $\begin{array}{l}1.362 \dagger \\
(0.796)\end{array}$ & $\begin{array}{l}1.401 \dagger \\
(0.760)\end{array}$ & $\begin{array}{l}0.084 \\
(0.075)\end{array}$ & - & - & - & - & - & - & - & - & - \\
\hline $\mathrm{R}^{2}$ & 0.096 & & & 0.216 & & & 0.063 & & & 0.156 & & \\
\hline $\operatorname{Adj}^{2}$ & 0.050 & & & -0.146 & & & -0.004 & & & 0.059 & & \\
\hline Log likelihood & & -479.36 & -501.05 & & -60.76 & -68.05 & & -259.49 & -271.32 & & -144.32 & -158.29 \\
\hline $\mathrm{N}$ & 175 & 175 & 175 & 21 & 21 & 21 & 91 & 91 & 91 & 63 & 63 & 63 \\
\hline Max. VIF & 1.885 & & & 2.042 & & & 1.580 & & & 1.398 & & \\
\hline
\end{tabular}

Standard errors in parentheses, all $t$ tests are two-tailed, $\uparrow p<.10, * p<.05, * * p<.01$ 
Table 7.32b: Regression estimates for equation 17b (high normative institutional distance)

\begin{tabular}{|c|c|c|c|c|c|c|c|c|c|c|c|c|}
\hline & \multicolumn{3}{|c|}{ Complete data set } & \multicolumn{3}{|c|}{ AU/NZ } & \multicolumn{3}{|c|}{ US/CA } & \multicolumn{3}{|c|}{ UK/IR } \\
\hline & OLS & Tobit & $\begin{array}{l}\text { Negative } \\
\text { binomial } \\
\end{array}$ & OLS & Tobit & $\begin{array}{l}\text { Negative } \\
\text { binomial }\end{array}$ & OLS & Tobit & $\begin{array}{l}\text { Negative } \\
\text { binomial } \\
\end{array}$ & OLS & Tobit & $\begin{array}{l}\text { Negative } \\
\text { binomial }\end{array}$ \\
\hline Intercept & $\begin{array}{l}8.395 * * \\
(1.956)\end{array}$ & $\begin{array}{l}8.126 * * \\
(1.749)\end{array}$ & $\begin{array}{l}2.096 * * \\
(0.118)\end{array}$ & $\begin{array}{l}14.967 * * \\
(2.703)\end{array}$ & $\begin{array}{l}15.336 * * \\
(2.343)\end{array}$ & $\begin{array}{l}2.725^{* *} \\
(0.159)\end{array}$ & $\begin{array}{l}\text { 10.159* } \\
(4.653)\end{array}$ & $\begin{array}{l}6.436 \dagger \\
(3.626)\end{array}$ & $\begin{array}{l}1.635^{* *} \\
(0.331)\end{array}$ & $\begin{array}{l}5.241 \dagger \\
(2.634)\end{array}$ & $\begin{array}{l}\text { 6.270** } \\
(2.219)\end{array}$ & $\begin{array}{l}1.976 * * \\
(0.347)\end{array}$ \\
\hline $\begin{array}{l}\text { Financial resources } \\
(\mathrm{H} 7 \mathrm{e})\end{array}$ & $\begin{array}{l}-0.303 \\
(0.195)\end{array}$ & $\begin{array}{l}-0.301 \dagger \\
(0.173)\end{array}$ & $\begin{array}{l}-0.019 \\
(0.015)\end{array}$ & $\begin{array}{l}-0.102 \\
(0.291)\end{array}$ & $\begin{array}{l}-0.094 \\
(0.265)\end{array}$ & $\begin{array}{l}-0.005 \\
(0.027)\end{array}$ & $\begin{array}{l}-0.719 \dagger \\
(0.418)\end{array}$ & $\begin{array}{l}-0.624 \dagger \\
(0.375)\end{array}$ & $\begin{array}{l}-0.045 \\
(0.041)\end{array}$ & $\begin{array}{l}0.157 \\
(0.314)\end{array}$ & $\begin{array}{l}0.066 \\
(0.232)\end{array}$ & $\begin{array}{l}0.003 \\
(0.048)\end{array}$ \\
\hline Age & $\begin{array}{l}0.006 \\
(0.007)\end{array}$ & $\begin{array}{l}0.005 \\
(0.006)\end{array}$ & $\begin{array}{l}0.0003 \\
(0.0005)\end{array}$ & - & - & - & $\begin{array}{l}0.016 \\
(0.017)\end{array}$ & $\begin{array}{l}0.019 \\
(0.014)\end{array}$ & $\begin{array}{l}0.001 \\
(0.001)\end{array}$ & $\begin{array}{l}0.000 \\
(0.008)\end{array}$ & $\begin{array}{l}-0.002 \\
(0.006)\end{array}$ & $\begin{array}{l}-0.0001 \\
(0.001)\end{array}$ \\
\hline Size (Staff) & $\begin{array}{l}0.000 \\
(0.000)\end{array}$ & $\begin{array}{l}0.0004 \\
(0.0002)\end{array}$ & $\begin{array}{l}0.267 \mathrm{E}-4 \\
(0.247 \mathrm{E}-4)\end{array}$ & $\begin{array}{l}-0.000 \\
(0.000)\end{array}$ & $\begin{array}{l}-0.664 \mathrm{E}-4 \\
(0.0004)\end{array}$ & $\begin{array}{l}-0.402 \mathrm{E}-5 \\
(0.460 \mathrm{E}-4)\end{array}$ & $\begin{array}{l}0.001 \\
(0.001)\end{array}$ & $\begin{array}{l}0.0006 \\
(0.0006)\end{array}$ & $\begin{array}{l}0.444 \mathrm{E}-4 \\
(0.480 \mathrm{E}-4)\end{array}$ & $\begin{array}{l}-0.000 \\
(0.000)\end{array}$ & $\begin{array}{l}0.331 \mathrm{E}-4 \\
(0.0003)\end{array}$ & $\begin{array}{l}0.252 \mathrm{E}-5 \\
(0.571 \mathrm{E}-4)\end{array}$ \\
\hline Offshore policy & $\begin{array}{l}\text { 7.984** } \\
(1.236)\end{array}$ & $\begin{array}{l}\text { 8.061** } \\
(1.172)\end{array}$ & $\begin{array}{l}0.679 * * \\
(0.071)\end{array}$ & $\begin{array}{l}2.200 \\
(2.215)\end{array}$ & $\begin{array}{l}2.264 \\
(2.059)\end{array}$ & $\begin{array}{l}0.143 \\
(0.139)\end{array}$ & $\begin{array}{l}10.143^{* *} \\
(2.394)\end{array}$ & $\begin{array}{l}10.059 * * \\
(2.174)\end{array}$ & $\begin{array}{l}1.148 * * \\
(0.201)\end{array}$ & $\begin{array}{l}\text { 8.973** } \\
(2.045)\end{array}$ & $\begin{array}{l}9.025 * * \\
(1.825)\end{array}$ & $\begin{array}{l}0.755^{* *} \\
(0.166)\end{array}$ \\
\hline $\begin{array}{l}\text { Internationalisation } \\
\text { at home strategy }\end{array}$ & $\begin{array}{l}-0.180 \\
(0.252)\end{array}$ & $\begin{array}{l}-0.0008 \\
(0.001)\end{array}$ & $\begin{array}{l}-0.478 \mathrm{E}-4 \\
(0.0001)\end{array}$ & $\begin{array}{l}0.175 \\
(0.446)\end{array}$ & $\begin{array}{l}-0.001 \\
(0.002)\end{array}$ & $\begin{array}{l}-0.736 \mathrm{E}-4 \\
(0.0003)\end{array}$ & $\begin{array}{l}-0.770 \\
(0.492)\end{array}$ & $\begin{array}{l}-0.005 \\
(0.004)\end{array}$ & $\begin{array}{l}-0.000 \\
(0.019)\end{array}$ & $\begin{array}{l}0.094 \\
(0.382)\end{array}$ & $\begin{array}{l}0.0008 \\
(0.001)\end{array}$ & $\begin{array}{l}0.500 \mathrm{E}-4 \\
(0.0002)\end{array}$ \\
\hline $\begin{array}{l}\text { Host country } \\
\text { demand }\end{array}$ & $\begin{array}{l}5.468 \\
(3.780)\end{array}$ & $\begin{array}{l}4.923 \\
(3.500)\end{array}$ & $\begin{array}{l}0.325 \\
(0.354)\end{array}$ & $\begin{array}{l}-1.355 \\
(5.445)\end{array}$ & $\begin{array}{l}0.049 \\
(4.828)\end{array}$ & $\begin{array}{l}0.001 \\
(0.528)\end{array}$ & $\begin{array}{l}5.246 \\
(10.432)\end{array}$ & $\begin{array}{l}7.527 \\
(9.385)\end{array}$ & $\begin{array}{l}0.642 \\
(1.038)\end{array}$ & $\begin{array}{l}\text { 13.005* } \\
(6.052)\end{array}$ & $\begin{array}{l}11.860 * \\
(5.236)\end{array}$ & $\begin{array}{l}0.701 \\
(1.120)\end{array}$ \\
\hline AU/NZ dummy & $\begin{array}{l}1.209 \\
(1.048)\end{array}$ & $\begin{array}{l}1.086 \\
(0.981)\end{array}$ & $\begin{array}{l}0.074 \\
(0.081)\end{array}$ & - & - & - & - & - & - & - & - & - \\
\hline UK/IR dummy & $\begin{array}{l}0.724 \\
(1.053)\end{array}$ & $\begin{array}{l}0.479 \\
(0.978)\end{array}$ & $\begin{array}{l}0.033 \\
(0.082)\end{array}$ & - & - & - & - & - & - & - & - & - \\
\hline $\mathrm{R}^{2}$ & 0.348 & & & 0.029 & & & 0.619 & & & 0.477 & & \\
\hline $\operatorname{AdjR}^{2}$ & 0.303 & & & -0.068 & & & 0.523 & & & 0.368 & & \\
\hline Log likelihood & & -351.68 & -371.76 & & -155.05 & -160.64 & & -88.71 & -94.56 & & -95.07 & -105.15 \\
\hline $\mathrm{N}$ & 132 & 132 & 132 & 59 & 59 & 59 & 32 & 32 & 32 & 41 & 41 & 41 \\
\hline Max. VIF & 2.501 & & & 1.539 & & & 1.325 & & & 1.840 & & \\
\hline
\end{tabular}


Table 7.33a: Regression estimates for equation 18a (low normative institutional distance)

\begin{tabular}{|c|c|c|c|c|c|c|c|c|c|c|c|c|}
\hline & \multicolumn{3}{|c|}{ Complete data set } & \multicolumn{3}{|c|}{ AU/NZ } & \multicolumn{3}{|c|}{ US/CA } & \multicolumn{3}{|c|}{ UK/IR } \\
\hline & OLS & Tobit & $\begin{array}{l}\text { Negative } \\
\text { binomial }\end{array}$ & OLS & Tobit & $\begin{array}{l}\text { Negative } \\
\text { binomial }\end{array}$ & OLS & Tobit & $\begin{array}{l}\text { Negative } \\
\text { binomial }\end{array}$ & OLS & Tobit & $\begin{array}{l}\text { Negative } \\
\text { binomial }\end{array}$ \\
\hline Intercept & $\begin{array}{l}23.250 * * \\
(2.335)\end{array}$ & $\begin{array}{l}24.158 * * \\
(2.168)\end{array}$ & $\begin{array}{l}3.266 * * \\
(0.209)\end{array}$ & $\begin{array}{l}31.345^{* *} \\
(9.801)\end{array}$ & $\begin{array}{l}32.042 * * \\
(7.783)\end{array}$ & $\begin{array}{l}3.721 * * \\
(0.551)\end{array}$ & $\begin{array}{l}\text { 26.093** } \\
(3.732)\end{array}$ & $\begin{array}{l}\text { 28.182** } \\
(3.190)\end{array}$ & $\begin{array}{l}3.543 * * \\
(0.286)\end{array}$ & $\begin{array}{l}20.920 * * \\
(2.670)\end{array}$ & $\begin{array}{l}\text { 20.206** } \\
(2.551)\end{array}$ & $\begin{array}{l}3.019 * * \\
(0.414)\end{array}$ \\
\hline $\begin{array}{l}\text { Reputation (Factor) } \\
\text { (H7f) }\end{array}$ & $\begin{array}{l}-1.635 * * \\
(0.279)\end{array}$ & $\begin{array}{l}-1.594^{* *} \\
(0.271)\end{array}$ & $\begin{array}{l}-0.099 * * \\
(0.027)\end{array}$ & $\begin{array}{l}-2.880 \dagger \\
(1.415)\end{array}$ & $\begin{array}{l}-2.968 * * \\
(1.167)\end{array}$ & $\begin{array}{l}-0.187 * \\
(0.085)\end{array}$ & $\begin{array}{l}-1.972^{* *} \\
(0.406)\end{array}$ & $\begin{array}{l}-2.042 * * \\
(0.386)\end{array}$ & $\begin{array}{l}-0.131 * * \\
(0.035)\end{array}$ & $\begin{array}{l}-0.909^{*} \\
(0.346)\end{array}$ & $\begin{array}{l}-0.677^{*} \\
(0.323)\end{array}$ & $\begin{array}{l}-0.039 \\
(0.050)\end{array}$ \\
\hline Age & $\begin{array}{l}0.002 \\
(0.005)\end{array}$ & $\begin{array}{l}-0.564 \mathrm{E}-4 \\
(0.004)\end{array}$ & $\begin{array}{l}-0.128 \mathrm{E}-4 \\
(0.0004)\end{array}$ & $\begin{array}{l}-0.026 \\
(0.045)\end{array}$ & $\begin{array}{l}-0.024 \\
(0.032)\end{array}$ & $\begin{array}{l}-0.001 \\
(0.005)\end{array}$ & $\begin{array}{l}-0.004 \\
(0.008)\end{array}$ & $\begin{array}{l}-0.004 \\
(0.007)\end{array}$ & $\begin{array}{l}-0.0003 \\
(0.0005)\end{array}$ & $\begin{array}{l}0.004 \\
(0.005)\end{array}$ & $\begin{array}{l}0.002 \\
(0.005)\end{array}$ & $\begin{array}{l}0.0001 \\
(0.0008)\end{array}$ \\
\hline Size (Staff) & $\begin{array}{l}-0.000 \\
(0.000)\end{array}$ & $\begin{array}{l}-0.344 \mathrm{E}-5 \\
(0.0002)\end{array}$ & $\begin{array}{l}0.382 \mathrm{E}-6 \\
(0.223 \mathrm{E}-4)\end{array}$ & $\begin{array}{l}0.000 \\
(0.001)\end{array}$ & $\begin{array}{l}-0.0004 \\
(0.0008)\end{array}$ & $\begin{array}{l}-0.266 \mathrm{E}-4 \\
(0.848 \mathrm{E}-4)\end{array}$ & $\begin{array}{l}8.021 \mathrm{E}-5 \\
(0.000)\end{array}$ & $\begin{array}{l}0.534 \mathrm{E}-4 \\
(0.0003)\end{array}$ & $\begin{array}{l}0.365 E-5 \\
(0.319 E-4)\end{array}$ & $\begin{array}{l}0.000 \\
(0.000)\end{array}$ & $\begin{array}{l}0.500 \mathrm{E}-4 \\
(0.0002)\end{array}$ & $\begin{array}{l}0.282 \mathrm{E}-5 \\
(0.479 \mathrm{E}-4)\end{array}$ \\
\hline Offshore policy & $\begin{array}{l}1.567 \\
(1.036)\end{array}$ & $\begin{array}{l}1.512 \\
(1.014)\end{array}$ & $\begin{array}{l}0.102 \\
(0.063)\end{array}$ & $\begin{array}{l}4.723 \\
(4.075)\end{array}$ & $\begin{array}{l}4.451 \\
(3.334)\end{array}$ & $\begin{array}{l}0.327 \\
(0.206)\end{array}$ & $\begin{array}{l}1.399 \\
(1.677)\end{array}$ & $\begin{array}{l}1.003 \\
(1.584)\end{array}$ & $\begin{array}{l}0.071 \\
(0.093)\end{array}$ & $\begin{array}{l}0.107 \\
(1.310)\end{array}$ & $\begin{array}{l}0.577 \\
(1.250)\end{array}$ & $\begin{array}{l}0.034 \\
(0.260)\end{array}$ \\
\hline $\begin{array}{l}\text { Internationalisation } \\
\text { at home strategy }\end{array}$ & $\begin{array}{l}0.324 \\
(0.198)\end{array}$ & $\begin{array}{l}0.0005 \\
(0.001)\end{array}$ & $\begin{array}{l}0.420 \mathrm{E}-4 \\
(0.0001)\end{array}$ & $\begin{array}{l}-0.041 \\
(0.977)\end{array}$ & $\begin{array}{l}-0.0003 \\
(0.005)\end{array}$ & $\begin{array}{l}0.188 \mathrm{E}-4 \\
(0.057)\end{array}$ & $\begin{array}{l}0.387 \\
(0.318)\end{array}$ & $\begin{array}{l}0.001 \\
(0.003)\end{array}$ & $\begin{array}{l}0.844 \mathrm{E}-4 \\
(0.013)\end{array}$ & $\begin{array}{l}0.343 \\
(0.245)\end{array}$ & $\begin{array}{l}0.0001 \\
(0.001)\end{array}$ & $\begin{array}{l}0.107 \mathrm{E}-4 \\
(0.0001)\end{array}$ \\
\hline $\begin{array}{l}\text { Host country } \\
\text { demand }\end{array}$ & $\begin{array}{l}\text { 7.151* } \\
(2.955)\end{array}$ & $\begin{array}{l}6.447 * \\
(2.799)\end{array}$ & $\begin{array}{l}0.384 \\
(0.280)\end{array}$ & $\begin{array}{l}11.001 \\
(10.100)\end{array}$ & $\begin{array}{l}11.705 \\
(8.286)\end{array}$ & $\begin{array}{l}0.713 \\
(0.739)\end{array}$ & $\begin{array}{l}1.897 \\
(4.511)\end{array}$ & $\begin{array}{l}2.265 \\
(4.336)\end{array}$ & $\begin{array}{l}0.157 \\
(0.359)\end{array}$ & $\begin{array}{l}\text { 11.073** } \\
\text { (3.994) }\end{array}$ & $\begin{array}{l}6.757 * \\
(3.449)\end{array}$ & $\begin{array}{l}0.377 \\
(0.609)\end{array}$ \\
\hline AU/NZ dummy & $\begin{array}{l}0.355 \\
(0.964)\end{array}$ & $\begin{array}{l}0.065 \\
(0.918)\end{array}$ & $\begin{array}{l}0.008 \\
(0.082)\end{array}$ & - & - & - & - & - & - & - & - & - \\
\hline UK/IR dummy & $\begin{array}{l}1.357 * \\
(0.610)\end{array}$ & $\begin{array}{l}1.277^{*} \\
(0.591)\end{array}$ & $\begin{array}{l}0.078 \\
(0.065)\end{array}$ & - & - & - & - & - & - & - & - & - \\
\hline $\mathrm{R}^{2}$ & 0.255 & & & 0.400 & & & 0.270 & & & 0.221 & & \\
\hline $\operatorname{AdjR}^{2}$ & 0.218 & & & 0.124 & & & 0.217 & & & 0.131 & & \\
\hline Log likelihood & & -463.56 & -488.41 & & -58.04 & -65.50 & & -247.64 & -259.82 & & -142.82 & -157.80 \\
\hline $\mathrm{N}$ & 175 & 175 & 175 & 21 & 21 & 21 & 91 & 91 & 91 & 63 & 63 & 63 \\
\hline Max. VIF & 1.337 & & & 1.850 & & & 1.090 & & & 1.348 & & \\
\hline
\end{tabular}


Table 7.33b: Regression estimates for equation 18b (high normative institutional distance)

\begin{tabular}{|c|c|c|c|c|c|c|c|c|c|c|c|c|}
\hline & \multicolumn{3}{|c|}{ Complete data set } & \multicolumn{3}{|c|}{ AU/NZ } & \multicolumn{3}{|c|}{ US/CA } & \multicolumn{3}{|c|}{ UK/IR } \\
\hline & OLS & Tobit & $\begin{array}{l}\text { Negative } \\
\text { binomial }\end{array}$ & OLS & Tobit & $\begin{array}{l}\text { Negative } \\
\text { binomial }\end{array}$ & OLS & Tobit & $\begin{array}{l}\text { Negative } \\
\text { binomial }\end{array}$ & OLS & Tobit & $\begin{array}{l}\text { Negative } \\
\text { binomial }\end{array}$ \\
\hline Intercept & $\begin{array}{l}15.259 * * \\
(3.019)\end{array}$ & $\begin{array}{l}14.693 * * \\
(2.590)\end{array}$ & $\begin{array}{l}2.497 * * \\
(0.241)\end{array}$ & $\begin{array}{l}20.556 * * \\
(4.392)\end{array}$ & $\begin{array}{l}20.970 * * \\
(3.750)\end{array}$ & $\begin{array}{l}3.058 * * \\
(0.338)\end{array}$ & $\begin{array}{l}28.174 * * \\
(7.030)\end{array}$ & $\begin{array}{l}22.553 * * \\
(6.143)\end{array}$ & $\begin{array}{l}2.827 * * \\
(0.529)\end{array}$ & $\begin{array}{l}6.973 \\
(4.342)\end{array}$ & $\begin{array}{l}9.540 * * \\
(3.475)\end{array}$ & $\begin{array}{l}2.168 * * \\
(0.565)\end{array}$ \\
\hline $\begin{array}{l}\text { Reputation (Factor) } \\
\text { (H7f) }\end{array}$ & $\begin{array}{l}-1.118 * * \\
(0.339)\end{array}$ & $\begin{array}{l}-1.162 * * \\
(0.306)\end{array}$ & $\begin{array}{l}-0.071 * \\
(0.031)\end{array}$ & $\begin{array}{l}-0.868 \\
(0.530)\end{array}$ & $\begin{array}{l}-0.923^{*} \\
(0.476)\end{array}$ & $\begin{array}{l}-0.054 \\
(0.052)\end{array}$ & $\begin{array}{l}-2.970 * * \\
(\mathbf{0 . 8 1 0 )}\end{array}$ & $\begin{array}{l}-2.697 * * \\
(0.754)\end{array}$ & $\begin{array}{l}-0.200 * * \\
(0.061)\end{array}$ & $\begin{array}{l}-0.176 \\
(0.485)\end{array}$ & $\begin{array}{l}-0.413 \\
(0.399)\end{array}$ & $\begin{array}{l}-0.025 \\
(0.070)\end{array}$ \\
\hline Age & $\begin{array}{l}0.003 \\
(0.006)\end{array}$ & $\begin{array}{l}0.003 \\
(0.005)\end{array}$ & $\begin{array}{l}0.0002 \\
(0.0005)\end{array}$ & $\begin{array}{l}-0.003 \\
(0.011)\end{array}$ & $\begin{array}{l}-0.002 \\
(0.009)\end{array}$ & $\begin{array}{l}-0.0001 \\
(0.001)\end{array}$ & $\begin{array}{l}0.020 \\
(0.014)\end{array}$ & $\begin{array}{l}0.023 \dagger \\
(0.012)\end{array}$ & $\begin{array}{l}0.001 \dagger \\
(0.001)\end{array}$ & $\begin{array}{l}0.000 \\
(0.000)\end{array}$ & $\begin{array}{l}-0.002 \\
(0.006)\end{array}$ & $\begin{array}{l}-0.0001 \\
(0.001)\end{array}$ \\
\hline Size (Staff) & $\begin{array}{l}0.000 \\
(0.000)\end{array}$ & $\begin{array}{l}0.0002 \\
(0.0002)\end{array}$ & $\begin{array}{l}0.168 \mathrm{E}-4 \\
(0.234 \mathrm{E}-4)\end{array}$ & $\begin{array}{l}5.288 \mathrm{E}-5 \\
(0.000)\end{array}$ & $\begin{array}{l}0.413 E-4 \\
(0.0003)\end{array}$ & $\begin{array}{l}0.247 \mathrm{E}-5 \\
(0.417 \mathrm{E}-4)\end{array}$ & $\begin{array}{l}-5.41 \mathrm{E}-5 \\
(0.001)\end{array}$ & $\begin{array}{l}0.429 \mathrm{E}-4 \\
(0.0005)\end{array}$ & $\begin{array}{l}0.200 \mathrm{E}-5 \\
(0.401 \mathrm{E}-4)\end{array}$ & $\begin{array}{l}6.127 \mathrm{E}-5 \\
(0.000)\end{array}$ & $\begin{array}{l}0.607 \mathrm{E}-4 \\
(0.0002)\end{array}$ & $\begin{array}{l}0.396 \mathrm{E}-5 \\
(0.530 \mathrm{E}-4)\end{array}$ \\
\hline Offshore policy & $\begin{array}{l}\text { 6.796** } \\
(1.242)\end{array}$ & $\begin{array}{l}\text { 6.784** } \\
(1.170)\end{array}$ & $\begin{array}{l}0.597 * * \\
(0.070)\end{array}$ & $\begin{array}{l}1.630 \\
(2.162)\end{array}$ & $\begin{array}{l}1.624 \\
(1.977)\end{array}$ & $\begin{array}{l}0.104 \\
(0.130)\end{array}$ & $\begin{array}{l}\text { 8.134** } \\
(2.118)\end{array}$ & $\begin{array}{l}\text { 8.209** } \\
(1.997)\end{array}$ & $\begin{array}{l}1.003 * * \\
(0.162)\end{array}$ & $\begin{array}{l}8.913 * * \\
(2.095)\end{array}$ & $\begin{array}{l}\text { 8.644** } \\
(1.843)\end{array}$ & $\begin{array}{l}0.732 * * \\
(0.169)\end{array}$ \\
\hline $\begin{array}{l}\text { Internationalisation } \\
\text { at home strategy }\end{array}$ & $\begin{array}{l}-0.328 \\
(0.238)\end{array}$ & $\begin{array}{l}-0.0005 \\
(0.001)\end{array}$ & $\begin{array}{l}-0.348 \mathrm{E}-4 \\
(0.0002)\end{array}$ & $\begin{array}{l}0.041 \\
(0.437)\end{array}$ & $\begin{array}{l}-0.0006 \\
(0.002)\end{array}$ & $\begin{array}{l}-0.329 \mathrm{E}-4 \\
(0.0003)\end{array}$ & $\begin{array}{l}-0.885^{*} \\
(0.418)\end{array}$ & $\begin{array}{l}0.0003 \\
(0.003)\end{array}$ & $\begin{array}{l}0.510 \mathrm{E}-4 \\
(0.014)\end{array}$ & $\begin{array}{l}0.167 \\
(0.351)\end{array}$ & $\begin{array}{l}0.0006 \\
(0.001)\end{array}$ & $\begin{array}{l}0.372 \mathrm{E}-4 \\
(0.0002)\end{array}$ \\
\hline $\begin{array}{l}\text { Host country } \\
\text { demand }\end{array}$ & $\begin{array}{l}5.802 \\
(3.643)\end{array}$ & $\begin{array}{l}5.557 \dagger \\
(3.357)\end{array}$ & $\begin{array}{l}0.367 \\
(0.381)\end{array}$ & $\begin{array}{l}0.487 \\
(5.489)\end{array}$ & $\begin{array}{l}1.429 \\
(4.681)\end{array}$ & $\begin{array}{l}0.082 \\
(0.586)\end{array}$ & $\begin{array}{l}-4.003 \\
(9.350)\end{array}$ & $\begin{array}{l}-0.718 \\
(8.701)\end{array}$ & $\begin{array}{l}0.018 \\
(0.923)\end{array}$ & $\begin{array}{l}12.374 * \\
(5.976)\end{array}$ & $\begin{array}{l}11.376^{*} \\
(5.030)\end{array}$ & $\begin{array}{l}0.682 \\
(0.985)\end{array}$ \\
\hline AU/NZ dummy & $\begin{array}{l}1.676 \dagger \\
(0.933)\end{array}$ & $\begin{array}{l}1.653 \dagger \\
(0.861)\end{array}$ & $\begin{array}{l}0.111 \\
(0.077)\end{array}$ & - & - & - & - & - & - & - & - & - \\
\hline UK/IR dummy & $\begin{array}{l}1.175 \\
(0.921)\end{array}$ & $\begin{array}{l}1.140 \\
(0.838)\end{array}$ & $\begin{array}{l}0.076 \\
(0.077)\end{array}$ & - & - & - & - & - & - & - & - & - \\
\hline $\mathrm{R}^{2}$ & 0.393 & & & 0.077 & & & 0.726 & & & 0.475 & & \\
\hline $\operatorname{Adj}^{2}$ & 0.350 & & & -0.036 & & & 0.657 & & & 0.366 & & \\
\hline Log likelihood & & -346.37 & -368.06 & & -153.30 & -159.50 & & -84.66 & -90.73 & & -94.58 & -104.96 \\
\hline $\mathrm{N}$ & 132 & 132 & 132 & 59 & 59 & 59 & 32 & 32 & 32 & 41 & 41 & 41 \\
\hline Max. VIF & 2.128 & & & 1.188 & & & 1.517 & & & 1.178 & & \\
\hline
\end{tabular}

Standard errors in parentheses, all $t$ tests are two-tailed, $\dagger p<.10, * p<.05, * * p<.01$ 


\subsection{TESTING THE ROBUSTNESS OF IMD REGULATIVE ID MEASURE USING EFI REGULATIVE ID MEASURE}

To test for the robustness of the regulative institutional distance measure, equations 1-9 (see tables 7.10-7.24) are re-estimated replacing the IMD regulative institutional distance measure with data from the Economic Freedom Index (EFI) (see Chapter 5). Equations 19-21 (see tables 7.34-7.36) are used to test the direct hypotheses of H1-6 (excluding $\mathrm{H} 4$ ). With $\mathrm{R}^{2}$ values ranging between 0.40 and 0.69 , equations $19-21$ have reasonable explanatory power.

\subsubsection{Direct hypotheses of H1-6 (excluding H4)}

H1a, which pertains to geographic experience, receives no support across any of the full samples tested in equations 19-21 (see tables 7.34-7.36). Some support $(p<0.10)$ is found for geographic experience - knowledge of education sector (Tobit for AU/NZ in tables 7.34, 7.35 and 7.36), geographic experience - academic capabilities (OLS for AU/NZ in table 7.34), geographic experience - financial capabilities (OLS for US/CA in tables 7.35) and geographic experience - international recruitment (OLS for AU/NZ in table 7.34, Tobit for AU/NZ in table 7.35). Support $(p<0.05)$ is also found for geographic experience - academic capabilities for AU/NZ (Tobit in table 7.36). Significant findings (at least $p<0.10$ ) are also found for geographic experience international recruitment (Tobit and negative binomial in table 7.34, OLS and Tobit in tables 7.35 and 7.36 all for the US/CA subsample) and geographic experience financial capabilities (Tobit for AU/NZ in table 7.36). The associated negative coefficients to these latter two variables, however, do not support H1a.

H1b, with respect to industry experience, receives some support $(p<0.10)$ for industry experience - international recruitment (OLS for full sample in table 7.35). Support ( $p<$ 0.05) for $\mathrm{H} 1 \mathrm{~b}$ is also obtained with respect to industry experience - international recruitment (Tobit for full sample and US/CA in table 7.34). Industry experience undergraduate marketing is also significant $(p<0.10)$ (Tobit for AU/NZ in table 7.34). The associated negative coefficient is, however, not supportive of H1b. 
H1c, which deals with transfer experience, is supported (at least $p<0.05$ ) in table 7.34 for the OLS models using the aggregate sample and all three geographical groupings.

H2, with respect to know-how, is not supported in table 7.35. Although significant findings are obtained across all models examined (except for UK/IR negative binomial), the associated negative coefficients are opposite to the hypothesised positive relationship between tacit know-how and equity stake.

Furthermore, the observed results in table 7.36 do not support H3 with respect to organisation culture. Although very strong findings $(p<0.01)$ are obtained (OLS, Tobit and negative binomial for the full sample, AU/NZ and US/CA), the negative coefficients associated with the organisation culture factor do not support the hypothesised positive relationship between organisation culture and equity stake. In the UK/IR grouping, some support $(p<0.10)$ is obtained for org. culture - no status distinction (OLS and Tobit in table 7.36). Support $(p<0.05)$ is found for: org. culture open discussion (Tobit in table 7.36) and org. culture - offshore development (OLS and Tobit in table 7.36). The associated coefficients with the latter three aspects of organisation culture are, however, negative, lending no support to H3.

$\mathrm{H} 5$, with respect to financial resources, receives some support $(p<0.10)$ for AU/NZ when using OLS (see tables 7.34 and 7.35). H5 receives support $(p<0.05)$ when using Tobit for AU/NZ (see table 7.35). The findings in table 7.36 do not support H5.

H6, with respect to reputation, is not supported in tables 7.34-7.36. Significant findings (at least $p<0.10$ ) are obtained for reputation in tables 7.34 and 7.36. However, the negative coefficients lend no support to the hypothesised positive relationship.

\subsubsection{Findings for moderating hypotheses $\mathrm{H8}$}

Similar to equations 4-9 (see tables 7.19-7.24), in order to test for the moderating relationship hypothesised in $\mathrm{H}$, the data are stratified using the median of the regulative institutional distance measure. It is hypothesised that, for lower values of institutional distance, positive relationships between specific resources and equity stake 
will be observed, while the relationships will be negative for larger values of institutional distance.

\subsubsection{Hypothesis 8a}

H8a, which pertains to the moderating role of regulative institutional distance on the hypothesised relationship between geographic experience and equity stake, is not supported. At low levels of institutional distance, a positive relationship is hypothesised. Some support $(p<0.10)$ is obtained for such a positive relationship with respect to geographic experience - knowledge of the education sector (Tobit for AU/NZ in table 7.37a). However, at higher levels of institutional distance, the associated negative hypothesised relationship is not found (see table 7.37b). Similarly, negative findings are found in table 7.37b for geographic experience - managerial capabilities (Tobit for AU/NZ) and geographic experience - financial capabilities (OLS and Tobit for UK/IR). However, the associated positive relationship at low levels of institutional distance is not supported. Hence, the combined results from tables 7.37a and 7.37b do not provide support to H8a.

\subsubsection{Hypothesis $8 b$}

H8b, which pertains to the role of regulative institutional distance on the hypothesised relationship between industry experience and equity stake, is not supported. Although, as hypothesised, significant positive findings (at least $p<0.10$ ) are found at low levels of institutional distance (industry experience - international recruitment for US/CA OLS and Tobit, industry experience - postgraduate marketing for UK/IR OLS and Tobit in table 7.38a), the associated negative findings at higher levels of institutional distance are not found (see table 7.38b). Similarly, industry experience - undergraduate offshore education is found to be negatively significant in table 7.38b (Tobit for $\mathrm{AU} / \mathrm{NZ}$ ), but the associated positive finding at low levels of institutional distance is not observed.

\subsubsection{Hypothesis 8c}

H8c, which deals with the moderating role of regulative institutional distance on the hypothesised relationship between know-how and equity stake, is not supported. While significant findings are obtained across all models estimated (at least $p<0.05$ ) in table 
7.39a, the negative coefficients do not support the hypothesised positive relationship at low levels of regulative institutional distance. At higher levels of regulative institutional distance, a negative relationship is hypothesised. While the findings from table 7.39b demonstrate support (at least $p<0.10$ ) for this hypothesis (except for OLS and Tobit for AU/NZ and UK/IR negative binomial), the combined findings from tables 7.39a and 7.39b suggest that regardless of the level of regulative institutional distance, know-how is mostly negatively associated with equity stake, after accounting for the other variables examined in this study.

\subsubsection{Hypothesis 8d}

H8d, which pertains to the moderating role of regulative institutional distance on the hypothesised relationship between organisation culture and equity stake, is not supported. While a positive relationship is hypothesised at low levels of regulative institutional distance, the findings from table 7.40a, demonstrate significant negative findings $(p<0.01)$ for the full sample, AU/NZ and US/CA sub-samples. At higher levels of regulative institutional distance, a negative relationship is hypothesised between organisation culture and equity stake. This hypothesis gains strong support ( $p<$ 0.01 ) for the full sample and US/CA (OLS, Tobit and negative binomial). Some support $(p<0.10)$ for this negative relationship at higher levels of regulative institutional distance is also obtained for the UK/IR grouping for: org. culture - experimentation (Tobit) and org. culture - offshore development (OLS and Tobit). The combined findings from tables 7.40a and 7.40b, however, do not support H8d. Rather than the hypothesised inverted relationship, the findings from tables 7.40a and 7.40b suggest that organisation culture is mostly negatively associated with equity stake regardless of the levels of regulative institutional distance and after accounting for the other variables examined in this study.

\subsubsection{Hypothesis 8 e}

H8e, which deals with the moderating role of regulative institutional distance on the hypothesised relationship between financial resources and equity stake, is not supported. At low levels of regulative institutional distance, a positive relationship is hypothesised. The insignificant findings in table 7.41a challenge this hypothesis. At 
higher levels of regulative institutional distance, a negative relationship is hypothesised. The findings in table 7.41b find support for this hypothesis $(p<0.10)$ in the UK/IR OLS and Tobit models. The combined findings from tables 7.41a and 7.41b, however, do not lend support to H8e.

\subsubsection{Hypothesis $8 f$}

H8f, which pertains to the moderating role of regulative institutional distance on the hypothesised relationship between reputation and equity, is not supported. While a positive relationship is hypothesised at low levels of regulative institutional distance, the findings from tables 7.42a suggest a significant negative association between reputation and equity stake (at least $p<0.05$ and except for UK/IR). At higher levels of regulative institutional distance, a negative relationship is hypothesised between reputation and equity stake. The findings from table $7.42 \mathrm{~b}$ support this hypothesis (at least $p<0.10$ ) in the aggregate (OLS, Tobit and negative binomial), US/CA (OLS and Tobit) and UK/IR (OLS and Tobit) groupings. The combined findings of equations 27a and 27b, thus, do not indicate an inverted relationship between reputation and equity stake. Rather, the combined results suggest that regardless of the levels of institutional distance, reputation is mostly negatively associated with equity stake in the models examined and after accounting for the other variables examined in this study. 
Table 7.34: Regression estimates for equation 19

\begin{tabular}{|c|c|c|c|c|c|c|c|c|c|c|c|c|}
\hline & \multicolumn{3}{|c|}{ Complete data set } & \multicolumn{3}{|c|}{ AU/NZ } & \multicolumn{3}{|c|}{ US/CA } & \multicolumn{3}{|c|}{ 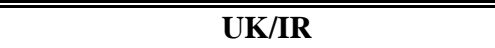 } \\
\hline & OLS & Tobit & $\begin{array}{l}\text { Negative } \\
\text { binomial }\end{array}$ & OLS & Tobit & $\begin{array}{l}\text { Negative } \\
\text { binomial }\end{array}$ & OLS & Tobit & $\begin{array}{l}\text { Negative } \\
\text { binomial }\end{array}$ & OLS & Tobit & $\begin{array}{l}\text { Negative } \\
\text { binomia }\end{array}$ \\
\hline Intercept & $\begin{array}{l}12.388 * * \\
(3.581)\end{array}$ & $\begin{array}{l}17.407 * * \\
(3.466)\end{array}$ & $\begin{array}{l}2.822 * * \\
(0.369)\end{array}$ & $\begin{array}{l}4.739 \\
(6.835)\end{array}$ & $\begin{array}{l}14.041^{*} \\
(6.560)\end{array}$ & $\begin{array}{l}2.668 * * \\
(0.935)\end{array}$ & $\begin{array}{l}13.926 * \\
(6.033)\end{array}$ & $\begin{array}{l}18.935 * * \\
(6.342)\end{array}$ & $\begin{array}{l}2.903 * * \\
(0.560)\end{array}$ & $\begin{array}{l}16.162 * \\
(6.361)\end{array}$ & $\begin{array}{l}17.567 * * \\
(4.597)\end{array}$ & $\begin{array}{l}2.832 * * \\
(0.914)\end{array}$ \\
\hline $\begin{array}{l}\text { Geo exp - } \\
\text { knowledge of edu } \\
\text { sector (HIa) }\end{array}$ & $\begin{array}{l}0.005 \\
(0.136)\end{array}$ & $\begin{array}{l}-0.132 \\
(0.135)\end{array}$ & $\begin{array}{l}-0.009 \\
(0.013)\end{array}$ & $\begin{array}{l}0.501 \\
(0.301)\end{array}$ & $\begin{array}{l}0.528 \dagger \\
(0.306)\end{array}$ & $\begin{array}{l}0.029 \\
(0.046)\end{array}$ & $\begin{array}{l}0.082 \\
(0.218)\end{array}$ & $\begin{array}{l}-0.122 \\
(0.227)\end{array}$ & $\begin{array}{l}-0.007 \\
(0.019)\end{array}$ & $\begin{array}{l}-0.223 \\
(0.255)\end{array}$ & $\begin{array}{l}-0.274 \\
(0.184)\end{array}$ & $\begin{array}{l}-0.016 \\
(0.039)\end{array}$ \\
\hline $\begin{array}{l}\text { Geo exp - academic } \\
\text { capabilities }(H 1 a)\end{array}$ & $\begin{array}{l}0.057 \\
(0.130)\end{array}$ & $\begin{array}{l}0.103 \\
(0.130)\end{array}$ & $\begin{array}{l}0.006 \\
(0.011)\end{array}$ & $\begin{array}{l}0.508 \dagger \\
(0.295)\end{array}$ & $\begin{array}{l}0.191 \\
(0.290)\end{array}$ & $\begin{array}{l}0.011 \\
(0.038)\end{array}$ & $\begin{array}{l}-0.004 \\
(0.220)\end{array}$ & $\begin{array}{l}0.239 \\
(0.230)\end{array}$ & $\begin{array}{l}0.014 \\
(0.017)\end{array}$ & $\begin{array}{l}0.103 \\
(0.222)\end{array}$ & $\begin{array}{l}0.020 \\
(0.172)\end{array}$ & $\begin{array}{l}0.001 \\
(0.031)\end{array}$ \\
\hline $\begin{array}{l}\text { Geo exp - } \\
\text { managerial } \\
\text { capabilities (H1a) }\end{array}$ & $\begin{array}{l}0.120 \\
(0.157)\end{array}$ & $\begin{array}{l}0.034 \\
(0.160)\end{array}$ & $\begin{array}{l}0.001 \\
(0.016)\end{array}$ & $\begin{array}{l}0.012 \\
(0.345)\end{array}$ & $\begin{array}{l}0.073 \\
(0.341)\end{array}$ & $\begin{array}{l}0.003 \\
(0.054)\end{array}$ & $\begin{array}{l}0.271 \\
(0.256)\end{array}$ & $\begin{array}{l}-0.042 \\
(0.230)\end{array}$ & $\begin{array}{l}-0.006 \\
(0.024)\end{array}$ & $\begin{array}{l}-0.078 \\
(0.265)\end{array}$ & $\begin{array}{l}-0.109 \\
(0.213)\end{array}$ & $\begin{array}{l}-0.006 \\
(0.041)\end{array}$ \\
\hline $\begin{array}{l}\text { Geo exp - financial } \\
\text { capabilities }(H 1 a)\end{array}$ & $\begin{array}{l}4.749 \mathrm{E}-5 \\
(0.146)\end{array}$ & $\begin{array}{l}0.046 \\
(0.147)\end{array}$ & $\begin{array}{l}0.002 \\
(0.014)\end{array}$ & $\begin{array}{l}-0.126 \\
(0.332)\end{array}$ & $\begin{array}{l}-0.295 \\
(0.317)\end{array}$ & $\begin{array}{l}-0.017 \\
(0.050)\end{array}$ & $\begin{array}{l}0.361 \\
(0.224)\end{array}$ & $\begin{array}{l}0.367 \\
(0.237)\end{array}$ & $\begin{array}{l}0.024 \\
(0.022)\end{array}$ & $\begin{array}{l}-0.392 \\
(0.266)\end{array}$ & $\begin{array}{l}-0.267 \\
(0.215)\end{array}$ & $\begin{array}{l}-0.016 \\
(0.039)\end{array}$ \\
\hline $\begin{array}{l}\text { Geo exp - intl } \\
\text { recruitment }(H 1 a)\end{array}$ & $\begin{array}{l}-0.043 \\
(0.163)\end{array}$ & $\begin{array}{l}-0.136 \\
(0.167)\end{array}$ & $\begin{array}{l}-0.008 \\
(0.014)\end{array}$ & $\begin{array}{l}0.517 \dagger \\
(0.287)\end{array}$ & $\begin{array}{l}0.376 \\
(0.321)\end{array}$ & $\begin{array}{l}0.022 \\
(0.043)\end{array}$ & $\begin{array}{l}-0.424 \\
(0.283)\end{array}$ & $\begin{array}{l}-0.642 * \\
(0.290)\end{array}$ & $\begin{array}{l}-0.042 \dagger \\
(0.025)\end{array}$ & $\begin{array}{l}0.016 \\
(0.313)\end{array}$ & $\begin{array}{l}0.105 \\
(0.232)\end{array}$ & $\begin{array}{l}0.006 \\
(0.045)\end{array}$ \\
\hline $\begin{array}{l}\text { Ind exp - UG } \\
\text { offshore edu (H1b) }\end{array}$ & $\begin{array}{l}0.052 \\
(0.126)\end{array}$ & $\begin{array}{l}0.050 \\
(0.128)\end{array}$ & $\begin{array}{l}0.003 \\
(0.011)\end{array}$ & $\begin{array}{l}-0.266 \\
(0.254)\end{array}$ & $\begin{array}{l}-0.121 \\
(0.273)\end{array}$ & $\begin{array}{l}-0.006 \\
(0.036)\end{array}$ & $\begin{array}{l}0.087 \\
(0.226)\end{array}$ & $\begin{array}{l}0.086 \\
(0.236)\end{array}$ & $\begin{array}{l}0.007 \\
(0.022)\end{array}$ & $\begin{array}{l}0.031 \\
(0.208)\end{array}$ & $\begin{array}{l}0.048 \\
(0.166)\end{array}$ & $\begin{array}{l}0.002 \\
(0.032)\end{array}$ \\
\hline $\begin{array}{l}\text { Ind exp - PG } \\
\text { offshore edu }(H 1 b)\end{array}$ & $\begin{array}{l}0.044 \\
(0.143)\end{array}$ & $\begin{array}{l}0.053 \\
(0.146)\end{array}$ & $\begin{array}{l}0.003 \\
(0.013)\end{array}$ & $\begin{array}{l}0.013 \\
(0.302)\end{array}$ & $\begin{array}{l}-0.007 \\
(0.306)\end{array}$ & $\begin{array}{l}-0.0007 \\
(0.038)\end{array}$ & $\begin{array}{l}0.029 \\
(0.228)\end{array}$ & $\begin{array}{l}-0.002 \\
(0.237)\end{array}$ & $\begin{array}{l}-0.001 \\
(0.018)\end{array}$ & $\begin{array}{l}0.152 \\
(0.257)\end{array}$ & $\begin{array}{l}-0.033 \\
(0.214)\end{array}$ & $\begin{array}{l}-0.001 \\
(0.047)\end{array}$ \\
\hline $\begin{array}{l}\text { Ind exp - intl } \\
\text { recruitment (Factor) } \\
(\text { H1b) }\end{array}$ & $\begin{array}{l}0.266 \\
(0.167)\end{array}$ & $\begin{array}{l}0.328^{*} \\
(0.162)\end{array}$ & $\begin{array}{l}0.019 \\
(0.017)\end{array}$ & $\begin{array}{l}0.100 \\
(0.361)\end{array}$ & $\begin{array}{l}0.194 \\
(0.339)\end{array}$ & $\begin{array}{l}0.010 \\
(0.045)\end{array}$ & $\begin{array}{l}0.409 \\
(0.285)\end{array}$ & $\begin{array}{l}0.576^{*} \\
(0.283)\end{array}$ & $\begin{array}{l}0.039 \\
(0.031)\end{array}$ & $\begin{array}{l}0.377 \\
(0.289)\end{array}$ & $\begin{array}{l}0.205 \\
(0.211)\end{array}$ & $\begin{array}{l}0.012 \\
(0.041)\end{array}$ \\
\hline $\begin{array}{l}\text { Ind exp - UG } \\
\text { marketing (H1b) }\end{array}$ & $\begin{array}{l}-0.058 \\
(0.129)\end{array}$ & $\begin{array}{l}-0.023 \\
(0.128)\end{array}$ & $\begin{array}{l}-0.002 \\
(0.013)\end{array}$ & $\begin{array}{l}-0.328 \\
(0.329)\end{array}$ & $\begin{array}{l}-0.599 \dagger \\
(0.320)\end{array}$ & $\begin{array}{l}-0.035 \\
(0.039)\end{array}$ & $\begin{array}{l}-0.186 \\
(0.196)\end{array}$ & $\begin{array}{l}0.073 \\
(0.204)\end{array}$ & $\begin{array}{l}0.005 \\
(0.018)\end{array}$ & $\begin{array}{l}0.145 \\
(0.226)\end{array}$ & $\begin{array}{l}0.142 \\
(0.164)\end{array}$ & $\begin{array}{l}0.008 \\
(0.036)\end{array}$ \\
\hline $\begin{array}{l}\text { Ind exp - PG } \\
\text { marketing (H1b) }\end{array}$ & $\begin{array}{l}0.062 \\
(0.124)\end{array}$ & $\begin{array}{l}0.028 \\
(0.124)\end{array}$ & $\begin{array}{l}0.002 \\
(0.011)\end{array}$ & $\begin{array}{l}0.323 \\
(0.242)\end{array}$ & $\begin{array}{l}0.179 \\
(0.264)\end{array}$ & $\begin{array}{l}0.009 \\
(0.034)\end{array}$ & $\begin{array}{l}-0.145 \\
(0.204)\end{array}$ & $\begin{array}{l}-0.129 \\
(0.202)\end{array}$ & $\begin{array}{l}-0.008 \\
(0.018)\end{array}$ & $\begin{array}{l}0.364 \\
(0.227)\end{array}$ & $\begin{array}{l}0.184 \\
(0.179)\end{array}$ & $\begin{array}{l}0.010 \\
(0.035)\end{array}$ \\
\hline $\begin{array}{l}\text { Transfer experience } \\
\text { (H1c) }\end{array}$ & $\begin{array}{l}-7.741 * * \\
(0.704)\end{array}$ & $\begin{array}{l}-0.0004 \\
(0.0005)\end{array}$ & $\begin{array}{l}-0.261 \mathrm{E}-4 \\
(0.689 \mathrm{E}-4)\end{array}$ & $\begin{array}{l}-10.241 * * \\
(1.596)\end{array}$ & $\begin{array}{l}-0.001 \\
(0.001)\end{array}$ & $\begin{array}{l}-0.717 \\
(0.0001)\end{array}$ & $\begin{array}{l}-8.879 * * \\
(1.129)\end{array}$ & $\begin{array}{l}-0.001 \\
(0.0009)\end{array}$ & $\begin{array}{l}-0.921 \mathrm{E}-4 \\
(0.0001)\end{array}$ & $\begin{array}{l}-4.512^{*} \\
(1.391)\end{array}$ & $\begin{array}{l}0.0002 \\
(0.0007)\end{array}$ & $\begin{array}{l}-0.004 \\
(0.033)\end{array}$ \\
\hline $\begin{array}{l}\text { Financial resources } \\
\text { (H5) }\end{array}$ & $\begin{array}{l}0.025 \\
(0.112)\end{array}$ & $\begin{array}{l}-0.052 \\
(0.108)\end{array}$ & $\begin{array}{l}-0.003 \\
(0.010)\end{array}$ & $\begin{array}{l}\mathbf{0 . 5 0 7 \dagger} \\
\mathbf{( 0 . 2 8 1 )}\end{array}$ & $\begin{array}{l}0.223 \\
(0.299)\end{array}$ & $\begin{array}{l}0.013 \\
(0.044)\end{array}$ & $\begin{array}{l}0.105 \\
(0.174)\end{array}$ & $\begin{array}{l}-0.010 \\
(0.165)\end{array}$ & $\begin{array}{l}-0.003 \\
(0.014)\end{array}$ & $\begin{array}{l}-0.077 \\
(0.202)\end{array}$ & $\begin{array}{l}-0.075 \\
(0.152)\end{array}$ & $\begin{array}{l}-0.004 \\
(0.033)\end{array}$ \\
\hline $\begin{array}{l}\text { Reputation (Factor) } \\
\text { (H6) }\end{array}$ & $\begin{array}{l}-0.301 \\
(0.235)\end{array}$ & $\begin{array}{l}-1.449 * * \\
(0.210)\end{array}$ & $\begin{array}{l}-0.090 * * \\
(0.020)\end{array}$ & $\begin{array}{l}0.086 \\
(0.506)\end{array}$ & $\begin{array}{l}-1.052 * \\
(0.510)\end{array}$ & $\begin{array}{l}-0.065 \\
(0.072)\end{array}$ & $\begin{array}{l}-0.380 \\
(0.413)\end{array}$ & $\begin{array}{l}-2.190 * * \\
(0.340)\end{array}$ & $\begin{array}{l}-0.152 * * \\
(0.031)\end{array}$ & $\begin{array}{l}-0.499 \\
(0.401)\end{array}$ & $\begin{array}{l}-0.624^{*} \\
(0.299)\end{array}$ & $\begin{array}{l}-0.036 \\
(0.055)\end{array}$ \\
\hline $\begin{array}{l}\text { IMD Normative ID } \\
\text { (lag 1) }\end{array}$ & $\begin{array}{l}-0.112 \\
(0.171)\end{array}$ & $\begin{array}{l}-0.004 \\
(0.003)\end{array}$ & $\begin{array}{l}-0.0002 \\
(0.011)\end{array}$ & $\begin{array}{l}-0.304 \\
(0.335)\end{array}$ & $\begin{array}{l}-0.022 \\
(0.348)\end{array}$ & $\begin{array}{l}-0.002 \\
(0.043)\end{array}$ & $\begin{array}{l}-0.456 \\
(0.323)\end{array}$ & $\begin{array}{l}-0.004 \\
(0.003)\end{array}$ & $\begin{array}{l}-0.0002 \\
(0.023)\end{array}$ & $\begin{array}{l}-0.174 \\
(0.329)\end{array}$ & $\begin{array}{l}0.150 \\
(0.245)\end{array}$ & $\begin{array}{l}0.009 \\
(0.055)\end{array}$ \\
\hline
\end{tabular}




\begin{tabular}{|c|c|c|c|c|c|c|c|c|c|c|c|c|}
\hline $\begin{array}{l}\text { EFI Regulative ID } \\
\text { (lag 1) }\end{array}$ & $\begin{array}{l}0.147 \\
(0.109)\end{array}$ & $\begin{array}{l}0.335 * * \\
(0.109)\end{array}$ & $\begin{array}{l}\text { 0.021* } \\
(0.009)\end{array}$ & $\begin{array}{l}-0.268 \\
(0.204)\end{array}$ & $\begin{array}{l}0.561 * * \\
(0.193)\end{array}$ & $\begin{array}{l}0.033 \\
(0.030)\end{array}$ & $\begin{array}{l}0.164 \\
(0.137)\end{array}$ & $\begin{array}{l}0.608 * * \\
(0.206)\end{array}$ & $\begin{array}{l}0.045 * * \\
(0.015)\end{array}$ & $\begin{array}{l}-0.212 \\
(0.170)\end{array}$ & $\begin{array}{l}-0.082 \\
(0.138)\end{array}$ & $\begin{array}{l}-0.004 \\
(0.025)\end{array}$ \\
\hline Age & $\begin{array}{l}0.005 \\
(0.004)\end{array}$ & $\begin{array}{l}0.002 \\
(0.003)\end{array}$ & $\begin{array}{l}0.0001 \\
(0.0003)\end{array}$ & $\begin{array}{l}-0.009 \\
(-0.014)\end{array}$ & $\begin{array}{l}-0.006 \\
(0.011)\end{array}$ & $\begin{array}{l}-0.0004 \\
(0.002)\end{array}$ & $\begin{array}{l}0.004 \\
(0.006)\end{array}$ & $\begin{array}{l}0.003 \\
(0.006)\end{array}$ & $\begin{array}{l}0.0003 \\
(0.0005)\end{array}$ & $\begin{array}{l}0.012^{*} \\
(0.006)\end{array}$ & $\begin{array}{l}0.005 \\
(0.004)\end{array}$ & $\begin{array}{l}0.0003 \\
(0.0007)\end{array}$ \\
\hline Size (Staff) & $\begin{array}{l}9.466 \mathrm{E}-5 \\
(0.000)\end{array}$ & $\begin{array}{l}0.0001 \\
(0.0001)\end{array}$ & $\begin{array}{l}0.116 \mathrm{E}-4 \\
(0.169 \mathrm{E}-4)\end{array}$ & - & - & - & $\begin{array}{l}0.000 \\
(0.000)\end{array}$ & $\begin{array}{l}0.0002 \\
(0.0002)\end{array}$ & $\begin{array}{l}0.166 \mathrm{E}-4 \\
(0.235 \mathrm{E}-4)\end{array}$ & $\begin{array}{l}0.000 \\
(0.000)\end{array}$ & $\begin{array}{l}0.0001 \\
(0.0002)\end{array}$ & $\begin{array}{l}0.911 \mathrm{E}-5 \\
(0.480 \mathrm{E}-4)\end{array}$ \\
\hline Offshore policy & $\begin{array}{l}1.935 \dagger \\
(0.716)\end{array}$ & $\begin{array}{l}3.482 * * \\
(0.764)\end{array}$ & $\begin{array}{l}0.265 * * \\
(0.046)\end{array}$ & $\begin{array}{l}1.910 \\
(1.456)\end{array}$ & $\begin{array}{l}3.600^{*} \\
(1.565)\end{array}$ & $\begin{array}{l}0.241 \\
(0.206)\end{array}$ & $\begin{array}{l}1.767 \\
(1.114)\end{array}$ & $\begin{array}{l}3.790 * * \\
(1.203)\end{array}$ & $\begin{array}{l}0.341 * * \\
(0.076)\end{array}$ & $\begin{array}{l}3.130^{*} \\
(1.399)\end{array}$ & $\begin{array}{l}\text { 3.853** } \\
(1.190)\end{array}$ & $\begin{array}{l}0.252 \\
(0.181)\end{array}$ \\
\hline $\begin{array}{l}\text { Internationalisation } \\
\text { at home strategy }\end{array}$ & $\begin{array}{l}0.069 \\
(0.145)\end{array}$ & $\begin{array}{l}-0.294 \mathrm{E}-4 \\
(0.0009)\end{array}$ & $\begin{array}{l}0.259 \mathrm{E}-5 \\
(0.0001)\end{array}$ & $\begin{array}{l}0.008 \\
(0.363)\end{array}$ & $\begin{array}{l}-0.0004 \\
(0.001)\end{array}$ & $\begin{array}{l}-0.205 \mathrm{E}-4 \\
(0.0003)\end{array}$ & $\begin{array}{l}-0.078 \\
(0.226)\end{array}$ & $\begin{array}{l}-0.0003 \\
(0.002)\end{array}$ & $\begin{array}{l}-0.155 \mathrm{E}-4 \\
(0.0001)\end{array}$ & $\begin{array}{l}0.000 \\
(0.266)\end{array}$ & $\begin{array}{l}0.0005 \\
(0.0009)\end{array}$ & $\begin{array}{l}0.340 \mathrm{E}-4 \\
(0.0001)\end{array}$ \\
\hline $\begin{array}{l}\text { Host country } \\
\text { demand }\end{array}$ & $\begin{array}{l}3.753 \\
(2.484)\end{array}$ & $\begin{array}{l}8.371^{* *} \\
(2.444)\end{array}$ & $\begin{array}{l}\text { 0.518* } \\
(0.234)\end{array}$ & $\begin{array}{l}4.650 \\
(4.319)\end{array}$ & $\begin{array}{l}\text { 8.745* } \\
\text { (4.118) }\end{array}$ & $\begin{array}{l}0.499 \\
(0.679)\end{array}$ & - & - & - & - & - & - \\
\hline AU/NZ dummy & $\begin{array}{l}0.361 \\
(0.606\end{array}$ & $\begin{array}{l}0.724 \\
(0.609)\end{array}$ & $\begin{array}{l}0.044 \\
(0.059)\end{array}$ & - & - & - & - & - & - & - & - & - \\
\hline UK/IR dummy & $\begin{array}{l}0.306 \\
(0.549)\end{array}$ & $\begin{array}{l}0.889 \\
(0.553)\end{array}$ & $\begin{array}{l}0.054 \\
(0.055)\end{array}$ & - & - & - & - & - & - & - & - & - \\
\hline $\mathrm{R}^{2}$ & 0.566 & & & 0.647 & & & 0.697 & & & 0.403 & & \\
\hline $\operatorname{AdjR}^{2}$ & 0.525 & & & 0.504 & & & 0.630 & & & 0.214 & & \\
\hline Log likelihood & & -811.05 & -860.53 & & -207.61 & -222.15 & & -331.21 & -354.50 & & -242.47 & -265.50 \\
\hline $\mathrm{N}$ & 308 & 308 & 308 & 80 & 80 & 80 & 124 & 124 & 124 & 104 & 104 & 104 \\
\hline Max. VIF & 2.069 & & & 2.443 & & & 2.451 & & & 1.811 & & \\
\hline
\end{tabular}

Standard errors in parentheses, all $t$ tests are two-tailed, $\dagger p<.10,{ }^{*} p<.05,{ }^{* *} p<.01$

Table 7.35: Regression estimates for equation 20

\begin{tabular}{|c|c|c|c|c|c|c|c|c|c|c|c|c|}
\hline & \multicolumn{3}{|c|}{ Complete data set } & \multicolumn{3}{|c|}{ AU/NZ } & \multicolumn{3}{|c|}{$\overline{\text { US/CA }}$} & \multicolumn{3}{|c|}{ UK/IR } \\
\hline & OLS & Tobit & $\begin{array}{l}\text { Negative } \\
\text { binomial }\end{array}$ & OLS & Tobit & $\begin{array}{l}\text { Negative } \\
\text { binomial }\end{array}$ & OLS & Tobit & $\begin{array}{l}\text { Negative } \\
\text { binomial }\end{array}$ & OLS & Tobit & $\begin{array}{l}\text { Negative } \\
\text { binomial }\end{array}$ \\
\hline Intercept & $\begin{array}{l}17.075 * * \\
(3.036)\end{array}$ & $\begin{array}{l}17.883 * * \\
(2.739)\end{array}$ & $\begin{array}{l}2.962 * * \\
(0.415)\end{array}$ & $\begin{array}{l}12.640 * \\
(6.154)\end{array}$ & $\begin{array}{l}15.215^{* *} \\
(5.052)\end{array}$ & $\begin{array}{l}\text { 2.881** } \\
\text { (1.081) }\end{array}$ & $\begin{array}{l}17.474 * \\
(3.133)\end{array}$ & $\begin{array}{l}16.902^{* *} \\
(4.974)\end{array}$ & $\begin{array}{l}2.897 * * \\
(0.751)\end{array}$ & $\begin{array}{l}20.351 * * \\
(4.499)\end{array}$ & $\begin{array}{l}19.613^{* *} \\
(3.840)\end{array}$ & $\begin{array}{l}\text { 3.041** } \\
(0.954)\end{array}$ \\
\hline $\begin{array}{l}\text { Geo exp - } \\
\text { knowledge of edu } \\
\text { sector (HIa) }\end{array}$ & $\begin{array}{l}-0.040 \\
(0.116)\end{array}$ & $\begin{array}{l}-0.047 \\
(0.107)\end{array}$ & $\begin{array}{l}-0.004 \\
(0.015)\end{array}$ & $\begin{array}{l}0.460 \\
(0.276)\end{array}$ & $\begin{array}{l}0.462 \dagger \\
(0.237)\end{array}$ & $\begin{array}{l}0.025 \\
(0.050)\end{array}$ & $\begin{array}{l}-0.094 \\
(0.194)\end{array}$ & $\begin{array}{l}-0.077 \\
(0.177)\end{array}$ & $\begin{array}{l}-0.006 \\
(0.027)\end{array}$ & $\begin{array}{l}-0.201 \\
(0.189)\end{array}$ & $\begin{array}{l}-0.177 \\
(0.155)\end{array}$ & $\begin{array}{l}-0.011 \\
(0.042)\end{array}$ \\
\hline
\end{tabular}




\begin{tabular}{|c|c|c|c|c|c|c|c|c|c|c|c|c|}
\hline $\begin{array}{l}\text { Geo exp - academic } \\
\text { capabilities }(H 1 a)\end{array}$ & $\begin{array}{l}0.011 \\
(0.112)\end{array}$ & $\begin{array}{l}-0.021 \\
(0.103)\end{array}$ & $\begin{array}{l}-0.002 \\
(0.014)\end{array}$ & $\begin{array}{l}0.271 \\
(0.261)\end{array}$ & $\begin{array}{l}0.252 \\
(0.224)\end{array}$ & $\begin{array}{l}0.010 \\
(0.041)\end{array}$ & $\begin{array}{l}0.073 \\
(0.197)\end{array}$ & $\begin{array}{l}0.043 \\
(0.180)\end{array}$ & $\begin{array}{l}0.001 \\
(0.025)\end{array}$ & $\begin{array}{l}-0.015 \\
(0.170)\end{array}$ & $\begin{array}{l}-0.045 \\
(0.146)\end{array}$ & $\begin{array}{l}-0.0023 \\
(0.035)\end{array}$ \\
\hline $\begin{array}{l}\text { Geo exp - } \\
\text { managerial } \\
\text { capabilities }(H 1 a)\end{array}$ & $\begin{array}{l}0.066 \\
(0.134)\end{array}$ & $\begin{array}{l}0.075 \\
(0.127)\end{array}$ & $\begin{array}{l}0.004 \\
(0.018)\end{array}$ & $\begin{array}{l}-0.068 \\
(0.302)\end{array}$ & $\begin{array}{l}-0.044 \\
(0.262)\end{array}$ & $\begin{array}{l}-0.001 \\
(0.050)\end{array}$ & $\begin{array}{l}0.259 \\
(0.233)\end{array}$ & $\begin{array}{l}0.289 \\
(0.214)\end{array}$ & $\begin{array}{l}0.016 \\
(0.028)\end{array}$ & $\begin{array}{l}-0.105 \\
(0.201)\end{array}$ & $\begin{array}{l}-0.060 \\
(0.177)\end{array}$ & $\begin{array}{l}-0.005 \\
(0.043)\end{array}$ \\
\hline $\begin{array}{l}\text { Geo exp - financial } \\
\text { capabilities }(H 1 a)\end{array}$ & $\begin{array}{l}0.053 \\
(0.128)\end{array}$ & $\begin{array}{l}0.023 \\
(0.116)\end{array}$ & $\begin{array}{l}0.0007 \\
(0.015)\end{array}$ & $\begin{array}{l}-0.052 \\
(0.302)\end{array}$ & $\begin{array}{l}-0.219 \\
(0.246)\end{array}$ & $\begin{array}{l}-0.014 \\
(0.048)\end{array}$ & $\begin{array}{l}0.351 \dagger \\
(0.207)\end{array}$ & $\begin{array}{l}0.285 \\
(0.187)\end{array}$ & $\begin{array}{l}0.020 \\
(0.024)\end{array}$ & $\begin{array}{l}-0.332 \\
(0.214)\end{array}$ & $\begin{array}{l}-0.267 \\
(0.183)\end{array}$ & $\begin{array}{l}-0.019 \\
(0.047)\end{array}$ \\
\hline $\begin{array}{l}\text { Geo exp - intl } \\
\text { recruitment }(H 1 a)\end{array}$ & $\begin{array}{l}-0.058 \\
(0.141)\end{array}$ & $\begin{array}{l}-0.030 \\
(0.132)\end{array}$ & $\begin{array}{l}-0.001 \\
(0.018)\end{array}$ & $\begin{array}{l}0.354 \\
(0.273)\end{array}$ & $\begin{array}{l}0.424 \dagger \\
(0.248)\end{array}$ & $\begin{array}{l}0.028 \\
(0.051)\end{array}$ & $\begin{array}{l}-0.461 \dagger \\
(0.253)\end{array}$ & $\begin{array}{l}-0.410 \dagger \\
(0.229)\end{array}$ & $\begin{array}{l}-0.029 \\
(0.032)\end{array}$ & $\begin{array}{l}0.021 \\
(0.228)\end{array}$ & $\begin{array}{l}0.085 \\
(0.197)\end{array}$ & $\begin{array}{l}0.007 \\
(0.053)\end{array}$ \\
\hline $\begin{array}{l}\text { Ind exp - UG } \\
\text { offshore edu }(H 1 b)\end{array}$ & $\begin{array}{l}0.054 \\
(0.108)\end{array}$ & $\begin{array}{l}0.043 \\
(0.101)\end{array}$ & $\begin{array}{l}0.003 \\
(0.013)\end{array}$ & $\begin{array}{l}-0.111 \\
(0.243)\end{array}$ & $\begin{array}{l}-0.200 \\
(0.211)\end{array}$ & $\begin{array}{l}-0.010 \\
(0.034)\end{array}$ & $\begin{array}{l}0.035 \\
(0.203)\end{array}$ & $\begin{array}{l}0.034 \\
(0.186)\end{array}$ & $\begin{array}{l}0.004 \\
(0.026)\end{array}$ & $\begin{array}{l}0.075 \\
(0.162)\end{array}$ & $\begin{array}{l}0.115 \\
(0.142)\end{array}$ & $\begin{array}{l}0.005 \\
(0.033)\end{array}$ \\
\hline $\begin{array}{l}\text { Ind exp - PG } \\
\text { offshore edu (H1b) }\end{array}$ & $\begin{array}{l}-0.040 \\
(0.123)\end{array}$ & $\begin{array}{l}-0.035 \\
(0.115)\end{array}$ & $\begin{array}{l}-0.003 \\
(0.015)\end{array}$ & $\begin{array}{l}0.125 \\
(0.275)\end{array}$ & $\begin{array}{l}0.209 \\
(0.239)\end{array}$ & $\begin{array}{l}0.013 \\
(0.041)\end{array}$ & $\begin{array}{l}-0.062 \\
(0.203)\end{array}$ & $\begin{array}{l}-0.040 \\
(0.186)\end{array}$ & $\begin{array}{l}-0.004 \\
(0.024)\end{array}$ & $\begin{array}{l}-0.177 \\
(0.208)\end{array}$ & $\begin{array}{l}-0.230 \\
(0.182)\end{array}$ & $\begin{array}{l}-0.014 \\
(0.048)\end{array}$ \\
\hline $\begin{array}{l}\text { Ind exp - intl } \\
\text { recruitment (Factor) } \\
\text { (H1b) }\end{array}$ & $\begin{array}{l}0.250 \dagger \\
(0.142)\end{array}$ & $\begin{array}{l}0.198 \\
(0.128)\end{array}$ & $\begin{array}{l}0.012 \\
(0.019)\end{array}$ & $\begin{array}{l}0.213 \\
(0.326)\end{array}$ & $\begin{array}{l}0.029 \\
(0.264)\end{array}$ & $\begin{array}{l}0.003 \\
(0.047)\end{array}$ & $\begin{array}{l}0.272 \\
(0.250)\end{array}$ & $\begin{array}{l}0.189 \\
(0.226)\end{array}$ & $\begin{array}{l}0.013 \\
(0.033)\end{array}$ & $\begin{array}{l}0.331 \\
(0.221)\end{array}$ & $\begin{array}{l}0.214 \\
(0.179)\end{array}$ & $\begin{array}{l}0.014 \\
(0.043)\end{array}$ \\
\hline $\begin{array}{l}\text { Ind exp - UG } \\
\text { marketing }(H 1 b)\end{array}$ & $\begin{array}{l}0.021 \\
(0.108)\end{array}$ & $\begin{array}{l}0.014 \\
(0.100)\end{array}$ & $\begin{array}{l}0.122 \mathrm{E}-4 \\
(0.015)\end{array}$ & $\begin{array}{l}-0.433 \\
(0.294)\end{array}$ & $\begin{array}{l}-0.365 \\
(0.250)\end{array}$ & $\begin{array}{l}-0.026 \\
(0.050)\end{array}$ & $\begin{array}{l}0.157 \\
(0.174)\end{array}$ & $\begin{array}{l}0.128 \\
(0.159)\end{array}$ & $\begin{array}{l}0.010 \\
(0.024)\end{array}$ & $\begin{array}{l}0.087 \\
(0.159)\end{array}$ & $\begin{array}{l}0.111 \\
(0.135)\end{array}$ & $\begin{array}{l}0.005 \\
(0.039)\end{array}$ \\
\hline $\begin{array}{l}\text { Ind exp - PG } \\
\text { marketing }(H 1 b)\end{array}$ & $\begin{array}{l}0.011 \\
(0.106)\end{array}$ & $\begin{array}{l}-0.038 \\
(0.098)\end{array}$ & $\begin{array}{l}-0.002 \\
(0.013)\end{array}$ & $\begin{array}{l}0.162 \\
(0.230)\end{array}$ & $\begin{array}{l}0.157 \\
(0.205)\end{array}$ & $\begin{array}{l}0.006 \\
(0.042)\end{array}$ & $\begin{array}{l}-0.071 \\
(0.173)\end{array}$ & $\begin{array}{l}-0.069 \\
(0.158)\end{array}$ & $\begin{array}{l}-0.004 \\
(0.023)\end{array}$ & $\begin{array}{l}0.166 \\
(0.183)\end{array}$ & $\begin{array}{l}0.041 \\
(0.152)\end{array}$ & $\begin{array}{l}0.002 \\
(0.042)\end{array}$ \\
\hline $\begin{array}{l}\text { Know-how (Factor) } \\
\text { (H2) }\end{array}$ & $\begin{array}{l}-1.516 * * \\
(0.118)\end{array}$ & $\begin{array}{l}-1.504 * * \\
(0.110)\end{array}$ & $\begin{array}{l}-0.117 * * \\
(0.014)\end{array}$ & $\begin{array}{l}-1.677 * * \\
(0.257)\end{array}$ & $\begin{array}{l}-1.628 * * \\
(0.221)\end{array}$ & $\begin{array}{l}-0.124 * * \\
(0.032)\end{array}$ & $\begin{array}{l}-1.617 * * \\
(0.197)\end{array}$ & $\begin{array}{l}-1.574 * * \\
(0.176)\end{array}$ & $\begin{array}{l}-0.124 * * \\
(0.025)\end{array}$ & $\begin{array}{l}-1.251 * * \\
(0.227)\end{array}$ & $\begin{array}{l}-1.290 * * \\
(0.203)\end{array}$ & $\begin{array}{l}-0.092 \\
(0.057)\end{array}$ \\
\hline $\begin{array}{l}\text { Financial resources } \\
\text { (H5) }\end{array}$ & $\begin{array}{l}0.019 \\
(0.095)\end{array}$ & $\begin{array}{l}0.043 \\
(0.086)\end{array}$ & $\begin{array}{l}0.001 \\
(0.012)\end{array}$ & $\begin{array}{l}0.452 \dagger \\
(0.262)\end{array}$ & $\begin{array}{l}0.467^{*} \\
(0.234)\end{array}$ & $\begin{array}{l}0.029 \\
(0.048)\end{array}$ & $\begin{array}{l}0.046 \\
(0.135)\end{array}$ & $\begin{array}{l}0.093 \\
(0.121)\end{array}$ & $\begin{array}{l}0.003 \\
(0.018)\end{array}$ & $\begin{array}{l}-0.094 \\
(0.149)\end{array}$ & $\begin{array}{l}-0.049 \\
(0.118)\end{array}$ & $\begin{array}{l}-0.004 \\
(0.033)\end{array}$ \\
\hline $\begin{array}{l}\text { Reputation (Factor) } \\
\text { (H6) }\end{array}$ & $\begin{array}{l}-0.220 \\
(0.206)\end{array}$ & $\begin{array}{l}-0.262 \\
(0.186)\end{array}$ & $\begin{array}{l}-0.020 \\
(0.028)\end{array}$ & $\begin{array}{l}-0.078 \\
(0.480)\end{array}$ & $\begin{array}{l}-0.143 \\
(0.406)\end{array}$ & $\begin{array}{l}-0.011 \\
(0.084)\end{array}$ & $\begin{array}{l}-0.329 \\
(0.372)\end{array}$ & $\begin{array}{l}-0.359 \\
(0.337)\end{array}$ & $\begin{array}{l}-0.036 \\
(0.054)\end{array}$ & $\begin{array}{l}-0.186 \\
(0.322)\end{array}$ & $\begin{array}{l}-0.161 \\
(0.265)\end{array}$ & $\begin{array}{l}-0.010 \\
(0.065)\end{array}$ \\
\hline $\begin{array}{l}\text { IMD Normative ID } \\
\text { (lag 1) }\end{array}$ & $\begin{array}{l}-0.080 \\
(0.151)\end{array}$ & $\begin{array}{l}-0.002 \\
(0.002)\end{array}$ & $\begin{array}{l}-0.0001 \\
(0.020)\end{array}$ & $\begin{array}{l}-0.243 \\
(0.315)\end{array}$ & $\begin{array}{l}-0.217 \\
(0.271)\end{array}$ & $\begin{array}{l}-0.012 \\
(0.057)\end{array}$ & $\begin{array}{l}-0.407 \\
(0.283)\end{array}$ & $\begin{array}{l}-0.002 \\
(0.002)\end{array}$ & $\begin{array}{l}-0.0001 \\
(0.016)\end{array}$ & $\begin{array}{l}0.072 \\
(0.265)\end{array}$ & $\begin{array}{l}0.161 \\
(0.208)\end{array}$ & $\begin{array}{l}0.010 \\
(0.064)\end{array}$ \\
\hline $\begin{array}{l}\text { EFI Regulative ID } \\
\text { (lag 1) }\end{array}$ & $\begin{array}{l}0.165 \dagger \\
(0.094)\end{array}$ & $\begin{array}{l}0.187 * \\
(0.086)\end{array}$ & $\begin{array}{l}0.012 \\
(0.012)\end{array}$ & $\begin{array}{l}0.297 \\
(0.181)\end{array}$ & $\begin{array}{l}0.350 * \\
(0.152)\end{array}$ & $\begin{array}{l}0.022 \\
(0.033)\end{array}$ & $\begin{array}{l}0.220 \\
(0.184)\end{array}$ & $\begin{array}{l}0.209 \\
(0.166)\end{array}$ & $\begin{array}{l}0.015 \\
(0.020)\end{array}$ & $\begin{array}{l}-0.130 \\
(0.134)\end{array}$ & $\begin{array}{l}-0.065 \\
(0.117)\end{array}$ & $\begin{array}{l}-0.003 \\
(0.031)\end{array}$ \\
\hline Age & $\begin{array}{l}0.003 \\
(0.003)\end{array}$ & $\begin{array}{l}0.002 \\
(0.003)\end{array}$ & $\begin{array}{l}0.0001 \\
(0.0004)\end{array}$ & $\begin{array}{l}-0.009 \\
(0.012)\end{array}$ & $\begin{array}{l}-0.008 \\
(0.008)\end{array}$ & $\begin{array}{l}-0.0005 \\
(0.002)\end{array}$ & $\begin{array}{l}0.003 \\
(0.006)\end{array}$ & $\begin{array}{l}0.002 \\
(0.005)\end{array}$ & $\begin{array}{l}0.0002 \\
(0.0007)\end{array}$ & $\begin{array}{l}\mathbf{0 . 0 0 7 \dagger} \\
(\mathbf{0 . 0 0 4 )}\end{array}$ & $\begin{array}{l}0.005 \\
(0.003)\end{array}$ & $\begin{array}{l}0.0003 \\
(0.0008)\end{array}$ \\
\hline Size (Staff) & $\begin{array}{l}0.000 \\
(0.000)\end{array}$ & $\begin{array}{l}0.0001 \\
(0.0001)\end{array}$ & $\begin{array}{l}0.790 \mathrm{E}-5 \\
(0.175 \mathrm{E}-4)\end{array}$ & - & - & - & - & - & - & - & - & - \\
\hline Offshore policy & $\begin{array}{l}1.284 * \\
(0.663)\end{array}$ & $\begin{array}{l}1.304 * \\
(0.624)\end{array}$ & $\begin{array}{l}0.108 \dagger \\
(0.063)\end{array}$ & $\begin{array}{l}0.842 \\
(1.465)\end{array}$ & $\begin{array}{l}0.719 \\
(1.279)\end{array}$ & $\begin{array}{l}0.071 \\
(0.200)\end{array}$ & $\begin{array}{l}1.782 \dagger \\
(1.058)\end{array}$ & $\begin{array}{l}2.074^{*} \\
(0.948)\end{array}$ & $\begin{array}{l}0.207 * \\
(0.088)\end{array}$ & $\begin{array}{l}\text { 2.639* } \\
(1.146)\end{array}$ & $\begin{array}{l}2.612^{*} \\
(1.022)\end{array}$ & $\begin{array}{l}0.173 \\
(0.284)\end{array}$ \\
\hline $\begin{array}{l}\text { Internationalisation } \\
\text { at home strategy }\end{array}$ & $\begin{array}{l}0.030 \\
(0.125)\end{array}$ & $\begin{array}{l}-0.0007 \\
(0.0007)\end{array}$ & $\begin{array}{l}-0.464 \mathrm{E}-4 \\
(0.0001)\end{array}$ & $\begin{array}{l}0.140 \\
(0.328)\end{array}$ & $\begin{array}{l}-0.001 \\
(0.001)\end{array}$ & $\begin{array}{l}-0.874 \mathrm{E}-4 \\
(0.0002)\end{array}$ & $\begin{array}{l}-0.049 \\
(0.203)\end{array}$ & $\begin{array}{l}-0.0009 \\
(0.002)\end{array}$ & $\begin{array}{l}-0.639 \mathrm{E}-4 \\
(0.0002)\end{array}$ & $\begin{array}{l}-0.023 \\
(0.201)\end{array}$ & $\begin{array}{l}0.0003 \\
(0.0008)\end{array}$ & $\begin{array}{l}0.139 \mathrm{E}-4 \\
(0.0002)\end{array}$ \\
\hline
\end{tabular}




\begin{tabular}{|c|c|c|c|c|c|c|c|c|c|c|c|c|}
\hline $\begin{array}{l}\text { Host country } \\
\text { demand }\end{array}$ & $\begin{array}{l}4.064^{*} \\
(2.118)\end{array}$ & $\begin{array}{l}\text { 4.393* } \\
(1.951)\end{array}$ & $\begin{array}{l}0.299 \\
(0.268)\end{array}$ & $\begin{array}{l}\text { 7.838* } \\
\text { (3.928) }\end{array}$ & $\begin{array}{l}\text { 9.578** } \\
(3.198)\end{array}$ & $\begin{array}{l}0.581 \\
(0.635)\end{array}$ & $\begin{array}{l}0.040 \\
(4.231)\end{array}$ & $\begin{array}{l}-0.102 \\
(3.871)\end{array}$ & $\begin{array}{l}0.038 \\
(0.483)\end{array}$ & - & - & - \\
\hline AU/NZ dummy & $\begin{array}{l}0.437 \\
(0.514)\end{array}$ & $\begin{array}{l}0.488 \\
(0.481)\end{array}$ & $\begin{array}{l}0.028 \\
(0.065)\end{array}$ & - & - & - & - & - & - & - & - & - \\
\hline UK/IR dummy & $\begin{array}{l}0.185 \\
(0.475)\end{array}$ & $\begin{array}{l}0.218 \\
(0.440)\end{array}$ & $\begin{array}{l}0.012 \\
(0.063)\end{array}$ & - & - & - & - & - & - & - & - & - \\
\hline $\mathrm{R}^{2}$ & 0.577 & & & 0.605 & & & 0.683 & & & 0.479 & & \\
\hline $\operatorname{AdjR}^{2}$ & 0.543 & & & 0.471 & & & 0.623 & & & 0.355 & & \\
\hline Log likelihood & & -738.56 & -808.25 & & -187.37 & -208.88 & & -301.29 & -329.97 & & -225.79 & -259.17 \\
\hline $\mathrm{N}$ & 308 & 308 & 308 & 80 & 80 & 80 & 124 & 124 & 124 & 104 & 104 & 104 \\
\hline Max. VIF & 1.983 & & & 2.355 & & & 2.511 & & & 1.821 & & \\
\hline
\end{tabular}

Standard errors in parentheses, all $t$ tests are two-tailed, $\uparrow p<.10,{ }^{*} p<.05,{ }^{* *} p<.01$

Table 7.36: Regression estimates for equation 21

\begin{tabular}{|c|c|c|c|c|c|c|c|c|c|c|c|c|}
\hline & \multicolumn{3}{|c|}{ Complete data set } & \multicolumn{3}{|c|}{ AU/NZ } & \multicolumn{3}{|c|}{ US/CA } & \multicolumn{3}{|c|}{$\overline{\text { UK/IR }}$} \\
\hline & OLS & Tobit & $\begin{array}{l}\text { Negative } \\
\text { binomial }\end{array}$ & OLS & Tobit & $\begin{array}{l}\text { Negative } \\
\text { binomial }\end{array}$ & OLS & Tobit & $\begin{array}{l}\text { Negative } \\
\text { binomial }\end{array}$ & OLS & Tobit & $\begin{array}{l}\text { Negative } \\
\text { binomial }\end{array}$ \\
\hline Intercept & $\begin{array}{l}\text { 20.190** } \\
(3.282)\end{array}$ & $\begin{array}{l}21.509 * * \\
(2.979)\end{array}$ & $\begin{array}{l}3.188 * * \\
(0.393)\end{array}$ & $\begin{array}{l}\text { 20.866* } \\
(6.547)\end{array}$ & $\begin{array}{l}22.313 * \\
(5.290)\end{array}$ & $\begin{array}{l}3.268 * * \\
(1.037)\end{array}$ & $\begin{array}{l}20.452^{* *} \\
(6.026)\end{array}$ & $\begin{array}{l}20.434 * * \\
(5.356)\end{array}$ & $\begin{array}{l}3.116 * * \\
(0.645)\end{array}$ & $\begin{array}{l}22.710 * * \\
(5.100)\end{array}$ & $\begin{array}{l}\text { 21.601** } \\
(4.227)\end{array}$ & $\begin{array}{l}3.142 * * \\
(0.999)\end{array}$ \\
\hline $\begin{array}{l}\text { Geo exp - } \\
\text { knowledge of edu } \\
\text { sector (HIa) }\end{array}$ & $\begin{array}{l}-0.041 \\
(0.125)\end{array}$ & $\begin{array}{l}-0.055 \\
(0.115)\end{array}$ & $\begin{array}{l}-0.004 \\
(0.015)\end{array}$ & $\begin{array}{l}0.476 \\
(0.286)\end{array}$ & $\begin{array}{l}0.414 \dagger \\
(0.233)\end{array}$ & $\begin{array}{l}0.023 \\
(0.049)\end{array}$ & $\begin{array}{l}-0.098 \\
(0.214)\end{array}$ & $\begin{array}{l}-0.087 \\
(0.195)\end{array}$ & $\begin{array}{l}-0.006 \\
(0.023)\end{array}$ & $\begin{array}{l}-0.188 \\
(0.213)\end{array}$ & $\begin{array}{l}-0.180 \\
(0.167)\end{array}$ & $\begin{array}{l}-0.011 \\
(0.041)\end{array}$ \\
\hline $\begin{array}{l}\text { Geo exp - academic } \\
\text { capabilities (H1a) }\end{array}$ & $\begin{array}{l}0.120 \\
(0.120)\end{array}$ & $\begin{array}{l}0.074 \\
(0.111)\end{array}$ & $\begin{array}{l}0.006 \\
(0.012)\end{array}$ & $\begin{array}{l}0.632 \\
(0.281)\end{array}$ & $\begin{array}{l}\text { 0.586* } \\
(0.238)\end{array}$ & $\begin{array}{l}0.036 \\
(0.045)\end{array}$ & $\begin{array}{l}0.103 \\
(0.217)\end{array}$ & $\begin{array}{l}0.074 \\
(0.199)\end{array}$ & $\begin{array}{l}0.004 \\
(0.020)\end{array}$ & $\begin{array}{l}0.088 \\
(0.186)\end{array}$ & $\begin{array}{l}-0.004 \\
(0.155)\end{array}$ & $\begin{array}{l}0.001 \\
(0.032)\end{array}$ \\
\hline $\begin{array}{l}\text { Geo exp - } \\
\text { managerial } \\
\text { capabilities (H1a) }\end{array}$ & $\begin{array}{l}0.140 \\
(0.145)\end{array}$ & $\begin{array}{l}0.145 \\
(0.137)\end{array}$ & $\begin{array}{l}0.008 \\
(0.017)\end{array}$ & $\begin{array}{l}-0.051 \\
(0.313)\end{array}$ & $\begin{array}{l}-0.029 \\
(0.267)\end{array}$ & $\begin{array}{l}-0.0002 \\
(0.048)\end{array}$ & $\begin{array}{l}0.323 \\
(0.257)\end{array}$ & $\begin{array}{l}0.336 \\
(0.236)\end{array}$ & $\begin{array}{l}0.020 \\
(0.025)\end{array}$ & $\begin{array}{l}-0.113 \\
(0.233)\end{array}$ & $\begin{array}{l}-0.032 \\
(0.201)\end{array}$ & $\begin{array}{l}-0.003 \\
(0.041)\end{array}$ \\
\hline $\begin{array}{l}\text { Geo exp - financial } \\
\text { capabilities (H1a) }\end{array}$ & $\begin{array}{l}-0.012 \\
(0.137)\end{array}$ & $\begin{array}{l}-0.040 \\
(0.126)\end{array}$ & $\begin{array}{l}-0.003 \\
(0.016)\end{array}$ & $\begin{array}{l}-0.343 \\
(0.313)\end{array}$ & $\begin{array}{l}-0.462 \dagger \\
(0.244)\end{array}$ & $\begin{array}{l}-0.032 \\
(0.045)\end{array}$ & $\begin{array}{l}0.233 \\
(0.227)\end{array}$ & $\begin{array}{l}0.174 \\
(0.205)\end{array}$ & $\begin{array}{l}0.011 \\
(0.023)\end{array}$ & $\begin{array}{l}-0.336 \\
(0.242)\end{array}$ & $\begin{array}{l}-0.307 \\
(0.207)\end{array}$ & $\begin{array}{l}-0.019 \\
(0.049)\end{array}$ \\
\hline $\begin{array}{l}\text { Geo exp - intl } \\
\text { recruitment (H1a) }\end{array}$ & $\begin{array}{l}-0.164 \\
(0.152)\end{array}$ & $\begin{array}{l}-0.153 \\
(0.142)\end{array}$ & $\begin{array}{l}-0.009 \\
(0.017)\end{array}$ & $\begin{array}{l}0.304 \\
(0.283)\end{array}$ & $\begin{array}{l}0.336 \\
(0.242)\end{array}$ & $\begin{array}{l}0.022 \\
(0.042)\end{array}$ & $\begin{array}{l}-0.605^{*} \\
(0.278)\end{array}$ & $\begin{array}{l}-0.570 * \\
(0.251)\end{array}$ & $\begin{array}{l}-0.040 \\
(0.029)\end{array}$ & $\begin{array}{l}0.022 \\
(0.253)\end{array}$ & $\begin{array}{l}0.060 \\
(0.217)\end{array}$ & $\begin{array}{l}0.004 \\
(0.053)\end{array}$ \\
\hline $\begin{array}{l}\text { Ind exp - UG } \\
\text { offshore edu (H1b) }\end{array}$ & $\begin{array}{l}0.008 \\
(0.116)\end{array}$ & $\begin{array}{l}-0.001 \\
(0.109)\end{array}$ & $\begin{array}{l}0.0001 \\
(0.012)\end{array}$ & $\begin{array}{l}-0.237 \\
(0.254)\end{array}$ & $\begin{array}{l}-0.270 \\
(0.214)\end{array}$ & $\begin{array}{l}-0.014 \\
(0.035)\end{array}$ & $\begin{array}{l}0.026 \\
(0.224)\end{array}$ & $\begin{array}{l}0.021 \\
(0.206)\end{array}$ & $\begin{array}{l}0.002 \\
(0.023)\end{array}$ & $\begin{array}{l}0.130 \\
(0.187)\end{array}$ & $\begin{array}{l}0.140 \\
(0.158)\end{array}$ & $\begin{array}{l}0.007 \\
(0.037)\end{array}$ \\
\hline
\end{tabular}




\begin{tabular}{|c|c|c|c|c|c|c|c|c|c|c|c|c|}
\hline $\begin{array}{l}\text { Ind exp - PG } \\
\text { offshore edu (H1b) }\end{array}$ & $\begin{array}{l}-0.029 \\
(0.132)\end{array}$ & $\begin{array}{l}-0.029 \\
(0.124)\end{array}$ & $\begin{array}{l}-0.002 \\
(0.014)\end{array}$ & $\begin{array}{l}-0.043 \\
(0.283)\end{array}$ & $\begin{array}{l}0.019 \\
(0.239)\end{array}$ & $\begin{array}{l}0.0004 \\
(0.039)\end{array}$ & $\begin{array}{l}-0.022 \\
(0.225)\end{array}$ & $\begin{array}{l}-0.009 \\
(0.206)\end{array}$ & $\begin{array}{l}-0.001 \\
(0.023)\end{array}$ & $\begin{array}{l}-0.020 \\
(0.234)\end{array}$ & $\begin{array}{l}-0.034 \\
(0.199)\end{array}$ & $\begin{array}{l}-0.0007 \\
(0.047)\end{array}$ \\
\hline $\begin{array}{l}\text { Ind exp - intl } \\
\text { recruitment (Factor) } \\
\text { (H1b) }\end{array}$ & $\begin{array}{l}0.191 \\
(0.153)\end{array}$ & $\begin{array}{l}0.163 \\
(0.139)\end{array}$ & $\begin{array}{l}0.010 \\
(0.018)\end{array}$ & $\begin{array}{l}-0.187 \\
(0.347)\end{array}$ & $\begin{array}{l}-0.288 \\
(0.278)\end{array}$ & $\begin{array}{l}-0.019 \\
(0.045)\end{array}$ & $\begin{array}{l}0.350 \\
(0.275)\end{array}$ & $\begin{array}{l}0.271 \\
(0.250)\end{array}$ & $\begin{array}{l}0.019 \\
(0.028)\end{array}$ & $\begin{array}{l}0.302 \\
(0.246)\end{array}$ & $\begin{array}{l}0.270 \\
(0.195)\end{array}$ & $\begin{array}{l}0.016 \\
(0.049)\end{array}$ \\
\hline $\begin{array}{l}\text { Ind exp - UG } \\
\text { marketing }(H 1 b)\end{array}$ & $\begin{array}{l}0.057 \\
(0.116)\end{array}$ & $\begin{array}{l}0.049 \\
(0.108)\end{array}$ & $\begin{array}{l}0.022 \\
(0.015)\end{array}$ & $\begin{array}{l}-0.275 \\
(0.308)\end{array}$ & $\begin{array}{l}-0.206 \\
(0.260)\end{array}$ & $\begin{array}{l}-0.015 \\
(0.054)\end{array}$ & $\begin{array}{l}0.265 \\
(0.194)\end{array}$ & $\begin{array}{l}0.231 \\
(0.177)\end{array}$ & $\begin{array}{l}0.017 \\
(0.022)\end{array}$ & $\begin{array}{l}0.054 \\
(0.177)\end{array}$ & $\begin{array}{l}0.087 \\
(0.147)\end{array}$ & $\begin{array}{l}0.003 \\
(0.039)\end{array}$ \\
\hline $\begin{array}{l}\text { Ind exp - PG } \\
\text { marketing }(H 1 b)\end{array}$ & $\begin{array}{l}0.026 \\
(0.114)\end{array}$ & $\begin{array}{l}-0.031 \\
(0.105)\end{array}$ & $\begin{array}{l}-0.002 \\
(0.012)\end{array}$ & $\begin{array}{l}0.277 \\
(0.239)\end{array}$ & $\begin{array}{l}0.264 \\
(0.201)\end{array}$ & $\begin{array}{l}0.014 \\
(0.036)\end{array}$ & $\begin{array}{l}-0.143 \\
(0.191)\end{array}$ & $\begin{array}{l}-0.144 \\
(0.175)\end{array}$ & $\begin{array}{l}-0.010 \\
(0.018)\end{array}$ & $\begin{array}{l}0.231 \\
(0.203)\end{array}$ & $\begin{array}{l}0.091 \\
(0.169)\end{array}$ & $\begin{array}{l}0.005 \\
(0.041)\end{array}$ \\
\hline $\begin{array}{l}\text { Org culture (Factor) } \\
-(H 3)\end{array}$ & $\begin{array}{l}-1.820 * * \\
(0.176)\end{array}$ & $\begin{array}{l}-1.755^{* *} \\
(0.162)\end{array}$ & $\begin{array}{l}-0.130 * * \\
(0.019)\end{array}$ & $\begin{array}{l}-2.107 * * \\
(0.352)\end{array}$ & $\begin{array}{l}-2.075^{* *} \\
(0.301)\end{array}$ & $\begin{array}{l}-0.151 * * \\
(0.045)\end{array}$ & $\begin{array}{l}-1.970^{* *} \\
(0.303)\end{array}$ & $\begin{array}{l}-1.873 * * \\
(0.265)\end{array}$ & $\begin{array}{l}-0.142 * * \\
(0.030)\end{array}$ & (20) & 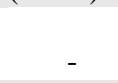 & - \\
\hline $\begin{array}{l}\text { Org culture - open } \\
\text { discussion (H3) }\end{array}$ & - & - & - & - & - & - & - & - & - & $\begin{array}{l}-0.387 \\
(0.239)\end{array}$ & $\begin{array}{l}-0.405^{*} \\
(0.203)\end{array}$ & $\begin{array}{l}-0.026 \\
(0.044)\end{array}$ \\
\hline $\begin{array}{l}\text { Org culture - no } \\
\text { status distinction } \\
\text { (H3) }\end{array}$ & - & - & - & - & - & - & - & - & - & $\begin{array}{l}-0.425 \dagger \\
(0.248)\end{array}$ & $\begin{array}{l}-0.368 \dagger \\
(0.210)\end{array}$ & $\begin{array}{l}-0.024 \\
(0.041)\end{array}$ \\
\hline $\begin{array}{l}\text { Org culture - } \\
\text { experimentation } \\
\text { (H3) }\end{array}$ & - & - & - & - & - & - & - & - & - & $\begin{array}{l}-0.175 \\
(0.215)\end{array}$ & $\begin{array}{l}-0.252 \\
(0.172)\end{array}$ & $\begin{array}{l}-0.016 \\
(0.040)\end{array}$ \\
\hline $\begin{array}{l}\text { Org culture - } \\
\text { offshore } \\
\text { development (H3) }\end{array}$ & - & - & - & - & - & - & - & - & - & $\begin{array}{l}-0.497^{*} \\
(0.232)\end{array}$ & $\begin{array}{l}-0.394^{*} \\
(0.187)\end{array}$ & $\begin{array}{l}-0.024 \\
(0.052)\end{array}$ \\
\hline $\begin{array}{l}\text { Org culture - } \\
\text { offshore } \\
\text { responsiveness (H3) }\end{array}$ & - & - & - & - & - & - & - & - & - & $\begin{array}{l}-0.041 \\
(0.225)\end{array}$ & $\begin{array}{l}0.047 \\
(0.186)\end{array}$ & $\begin{array}{l}0.001 \\
(0.040)\end{array}$ \\
\hline $\begin{array}{l}\text { Financial resources } \\
\text { (H5) }\end{array}$ & $\begin{array}{l}0.116 \\
(0.104)\end{array}$ & $\begin{array}{l}0.111 \\
(0.094)\end{array}$ & $\begin{array}{l}0.006 \\
(0.011)\end{array}$ & $\begin{array}{l}0.297 \\
(0.268)\end{array}$ & $\begin{array}{l}0.208 \\
(0.205)\end{array}$ & $\begin{array}{l}0.012 \\
(0.043)\end{array}$ & $\begin{array}{l}0.118 \\
(0.150)\end{array}$ & $\begin{array}{l}0.157 \\
(0.135)\end{array}$ & $\begin{array}{l}0.007 \\
(0.014)\end{array}$ & $\begin{array}{l}0.025 \\
(0.165)\end{array}$ & $\begin{array}{l}-0.003 \\
(0.128)\end{array}$ & $\begin{array}{l}-0.0005 \\
(0.039)\end{array}$ \\
\hline $\begin{array}{l}\text { Reputation (Factor) } \\
\text { (H6) }\end{array}$ & $\begin{array}{l}-0.389 \dagger \\
(0.221)\end{array}$ & $\begin{array}{l}-0.493^{*} \\
(0.199)\end{array}$ & $\begin{array}{l}-0.034 \\
(0.026)\end{array}$ & $\begin{array}{l}-0.423 \\
(0.485)\end{array}$ & $\begin{array}{l}-0.441 \\
(0.401)\end{array}$ & $\begin{array}{l}-0.031 \\
(0.058)\end{array}$ & $\begin{array}{l}-0.531 \\
(0.425)\end{array}$ & $\begin{array}{l}-0.612 \\
(0.380)\end{array}$ & $\begin{array}{l}-0.047 \\
(0.049)\end{array}$ & $\begin{array}{l}-0.302 \\
(0.351)\end{array}$ & $\begin{array}{l}-0.365 \\
(0.280)\end{array}$ & $\begin{array}{l}-0.023 \\
(0.070)\end{array}$ \\
\hline $\begin{array}{l}\text { IMD Normative ID } \\
\text { (lag 1) }\end{array}$ & $\begin{array}{l}-0.047 \\
(0.162)\end{array}$ & $\begin{array}{l}-0.001 \\
(0.002)\end{array}$ & $\begin{array}{l}-0.964 \mathrm{E}-4 \\
(0.018)\end{array}$ & $\begin{array}{l}-0.096 \\
(0.324)\end{array}$ & $\begin{array}{l}-0.012 \\
(0.270)\end{array}$ & $\begin{array}{l}0.003 \\
(0.058)\end{array}$ & $\begin{array}{l}-0.454 \\
(0.318)\end{array}$ & $\begin{array}{l}-0.002 \\
(0.003)\end{array}$ & $\begin{array}{l}-0.0001 \\
(0.016)\end{array}$ & $\begin{array}{l}-0.047 \\
(0.296)\end{array}$ & $\begin{array}{l}0.142 \\
(0.233)\end{array}$ & $\begin{array}{l}0.009 \\
(0.054)\end{array}$ \\
\hline $\begin{array}{l}\text { EFI Regulative ID } \\
\text { (lag 1) }\end{array}$ & $\begin{array}{l}\text { 0.201* } \\
(0.101)\end{array}$ & $\begin{array}{l}0.216^{*} \\
(0.093)\end{array}$ & $\begin{array}{l}0.014 \\
(0.011)\end{array}$ & $\begin{array}{l}0.274 \\
(0.189)\end{array}$ & $\begin{array}{l}\text { 0.311* } \\
(0.157)\end{array}$ & $\begin{array}{l}0.019 \\
(0.029)\end{array}$ & $\begin{array}{l}0.240 \\
(0.138)\end{array}$ & $\begin{array}{l}0.240 \dagger \\
(0.125)\end{array}$ & $\begin{array}{l}0.016 \\
(0.014)\end{array}$ & $\begin{array}{l}-0.132 \\
(0.149)\end{array}$ & $\begin{array}{l}-0.052 \\
(0.128)\end{array}$ & $\begin{array}{l}-0.002 \\
(0.027)\end{array}$ \\
\hline Age & $\begin{array}{l}0.002 \\
(0.004)\end{array}$ & $\begin{array}{l}0.001 \\
(0.003)\end{array}$ & $\begin{array}{l}0.0001 \\
(0.0004)\end{array}$ & $\begin{array}{l}-0.002 \\
(0.012)\end{array}$ & $\begin{array}{l}0.0004 \\
(0.009)\end{array}$ & $\begin{array}{l}0.421 \mathrm{E}-4 \\
(0.002)\end{array}$ & $\begin{array}{l}0.003 \\
(0.006)\end{array}$ & $\begin{array}{l}0.002 \\
(0.005)\end{array}$ & $\begin{array}{l}0.0002 \\
(0.0006)\end{array}$ & $\begin{array}{l}0.004 \\
(0.005)\end{array}$ & $\begin{array}{l}0.002 \\
(0.004)\end{array}$ & $\begin{array}{l}0.0001 \\
(0.001)\end{array}$ \\
\hline Size (Staff) & $\begin{array}{l}1.025 \mathrm{E}-5 \\
(0.000)\end{array}$ & $\begin{array}{l}0.202 \mathrm{E}-4 \\
(0.0001)\end{array}$ & $\begin{array}{l}0.141 \mathrm{E}-5 \\
(0.185 \mathrm{E}-4)\end{array}$ & - & - & - & - & - & - & - & - & - \\
\hline Offshore policy & $\begin{array}{l}\text { 1.606* } \\
(0.713)\end{array}$ & $\begin{array}{l}1.669 * \\
(0.672)\end{array}$ & $\begin{array}{l}\text { 0.138* } \\
(0.058)\end{array}$ & $\begin{array}{l}1.356 \\
(1.499)\end{array}$ & $\begin{array}{l}1.252 \\
(1.291)\end{array}$ & $\begin{array}{l}0.112 \\
(0.218)\end{array}$ & $\begin{array}{l}2.282 \dagger \\
\text { (1.169) }\end{array}$ & $\begin{array}{l}2.557 * \\
(1.044)\end{array}$ & $\begin{array}{l}0.241^{* *} \\
(\mathbf{0 . 0 8 0 )}\end{array}$ & $\begin{array}{l}\text { 2.530* } \\
(1.274)\end{array}$ & $\begin{array}{l}\text { 2.694* } \\
\text { (1.115) }\end{array}$ & $\begin{array}{l}0.180 \\
(0.262)\end{array}$ \\
\hline
\end{tabular}




\begin{tabular}{|c|c|c|c|c|c|c|c|c|c|c|c|c|}
\hline $\begin{array}{l}\text { Internationalisation } \\
\text { at home strategy }\end{array}$ & $\begin{array}{l}0.016 \\
(0.135)\end{array}$ & $\begin{array}{l}0.0001 \\
(0.0007)\end{array}$ & $\begin{array}{l}0.167 \mathrm{E}-4 \\
(0.0001)\end{array}$ & $\begin{array}{l}-0.060 \\
(0.341)\end{array}$ & $\begin{array}{l}-0.0004 \\
(0.001)\end{array}$ & $\begin{array}{l}-0.584 \mathrm{E}-5 \\
(0.0002)\end{array}$ & $\begin{array}{l}0.047 \\
(0.225)\end{array}$ & $\begin{array}{l}-0.0008 \\
(0.002)\end{array}$ & $\begin{array}{l}-0.565 E-4 \\
(0.0002)\end{array}$ & $\begin{array}{l}-0.117 \\
(0.231)\end{array}$ & $\begin{array}{l}0.001 \\
(0.009)\end{array}$ & $\begin{array}{l}0.647 \mathrm{E}-4 \\
(0.0001)\end{array}$ \\
\hline $\begin{array}{l}\text { Host country } \\
\text { demand }\end{array}$ & $\begin{array}{l}4.636^{*} \\
(2.279)\end{array}$ & $\begin{array}{l}4.841 * \\
(2.107)\end{array}$ & $\begin{array}{l}0.308 \\
(0.247)\end{array}$ & $\begin{array}{l}\text { 8.948* } \\
(4.072)\end{array}$ & $\begin{array}{l}\text { 9.854** } \\
(3.289)\end{array}$ & $\begin{array}{l}0.597 \\
(0.599)\end{array}$ & - & - & - & - & - & - \\
\hline AU/NZ dummy & $\begin{array}{l}0.765 \\
(0.553)\end{array}$ & $\begin{array}{l}0.756 \\
(0.519)\end{array}$ & $\begin{array}{l}0.049 \\
(0.064)\end{array}$ & - & - & - & - & - & - & - & - & - \\
\hline UK/IR dummy & $\begin{array}{l}0.501 \\
(0.509)\end{array}$ & $\begin{array}{l}0.446 \\
(0.473)\end{array}$ & $\begin{array}{l}0.028 \\
(0.060)\end{array}$ & - & - & - & - & - & - & - & - & - \\
\hline $\mathrm{R}^{2}$ & 0.511 & & & 0.576 & & & 0.607 & & & 0.417 & & \\
\hline $\operatorname{AdjR}^{2}$ & 0.471 & & & 0.432 & & & 0.538 & & & 0.238 & & \\
\hline Log likelihood & & -761.89 & -823.94 & & -189.55 & -210.47 & & -314.03 & -339.72 & & -232.57 & -261.75 \\
\hline $\mathrm{N}$ & 308 & 308 & 308 & 80 & 80 & 80 & 124 & 124 & 124 & 104 & 104 & 104 \\
\hline Max. VIF & 2.050 & & & 2.296 & & & 2.384 & & & 1.935 & & \\
\hline
\end{tabular}

Standard errors in parentheses, all $t$ tests are two-tailed, $\uparrow p<.10,{ }^{*} p<.05,{ }^{* *} p<.01$

Table 7.37a: Regression estimates for equation 22a (low regulative institutional distance)

\begin{tabular}{|c|c|c|c|c|c|c|c|c|c|c|c|c|}
\hline & \multicolumn{3}{|c|}{ Complete data set } & \multicolumn{3}{|c|}{ AU/NZ } & \multicolumn{3}{|c|}{$\overline{\mathrm{US} / \mathrm{CA}}$} & \multicolumn{3}{|c|}{ 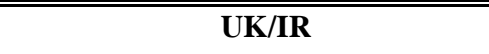 } \\
\hline & OLS & Tobit & $\begin{array}{l}\text { Negative } \\
\text { binomial }\end{array}$ & OLS & Tobit & $\begin{array}{l}\text { Negative } \\
\text { binomial }\end{array}$ & OLS & Tobit & $\begin{array}{l}\text { Negative } \\
\text { binomial }\end{array}$ & OLS & Tobit & $\begin{array}{l}\text { Negative } \\
\text { binomial }\end{array}$ \\
\hline Intercept & $\begin{array}{l}3.773 \\
(4.502)\end{array}$ & $\begin{array}{l}5.165 \\
(4.019)\end{array}$ & $\begin{array}{l}1.887 * * \\
(0.327)\end{array}$ & $\begin{array}{l}4.260 \\
(9.270)\end{array}$ & $\begin{array}{l}5.434 \\
(7.456)\end{array}$ & $\begin{array}{l}2.030 * \\
(0.791)\end{array}$ & $\begin{array}{l}6.698 \\
(7.695)\end{array}$ & $\begin{array}{l}5.502 \\
(6.628)\end{array}$ & $\begin{array}{l}1.635 * * \\
(0.594)\end{array}$ & $\begin{array}{l}7.049 \\
(6.714)\end{array}$ & $\begin{array}{l}7.957 \\
(5.669)\end{array}$ & $\begin{array}{l}2.234 * * \\
(1.089)\end{array}$ \\
\hline $\begin{array}{l}\text { Geo exp - } \\
\text { knowledge of edu } \\
\text { sector }(H 8 a)\end{array}$ & $\begin{array}{l}0.224 \\
(0.244)\end{array}$ & $\begin{array}{l}0.199 \\
(0.221)\end{array}$ & $\begin{array}{l}0.011 \\
(0.015)\end{array}$ & $\begin{array}{l}0.830 \\
(0.579)\end{array}$ & $\begin{array}{l}0.758 \dagger \\
(0.457)\end{array}$ & $\begin{array}{l}0.044 \\
(0.038)\end{array}$ & $\begin{array}{l}0.121 \\
(0.431)\end{array}$ & $\begin{array}{l}0.151 \\
(0.387)\end{array}$ & $\begin{array}{l}0.012 \\
(0.037)\end{array}$ & $\begin{array}{l}-0.323 \\
(0.336)\end{array}$ & $\begin{array}{l}-0.289 \\
(0.272)\end{array}$ & $\begin{array}{l}-0.016 \\
(0.047)\end{array}$ \\
\hline $\begin{array}{l}\text { Geo exp - academic } \\
\text { capabilities }(H 8 a)\end{array}$ & $\begin{array}{l}0.055 \\
(0.219)\end{array}$ & $\begin{array}{l}-0.001 \\
(0.200)\end{array}$ & $\begin{array}{l}0.0002 \\
(0.014)\end{array}$ & $\begin{array}{l}-0.536 \\
(0.478)\end{array}$ & $\begin{array}{l}-0.533 \\
(0.393)\end{array}$ & $\begin{array}{l}-0.030 \\
(0.035)\end{array}$ & $\begin{array}{l}0.405 \\
(0.416)\end{array}$ & $\begin{array}{l}0.417 \\
(0.377)\end{array}$ & $\begin{array}{l}0.023 \\
(0.029)\end{array}$ & $\begin{array}{l}0.165 \\
(0.287)\end{array}$ & $\begin{array}{l}0.040 \\
(0.242)\end{array}$ & $\begin{array}{l}0.002 \\
(0.040)\end{array}$ \\
\hline $\begin{array}{l}\text { Geo exp - } \\
\text { managerial } \\
\text { capabilities (H8a) }\end{array}$ & $\begin{array}{l}0.125 \\
(0.252)\end{array}$ & $\begin{array}{l}0.150 \\
(0.236)\end{array}$ & $\begin{array}{l}0.010 \\
(0.018)\end{array}$ & $\begin{array}{l}0.574 \\
(0.676)\end{array}$ & $\begin{array}{l}0.546 \\
(0.567)\end{array}$ & $\begin{array}{l}0.034 \\
(0.061)\end{array}$ & $\begin{array}{l}-0.273 \\
(0.442)\end{array}$ & $\begin{array}{l}-0.263 \\
(0.400)\end{array}$ & $\begin{array}{l}-0.013 \\
(0.031)\end{array}$ & $\begin{array}{l}0.148 \\
(0.381)\end{array}$ & $\begin{array}{l}0.268 \\
(0.324)\end{array}$ & $\begin{array}{l}0.014 \\
(0.056)\end{array}$ \\
\hline $\begin{array}{l}\text { Geo exp - financial } \\
\text { capabilities }(H 8 a)\end{array}$ & $\begin{array}{l}-0.005 \\
(0.250)\end{array}$ & $\begin{array}{l}-0.037 \\
(0.224)\end{array}$ & $\begin{array}{l}-0.002 \\
(0.020)\end{array}$ & $\begin{array}{l}-0.421 \\
(0.610)\end{array}$ & $\begin{array}{l}-0.311 \\
(0.437)\end{array}$ & $\begin{array}{l}-0.018 \\
(0.046)\end{array}$ & $\begin{array}{l}0.049 \\
(0.428)\end{array}$ & $\begin{array}{l}0.052 \\
(0.388)\end{array}$ & $\begin{array}{l}0.002 \\
(0.042)\end{array}$ & $\begin{array}{l}0.028 \\
(0.383)\end{array}$ & $\begin{array}{l}0.079 \\
(0.316)\end{array}$ & $\begin{array}{l}0.003 \\
(0.056)\end{array}$ \\
\hline $\begin{array}{l}\text { Geo exp - intl } \\
\text { recruitment }(\mathrm{H} 8 \mathrm{a})\end{array}$ & $\begin{array}{l}-0.237 \\
(0.276)\end{array}$ & $\begin{array}{l}-0.239 \\
(0.257)\end{array}$ & $\begin{array}{l}-0.015 \\
(0.017)\end{array}$ & $\begin{array}{l}0.073 \\
(0.616)\end{array}$ & $\begin{array}{l}0.146 \\
(0.521)\end{array}$ & $\begin{array}{l}0.008 \\
(0.044)\end{array}$ & $\begin{array}{l}-0.685 \\
(0.489)\end{array}$ & $\begin{array}{l}-0.660 \\
(0.441)\end{array}$ & $\begin{array}{l}-0.039 \\
(0.040)\end{array}$ & $\begin{array}{l}0.126 \\
(0.393)\end{array}$ & $\begin{array}{l}0.169 \\
(0.337)\end{array}$ & $\begin{array}{l}0.010 \\
(0.065)\end{array}$ \\
\hline Age & 0.008 & 0.0006 & 0.0003 & 0.014 & 0.009 & 0.0005 & 0.008 & 0.008 & 0.0006 & 0.004 & 0.002 & 0.0001 \\
\hline
\end{tabular}




\begin{tabular}{|c|c|c|c|c|c|c|c|c|c|c|c|c|}
\hline & $(0.006)$ & $(0.005)$ & $(0.0004)$ & $(0.021)$ & $(0.016)$ & $(0.0018)$ & $(0.011)$ & (0.009) & $(0.0007)$ & $(0.008)$ & $(0.006)$ & $(0.001)$ \\
\hline Size (Staff) & $\begin{array}{l}0.000 \\
(0.000)\end{array}$ & $\begin{array}{l}0.0001 \\
(0.0002)\end{array}$ & $\begin{array}{l}0.892 \mathrm{E}-5 \\
(0.193 \mathrm{E}-4)\end{array}$ & $\begin{array}{l}0.000 \\
(0.001)\end{array}$ & $\begin{array}{l}-0.0004 \\
(0.0004)\end{array}$ & $\begin{array}{l}-0.261 \mathrm{E}-4 \\
(0.385 \mathrm{E}-4)\end{array}$ & $\begin{array}{l}0.001 \\
(0.000)\end{array}$ & $\begin{array}{l}0.0004 \\
(0.0004)\end{array}$ & $\begin{array}{l}0.308 \mathrm{E}-4 \\
(0.474 \mathrm{E}-4)\end{array}$ & $\begin{array}{l}-2.861 \mathrm{E}-5 \\
(0.000)\end{array}$ & $\begin{array}{l}0.0001 \\
(0.0002)\end{array}$ & $\begin{array}{l}0.738 \mathrm{E}-5 \\
(0.499 \mathrm{E}-4)\end{array}$ \\
\hline Offshore policy & $\begin{array}{l}\text { 8.087** } \\
(1.174)\end{array}$ & $\begin{array}{l}\text { 8.178** } \\
(1.111)\end{array}$ & $\begin{array}{l}0.704 * * \\
(0.061)\end{array}$ & $\begin{array}{l}6.300^{*} \\
(2.744)\end{array}$ & $\begin{array}{l}6.063 * * \\
(2.311)\end{array}$ & $\begin{array}{l}0.460 * * \\
(0.160)\end{array}$ & $\begin{array}{l}8.917 * * \\
(2.109)\end{array}$ & $\begin{array}{l}\text { 8.875** } \\
(1.913)\end{array}$ & $\begin{array}{l}0.990 * * \\
(0.151)\end{array}$ & $\begin{array}{l}\text { 6.591** } \\
(1.905)\end{array}$ & $\begin{array}{l}\text { 6.532** } \\
(1.667)\end{array}$ & $\begin{array}{l}0.457 * * \\
(0.143)\end{array}$ \\
\hline $\begin{array}{l}\text { Internationalisation } \\
\text { at home strategy }\end{array}$ & $\begin{array}{l}0.151 \\
(0.245)\end{array}$ & $\begin{array}{l}-0.0006 \\
(0.0012)\end{array}$ & $\begin{array}{l}-0.302 \mathrm{E}-4 \\
(0.0001)\end{array}$ & $\begin{array}{l}0.352 \\
(0.677)\end{array}$ & $\begin{array}{l}-0.002 \\
(0.002)\end{array}$ & $\begin{array}{l}-0.0001 \\
(0.0003)\end{array}$ & $\begin{array}{l}-0.197 \\
(0.388)\end{array}$ & $\begin{array}{l}-0.006 \\
(0.004)\end{array}$ & $\begin{array}{l}-0.0003 \\
(0.025)\end{array}$ & $\begin{array}{l}0.438 \\
(0.401)\end{array}$ & $\begin{array}{l}0.001 \\
(0.001)\end{array}$ & $\begin{array}{l}0.814 \mathrm{E}-4 \\
(0.0002)\end{array}$ \\
\hline $\begin{array}{l}\text { Host country } \\
\text { demand }\end{array}$ & $\begin{array}{l}8.729 * \\
(3.402)\end{array}$ & $\begin{array}{l}\text { 7.994* } \\
\text { (3.119) }\end{array}$ & $\begin{array}{l}0.499 * \\
(0.251)\end{array}$ & $\begin{array}{l}9.697 \\
(7.179)\end{array}$ & $\begin{array}{l}9.072 \\
(5.783)\end{array}$ & $\begin{array}{l}0.530 \\
(0.552)\end{array}$ & $\begin{array}{l}\text { 12.932* } \\
(6.354)\end{array}$ & $\begin{array}{l}\text { 12.937* } \\
(5.769)\end{array}$ & $\begin{array}{l}0.824 \dagger \\
(0.470)\end{array}$ & $\begin{array}{l}9.427 \\
(6.447)\end{array}$ & $\begin{array}{l}5.800 \\
(4.709)\end{array}$ & $\begin{array}{l}0.322 \\
(0.702)\end{array}$ \\
\hline AU/NZ dummy & $\begin{array}{l}1.382 \\
(0.920)\end{array}$ & $\begin{array}{l}1.285 \\
(0.848)\end{array}$ & $\begin{array}{l}0.086 \\
(0.067)\end{array}$ & - & - & - & - & - & - & - & - & - \\
\hline UK/IR dummy & $\begin{array}{l}2.152 * * \\
(0.804)\end{array}$ & $\begin{array}{l}1.833^{*} \\
(0.741)\end{array}$ & $\begin{array}{l}0.114 \dagger \\
(0.059)\end{array}$ & - & - & - & - & - & - & - & - & - \\
\hline $\mathrm{R}^{2}$ & 0.343 & & & 0.271 & & & 0.456 & & & 0.317 & & \\
\hline $\operatorname{AdjR}^{2}$ & 0.289 & & & 0.050 & & & 0.357 & & & 0.133 & & \\
\hline Log likelihood & & -467.20 & -498.53 & & -132.77 & -142.81 & & -189.07 & -202.56 & & -130.02 & -141.15 \\
\hline $\mathrm{N}$ & 169 & 169 & 169 & 48 & 48 & 48 & 67 & 67 & 67 & 54 & 54 & 54 \\
\hline Max. VIF & 1.621 & & & 1.451 & & & 1.388 & & & 1.666 & & \\
\hline
\end{tabular}

Standard errors in parentheses, all $t$ tests are two-tailed, $\dagger p<.10,{ }^{*} p<.05,{ }^{* *} p<.01$

Table 7.37b: Regression estimates for equation 22b (high regulative institutional distance)

\begin{tabular}{|c|c|c|c|c|c|c|c|c|c|c|c|c|}
\hline & \multicolumn{3}{|c|}{ Complete data set } & \multicolumn{3}{|c|}{ AU/NZ } & \multicolumn{3}{|c|}{$\overline{\text { US/CA }}$} & \multicolumn{3}{|c|}{ 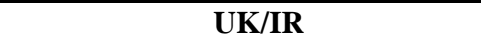 } \\
\hline & OLS & Tobit & $\begin{array}{l}\text { Negative } \\
\text { binomial } \\
\end{array}$ & OLS & Tobit & $\begin{array}{l}\text { Negative } \\
\text { binomial } \\
\end{array}$ & OLS & Tobit & $\begin{array}{l}\text { Negative } \\
\text { binomial }\end{array}$ & OLS & Tobit & $\begin{array}{l}\text { Negative } \\
\text { binomial }\end{array}$ \\
\hline Intercept & $\begin{array}{l}17.437 * * \\
(3.600)\end{array}$ & $\begin{array}{l}17.723 * * \\
(3.287)\end{array}$ & $\begin{array}{l}2.873 * * \\
(0.414)\end{array}$ & $\begin{array}{l}\begin{array}{l}11.921 \dagger \\
(5.901)\end{array}\end{array}$ & $\begin{array}{l}\text { 11.921* } \\
\text { (4.779) }\end{array}$ & $\begin{array}{l}2.566 \\
(2.003)\end{array}$ & $\begin{array}{l}23.024 * * \\
(7.018)\end{array}$ & $\begin{array}{l}20.737 * * \\
(6.011)\end{array}$ & $\begin{array}{l}3.060 * * \\
(0.922)\end{array}$ & $\begin{array}{l}21.014 * * \\
(5.837)\end{array}$ & $\begin{array}{l}19.799 * * \\
(4.950)\end{array}$ & $\begin{array}{l}2.989 * \\
(1.244)\end{array}$ \\
\hline $\begin{array}{l}\text { Geo exp - knowledge } \\
\text { of edu sector }(H 8 a)\end{array}$ & $\begin{array}{l}-0.219 \\
(0.180)\end{array}$ & $\begin{array}{l}-0.251 \\
(0.165)\end{array}$ & $\begin{array}{l}-0.015 \\
(0.021)\end{array}$ & $\begin{array}{l}0.033 \\
(0.348)\end{array}$ & $\begin{array}{l}0.032 \\
(0.281)\end{array}$ & $\begin{array}{l}0.002 \\
(0.124)\end{array}$ & $\begin{array}{l}-0.400 \\
(0.298)\end{array}$ & $\begin{array}{l}-0.394 \\
(0.268)\end{array}$ & $\begin{array}{l}-0.024 \\
(0.036)\end{array}$ & $\begin{array}{l}-0.312 \\
(0.328)\end{array}$ & $\begin{array}{l}-0.340 \\
(0.268)\end{array}$ & $\begin{array}{l}-0.020 \\
(0.062)\end{array}$ \\
\hline $\begin{array}{l}\text { Geo exp - academic } \\
\text { capabilities }(H 8 a)\end{array}$ & $\begin{array}{l}0.294 \\
(0.181)\end{array}$ & $\begin{array}{l}\mathbf{0 . 3 2 7} \dagger \\
(\mathbf{0 . 1 6 8 )}\end{array}$ & $\begin{array}{l}0.019 \\
(0.020)\end{array}$ & $\begin{array}{l}0.676^{*} \\
(0.296)\end{array}$ & $\begin{array}{l}0.675 * * \\
(0.239)\end{array}$ & $\begin{array}{l}0.038 \\
(0.091)\end{array}$ & $\begin{array}{l}0.373 \\
(0.324)\end{array}$ & $\begin{array}{l}0.328 \\
(0.289)\end{array}$ & $\begin{array}{l}0.020 \\
(0.036)\end{array}$ & $\begin{array}{l}0.114 \\
(0.290)\end{array}$ & $\begin{array}{l}0.224 \\
(0.246)\end{array}$ & $\begin{array}{l}0.014 \\
(0.072)\end{array}$ \\
\hline $\begin{array}{l}\text { Geo exp - managerial } \\
\text { capabilities (H8a) }\end{array}$ & $\begin{array}{l}-0.002 \\
(0.230)\end{array}$ & $\begin{array}{l}-0.017 \\
(0.216)\end{array}$ & $\begin{array}{l}-0.001 \\
(0.028)\end{array}$ & $\begin{array}{l}-0.557 \\
(0.368)\end{array}$ & $\begin{array}{l}-0.557 \dagger \\
(0.298)\end{array}$ & $\begin{array}{l}-0.033 \\
(0.116)\end{array}$ & $\begin{array}{l}0.765 \\
(0.456)\end{array}$ & $\begin{array}{l}0.658 \\
(0.400)\end{array}$ & $\begin{array}{l}0.040 \\
(0.052)\end{array}$ & $\begin{array}{l}-0.264 \\
(0.313)\end{array}$ & $\begin{array}{l}-0.318 \\
(0.267)\end{array}$ & $\begin{array}{l}-0.019 \\
(0.077)\end{array}$ \\
\hline
\end{tabular}




\begin{tabular}{|c|c|c|c|c|c|c|c|c|c|c|c|c|}
\hline $\begin{array}{l}\text { Geo exp - financial } \\
\text { capabilities (H8a) }\end{array}$ & $\begin{array}{l}-0.246 \\
(0.210)\end{array}$ & $\begin{array}{l}-0.206 \\
(0.195)\end{array}$ & $\begin{array}{l}-0.012 \\
(0.025)\end{array}$ & $\begin{array}{l}0.151 \\
(0.394)\end{array}$ & $\begin{array}{l}0.151 \\
(0.319)\end{array}$ & $\begin{array}{l}0.006 \\
(0.098)\end{array}$ & $\begin{array}{l}-0.284 \\
(0.373)\end{array}$ & $\begin{array}{l}-0.270 \\
(0.335)\end{array}$ & $\begin{array}{l}-0.017 \\
(0.048)\end{array}$ & $\begin{array}{l}-0.786 * \\
(0.319)\end{array}$ & $\begin{array}{l}-0.654 * \\
(0.273)\end{array}$ & $\begin{array}{l}-0.039 \\
(0.063)\end{array}$ \\
\hline $\begin{array}{l}\text { Geo exp - intl } \\
\text { recruitment (H8a) }\end{array}$ & $\begin{array}{l}0.078 \\
(0.233)\end{array}$ & $\begin{array}{l}0.072 \\
(0.217)\end{array}$ & $\begin{array}{l}0.004 \\
(0.027)\end{array}$ & $\begin{array}{l}0.196 \\
(0.376)\end{array}$ & $\begin{array}{l}0.196 \\
(0.304)\end{array}$ & $\begin{array}{l}0.011 \\
(0.127)\end{array}$ & $\begin{array}{l}0.063 \\
(0.453)\end{array}$ & $\begin{array}{l}0.083 \\
(0.407)\end{array}$ & $\begin{array}{l}0.004 \\
(0.048)\end{array}$ & $\begin{array}{l}0.169 \\
(0.395)\end{array}$ & $\begin{array}{l}0.177 \\
(0.341)\end{array}$ & $\begin{array}{l}0.011 \\
(0.067)\end{array}$ \\
\hline Age & $\begin{array}{l}-0.003 \\
(0.005)\end{array}$ & $\begin{array}{l}-0.003 \\
(0.004)\end{array}$ & $\begin{array}{l}-0.0001 \\
(0.0005)\end{array}$ & $\begin{array}{l}-0.010 \\
(0.011)\end{array}$ & $\begin{array}{l}-0.010 \\
(0.009)\end{array}$ & $\begin{array}{l}-0.0006 \\
(0.003)\end{array}$ & $\begin{array}{l}-0.008 \\
(0.009)\end{array}$ & $\begin{array}{l}-0.008 \\
(0.008)\end{array}$ & $\begin{array}{l}-0.0005 \\
(0.0009)\end{array}$ & $\begin{array}{l}0.005 \\
(0.007)\end{array}$ & $\begin{array}{l}0.005 \\
(0.005)\end{array}$ & $\begin{array}{l}0.0003 \\
(0.001)\end{array}$ \\
\hline Size (Staff) & $\begin{array}{l}0.000 \\
(0.000)\end{array}$ & $\begin{array}{l}0.0001 \\
(0.0002)\end{array}$ & $\begin{array}{l}0.107 \mathrm{E}-4 \\
(0.298 \mathrm{E}-4)\end{array}$ & $\begin{array}{l}0.002 * \\
(0.001)\end{array}$ & $\begin{array}{l}\text { 0.001** } \\
(0.0004)\end{array}$ & $\begin{array}{l}0.908 \mathrm{E}-4 \\
(0.0001)\end{array}$ & $\begin{array}{l}-0.000 \\
(0.000)\end{array}$ & $\begin{array}{l}-0.157 \mathrm{E}-4 \\
(0.0003)\end{array}$ & $\begin{array}{l}-0.175 E-5 \\
(0.402 E-4)\end{array}$ & $\begin{array}{l}0.000 \\
(0.000)\end{array}$ & $\begin{array}{l}0.0001 \\
(0.0002)\end{array}$ & $\begin{array}{l}0.908 E-5 \\
(0.898 E-4)\end{array}$ \\
\hline Offshore policy & $\begin{array}{l}-0.965 \\
(1.082)\end{array}$ & $\begin{array}{l}-1.030 \\
(1.016)\end{array}$ & $\begin{array}{l}-0.060 \\
(0.115)\end{array}$ & $\begin{array}{l}-0.662 \\
(1.847)\end{array}$ & $\begin{array}{l}-0.661 \\
(1.496)\end{array}$ & $\begin{array}{l}-0.037 \\
(0.367)\end{array}$ & $\begin{array}{l}-4.061 \dagger \\
(2.206)\end{array}$ & $\begin{array}{l}-3.004 \dagger \\
(1.772)\end{array}$ & $\begin{array}{l}-0.180 \\
(0.174)\end{array}$ & $\begin{array}{l}2.116 \\
(1.643)\end{array}$ & $\begin{array}{l}1.789 \\
(1.408)\end{array}$ & $\begin{array}{l}0.109 \\
(0.774)\end{array}$ \\
\hline $\begin{array}{l}\text { Internationalisation at } \\
\text { home strategy }\end{array}$ & $\begin{array}{l}0.121 \\
(0.200)\end{array}$ & $\begin{array}{l}-0.001 \\
(0.001)\end{array}$ & $\begin{array}{l}-0.668 \mathrm{E}-4 \\
(0.0003)\end{array}$ & $\begin{array}{l}0.039 \\
(0.324)\end{array}$ & $\begin{array}{l}0.039 \\
(0.262)\end{array}$ & $\begin{array}{l}0.001 \\
(0.124)\end{array}$ & $\begin{array}{l}-0.468 \\
(0.437)\end{array}$ & $\begin{array}{l}-0.002 \\
(0.003)\end{array}$ & $\begin{array}{l}-0.0001 \\
(0.027)\end{array}$ & $\begin{array}{l}-0.063 \\
(0.299)\end{array}$ & $\begin{array}{l}-0.001 \\
(0.001)\end{array}$ & $\begin{array}{l}-0.922 \mathrm{E}-4 \\
(0.0003)\end{array}$ \\
\hline Host country demand & $\begin{array}{l}2.912 \\
(6.586)\end{array}$ & $\begin{array}{l}3.793 \\
(6.117)\end{array}$ & $\begin{array}{l}0.222 \\
(0.914)\end{array}$ & $\begin{array}{l}5.449 \\
(5.453)\end{array}$ & $\begin{array}{l}5.449 \\
(4.417)\end{array}$ & $\begin{array}{l}0.307 \\
(1.869)\end{array}$ & $\begin{array}{l}-279.990 * \\
(113.298)\end{array}$ & $\begin{array}{l}-244.50 * \\
(97.43)\end{array}$ & $\begin{array}{l}-14.879 \\
(11.516)\end{array}$ & $\begin{array}{l}43.215 \\
(82.872)\end{array}$ & $\begin{array}{l}53.246 \\
(72.780)\end{array}$ & $\begin{array}{l}3.146 \\
(19.327)\end{array}$ \\
\hline AU/NZ dummy & $\begin{array}{l}1.046 \\
(0.866)\end{array}$ & $\begin{array}{l}0.904 \\
(0.789)\end{array}$ & $\begin{array}{l}0.053 \\
(0.110)\end{array}$ & (J.To) & 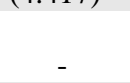 & (1.000) & 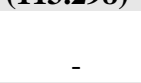 & (5) (ס) & (ע) & $(02.072)$ & $(1,2.70)$ & (10.021) \\
\hline UK/IR dummy & $\begin{array}{l}0.262 \\
(0.683)\end{array}$ & $\begin{array}{l}0.253 \\
(0.636)\end{array}$ & $\begin{array}{l}0.016 \\
(0.088)\end{array}$ & - & - & - & - & - & - & - & - & - \\
\hline $\mathrm{R}^{2}$ & 0.096 & & & 0.394 & & & 0.266 & & & 0.226 & & \\
\hline $\operatorname{Adj}^{2}$ & 0.007 & & & 0.105 & & & 0.103 & & & 0.011 & & \\
\hline Log likelihood & & -345.78 & -362.76 & & -65.87 & -78.61 & & -147.33 & -153.33 & & -109.68 & -123.54 \\
\hline $\mathrm{N}$ & 139 & 139 & 139 & 32 & 32 & 32 & 57 & 57 & 57 & 50 & 50 & 50 \\
\hline Max. VIF & 1.919 & & & 1.806 & & & 1.465 & & & 1.811 & & \\
\hline
\end{tabular}

Table 7.38a: Regression estimates for equation 23a (low regulative institutional distance)

\begin{tabular}{|c|c|c|c|c|c|c|c|c|c|c|c|c|}
\hline & \multicolumn{3}{|c|}{ Complete data set } & \multicolumn{3}{|c|}{ AU/NZ } & \multicolumn{3}{|c|}{$\overline{\text { US/CA }}$} & \multicolumn{3}{|c|}{ 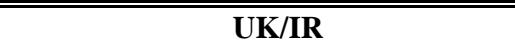 } \\
\hline & OLS & Tobit & $\begin{array}{l}\text { Negative } \\
\text { binomial }\end{array}$ & OLS & Tobit & $\begin{array}{l}\text { Negative } \\
\text { binomial }\end{array}$ & OLS & Tobit & $\begin{array}{l}\text { Negative } \\
\text { binomial }\end{array}$ & OLS & Tobit & $\begin{array}{l}\text { Negative } \\
\text { binomial }\end{array}$ \\
\hline Intercept & $\begin{array}{l}3.517 \\
(3.090)\end{array}$ & $\begin{array}{l}4.950 \dagger \\
(2.751)\end{array}$ & $\begin{array}{l}1.892^{* *} \\
(0.172)\end{array}$ & $\begin{array}{l}11.211 \\
(7.479)\end{array}$ & $\begin{array}{l}\text { 13.994* } \\
(5.620)\end{array}$ & $\begin{array}{l}2.548 * * \\
(0.468)\end{array}$ & $\begin{array}{l}-1.697 \\
(5.478)\end{array}$ & $\begin{array}{l}-2.641 \\
(4.759)\end{array}$ & $\begin{array}{l}1.134 * * \\
(0.346)\end{array}$ & $\begin{array}{l}6.713 \dagger \\
(3.945)\end{array}$ & $\begin{array}{l}7.499 * \\
(3.407)\end{array}$ & $\begin{array}{l}2.204 * * \\
(0.531)\end{array}$ \\
\hline $\begin{array}{l}\text { Ind exp - UG } \\
\text { offshore edu (H8b) }\end{array}$ & $\begin{array}{l}0.038 \\
(0.198)\end{array}$ & $\begin{array}{l}0.007 \\
(0.183)\end{array}$ & $\begin{array}{l}0.001 \\
(0.014)\end{array}$ & $\begin{array}{l}0.018 \\
(0.514)\end{array}$ & $\begin{array}{l}-0.147 \\
(0.416)\end{array}$ & $\begin{array}{l}-0.008 \\
(0.038)\end{array}$ & $\begin{array}{l}-0.136 \\
(0.342)\end{array}$ & $\begin{array}{l}-0.103 \\
(0.307)\end{array}$ & $\begin{array}{l}-0.006 \\
(0.033)\end{array}$ & $\begin{array}{l}0.066 \\
(0.284)\end{array}$ & $\begin{array}{l}0.078 \\
(0.237)\end{array}$ & $\begin{array}{l}0.004 \\
(0.046)\end{array}$ \\
\hline $\begin{array}{l}\text { Ind exp - PG } \\
\text { offshore edu (H8b) }\end{array}$ & $\begin{array}{l}0.046 \\
(0.242)\end{array}$ & $\begin{array}{l}0.026 \\
(0.225)\end{array}$ & $\begin{array}{l}0.002 \\
(0.017)\end{array}$ & $\begin{array}{l}0.031 \\
(0.604)\end{array}$ & $\begin{array}{l}0.045 \\
(0.491)\end{array}$ & $\begin{array}{l}0.004 \\
(0.052)\end{array}$ & $\begin{array}{l}0.141 \\
(0.417)\end{array}$ & $\begin{array}{l}0.167 \\
(0.376)\end{array}$ & $\begin{array}{l}0.006 \\
(0.030)\end{array}$ & $\begin{array}{l}-0.143 \\
(0.349)\end{array}$ & $\begin{array}{l}-0.225 \\
(0.297)\end{array}$ & $\begin{array}{l}-0.012 \\
(0.070)\end{array}$ \\
\hline
\end{tabular}




\begin{tabular}{|c|c|c|c|c|c|c|c|c|c|c|c|c|}
\hline $\begin{array}{l}\text { Ind exp - intl } \\
\text { recruitment (Factor) } \\
(H 8 b)\end{array}$ & $\begin{array}{l}0.278 \\
(0.274)\end{array}$ & $\begin{array}{l}0.256 \\
(0.252)\end{array}$ & $\begin{array}{l}0.013 \\
(0.021)\end{array}$ & $\begin{array}{l}0.350 \\
(0.727)\end{array}$ & $\begin{array}{l}0.060 \\
(0.553)\end{array}$ & $\begin{array}{l}0.003 \\
(0.051)\end{array}$ & $\begin{array}{l}0.942 \dagger \\
(0.474)\end{array}$ & $\begin{array}{l}0.926 * \\
(0.430)\end{array}$ & $\begin{array}{l}0.061 \\
(0.041)\end{array}$ & $\begin{array}{l}-0.007 \\
(0.387)\end{array}$ & $\begin{array}{l}0.150 \\
(0.325)\end{array}$ & $\begin{array}{l}0.008 \\
(0.066)\end{array}$ \\
\hline $\begin{array}{l}\text { Ind exp - UG } \\
\text { marketing (H8b) }\end{array}$ & $\begin{array}{l}-0.195 \\
(0.225)\end{array}$ & $\begin{array}{l}-0.226 \\
(0.204)\end{array}$ & $\begin{array}{l}-0.014 \\
(0.018)\end{array}$ & $\begin{array}{l}-0.862 \\
(0.647)\end{array}$ & $\begin{array}{l}-0.697 \\
(0.528)\end{array}$ & $\begin{array}{l}-0.041 \\
(0.047)\end{array}$ & $\begin{array}{l}-0.052 \\
(0.356)\end{array}$ & $\begin{array}{l}-0.057 \\
(0.323)\end{array}$ & $\begin{array}{l}-0.003 \\
(0.032)\end{array}$ & $\begin{array}{l}0.166 \\
(0.313)\end{array}$ & $\begin{array}{l}0.139 \\
(0.248)\end{array}$ & $\begin{array}{l}0.008 \\
(0.047)\end{array}$ \\
\hline $\begin{array}{l}\text { Ind exp - PG } \\
\text { marketing (H8b) }\end{array}$ & $\begin{array}{l}0.276 \\
(0.204)\end{array}$ & $\begin{array}{l}0.198 \\
(0.189)\end{array}$ & $\begin{array}{l}0.012 \\
(0.014)\end{array}$ & $\begin{array}{l}0.327 \\
(0.513)\end{array}$ & $\begin{array}{l}0.319 \\
(0.432)\end{array}$ & $\begin{array}{l}0.018 \\
(0.041)\end{array}$ & $\begin{array}{l}-0.196 \\
(0.352)\end{array}$ & $\begin{array}{l}-0.171 \\
(0.317)\end{array}$ & $\begin{array}{l}-0.011 \\
(0.024)\end{array}$ & $\begin{array}{l}0.533 \dagger \\
(0.297)\end{array}$ & $\begin{array}{l}0.472 \dagger \\
(0.241)\end{array}$ & $\begin{array}{l}0.026 \\
(0.045)\end{array}$ \\
\hline Age & $\begin{array}{l}0.006 \\
(0.006)\end{array}$ & $\begin{array}{l}0.004 \\
(0.005)\end{array}$ & $\begin{array}{l}0.0002 \\
(0.0004)\end{array}$ & $\begin{array}{l}0.001 \\
(0.019)\end{array}$ & $\begin{array}{l}0.002 \\
(0.015)\end{array}$ & $\begin{array}{l}0.0001 \\
(0.001)\end{array}$ & $\begin{array}{l}0.005 \\
(0.011)\end{array}$ & $\begin{array}{l}0.006 \\
(0.010)\end{array}$ & $\begin{array}{l}0.0004 \\
(0.0008)\end{array}$ & $\begin{array}{l}0.004 \\
(0.008)\end{array}$ & $\begin{array}{l}0.001 \\
(0.006)\end{array}$ & $\begin{array}{l}0.0001 \\
(0.0009)\end{array}$ \\
\hline Size (Staff) & $\begin{array}{l}0.000 \\
(0.000)\end{array}$ & $\begin{array}{l}0.0001 \\
(0.0002)\end{array}$ & $\begin{array}{l}0.786 \mathrm{E}-5 \\
(0.194 \mathrm{E}-4)\end{array}$ & $\begin{array}{l}0.000 \\
(0.001)\end{array}$ & $\begin{array}{l}-0.0004 \\
(0.0004)\end{array}$ & $\begin{array}{l}-0.248 \mathrm{E}-4 \\
(0.358 \mathrm{E}-4)\end{array}$ & $\begin{array}{l}0.001 \\
(0.000)\end{array}$ & $\begin{array}{l}0.0005 \\
(0.0004)\end{array}$ & $\begin{array}{l}0.384 E-4 \\
(0.393 E-4)\end{array}$ & $\begin{array}{l}2.170 \mathrm{E}-5 \\
(0.000)\end{array}$ & $\begin{array}{l}0.650 \mathrm{E}-4 \\
(0.0002)\end{array}$ & $\begin{array}{l}0.453 \mathrm{E}-5 \\
(0.566 \mathrm{E}-4)\end{array}$ \\
\hline Offshore policy & $\begin{array}{l}\text { 8.015** } \\
(1.170)\end{array}$ & $\begin{array}{l}\text { 8.086** } \\
(1.108)\end{array}$ & $\begin{array}{l}0.700 * * \\
(0.058)\end{array}$ & $\begin{array}{l}\text { 6.571* } \\
(2.844)\end{array}$ & $\begin{array}{l}6.630 * * \\
(2.437)\end{array}$ & $\begin{array}{l}0.496 * \\
(0.250)\end{array}$ & $\begin{array}{l}9.438 * * \\
(1.919)\end{array}$ & $\begin{array}{l}9.362 * * \\
(1.740)\end{array}$ & $\begin{array}{l}1.016 * * \\
(0.126)\end{array}$ & $\begin{array}{l}\text { 6.857** } \\
(2.070)\end{array}$ & $\begin{array}{l}\text { 6.618** } \\
(1.797)\end{array}$ & $\begin{array}{l}0.459 * \\
(0.229)\end{array}$ \\
\hline $\begin{array}{l}\text { Internationalisation } \\
\text { at home strategy }\end{array}$ & $\begin{array}{l}0.127 \\
(0.245)\end{array}$ & $\begin{array}{l}-0.0001 \\
(0.001)\end{array}$ & $\begin{array}{l}-0.294 \mathrm{E}-5 \\
(0.0001)\end{array}$ & $\begin{array}{l}0.386 \\
(0.736)\end{array}$ & $\begin{array}{l}-0.001 \\
(0.002)\end{array}$ & $\begin{array}{l}-0.0001 \\
(0.0003)\end{array}$ & $\begin{array}{l}-0.233 \\
(0.387)\end{array}$ & $\begin{array}{l}-0.006 \\
(0.004)\end{array}$ & $\begin{array}{l}-0.0003 \\
(0.023)\end{array}$ & $\begin{array}{l}0.252 \\
(0.404)\end{array}$ & $\begin{array}{l}0.001 \\
(0.001)\end{array}$ & $\begin{array}{l}0.970 \mathrm{E}-4 \\
(0.0002)\end{array}$ \\
\hline $\begin{array}{l}\text { Host country } \\
\text { demand }\end{array}$ & $\begin{array}{l}\text { 8.138* } \\
(3.418)\end{array}$ & $\begin{array}{l}\text { 7.651* } \\
\text { (3.112) }\end{array}$ & $\begin{array}{l}0.481 \dagger \\
(0.263)\end{array}$ & $\begin{array}{l}5.141 \\
(7.217)\end{array}$ & $\begin{array}{l}7.481 \\
(5.875)\end{array}$ & $\begin{array}{l}0.433 \\
(0.622)\end{array}$ & $\begin{array}{l}\text { 13.363* } \\
\text { (5.998) }\end{array}$ & $\begin{array}{l}\text { 13.371* } \\
\text { (5.450) }\end{array}$ & $\begin{array}{l}0.906 \dagger \\
(0.477)\end{array}$ & $\begin{array}{l}4.483 \\
(6.577)\end{array}$ & $\begin{array}{l}1.973 \\
(4.487)\end{array}$ & $\begin{array}{l}0.098 \\
(0.841)\end{array}$ \\
\hline AU/NZ dummy & $\begin{array}{l}1.224 \\
(0.921)\end{array}$ & $\begin{array}{l}1.257 \\
(0.844)\end{array}$ & $\begin{array}{l}0.084 \\
(0.067)\end{array}$ & - & - & - & - & - & - & - & - & - \\
\hline UK/IR dummy & $\begin{array}{l}\text { 2.218** } \\
(0.797)\end{array}$ & $\begin{array}{l}\text { 1.871* } \\
(0.735)\end{array}$ & $\begin{array}{l}\mathbf{0 . 1 1 7} \dagger \\
\mathbf{( 0 . 0 6 0 )}\end{array}$ & - & - & - & - & - & - & - & - & - \\
\hline $\mathrm{R}^{2}$ & 0.351 & & & 0.256 & & & 0.466 & & & 0.352 & & \\
\hline $\operatorname{Adj}^{2}$ & 0.297 & & & 0.030 & & & 0.369 & & & 0.177 & & \\
\hline Log likelihood & & -466.59 & -498.01 & & -133.58 & -143.50 & & -188.51 & -201.76 & & -128.48 & -140.56 \\
\hline $\mathrm{N}$ & 169 & 169 & 169 & 48 & 48 & 48 & 67 & 67 & 67 & 54 & 54 & 54 \\
\hline Max. VIF & 1.643 & & & 1.797 & & & 1.225 & & & 1.777 & & \\
\hline
\end{tabular}

Standard errors in parentheses, all $t$ tests are two-tailed, $\dagger p<.10,{ }^{*} p<.05,{ }^{* *} p<.01$

Table 7.38b: Regression estimates for equation 23b (high regulative institutional distance)

\begin{tabular}{|c|c|c|c|c|c|c|c|c|c|c|c|c|}
\hline & \multicolumn{3}{|c|}{ Complete data set } & \multicolumn{3}{|c|}{ AU/NZ } & \multicolumn{3}{|c|}{$\overline{\mathrm{US} / \mathrm{CA}}$} & \multicolumn{3}{|c|}{$\overline{\mathrm{UK} / \mathrm{IR}}$} \\
\hline & OLS & Tobit & $\begin{array}{l}\text { Negative } \\
\text { binomial } \\
\end{array}$ & OLS & Tobit & $\begin{array}{l}\text { Negative } \\
\text { binomial } \\
\end{array}$ & OLS & Tobit & $\begin{array}{l}\text { Negative } \\
\text { binomial } \\
\end{array}$ & OLS & Tobit & $\begin{array}{l}\text { Negative } \\
\text { binomial }\end{array}$ \\
\hline Intercept & $\begin{array}{l}13.620^{* *} \\
(2.807)\end{array}$ & $\begin{array}{l}14.756^{* *} \\
(2.550)\end{array}$ & $\begin{array}{l}\text { 2.697** } \\
(0.322)\end{array}$ & $\begin{array}{l}\text { 12.546* } \\
(5.859)\end{array}$ & $\begin{array}{l}12.545^{* *} \\
(4.746)\end{array}$ & $\begin{array}{l}2.581 \\
(2.303)\end{array}$ & $\begin{array}{l}\text { 23.181** } \\
(7.426)\end{array}$ & $\begin{array}{l}22.060 * * \\
(5.712)\end{array}$ & $\begin{array}{l}3.140 * * \\
(0.613)\end{array}$ & $\begin{array}{l}12.468 * * \\
(3.788)\end{array}$ & $\begin{array}{l}\text { 13.902** } \\
(3.186)\end{array}$ & $\begin{array}{l}2.643^{* *} \\
(0.809)\end{array}$ \\
\hline
\end{tabular}




\begin{tabular}{|c|c|c|c|c|c|c|c|c|c|c|c|c|}
\hline $\begin{array}{l}\text { Ind exp - UG } \\
\text { offshore edu (H8b) }\end{array}$ & $\begin{array}{l}-0.111 \\
(0.188)\end{array}$ & $\begin{array}{l}-0.138 \\
(0.176)\end{array}$ & $\begin{array}{l}-0.008 \\
(0.022)\end{array}$ & $\begin{array}{l}-0.470 \\
(0.329)\end{array}$ & $\begin{array}{l}-0.470 \dagger \\
(0.266)\end{array}$ & $\begin{array}{l}-0.027 \\
(0.117)\end{array}$ & $\begin{array}{l}-0.214 \\
(0.443)\end{array}$ & $\begin{array}{l}-0.215 \\
(0.394)\end{array}$ & $\begin{array}{l}-0.013 \\
(0.037)\end{array}$ & $\begin{array}{l}0.077 \\
(0.249)\end{array}$ & $\begin{array}{l}0.031 \\
(0.215)\end{array}$ & $\begin{array}{l}0.001 \\
(0.041)\end{array}$ \\
\hline $\begin{array}{l}\text { Ind exp - PG } \\
\text { offshore edu (H8b) }\end{array}$ & $\begin{array}{l}0.029 \\
(0.204)\end{array}$ & $\begin{array}{l}0.065 \\
(0.189)\end{array}$ & $\begin{array}{l}0.003 \\
(0.024)\end{array}$ & $\begin{array}{l}0.248 \\
(0.329)\end{array}$ & $\begin{array}{l}0.247 \\
(0.266)\end{array}$ & $\begin{array}{l}0.014 \\
(0.095)\end{array}$ & $\begin{array}{l}-0.456 \\
(0.397)\end{array}$ & $\begin{array}{l}-0.457 \\
(0.353)\end{array}$ & $\begin{array}{l}-0.029 \\
(0.029)\end{array}$ & $\begin{array}{l}-0.096 \\
(0.342)\end{array}$ & $\begin{array}{l}-0.094 \\
(0.294)\end{array}$ & $\begin{array}{l}-0.005 \\
(0.064)\end{array}$ \\
\hline $\begin{array}{l}\text { Ind exp - intl } \\
\text { recruitment (Factor) } \\
(\mathrm{H} 8 b)\end{array}$ & $\begin{array}{l}0.336 \\
(0.245)\end{array}$ & $\begin{array}{l}0.254 \\
(0.218)\end{array}$ & $\begin{array}{l}0.015 \\
(0.031)\end{array}$ & $\begin{array}{l}0.622 \\
(0.472)\end{array}$ & $\begin{array}{l}0.621 \\
(0.382)\end{array}$ & $\begin{array}{l}0.034 \\
(0.207)\end{array}$ & $\begin{array}{l}0.415 \\
(0.533)\end{array}$ & $\begin{array}{l}0.426 \\
(0.472)\end{array}$ & $\begin{array}{l}0.027 \\
(0.056)\end{array}$ & $\begin{array}{l}0.002 \\
(0.349)\end{array}$ & $\begin{array}{l}-0.098 \\
(0.268)\end{array}$ & $\begin{array}{l}-0.005 \\
(0.069)\end{array}$ \\
\hline $\begin{array}{l}\text { Ind exp - UG } \\
\text { marketing }(H 8 b)\end{array}$ & $\begin{array}{l}0.102 \\
(0.174)\end{array}$ & $\begin{array}{l}0.128 \\
(0.163)\end{array}$ & $\begin{array}{l}0.007 \\
(0.021)\end{array}$ & $\begin{array}{l}0.125 \\
(0.451)\end{array}$ & $\begin{array}{l}0.125 \\
(0.365)\end{array}$ & $\begin{array}{l}0.007 \\
(0.137)\end{array}$ & $\begin{array}{l}0.032 \\
(0.344)\end{array}$ & $\begin{array}{l}0.050 \\
(0.300)\end{array}$ & $\begin{array}{l}0.002 \\
(0.035)\end{array}$ & $\begin{array}{l}0.225 \\
(0.246)\end{array}$ & $\begin{array}{l}0.243 \\
(0.209)\end{array}$ & $\begin{array}{l}0.014 \\
(0.049)\end{array}$ \\
\hline $\begin{array}{l}\text { Ind exp - PG } \\
\text { marketing (H8b) }\end{array}$ & $\begin{array}{l}0.132 \\
(0.179)\end{array}$ & $\begin{array}{l}0.083 \\
(0.164)\end{array}$ & $\begin{array}{l}0.004 \\
(0.019)\end{array}$ & $\begin{array}{l}0.010 \\
(0.317)\end{array}$ & $\begin{array}{l}0.010 \\
(0.256)\end{array}$ & $\begin{array}{l}-0.531 \mathrm{E}-4 \\
(0.100)\end{array}$ & $\begin{array}{l}0.152 \\
(0.329)\end{array}$ & $\begin{array}{l}0.148 \\
(0.292)\end{array}$ & $\begin{array}{l}0.009 \\
(0.030)\end{array}$ & $\begin{array}{l}0.150 \\
(0.298)\end{array}$ & $\begin{array}{l}-0.001 \\
(0.238)\end{array}$ & $\begin{array}{l}-0.0006 \\
(0.060)\end{array}$ \\
\hline Age & $\begin{array}{l}-0.002 \\
(0.005)\end{array}$ & $\begin{array}{l}-0.002 \\
(0.004)\end{array}$ & $\begin{array}{l}-0.0001 \\
(0.0005)\end{array}$ & $\begin{array}{l}-0.011 \\
(0.10)\end{array}$ & $\begin{array}{l}-0.011 \\
(0.008)\end{array}$ & $\begin{array}{l}-0.0006 \\
(0.003)\end{array}$ & $\begin{array}{l}-0.008 \\
(0.010)\end{array}$ & $\begin{array}{l}-0.008 \\
(0.008)\end{array}$ & $\begin{array}{l}-0.0005 \\
(0.0009)\end{array}$ & $\begin{array}{l}0.005 \\
(0.007)\end{array}$ & $\begin{array}{l}0.005 \\
(0.006)\end{array}$ & $\begin{array}{l}0.0003 \\
(0.001)\end{array}$ \\
\hline Size (Staff) & $\begin{array}{l}0.000 \\
(0.000)\end{array}$ & $\begin{array}{l}0.0001 \\
(0.0002)\end{array}$ & $\begin{array}{l}0.111 \mathrm{E}-4 \\
(0.298 \mathrm{E}-4)\end{array}$ & $\begin{array}{l}\mathbf{0 . 0 0 1 \dagger} \\
(\mathbf{0 . 0 0 1 )}\end{array}$ & $\begin{array}{l}\text { 0.001* } \\
(0.0004)\end{array}$ & $\begin{array}{l}0.587 \mathrm{E}-4 \\
(0.0002)\end{array}$ & $\begin{array}{l}-0.000 \\
(0.000)\end{array}$ & $\begin{array}{l}-0.717 \mathrm{E}-4 \\
(0.0003)\end{array}$ & $\begin{array}{l}-0.481 \mathrm{E}-5 \\
(0.388 \mathrm{E}-4)\end{array}$ & $\begin{array}{l}0.000 \\
(0.000)\end{array}$ & $\begin{array}{l}0.0001 \\
(0.0002)\end{array}$ & $\begin{array}{l}0.115 E-4 \\
(0.862 E-4)\end{array}$ \\
\hline Offshore policy & $\begin{array}{l}-0.709 \\
(1.089)\end{array}$ & $\begin{array}{l}-0.773 \\
(1.029)\end{array}$ & $\begin{array}{l}-0.045 \\
(0.099)\end{array}$ & $\begin{array}{l}-0.242 \\
(2.025)\end{array}$ & $\begin{array}{l}-0.242 \\
(1.640)\end{array}$ & $\begin{array}{l}-0.010 \\
(0.572)\end{array}$ & $\begin{array}{l}-2.580 \\
(2.378)\end{array}$ & $\begin{array}{l}-2.239 \\
(1.860)\end{array}$ & $\begin{array}{l}-0.137 \\
(0.162)\end{array}$ & $\begin{array}{l}1.080 \\
(1.685)\end{array}$ & $\begin{array}{l}1.022 \\
(1.429)\end{array}$ & $\begin{array}{l}0.064 \\
(0.374)\end{array}$ \\
\hline $\begin{array}{l}\text { Internationalisation } \\
\text { at home strategy }\end{array}$ & $\begin{array}{l}0.150 \\
(0.198)\end{array}$ & $\begin{array}{l}-0.001 \\
(0.001)\end{array}$ & $\begin{array}{l}-0.613 \mathrm{E}-4 \\
(0.0002)\end{array}$ & $\begin{array}{l}0.210 \\
(0.365)\end{array}$ & $\begin{array}{l}0.210 \\
(0.295)\end{array}$ & $\begin{array}{l}0.011 \\
(0.201)\end{array}$ & $\begin{array}{l}-0.138 \\
(0.456)\end{array}$ & $\begin{array}{l}-0.001 \\
(0.003)\end{array}$ & $\begin{array}{l}-0.659 \mathrm{E}-4 \\
(0.037)\end{array}$ & $\begin{array}{l}-0.022 \\
(0.306)\end{array}$ & $\begin{array}{l}-0.001 \\
(0.001)\end{array}$ & $\begin{array}{l}-0.816 \mathrm{E}-4 \\
(0.0003)\end{array}$ \\
\hline $\begin{array}{l}\text { Host country } \\
\text { demand }\end{array}$ & $\begin{array}{l}1.919 \\
(6.813)\end{array}$ & $\begin{array}{l}2.950 \\
(6.387)\end{array}$ & $\begin{array}{l}0.166 \\
(1.033)\end{array}$ & $\begin{array}{l}4.073 \\
(6.874)\end{array}$ & $\begin{array}{l}4.072 \\
(5.568)\end{array}$ & $\begin{array}{l}0.233 \\
(3.373)\end{array}$ & $\begin{array}{l}-259.563 * \\
(123.291)\end{array}$ & $\begin{array}{l}-248.36^{*} \\
(104.53)\end{array}$ & $\begin{array}{l}-15.084 \\
(14.422)\end{array}$ & $\begin{array}{l}4.744 \\
(90.081)\end{array}$ & $\begin{array}{l}2.063 \\
(78.312)\end{array}$ & $\begin{array}{l}0.177 \\
(16.955)\end{array}$ \\
\hline AU/NZ dummy & $\begin{array}{l}0.952 \\
(0.860)\end{array}$ & $\begin{array}{l}0.840 \\
(0.794)\end{array}$ & $\begin{array}{l}0.050 \\
(0.110)\end{array}$ & - & - & - & - & - & - & - & - & - \\
\hline UK/IR dummy & $\begin{array}{l}0.087 \\
(0.669)\end{array}$ & $\begin{array}{l}0.116 \\
(0.630)\end{array}$ & $\begin{array}{l}0.007 \\
(0.079)\end{array}$ & - & - & - & - & - & - & - & - & - \\
\hline $\mathrm{R}^{2}$ & 0.077 & & & 0.347 & & & 0.173 & & & 0.083 & & \\
\hline $\operatorname{AdjR}^{2}$ & -0.014 & & & 0.037 & & & -0.011 & & & -0.171 & & \\
\hline Log likelihood & & -348.06 & -363.94 & & -67.05 & -78.85 & & -150.06 & -155.13 & & -113.29 & -124.64 \\
\hline $\mathrm{N}$ & 139 & 139 & 139 & 32 & 32 & 32 & 57 & 57 & 57 & 50 & 50 & 50 \\
\hline Max. VIF & 1.852 & & & 1.871 & & & 1.466 & & & 1.368 & & \\
\hline
\end{tabular}


Table 7.39a: Regression estimates for equation 24a (low regulative institutional distance)

\begin{tabular}{|c|c|c|c|c|c|c|c|c|c|c|c|c|}
\hline & \multicolumn{3}{|c|}{ Complete data set } & \multicolumn{3}{|c|}{ AU/NZ } & \multicolumn{3}{|c|}{ US/CA } & \multicolumn{3}{|c|}{ UK/IR } \\
\hline & OLS & Tobit & $\begin{array}{l}\text { Negative } \\
\text { binomial }\end{array}$ & OLS & Tobit & $\begin{array}{l}\text { Negative } \\
\text { binomial } \\
\end{array}$ & OLS & Tobit & $\begin{array}{l}\text { Negative } \\
\text { binomial } \\
\end{array}$ & OLS & Tobit & $\begin{array}{l}\text { Negative } \\
\text { binomial }\end{array}$ \\
\hline Intercept & $\begin{array}{l}\text { 16.913** } \\
(1.521)\end{array}$ & $\begin{array}{l}\text { 17.315** } \\
(1.333)\end{array}$ & $\begin{array}{l}2.792^{* *} \\
(0.145)\end{array}$ & $\begin{array}{l}\text { 18.353** } \\
(2.817)\end{array}$ & $\begin{array}{l}19.412^{* *} \\
(2.215)\end{array}$ & $\begin{array}{l}3.008 * * \\
(0.252)\end{array}$ & $\begin{array}{l}18.579 * * \\
(2.658)\end{array}$ & $\begin{array}{l}18.248 * * \\
(2.369)\end{array}$ & $\begin{array}{l}2.730 * * \\
(0.240)\end{array}$ & $\begin{array}{l}\text { 16.806** } \\
(2.529)\end{array}$ & $\begin{array}{l}\text { 18.193** } \\
(2.050)\end{array}$ & $\begin{array}{l}2.880 * * \\
(0.422)\end{array}$ \\
\hline $\begin{array}{l}\text { Know-how (Factor) } \\
\text { (H8c) }\end{array}$ & $\begin{array}{l}-1.706 * * \\
(0.143)\end{array}$ & $\begin{array}{l}-1.714 * * \\
(0.138)\end{array}$ & $\begin{array}{l}-0.141 * * \\
(0.013)\end{array}$ & $\begin{array}{l}-1.663^{* *} \\
(0.248)\end{array}$ & $\begin{array}{l}-1.666^{* *} \\
(0.228)\end{array}$ & $\begin{array}{l}-0.129 * * \\
(0.023)\end{array}$ & $\begin{array}{l}-1.889 * * \\
(0.254)\end{array}$ & $\begin{array}{l}-1.892^{* *} \\
(0.238)\end{array}$ & $\begin{array}{l}-0.161 * * \\
(0.021)\end{array}$ & $\begin{array}{l}-1.624 * * \\
(0.324)\end{array}$ & $\begin{array}{l}-1.642^{* *} \\
(0.297)\end{array}$ & $\begin{array}{l}-0.119^{*} \\
(0.059)\end{array}$ \\
\hline Age & $\begin{array}{l}0.007 \\
(0.004)\end{array}$ & $\begin{array}{l}0.005 \\
(0.004)\end{array}$ & $\begin{array}{l}0.0004 \\
(0.0004)\end{array}$ & $\begin{array}{l}0.008 \\
(0.013)\end{array}$ & $\begin{array}{l}0.006 \\
(0.011)\end{array}$ & $\begin{array}{l}0.0004 \\
(0.001)\end{array}$ & $\begin{array}{l}0.005 \\
(0.008)\end{array}$ & $\begin{array}{l}0.005 \\
(0.007)\end{array}$ & $\begin{array}{l}0.0003 \\
(0.0007)\end{array}$ & $\begin{array}{l}0.006 \\
(0.006)\end{array}$ & $\begin{array}{l}0.003 \\
(0.005)\end{array}$ & $\begin{array}{l}0.0002 \\
(0.001)\end{array}$ \\
\hline Size (Staff) & $\begin{array}{l}8.191 \mathrm{E}-5 \\
(0.000)\end{array}$ & $\begin{array}{l}0.744 \mathrm{E}-4 \\
(0.0001)\end{array}$ & $\begin{array}{l}0.698 E-5 \\
(0.212 E-4)\end{array}$ & $\begin{array}{l}7.096 \mathrm{E}-5 \\
(0.000)\end{array}$ & $\begin{array}{l}0.147 \mathrm{E}-4 \\
(0.0003)\end{array}$ & $\begin{array}{l}-0.159 \mathrm{E}-5 \\
(0.416 \mathrm{E}-4)\end{array}$ & $\begin{array}{l}-0.000 \\
(0.000)\end{array}$ & $\begin{array}{l}-0.155 E-4 \\
(0.0003)\end{array}$ & $\begin{array}{l}-0.273 \mathrm{E}-5 \\
(0.377 \mathrm{E}-4)\end{array}$ & $\begin{array}{l}-6.964 \mathrm{E}-5 \\
(0.000)\end{array}$ & $\begin{array}{l}-0.661 \mathrm{E}-5 \\
(0.0002)\end{array}$ & $\begin{array}{l}0.420 \mathrm{E}-5 \\
(0.434 \mathrm{E}-4)\end{array}$ \\
\hline Offshore policy & $\begin{array}{l}2.776 * * \\
(0.932)\end{array}$ & $\begin{array}{l}2.776 * * \\
(0.905)\end{array}$ & $\begin{array}{l}0.294 * * \\
(0.082)\end{array}$ & $\begin{array}{l}0.878 \\
(1.854)\end{array}$ & $\begin{array}{l}0.753 \\
(1.705)\end{array}$ & $\begin{array}{l}0.082 \\
(0.170)\end{array}$ & $\begin{array}{l}3.706 * \\
(1.567)\end{array}$ & $\begin{array}{l}3.664 * \\
(1.469)\end{array}$ & $\begin{array}{l}0.509 * * \\
(0.123)\end{array}$ & $\begin{array}{l}3.236 * \\
(1.563)\end{array}$ & $\begin{array}{l}3.130 * \\
(1.459)\end{array}$ & $\begin{array}{l}0.240 \\
(0.266)\end{array}$ \\
\hline $\begin{array}{l}\text { Internationalisation } \\
\text { at home strategy }\end{array}$ & $\begin{array}{l}0.061 \\
(0.173)\end{array}$ & $\begin{array}{l}-0.0007 \\
(0.0008)\end{array}$ & $\begin{array}{l}-0.561 \mathrm{E}-4 \\
(0.0001)\end{array}$ & $\begin{array}{l}0.309 \\
(0.452)\end{array}$ & $\begin{array}{l}-0.001 \\
(0.001)\end{array}$ & $\begin{array}{l}-0.0001 \\
(0.0002)\end{array}$ & $\begin{array}{l}-0.110 \\
(0.273)\end{array}$ & $\begin{array}{l}-0.002 \\
(0.003)\end{array}$ & $\begin{array}{l}-0.0001 \\
(0.024)\end{array}$ & $\begin{array}{l}0.256 \\
(0.298)\end{array}$ & $\begin{array}{l}0.0004 \\
(0.0009)\end{array}$ & $\begin{array}{l}0.135 \mathrm{E}-4 \\
(0.0001)\end{array}$ \\
\hline $\begin{array}{l}\text { Host country } \\
\text { demand }\end{array}$ & $\begin{array}{l}2.965 \\
(2.462)\end{array}$ & $\begin{array}{l}2.915 \\
(2.294)\end{array}$ & $\begin{array}{l}0.216 \\
(0.288)\end{array}$ & $\begin{array}{l}7.168 \\
(4.486)\end{array}$ & $\begin{array}{l}8.426^{*} \\
(3.903)\end{array}$ & $\begin{array}{l}0.541 \\
(0.597)\end{array}$ & $\begin{array}{l}-3.403 \\
(4.733)\end{array}$ & $\begin{array}{l}-3.403 \\
(4.447)\end{array}$ & $\begin{array}{l}-0.184 \\
(0.488)\end{array}$ & $\begin{array}{l}4.957 \\
(4.826)\end{array}$ & $\begin{array}{l}2.372 \\
(3.683)\end{array}$ & $\begin{array}{l}0.156 \\
(0.813)\end{array}$ \\
\hline AU/NZ dummy & $\begin{array}{l}1.102 \dagger \\
(0.641)\end{array}$ & $\begin{array}{l}1.063 \dagger \\
(0.605)\end{array}$ & $\begin{array}{l}0.076 \\
(0.074)\end{array}$ & - & - & - & - & - & - & - & - & - \\
\hline UK/IR dummy & $\begin{array}{l}1.050 \dagger \\
(0.572)\end{array}$ & $\begin{array}{l}0.841 \\
(0.540)\end{array}$ & $\begin{array}{l}0.058 \\
(0.070)\end{array}$ & - & - & - & - & - & - & - & - & - \\
\hline $\mathrm{R}^{2}$ & 0.659 & & & 0.628 & & & 0.702 & & & 0.556 & & \\
\hline $\operatorname{Adj}^{2}$ & 0.641 & & & 0.568 & & & 0.672 & & & 0.491 & & \\
\hline Log likelihood & & -413.60 & -452.53 & & -117.56 & -129.74 & & -168.82 & -181.94 & & -119.12 & -136.51 \\
\hline $\mathrm{N}$ & 169 & 169 & 169 & 48 & 48 & 48 & 67 & 67 & 67 & 54 & 54 & 54 \\
\hline Max. VIF & 1.558 & & & 1.293 & & & 1.881 & & & 1.566 & & \\
\hline
\end{tabular}

Standard errors in parentheses, all $t$ tests are two-tailed, $\dagger p<.10,{ }^{*} p<.05, * * p<.01$ 
Table 7.39b: Regression estimates for equation 24b (high regulative institutional distance)

\begin{tabular}{|c|c|c|c|c|c|c|c|c|c|c|c|c|}
\hline & \multicolumn{3}{|c|}{ Complete data set } & \multicolumn{3}{|c|}{ AU/NZ } & \multicolumn{3}{|c|}{ US/CA } & \multicolumn{3}{|c|}{ UK/IR } \\
\hline & OLS & Tobit & $\begin{array}{l}\text { Negative } \\
\text { binomial }\end{array}$ & OLS & Tobit & $\begin{array}{l}\text { Negative } \\
\text { binomial }\end{array}$ & OLS & Tobit & $\begin{array}{l}\text { Negative } \\
\text { binomial }\end{array}$ & OLS & Tobit & $\begin{array}{l}\text { Negative } \\
\text { binomial }\end{array}$ \\
\hline Intercept & $\begin{array}{l}\text { 21.483** } \\
(1.206)\end{array}$ & $\begin{array}{l}\text { 21.690** } \\
(1.034)\end{array}$ & $\begin{array}{l}\text { 3.151** } \\
(0.212)\end{array}$ & $\begin{array}{l}\text { 20.755** } \\
(3.065)\end{array}$ & $\begin{array}{l}\text { 20.755** } \\
(2.708)\end{array}$ & $\begin{array}{l}3.040 * * \\
(0.626)\end{array}$ & $\begin{array}{l}25.323 * * \\
(2.589)\end{array}$ & $\begin{array}{l}\text { 24.189** } \\
(1.712)\end{array}$ & $\begin{array}{l}\text { 3.312** } \\
(0.514)\end{array}$ & $\begin{array}{l}\text { 17.866** } \\
(2.186)\end{array}$ & $\begin{array}{l}\text { 17.889** } \\
(1.964)\end{array}$ & $\begin{array}{l}2.907 * * \\
(0.632)\end{array}$ \\
\hline $\begin{array}{l}\text { Know-how (Factor) } \\
(\mathrm{H} 8 \mathrm{c})\end{array}$ & $\begin{array}{l}-1.267^{* *} \\
(0.131)\end{array}$ & $\begin{array}{l}-1.272 * * \\
(0.125)\end{array}$ & $\begin{array}{l}-0.089 * * \\
(0.024)\end{array}$ & $\begin{array}{l}-1.677 \\
(1.048)\end{array}$ & $\begin{array}{l}-1.676 \dagger \\
(0.926)\end{array}$ & $\begin{array}{l}-0.094 \\
(0.212)\end{array}$ & $\begin{array}{l}-1.346^{* *} \\
(0.160)\end{array}$ & $\begin{array}{l}-1.345^{* *} \\
(0.149)\end{array}$ & $\begin{array}{l}-0.096^{* *} \\
(0.029)\end{array}$ & $\begin{array}{l}-0.948 * * \\
(0.278)\end{array}$ & $\begin{array}{l}-0.963^{* *} \\
(0.253)\end{array}$ & $\begin{array}{l}-0.064 \\
(0.065)\end{array}$ \\
\hline Age & $\begin{array}{l}-0.002 \\
(0.004)\end{array}$ & $\begin{array}{l}-0.002 \\
(0.003)\end{array}$ & $\begin{array}{l}-0.0001 \\
(0.0007)\end{array}$ & $\begin{array}{l}-0.017 \\
(0.010)\end{array}$ & $\begin{array}{l}-0.016 \dagger \\
(0.008)\end{array}$ & $\begin{array}{l}-0.0009 \\
(0.002)\end{array}$ & $\begin{array}{l}-0.003 \\
(0.006)\end{array}$ & $\begin{array}{l}-0.003 \\
(0.005)\end{array}$ & $\begin{array}{l}-0.0002 \\
(0.0013)\end{array}$ & $\begin{array}{l}0.006 \\
(0.006)\end{array}$ & $\begin{array}{l}0.006 \\
(0.005)\end{array}$ & $\begin{array}{l}0.0003 \\
(0.001)\end{array}$ \\
\hline Size (Staff) & $\begin{array}{l}0.000 \\
(0.000)\end{array}$ & $\begin{array}{l}0.0002 \\
(0.0001)\end{array}$ & $\begin{array}{l}0.160 \mathrm{E}-4 \\
(0.327 \mathrm{E}-4)\end{array}$ & $\begin{array}{l}0.001 \\
(0.001)\end{array}$ & $\begin{array}{l}\text { 0.001** } \\
(0.0004)\end{array}$ & $\begin{array}{l}0.794 \mathrm{E}-4 \\
(0.0001)\end{array}$ & $\begin{array}{l}\text { 4.339E-5 } \\
(0.000)\end{array}$ & $\begin{array}{l}0.675 E-4 \\
(0.0002)\end{array}$ & $\begin{array}{l}0.510 \mathrm{E}-5 \\
(0.487 \mathrm{E}-4)\end{array}$ & $\begin{array}{l}0.000 \\
(0.000)\end{array}$ & $\begin{array}{l}0.0001 \\
(0.0002)\end{array}$ & $\begin{array}{l}0.838 E-5 \\
(0.662 E-4)\end{array}$ \\
\hline Offshore policy & $\begin{array}{l}-1.237 \\
(0.822)\end{array}$ & $\begin{array}{l}-1.263 \\
(0.788)\end{array}$ & $\begin{array}{l}-0.080 \\
(0.159)\end{array}$ & $\begin{array}{l}-1.636 \\
(1.771)\end{array}$ & $\begin{array}{l}-1.635 \\
(1.565)\end{array}$ & $\begin{array}{l}-0.091 \\
(0.347)\end{array}$ & $\begin{array}{l}-2.568 \dagger \\
(1.403)\end{array}$ & $\begin{array}{l}-2.183 \dagger \\
(1.172)\end{array}$ & $\begin{array}{l}-0.132 \\
(0.299)\end{array}$ & $\begin{array}{l}0.871 \\
(1.423)\end{array}$ & $\begin{array}{l}0.768 \\
(1.268)\end{array}$ & $\begin{array}{l}0.043 \\
(0.440)\end{array}$ \\
\hline $\begin{array}{l}\text { Internationalisation } \\
\text { at home strategy }\end{array}$ & $\begin{array}{l}0.053 \\
(0.149)\end{array}$ & $\begin{array}{l}-0.0007 \\
(0.001)\end{array}$ & $\begin{array}{l}-0.496 \mathrm{E}-4 \\
(0.0002)\end{array}$ & $\begin{array}{l}0.249 \\
(0.327)\end{array}$ & $\begin{array}{l}0.249 \\
(0.289)\end{array}$ & $\begin{array}{l}0.013 \\
(0.090)\end{array}$ & $\begin{array}{l}-0.173 \\
(0.279)\end{array}$ & $\begin{array}{l}0.0005 \\
(0.002)\end{array}$ & $\begin{array}{l}0.201 \mathrm{E}-4 \\
(0.037)\end{array}$ & $\begin{array}{l}-0.075 \\
(0.253)\end{array}$ & $\begin{array}{l}-0.001 \\
(0.001)\end{array}$ & $\begin{array}{l}-0.0001 \\
(0.0003)\end{array}$ \\
\hline $\begin{array}{l}\text { Host country } \\
\text { demand }\end{array}$ & $\begin{array}{l}5.127 \\
(4.932)\end{array}$ & $\begin{array}{l}5.387 \\
(4.695)\end{array}$ & $\begin{array}{l}0.333 \\
(0.947)\end{array}$ & $\begin{array}{l}4.012 \\
(5.360)\end{array}$ & $\begin{array}{l}4.011 \\
(4.737)\end{array}$ & $\begin{array}{l}0.227 \\
(1.633)\end{array}$ & $\begin{array}{l}-71.653 \\
(74.531)\end{array}$ & $\begin{array}{l}-58.359 \\
(66.465)\end{array}$ & $\begin{array}{l}-3.925 \\
(16.369)\end{array}$ & $\begin{array}{l}18.539 \\
(72.960)\end{array}$ & $\begin{array}{l}-19.967 \\
(66.958)\end{array}$ & $\begin{array}{l}-1.366 \\
(14.947)\end{array}$ \\
\hline AU/NZ dummy & $\begin{array}{l}-1.267 * * \\
(0.131)\end{array}$ & $\begin{array}{l}-0.374 \\
(0.616)\end{array}$ & $\begin{array}{l}-0.023 \\
(0.119)\end{array}$ & - & - & - & - & - & - & - & - & - \\
\hline UK/IR dummy & $\begin{array}{l}-0.915 \dagger \\
(0.508)\end{array}$ & $\begin{array}{l}-0.873 \dagger \\
(0.484)\end{array}$ & $\begin{array}{l}-0.053 \\
(0.097)\end{array}$ & - & - & - & - & - & - & - & - & - \\
\hline $\mathrm{R}^{2}$ & 0.455 & & & 0.267 & & & 0.645 & & & 0.265 & & \\
\hline $\operatorname{AdjR}^{2}$ & 0.421 & & & 0.091 & & & 0.602 & & & 0.155 & & \\
\hline Log likelihood & & -311.12 & -347.04 & & -68.92 & -79.29 & & -126.11 & -142.29 & & -107.65 & -122.87 \\
\hline $\mathrm{N}$ & 139 & 139 & 139 & 32 & 32 & 32 & 57 & 57 & 57 & 50 & 50 & 50 \\
\hline Max. VIF & 1.906 & & & 1.617 & & & 1.351 & & & 1.301 & & \\
\hline
\end{tabular}

Standard errors in parentheses, all $t$ tests are two-tailed, $\uparrow p<.10, * p<.05, * * p<.01$ 
Table 7.40a: Regression estimates for equation 25a (low regulative institutional distance)

\begin{tabular}{|c|c|c|c|c|c|c|c|c|c|c|c|c|}
\hline & \multicolumn{3}{|c|}{ Complete data set } & \multicolumn{3}{|c|}{ AU/NZ } & \multicolumn{3}{|c|}{$\overline{\text { US/CA }}$} & \multicolumn{3}{|c|}{ UK/IR } \\
\hline & OLS & Tobit & $\begin{array}{l}\text { Negative } \\
\text { binomial }\end{array}$ & OLS & Tobit & $\begin{array}{l}\text { Negative } \\
\text { binomial }\end{array}$ & OLS & Tobit & $\begin{array}{l}\text { Negative } \\
\text { binomial }\end{array}$ & OLS & Tobit & $\begin{array}{l}\text { Negative } \\
\text { binomial }\end{array}$ \\
\hline$\overline{\text { Intercept }}$ & $\begin{array}{l}19.452 * * \\
(1.912)\end{array}$ & $\begin{array}{l}19.532^{* *} \\
(1.741)\end{array}$ & $\begin{array}{l}2.918 * * \\
(0.161)\end{array}$ & $\begin{array}{l}22.018 * * \\
(3.655)\end{array}$ & $\begin{array}{l}22.696 * * \\
(2.978)\end{array}$ & $\begin{array}{l}3.205^{* *} \\
(0.290)\end{array}$ & $\begin{array}{l}\text { 20.564** } \\
(3.084)\end{array}$ & $\begin{array}{l}20.726 * * \\
(2.837)\end{array}$ & $\begin{array}{l}2.889 * * \\
(0.259)\end{array}$ & $\begin{array}{l}18.837 * * \\
(3.884)\end{array}$ & $\begin{array}{l}18.370 * * \\
(3.055)\end{array}$ & $\begin{array}{l}2.878 * * \\
(0.583)\end{array}$ \\
\hline $\begin{array}{l}\text { Org culture (Factor) } \\
-(H 8 d)\end{array}$ & $\begin{array}{l}-2.117 * * \\
(0.213)\end{array}$ & $\begin{array}{l}-2.058 * * \\
(0.208)\end{array}$ & $\begin{array}{l}-0.155 * * \\
(0.019)\end{array}$ & $\begin{array}{l}-2.051 * * \\
(0.388)\end{array}$ & $\begin{array}{l}-2.033^{* *} \\
(0.355)\end{array}$ & $\begin{array}{l}-0.144^{* *} \\
(0.037)\end{array}$ & $\begin{array}{l}-2.443^{* *} \\
(0.360)\end{array}$ & $\begin{array}{l}-2.431 * * \\
(0.335)\end{array}$ & $\begin{array}{l}-0.197 * * \\
(0.029)\end{array}$ & - & - & - \\
\hline $\begin{array}{l}\text { Org culture - open } \\
\text { discussion }(H 8 d)\end{array}$ & (1) & ( & ( & ( & (a & ( & ( & ( & ( & $\begin{array}{l}-0.279 \\
(0.332)\end{array}$ & $\begin{array}{l}-0.351 \\
(0.299)\end{array}$ & $\begin{array}{l}-0.022 \\
(0.056)\end{array}$ \\
\hline $\begin{array}{l}\text { Org culture - no } \\
\text { status distinction } \\
(H 8 d)\end{array}$ & - & - & - & - & - & - & - & - & - & $\begin{array}{l}-0.497 \\
(0.309)\end{array}$ & $\begin{array}{l}-0.324 \\
(0.267)\end{array}$ & $\begin{array}{l}-0.021 \\
(0.050)\end{array}$ \\
\hline $\begin{array}{l}\text { Org culture - } \\
\text { experimentation } \\
\text { (H8d) }\end{array}$ & - & - & - & - & - & - & - & - & - & $\begin{array}{l}-0.182 \\
(0.282)\end{array}$ & $\begin{array}{l}-0.317 \\
(0.235)\end{array}$ & $\begin{array}{l}-0.021 \\
(0.036)\end{array}$ \\
\hline $\begin{array}{l}\text { Org culture - } \\
\text { offshore } \\
\text { development (H8d) }\end{array}$ & - & - & - & - & - & - & - & - & - & $\begin{array}{l}-0.546 \\
(0.346)\end{array}$ & $\begin{array}{l}-0.307 \\
(0.287)\end{array}$ & $\begin{array}{l}-0.019 \\
(0.064)\end{array}$ \\
\hline $\begin{array}{l}\text { Org culture - } \\
\text { offshore } \\
\text { responsiveness } \\
(\text { H8d) }\end{array}$ & - & - & - & - & - & - & - & - & - & $\begin{array}{l}-0.216 \\
(0.302)\end{array}$ & $\begin{array}{l}-0.113 \\
(0.266)\end{array}$ & $\begin{array}{l}-0.008 \\
(0.046)\end{array}$ \\
\hline Age & $\begin{array}{l}0.005 \\
(0.005)\end{array}$ & $\begin{array}{l}0.004 \\
(0.004)\end{array}$ & $\begin{array}{l}0.0002 \\
(0.004)\end{array}$ & $\begin{array}{l}0.008 \\
(0.014)\end{array}$ & $\begin{array}{l}0.008 \\
(0.012)\end{array}$ & $\begin{array}{l}0.0004 \\
(0.0014)\end{array}$ & $\begin{array}{l}0.009 \\
(0.008)\end{array}$ & $\begin{array}{l}0.008 \\
(0.007)\end{array}$ & $\begin{array}{l}0.0006 \\
(0.0007)\end{array}$ & $\begin{array}{l}0.001 \\
(0.007)\end{array}$ & $\begin{array}{l}-0.001 \\
(0.005)\end{array}$ & $\begin{array}{l}-0.493 \mathrm{E}-4 \\
(0.001)\end{array}$ \\
\hline Size (Staff) & $\begin{array}{l}2.155 E-5 \\
(0.000)\end{array}$ & $\begin{array}{l}0.114 \mathrm{E}-4 \\
(0.0001)\end{array}$ & $\begin{array}{l}0.203 E-5 \\
(0.218 E-4)\end{array}$ & $\begin{array}{l}-0.000 \\
(0.000)\end{array}$ & $\begin{array}{l}-0.563 \mathrm{E}-4 \\
(0.0003)\end{array}$ & $\begin{array}{l}-0.476 \mathrm{E}-5 \\
(0.358 \mathrm{E}-4)\end{array}$ & $\begin{array}{l}-0.000 \\
(0.000)\end{array}$ & $\begin{array}{l}-0.452 \mathrm{E}-4 \\
(0.0003)\end{array}$ & $\begin{array}{l}-0.597 \mathrm{E}-5 \\
(0.416 \mathrm{E}-4)\end{array}$ & $\begin{array}{l}0.000 \\
(0.000)\end{array}$ & $\begin{array}{l}-0.001 \\
(0.002)\end{array}$ & $\begin{array}{l}-0.774 \mathrm{E}-5 \\
(0.609 \mathrm{E}-4)\end{array}$ \\
\hline Offshore policy & $\begin{array}{l}3.649 * * \\
(0.994)\end{array}$ & $\begin{array}{l}\text { 3.792** } \\
(0.978)\end{array}$ & $\begin{array}{l}0.392 * * \\
(0.075)\end{array}$ & $\begin{array}{l}1.101 \\
(2.103)\end{array}$ & $\begin{array}{l}1.015 \\
(1.933)\end{array}$ & $\begin{array}{l}0.105 \\
(0.160)\end{array}$ & $\begin{array}{l}4.469 * * \\
(1.600)\end{array}$ & $\begin{array}{l}4.515^{* *} \\
(1.493)\end{array}$ & $\begin{array}{l}0.613^{* *} \\
(0.126)\end{array}$ & $\begin{array}{l}4.480 * \\
(1.812)\end{array}$ & $\begin{array}{l}4.595^{* *} \\
(1.655)\end{array}$ & $\begin{array}{l}0.339 \\
(0.232)\end{array}$ \\
\hline $\begin{array}{l}\text { Internationalisation } \\
\text { at home strategy }\end{array}$ & $\begin{array}{l}0.090 \\
(0.187)\end{array}$ & $\begin{array}{l}0.0004 \\
(0.0009)\end{array}$ & $\begin{array}{l}0.405 E-4 \\
(0.884 E-4)\end{array}$ & $\begin{array}{l}0.256 \\
(0.508)\end{array}$ & $\begin{array}{l}-0.001 \\
(0.001)\end{array}$ & $\begin{array}{l}-0.578 \mathrm{E}-4 \\
(0.0002)\end{array}$ & $\begin{array}{l}0.074 \\
(0.287)\end{array}$ & $\begin{array}{l}-0.001 \\
(0.003)\end{array}$ & $\begin{array}{l}-0.853 \mathrm{E}-4 \\
(0.026)\end{array}$ & $\begin{array}{l}0.206 \\
(0.360)\end{array}$ & $\begin{array}{l}0.001 \\
(0.001)\end{array}$ & $\begin{array}{l}0.830 \mathrm{E}-4 \\
(0.0001)\end{array}$ \\
\hline $\begin{array}{l}\text { Host country } \\
\text { demand }\end{array}$ & $\begin{array}{l}3.234 \\
(2.675)\end{array}$ & $\begin{array}{l}3.295 \\
(2.528)\end{array}$ & $\begin{array}{l}0.217 \\
(0.257)\end{array}$ & $\begin{array}{l}4.884 \\
(5.034)\end{array}$ & $\begin{array}{l}6.503 \\
(4.378)\end{array}$ & $\begin{array}{l}0.413 \\
(0.476)\end{array}$ & $\begin{array}{l}-0.902 \\
(4.826)\end{array}$ & $\begin{array}{l}-0.854 \\
(4.528)\end{array}$ & $\begin{array}{l}-0.048 \\
(0.499)\end{array}$ & $\begin{array}{l}4.425 \\
(5.939)\end{array}$ & $\begin{array}{l}2.535 \\
(4.478)\end{array}$ & $\begin{array}{l}0.138 \\
(0.797)\end{array}$ \\
\hline AU/NZ dummy & $\begin{array}{l}1.308 \dagger \\
(0.695)\end{array}$ & $\begin{array}{l}1.286 \dagger \\
(0.665)\end{array}$ & $\begin{array}{l}0.087 \\
(0.069)\end{array}$ & - & - & - & - & - & - & - & - & - \\
\hline UK/IR dummy & $\begin{array}{l}1.113 \dagger \\
(0.621)\end{array}$ & $\begin{array}{l}0.894 \\
(0.595)\end{array}$ & $\begin{array}{l}0.060 \\
(0.066)\end{array}$ & - & - & - & - & - & - & - & - & - \\
\hline
\end{tabular}




\begin{tabular}{|c|c|c|c|c|c|c|c|c|c|c|c|c|}
\hline $\mathrm{R}^{2}$ & 0.599 & & & 0.531 & & & 0.676 & & & 0.453 & & \\
\hline $\operatorname{Adj}^{2}$ & 0.577 & & & 0.455 & & & 0.643 & & & 0.306 & & \\
\hline Log likelihood & & -429.59 & -465.49 & & -123.01 & -134.07 & & -171.59 & -184.66 & & -126.47 & -139.52 \\
\hline $\mathrm{N}$ & 169 & 169 & 169 & 48 & 48 & 48 & 67 & 67 & 67 & 54 & 54 & 54 \\
\hline Max. VIF & 1.557 & & & 1.320 & & & 1.741 & & & 1.704 & & \\
\hline
\end{tabular}

Standard errors in parentheses, all $t$ tests are two-tailed, $\uparrow p<.10,{ }^{*} p<.05,{ }^{* *} p<.01$

Table 7.40b: Regression estimates for equation 25b (high regulative institutional distance)

\begin{tabular}{|c|c|c|c|c|c|c|c|c|c|c|c|c|}
\hline & \multicolumn{3}{|c|}{ Complete data set } & \multicolumn{3}{|c|}{$\overline{\mathrm{AU} / \mathrm{NZ}}$} & \multicolumn{3}{|c|}{$\overline{\mathrm{US} / \mathrm{CA}}$} & \multicolumn{3}{|c|}{$\overline{\mathrm{UK} / \mathrm{IR}}$} \\
\hline & OLS & Tobit & $\begin{array}{l}\text { Negative } \\
\text { binomial }\end{array}$ & OLS & Tobit & $\begin{array}{l}\text { Negative } \\
\text { binomial }\end{array}$ & OLS & Tobit & $\begin{array}{l}\text { Negative } \\
\text { binomial }\end{array}$ & OLS & Tobit & $\begin{array}{l}\text { Negative } \\
\text { binomial }\end{array}$ \\
\hline Intercept & $\begin{array}{l}\text { 23.715** } \\
(1.506)\end{array}$ & $\begin{array}{l}\text { 23.983** } \\
(1.308)\end{array}$ & $\begin{array}{l}3.286 * * \\
(0.196)\end{array}$ & $\begin{array}{l}18.076 * * \\
(3.873)\end{array}$ & $\begin{array}{l}18.075 * * \\
(3.423)\end{array}$ & $\begin{array}{l}2.892 * * \\
(0.618)\end{array}$ & $\begin{array}{l}\text { 27.504** } \\
(3.284)\end{array}$ & $\begin{array}{l}\text { 26.803** } \\
(2.217)\end{array}$ & $\begin{array}{l}3.471 * * \\
(0.445)\end{array}$ & $\begin{array}{l}\text { 20.807** } \\
(2.860)\end{array}$ & $\begin{array}{l}\text { 21.165** } \\
(2.385)\end{array}$ & $\begin{array}{l}3.117 * * \\
(0.690)\end{array}$ \\
\hline $\begin{array}{l}\text { Org culture (Factor) } \\
-(H 8 d)\end{array}$ & $\begin{array}{l}-1.503^{* *} \\
(0.194)\end{array}$ & $\begin{array}{l}-1.520^{* *} \\
(0.184)\end{array}$ & $\begin{array}{l}-0.101 * * \\
(0.027)\end{array}$ & $\begin{array}{l}-0.314 \\
(1.046)\end{array}$ & $\begin{array}{l}-0.313 \\
(0.924)\end{array}$ & $\begin{array}{l}-0.018 \\
(0.197)\end{array}$ & $\begin{array}{l}-1.471^{* *} \\
(0.278)\end{array}$ & $\begin{array}{l}-1.473^{* *} \\
(0.258)\end{array}$ & $\begin{array}{l}-0.101 * * \\
(0.033)\end{array}$ & - & - & - \\
\hline $\begin{array}{l}\text { Org culture - open } \\
\text { discussion (H8d) }\end{array}$ & - & - & - & - & - & - & - & - & - & $\begin{array}{l}-0.089 \\
(0.307)\end{array}$ & $\begin{array}{l}-0.193 \\
(0.256)\end{array}$ & $\begin{array}{l}-0.013 \\
(0.056)\end{array}$ \\
\hline $\begin{array}{l}\text { Org culture - no } \\
\text { status distinction } \\
(\text { H8d) }\end{array}$ & - & - & - & - & - & - & - & - & - & $\begin{array}{l}-0.248 \\
(0.333)\end{array}$ & $\begin{array}{l}-0.318 \\
(0.271)\end{array}$ & $\begin{array}{l}-0.020 \\
(0.069)\end{array}$ \\
\hline $\begin{array}{l}\text { Org culture - } \\
\text { experimentation } \\
(H 8 d)\end{array}$ & - & - & - & - & - & - & - & - & - & $\begin{array}{l}-0.280 \\
(0.290)\end{array}$ & $\begin{array}{l}-0.384 \dagger \\
(0.227)\end{array}$ & $\begin{array}{l}-0.023 \\
(0.078)\end{array}$ \\
\hline $\begin{array}{l}\text { Org culture - } \\
\text { offshore } \\
\text { development (H8d) }\end{array}$ & - & - & - & - & - & - & - & - & - & $\begin{array}{l}-0.480 \dagger \\
(0.276)\end{array}$ & $\begin{array}{l}-0.451 \dagger \\
(0.231)\end{array}$ & $\begin{array}{l}-0.029 \\
(0.079)\end{array}$ \\
\hline $\begin{array}{l}\text { Org culture - } \\
\text { offshore } \\
\text { responsiveness } \\
(\text { H8d) }\end{array}$ & - & - & - & - & - & - & - & - & - & $\begin{array}{l}-0.274 \\
(0.341)\end{array}$ & $\begin{array}{l}-0.142 \\
(0.265)\end{array}$ & $\begin{array}{l}-0.010 \\
(0.070)\end{array}$ \\
\hline Age & $\begin{array}{l}-0.002 \\
(0.004)\end{array}$ & $\begin{array}{l}-0.002 \\
(0.003)\end{array}$ & $\begin{array}{l}-0.0001 \\
(0.0007)\end{array}$ & $\begin{array}{l}-0.010 \\
(0.010)\end{array}$ & $\begin{array}{l}-0.009 \\
(0.008)\end{array}$ & $\begin{array}{l}-0.0005 \\
(0.002)\end{array}$ & $\begin{array}{l}-0.004 \\
(0.007)\end{array}$ & $\begin{array}{l}-0.004 \\
(0.006)\end{array}$ & $\begin{array}{l}-0.0002 \\
(0.0009)\end{array}$ & $\begin{array}{l}0.003 \\
(0.006)\end{array}$ & $\begin{array}{l}0.003 \\
(0.005)\end{array}$ & $\begin{array}{l}0.0001 \\
(0.001)\end{array}$ \\
\hline Size (Staff) & $\begin{array}{l}0.000 \\
(0.000)\end{array}$ & $\begin{array}{l}0.0002 \\
(0.0001)\end{array}$ & $\begin{array}{l}0.143 E-4 \\
(0.317 E-4)\end{array}$ & $\begin{array}{l}\text { 0.001† } \\
(\mathbf{0 . 0 0 1 )}\end{array}$ & $\begin{array}{l}0.0009 * \\
(0.0004)\end{array}$ & $\begin{array}{l}0.538 \mathrm{E}-4 \\
(0.0001)\end{array}$ & $\begin{array}{l}0.000 \\
(0.000)\end{array}$ & $\begin{array}{l}0.0001 \\
(0.0003)\end{array}$ & $\begin{array}{l}0.917 \mathrm{E}-5 \\
(0.397 \mathrm{E}-4)\end{array}$ & $\begin{array}{l}0.000 \\
(0.000)\end{array}$ & $\begin{array}{l}0.0002 \\
(0.0002)\end{array}$ & $\begin{array}{l}0.152 \mathrm{E}-4 \\
(0.900 \mathrm{E}-4)\end{array}$ \\
\hline Offshore policy & -1.331 & -1.358 & -0.082 & -1.164 & -1.163 & -0.065 & $-3.117 \dagger$ & $-2.877^{*}$ & -0.181 & 0.178 & 0.109 & -0.0004 \\
\hline
\end{tabular}




\begin{tabular}{|c|c|c|c|c|c|c|c|c|c|c|c|c|}
\hline & $(0.894)$ & $(0.853)$ & $(0.135)$ & $(2.000)$ & $(1.767)$ & $(0.312)$ & (1.750) & (1.458) & $(0.272)$ & $(1.490)$ & (1.261) & $(0.413)$ \\
\hline $\begin{array}{l}\text { Internationalisation } \\
\text { at home strategy }\end{array}$ & $\begin{array}{l}0.053 \\
(0.162)\end{array}$ & $\begin{array}{l}-0.0003 \\
(0.0012)\end{array}$ & $\begin{array}{l}-0.174 \mathrm{E}-4 \\
(0.0003)\end{array}$ & $\begin{array}{l}0.130 \\
(0.347)\end{array}$ & $\begin{array}{l}0.1300 \\
(0.306)\end{array}$ & $\begin{array}{l}0.007 \\
(0.109)\end{array}$ & $\begin{array}{l}-0.109 \\
(0.349)\end{array}$ & $\begin{array}{l}-0.179 \mathrm{E}-4 \\
(0.002)\end{array}$ & $\begin{array}{l}-0.109 \mathrm{E}-4 \\
(0.042)\end{array}$ & $\begin{array}{l}-0.128 \\
(0.274)\end{array}$ & $\begin{array}{l}-0.0004 \\
(0.001)\end{array}$ & $\begin{array}{l}-0.196 \mathrm{E}-4 \\
(0.0004)\end{array}$ \\
\hline $\begin{array}{l}\text { Host country } \\
\text { demand }\end{array}$ & $\begin{array}{l}4.044 \\
(5.350)\end{array}$ & $\begin{array}{l}4.313 \\
(5.069)\end{array}$ & $\begin{array}{l}0.251 \\
(0.931)\end{array}$ & $\begin{array}{l}2.267 \\
(1.046)\end{array}$ & $\begin{array}{l}2.266 \\
(4.871)\end{array}$ & $\begin{array}{l}0.130 \\
(1.570)\end{array}$ & $\begin{array}{l}-76.168 \\
(95.488)\end{array}$ & $\begin{array}{l}-67.506 \\
(84.772)\end{array}$ & $\begin{array}{l}-3.854 \\
(11.975)\end{array}$ & $\begin{array}{l}9.666 \\
(77.205)\end{array}$ & $\begin{array}{l}2.273 \\
(66.118)\end{array}$ & $\begin{array}{l}0.218 \\
(17.507)\end{array}$ \\
\hline AU/NZ dummy & $\begin{array}{l}-0.154 \\
(0.716)\end{array}$ & $\begin{array}{l}-0.212 \\
(0.665)\end{array}$ & $\begin{array}{l}-0.012 \\
(0.110)\end{array}$ & - & - & - & - & - & - & - & - & - \\
\hline UK/IR dummy & $\begin{array}{l}-0.772 \\
(0.551)\end{array}$ & $\begin{array}{l}-0.760 \\
(0.523)\end{array}$ & $\begin{array}{l}-0.044 \\
(0.092)\end{array}$ & - & - & - & - & - & - & - & - & - \\
\hline $\mathrm{R}^{2}$ & 0.358 & & & 0.194 & & & 0.448 & & & 0.308 & & \\
\hline $\operatorname{Adj}^{2}$ & 0.317 & & & 0.001 & & & 0.380 & & & 0.115 & & \\
\hline Log likelihood & & -321.90 & -351.48 & & -70.42 & -79.67 & & -138.55 & -147.82 & & -105.89 & -122.43 \\
\hline $\mathrm{N}$ & 139 & 139 & 139 & 32 & 32 & 32 & 57 & 57 & 57 & 50 & 50 & 50 \\
\hline Max. VIF & 1.908 & & & 1.385 & & & 1.352 & & & 2.018 & & \\
\hline
\end{tabular}

Standard errors in parentheses, all $t$ tests are two-tailed, $\dagger p<.10, * p<.05, * * p<.01$

Table 7.41a: Regression estimates for equation 26a (low regulative institutional distance)

\begin{tabular}{|c|c|c|c|c|c|c|c|c|c|c|c|c|}
\hline & \multicolumn{3}{|c|}{ Complete data set } & \multicolumn{3}{|c|}{$\overline{\mathrm{AU} / \mathrm{NZ}}$} & \multicolumn{3}{|c|}{$\overline{\mathrm{US} / \mathrm{CA}}$} & \multicolumn{3}{|c|}{$\overline{\text { UK/IR }}$} \\
\hline & OLS & Tobit & $\begin{array}{l}\text { Negative } \\
\text { binomial }\end{array}$ & OLS & Tobit & $\begin{array}{l}\text { Negative } \\
\text { binomial }\end{array}$ & OLS & Tobit & $\begin{array}{l}\text { Negative } \\
\text { binomial }\end{array}$ & OLS & Tobit & $\begin{array}{l}\text { Negative } \\
\text { binomial }\end{array}$ \\
\hline Intercept & $\begin{array}{l}5.780 * * \\
(1.837)\end{array}$ & $\begin{array}{l}6.438 * * \\
(1.603)\end{array}$ & $\begin{array}{l}1.977 * * \\
(0.100)\end{array}$ & $\begin{array}{l}10.499 * * \\
(3.738)\end{array}$ & $\begin{array}{l}11.802 * * \\
(2.888)\end{array}$ & $\begin{array}{l}2.421 * * \\
(0.161)\end{array}$ & $\begin{array}{l}3.892 \\
(2.852)\end{array}$ & $\begin{array}{l}3.374 \\
(2.446)\end{array}$ & $\begin{array}{l}1.529 * * \\
(0.192)\end{array}$ & $\begin{array}{l}8.677 * * \\
(2.505)\end{array}$ & $\begin{array}{l}10.601 * * \\
(2.072)\end{array}$ & $\begin{array}{l}2.385 * * \\
(0.277)\end{array}$ \\
\hline $\begin{array}{l}\text { Financial resources } \\
\text { (H8e) }\end{array}$ & $\begin{array}{l}-0.128 \\
(0.169)\end{array}$ & $\begin{array}{l}-0.114 \\
(0.156)\end{array}$ & $\begin{array}{l}-0.007 \\
(0.012)\end{array}$ & $\begin{array}{l}-0.589 \\
(0.530)\end{array}$ & $\begin{array}{l}-0.509 \\
(0.435)\end{array}$ & $\begin{array}{l}-0.029 \\
(0.038)\end{array}$ & $\begin{array}{l}-0.047 \\
(0.262)\end{array}$ & $\begin{array}{l}-0.053 \\
(0.245)\end{array}$ & $\begin{array}{l}-0.006 \\
(0.026)\end{array}$ & $\begin{array}{l}-0.069 \\
(0.272)\end{array}$ & $\begin{array}{l}-0.012 \\
(0.216)\end{array}$ & $\begin{array}{l}-0.0009 \\
(0.040)\end{array}$ \\
\hline Age & $\begin{array}{l}0.008 \\
(0.006)\end{array}$ & $\begin{array}{l}0.005 \\
(0.005)\end{array}$ & $\begin{array}{l}0.0003 \\
(0.0004)\end{array}$ & $\begin{array}{l}0.022 \\
(0.023)\end{array}$ & $\begin{array}{l}0.018 \\
(0.018)\end{array}$ & $\begin{array}{l}0.001 \\
(0.002)\end{array}$ & $\begin{array}{l}0.008 \\
(0.012)\end{array}$ & $\begin{array}{l}0.008 \\
(0.010)\end{array}$ & $\begin{array}{l}0.0007 \\
(0.0008)\end{array}$ & $\begin{array}{l}0.003 \\
(0.007)\end{array}$ & $\begin{array}{l}0.001 \\
(0.006)\end{array}$ & $\begin{array}{l}0.906 \mathrm{E}-4 \\
(0.0009)\end{array}$ \\
\hline Size (Staff) & $\begin{array}{l}0.000 \\
(0.000)\end{array}$ & $\begin{array}{l}0.0002 \\
(0.0002)\end{array}$ & $\begin{array}{l}0.139 \mathrm{E}-4 \\
(0.196 \mathrm{E}-4)\end{array}$ & $\begin{array}{l}1.525 \mathrm{E}-5 \\
(0.001)\end{array}$ & $\begin{array}{l}-0.564 \mathrm{E}-4 \\
(0.0005)\end{array}$ & $\begin{array}{l}-0.645 E-5 \\
(0.469 E-4)\end{array}$ & $\begin{array}{l}0.000 \\
(0.000)\end{array}$ & $\begin{array}{l}0.0004 \\
(0.0004)\end{array}$ & $\begin{array}{l}0.329 \mathrm{E}-4 \\
(0.450 \mathrm{E}-4)\end{array}$ & $\begin{array}{l}-0.000 \\
(0.000)\end{array}$ & $\begin{array}{l}0.825 \mathrm{E}-4 \\
(0.0002)\end{array}$ & $\begin{array}{l}0.474 \mathrm{E}-5 \\
(0.406 \mathrm{E}-4)\end{array}$ \\
\hline Offshore policy & $\begin{array}{l}\text { 8.024** } \\
(1.153)\end{array}$ & $\begin{array}{l}\text { 8.085** } \\
(1.106)\end{array}$ & $\begin{array}{l}0.699 * * \\
(0.057)\end{array}$ & $\begin{array}{l}5.992^{*} \\
(2.562)\end{array}$ & $\begin{array}{l}5.858^{*} \\
(2.309)\end{array}$ & $\begin{array}{l}0.449 * * \\
(0.132)\end{array}$ & $\begin{array}{l}9.341 * * \\
(1.916)\end{array}$ & $\begin{array}{l}9.285 * * \\
(1.796)\end{array}$ & $\begin{array}{l}1.010^{* *} \\
(0.125)\end{array}$ & $\begin{array}{l}\text { 6.377** } \\
(1.876)\end{array}$ & $\begin{array}{l}6.260 * * \\
(1.717)\end{array}$ & $\begin{array}{l}0.440 * * \\
(0.133)\end{array}$ \\
\hline $\begin{array}{l}\text { Internationalisation } \\
\text { at home strategy }\end{array}$ & $\begin{array}{l}0.141 \\
(0.242)\end{array}$ & $\begin{array}{l}-0.0005 \\
(0.001)\end{array}$ & $\begin{array}{l}-0.243 \mathrm{E}-4 \\
(0.0001)\end{array}$ & $\begin{array}{l}0.476 \\
(0.679)\end{array}$ & $\begin{array}{l}-0.002 \\
(0.002)\end{array}$ & $\begin{array}{l}-0.001 \\
(0.0004)\end{array}$ & $\begin{array}{l}-0.173 \\
(0.380)\end{array}$ & $\begin{array}{l}-0.004 \\
(0.004)\end{array}$ & $\begin{array}{l}-0.0002 \\
(0.025)\end{array}$ & $\begin{array}{l}0.516 \\
(0.400)\end{array}$ & $\begin{array}{l}0.0010 \\
(0.001)\end{array}$ & $\begin{array}{l}0.582 \mathrm{E}-4 \\
(0.0002)\end{array}$ \\
\hline $\begin{array}{l}\text { Host country } \\
\text { demand }\end{array}$ & $\begin{array}{l}8.605^{*} \\
(3.383)\end{array}$ & $\begin{array}{l}\text { 7.891* } \\
(3.140)\end{array}$ & $\begin{array}{l}0.489 * \\
(0.243)\end{array}$ & $\begin{array}{l}4.660 \\
(6.639)\end{array}$ & $\begin{array}{l}5.988 \\
(5.703)\end{array}$ & $\begin{array}{l}0.361 \\
(0.590)\end{array}$ & $\begin{array}{l}\text { 12.326* } \\
(5.906)\end{array}$ & $\begin{array}{l}\text { 12.353* } \\
\text { (5.550) }\end{array}$ & $\begin{array}{l}0.848 \dagger \\
(0.475)\end{array}$ & $\begin{array}{l}8.976 \\
(6.060)\end{array}$ & $\begin{array}{l}4.232 \\
(4.623)\end{array}$ & $\begin{array}{l}0.243 \\
(0.704)\end{array}$ \\
\hline
\end{tabular}




\begin{tabular}{|c|c|c|c|c|c|c|c|c|c|c|c|c|}
\hline AU/NZ dummy & $\begin{array}{l}1.065 \\
(0.948)\end{array}$ & $\begin{array}{l}1.030 \\
(0.895)\end{array}$ & $\begin{array}{l}0.069 \\
(0.067)\end{array}$ & - & - & - & - & - & - & - & - & - \\
\hline UK/IR dummy & $\begin{array}{l}\text { 1.861* } \\
(0.875)\end{array}$ & $\begin{array}{l}1.570 \dagger \\
(\mathbf{0 . 8 2 4})\end{array}$ & $\begin{array}{l}0.097 \\
(0.068)\end{array}$ & - & - & - & - & - & - & - & - & - \\
\hline $\mathrm{R}^{2}$ & 0.336 & & & 0.204 & & & 0.423 & & & 0.284 & & \\
\hline $\operatorname{AdjR}^{2}$ & 0.301 & & & 0.075 & & & 0.365 & & & 0.180 & & \\
\hline Log likelihood & & -468.03 & -499.29 & & -134.91 & -144.74 & & -190.98 & -203.80 & & -131.21 & -141.66 \\
\hline $\mathrm{N}$ & 169 & 169 & 169 & 48 & 48 & 48 & 67 & 67 & 67 & 54 & 54 & 54 \\
\hline Max. VIF & 1.752 & & & 2.289 & & & 1.374 & & & 1.747 & & \\
\hline
\end{tabular}

Table 7.41b: Regression estimates for equation $26 \mathrm{~b}$ (high regulative institutional distance)

\begin{tabular}{|c|c|c|c|c|c|c|c|c|c|c|c|c|}
\hline & \multicolumn{3}{|c|}{ Complete data set } & \multicolumn{3}{|c|}{ AU/NZ } & \multicolumn{3}{|c|}{ US/CA } & \multicolumn{3}{|c|}{$\overline{\mathrm{UK} / \mathrm{IR}}$} \\
\hline & OLS & Tobit & $\begin{array}{l}\text { Negative } \\
\text { binomial } \\
\end{array}$ & OLS & Tobit & $\begin{array}{l}\text { Negative } \\
\text { binomial } \\
\end{array}$ & OLS & Tobit & $\begin{array}{l}\text { Negative } \\
\text { binomial } \\
\end{array}$ & OLS & Tobit & $\begin{array}{l}\text { Negative } \\
\text { binomial }\end{array}$ \\
\hline Intercept & $\begin{array}{l}17.246 * * \\
(1.577)\end{array}$ & $\begin{array}{l}17.658 * * \\
(1.443)\end{array}$ & $\begin{array}{l}2.871 * * \\
(0.178)\end{array}$ & $\begin{array}{l}17.091 * * \\
(2.175)\end{array}$ & $\begin{array}{l}17.091 * * \\
(1.922)\end{array}$ & $\begin{array}{l}2.834 * * \\
(0.372)\end{array}$ & $\begin{array}{l}24.553 * * \\
(4.172)\end{array}$ & $\begin{array}{l}23.813 * * \\
(3.073)\end{array}$ & $\begin{array}{l}3.244 * * \\
(0.404)\end{array}$ & $\begin{array}{l}14.767 * * \\
(2.074)\end{array}$ & $\begin{array}{l}14.854 * * \\
(1.918)\end{array}$ & $\begin{array}{l}2.693 * * \\
(0.644)\end{array}$ \\
\hline $\begin{array}{l}\text { Financial resources } \\
(\mathrm{H} 8 \mathrm{e})\end{array}$ & $\begin{array}{l}-0.198 \\
(0.170)\end{array}$ & $\begin{array}{l}-0.123 \\
(0.155)\end{array}$ & $\begin{array}{l}-0.007 \\
(0.020)\end{array}$ & $\begin{array}{l}0.019 \\
(0.366)\end{array}$ & $\begin{array}{l}0.019 \\
(0.323)\end{array}$ & $\begin{array}{l}0.0009 \\
(0.089)\end{array}$ & $\begin{array}{l}-0.088 \\
(0.294)\end{array}$ & $\begin{array}{l}-0.107 \\
(0.265)\end{array}$ & $\begin{array}{l}-0.006 \\
(0.033)\end{array}$ & $\begin{array}{l}-0.449 \dagger \\
(0.238)\end{array}$ & $\begin{array}{l}-0.368 \dagger \\
(0.213)\end{array}$ & $\begin{array}{l}-0.023 \\
(0.046)\end{array}$ \\
\hline Age & $\begin{array}{l}-0.001 \\
(0.005)\end{array}$ & $\begin{array}{l}-0.002 \\
(0.005)\end{array}$ & $\begin{array}{l}-0.0001 \\
(0.0005)\end{array}$ & $\begin{array}{l}-0.011 \\
(0.012)\end{array}$ & $\begin{array}{l}-0.011 \\
(0.010)\end{array}$ & $\begin{array}{l}-0.0006 \\
(0.0029)\end{array}$ & $\begin{array}{l}-0.006 \\
(0.010)\end{array}$ & $\begin{array}{l}-0.005 \\
(0.009)\end{array}$ & $\begin{array}{l}-0.0003 \\
(0.0007)\end{array}$ & $\begin{array}{l}0.009 \\
(0.007)\end{array}$ & $\begin{array}{l}0.007 \\
(0.006)\end{array}$ & $\begin{array}{l}0.0004 \\
(0.001)\end{array}$ \\
\hline Size (Staff) & $\begin{array}{l}0.000 \\
(0.000)\end{array}$ & $\begin{array}{l}0.0003 \\
(0.0002)\end{array}$ & $\begin{array}{l}0.193 \mathrm{E}-4 \\
(0.302 \mathrm{E}-4)\end{array}$ & $\begin{array}{l}0.001 \\
(0.001)\end{array}$ & $\begin{array}{l}\mathbf{0 . 0 0 0 9} \dagger \\
(\mathbf{0 . 0 0 0 5 )}\end{array}$ & $\begin{array}{l}0.542 \mathrm{E}-4 \\
(0.0001)\end{array}$ & $\begin{array}{l}0.000 \\
(0.000)\end{array}$ & $\begin{array}{l}0.0001 \\
(0.0004)\end{array}$ & $\begin{array}{l}0.971 \mathrm{E}-5 \\
(0.423 \mathrm{E}-4)\end{array}$ & $\begin{array}{l}0.000 \\
(0.000)\end{array}$ & $\begin{array}{l}0.0004 \\
(0.0002)\end{array}$ & $\begin{array}{l}0.289 \mathrm{E}-4 \\
(0.752 \mathrm{E}-4)\end{array}$ \\
\hline Offshore policy & $\begin{array}{l}-0.616 \\
(1.090)\end{array}$ & $\begin{array}{l}-0.769 \\
(1.044)\end{array}$ & $\begin{array}{l}-0.045 \\
(0.101)\end{array}$ & $\begin{array}{l}-1.392 \\
(1.853)\end{array}$ & $\begin{array}{l}-1.392 \\
(1.637)\end{array}$ & $\begin{array}{l}-0.077 \\
(0.280)\end{array}$ & $\begin{array}{l}-2.786 \\
(2.203)\end{array}$ & $\begin{array}{l}-2.495 \\
(1.823)\end{array}$ & $\begin{array}{l}-0.151 \\
(0.134)\end{array}$ & $\begin{array}{l}1.866 \\
(1.608)\end{array}$ & $\begin{array}{l}1.917 \\
(1.474)\end{array}$ & $\begin{array}{l}0.124 \\
(0.496)\end{array}$ \\
\hline $\begin{array}{l}\text { Internationalisation } \\
\text { at home strategy }\end{array}$ & $\begin{array}{l}0.228 \\
(0.201)\end{array}$ & $\begin{array}{l}-0.0009 \\
(0.001)\end{array}$ & $\begin{array}{l}-0.563 \mathrm{E}-4 \\
(0.0002)\end{array}$ & $\begin{array}{l}0.149 \\
(0.351)\end{array}$ & $\begin{array}{l}0.149 \\
(0.310)\end{array}$ & $\begin{array}{l}0.008 \\
(0.108)\end{array}$ & $\begin{array}{l}-0.132 \\
(0.448)\end{array}$ & $\begin{array}{l}-0.001 \\
(0.003)\end{array}$ & $\begin{array}{l}-0.878 \mathrm{E}-4 \\
(0.042)\end{array}$ & $\begin{array}{l}0.153 \\
(0.281)\end{array}$ & $\begin{array}{l}-0.001 \\
(0.001)\end{array}$ & $\begin{array}{l}-0.944 \mathrm{E}-4 \\
(0.0002)\end{array}$ \\
\hline $\begin{array}{l}\text { Host country } \\
\text { demand }\end{array}$ & $\begin{array}{l}2.117 \\
(6.469)\end{array}$ & $\begin{array}{l}2.879 \\
(6.193)\end{array}$ & $\begin{array}{l}0.165 \\
(0.870)\end{array}$ & $\begin{array}{l}2.022 \\
(5.556)\end{array}$ & $\begin{array}{l}2.022 \\
(4.910)\end{array}$ & $\begin{array}{l}0.117 \\
(1.539)\end{array}$ & $\begin{array}{l}-267.298 * \\
(110.586)\end{array}$ & $\begin{array}{l}-257.431 * * \\
(97.691)\end{array}$ & $\begin{array}{l}-15.610 \\
(11.630)\end{array}$ & $\begin{array}{l}14.068 \\
(78.319)\end{array}$ & $\begin{array}{l}14.883 \\
(72.973)\end{array}$ & $\begin{array}{l}0.998 \\
(16.026)\end{array}$ \\
\hline AU/NZ dummy & $\begin{array}{l}0.589 \\
(0.959)\end{array}$ & $\begin{array}{l}0.601 \\
(0.917)\end{array}$ & $\begin{array}{l}0.035 \\
(0.122)\end{array}$ & - & - & - & (1-tionos) & $(-100-1$ & - & (20) & (- & - \\
\hline UK/IR dummy & $\begin{array}{l}-0.422 \\
(0.811)\end{array}$ & $\begin{array}{l}-0.180 \\
(0.759)\end{array}$ & $\begin{array}{l}-0.010 \\
(0.102)\end{array}$ & - & - & - & - & - & - & - & - & - \\
\hline $\mathrm{R}^{2}$ & 0.061 & & & 0.192 & & & 0.135 & & & 0.129 & & \\
\hline $\operatorname{Adj}^{2}$ & 0.002 & & & -0.002 & & & 0.029 & & & -0.001 & & \\
\hline
\end{tabular}




\begin{tabular}{|c|c|c|c|c|c|c|c|c|c|c|c|c|}
\hline Log likelihood & & -349.26 & -364.57 & & -70.48 & -79.69 & & -151.34 & -155.97 & & -112.56 & -124.39 \\
\hline $\mathrm{N}$ & 139 & 139 & 139 & 32 & 32 & 32 & 57 & 57 & 57 & 50 & 50 & 50 \\
\hline Max. VIF & 2.447 & & & 2.593 & & & 1.549 & & & 1.667 & & \\
\hline
\end{tabular}

Table 7.42a: Regression estimates for equation 27a (low regulative institutional distance)

\begin{tabular}{|c|c|c|c|c|c|c|c|c|c|c|c|c|}
\hline & \multicolumn{3}{|c|}{ Complete data set } & \multicolumn{3}{|c|}{$\overline{\mathrm{AU} / \mathrm{NZ}}$} & \multicolumn{3}{|c|}{ US/CA } & \multicolumn{3}{|c|}{$\overline{\mathrm{UK} / \mathrm{IR}}$} \\
\hline & OLS & Tobit & $\begin{array}{l}\text { Negative } \\
\text { binomial }\end{array}$ & OLS & Tobit & $\begin{array}{l}\text { Negative } \\
\text { binomial }\end{array}$ & OLS & Tobit & $\begin{array}{l}\text { Negative } \\
\text { binomial }\end{array}$ & OLS & Tobit & $\begin{array}{l}\text { Negative } \\
\text { binomial }\end{array}$ \\
\hline Intercept & $\begin{array}{l}16.759 * * \\
(2.906)\end{array}$ & $\begin{array}{l}17.285^{* *} \\
(2.523)\end{array}$ & $\begin{array}{l}2.648 * * \\
(0.208)\end{array}$ & $\begin{array}{l}22.973 * * \\
(5.703)\end{array}$ & $\begin{array}{l}23.697 * * \\
(4.590)\end{array}$ & $\begin{array}{l}3.146 * * \\
(0.345)\end{array}$ & $\begin{array}{l}21.393 * * \\
(4.904)\end{array}$ & $\begin{array}{l}20.388 * * \\
(4.441)\end{array}$ & $\begin{array}{l}2.720 * * \\
(0.338)\end{array}$ & $\begin{array}{l}8.453 \dagger \\
(4.519)\end{array}$ & $\begin{array}{l}12.405 * * \\
(3.555)\end{array}$ & $\begin{array}{l}2.489 * * \\
(0.562)\end{array}$ \\
\hline $\begin{array}{l}\text { Reputation (Factor) } \\
\text { (H8f) }\end{array}$ & $\begin{array}{l}-1.540 * * \\
(0.329)\end{array}$ & $\begin{array}{l}-1.560 * * \\
(0.299)\end{array}$ & $\begin{array}{l}-0.098 * * \\
(0.025)\end{array}$ & $\begin{array}{l}-2.094 * * \\
(0.723)\end{array}$ & $\begin{array}{l}-2.132 * * \\
(0.628)\end{array}$ & $\begin{array}{l}-0.130 * \\
(0.056)\end{array}$ & $\begin{array}{l}-2.267 * * \\
(0.563)\end{array}$ & $\begin{array}{l}-2.244 * * \\
(0.529)\end{array}$ & $\begin{array}{l}-0.161 * * \\
(0.040)\end{array}$ & $\begin{array}{l}0.009 \\
(0.484)\end{array}$ & $\begin{array}{l}-0.245 \\
(0.398)\end{array}$ & $\begin{array}{l}-0.014 \\
(0.071)\end{array}$ \\
\hline Age & $\begin{array}{l}0.008 \\
(0.006)\end{array}$ & $\begin{array}{l}0.005 \\
(0.005)\end{array}$ & $\begin{array}{l}0.0003 \\
(0.0004)\end{array}$ & $\begin{array}{l}0.001 \\
(0.017)\end{array}$ & $\begin{array}{l}0.0001 \\
(0.014)\end{array}$ & $\begin{array}{l}-0.101 E-4 \\
(0.001)\end{array}$ & $\begin{array}{l}0.012 \\
(0.010)\end{array}$ & $\begin{array}{l}0.012 \\
(0.009)\end{array}$ & $\begin{array}{l}0.001 \dagger \\
(0.0006)\end{array}$ & $\begin{array}{l}0.003 \\
(0.007)\end{array}$ & $\begin{array}{l}0.001 \\
(0.006)\end{array}$ & $\begin{array}{l}0.836 \mathrm{E}-4 \\
(0.0008)\end{array}$ \\
\hline Size (Staff) & $\begin{array}{l}0.000 \\
(0.000)\end{array}$ & $\begin{array}{l}0.001 \\
(0.002)\end{array}$ & $\begin{array}{l}0.102 \mathrm{E}-4 \\
(0.188 \mathrm{E}-4)\end{array}$ & $\begin{array}{l}0.000 \\
(0.001)\end{array}$ & $\begin{array}{l}0.884 \mathrm{E}-4 \\
(0.0004)\end{array}$ & $\begin{array}{l}0.533 E-5 \\
(0.386 E-4)\end{array}$ & $\begin{array}{l}0.000 \\
(0.000)\end{array}$ & $\begin{array}{l}0.0002 \\
(0.0003)\end{array}$ & $\begin{array}{l}0.166 E-4 \\
(0.325 E-4)\end{array}$ & $\begin{array}{l}-0.000 \\
(0.000)\end{array}$ & $\begin{array}{l}0.628 \mathrm{E}-4 \\
(0.0002)\end{array}$ & $\begin{array}{l}0.348 \mathrm{E}-5 \\
(0.423 \mathrm{E}-4)\end{array}$ \\
\hline Offshore policy & $\begin{array}{l}\text { 6.325** } \\
(1.131)\end{array}$ & $\begin{array}{l}\text { 6.353** } \\
(1.074)\end{array}$ & $\begin{array}{l}0.590 * * \\
(0.059)\end{array}$ & $\begin{array}{l}4.768^{*} \\
(2.325)\end{array}$ & $\begin{array}{l}4.691 * \\
(2.079)\end{array}$ & $\begin{array}{l}0.380 * * \\
(0.132)\end{array}$ & $\begin{array}{l}6.706^{* *} \\
(1.813)\end{array}$ & $\begin{array}{l}\text { 6.659** } \\
(1.708)\end{array}$ & $\begin{array}{l}0.816 * * \\
(0.107)\end{array}$ & $\begin{array}{l}6.280 * * \\
(1.888)\end{array}$ & $\begin{array}{l}\text { 6.006** } \\
(1.721)\end{array}$ & $\begin{array}{l}0.425^{* *} \\
(0.137)\end{array}$ \\
\hline $\begin{array}{l}\text { Internationalisation } \\
\text { at home strategy }\end{array}$ & $\begin{array}{l}0.029 \\
(0.226)\end{array}$ & $\begin{array}{l}0.002 \\
(0.001)\end{array}$ & $\begin{array}{l}0.227 \mathrm{E}-4 \\
(0.0001)\end{array}$ & $\begin{array}{l}0.105 \\
(0.611)\end{array}$ & $\begin{array}{l}0.399 \mathrm{E}-4 \\
(0.002)\end{array}$ & $\begin{array}{l}0.159 \mathrm{E}-4 \\
(0.0006)\end{array}$ & $\begin{array}{l}-0.250 \\
(0.336)\end{array}$ & $\begin{array}{l}-0.001 \\
(0.003)\end{array}$ & $\begin{array}{l}-0.835 E-4 \\
(0.018)\end{array}$ & $\begin{array}{l}0.482 \\
(0.378)\end{array}$ & $\begin{array}{l}0.001 \\
(0.001)\end{array}$ & $\begin{array}{l}0.579 \mathrm{E}-4 \\
(0.0002)\end{array}$ \\
\hline $\begin{array}{l}\text { Host country } \\
\text { demand }\end{array}$ & $\begin{array}{l}\text { 8.114* } \\
\text { (3.150) }\end{array}$ & $\begin{array}{l}7.479 * * \\
(2.896)\end{array}$ & $\begin{array}{l}0.483 \dagger \\
(0.251)\end{array}$ & $\begin{array}{l}11.421 \dagger \\
(6.321)\end{array}$ & $\begin{array}{l}11.608 * \\
(5.271)\end{array}$ & $\begin{array}{l}0.688 \\
(0.606)\end{array}$ & $\begin{array}{l}7.516 \\
(5.352)\end{array}$ & $\begin{array}{l}7.627 \\
(5.043)\end{array}$ & $\begin{array}{l}0.593 \\
(0.427)\end{array}$ & $\begin{array}{l}8.887 \\
(6.098)\end{array}$ & $\begin{array}{l}4.001 \\
(4.592)\end{array}$ & $\begin{array}{l}0.232 \\
(0.692)\end{array}$ \\
\hline AU/NZ dummy & $\begin{array}{l}1.453 \dagger \\
(0.836)\end{array}$ & $\begin{array}{l}1.330 \dagger \\
(0.776)\end{array}$ & $\begin{array}{l}0.092 \\
(0.066)\end{array}$ & - & - & - & - & - & $(0.721)$ & $(0.000)$ & 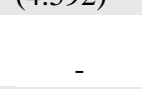 & $(0.002)$ \\
\hline UK/IR dummy & $\begin{array}{l}2.093 \dagger \\
(0.736)\end{array}$ & $\begin{array}{l}1.884 * * \\
(0.685)\end{array}$ & $\begin{array}{l}\text { 0.119* } \\
(0.055)\end{array}$ & - & - & - & - & - & - & - & - & - \\
\hline $\mathrm{R}^{2}$ & 0.419 & & & 0.329 & & & 0.547 & & & 0.283 & & \\
\hline $\operatorname{AdjR}^{2}$ & 0.388 & & & 0.221 & & & 0.501 & & & 0.178 & & \\
\hline Log likelihood & & -455.68 & -488.38 & & -130.42 & -140.60 & & -183.06 & -195.79 & & -131.02 & -141.58 \\
\hline $\mathrm{N}$ & 169 & 169 & 169 & 48 & 48 & 48 & 67 & 67 & 67 & 54 & 54 & 54 \\
\hline Max. VIF & 1.559 & & & 1.300 & & & 1.338 & & & 1.559 & & \\
\hline
\end{tabular}

Standard errors in parentheses, all $t$ tests are two-tailed, $\dagger p<.10,{ }^{*} p<.05,{ }^{* *} p<.01$ 
Table 7.42b: Regression estimates for equation 27b (high regulative institutional distance)

\begin{tabular}{|c|c|c|c|c|c|c|c|c|c|c|c|c|}
\hline & \multicolumn{3}{|c|}{ Complete data set } & \multicolumn{3}{|c|}{ AU/NZ } & \multicolumn{3}{|c|}{$\overline{\text { US/CA }}$} & \multicolumn{3}{|c|}{ 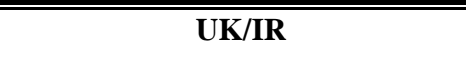 } \\
\hline & OLS & Tobit & $\begin{array}{l}\text { Negative } \\
\text { binomial }\end{array}$ & OLS & Tobit & $\begin{array}{l}\text { Negative } \\
\text { binomial }\end{array}$ & OLS & Tobit & $\begin{array}{l}\text { Negative } \\
\text { binomial }\end{array}$ & OLS & Tobit & $\begin{array}{l}\text { Negative } \\
\text { binomial }\end{array}$ \\
\hline Intercept & $\begin{array}{l}22.772 * * \\
(2.143)\end{array}$ & $\begin{array}{l}23.129 * * \\
(1.946)\end{array}$ & $\begin{array}{l}3.202^{* *} \\
(0.245)\end{array}$ & $\begin{array}{l}16.296 * * \\
(3.788)\end{array}$ & $\begin{array}{l}16.296 * * \\
(3.348)\end{array}$ & $\begin{array}{l}2.787 * * \\
(0.783)\end{array}$ & $\begin{array}{l}32.668 * * \\
(4.657)\end{array}$ & $\begin{array}{l}30.423 * * \\
(3.369)\end{array}$ & $\begin{array}{l}\text { 3.668** } \\
(0.460)\end{array}$ & $\begin{array}{l}21.203 * * \\
(2.832)\end{array}$ & $\begin{array}{l}20.661^{* *} \\
(2.620)\end{array}$ & $\begin{array}{l}3.054 * * \\
(0.580)\end{array}$ \\
\hline $\begin{array}{l}\text { Reputation (Factor) } \\
\text { (H8f) }\end{array}$ & $\begin{array}{l}-0.996 * * \\
(0.262)\end{array}$ & $\begin{array}{l}-0.971 * * \\
(0.249)\end{array}$ & $\begin{array}{l}-0.058 \dagger \\
(0.033)\end{array}$ & $\begin{array}{l}0.133 \\
(0.511)\end{array}$ & $\begin{array}{l}0.132 \\
(0.451)\end{array}$ & $\begin{array}{l}0.007 \\
(0.105)\end{array}$ & $\begin{array}{l}-1.370 * * \\
(0.456)\end{array}$ & $\begin{array}{l}-1.327^{* *} \\
(0.422)\end{array}$ & $\begin{array}{l}-0.083 \\
(0.058)\end{array}$ & $\begin{array}{l}-1.231 * * \\
(0.363)\end{array}$ & $\begin{array}{l}-1.100^{* *} \\
(0.329)\end{array}$ & $\begin{array}{l}-0.066 \\
(0.058)\end{array}$ \\
\hline Age & $\begin{array}{l}-0.005 \\
(0.005)\end{array}$ & $\begin{array}{l}-0.005 \\
(0.004)\end{array}$ & $\begin{array}{l}-0.0003 \\
(0.0005)\end{array}$ & $\begin{array}{l}-0.010 \\
(0.010)\end{array}$ & $\begin{array}{l}-0.009 \\
(0.009)\end{array}$ & $\begin{array}{l}-0.0005 \\
(0.001)\end{array}$ & $\begin{array}{l}-0.009 \\
(0.009)\end{array}$ & $\begin{array}{l}-0.008 \\
(0.007)\end{array}$ & $\begin{array}{l}-0.0005 \\
(0.0008)\end{array}$ & $\begin{array}{l}0.007 \\
(0.006)\end{array}$ & $\begin{array}{l}0.006 \\
(0.005)\end{array}$ & $\begin{array}{l}0.0003 \\
(0.001)\end{array}$ \\
\hline Size (Staff) & $\begin{array}{l}0.000 \\
(0.000)\end{array}$ & $\begin{array}{l}0.0002 \\
(0.002)\end{array}$ & $\begin{array}{l}0.165 E-4 \\
(0.301 E-4)\end{array}$ & $\begin{array}{l}0.001 \dagger \\
(0.001)\end{array}$ & $\begin{array}{l}0.0009 * \\
(0.0004)\end{array}$ & $\begin{array}{l}0.546 \mathrm{E}-4 \\
(0.0001)\end{array}$ & $\begin{array}{l}-0.000 \\
(0.000)\end{array}$ & $\begin{array}{l}0.340 \mathrm{E}-4 \\
(0.0003)\end{array}$ & $\begin{array}{l}0.292 \mathrm{E}-5 \\
(0.392 \mathrm{E}-4)\end{array}$ & $\begin{array}{l}0.000 \\
(0.000)\end{array}$ & $\begin{array}{l}0.0003 \\
(0.0002)\end{array}$ & $\begin{array}{l}0.214 \mathrm{E}-4 \\
(0.705 \mathrm{E}-4)\end{array}$ \\
\hline Offshore policy & $\begin{array}{l}-0.585 \\
(1.028)\end{array}$ & $\begin{array}{l}-0.653 \\
(0.987)\end{array}$ & $\begin{array}{l}-0.039 \\
(0.108)\end{array}$ & $\begin{array}{l}-1.458 \\
(1.867)\end{array}$ & $\begin{array}{l}-1.457 \\
(1.650)\end{array}$ & $\begin{array}{l}-0.081 \\
(0.285)\end{array}$ & $\begin{array}{l}-2.216 \\
(2.026)\end{array}$ & $\begin{array}{l}-1.562 \\
(1.712)\end{array}$ & $\begin{array}{l}-0.097 \\
(0.165)\end{array}$ & $\begin{array}{l}0.551 \\
(1.431)\end{array}$ & $\begin{array}{l}0.844 \\
(1.300)\end{array}$ & $\begin{array}{l}0.049 \\
(0.365)\end{array}$ \\
\hline $\begin{array}{l}\text { Internationalisation } \\
\text { at home strategy }\end{array}$ & $\begin{array}{l}0.150 \\
(0.186)\end{array}$ & $\begin{array}{l}-0.0006 \\
(0.001)\end{array}$ & $\begin{array}{l}-0.392 \mathrm{E}-4 \\
(0.0002)\end{array}$ & $\begin{array}{l}0.159 \\
(0.338)\end{array}$ & $\begin{array}{l}0.159 \\
(0.298)\end{array}$ & $\begin{array}{l}0.009 \\
(0.111)\end{array}$ & $\begin{array}{l}-0.309 \\
(0.404)\end{array}$ & $\begin{array}{l}-0.0001 \\
(0.003)\end{array}$ & $\begin{array}{l}-0.182 \mathrm{E}-4 \\
(0.030)\end{array}$ & $\begin{array}{l}0.183 \\
(0.256)\end{array}$ & $\begin{array}{l}-0.001 \\
(0.001)\end{array}$ & $\begin{array}{l}-0.825 \mathrm{E}-4 \\
(0.0002)\end{array}$ \\
\hline $\begin{array}{l}\text { Host country } \\
\text { demand }\end{array}$ & $\begin{array}{l}0.968 \\
(6.150)\end{array}$ & $\begin{array}{l}1.667 \\
(5.871)\end{array}$ & $\begin{array}{l}0.087 \\
(0.970)\end{array}$ & $\begin{array}{l}2.185 \\
(5.492)\end{array}$ & $\begin{array}{l}2.185 \\
(4.854)\end{array}$ & $\begin{array}{l}0.127 \\
(1.552)\end{array}$ & $\begin{array}{l}-232.771 * \\
(102.331)\end{array}$ & $\begin{array}{l}-210.489 * \\
(91.479)\end{array}$ & $\begin{array}{l}-12.893 \\
(11.403)\end{array}$ & $\begin{array}{l}39.194 \\
(71.490)\end{array}$ & $\begin{array}{l}36.865 \\
(67.330)\end{array}$ & $\begin{array}{l}2.274 \\
(14.675)\end{array}$ \\
\hline AU/NZ dummy & $\begin{array}{l}0.804 \\
(0.807)\end{array}$ & $\begin{array}{l}0.672 \\
(0.757)\end{array}$ & $\begin{array}{l}0.040 \\
(0.101)\end{array}$ & - & - & - & - & - & - & - & - & - \\
\hline UK/IR dummy & $\begin{array}{l}-0.106 \\
(0.623)\end{array}$ & $\begin{array}{l}-0.070 \\
(0.596)\end{array}$ & $\begin{array}{l}-0.003 \\
(0.087)\end{array}$ & - & - & - & - & - & - & - & - & - \\
\hline $\mathrm{R}^{2}$ & 0.149 & & & 0.194 & & & 0.268 & & & 0.263 & & \\
\hline $\operatorname{Adj}^{2}$ & 0.095 & & & 0.000 & & & 0.178 & & & 0.152 & & \\
\hline Log likelihood & & -342.39 & -361.04 & & -70.44 & -76.68 & & -146.87 & -152.97 & & -108.95 & -123.34 \\
\hline $\mathrm{N}$ & 139 & 139 & 139 & 32 & 32 & 32 & 57 & 57 & 57 & 50 & 50 & 50 \\
\hline Max. VIF & 1.827 & & & 1.170 & & & 1.371 & & & 1.320 & & \\
\hline
\end{tabular}

Standard errors in parentheses, all $t$ tests are two-tailed, $\uparrow p<.10, * p<.05, * * p<.01$ 


\subsection{TESTING FOR HYPOTHESIS 4}

As indicated above, H4, with respect to learning intent, is not included in equations 127 (see tables 7.10-7.42) since it applies only to joint ventures. Rather, to test H4, t-tests are conducted to compare mean levels of learning intent between majority and minority JVs. Equal ownership is used as the delineation, so that joint ventures with 50/50 ownership are classified with JVs having minority ownership. The results presented below do not support $\mathrm{H} 4$. The independent sample t-tests suggest no significant differences in the average learning intents between majority and minority JVs.

\section{Table 7.43: Independent Sample t-test (equal variances assumed based on}

\section{Levene's test)}

\begin{tabular}{|c|c|c|c|c|c|c|c|}
\hline \multicolumn{8}{|c|}{ Independent Samples Test } \\
\hline & & \multicolumn{2}{|c|}{$\begin{array}{c}\text { Levene's Test for } \\
\text { Equality of Variances }\end{array}$} & \multicolumn{4}{|c|}{ t-test for Equality of Means } \\
\hline & & $\mathrm{F}$ & Sig. & $\mathrm{t}$ & df & Sig. (2-tailed) & $\begin{array}{l}\text { Std. Error } \\
\text { Difference }\end{array}$ \\
\hline $\begin{array}{l}\begin{array}{l}\text { Learning Intent } \\
\text { (time } \\
\text { considerations) }\end{array} \\
\end{array}$ & $\begin{array}{l}\text { Equal variances } \\
\text { assumed }\end{array}$ & .010 & .922 & .664 & 21 & .514 & .309 \\
\hline $\begin{array}{l}\text { Learning Intent } \\
\text { (cost } \\
\text { considerations) }\end{array}$ & $\begin{array}{l}\text { Equal variances } \\
\text { assumed }\end{array}$ & .410 & .529 & -.023 & 21 & .982 & .391 \\
\hline $\begin{array}{l}\text { Learning Intent } \\
\text { (profit } \\
\text { considerations) }\end{array}$ & $\begin{array}{l}\text { Equal variances } \\
\text { assumed }\end{array}$ & .008 & .928 & .102 & 21 & .919 & .349 \\
\hline $\begin{array}{l}\text { Learning Intent } \\
\text { (skills } \\
\text { development } \\
\text { considerations) } \\
\end{array}$ & \begin{tabular}{|l|}
$\begin{array}{l}\text { Equal variances } \\
\text { assumed }\end{array}$ \\
\end{tabular} & .256 & .618 & 1.214 & 21 & .238 & .294 \\
\hline $\begin{array}{l}\begin{array}{l}\text { Learning Intent } \\
\text { (deeper } \\
\text { understanding } \\
\text { of partner's } \\
\text { expertise) }\end{array} \\
\end{array}$ & $\begin{array}{l}\text { Equal variances } \\
\text { assumed }\end{array}$ & .154 & .699 & .420 & 21 & .679 & .362 \\
\hline \begin{tabular}{l|} 
Learning Intent \\
(skills \\
duplication \\
considerations)
\end{tabular} & \begin{tabular}{|l|} 
Equal variances \\
assumed
\end{tabular} & .801 & .381 & .784 & 21 & .442 & .330 \\
\hline $\begin{array}{l}\text { Learning Intent } \\
\text { (understanding } \\
\text { of partner's } \\
\text { perspective, } \\
\text { systems and } \\
\text { know-how) }\end{array}$ & $\begin{array}{l}\text { Equal variances } \\
\text { assumed }\end{array}$ & 2.482 & .130 & -.442 & 21 & .663 & .404 \\
\hline
\end{tabular}




\subsection{SUMMARY OF FINDINGS}

From the results, I conclude that at best mixed support for H1a (Geographic experience) is observed. As summarised in table 7.44, while significant positive findings are found in support of H1a across the different equations and estimation techniques (at least $p<$ 0.10), significant negative findings are also obtained. For example, for AU/NZ, significant positive coefficients are obtained for geographic experiences - international recruitment while significant negative findings are found with respect to this same aspect of geographic experience for US/CA. Similarly, for geographic experience financial capabilities, significant positive findings are obtained for US/CA while significant negative coefficients are found for UK/IR. These geographical differences highlight the benefit of analysing the data across geographical clusters rather than in an aggregate format.

Similarly, only partial support is also obtained with respect to H1b (Industry experience). While a positive relationship between industry experience and equity stake is hypothesised, both significant positive and negative findings are found across the different equations and estimation techniques (at least $p<0.10$ ) (see table 7.45).

From the findings outlined in tables 7.44 and 7.45, I conjecture, in contrast to the hypothesised positive relationship, that the negative coefficients obtained with respect to geographic and industry experience, can be explained as follows. In the education context, less resource intensive entry modes may be riskier than more resource intensive entry modes such that, with more experience comes the ability to deal with the uncertainties associated with less resource intensive modes of entry. As indicated in Section 7.3.6, non-equity arrangements in the education services context is interpreted by the respondents to include academic investments and not just financial capital investment as in the more commercial sense. Therefore, it can be postulated that given such academic investments, there is an associated risk in being involved in non-equity arrangements given less academic control over jointly delivered programmes. For example, although quality assurance mechanisms can be incorporated in twinning arrangement contracts, an issue of enforcement arises as institutions do not have control 
over the delivery of academic courses at their partners' campuses. Given this lack of enforcement control, a student from a local institution transferring to a twinning partner overseas might not be equipped with sufficient academic preparation even if, on paper, s/he has taken the equivalent prerequisite courses to gain advanced credit standing. In this respect, it can be suggested that experience is required for institutions to adequately manage the academic investments associated with non-equity arrangements. It may be that, if institutions do not possess such experience, they might prefer to engage in offshore education developments via more resource intensive modes of entry in order to maintain control over the academic delivery process.

The interviews shed some light on this. Quality assurance is a key theme that emerged during the exploratory qualitative phase of this research (see Chapter 6). The following comments from UK1, is representative of the many others that highlight the importance of quality assurance:

Ensuring the quality assurance of our courses is extremely important to us. Therefore, our due diligence process is very strict when we decide to partner with a foreign institution to deliver our courses offshore. The stance we often take is that we will not partner with anyone in an offshore campus operation in order to preserve our reputation.... This way, we are able to strictly ensure the quality of who gets into our programme....

Furthermore, I conjecture that, with increased geographic and industry experience, universities learn about how to deal with the local institutional environment, and potentially more importantly, they have the prospect to develop relationships with local institutions. As highlighted during the interviews outlined in Chapter 6, such relationships may evolve into offshore education developments:

Often, these offshore engagements develop from somebody you know at the overseas institution. They are ad hoc approaches, really. (NZ1)

It can, thus, be postulated that longer geographic and industry experience is associated with more solid foundations for these relationships to evolve into offshore 
collaboration. Therefore, it may be plausible that the longer the experience, the less equity universities need to invest in developing offshore programmes, since they possess the local relationships to do so collaboratively. This could explain the observed negative relationships between geographic/industry experience and equity stake, contrary to the positive hypothesis. However, as discussed above, since significant positive findings are also found in tables 7.44 and 7.45 with respect to different aspects of geographic and industry experience, such a conclusion cannot be drawn at an aggregate level. Rather, the mixed findings from tables 7.44 and 7.45 reinforce the importance of analysing the three geographical clusters (i.e. AU/NZ, US/CA and UK/IR) independently of each other.

The findings in table 7.46 show that H1c, which deals with transfer experience, is supported in the OLS models estimated across the aggregate sample and all three geographical groupings. As hypothesised, the sampled education services providers seem to develop routine responses to choosing an entry mode, such that the more extensive their transfer experience with a particular mode of entry, the less likely they seem to favour a higher level of ownership. However, since these observed findings are not replicated across the tobit and negative binomial models, it would be useful future research to further explore how transfer experience is related to the level of equity investment in the offshore education context.

Table 7.47 shows that H2, which pertains to know-how, is not supported across any of the models. While a positive relationship is hypothesised between know-how and equity stake, a highly significant and consistent negative relationship is found $(p<0.01$ across all models except UK/IR negative binomial). In the education context, as pointed out in the exploratory qualitative phase of this research (see Chapter 6), quality assurance is an important theme for universities involved in offshore education. An important aspect of ensuring the quality of education is informed by the need to enforce consistent academic standards between home and host country programmes. Since the delivery of education programmes is often dictated by unofficial behavioural scripts of home-based academics (i.e. tacit know-how), it is important to replicate this tacit know-how 
overseas to ensure consistency of academic standards. However, given the difficulty of transferring an organisation's routines/human capital in a foreign location (Teece, 1981, 1988), it is hypothesised in Chapter 4, that due to low codifiability of know-how, universities involved in offshore education may choose more resource intensive modes of entry to facilitate the transfer of such tacit know-how. The unexpected observed relationship in the sample, marginal to the other variables in the models, requires additional research. Comments made by the representative of US2 in the exploratory qualitative phase of this study could be a point of departure for such future research: “....the totality of what constitutes a top quality institution as an organisational organism is not mobile”, indicating the possibility that even more resource intensive modes of entry may not be conducive to tacit know-how transfer.

Table 7.48 shows that H3, dealing with organisational culture, is not supported by the sample. Significant negative findings (at least $p<0.10$ ) are obtained across the different models. While it is reasoned that the more entrepreneurial the organisational culture of a university is, the more that university would be likely to invest offshore through resource intensive modes of entry, the empirical findings do not support this hypothesis. As discussed previously, in the education context, less resource intensive ownership forms are not necessarily associated with less risk. The academic input that universities often provide in non-equity arrangements offshore may be as important as a financial investment. Knowledge creation and sharing are after all, the main tenets of academe. Therefore, less resource intensive modes of entry can be perceived by universities to be risky in terms of their academic and reputational impact should the collaboration fail. The emphasis on quality assurance and reputation was strongly communicated during the interview phase of this study (see Chapter 6); less resource intensive ownership forms may be more risky in the education context, as they provide less control over the academic content of the offshore programme. Thus, more risk averse universities may opt for more resource intensive engagement in offshore education. In Chapter 4, it is theorised that the more entrepreneurial the organisational culture of a university is, the more accepting of risks it will be in offshore education developments. It appears that the education context may be a special case, such that more entrepreneurial universities are 
more likely to be at ease in managing the academic and reputational risks involved in less resource intensive modes of entry.

Table 7.49 shows that $\mathrm{H} 4$, pertaining to learning intent, is not supported. It is hypothesised in this study that the stronger the learning intent of a university, the more likely that university is to prefer international joint ventures with majority equity. The obtained empirical findings indicate a lack of support for this hypothesis. Learning intent, in fact, did not emerge as a theme during the exploratory qualitative phase of this study (see Chapter 6), indicating that the learning intent of universities might not play a determining role in offshore entry mode selection in the education context.

From the results presented in table 7.50, mixed support for H5, which deals with financial resources, is obtained. While positive significant findings (at least $p<0.10$ ) are obtained across some of the models/estimation techniques analysed, there are also a considerable number of model/estimation combinations which do not show a significant relationship between financial resources and equity stake. Similarly, a negative coefficient is obtained for H5 in the aggregate OLS model when using Hofstede data, although this finding is not significant. I conjecture that this mixed support can be explained by the fact that the survey respondents might have had different interpretations of equity investment. As per our previous discussion, equity investment in the education context, is not necessarily understood as financial capital investment, but also includes academic investment. Consequently, access to finance in the education context, might not feature strongly as a key determinant of offshore education engagement marginal to the other variables in the models. The following statement from the representative of AU1 during the exploratory qualitative phase of this study (see Chapter 6) captures this:

...cash is not the only thing important in determining our type of engagement offshore. Very often, host governments will provide substantial financial benefits for our university to set up campuses offshore. (AU1) 
However, the above statement does not completely negate finance as a determinant of the level of offshore investment. Rather, it positions finance to be context specific in terms of its influence on the entry mode decision since equity investment is not solely undertaken through financial capital investment.

Table 7.51 shows that H6, which deals with reputation, is not supported. While a positive relationship is hypothesised between reputation and equity stake, significant negative findings (at least $p<0.10$ ) are observed across a number of the models/estimation combinations. Informed by extant theory, it is hypothesised that, because more resource intensive modes of entry should provide control over brand protection (which is important for reputable institutions), universities with stronger reputations will tend to prefer to protect their 'brands' with higher ownership levels. However, the empirical findings do not support this hypothesis. It may be that, because universities are "not-for-profit" organisations, their international operations might be different from those of multinational firms, on which mainstream international business and strategy theoretical frameworks tend to focus and develop from. For example, highly reputable universities such as the Ivy League institutions in the US, might not have a financial need, one of the primary motives for offshore investment, to operate overseas. Doing so might even dilute their brand value, since being smaller and more exclusive is better from a reputational perspective. Smallness and exclusivity can also provide better control over academic standards. Reputable universities might, thus, opt for less resource-intensive entry modes, should they seek to engage in offshore investment for academic rather than purely financial motives. For example, the Wharton School of Business (ISB) collaborates with the Indian School of Business in Hyderabad on a purely academic alliance model which involves having Wharton faculty involved in teaching at ISB and using their networks to recruit other high-quality academics from other universities (see www.isb.edu). Similarly, Harvard University's Kennedy School of Government has established a Public Policy programme with The National University of Singapore on academic grounds. On the other hand, other less prestigious universities may need to go abroad to survive, partly because they are dominated by the leading domestic universities. This financial motive might push these universities to be 
more aggressive in their overseas investment, opting for more resource intensive entry modes. In addition, it is also possible that less prestigious universities may not have the bargaining power of entering overseas markets without some sort of equity investment, thus requiring more resource-intensive entry modes.

H7a, which deals with the moderating role of normative institutional distance on the geographic experience-equity stake relationship, is not supported across any of the models/estimation combinations in table 7.52. An inverted relationship is hypothesised, specifically, a positive relationship at low levels of normative institutional distance and a negative one at higher levels of normative institutional distance. Of the significant findings outlined in table 7.52, only one variable, namely geographic experience knowledge of the education sector in the aggregate Tobit model, demonstrates a significant relationship (at least $p<0.05$ ) at both low and higher levels of distance. However, this finding is opposite to H7a. Rather than the hypothesised positive relationship at low levels of normative institutional distance, a negative significant finding is found $(p<0.01)$ and at higher levels of normative institutional distance, a positively significant finding is found $(p<0.05)$ as opposed to the hypothesised negative relationship. As per previous discussions for H1a, I conjecture that possible explanations for a negative relationship might be that more geographic experience brings the ability to deal with the academic risks associated with non-equity arrangements. Similarly, more geographic experience could lead to better relationships with local institutions, in turn providing a better foundation for collaborative offshore engagements to take place. However, the negative relationship between geographic experience and equity stake that these explanations suggest might only hold true at low levels of normative institutional distance. At higher levels of distance, universities might have to increase their equity stake in order to maintain control over their offshore engagements and avoid the academic and reputational pitfalls of failure. With more normative institutional distance, besides the associated academic and reputational risks of operating offshore, universities might also have to face up to the challenges of operating in a different institutional environment. These challenges may bring an added 
level of complexity in operating offshore which may encourage universities to regain control over their operations.

Table 7.53 shows that H7b, dealing with the moderating impact of normative institutional distance on the industry experience-equity stake relationship, is not supported. An inverted relationship is hypothesised with a positive relationship expected at low levels of normative institutional distance and a negative relationship at higher levels of normative institutional distance. None of the significant findings (at least $p<0.10$ ) obtained in the various models/estimation combinations lend support to this hypothesis. The aspects of industry experience analysed which demonstrate significant positive relationships at low levels of normative institutional distance do not have significant negative relationships at higher levels of normative institutional distance. For example, the coefficient associated with industry experience international recruitment is positively significant for the AU/NZ OLS and Tobit models at low levels of institutional distance, but is not significant at higher levels of distance. Of interest are the significant negative findings for industry experience - undergraduate marketing at low levels of distance for the AU/NZ models; these negative findings are associated with the only aspect of industry experience analysed which contradict the hypothesised positive relationship at low levels of distance. These findings are not replicated when the Hofstede measure of distance is used. Similarly, industry experience - international recruitment is the only aspect of industry experience analysed with a significant positive relationship to equity stake at higher levels of distance, opposing the hypothesised negative relationship. These significant positive findings are only obtained when the Hofstede measure of distance is used. Therefore, these two aspects of industry experience (undergraduate marketing and international recruitment) present an opportunity for future research.

Table 7.54 shows that $\mathrm{H7c}$, which pertains to the moderating role of normative institutional distance on the tacit know-how-equity stake relationship, is not supported. An inverted relationship is hypothesised with a positive relationship expected at low levels of normative institutional distance and a negative relationship at higher levels of 
normative institutional distance. However, the significant negative findings $(p<0.01)$ across all the models and estimation combinations, at both low and higher levels of distance, indicate that know-how appears to be negatively associated with equity stake, regardless of the levels of distance and marginal to the other variables analysed. This finding is highly consistent with the finding for $\mathrm{H} 2$ and similar to the conjecture for that particular hypothesis, I advance that in the education context, more resource intensive modes of entry may not be conducive to tacit know-how transfer. Future research is necessary to address this unexpected observed relationship.

Table 7.55 shows that H7d, which deals with the moderating role of normative institutional distance on the organisation culture-equity stake relationship, is not supported. An inverted relationship is hypothesised with a positive relationship expected at low levels of normative institutional distance and a negative relationship at higher levels of normative institutional distance. However, the significant negative findings $(p<0.01)$ for the aggregate, AU/NZ, US/CA groupings across all the models and estimation combinations and at both low and higher levels of normative institutional distance, indicate that organisational culture appears to be negatively associated with equity stake, regardless of the levels of distance and marginal to the other variables analysed. This finding is similar to the finding of H3 and I, therefore, conjecture likewise to $\mathrm{H} 3$, that the more entrepreneurial a university is, the more it is likely to be at ease in managing the academic and reputational risks involved with less resource intensive modes of entry. Furthermore, in the UK/IR grouping, a V-shaped relationship is found contrary to the hypothesised inverted relationship. For the organisational culture - open discussion variable, significant negative relationships $(p<$ 0.01) are obtained at low levels of normative institutional distance in the OLS and Tobit models. These relationships become positive and significant $(p<0.01)$ at higher levels of normative institutional distance. The source of this geographic distinction presents an opportunity for future research.

Table 7.56 shows that H7e, dealing with the moderating role of normative institutional distance on the financial resources-equity stake relationship, is not supported. An 
inverted relationship is hypothesised with a positive relationship expected at low levels of normative institutional distance and a negative relationship at higher levels of normative institutional distance. However, the findings across all the models/estimation combinations do not lend support to H7e. For example, while at higher levels of institutional distance, the relationship between financial resources and equity stake is negatively significant for the aggregate Tobit, US/CA OLS and Tobit models, the associated positive relationship is not observed at low levels of distance. Furthermore, for the UK/IR OLS model, a significant negative relationship is observed contrary to the positive hypothesised relationship at low levels of distance. Similar to the previous discussions for H5, I postulate that these findings can be partly explained by the fact that, in the education context, equity investment is not necessarily understood as financial capital investment but can also include academic investment.

H7f, which deals with the moderating role of normative institutional distance on the reputation-equity stake relationship, is not supported across any of the models/estimation combinations in table 7.57. An inverted relationship is hypothesised with a positive relationship expected at low levels of normative institutional distance and a negative relationship at higher levels of normative institutional distance. However, the findings suggest a negatively significant relationship (at least $p<0.10$ ) at both low and higher levels of normative institutional distance. These findings are consistent with the findings for $\mathrm{H} 6$ and likewise to that hypothesis, I conjecture that in the education context, more reputable universities might have less of a financial need to engage offshore, in turn, making them less aggressive in terms of the resource intensity of their entry modes. Less reputable universities, on the other hand, may need to go overseas to survive from competition by leading domestic universities. This financial motive might push these universities to be more aggressive in their overseas investment, opting for more resource intensive entry modes.

H8a, which deals with the moderating role of regulative institutional distance on the geographic experience-equity stake relationship, is not supported across any of the models/estimation combinations in table 7.58. An inverted relationship is hypothesised 
with a positive relationship expected at low levels of regulative institutional distance and a negative relationship at higher levels of regulative institutional distance. However, none of the significant findings (at least $p<0.10$ ) across all the models and estimation techniques support this hypothesis. For example, while geographic experience - knowledge of education sector is positively significant in the AU/NZ Tobit models $(p<0.10)$ at low levels of distance, the associated negative finding is not observed at higher levels of distance. Furthermore, of the significant variables at low levels of distance, the significant negative relationship $(p<0.10)$ for geographic experience - financial capabilities in the UK/IR OLS model is particularly interesting given that it is the only such negative finding at low levels of distance. As per the previous discussion for $\mathrm{H} 1 \mathrm{a}$, I conjecture that a possible explanation for this negative relationship might be that more geographic experience brings the ability to deal with the academic risks associated with non-equity arrangements. Similarly, more geographic experience could lead to better relationships with local institutions, in turn, providing a better foundation for collaborative offshore engagements to take place.

H8b, which deals with the moderating role of regulative institutional distance on the industry experience-equity stake relationship, is not supported across any of the models/estimation combinations in table 7.59. An inverted relationship is hypothesised with a positive relationship expected at low levels of regulative institutional distance and a negative relationship at higher levels of regulative institutional distance. However, none of the significant findings (at least $p<0.10$ ) across all the models and estimation techniques analysed support this hypothesis. The aspects of industry experience analysed which demonstrate significant positive relationship at low levels of institutional distance, do not have significant negative relationships at higher levels of distance. For example, the coefficient associated with industry experience international recruitment is positively significant for the US/CA OLS and Tobit models when using the Hofstede measure of distance. The associated hypothesis at higher levels of distance is not supported for industry experience - international recruitment in the US/CA grouping. Also, of interest, are the negative significant findings for industry experience - undergraduate marketing at low levels of distance for the US/CA models 
when a positive relationship is hypothesised. These findings are not replicated when the Hofstede measure of distance is used. Similarly, industry experience - international recruitment is the only aspect of industry experience analysed with a significant positive relationship to equity stake at higher levels of distance, thereby opposing the hypothesised negative relationship. These significant negative findings are only obtained when using the IMD measure of distance. Therefore, similar to the discussion for H7b, these two aspects of industry experience (undergraduate marketing and international recruitment) present an opportunity for future research.

Table 7.60 shows that H8c, which pertains to the moderating role of regulative institutional distance on the tacit know-how-equity stake relationship, is not supported. An inverted relationship is hypothesised with a positive relationship expected at low levels of regulative institutional distance and a negative relationship at higher levels of regulative institutional distance. However, highly significant negative findings $(p<$ 0.01) are observed at both low and higher levels of regulative institutional distance in the aggregate and US/CA groupings across all the models and estimation combinations. In the AU/NZ grouping, a significant negative finding (at least $p<0.10$ ) is found when using Tobit estimation at both low and higher levels of regulative institutional distance. In the UK/IR grouping, a negative significant finding $(p<0.01)$ is found at both low and higher levels of regulative institutional distance, when using both OLS and Tobit estimation. These findings are consistent with the finding for $\mathrm{H} 2$ and similar to the conjecture for that particular hypothesis, I advance that in the education context, more resource intensive entry modes may not be conducive to tacit know-how transfer. Future research is necessary to address this unexpected observed relationship.

Table 7.61 shows that H8d, which deals with the moderating role of regulative institutional distance on the organisation culture-equity stake relationship, is not supported. An inverted relationship is hypothesised with a positive relationship expected at low levels of regulative institutional distance and a negative relationship at higher levels of regulative institutional distance. However, the significant negative findings $(p<0.01)$ for the aggregate and US/CA groupings across all the 
models/estimation combinations and at both low and higher levels of regulative institutional distance, indicate that organisational culture is negatively associated with equity stake, regardless of the levels of distance and marginal to the other variables in the models. This finding is similar to the finding of $\mathrm{H} 3$ and I, therefore, conjecture likewise to $\mathrm{H} 3$, that the more entrepreneurial a university is, the more it is likely to be at ease in managing the academic and reputational risks involved in less resource intensive modes of entry. Furthermore, in the AU/NZ grouping, evidence for a significant negative relationship is observed at low levels of distance but not replicated at higher levels of distance. For the UK/IR grouping, although negative significant findings are obtained at both low and higher levels of distance for organisational culture - offshore development, these findings do not relate to the same measure of distance. At low levels of distance, significant negative findings are found when using the IMD measure while at higher levels of distance, significant negative findings are observed when using the EFI measure. These observed results for both the AU/NZ and UK/IR groupings suggest a lack of support for H8d.

Table 7.62 shows that H8e, dealing with the moderating role of regulative institutional distance on the financial resources-equity stake relationship, is not supported. An inverted relationship is hypothesised with a positive relationship expected at low levels of regulative institutional distance and a negative relationship at higher levels of regulative institutional distance. However, the findings across all the models/estimation combinations and at both low and higher levels of regulative institutional distance do not lend support to H8e. For example, while negative significant relationships are observed at higher levels of distance for the aggregate OLS and Tobit models as well as the UK/IR OLS and Tobit models, the associated hypothesised positive relationship is not found at low levels of distance. Similar to the previous discussion for H5, I postulate that these findings can be partly explained by the fact that, in the education context, equity investment is not necessarily understood as financial capital investment but can also include academic investment. 
H8f, which deals with the moderating influence of regulative institutional distance on the reputation-equity stake relationship, is not supported across any of the models/estimation combinations in table 7.63. An inverted relationship is hypothesised with a positive relationship expected at low levels of regulative institutional distance and a negative relationship at higher levels of regulative institutional distance. However, the findings suggest a negatively significant relationship (at least $p<0.10$ ) at both low and higher levels of regulative institutional distance in the aggregate and US/CA groupings. The observed results for the AU/NZ grouping at lower levels of distance and for the UK/IR grouping at higher levels of distance also indicate a lack of support for H8f. These findings are fairly consistent with the findings for H6 and likewise to that hypothesis, I conjecture that in the education context, more reputable universities might have less of a financial need to engage offshore, in turn, making them less aggressive in terms of the resource intensity of their entry modes. Less reputable universities, on the other hand, may need to go overseas to survive from competition by leading domestic universities. This financial motive might push these universities to be more aggressive in their overseas investment, opting for more resource intensive entry modes. 
Table 7.44: Summary of Significant Findings for H1a (Geographic Experience; Positive Coefficient Sign Hypothesised) Using the Three measures of Distance

\begin{tabular}{|c|c|c|c|c|c|c|c|c|c|c|c|c|}
\hline & \multicolumn{3}{|c|}{ Complete data set } & \multicolumn{3}{|c|}{ AU/NZ } & \multicolumn{3}{|c|}{ US/CA } & \multicolumn{3}{|c|}{ UK/IR } \\
\hline & OLS & Tobit & $\begin{array}{l}\text { Negative } \\
\text { binomial } \\
\end{array}$ & OLS & Tobit & $\begin{array}{l}\text { Negative } \\
\text { binomial } \\
\end{array}$ & OLS & Tobit & $\begin{array}{l}\text { Negative } \\
\text { binomial } \\
\end{array}$ & OLS & Tobit & $\begin{array}{l}\text { Negative } \\
\text { binomial }\end{array}$ \\
\hline \multicolumn{13}{|l|}{$\begin{array}{l}\text { Equations 1/10/19 } \\
\text { (IMD/Hofstede/ } \\
\text { EFI) }\end{array}$} \\
\hline $\begin{array}{l}\text { Geo exp - } \\
\text { knowledge of edu } \\
\text { sector }\end{array}$ & & & & & $\begin{array}{c}(+) /(+) / \\
(+) \dagger\end{array}$ & & & & & & & \\
\hline $\begin{array}{l}\text { Geo exp - academic } \\
\text { capabilities }\end{array}$ & & & & $\begin{array}{c}(+) \dagger /(+)^{* /} \\
(+) \dagger\end{array}$ & & & & & & & & \\
\hline \multicolumn{13}{|l|}{$\begin{array}{l}\text { Geo exp - } \\
\text { managerial } \\
\text { capabilities }\end{array}$} \\
\hline $\begin{array}{l}\text { Geo exp - financial } \\
\text { capabilities }\end{array}$ & & & & & & & & & & $\begin{array}{c}(-) \dagger /(-) \dagger / \\
(-)\end{array}$ & $\begin{array}{c}(-) /(-) \dagger / \\
(-)\end{array}$ & \\
\hline $\begin{array}{l}\text { Geo exp - intl } \\
\text { recruitment }\end{array}$ & & & & $\begin{array}{c}(+) \dagger /(+) / \\
(+) \dagger\end{array}$ & & & & $\begin{array}{c}(-)^{* /(-) * /} \\
(-)^{*}\end{array}$ & $\begin{array}{c}(-) \dagger /(-) \dagger / \\
(-) \dagger\end{array}$ & & & \\
\hline \multicolumn{13}{|l|}{$\begin{array}{l}\text { Equations 2/11/20 } \\
\text { (IMD/Hofstede/ } \\
\text { EFI) }\end{array}$} \\
\hline $\begin{array}{l}\text { Geo exp - } \\
\text { knowledge of edu } \\
\text { sector }\end{array}$ & & & & & $\begin{array}{c}(+) /(+) / \\
(+) \dagger\end{array}$ & & & & & & & \\
\hline \multicolumn{13}{|l|}{$\begin{array}{l}\text { Geo exp - academic } \\
\text { capabilities }\end{array}$} \\
\hline $\begin{array}{l}\text { Geo exp - } \\
\text { managerial } \\
\text { capabilities }\end{array}$ & & & & & & & & $\begin{array}{c}(+) \dagger /(+) / \\
(+)\end{array}$ & & & & \\
\hline $\begin{array}{l}\text { Geo exp - financial } \\
\text { capabilities }\end{array}$ & & & & & & & $\begin{array}{c}(+) /(+) / \\
(+) \dagger\end{array}$ & $\begin{array}{c}(+) \dagger /(+) / \\
(+)\end{array}$ & & $\begin{array}{c}(-) \dagger /(-)^{* /} \\
(-)\end{array}$ & $\begin{array}{c}(-) /(-)^{* /} \\
(-)\end{array}$ & \\
\hline $\begin{array}{l}\text { Geo exp - intl } \\
\text { recruitment }\end{array}$ & & & & & $\begin{array}{c}(+) /(+) / \\
(+) \dagger\end{array}$ & & $\begin{array}{c}(-) \dagger /(-) \dagger / \\
(-) \dagger\end{array}$ & $\begin{array}{c}(-) \dagger /(-) \dagger / \\
(-) \dagger\end{array}$ & & & & \\
\hline
\end{tabular}




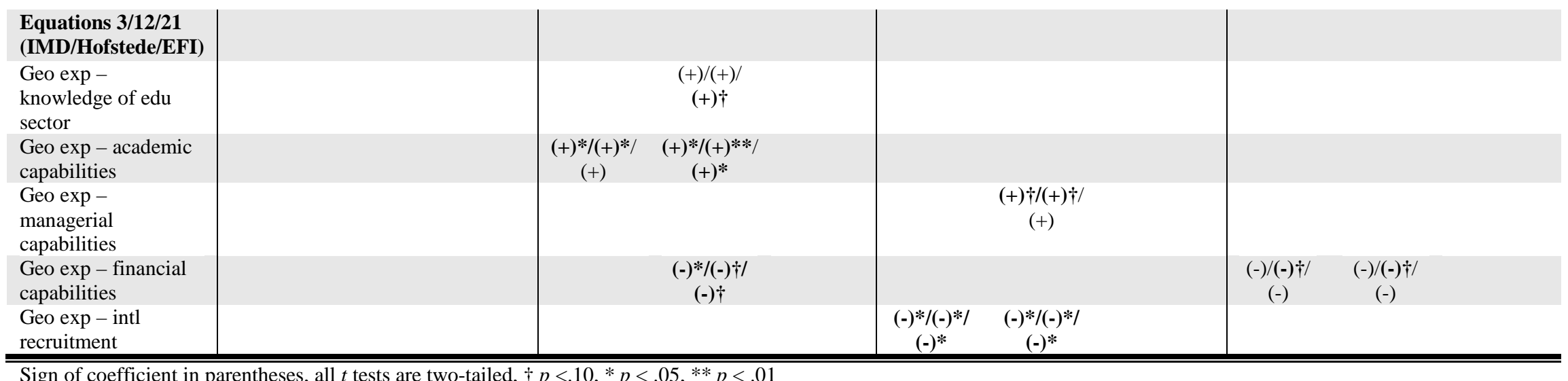

Sign of coefficient in parentheses, all $t$ tests are two-tailed, $\dagger p<.10, * p<.05,{ }^{* *} p<.01$

Table 7.45: Summary of Significant Findings for H1b (Industry Experience; Positive Coefficient Sign Hypothesised) Using the Three measures of Distance

\begin{tabular}{|c|c|c|c|c|c|c|c|c|c|c|c|c|}
\hline & \multicolumn{3}{|c|}{ Complete data set } & \multicolumn{3}{|c|}{$\overline{\mathrm{AU} / \mathrm{NZ}}$} & \multicolumn{3}{|c|}{$\overline{\text { US/CA }}$} & \multicolumn{3}{|c|}{$\overline{\text { UK/IR }}$} \\
\hline & OLS & Tobit & $\begin{array}{l}\text { Negative } \\
\text { binomial } \\
\end{array}$ & OLS & Tobit & $\begin{array}{l}\text { Negative } \\
\text { binomial }\end{array}$ & OLS & Tobit & $\begin{array}{l}\text { Negative } \\
\text { binomial }\end{array}$ & OLS & Tobit & $\begin{array}{l}\text { Negative } \\
\text { binomial }\end{array}$ \\
\hline \multicolumn{13}{|l|}{$\begin{array}{l}\text { Equations 1/10/19 } \\
\text { (IMD/Hofstede/ } \\
\text { EFI) }\end{array}$} \\
\hline \multicolumn{13}{|l|}{$\begin{array}{l}\text { Ind exp - UG } \\
\text { offshore edu }\end{array}$} \\
\hline \multicolumn{13}{|l|}{$\begin{array}{l}\text { Ind exp - PG } \\
\text { offshore edu }\end{array}$} \\
\hline $\begin{array}{l}\text { Ind exp - intl } \\
\text { recruitment (Factor) }\end{array}$ & $\begin{array}{c}(+) \dagger /(+) \dagger / \\
(+)\end{array}$ & $\begin{array}{c}(+) \dagger /(+)^{* /} \\
(+)^{*}\end{array}$ & & & & & & $\begin{array}{c}(+)^{* /(+)^{* /}} \\
(+)^{*}\end{array}$ & & & & \\
\hline $\begin{array}{l}\text { Ind exp - UG } \\
\text { marketing }\end{array}$ & & & & & $\begin{array}{c}(-) \dagger /(-) \dagger / \\
(-) \dagger\end{array}$ & & & & & & & \\
\hline \multicolumn{13}{|l|}{$\begin{array}{l}\text { Ind exp - PG } \\
\text { marketing }\end{array}$} \\
\hline Equations 2/11/20 & & & & & & & & & & & & \\
\hline
\end{tabular}




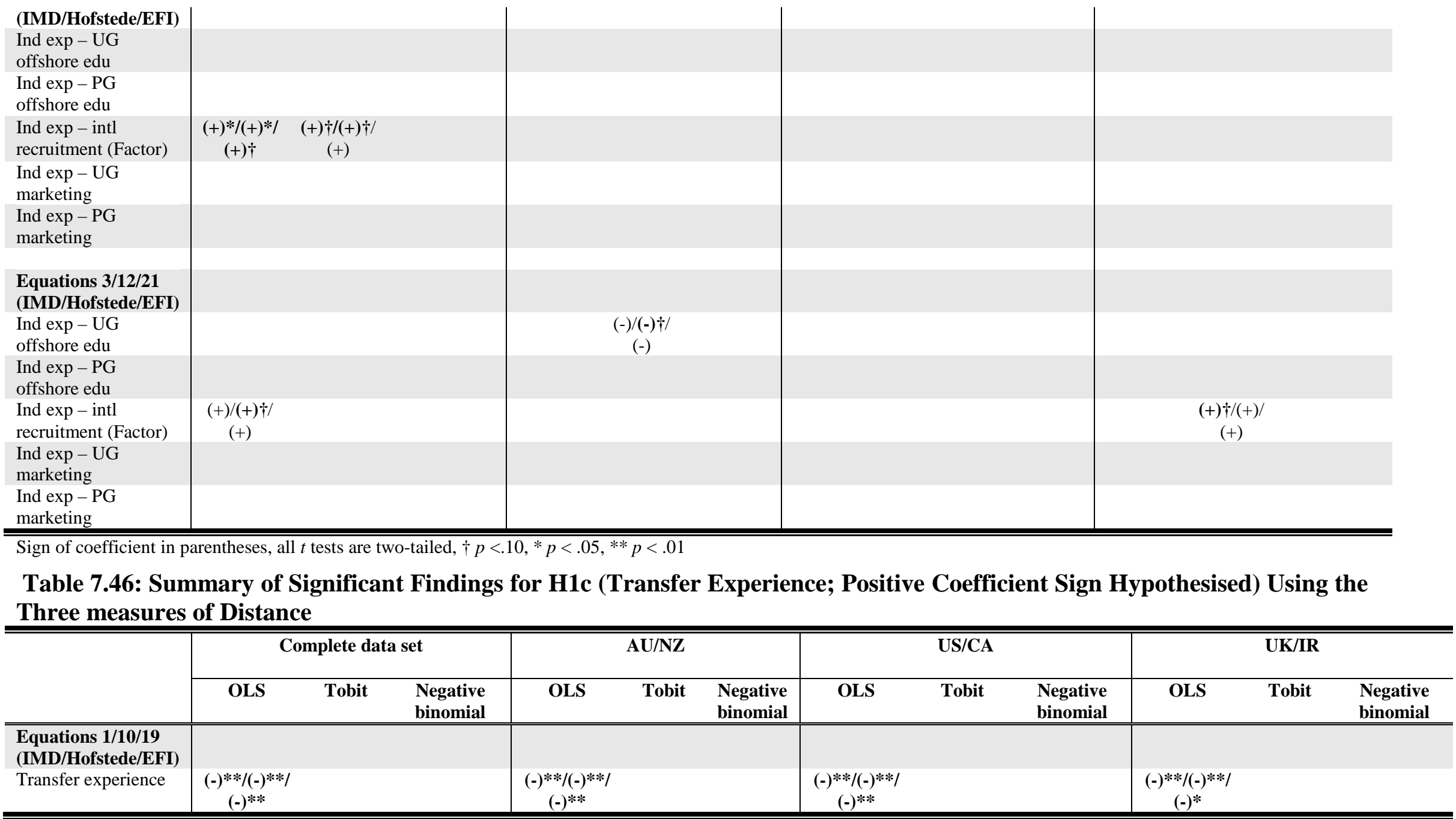

Sign of coefficient in parentheses, all $t$ tests are two-tailed, $\dagger p<.10,{ }^{*} p<.05$, ** $p<.01$ 
Table 7.47: Summary of Significant Findings for H2 (Know-how; Positive Coefficient Sign Hypothesised) Using the Three measures of Distance

\begin{tabular}{|c|c|c|c|c|c|c|c|c|c|c|c|c|}
\hline & \multicolumn{3}{|c|}{ Complete data set } & \multicolumn{3}{|c|}{$\overline{\mathrm{AU} / \mathrm{NZ}}$} & \multicolumn{3}{|c|}{ US/CA } & \multicolumn{3}{|c|}{ UK/IR } \\
\hline & OLS & Tobit & $\begin{array}{l}\text { Negative } \\
\text { binomial } \\
\end{array}$ & OLS & Tobit & $\begin{array}{l}\text { Negative } \\
\text { binomial } \\
\end{array}$ & OLS & Tobit & $\begin{array}{l}\text { Negative } \\
\text { binomial } \\
\end{array}$ & OLS & Tobit & $\begin{array}{l}\text { Negative } \\
\text { binomial }\end{array}$ \\
\hline \multicolumn{13}{|l|}{$\begin{array}{l}\text { Equations 2/11/20 } \\
\text { (IMD/Hofstede/EFI) }\end{array}$} \\
\hline Know-how (Factor) & $\begin{array}{c}(-)^{* * /} \\
(-)^{* * /} \\
(-)^{* *}\end{array}$ & $\begin{array}{l}(-)^{* * /} \\
(-)^{* * /} \\
(-)^{* *}\end{array}$ & $\begin{array}{l}(-)^{* * /} \\
(-)^{* * /} \\
(-)^{* *}\end{array}$ & $\begin{array}{c}(-)^{* * /} \\
(-)^{* * /} \\
(-)^{* *}\end{array}$ & $\begin{array}{c}(-)^{* * /} \\
(-)^{* * /} \\
(-)^{* *}\end{array}$ & $\begin{array}{l}(-)^{* * /} \\
(-)^{* * /} \\
(-)^{* *}\end{array}$ & $\begin{array}{c}(-)^{* * /} \\
(-)^{* * /} \\
(-)^{* *}\end{array}$ & $\begin{array}{l}(-)^{* * /} \\
(-)^{* * /} \\
(-)^{* *}\end{array}$ & $\begin{array}{l}(-)^{* * /} \\
(-)^{* * /} \\
(-)^{* *}\end{array}$ & $\begin{array}{l}(-)^{* * /} \\
(-)^{* * /} \\
(-)^{* *}\end{array}$ & $\begin{array}{l}(-)^{* * /} \\
(-)^{* * /} \\
(-)^{* *}\end{array}$ & \\
\hline
\end{tabular}

Sign of coefficient in parentheses, all $t$ tests are two-tailed, $\dagger p<.10, * p<.05, * * p<.01$

Table 7.48: Summary of Significant Findings for H3 (Entrepreneurial Organisational Culture; Positive Coefficient Sign Hypothesised) Using the Three measures of Distance

\begin{tabular}{|c|c|c|c|c|c|c|c|c|c|c|c|c|}
\hline & \multicolumn{3}{|c|}{ "Complete data set } & \multicolumn{3}{|c|}{ AU/NZ } & \multicolumn{3}{|c|}{ US/CA } & \multicolumn{3}{|c|}{ 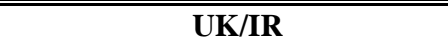 } \\
\hline & OLS & Tobit & $\begin{array}{l}\text { Negative } \\
\text { binomial }\end{array}$ & OLS & Tobit & $\begin{array}{l}\text { Negative } \\
\text { binomial }\end{array}$ & OLS & Tobit & $\begin{array}{l}\text { Negative } \\
\text { binomial }\end{array}$ & OLS & Tobit & $\begin{array}{l}\text { Negative } \\
\text { binomial }\end{array}$ \\
\hline \multicolumn{13}{|l|}{$\begin{array}{l}\text { Equations 3/12/21 } \\
\text { (IMD/Hofstede/EFI) }\end{array}$} \\
\hline Org culture (Factor) & $\begin{array}{l}(-)^{* * /} \\
(-)^{* * /} \\
(-)^{* *}\end{array}$ & $\begin{array}{l}(-)^{* * /} \\
(-)^{* * /} \\
(-)^{* *}\end{array}$ & $\begin{array}{l}(-)^{* * /} \\
(-)^{* * /} \\
(-)^{* *}\end{array}$ & $\begin{array}{l}(-)^{* * /} \\
(-)^{* * /} \\
(-)^{* *}\end{array}$ & $\begin{array}{l}(-)^{* * /} \\
(-)^{* * /} \\
(-)^{* *}\end{array}$ & $\begin{array}{l}(-)^{* * /} \\
(-)^{* * /} \\
(-)^{* *}\end{array}$ & $\begin{array}{l}(-)^{* * /} \\
(-)^{* * /} \\
(-)^{* *}\end{array}$ & $\begin{array}{l}(-)^{* * /} \\
(-)^{* * /} \\
(-)^{* *}\end{array}$ & $\begin{array}{l}(-)^{* * /} \\
(-)^{* * /} \\
(-)^{* *}\end{array}$ & - & - & - \\
\hline $\begin{array}{l}\text { Org culture - open } \\
\text { discussion }\end{array}$ & - & - & - & - & - & - & - & - & - & $\begin{array}{c}(-) \dagger /(-) \dagger / \\
(-)\end{array}$ & $\begin{array}{c}(-)^{*} /(-)^{* /} \\
(-)^{*}\end{array}$ & \\
\hline $\begin{array}{l}\text { Org culture - no } \\
\text { status distinction }\end{array}$ & - & - & - & - & - & - & - & - & - & $\begin{array}{c}(-) \dagger /(-) \dagger / \\
(-) \dagger\end{array}$ & $\begin{array}{c}(-) \dagger /(-) / \\
(-) \dagger\end{array}$ & \\
\hline $\begin{array}{l}\text { Org culture - } \\
\text { experimentation }\end{array}$ & - & - & - & - & - & - & - & - & - & & & \\
\hline $\begin{array}{l}\text { Org culture - } \\
\text { offshore } \\
\text { development }\end{array}$ & - & - & - & - & - & - & - & - & - & $\begin{array}{c}(-)^{*} /(-)^{* /} \\
(-)^{*}\end{array}$ & $\begin{array}{c}(-)^{*} /(-) \dagger / \\
(-)^{*}\end{array}$ & \\
\hline $\begin{array}{l}\text { Org culture - } \\
\text { offshore } \\
\text { responsiveness }\end{array}$ & - & - & - & - & - & - & - & - & - & & & \\
\hline
\end{tabular}

Sign of coefficient in parentheses, all $t$ tests are two-tailed, $\uparrow p<.10, * p<.05, * * p<.01$ 
Table 7.49: Summary of Findings for H4 (Learning Intent; Positive Relationship Hypothesised for Joint Ventures)

\begin{tabular}{|c|c|c|c|}
\hline & \multirow[b]{2}{*}{ Sig. (2-tailed) } & \multicolumn{2}{|c|}{ 95\% Confidence Interval of the Difference } \\
\hline & & Lower & Upper \\
\hline Learning Intent (time considerations) & 0.514 & -0.438 & 0.849 \\
\hline Learning Intent (cost considerations) & 0.982 & -0.823 & 0.805 \\
\hline Learning Intent (profit considerations) & 0.919 & -0.689 & 0.761 \\
\hline Learning Intent (skills development considerations) & 0.238 & -0.255 & 0.969 \\
\hline Learning Intent (deeper understanding of partner’s expertise) & 0.679 & -0.600 & 0.904 \\
\hline Learning Intent (skills duplication considerations) & 0.442 & -0.428 & 0.946 \\
\hline $\begin{array}{l}\text { Learning Intent (understanding of partner's perspective, systems } \\
\text { and know-how) }\end{array}$ & 0.663 & -1.019 & 0.662 \\
\hline
\end{tabular}

Table 7.50: Summary of Significant Findings for H5 (Financial resources; Positive Coefficient Sign Hypothesised) Using the Three measures of Distance

\begin{tabular}{|c|c|c|c|c|c|c|c|c|c|c|c|c|}
\hline & \multicolumn{3}{|c|}{ Complete data set } & \multicolumn{3}{|c|}{ AU/NZ } & \multicolumn{3}{|c|}{ US/CA } & \multicolumn{3}{|c|}{ 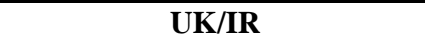 } \\
\hline & OLS & Tobit & $\begin{array}{l}\text { Negative } \\
\text { binomial } \\
\end{array}$ & OLS & Tobit & $\begin{array}{l}\text { Negative } \\
\text { binomial } \\
\end{array}$ & OLS & Tobit & $\begin{array}{l}\text { Negative } \\
\text { binomial } \\
\end{array}$ & OLS & Tobit & $\begin{array}{l}\text { Negative } \\
\text { binomial }\end{array}$ \\
\hline \multicolumn{13}{|l|}{$\begin{array}{l}\text { Equations 1/10/19 } \\
\text { (IMD/Hofstede/EFI) }\end{array}$} \\
\hline Financial resources & & & & $\begin{array}{c}(+) /(+) / \\
(+) \dagger\end{array}$ & & & & & & & & \\
\hline \multicolumn{13}{|l|}{$\begin{array}{l}\text { Equations } 2 / 11 / 20 \\
\text { (IMD/Hofstede/EFI) }\end{array}$} \\
\hline Financial resources & $\begin{array}{c}(+) \dagger /(-) / \\
(+)\end{array}$ & & & $\begin{array}{c}(+) /(+) / \\
(+) \dagger\end{array}$ & $\begin{array}{c}(+) /(+) / \\
(+)^{*}\end{array}$ & & & & & & & \\
\hline \multicolumn{13}{|l|}{$\begin{array}{l}\text { Equations 3/12/21 } \\
\text { (IMD/Hofstede/EFI) }\end{array}$} \\
\hline Financial resources & & & & & & & & & & & & \\
\hline
\end{tabular}

Sign of coefficient in parentheses, all $t$ tests are two-tailed, $\uparrow p<.10, * p<.05, * * p<.01$ 
Table 7.51: Summary of Significant Findings for H6 (Reputation; Positive Coefficient Sign Hypothesised) Using the Three measures of Distance

\begin{tabular}{|c|c|c|c|c|c|c|c|c|c|c|c|c|}
\hline & \multicolumn{3}{|c|}{ Complete data set } & \multicolumn{3}{|c|}{ AU/NZ } & \multicolumn{3}{|c|}{ US/CA } & \multicolumn{3}{|c|}{$\overline{~ U K / I R ~}$} \\
\hline & OLS & Tobit & $\begin{array}{l}\text { Negative } \\
\text { binomial }\end{array}$ & OLS & Tobit & $\begin{array}{l}\text { Negative } \\
\text { binomial }\end{array}$ & OLS & Tobit & $\begin{array}{l}\text { Negative } \\
\text { binomial }\end{array}$ & OLS & Tobit & $\begin{array}{l}\text { Negative } \\
\text { binomial }\end{array}$ \\
\hline \multicolumn{13}{|l|}{$\begin{array}{l}\text { Equations 1/10/19 } \\
\text { (IMD) }\end{array}$} \\
\hline Reputation (Factor) & & $\begin{array}{l}(-)^{* * /} \\
(-)^{* * /} \\
(-)^{* *}\end{array}$ & $\begin{array}{l}(-)^{* * /} \\
(-)^{* * /} \\
(-)^{* *}\end{array}$ & & $\begin{array}{l}(-) \dagger / \\
(-) \dagger / \\
(-)^{*}\end{array}$ & & & $\begin{array}{l}(-)^{* * /} \\
(-)^{* * /} \\
(-)^{* *}\end{array}$ & $\begin{array}{l}(-)^{* * /} \\
(-)^{* * /} \\
(-)^{* *}\end{array}$ & & $\begin{array}{l}(-)^{* /} \\
(-)^{* /} \\
(-)^{*}\end{array}$ & \\
\hline \multicolumn{13}{|l|}{$\begin{array}{l}\text { Equations 2/11/20 } \\
\text { (IMD) }\end{array}$} \\
\hline Reputation (Factor) & & & & & & & & & & & & \\
\hline \multicolumn{13}{|l|}{$\begin{array}{l}\text { Equations 3/12/21 } \\
\text { (IMD) }\end{array}$} \\
\hline Reputation (Factor) & $\begin{array}{c}(-) \dagger / \\
(-) \dagger / \\
(-) \dagger\end{array}$ & $\begin{array}{l}(-)^{* /} \\
(-)^{* /} \\
(-)^{*}\end{array}$ & & & & & & $\begin{array}{c}(-) \dagger /(-) / \\
(-)\end{array}$ & & & $\begin{array}{c}(-) /(-) \dagger / \\
(-)\end{array}$ & \\
\hline
\end{tabular}

Sign of coefficient in parentheses, all $t$ tests are two-tailed, $\uparrow p<.10, * p<.05, * * p<.01$

Table 7.52: Summary of Significant Findings for H7a (Normative Institutional Distance \& Geographic Experience; Inverted Relationship Hypothesised With Positive Values at Low Levels of Distance and Negative Values at Higher Levels) Using the Three measures of Distance

\begin{tabular}{|c|c|c|c|c|c|c|c|c|c|c|c|c|}
\hline & \multicolumn{3}{|c|}{ Complete data set } & \multicolumn{3}{|c|}{ AU/NZ } & \multicolumn{3}{|c|}{ 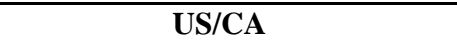 } & \multicolumn{3}{|c|}{ UK/IR } \\
\hline & OLS & Tobit & $\begin{array}{l}\text { Negative } \\
\text { binomial }\end{array}$ & OLS & Tobit & $\begin{array}{l}\text { Negative } \\
\text { binomial }\end{array}$ & OLS & Tobit & $\begin{array}{l}\text { Negative } \\
\text { binomial }\end{array}$ & OLS & Tobit & $\begin{array}{l}\text { Negative } \\
\text { binomial }\end{array}$ \\
\hline \multicolumn{13}{|l|}{$\begin{array}{l}\text { Equations 4a/13a } \\
\text { (low-median ID) } \\
\text { (IMD/Hofstede) }\end{array}$} \\
\hline $\begin{array}{l}\text { Geo exp - } \\
\text { knowledge of edu } \\
\text { sector }\end{array}$ & & $(-)^{* * /(-)}$ & $(-) \dagger /(-)$ & & & & & $(-) \dagger /(-)$ & & & $(-) /(-)^{*}$ & \\
\hline $\begin{array}{l}\text { Geo exp - } \\
\text { academic }\end{array}$ & & & & & & & & & & & & \\
\hline
\end{tabular}




\begin{tabular}{|c|c|c|c|c|}
\hline \multicolumn{5}{|l|}{ capabilities } \\
\hline $\begin{array}{l}\text { Geo exp - } \\
\text { managerial } \\
\text { capabilities }\end{array}$ & & & $(+) /(+) \dagger$ & \\
\hline $\begin{array}{l}\text { Geo exp - financial } \\
\text { capabilities }\end{array}$ & $(-) \dagger /(-)$ & $(-) /(-) \dagger$ & & $(-) \dagger /(-)$ \\
\hline $\begin{array}{l}\text { Geo exp - intl } \\
\text { recruitment }\end{array}$ & & $(+) /(+) \dagger \quad(+) /(+)^{* *}$ & & \\
\hline \multicolumn{5}{|l|}{$\begin{array}{l}\text { Equations 4b/13b } \\
\text { (median-high ID) } \\
\text { (IMD/Hofstede) }\end{array}$} \\
\hline $\begin{array}{l}\text { Geo exp - } \\
\text { knowledge of edu } \\
\text { sector }\end{array}$ & $(+) \dagger /(+) \quad(+)^{* /(+)}$ & $(+)^{* /(+)} \quad(+)^{* * /(+)}$ & & \\
\hline \multicolumn{5}{|l|}{$\begin{array}{l}\text { Geo exp - } \\
\text { academic } \\
\text { capabilities }\end{array}$} \\
\hline $\begin{array}{l}\text { Geo exp - } \\
\text { managerial } \\
\text { capabilities }\end{array}$ & & & & $(+) \dagger /(+)$ \\
\hline $\begin{array}{l}\text { Geo exp - financial } \\
\text { capabilities }\end{array}$ & & & & $(+) /(-) \dagger$ \\
\hline $\begin{array}{l}\text { Geo exp - intl } \\
\text { recruitment }\end{array}$ & & & & \\
\hline
\end{tabular}


Table 7.53: Summary of Significant Findings for H7b (Normative Institutional Distance \& Industry Experience; Inverted Relationship Hypothesised With Positive Values at Low Levels of Distance and Negative Values at Higher Levels) Using the Three measures of Distance

\begin{tabular}{|c|c|c|c|c|c|c|c|c|c|c|c|c|}
\hline & \multicolumn{3}{|c|}{ "Complete data set } & \multicolumn{3}{|c|}{ AU/NZ } & \multicolumn{3}{|c|}{ US/CA } & \multicolumn{3}{|c|}{ 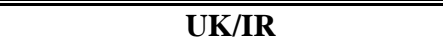 } \\
\hline & OLS & Tobit & $\begin{array}{l}\text { Negative } \\
\text { binomial }\end{array}$ & OLS & Tobit & $\begin{array}{l}\text { Negative } \\
\text { binomial }\end{array}$ & OLS & Tobit & $\begin{array}{l}\text { Negative } \\
\text { binomial }\end{array}$ & OLS & Tobit & $\begin{array}{l}\text { Negative } \\
\text { binomial }\end{array}$ \\
\hline $\begin{array}{l}\text { Equations 5a/14a } \\
\text { (low-median ID) } \\
\text { (IMD/Hofstede) }\end{array}$ & & & & & & & & & & & & \\
\hline $\begin{array}{l}\text { Ind exp - UG } \\
\text { offshore edu }\end{array}$ & & & & & & & & & & & & \\
\hline $\begin{array}{l}\text { Ind exp - PG } \\
\text { offshore edu }\end{array}$ & & & & & & & & & & & & \\
\hline $\begin{array}{l}\text { Ind exp - intl } \\
\text { recruitment } \\
\text { (Factor) }\end{array}$ & $(+)^{* * /(+) *}$ & $(+)^{* * /(+) *}$ & $(+) \dagger /(+)$ & $(+) * /(+)$ & $(+)^{* /(+)}$ & & $(+) * /(+)^{*}$ & $(+)^{* /(+) \dagger}$ & $(+) \dagger /(+)$ & & & \\
\hline $\begin{array}{l}\text { Ind exp - UG } \\
\text { marketing }\end{array}$ & & & & $(-)^{* /(-)}$ & $(-)^{* * /(-)}$ & $(-) \dagger /(-)$ & & & & $(+) /(+) \dagger$ & $(+) /(+) \dagger$ & \\
\hline $\begin{array}{l}\text { Ind exp - PG } \\
\text { marketing }\end{array}$ & & & & & $(+) /(+) \dagger$ & & & & & $(+) \dagger /(+)$ & & \\
\hline $\begin{array}{l}\text { Equations 5b/14b } \\
\text { (median-high ID) } \\
\text { (IMD/Hofstede) }\end{array}$ & & & & & & & & & & & & \\
\hline $\begin{array}{l}\text { Ind exp - UG } \\
\text { offshore edu }\end{array}$ & $(+) /(-) \dagger$ & $(+) /(-) *$ & & & $(-) /(-)^{*}$ & & & & & & & \\
\hline $\begin{array}{l}\text { Ind exp - PG } \\
\text { offshore edu }\end{array}$ & & & & & & & $(-) /(-) \dagger$ & $(-) /(-)^{*}$ & & & & \\
\hline $\begin{array}{l}\text { Ind exp - intl } \\
\text { recruitment } \\
\text { (Factor) }\end{array}$ & & & & $(+) /(+) \dagger$ & $(-) /(+) \dagger$ & & & & & & & \\
\hline $\begin{array}{l}\text { Ind exp - UG } \\
\text { marketing }\end{array}$ & $(-) /(-) \dagger$ & $(-) /(-)^{*}$ & & & & & & $(-) /(-) \dagger$ & & & & \\
\hline $\begin{array}{l}\text { Ind exp - PG } \\
\text { marketing }\end{array}$ & & & & & & & & & & & & \\
\hline
\end{tabular}

Sign of coefficient in parentheses, all $t$ tests are two-tailed, $\dagger p<.10,{ }^{*} p<.05,{ }^{* *} p<.01$ 
Table 7.54: Summary of Significant Findings for H7c (Normative Institutional Distance \& Know-how; Inverted Relationship Hypothesised With Positive Values at Low Levels of Distance and Negative Values at Higher Levels) Using the Three measures of Distance

\begin{tabular}{|c|c|c|c|c|c|c|c|c|c|c|c|c|}
\hline & \multicolumn{3}{|c|}{ Complete data set } & \multicolumn{3}{|c|}{ AU/NZ } & \multicolumn{3}{|c|}{$\overline{\text { US/CA }}$} & \multicolumn{3}{|c|}{$\overline{~ U K / I R ~}$} \\
\hline & OLS & Tobit & $\begin{array}{l}\text { Negative } \\
\text { binomial }\end{array}$ & OLS & Tobit & $\begin{array}{l}\text { Negative } \\
\text { binomial } \\
\end{array}$ & OLS & Tobit & $\begin{array}{l}\text { Negative } \\
\text { binomial } \\
\end{array}$ & OLS & Tobit & $\begin{array}{l}\text { Negative } \\
\text { binomial }\end{array}$ \\
\hline $\begin{array}{l}\text { Equations 6a/15a } \\
\text { (low-median ID) } \\
\text { (IMD/Hofstede) }\end{array}$ & & & & & & & & & & & & \\
\hline Know-how (Factor) & $\begin{array}{l}(-)^{* * /} \\
(-)^{* *}\end{array}$ & $\begin{array}{l}(-)^{* * /} \\
(-)^{* *}\end{array}$ & $\begin{array}{l}(-)^{* * /} \\
(-)^{* *}\end{array}$ & $\begin{array}{l}(-)^{* * /} \\
(-)^{* *}\end{array}$ & $\begin{array}{l}(-)^{* * /} \\
(-)^{* *}\end{array}$ & $\begin{array}{l}(-)^{* * /} \\
(-)^{* *}\end{array}$ & $\begin{array}{l}(-)^{* * /} \\
(-)^{* *}\end{array}$ & $\begin{array}{l}(-)^{* * /} \\
(-)^{* *}\end{array}$ & $\begin{array}{l}(-)^{* * /} \\
(-)^{* *}\end{array}$ & $\begin{array}{l}(-)^{* * /} \\
(-)^{* *}\end{array}$ & $\begin{array}{l}(-)^{* * /} \\
(-)^{* *}\end{array}$ & \\
\hline $\begin{array}{l}\text { Equations 6b/15b } \\
\text { (median-high ID) } \\
\text { (IMD/Hofstede) }\end{array}$ & & & & & & & & & & & & \\
\hline Know-how (Factor) & $\begin{array}{l}(-)^{* * /} \\
(-)^{* *}\end{array}$ & $\begin{array}{l}(-)^{* * 1} \\
(-)^{* *}\end{array}$ & $\begin{array}{l}(-)^{* * /} \\
(-)^{* *}\end{array}$ & $\begin{array}{l}(-)^{* * /} \\
(-)^{* *}\end{array}$ & $\begin{array}{l}(-)^{* * /} \\
(-)^{* *}\end{array}$ & $\begin{array}{l}(-)^{* * /} \\
(-)^{* *}\end{array}$ & $\begin{array}{l}(-)^{* * /} \\
(-)^{* *}\end{array}$ & $\begin{array}{l}(-)^{* * /} \\
(-)^{* *}\end{array}$ & $\begin{array}{l}(-)^{* * /} \\
(-)^{* *}\end{array}$ & $\begin{array}{c}(-) / \\
(-)^{* *}\end{array}$ & $\begin{array}{c}(-) / \\
(-)^{* *}\end{array}$ & $\begin{array}{l}(-) / \\
(-)\end{array}$ \\
\hline
\end{tabular}

Sign of coefficient in parentheses, all $t$ tests are two-tailed, $\dagger p<.10, * p<.05, * * p<.01$

Table 7.55: Summary of Significant Findings for H7d (Normative Institutional Distance \& Entrepreneurial Organisational Culture; Inverted Relationship Hypothesised With Positive Values at Low Levels of Distance and Negative Values at Higher Levels) Using the Three measures of Distance

\begin{tabular}{|c|c|c|c|c|c|c|c|c|c|c|c|c|}
\hline & \multicolumn{3}{|c|}{ Complete data set } & \multicolumn{3}{|c|}{ AU/NZ } & \multicolumn{3}{|c|}{ US/CA } & \multicolumn{3}{|c|}{ UK/IR } \\
\hline & OLS & Tobit & $\begin{array}{l}\text { Negative } \\
\text { binomial }\end{array}$ & OLS & Tobit & $\begin{array}{l}\text { Negative } \\
\text { binomial }\end{array}$ & OLS & Tobit & $\begin{array}{l}\text { Negative } \\
\text { binomial }\end{array}$ & OLS & Tobit & $\begin{array}{l}\text { Negative } \\
\text { binomial }\end{array}$ \\
\hline \multicolumn{13}{|l|}{$\begin{array}{l}\text { Equations 7a/16a } \\
\text { (low-median ID) } \\
\text { (IMD/Hofstede) }\end{array}$} \\
\hline $\begin{array}{l}\text { Org culture } \\
\text { (Factor) }\end{array}$ & $\begin{array}{l}(-)^{* * /} \\
(-)^{* *}\end{array}$ & $\begin{array}{l}(-)^{* * /} \\
(-)^{* *}\end{array}$ & $\begin{array}{l}(-)^{* * /} \\
(-)^{* *}\end{array}$ & $\begin{array}{l}(-)^{* * /} \\
(-)^{* *}\end{array}$ & $\begin{array}{l}(-)^{* * /} \\
(-)^{* *}\end{array}$ & $\begin{array}{l}(-)^{* * /} \\
(-)^{* *}\end{array}$ & $\begin{array}{l}(-)^{* * /} \\
(-)^{* *}\end{array}$ & $\begin{array}{l}(-)^{* * /} \\
(-)^{* *}\end{array}$ & $\begin{array}{l}(-)^{* * /} \\
(-)^{* *}\end{array}$ & - & - & - \\
\hline $\begin{array}{l}\text { Org culture - open } \\
\text { discussion }\end{array}$ & ( & - & - & (1) & (1) & - & (I) & (1) & (1) & $(-)^{* * /(-)}$ & $(-)^{* * /(-)}$ & \\
\hline $\begin{array}{l}\text { Org culture - no } \\
\text { status distinction }\end{array}$ & - & - & - & - & - & - & - & - & - & & $(-)^{* /(-)}$ & \\
\hline
\end{tabular}




\begin{tabular}{|c|c|c|c|c|c|c|c|c|c|c|c|c|}
\hline $\begin{array}{l}\text { Org culture - } \\
\text { experimentation }\end{array}$ & - & - & - & - & - & - & - & - & - & & & \\
\hline $\begin{array}{l}\text { Org culture - } \\
\text { offshore } \\
\text { development }\end{array}$ & - & - & - & - & - & - & - & - & - & $(-) /(-)^{*}$ & $(-)^{* /(-) * *}$ & \\
\hline $\begin{array}{l}\text { Org culture - } \\
\text { offshore } \\
\text { responsiveness }\end{array}$ & - & - & - & - & - & - & - & - & - & & & \\
\hline \multicolumn{13}{|l|}{$\begin{array}{l}\text { Equations 7b/16b } \\
\text { (median-high ID) } \\
\text { (IMD/Hofstede) }\end{array}$} \\
\hline $\begin{array}{l}\text { Org culture } \\
\text { (Factor) }\end{array}$ & $\begin{array}{l}(-)^{* * /} \\
(-)^{* *}\end{array}$ & $\begin{array}{l}(-)^{* * /} \\
(-)^{* *}\end{array}$ & $\begin{array}{l}(-)^{* * /} \\
(-)^{* *}\end{array}$ & $\begin{array}{l}(-)^{* * /} \\
(-)^{* *}\end{array}$ & $\begin{array}{l}(-)^{* * /} \\
(-)^{* *}\end{array}$ & $\begin{array}{l}(-)^{* * /} \\
(-)^{* *}\end{array}$ & $\begin{array}{l}(-)^{* * /} \\
(-)^{* *}\end{array}$ & $\begin{array}{l}(-)^{* * /} \\
(-)^{* *}\end{array}$ & $\begin{array}{l}(-)^{* * /} \\
(-)^{* *}\end{array}$ & - & - & - \\
\hline $\begin{array}{l}\text { Org culture - open } \\
\text { discussion }\end{array}$ & - & - & - & - & - & - & - & - & - & $(+)^{* * /(-)}$ & $(+)^{* * /(-)}$ & \\
\hline $\begin{array}{l}\text { Org culture - no } \\
\text { status distinction }\end{array}$ & - & - & - & - & - & - & - & - & - & & & \\
\hline $\begin{array}{l}\text { Org culture - } \\
\text { experimentation }\end{array}$ & - & - & - & - & - & - & - & - & - & & & \\
\hline $\begin{array}{l}\text { Org culture - } \\
\text { offshore } \\
\text { development }\end{array}$ & - & - & - & - & - & - & - & - & - & & & \\
\hline $\begin{array}{l}\text { Org culture - } \\
\text { offshore } \\
\text { responsiveness }\end{array}$ & - & - & - & - & - & - & - & - & - & & $(+) \dagger /(-)$ & \\
\hline
\end{tabular}

Sign of coefficient in parentheses, all $t$ tests are two-tailed, $\dagger p<.10,{ }^{*} p<.05$, ** $p<.01$ 
Table 7.56: Summary of Significant Findings for H7e (Normative Institutional Distance \& Financial Resources; Inverted Relationship Hypothesised With Positive Values at Low Levels of Distance and Negative Values at Higher Levels) Using the Three measures of Distance

\begin{tabular}{|c|c|c|c|c|c|c|c|c|c|c|c|c|}
\hline & \multicolumn{3}{|c|}{ Complete data set } & \multicolumn{3}{|c|}{ AU/NZ } & \multicolumn{3}{|c|}{ US/CA } & \multicolumn{3}{|c|}{$\overline{~ U K / I R ~}$} \\
\hline & OLS & Tobit & $\begin{array}{l}\text { Negative } \\
\text { binomial }\end{array}$ & OLS & Tobit & $\begin{array}{l}\text { Negative } \\
\text { binomial }\end{array}$ & OLS & Tobit & $\begin{array}{l}\text { Negative } \\
\text { binomial }\end{array}$ & OLS & Tobit & $\begin{array}{l}\text { Negative } \\
\text { binomial }\end{array}$ \\
\hline \multicolumn{13}{|l|}{$\begin{array}{l}\text { Equations 8a/17a } \\
\text { (low-median ID) } \\
\text { (IMD/Hofstede) }\end{array}$} \\
\hline Financial Resources & & & & & & & & & & $(-) \dagger /(-)$ & & \\
\hline \multicolumn{13}{|l|}{$\begin{array}{l}\text { Equations 8b/17b } \\
\text { (median-high ID) } \\
\text { (IMD/Hofstede) }\end{array}$} \\
\hline Financial Resources & & $(-) \dagger /(-) \dagger$ & & & & & $(-) /(-) \dagger$ & $(-) /(-) \dagger$ & & & & \\
\hline
\end{tabular}

Sign of coefficient in parentheses, all $t$ tests are two-tailed, $\uparrow p<.10,{ }^{*} p<.05,{ }^{* *} p<.01$

Table 7.57: Summary of Significant Findings for H7f (Normative Institutional Distance \& Reputation; Inverted Relationship Hypothesised With Positive Values at Low Levels of Distance and Negative Values at Higher Levels) Using the Three measures of Distance

\begin{tabular}{|c|c|c|c|c|c|c|c|c|c|c|c|c|}
\hline & \multicolumn{3}{|c|}{ Complete data set } & \multicolumn{3}{|c|}{$\overline{\mathrm{AU} / \mathrm{NZ}}$} & \multicolumn{3}{|c|}{ US/CA } & \multicolumn{3}{|c|}{$\begin{array}{l}\text { UK/IR } \\
\end{array}$} \\
\hline & OLS & Tobit & $\begin{array}{l}\text { Negative } \\
\text { binomial }\end{array}$ & OLS & Tobit & $\begin{array}{l}\text { Negative } \\
\text { binomial }\end{array}$ & OLS & Tobit & $\begin{array}{l}\text { Negative } \\
\text { binomial }\end{array}$ & OLS & Tobit & $\begin{array}{l}\text { Negative } \\
\text { binomial }\end{array}$ \\
\hline $\begin{array}{l}\text { Equations 9a/18a } \\
\text { (low-median ID) } \\
\text { (IMD/Hofstede) }\end{array}$ & & & & & & & & & & & & \\
\hline Reputation (Factor) & $\begin{array}{l}(-)^{* * /} \\
(-)^{* *}\end{array}$ & $\begin{array}{l}(-)^{* * /} \\
(-)^{* *}\end{array}$ & $\begin{array}{l}(-)^{* * /} \\
(-)^{* *}\end{array}$ & $\begin{array}{l}(-) / \\
(-) \dagger\end{array}$ & $\begin{array}{c}(-) / \\
(-)^{* *}\end{array}$ & $\begin{array}{l}(-) / \\
(-)^{*}\end{array}$ & $\begin{array}{l}(-)^{* * /} / \\
(-)^{* *}\end{array}$ & $\begin{array}{l}(-)^{* *} / \\
(-)^{* *}\end{array}$ & $\begin{array}{l}(-)^{* * /} \\
(-)^{* *}\end{array}$ & $\begin{array}{c}(-) \dagger / \\
(-)^{*}\end{array}$ & $\begin{array}{l}(-) / \\
(-)^{*}\end{array}$ & \\
\hline
\end{tabular}

Equations 9b/18b

(median-high ID) 
(IMD/Hofstede)

\begin{tabular}{|c|c|c|c|c|c|c|c|}
\hline Reputation (Factor) & $\begin{array}{l}(-)^{* * /} \\
(-)^{* *}\end{array}$ & $\begin{array}{l}(-)^{* * /} \\
(-)^{* *}\end{array}$ & $\begin{array}{l}(-)^{* * /} \\
(-)^{*}\end{array}$ & $\begin{array}{cc}(-)^{* * /} & (-)^{* * /} / \\
(-) & (-)^{*}\end{array}$ & $\begin{array}{l}(-)^{* * /} \\
(-)^{* *}\end{array}$ & $\begin{array}{l}(-)^{* * /} \\
(-)^{* *}\end{array}$ & $\begin{array}{l}(-)^{* * /} \\
(-)^{* *}\end{array}$ \\
\hline
\end{tabular}

Sign of coefficient in parentheses, all $t$ tests are two-tailed, $\dagger p<.10, * p<.05$, ** $p<.01$

Table 7.58: Summary of Significant Findings for H8a (Regulative Institutional Distance \& Geographic Experience; Inverted Relationship Hypothesised With Positive Values at Low Levels of Distance and Negative Values at Higher Levels) Using the Three measures of Distance

\begin{tabular}{|c|c|c|c|c|c|c|c|c|c|c|c|c|}
\hline & \multicolumn{3}{|c|}{ Complete data set } & \multicolumn{3}{|c|}{ AU/NZ } & \multicolumn{3}{|c|}{ US/CA } & \multicolumn{3}{|c|}{$\overline{\text { UK/IR }}$} \\
\hline & OLS & Tobit & $\begin{array}{l}\text { Negative } \\
\text { binomial }\end{array}$ & OLS & Tobit & $\begin{array}{l}\text { Negative } \\
\text { binomial }\end{array}$ & OLS & Tobit & $\begin{array}{l}\text { Negative } \\
\text { binomial }\end{array}$ & OLS & Tobit & $\begin{array}{l}\text { Negative } \\
\text { binomial }\end{array}$ \\
\hline \multicolumn{13}{|l|}{$\begin{array}{l}\text { Equations 4c/22a } \\
\text { (low-median ID) } \\
\text { (IMD/EFI) }\end{array}$} \\
\hline $\begin{array}{l}\text { Geo exp - } \\
\text { knowledge of edu } \\
\text { sector }\end{array}$ & & & & & $(+) /(+) \dagger$ & & & & & & & \\
\hline $\begin{array}{l}\text { Geo exp - } \\
\text { academic } \\
\text { capabilities }\end{array}$ & & & & & & & $(+) * /(+)$ & $(+)^{* * /(+)}$ & $(+) * /(+)$ & & & \\
\hline \multicolumn{13}{|l|}{$\begin{array}{l}\text { Geo exp - } \\
\text { managerial } \\
\text { capabilities }\end{array}$} \\
\hline $\begin{array}{l}\text { Geo exp - financial } \\
\text { capabilities }\end{array}$ & & & & & & & & & & $(-) \dagger /(+)$ & & \\
\hline \multicolumn{13}{|l|}{$\begin{array}{l}\text { Geo exp - intl } \\
\text { recruitment }\end{array}$} \\
\hline \multicolumn{13}{|l|}{$\begin{array}{l}\text { Equations 4d/22b } \\
\text { (median-high ID) } \\
\text { (IMD/EFI) }\end{array}$} \\
\hline $\begin{array}{l}\text { Geo exp - } \\
\text { knowledge of edu } \\
\text { sector }\end{array}$ & & & & $(+) \dagger /(+)$ & $(+) * /(+)$ & & & & & & & \\
\hline
\end{tabular}




\begin{tabular}{l|c|c|c|}
$\begin{array}{l}\text { Geo exp - } \\
\text { academic } \\
\text { capabilities }\end{array}$ & $(+) /(+) \dagger$ & $(+) /(+) *$ & $(+) /(+) * *$ \\
$\begin{array}{l}\text { Geo exp - } \\
\text { managerial } \\
\text { capabilities }\end{array}$ \\
$\begin{array}{l}\text { Geo exp - financial } \\
\text { capabilities }\end{array}$ \\
$\begin{array}{l}\text { Geo exp - intl } \\
\text { recruitment }\end{array}$ & $(-) /(-) \dagger$ & $(+) \dagger /(+)$ \\
\hline \hline
\end{tabular}

Sign of coefficient in parentheses, all $t$ tests are two-tailed, $\dagger p<.10,{ }^{*} p<.05,{ }^{* *} p<.01$

Table 7.59: Summary of Significant Findings for H8b (Regulative Institutional Distance \& Industry Experience; Inverted Relationship Hypothesised With Positive Values at Low Levels of Distance and Negative Values at Higher Levels) Using the Three measures of Distance

\begin{tabular}{|c|c|c|c|c|c|c|c|c|c|c|c|c|}
\hline & \multicolumn{3}{|c|}{ Complete data set } & \multicolumn{3}{|c|}{ AU/NZ } & \multicolumn{3}{|c|}{ US/CA } & \multicolumn{3}{|c|}{ UK/IR } \\
\hline & OLS & Tobit & $\begin{array}{l}\text { Negative } \\
\text { binomial }\end{array}$ & OLS & Tobit & $\begin{array}{l}\text { Negative } \\
\text { binomial }\end{array}$ & OLS & Tobit & $\begin{array}{l}\text { Negative } \\
\text { binomial }\end{array}$ & OLS & Tobit & $\begin{array}{l}\text { Negative } \\
\text { binomial }\end{array}$ \\
\hline \multicolumn{13}{|l|}{$\begin{array}{l}\text { Equations 5c/23a } \\
\text { (low-median ID) } \\
\text { (IMD/Hofstede) }\end{array}$} \\
\hline \multicolumn{13}{|l|}{$\begin{array}{l}\text { Ind exp - UG } \\
\text { offshore edu }\end{array}$} \\
\hline \multicolumn{13}{|l|}{$\begin{array}{l}\text { Ind exp - PG } \\
\text { offshore edu }\end{array}$} \\
\hline $\begin{array}{l}\text { Ind exp - intl } \\
\text { recruitment } \\
\text { (Factor) }\end{array}$ & & & & & $(+) \dagger /(+)$ & & $(+) /(+) \dagger$ & $(+) /(+)^{*}$ & & & & \\
\hline $\begin{array}{l}\text { Ind exp - UG } \\
\text { marketing }\end{array}$ & & & & & & & $(-) \dagger /(-)$ & $(-) \dagger /(-)$ & & & & \\
\hline $\begin{array}{l}\text { Ind exp - PG } \\
\text { marketing }\end{array}$ & & & & & $(+) \dagger /(+)$ & & & & & $(+) /(+) \dagger$ & $(+) /(+) \dagger$ & \\
\hline $\begin{array}{l}\text { Equations } 5 d / 23 b \\
\text { (median-high ID) } \\
\text { (IMD/EFI) }\end{array}$ & & & & & & & & & & & & \\
\hline
\end{tabular}




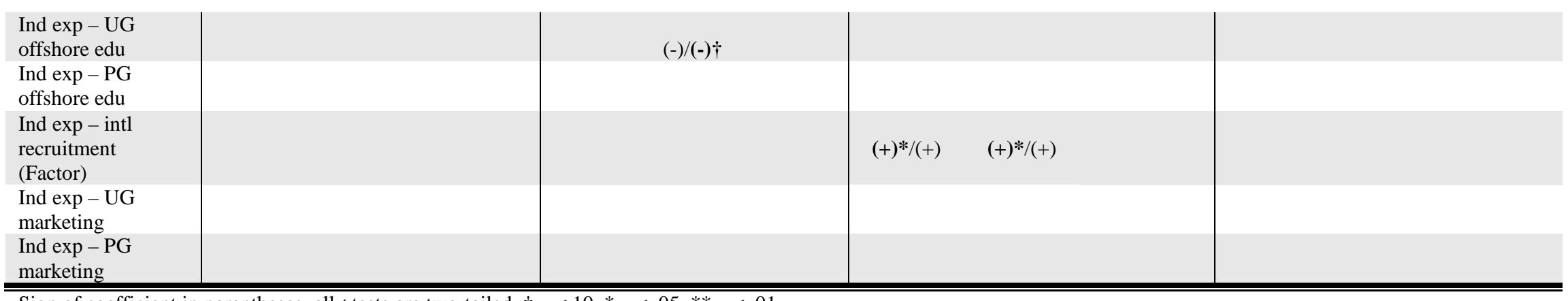

Sign of coefficient in parentheses, all $t$ tests are two-tailed, $\dagger p<.10,{ }^{*} p<.05,{ }^{* *} p<.01$

Table 7.60: Summary of Significant Findings for H8c (Regulative Institutional Distance \& Know-how; Inverted Relationship Hypothesised With Positive Values at Low Levels of Distance and Negative Values at Higher Levels) Using the Three measures of Distance

\begin{tabular}{|c|c|c|c|c|c|c|c|c|c|c|c|c|}
\hline & \multicolumn{3}{|c|}{ Complete data set } & \multicolumn{3}{|c|}{ AU/NZ } & \multicolumn{3}{|c|}{ US/CA } & \multicolumn{3}{|c|}{ UK/IR } \\
\hline & OLS & Tobit & $\begin{array}{l}\text { Negative } \\
\text { binomial } \\
\end{array}$ & OLS & Tobit & $\begin{array}{l}\text { Negative } \\
\text { binomial }\end{array}$ & OLS & Tobit & $\begin{array}{l}\text { Negative } \\
\text { binomial }\end{array}$ & OLS & Tobit & $\begin{array}{l}\text { Negative } \\
\text { binomial }\end{array}$ \\
\hline \multicolumn{13}{|l|}{$\begin{array}{l}\text { Equations 6c/24a } \\
\text { (low-median ID) } \\
\text { (IMD/EFI) }\end{array}$} \\
\hline Know-how (Factor) & $\begin{array}{c}(-)^{* * /} \\
(-)^{* *}\end{array}$ & $\begin{array}{l}(-)^{* * /} \\
(-)^{* *}\end{array}$ & $\begin{array}{l}(-)^{* * /} \\
(-)^{* *}\end{array}$ & $\begin{array}{c}(-)^{* * /} \\
(-)^{* *}\end{array}$ & $\begin{array}{c}(-)^{* * /} \\
(-)^{* *}\end{array}$ & $\begin{array}{l}(-)^{* * /} \\
(-)^{* *}\end{array}$ & $\begin{array}{c}(-)^{* * /} \\
(-)^{* *}\end{array}$ & $\begin{array}{c}(-)^{* * /} \\
(-)^{* *}\end{array}$ & $\begin{array}{c}(-)^{* * /} \\
(-)^{* *}\end{array}$ & $\begin{array}{l}(-)^{* * /} \\
(-)^{* *}\end{array}$ & $\begin{array}{l}(-)^{* * /} \\
(-)^{* *}\end{array}$ & $\begin{array}{l}(-)^{* /} \\
(-)^{*}\end{array}$ \\
\hline \multicolumn{13}{|l|}{$\begin{array}{l}\text { Equations 6d/24b } \\
\text { (median-high ID) } \\
\text { (IMD/EFI) }\end{array}$} \\
\hline Know-how (Factor) & $\begin{array}{c}(-)^{* *} / \\
(-)^{* *} \\
\end{array}$ & $\begin{array}{c}(-)^{* *} / \\
(-)^{* *} \\
\end{array}$ & $\begin{array}{l}(-)^{* * /} \\
(-)^{* *} \\
\end{array}$ & & $\begin{array}{l}(-) / \\
(-) \dagger \\
\end{array}$ & & $\begin{array}{c}(-)^{* * /} \\
(-)^{* *} \\
\end{array}$ & $\begin{array}{l}(-)^{* * /} \\
(-)^{* *} \\
\end{array}$ & $\begin{array}{c}(-)^{* * /} \\
(-)^{* *} \\
\end{array}$ & $\begin{array}{l}(-)^{* /} \\
(-)^{* *} \\
\end{array}$ & $\begin{array}{l}(-)^{* /} \\
(-)^{* *} \\
\end{array}$ & \\
\hline
\end{tabular}

Sign of coefficient in parentheses, all $t$ tests are two-tailed, $\uparrow p<.10, * p<.05, * * p<.01$ 
Table 7.61: Summary of Significant Findings for H8d (Regulative Institutional Distance \& Entrepreneurial Organisational Culture; Inverted Relationship Hypothesised With Positive Values at Low Levels of Distance and Negative Values at Higher Levels) Using the Three measures of Distance

\begin{tabular}{|c|c|c|c|c|c|c|c|c|c|c|c|c|}
\hline & \multicolumn{3}{|c|}{ Complete data set } & \multicolumn{3}{|c|}{ AU/NZ } & \multicolumn{3}{|c|}{ US/CA } & \multicolumn{3}{|c|}{ UK/IR } \\
\hline & OLS & Tobit & $\begin{array}{l}\text { Negative } \\
\text { binomial } \\
\end{array}$ & OLS & Tobit & $\begin{array}{l}\text { Negative } \\
\text { binomial }\end{array}$ & OLS & Tobit & $\begin{array}{l}\text { Negative } \\
\text { binomial } \\
\end{array}$ & OLS & Tobit & $\begin{array}{l}\text { Negative } \\
\text { binomial }\end{array}$ \\
\hline \multicolumn{13}{|l|}{$\begin{array}{l}\text { Equations 7c/25a } \\
\text { (low-median ID) } \\
\text { (IMD/EFI) }\end{array}$} \\
\hline $\begin{array}{l}\text { Org culture } \\
\text { (Factor) }\end{array}$ & $\begin{array}{l}(-)^{* * /} \\
(-)^{* *}\end{array}$ & $\begin{array}{l}(-)^{* * /} \\
(-)^{* *}\end{array}$ & $\begin{array}{l}(-)^{* * /} \\
(-)^{* *}\end{array}$ & $\begin{array}{l}(-)^{* * /} \\
(-)^{* *}\end{array}$ & $\begin{array}{l}(-)^{* * /} \\
(-)^{* *}\end{array}$ & $\begin{array}{l}(-)^{* * /} \\
(-)^{* *}\end{array}$ & $\begin{array}{l}(-)^{* * /} \\
(-)^{* *}\end{array}$ & $\begin{array}{l}(-)^{* * /} \\
(-)^{* *}\end{array}$ & $\begin{array}{l}(-)^{* * /} \\
(-)^{* *}\end{array}$ & - & - & - \\
\hline $\begin{array}{l}\text { Org culture - open } \\
\text { discussion }\end{array}$ & - & . & - & - & - & . & ( & . & - & & $(-) \dagger /(-)$ & \\
\hline $\begin{array}{l}\text { Org culture - no } \\
\text { status distinction }\end{array}$ & - & - & - & - & - & - & - & - & - & $(-)^{* /(-)}$ & $(-)^{* /(-)}$ & \\
\hline $\begin{array}{l}\text { Org culture - } \\
\text { experimentation }\end{array}$ & - & - & - & - & - & - & - & - & - & & & \\
\hline $\begin{array}{l}\text { Org culture - } \\
\text { offshore } \\
\text { development }\end{array}$ & - & - & - & - & - & - & - & - & - & $(-) \dagger /(-)$ & $(-) * /(-)$ & \\
\hline $\begin{array}{l}\text { Org culture - } \\
\text { offshore } \\
\text { responsiveness }\end{array}$ & - & - & - & - & - & - & - & - & - & & & \\
\hline \multicolumn{13}{|l|}{$\begin{array}{l}\text { Equations 7d/25b } \\
\text { (median-high ID) } \\
\text { (IMD/EFI) }\end{array}$} \\
\hline $\begin{array}{l}\text { Org culture } \\
\text { (Factor) }\end{array}$ & $\begin{array}{l}(-)^{* * /} \\
(-)^{* *}\end{array}$ & $\begin{array}{l}(-)^{* * /} \\
(-)^{* *}\end{array}$ & $\begin{array}{l}(-)^{* * /} \\
(-)^{* *}\end{array}$ & & & & $\begin{array}{l}(-)^{* * /} \\
(-)^{* *}\end{array}$ & $\begin{array}{l}(-)^{* * /} \\
(-)^{* *}\end{array}$ & $\begin{array}{l}(-)^{* * /} \\
(-)^{* *}\end{array}$ & - & - & - \\
\hline $\begin{array}{l}\text { Org culture - open } \\
\text { discussion }\end{array}$ & - & - & - & - & - & - & - & - & - & & & \\
\hline $\begin{array}{l}\text { Org culture - no } \\
\text { status distinction }\end{array}$ & - & - & - & - & - & - & - & - & - & & & \\
\hline $\begin{array}{l}\text { Org culture - } \\
\text { experimentation }\end{array}$ & - & - & - & - & - & - & - & - & - & & $(-) /(-) \dagger$ & \\
\hline $\begin{array}{l}\text { Org culture - } \\
\text { offshore } \\
\text { development }\end{array}$ & - & - & - & - & - & - & - & - & - & $(-) /(-) \dagger$ & $(-) /(-) \dagger$ & \\
\hline
\end{tabular}


Org culture -

offshore

responsiveness

Sign of coefficient in parentheses, all $t$ tests are two-tailed, $\uparrow p<.10, * p<.05, * * p<.01$

Table 7.62: Summary of Significant Findings for H8e (Regulative Institutional Distance \& Financial Resources; Inverted Relationship Hypothesised With Positive Values at Low Levels of Distance and Negative Values at Higher Levels) Using the Three measures of Distance

\begin{tabular}{|c|c|c|c|c|c|c|c|c|c|c|c|c|}
\hline & \multicolumn{3}{|c|}{ Complete data set } & \multicolumn{3}{|c|}{$\overline{\mathrm{AU} / \mathrm{NZ}}$} & \multicolumn{3}{|c|}{ US/CA } & \multicolumn{3}{|c|}{ UK/IR } \\
\hline & OLS & Tobit & $\begin{array}{l}\text { Negative } \\
\text { binomial }\end{array}$ & OLS & Tobit & $\begin{array}{l}\text { Negative } \\
\text { binomial }\end{array}$ & OLS & Tobit & $\begin{array}{l}\text { Negative } \\
\text { binomial }\end{array}$ & OLS & Tobit & $\begin{array}{l}\text { Negative } \\
\text { binomial }\end{array}$ \\
\hline $\begin{array}{l}\text { Equations 8c/26a } \\
\text { (low-median ID) } \\
\text { (IMD/EFI) }\end{array}$ & & & & & & & & & & & & \\
\hline $\begin{array}{l}\text { Financial } \\
\text { Resources }\end{array}$ & & & & & & & & & & & & \\
\hline $\begin{array}{l}\text { Equations 8d/26b } \\
\text { (median-high ID) } \\
\text { (IMD/EFI) }\end{array}$ & & & & & & & & & & & & \\
\hline $\begin{array}{l}\text { Financial } \\
\text { Resources }\end{array}$ & $(-) \dagger /(-)$ & $(-) \dagger /(-)$ & & & & & & & & $(-) /(-) \dagger$ & $(-) /(-) \dagger$ & \\
\hline
\end{tabular}

Sign of coefficient in parentheses, all $t$ tests are two-tailed, $\dagger p<.10,{ }^{*} p<.05$, ** $p<.01$ 
Table 7.63: Summary of Significant Findings for H8f (Regulative Institutional Distance \& Reputation; Inverted Relationship Hypothesised With Positive Values at Low Levels of Distance and Negative Values at Higher Levels) Using the Three measures of Distance

\begin{tabular}{|c|c|c|c|c|c|c|c|c|c|c|c|c|}
\hline & \multicolumn{3}{|c|}{ Complete data set } & \multicolumn{3}{|c|}{ AU/NZ } & \multicolumn{3}{|c|}{ US/CA } & \multicolumn{3}{|c|}{ UK/IR } \\
\hline & OLS & Tobit & $\begin{array}{l}\text { Negative } \\
\text { binomial }\end{array}$ & OLS & Tobit & $\begin{array}{l}\text { Negative } \\
\text { binomial } \\
\end{array}$ & OLS & Tobit & $\begin{array}{l}\text { Negative } \\
\text { binomial }\end{array}$ & OLS & Tobit & $\begin{array}{l}\text { Negative } \\
\text { binomial }\end{array}$ \\
\hline $\begin{array}{l}\text { Equations 9c/27a } \\
\text { (low-median ID) } \\
\text { (IMD/EFI) }\end{array}$ & & & & & & & & & & & & \\
\hline Reputation (Factor) & $\begin{array}{l}(-)^{* * /} \\
(-)^{* *}\end{array}$ & $\begin{array}{l}(-)^{* * /} \\
(-)^{* *}\end{array}$ & $\begin{array}{l}(-)^{* *} / \\
(-)^{* *}\end{array}$ & $\begin{array}{l}(-)^{* /} \\
(-)^{* *}\end{array}$ & $\begin{array}{l}(-)^{* /} \\
(-)^{* *}\end{array}$ & $\begin{array}{l}(-) / \\
(-)^{*}\end{array}$ & $\begin{array}{l}(-)^{* * /} \\
(-)^{* *}\end{array}$ & $\begin{array}{l}(-)^{* * /} \\
(-)^{* *}\end{array}$ & $\begin{array}{l}(-)^{* * /} \\
(-)^{* *}\end{array}$ & & & \\
\hline $\begin{array}{l}\text { Equations 9d/27b } \\
\text { (median-high ID) } \\
\text { (IMD/EFI) }\end{array}$ & & & & & & & & & & & & \\
\hline Reputation (Factor) & $\begin{array}{l}(-)^{* * /} \\
(-)^{* *}\end{array}$ & $\begin{array}{l}(-)^{* * /} \\
(-)^{* *}\end{array}$ & $\begin{array}{c}(-)^{* *} / \\
(-) \dagger \\
\end{array}$ & & & & $\begin{array}{l}(-)^{* * /} \\
(-)^{* *}\end{array}$ & $\begin{array}{l}(-)^{* *} / \\
(-)^{* *}\end{array}$ & $\begin{array}{c}(-) * / \\
(-)\end{array}$ & $\begin{array}{l}(-)^{* * /} \\
(-)^{* *}\end{array}$ & $\begin{array}{l}(-)^{* * /} \\
(-)^{* *}\end{array}$ & \\
\hline
\end{tabular}

Sign of coefficient in parentheses, all $t$ tests are two-tailed, $\uparrow p<.10, * p<.05, * * p<.01$ 


\subsection{CHAPTER SUMMARY}

This chapter presents the results of the data analysis conducted from the survey administered with the most knowledgeable executives familiar with the foreign market entry of their respective universities. A final response rate of $59.7 \%$ was obtained representing a final data set of 308 observations. These observations cover the three types of entry modes examined: wholly-owned subsidiary, joint venture and non-equity arrangements. In sum, the empirical analysis highlighted through equations 1-27 (see tables 7.10-7.42) suggests that support is obtained for H1c (Transfer experience), but only when using OLS models. Mixed support is also obtained for H1a (Geographic experience), H1b (Industry experience) and H5 (Financial resources). All the other hypotheses examined in this study are not supported. Of particular interest are H2 (Know-how), H3 (Organisational culture) and H6 (Reputation) which are found to be significant (at least $p<0.10$ ) but with negative coefficients while a positive relationship is hypothesised. Similarly, for the moderating hypotheses of H7c (Know-how and normative institutional distance), $\mathrm{H} 7 \mathrm{~d}$ (Organisational culture and normative institutional distance), H7f (Reputation and normative institutional distance), H8c (Know-how and regulative institutional distance), H8d (Organisational culture and regulative institutional distance) and H8f (Reputation and regulative institutional distance), contrary to an inverted hypothesised relationship, negative significant coefficients are mostly observed at both low and higher levels of institutional distance. Possible explanations for these contradicting relationships are advanced based on the interviews conducted during the exploratory qualitative phase of this study (see Chapter 6). Last, but not least, a key objective of the analysis highlighted in equations 1-27 above is to assess the sensitivity and robustness of the institutional distance measure using three measures: the IMD World Competitiveness Yearbook, Hofstede cultural indices and data from the Economic Freedom Index. Overall, the findings are fairly consistent across the different measures, indicating a fairly stable robustness of the observed findings. 


\section{Chapter Eight}

\section{SUMMARY AND CONCLUSION}

\subsection{INTRODUCTION}

This study examines trade in education services. Estimated at roughly US\$65 billion and representing roughly $3 \%$ of the world's services export (Alderman, 2001), trade in education services is fast becoming a global business (Czinkota, 2006). To date, growth in the trade of education services has largely developed through international student mobility or what is termed in the services literature as Mode 2 trade, one of the four "Modes of Supply" through which services can be traded (see Box 8.1 and Chapter 2 for a more detailed discussion).

\section{Box 8.1: The different modes of services trade according to the GATS classification}

Mode 1: Cross-border supply corresponds to the common form of trade in goods; only the service itself crosses the border (e.g. online learning).

Mode 2: Consumption abroad refers to a situation in which a service consumer moves to another country to obtain the service (e.g. a student who travels abroad to study).

Mode 3: Commercial presence of educational services refers to the commercial establishment of facilities abroad by education providers (e.g. 'local branch campuses' or partnerships with domestic education institutions).

Mode 4: Presence of natural persons consists of a natural person (e.g. a professor, researcher, teacher, etc.) travelling to another country on a temporary basis to provide an educational service.

Source: Adapted from Larsen et al (2002), Knight (2002a) \& World Trade Organisation (2004)

While Mode 2 currently constitutes the bulk of trade in education services, it is highlighted in Chapter 2 how trade in education services is currently evolving via the other "Modes of supply". This study focuses specifically on the development of Mode 3 (Commercial Presence) trade in education services. Based on the overall trend of services trade, growth in the trade of education services is postulated to take place 
primarily via Mode 3 and to a lesser extent Mode 1 (OECD, 2004). Mode 3 developments are referred to as 'offshore' or 'transnational', ${ }^{38}$ education (used interchangeably in this study). To position the growth that is currently taking place in offshore education, Chapter 2 presents a snapshot of the vast array of Mode 3 transnational developments. It is highlighted in Section 2.2, how the existing conventions used in the education literature to describe Mode 3 transnational education are multidimensional. Twinning programmes, articulation programmes, franchise (or license programmes), joint award programmes are just some of the terms currently used in the literature, often inconsistently, to describe the complex range of Mode 3 activity (Davis et al, 2000). In an attempt to bring structure to the debates pertaining to categorising and defining offshore education, this study develops a typology by building on concepts from the international business and strategic management literature. In particular, the literature on three ownership forms - equity, joint ventures and nonequity arrangements - is employed to explain transnational education developments. Given that growth in offshore education is taking place through both equity and nonequity arrangements, the research question that this study addresses is: What explains entry mode ${ }^{39}$ choice for education providers entering overseas markets? From a review of the mode of entry literature, the resource-based view, organisational capability theory ${ }^{40}$ and institutional theory are employed as the theoretical perspectives particularly applicable for addressing the offshore education phenomenon. This approach is particularly applicable because of the importance of resources (defined as both capabilities and rent-generating assets) in the education context. For example, the

\footnotetext{
${ }^{38}$ As discussed in Chapter 1, the term 'transnational' employed in this study is not to be confused with Bartlett and Ghoshal's use of the term, (Bartlett and Ghoshal, 1987). Section 1.2 provides a definition of 'transnational' as used in the education service context, the focus of this study. Similarly, the terms 'global' and 'international' as employed in this study, are not to be confused with Bartlett and Goshal's use of the term.

${ }^{39}$ Following previous studies (e.g. Rajan and Pangarkar, 2000; Rose and Ito, 2004), the dependent variable in this study is the focal university's equity stake for a single overseas investment.

${ }^{40}$ As discussed in Chapter 3, there is debate in the literature as to whether Organisational Capability is a separate paradigm to the RBV. This study treats the two paradigms separately in order to explore their theoretical underpinnings individually. The study, however, acknowledges that there is a great deal of overlap between the two theoretical perspectives and views the capabilities approach as an extension of the RBV.
} 
service delivered by education providers is embedded in human resources (i.e. academics/faculty), without whom, offshore investment cannot take place. The education services context is also a good fit to institutional theory, since the offshore environment in which the education provider operates may have a moderating role on the influence of resources on entry modes, by adding or reducing the transaction costs of the entry mode process. The education sector in most countries is a regulated sector, where authorities monitor the delivery of quality education. Therefore, when investing offshore, education service providers are likely to operate around some form of regulated institutional environments that are likely to affect their mode of entry decisions.

A conceptual model for investigating the influence of key resources on entry modes in the education context is presented in Chapter 4. Building on Grant's (1991) typology of resources (and capabilities), the conceptual model focuses on financial, human, reputation and organisational resources. The model builds on two assumptions of the resource-based approach to entry mode selection. First, under the RBV, a higher level of ownership is presumed to be the preferred entry mode until proven otherwise, since it provides the organisation the most control in protecting its rent-generating assets and capabilities (Stopford and Wells, 1972; Ekeledo and Sivakumar, 2004). As previously discussed, this assumption is consistent with empirical studies that have found that US firms tend to prefer sole ownership as a mode of entry (Anderson and Gatignon, 1986; Erramilli and Rao, 1993). There is also documented evidence in the literature that Japanese firms generally view alliance as a second-best alternative to sole ownership (Hamel, 1991). This fundamental assumption of the resource-based view differs to that of the transaction cost approach, which typically views shared-control modes as the default mode of entry (Anderson and Gatignon, 1986). Second, implicit in the first assumption, is the condition that the target foreign market has enough current or potential demand to make a higher level of ownership a viable entry mode. Otherwise, a higher level of ownership would not merit consideration, since it is more resource intensive (Anderson and Gatignon, 1986). These two assumptions provide the foundation for the analytical framework used in this study. Building on these two 
assumptions, the basic premise of the conceptual model developed in Chapter 4, therefore, assumes that an education service provider in possession of resources that are potential sources of competitive advantage in a target market, would favour a mode of entry that facilitates control over and protection of the resources. The conceptual model further postulates that this relationship is moderated by aspects of institutional distance between the home and host country.

A multi-method research design consisting of two stages is employed to test the hypotheses developed from the conceptual model. In the first stage, exploratory interviews are conducted with representatives of universities operating offshore. The interviews allow for validation and refinement of the research model (Churchill, 1979) as well as to gain a better understanding of the subject matter at hand (Denzin and Lincoln, 2000). Lasting about one hour each, the interviews are conducted with 10 senior university representatives responsible to international operations and took place over a three-month period between January and March 2008. A conscious decision is made to target universities across all six English-speaking home countries (i.e. Canada, United States, United Kingdom, Ireland, Australia, and New Zealand) and all three modes of entry under investigation in this study: wholly-owned subsidiaries, joint ventures and non-equity arrangements. This purposeful and maximum variation sampling approach is used to obtain access to a diverse set of opinions and perspectives about foreign market entry in the educational sector. The qualitative data gathered from the interviews are subjected to content analysis using QSR*NUDIST, allowing for the exploration and explanation of the inter-relationships among the generated constructs. Chapter 6 highlights the findings that emerged from the interviews.

Post interviewing, surveys are pre-tested and then administered with university officials familiar with the foreign market entry of their respective universities (see Chapter 5). A usable response rate of $59.7 \%$ is obtained, representing 154 universities from the 258 identified in the sampling frame outlined in Appendix J. With each institution responding to the questionnaire with respect to two recent offshore education 
operations, a final data set of 308 instances of foreign market entry is obtained. Chapter 7 presents the analysis of and the findings from this data set.

Ordinary least squares (OLS) regression is used to derive initial results. However, because the dependent variable of equity stake variable employed in this study is censored - it can only assume values between 0 and 100 - and ordinary least squares with a censored dependent variable tends to yield coefficient estimators that are biased toward zero (Rose and Ito, 2004), Tobit analysis is also used to test the robustness of the OLS findings. Similarly, because the dependent equity stake variable is also operationalised as an ordinal variable, which is similar to a count variable, a negative binomial regression approach is also employed to test the hypotheses of interest. Using these three different regression approaches allows for sensitivity and robustness assessment of the results.

Furthermore, to assess the robustness of IMD's measure of normative and regulative institutional distance (see Section 5.3.3), two alternative measures of distance are also analysed: Hofstede’s (1980) cultural distance and EFI regulative institutional distance. With nine equations analysed to test hypotheses H1-H8 and with these nine equations re-estimated using the two alternative measures of distance, 27 equations are examined in total. Since each equation is analysed with respect to four geographical clusters (AU/NZ, US/CA, UK/IR and the aggregate data set), and each of these estimated using three approaches (OLS, Tobit and negative binomial), 324 models are estimated in total (27 equations x [4 geographical clusters x 3 regression methods for each cluster] - see Section 7.6). The findings from these analyses are presented in Chapter 7.

\subsection{DISCUSSION AND IMPLICATIONS}

As indicated above, based on two key assumptions of the resource-based view, the conceptual model developed in this dissertation, postulates that an education service provider in possession of resources that are potential sources of competitive advantage in a target market, would favour a mode of entry that facilitates control over and protection of the resources. However, as discussed in Chapter 3, because of the diversity of services, any theoretical assumptions can only serve as a starting point in the 
development of context and service-industry specific analytical frameworks (Richardson, 1987). The results observed in this study lend credence to this position. Of the hypotheses tested, only H1c (Transfer experience) is supported, but only when using OLS models. Mixed results are observed for H1a (Geographic experience), H1b (Industry experience), and H5 (Financial resources), indicating both positive and negative relationships between ownership levels and these respective types of resources. Likewise, the negative and significant findings for H2 (Know-how), H3 (Organisational culture) and H6 (Reputation) contradict the hypothesised positive relationship with equity stake, marginal to the other variables analysed. Institutional distance, as operationalised in this study, is also not found to be a moderating variable in the modelling. Possible explanations for these empirical findings are conjectured in the previous chapter (see section 7.10) and are summarised in Box 8.2 below.

\section{Box 8.2: Summary of Findings}

H1a (Geographical experience) and H1b (Industry experience): Positive relationship hypothesised, both positive and negative results observed.

In the education context, less resource intensive entry modes may be riskier than more resource intensive entry modes, such that, with more experience comes the ability to deal with the uncertainties associated with less resource intensive entry modes. As indicated in Section 7.3.6, non-equity arrangements in the education services context was interpreted by the respondents to include academic investments and not just financial capital investment as in the more commercial sense. Therefore, it can be postulated that, given such academic investment, there is an associated risk in being involved in less resource intensive entry modes, given less control over the academic delivery process.

H1c (Transfer Experience): Negative relationship hypothesised, negative results observed in OLS models.

As hypothesised, the sampled education service providers seem to develop routine responses to choosing an entry mode. These routines can result in a "locked-in" situation whereby managers might not adopt a more resource intensive entry mode, given that their accustomed level sets a benchmark of familiarity, effectively discouraging higher levels of resource commitments in subsequent foreign market entries. The observed findings in the OLS models support this hypothesis. However, since these results are not replicated in the Tobit and negative binomial models, it would be appropriate for future research to further explore how transfer experience is related to the level of equity investment in the offshore education context. 


\section{Box 8.2: Summary of Findings (continued)}

H2 (Know-how): Positive relationship hypothesised, negative results observed.

The unexpected observed relationship in the sample, marginal to the other variables in the models, requires additional research. Comments made by the representative of US2 in the qualitative phase of this study could be a point of departure for such future research: "....the totality of what constitutes a top quality institution as an organisational organism is not mobile”, indicating the possibility that even more resource intensive modes of entry may not be conducive to tacit know-how transfer.

H3 (Organisational culture): Positive relationship hypothesised, negative results observed.

As discussed previously, in the education context, less resource intensive ownership forms are not necessarily associated with lower risk. In Chapter 4, it is theorised that the more entrepreneurial the organisational culture of a university is, the more accepting of risks it will be in offshore education developments. It appears that the education context may be a special case, such that more entrepreneurial universities are more likely to be at ease in managing the academic and reputational risks involved in less resource intensive modes of entry.

H4 (Learning intent): Positive relationship hypothesised for joint ventures, no significant findings observed.

The obtained empirical findings indicate a lack of support for this hypothesis. Learning intent, in fact, did not emerge as a theme during the exploratory qualitative phase of this study (see Chapter 6), indicating that the learning intent of universities might not play a determining role in offshore entry mode selection in the education context.

H5 (Financial resources): Positive relationship hypothesised, both positive and negative results observed.

I conjecture that this mixed support can be explained by the fact that the survey respondents might have had different interpretations of equity investment. As per noted earlier, equity investment in the education context, is not necessarily understood as financial capital investment, but also includes academic investment. Consequently, access to finance might be context-specific in terms of its relationship on the entry mode decision since equity investment is not solely undertaken through financial capital investment.

H6 (Reputation): Positive relationship hypothesised, negative results observed.

Informed by extant theory, it is hypothesised that more resource intensive modes of entry would provide control over brand protection. However, the empirical findings contradict this hypothesis. It is conjectured that, in the education context, more reputable universities might have less of a financial need to engage offshore, in turn, making them less aggressive in terms of the resource intensity of their entry modes. On the other hand, less reputable universities may need to go overseas to survive from competition by leading domestic universities. This financial motive might push these universities to be more aggressive in their overseas investment, opting for more resource-intensive entry modes. 


\section{Box 8.2: Summary of Findings (continued)}

H7a (Geographical experience and normative institutional distance): inverted relationship hypothesised, hypothesis not supported.

As per previous discussions for $\mathrm{H} 1 \mathrm{a}$, I conjecture that possible explanations for a negative relationship might be that more geographic experience brings the ability to deal with the academic risks associated with non-equity arrangements. Similarly, more geographic experience could lead to better relationships with local institutions, in turn providing a better foundation for collaborative offshore engagements to take place. However, the negative relationship between geographic experience and equity stake that these explanations suggest, appears applicable only at lower levels of normative institutional distance. At higher levels of distance, universities might have to increase their equity stake in order to maintain control over their offshore engagements and avoid the academic and reputational pitfalls of failure. With more normative institutional distance, besides the associated academic and reputational risks of operating offshore, universities might also have to face up to the challenges of operating in a different institutional environment. These challenges may bring an added level of complexity in operating offshore which may encourage universities to regain control over their operations.

H7b (Industry experience and normative institutional distance): inverted relationship hypothesised, hypothesis not supported.

Based on extant theory and the interviews conducted (see Chapter 6), it proved difficult to develop a convincing conjecture to explain the observed findings with respect to the moderating influence of normative institutional distance on the industry experience-equity stake relationship. Therefore, this finding presents an opportunity for future research.

H7c (Know-how and normative institutional distance): inverted relationship hypothesised, hypothesis not supported.

This finding is highly consistent with the finding for H2. Similar to the conjecture for that hypothesis, I advance that, in the education context, more resource intensive modes of entry may not be conducive to tacit know-how transfer.

H7d (Organisation culture and normative institutional distance): inverted relationship hypothesised, hypothesis not supported.

This finding is similar to the finding of $\mathrm{H} 3$ and I, therefore, conjecture likewise to H3, that the more entrepreneurial a university is, the more it is likely to be at ease in managing the academic and reputational risks involved with less resource intensive modes of entry. Furthermore, in the UK/IR grouping, a V-shaped relationship is found contrary to the hypothesised inverted relationship. The source of this geographic distinction presents an opportunity for future research.

H7e (Financial resources and normative institutional distance): inverted relationship hypothesised, hypothesis not supported.

Similar to the previous discussions for $\mathrm{H} 5$, I postulate that these findings can be partly explained by the fact that, in the education context, equity investment is not necessarily understood as financial capital investment but can also include academic investment. 


\section{Box 8.2: Summary of Findings (continued)}

H7f (Reputation and normative institutional distance): inverted relationship hypothesised, hypothesis not supported.

These findings are consistent with the findings for $\mathrm{H} 6$ and, likewise to that hypothesis, I conjecture that in the education context, more reputable universities might have less of a financial need to engage offshore, in turn, making them less aggressive in terms of the resource intensity of their entry modes. On the other hand, less reputable universities may need to go overseas to survive from competition by leading domestic universities. This financial motive might push these universities to be more aggressive in their overseas investment, opting for more resource-intensive entry modes.

H8a (Geographical experience and regulative institutional distance): inverted relationship hypothesised, hypothesis not supported.

For those positive coefficients at low levels of distance, the associated negative finding is not observed at higher levels of distance. Furthermore, of the significant variables at low levels of distance, the coefficients associated with geographic experience - financial capabilities are significantly negative in the UK/IR OLS model. This finding is particularly interesting given that it is the only such negative finding at low levels of distance. As per the previous discussion for $\mathrm{H} 1 \mathrm{a}$, I conjecture that a possible explanation for this negative relationship might be that more geographic experience brings the ability to deal with the academic risks associated with non-equity arrangements.

H8b (Industry experience and regulative institutional distance): inverted relationship hypothesised, hypothesis not supported.

Similar to the findings for H7b, it proved difficult to develop a convincing conjecture, based on extant theory and the exploratory interviews conducted (see Chapter 6), to explain the observed findings with respect to the moderating influence of regulative institutional distance on the industry experience-equity stake relationship. Therefore, this finding presents an opportunity for future research.

H8c (Know-how and regulative institutional distance): inverted relationship hypothesised, hypothesis not supported.

These findings are consistent with the finding for $\mathrm{H} 2$ and similar to the conjecture for that particular hypothesis, I advance that, in the education context, more resource intensive entry modes may not be conducive to tacit know-how transfer.

H8d (Organisational culture and regulative institutional distance): inverted relationship hypothesised, hypothesis not supported.

These findings are similar to the finding of $\mathrm{H} 3$ and I, therefore, conjecture, likewise to H3, that the more entrepreneurial a university is, the more it is likely to be at ease in managing the academic and reputational risks involved in less resource intensive modes of entry. 


\section{Box 8.2: Summary of Findings (continued)}

H8e (Finance and regulative institutional distance): inverted relationship hypothesised, hypothesis not supported.

Similar to the previous discussion for H5, I postulate that these findings can be partly explained by the fact that, in the education context, equity investment is not necessarily understood as financial capital investment but can also include academic investment.

H8f (Reputation and regulative institutional distance): inverted relationship hypothesised, hypothesis not supported.

These findings are fairly consistent with the findings for $\mathrm{H} 6$ and, likewise to that hypothesis, I conjecture that in the education context, more reputable universities might have less of a financial need to engage offshore, in turn, making them less aggressive in terms of the resource intensity of their entry modes. On the other hand, less reputable universities may need to go overseas to survive from competition by leading domestic universities. This financial motive might push these universities to be more aggressive in their overseas investment, opting for more resource-intensive entry modes.

Taken as a whole, the above results indicate several significant contributions. These are classified below in terms of theoretical and managerial contributions.

\subsection{CONTRIBUTIONS}

\subsubsection{Theoretical contributions}

As mentioned in Chapter 1, one of the most critical issues in international strategy is the selection of an entry mode (Wind and Perlmuter, 1977; Terpstra and Sarathy, 2000). With ownership form being a relatively important determinant of performance (Sharma, 1993; Root, 1994; Li, 1995), it is crucial to gain an understanding of when particular equity channels are appropriate. This study is motivated by the importance of the international entry mode phenomenon, especially as it applies to the education services sector. Erramilli and Rao (1990:136) highlight how "little is known about how service firms enter foreign markets”. Clark, Rajaratnam and Smith (1996:9) further elaborate that "for international services, theory lags practice by a considerable degree and many important questions await answers”. More recently, Contractor, Kundu and Hsu (2003:9) state that “...there is little research on the growth and internationalisation of service firms...” and further suggest that “...there are substantial differences among 
different types of services". This study contributes to the theoretical literature by seeking to understand entry decisions, in the specific context of education services.

The observed findings indicate that entry decisions in the case of education services do not conform to mainstream international business and strategic management theories. Traditional international business research has largely focused on for-profit firms. Due to diminishing public funding, it was discussed in Chapter 2 how universities are increasingly being run along business lines and subsequently in Chapter 4, I applied mainstream international business theories to the context of a commercialised higher education sector. However, as observed from the findings, there appears to be some important distinctions between universities and for-profit businesses in the sense that although commercially oriented, universities are largely still operating as not-for-profit service organisations. As such, it needs to be recognised that the international operations of universities are different from those of regular multinational firms. For example, in contrast to business enterprises, there are a number of non-profit maximisation drivers for universities to engage in off-shoring. As identified in Chapter 6 , these include the universities' civic duties, the internationalisation of their curriculum and professional development of academic staff, among others. As such, the above findings contribute to further our understanding of entry modes in services industries, particularly not-for-profit service organisations.

How universities as not-for-profit service organisations view the notion of equity investment is also an interesting theoretical contribution. As discussed in Section 7.3.6, universities view ideas of equity in a broader context than capital alone. This is in sharp contrast to the traditional focus of mainstream international business literature on financial offshore equity investment. This broader understanding of offshore equity investment, which may not be unique to the context of education services, but also pertain to not-for-profit organisations in general (e.g. non-governmental organisations), is an avenue that deserves further research from the international business community. 
The study further contributes to the literature through the use of a mixed research design. By employing information captured during in-depth interviews with senior university officials experienced with foreign market entry, this study provides additional and richer insight into findings that contradict conventional theoretical wisdom. In doing so, this study begins to answer Erramilli and Rao’s (1990) call for the development of and investigation into the behavioural approach of foreign market entry complimentary to the more often used transaction cost approach. Penrose (1959) distinguishes between the objective and subjective opportunity set of a firm, postulating that decision maker's perceptions, rather than objective data, might be influential for strategic actions. Thus, in the context of market entry decisions, the mindset of managers influences organisations’ approaches to offshore activities. The data captured in this study through the use of a mixed research design allows for a richer understanding of entry mode decisions, relative to a purely qualitative or quantitative study. Similarly, the use of multiple approaches to estimation is not yet common in the international business literature. The thoroughness of the estimation approach adopted in this study, therefore, also represents another contribution to the literature.

Another major theoretical contribution of this study is that it draws from and integrates several disciplinary areas - international business, strategic management and education - to help understand an emerging global phenomenon in the form of trade in education services. Doing so has allowed for traditional concepts coming from relatively independent established areas to be linked and leveraged, in turn leading to new understandings in the area of services internationalisation. For example, an important finding of this study is that investment as a concept, is not necessarily understood in the context of education services as the more commercial interpretation of the term. As discussed above, investment in the education services context includes both academic and financial investment rather than having a pure financial focus. This definition of equity is in sharp contrast to traditional business theories. As services internationalisation becomes more established in the global business community and as more diverse service sectors become more internationally tradable, the scholarly community needs to adapt the understanding of investment to different 
contexts/industries. Similarly, this broader definition of equity could have implications for our understanding of environmental sustainability in business contexts and corporate social responsibility where firms might not be acting just to maximise shareholder wealth, but thinking more broadly when investing offshore.

Furthermore, as suggested by Cudeck and Browne (1983), an important challenge of model development is that any proposed hypothesized model must "be regarded as one of many formations for describing behavioural theory, some of which are reasonable" (pg. 50). The observed findings, which do not fully conform to the resource-based framework, exemplify how traditional theories can only present a partial view of the complexity of organisations. This is particularly true in the case of service industries, which because of their diversity, mean that any theoretical assumption can only serve as a starting point. As described in Chapter 3, while the resource-based view was adopted as the theoretical foundation for this study, there are other frameworks that have been used in the literature to explore the entry mode phenomenon. Hence, the use of other theoretical dimensions to more fully understand entry mode in the case of education services would be complimentary to this study.

Another theoretical contribution of the study is the conceptual development and operational use of the concept of distance. As discussed above, there has been a great deal of debate around the applicability of institutional versus cultural distance in international business research (e.g. Kostova and Zaheer, 1999; Shenkar, 2001). Cultural distance has taken centre stage in cross-cultural international business research, while still being prone to controversies. Some (e.g. Kostova and Zaheer, 1999) have argued that reliance on cultural distance as a measure of cross-country differences is an over-simplification, as other more complex environmental variations are ignored. Others (e.g. Shenkar, 2001) have suggested that the widely-employed Hofstede (1980) indices are outdated given that the data were collected over three decades ago and culture is an evolving concept. Such claims have led some scholars (e.g. Kostova and Zaheer, 1999) to suggest that institutional distance may be a more complete construct, compared to focusing purely on cultural distance. Institutional distance covers two 
domains not addressed by cultural distance: the regulative and normative dimensions of environmental variation. In this study, the fairly consistent findings between cultural and institutional distances, suggest that, in the context of education services, the relative importance of the two concepts of distance on the resource-equity stake relationship might be consistent with each other. This view is further supported through the stability of the results across multiple approaches to estimation (i.e. OLS, Tobit and negative binomial).

Finally, few studies in the international business and strategic management literatures have examined the influence of transfer experience on entry modes. As outlined in Chapter 3, Davidson and McFetridge (1985) is the only study that I identified as having previously investigated this relationship. Similar to the latter authors, I hypothesize that the more extensive the transfer experience of an education service provider with a particular mode of entry, the less likely it will favour a higher level of ownership. The observed findings of this study with respect to H1c add to our body of knowledge on how transfer experience is related to the choice of entry mode.

\subsubsection{Managerial contributions}

In addition to theoretical advances, this study also makes several managerial contributions. First of all, it is amongst the first studies to investigate the emerging phenomenon of trade in education services from a business (as opposed to an educational) perspective. As discussed in Chapter 1, transnational education is an under-researched area and little has been written about the nature of the forces affecting its development. Existing publications in this area tend to be grounded in the educational literature. With transnational education taking more of a market and trade approach in its expansion, there is a growing need to start addressing these developments from a business and managerial perspective, rather than purely through educational lenses. Suspicion of the commercial aspects of what is often viewed as a public good is suggested as a possible reason for this absence of research (Czinkota, 2006). However, with the effective commercialisation of education services growing at its current phenomenal rate, and all trends pointing to that continued growth, it is time 
that research addresses the commercial realities and challenges that are being experienced by managers working in the business of education. This study is a step in that direction.

Another key contribution of this study is to highlight that the action of managers tends to contradict the key assumption of the resource-based view, which suggests that organisations will favour a resource-intensive entry mode to facilitate control over, and protection of resources that are potential sources of competitive advantage in a target market. From the observed findings, it would rather appear that the foreign entry decision making process is subject to context-specific factors. Consequently, managers need to carefully examine each opportunity for offshore business activities based on the specific environment they are dealing with along with the difference between the home and host country environment. This is not altogether a surprising result. During the interviews (see Chapter 6), it was communicated by the representative of AU3 that there are a number of variables which might impact on the entry mode decision, thereby highlighting how each offshore investment opportunity need to be considered on a caseby-case basis.

The negative relationship observed between experience - geographic (H1a) and industry (H1b) - and equity investment is also worthy of mention given that mainstream international business and strategic management literatures tend to attribute a positive relationship between these two concepts. However, as observed from the findings, it would appear that, in the context of education services, more experienced universities tend to undertake less resource-intensive investment. It is conjectured that this negative relationship can be attributed to the ability of experienced universities to manage the higher academic risks associated with less resource intensive entry modes. This indicates that experienced universities might have come to the realisation that what is considered a low risk investment from a financial point of view might prove to be more risky from an academic perspective. Since offshoring in education services is a fairly new phenomenon (see Chapter 2), and consequently most universities are still fairly inexperienced with their offshoring activities, this finding has a major cautionary 
implication. As highlighted in Chapter 2, the majority of universities currently involved in offshoring activities tend to adopt low equity entry modes. This low equity approach, as mentioned in Chapter 3 and outlined in figure 3.2, is usually explained by universities starting their offshoring activities on a small scale before commitment is deepened, all in the hope of minimising risks. This gradual internationalisation process however focuses purely on financial risks and does not take into account the academic risks involved with less resource intensive entry modes. The observed findings in this study suggest that universities ought to adopt a more strategic approach to offshoring rather than one focused purely on financial motivations. Unfortunately, such a strategic focus is not mainstream among universities. As highlighted in Chapters 4 and 6, involvement in offshore education for many universities often arises from an opportunity presenting itself, often through personal contacts of academic staff (see the comment obtained from the representative of NZ1 during the interviews - Chapter 6). Consequently, a key takeaway from the observed findings is that universities need to be strategic in their offshore engagement rather than considering these opportunities as they arise in an ad-hoc manner. What might be perceived as a low-risk offshore engagement from a financial perspective might involve high academic risks such as loss of reputation. As such, it is recommended that universities develop an offshore education policy as part of their broader internationalisation strategy in order to carefully and comprehensively assess offshoring opportunities as they arise.

Furthermore, when it comes to the transfer of tacit know-how (H2), the observed findings suggest that there is an inverse relationship with equity investment. It was hypothesized in Chapter 4 that due to low codifiability of know-how, universities involved in offshore education may choose more resource intensive mode of entry to facilitate the transfer of such tacit knowledge. The unexpected observed results indicate, as described by the representative of US2 in Chapter 6, that what constitutes a top quality institution as an organisational organism is not mobile. From a recruitment/marketing perspective, many universities that are setting up campuses offshore hint at the equivalency between their educational offering offshore and homebased programmes in their marketing materials. The observed findings from this study 
suggest that this might not actually be the case. While it might be appropriate to suggest that offshore and home-based courses are developed using the same teaching and evaluation standards, it would seem a bit of a stretch to argue that both courses are equivalent even through the use of resource intensive entry modes. As such, a key takeaway from the observed findings is that universities need to realise that replicating their institution offshore through a brick and mortar strategy does not guarantee equivalent academic standards with the home-based campus, since the tacit know-how of home-based academics might not be replicated offshore. Practically, the offshore campus might even be viewed as a second best alternative to attending the main homebased campus since the ethos of the latter campus are not easily transferred to the former campus. As such, in the development of any recruitment/marketing strategy for offshore programmes, universities need to take account of that differential/segmentation aspect. For example, in setting tuition fees, the universities may consider not charging international students attending the offshore campus the same fees they would charge international students enrolling at the home-based campus.

Another managerial implication of this study relates to the observed findings for H3 (an entrepreneurial organisational culture). In many Western countries, governments are increasingly finding it difficult to provide an adequate level of funding for tertiary education against a backdrop of increasing costs in other areas of social importance (e.g. school education, health care, infrastructure, etc). This has led to a number of higher education institutions adopting a more entrepreneurial mindset in order to develop an alternative funding stream to public funds. This, as discussed in Chapter 2, has seen the development of an increasingly commercial world of higher education and in some countries has even led to the rise of export education as an industry (e.g. Australia and New Zealand), of which offshore education is one aspect. For individual universities, a symptom of being more entrepreneurial has traditionally been hypothesized to involve taking more risks and, in the case of offshore education, making more resource-intensive investments, all in the hope of realising the most return (see Chapter 4). However, the observed findings suggest that this assumption of a positive relationship between entrepreneurialism and the resource-intensity of offshore 
investments does not necessarily hold true in the education services context. As such, and contrary to theory, this implies that universities which have a high entrepreneurial orientation should not necessarily dismiss low-resource intensive offshore opportunities on the premise that they only conduct high-resource intensive offshore investments to achieve better returns. Rather, it would be appropriate for all offshore opportunities to be assessed in the context of a broader internationalisation strategy, as previously discussed.

A further implication of note is with respect to the findings for H5 (financial resources). From a public policy perspective, this rise in the commercial aspect of higher education has been both welcomed and cautioned. Many governments have, indeed, been encouraging of the development of commercial activities within their higher education sector as it eases the burden on public funding. At the same time, however, many governments have also been concerned about the need to ensure that these commercial activities do not take place at the expense of the domestic higher education needs. For example, in the offshore education context, there has been a conflict within the education public policy sphere of both encouraging and managing the offshore activities of higher education institutions such as universities. Traditionally, this conflict has been resolved by governments focusing on the financial risks posed by the offshore activities of universities. This financial focus is meant to ensure that the already diminishing public funds supporting domestic-based higher education are not diverted to offshore operations. However, the observed findings for H5 suggest that a focus on financial resources might be too narrow a criterion for adequately regulating the offshore activities of universities since it would appear that equity investment in the context of education service is not solely undertaken through financial capital investment. An effective regulation policy ought to, therefore, consider both the financial as well as the academic risks associated with offshore education activities.

Lastly, when it comes to the observed findings for H6 (reputation), it was conjectured from the observed findings that the more reputable universities might have less of a financial need to engage offshore, in turn, making them less aggressive in terms of the 
resource intensity of their entry modes. It was also conjectured that lower ranking universities may need to go overseas to survive from competition by leading domestic universities. Thus, from a regulatory perspective, reputation might provide public policy managers a criterion for predicting between those universities that might develop an interest in operating offshore and those which are unlikely to. Such predictive ability might assist public policy managers in targeting their quality assurance/audit frameworks to only specific universities of interest.

\subsection{LIMITATIONS}

As discussed in the preceding section, this study has substantial theoretical and managerial contributions. However, it does have limitations which need to be raised and addressed in future research.

First, as outlined in Section 1.4, this study investigates specific approaches to investment, particularly as they relate to not-for-profit universities. For-profit education companies, such as the Apollo group (the owner of the University of Phoenix), are outside the scope of this study. These for-profit companies tend to enter the offshore education landscape mostly through acquisition rather than greenfield investments (Vincent-Lancrin, 2004). This study does not examine acquisition as a mode of entry. With for-profit education companies becoming increasingly active in the offshore education market, an understanding of acquisition as a mode of entry could be beneficial for a greater understanding of this emerging phenomenon. Similarly, strategic alliances between education institutions are also appearing on the education landscape (for example, the Association of Pacific Rim Universities and Universitas 21). These alliances could offer the advantage of collaboratively entering the offshore education market with greater resource endowment and thus greater competitive advantage (Bannerman et al, 2005). Therefore, strategic alliances as a mode of entry also warrant attention in future research.

Second, in the current study, entry mode is treated as a static event, rather than a process. In doing so, issues such as the existence of prior relationships in the offshore 
market are not examined. As discussed in Chapter 6, offshore education developments are often founded on the basis of prior relationships at an overseas institution. Such relationships can provide the basis for trust in engaging in a collaborative non-equity arrangement as opposed to going it alone through a wholly-owned subsidiary. Therefore, an examination of prior relationships in the context of entry modes would be a fruitful area for future research.

Third, in examining whether/how institutional distance moderates the resource-equity stake relationship, it is important to recognise that governments can use policy incentives to influence investment decisions. For example, as noted in Chapter 2, both Singapore and the Middle East are strongly encouraging investment in their education sectors. Conversely, other countries (e.g. Malaysia) are restrictive, in terms of their education sector investment policies. Therefore, governmental policy factors are of interest for understanding entry mode decisions. While governmental factors are partially captured by the institutional distance construct used in this study, future research should ideally incorporate policy controls to differentiate institutional factors from evolving policy effects. In this study, subjective measures were used to capture some of this issue (see survey question $\mathrm{D}$ (iii) in Appendix M); however, the findings are inconclusive and are not presented as part of the findings reported in Chapter 7 . The use of more objective secondary measures might provide stronger insights for future studies.

Fourth, the caveats concerning self-reported, questionnaire-based responses apply to this study. There could, thus, be some response bias when the respondents provided their perceptual responses to the survey. For example, with the ranking measure, it is highly probable that respondents would have biased their answers towards better rankings for their respective universities.

Another limitation of the study is related to its generalisability. With this study having been conducted with universities from six English-speaking countries, there needs to be additional research investigating universities in other cultural contexts. This is 
particularly important as universities from non English-speaking backgrounds become more active in the offshore education landscape (see Chapter 2).

Lastly, another line of enquiry worth further investigation is the entry modeperformance relationship. While performance has not been a focus of this study, ownership form is an important determinant of performance (Sharma 1993; Root 1994; $\mathrm{Li}, 1995)$. It would be useful for future research to link the two concepts. Contemporary offshore education developments are still in their infancy and objective performance data are difficult to obtain. However, as the sector matures and data become more readily available, future research should be able to address this gap.

\subsection{CONCLUDING REMARKS}

In the last decade or so, we have witnessed a shift from an aid to a trade approach of education services. As a major industry in a number of countries, this sector has grown to include both international student mobility as well as the mobility of educational programmes and institutions across borders. Until now, there has been little research grounded in the business literature that has addressed the dynamics of this new and increasingly important part of the service sector. This study aims to partially fill this gap. Grounded in the resource-based perspective, organisational capability theory and institutional theory, a conceptual model linking specific types of resources (and capabilities) and equity stake (the operational measure of entry modes used in this study) was tested on a data set of 308 instances of foreign market entry. To ensure robustness of the measures and sensitivity of the findings, in total, 324 regression models are estimated across 27 equations (see Box 7.1 in Chapter 7), four geographical clusters (AU/NZ, US/CA, UK/IR and aggregate data set) and three methodologies (OLS, Tobit and negative binomial). The observed results are fairly consistent across the different models. From the richness of these findings, the greatest takeaway is that the results, which do not fully conform to mainstream international business and strategic management theories, can be attributed to context/industry specific conditions. As previously discussed, traditional international business research has largely focused on for-profit firms. Given that universities, the unit of analysis of this study, are "notfor-profit” organisations, it needs to be recognised that their international operations are 
different from those of regular multinational firms. These findings provide initial steps in improving our understanding of the internationalisation of the education services sector. It is hoped that other business researchers will pick up the challenge of further investigating the dynamics of the education services sector. With the sector still in its infancy, there is plenty of scope for the investigation of this phenomenon. 
Aaker, D.A. 1989. Managing Assets and Skills: The Key to a Sustainable Competitive Advantage. California Management Review, winter: 91-106.

Adam, S. 2001. Transnational Education Project Report and Recommendations. Confederation of European Union Rector's Conference. Available: www.esib.org [Retrieved 05 January 2005].

Adams, T. 1998.The Operation of Transnational Degree and Diploma Programs: The Australian Case. Journal of Studies in International Education, 2(1): 3-22.

Agarwal, S. 1994. Socio-Cultural Distance and the Choice of Joint Ventures: A Contingency Perspective. Journal of International Marketing, 2(2): 63-80.

Agarwal, S. and Ramaswami, S. 1992. Choice of Foreign Market Entry Mode: Impact of Ownership, Location and Internationalisation Factors. Journal of International Business Studies, 23(1): 1-27.

Aharoni, Y. 1993. "Globalisation of Professional Business Services” in Y. Aharoni (Ed.), Coalitions and Competition: The Globalisation of Professional Business Services, New York: Routeledge.

Aharoni, Y. and Nachum, L. (Eds). 2000. Globalization of Services: Some Implications for Theory and Practice, Routeledge, London.

Akerlof, G.A. 1970. The Market for Lemons: Qualitative Uncertainty and the Market Mechanism. Quarterly Journal of Economics, 84(3): 488-500.

Akkari, A. 2004. Education in the Middle East and North Africa: The Current Situation and Future Challenges. International Education Journal, 5(2): 144-153.

Alchian, A.A. and Demsetz, H. 1972. Production, Information Costs and Economic Organisation. American Economic Review, 62(5): 777-795.

Alderman, G. 2001. The Globalization of Higher Education: Some Observations Regarding the Free Market and the National Interest. Higher Education in Europe, XXVI (1): 47-52.

Aldrich, H.E. 1979. Organisations and Environment. Englewood Cliffs, NJ: PrenticeHall.

Aldrich, H.E. and Fiol, C.M. 1994. Fools Rush In? The Institutional Context of Industry Creation. Academy of Management Review, 19: 645-670. 
Aliber, R. 1970. “A Theory of Direct Investment” in C. Kindleberger (Ed.) The International Corporation, Cambridge, MA: MIT Press. Pg. 17-34.

Alon, I. and McKee, D.L. 1999. The Internationalisation of Professional Business Service Franchises. Journal of Consumer Marketing, 16(1): 74-85.

Altbach, P. 2007. Branch Campuses and Twinning: The Professional Obstacle. International Higher Education, 48:2-3.

Amit R. and Shoemaker, P.J.H. 1993. Strategic Assets and Organisational Rent. Strategic Management Journal, 14(1), 33-46

Andersen, O. 1997. Internationalisation and Market Entry Mode: A Review of Theories and Conceptual Frameworks. Management International Review, (37): 27-42.

Anderson, E. 1985. The Salesperson as Outside Agent or Employee: A Transaction Cost Analysis. Marketing Science, 4(3): 134-154.

Anderson, E. 1990. Two Firms, One Frontier: On Assessing Joint Venture Performance. Sloan Management Review, 18(Winter): 19-30.

Anderson, E. and Schmittlein, D. 1984. Integration of the Sales Force: An Empirical Examination. Rand Journal of Economics, 15(3): 385-395.

Anderson, E. and Gatignon, H. 1986. Modes of Foreign Entry: A Transaction Cost Analysis and Propositions. Journal of International Business Studies, 17(Fall): $1-26$.

Anderson, O. 1993. On the Internationalisation Process of Firms: A Critical Analysis. Journal of International Business Studies, 24(2): 203-209.

Anderson, S. and Wictor, I. 2003. Innovative Internationalisation in New Firms: Born Globals - The Swedish Case. Journal of International Entrepreneurship, 1(3): $249-275$.

Apollo Group. 2005. Annual Report. Available: www.apollogrp.edu. [Retrieved 24 December 2006].

Ardichvili, A., Cardozo, R. and Ray, S. 2003. A Theory of Entrepreneurial Opportunity Identification and Development. Journal of Business Venturing, 18: 105-123.

Argote, L. 1982. Input Uncertainty and Organizational Coordination in Hospital Emergency Units. Administrative Science Quarterly, 27(3): 420-434.

Arogyaswamy, D. and Byles, C.M. 1987. Organizational Culture: Internal and External 
Fits. Journal of Management, 13(4): 647-659.

Arrow, K.J. 1974. The Limits of Organisation, New York: W.W. Norton \& Company.

Arrow, K.J. 1985. "The Economics of Agency” in Pratt, J. \& Zechhauser (Eds.), Principals and Agents: The Structure of Business. Boston: Harvard Business School Press. Pg. 37-51.

Arvidson, N. 1997. "Internationalisation of Service Firms: Strategic Considerations" in G. Chryssochoidis, C. Miller and J. Clegg (Eds.) Internationalisation Strategies. St Martin's Press, New York, Pg. 71-89.

Asian Development Bank. 2002. Policy on Education. Available: www.adb.org [Retrieved 06 March 2004].

Aulakh, P.S. and Kotabe, M. 1997. Antecedents and Performance Implications of Channel Integration in Foreign Markets. Journal of International Business Studies, 28(1): 145-175.

Australian Vice-Chancellors' Committee. 2003. Offshore Programmes of Australian Universities. Available: www.avcc.edu.au [Retrieved 25 December 2006].

Autio, E., Sapienza, H.J. and Almeida, J.G. 2000. Effects of Age at Entry, Knowledge Intensity and Inimitability on International Growth. Academy of Management Journal, 43: 909-925.

Avalos, B. 1992. Education for the Poor: Quality or Relevance? British Journal of Sociology of Education, 13 (4): 419-436

Badaracco, J.L. 1991. The Knowledge Link, Boston, MA: Harvard Business School Press.

Bain, J. 1956. Barriers to New Competition. Harvard University Press, Cambridge, MA.

Ball, C.A. and Tschoegl, A.E. 1982. The Decision to Establish a Foreign Bank Branch or Subsidiary: An Application of Binary Classification Procedures. Journal of Financial and Quantitative Analysis, XVII(3): 411-424.

Bannerman, P., Spiller, J., Yetton, P. and Davis, J. 2005. Strategic Alliances in Education and Training: A Literature Review. DEST. Canberra.

Barney, J. 1986. Organisational Culture: Can it be a Source of Competitive Advantage? Academy of Management Review. 11(3): 656-665.

Barney, J. 1991. Firm Resources and Sustained Competitive Advantage. Journal of 
Management, 17(1): 99-120.

Barro, R.J. and Lee, J-W. 2000. International Data on Educational Attainment: Updates and Implications. NBER Working Paper No 7911. Data available at the Harvard's Centre for International Development: www.cid.harvard.edu [Retrieved 13 June 2007]

Bartlett, C.A. and Ghoshal, S. 1987. Managing Across Borders: New Strategic Requirements. Sloan Management Review, 28(4): 7-17.

Bartol, K.M and Martin, D.C. 1998. Management. Third Edition. Illinois: Irwin McGraw-Hill.

Bateson, J.E. G. 1979. "Why We Need Service Marketing” in O.C. Ferrell, S.W. Brown \& C.W. Lamb, Jr. (Eds.), Conceptual and Theoretical Developments in Marketing. Chicago: American Marketing. Pg. 131-146.

Bateson, J.E.G. Managing Services Marketing. Second Edition. Fort Worth, TX: Dryden Press.

Bauershmidt, A., Sullivan, D. and Gillespie, K. 1985. Common Factors Underlying Barriers to Export: Studies in the US Paper Industry. Journal of International Business Studies, 16(Fall): 111-123.

Beamish, P.W. 1988. Multinational Joint Ventures in Developing Countries. London: Routeledge.

Beamish, P.W. and Banks, J.C. 1987. Equity Joint Ventures and the Theory of the Multinational Enterprise. Journal of International Business Studies, 18(2): 116.

Becker, G.S. 1964. Human Capital: A Theoretical and Empirical Analysis, with Special Reference to Education. Columbia University Press, New York. Chap $1-3$.

Becker, M. 1970. Sociometric Location and Innovativeness. American Sociology Review, 35: 267-304.

Belderbos, R. 2003. Entry Mode, Organizational Learning, and R\&D in Foreign Affiliates: Evidence From Japanese Firms. Strategic Management Journal, 24: 235-259.

Bell, J. and Young, S. 1998. Towards An Integrative Framework of the Internationalisation of the Firm. In Hooley R., Loveridge R. and Wilson, D. (Eds.). Internationalisation: Process, Context and Markets. London: MacMillan Press. Pg 5-28. 
Bennell, P. \& Pearce, T. 2003. The Internationalisation of Higher Education: Exporting Education to Developing and Transnational Economies. International Journal of Educational Development, 23: 215-232.

Benito, G.R. 1996 Ownership of Norwegian Foreign Subsidiaries in Manufacturing. International Trade Journal, 10(2): 157-198.

Bergen, M., Dutta, S. and Walker, O.C. 1992. Agency Relationships in Marketing: A Review of the Implications and Applications of Agency and Related Theories. Journal of Marketing, 56(3): 1-24.

Berle, A.A. and Means, G. C. 1932. The Modern Corporation and Private Property. New York: Macmillan.

Berry, L.L. 1980. Services Marketing is Different. Business. 30(3): 24-29.

Bessom, R.M. and Jackson, D.W. 1975. Services Retailing - A Strategic Marketing Approach. Journal of Retailing, 8 (Summer): 137-149.

Bharadwai, S.G., Varadarajan, P.R. and Fahy, J. 1993. Sustainable Competitive Advantage in Service Industries: A Conceptual Model and Research Propositions. Journal of Marketing, 58(October): 83-99.

Bhushan, S. 2006. Foreign Education Providers in India: Mapping the Extent and Regulation. The Observatory of Borderless Higher Education. Available: www.obhe.ac.uk [Retrieved 02 January 2007].

Billaterals.Org. 2006. Jordan, GCC Make Bid Advancements in FTA Negotiations. Available: www.bilaterals.org [Retrieved 24 October 2008].

Bitner, M.J., Booms, B.H. and Tetreault, M.S. 1990. The Service Encounter: Diagnosing Favourable and Unfavourable Incidents. Journal of Marketing, 54(January), 71-84.

Bjarnason, S. 2006. Emerging Trends in Cross-Border Higher Education for Capacity Development. Available: www.obhe.ac.uk. [Retrieved: 02 January 2007].

Blomstermo, A, Sharma, D.D. Sallis, J. 2006. Choice of Foreign Market Entry Mode in Service Firms. International Marketing Review, 23(2): 211-229.

Bloodgood, J.M., Sapienza, H.J. and Alemida, J.G. 1996. The Internationalisation of New High Potential US Ventures: Antecedents and Outcomes.

Entrepreneurship: Theory and Practice, 20: 61-76.

Blumenstyk, G. 2006. Laureate Education Takes First Step Into Middle East. The Chronicles of Higher Education, 8 December issue. 
Boddewyn, J.J. Halbrich, M.B. and Perry, A.C. 1986. Service Multinationals: Conceptualisation, Measurement and Theory, Journal of International Business Studies, 17(3):41-57.

Bohm, A., Davis, D., Meares, D. \& Pearce, D. 2002. Global Student Mobility 2025: Forecasts of the Global Demand for International Higher Education. IDP Education Australia, Canberra.

Bouquet, C., Hebert, L. and Delios, A. 2004. Foreign Expansion in Service Industries: Separability and Human Capital Intensity. Journal of Business Research. 57(1): 35-46.

Bowen, K. 1990. Development of a Taxonomy of Services to Gain Strategic Marketing Insights. Journal of the Academy of Marketing Insights, 18(1): 43-49.

Bowen, D.E. and Jones, G.R. 1986. Transaction Cost Analysis of Service Organization - Customer Exchange. Academy of Management Review, 11(2): 428-441.

Brewer, J. and Hunter, A. 1989. Multimethod Research: A Synthesis of Styles. Newbury Park, CA: Sage Publications.

Brickley, J.A. and Dark, F.H. 1987. The Choice of Organisation Form: The Case of Franchising. Journal of Financial Economics, 18(2): 401-420.

British Council. 2004. Vision 2020: Forecasting International Student Mobility: A UK Perspective. London, British Council.

British Council. 2006. The Prime Minister's Initiative. Available: www.britishcouncil.org/promotion/pmi.htm. [Retrieved 25 December 2006].

Brouthers, K.D. and Brouthers, L.E. 2001. Explaining the National Cultural Distance Paradox. Journal of International Business Studies, 32(1): 177-189.

Brouthers, K.D., Brouthers, L.E. and Werner, S. 2003. Transaction Cost-Enhanced Entry Mode Choices and Firm Performance. Strategic Management Journal, 24: 1239-1248.

Brown, J.R., Dev, C.S. and Zhou, Z. 2003. Broadening the Foreign Market Entry Mode Decision: Separating Ownership and Control. Journal of International Business Studies, 34: 473-488.

Buckley, P.J. and Cason, M.C. 1976. The Future of Multinational Enterprise. New York: Holmer and Meier Publishers.

Buckley, P.J. and Pearce, R.D. 1979. Overseas Production and Exporting by the World's Largest Enterprises: A Study in Sourcing Policy. Journal of 
International Business Studies, 10(1): 9-20.

Buckley, P.J. and Prescott, K. 1989. The Structure of British Industry's Sales in Foreign Markets. Managerial and Decision Economics, 10(3): 189-208.

Buckley, P. J. Pass, C.L. and Prescott, K. 1992. The Internationalisation of Service Firms: A Comparison with the Manufacturing Sector. Scandinavian International Business Review, 1(1): 39-56.

Burgel, O. and Murray, G.C. 2000. The International Market Entry Choices of Start Up Companies in High-Technology Performance. Journal of International Marketing, 8: 33-62.

Campron, L. and Hulland, J. 1999. Redeployment of Brands, Sales Forces and General Marketing Management Expertise Following Horizontal Acquisition: A Resource-Based View. Journal of Marketing, 63: 41-54.

Campbell, A.J. and Verbeke, A. 1994. The Globalisation of Service Multinationals. Long Range Planning, 27(2): 95-102.

Cantwell, J. 1991. "The Theory of Technological Competence and its Application to International Production" in D. McFetridge (Ed.). Foreign Investment, Technology and Economic Growth. Calgary: University of Calgary Press. Pg. 33-67.

Career Education Corporation. 2005. Annual Report. Available: www.careered.com [Retrieved 24 December 2006].

Carmen, M. and Langeard, E. 1980. Growth Strategies of Service Firms. Strategic Management Journal, 1(1): 7-22.

Carney, M. and Gedajlovic, E. 1991. Vertical Integration in Franchise Systems: Agency Theory and Resource Explanations. Strategic Management Journal, 12(8): 607-629.

Casson, R. 1981. Language, Culture and Cognition: Anthropological Perspectives. New York: Macmillan.

Caves, R.E. 1971. International Corporations: The Industrial Economics of Foreign Investment. Economica, 38: 1-27.

Caves, R.E. 1996. Multinational Enterprise and Economic Analysis, ( ${ }^{\text {nd }}$ edition), Cambridge University Press, Cambridge, UK.

Caves, R.E. and Porter, M.E. 1977. From Entry Barriers to Mobility Barriers: Conjectural Decisions and Contrived Deterrence to New Competition. 
Quarterly Journal of Economics, 91(2): 241-261.

Caves, R.E. and Mehra, K. 1986. "Entry of Foreign Multinationals into U.S. Manufacturing Industries” in Porter, M.E. (Ed.) Competition in Global Industries. Boston: Harvard Business School Press.

Cavusgil, T. 1980. On the Internationalisation Process of Firms. European Research, November: 273-281.

Cavusgil, T. 1982. "Some Observations on the Relevance of the Critical Variables for Internationalisation Stages” in M.R. Czinkota (Ed.), Export management, New York: Praeger. Pg. 276-286.

Cavusgil, E., Seggie, S.H. and Talay, M.B. 2007. Dynamic Capabilities View: Foundations and Research Agenda. Journal of Marketing Theory and Practice, 15(2): 159-166.

Cervantes, M. and Guellec, D. 2002. International Mobility of Highly Skilled Workers. OECD, Paris, Pg. 71-98.

Chase, R.B. 1978. Where Does the Customer Fit in a Service Operation? Harvard Business Review, 56(6): 137-142.

Chase, R.B. 1983. The Customer Contact Approach to Services: Theoretical Bases and Practical Extensions. Operations Research, 21(4): 698-705.

Chase, R.B and Apte, U.M. 2007. A History of Research in Service Operations: What's the Big Idea? Journal of Operations Management, 25: 375-386.

CHEA. 2006. Database of US Institutions and Programs Accredited By US Recognized Accrediting Organisations. Available: www.chea.org [Retrieved 28 December 2006].

Cheah, Y.J. 2002. Fundamental Analysis and Conceptual Model for Corporate Strategy in Global Engineering and Construction Markets. Unpublished $\mathrm{PhD}$ Dissertation. Massachusetts Institute of Technology, Cambridge. M.A.

Child, J. 1972. Organisational Structure, Environment and Performance: The Role of Strategic Choice. Sociology, 6(1): 1-22.

China Ministry of Education. 2006. List of Approved Higher Education Joint Programmes Leading to the Award of Overseas Degrees. Available: www.moe.edu.cn (Chinese version) [Retrieved 25 December 2006]

Churchill, G. A. 1979. A Paradigm for Developing Better Measures of Marketing Constructs. Journal of Marketing Research, 16: 64-73. 
Churchill, G.A. and Iacobucci, D. 2002. Marketing Research: Methodological Foundations, $8^{\text {th }}$ edition. Harcourt College Publishers. Forth Worth, Texas.

Chung, H.F. L. and Enderwick P. 2001. An Investigation of Market Entry Strategy Selection: Exporting vs. Foreign Direct Investment Modes - A Home-Host Country Scenario. Asia Pacific Journal of Management, 18(4): 443-460.

CIA Fact book, 2008. Jordan, Bahrain, UAE and Qatar Country Profiles. Available: www.cia.gov/library/publications/the-world-factbook/ [Retrieved 24 October 2008]

Clark, T., Rajaratnam, D. and Smith, T. 1996. Toward a Theory of International Services: Marketing Intangibles in a World of Nations. Journal of International Marketing, 4(2): 9-28.

Cloninger, P. 2000. The Influence of Service Characteristics on Internationalisation: Extending the Eclectic Paradigm. Unpublished PhD Dissertation. Georgia State University.

Cloninger, P. 2004. The Effect of Service Intangibility on Revenue from Foreign Markets. Journal of International Management, 10: 125-146.

Coase, R.H. 1937. The Nature of the Firm. Economica, 4(16): 386-405.

Cohen, W.M. and Levinthal, D.A. 1990. Absorptive Capacity: A New Perspective on Learning and Innovation. Administrative Science Quarterly, 35(1): 128-152.

Collis, D.J. 1991. A Resource-Based Analysis of Global Competition: The Case of the Bearings Industry. Strategic Management Journal, 12: 49-68.

Committee of Vice-Chancellors and Principals. 2000. The Business of Borderless Education: UK Perspectives. Vol 1-3: Universities UK, London.

Conner. K.R. 1991. A Historical Comparison of Resource-Based Theory and Five Schools of Thought within Industrial Organization Economics: Do We Have a New Theory of the Firm? Journal of Management, 17(1): 121-154.

Constant, D., Sproull, L. and Kiesler S. 1996. The Kindness of Strangers: The Usefulness of Electronic Weak Ties for Technical Advice. Organization Science, 7(2): 119-135.

Contractor, F.J. 1990. Contractual and Cooperative Forms of International Business: Towards a Unified Theory of Modal Choice. Management International Review, 30(1): 31-54.

Contractor, F.J. and Kundu, S.K. 1998. Franchising Versus Company-Run Operations: 
Modal Choice in the Global Hotel Sector. Journal of International Marketing, 6(2): 28-53.

Contractor, F.J., Kundu, S.K. and Hsu, C-C. 2003. A Three-Stage Theory of International Expansion: The Link between Multinationality and Performance in the Service Sector. Journal of International Business Studies, 34(1): 5-18.

Cool, K. and Schendel, D. 1988. Performance Differences Among Strategic Group Members. Strategic Management Journal, 9: 207-223.

Coviello, N. and Munro, H. 1995. Growing the Entrepreneurial Firm: Networking for International Market Development. European Journal of Marketing, 29: 4961.

Coviello, N. and Munro, H. 1997. Network Relationships and the Internationalisation Process of the Small Software Firm. International Business Review, 6(4): 361386.

Coviello, N. and McAuley, A. 1999. Internationalisation Processes and the Smaller Firm: A Review of Contemporary Empirical Research. Management International Review, 39(3): 223-256.

Covin, J.G. and Slevin, D.P. 1989. Strategic Management of Small Firms in Hostile and Benign Environments. Strategic Management Journal, 10(1): 75-87.

Cox, E.P. 1980. The Optimal Number of Response Alternatives for a Scale: A Review. Journal of Marketing Research, 17 (November): 407-422.

Coyte, P.C. 1984. Specific Human Capital and Sorting Mechanisms in Labour Markets. Southern Economic Journal, 51(2): 469-480.

Cudeck, R. \& Browne, M.W. 1983. Cross-Validation of Covariance Structures. Multivariate Behavioural Research, 18: 147-167.

Cyert, R. M. and March, J.G. 1963. A Behavioural Theory of the Firm. Englewood Cliffs, NJ: Prentice-Hall.

Czinkota, M. 2006. Academic Freedom for All in Higher Education: The Role of the General Agreement on Trade in Services. Journal of World Business. 41: 149160.

Dacin, M.T. 1997. Isomorphism in Context: The Power and Prescription of Institutional Norms. Academy of Management Journal, 40(1): 46-81.

Davidson, W.H. 1980. The Location of Foreign Direct Investment Activity: Country Characteristics and Experience Effects. Journal of International Business 
Studies, 11(2): 9-22.

Davidson, W.H. 1982. Global Strategic Management. New York: John Wiler \& Sons.

Davidson, W.H. \& McFetridge, D.G. 1985. Key Characteristics in the Choice of International Technology Transfer Mode. Journal of International Business Studies, 16(2): 5-21.

Davis, S.M. 1984. Managing Corporate Culture, Cambridge, MA: Ballinger Publishing Co.

Davis, P.S., Desai, A.B. and Francis, J.D. 2000. Mode of International Entry: An Isomorphism Perspective. Journal of International Business Studies, 31(2): 239-258.

Davis, D., Olsen, A. \& Bohm, A. 2000. Transnational Education: Providers, Partners and Policy: Challenges for Australian Institutions Offshore, Brisbane, IDP.

Day, G. 1986. Analysis for Strategic Marketing Decisions. West, St. Paul.

Day, G. and Wensley, R. 1988. Assessing Advantage: A Framework for Diagnosing Competitive Superiority. Journal of Marketing, 52(April): 1-20.

D’Aunno, T., Sutton, R.I. and Price, R.H. 1991. Isomorphism and External Support in Conflicting Institutional Environments: A Study of Drug Abuse Treatment Units. Academy of Management Journal, 34: 636-661.

Delios, A. and Beamish, P.W. 1999. Ownership Strategies of Japanese Firms: Transactional, Institutional and Experience Influences. Strategic Management Journal, 20: 915-933.

Delios, A. and Beamish, P.W. 2001. Survival and Profitability: The Roles of Experience and Intangible Assets in Foreign Subsidiary Performance. Academy of Management Journal, 44: 1028-1038.

Delios, A. and Henisz, W. 2003. Political Hazards, Experience and Sequential Entry Strategies: The International Expansion of Japanese Firms, 1980-1998. Strategic Management Journal, 24:1153-1164.

Deephouse, D.L. 1996. Does Isomorphism Legitimate? Academy of Management Journal, 39(4): 1024-1039.

Deephouse, D.L. 2000. Media Reputation as a Strategic Resource: An Integration of Mass Communication and Resource-Based Theories. Journal of Management, 26: 1091-1112. 
Denzin, N.K. and Lincoln, Y.S. 2000. Handbook of Qualitative Research, $2^{\text {nd }}$ edition, Thousand Oaks: Sage. Pg. 1-28.

Deshpande, R. and Webster, F.E. Jr. 1989. Organisational Culture and Marketing: Defining the Research Agenda. Journal of Marketing, 53(January): 3-15.

Dess, G., Lumpkin, G.T. and Covin, J.G. 1997. Entrepreneurial Strategy Making and Firm Performance: Tests of Contingency and Configurational Models. Strategic Management Journal, 18(9): 677-695.

DEST (Department of Education, Science and Training). Various. Commonwealth of Australia Higher Education Statistics Collection. Available: www.dest.gov.au/highered/statinfo.htm [Retrieved 24 December 2006].

DeVry Inc. 2006. Annual Report. Available: www.devryinc.com [Retrieved 24 December 2006].

Dierickx, I. and Cool, K. 1989. Asset Stock Accumulation and Sustainability of Competitive Advantage. Management Science, 12: 1504-1513.

Dillman, D.A. 1978. Mail and Telephone Surveys: The Total Design Method, New York: Wiley

Didou Aupetit, S. 2006. Borderless Higher Education in Latin America: The Issues of Expansion. Available: www.obhe.ac.uk. [Retrieved 02 July 2007].

DiMaggio, P.J. and Powell, W.W. 1983. The Iron Cage Revisited: Institutional Isomorphism and Collective Rationality in Organisational Fields. American Sociological Review, 48:147-160.

DiMaggio, P.J. and Powell, W.W. 1991. "Introduction" in W.W. Powell and P.J. DiMaggio (Eds.). The New Institutionalism in Organisational Analysis. Chicago, University of Chicago University Press. Pg. 1-38.

Dollinger, M.J., Golden, P.A and Saxton, T. 1997. The Effect of Reputation on the Decision to Joint Venture. Strategic Management Journal, 18(2): 127-140.

Domke-Damonte, D. 2000. Interactive Effects of International Strategy and Throughput Technology on Entry Mode for Service Firms. Management International Review, 40(1): 41-59.

Douglas, M. 1986. How Institutions Think. Syracuse, New York: Syracuse University Press.

Douglas, S. P. and Craig, C.S. 1995. Global Marketing Strategy. New York: McGraw-Hill, Inc. 
Duncan, R. and Weiss, A. 1979. "Organisational Learning: Implications for Organisational Design” in B.M. Shaw (Ed.), Research in Organisational Behaviour. Greenwich, CT: JAI Press. Pg. 75-123.

Dunning, J.H. 1977. "Trade, Location of Economic Activity and the MNE: A Search for an Eclectic Approach" in B. Ohlin, P.O. Hesselborn, and P.M. Wijkman (Eds), The International Allocation of Economic Activity, London: Macmillan.

Dunning, J.H. 1979. Explaining Changing Patterns of International Production: In Defense of the Eclectic Theory. Oxford Bulletin of Economics and Statistics, 41(4), 269-295.

Dunning, J.H. 1980. Toward an Eclectic Theory of International Production: Some Empirical Tests. Journal of International Business Studies, 11(spring/summer): 9-31.

Dunning, J.H. 1988. The Eclectic Paradigm of International Production: A Restatement and Some Possible Extensions. Journal of International Business Studies, 19: $1-31$.

Dunning, J.H. 1989. Multinational Enterprises and the Growth of Services: Some Conceptual and Theoretical Issues. Services Industries Journal, 9(1): 5-39.

Dunning, J.H. 1995. Reappraising the Eclectic Paradigm in the Age of Alliance Capitalism. Journal of International Business Studies, 26(3): 461-93.

Dunning, J.H. 2000. The Eclectic Paradigm as an Envelope for Economic and Business Theories of MNE Activity. International Business Review, 9(1): 163-90.

Dunning, J.H. 2001. The Eclectic (OLI) Paradigm of International Production: Past, Present and Future. International Journal of the Economics of Business, 8(2): 173-190.

Dunning, J.H and Kundu, S.K. 1995. The Internationalisation of the Hotel Industry: Some New Findings from a Field Study. Management International Review, 35(2): 101-133.

Dunning, J.H. and McQueen, M. 1982. "The Eclectic Theory of the Multinational Enterprise and the International Hotel Industry” in Rugman, A.M. (Ed) New Theories of Multinational Enterprise. New York: St. Martin's.

Dutta, S., Narasimhan, O. and Rajiv, S, 2005. Conceptualizing and Measuring Capabilities: Methodology and Empirical Application. Strategic Management Journal, 26: 277-285. 
Enderwick, P. 1989. "Some Economics of Service-Sector Multinational Enterprises” in P. Enderwick (Ed.), Multinational Service Firms, London: Routledge, Chapter 1.

Education and Manpower Bureau. 2006. List of Non-Local Higher and Professional Education Courses. Available: www.emb.gov.hk [Retrieved 25 December 2006].

Education New Zealand. 2006. Offshore Education: Stock take and Analysis. Wellington.

Education UK. 2006. Study in Your Country: Course Search Database. Available: www.educationuk.org [Retrieved 29 December 2006].

Eisenhardt, K.M. 1988. Agency and Institutional Theory Explanations: The Case of Retail Sales Compensation. Academy of Management Journal, 31(3): 488-511.

Eisenhardt, K.M. 1989. Agency Theory: An Assessment and Review. Academy of Management Review, 14(1): 57-74.

Eisenhardt, K.M. 1989b. Building Theories from Case Study Research. Academy of Management Review, 14(4): 532-550.

Eisenhardt, K.M. and Tabrizi, B.N. 1995. Accelerating Adaptive Process: Product Innovation in the Global Computer Industry. Administrative Science Quarterly, 40 (1):84-109.

Ekeledo, I. and Sivakumar, K. 1998. Foreign Market Entry Mode Choice of Service Firms: A Contingency Perspective. Journal of the Academy of Marketing Science, 26(4): 274-292.

Ekeledo, I. and Sivakumar, K. 2004. International Market Entry Mode Strategies of Manufacturing Firms and Service Firms: A Resource-Based Perspective. International Marketing Review, 21(1): 68-101.

Emerson, R.M. 1962. Power-Dependence Relations. American Sociological Review, 27(1): 31-41.

Enders, J. 2004. Higher Education, Internationalisation and the Nation State: Recent Developments and Challenges to Governance Theory. Higher Education, 47(3): 361-382.

Erramilli M.K. 1987. Understanding the Foreign Market Entry Mode Choice in Service Firms: An Exploratory Study. Unpublished PhD. Dissertation, University of Arkansas, Fayetteville, AR. 
Erramilli, M.K. 1990. Entry Mode Choice in Service Industries. International Marketing Review, 7(5): 50-62.

Erramilli, M.K. 1991. The Experience Factor in Foreign Market Entry Behaviour of Service Firms. Journal of International Business Studies, 22(3): 479-501.

Erramilli, M.K. 1992. Influence of Some External and Internal Environmental Factors on Foreign Market Entry Mode Choice in Service Firms. Journal of Business Research, 25(4): 263-276.

Erramilli, M.K. and Rao, C.P. 1990. Choice of Foreign Market Entry Modes by Service Firms: Role of Market Knowledge. Management International Review, 30(2):135-150.

Erramilli, M.K. and Rao, C.P. 1993. Service Firms’ International Entry-Mode Choice: A Modified Transaction Cost Analysis Approach. Journal of Marketing, 57(July): 19-38.

Feng, G. 2006. Sino-Foreign Joint Education Ventures: A National, Regional and Institutional Analysis. The Observatory of Borderless Higher Education. Available: www.obhe.ac.uk [Retrieved 02 January 2007]

Finn, M.G. 2003. Stay Rates of Foreign Doctorate Recipients from U.S. Universities, 2001. Oak Ridge Institute for Science and Education. Available: www.orau.gov/orise/pubs/stayrate03.pdf [Retrieved 05 January 2007].

Fladmoe-Lindquist, K. 1991. Ownership and Control Patterns of International Service Companies: A Principal-Agent Framework. Unpublished PhD Dissertation, University of Minnesota, Minneapolis, MN.

Fladmoe-Lindquist, K. and Jacque, L.L. 1995. Control Modes in International Service Operations: The Propensity to Franchise. Management Science, 41(7): 12381249.

Flowers, E.B. 1976. Oligopolistic Reactions in European and Canadian Direct Investment in the United States. Journal of International Business Studies, Fall/Winter: 43-55.

Foil, C.M. 1991. Managing Culture as Competitive Resource: An Identity-Based View of Sustainable Competitive Advantage. Journal of Management, 17(1): 191211.

Fombrun, C.J. 1996. Reputation: Realizing Value from the Corporate Image. Boston, MA: Harvard Business School Press.

Fombrun, C.J. and Van Riel, C.B.M. 2004. Fame and Fortune. Upper Saddle River, 
NJ: Prentice Hall.

Forsgren, M. 1990. Managing the Internationalisation Process: The Swedish Case. Routeledge, London.

Franko, L.G. 1971. Joint Venture Survival in Multinational Cooperations. New York, Praeger.

Galbraith, J.R. Designing Complex Organisations. Reading, MA : Addison-Wesley.

García-Guadilla, C., Didou Aupetit, S. \& Marquis C. 2002. New Providers, Transnational Education and Accreditation of Higher Education in Latin America. Available: www.iesalc.unesco.org.ve [Retrieved 02 January 2007].

Garrett, R. \& Verbik, L. 2003. Transnational Higher Education, Part 1: The Major Markets - Hong Kong \& Singapore. Available: www.obhe.ac.uk [Retrieved 02 January 2007].

Garrett, R. \& Verbik, L. 2004. Transnational Delivery by UK Higher Education, Part 1: Data \& Missing Data. Available: www.obhe.ac.uk [Retrieved 02 January 2007].

Gattigon, H. \& Anderson, E. 1988. The Multinational Corporation's Degree of Control Over Foreign Subsidiaries: An Empirical Explanation of a Transaction Cost Explanation. Journal of Law, Economics and Organization, 4(2): 305-336.

Gaur, A. and Lu, J.W. 2007. Ownership Strategies and Survival of Foreign Subsidiaries: Impacts of Institutional Distance and Experience. Journal of Management, 33(1): 84-110.

Geringer, J.M. 1991. Strategic Determinants of Partner Selection Criteria in International Joint Ventures. Journal of International Business Studies, 3 (Spring): 33-60.

Ghosh, M. and John, G. 1999. Governance Value Analysis and Marketing Strategy. Journal of Marketing, 63(Oct- Special Issue): 131-45.

Ghoshal, S. 1987. Global Strategy: An Organising Framework. Strategic Management Journal, 8: 425-440.

Ginsberg, A. and Venkatraman, N. 1985. Contingency Perspectives of Organisational Strategy: A Critical Review of the Empirical Research. Academy of Management Review, 10(3): 421-434.

Glaser, B. G. 1978. Theoretical Sensitivity: Advances in the Methodology of Grounded Theory. Mill Valley, CA: Sociology Press. 
Goldberg, M.E. and Hartwick, J. 1990. The Effects of Advertiser Reputation and Extremity of Advertising Claim on Advertising Effectiveness. Journal of Consumer Research, 17: 172-179.

Goodnow, J.D. 1985. Developments in International Mode of Entry Analysis. International Marketing Review, 2(Fall): 17-30.

Goodnow, J.D. and Hansz, J.H. 1972. Environmental Determinants of Overseas Market Entry Strategies. Journal of International Business Studies, 3(1): 33-50.

Gomes-Caserres, B. 1985. Multinational Ownership Strategies, Unpublished DBA Dissertation. Harvard University.

Gomes-Caserres, B. 1989. Ownership Structures of Foreign Subsidiaries: Theory and Evidence. Journal of Economic Behaviour and Organisation, 11: 1-25.

Gomes-Caserres, B. 1990. Firm Ownership Preferences and Host Government Restrictions: An Integrated Approach. Journal of International Business Studies, 21(1): 1-22.

Gomes, L. \& Murphy, J. 2003. An Exploratory Study of Marketing International Education Online. International Journal of Educational Management, 17(3): 116-125.

Goodnow, J. D. 1985. Development in International Mode of Entry Analysis. International Marketing Review, 2 (Autumn), 17-30.

Graham, E.M. 1978. Transatlantic Investment by Multinational Firms: A Rivalistic Phenomenon. Journal of Post-Keynesian Economics, Fall: 82-84

Graham, E.M. 1998. Market Structure and the Multinational Enterprise: A GameTheoretic Approach. Journal of International Business Studies, 29: 67-83.

Grant, R.M. 1991. The Resource-Based Theory of Competitive Advantage: Implications for Strategy Formulation. California Management Review, Spring: 114-135.

Grant, R.M. 1996. Towards a Knowledge-Based Theory of the Firm. Strategic Management Journal, 17: 109-122.

Grönroos, C. 1978. A Service Oriented Approach to Marketing of Services. European Journal of Marketing, 12(8) 588-601.

Grönroos, C. 1999. Internationalisation Strategies for Services. Journal of Services Marketing, 13(4/5): 290-297. 
Guoqing, Z. 2003. Study Abroad, Study Abroad! Chinese Education and Society, 36(4): 85-90.

Gupta, A.K. and Govindarajan, V. 2002. Cultivating Global Mindset. Academy of Management Executive, 16(1): 116-126.

Gupta, V., Hanges, P.J. and Dorfman, P. 2002. Cultural Clusters: Methodology and Findings. Journal of World Business, 37: 11-15.

Hair, J.E., Anderson, E., Rolph, E., Tatham, R.L. and Black, W.C. 1998. Multivariate Data Analysis. Prentice Hall, Upper Saddle River, New Jersey.

Hall, R. 1992. The Strategic Analysis of Intangible Resources. Strategic Management Journal, 13: 135-144.

Hamel, G. 1991. Competition for Competence and Inter-partner Learning Within International Strategic Alliances. Strategic Management Journal, 12(Special Issue): 83-103.

Hamel, G. and Prahalad, C.K. 1994. Competing for the Future, Harvard Business School Press, Boston, MA.

Harman, G. 2005. Australian Social Scientists and Transition to a More Commercial University Environment. Higher Education Research and Development, 24(1): 79-94.

Harrigan, K.R. 1983. Strategies for Vertical Integration. Lexington, MA: D.C. Health.

Harrigan, K.R. 1985a. Strategic Flexibility. Lexington, MA: D.C. Health.

Harrigan, K.R. 1985b. A Framework for Looking at Vertical Integration. Journal of Business Strategy, 3(3): 30-37.

Harrigan, K.R. 1985c. Strategies for Joint Ventures, Lexington, MA: D.C. Heath.

Harrigan, K.R. 1986. Matching Vertical Integration Strategies. Academy of Management Review, 9(4): 638-652.

Harrigan, K.R. 1988. Joint Ventures and Competitive Strategy. Strategic Management Journal, 9: 141-158.

Harris, M. \& Raviv, A. 1978. Some Results on Incentive Contracts with Applications to Education and Employment, Health Insurance and Law Enforcement.

American Economic Review, 68(1): 20-30. 
Haverman, H.H. 1993. Follow the Leader: Mimetic Isomorphism and Entry into New Markets. Administrative Science Quarterly, 38:593-627.

Heide, J.B. 1994. Interorganisational Governance in Marketing Channels. Journal of Marketing, 58(1): 71-85.

Helfaf, C.E. and Peteraf, M.A. 2003. The Dynamic Resource-Based View: Capability Lifecycles. Strategic Management Journal, 24: 997-1010.

Henisz, W. and Williamson, O. 1999. Comparative Economic Organization - Within and Between Countries. Business and Politics, 1: 261-276.

Hennart, J.F. 1988. Can the 'New Forms of Investment' Substitute for the 'Old Forms': A Transaction Costs Perspective. Journal of International Business Studies, 20(2): 211-234.

Hennart, J.F. 1991. The Transactions Costs Theory of Joint Ventures: An Empirical Study of Japanese Subsidiaries in the United States. Management Science, 37(4): 483-497.

Hennart, J.F. and Park, Y.R. 1993. Greenfield v/s Acquisition. The Strategies of Japanese Investors in the United States. Management Science, 39(9): 10541070.

Heskett, J.L. 1986. Managing in the Service Economy. Boston: Harvard University Press.

Higher Education Commission of Pakistan. 2006. List of Accredited Foreign Programmes. Available: www.hec.gov.pk [Retrieved 30 December 2006].

Hill, C.W. L. 1990. Cooperation, Opportunism and the Invisible Hand: Implications for Transaction Cost Theory. Academy of Management Review, 15(3): 500-513.

Hill, C.W.L. 1997. International Business - Competing in the Global Marketplace, Irwin McGraw-Hill.

Hill, C.W.L., Hwang, P. and Kim, W.C. 1990. An Eclectic Theory of the Choice of International Entry Mode. Strategic Management Journal, 11(2): 117-28.

Hinings, B. and Greenwood, R. 1988. The Normative Prescription of Organisations in L.G. Zucker (Ed.), Institutional Patterns and Organisations: Culture and Environment. Cambridge, MA: Ballinger.

Hobbs, J.E. 1996. A Transaction Cost Approach to Supply Chain Management. Supply Chain Management, 1(2): 15-27. 
Hofstede, G. 1980. Culture’s Consequences. New York: Sage.

Hofstede, G. 1991. Cultures and Organisations. London: McGraw-Hill.

Hölmstrom, B. 1985. “The Provision of Services in a Market Economy”, in R.P. Inman (Ed.), Managing the Service Economy: Prospects and Problems.

Cambridge: Cambridge University Press. Pg. 183-213.

Hong Kong Education and Manpower Bureau. 2006. List of Registered Non-Local Higher and Professional Education Courses. Available: www.emb.gov.hk [Retrieved 25 December 2006]

Huber, G.P. 1991. Organisational Learning: The Processes and The Literatures. Organisation Science, 2: 88-115.

Hunt, S.D. and Morgan, R.M. 1995. The Comparative Advantage Theory of Competition. Journal of Marketing, 59: 1-15.

Hutchinson, P. 1999. Small Enterprise: Finance, Ownership and Control. International Journal of Management Reviews, 1(3): 343-365.

Illuminate Consulting Group. 2008. Imperial College London-Dubai Branch Campus. Opportunity Assessment. San Francisco Bay Area. [Confidential report not for public circulation]

Indian National Institute of Educational Planning and Administration. 2006. Directory on Foreign Education Providers in India. New Delhi, India.

Inkpen, A.C. 1992. Learning and Collaboration: An Examination of North AmericanJapanese Joint Ventures. Unpublished PhD dissertation. University of Western Ontario.

International Association of Universities and UNESCO. Various. International Handbook of Universities. Paris. UNESCO.

International Monetary Fund. 1993. Balance of Payment Manual, Washington D.C., IMF Publications.

Ionascu, D., Meyer, K., Erstin, S. 2004. Institutional Distance and International Business Strategies in Emerging Economies. William Davidson Institute Working Paper Number 728, University of Michigan Business School. Available: www.wdi.bus.umich.edu [Retrieved 14 May 2007].

Itami, T. 1987. Mobilising Invisible Assets. Cambridge, MA.. Harvard University Press. 
Jamaica University Council, 2007. List of Overseas Institutions with Accredited Programmes in Jamaica. Available: www.ucjamaica.com [Retrieved 02 January 2007].

Javalgi, R.G. and White, D.S. 2002. Strategic Challenges for the Marketing of Services Internationally. International Marketing Review, 19(6): 563-581.

Jensen, M.C. 1983. Organization Theory and Methodology. Accounting Review, 58(2): 319-339.

Jensen, M.C. 1989. Eclipse of the Public Corporation. Harvard Business Review, 67(5): 61-74.

Jensen, M.C. and Meckling, W.H. 1976. Theory of the Firm: Managerial Behaviour, Agency Costs and Ownership Structure. Journal of Financial Economics, 3(4): 305-360.

Jensen, J., Rutherford, T. and Tarr, D. 2007. The Impact of Liberalising Barriers to Foreign Direct Investment in Services: The Case of Russian Accession to the World Trade Organisation. Review of Development Economics, 11(3): 482506.

Jobber, D. and O’Reilly, D. 1998. Industrial Mail Surveys: A Methodological Update. Industrial Marketing Management, 27: 95-107.

Johanson, J. and Vahlne, J. 1977. The Internationalisation Process of the Firm - A Model of Knowledge Development and Increasing Foreign Market Commitments. Journal of International Business studies, 4:23-32.

Johanson, J. and Vahlne, J. 1990. The Mechanisms of Internationalisation. International Marketing Review, 7(4):11-24.

Johanson, J. and Wierdersheim-Paul, F. 1975. The Internationalisation of the Firm: Four Swedish Cases. Journal of Management Studies, 12(3): 305-322.

Johnston, D. J. 2002. Opening Remarks by the Honourable Donald J. Johnston, Secretary-General of the OECD. Paper presented at the OECD/US Forum on Trade in Educational Services, 23-24 May Washington D.C. Available: www.oecd.org [Retrieved 10 February 2004]

Jokivirta, L. 2005. Higher Education Crossing Borders in Francophone Africa Part 1: Opportunities, Challenges and Implications. Available: www.obhe.ac.uk [Retrieved 02 January 2007].

Jones, G.R. and Hill, C.W.L. 1988. Transaction Cost Analysis of Strategy-Structure Choice. Strategic Management Journal, 9(2): 159-172. 
Kanter, R.M. 1995. World Class: Thriving Locally in the Global Economy. New York: Simon \& Schuster. Chapter 1-3.

Karakaya, F. and Stahl, M.J. 1991. Entry Barriers and Market Entry Decision: A Guide for Marketing Executives, Greenwood Publishing Group, Inc., New York.

Karnani, A. and Wernerfelt, B. 1985. Research Note and Communication: Multiple Point Competition. Strategic Management Journal, 6:87-96.

Keegan, W. J. and Green, M.C. 1997. Principles of Global Marketing. Upper Saddle River, NJ: Prentice Hall.

Killing, J.P. 1982. How to Make a Global Joint Venture Work? Harvard Business Review, 60(3): 120-127.

Kilman, R., Saxton, M.J., Serpa, R. 1986. Issues in Understanding and Changing Culture. California Management Review, 28: 87-94.

Kim, W.C and Hwang, P. 1992. Global Strategy and Multinationals’ Entry Mode Choice. Journal of International Business Studies, 23( $1^{\text {st }}$ Quarter): 29-54.

Kimberly, J. and Evanisko, M. 1981. Organizational Innovation: The Influence of the Individual, Organizational and Contextual Factors on Hospital Adoption of Technological and Administrative Innovations. Academy of Management Journal, 24: 689-713.

Kimura, Y. 1989. Firm Specific Strategic Advantages and Foreign Direct Investment Behaviour of Firms: The Case of Japanese Semi-Conductor Firms. Journal of International Business Studies, 20(Summer): 296-314.

Klein, B., Crawford, R.G., Alchian, A.A. 1978. Vertical Integration, Appropriable Quasi-Rents and the Competitive Contracting Process. Journal of Law and Economics, 21(2): 297-326.

Klein, S., Frazier, G. and Roth, V.J. 1990. A Transaction Cost Analysis Model of Channel Integration in International Markets. Journal of Marketing Research, May Issue: 196-208.

Knickerbocker, F. T. 1973. Oligopolistic Reaction and Multinational Enterprise. Boston, MA: Division of Research, Graduate School of Business Administration, Harvard University.

Knight, G. 1999. International Services Marketing: Review of Research, 1980-1998. Journal of Services Marketing, 13(4/5): 347-360. 
Knight, J. 1999. Internationalisation of Higher Education in J. Knight (Ed.), Quality of Internationalisation in Higher Education. Paris: OECD. Pg. 13-28.

Knight, J. 2000. Progress and Promise: The 2000 AUCC Report on Internationalisation at Canadian Universities. AUCC, Ottawa, Canada.

Knight, J. 2002a. The Impact of Trade Liberalization on Higher Education: Policy Implications. Paper presented at the "Globalisation: What Issues are at Stake for Universities?" Conference, University of Laval, Quebec, Canada. 20th September: Available:www.ulaval.ca/BI/Globalisation-Universities/ index.htm. [Retrieved 10 January 2004].

Knight, J. 2003. GATS, Trade and Higher Education. Perspectives 2003: Where are We? Available: www.obhe.ac.uk [Retrieved 25 May 2005].

Knight, J. 2004. Internationalisation Remodelled: Definitions, Approaches and Rationales. Journal of Studies in International Education, 8(1): 5-31.

Knight, J. 2005a. Borderless, Offshore, Transnational and Cross-Border Education: Definition and Data Dilemmas. Available: www.obhe.ac.uk [Retrieved 04 January 2007].

Knight, J. 2005b. Internationalisation - the New World of Cross-Border Education Development, Complexities and Challenges. Paper delivered at the Institute of Technology and Polytechnics of New Zealand Conference. Available: www.itpnz.ac.nz [Retrieved 02 January 2007].

Knight, J. 2005c. Cross-Border Education: Programs and Providers on the Move. Canadian Bureau for International Education, Research Paper No 10.

Knight, G.A. and Cavusgill, S.T. 2004. Innovation, Organisational Capabilities and the Born-Global Firm. Journal of International Business Studies, 35(2): 124-141

Knisely, G. 1979. Financial Services Marketers Must Learn Packaged Goods Selling Tools. Advertising Age, 50:58-62.

Kobrin, S.J. 1979. Political Risk: A Review and Reconsideration. Journal of International Business Studies, 10: 67-80.

Kobrin, S.J. 1983. Selective Vulnerability and Corporate Management” in T.H. Moran (Ed.), International Political Risk Assessment: The State of the Art. Washington D.C.: Georgetown University Press.

Kobrin, S.J. 1991. An Empirical Analysis of the Determinants of Global Integration. Strategic Management Journal, 12: 17-31. 
Kogut, B. 1988. A Study of the Life Cycle of Joint Ventures in Contractor F.J. and Lorange P. (Eds.) Cooperative Strategies in International Business, Lexington, M.A.: Lexington Books. Pg. 169-186.

Kogut, B. 1988b. Joint Ventures: Theoretical and Empirical Perspectives. Strategic Management Journal, 9: 319-322.

Kogut, B. and Singh, H. 1988. The Effect of National Culture on the Choice of Entry Mode. Journal of International Business Studies, 19(3): 411-432.

Kogut, B. and Zander, U. 1993. Knowledge of the Firm and the Evolutionary Theory of the Multinational Corporation. Journal of International Business Studies, Fourth Quarter, 625-645.

Kostova, T. 1996. Success of the Transnational Transfer of Organizational Practices Within Multinational Companies. Unpublished PhD Dissertation. University of Minnesota, Minneapolis.

Kostova, T. 1999. Transnational Transfer of Strategic Organizational Practices: A Contextual Perspective. Academy of Management Review, 24(2): 308-324.

Kostova, T. and Zaheer, S. 1999. Organizational Legitimacy Under Conditions of Complexity: The Case of the Multinational Enterprise. Academy of Management Review, 24(1): 64-81.

Kostova, T. and Roth, K. 2002. Adoption of an Organizational Practice by Subsidiaries of Multinational Corporations: Institutional and Relational Effects, Academy of Management Journal, 45(1): 215-233.

Kotabe, M., Murray, J.Y., and Javalgi, R.G. 1998. Global Sourcing of Services and Market Performance: An Empirical Investigation. Journal of International Marketing, 6(4): 10-31.

Kotha, S. Rindova, V.P. and Rothaermel, F.T. 2001. Assets and Actions: Firm Specific Factors in the Internationalization of US Internet Firms. Journal of

International Business Studies, 32: 769-791.

Kotler, P. and Armstrong, G. 1991. Principles of Marketing (5 ${ }^{\text {th }}$ ed.). Englewoods Cliffs, NJ: Prentice-Hall.

Kreiser, P. and Marino, L. 2002. Analysing the Historical Development of the Environmental Uncertainty Construct. Management Decision, 40(9): 895-905.

Kshetri, N. and Ajami, R. 2008. Institutional Reforms in the Gulf Cooperation Council Economies: A Conceptual Framework. Journal of International Management, 14: 300-318. 
Kumar, V. and Subramaniam, V. 1997. A Contingency Framework for the Mode of Entry Decision. Journal of World Business, 32(1): 53-72.

Lado, A.A., Boyd, N.G. and Wright, P. 1992. A Competency Based Model of Sustainable Competitive Advantage: Toward a Conceptual Integration. Journal of Management, 18(1): 77-91.

LAN. 2006. List of Accredited Courses Awarded by Private Higher Education Institutions. Available: www.lan.gov.my [Retrieved 25 December 2006].

Langeard, E., Bateson, J.E.G., Lovelock, C.H. and Eiglier, P. 1981. Services Marketing: New Insights from Consumers and Managers. Cambridge, MA: Marketing Science Institute.

Larsen, K. \& Vincent-Lancrin. 2002. The Learning Business: Can Trade in Interna tional Education Work? The OECD Observer, 235(December):26-29.

Larsen, K., Martin, J.P., \& Morris, R. 2002. Trade in Educational Services: Trends and Emerging Issues. OECD Working Paper. Available: www.oecd.org [Retrieved 09 February 2004].

Larsen, K., Momii, K. \& Vincent-Lancrin, S. 2004. Cross-Border Higher Education: An Analysis of Current Trends, Policy Strategies and Future Scenarios. The Observatory for Borderless Higher Education. Available: www.obhe.ac.uk [Retrieved 02 January 2007]

Larson, A. 1992. Network Dyads in Entrepreneurial Settings: A Study of the Governance of Exchange Relationships. Administrative Science Quarterly, 37:76-104.

Laureate Education Inc. 2006. Laureate Education, Inc. Reports Record Student Enrolment and Third Quarter 2006 Results. Available: www.laureate-inc.com [Retrieved 10 September 2006].

Lawrence, P.R. and Lorsch, J.W. 1967. Organisation and Environment: Managing Differentiation and Integration. Boston: Harvard Business School Press.

Lenn, M.P. 2003. Strengthening World Bank Support for Quality Assurance and Accreditation in Higher Education in East Asia and the Pacific. Report Commissioned by the World Bank. Available: www.portal.unesco.org [Retrieved 06 January 2007].

Levinthal, D. 1988. A Survey of Agency Models of Organization. Journal of Economic Behaviour and Organisation, 9(2): 153-185.

Levitt, B. and March, J.G. Organisational Learning. Annual Review of Sociology, 14: 
319-340.

Li, J. 1995. Foreign Entry and Survival: Effects of Strategic Choices on Performance in International Markets. Strategic Management Journal, 16: 333-351.

Lindblom, C. 1959. The Science of "Muddling Through". Public Administration Review, 19(2): 79-88.

Lovelock, C.H. 1981. "Why Marketing Management Needs to be Different for Services”, in J.H. Donnelly \& W.R. George (Eds.). Marketing of Services. Chicago: American Marketing. Pg. 5-9.

Lovelock, C.H. 1983. Classifying Services to Gain Strategic Marketing Insights. Journal of Marketing, 47(3): 9-20.

Lovelock, C.H. 1996. Services Marketing ( $3^{\text {rd }}$ edition). Upper Saddle River, NJ: Prentice-Hall.

Lovelock, C.H. and Yip, G.S. 1996. Developing Global Strategies for Service Business. California Management Review, 38(2): 64-86.

Lowendahl, B.R. 1997. Strategic Management of Professional Service Firms ( $2^{\text {nd }}$ Edition). Copenhagen: Copenhagen Business School Press.

Lowendahl, B.R and Revang, O. 1998. Challenges to Existing Strategy Theory in a Post Industrial Society. Strategic Management Journal, 19: 755-73.

Lu, J.W. and Beamish, P.W. 2001. The Internationalisation and Performance of SMEs. Strategic Management Journal, 22: 565-586.

Luo, Y. 2001. Equity Sharing in International Joint Ventures: An Empirical Analysis of Strategic and Environmental Determinants. Journal of International Management, 7: 31-58.

Lumpkin, G.T. and Dess, G.G. 1996. Clarifying the Entrepreneurial Orientation Construct and Linking it to Performance. Academy of Management Review, 21(1): 135-172.

Madhok, A. 1997. Cost, Value and Foreign Market Entry Mode: The Transaction and the Firm. Strategic Management Journal, 18: 39-61.

Madhok, A. 1998. The Nature of Multinational Firm Boundaries: Transactions Costs, Firm Capabilities and Foreign Market Entry Mode: International Business Review, 7(3): 259-290.

Mahoney, J.T. and Pandian, R. 1992. The Resource-Based View Within the 
Conversation of Strategic Management. Strategic Management Journal, 13(June): 363-380.

Mani, S., Antia, K.D. and Rindfleisch, A. 2007. Entry Modes and Equity Level: A Multilevel Examination of FDI Ownership Structure. Strategic Management Journal, 28: 857-866.

March, J.G. 1991. Exploration and Exploitation in Organisational Learning. Organisation Science, 2: 71-87.

March, J.G. and Simon, H.A. 1958. Organisations. New York: John Wiley \& Sons.

Marginson, S. and Considine, M. 2000. The Enterprise University: Power, Governance and Reinvention in Australia. Melbourne: Cambridge University Press.

Marginson, S. and Van der Wende, M. 2007. Globalization and Higher Education. Paper prepared for the OECD. Available: www.cshe.unimelb.edu.au [Retrieved 13 July 2007].

Martin, R.E. 1988. Franchising and Risk Management. American Economic Review, 78(5): 954-968.

Mathewson, G. and Winter, R.A. 1985. The Economics of Franchise Contracts. Journal of Law and Economics, 28(3): 503-526.

Mazzarol, T.W. and Soutar, G.N. 2001. The Global Market for Higher Education: Sustainable Competitive Strategies for the New Millennium, Cheltenham, UK, Edward Elgar Publishing.

Mazzarol, T. W., \& Soutar, G. N. 2002. "Push-Pull" Factors Influencing International Student Destination Choice. The International Journal of Educational Management, 16(2/3): 82-90

Mazzarol, T.W., Soutar, G.N. and Sim, M.Y-S. 2003. The Third Wave: Future Trends in International Education, International Journal of Educational Management, 17(3): 90-99.

McDougall, P.P., Shane, S. and Oviatt, B.M. 1994. Explaining the Formation of International New Ventures: The Limits of Theories of International Business Research. Journal of Business Venturing, 9: 469-487.

McGahan, A. M. and Porter, M.E. 1997. How Much Does Industry Matter, Really? Strategic Management Journal, 18 (Summer Special Issue): 15-30.

McGee, J. and Thomas, H. 1985. Strategic Groups: Theory, Research and Taxonomy. Strategic Management Journal, 7: 141-160. 
McLaughlin, C. and Fitzsimmons, J. 1996. Strategies for Globalising Service Operations. International Journal of Service Industry Management, 7(4): 4357.

McMahon, M. E. 1988. Knowledge Acquisition in the Global Market: Third World Participation in International Study. Unpublished PhD Dissertation, Stanford University.

McNaughton, R.B., 2003. The Number of Export Markets that a Firm Serves: Process Models versus the Born-Global Phenomenon. Journal of International Entrepreneurship, 1: 297-311.

Melin, L. 1992. Internationalisation as a Strategy Process. Strategic Management Journal, 13: 99-118.

Merton, R.K. 1957. Social Theory and Social Structure. Glencoe: The Free Press.

Meyer, J.W., Scott, W.R. and Strange, D. 1987. Centralization, Fragmentation and School District Complexity. Administrative Science Quarterly, 32: 186-201.

Miles, M.B and Huberman, A.M. 1994. Qualitative Data Analysis: An Expanded Sourcebook ( ${ }^{\text {nd }}$ edition). Thousand Oaks: Sage.

Millington, A.I. and Bayliss, B.I. 1990. The Process of Internationalisation: UK Companies in the E.C. Management International Review, 30(9): 788-804.

Miller, D. and Friesen, P.H. 1984. Organisations: A Quantum View. Englewood Cliffs, NJ: Prentice-Hall

Miller, D. and Shamsie, J. 1996. The Resource-Based View of the Firm in Two Environments: The Hollywood Film Studios from 1936 to 1965. Academy of Management Journal, 39(3): 519-543.

Mills, P.K. 1986. Managing Service Industries: Organizational Practices in PostIndustrial Economy. Cambridge, MA: Ballinger Publishing.

Mintzberg, H. 1979. The Structure of Organisations. Englewoods Cliffs, NJ: Prentice-Hall.

Moe, T.M. 1984. The New Economics of Organisation. Journal of Political Science, 28(November), 739-777.

Mohan, R. and Balendra, K. 2003. Joint Study Group Report on India-Sri Lanka Comprehensive Economic Partnership Agreement. Available: www.ips.lk [Retrieved 16 September 2008]. 
Naidoo,V. 2006a. A Case Study of New Zealand's Trade Commitments in Education Services: The Pros and Cons of GATS. Proceedings of the Australia New Zealand International Business Academy, 16-18 November, Wellington, New Zealand.

Naidoo, V. 2006b. Global Trade in Education Services: The Need to Move Out of the Current Impasse. Conference Proceedings of the Academy of International Business, June 23-26. Beijing, China

Nelson, R. and Winter, S. 1982. An Evolutionary Theory of Economic Change, Cambridge, MA: Harvard University Press.

Newbert, S. 2007. Empirical Research on the Resource-Based View of the Firm: An Assessment and Suggestions for Future Research. Strategic management Journal, 28: 121-146.

New China News Agency. 2003. Regulation of the People's Republic of China on Sino-Foreign Cooperation in the Running of Schools. Available: www.chinaview.cn [Retrieved 24 March 2003].

New Zealand Ministry of Education. 2004. New Zealand's Offshore Public Tertiary Education Programmes: Stock-take for the 2003 Academic Year. Wellington.

Nigh, D., Cho, K. and Krishnan, S. 1986. The Role of Location-Related Factors in US Banking Involvement Abroad: An Empirical Examination, Journal of International Business Studies, 17(3): 59-72.

Nitsch, D. 1999. The Determinants of Foreign Entry Mode. Unpublished PhD Dissertation. The University of Western Ontario.

Norman, P. Protecting Capabilities in Strategic Alliances: The Role of Isolation. Unpublished PhD Dissertation. University of North Carolina at Chapel Hill.

North, D.C. 1990. Institutions, Institutional Change and Economic Performance. Cambridge, UK: Cambridge University Press.

Norton, S.W. 1988a. An Empirical Look at Franchising as an Organizational Form. Journal of Business, 61(2): 197-217.

Norton, S.W. 1988b. Franchising, Brand Name Capital and Entrepreneurial Capacity Problem. Strategic Management Journal, 9 (Summer Special Issue): 105-114.

Nummella, N. 2004. Cultural Integration in Cross-Border Acquisition: A Case Study in Nummella, N. (Ed.) Many Faces of Cross-Border Mergers and Acquisitions. Publications of the Turku School of Economics and Business Administration. 
OBHE. 2003a. Sylvan Sells K-12 and Venture Arms To Focus on Higher Education. Available: www.obhe.ac.uk [Retrieved 27 December 2006].

OBHE 2003b. The 'World's Last Great Emerging Economy'- Russia Plans To Become A Major Player in Transnational Higher Education. Available: www.obhe.ac.uk [Retrieved 02 January 2007].

OBHE. 2004. The Swiss-German University in Indonesia Graduates its First Bachelors Students - Is Indonesia a Growth Market for Transnational Higher Education? Available: www.obhe.ac.uk [Retrieved 27 December 2006].

OBHE. 2005a. Australia Considers a National Quality Strategy for Transnational Higher Education- Will This Leave the Competition Behind? Available: www.obhe.ac.uk [Retrieved 01 December 2006].

OBHE. 2005b. The Dominican Republic to Encourage Transnational Activity en Mass in Pursuit of 'Cyber Island Status'. Available: www.obhe.ac.uk [Retrieved 02 December 2006].

OBHE. 2005c. Recent Developments in Nigeria: International Recruitment, SectorWide Accreditation Process and Latest Transnational Establishments. Available: www.obhe.ac.uk [Retrieved 02 January 2007].

OBHE. 2006a. Bigger, Broader, Better? UK Launches the Second Phase of the Prime Minister's Initiative for International Education. Available: www. obhe.ac.uk [Retrieved 02 January 2007].

OBHE. 2006b. Next Stop India? A Round-Up of Recent Developments in the Country's International Higher Education Sector. Available: www.obhe.ac.uk [Retrieved 27 December 2007].

OECD. 2004. Internationalisation and Trade in Higher Education: Opportunities and Challenges. Paris, OECD.

OECD Education Database. Annual. OECD Education Database. Available: www.sourceoecd.org. [Retrieved 10 February 2004].

OECD, 2006. Education at a Glance: OECD Indicators 2006, Paris: OECD.

O’Farrell, F.N., Wood, P.A. and Zheng, J. 1996. Internationlization of Business Service Firms: An Inter-Regional Analysis. International Small Business Journal, 16(2): 13-33.

Oliver, C. 1991. Strategic Responses to Institutional Processes. Academy of Management Review, 16(1): 145-179. 
Oliver, C. 1997. Sustainable Competitive Advantage: Combining Institutional and Resource-Based Views. Strategic Management Journal, 18(9): 697-713.

Osborn, R.N. and Baughn, C.C. 1990. Forms of Inter-organizational Governance for Multinational Alliances. Academy of Management Journal, 33(3): 503-519.

Oviatt, B.M. and McDougall, P.P. 1994. Toward a Theory of International New Ventures. Journal of International Business Studies, 25(1): 45-64.

Oviatt, B.M. and McDougall, P.P. 1995. Global Start-Ups: Entrepreneurs on a WorldWide Stage. Academy of Management Executive, 9(2): 30-44.

Oviatt, B.M. and McDougall, P.P. 1997. Challenges for Internationalisation Process Theory: The Case of International New Ventures. Management International Review, 37(2): 85-99.

Palay, T. 1984. Comparative Institutional Economics: The Governance of Rail Freight Contracting. Journal of Legal Studies, 13(June): 265-288.

Palmer, A. and Cole, C. 1995. Services Marketing: Principles and Practice. Englewood Cliffs, NJ: Prentice-Hall.

Palloff, R., \& Pratt, K. (2000). Making the Transition: Helping Teachers to Teach Online. Paper presented at the 'EDUCAUSE: Thinking it through' conference. Nashville, Tennessee. (ERIC Document Reproduction Service No. ED 452 806).

Pan, Y.G. 1996. Influences on Foreign Equity Ownership Level in Joint Ventures in China. Journal of International Business Studies, 77(1): 1-26.

Pan, Y.G. and Tse, D. K. 2000. The Hierarchical Model of Market Entry Modes. Journal of International Business Studies, 31 (4): 535-554.

Parasuraman, A., Berry, L. and Zeithaml, V. 1985. A Conceptual Model of Service Quality and Its Implications for Future Research. Journal of Marketing, 49(Fall): 41-50.

Park, S.H., Chen, R., Gallagher, S. 2002. Firm Resources as Moderators of the Relationship between Market Growth and Strategic Alliances in Semiconductor Start Ups. Academy of Management Journal, 45(3): 527-545.

Paterson, P.G. and Cicic, M. 1995. A Typology of Service Firms in International Markets: An Empirical Investigation. Journal of International Marketing, 3(4): 57-83.

Patton, M. Q. 1990. Qualitative Evaluation and Research Methods. Newbury Park, CA: Sage. 
Penrose, E.T. 1959. The Theory of the Growth of the Firm. London: Basil Blackwell.

Peter, J.P. 1979. Reliability: A Review of Psychometric Basics and Recent Marketing Practices. Journal of Marketing Research, 16(February): 6-17.

Peteraf, M.A. 1993. The Cornerstones of Competitive Advantage: A Resource-Based View. Strategic Management Journal, 14(3): 179-191.

Pfeffer, J. 1972. Merger as a Response to Organisational Interdependence. Administrative Science Quarterly, 17(3): 382-294.

Pfeffer, J. and Salancik, G.R. 1978. The External Control of Organisations: A Resource Dependence Perspective. New York: Harper and Row.

Picard, P. 1987. On the Design of Incentive Schemes under Moral Hazard and Averse Selection. Journal of Public Economics, 33(3): 305-332.

Pissano, G.P. 1988. Innovation through Markets, Hierarchies and Joint Ventures: Technology Strategy and Collaborative Arrangements in the Biotechnology Industry. Unpublished PhD Dissertation, University of California, Berkeley.

Porter, M. E. 1980. Competitive Strategy: Techniques for Analysing Industries and Competitors, The Free Press, New York.

Powell, W. W. 1988. Hybrid Organisational Arrangements. California Management Review, 30(1): 67-87.

Prebble, J.F. 1992. Global Expansion: The Case of US Fast-Food Franchisors. Journal of Global Marketing, 6(1/2): 185-205.

Prescott, E. and Visscher, M. 1980. Organisational Capital. Journal of Political Economy, 88(3): 446-461.

Priess, D. 1998. The Gulf Cooperation Council: Prospects For Expansion. Middle East Policy, 5(4): 17-26.

Prince, C. 2004. Commercial Activity in New University Business Schools. Higher Education, 18(2): 16-18.

Pucik, V. 1988. Strategic Alliances, Organisational Learning and Competitive Advantage: The HRM Agenda. Human Resource Management, 27(1): 77-93.

Pucik, V. 1991. Technology Transfer in Strategic Alliances: Competitive Collaboration and Organisational Learning in Agmon, T. and Von Glinow M.A. (Eds.) Technology Transfer in International Business. New York: Oxford 
University Press. Pg. 121-138

Rajan, K.S. and Pangarkar, N. 2000. Mode of Entry Choice: An Empirical Study of Singaporean Multinationals. Asia Pacific Journal of Management, 17: 49-66.

Rathmell, J.M. 1966. What is Meant by Services? Journal of Marketing, 30(October): 32-36.

Reid, S. 1983. Firm Internationalisation, Transaction Costs and Strategic Choice. International Marketing Review, 1(2): 44-56

Regan, W.J. 1963. The Service Revolution. Journal of Marketing, 47(July): 57-62.

Reuber, A.R. and Fischer, R. 1997. The Influence of the Management Team's International Experience on the Internationalisation Behaviours of SME's. Journal of International Business Studies, 28: 807-825.

Richardson, J. 1987. “A Sub-Sectoral Approach to Services’ Trade Theory”, in Grarini, O. (Ed.), The Emerging Service Economy. New York: Pergamon Press.

Riddle, D.I. 1986. Service-Led Growth: The Role of the Service Sector in World Development. New York: Praeger Publishers.

Rindfleisch, A. and Heide, J.B. 1997. Transaction Cost Analysis: Past, Present and Future Applications. Journal of Marketing, 61(4): 30-54.

Roberts, P. and Greenwood, R. 1997. Integrating Transaction Cost and Institutional Theories: Toward a Constrained-Efficiency Framework for Understanding Organisational Design Adoption. Academy of Management Review, 22: 346373.

Rogers, E.M. and Shoemaker, F.F. 1971. Communication of Innovations, New York: Free Press.

Romer, P. M. 1986. Increasing Returns and Long-Run Growth. Journal of Political Economy, 94: 1002-1037.

Root, F.R. 1987. Foreign Market Entry Strategies, New York: AMACOM.

Root, F. R. 1994. Entry Strategies for International Markets, John Wiley \& Sons, Inc. San Francisco.

Rose, E.L. and Ito, K. 2004. On the Relationship Between Japanese Manufacturing Firms and Their Subsidiaries. Asia Pacific Journal of Management, 21: 469490. 
Rosenzweig, P.M. and Singh, J.V. 1991. Organizational Environments and the Multinational Enterprise. Academy of Management Review, 16(2): 340-361.

Ross, S.A. 1973. The Economic Theory of Agency: The Principal's Problem. American Economic Review, 63(2): 134-139.

Roth, A.V. and Menor, L.J. 2003. Insights Into Service Operations Management: A Research Agenda. Production and Operations Management, 12(2): 145-164.

Rubin, P.H. 1973. The Expansion of Firms. Journal of Political Economy, 84: 936949.

Rubin, P.H. 1990. Managing Business Transactions. New York: The Free Press.

Rugman, A.M. 1980. Internalisation as a General Theory of Foreign Direct Investment: A Reappraisal of the Literature. Weltwirtschaftliches Archiv, 116(2): 365-379.

Rumelt, R. P. 1984. "Toward a Strategic Theory of the Firm” in R. Lamb (Ed.) Competitive Strategic Management, Englewood Cliffs, NJ: Prentice Hall, Inc. Pg. 556-570.

Rumelt, R. P. 1987. “Theory, Strategy and Entrepreneurship” in Teece, D. (Ed.) The Competitive Challenge: Strategies for Industrial Innovation and Renewal, Cambridge, MA: Ballinger Publishing Co.

Rumelt, R.P., Schendel, D. and Teece, D.J. 1991. Strategic Management and Economics. Strategic Management Journal, 12: 5-29.

Sampson, G.P. and Snape, R.H. 1985. Identifying the Issues of Trade in Services. World Economy, 8(June): 171-182.

Sarkar, M. and Cavusgil, T.S. 1996. Trends in International Business Thought and Literature: A Review of International Market Entry Mode Research: Integration and Synthesis. International Executive, 38(6): 825-847.

Schemenner, R.W. 1986. How Can Service Businesses Survive and Prosper? Sloan Management Review, 27(3): 21-32.

Scott, P. 1998. Massification, Internationalization and Globalization. In P. Scott (Ed.), The Globalization of Higher Education: 108-129. Buckingham: Society for Research into Higher Education \& Open University Press.

Scott, W.R. 1995. Institutions and Organisations. Thousand Oaks. CA: Sage.

Scott, W. R. 2004. “Institutional Theory: Contributing to a Theoretical Research 
Paradigm" in Smith, K.G. and Hill, M.A. (Eds) Great Minds in Management: The Process of Theory Development. Oxford, Oxford University Press.

Scott, P. 2000. Globalisation and Higher Education: Challenges for the 21st Century. Journal of Studies in International Education, 4, No. 1, 3-10.

Selingo, J. 2007. Cornell Courts a Subcontinent: A Whirlwind Tour of India Highlights US Institutions' Haste to Find Global Partners. The Chronicle of Higher Education, March 2, A38-A41.

Selznick, P. 1996. Institutionalism "Old" and "New". Administrative Science Quarterly, 41(2): 270.

Shane, S.A. 1996. Hybrid Organisational Arrangements and Their Implications for Firm Growth and Survival: A Study of New Franchisors. Academy of Management Journal, 39(1): 216-234.

Shane, S. 2000. Prior Knowledge and the Discovery of Entrepreneurial Opportunities. Organisation Science, 11: 448-469.

Sharma, A. 1993. Diversifying Entry: Performance Implications of Industry and Firm Level Factors. Unpublished PhD Dissertation, University of North Carolina at Chapel Hill.

Sharma, V. M. 1995. Development and Testing of a Resource-Based Theory of International Entry Mode Choice. Unpublished PhD Dissertation, University of North Texas.

Sharma, V.M. and Erramilli, M.K. 2004. Resource-Based Explanation of Entry Mode Choice. Journal of Marketing Theory and Practice, 12(1): 1-18.

Shenkar, O. 2001. Cultural Distance Revisited: Towards a More Rigorous Conceptualization and Measurement of Cultural Differences. Journal of International Business Studies, 32(3): 519-535.

Shenkar, O. \& Zehra, Y. 1987. Human Resource Management in International Joint Ventures: Directions for Research. Academy of Management Review, 12(3): 546-557.

Shepherd, D.A. and DeTienne, D.R. 2005. Prior Knowledge, Potential Financial Reward and Opportunity Identification. Entrepreneurship: Theory and Practice, 29(1): 91-112.

Shoestack, G.L. 1977. Breaking Free From Product Marketing. Journal of Marketing, 41(2): 73-80. 
Shrader, R.C., Oviatt, B.M. and McDougall, P.P. 2000. How New Ventures Exploit Trade-Offs Among International Risk Factors: Lessons for the Accelerated Internationalisation of the $21^{\text {st }}$ Century. Academy of Management Journal, 43(6): 1227-1247.

Simerly, R. L. and Li, M. 2000. Environmental Dynamism, Capital Structure and Performance: A Theoretical Integration and an Empirical Test. Strategic Management Journal, 21(1): 31-49.

Simon, H. 1945. Administrative Behaviour. New York: The Free Press.

Singapore Ministry of Education. 2006. List of External Degree Programmes. Available: www.moe.gov.sg [Retrieved 27 December 2006].

Singh, J.V., House, R.J. and Tucker, D.J. 1986. Organizational Change and Organizational Maturity. Administrative Science Quarterly, 31: 587-611.

Smart, D., \& Ang, G. 1993. Exporting Education: From Trade to Internationalization. IPA Review, 46(1): 31-33.

Smith, K.G., Smith, K.A., Olian, J.D., Sims Jr. H.P., O’Bannon, D.P. and Scully, J.A. 1994. Top Management Demography and Process: The Role of Social Integration and Communication. Administrative Science Quarterly, 39 (3): 412-438

Sobel, I. 1982. Human Capital and Institutional Theories of the Labor Market: Rivals or Complements. Journal of Economic Issues, 16(1): 255-272.

South African Qualifications Authority. 2006. Registry for Private Higher Education Institutions. Available: www.saqa.org.za [Retrieved 30 December 2006].

Stopford, J.M. and Wells, L.T. 1972. Managing the Multinational Enterprise. New York, Basic Books.

Stöttinger, B. 2001. Strategic Export Pricing: A Long and Winding Road. Journal of International Marketing, 9(1): 40-63.

Strauss, A. and Corbin, J. 1998. Basics of Qualitative Research: Techniques and Procedures for Developing Grounded Theory. Beverly Hills. CA: Sage.

Tallman, S.B. 1991. Strategic Management Models and Resource-Based Strategies Among MNEs in a Host Market. Strategic Management Journal, 12:69-82.

Tallman, S.B. 1992. A Strategic Management Perspective on the Host Country Structures of MNEs. Journal of Management, 18(3): 455-471. 
Tallman, S.B. and Shenkar, O. 1994. A Managerial Decision Model of International Cooperative Venture Formation. Journal of International Business Studies, First Quarter: 91-113.

Teece, D.J. 1981. The Market for Know-How and the Efficient International Transfer of Technology. Annals of the American Academy of Political and Social Science, 458(November): 81-96.

Teece, D.J. 1988. Capturing Value from Technological Innovation: Integration, Strategic Partnering and Licensing Decision. Interfaces, 18(May-June): 46-61.

Teece, D.J. and Winter, S.G. 1984. The Limits of Neoclassical Theory in Management Education. American Economic Review, 74: 116-121.

Terpstra, V. and Yu, C-M. 1988. Determinants of Foreign Investment of US Advertising Agencies. Journal of International Business Studies, 19(1): 3346.

Terpstra, V. and Sarathy, R. 1994. International Marketing ( $6^{\text {th }}$ edition). Fort Worth, TX: Dryden Press.

Thai Commission on Higher Education. 2006. List of Accredited Foreign Degrees. Available: www.inter.mua.go.th [Retrieved 30 December 2006].

The Australian. 2005a. Global reach a Growth Key. 05 April issue.

The Australian. 2005b. It's a Whole New Playing Field. 20 April issue.

The Australian. 2005c. Monash Loses More on South Africa Campus. 26 May issue.

The Australian. 2005d. AVCC Wary of an Offshore Learning Watchdog. 15 June issue.

The Australian. 2005e. Overseas Audit. 07 September issue.

The Australian. 2005f. Move to UAE, the Best of Both Worlds. 28 Sept issue.

The Australian. 2006. Monash to welcome China on Campus. 28 August issue.

The Chronicles of Higher Education. 2005. Boom in Online Programs Will Drive Growth of For-Profit Education, Analysts Predict. 13 December issue.

The Chronicles of Higher Education. 2006. Colleges Rush to Open Degree Programs Overseas, for Both Academic and Business Reasons. 17 February issue.

The Times Higher Education Supplement. 2004. Beijing Makes Those Tills Ring. 3 
December issue.

The Times Higher Education Supplement. 2004. Uncle Sam no Longer a Favourite. 10 December issue.

The Times Higher Education Supplement. 2004. The Challenges of Change. 17 December issue.

Thomas, D.R.E. 1978. Strategy is Different in Service Businesses. Harvard Business Review, 56(4): 158-165.

Thompson, J.D. 1967. Organisation in Action. New York: McGraw-Hill.

Thompson, C. J., Locander, W.B. and Polio, H. R. 1989. Putting Consumer Experience Back Into Consumer Research: The Philosophy and Method of ExistentialPhenomenology. Journal of Consumer Research, 16: 133-147.

Tse, A.C.B. 1998. Comparing the Response Rate, Response Speed and Response Quality of Two Methods of Sending Questionnaires: E-Mail vs. Mail. Journal of the Market Research Society, 40(4): 353-361.

Tse, D.K., Pan, Y.G. and Au, K.Y. 1997. How MNEs Choose Entry modes and Form Alliances; the China Experience. Journal of International Business Studies, 28(4): 779-805.

Tsui, A. 1984. A Role Set Analysis of Managerial Reputation. Organisational Behaviour and Human Performance, 34: 64-96.

Turnbull, P.W. 1987. “A Challenge to the Stages Theory of the Internationalisation Process” in Reid, S. and Rosson, P. (Eds). Managing Export Entry and Expansion, Praeger, New York.

Ulrich, D. and Barney, J.B. 1984. Perspective in Organisations: Resource Dependence, Efficiency and Population. Academy of Management Review, 9(3): 471-481.

UNCTAD. 2004. World Investment Report 2004: The Shift Towards Services, New York and Geneva, United Nations.

UNCTAD. Various. Handbook of Statistics. Available: http://stats.unctad.org [Retrieved 14 January 2007].

UNESCO. Annual. UNESCO Statistical Yearbook. Paris: UNESCO.

UNESCO. 1971. Statistics of Students Studying Abroad: 1962-1968. Paris: UNESCO

UNESCO. 1976. Statistics of Students Studying Abroad: 1969-1973. Paris: UNESCO 
UNESCO/Council of Europe. 2000. Code of Good Practice in the Provision of Transnational Education. Paris. UNESCO.

University Council of Jamaica. 2006. List of Accredited Courses. Available: www.ucjamaica.com [Retrieved 30 December 2006].

Usher, A. and Savino, M. 2006. A World of Difference: A Global Survey of University League Tables. Available: http://www.educationalpolicy.org [Retrieved 2 April 2006].

Vandermerwe, S. and Chadwick, M. 1989. The Internationalisation of Services. The Service Industries Journal, 9(1): 79-83.

Venkatraman, N. and Ramanujam, V. 1987. Measurement of Business Economic Performance: An Examination of Method Convergence. Journal of Management, 13(1): 109-122.

Verbik, L. \& Jokivirta. 2005. National Regulatory Frameworks for Transnational Higher Education: Models and Trends. The Observatory of Borderless Higher Education. Available: www.obhe.ac.uk. [Retrieved 02 January 2007].

Verbik, L. \& Merkley, C.2006. The International Branch Campus - Models and Trends. London, The Observatory for Borderless Higher Education.

Verikios, G. and Zhang, X-G. 2004. The Economic Effects of Removing Barriers to Trade in Telecommunications. The World Economy, 27(3): 435-458.

Vernon, R. 1966. International Investments and International Trade in the Product Life Cycle. Quarterly Journal of Economics, May issue: 190-207.

Vernon, R. 1983. “Organisational and Institutional Responses to International Risk” in R. Herring (Ed.), Managing International Risk. Cambridge MA: Cambridge University Press.

Vernon, R. and Wells, L.T. 1986. The Economic Environment of International Business. Englewood Cliffs, NJ: Prentice-Hall.

Vincent-Lancrin, S. 2004. Cross-border Education: An Overview in OECD (Eds.) Internationalisation and Trade in Higher Education: Opportunities and Challenges, Paris, OECD.

Vincent-Lancrin, S. 2005. Building Capacity Through Cross-Border Tertiary Education. The Observatory for Borderless Higher Education. Available: www.obhe.ac.uk. [Retrieved 02 January 2007]. 
Voss, C.A., Roth, A.V., Rosenzweig, E.D., Blackmon, K. and Chase, R.B. 2004. A Tale of Two Countries' Conservatism, Service Quality and Feedback on Customer Satisfaction. Journal of Service Research, 6(3): 212-230.

Weigelt, K and Camerer, C. 1988. Reputation and Corporate Strategy: A Review of Recent Theory of Applications. Strategic Management Journal, 9: 443-454.

Weinstein, A.K. 1977. Foreign Investments by Service Firms: The Case of Multinational Advertising Agencies. Journal of International Business Studies, 8(1): 83-91.

Wernerfelt, B. 1984. A Resource-Based View of the Firm. Strategic Management Journal, 5: 171-180.

Wernerfelt, B. 1989. From Critical Resources to Corporate Strategy. Journal of General Management, 14(3): 4-12.

Wernerfelt, B. and Karnani, A. 1987. Research Notes and Communications: Competitive Strategy Under Uncertainty. Strategic Management Journal, 8: 187-194.

Westney, D. E. 1983. Institutional Theory and the Multinational Corporation in S. Ghoshal and D.E. Westney (Eds) Organization Theory and the Multinational Corporation. New York: St Martins Press.

Westney, D.E. 1988. Domestic and Foreign Learning Curves in Managing International Cooperative Strategies in Contractor, F. and Lorange, P. (Eds.) Cooperative Strategies in International Business. Toronto: Lexington Books. Pg. 339-346

Wiersema, M.F. and Bantel, K.A. 1992. Top Management Team Demography and Corporate Strategic Change. Academy of Management Journal, 35(1): 91-121.

Wille, J.R. 1988. “Joint Venturing Strategies” in J.D. Carter, R.F. Cushman and C.S. Hartz (Eds.) The Handbook of Joint Venturing. Homewood, IL: Dow JonesIrwin.

Williams, J.R. 1992. How Sustainable is Your Competitive Advantage? California Management Review, Spring: 29-51.

Williamson, O.E. 1975. Markets and Hierarchies: Analysis and Antitrust Implications. New York: Free Press.

Williamson, O.E. 1979. Transaction Cost Economics: The Governance of Contractual Relations. Journal of Law and Economics, 22 (October), 233-262.

Williamson, O.E. 1981a. The Economics of Organisation: The Transaction Cost 
Approach. American Journal of Sociology, 87(3): 548-577.

Williamson, O.E. 1981b. The Modern Corporation: Origins, Evolution, Attributes. Journal of Economics Literature, 19(December): 1537-1568.

Williamson, O.E. 1985. The Economic Institutions of Capitalism. New York: Free Press.

Williamson, O.E. 1986. Economic Organisation. New York: New York University Press.

Williamson, O.E. and Ouchi, W.G. 1981. "The Markets and Hierarchies Program of Research: Origins, Implications, Prospects” in A.H.Van de Ven \& F.W. Joyce (Eds.) Perspectives on Organisation Design and Behaviour, New York: John Wiley \& Sons. Pg. 347-370.

Wind, Y. and Perlumutter, H.V. 1977. On the identification of the Frontier Issues of International Marketing. Columbia Journal of World Business, 12(Winter): 131-145.

Wood, B.J.G., Tapsell, S. and Soutar, G.N. 2005. Borderless Education: Some Implications for Management, International Journal of Management Education, 19(5): 428-436

Woodcock, C.P., Beamish, P. and Makino, S. 1994. Ownership-Based Entry Mode Strategies and International Performance. Journal of International Business Studies, 25(2): 253-273.

World Bank. 2002. Constructing Knowledge Societies: New Challenges for Tertiary Education. Available:www1.worldbank.org/education/tertiary [Retrieved 04 February 2004].

World Bank. 2003. World Development Indicators, Washington, D.C., World Bank.

World Bank. Various years. World Bank Education Statistics Database. Available: www.worldbank.org [Retrieved 28 September 2008].

World Trade Organisation, 2004. International Trade Statistics Report 2004, Geneva, World Trade Organisation.

Young, S., Hamill, J. Wheeler, C. and Davies, J.R. 1989. International Market Entry and Development: Strategies and Management. Englewood Cliffs: Prentice Hall

Yu, C-M. and Ito, K. 1988. Oligopolistic Reaction and Foreign Direct Investment: The Case of the U.S. Tyre and Textile Industries. Journal of International 
Business Studies, 19(Fall): 449-460.

Xinhua News Agency, 2002. 70 Percent of Students Want to Go Abroad. Available: www1.china.org.cn. [Retrieved 17 May, 2008]

Xu, D. and Shenkar, O. 2002. Institutional Distance and the Multinational Enterprise. Academy of Management Review, 27(4): 608-618.

Zahra, S.A., Neubaum, D.O. and Huse, M. 1997. The Effect of the Environment on Export Performance Among Telecommunications New Ventures. Entrepreneurship: Theory and Practice, 26: 25-46.

Zahra, S.A., Ireland, R.D. and Hitt, M.A. 2000. International Expansion by New Venture Firms: International Diversity, Mode of Market Entry, Technological Learning and Performance. Academy of Management Journal, 43: 925-951.

Zaheer, S. 1995. Overcoming the Liability of Foreignness. Academy of Management Journal, 38(2): 341-363.

Zeithaml, V.A., Parasuraman, A. and Berry, L.L. 1985. Problems and Strategies in Services Marketing. Journal of Marketing, 49(2): 33-46.

Zimmerman, A. 1999. Impacts of Service Trade Barriers: A Study of Insurance Industry. Journal of Service Marketing, 14(3): 211-218.

Zou, S and Cavusgil, S. T. 1996. Global Strategy: A Review and An Integrated Conceptual Framework. European Journal of Marketing, 30(1): 52-69.

Zucker, L. 1983. Organizations as Institutions, in S.B. Bacharach (Ed.) Research in the Sociology of Organisations, Greenwich, CN: Jai Press. Pg. 1-4 


\section{Appendix A \\ LIST OF WHOLLY-OWNED SUBSIDIARY BRANCH CAMPUSES}

\begin{tabular}{|c|c|c|c|c|}
\hline Institution & Home country & Branch location & $\begin{array}{c}\text { Year } \\
\text { Opened }\end{array}$ & Level \\
\hline $\begin{array}{l}\text { Charles Sturt } \\
\text { University }\end{array}$ & Australia & Canada & 2005 & Bachelors \\
\hline $\begin{array}{c}\text { Central Queensland } \\
\text { University }\end{array}$ & Australia & Fiji & 1998 & $\begin{array}{l}\text { Bachelors, } \\
\text { Masters }\end{array}$ \\
\hline Monash University & Australia & South Africa & 2001 & Bachelors \\
\hline $\begin{array}{l}\text { Dublin Business } \\
\text { School }\end{array}$ & Ireland & Malaysia & ? & $\begin{array}{l}\text { Bachelors } \\
\text { (final year in } \\
\text { Dublin) }\end{array}$ \\
\hline $\begin{array}{l}\text { Systems Technology } \\
\text { Institute }\end{array}$ & Philippines & Indonesia & ? & Diploma \\
\hline $\begin{array}{l}\text { Systems Technology } \\
\text { Institute }\end{array}$ & Philippines & Indonesia & ? & Diploma \\
\hline Webster University & USA & Austria & 1981 & $\begin{array}{l}\text { Bachelor, } \\
\text { Masters }\end{array}$ \\
\hline Webster University & USA & Netherlands & 1983 & $\begin{array}{l}\text { Bachelors, } \\
\text { Masters }\end{array}$ \\
\hline Webster University & USA & Switzerland & 1978 & $\begin{array}{l}\text { Bachelors, } \\
\text { Masters }\end{array}$ \\
\hline Webster University & USA & Thailand & 1999 & $\begin{array}{l}\text { Bachelors, } \\
\text { Masters }\end{array}$ \\
\hline Boston University & USA & Belgium & 1972 & Masters \\
\hline $\begin{array}{c}\text { Fairleigh Dickinson } \\
\text { University }\end{array}$ & USA & Canada & 2007 & Bachelors \\
\hline $\begin{array}{c}\text { University of } \\
\text { Northern Virginia }\end{array}$ & USA & Czech Republic & 2005(?) & $\begin{array}{l}\text { Bachelor, } \\
\text { Masters }\end{array}$ \\
\hline $\begin{array}{l}\text { Georgia Institute of } \\
\text { Technology }\end{array}$ & USA & France & 1990 & $\begin{array}{l}\text { Bachelors, } \\
\text { Masters }\end{array}$ \\
\hline $\begin{array}{l}\text { University of } \\
\text { Indianapolis }\end{array}$ & USA & Greece & 1989 & $\begin{array}{l}\text { Bachelors, } \\
\text { Masters }\end{array}$ \\
\hline $\begin{array}{c}\text { University of New } \\
\text { Orleans }\end{array}$ & USA & Jamaica & ? & $\begin{array}{l}\text { Executive } \\
\text { MBA }\end{array}$ \\
\hline $\begin{array}{l}\text { Alliant International } \\
\text { University }\end{array}$ & USA & Mexico & 1970 & $\begin{array}{l}\text { Bachelors, } \\
\text { Masters }\end{array}$ \\
\hline $\begin{array}{l}\text { Chicago Business } \\
\text { School }\end{array}$ & USA & Singapore & ? & Masters \\
\hline $\begin{array}{c}\text { University of Nevada, } \\
\text { Las Vegas }\end{array}$ & USA & Singapore & 2006 & $\begin{array}{l}\text { Bachelors, } \\
\text { Masters }\end{array}$ \\
\hline $\begin{array}{l}\text { Chicago Business } \\
\text { School }\end{array}$ & USA & UK & 2005 & $\begin{array}{l}\text { Executive } \\
\text { MBA }\end{array}$ \\
\hline Endicott College & USA & Mexico & 1996 & $\begin{array}{l}\text { Bachelors, } \\
\text { Masters }\end{array}$ \\
\hline
\end{tabular}




\section{Appendix B \\ LIST OF JOINT VENTURE BRANCH CAMPUSES}

\begin{tabular}{|c|c|c|c|c|}
\hline Institution & Home country & Branch location & $\begin{array}{c}\text { Year } \\
\text { Opened }\end{array}$ & Level \\
\hline Curtin University & Australia & Malaysia & 1999 & $\begin{array}{l}\text { Bachelors, } \\
\text { Masters, PhD } \\
\text { (limited) }\end{array}$ \\
\hline Monash University & Australia & Malaysia & 1998 & $\begin{array}{l}\text { Bachelors, } \\
\text { Masters, PhD } \\
\text { (limited) }\end{array}$ \\
\hline $\begin{array}{l}\text { Swinburne University } \\
\text { of Technology }\end{array}$ & Australia & Malaysia & 2001 & $\begin{array}{l}\text { Bachelors, } \\
\text { Masters, PhD } \\
\text { (limited) }\end{array}$ \\
\hline $\begin{array}{c}\text { James Cook } \\
\text { University }\end{array}$ & Australia & Singapore & 2003 & $\begin{array}{l}\text { Bachelors, } \\
\text { Masters }\end{array}$ \\
\hline $\begin{array}{l}\text { University of New } \\
\text { South Wales }\end{array}$ & Australia & Singapore & 2007 & $\begin{array}{l}\text { Bachelors, } \\
\text { Masters }\end{array}$ \\
\hline $\begin{array}{l}\text { University of } \\
\text { Wollongong }\end{array}$ & Australia & UAE & 1993 & $\begin{array}{l}\text { Bachelors, } \\
\text { Masters }\end{array}$ \\
\hline RMIT & Australia & Vietnam & ? & $\begin{array}{l}\text { Bachelors, } \\
\text { Masters }\end{array}$ \\
\hline $\begin{array}{c}\text { EHSAL European } \\
\text { University College } \\
\text { Brussels }\end{array}$ & Belgium & UAE & ? & $\begin{array}{l}\text { Bachelors, } \\
\text { Masters }\end{array}$ \\
\hline Centennial College & Canada & UAE & 2006 & ? \\
\hline $\begin{array}{c}\text { College of the North } \\
\text { Atlantic }\end{array}$ & Canada & Qatar & 2002 & Sub-degree \\
\hline $\begin{array}{l}\text { University of New } \\
\text { Brunswick }\end{array}$ & Canada & UAE & 2006 & Bachelors \\
\hline INSEAD & France & Singapore & 2000 & Masters \\
\hline $\begin{array}{l}\text { SP Jain Centre of } \\
\text { Management }\end{array}$ & India & UAE & 2005 & Masters \\
\hline $\begin{array}{l}\text { Manipal Academy of } \\
\text { Higher Education }\end{array}$ & India & UAE & 2003 & $\begin{array}{l}\text { Bachelors, } \\
\text { Masters }\end{array}$ \\
\hline $\begin{array}{l}\text { Birla Institute of } \\
\text { Technology and } \\
\text { Science }\end{array}$ & India & UAE & 2000 & Bachelors \\
\hline $\begin{array}{l}\text { Mahatma Gandhi } \\
\text { University }\end{array}$ & India & UAE & 2001 & $\begin{array}{l}\text { Bachelors, } \\
\text { Masters }\end{array}$ \\
\hline $\begin{array}{l}\text { Islamic Azad } \\
\text { University }\end{array}$ & Iran & UAE & 2004 & $\begin{array}{l}\text { Bachelors, } \\
\text { Masters }\end{array}$ \\
\hline $\begin{array}{l}\text { Royal College of } \\
\text { Surgeons }\end{array}$ & Ireland & UAE & 2005 & Masters \\
\hline Aga Khan University & Pakistan & Kenya & 2003 & $\begin{array}{c}\text { Diplomas and } \\
\text { Bachelors }\end{array}$ \\
\hline
\end{tabular}




\begin{tabular}{|c|c|c|c|c|}
\hline $\begin{array}{l}\text { Shaheed Sulfikar Ali } \\
\text { Bhutto Institute of } \\
\text { Science and Tech }\end{array}$ & Pakistan & UAE & 2003 & $\begin{array}{l}\text { Bachelors, } \\
\text { Masters }\end{array}$ \\
\hline $\begin{array}{l}\text { Stockholm School of } \\
\text { Economics }\end{array}$ & Sweden & Russia & 1997 & $\begin{array}{l}\text { Executive } \\
\text { MBA }\end{array}$ \\
\hline $\begin{array}{l}\text { University of } \\
\text { Nottingham }\end{array}$ & UK & China & 2004 & $\begin{array}{c}\text { Bachelors, } \\
\text { Masters }\end{array}$ \\
\hline $\begin{array}{l}\text { University of } \\
\text { Nottingham }\end{array}$ & UK & Malaysia & 2000 & $\begin{array}{c}\text { Bachelors, } \\
\text { Masters }\end{array}$ \\
\hline $\begin{array}{l}\text { Heriot Watt } \\
\text { University }\end{array}$ & UK & UAE & 2005 (?) & $\begin{array}{c}\text { Bachelors, } \\
\text { Masters }\end{array}$ \\
\hline Middlesex University & UK & UAE & 2005 & $\begin{array}{c}\text { Bachelors, } \\
\text { Masters }\end{array}$ \\
\hline $\begin{array}{l}\text { Carnegie Mellon } \\
\text { University }\end{array}$ & USA & Australia & 2006 & $\begin{array}{c}\text { Bachelors, } \\
\text { Masters }\end{array}$ \\
\hline $\begin{array}{c}\text { Florida International } \\
\text { University }\end{array}$ & USA & China & 2006 & ? \\
\hline Kean University & USA & China & 2007 & $\begin{array}{c}\text { Bachelors, } \\
\text { Masters }\end{array}$ \\
\hline $\begin{array}{l}\text { Missouri State } \\
\text { University }\end{array}$ & USA & China & 2000 & $\begin{array}{l}\text { Diploma, } \\
\text { Bachelors }\end{array}$ \\
\hline McDaniel College & USA & Hungary & 1994 & Masters \\
\hline Temple University & USA & Japan & 1982 & $\begin{array}{l}\text { Bachelors, } \\
\text { Masters, PhD } \\
\text { (limited) }\end{array}$ \\
\hline Columbia University & USA & Jordan & 2009 & ? \\
\hline DePaul University & USA & Jordan & 2005 & Masters \\
\hline $\begin{array}{l}\text { Florida State } \\
\text { University }\end{array}$ & USA & Panama & 1999 & Bachelors \\
\hline Clark University & USA & Poland & 2004 & Masters \\
\hline $\begin{array}{l}\text { Carnegie Mellon } \\
\text { University }\end{array}$ & USA & Qatar & 2004 & Bachelors \\
\hline Cornell University & USA & Qatar & 2002 & Masters \\
\hline $\begin{array}{l}\text { Georgetown } \\
\text { University }\end{array}$ & USA & Qatar & 2005 & Bachelors \\
\hline $\begin{array}{c}\text { Texas A\&M } \\
\text { University }\end{array}$ & USA & Qatar & 2003 & $\begin{array}{l}\text { Bachelors, } \\
\text { Masters }\end{array}$ \\
\hline $\begin{array}{c}\text { Virginia } \\
\text { Commonwealth } \\
\text { University }\end{array}$ & USA & Qatar & 1997 & Bachelors \\
\hline $\begin{array}{l}\text { George Mason } \\
\text { University }\end{array}$ & USA & UAE & 2006 & Bachelors \\
\hline $\begin{array}{l}\text { Harvard Medical } \\
\text { International }\end{array}$ & USA & UAE & 2004 & $\begin{array}{l}\text { Postgraduate } \\
\text { Programmes }\end{array}$ \\
\hline $\begin{array}{c}\text { American } \\
\text { Intercontinental } \\
\text { University }\end{array}$ & USA & UK & 1973 & $\begin{array}{l}\text { Bachelors, } \\
\text { Masters }\end{array}$ \\
\hline
\end{tabular}


Appendix C

\section{LIST OF NON-EQUITY MODE 3 \\ TRANSNATIONAL EDUCATION PROGRAMMES IN CHINA}

\begin{tabular}{|c|c|c|}
\hline "Source Country & "Education Institution & $\begin{array}{c}\text { No of Joint Programmes on } \\
\text { Offer }\end{array}$ \\
\hline \multirow[t]{24}{*}{ Australia } & Australian National University & 2 \\
\hline & Charles Sturt University & 37 \\
\hline & Curtin University of Technology & 3 \\
\hline & Deakin University & 2 \\
\hline & Edith Cowan University & 3 \\
\hline & Flinders University & 6 \\
\hline & Griffith University & 2 \\
\hline & La Trobe University & 13 \\
\hline & Macquarie University & 1 \\
\hline & Queensland University of Tech & 1 \\
\hline & RMIT University & 7 \\
\hline & Southern Cross University & 6 \\
\hline & University of Ballarat & 2 \\
\hline & University of Canberra & 8 \\
\hline & University of New England & 5 \\
\hline & University of South Australia & 1 \\
\hline & Uni of Southern Queensland & 39 \\
\hline & University of Sydney & 7 \\
\hline & University of Tasmania & 3 \\
\hline & University of Tech, Sydney & 13 \\
\hline & University of Western Sydney & 6 \\
\hline & University of Wollongong & 2 \\
\hline & Victoria University of Tech & 30 \\
\hline & Total & 199 \\
\hline Belgium & Louvain Institute of Technology & 1 \\
\hline \multirow[t]{10}{*}{ Canada } & Brock University & 1 \\
\hline & Carleton University & 1 \\
\hline & Capilano College & 1 \\
\hline & $\begin{array}{l}\text { Lambton College of Applied } \\
\text { Arts \& Tech }\end{array}$ & 2 \\
\hline & Newfoundland Memorial Uni & 1 \\
\hline & $\begin{array}{l}\text { Northern Alberta Institute of } \\
\text { Tech }\end{array}$ & 1 \\
\hline & Simon Fraser University & 1 \\
\hline & University of British Columbia & 1 \\
\hline & University of New Brunswick & 1 \\
\hline & $\begin{array}{l}\text { University of Quebec, } \\
\text { Chicoutimi }\end{array}$ & 4 \\
\hline
\end{tabular}




\begin{tabular}{|c|c|c|}
\hline & Total & 14 \\
\hline \multirow[t]{6}{*}{ France } & $\begin{array}{l}\text { Ecole Nationale des Ponts et } \\
\text { Chausses }\end{array}$ & 1 \\
\hline & $\begin{array}{l}\text { Ecole Nationale de l'Aviation } \\
\text { Civile }\end{array}$ & 1 \\
\hline & $\begin{array}{l}\text { Institut National Polytechnique } \\
\text { de Grenoble }\end{array}$ & 1 \\
\hline & $\begin{array}{l}\text { The National Enterprise Mgmt } \\
\text { Edu Foundation }\end{array}$ & 2 \\
\hline & University of Grenoble & 2 \\
\hline & Total & 7 \\
\hline Germany & Dresden Technical University & 1 \\
\hline \multirow[t]{5}{*}{ Hong Kong } & Chinese Uni of Hong Kong & 2 \\
\hline & Hong Kong Polytech University & 14 \\
\hline & $\begin{array}{l}\text { Hong Kong Uni of Science \& } \\
\text { Tech }\end{array}$ & 2 \\
\hline & University of Hong Kong & 4 \\
\hline & Total & 22 \\
\hline \multirow[t]{4}{*}{ Ireland } & Dublin Institute of Technology & 1 \\
\hline & University College Dublin & 1 \\
\hline & University of Limerick & 1 \\
\hline & Total & 3 \\
\hline Korea & Taebu University & 1 \\
\hline \multirow[t]{11}{*}{ New Zealand } & $\begin{array}{l}\text { Auckland Institute of Studies- St } \\
\text { Helens }\end{array}$ & 5 \\
\hline & Auckland Uni of Technology & 5 \\
\hline & Christchurch Polytechnic & 6 \\
\hline & Massey & 2 \\
\hline & University of Auckland & 1 \\
\hline & University of Waikato & 2 \\
\hline & UNITEC & 2 \\
\hline & $\begin{array}{l}\text { Victoria University of } \\
\text { Wellington }\end{array}$ & 3 \\
\hline & Waikato Institute of Technology & 2 \\
\hline & $\begin{array}{l}\text { Western Institute of Tech at } \\
\text { Taranaki }\end{array}$ & 3 \\
\hline & Total & 31 \\
\hline Netherlands & Maastricht School of Mgmt & 2 \\
\hline Norway & Norway School of Mgmt & 2 \\
\hline \multirow[t]{3}{*}{ Singapore } & Nanyang Technological Uni & 1 \\
\hline & National University of Singapore & 1 \\
\hline & Total & 2 \\
\hline Sweden & World Maritime University & 2 \\
\hline \multirow[t]{2}{*}{ UK } & Middlesex University & 1 \\
\hline & Northumbria University & 20 \\
\hline
\end{tabular}




\begin{tabular}{|c|c|c|}
\hline & Oxford Brookes University & 2 \\
\hline & Staffordshire University & 14 \\
\hline & University of Abertay Dundee & 2 \\
\hline & UCE Birmingham & 14 \\
\hline & $\begin{array}{l}\text { University of Central } \\
\text { Lancanshire }\end{array}$ & 1 \\
\hline & University of Lancaster & 2 \\
\hline & University of Leeds & 1 \\
\hline & University of Wales & 16 \\
\hline & Total & 73 \\
\hline \multirow[t]{34}{*}{ USA } & Benedictine College & 2 \\
\hline & City University of New York & 1 \\
\hline & Eastern Michigan University & 1 \\
\hline & Florida Intl University & 2 \\
\hline & Fordham University & 1 \\
\hline & Fort Hays State University & 3 \\
\hline & George Washington University & 1 \\
\hline & Harper Adams Uni College & 1 \\
\hline & Indiana Wesleyan University & 2 \\
\hline & Keoka College & 4 \\
\hline & Maharishi University of Mgmt & 1 \\
\hline & Michigan State University & 1 \\
\hline & New England Optometry College & 1 \\
\hline & New York Institute of Tech & 1 \\
\hline & Ohio University & 1 \\
\hline & Pace University & 1 \\
\hline & San Diego State University & 1 \\
\hline & Southwest Missouri State Uni & 1 \\
\hline & $\begin{array}{l}\text { State University of New York, } \\
\text { Buffalo }\end{array}$ & 1 \\
\hline & Stephens Institute of Tech & 4 \\
\hline & Temple University & 1 \\
\hline & Towson University & 1 \\
\hline & Uni of Colorado, Denver & 1 \\
\hline & University of Maryland & 2 \\
\hline & University of Michigan & 3 \\
\hline & University of Minnesota & 1 \\
\hline & University of Oklahoma & 1 \\
\hline & $\begin{array}{l}\text { University of Southern } \\
\text { California }\end{array}$ & 1 \\
\hline & Uni of Texas at Arlington & 2 \\
\hline & University of Washington & 1 \\
\hline & Uni of Wisconsin, Platteville & 1 \\
\hline & Utah State University & 2 \\
\hline & Webster University & 2 \\
\hline & Total & 50 \\
\hline Grand Total & & 410 \\
\hline
\end{tabular}

Source: Chinese Ministry of Education (2006), AVCC (2003), ENZ (2006), Education UK (2006) 


\section{Appendix D}

\section{LIST OF NON-EQUITY MODE 3 TRANSNATIONAL EDUCATION PROGRAMMES IN HONG KONG}

\begin{tabular}{|c|c|c|}
\hline Source Country & Education Institution & $\begin{array}{c}\text { No of Joint Programmes on } \\
\text { Offer } \\
\end{array}$ \\
\hline \multirow[t]{30}{*}{ Australia } & "Australian Catholic University & 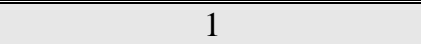 \\
\hline & Central Queensland University & 5 \\
\hline & Charles Sturt University & 12 \\
\hline & Curtin University of Technology & 23 \\
\hline & Deakin University & 5 \\
\hline & Edith Cowan University & 11 \\
\hline & Griffith University & 5 \\
\hline & James Cook University & 1 \\
\hline & La Trobe University & 2 \\
\hline & Macquarie University & 16 \\
\hline & Monash University & 24 \\
\hline & Murdoch University & 1 \\
\hline & Queensland University of Tech & 4 \\
\hline & RMIT University & 15 \\
\hline & Southern Cross University & 4 \\
\hline & Swinburne University of Tech & 4 \\
\hline & University of Ballarat & 12 \\
\hline & University of Canberra & 3 \\
\hline & University of Melbourne & 2 \\
\hline & University of New England & 15 \\
\hline & University of New South Wales & 5 \\
\hline & University of South Australia & 50 \\
\hline & Uni of Southern Queensland & 11 \\
\hline & University of Sydney & 3 \\
\hline & University of Tech, Sydney & 7 \\
\hline & University of Western Sydney & 11 \\
\hline & University of Western Australia & 4 \\
\hline & University of Wollongong & 18 \\
\hline & Victoria University of Tech & 17 \\
\hline & Total & 291 \\
\hline \multirow[t]{8}{*}{ Canada } & Dalhousie University & 1 \\
\hline & Senecca College & 1 \\
\hline & Ottawa University & 2 \\
\hline & Royal Roads University & 1 \\
\hline & Simon Fraser University & 1 \\
\hline & University of Alberta & 1 \\
\hline & University of British Columbia & 1 \\
\hline & University of Waterloo & 1 \\
\hline
\end{tabular}




\begin{tabular}{|c|c|c|}
\hline & University of Western Ontario & 1 \\
\hline & Total & 10 \\
\hline \multirow[t]{13}{*}{ China } & Beijing Normal University & 1 \\
\hline & Beijing Sport University & 3 \\
\hline & Beijing U of Chinese Medicine & 10 \\
\hline & $\begin{array}{l}\text { Capital University of Economics } \\
\text { \& Business }\end{array}$ & 2 \\
\hline & Communication Uni of China & 1 \\
\hline & $\begin{array}{l}\text { Dongbei University of Finance \& } \\
\text { Economics }\end{array}$ & 2 \\
\hline & East China Normal University & 4 \\
\hline & Jinan University & 1 \\
\hline & Tsinghua University & 3 \\
\hline & U of Political Science \& Law & 1 \\
\hline & $\begin{array}{l}\text { The University of Intl Business } \\
\text { and Economics }\end{array}$ & 1 \\
\hline & Unspecified institution(s) & 52 \\
\hline & Total & 81 \\
\hline Ireland & National University of Ireland & 3 \\
\hline Japan & Osaka U of Foreign Studies & 1 \\
\hline Macau & Asia Intl Open University & 3 \\
\hline \multirow[t]{3}{*}{ New Zealand } & Massey University & 4 \\
\hline & $\begin{array}{l}\text { Victoria University of } \\
\text { Wellington }\end{array}$ & 1 \\
\hline & Total & 5 \\
\hline Philippines & Philippines Women’s University & 1 \\
\hline \multirow[t]{23}{*}{ UK } & Binghampton University & 1 \\
\hline & Brunel University & 2 \\
\hline & Coventry University & 7 \\
\hline & DeMonfort University & 2 \\
\hline & Henley Management College & 4 \\
\hline & Heriot-Watt University & 31 \\
\hline & Kingston University & 1 \\
\hline & Lancaster University & 4 \\
\hline & Leeds Metropolitan University & 7 \\
\hline & Liverpool John-Moores Uni & 3 \\
\hline & London Metropolitan University & 2 \\
\hline & Manchester Metropolitan Uni & 2 \\
\hline & Middlesex University & 31 \\
\hline & Napier University & 18 \\
\hline & Northumbria University & 2 \\
\hline & Nottingham Trent University & 4 \\
\hline & Oxford Brookes University & 7 \\
\hline & Queen Mary Uni of London & 3 \\
\hline & Sheffield Hallam University & 23 \\
\hline & Thames Valley University & 1 \\
\hline & Trinity College, London & 1 \\
\hline & University of Bath & 3 \\
\hline & University of Birmingham & 4 \\
\hline
\end{tabular}




\begin{tabular}{|c|c|c|}
\hline & University of Bolton & 4 \\
\hline & University of Bradford & 4 \\
\hline & University of Bristol & 2 \\
\hline & University of Central England & 5 \\
\hline & University of Central Lancashire & 17 \\
\hline & University of Durham & 4 \\
\hline & University of East Anglia & 2 \\
\hline & University of Exeter & 2 \\
\hline & University of Glamorgan & 3 \\
\hline & University of Greenwich & 8 \\
\hline & University of Huddersfield & 6 \\
\hline & University of Hull & 5 \\
\hline & University of Leicester & 38 \\
\hline & University of London & 1 \\
\hline & University of Manchester & 2 \\
\hline & University of Newcastle & 5 \\
\hline & University of Northumbria & 20 \\
\hline & University of Nottingham & 3 \\
\hline & University of Portsmouth & 18 \\
\hline & University of Reading & 8 \\
\hline & University of Sunderland & 11 \\
\hline & University of Staffordshire & 8 \\
\hline & University of Surrey & 2 \\
\hline & University of Ulster & 14 \\
\hline & University of Wales & 12 \\
\hline & University of Warwick & 16 \\
\hline & University of Wolverhampton & 7 \\
\hline & University of York & 1 \\
\hline & Total & 391 \\
\hline \multirow[t]{24}{*}{ USA } & Alliant Intl University & 1 \\
\hline & $\begin{array}{l}\text { Baruch College, City U of New } \\
\text { York }\end{array}$ & 3 \\
\hline & Benedictine College & 3 \\
\hline & Bulacan State University & 1 \\
\hline & Clark University & 1 \\
\hline & Cal State University, East Bay & 1 \\
\hline & Cal State University, Fullerton & 1 \\
\hline & Columbia Southern University & 1 \\
\hline & Indiana Uni at Bloomington & 2 \\
\hline & Louisiana University at Munroe & 1 \\
\hline & Northwestern University & 1 \\
\hline & Ohio University & 7 \\
\hline & Olivet Nazarene University & 1 \\
\hline & Southern Illinois University & 1 \\
\hline & The George Washington Uni & 1 \\
\hline & University of Alabama & 1 \\
\hline & University of Dubuque & 1 \\
\hline & University of Iowa & 1 \\
\hline & $\begin{array}{l}\text { University of Michigan- } \\
\text { Dearborn }\end{array}$ & 1 \\
\hline & University of Minnesota & 1 \\
\hline & University of North Alabama & 1 \\
\hline & University of Northern Iowa & 1 \\
\hline & University of Northern Virginia & 2 \\
\hline & University of Oklahoma & 1 \\
\hline
\end{tabular}




\begin{tabular}{|c|c|c|}
\hline & Upper Iowa University & 1 \\
\hline & Utah State University & 2 \\
\hline & Weber State University & 2 \\
\hline & Western Michigan University & 1 \\
\hline & Total & 41 \\
\hline Grand Total & & 827 \\
\hline
\end{tabular}

Source: Education and Manpower Bureau (2006) 


\section{Appendix E}

\section{LIST OF NON-EQUITY MODE 3 TRANSNATIONAL EDUCATION PROGRAMMES IN INDIA}

\begin{tabular}{|c|c|c|}
\hline Source Country & "Education Institution & $\begin{array}{c}\text { No of Joint Programmes on } \\
\text { Offer } \\
\end{array}$ \\
\hline \multirow{10}{*}{ Australia } & Charles Sturt University & 21 \\
\hline & Edith Cowan University & 1 \\
\hline & Griffith University & 1 \\
\hline & Northern Territory University & 1 \\
\hline & Southern Cross University & 1 \\
\hline & University of New England & 1 \\
\hline & University of Canberra & 2 \\
\hline & Uni of Southern Queensland & 12 \\
\hline & University of Western Sydney & 1 \\
\hline & Total & 41 \\
\hline Bangladesh & Intl Uni of Science and Tech & 1 \\
\hline \multirow[t]{3}{*}{ Canada } & Centennial College & 1 \\
\hline & McMaster University & 1 \\
\hline & Total & 2 \\
\hline Germany & European Uni of Viadrina & 1 \\
\hline \multirow[t]{3}{*}{ New Zealand } & Massey University & 1 \\
\hline & UCOL & 1 \\
\hline & Total & 2 \\
\hline Thailand & Assumption University & 1 \\
\hline \multirow[t]{15}{*}{ UK } & Binghampton University & 2 \\
\hline & Brunel University & 1 \\
\hline & Coventry University & 2 \\
\hline & DeMonfort University & 1 \\
\hline & Henley Management College & 2 \\
\hline & Heriot-Watt University & 4 \\
\hline & Kingston University & 3 \\
\hline & Lancaster University & 2 \\
\hline & Leeds Metropolitan University & 2 \\
\hline & Liverpool John-Moores Uni & 2 \\
\hline & London Metropolitan University & 1 \\
\hline & Manchester Metropolitan Uni & 2 \\
\hline & Middlesex University & 4 \\
\hline & Napier University & 2 \\
\hline & Northumbria University & 2 \\
\hline
\end{tabular}




\begin{tabular}{|c|c|c|}
\hline & Nottingham Trent University & 3 \\
\hline & Oxford Brookes University & 3 \\
\hline & Queen Mary Uni of London & 1 \\
\hline & Sheffield Hallam University & 2 \\
\hline & Thames Valley University & 1 \\
\hline & Trinity College, London & 2 \\
\hline & University of Bath & 2 \\
\hline & University of Birmingham & 3 \\
\hline & University of Bolton & 2 \\
\hline & University of Bradford & 2 \\
\hline & University of Bristol & 1 \\
\hline & University of Central England & 3 \\
\hline & University of Central Lancashire & 3 \\
\hline & University of Durham & 2 \\
\hline & University of East Anglia & 3 \\
\hline & University of Exeter & 2 \\
\hline & University of Glamorgan & 1 \\
\hline & University of Greenwich & 1 \\
\hline & University of Huddersfield & 2 \\
\hline & University of Hull & 1 \\
\hline & University of Leicester & 3 \\
\hline & University of London & 2 \\
\hline & University of Manchester & 1 \\
\hline & University of Newcastle & 2 \\
\hline & University of Northumbria & 3 \\
\hline & University of Nottingham & 1 \\
\hline & University of Portsmouth & 2 \\
\hline & University of Reading & 3 \\
\hline & University of Sunderland & 1 \\
\hline & University of Staffordshire & 4 \\
\hline & University of Surrey & 1 \\
\hline & University of Ulster & 2 \\
\hline & University of Wales & 1 \\
\hline & University of Warwick & 2 \\
\hline & University of Wolverhampton & 3 \\
\hline & University of York & 2 \\
\hline & Total & 105 \\
\hline \multirow[t]{17}{*}{ USA } & Adams State College & 1 \\
\hline & Alliant Intl University & 1 \\
\hline & Andrew Jackson University & 2 \\
\hline & Aspen University & 1 \\
\hline & Auburn University & 2 \\
\hline & Aurora University & 1 \\
\hline & $\begin{array}{l}\text { Baruch College, City U of New } \\
\text { York }\end{array}$ & 2 \\
\hline & Benedictine College & 2 \\
\hline & Bulacan State University & 2 \\
\hline & Clark University & 1 \\
\hline & Cal State University, East Bay & 2 \\
\hline & Cal State University, Fullerton & 1 \\
\hline & Clemson University & 1 \\
\hline & Coastal Carolina University & 2 \\
\hline & Columbia Southern University & 1 \\
\hline & Columbus State University & 1 \\
\hline & Cornerstone University & 2 \\
\hline
\end{tabular}




\begin{tabular}{|c|c|c|}
\hline & George Washington University & 1 \\
\hline & Golden Gate University & 1 \\
\hline & Grand Valley State University & 1 \\
\hline & Indiana Uni at Bloomington & 2 \\
\hline & Johnson \& Wales University & 2 \\
\hline & Louisiana University at Munroe & 2 \\
\hline & Loyolla College & 1 \\
\hline & Northern Illinois University & 1 \\
\hline & North West Missouri State Uni & 2 \\
\hline & North Dakota State University & 2 \\
\hline & Northwestern University & 1 \\
\hline & Nova Southeastern University & 2 \\
\hline & Oklahoma City University & 1 \\
\hline & Oklahoma State University & 2 \\
\hline & Ohio State University & 1 \\
\hline & Pittsburgh State University & 2 \\
\hline & Rutgers University & 1 \\
\hline & $\begin{array}{l}\text { Savanah College of Art and } \\
\text { Design }\end{array}$ & 2 \\
\hline & San Diego State University & 1 \\
\hline & Saint Joseph University & 2 \\
\hline & Shenandoah University & 2 \\
\hline & Southeastern University & 2 \\
\hline & Southern Illinois University & 1 \\
\hline & Southern New Hampshire Uni & 1 \\
\hline & $\begin{array}{l}\text { State University of New York, } \\
\text { Buffalo }\end{array}$ & 2 \\
\hline & $\begin{array}{l}\text { State University of New York, } \\
\text { Empire State College }\end{array}$ & 1 \\
\hline & Syracuse University & 1 \\
\hline & Tarleton State University & 2 \\
\hline & Troy State University & 2 \\
\hline & University of Alabama & 1 \\
\hline & University of Central Michigan & 3 \\
\hline & University of Dubuque & 1 \\
\hline & University of Iowa & 2 \\
\hline & $\begin{array}{l}\text { University of Michigan- } \\
\text { Dearborn }\end{array}$ & 1 \\
\hline & University of Minnesota & 2 \\
\hline & University of North Alabama & 1 \\
\hline & University of Northern Iowa & 1 \\
\hline & University of Northern Virginia & 1 \\
\hline & University of Oklahoma & 2 \\
\hline & University of San Francisco & 1 \\
\hline & University of Wisconsin - Stout & 1 \\
\hline & Upper Iowa University & 1 \\
\hline & Utah State University & 1 \\
\hline & Weber State University & 2 \\
\hline & Western Michigan University & 3 \\
\hline & Wheelock College & 1 \\
\hline & Wilmington College & 2 \\
\hline & Total & 96 \\
\hline Grand Total & & 249 \\
\hline
\end{tabular}

Source: NIEPA (2006) 


\section{Appendix $F$ \\ LIST OF NON-EQUITY MODE 3 \\ TRANSNATIONAL EDUCATION PROGRAMMES IN MALAYSIA}

\begin{tabular}{|c|c|c|}
\hline Pource Country & Education Institution & $\begin{array}{c}\text { No of Joint Programmes on } \\
\text { Offer } \\
\end{array}$ \\
\hline \multirow[t]{29}{*}{ Australia } & "Bond University & 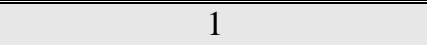 \\
\hline & Central Queensland University & 4 \\
\hline & Charles Sturt University & 54 \\
\hline & Curtin University of Technology & 54 \\
\hline & Deakin University & 9 \\
\hline & Intl College of Hospitality Mgmt & 1 \\
\hline & Edith Cowan University & 31 \\
\hline & James Cook University & 2 \\
\hline & La Trobe University & 6 \\
\hline & Monash University* & 2 \\
\hline & Murdoch University & 6 \\
\hline & Northern Territory University & 9 \\
\hline & RMIT University & 7 \\
\hline & Swinburne University of Tech & 9 \\
\hline & University of Adelaide & 12 \\
\hline & University of Ballarat & 12 \\
\hline & University of Canberra & 2 \\
\hline & University of Newcastle & 1 \\
\hline & University of New England & 13 \\
\hline & University of South Australia & 18 \\
\hline & Uni of Southern Queensland & 39 \\
\hline & University of Sunshine Coast & 1 \\
\hline & University of Tech, Sydney & 3 \\
\hline & University of Tasmania & 2 \\
\hline & University of Western Australia & 1 \\
\hline & University of Wollongong & 5 \\
\hline & Victoria University of Tech & 11 \\
\hline & Unspecified institution(s) & 1 \\
\hline & Total & 315 \\
\hline \multirow[t]{4}{*}{ Canada } & Ontario Ministry of Education & 1 \\
\hline & Uni College of The Cariboo & 2 \\
\hline & University of Lethbridge & 1 \\
\hline & Total & 4 \\
\hline France & University of Toulouse & 6 \\
\hline Germany & U of Applied Sciences, Manheim & 1 \\
\hline India & U.M.R.F. Deemed University & 1 \\
\hline
\end{tabular}




\begin{tabular}{|c|c|c|}
\hline Ireland & National University of Ireland & 1 \\
\hline Jordan & Yarmouk University & 1 \\
\hline \multirow[t]{5}{*}{ New Zealand } & $\begin{array}{l}\text { Auckland Institute of Studies - } \\
\text { St Helens }\end{array}$ & 1 \\
\hline & University of Auckland & 1 \\
\hline & $\begin{array}{l}\text { Victoria University of } \\
\text { Wellington }\end{array}$ & 9 \\
\hline & Unspecified institution(s) & 2 \\
\hline & Total & 16 \\
\hline Switzerland & Intl Hotel Mgmt Institute & 1 \\
\hline \multirow[t]{25}{*}{ UK } & Anglia Ruskin University & 4 \\
\hline & Bolton University & 1 \\
\hline & Bradford University & 8 \\
\hline & Coventry University & 11 \\
\hline & Heriot-Watt University & 2 \\
\hline & Herfordshire University & 9 \\
\hline & Liverpool John-Moores Uni & 4 \\
\hline & Napier University & 2 \\
\hline & Northumbria University & 8 \\
\hline & Nottingham Trent University & 10 \\
\hline & Oxford Brookes University & 3 \\
\hline & Sheffield Hallam University & 3 \\
\hline & Staffordshire University & 8 \\
\hline & Teeside University & 1 \\
\hline & University of Abertay, Dundee & 5 \\
\hline & University of Birmingham & 3 \\
\hline & University of East London & 17 \\
\hline & University of London & 2 \\
\hline & University of Luton & 1 \\
\hline & University of Manchester & 3 \\
\hline & Uni of Wales College, Newport & 6 \\
\hline & University of West of England & 2 \\
\hline & University of Westminster & 2 \\
\hline & Unspecified institution(s) & 6 \\
\hline & Total & 121 \\
\hline \multirow[t]{9}{*}{ USA } & California State U, Long Beach & 3 \\
\hline & Indiana University & 1 \\
\hline & Northwood University & 2 \\
\hline & Southern New Hampshire Uni & 1 \\
\hline & Troy State & 2 \\
\hline & Upper Iowa University & 1 \\
\hline & $\begin{array}{l}\text { U of the State of New York - } \\
\text { Regent College }\end{array}$ & 1 \\
\hline & Unspecified institution(s) & 12 \\
\hline & Total & 23 \\
\hline Grand Total & & 490 \\
\hline
\end{tabular}

Source: Malaysia Accreditation Board (LAN) (2006)

* Exclusive of education programmes run at the Monash campus in Malaysia. 
Appendix $G$

\section{LIST OF NON-EQUITY MODE 3 TRANSNATIONAL EDUCATION PROGRAMMES IN SINGAPORE}

\begin{tabular}{|c|c|c|}
\hline "Source Country & Education Institution & $\begin{array}{c}\text { No of Joint Programmes on } \\
\text { Offer } \\
\end{array}$ \\
\hline \multirow[t]{39}{*}{ Australia } & Australian Catholic University & 1 \\
\hline & Australian Intl Hotel School & 1 \\
\hline & Australian Maritime College & 5 \\
\hline & Australian National University & 1 \\
\hline & Central Queensland University & 12 \\
\hline & Charles Sturt University & 12 \\
\hline & Curtin University of Technology & 49 \\
\hline & Deakin University & 20 \\
\hline & Edith Cowan University & 28 \\
\hline & $\begin{array}{l}\text { Entrepreneurship Institute of } \\
\text { Australia }\end{array}$ & 15 \\
\hline & Flinders University & 9 \\
\hline & Griffith University & 4 \\
\hline & Institute of Technology of & 1 \\
\hline & Australia & \\
\hline & James Cook University & 26 \\
\hline & KvB Institute of Technology & 2 \\
\hline & La Trobe University & 14 \\
\hline & Macquarie University & 11 \\
\hline & $\begin{array}{l}\text { Mitchell College of Advanced } \\
\text { Education }\end{array}$ & 2 \\
\hline & Monash University & 35 \\
\hline & Murdoch University & 5 \\
\hline & Open Learning Institute & 4 \\
\hline & Qantam College & 2 \\
\hline & Queensland University of Tech & 7 \\
\hline & RMIT University & 25 \\
\hline & Southern Cross University & 21 \\
\hline & Swinburne University of Tech & 10 \\
\hline & Tourism Institute of Australia & 2 \\
\hline & University of Adelaide & 8 \\
\hline & University of Ballarat & 7 \\
\hline & University of Canberra & 8 \\
\hline & University of Central Queensland & 1 \\
\hline & University of Melbourne & 6 \\
\hline & University of New England & 3 \\
\hline & University of New South Wales & 32 \\
\hline & University of Queensland & 9 \\
\hline & University of South Australia & 24 \\
\hline & Uni of Southern Queensland & 12 \\
\hline & University of Sunshine Coast & 1 \\
\hline
\end{tabular}




\begin{tabular}{|c|c|c|}
\hline & University of Sydney & 6 \\
\hline & University of Tasmania & 1 \\
\hline & University of Tech, Sydney & 4 \\
\hline & University of Western Sydney & 12 \\
\hline & University of Western Australia & 10 \\
\hline & University of Wollongong & 13 \\
\hline & Victoria University of Tech & 10 \\
\hline & Total & 491 \\
\hline \multirow[t]{6}{*}{ Canada } & Malaspina University College & 1 \\
\hline & Ottawa University & 3 \\
\hline & Royal Roads University & 1 \\
\hline & Thompson Rivers University & 1 \\
\hline & University of New Brunswick & 3 \\
\hline & Total & 9 \\
\hline \multirow[t]{13}{*}{ China } & Beijing Language \& Culture Uni & 1 \\
\hline & Beijing Normal University & 1 \\
\hline & Capital Normal Uni, Beijing & 2 \\
\hline & Central China Normal University & 2 \\
\hline & $\begin{array}{l}\text { East China Normal Uni, } \\
\text { Shanghai }\end{array}$ & 3 \\
\hline & Fudan University & 4 \\
\hline & Jinan University & 1 \\
\hline & Nanjing Normal University & 1 \\
\hline & Nanjing University & 3 \\
\hline & Shanghai Jiao Tong University & 3 \\
\hline & Soochow University & 1 \\
\hline & South China Normal University & 1 \\
\hline & Total & 23 \\
\hline \multirow[t]{5}{*}{ France } & Grenoble Ecole de Management & 1 \\
\hline & INSEAD & 1 \\
\hline & Paris Graduate School of Mgmt & 1 \\
\hline & University of La Rochelle & 1 \\
\hline & Total & 4 \\
\hline Finland & Helsinki School of Economics & 1 \\
\hline Germany & Technische Universitat Munchen & 1 \\
\hline Hungary & Central European University & 1 \\
\hline India & Xavier Labour Relations Institute & 1 \\
\hline \multirow[t]{3}{*}{ Ireland } & National University of Ireland & 9 \\
\hline & University College Dublin & 6 \\
\hline & Total & 15 \\
\hline Italy & Libera Academia di Bella Arti & 6 \\
\hline \multirow[t]{3}{*}{ Macau } & Asia Intl Open University & 2 \\
\hline & Macau Uni of Science and Tech & 1 \\
\hline & Total & 3 \\
\hline Malaysia & Multimedia University & 2 \\
\hline
\end{tabular}




\begin{tabular}{|c|c|c|}
\hline \multirow[t]{5}{*}{ Netherlands } & Masstricht School of Mgmt & 1 \\
\hline & RVB Intl Institute of Mgmt & 2 \\
\hline & Technische Universiteit & \\
\hline & Eindhoven & 1 \\
\hline & Total & 4 \\
\hline \multirow[t]{6}{*}{ New Zealand } & Christchurch Polytechnic & 1 \\
\hline & Institute of Tech & \\
\hline & Lincoln University & 2 \\
\hline & University of Waikato & 1 \\
\hline & Unspecified institution(s) & 7 \\
\hline & Total & 11 \\
\hline \multirow[t]{3}{*}{ Philippines } & Asian Institute of Management & 1 \\
\hline & Polytechnic Uni of University & 2 \\
\hline & Total & 3 \\
\hline Pakistan & Preston University & 2 \\
\hline \multirow[t]{4}{*}{ Switzerland } & $\begin{array}{l}\text { KS Graduate Business School \& } \\
\text { the Federal Uni of Applied } \\
\text { Sciences, Berne }\end{array}$ & 3 \\
\hline & Marketing Development Institute & 1 \\
\hline & European University & 10 \\
\hline & Total & 14 \\
\hline Taiwan & Taiwan National Chin Nan Uni & 1 \\
\hline \multirow[t]{25}{*}{ UK } & Brunel University & 4 \\
\hline & $\begin{array}{l}\text { Buckinghamshire Chilterns Uni } \\
\text { College }\end{array}$ & 4 \\
\hline & Coventry University & 8 \\
\hline & Cranfield School of Management & 1 \\
\hline & DeMonfort University & 3 \\
\hline & Glasgow Caledonian University & 2 \\
\hline & Henley Management College & 3 \\
\hline & Heriot-Watt University & 16 \\
\hline & Imperial College, London & 1 \\
\hline & Lancaster University & 1 \\
\hline & Leeds Metropolitan University & 3 \\
\hline & Liverpool John-Moores Uni & 6 \\
\hline & Loughborough University & 3 \\
\hline & Manchester University & 5 \\
\hline & Middlesex University & 3 \\
\hline & North East London Polytechnic & 1 \\
\hline & Northumbria University & 23 \\
\hline & Nottingham Trent University & 5 \\
\hline & Oxford Brookes University & 2 \\
\hline & Queen Margaret U College & 6 \\
\hline & Roehampton University & 1 \\
\hline & Royal Holloway, U of London & 1 \\
\hline & Sheffield City Polytechnic & 1 \\
\hline & Sheffield Hallam University & 5 \\
\hline & South Bank University & 2 \\
\hline
\end{tabular}




\begin{tabular}{|c|c|c|}
\hline & Thames Valley University & 4 \\
\hline & University of Bath & 2 \\
\hline & University of Birmingham & 2 \\
\hline & University of Bradford & 9 \\
\hline & University College Chester & 1 \\
\hline & University of Durham & 1 \\
\hline & University of East London & 24 \\
\hline & University of Glamorgan & 6 \\
\hline & University of Greenwich & 3 \\
\hline & University of Huddersfield & 19 \\
\hline & University of Hull & 6 \\
\hline & University of Keele & 1 \\
\hline & University of Leads & 4 \\
\hline & University of Leicester & 12 \\
\hline & University of Lincoln & 1 \\
\hline & University of Luton & 2 \\
\hline & $\begin{array}{l}\text { Uni of Manchester Institute of } \\
\text { Science and Tech }\end{array}$ & 2 \\
\hline & University of Nottingham & 2 \\
\hline & University of Portsmouth & 2 \\
\hline & University of Salford & 1 \\
\hline & University of Sheffield & 2 \\
\hline & University of Stirling & 10 \\
\hline & University of Sunderland & 18 \\
\hline & University of Surrey & 6 \\
\hline & University of Wales & 17 \\
\hline & University of Westminster & 2 \\
\hline & University of Warwick & 1 \\
\hline & University of Wolverhampton & 14 \\
\hline & University of York & 1 \\
\hline & Wolsey Hall & 3 \\
\hline & Total & 288 \\
\hline \multirow[t]{21}{*}{ USA } & Adams State College & 3 \\
\hline & Andrew Jackson University & 2 \\
\hline & Aspen University & 1 \\
\hline & Aurora University & 1 \\
\hline & $\begin{array}{l}\text { Baruch College, City Uni of New } \\
\text { York }\end{array}$ & 3 \\
\hline & Benedictine College & 1 \\
\hline & Cal State University, Fresno & 1 \\
\hline & Cal State University, Long Beach & 1 \\
\hline & Columbia Southern University & 3 \\
\hline & Columbus State University & 1 \\
\hline & Cornerstone University & 2 \\
\hline & George Washington University & 2 \\
\hline & Georgian Institute of Tech & 1 \\
\hline & Golden Gate University & 4 \\
\hline & Maharishi University of Mgmt & 2 \\
\hline & MIT & 1 \\
\hline & National American University & 6 \\
\hline & North West Missouri State Uni & 1 \\
\hline & Nova Southeastern University & 1 \\
\hline & Oklahoma City University & 5 \\
\hline & $\begin{array}{l}\text { Pennsylvania College of } \\
\text { Optometry }\end{array}$ & 2 \\
\hline
\end{tabular}




\begin{tabular}{|c|c|c|}
\hline & Revans University & 3 \\
\hline & Rutgers University & 1 \\
\hline & Salem International University & 4 \\
\hline & San Diego State University & 3 \\
\hline & Southeastern University & 1 \\
\hline & Southern Illinois University & 1 \\
\hline & Southern New Hampshire Uni & 1 \\
\hline & $\begin{array}{l}\text { State University of New York, } \\
\text { Buffalo }\end{array}$ & 6 \\
\hline & $\begin{array}{l}\text { State University of New York, } \\
\text { Empire State College }\end{array}$ & 1 \\
\hline & Syracuse University & 2 \\
\hline & University of Central Michigan & 1 \\
\hline & University of Chicago & 1 \\
\hline & University of Hawaii & 1 \\
\hline & University of Louisville & 1 \\
\hline & University of Massachusetts & 1 \\
\hline & University of Northern Virginia & 2 \\
\hline & University of Pennsylvania & 1 \\
\hline & University of San Francisco & 1 \\
\hline & University of Wisconsin - Stout & 1 \\
\hline & Upper Iowa University & 2 \\
\hline & Utah State University & 1 \\
\hline & Western Michigan University & 2 \\
\hline & Wheelock College & 2 \\
\hline & Wilmington College & 2 \\
\hline & Total & 86 \\
\hline Grand Total & & 966 \\
\hline
\end{tabular}

Source: Singapore Ministry of Education (2006) 


\section{Appendix H}

\section{INFORMAL CORRESPONDENCE WITH UNIVERSITY SENIOR REPRESENTATIVES}

As part of my professional experience, I informally corresponded with 40 senior university representatives from 28 universities of the Association of Pacific Rim Universities (APRU) (see www.apru.org) in March 2005 when APRU held one of its senior staff meetings at the University of Auckland in New Zealand. At the time I was working in the Office of the Pro Vice-Chancellor (International) at the University of Auckland in the capacity of International Relations Officer and was directly involved in the day to day running of the senior staff meeting. This involvement allowed me to informally talk to a number of Pro Vice-Chancellors/Vice-Presidents (International) and/or Directors of International Offices who were present at the meeting. The table below is a geographical representation of the universities who had representatives at the senior staff meeting with whom I have had informal discussion about the proposed $\mathrm{PhD}$ topic.

Table 1: Geographical Representation of Respondent Universities ( $N=28)$

\begin{tabular}{lcc}
\hline Location & Number of Universities & Percentage \\
\hline USA & 8 & 29 \\
Japan & 5 & 18 \\
China & 3 & 11 \\
Australia & 2 & 7 \\
Canada & 1 & 3.5 \\
New Zealand & 1 & 3.5 \\
Russia & 1 & 3.5 \\
Chile & 1 & 3.5 \\
H Kong & 1 & 3.5 \\
Taiwan & 1 & 3.5 \\
South Korea & 1 & 3.5 \\
Thailand & 1 & 3.5 \\
Singapore & 1 & 3.5 \\
Philippines & 1 & 3.5 \\
\hline Total & $\mathbf{2 8}$ & $\mathbf{1 0 0}$ \\
\hline
\end{tabular}




\section{Appendix 1}

\section{OPERATIONALISATION OF RESOURCES IN THE LITERATURE}

Independent Variable

Resource

Human Capital

Knowledge

Experience

Social Capital

Innovation

Reputation

Service climate

Economies of scale

Financial

Culture

Physical

Entrepreneurial

Customer-related

Organisational

Racial diversity

Top management team

Property-based

Business

Environmental performance

Intangible

Managerial

Price

Tangible

Work-family policy

Technological

Tenure

Capability

Human resource

Innovative

Information technology

Technological

Entrepreneurial

Learning

Cost reduction

Product development

Quality

Client retention

Customer relationship building
\# of Articles

\% Total Articles ${ }^{\mathrm{a}}$ 
Appendix I (continued)

\begin{tabular}{lcc}
\hline Independent Variable & \# of Articles & \% Total Articles $^{\mathrm{a}}$ \\
\hline Information acquisition & 1 & $2 \%$ \\
Knowledge & 1 & $2 \%$ \\
Market orientation & 1 & $2 \%$ \\
Negotiation & 1 & $2 \%$ \\
Specialisation & 1 & $2 \%$ \\
Supplier relationship building & 1 & $2 \%$ \\
Title-taking & 1 & $2 \%$ \\
Communication & 1 & $2 \%$ \\
Distribution & 1 & $2 \%$ \\
Research and development & 1 & $2 \%$ \\
Ancillary & 1 & $2 \%$ \\
Change & 1 & $2 \%$ \\
Leveraging & 1 & $2 \%$ \\
Merger and acquisition & 1 & $2 \%$ \\
Medical & 1 & $2 \%$ \\
Pricing & 1 & $2 \%$ \\
Core competence & & $2 \%$ \\
Marketing & 1 & $2 \%$ \\
Technological & 1 & \\
Architectural & 1 & $2 \%$ \\
Regulatory & 1 & $2 \%$ \\
Component & 1 & \\
Integrative & 1 & \\
\hline
\end{tabular}

Source: Newbert (2007)

a The total number of articles is 55 


\section{Appendix J}

\section{SAMPLING FRAME}

\begin{tabular}{|c|c|c|}
\hline Source Country & Education Institution & Mode of Entry \\
\hline A Australia & Charles Sturt University & "Wholly Owned \\
\hline Australia & Central Queensland University & Wholly Owned \\
\hline Australia & Monash University & Wholly Owned \\
\hline Ireland & Dublin Business School & Wholly Owned \\
\hline USA & Webster University & Wholly Owned \\
\hline USA & Boston University & Wholly Owned \\
\hline USA & Fairleigh Dickinson University & Wholly Owned \\
\hline USA & University of Northern Virginia & Wholly Owned \\
\hline USA & Georgia Institute of Technology & Wholly Owned \\
\hline USA & University of Indianapolis & Wholly Owned \\
\hline USA & University of New Orleans & Wholly Owned \\
\hline USA & Alliant International University & Wholly Owned \\
\hline USA & Chicago Business School & Wholly Owned \\
\hline USA & University of Nevada Las Vegas & Wholly Owned \\
\hline USA & Endicott College & Wholly Owned \\
\hline \multicolumn{3}{|c|}{ Total Number of wholly owned operations $=15$} \\
\hline Australia & Curtin University of Technology & Joint Venture \\
\hline Australia & $\begin{array}{l}\text { Swinburne University of } \\
\text { Technology }\end{array}$ & Joint Venture \\
\hline Australia & James Cook University & Joint Venture \\
\hline Australia & University of New South Wales ${ }^{41}$ & Joint Venture \\
\hline Australia & University of Wollongong & Joint Venture \\
\hline Australia & RMIT University & Joint Venture \\
\hline Canada & University of New Brunswick & Joint Venture \\
\hline UK & University of Nottingham & Joint Venture \\
\hline UK & Heriot Watt University & Joint Venture \\
\hline UK & Middlesex University & Joint Venture \\
\hline USA & Carnegie Mellon University & Joint Venture \\
\hline USA & Florida International University & Joint Venture \\
\hline USA & Kean University & Joint Venture \\
\hline USA & Missouri State University & Joint Venture \\
\hline USA & McDaniel College & Joint Venture \\
\hline USA & Temple University & Joint Venture \\
\hline USA & Columbia University & Joint Venture \\
\hline USA & DePaul University & Joint Venture \\
\hline USA & Florida State University & Joint Venture \\
\hline USA & Clark University & Joint Venture \\
\hline USA & Cornell University & Joint Venture \\
\hline USA & Georgetown University & Joint Venture \\
\hline USA & Texas A\&M University & Joint Venture \\
\hline USA & Virginia Commonwealth & Joint Venture \\
\hline
\end{tabular}

${ }^{41}$ Although UNSW has closed its joint venture operation in Singapore in June 2007, it is still examined as part of this study since we are interested with mode of entry decision making process rather than performance - see section 1.4 in Chapter 1. 


\begin{tabular}{|c|c|c|}
\hline & University & \\
\hline USA & George Mason University & Joint Venture \\
\hline USA & Harvard Medical International & Joint Venture \\
\hline USA & $\begin{array}{l}\text { American Intercontinental } \\
\text { University }\end{array}$ & Joint Venture \\
\hline \multicolumn{3}{|c|}{ Total Joint venture operations $=27$} \\
\hline Australia & Australian Catholic University & Non-equity arrangement \\
\hline Australia & Australian National University & Non-equity arrangement \\
\hline Australia & Bond University & Non-equity arrangement \\
\hline Australia & Deakin University & Non-equity arrangement \\
\hline Australia & Edith Cowan University & Non-equity arrangement \\
\hline Australia & Flinders University & Non-equity arrangement \\
\hline Australia & Griffith University & Non-equity arrangement \\
\hline Australia & La Trobe University & Non-equity arrangement \\
\hline Australia & Macquarie University & Non-equity arrangement \\
\hline Australia & Murdoch University & Non-equity arrangement \\
\hline Australia & Northern Territory University & Non-equity arrangement \\
\hline Australia & Queensland Uni of Tech & Non-equity arrangement \\
\hline Australia & Southern Cross University & Non-equity arrangement \\
\hline Australia & University of Adelaide & Non-equity arrangement \\
\hline Australia & University of Ballarat & Non-equity arrangement \\
\hline Australia & University of Canberra & Non-equity arrangement \\
\hline Australia & University of Queensland & Non-equity arrangement \\
\hline Australia & University of Melbourne & Non-equity arrangement \\
\hline Australia & University of Newcastle & Non-equity arrangement \\
\hline Australia & University of New England & Non-equity arrangement \\
\hline Australia & University of South Australia & Non-equity arrangement \\
\hline Australia & $\begin{array}{l}\text { University of Southern } \\
\text { Queensland }\end{array}$ & Non-equity arrangement \\
\hline Australia & University of the Sunshine Coast & Non-equity arrangement \\
\hline Australia & University of Sydney & Non-equity arrangement \\
\hline Australia & University of Tech Sydney & Non-equity arrangement \\
\hline Australia & University of Tasmania & Non-equity arrangement \\
\hline Australia & University of Western Sydney & Non-equity arrangement \\
\hline Australia & University of Western Australia & Non-equity arrangement \\
\hline Australia & $\begin{array}{l}\text { Victoria University of } \\
\text { Technology }\end{array}$ & Non-equity arrangement \\
\hline Canada & Brock University & Non-equity arrangement \\
\hline Canada & Carleton University & Non-equity arrangement \\
\hline Canada & Dalhousie University & Non-equity arrangement \\
\hline Canada & Malaspina University College & Non-equity arrangement \\
\hline Canada & McMaster University & Non-equity arrangement \\
\hline Canada & $\begin{array}{l}\text { Newfoundland Memorial } \\
\text { University }\end{array}$ & Non-equity arrangement \\
\hline Canada & Royal Roads University & Non-equity arrangement \\
\hline Canada & Simon Fraser University & Non-equity arrangement \\
\hline Canada & University of Alberta & Non-equity arrangement \\
\hline Canada & University of British Columbia & Non-equity arrangement \\
\hline Canada & University Quebec, Chicoutimi & Non-equity arrangement \\
\hline Canada & University of Ottawa & Non-equity arrangement \\
\hline Canada & University of Waterloo & Non-equity arrangement \\
\hline Canada & University of Western Ontario & Non-equity arrangement \\
\hline Canada & University college of the Cariboo & Non-equity arrangement \\
\hline Canada & University Lethbridge & Non-equity arrangement \\
\hline Canada & Thompson Rivers University & Non-equity arrangement \\
\hline
\end{tabular}




\begin{tabular}{|c|c|c|}
\hline Ireland & Dublin Institute of Technology & Non-equity arrangement \\
\hline Ireland & University College Dublin & Non-equity arrangement \\
\hline Ireland & University of Limerick & Non-equity arrangement \\
\hline Ireland & National University of Ireland & Non-equity arrangement \\
\hline New Zealand & $\begin{array}{l}\text { Auckland University of } \\
\text { Technology }\end{array}$ & Non-equity arrangement \\
\hline New Zealand & Massey university & Non-equity arrangement \\
\hline New Zealand & University of Auckland & Non-equity arrangement \\
\hline New Zealand & University of Waikato & Non-equity arrangement \\
\hline New Zealand & $\begin{array}{l}\text { Victoria University of } \\
\text { Wellington }\end{array}$ & Non-equity arrangement \\
\hline New Zealand & Waikato Institute of Technology & Non-equity arrangement \\
\hline New Zealand & Western Institute of Technology & Non-equity arrangement \\
\hline New Zealand & Lincoln University & Non-equity arrangement \\
\hline UK & Anglia Ruskin University & Non-equity arrangement \\
\hline UK & Binghampton University & Non-equity arrangement \\
\hline UK & Brunel University & Non-equity arrangement \\
\hline UK & $\begin{array}{l}\text { Buckinghamshire Chilterns Uni } \\
\text { College }\end{array}$ & Non-equity arrangement \\
\hline UK & Coventry University & Non-equity arrangement \\
\hline UK & Cranfield School of Management & Non-equity arrangement \\
\hline UK & De Montfort University & Non-equity arrangement \\
\hline UK & Glasgow Caledonia University & Non-equity arrangement \\
\hline UK & Henley Management College & Non-equity arrangement \\
\hline UK & Kingston University & Non-equity arrangement \\
\hline UK & Imperial College, London & Non-equity arrangement \\
\hline UK & Lancaster University & Non-equity arrangement \\
\hline UK & Leeds Metropolitan University & Non-equity arrangement \\
\hline UK & $\begin{array}{l}\text { Liverpool John Moores } \\
\text { University }\end{array}$ & Non-equity arrangement \\
\hline UK & Loughborough University & Non-equity arrangement \\
\hline UK & London Metropolitan University & Non-equity arrangement \\
\hline UK & $\begin{array}{l}\text { Manchester Metropolitan } \\
\text { University }\end{array}$ & Non-equity arrangement \\
\hline UK & Napier University & Non-equity arrangement \\
\hline UK & Northumbria University & Non-equity arrangement \\
\hline UK & Nottingham Trent University & Non-equity arrangement \\
\hline UK & Oxford Brookes University & Non-equity arrangement \\
\hline UK & Queen Margaret Uni College & Non-equity arrangement \\
\hline UK & Queen Mary Uni of London & Non-equity arrangement \\
\hline UK & Roehampton University & Non-equity arrangement \\
\hline UK & Royal Holloway, Uni of London & Non-equity arrangement \\
\hline UK & Sheffield Hallam University & Non-equity arrangement \\
\hline UK & South Bank University & Non-equity arrangement \\
\hline UK & Teeside University & Non-equity arrangement \\
\hline UK & Thames Valley University & Non-equity arrangement \\
\hline UK & Trinity College, London & Non-equity arrangement \\
\hline UK & University of Abertay, Dundee & Non-equity arrangement \\
\hline UK & University of Bath & Non-equity arrangement \\
\hline UK & University of Birmingham & Non-equity arrangement \\
\hline UK & University of Bolton & Non-equity arrangement \\
\hline UK & University of Bradford & Non-equity arrangement \\
\hline UK & University of Bristol & Non-equity arrangement \\
\hline UK & University of Chester & Non-equity arrangement \\
\hline UK & University of Central England & Non-equity arrangement \\
\hline UK & University of Central Lancashire & Non-equity arrangement \\
\hline
\end{tabular}




\begin{tabular}{|c|c|c|}
\hline UK & University of Durham & Non-equity arrangement \\
\hline UK & University of East Anglia & Non-equity arrangement \\
\hline UK & University of East London & Non-equity arrangement \\
\hline UK & University of Exeter & Non-equity arrangement \\
\hline UK & University of Glamorgan & Non-equity arrangement \\
\hline UK & University of Greenwich & Non-equity arrangement \\
\hline UK & University of Huddersfield & Non-equity arrangement \\
\hline UK & University of Hull & Non-equity arrangement \\
\hline UK & University of Keele & Non-equity arrangement \\
\hline UK & University of Leeds & Non-equity arrangement \\
\hline UK & University of Leicester & Non-equity arrangement \\
\hline UK & University of Luton & Non-equity arrangement \\
\hline UK & University of Lincoln & Non-equity arrangement \\
\hline UK & University of Manchester & Non-equity arrangement \\
\hline UK & University of Northumbria & Non-equity arrangement \\
\hline UK & University of Portsmouth & Non-equity arrangement \\
\hline UK & University of Reading & Non-equity arrangement \\
\hline UK & University of Salford & Non-equity arrangement \\
\hline UK & University of Sheffield & Non-equity arrangement \\
\hline UK & University of Stirling & Non-equity arrangement \\
\hline UK & University of Sunderland & Non-equity arrangement \\
\hline UK & University of Staffordshire & Non-equity arrangement \\
\hline UK & University of Surrey & Non-equity arrangement \\
\hline UK & University of Ulster & Non-equity arrangement \\
\hline UK & University of Wales, Newport & Non-equity arrangement \\
\hline UK & University of West England & Non-equity arrangement \\
\hline UK & University of Warwick & Non-equity arrangement \\
\hline UK & University of Westminster & Non-equity arrangement \\
\hline UK & University of Wolverhampton & Non-equity arrangement \\
\hline UK & University of York & Non-equity arrangement \\
\hline USA & Adams State College & Non-equity arrangement \\
\hline USA & Andrew Jackson University & Non-equity arrangement \\
\hline USA & Aspen University & Non-equity arrangement \\
\hline USA & Auburn University & Non-equity arrangement \\
\hline USA & Aurora University & Non-equity arrangement \\
\hline USA & $\begin{array}{l}\text { Baruch College, City U of New } \\
\text { York }\end{array}$ & Non-equity arrangement \\
\hline USA & Benedictine College & Non-equity arrangement \\
\hline USA & Bulacan State University & Non-equity arrangement \\
\hline USA & Clark University & Non-equity arrangement \\
\hline USA & Cal State U, Long Beach & Non-equity arrangement \\
\hline USA & Cal State U, East Bay & Non-equity arrangement \\
\hline USA & Cal State U, Fullerton & Non-equity arrangement \\
\hline USA & Cal State U, Fresno & Non-equity arrangement \\
\hline USA & Clemson University & Non-equity arrangement \\
\hline USA & Coastal Carolina University & Non-equity arrangement \\
\hline USA & Columbia Southern University & Non-equity arrangement \\
\hline USA & Columbus State University & Non-equity arrangement \\
\hline USA & Cornerstone University & Non-equity arrangement \\
\hline USA & Eastern Michigan University & Non-equity arrangement \\
\hline USA & Fordham University & Non-equity arrangement \\
\hline USA & Fort Hays State University & Non-equity arrangement \\
\hline USA & George Washington University & Non-equity arrangement \\
\hline USA & Golden Gate University & Non-equity arrangement \\
\hline USA & Grand Valley State University & Non-equity arrangement \\
\hline USA & Indiana University, Bloomington & Non-equity arrangement \\
\hline
\end{tabular}




\begin{tabular}{|c|c|c|}
\hline USA & Indiana Wesleyan University & Non-equity arrangement \\
\hline USA & Johnson and Wales University & Non-equity arrangement \\
\hline USA & Louisiana University, Munroe & Non-equity arrangement \\
\hline USA & Loyolla College & Non-equity arrangement \\
\hline USA & Maharishi Uni of Management & Non-equity arrangement \\
\hline USA & Michigan State University & Non-equity arrangement \\
\hline USA & New York Institute of Tech & Non-equity arrangement \\
\hline USA & Massachusetts Institute of Tech & Non-equity arrangement \\
\hline USA & National American University & Non-equity arrangement \\
\hline USA & Northern Illinois University & Non-equity arrangement \\
\hline USA & $\begin{array}{l}\text { Northwest Missouri State } \\
\text { University }\end{array}$ & Non-equity arrangement \\
\hline USA & Northwood University & Non-equity arrangement \\
\hline USA & North Dakota State University & Non-equity arrangement \\
\hline USA & Northwestern University & Non-equity arrangement \\
\hline USA & Nova Southeastern University & Non-equity arrangement \\
\hline USA & Oklahoma City University & Non-equity arrangement \\
\hline USA & Oklahoma State University & Non-equity arrangement \\
\hline USA & Ohio State University & Non-equity arrangement \\
\hline USA & Olivet Nazarene University & Non-equity arrangement \\
\hline USA & Pace University & Non-equity arrangement \\
\hline USA & Pittsburgh State University & Non-equity arrangement \\
\hline USA & Rutgers University & Non-equity arrangement \\
\hline USA & Revans University & Non-equity arrangement \\
\hline USA & Salem International University & Non-equity arrangement \\
\hline USA & San Diego State University & Non-equity arrangement \\
\hline USA & Saint Joseph University & Non-equity arrangement \\
\hline USA & Shenandoah University & Non-equity arrangement \\
\hline USA & Southeastern University & Non-equity arrangement \\
\hline USA & $\begin{array}{l}\text { South-western Missouri State } \\
\text { University }\end{array}$ & Non-equity arrangement \\
\hline USA & Southern Illinois University & Non-equity arrangement \\
\hline USA & $\begin{array}{l}\text { Southern New Hampshire } \\
\text { University }\end{array}$ & Non-equity arrangement \\
\hline USA & State University of NY, Buffalo & Non-equity arrangement \\
\hline USA & $\begin{array}{l}\text { State U of NY, Empire State } \\
\text { College }\end{array}$ & Non-equity arrangement \\
\hline USA & Syracuse University & Non-equity arrangement \\
\hline USA & Tarleton State University & Non-equity arrangement \\
\hline USA & Towson University & Non-equity arrangement \\
\hline USA & Troy State University & Non-equity arrangement \\
\hline USA & University of Alabama & Non-equity arrangement \\
\hline USA & University of Central Michigan & Non-equity arrangement \\
\hline USA & University of Colorado, Denver & Non-equity arrangement \\
\hline USA & University of Dubuque & Non-equity arrangement \\
\hline USA & University of Hawaii & Non-equity arrangement \\
\hline USA & University of Iowa & Non-equity arrangement \\
\hline USA & University of Louisville & Non-equity arrangement \\
\hline USA & University of Massachusetts & Non-equity arrangement \\
\hline USA & University of Maryland & Non-equity arrangement \\
\hline USA & $\begin{array}{l}\text { University of Michigan, } \\
\text { Dearborn }\end{array}$ & Non-equity arrangement \\
\hline USA & University of Minnesota & Non-equity arrangement \\
\hline USA & University North Alabama & Non-equity arrangement \\
\hline USA & University Northern Iowa & Non-equity arrangement \\
\hline USA & University of Pennsylvania & Non-equity arrangement \\
\hline
\end{tabular}




\begin{tabular}{lll}
\hline \hline USA & University of Oklahoma & Non-equity arrangement \\
USA & University of San Francisco & Non-equity arrangement \\
USA & University of Southern California & Non-equity arrangement \\
USA & University of Texas, Arlington & Non-equity arrangement \\
USA & University of Washington & Non-equity arrangement \\
USA & University of Wisconsin, & Non-equity arrangement \\
& Platteville & \\
USA & University of Wisconsin, Stout & Non-equity arrangement \\
USA & Upper Iowa University & Non-equity arrangement \\
USA & Utah State University & Non-equity arrangement \\
USA & Weber State University & Non-equity arrangement \\
USA & Western Michigan University & Non-equity arrangement \\
USA & Wheelock college & Non-equity arrangement \\
USA & Wilmington College & Non-equity arrangement \\
Total Non-equity arrangements & $\mathbf{2 1 6}$ & \\
Total Sample Size $=\mathbf{2 5 8}$ & & \\
\hline \hline
\end{tabular}

Source: Appendices A-G

Note: As outlined in section 1.4 of Chapter 1, only universities (and 4 year degree granting colleges in the US system) constitute the focus of this study as opposed to other institutions of higher education (e.g. community colleges, polytechnics, etc). Three polytechnics/institutes of technology, in the case of New Zealand and Ireland, are however, included in the sampling frame given that they are delivering applied degree programmes on behalf of university partners. 


\section{Appendix $K^{42}$ PROPOSED INTERVIEW PROTOCOL}

Main research question 1: What types of resources are associated with whether an education service provider enters a foreign market to provide offshore education via equity or non-equity arrangements?

Main research question 2: What role does distance play in moderating the link between resources and the mode of entry in the offshore education market?

The questions listed below will direct the interview conversation. They are written broadly, and may be asked in a different order, according to the conversation with the interviewee. The outlined protocol is semi-structured, and emerging questions not listed below may be asked as judged appropriate by the interviewer. Each interview is anticipated to last about one hour and this time limit will be strictly respected.

Part 1 - Introduction questions to warm up the conversation (Limited to 5-10 minutes)

1. Is your institution currently involved in offshore education?

2. Does your institution have a policy on offshore education?

3. What do you think of the future of offshore education developments?

Part 2 - Exploring general involvement in offshore education (Limited to 10-15 minutes)

1. If your institution is currently involved in offshore education, what are the reasons for that involvement?

(If the interviewee needs cueing, the following can be given as possible answers: financial gain, diversification, internationalisation, pathway to onshore provision, etc)

2. Does your institution intend to increase its involvement in offshore education in the future?

What types of further development in offshore education do you see for the future of your institution?

42 VUW Ethics approval obtained in November 2007. VUW's Human Ethics Committee Reference Number: 2007-101 
3. Do you have any further comments about general involvement in offshore education (e.g. successes and failures)?

Part 3 - Exploring the influence of resources and distance in offshore education developments (Limited to 15-25 minutes)

1. Broadly speaking, what types of resources do you think are crucial for your institution to develop offshore education programmes?

2. Would the influence of these resources differ, according to where the offshore education developments are taking place?

3. What are some of the key impediments of your institution's offshore education development?

4. Is there a formal business development process that is undertaken when offshore education projects are proposed at your institution or is it more a case of serendipity?

5. How does your institution decide where to develop offshore education developments? (If interviewee needs cueing, the following can be provided as possible answers: a function of demand, a function of the regulatory environment)

\section{Part 4 - Ending the interview (Limited to 5-10 minutes)}

1. Do you have anything else you would like to add about:

- offshore education developments in general?

- the types of resources that are required for offshore education developments to take place at your institution?

- the influence of the environment in the host country in influencing offshore education developments?

Interviewer will thank the interviewee for his or her time and interest in participating in the study. 


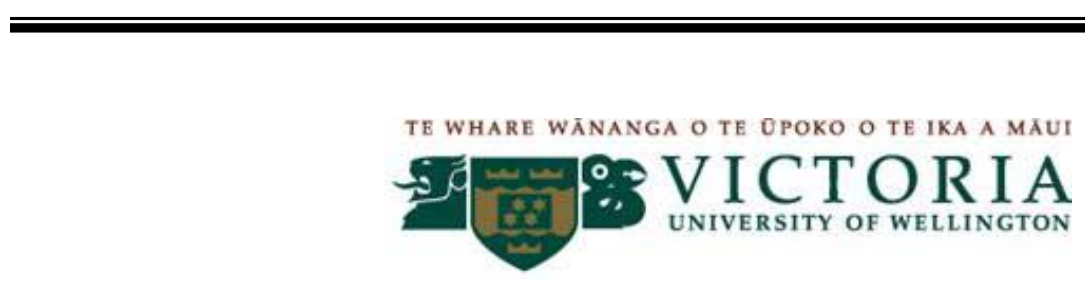

\section{Research Information Sheet \\ An Empirical Analysis of Ownership Forms in Offshore Higher Education \\ Markets: \\ A Resource-Based Perspective}

Greetings,

My name is Vik Naidoo, and I am a PhD student at Victoria University of Wellington, New Zealand. My thesis is entitled "An Empirical Analysis of Ownership Forms in Offshore Higher Education Markets: A Resource-Based Perspective”. This study aims to enhance our understanding of the types of resources that are important to universities that enter offshore education markets.

I would like to invite you to participate in this research, which will be conducted by telephone interview. The interview will take approximately one hour. All information provided in the interview will be maintained in strict confidentiality, and data from this study will only be released in aggregate form. Following the established procedures for research involving human subjects at Victoria University of Wellington, this study has been assessed and approved by the Faculty of Commerce and Administration's Human Ethics Committee. Here, I also attach the proposed interview script for your consideration. If you agree to participate in this research, please reply with the acceptance form provided, and email it to vik.naidoo@vuw.ac.nz. I will contact you and arrange an interview time at the most convenient time for you. I will be making notes during the interview (but not tape recorded) and you will have the right to review my interview notes should you choose to do so. The notes will be kept for three years and then destroyed upon completion of this study.

Thank you very much for your time and help in making this study possible. As a gesture of my appreciation for your contribution, I will happily forward a summary of our discussion, and report outlining the study's findings and conclusions in electronic form. The study is likely to be completed in December 2009. For additional information about this research, do not hesitate to contact me or my supervisor.

Vik Naidoo

PhD student, School of Marketing and International Business

Victoria University of Wellington, email: vik.naidoo@vuw.ac.nz

Dr. Elizabeth Rose

Senior Research Fellow, School of Marketing and International Business

Victoria University of Wellington, email: elizabeth.rose@vuw.ac.nz 


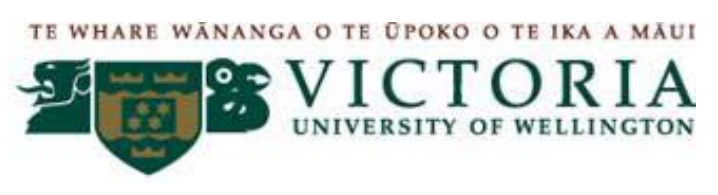

\section{Interview Consent Form \\ An Empirical Analysis of Ownership Forms in Offshore Higher Education Markets: \\ A Resource-Based Perspective}

This consent form outlines my rights as a participant in the study entitled "An Empirical Analysis of Ownership Forms in Offshore Higher Education Markets: A ResourceBased Perspective”, conducted by Vik Naidoo, PhD Student in International Business, Victoria University of Wellington, New Zealand.

The interview will explore my knowledge about how my university decides to enter international markets and the types of resources that influence that decision-making process.

I understand that (please tick as appropriate):

I agree to be interviewed for the purpose of the study.

The purpose and nature of the interview have been explained to me, and I have read the information sheet as provided by the researcher. I have understood that information, and have been given the opportunity to seek further clarification.

I agree that the interview may be electronically recorded.

Any questions that I have asked about the purpose and nature of the interview and research have been answered satisfactorily.

I have the right to decline to answer any questions asked.

I have the right to withdraw my consent to participate in this study within two weeks from when the interview is to be conducted, without penalty.

I understand that, if I withdraw from this study, any data I have provided will be destroyed.

I understand that any information or opinions I provide will be kept confidential and reported only in an aggregated/non-attributable form

I understand that the information I have provided will be used only for this research project, and that any further use will require my written consent.

I understand that, when this research is completed, the information obtained will be destroyed within three years of the completion of this study.

Please sign and return this form to Vik Naidoo (vik.naidoo@vuw.ac.nz) prior to scheduled interview. 
Name of interviewee:

Signature of interviewee:

Date:......................................

This study is aimed to be finalised in November 2009. Please indicate whether you would want a copy of the study's findings and conclusions.

$\square$ Yes

$\square$ No 


\section{Appendix L \\ COVER LETTER}

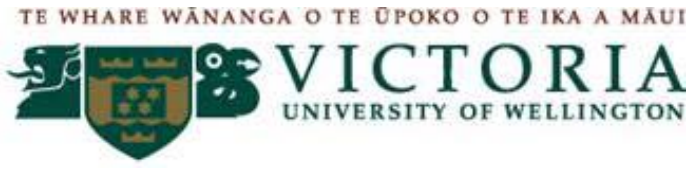

School of Marketing and International Business

PO Box 600

Victoria University of Wellington

Wellington

New Zealand

Date: ----

Dear: ----

My name is Vik Naidoo, and I am currently pursuing a $\mathrm{PhD}$ in International Business at Victoria University of Wellington, New Zealand. I am writing to ask for your assistance in helping me complete my doctoral studies by responding to the enclosed survey.

My study focuses on offshore education developments, an area of growing importance in both the education and international business contexts. For my research, I am considering offshore education to consist of any teaching-related activity in which the students are in a different country (the host country) from the home country of the institution providing the education, and requiring that national boundaries be crossed.

The enclosed survey is an important part of my doctoral study, which is expected to be of use in helping education institutions involved in offshore education to develop appropriate foreign market entry strategies. In particular, the study is aiming to identify the role of resources, with respect to their impact on entry mode choices in offshore education. As more and more educational institutions engage in foreign operations, it is important to identify the types of resources that are associated with the types of foreign market entries in the offshore education environment.

Following a search of both the academic and professional literatures, your institution has been identified as currently involved in offshore education developments. I would, therefore, be very grateful if you would assist me by taking the time to complete the questionnaire and return it in the enclosed, self-addressed, postage-paid envelope. The survey should take no more than 45 minutes to complete, and neither proprietary nor personal information has been requested in this questionnaire. All responses that you 
provide will be held in strictest anonymity, and under no circumstance will a respondent or the respondent's institution be identified as having provided a particular response. For your information, following the established procedures for research involving human subjects at Victoria University of Wellington, this study has also been assessed and approved by the Faculty of Commerce and Administration's Human Ethics Committee.

Please respond to all the survey items as objectively as you can. There is no correct or incorrect response to any of the items. Also, please feel free to make any additional explanatory or qualifying comments regarding any of the questions, if you feel that such comments will help to clarify your response. I would be most grateful to have your completed survey returned to me by 15 August, 2008. The current population for this study is limited and, therefore, your response will be particularly valuable. Last but not least, the data collected from this questionnaire will be kept for three years and then destroyed upon completion of this study. If you have any questions or concerns, please feel free to call me at $(+64)$--------- or my supervisor, Dr Elizabeth Rose, at (+64) --------; she can also be reached via email (elizabeth.rose@vuw.ac.nz).

I will be glad to send you a comprehensive report of this study. Please send me a separate email at vik.naidoo@vuw.ac.nz if you would like to receive a copy of the results of this study as soon as they are ready.

Sincerely, Vik Naidoo

PhD Student

School of Marketing and International Business 


\section{Appendix $M^{43}$}

TE WHARE WANANGa O TE OPOKO O TE IKA A MAUT

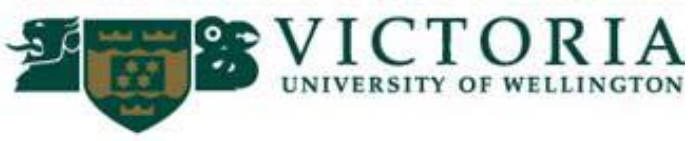

SCHOOL OF MARKETING AND INTERNATIONAL BUSINESS

\section{UNDERSTANDING FOREIGN MARKET ENTRY STRATEGIES: A SURVEY OF OFFSHORE EDUCATION PROVIDERS}

\section{INSTRUCTIONS AND DEFINITIONS}

1. Please consider two foreign market entries (i.e. offshore education developments), undertaken by your institution in the past ten years, and with which you are very familiar. Kindly respond to all of the questions in this questionnaire with these particular foreign market entries in mind, based on information that was available at the time the decision to undertake the entries was made.

2. Throughout the questionnaire, 'foreign market entry' refers to offshore education development, which denotes teaching-related activities in which the students are in a different country (the host country) from that in which the institution providing the education is based (the home country).

3. Please respond to all the questions as objectively as you can. There is no correct or incorrect response to any of the questions.

4. Feel free to make any additional explanatory or qualifying comments related to any of the questions, if you feel that such comments will help clarify your response.

5. All survey responses are completely anonymous and cannot be linked back to you or your institution. The findings of this study will only be reported in aggregate form.

THANK YOU FOR YOUR COOPERATION IN THIS RESEARCH.

If you would like a summary report of the findings of the research, please contact me at vik.naidoo@vuw.ac.nz.

\footnotetext{
${ }^{43}$ VUW Ethics approval obtained in June 2008. VUW's Human Ethics Committee Reference Number: 2007-101
} 
A. Please reflect on two recent foreign market entries (i.e. offshore education developments) by your university, with which you are very familiar, and provide information about these particular developments in the questions that follow.

Name of the host country (Please tick the most appropriate box.):

\begin{tabular}{l|l|l}
\hline \hline & Foreign Entry One & Foreign Entry Two \\
\hline China & $\square$ & $\square$ \\
\hline India & $\square$ & $\square$ \\
\hline Hong Kong & $\square$ & $\square$ \\
\hline Indonesia & $\square$ & $\square$ \\
\hline Malaysia & $\square$ & $\square$ \\
\hline Qatar & $\square$ & $\square$ \\
\hline South Africa & $\square$ & $\square$ \\
\hline UAE - Dubai & $\square$ & $\square$ \\
\hline UAE - Other (Please specify.) & & \\
\hline Vietnam & $\square$ & $\square$ \\
\hline Other (Please specify.) & & \\
\hline \hline
\end{tabular}

Year of foreign entry into the named host country (Please tick the most appropriate box.):

\begin{tabular}{l|l|l}
\hline \hline & Foreign Entry One & Foreign Entry Two \\
\hline 2008 & $\square$ & $\square$ \\
\hline 2007 & $\square$ & $\square$ \\
\hline 2006 & $\square$ & $\square$ \\
\hline 2005 & $\square$ & $\square$ \\
\hline 2004 & $\square$ & $\square$ \\
\hline 2003 & $\square$ & $\square$ \\
\hline 2002 & $\square$ & $\square$ \\
\hline 2001 & $\square$ & $\square$ \\
\hline 2000 & $\square$ & $\square$ \\
\hline 1999 & $\square$ & $\square$ \\
\hline Other (Please specify.) & & \\
\hline \hline
\end{tabular}

B. What types of academic programmes does/did your institution offer as a result of these particular market entries? (Please tick all that apply.) 
By academic discipline area:

\begin{tabular}{|c|c|c|}
\hline & Foreign Entry One & Foreign Entry Two \\
\hline Arts and Social Sciences & $\square$ & $\square$ \\
\hline Business and Management & $\square$ & $\square$ \\
\hline Engineering & $\square$ & $\square$ \\
\hline $\begin{array}{l}\text { Sciences (including Life } \\
\text { Sciences) }\end{array}$ & $\square$ & $\square$ \\
\hline Law & $\square$ & $\square$ \\
\hline $\begin{array}{l}\text { Information Technology } \\
\text { (including Computer Science) }\end{array}$ & $\square$ & $\square$ \\
\hline Education & $\square$ & $\square$ \\
\hline $\begin{array}{l}\text { Creative Arts (e.g. Fine Arts, } \\
\text { Music, Dance) }\end{array}$ & $\square$ & $\square$ \\
\hline $\begin{array}{l}\text { Architecture, Planning and } \\
\text { Design }\end{array}$ & $\square$ & $\square$ \\
\hline $\begin{array}{l}\text { Interdisciplinary (Please } \\
\text { specify.) }\end{array}$ & & \\
\hline Other (Please specify.) & & \\
\hline
\end{tabular}

By level of programme:

\begin{tabular}{l|l|l}
\hline \hline & $\begin{array}{l}\text { Foreign Entry } \\
\text { One }\end{array}$ & $\begin{array}{l}\text { Foreign Entry } \\
\text { Two }\end{array}$ \\
\hline PhD & $\square$ & $\square$ \\
\hline $\begin{array}{l}\text { Professional Doctorates (e.g. DBA, } \\
\text { EdD) }\end{array}$ & $\square$ & $\square$ \\
\hline Doctorates by coursework only & $\square$ & $\square$ \\
\hline Master's by research & $\square$ & $\square$ \\
\hline Master's by coursework only & $\square$ & $\square$ \\
\hline $\begin{array}{l}\text { Master's by both coursework and } \\
\text { research }\end{array}$ & $\square$ & $\square$ \\
\hline $\begin{array}{l}\text { Undergraduate degree (three or four } \\
\text { years in duration) }\end{array}$ & $\square$ & $\square$ \\
\hline $\begin{array}{l}\text { Diploma (two years in duration) } \\
\text { Associate Degree (two years in } \\
\text { duration) }\end{array}$ & $\square$ & $\square$ \\
\hline $\begin{array}{l}\text { Certificate (at least one year in } \\
\text { duration) }\end{array}$ & $\square$ & $\square$ \\
\hline $\begin{array}{l}\text { Executive/Professional short } \\
\text { programme (less than one year in } \\
\text { duration) }\end{array}$ & $\square$ & $\square$ \\
\hline
\end{tabular}


C. Between 0 and 100, with 0 representing no ownership and 100 representing majority ownership, what ownership did your institution have in the foreign market entries you have in mind at the time of entry?

Foreign Entry One:

\begin{tabular}{ll|ll|ll|l|l}
\hline \hline $100 \%$ & $\square$ & $95-99 \%$ & $\square$ & $90-94 \%$ & $\square$ & $85-89 \%$ & $\square$ \\
\hline $80-84 \%$ & $\square$ & $75-79 \%$ & $\square$ & $70-74 \%$ & $\square$ & $65-69 \%$ & $\square$ \\
\hline $60-64 \%$ & $\square$ & $55-59 \%$ & $\square$ & $51-54 \%$ & $\square$ & $50 \%$ & $\square$ \\
\hline $45-49 \%$ & $\square$ & $40-44 \%$ & $\square$ & $35-39 \%$ & $\square$ & $30-34 \%$ & $\square$ \\
\hline $25-29 \%$ & $\square$ & $20-24 \%$ & $\square$ & $15-19 \%$ & $\square$ & $10-14 \%$ & $\square$ \\
\hline $5-9 \%$ & $\square$ & $1-4 \%$ & $\square$ & $0 \%$ & $\square$ & & \\
\hline \hline
\end{tabular}

\section{Foreign Entry Two:}

\begin{tabular}{ll|ll|ll|l|l}
\hline \hline $100 \%$ & $\square$ & $95-99 \%$ & $\square$ & $90-94 \%$ & $\square$ & $85-89 \%$ & $\square$ \\
\hline $80-84 \%$ & $\square$ & $75-79 \%$ & $\square$ & $70-74 \%$ & $\square$ & $65-69 \%$ & $\square$ \\
\hline $60-64 \%$ & $\square$ & $55-59 \%$ & $\square$ & $51-54 \%$ & $\square$ & $50 \%$ & $\square$ \\
\hline $45-49 \%$ & $\square$ & $40-44 \%$ & $\square$ & $35-39 \%$ & $\square$ & $30-34 \%$ & $\square$ \\
\hline $25-29 \%$ & $\square$ & $20-24 \%$ & $\square$ & $15-19 \%$ & $\square$ & $10-14 \%$ & $\square$ \\
\hline $5-9 \%$ & $\square$ & $1-4 \%$ & $\square$ & $0 \%$ & $\square$ & & \\
\hline \hline
\end{tabular}

D. (i) Which of the following modes of operation best describes the foreign market entries you have in mind? (Please tick the most appropriate mode of entry.)

\begin{tabular}{l|l|l}
\hline \hline & $\begin{array}{l}\text { Foreign } \\
\text { Entry } \\
\text { One }\end{array}$ & $\begin{array}{l}\text { Foreign } \\
\text { Entry } \\
\text { Two }\end{array}$ \\
\hline $\begin{array}{l}\text { Non-equity arrangements (e.g. twinning programmes of } \\
\text { the 2+2 type, programme articulations, franchise } \\
\text { programmes) [Please skip to question D (iii).] }\end{array}$ & $\square$ & $\square$ \\
\hline $\begin{array}{l}\text { Joint venture with minority equity share (less than 50\% } \\
\text { equity share) [Continue to question D (ii).] }\end{array}$ & $\square$ & $\square$ \\
\hline $\begin{array}{l}\text { Joint venture with 50/50 equity share [Continue to } \\
\text { question D (ii).] }\end{array}$ & $\square$ & $\square$ \\
\hline $\begin{array}{l}\text { Joint venture with majority equity share (between 50-95 \% } \\
\text { equity share) [Continue to question D (ii).] }\end{array}$ & $\square$ & $\square$ \\
\hline $\begin{array}{l}\text { Sole ownership (i.e. greater than 95\% ownership of the } \\
\text { offshore education development) [Please skip to question } \\
D \text { (iii).] }\end{array}$ & $\square$ & $\square$ \\
\hline
\end{tabular}


Another mode of operation (Please specify.)

[Please skip to question D (iii).]

Foreign Entry One :

Foreign Entry Two:

(ii) Thinking from the perspective of the time at which this joint venture was established, to what extent do you agree or disagree with each of the following statements? (Please circle the number that best corresponds to your level of agreement for each statement.)

\begin{tabular}{|c|c|}
\hline & $\begin{array}{l}\text { Strongly } \\
\text { disagree }\end{array}$ \\
\hline $\begin{array}{l}\text { Collaboration was preferred by my } \\
\text { institution primarily because it was a } \\
\text { way to attain our objectives more } \\
\text { quickly. }\end{array}$ & $\begin{array}{l}\text { Foreign Entry One: } \\
1 \ldots .2 \ldots 3 \ldots . \ldots 4 \ldots 5 \ldots \ldots 6 \ldots 7 \ldots 8 \ldots 9 \\
\text { Foreign Entry Two: } \\
1 \ldots . \ldots .63 \ldots 4 \ldots \ldots 5 \ldots \ldots 6 \ldots 7 \ldots 8 \ldots .9\end{array}$ \\
\hline $\begin{array}{l}\text { Collaboration was preferred by my } \\
\text { institution primarily because it was a } \\
\text { way to attain our objectives at a lower } \\
\text { cost. }\end{array}$ & $\begin{array}{l}\text { Foreign Entry One: } \\
1 \ldots .2 \ldots 3 \ldots . \ldots 4 \ldots 5 \ldots 6 \ldots 7 \ldots 8 \ldots .9 \\
\text { Foreign Entry Two: } \\
1 \ldots .2 \ldots 3 \ldots . .4 \ldots 5 \ldots \ldots 6 \ldots 7 \ldots 8 \ldots 9\end{array}$ \\
\hline $\begin{array}{l}\text { My institution considered short term } \\
\text { profitability criteria to be critical } \\
\text { indicators for evaluating the success } \\
\text { of the offshore operation. }\end{array}$ & $\begin{array}{l}\text { Foreign Entry One: } \\
1 \ldots .2 \ldots 3 \ldots . \ldots 4 \ldots 5 \ldots . .6 \ldots 7 \ldots 8 \ldots .9 \\
\text { Foreign Entry Two: } \\
1 \ldots .2 \ldots 3 \ldots .4 \ldots . .5 \ldots 6 \ldots . \ldots 8 \ldots 9\end{array}$ \\
\hline $\begin{array}{l}\text { My institution’s decision to } \\
\text { collaborate was driven by a desire to } \\
\text { gradually and systematically develop } \\
\text { our own skills and capabilities in } \\
\text { particular directions. }\end{array}$ & 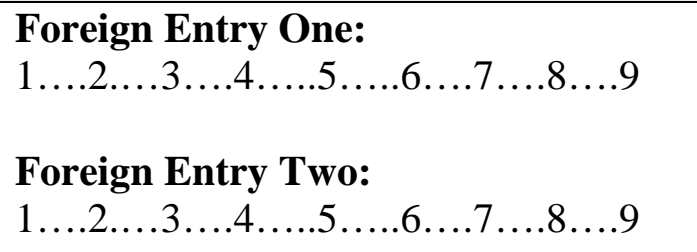 \\
\hline $\begin{array}{l}\text { My institution's decision to } \\
\text { collaborate was driven by a desire to } \\
\text { develop a deeper understanding of } \\
\text { our partner's expertise. }\end{array}$ & $\begin{array}{l}\text { Foreign Entry One: } \\
1 \ldots .2 \ldots 3 \ldots . \ldots 4 \ldots 5 \ldots .6 \ldots 7 \ldots 8 \ldots .9 \\
\text { Foreign Entry Two: } \\
1 \ldots .2 \ldots 3 \ldots .4 \ldots .5 \ldots 6 \ldots . \ldots 8 \ldots 9\end{array}$ \\
\hline $\begin{array}{l}\text { Entering this market on our own } \\
\text { would have been a waste of resources }\end{array}$ & $\begin{array}{l}\text { Foreign Entry One: } \\
1 \ldots 2 \ldots 3 \ldots .4 \ldots .5 \ldots 6 \ldots 7 \ldots . .69\end{array}$ \\
\hline
\end{tabular}


for my institution, since we would only have been duplicating skills already possessed by our partner. In addition to the specific know-how, my institution placed a high priority on understanding our partner's general perspective, management systems and procedures related to the know-how.

\section{Foreign Entry Two:}

$1 \ldots .2 \ldots 3 \ldots 4 \ldots 5 \ldots 6 \ldots 7 \ldots 8 \ldots 9$

Foreign Entry One:

$1 \ldots . . .3 \ldots 4 \ldots . .5 \ldots 6 \ldots 7 \ldots 8 \ldots 9$

Foreign Entry Two:

$1 \ldots .2 \ldots . . .4 \ldots . .5 \ldots 6 \ldots . . . .8 \ldots . .9$

\section{Strongly}

disagree
Strongly agree

(iii) Thinking from the perspective of the time at which these two international operations were established, to what extent do you agree or disagree with each of the following statements? (Please circle the number that best corresponds to your level of agreement for each statement.)

\begin{tabular}{|c|c|}
\hline & $\begin{array}{l}\text { Strongly } \\
\text { disagree }\end{array}$ \\
\hline $\begin{array}{l}\text { Host country regulations (e.g. } \\
\text { government investment laws) } \\
\text { restricted how my institution chose to } \\
\text { enter this particular foreign market. }\end{array}$ & 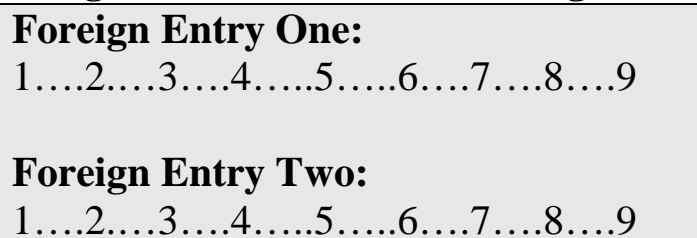 \\
\hline $\begin{array}{l}\text { Home country regulations (e.g. union } \\
\text { rules) restricted how my institution } \\
\text { chose to enter this particular foreign } \\
\text { market. }\end{array}$ & $\begin{array}{l}\text { Foreign Entry One: } \\
1 \ldots 2 \ldots . \ldots 4 \ldots \ldots 5 \ldots 6 \ldots 7 \ldots 8 \ldots .9 \\
\text { Foreign Entry Two: } \\
1 \ldots . \ldots .3 \ldots 4 \ldots \ldots 5 \ldots . .6 \ldots 7 \ldots 8 \ldots .9\end{array}$ \\
\hline $\begin{array}{l}\text { Proactive liberalisation of the } \\
\text { education sector in the host country } \\
\text { influenced my institution's entry } \\
\text { mode decision. }\end{array}$ & $\begin{array}{l}\text { Foreign Entry One: } \\
1 \ldots . \ldots .3 \ldots 4 \ldots \ldots 5 \ldots .6 \ldots 7 \ldots 8 \ldots 9 \\
\text { Foreign Entry Two: } \\
1 \ldots . \ldots .3 \ldots 4 \ldots \ldots 5 \ldots . .6 \ldots 7 \ldots 8 \ldots 9\end{array}$ \\
\hline
\end{tabular}

(iv) For these two particular offshore educational developments, are the students enrolled in programmes offered by your institution required to undertake any of their studies at one of your institution's home-country campuses?

\begin{tabular}{l|ll|ll|ll}
\hline \hline $\begin{array}{l}\text { Foreign } \\
\text { Entry One } \\
\begin{array}{l}\text { Foreign } \\
\text { Entry Two }\end{array}\end{array}$ & Yes & $\square$ & No & $\square$ & Not required, but strongly encouraged & $\square$ \\
\hline \hline
\end{tabular}


E. In which country is the main campus of your institution located? (Please tick the most appropriate response.)

\begin{tabular}{l|l}
\hline \hline Australia & $\square$ \\
\hline Canada & $\square$ \\
\hline Ireland & $\square$ \\
\hline New Zealand & $\square$ \\
\hline United Kingdom & $\square$ \\
\hline United States & $\square$ \\
\hline \hline
\end{tabular}

F. These statements pertain to your institution at the time of these two particular foreign market entries. To what extent do you agree or disagree with each of the following statements? (Please circle the number that best corresponds to your response for each statement.)

\begin{tabular}{|c|c|}
\hline & $\begin{array}{l}\text { Strongly } \\
\text { disagree }\end{array}$ \\
\hline $\begin{array}{l}\text { My institution had previously been } \\
\text { recruiting international students from this } \\
\text { foreign country, and had extensive } \\
\text { knowledge of its education sector at the } \\
\text { time of entry. }\end{array}$ & $\begin{array}{l}\text { Foreign Entry One: } \\
1 \ldots .2 \ldots 3 \ldots .4 \ldots . . \ldots 6 \ldots 7 \ldots 8 \ldots 9 \\
\text { Foreign Entry Two: } \\
1 \ldots . . .3 \ldots 4 \ldots . .5 \ldots 6 \ldots 7 \ldots 8 \ldots 9\end{array}$ \\
\hline $\begin{array}{l}\text { My institution was able to handle } \\
\text { international expansion, in terms of } \\
\text { academic capabilities. }\end{array}$ & 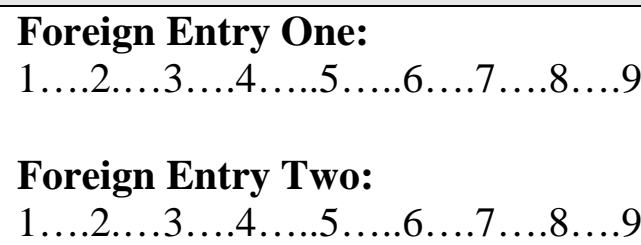 \\
\hline $\begin{array}{l}\text { My institution was able to handle } \\
\text { international expansion, in terms of } \\
\text { managerial capabilities. }\end{array}$ & $\begin{array}{l}\text { Foreign Entry One: } \\
1 \ldots .2 \ldots 3 \ldots .4 \ldots . . \ldots 6 \ldots 7 \ldots 8 \ldots 9 \\
\text { Foreign Entry Two: } \\
1 \ldots .2 \ldots 3 \ldots .4 \ldots . . .6 \ldots .7 \ldots 8 \ldots 9\end{array}$ \\
\hline $\begin{array}{l}\text { My institution was able to handle } \\
\text { international expansion, in terms of } \\
\text { financial capabilities. }\end{array}$ & 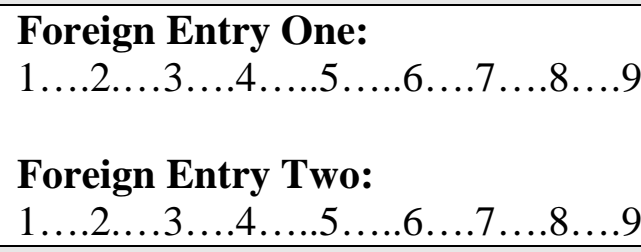 \\
\hline $\begin{array}{l}\text { The region of the world in which this } \\
\text { foreign market is located was one of my } \\
\text { institution's key markets for recruiting } \\
\text { international students. }\end{array}$ & $\begin{array}{l}\text { Foreign Entry One: } \\
1 \ldots .2 \ldots 3 \ldots .4 \ldots . \ldots 5 \ldots . \ldots 7 \ldots 8 \ldots 9 \\
\text { Foreign Entry Two: } \\
1 \ldots . \ldots 3 \ldots 4 \ldots . \ldots 5 \ldots 6 \ldots 7 \ldots 8 \ldots 9\end{array}$ \\
\hline
\end{tabular}


G. The following items pertain to your institution's international experience at the time of these two specific foreign market entries. (Please tick the most appropriate box for each item.)

\begin{tabular}{|c|c|c|c|c|c|c|c|c|c|c|c|c|c|}
\hline & \multicolumn{13}{|c|}{ Number of Years } \\
\hline & & 0 & 1 & 2 & 3 & 4 & 5 & 6 & 7 & 8 & 9 & 10 & $>10$ \\
\hline \multirow{2}{*}{$\begin{array}{l}\text { For approximately } \\
\text { how many years } \\
\text { had your institution } \\
\text { been engaged in } \\
\text { offering offshore } \\
\text { education } \\
\text { programmes at the } \\
\text { undergraduate } \\
\text { level? (If none, } \\
\text { please answer ' } 0 \text { '.) }\end{array}$} & $\begin{array}{l}\text { Foreign } \\
\text { Entry } \\
\text { One }\end{array}$ & $\square$ & $\square$ & $\square$ & $\square$ & $\square$ & $\square$ & $\square$ & $\square$ & $\square$ & $\square$ & $\square$ & $\square$ \\
\hline & $\begin{array}{l}\text { Foreign } \\
\text { Entry } \\
\text { Two }\end{array}$ & $\square$ & $\square$ & $\square$ & $\square$ & $\square$ & $\square$ & $\square$ & $\square$ & $\square$ & $\square$ & $\square$ & $\square$ \\
\hline \multirow{2}{*}{$\begin{array}{l}\text { For approximately } \\
\text { how many years } \\
\text { had your institution } \\
\text { been engaged in } \\
\text { offering offshore } \\
\text { education } \\
\text { programmes at the } \\
\text { graduate or } \\
\text { postgraduate } \\
\text { level? (If none, } \\
\text { please answer '0’.) }\end{array}$} & $\begin{array}{l}\text { Foreign } \\
\text { Entry } \\
\text { One }\end{array}$ & $\square$ & $\square$ & $\square$ & $\square$ & $\square$ & $\square$ & $\square$ & $\square$ & $\square$ & $\square$ & $\square$ & $\square$ \\
\hline & $\begin{array}{l}\text { Foreign } \\
\text { Entry } \\
\text { Two }\end{array}$ & $\square$ & $\square$ & $\square$ & $\square$ & $\square$ & $\square$ & $\square$ & $\square$ & $\square$ & $\square$ & $\square$ & $\square$ \\
\hline \multirow{2}{*}{$\begin{array}{l}\text { For approximately } \\
\text { how many years } \\
\text { had your institution } \\
\text { been actively } \\
\text { recruiting } \\
\text { undergraduate } \\
\text { international } \\
\text { students to study at } \\
\text { the main campus? } \\
\text { (If none, please } \\
\text { answer '0'.) }\end{array}$} & $\begin{array}{l}\text { Foreign } \\
\text { Entry } \\
\text { One }\end{array}$ & $\square$ & $\square$ & $\square$ & $\square$ & $\square$ & $\square$ & $\square$ & $\square$ & $\square$ & $\square$ & $\square$ & $\square$ \\
\hline & $\begin{array}{l}\text { Foreign } \\
\text { Entry } \\
\text { Two }\end{array}$ & $\square$ & $\square$ & $\square$ & $\square$ & $\square$ & $\square$ & $\square$ & $\square$ & $\square$ & $\square$ & $\square$ & $\square$ \\
\hline \multirow{2}{*}{$\begin{array}{l}\text { For approximately } \\
\text { how many years } \\
\text { had your institution } \\
\text { been actively } \\
\text { recruiting } \\
\text { graduate or } \\
\text { postgraduate } \\
\text { international } \\
\text { students to study at }\end{array}$} & $\begin{array}{l}\text { Foreign } \\
\text { Entry } \\
\text { One }\end{array}$ & $\square$ & $\square$ & $\square$ & $\square$ & $\square$ & $\square$ & $\square$ & $\square$ & $\square$ & $\square$ & $\square$ & $\square$ \\
\hline & $\begin{array}{l}\text { Foreign } \\
\text { Entry } \\
\text { Two }\end{array}$ & $\square$ & $\square$ & $\square$ & $\square$ & $\square$ & $\square$ & $\square$ & $\square$ & $\square$ & $\square$ & $\square$ & $\square$ \\
\hline
\end{tabular}




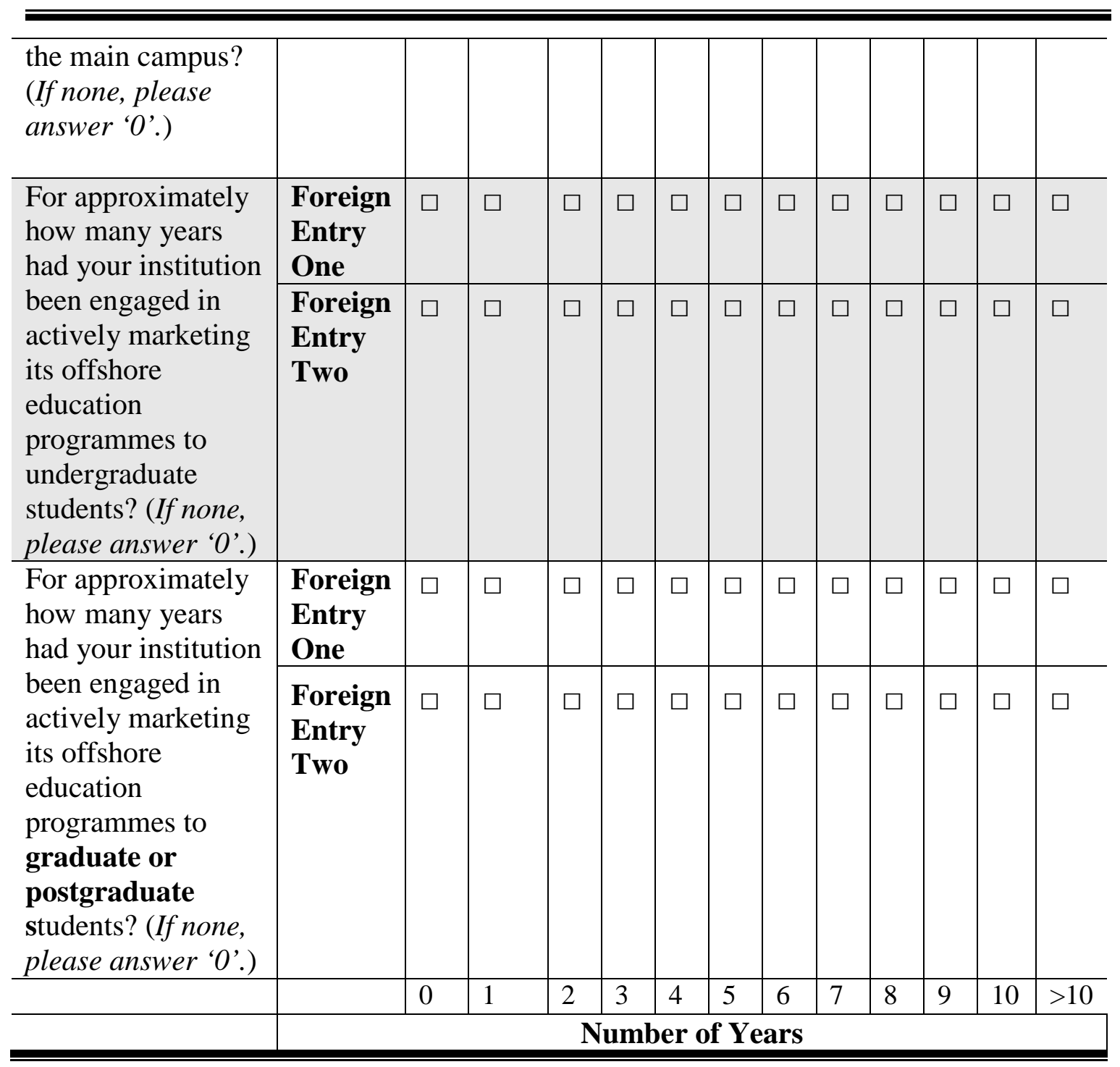

H. (i) Was either of these two particular foreign market entries your institution's first offshore education development

\begin{tabular}{l|ll|ll}
\hline \hline Foreign Entry One & $\square$ & Yes & $\square$ & No \\
\hline Foreign Entry Two & $\square$ & Yes & $\square$ & No \\
\hline \hline
\end{tabular}

If you have answered YES to any one of the two entries above, please skip to question I for that respective entry. Otherwise, continue to question $H$ (ii) for the respective foreign entry for which you have answered NO above.

(ii) At the time of these particular foreign market entries, had your institution previously used this entry mode for another international education development? 


\begin{tabular}{l|ll|ll}
\hline \hline Foreign Entry One & $\square$ & Yes & $\square$ & No \\
\hline Foreign Entry Two & $\square$ & Yes & $\square$ & No \\
\hline \hline
\end{tabular}

I. For these particular foreign market entries, please indicate the extent to which you agree or disagree with each of the following statements, with regard to your institution at the time of the offshore development. (Please circle the number that best corresponds to your response for each statement.)

\begin{tabular}{|c|c|}
\hline & $\begin{array}{lr}\text { Strongly } & \text { Strongly } \\
\text { disagree } & \text { agree }\end{array}$ \\
\hline $\begin{array}{l}\text { We found it difficult to write a useful } \\
\text { manual that perfectly captures the teaching } \\
\text { methods used at our home institution. }\end{array}$ & $\begin{array}{l}\text { Foreign Entry One: } \\
1 \ldots .2 \ldots 3 \ldots . \ldots 4 \ldots 5 \ldots . \ldots \ldots 7 \ldots 8 \ldots .9 \\
\text { Foreign Entry Two: } \\
1 \ldots .2 \ldots 3 \ldots .4 \ldots . .5 \ldots 6 \ldots 7 \ldots 8 \ldots .9\end{array}$ \\
\hline $\begin{array}{l}\text { We anticipated that it would be difficult to } \\
\text { apply the teaching methods used at our main } \\
\text { campus in our offshore development, due to } \\
\text { local sensitivities. }\end{array}$ & $\begin{array}{l}\text { Foreign Entry One: } \\
1 \ldots .2 \ldots 3 \ldots .4 \ldots . \ldots 5 \ldots 6 \ldots 7 \ldots 8 \ldots .9 \\
\text { Foreign Entry Two: } \\
1 \ldots . . \ldots 3 \ldots . \ldots 4 \ldots 5 \ldots 6 \ldots 7 \ldots 8 \ldots .9\end{array}$ \\
\hline $\begin{array}{l}\text { We found it difficult to document how } \\
\text { academics (i.e. faculty) at our main campus } \\
\text { deliver their lectures, in terms of their } \\
\text { interactions with students. }\end{array}$ & $\begin{array}{l}\text { Foreign Entry One: } \\
1 \ldots . \ldots . \ldots .4 \ldots .5 \ldots 6 \ldots 7 \ldots 8 \ldots .9 \\
\text { Foreign Entry Two: } \\
1 \ldots .2 \ldots 3 \ldots .4 \ldots .5 \ldots .6 \ldots 7 \ldots 8 \ldots 9 \\
\end{array}$ \\
\hline $\begin{array}{l}\text { We anticipated that it would be difficult for } \\
\text { academics (i.e. faculty) hired specifically to } \\
\text { teach on the offshore programmes, and based } \\
\text { offshore, to acquire the teaching ethos on our } \\
\text { main campus by simply talking to a few key } \\
\text { personnel (e.g. key academics/faculty on our } \\
\text { main campus). }\end{array}$ & $\begin{array}{l}\text { Foreign Entry One: } \\
1 \ldots .2 \ldots 3 \ldots .4 \ldots .5 \ldots 6 \ldots . \ldots 7 \ldots 8 \ldots 9 \\
\text { Foreign Entry Two: } \\
1 \ldots . . \ldots 3 \ldots .4 \ldots .5 \ldots .6 \ldots 7 \ldots 8 \ldots .9\end{array}$ \\
\hline $\begin{array}{l}\text { We anticipated that it would be difficult for } \\
\text { academics (i.e. faculty) hired specifically to } \\
\text { teach on the offshore programmes, and based } \\
\text { offshore, to acquire the teaching ethos on our } \\
\text { main campus by simply studying the } \\
\text { curriculum content of specified courses. }\end{array}$ & $\begin{array}{l}\text { Foreign Entry One: } \\
1 \ldots .2 \ldots 3 \ldots .4 \ldots .5 \ldots 6 \ldots 7 \ldots 8 \ldots 9 \\
\text { Foreign Entry Two: } \\
1 \ldots . . \ldots 3 \ldots . \ldots \ldots 5 \ldots \ldots 6 \ldots 7 \ldots 8 \ldots .9\end{array}$ \\
\hline
\end{tabular}

J. At the time of the entries you have in mind, to what extent would you have agreed or disagreed with each of the following statements? (Please circle the number that best corresponds to your level of agreement for each statement.) 


\begin{tabular}{|c|c|}
\hline & $\begin{array}{l}\text { Strongly } \\
\text { disagree }\end{array}$ \\
\hline $\begin{array}{l}\text { My institution encourages open } \\
\text { discussion between academic (i.e. } \\
\text { faculty) and non-academic staff, } \\
\text { including senior management. }\end{array}$ & $\begin{array}{l}\text { Foreign Entry One: } \\
1 \ldots 2 \ldots 3 \ldots .4 \ldots 5 \ldots \ldots 6 \ldots 7 \ldots 8 \ldots 9 \\
\text { Foreign Entry Two: } \\
1 \ldots .2 \ldots 3 \ldots .4 \ldots . . \ldots 6 \ldots 7 \ldots 8 \ldots 9\end{array}$ \\
\hline $\begin{array}{l}\text { My institution de-emphasises status } \\
\text { distinctions. }\end{array}$ & 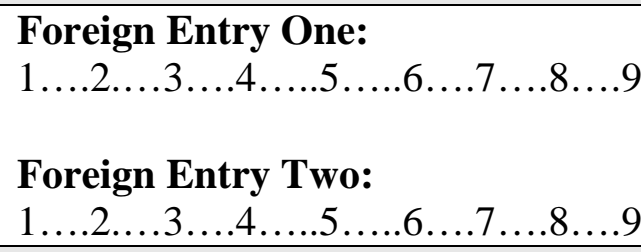 \\
\hline $\begin{array}{l}\text { My institution encourages } \\
\text { experimentation and tolerates mistakes. }\end{array}$ & $\begin{array}{l}\text { Foreign Entry One: } \\
1 \ldots 2 \ldots 3 \ldots \ldots 4 \ldots \ldots 5 \ldots \ldots 6 \ldots 7 \ldots 8 \ldots .9 \\
\text { Foreign Entry Two: } \\
1 \ldots 2 \ldots 3 \ldots .4 \ldots \ldots 5 \ldots 6 \ldots 7 \ldots 8 \ldots 9\end{array}$ \\
\hline $\begin{array}{l}\text { My institution encourages academics (i.e. } \\
\text { faculty) to develop offshore education } \\
\text { programmes through their international } \\
\text { networks. }\end{array}$ & $\begin{array}{l}\text { Foreign Entry One: } \\
1 \ldots .2 \ldots 3 \ldots .4 \ldots . . \ldots 6 \ldots 7 \ldots 8 \ldots 9 \\
\text { Foreign Entry Two: } \\
1 \ldots .2 \ldots 3 \ldots .4 \ldots . . \ldots 6 \ldots 7 \ldots 8 \ldots 9\end{array}$ \\
\hline $\begin{array}{l}\text { My institution is responsive to offshore } \\
\text { education projects that arise out of } \\
\text { academics' (i.e. faculty) international } \\
\text { networks. }\end{array}$ & $\begin{array}{l}\text { Foreign Entry One: } \\
1 \ldots .2 \ldots 3 \ldots .4 \ldots .5 \ldots 6 \ldots 7 \ldots 8 \ldots 9 \\
\text { Foreign Entry Two: } \\
1 \ldots 2 \ldots 3 \ldots .4 \ldots .5 \ldots 6 \ldots 7 \ldots 8 \ldots 9\end{array}$ \\
\hline
\end{tabular}

K. At the time of these particular foreign market entries, what was your institution's approximate financial endowment in US\$ (i.e. cash in the bank)? (Please tick the most appropriate box.)

\begin{tabular}{l|l|l}
\hline \hline & $\begin{array}{l}\text { Foreign } \\
\text { Entry } \\
\text { One }\end{array}$ & $\begin{array}{l}\text { Foreign } \\
\text { Entry } \\
\text { Two }\end{array}$ \\
\hline Less than US\$100,000 & $\square$ & $\square$ \\
\hline Between US\$ 100,001 and US\$ 500,000 & $\square$ & $\square$ \\
\hline Between US\$ 500,001 and US\$ 1 million & $\square$ & $\square$ \\
\hline Between US\$ 1,000,001 and US\$ 2 million & $\square$ & $\square$ \\
\hline Between US\$ 2,000,001 and US\$ 5 million & $\square$ & $\square$ \\
\hline Between US\$ 5,000,001 and US\$ 10 million & $\square$ & $\square$ \\
\hline Between US\$ 10,000,001 million and US\$ 20 million & $\square$ & $\square$ \\
\hline
\end{tabular}




\begin{tabular}{l|l|l}
\hline Between US\$ 20,000,001 million and US\$ 50 million & $\square$ & $\square$ \\
\hline Between US\$ 50,000,001 and US\$ 1 billion & $\square$ & $\square$ \\
\hline Between US\$ 100,000,001 and US\$ 10 billion & $\square$ & $\square$ \\
\hline Between US\$ 10,000,000,001 and US\$20 billion & $\square$ & $\square$ \\
\hline Between US\$ 20,000,000,001 and US\$ 50 billion & $\square$ & $\square$ \\
\hline Greater than US\$ 50 billion & $\square$ & $\square$ \\
\hline \hline
\end{tabular}

L. With respect to the two international developments you have in mind, how important was protecting your institution's reputation, with respect to the choice of entry mode? (Please circle the number that best corresponds to your view.)

\begin{tabular}{|c|c|}
\hline & $\begin{array}{l}\text { Not } \\
\text { important }\end{array}$ \\
\hline Foreign Entry One & $1 \ldots \ldots 2 \ldots \ldots 3 \ldots \ldots 4 \ldots \ldots 5 \ldots \ldots 6 \ldots \ldots 7 \ldots \ldots 8 \ldots \ldots 9$ \\
\hline Foreign Entry Two & $1 \ldots \ldots .2 \ldots \ldots 3 \ldots \ldots 4 \ldots \ldots 5 \ldots \ldots 6 \ldots \ldots 7 \ldots \ldots ., \ldots \ldots 9$ \\
\hline
\end{tabular}

M. At the time of each of these international developments, how would you rate your institution's reputation in the applicable foreign markets, with respect to the following aspects? (Please circle the number that best corresponds to your view for each aspect.)

\begin{tabular}{|c|c|}
\hline & $\begin{array}{lc}\text { Very } & \text { Very } \\
\text { low } & \text { high }\end{array}$ \\
\hline $\begin{array}{l}\text { Reputation for superior academic } \\
\text { management }\end{array}$ & $\begin{array}{l}\text { Foreign Entry One: } \\
1 \ldots . \ldots .3 \ldots 4 \ldots \ldots 5 \ldots \ldots 6 \ldots 7 \ldots 8 \ldots 9 \\
\text { Foreign Entry Two: } \\
1 \ldots .2 \ldots . .4 \ldots 4 \ldots 5 \ldots .6 \ldots 7 \ldots 8 \ldots 9\end{array}$ \\
\hline $\begin{array}{l}\text { Reputation for superior academic } \\
\text { service delivery }\end{array}$ & 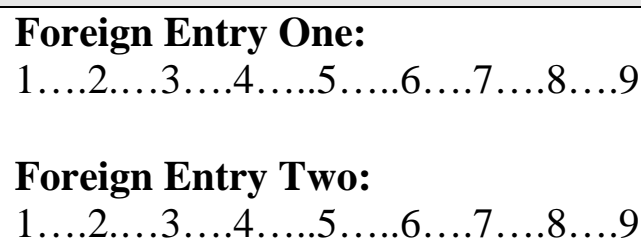 \\
\hline $\begin{array}{l}\text { Reputation for superior academic and } \\
\text { programme innovativeness }\end{array}$ & 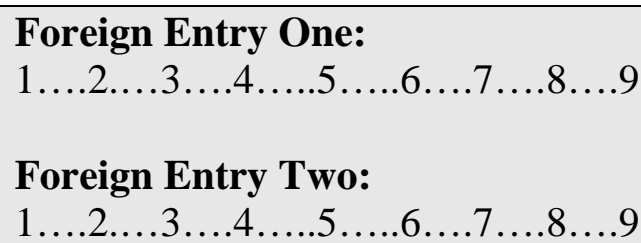 \\
\hline Reputation for student selectivity & $\begin{array}{l}\text { Foreign Entry One: } \\
1 \ldots 2 \ldots 3 \ldots 4 \ldots 5 \ldots \ldots 6 \ldots 7 \ldots 8 \ldots 9\end{array}$ \\
\hline
\end{tabular}


N. Approximately how many students and academic staff members (i.e. faculty) did your institution have at the times of these foreign market entries?

\begin{tabular}{l|l|l}
\hline \hline & Foreign Entry One & Foreign Entry Two \\
\hline Undergraduate students & & \\
\cline { 2 - 3 } $\begin{array}{l}\text { Graduate/Postgraduate } \\
\text { students }\end{array}$ & & \\
\hline Academic staff/Faculty & & \\
\hline \hline
\end{tabular}

O. (i) Did your institution have an official, documented policy on offshore education at the times of these two entries? (Please tick one box.)

\begin{tabular}{l|l|l}
\hline \hline Foreign Entry One & $\square$ Yes & $\square$ No \\
\hline Foreign Entry Two & $\square$ Yes & $\square$ No \\
\hline \hline
\end{tabular}

If you have answered YES to any one of the two entries above, please continue to question $O$ (ii) for that respective entry. Otherwise, skip to question $P$ for the respective foreign entry for which you have answered NO above.

(ii) At the times of the two foreign entries you have in mind, which of the following were included in your institution's policy? (Please tick all applicable boxes.)

\begin{tabular}{l|l|l}
\hline \hline & $\begin{array}{l}\text { Foreign } \\
\text { Entry } \\
\text { One }\end{array}$ & $\begin{array}{l}\text { Foreign } \\
\text { Entry } \\
\text { Two }\end{array}$ \\
\hline $\begin{array}{l}\text { A formal decision-making model specified on an } \\
\text { institution-wide basis }\end{array}$ & $\square$ \\
\hline $\begin{array}{l}\text { Compliance with institution-wide strategic considerations } \\
\text { (i.e. Why is this initiative in the strategic interests of the } \\
\text { institution?) }\end{array}$ & $\square$ & $\square$ \\
\hline $\begin{array}{l}\text { Compliance with institution-wide academic considerations } \\
\text { (i.e. Why is this initiative in the academic interests of the } \\
\text { institution?) }\end{array}$ & $\square$ & $\square$ \\
\hline $\begin{array}{l}\text { Compliance with institution-wide business considerations } \\
\text { (i.e. Why is this initiative in the business interests of the } \\
\text { institution?) }\end{array}$ & $\square$ & $\square$ \\
\hline An explicit, institution-wide quality assurance strategy & $\square$ & $\square$ \\
\hline
\end{tabular}




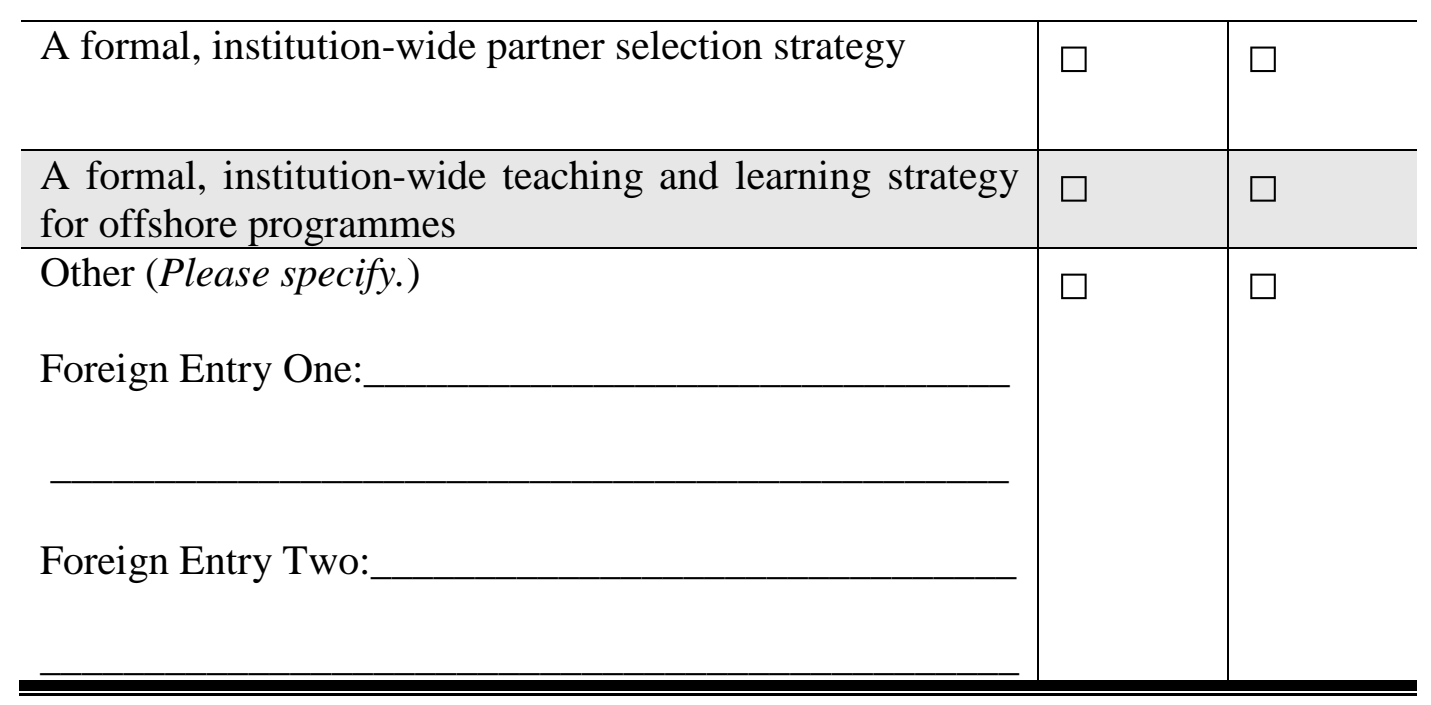

P. In what year was your home institution established?

Q. Thinking about rankings of academic institutions, approximately what percentage of institutions would have been ranked lower than your institution, at the time of these foreign market entries? (Please tick the boxes that best correspond to the worldwide and domestic ranking of your institution at that time.)

(i) Worldwide ranking

\begin{tabular}{l|l|l}
\hline \hline & $\begin{array}{l}\text { Foreign Entry } \\
\text { One }\end{array}$ & $\begin{array}{l}\text { Foreign Entry } \\
\text { Two }\end{array}$ \\
\hline $\begin{array}{l}\text { 90\% of other institutions would be } \\
\text { ranked lower than yours }\end{array}$ & $\square$ & $\square$ \\
\hline $\begin{array}{l}\text { 80\% of other institutions would be } \\
\text { ranked lower than yours }\end{array}$ & $\square$ & $\square$ \\
\hline $\begin{array}{l}70 \% \text { of other institutions would be } \\
\text { ranked lower than yours }\end{array}$ & $\square$ & $\square$ \\
\hline $\begin{array}{l}\text { 60\% of other institutions would be } \\
\text { ranked lower than yours }\end{array}$ & $\square$ & $\square$ \\
\hline $\begin{array}{l}50 \% \text { of other institutions would be } \\
\text { ranked lower than yours }\end{array}$ & $\square$ & $\square$ \\
\hline $\begin{array}{l}40 \% \text { of other institutions would be } \\
\text { ranked lower than yours }\end{array}$ & $\square$ & $\square$ \\
\hline $\begin{array}{l}\text { 30\% of other institutions would be } \\
\text { ranked lower than yours }\end{array}$ & $\square$ & $\square$ \\
\hline $\begin{array}{l}20 \% \text { of other institutions would be } \\
\text { ranked lower than yours }\end{array}$ & $\square$ & $\square$ \\
\hline $\begin{array}{l}\text { 10\% of other institutions would be } \\
\text { ranked lower than yours }\end{array}$ & $\square$ & $\square$ \\
\hline \hline
\end{tabular}


(ii) Domestic Ranking

\begin{tabular}{l|l|l}
\hline \hline & $\begin{array}{l}\text { Foreign Entry } \\
\text { One }\end{array}$ & $\begin{array}{l}\text { Foreign Entry } \\
\text { Two }\end{array}$ \\
\hline $\begin{array}{l}\text { 90\% of other institutions would be } \\
\text { ranked lower than yours }\end{array}$ & $\square$ & $\square$ \\
\hline $\begin{array}{l}\text { 80\% of other institutions would be } \\
\text { ranked lower than yours }\end{array}$ & $\square$ & $\square$ \\
\hline $\begin{array}{l}70 \% \text { of other institutions would be } \\
\text { ranked lower than yours }\end{array}$ & $\square$ & $\square$ \\
\hline $\begin{array}{l}\text { 60\% of other institutions would be } \\
\text { ranked lower than yours }\end{array}$ & $\square$ & $\square$ \\
\hline $\begin{array}{l}50 \% \text { of other institutions would be } \\
\text { ranked lower than yours }\end{array}$ & $\square$ & $\square$ \\
\hline $\begin{array}{l}40 \% \text { of other institutions would be } \\
\text { ranked lower than yours }\end{array}$ & $\square$ & $\square$ \\
\hline $\begin{array}{l}\text { 30\% of other institutions would be } \\
\text { ranked lower than yours }\end{array}$ & $\square$ & $\square$ \\
\hline $\begin{array}{l}20 \% \text { of other institutions would be } \\
\text { ranked lower than yours }\end{array}$ & $\square$ & $\square$ \\
\hline $\begin{array}{l}\text { 10\% of other institutions would be } \\
\text { ranked lower than yours }\end{array}$ & $\square$ & $\square$ \\
\hline \hline
\end{tabular}

R. At the time of these two international developments, how would you rate your institution's involvement in the foreign markets, with respect to the following aspects? (Please circle the number that best corresponds to your view for each aspect.)

\begin{tabular}{|c|c|}
\hline & $\begin{array}{ll}\text { Very } & \text { Very } \\
\text { low } & \text { high } \\
\end{array}$ \\
\hline Financial gain & 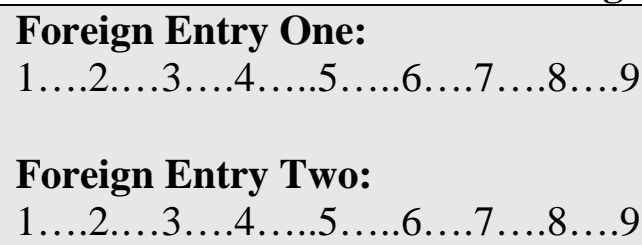 \\
\hline Investment opportunity & $\begin{array}{l}\text { Foreign Entry One: } \\
1 \ldots 2 \ldots 3 \ldots .4 \ldots .5 \ldots 6 \ldots .7 \ldots 8 \ldots .9 \\
\text { Foreign Entry Two: } \\
1 \ldots 2 \ldots . \ldots 4 \ldots \ldots 5 \ldots \ldots 6 \ldots 7 \ldots 8 \ldots .9\end{array}$ \\
\hline Growth of business & 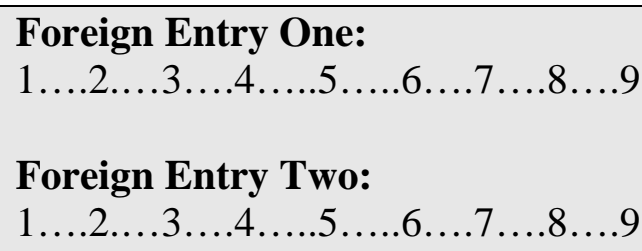 \\
\hline
\end{tabular}




\begin{tabular}{|c|c|}
\hline Diversification & $\begin{array}{l}\text { Foreign Entry One: } \\
1 \ldots . \ldots .3 \ldots 4 \ldots \ldots 5 \ldots . .6 \ldots 7 \ldots 8 \ldots .9 \\
\text { Foreign Entry Two: } \\
1 \ldots . \ldots .3 \ldots 4 \ldots \ldots 5 \ldots \ldots 6 \ldots 7 \ldots 8 \ldots .9\end{array}$ \\
\hline $\begin{array}{l}\text { Pathway to onshore provision (i.e. } \\
2+2 \text { type of programmes) }\end{array}$ & 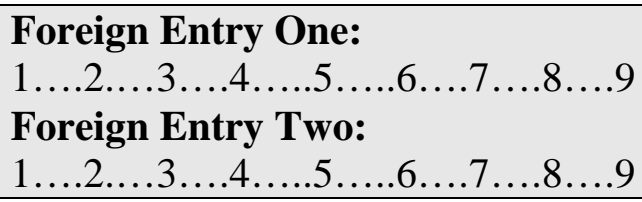 \\
\hline Internationalisation & $\begin{array}{l}\text { Foreign Entry One: } \\
1 \ldots . \ldots .3 \ldots 4 \ldots \ldots 5 \ldots . .6 \ldots 7 \ldots . . .9 \\
\text { Foreign Entry Two: } \\
1 \ldots . \ldots .3 \ldots 4 \ldots \ldots 5 \ldots \ldots 6 \ldots 7 \ldots . \ldots 9\end{array}$ \\
\hline Professional development of staff & $\begin{array}{l}\text { Foreign Entry One: } \\
1 \ldots . \ldots .6 \ldots 4 \ldots \ldots 5 \ldots .6 \ldots 7 \ldots . . .9 \\
\text { Foreign Entry Two: } \\
1 \ldots . \ldots .3 \ldots 4 \ldots \ldots 5 \ldots \ldots 6 \ldots 7 \ldots 8 \ldots .9\end{array}$ \\
\hline
\end{tabular}

S. Did your institution have an 'internationalisation at home' strategy at the time of this market entry?

\begin{tabular}{l|l|l}
\hline \hline Foreign Entry One & $\square$ Yes & $\square$ No \\
\hline Foreign Entry Two & $\square$ Yes & $\square$ No \\
\hline \hline
\end{tabular}

If you have answered YES to any one of the two entries above, please continue to question $T$ for that respective entry. Otherwise, skip to question $U$ for the respective foreign entry for which you have answered NO above.

T. At the time of these two market entries, to what extent did this internationalisation strategy influence your institution's involvement in offshore education?

\begin{tabular}{|c|c|}
\hline & $\begin{array}{c}\text { A great } \\
\text { deal }\end{array}$ \\
\hline Foreign Entry One & $1 \ldots \ldots .2 \ldots \ldots 3 \ldots \ldots 4 \ldots \ldots 5 \ldots \ldots 6 \ldots \ldots 7 \ldots \ldots 8 \ldots \ldots 9$ \\
\hline Foreign Entry Two & $1 \ldots \ldots . \ldots \ldots 3 \ldots \ldots 4 \ldots \ldots 5 \ldots \ldots 6 \ldots \ldots 7 \ldots \ldots 8 \ldots \ldots 9$ \\
\hline
\end{tabular}


U. At the time of the two entries you have in mind, how strong was your institution's intent to increase its involvement in offshore education?

\begin{tabular}{l|l}
\hline \hline & $\begin{array}{l}\text { Not strong } \\
\text { at all }\end{array}$ \\
\hline Foreign Entry One & $1 \ldots \ldots 2 \ldots \ldots 3 \ldots \ldots 4 \ldots \ldots 5 \ldots \ldots 6 \ldots \ldots 7 \ldots \ldots 8 \ldots \ldots 9$ \\
\hline Foreign Entry Two & $1 \ldots \ldots 2 \ldots \ldots 3 \ldots \ldots 4 \ldots \ldots 5 \ldots \ldots 6 \ldots \ldots 7 \ldots \ldots 8 \ldots \ldots 9$ \\
\hline \hline
\end{tabular}

WE THANK YOU FOR YOUR COOPERATION IN THIS RESEARCH.

Please send the completed questionnaire by 15 August, 2008 to:

Vik Naidoo, PhD Candidate

School of Marketing and International Business

PO Box 600

Victoria University of Wellington

Wellington

NEW ZEALAND

If you would like a summary report of the findings of the research, please contact me at vik.naidoo@vuw.ac.nz. 


\section{Appendix $N$ \\ COVER LETTER - FOLLOW UP 1}

TE WHARE WÃNANGA O TE OPOKO O TE IKA A MAUU

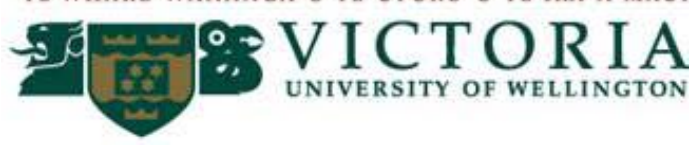

School of Marketing and International Business

PO Box 600

Victoria University of Wellington

Wellington

New Zealand

Date: ----

Dear: ----

A few weeks ago, I sent you a questionnaire entitled Understanding Foreign Market Entry Strategies: A Survey of Offshore Education Providers, requesting your participation on behalf of your institution. The questionnaire forms part of my $\mathrm{PhD}$ study investigating offshore education, and your institution was identified as being currently involved in such activities.

Many of the people to whom I sent questionnaires have responded, and their participation is very much appreciated. However, still more completed questionnaires are needed to ensure that the study is representative of current offshore education dynamics. If you have already responded to the questionnaire, thank you very much; your participation is greatly appreciated. If you have not had time to complete and return the questionnaire, please do so - it would be very helpful to have your completed questionnaire by 15, August, 2008.

Your participation is vital to the success of this research project. The information that you provide will assist in the development of a more in-depth understanding of entry into the offshore education market. If you have any questions about this survey, you can reach me by e-mail at vik.naidoo@vuw.ac.nz or by telephone at (+64) ---------. Thank you in advance for your help.

Sincerely, Vik Naidoo

PhD Student

School of Marketing and International Business 


\section{Appendix $\mathrm{O}$ \\ COVER LETTER - FOLLOW UP 2}

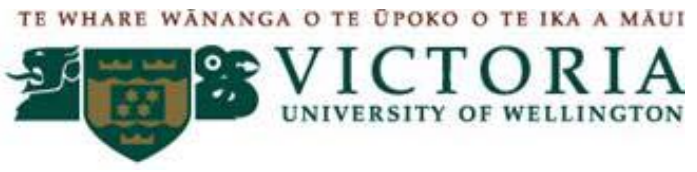

School of Marketing and International Business

PO Box 600

Victoria University of Wellington

Wellington

New Zealand

Date: ----

Dear: ----

About a week ago, I wrote to remind you about a questionnaire entitled Understanding Foreign Market Entry Strategies: A Survey of Offshore Education Providers, which I sent you on ----, 2008. If you have already completed and mailed the questionnaire, please disregard this letter, and I thank you very much for your participation. If you have not completed the questionnaire, I would be very grateful if you would do so as soon as possible. For this research to be completed by its deadline, it would be very helpful to receive your completed questionnaire by 15 August, 2008.

Your participation is very important, in order for this study to comprehensively reflect the collective wisdom of those institutions that have been involved in offshore education. The information you provide will be strictly anonymous. When made public, results of the survey will be reported in aggregate data, without identification of respondents or their institution.

In case you misplaced the questionnaire, I have enclosed another copy. If you have any questions or concerns about this survey, please feel free to contact me. I can be reached by email at vik.naidoo@vuw.ac.nz or at (+64) ---------.

Sincerely, Vik Naidoo

PhD Student

School of Marketing and International Business 


\section{Appendix $P$}

\section{DESCRIPTIVE STATISTICS - CROSS TABULATIONS}

\section{Home Country \& Host Country}

\begin{tabular}{|c|c|c|c|c|c|c|c|c|c|c|c|c|c|c|c|c|c|c|c|c|c|c|}
\hline & \multicolumn{22}{|c|}{ Host Country } \\
\hline & Australia & Bahrain & Canada & China & a Czech Rep & Dubai $\mathrm{Fi}_{\mathrm{i}}$ & & & еece & Hong Kong & Hungary & IIndial & Indonesia & Malaysia & Mexico & Poland & Qatar & Singapore & South Africa & Sri Lanka & Thailand & d Total \\
\hline Home Country AU & & 0 & & 24 & 0 & 1 & 0 & 0 & 0 & 6 & 0 & 9 & & 10 & & 0 & 0 & 16 & & 0 & & \begin{tabular}{|l|l|}
0 & 70 \\
\end{tabular} \\
\hline $\mathrm{CA}$ & 0 & 1 & 0 & 10 & 0 & 0 & 0 & 0 & 0 & 2 & & 7 & 2 & 2 & 0 & 0 & 0 & 6 & 0 & 0 & & $\begin{array}{lll}0 & 30 \\
\end{array}$ \\
\hline IR & 0 & 0 & 0 & 2 & 0 & 0 & 0 & 0 & 0 & 0 & 0 & 2 & 0 & 2 & 0 & 0 & 0 & 0 & 0 & 0 & & 0 \\
\hline $\mathrm{NZ}$ & 0 & 0 & 0 & 3 & 0 & 0 & 0 & 0 & 0 & 3 & 0 & 0 & 0 & 2 & 0 & 0 & 0 & 2 & 0 & 0 & 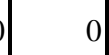 & 10 \\
\hline UK & 0 & 1 & 0 & 35 & 0 & 0 & 0 & 0 & 0 & 11 & 0 & 12 & 3 & 10 & 0 & 0 & 2 & 23 & 1 & 0 & & 98 \\
\hline US & 1 & 1 & 1 & 34 & 1 & 1 & 1 & 1 & 1 & 9 & 1 & 9 & 1 & 3 & 1 & 1 & 1 & 22 & 1 & 2 & & 94 \\
\hline Total & 1 & 3 & 2 & 108 & 1 & 2 & 1 & 1 & 1 & 31 & 1 & 39 & 8 & 29 & 1 & 1 & 3 & 69 & 3 & 2 & 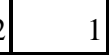 & 308 \\
\hline
\end{tabular}

\section{Home Country \& Academic Discipline}

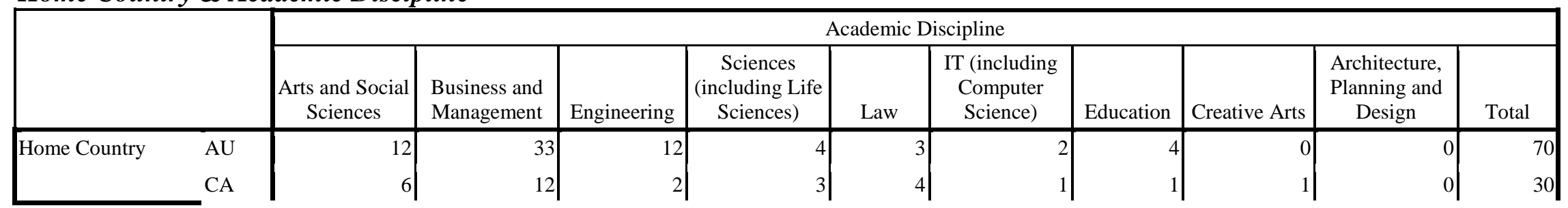




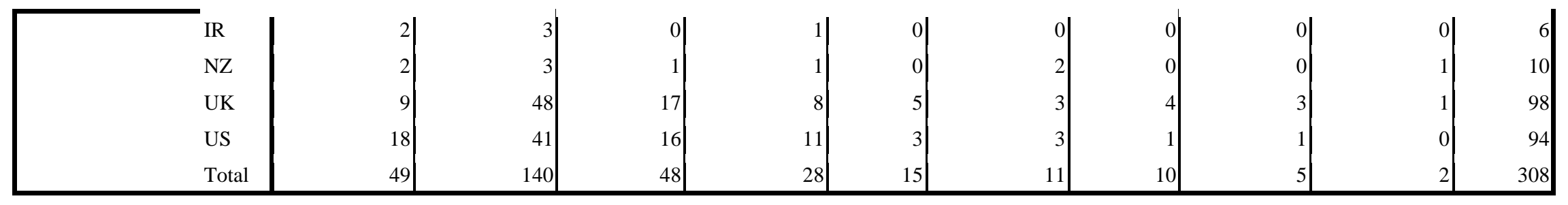

Home Country \& Programme Level

\begin{tabular}{|c|c|c|c|c|c|c|c|c|c|c|}
\hline & & \multicolumn{9}{|c|}{ Programme Level } \\
\hline & & $\begin{array}{c}\text { Professional } \\
\text { Doctorates }\end{array}$ & $\begin{array}{l}\text { Master's by } \\
\text { coursework } \\
\text { only }\end{array}$ & $\begin{array}{l}\text { Master's by } \\
\text { both } \\
\text { coursework and } \\
\text { research }\end{array}$ & $\begin{array}{c}\text { Undergraduate } \\
\text { degree }\end{array}$ & Diploma & $\begin{array}{l}\text { Associate } \\
\text { Degree }\end{array}$ & Certificate & $\begin{array}{l}\text { Executive/Professional } \\
\text { short programme }\end{array}$ & Total \\
\hline \multirow{7}{*}{ Home Country } & $\mathrm{AU}$ & 0 & 6 & 2 & 53 & 7 & 2 & 0 & 0 & 70 \\
\hline & CA & 0 & 1 & 0 & 23 & 4 & 2 & 0 & 0 & 30 \\
\hline & IR & 0 & 0 & 0 & 6 & 0 & 0 & 0 & 0 & 6 \\
\hline & $\mathrm{NZ}$ & 0 & 0 & 0 & 10 & 0 & 0 & 0 & 0 & 10 \\
\hline & UK & 2 & 14 & 3 & 64 & 9 & 4 & 2 & 0 & 98 \\
\hline & US & 3 & 15 & 3 & 62 & 4 & 5 & 1 & 1 & 94 \\
\hline & Total & 5 & 36 & 8 & 218 & 24 & 13 & 3 & 1 & 308 \\
\hline
\end{tabular}

\section{Home Country \& Year of Entry}

\begin{tabular}{|c|c|c|c|c|c|c|c|c|c|c|c|}
\hline & & \multicolumn{10}{|c|}{ Year of Entry } \\
\hline & & 1999 & 2000 & 2001 & 2002 & 2003 & 2004 & 2005 & 2006 & 2007 & Total \\
\hline \multirow[t]{4}{*}{ Home Country } & $\mathrm{AU}$ & 10 & 8 & 12 & 3 & 9 & 11 & 9 & 2 & 6 & 70 \\
\hline & CA & 4 & 1 & 2 & 4 & 2 & 6 & 2 & 5 & 4 & 30 \\
\hline & IR & 0 & 0 & 0 & 2 & 1 & 1 & 1 & 0 & 1 & 6 \\
\hline & $\mathrm{NZ}$ & 2 & 0 & 0 & 1 & 3 & 1 & 3 & 0 & 0 & 10 \\
\hline
\end{tabular}




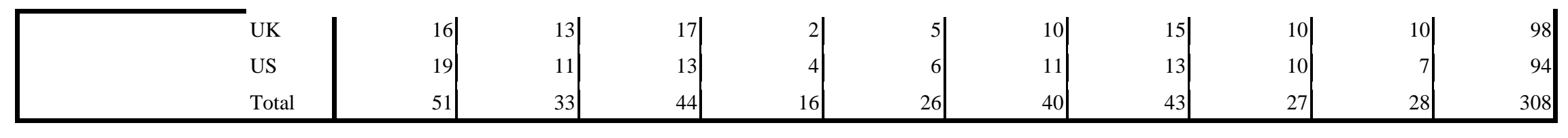

\section{Home Country \& Size (Undergraduate Students)}

\begin{tabular}{|c|c|c|c|c|c|c|c|c|c|c|c|c|}
\hline & \multicolumn{11}{|c|}{ Size (Undergraduate Students) } \\
\hline & & $<1000$ & $1001-5000$ & $5001-10000$ & $\begin{array}{c}10001- \\
15000\end{array}$ & $\begin{array}{c}15001- \\
20000\end{array}$ & $\begin{array}{c}20001- \\
25000\end{array}$ & $\begin{array}{c}25001- \\
30000\end{array}$ & $\begin{array}{c}30001- \\
35000\end{array}$ & $\begin{array}{c}35001- \\
40000\end{array}$ & $\begin{array}{c}45001- \\
50000\end{array}$ & Total \\
\hline \multirow[t]{7}{*}{ Home Country } & $\mathrm{AU}$ & c & 4 & 6 & 14 & 12 & 14 & 10 & 8 & 2 & 0 & 70 \\
\hline & CA & c & 2 & 4 & 6 & 2 & 8 & 6 & 0 & 2 & 0 & 30 \\
\hline & IR & c & 0 & 2 & 2 & 2 & 0 & 0 & 0 & 0 & 0 & 6 \\
\hline & $\mathrm{NZ}$ & c & 2 & 0 & 2 & 0 & 2 & 0 & 2 & 2 & 0 & 10 \\
\hline & UK & c & 0 & 25 & 25 & 30 & 10 & 6 & 2 & 0 & 0 & 98 \\
\hline & US & $\epsilon$ & 13 & 17 & 14 & 16 & 8 & 10 & 3 & 5 & 2 & 94 \\
\hline & Total & $\epsilon$ & 21 & 54 & 63 & 62 & 42 & 32 & 15 & 11 & 2 & 308 \\
\hline
\end{tabular}

\section{Home Country \& Size (Postgraduate Students)}

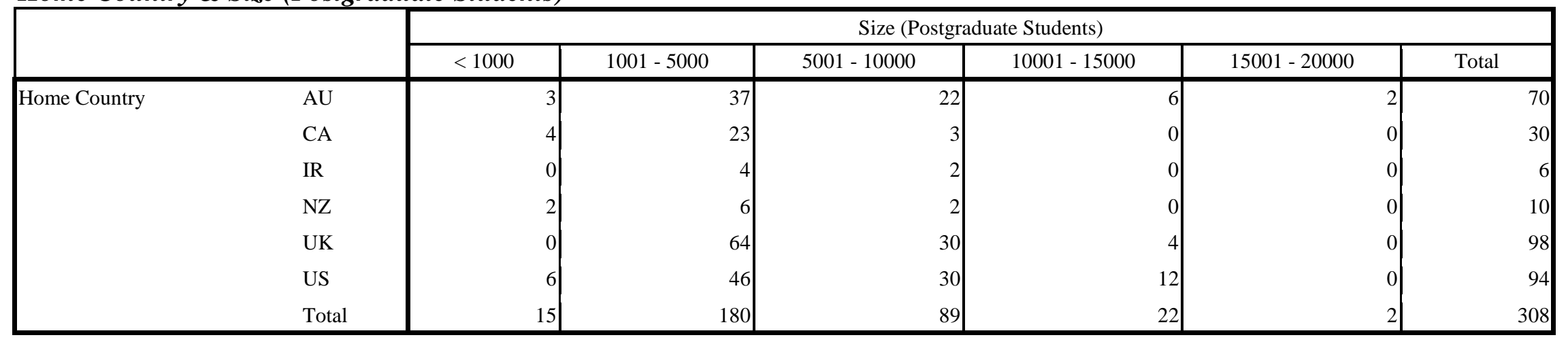




\section{Home Country \& Size (Staff)}

\begin{tabular}{|c|c|c|c|c|c|c|c|c|c|c|c|c|c|c|c|}
\hline & \multicolumn{14}{|c|}{ Size (Staff) } \\
\hline & & $<500$ & $\begin{array}{l}501- \\
1000\end{array}$ & $\begin{array}{c}1001- \\
1500\end{array}$ & $\begin{array}{c}1501- \\
2000\end{array}$ & $\begin{array}{c}2001- \\
2500\end{array}$ & $\begin{array}{c}2501- \\
3000\end{array}$ & $\begin{array}{c}3001- \\
3500\end{array}$ & $\begin{array}{c}3501- \\
4000\end{array}$ & $\begin{array}{c}4001- \\
4500\end{array}$ & $\begin{array}{c}4501- \\
5000\end{array}$ & $\begin{array}{c}5001- \\
5500\end{array}$ & $\begin{array}{c}5501- \\
6000\end{array}$ & $\begin{array}{c}7501- \\
8000\end{array}$ & Total \\
\hline \multirow[t]{7}{*}{ Home Country } & $\mathrm{AU}$ & 3 & 15 & 12 & 6 & 8 & 16 & 3 & 3 & 1 & 1 & 0 & 2 & 0 & 70 \\
\hline & CA & 4 & 7 & 7 & 2 & 0 & 0 & 4 & 4 & 0 & 2 & 0 & 0 & 0 & 30 \\
\hline & IR & 4 & 0 & 2 & 0 & 0 & 0 & 0 & 0 & 0 & 0 & 0 & 0 & 0 & 6 \\
\hline & NZ & 0 & 2 & 0 & 0 & 0 & 4 & 1 & 3 & 0 & 0 & 0 & 0 & 0 & 10 \\
\hline & UK & 2 & 17 & 15 & 28 & 10 & 10 & 2 & 8 & 0 & 4 & 0 & 0 & 2 & 98 \\
\hline & US & 21 & 21 & 14 & 10 & 7 & 13 & 2 & 2 & 0 & 2 & 2 & 0 & 0 & 94 \\
\hline & Total & 34 & 62 & 50 & 46 & 25 & 43 & 12 & 20 & 1 & 9 & 2 & 2 & 2 & 308 \\
\hline
\end{tabular}

\section{Home Country \& Age}

\begin{tabular}{|c|c|c|c|c|c|c|c|c|c|}
\hline & & \multicolumn{8}{|c|}{ Age (Years) } \\
\hline & & $<10$ & $11-50$ & $51-100$ & $101-150$ & $151-200$ & $201-250$ & $251-300$ & Total \\
\hline \multirow[t]{7}{*}{ Home Country } & $\mathrm{AU}$ & 0 & 40 & 18 & 8 & 4 & 0 & 0 & 70 \\
\hline & CA & 0 & 10 & 8 & 6 & 4 & 2 & 0 & 30 \\
\hline & IR & 0 & 4 & 0 & 0 & 2 & 0 & 0 & 6 \\
\hline & $\mathrm{NZ}$ & 2 & 4 & 2 & 2 & 0 & 0 & 0 & 10 \\
\hline & UK & 2 & 40 & 16 & 18 & 20 & 2 & 0 & 98 \\
\hline & US & 4 & 4 & 14 & 40 & 26 & 4 & 2 & 94 \\
\hline & Total & 8 & 102 & 58 & 74 & 56 & 8 & 2 & 308 \\
\hline
\end{tabular}

\section{Home Country \& Worldwide Rankings}

\begin{tabular}{|l|r|r|r|r|r|r|r|r|r|}
\hline & \multicolumn{8}{|c|}{ World Ranking } \\
\cline { 2 - 8 } & $90^{\text {th }}$ percentile & $80^{\text {th }}$ percentile & $70^{\text {th }}$ percentile & $60^{\text {th }}$ percentile & $50^{\text {th }}$ percentile & $40^{\text {th }}$ percentile & $30^{\text {th }}$ percentile & $20^{\text {th }}$ percentile & Total \\
\hline Home Country AU & 0 & 12 & 4 & 4 & 32 & & 14 & 4 & 0 \\
\hline
\end{tabular}


<smiles>[C-]1C=C1</smiles> 


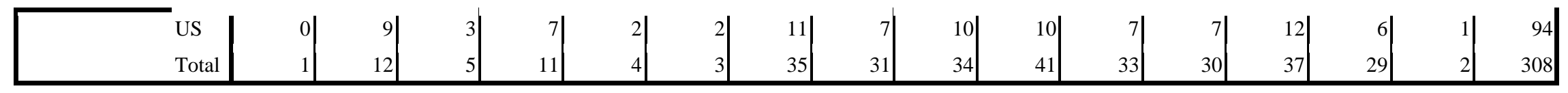

Home Country \& Mode of Entry (Categorical Measure)

\begin{tabular}{|c|c|c|c|c|c|c|c|}
\hline & & \multicolumn{6}{|c|}{ Mode of Entry (Categorical Measure) } \\
\hline & & Non-equity & $\begin{array}{c}\text { Joint venture (minority } \\
\text { equity) }\end{array}$ & $\begin{array}{l}\text { Joint venture (50/50 } \\
\text { equity) }\end{array}$ & $\begin{array}{c}\text { Joint venture (majority } \\
\text { equity) }\end{array}$ & Sole ownership & Total \\
\hline Home Country & $\mathrm{AU}$ & 62 & 1 & 2 & 2 & 3 & 70 \\
\hline & CA & 29 & 0 & 0 & 1 & 0 & 30 \\
\hline & IR & 5 & 0 & 0 & 0 & 1 & 6 \\
\hline & NZ & 10 & 0 & 0 & 0 & 0 & 10 \\
\hline & UK & 95 & 0 & 0 & 3 & 0 & 98 \\
\hline & US & 71 & 2 & 2 & 10 & 9 & 94 \\
\hline & Total & 272 & 3 & 4 & 16 & 13 & 308 \\
\hline
\end{tabular}

\section{Host Country \& Academic Discipline}

\begin{tabular}{|c|c|c|c|c|c|c|c|c|c|c|c|}
\hline & & \multicolumn{10}{|c|}{ Academic Discipline } \\
\hline & & $\begin{array}{c}\text { Arts and Social } \\
\text { Sciences }\end{array}$ & $\begin{array}{l}\text { Business and } \\
\text { Management }\end{array}$ & Engineering & \begin{tabular}{c|} 
Sciences \\
(including Life \\
Sciences)
\end{tabular} & Law & $\begin{array}{l}\text { IT (including } \\
\text { Computer } \\
\text { Science) }\end{array}$ & Education & Creative Arts & $\begin{array}{l}\text { Architecture, } \\
\text { Planning and } \\
\text { Design }\end{array}$ & Total \\
\hline \multirow[t]{8}{*}{ Host Country } & Australia & 0 & 1 & 0 & 0 & 0 & 0 & 0 & 0 & 0 & 1 \\
\hline & Bahrain & 2 & 0 & 0 & 0 & 0 & 0 & 0 & 1 & 0 & 3 \\
\hline & Canada & 0 & 0 & 1 & 0 & 0 & 0 & 1 & 0 & 0 & 2 \\
\hline & China & 15 & 53 & 14 & 13 & 6 & 1 & 2 & 4 & 0 & 108 \\
\hline & Czech Rep & 1 & 0 & 0 & 0 & 0 & 0 & 0 & 0 & 0 & 1 \\
\hline & Dubai & 1 & 0 & 0 & 1 & 0 & 0 & 0 & 0 & 0 & 2 \\
\hline & Fiji & 0 & 0 & 0 & 0 & 0 & 0 & 1 & 0 & 0 & 1 \\
\hline & France & 1 & 0 & 0 & 0 & 0 & 0 & 0 & 0 & 0 & 1 \\
\hline
\end{tabular}




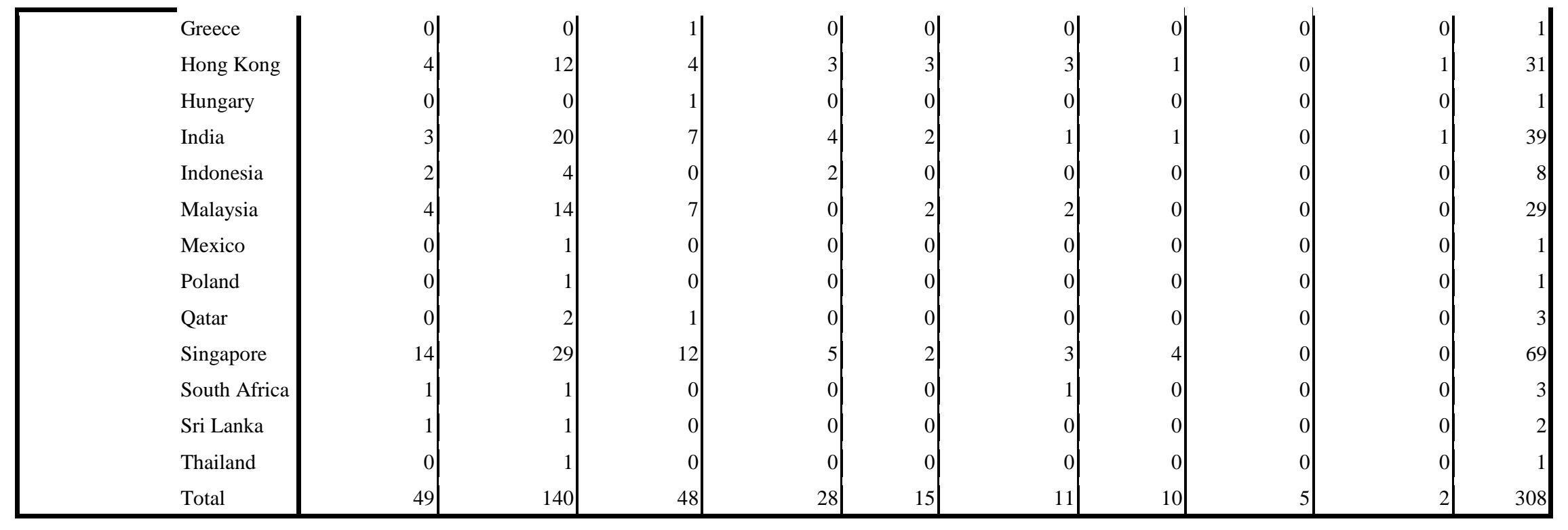

Host Country \& Programme Level

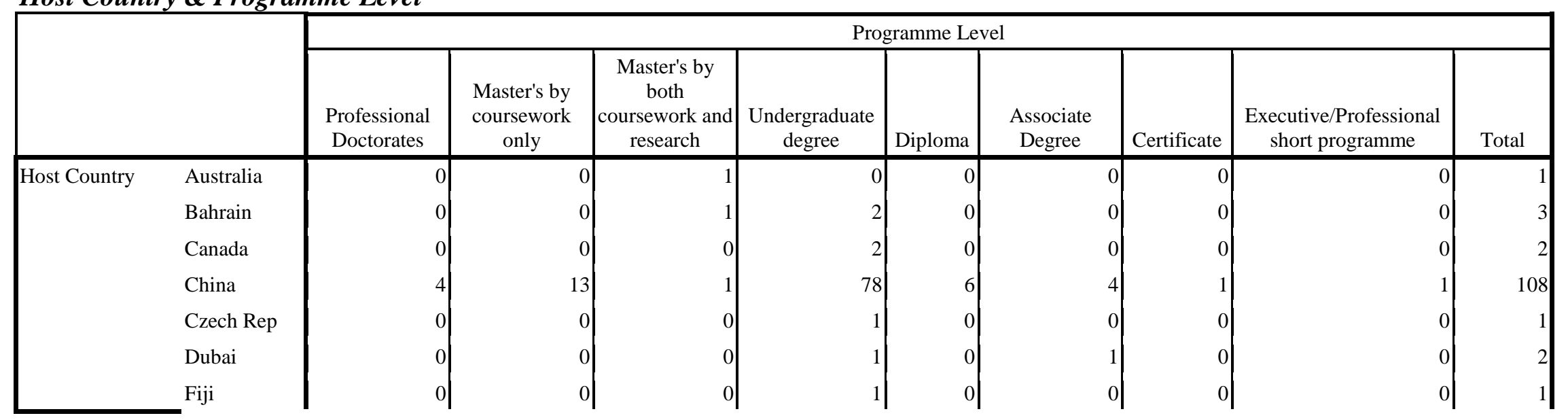




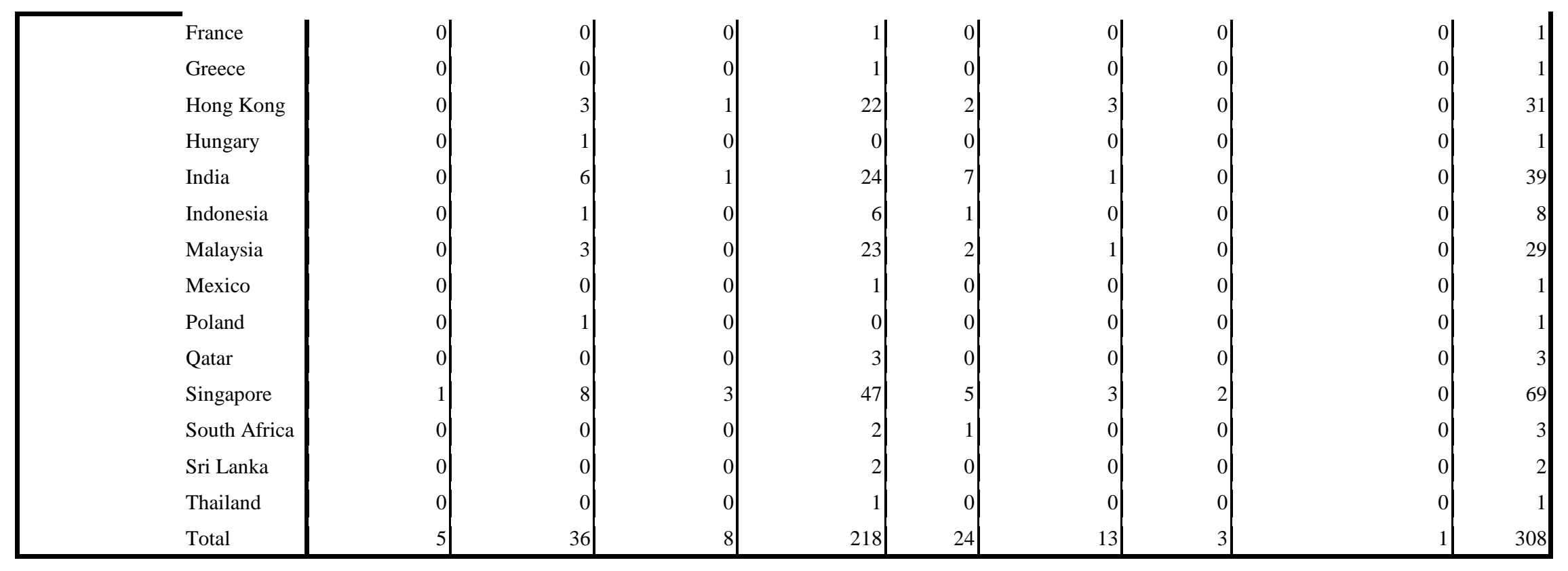

Host Country \& Year of Entry

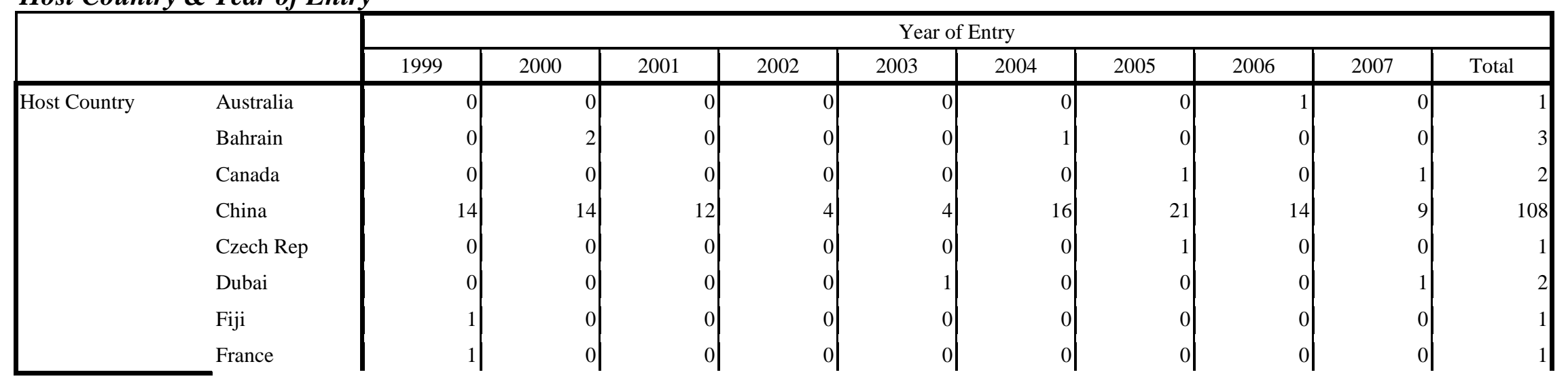




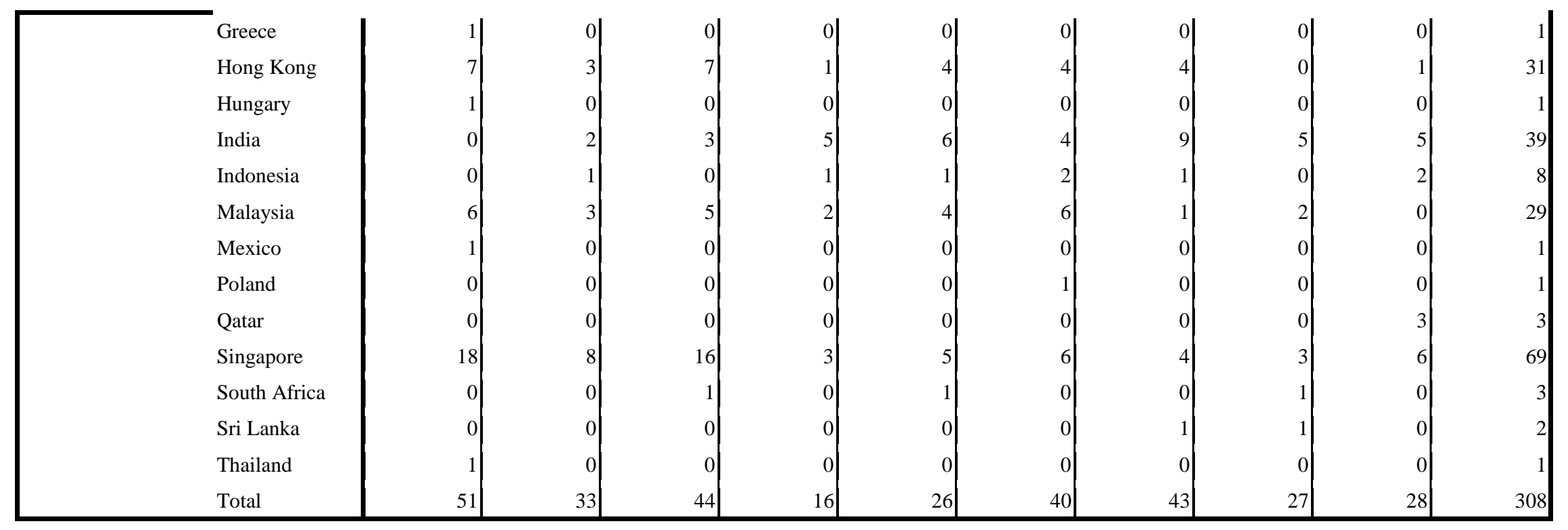

\section{Host Country \& Size (Undergraduate Students)}

\begin{tabular}{|c|c|c|c|c|c|c|c|c|c|c|c|c|}
\hline & & \multicolumn{11}{|c|}{ Size (Undergraduate Students) } \\
\hline & & $<1000$ & $1001-5000$ & $5001-10000$ & $\begin{array}{c}10001 \text { - } \\
15000\end{array}$ & $\begin{array}{c}15001- \\
20000\end{array}$ & $\begin{array}{c}20001- \\
25000\end{array}$ & $\begin{array}{c}25001- \\
30000\end{array}$ & $\begin{array}{c}30001- \\
35000\end{array}$ & $\begin{array}{c}35001- \\
40000\end{array}$ & $\begin{array}{c}45001- \\
50000\end{array}$ & Total \\
\hline \multirow[t]{9}{*}{ Host Country } & Australia & 0 & 0 & 0 & 0 & 0 & 1 & 0 & 0 & 0 & 0 & 1 \\
\hline & Bahrain & 0 & 0 & 0 & 1 & 0 & 1 & 1 & 0 & 0 & 0 & 3 \\
\hline & Canada & 0 & 0 & 1 & 0 & 0 & 1 & 0 & 0 & 0 & 0 & 2 \\
\hline & China & 2 & 9 & 20 & 19 & 22 & 12 & 13 & 9 & 1 & 1 & 108 \\
\hline & Czech Rep & 0 & 1 & 0 & 0 & 0 & 0 & 0 & 0 & 0 & 0 & 1 \\
\hline & Dubai & 0 & 0 & 1 & 0 & 1 & 0 & 0 & 0 & 0 & 0 & 2 \\
\hline & Fiji & 0 & 0 & 0 & 0 & 0 & 1 & 0 & 0 & 0 & 0 & 1 \\
\hline & France & 0 & 0 & 0 & 0 & 1 & 0 & 0 & 0 & 0 & 0 & 1 \\
\hline & Greece & 0 & 0 & 0 & 1 & 0 & 0 & 0 & 0 & 0 & 0 & 1 \\
\hline
\end{tabular}




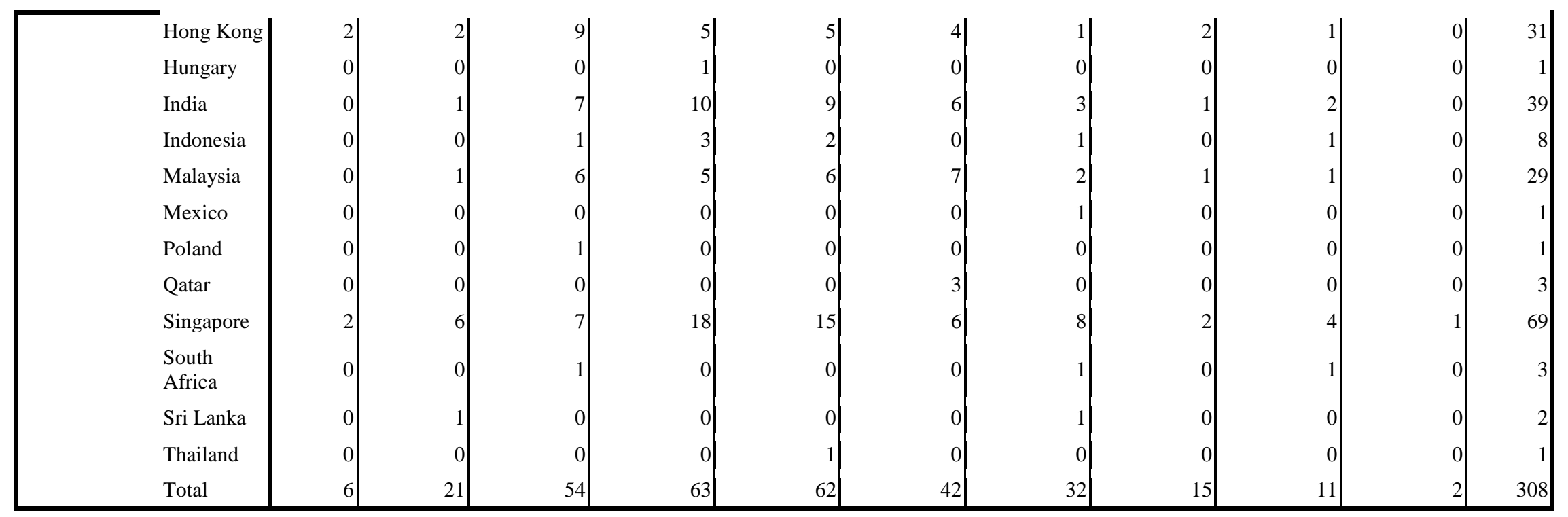

\section{Host Country \& Size (Postgraduate Students)}

\begin{tabular}{|c|c|c|c|c|c|c|c|}
\hline & & & & Size (Postg & te Students) & & \\
\hline & & $<1000$ & $1001-5000$ & $5001-10000$ & $10001-15000$ & $15001-20000$ & Total \\
\hline Host Country & Australia & & 0 & 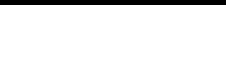 & & & \\
\hline & Bahrain & & 2 & & & & 3 \\
\hline & Canada & & 1 & & & & 2 \\
\hline & China & & 61 & 3 & & & 108 \\
\hline & Czech Rep & & 1 & & & & 1 \\
\hline & Dubai & & 2 & & & & 2 \\
\hline & Fiji & & 1 & & & & \\
\hline & France & & 0 & & & & 1 \\
\hline & Greece & & 0 & & & & 1 \\
\hline & Hong Kong & & 21 & & & & 31 \\
\hline
\end{tabular}




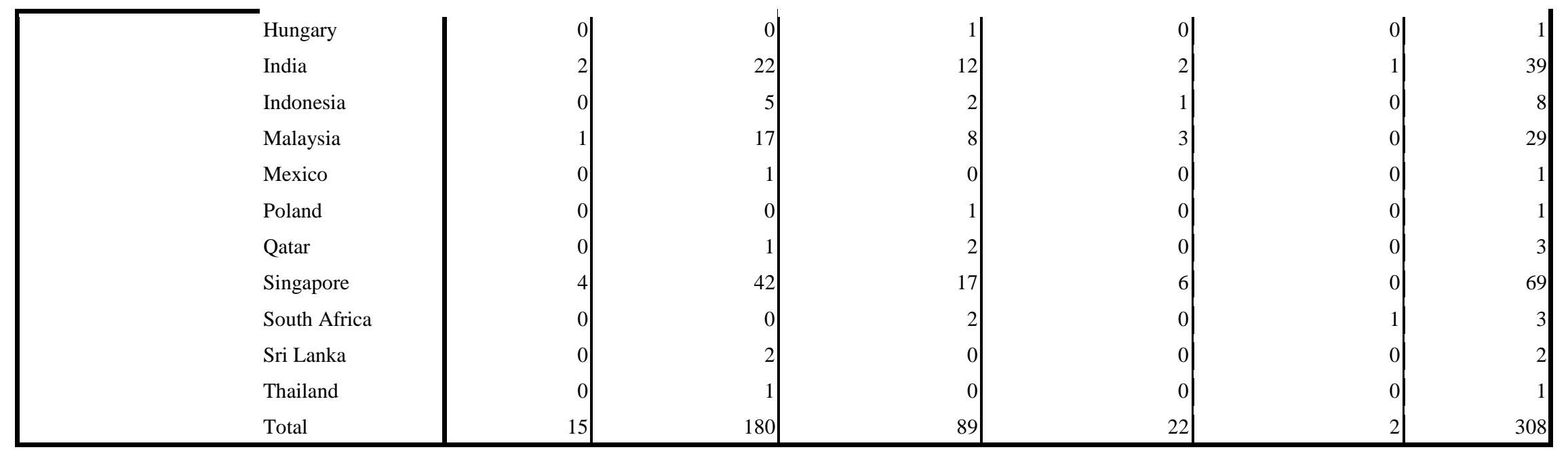

\section{Host Country \& Size (Staff)}

\begin{tabular}{|c|c|c|c|c|c|c|c|c|c|c|c|c|c|c|c|}
\hline & & \multicolumn{14}{|c|}{ Size (Staff) } \\
\hline & & $<500$ & $\begin{array}{l}501- \\
1000\end{array}$ & $\begin{array}{c}1001- \\
1500\end{array}$ & $\begin{array}{c}1501- \\
2000\end{array}$ & $\begin{array}{c}2001- \\
2500\end{array}$ & $\begin{array}{c}2501- \\
3000\end{array}$ & $\begin{array}{c}3001- \\
3500\end{array}$ & $\begin{array}{c}3501- \\
4000\end{array}$ & $\begin{array}{c}4001 \text { - } \\
4500\end{array}$ & $\begin{array}{c}4501- \\
5000\end{array}$ & $\begin{array}{c}5001- \\
5500\end{array}$ & $\begin{array}{c}5501- \\
6000\end{array}$ & $\begin{array}{c}7501- \\
8000\end{array}$ & Total \\
\hline \multirow[t]{10}{*}{ Host Country } & Australia & 0 & 0 & 0 & 1 & 0 & 0 & 0 & 0 & 0 & 0 & 0 & 0 & 0 & 1 \\
\hline & Bahrain & 0 & 0 & 0 & 1 & 0 & 0 & 0 & 2 & 0 & 0 & 0 & 0 & 0 & 3 \\
\hline & Canada & 0 & 1 & 0 & 0 & 0 & 1 & 0 & 0 & 0 & 0 & 0 & 0 & 0 & 2 \\
\hline & China & 14 & 22 & 18 & 14 & 10 & 16 & 4 & 7 & 0 & 2 & 1 & 0 & 0 & 108 \\
\hline & Czech Rep & 1 & 0 & 0 & 0 & 0 & 0 & 0 & 0 & 0 & 0 & 0 & 0 & 0 & 1 \\
\hline & Dubai & 0 & 1 & 0 & 1 & 0 & 0 & 0 & 0 & 0 & 0 & 0 & 0 & 0 & 2 \\
\hline & Fiji & 0 & 0 & 0 & 1 & 0 & 0 & 0 & 0 & 0 & 0 & 0 & 0 & 0 & 1 \\
\hline & France & 0 & 0 & 1 & 0 & 0 & 0 & 0 & 0 & 0 & 0 & 0 & 0 & 0 & 1 \\
\hline & Greece & 0 & 1 & 0 & 0 & 0 & 0 & 0 & 0 & 0 & 0 & 0 & 0 & 0 & 1 \\
\hline & $\begin{array}{l}\text { Hong } \\
\text { Kong }\end{array}$ & 4 & 6 & 7 & 3 & 1 & 4 & 2 & 3 & 0 & 1 & 0 & 0 & 0 & 31 \\
\hline
\end{tabular}




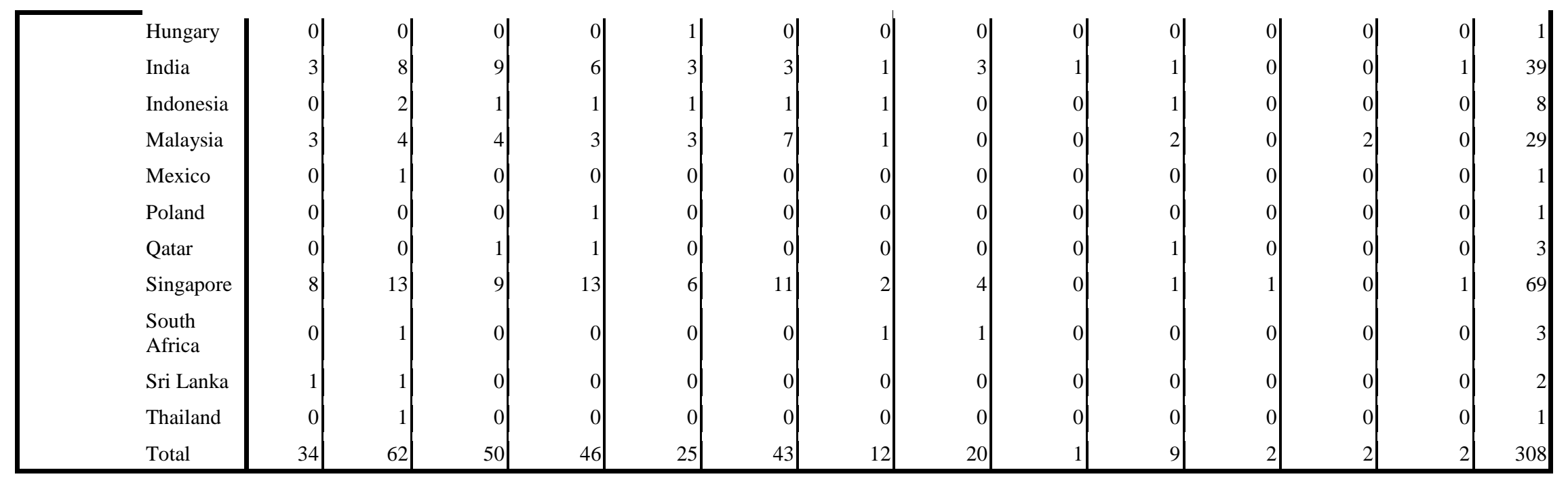

\section{Host Country \& Age}

\begin{tabular}{|c|c|c|c|c|c|c|c|c|c|}
\hline & & \multicolumn{8}{|c|}{ Age (Years) } \\
\hline & & $<10$ & $11-50$ & $51-100$ & $101-150$ & $151-200$ & $201-250$ & $251-300$ & Total \\
\hline \multirow[t]{11}{*}{ Host Country } & Australia & 0 & 0 & 0 & 1 & 0 & 0 & 0 & 1 \\
\hline & Bahrain & 0 & 1 & 0 & 1 & 1 & 0 & 0 & 3 \\
\hline & Canada & 0 & 2 & 0 & 0 & 0 & 0 & 0 & 2 \\
\hline & China & 1 & 35 & 18 & 27 & 23 & 3 & 1 & 108 \\
\hline & Czech Rep & 1 & 0 & 0 & 0 & 0 & 0 & 0 & 1 \\
\hline & Dubai & 0 & 1 & 1 & 0 & 0 & 0 & 0 & 2 \\
\hline & Fiji & 0 & 0 & 0 & 1 & 0 & 0 & 0 & 1 \\
\hline & France & 0 & 0 & 0 & 0 & 1 & 0 & 0 & 1 \\
\hline & Greece & 0 & 0 & 0 & 1 & 0 & 0 & 0 & 1 \\
\hline & Hong Kong & 1 & 9 & 8 & 5 & 7 & 0 & 1 & 31 \\
\hline & Hungary & 0 & 0 & 0 & 1 & 0 & 0 & 0 & 1 \\
\hline
\end{tabular}




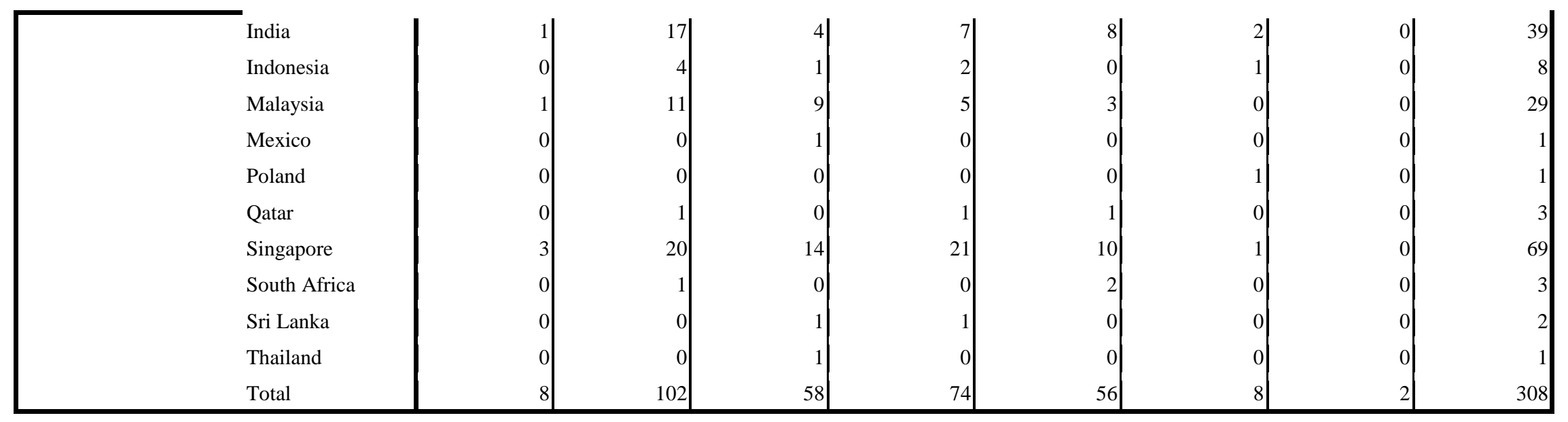

Host Country \& Worldwide Rankings

\begin{tabular}{|c|c|c|c|c|c|c|c|c|c|c|}
\hline & & \multicolumn{9}{|c|}{ World Ranking } \\
\hline & & $90^{\text {th }}$ percentile & $80^{\text {th }}$ percentile & $70^{\text {th }}$ percentile & $60^{\text {th }}$ percentile & $50^{\text {th }}$ percentile & $40^{\text {th }}$ percentile & $30^{\text {th }}$ percentile & $20^{\text {th }}$ percentile & Total \\
\hline \multirow[t]{12}{*}{ Host Country } & Australia & 0 & 1 & 0 & 0 & 0 & 0 & 0 & 0 & 1 \\
\hline & Bahrain & 0 & 0 & 1 & 1 & 0 & 1 & 0 & 0 & 3 \\
\hline & Canada & 0 & 0 & 0 & 0 & 2 & 0 & 0 & 0 & 2 \\
\hline & China & 3 & 6 & 11 & 17 & 33 & 22 & 15 & 1 & 108 \\
\hline & Czech Rep & 0 & 0 & 0 & 0 & 1 & 0 & 0 & 0 & 1 \\
\hline & Dubai & 0 & 0 & 0 & 1 & 0 & 0 & 1 & 0 & 2 \\
\hline & Fiji & 0 & 0 & 0 & 1 & 0 & 0 & 0 & 0 & 1 \\
\hline & France & 0 & 0 & 1 & 0 & 0 & 0 & 0 & 0 & 1 \\
\hline & Greece & 0 & 1 & 0 & 0 & 0 & 0 & 0 & 0 & 1 \\
\hline & Hong Kong & 3 & 2 & 1 & 5 & 6 & 10 & 4 & 0 & 31 \\
\hline & Hungary & 1 & 0 & 0 & 0 & 0 & 0 & 0 & 0 & 1 \\
\hline & India & 0 & 3 & 4 & 8 & 14 & 7 & 3 & 0 & 39 \\
\hline
\end{tabular}




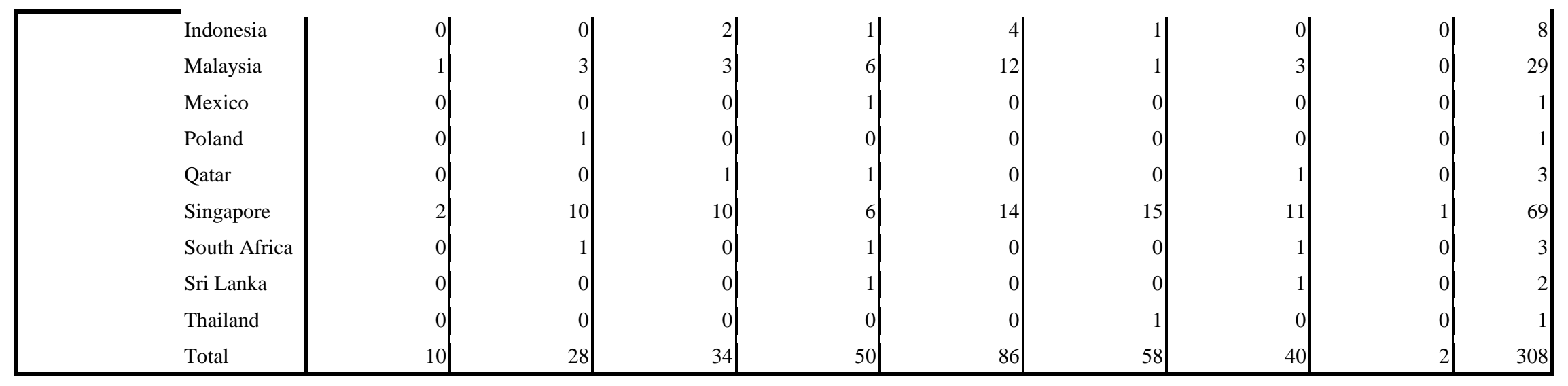

\section{Host Country \& Domestic Rankings}

\begin{tabular}{|c|c|c|c|c|c|c|c|c|c|c|c|c|}
\hline & & \multicolumn{11}{|c|}{ Domestic Ranking } \\
\hline & & $90^{\text {th }}$ percentile & $80^{\text {th }}$ percentile & $70^{\text {th }}$ percentil & $60^{\text {th }}$ percentile & $50^{\text {th }}$ percentil & & $10^{\text {th }}$ percentil & $30^{\text {th }}$ percentile & $20^{\text {th }}$ percentile & $90^{\text {th }}$ percentile & Total \\
\hline \multirow[t]{13}{*}{ Host Country } & Australia & 0 & & & 0 & & & & & 0 & 0 & \\
\hline & Bahrain & & & & 2 & & & & & 0 & 0 & \\
\hline & Canada & & & & 0 & s. & & & . & 0 & 0 & \\
\hline & China & & 14 & 1 & 20 & 3 & & 1 & 1 & 0 & 1 & 108 \\
\hline & Czech Rep & & & & 0 & 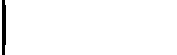 & 1 & & & 0 & 0 & 1 \\
\hline & Dubai & & c & & 1 & 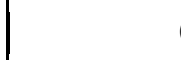 & & & ( & 0 & 0 & \\
\hline & & & c & & 0 & 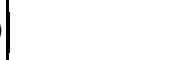 & & & ( & 0 & 0 & \\
\hline & France & & c & 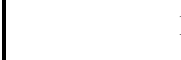 & 1 & 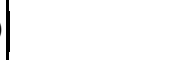 & & & c & 0 & 0 & 1 \\
\hline & Greece & 0 & 1 & & 0 & & & & c & 0 & 0 & \\
\hline & Hong Kong & & 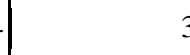 & & 3 & 1 & & & 3 & 1 & 0 & 31 \\
\hline & Hungary & & 1 & & 0 & & & & & 0 & 0 & 1 \\
\hline & India & & 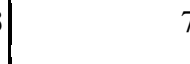 & & $\mathrm{s}$ & & 9 & & & 0 & 0 & 35 \\
\hline & Indonesia & c & | & 1 & 0 & & 4 & & c & 0 & 0 & \\
\hline
\end{tabular}




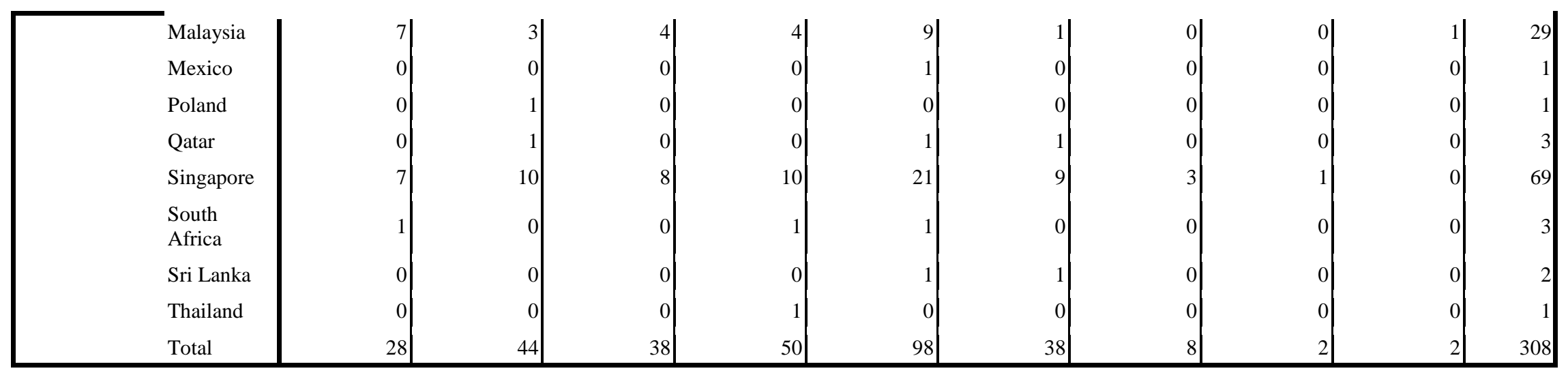

Host Country \& Mode of Entry (Continuous Measure)

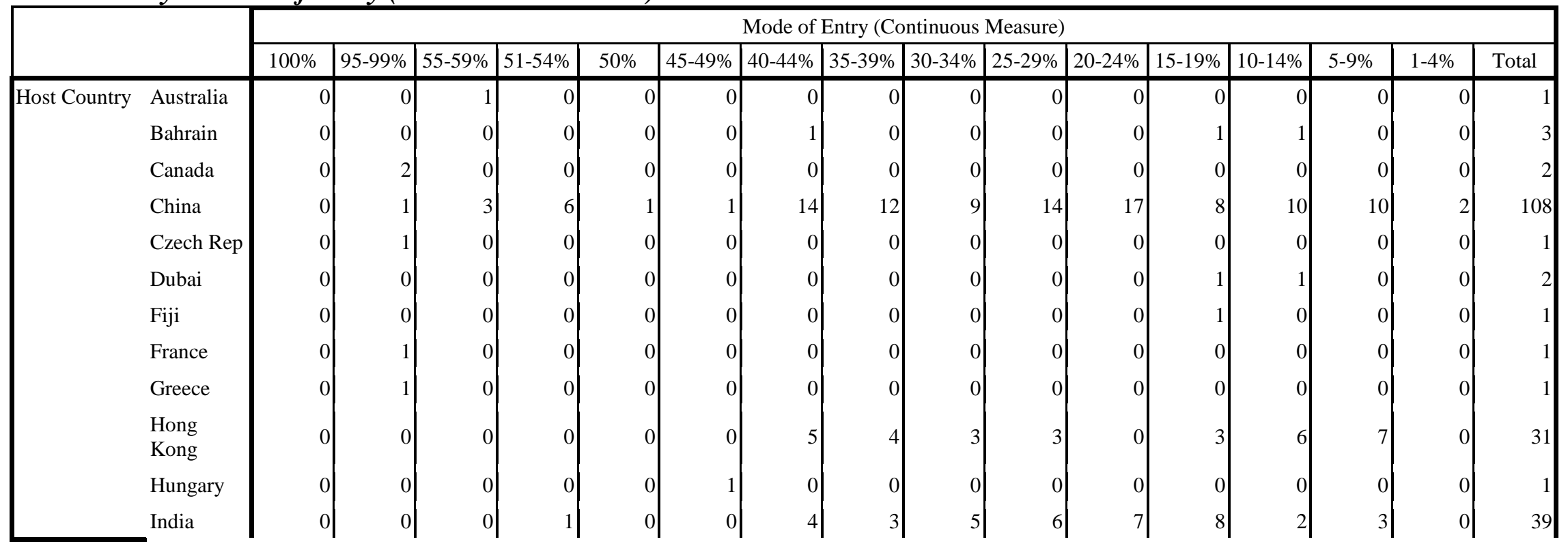

428 


\begin{tabular}{|c|c|c|c|c|c|c|c|c|c|c|c|c|c|c|c|c|}
\hline Indonesia & 0 & 0 & 0 & 0 & 0 & 0 & 2 & 0 & 1 & 3 & 2 & 0 & 0 & 0 & 0 & 8 \\
\hline Malaysia & 0 & 2 & 1 & 3 & 1 & 1 & 1 & 3 & 3 & 4 & 2 & 1 & 6 & 1 & 0 & 29 \\
\hline Mexico & 0 & 1 & 0 & 0 & 0 & 0 & 0 & 0 & 0 & 0 & 0 & 0 & 0 & 0 & 0 & 1 \\
\hline Poland & 0 & 0 & 0 & 0 & 1 & 0 & 0 & 0 & 0 & 0 & 0 & 0 & 0 & 0 & 0 & 1 \\
\hline Qatar & 0 & 0 & 0 & 0 & 0 & 0 & 0 & 0 & 1 & 0 & 0 & 0 & 0 & 2 & 0 & 3 \\
\hline Singapore & 0 & 2 & 0 & 1 & 1 & 0 & 8 & 9 & 10 & 11 & 4 & 7 & 10 & 6 & 0 & 69 \\
\hline $\begin{array}{l}\text { South } \\
\text { Africa }\end{array}$ & 1 & 0 & 0 & 0 & 0 & 0 & 0 & 0 & 1 & 0 & 1 & 0 & 0 & 0 & 0 & 3 \\
\hline Sri Lanka & 0 & 0 & 0 & 0 & 0 & 0 & 0 & 0 & 1 & 0 & 0 & 0 & 1 & 0 & 0 & 2 \\
\hline Thailand & 0 & 1 & 0 & 0 & 0 & 0 & 0 & 0 & 0 & 0 & 0 & 0 & 0 & 0 & 0 & 1 \\
\hline Total & 1 & 12 & 5 & 11 & 4 & 3 & 35 & 31 & 34 & 41 & 33 & 30 & 37 & 29 & 2 & 308 \\
\hline
\end{tabular}

\section{Host Country \& Mode of Entry (Categorical Measure)}

\begin{tabular}{|c|c|c|c|c|c|c|c|}
\hline & & \multicolumn{6}{|c|}{ Mode of Entry (Categorical Measure) } \\
\hline & & Non-equity & $\begin{array}{c}\text { Joint venture (minority } \\
\text { equity) }\end{array}$ & $\begin{array}{c}\text { Joint venture (50/50 } \\
\text { equity) }\end{array}$ & $\begin{array}{c}\text { Joint venture (majority } \\
\text { equity) }\end{array}$ & Sole ownership & Total \\
\hline Host Country & Australia & 0 & 0 & c & 1 & 0 & 1 \\
\hline & Bahrain & 3 & 0 & c & 0 & 0 & 3 \\
\hline & Canada & 0 & 0 & $c$ & 0 & 2 & 2 \\
\hline & China & 96 & 1 & 1 & 9 & 1 & 108 \\
\hline & Czech Rep & 0 & 0 & c & 0 & 1 & 1 \\
\hline & Dubai & 2 & 0 & $c$ & 0 & 0 & 2 \\
\hline & Fiji & 1 & 0 & c & 0 & 0 & 1 \\
\hline & France & 0 & 0 & $c$ & 0 & 1 & 1 \\
\hline & Greece & 0 & 0 & c & 0 & 1 & 1 \\
\hline & Hong Kong & 31 & 0 & c & 0 & 0 & 31 \\
\hline & Hungary & 0 & 1 & c & 0 & 0 & 1 \\
\hline
\end{tabular}




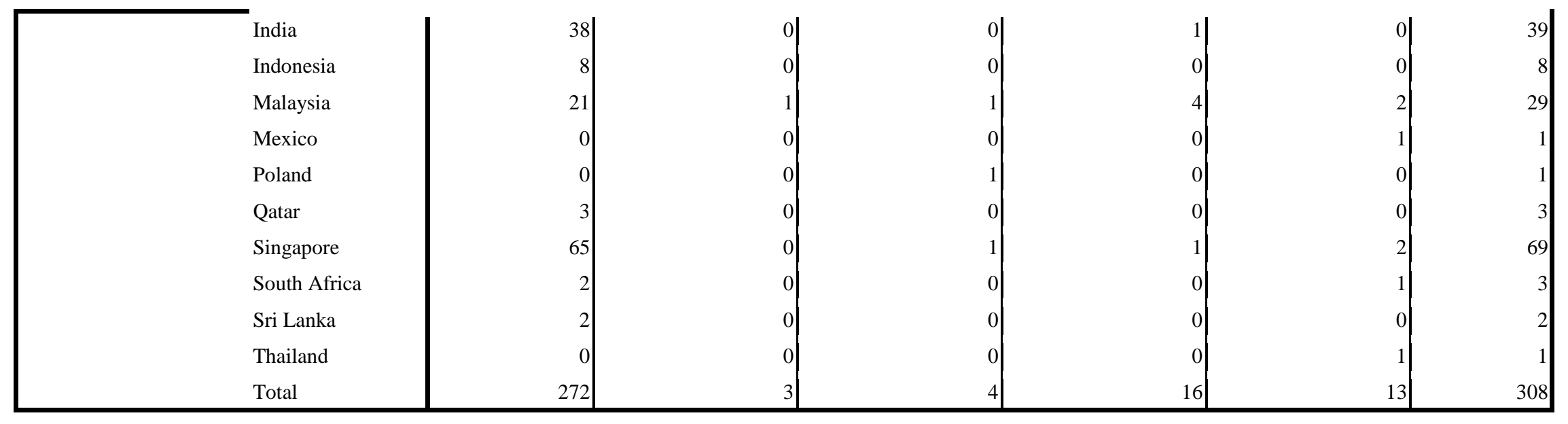

Academic Discipline \& Programme Level

\begin{tabular}{|c|c|c|c|c|c|c|c|c|c|c|}
\hline & & \multicolumn{9}{|c|}{ Programme Level } \\
\hline & & $\begin{array}{c}\text { Professional } \\
\text { Doctorates }\end{array}$ & $\begin{array}{c}\text { Master's by } \\
\text { coursework } \\
\text { only }\end{array}$ & $\begin{array}{c}\text { Master's by } \\
\text { both } \\
\text { coursework } \\
\text { and research }\end{array}$ & \begin{tabular}{|c} 
Undergraduate \\
degree
\end{tabular} & Diploma & $\begin{array}{l}\text { Associate } \\
\text { Degree }\end{array}$ & Certificate & $\begin{array}{c}\text { Executive/Professional } \\
\text { short programme }\end{array}$ & Total \\
\hline \multirow[t]{6}{*}{ Academic Discipline } & $\begin{array}{l}\text { Arts and Social } \\
\text { Sciences }\end{array}$ & 1 & 0 & 1 & 45 & 2 & 0 & 0 & 0 & 49 \\
\hline & $\begin{array}{l}\text { Business and } \\
\text { Management }\end{array}$ & 3 & 20 & 7 & 88 & 14 & 7 & 1 & 0 & 140 \\
\hline & Engineering & 0 & 9 & 0 & 34 & 3 & 1 & 1 & 0 & 48 \\
\hline & $\begin{array}{l}\text { Sciences (including } \\
\text { Life Sciences) }\end{array}$ & 0 & 5 & 0 & 18 & 1 & 3 & 0 & 1 & 28 \\
\hline & Law & 1 & 1 & 0 & 12 & 0 & 1 & 0 & 0 & 15 \\
\hline & $\begin{array}{l}\text { Information } \\
\text { Technology (including } \\
\text { Computer Science) }\end{array}$ & 0 & 1 & 0 & 7 & 1 & 1 & 1 & 0 & 11 \\
\hline
\end{tabular}




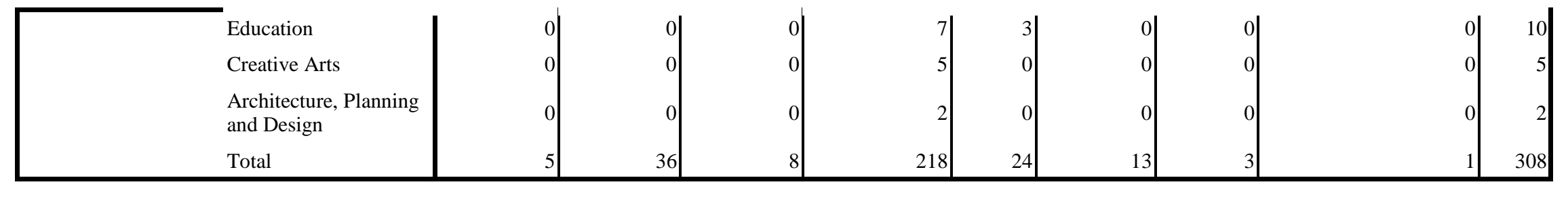

Academic Discipline \& Year of Entry

\begin{tabular}{|c|c|c|c|c|c|c|c|c|c|c|c|}
\hline \multicolumn{12}{|c|}{ Year of Entry } \\
\hline & & 1999 & 2000 & 2001 & 2002 & 2003 & 2004 & 2005 & 2006 & 2007 & Total \\
\hline \multirow[t]{10}{*}{ Academic Discipline } & Arts and Social Sciences & 6 & 7 & 9 & 2 & 3 & 4 & 8 & 7 & 3 & 49 \\
\hline & Business and Management & 19 & 12 & 18 & 10 & 8 & 23 & 19 & 14 & 17 & 140 \\
\hline & Engineering & 13 & 6 & 5 & 2 & 5 & 6 & 7 & 2 & 2 & 48 \\
\hline & $\begin{array}{l}\text { Sciences (including Life } \\
\text { Sciences) }\end{array}$ & 6 & 1 & 3 & 2 & 4 & 4 & 5 & 1 & 2 & 28 \\
\hline & Law & 1 & 3 & 4 & 0 & 2 & 1 & 1 & 3 & 0 & 15 \\
\hline & $\begin{array}{l}\text { Information Technology } \\
\text { (including Computer } \\
\text { Science) }\end{array}$ & 3 & 3 & 0 & 0 & 2 & 1 & 1 & 0 & 1 & 11 \\
\hline & Education & 2 & 0 & 5 & 0 & 1 & 0 & 1 & 0 & 1 & 10 \\
\hline & Creative Arts & 1 & 1 & 0 & 0 & 0 & 1 & 0 & 0 & 2 & 5 \\
\hline & $\begin{array}{l}\text { Architecture, Planning and } \\
\text { Design }\end{array}$ & 0 & 0 & 0 & 0 & 1 & 0 & 1 & 0 & 0 & 2 \\
\hline & Total & 51 & 33 & 44 & 16 & 26 & 40 & 43 & 27 & 28 & 308 \\
\hline
\end{tabular}

Academic Discipline \& Size (Undergraduate Students)

\begin{tabular}{|c|c|c|c|c|c|c|c|c|c|c|c|}
\hline \multirow{2}{*}{ 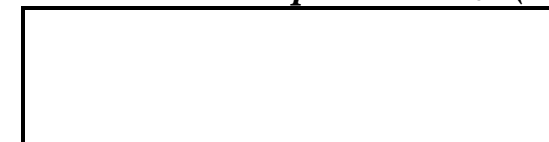 } & \multicolumn{11}{|c|}{ uate Stud } \\
\hline & $<1000$ & $\begin{array}{c}1001- \\
5000\end{array}$ & $\begin{array}{l}5001- \\
10000\end{array}$ & $\begin{array}{c}10001- \\
15000\end{array}$ & $\begin{array}{c}15001- \\
20000\end{array}$ & $\begin{array}{c}20001- \\
25000\end{array}$ & $\begin{array}{c}25001- \\
30000\end{array}$ & $\begin{array}{c}30001- \\
35000\end{array}$ & $\begin{array}{c}35001- \\
40000\end{array}$ & $\begin{array}{c}45001- \\
50000\end{array}$ & Total \\
\hline
\end{tabular}




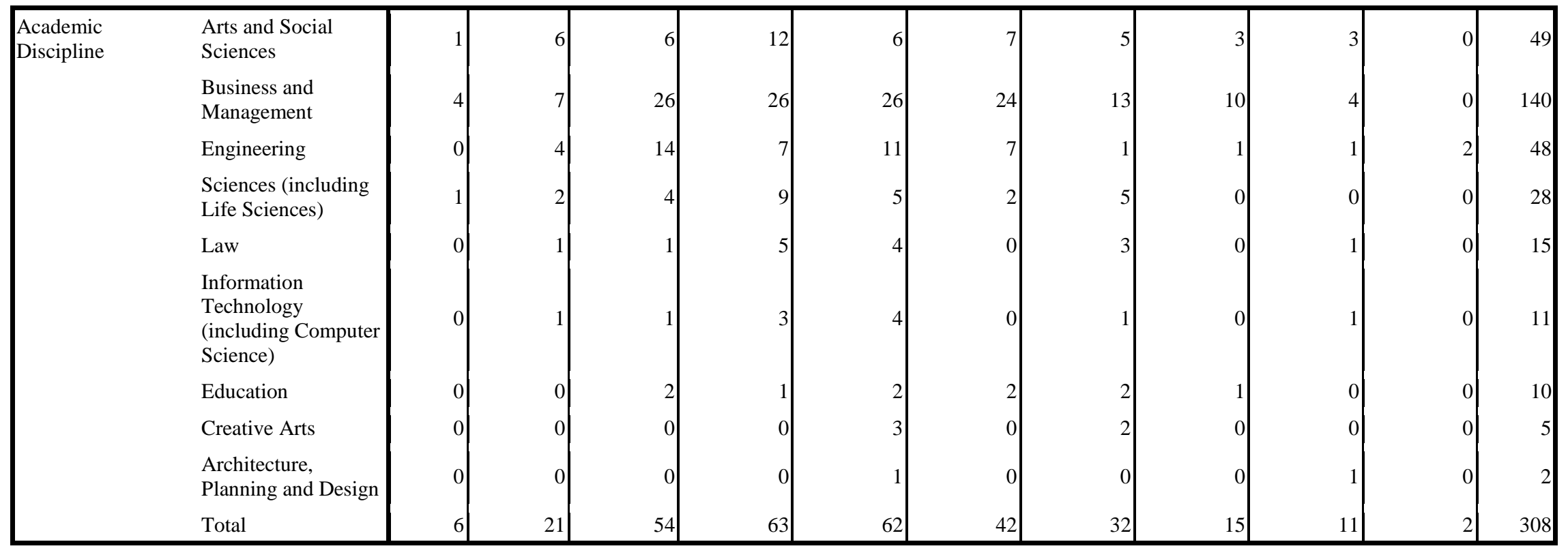

\section{Academic Discipline \& Size (Postgraduate Students)}

\begin{tabular}{|c|c|c|c|c|c|c|c|}
\hline \multicolumn{8}{|c|}{ Size (Postgraduate Students) } \\
\hline & & $<1000$ & $1001-5000$ & $5001-10000$ & $10001-15000$ & $15001-20000$ & Total \\
\hline \multirow[t]{6}{*}{ Academic Discipline } & Arts and Social Sciences & 2 & 32 & 10 & 4 & 1 & 49 \\
\hline & Business and Management & 4 & 85 & 40 & 10 & 1 & 140 \\
\hline & Engineering & 3 & 25 & 15 & 5 & 0 & 48 \\
\hline & $\begin{array}{l}\text { Sciences (including Life } \\
\text { Sciences) }\end{array}$ & 2 & 17 & 7 & 2 & 0 & 28 \\
\hline & Law & 1 & 9 & 5 & 0 & 0 & 15 \\
\hline & $\begin{array}{l}\text { Information Technology } \\
\text { (including Computer Science) }\end{array}$ & 1 & 7 & 3 & 0 & 0 & 11 \\
\hline
\end{tabular}




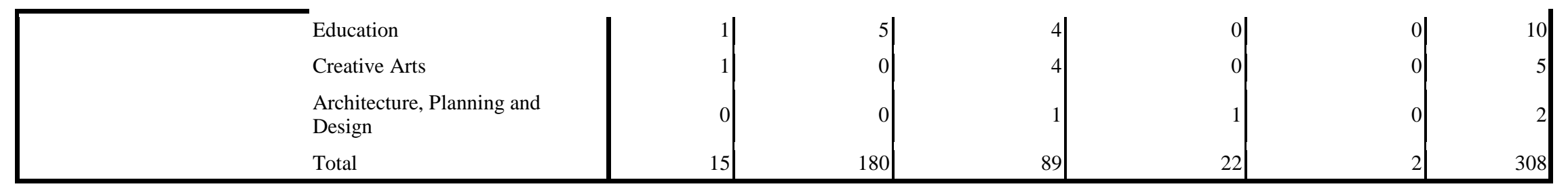

\section{Academic Discipline \& Size (Staff)}

\begin{tabular}{|c|c|c|c|c|c|c|c|c|c|c|c|c|c|c|c|}
\hline & \multicolumn{14}{|c|}{ Size (Staff) } \\
\hline & & $<500$ & $\begin{array}{l}501- \\
1000\end{array}$ & $\begin{array}{l}1001- \\
1500\end{array}$ & $\begin{array}{l}1501- \\
2000\end{array}$ & $\begin{array}{l}2001- \\
2500\end{array}$ & $\begin{array}{l}2501- \\
3000\end{array}$ & $\begin{array}{c}3001- \\
3500\end{array}$ & $\begin{array}{l}3501- \\
4000\end{array}$ & $\begin{array}{l}4001- \\
4500\end{array}$ & $\begin{array}{l}4501- \\
5000\end{array}$ & $\begin{array}{l}5001- \\
5500\end{array}$ & $\begin{array}{l}5501- \\
6000\end{array}$ & $\begin{array}{l}7501- \\
8000\end{array}$ & Total \\
\hline \multirow[t]{10}{*}{$\begin{array}{l}\text { Academic } \\
\text { Discipline }\end{array}$} & $\begin{array}{l}\text { Arts and Social } \\
\text { Sciences }\end{array}$ & 7 & 12 & 8 & 3 & 3 & 7 & 3 & 4 & 1 & 1 & 0 & 0 & 0 & 49 \\
\hline & $\begin{array}{l}\text { Business and } \\
\text { Management }\end{array}$ & 14 & 27 & 26 & 17 & 18 & 19 & 6 & 6 & 0 & 4 & 0 & 1 & 2 & 140 \\
\hline & Engineering & 5 & 15 & 3 & 9 & 3 & 5 & 1 & 2 & 0 & 2 & 2 & 1 & 0 & 48 \\
\hline & $\begin{array}{l}\text { Sciences } \\
\text { (including Life } \\
\text { Sciences) }\end{array}$ & 4 & 6 & 6 & 4 & 0 & 4 & 1 & 2 & 0 & 1 & 0 & 0 & 0 & 28 \\
\hline & Law & 1 & 1 & 5 & 4 & 1 & 1 & 0 & 1 & 0 & 1 & 0 & 0 & 0 & 15 \\
\hline & $\begin{array}{l}\text { Information } \\
\text { Technology } \\
\text { (including } \\
\text { Computer } \\
\text { Science) }\end{array}$ & 1 & 1 & 0 & 4 & 0 & 4 & 1 & 0 & 0 & 0 & 0 & 0 & 0 & 11 \\
\hline & Education & 1 & 0 & 2 & 2 & 0 & 3 & 0 & 2 & 0 & 0 & 0 & 0 & 0 & 10 \\
\hline & Creative Arts & 1 & 0 & 0 & 3 & 0 & 0 & 0 & 1 & 0 & 0 & 0 & 0 & 0 & 5 \\
\hline & $\begin{array}{l}\text { Architecture, } \\
\text { Planning and } \\
\text { Design }\end{array}$ & 0 & 0 & 0 & 0 & 0 & 0 & 0 & 2 & 0 & 0 & 0 & 0 & 0 & 2 \\
\hline & Total & 34 & 62 & 50 & 46 & 25 & 43 & 12 & 20 & 1 & 9 & 2 & 2 & 2 & 308 \\
\hline
\end{tabular}


Academic Discipline \& Age

\begin{tabular}{|c|c|c|c|c|c|c|c|c|c|}
\hline & & \multicolumn{8}{|c|}{ Age (Years) } \\
\hline & & $<10$ & $11-50$ & $51-100$ & $101-150$ & $151-200$ & $201-250$ & $251-300$ & Total \\
\hline \multirow[t]{10}{*}{ Academic Discipline } & Arts and Social Sciences & 2 & 21 & 5 & 10 & 9 & 1 & 1 & 49 \\
\hline & Business and Management & 5 & 37 & 31 & 36 & 28 & 2 & 1 & 140 \\
\hline & Engineering & 1 & 18 & 9 & 11 & 6 & 3 & 0 & 48 \\
\hline & $\begin{array}{l}\text { Sciences (including Life } \\
\text { Sciences) }\end{array}$ & 0 & 7 & 5 & 8 & 6 & 2 & 0 & 28 \\
\hline & Law & 0 & 7 & 2 & 4 & 2 & 0 & 0 & 15 \\
\hline & $\begin{array}{l}\text { Information Technology } \\
\text { (including Computer Science) }\end{array}$ & 0 & 4 & 3 & 3 & 1 & 0 & 0 & 11 \\
\hline & Education & 0 & 6 & 3 & 1 & 0 & 0 & 0 & 10 \\
\hline & Creative Arts & 0 & 2 & 0 & 0 & 3 & 0 & 0 & 5 \\
\hline & $\begin{array}{l}\text { Architecture, Planning and } \\
\text { Design }\end{array}$ & 0 & 0 & 0 & 1 & 1 & 0 & 0 & 2 \\
\hline & Total & 8 & 102 & 58 & 74 & 56 & 8 & 2 & 308 \\
\hline
\end{tabular}

Academic Discipline \& Worldwide Rankings

\begin{tabular}{|c|c|c|c|c|c|c|c|c|c|c|}
\hline & \multicolumn{9}{|c|}{ World Ranking } \\
\hline & & $\begin{array}{c}90^{\text {th }} \\
\text { percentile }\end{array}$ & $\begin{array}{c}80^{\text {th }} \\
\text { percentile }\end{array}$ & $\begin{array}{c}70^{\text {th }} \\
\text { percentile }\end{array}$ & $\begin{array}{c}60^{\text {th }} \\
\text { percentile }\end{array}$ & $\begin{array}{c}50^{\text {th }} \\
\text { percentile }\end{array}$ & $\begin{array}{c}40^{\text {th }} \\
\text { percentile }\end{array}$ & $\begin{array}{c}30^{\text {th }} \\
\text { percentile }\end{array}$ & $\begin{array}{c}20^{\text {th }} \\
\text { percentile }\end{array}$ & Total \\
\hline \multirow[t]{4}{*}{ Academic Discipline } & $\begin{array}{l}\text { Arts and Social } \\
\text { Sciences }\end{array}$ & 2 & 3 & 7 & 8 & 18 & 5 & 5 & 1 & 49 \\
\hline & $\begin{array}{l}\text { Business and } \\
\text { Management }\end{array}$ & 6 & 12 & 14 & 21 & 41 & 29 & 16 & 1 & 140 \\
\hline & Engineering & 1 & 4 & 7 & 5 & 12 & 12 & 7 & 0 & 48 \\
\hline & $\begin{array}{l}\text { Sciences (including } \\
\text { Life Sciences) }\end{array}$ & 0 & 2 & 3 & 8 & 5 & 5 & 5 & 0 & 28 \\
\hline
\end{tabular}




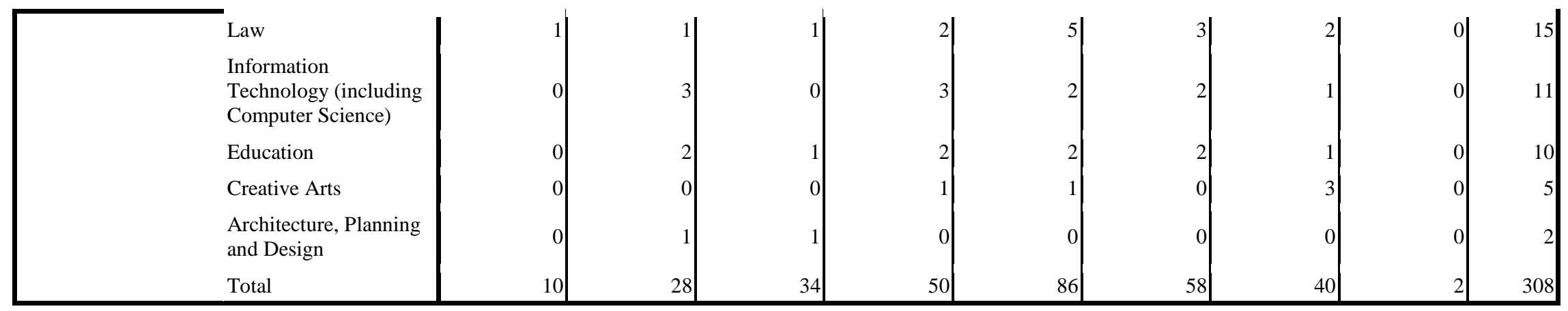

\section{Academic Discipline \& Domestic Rankings}

\begin{tabular}{|c|c|c|c|c|c|c|c|c|c|c|c|}
\hline & \multicolumn{10}{|c|}{ Domestic Ranking } \\
\hline & & $\begin{array}{c}90^{\text {th }} \\
\text { percentile }\end{array}$ & $\begin{array}{c}80^{\text {th }} \\
\text { percentile }\end{array}$ & $\begin{array}{c}70^{\text {th }} \\
\text { percentile }\end{array}$ & $\begin{array}{c}60^{\text {th }} \\
\text { percentile }\end{array}$ & $\begin{array}{c}50^{\text {th }} \\
\text { percentile }\end{array}$ & $\begin{array}{c}40^{\text {th }} \\
\text { percentile }\end{array}$ & $\begin{array}{c}30^{\text {th }} \\
\text { percentile }\end{array}$ & $\begin{array}{c}20^{\text {th }} \\
\text { percentile }\end{array}$ & $\begin{array}{c}90^{\text {th }} \\
\text { percentile }\end{array}$ & Total \\
\hline \multirow[t]{10}{*}{$\begin{array}{l}\text { Academic } \\
\text { Discipline }\end{array}$} & $\begin{array}{l}\text { Arts and Social } \\
\text { Sciences }\end{array}$ & & & & 10 & 15 & 7 & 0 & 0 & 1 & 49 \\
\hline & $\begin{array}{l}\text { Business and } \\
\text { Management }\end{array}$ & 12 & 24 & 15 & 22 & 48 & 15 & 2 & 2 & 0 & 140 \\
\hline & Engineering & 2 & 6 & 7 & 7 & 16 & 8 & 1 & 0 & 1 & 48 \\
\hline & $\begin{array}{l}\text { Sciences (including } \\
\text { Life Sciences) }\end{array}$ & 1 & 4 & 6 & 6 & 5 & 3 & 3 & 0 & 0 & 28 \\
\hline & Law & 2 & 2 & 1 & 2 & 5 & 1 & 2 & 0 & 0 & 15 \\
\hline & $\begin{array}{l}\text { Information } \\
\text { Technology } \\
\text { (including Computer } \\
\text { Science) }\end{array}$ & 3 & 0 & 2 & 2 & 2 & 2 & 0 & 0 & 0 & 11 \\
\hline & Education & 2 & 1 & 0 & 1 & 5 & 1 & 0 & 0 & 0 & 10 \\
\hline & Creative Arts & 0 & 0 & 2 & 0 & 2 & 1 & 0 & 0 & 0 & 5 \\
\hline & $\begin{array}{l}\text { Architecture, } \\
\text { Planning and Design }\end{array}$ & 1 & 1 & 0 & 0 & 0 & 0 & 0 & 0 & 0 & 2 \\
\hline & Total & 28 & 44 & 38 & 50 & 98 & 38 & 8 & 2 & 2 & 308 \\
\hline
\end{tabular}


Academic Discipline \& Mode of Entry (Continuous Measure)

\begin{tabular}{|c|c|c|c|c|c|c|c|c|c|c|c|c|c|c|c|c|c|}
\hline & & \multicolumn{16}{|c|}{ Mode of Entry (Continuous Measure) } \\
\hline & & $100 \%$ & $\begin{array}{c}95- \\
99 \%\end{array}$ & $\begin{array}{c}55- \\
59 \%\end{array}$ & $\begin{array}{c}51- \\
54 \%\end{array}$ & $50 \%$ & $\begin{array}{c}45- \\
49 \% \\
\end{array}$ & $\begin{array}{c}40- \\
44 \%\end{array}$ & $\begin{array}{c}35- \\
39 \%\end{array}$ & $\begin{array}{c}30- \\
34 \%\end{array}$ & $\begin{array}{c}25- \\
29 \%\end{array}$ & $\begin{array}{c}20- \\
24 \%\end{array}$ & $\begin{array}{c}15- \\
19 \%\end{array}$ & $\begin{array}{c}10- \\
14 \%\end{array}$ & $5-9 \%$ & $1-4 \%$ & Total \\
\hline \multirow[t]{10}{*}{$\begin{array}{l}\text { Academic } \\
\text { Discipline }\end{array}$} & $\begin{array}{l}\text { Arts and Social } \\
\text { Sciences }\end{array}$ & 1 & 2 & 0 & 1 & 0 & 0 & 4 & 6 & 3 & 9 & 6 & 6 & 7 & 4 & 0 & 49 \\
\hline & $\begin{array}{l}\text { Business and } \\
\text { Management }\end{array}$ & 0 & 7 & 2 & 6 & 4 & 1 & 14 & 11 & 20 & 17 & 20 & 14 & 13 & 10 & 1 & 140 \\
\hline & Engineering & 0 & 2 & 1 & 2 & 0 & 2 & 5 & 6 & 4 & 5 & 3 & 2 & 8 & 8 & 0 & 48 \\
\hline & $\begin{array}{l}\text { Sciences (including } \\
\text { Life Sciences) }\end{array}$ & 0 & 0 & 1 & 2 & 0 & 0 & 3 & 1 & 3 & 6 & 1 & 3 & 3 & 4 & 1 & 28 \\
\hline & Law & 0 & 0 & 0 & 0 & 0 & 0 & 4 & 4 & 2 & 1 & 2 & 1 & 1 & 0 & 0 & 15 \\
\hline & $\begin{array}{l}\text { Information } \\
\text { Technology } \\
\text { (including } \\
\text { Computer Science) }\end{array}$ & 0 & 0 & 0 & 0 & 0 & 0 & 4 & 0 & 1 & 3 & 0 & 2 & 1 & 0 & 0 & 11 \\
\hline & Education & 0 & 1 & 0 & 0 & 0 & 0 & 1 & 2 & 1 & 0 & 0 & 2 & 1 & 2 & 0 & 10 \\
\hline & Creative Arts & 0 & 0 & 1 & 0 & 0 & 0 & 0 & 0 & 0 & 0 & 1 & 0 & 3 & 0 & 0 & 5 \\
\hline & $\begin{array}{l}\text { Architecture, } \\
\text { Planning and } \\
\text { Design }\end{array}$ & 0 & 0 & 0 & 0 & 0 & 0 & 0 & 1 & 0 & 0 & 0 & 0 & 0 & 1 & 0 & 2 \\
\hline & Total & 1 & 12 & 5 & 11 & 4 & 3 & 35 & 31 & 34 & 41 & 33 & 30 & 37 & 29 & 2 & 308 \\
\hline
\end{tabular}

Academic Discipline \& Mode of Entry (Categorical Measure)

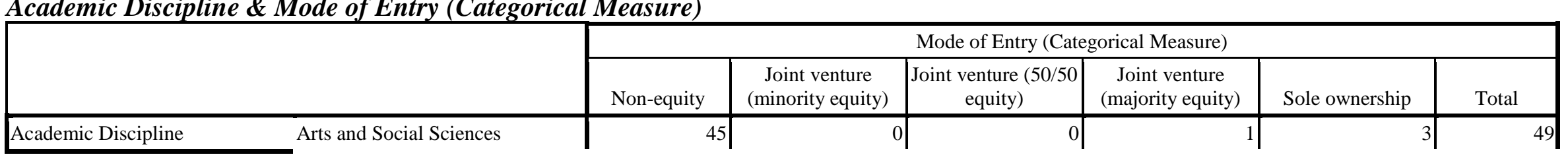




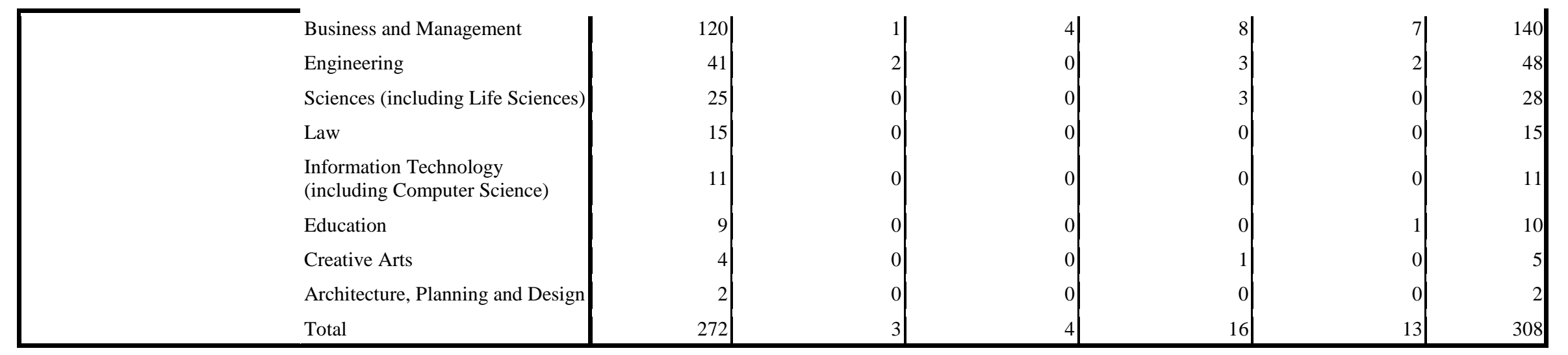

\section{Programme Level \& Year of Entry}

\begin{tabular}{|c|c|c|c|c|c|c|c|c|c|c|c|}
\hline & & \multicolumn{10}{|c|}{ Year of Entry } \\
\hline & & 1999 & 2000 & 2001 & 2002 & 2003 & 2004 & 2005 & 2006 & 2007 & Total \\
\hline \multirow[t]{9}{*}{ Programme Level } & Professional Doctorates & 1 & 0 & 2 & 0 & 0 & 1 & 1 & 0 & 0 & 5 \\
\hline & Master's by coursework only & 10 & 1 & 3 & 1 & 4 & 10 & 3 & 1 & 3 & 36 \\
\hline & $\begin{array}{l}\text { Master's by both coursework } \\
\text { and research }\end{array}$ & 0 & 1 & 3 & 0 & 0 & 2 & 1 & 1 & 0 & 8 \\
\hline & Undergraduate degree & 34 & 25 & 32 & 11 & 17 & 24 & 32 & 22 & 21 & 218 \\
\hline & Diploma & 2 & 3 & 3 & 3 & 2 & 2 & 4 & 2 & 3 & 24 \\
\hline & Associate Degree & 4 & 2 & 0 & 1 & 3 & 0 & 2 & 1 & 0 & 13 \\
\hline & Certificate & 0 & 1 & 1 & 0 & 0 & 0 & 0 & 0 & 1 & 3 \\
\hline & $\begin{array}{l}\text { Executive/Professional short } \\
\text { programme }\end{array}$ & 0 & 0 & 0 & 0 & 0 & 1 & 0 & 0 & 0 & 1 \\
\hline & Total & 51 & 33 & 44 & 16 & 26 & 40 & 43 & 27 & 28 & 308 \\
\hline
\end{tabular}


Programme Level \& Size (Undergraduate Students)

\begin{tabular}{|c|c|c|c|c|c|c|c|c|c|c|c|c|}
\hline & \multicolumn{11}{|c|}{ Size (Undergraduate Students) } \\
\hline & & $<1000$ & $\begin{array}{c}1001- \\
5000\end{array}$ & $\begin{array}{l}5001- \\
10000\end{array}$ & $\begin{array}{l}10001- \\
15000\end{array}$ & $\begin{array}{l}15001- \\
20000\end{array}$ & $\begin{array}{l}20001- \\
25000\end{array}$ & $\begin{array}{l}25001- \\
30000\end{array}$ & $\begin{array}{l}30001- \\
35000\end{array}$ & $\begin{array}{l}35001- \\
40000\end{array}$ & $\begin{array}{l}45001- \\
50000\end{array}$ & Total \\
\hline \multirow[t]{9}{*}{ Programme Level } & Professional Doctorates & 0 & 0 & 2 & 0 & 2 & 1 & 0 & 0 & 0 & 0 & 5 \\
\hline & $\begin{array}{l}\text { Master's by coursework } \\
\text { only }\end{array}$ & 1 & 0 & 12 & 6 & 6 & 2 & 7 & 2 & 0 & 0 & 36 \\
\hline & $\begin{array}{l}\text { Master's by both } \\
\text { coursework and research }\end{array}$ & 0 & 1 & 0 & 2 & 2 & 1 & 1 & 1 & 0 & 0 & 8 \\
\hline & Undergraduate degree & 4 & 17 & 32 & 45 & 43 & 32 & 21 & 11 & 11 & 2 & 218 \\
\hline & Diploma & 1 & 1 & 6 & 6 & 3 & 5 & 1 & 1 & 0 & 0 & 24 \\
\hline & Associate Degree & 0 & 0 & 1 & 3 & 6 & 1 & 2 & 0 & 0 & 0 & 13 \\
\hline & Certificate & 0 & 1 & 1 & 1 & 0 & 0 & 0 & 0 & 0 & 0 & 3 \\
\hline & $\begin{array}{l}\text { Executive/Professional } \\
\text { short programme }\end{array}$ & 0 & 1 & 0 & 0 & 0 & 0 & 0 & 0 & 0 & 0 & 1 \\
\hline & Total & 6 & 21 & 54 & 63 & 62 & 42 & 32 & 15 & 11 & 2 & 308 \\
\hline
\end{tabular}

Programme Level \& Size (Postgraduate Students)

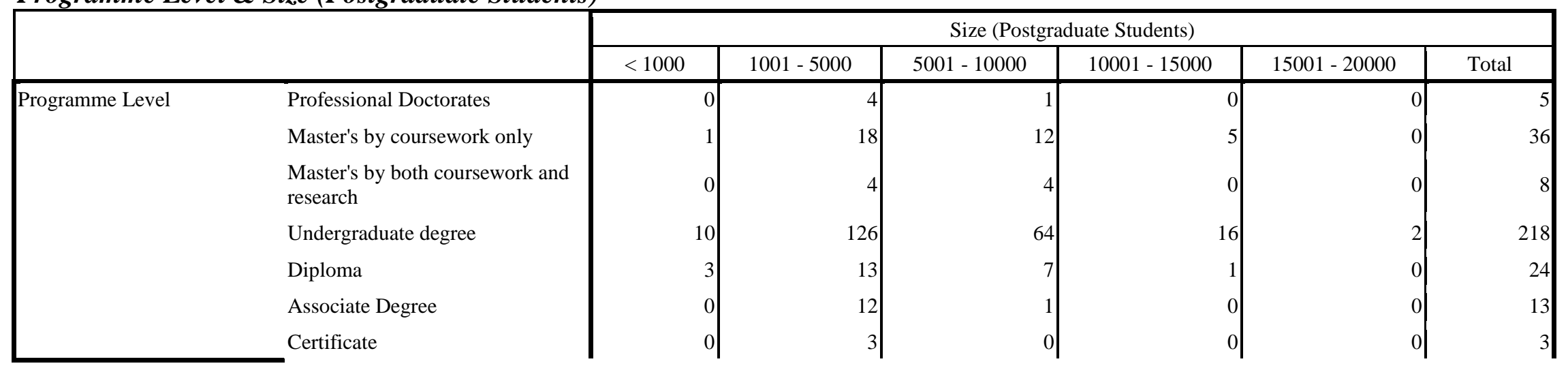




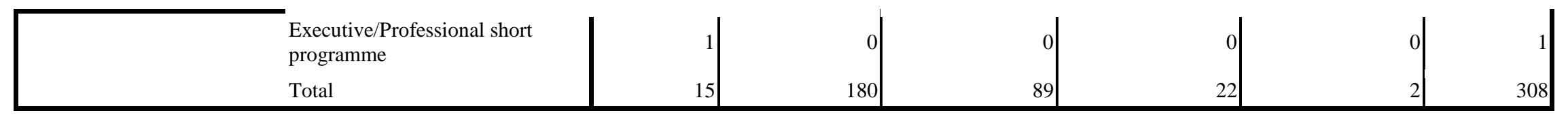

Programme Level \& Size (Staff)

\begin{tabular}{|c|c|c|c|c|c|c|c|c|c|c|c|c|c|c|c|}
\hline & & \multicolumn{14}{|c|}{ Size (Staff) } \\
\hline & & $<500$ & $\begin{array}{l}501- \\
1000\end{array}$ & $\begin{array}{c}1001- \\
1500\end{array}$ & $\begin{array}{l}1501- \\
2000\end{array}$ & $\begin{array}{l}2001- \\
2500\end{array}$ & $\begin{array}{c}2501- \\
3000\end{array}$ & $\begin{array}{c}3001- \\
3500\end{array}$ & $\begin{array}{c}3501- \\
4000\end{array}$ & $\begin{array}{c}4001- \\
4500\end{array}$ & $\begin{array}{c}4501- \\
5000\end{array}$ & $\begin{array}{c}5001- \\
5500\end{array}$ & $\begin{array}{c}5501- \\
6000\end{array}$ & $\begin{array}{c}7501- \\
8000\end{array}$ & Total \\
\hline \multirow{9}{*}{$\begin{array}{l}\text { Programme } \\
\text { Level }\end{array}$} & Professional Doctorates & 1 & 0 & 4 & 0 & 0 & 0 & 0 & 0 & 0 & 0 & 0 & 0 & 0 & 5 \\
\hline & $\begin{array}{l}\text { Master's by coursework } \\
\text { only }\end{array}$ & 2 & 7 & 5 & 7 & 6 & 5 & 1 & 0 & 0 & 1 & 0 & 0 & 2 & 36 \\
\hline & $\begin{array}{l}\text { Master's by both } \\
\text { coursework and research }\end{array}$ & 1 & 0 & 1 & 4 & 1 & 1 & 0 & 0 & 0 & 0 & 0 & 0 & 0 & 8 \\
\hline & Undergraduate degree & 22 & 50 & 31 & 27 & 16 & 33 & 9 & 18 & 1 & 8 & 2 & 1 & 0 & 218 \\
\hline & Diploma & 4 & 3 & 8 & 2 & 0 & 4 & 0 & 2 & 0 & 0 & 0 & 1 & 0 & 24 \\
\hline & Associate Degree & 2 & 2 & 0 & 5 & 2 & 0 & 2 & 0 & 0 & 0 & 0 & 0 & 0 & 13 \\
\hline & Certificate & 1 & 0 & 1 & 1 & 0 & 0 & 0 & 0 & 0 & 0 & 0 & 0 & 0 & 3 \\
\hline & $\begin{array}{l}\text { Executive/Professional } \\
\text { short programme }\end{array}$ & 1 & 0 & 0 & 0 & 0 & 0 & 0 & 0 & 0 & 0 & 0 & 0 & 0 & 1 \\
\hline & Total & 34 & 62 & 50 & 46 & 25 & 43 & 12 & 20 & 1 & 9 & 2 & 2 & 2 & 308 \\
\hline
\end{tabular}

Programme Level \& Age

\begin{tabular}{|c|c|c|c|c|c|c|c|c|c|}
\hline & & \multicolumn{8}{|c|}{ Age (Years) } \\
\hline & & $<10$ & $11-50$ & $51-100$ & $101-150$ & $151-200$ & $201-250$ & $251-300$ & Total \\
\hline \multirow[t]{4}{*}{ Programme Level } & Professional Doctorates & 0 & 0 & 1 & 2 & 2 & 0 & 0 & 5 \\
\hline & Master's by coursework only & 2 & 8 & 9 & 8 & 6 & 2 & 1 & 36 \\
\hline & $\begin{array}{l}\text { Master's by both coursework and } \\
\text { research }\end{array}$ & 0 & 1 & 1 & 4 & 2 & 0 & 0 & 8 \\
\hline & Undergraduate degree & 5 & 78 & 34 & 56 & 38 & 6 & 1 & 218 \\
\hline
\end{tabular}




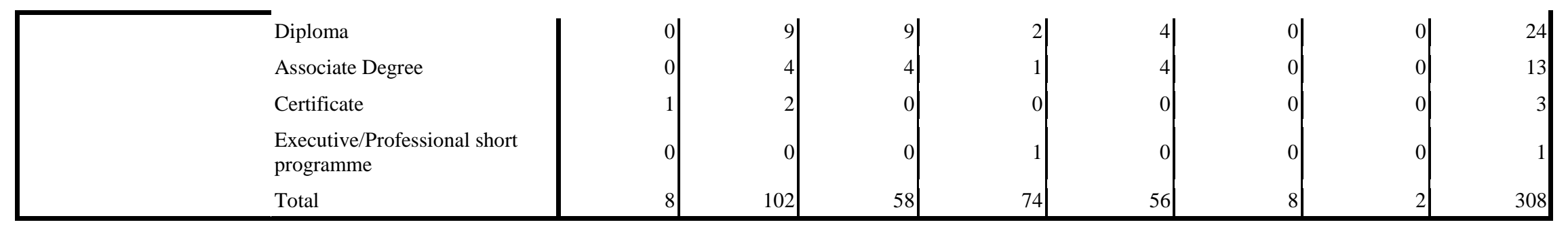

\section{Programme Level \& Worldwide Rankings}

\begin{tabular}{|c|c|c|c|c|c|c|c|c|c|c|}
\hline & \multicolumn{9}{|c|}{ World Ranking } \\
\hline & & $90^{\text {th }}$ percentile & $80^{\text {th }}$ percentile & $70^{\text {th }}$ percentile & $60^{\text {th }}$ percentile & $50^{\text {th }}$ percentile & $40^{\text {th }}$ percentile & $30^{\text {th }}$ percentile & $20^{\text {th }}$ percentile & Total \\
\hline \multirow[t]{9}{*}{ Programme Level } & Professional Doctorates & 0 & 0 & 0 & 2 & 2 & 0 & 1 & 0 & 5 \\
\hline & $\begin{array}{l}\text { Master's by coursework } \\
\text { only }\end{array}$ & 3 & 6 & 3 & 8 & 5 & 8 & 3 & 0 & 36 \\
\hline & $\begin{array}{l}\text { Master's by both } \\
\text { coursework and research }\end{array}$ & 0 & 3 & 0 & 0 & 3 & 2 & 0 & 0 & 8 \\
\hline & Undergraduate degree & 7 & 18 & 27 & 32 & 60 & 42 & 30 & 2 & 218 \\
\hline & Diploma & 0 & 1 & 2 & 4 & 11 & 2 & 4 & 0 & 24 \\
\hline & Associate Degree & 0 & 0 & 2 & 2 & 4 & 3 & 2 & 0 & 13 \\
\hline & Certificate & 0 & 0 & 0 & 1 & 1 & 1 & 0 & 0 & 3 \\
\hline & $\begin{array}{l}\text { Executive/Professional } \\
\text { short programme }\end{array}$ & 0 & 0 & 0 & 1 & 0 & 0 & 0 & 0 & 1 \\
\hline & Total & 10 & 28 & 34 & 50 & 86 & 58 & 40 & 2 & 308 \\
\hline
\end{tabular}

\section{Programme Level \& Domestic Rankings}

\begin{tabular}{|c|c|c|c|c|c|c|c|c|c|c|c|}
\hline & & \multicolumn{10}{|c|}{ Domestic Ranking } \\
\hline & & $\begin{array}{c}90^{\text {th }} \\
\text { percentile }\end{array}$ & $\begin{array}{c}80^{\text {th }} \\
\text { percentile }\end{array}$ & $\begin{array}{c}70^{\text {th }} \\
\text { percentile }\end{array}$ & $\begin{array}{c}60^{\text {th }} \\
\text { percentile }\end{array}$ & $\begin{array}{c}50^{\text {th }} \\
\text { percentile }\end{array}$ & $\begin{array}{c}40^{\text {th }} \\
\text { percentile }\end{array}$ & $\begin{array}{c}30^{\text {th }} \\
\text { percentile }\end{array}$ & $\begin{array}{c}20^{\text {th }} \\
\text { percentile }\end{array}$ & $\begin{array}{c}90^{\text {th }} \\
\text { percentile }\end{array}$ & Total \\
\hline Programme Level & Professional Doctorates & 0 & 0 & 2 & 0 & 3 & & 0 & & & \\
\hline
\end{tabular}




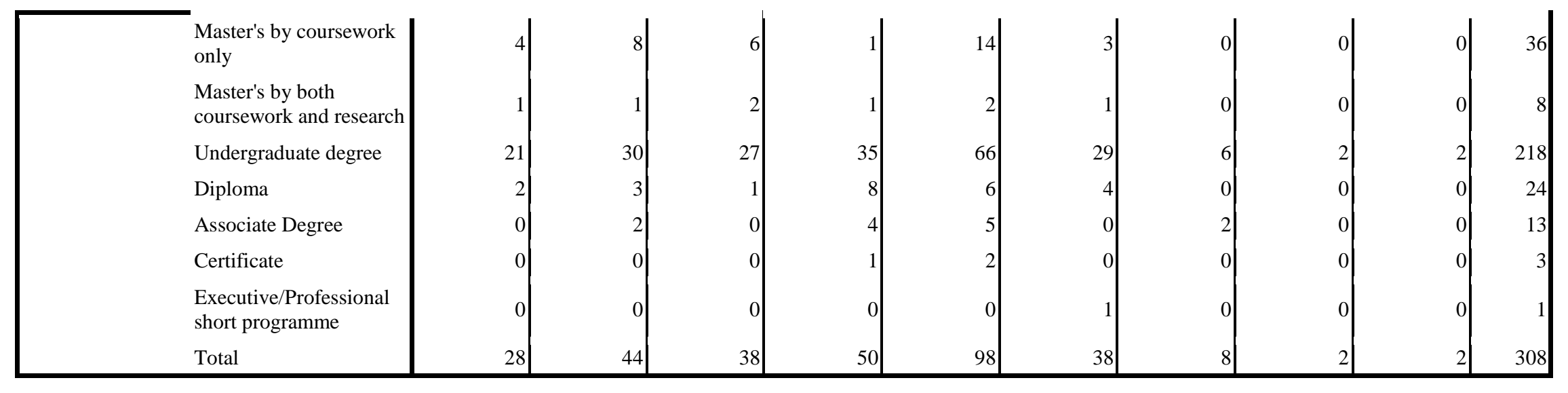

\section{Programme Level \& Mode of Entry (Continuous Measure)}

\begin{tabular}{|c|c|c|c|c|c|c|c|c|c|c|c|c|c|c|c|c|c|}
\hline & & \multicolumn{16}{|c|}{ Mode of Entry (Continuous Measure) } \\
\hline & & $100 \%$ & $\begin{array}{c}95- \\
99 \%\end{array}$ & $\begin{array}{c}55- \\
59 \%\end{array}$ & $\begin{array}{l}51- \\
54 \%\end{array}$ & $50 \%$ & $\begin{array}{l}45- \\
49 \%\end{array}$ & $\begin{array}{l}40- \\
44 \%\end{array}$ & $\begin{array}{c}35- \\
39 \%\end{array}$ & $\begin{array}{l}30- \\
34 \%\end{array}$ & $\begin{array}{l}25- \\
29 \%\end{array}$ & $\begin{array}{l}20- \\
24 \%\end{array}$ & $\begin{array}{l}15- \\
19 \%\end{array}$ & $\begin{array}{l}10- \\
14 \%\end{array}$ & $5-9 \%$ & $1-4 \%$ & Total \\
\hline \multirow{9}{*}{$\begin{array}{l}\text { Programme } \\
\text { Level }\end{array}$} & Professional Doctorates & 0 & 0 & 0 & 0 & 0 & 0 & 3 & 0 & 0 & 0 & 1 & 0 & 1 & 0 & 0 & 5 \\
\hline & $\begin{array}{l}\text { Master's by coursework } \\
\text { only }\end{array}$ & 0 & 1 & 0 & 4 & 1 & 1 & 3 & 4 & 4 & 3 & 6 & 1 & 5 & 2 & 1 & 36 \\
\hline & $\begin{array}{l}\text { Master's by both } \\
\text { coursework and research }\end{array}$ & 0 & 1 & 1 & 0 & 0 & 0 & 2 & 1 & 1 & 1 & 0 & 0 & 0 & 1 & 0 & 8 \\
\hline & Undergraduate degree & 1 & 9 & 4 & 6 & 3 & 1 & 21 & 22 & 23 & 32 & 20 & 25 & 27 & 23 & 1 & 218 \\
\hline & Diploma & 0 & 0 & 0 & 0 & 0 & 0 & 4 & 2 & 5 & 2 & 5 & 2 & 2 & 2 & 0 & 24 \\
\hline & Associate Degree & 0 & 1 & 0 & 0 & 0 & 1 & 2 & 1 & 1 & 2 & 1 & 2 & 1 & 1 & 0 & 13 \\
\hline & Certificate & 0 & 0 & 0 & 0 & 0 & 0 & 0 & 1 & 0 & 1 & 0 & 0 & 1 & 0 & 0 & 3 \\
\hline & $\begin{array}{l}\text { Executive/Professional } \\
\text { short programme }\end{array}$ & 0 & 0 & 0 & 1 & 0 & 0 & 0 & 0 & 0 & 0 & 0 & 0 & 0 & 0 & 0 & 1 \\
\hline & Total & 1 & 12 & 5 & 11 & 4 & 3 & 35 & 31 & 34 & 41 & 33 & 30 & 37 & 29 & 2 & 308 \\
\hline
\end{tabular}


Programme Level \& Mode of entry (Categorical Measure)

\begin{tabular}{|c|c|c|c|c|c|c|c|}
\hline & \multicolumn{6}{|c|}{ Mode of Entry (Categorical Measure) } \\
\hline & & Non-equity & $\begin{array}{c}\text { Joint venture } \\
\text { (minority equity) }\end{array}$ & $\begin{array}{l}\text { Joint venture } \\
\text { (50/50 equity) }\end{array}$ & $\begin{array}{l}\text { Joint venture } \\
\text { (majority equity) }\end{array}$ & Sole ownership & Total \\
\hline \multirow[t]{9}{*}{ Programme Level } & Professional Doctorates & 5 & 0 & 0 & 0 & 0 & 5 \\
\hline & Master's by coursework only & 29 & 1 & 1 & 4 & 1 & 36 \\
\hline & $\begin{array}{l}\text { Master's by both coursework and } \\
\text { research }\end{array}$ & 6 & 0 & 0 & 1 & 1 & 8 \\
\hline & Undergraduate degree & 194 & 1 & 3 & 10 & 10 & 218 \\
\hline & Diploma & 24 & 0 & 0 & 0 & 0 & 24 \\
\hline & Associate Degree & 11 & 1 & 0 & 0 & 1 & 13 \\
\hline & Certificate & 3 & 0 & 0 & 0 & 0 & 3 \\
\hline & $\begin{array}{l}\text { Executive/Professional short } \\
\text { programme }\end{array}$ & 0 & 0 & 0 & 1 & 0 & 1 \\
\hline & Total & 272 & 3 & 4 & 16 & 13 & 308 \\
\hline
\end{tabular}

Year of Entry \& Size (Undergraduate Students)

\begin{tabular}{|c|c|c|c|c|c|c|c|c|c|c|c|c|}
\hline & \multicolumn{11}{|c|}{ Size (Undergraduate Students) } \\
\hline & & $<1000$ & $1001-5000$ & $5001-10000$ & $\begin{array}{c}10001- \\
15000\end{array}$ & $\begin{array}{c}15001- \\
20000\end{array}$ & $\begin{array}{c}20001- \\
25000\end{array}$ & $\begin{array}{c}25001- \\
30000\end{array}$ & $\begin{array}{c}30001- \\
35000\end{array}$ & $\begin{array}{c}35001- \\
40000\end{array}$ & $\begin{array}{c}45001- \\
50000\end{array}$ & Total \\
\hline Year of Entry & 1999 & 0 & 2 & 10 & 10 & 7 & 6 & 8 & 2 & 4 & 2 & 51 \\
\hline & 2000 & 1 & 3 & 5 & 9 & 6 & 3 & 3 & 0 & 3 & 0 & 33 \\
\hline & 2001 & 0 & 4 & 8 & 8 & 13 & 4 & 4 & 2 & 1 & 0 & 44 \\
\hline & 2002 & 0 & 1 & 2 & 4 & 3 & 3 & 2 & 1 & 0 & 0 & 16 \\
\hline & 2003 & 0 & 0 & 3 & 6 & 5 & 4 & 5 & 2 & 1 & 0 & 26 \\
\hline & 2004 & 1 & 4 & 6 & 6 & 10 & 6 & 5 & 2 & 0 & 0 & 40 \\
\hline & 2005 & 2 & 4 & 10 & 9 & 9 & 7 & 1 & 1 & 0 & 0 & 43 \\
\hline
\end{tabular}




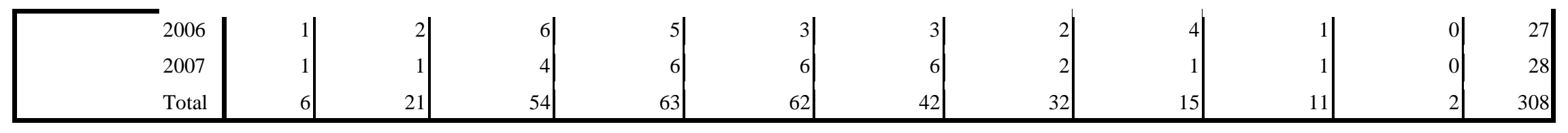

Year of Entry \& Size (Postgraduate Students)

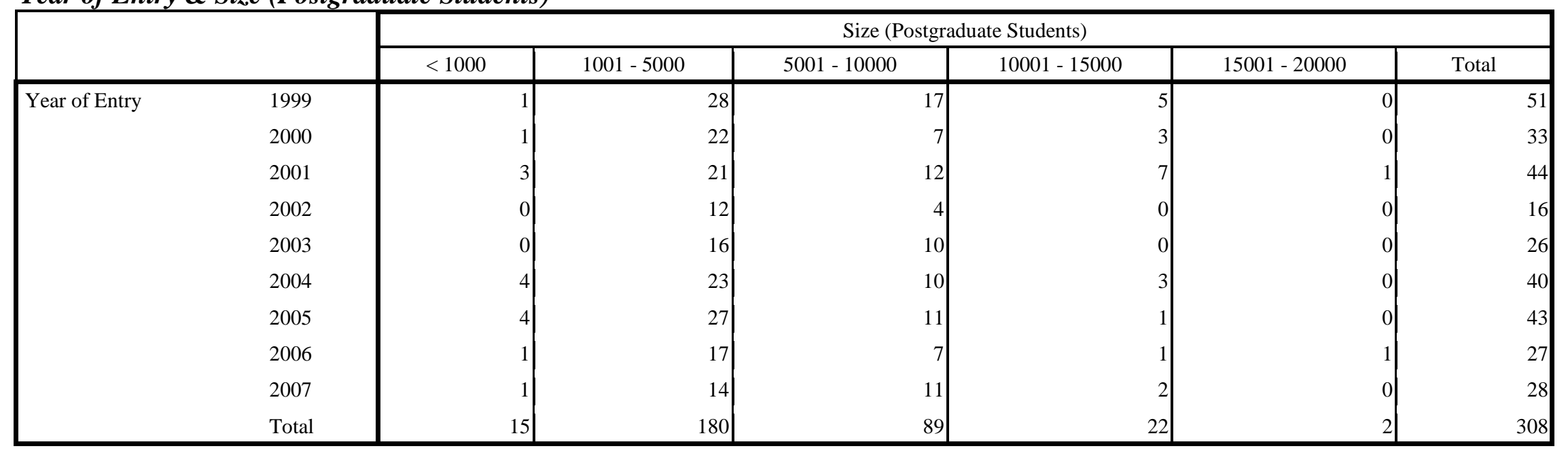

\section{Year of Entry \& Size (Staff)}

\begin{tabular}{|c|c|c|c|c|c|c|c|c|c|c|c|c|c|c|}
\hline & \multicolumn{14}{|c|}{ Size (Staff) } \\
\hline & $<500$ & $\begin{array}{l}501- \\
1000\end{array}$ & $\begin{array}{c}1001- \\
1500\end{array}$ & $\begin{array}{l}1501- \\
2000\end{array}$ & $\begin{array}{c}2001- \\
2500\end{array}$ & $\begin{array}{c}2501- \\
3000\end{array}$ & $\begin{array}{l}3001- \\
3500\end{array}$ & $\begin{array}{c}3501- \\
4000\end{array}$ & $\begin{array}{l}4001- \\
4500\end{array}$ & $\begin{array}{c}4501- \\
5000\end{array}$ & $\begin{array}{c}5001- \\
5500\end{array}$ & $\begin{array}{c}5501- \\
6000\end{array}$ & $\begin{array}{c}7501 \text { - } \\
8000\end{array}$ & Total \\
\hline Year of entry 1999 & 4 & 13 & 7 & 5 & 5 & 5 & 2 & 4 & 0 & 3 & 2 & 0 & 1 & 51 \\
\hline 2000 & 5 & 6 & 6 & 3 & 5 & 4 & 1 & 2 & 0 & 1 & 0 & 0 & 0 & 33 \\
\hline 2001 & 7 & 2 & 10 & 6 & 3 & 9 & 2 & 2 & 1 & 1 & 0 & 1 & 0 & 44 \\
\hline 2002 & 2 & 4 & 6 & 1 & 0 & 1 & 0 & 2 & 0 & 0 & 0 & 0 & 0 & 16 \\
\hline
\end{tabular}




\begin{tabular}{|r|r|r|r|r|r|r|r|r|r|r|r|r|r|r|}
\hline 2003 & 1 & 8 & 2 & 5 & 1 & 4 & 3 & 2 & 0 & 0 & 0 & 0 & 0 & 26 \\
2004 & 4 & 8 & 8 & 8 & 4 & 3 & 1 & 2 & 0 & 1 & 0 & 1 & 0 & 40 \\
2005 & 7 & 9 & 6 & 6 & 3 & 10 & 0 & 1 & 0 & 1 & 0 & 0 & 0 & 43 \\
2006 & 3 & 6 & 1 & 5 & 3 & 2 & 2 & 4 & 0 & 1 & 0 & 0 & 0 \\
2007 & 1 & 6 & 4 & 7 & 1 & 5 & 1 & 1 & 0 & 1 & 0 & 0 & 1 & 28 \\
Total & 34 & 62 & 50 & 46 & 25 & 43 & 12 & 20 & 1 & 9 & 2 & 2 & 2 \\
308 \\
\hline
\end{tabular}

\begin{tabular}{|c|c|c|c|c|c|c|c|c|c|}
\hline \multicolumn{10}{|c|}{ Age (Years) } \\
\hline & & $<10$ & $11-50$ & $51-100$ & $101-150$ & $151-200$ & $201-250$ & $251-300$ & Total \\
\hline \multirow[t]{10}{*}{ Year of Entry } & 1999 & 1 & 15 & 11 & 15 & 9 & 0 & 0 & 51 \\
\hline & 2000 & 0 & 10 & 6 & 8 & 7 & 2 & 0 & 33 \\
\hline & 2001 & & 13 & 12 & 6 & 11 & 0 & 1 & 44 \\
\hline & 2002 & & 5 & 3 & 5 & 2 & 1 & 0 & 16 \\
\hline & 2003 & & 11 & 5 & 6 & 3 & 0 & 0 & 26 \\
\hline & 2004 & 1 & 15 & 8 & 8 & 7 & 1 & 0 & 40 \\
\hline & 2005 & 2 & 15 & 6 & 12 & 5 & 3 & 0 & 43 \\
\hline & 2006 & 1 & 8 & 3 & 7 & 6 & 1 & 1 & 27 \\
\hline & 2007 & 1 & 10 & 4 & 7 & 6 & 0 & 0 & 28 \\
\hline & Total & 8 & 102 & 58 & 74 & 56 & 8 & 2 & 308 \\
\hline
\end{tabular}

Year of Entry \& Worldwide Rankings

\begin{tabular}{|c|c|c|c|c|c|c|c|c|c|}
\hline & \multicolumn{9}{|c|}{ World Ranking } \\
\hline & $90^{\text {th }}$ percentile & $80^{\text {th }}$ percentile & $70^{\text {th }}$ percentile & $60^{\text {th }}$ percentile & $50^{\text {th }}$ percentile & $40^{\text {th }}$ percentile & $30^{\text {th }}$ percentile & $20^{\text {th }}$ percentile & Total \\
\hline 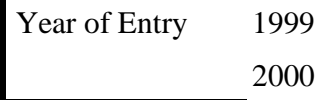 & 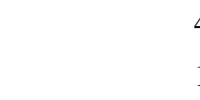 & & & 8 & 1 & & & 0 & 51 \\
\hline
\end{tabular}




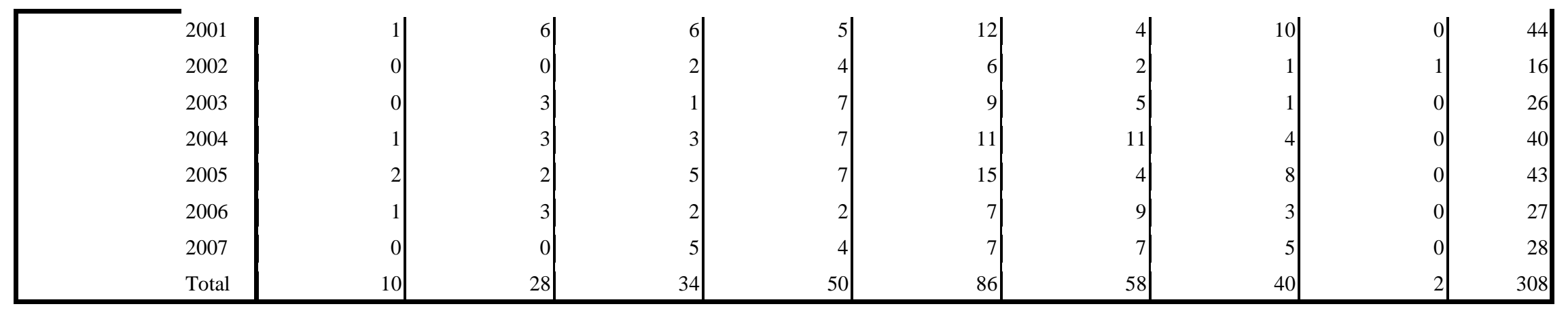

\section{Year of Entry \& Domestic Rankings}

\begin{tabular}{|c|c|c|c|c|c|c|c|c|c|c|c|}
\hline & & \multicolumn{10}{|c|}{ Domestic Ranking } \\
\hline & & $90^{\text {th }}$ percentile & $80^{\text {th }}$ percentile & $70^{\text {th }}$ percentile & $60^{\text {th }}$ percentile & $50^{\text {th }}$ percentile & $40^{\text {th }}$ percentile & $30^{\text {th }}$ percentile & $20^{\text {th }}$ percentile & $90^{\text {th }}$ percentile & Total \\
\hline \multirow[t]{10}{*}{ Year of Entry } & 1999 & 6 & 9 & 7 & 8 & 18 & 2 & 1 & 0 & 0 & 51 \\
\hline & 2000 & 3 & 3 & 5 & 7 & 10 & 2 & 3 & 0 & 0 & 33 \\
\hline & 2001 & 7 & 4 & 5 & 8 & 10 & 7 & 3 & 0 & 0 & 44 \\
\hline & 2002 & 1 & 3 & 4 & 2 & 4 & 2 & 0 & 0 & 0 & 16 \\
\hline & 2003 & 3 & 3 & 2 & 6 & 10 & 1 & 0 & 0 & 1 & 26 \\
\hline & 2004 & 3 & 5 & 8 & 5 & 11 & 7 & 0 & 1 & 0 & 40 \\
\hline & 2005 & 2 & 10 & 4 & 8 & 10 & 8 & 0 & 0 & 1 & 43 \\
\hline & 2006 & 2 & 4 & 2 & 4 & 9 & 4 & 1 & 1 & 0 & 27 \\
\hline & 2007 & 1 & 3 & 1 & 2 & 16 & 5 & 0 & 0 & 0 & 28 \\
\hline & Total & 28 & 44 & 38 & 50 & 98 & 38 & 8 & 2 & 2 & 308 \\
\hline
\end{tabular}

Year of Entry \& Mode of Entry (Continuous Measure)

\begin{tabular}{|c|c|c|c|c|c|c|c|c|c|c|c|c|c|c|c|c|}
\hline & \multicolumn{16}{|c|}{ Mode of Entry (Continuous Measure) } \\
\hline & $100 \%$ & $95-99 \%$ & $55-59 \%$ & $51-54 \%$ & $50 \%$ & $45-49 \%$ & $40-44 \%$ & $35-39 \%$ & $30-34 \%$ & $25-29 \%$ & $20-24 \%$ & $15-19 \%$ & $10-14 \%$ & $5-9 \%$ & $1-4 \%$ & Total \\
\hline Year of Entry 1999 & & 5 & 1 & 1 & & 1 & 6 & 2 & 8 & 3 & 4 & 7 & 10 & 3 & 0 & 51 \\
\hline
\end{tabular}




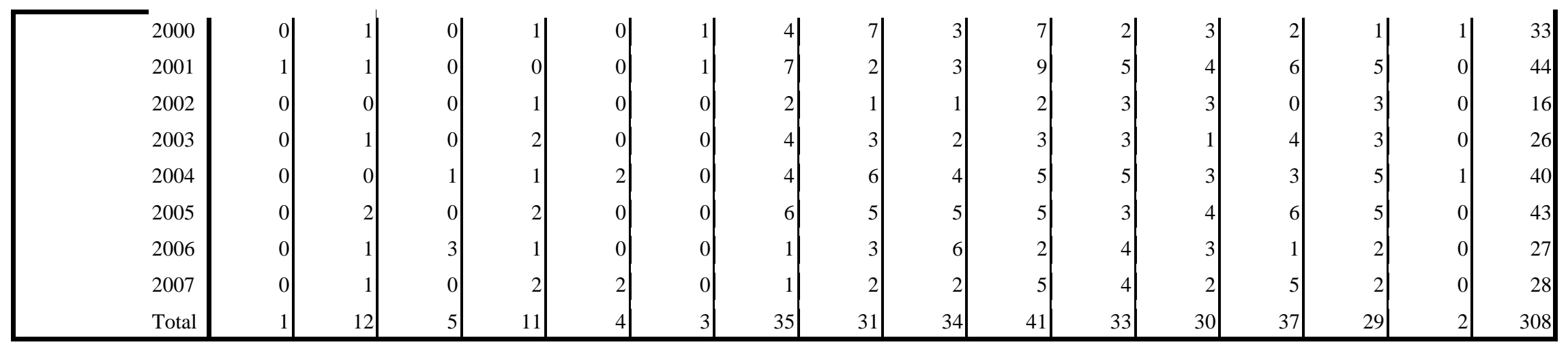

Year of Entry \& Mode of Entry (Categorical Measure)

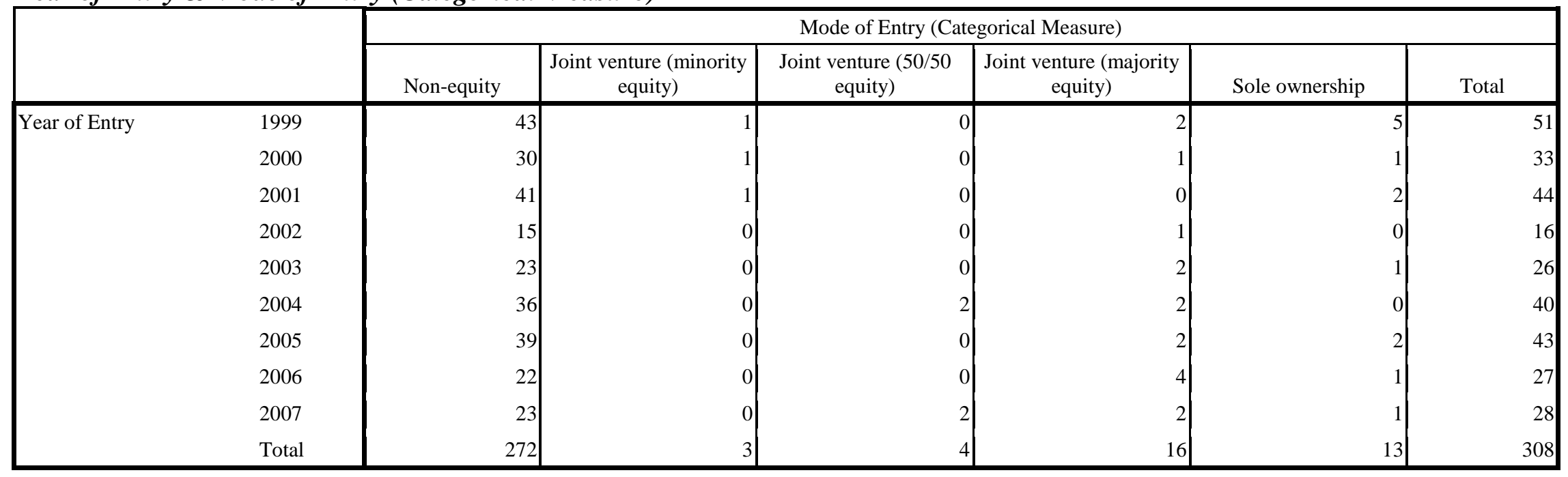

446 
Mode of Entry (Continuous Measure) and Size (Undergraduate Students)

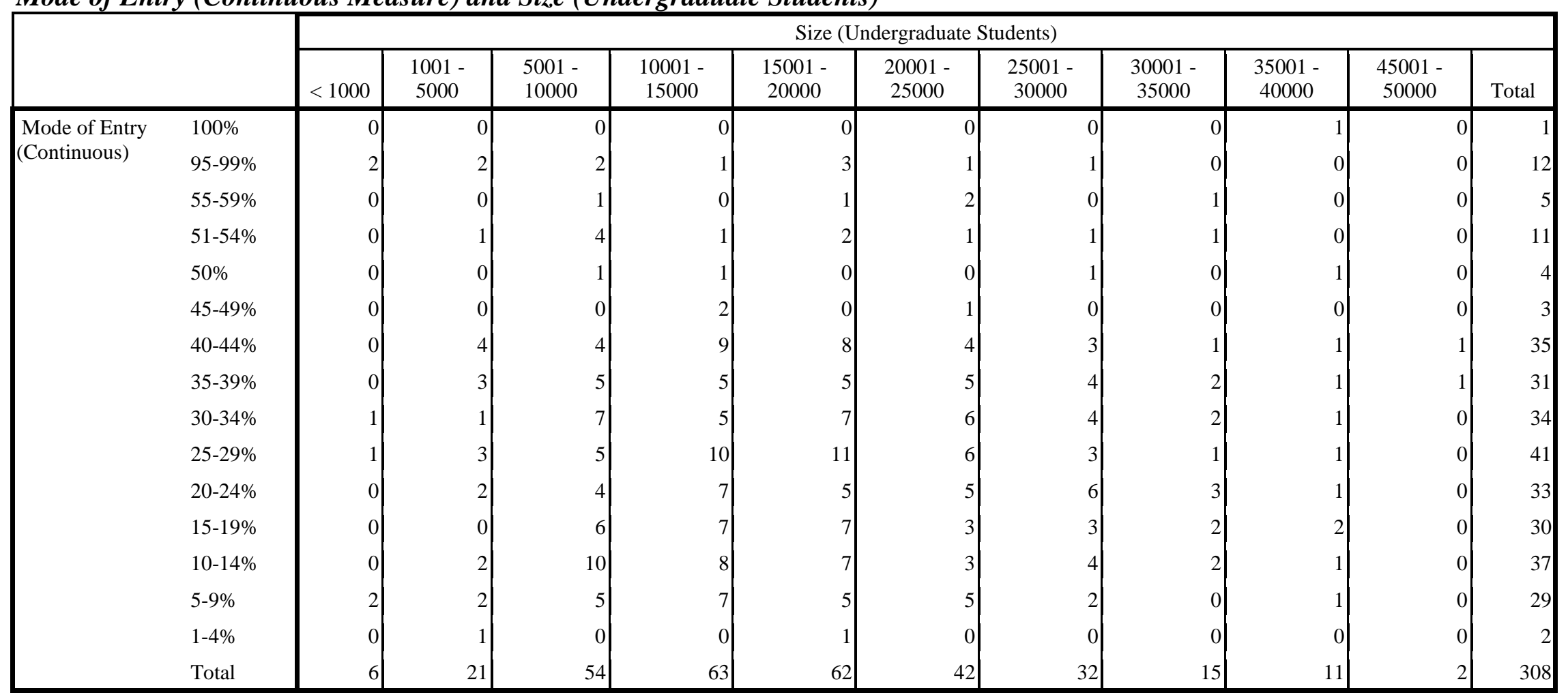

Mode of Entry (Continuous Measure) and Size (Postgraduate Students)

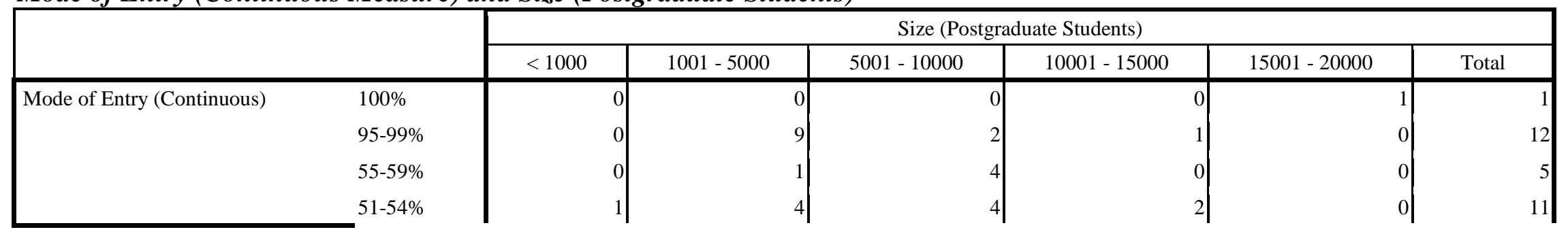




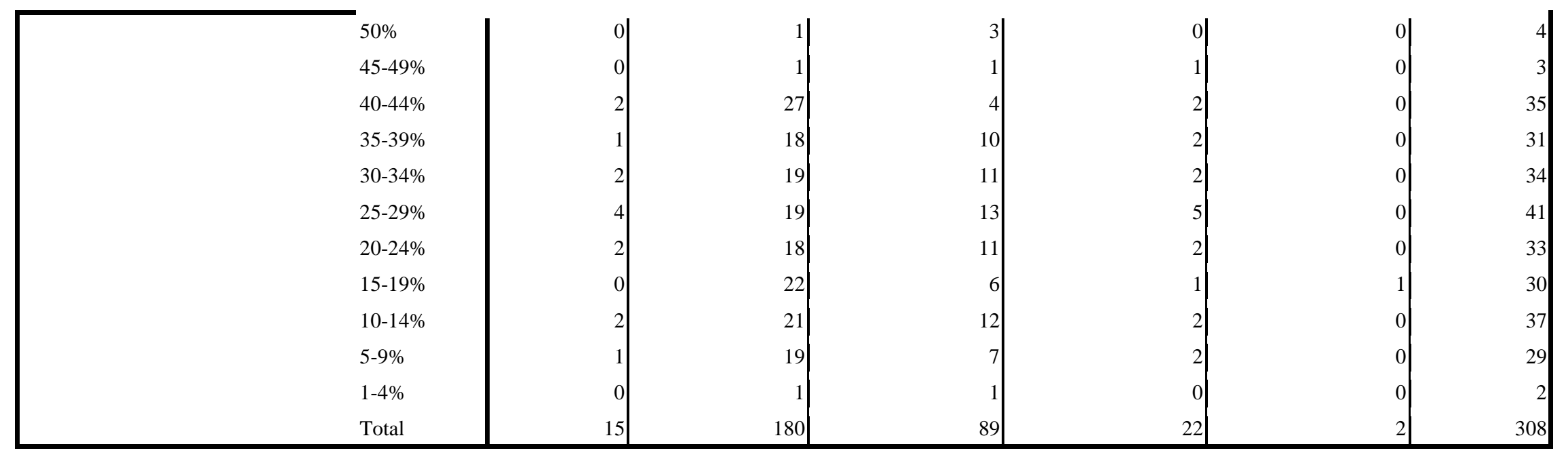

\section{Mode of Entry (Continuous Measure) and Size (Staff)}

\begin{tabular}{|c|c|c|c|c|c|c|c|c|c|c|c|c|c|c|c|}
\hline & & \multicolumn{14}{|c|}{ Size (Staff) } \\
\hline & & $<500$ & $\begin{array}{l}501- \\
1000\end{array}$ & $\begin{array}{c}1001- \\
1500\end{array}$ & $\begin{array}{c}1501- \\
2000\end{array}$ & $\begin{array}{c}2001- \\
2500\end{array}$ & $\begin{array}{c}2501- \\
3000\end{array}$ & $\begin{array}{c}3001- \\
3500\end{array}$ & $\begin{array}{c}3501- \\
4000\end{array}$ & $\begin{array}{c}4001- \\
4500\end{array}$ & $\begin{array}{c}4501- \\
5000\end{array}$ & $\begin{array}{c}5001- \\
5500\end{array}$ & $\begin{array}{c}5501- \\
6000\end{array}$ & $\begin{array}{c}7501- \\
8000\end{array}$ & Total \\
\hline \multirow{7}{*}{$\begin{array}{l}\text { Mode of Entry } \\
\text { (Continuous) }\end{array}$} & $100 \%$ & 0 & 0 & 0 & 0 & 0 & 0 & 1 & 0 & 0 & 0 & 0 & 0 & 0 & 1 \\
\hline & $\begin{array}{l}95- \\
99 \%\end{array}$ & 5 & 4 & 1 & 0 & 1 & 1 & 0 & 0 & 0 & 0 & 0 & 0 & 0 & 12 \\
\hline & $\begin{array}{l}55- \\
59 \%\end{array}$ & 0 & 1 & 0 & 2 & 1 & 1 & 0 & 0 & 0 & 0 & 0 & 0 & 0 & 5 \\
\hline & $\begin{array}{l}51- \\
54 \%\end{array}$ & 1 & 2 & 2 & 1 & 0 & 3 & 1 & 0 & 0 & 1 & 0 & 0 & 0 & 11 \\
\hline & $50 \%$ & 0 & 0 & 1 & 1 & 1 & 1 & 0 & 0 & 0 & 0 & 0 & 0 & 0 & 4 \\
\hline & $\begin{array}{l}45- \\
49 \%\end{array}$ & 1 & 0 & 0 & 0 & 1 & 0 & 0 & 0 & 0 & 0 & 0 & 1 & 0 & 3 \\
\hline & $\begin{array}{l}40- \\
44 \%\end{array}$ & 5 & 7 & 7 & 8 & 3 & 2 & 1 & 1 & 0 & 0 & 1 & 0 & 0 & 35 \\
\hline
\end{tabular}




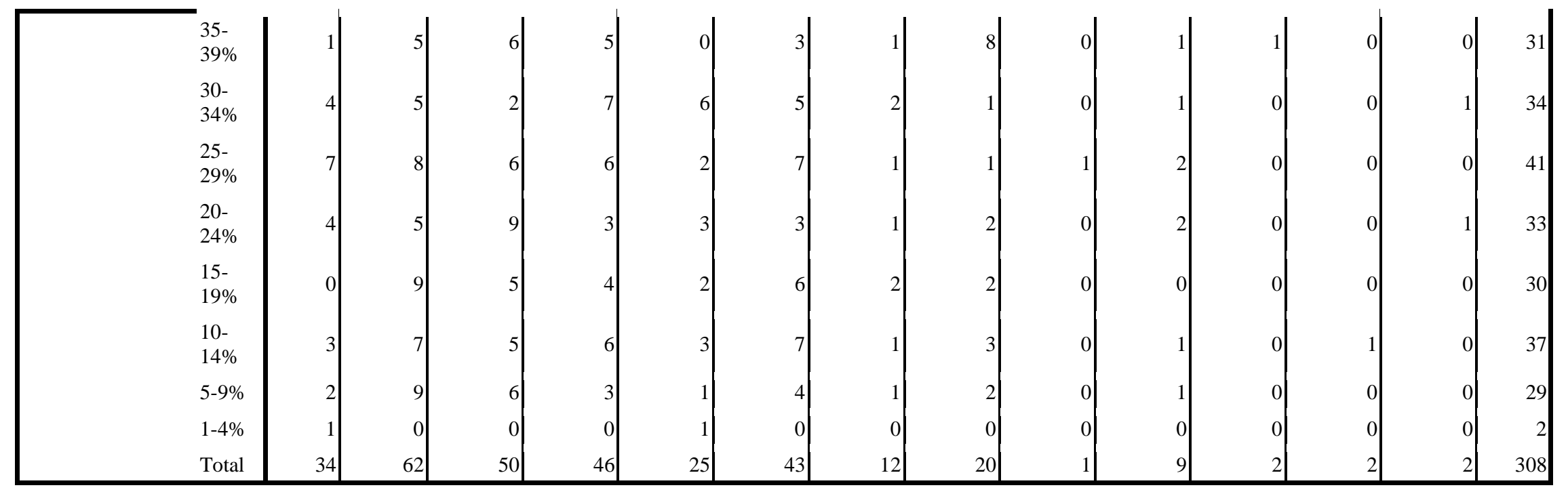

Mode of Entry (Continuous Measure) and Age

\begin{tabular}{|c|c|c|c|c|c|c|c|c|c|}
\hline & & \multicolumn{8}{|c|}{ Age (Years) } \\
\hline & & $<10$ & $11-50$ & $51-100$ & $101-150$ & $151-200$ & $201-250$ & $251-300$ & Total \\
\hline \multirow[t]{11}{*}{ Mode of Entry (Continuous) } & $100 \%$ & & 1 & 0 & 0 & 0 & 0 & 0 & 1 \\
\hline & $95-99 \%$ & & 4 & 2 & 3 & 1 & 0 & 0 & 12 \\
\hline & $55-59 \%$ & & 1 & 0 & 1 & 2 & 1 & 0 & 5 \\
\hline & $51-54 \%$ & & 2 & 2 & 4 & 1 & 1 & 1 & 11 \\
\hline & $50 \%$ & & 0 & 1 & 2 & 0 & 1 & 0 & 4 \\
\hline & $45-49 \%$ & & 0 & 1 & 1 & 1 & 0 & 0 & 3 \\
\hline & $40-44 \%$ & & 12 & 5 & 11 & 6 & 0 & 1 & 35 \\
\hline & $35-39 \%$ & & 17 & 4 & 5 & 5 & 0 & 0 & 31 \\
\hline & $30-34 \%$ & & 8 & 10 & 9 & 6 & 0 & 0 & 34 \\
\hline & $25-29 \%$ & & 9 & 7 & 10 & 11 & 3 & 0 & 41 \\
\hline & $20-24 \%$ & & 14 & 6 & 4 & 7 & 1 & 0 & 33 \\
\hline
\end{tabular}




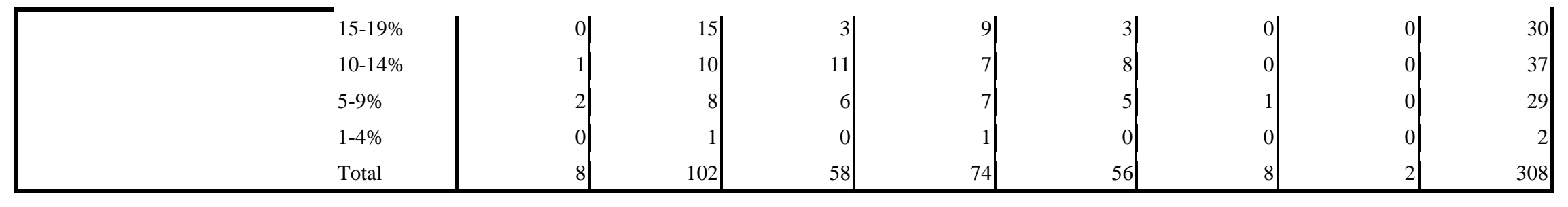

Mode of Entry (Continuous Measure) and Worldwide Rankings

\begin{tabular}{|c|c|c|c|c|c|c|c|c|c|c|}
\hline & \multicolumn{9}{|c|}{ World Ranking } \\
\hline & & $90^{\text {th }}$ percentile & $80^{\text {th }}$ percentile & $70^{\text {th }}$ percentile & $60^{\text {th }}$ percentile & $50^{\text {th }}$ percentile & $40^{\text {th }}$ percentile & $30^{\text {th }}$ percentile & $20^{\text {th }}$ percentile & Total \\
\hline \multirow{16}{*}{\begin{tabular}{|l} 
Mode of Entry \\
(Continuous)
\end{tabular}} & $100 \%$ & & 1 & ( & ( & c & 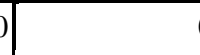 & 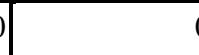 & ( & \\
\hline & $95-99 \%$ & & 2 & 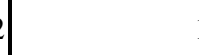 & 2 & 2 & 4 & 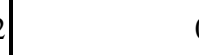 & ( & 12 \\
\hline & $55-59 \%$ & 0 & 1 & to & 2 & 2 & ( & ( & ( & 3 \\
\hline & $51-54 \%$ & 1 & 0 & : & 3 & 3 & 3 & ( & ( & 1 \\
\hline & $50 \%$ & 0 & 1 & 2 & ( & 1 & ( & ( & 0 & 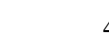 \\
\hline & $45-49 \%$ & 1 & 0 & 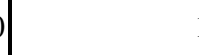 & ( & c & 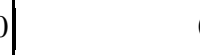 & 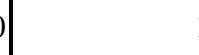 & ( & 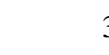 \\
\hline & $40-44 \%$ & 2 & 1 & 2 & 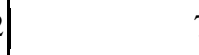 & 15 & 5 & 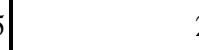 & 2 & 35 \\
\hline & $35-39 \%$ & 1 & 3 & 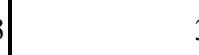 & 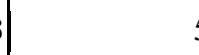 & $\varepsilon$ & 3 & 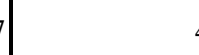 & ( & 3 \\
\hline & $30-34 \%$ & 1 & 3 & 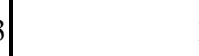 & 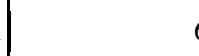 & $\varepsilon$ & s & 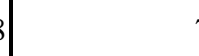 & ( & 3 \\
\hline & $25-29 \%$ & 0 & 4 & 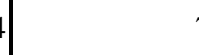 & 3 & 12 & & ( & 6 & 4 \\
\hline & $20-24 \%$ & 0 & 1 & se & s & 14 & 4 & 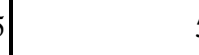 & 5 & 33 \\
\hline & $15-19 \%$ & 0 & 4 & 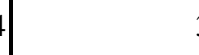 & 2 & 7 & 7 & 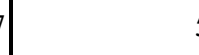 & ( & 30 \\
\hline & $10-14 \%$ & 2 & 2 & . & 16 & 5 & 5 & 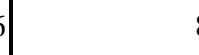 & ( & 37 \\
\hline & $5-9 \%$ & 1 & 4 & $i$ & 2 & $\epsilon$ & 6 & 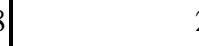 & 2 & 25 \\
\hline & $1-4 \%$ & 0 & 1 & ( & ( & 1 & 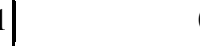 & ( & ( & 2 \\
\hline & Total & 10 & 28 & 3. & 56 & 86 & 5 & 4 & 0 & 308 \\
\hline
\end{tabular}


Mode of Entry (Continuous Measure) and Domestic Rankings

\begin{tabular}{|c|c|c|c|c|c|c|c|c|c|c|c|}
\hline & \multicolumn{10}{|c|}{ Domestic Ranking } \\
\hline & & $\begin{array}{c}90^{\text {th }} \\
\text { percentile }\end{array}$ & $\begin{array}{c}80^{\text {th }} \\
\text { percentile }\end{array}$ & $\begin{array}{c}70^{\text {th }} \\
\text { percentile }\end{array}$ & $\begin{array}{c}60^{\text {th }} \\
\text { percentile }\end{array}$ & $\begin{array}{c}50^{\text {th }} \\
\text { percentile }\end{array}$ & $\begin{array}{c}40^{\text {th }} \\
\text { percentile }\end{array}$ & $\begin{array}{c}30^{\text {th }} \\
\text { percentile }\end{array}$ & $\begin{array}{c}20^{\text {th }} \\
\text { percentile }\end{array}$ & $\begin{array}{c}90^{\text {th }} \\
\text { percentile }\end{array}$ & Total \\
\hline \multirow{16}{*}{$\begin{array}{l}\text { Mode of Entry } \\
\text { (Continuous) }\end{array}$} & $100 \%$ & 1 & 0 & 0 & 0 & 0 & 0 & 0 & 0 & 0 & 1 \\
\hline & $95-99 \%$ & 2 & 1 & 2 & 1 & 5 & 0 & 0 & 1 & 0 & 12 \\
\hline & $55-59 \%$ & 0 & 1 & 2 & 0 & 2 & 0 & 0 & 0 & 0 & 5 \\
\hline & $51-54 \%$ & 1 & 3 & 0 & 1 & 4 & 2 & 0 & 0 & 0 & 11 \\
\hline & $50 \%$ & 0 & 2 & 1 & 1 & 0 & 0 & 0 & 0 & 0 & 4 \\
\hline & $45-49 \%$ & 1 & 1 & 0 & 0 & 0 & 0 & 1 & 0 & 0 & 3 \\
\hline & $40-44 \%$ & 3 & 3 & 5 & 13 & 6 & 4 & 1 & 0 & 0 & 35 \\
\hline & $35-39 \%$ & 2 & 4 & 5 & 6 & 9 & 3 & 2 & 0 & 0 & 31 \\
\hline & $30-34 \%$ & 2 & 5 & 5 & 5 & 10 & 6 & 1 & 0 & 0 & 34 \\
\hline & $25-29 \%$ & 4 & 7 & 4 & 6 & 13 & 7 & 0 & 0 & 0 & 41 \\
\hline & $20-24 \%$ & 0 & 5 & 3 & 8 & 12 & 5 & 0 & 0 & 0 & 33 \\
\hline & $15-19 \%$ & 3 & 3 & 3 & 4 & 12 & 4 & 1 & 0 & 0 & 30 \\
\hline & $10-14 \%$ & 3 & 6 & 3 & 2 & 16 & 4 & 2 & 0 & 1 & 37 \\
\hline & $5-9 \%$ & 6 & 3 & 3 & 3 & 9 & 3 & 0 & 1 & 1 & 29 \\
\hline & $1-4 \%$ & 0 & 0 & 2 & 0 & 0 & 0 & 0 & 0 & 0 & 2 \\
\hline & Total & 28 & 44 & 38 & 50 & 98 & 38 & 8 & 2 & 2 & 308 \\
\hline
\end{tabular}

\section{Mode of Entry (Categorical Measure) and Size (Undergraduate Students)}

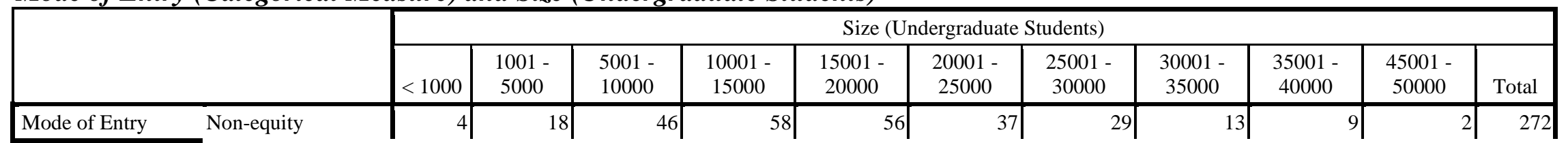




\begin{tabular}{|c|c|c|c|c|c|c|c|c|c|c|c|c|}
\hline \multirow[t]{5}{*}{ (Categorical) } & $\begin{array}{l}\text { Joint venture } \\
\text { (minority equity) }\end{array}$ & 0 & 0 & 0 & 2 & 0 & 1 & 0 & 0 & 0 & 0 & 3 \\
\hline & $\begin{array}{l}\text { Joint venture (50/50 } \\
\text { equity) }\end{array}$ & 0 & 0 & 1 & 1 & 0 & 0 & 1 & 0 & 1 & 0 & 4 \\
\hline & $\begin{array}{l}\text { Joint venture } \\
\text { (majority equity) }\end{array}$ & 0 & 1 & 5 & 1 & 3 & 3 & 1 & 2 & 0 & 0 & 16 \\
\hline & Sole ownership & 2 & 2 & 2 & 1 & 3 & 1 & 1 & 0 & 1 & 0 & 13 \\
\hline & Total & 6 & 21 & 54 & 63 & 62 & 42 & 32 & 15 & 11 & 2 & 308 \\
\hline
\end{tabular}

\section{Mode of Entry (Categorical Measure) and Size (Postgraduate Students)}

\begin{tabular}{|c|c|c|c|c|c|c|c|}
\hline & \multicolumn{6}{|c|}{ Size (Postgraduate Students) } \\
\hline & & $<1000$ & $1001-5000$ & $5001-10000$ & $10001-15000$ & $15001-20000$ & Total \\
\hline \multirow[t]{6}{*}{ Mode of Entry (Categorical) } & Non-equity & 14 & 164 & 75 & 18 & 1 & 272 \\
\hline & Joint venture (minority equity) & 0 & 1 & 1 & 1 & 0 & 3 \\
\hline & Joint venture (50/50 equity) & 0 & 1 & 3 & 0 & 0 & 4 \\
\hline & Joint venture (majority equity) & 1 & 5 & 8 & 2 & 0 & 16 \\
\hline & Sole ownership & 0 & 9 & 2 & 1 & 1 & 13 \\
\hline & Total & 15 & 180 & 89 & 22 & 2 & 308 \\
\hline
\end{tabular}

Mode of Entry (Categorical Measure) and Size (Staff)

\begin{tabular}{|c|c|c|c|c|c|c|c|c|c|c|c|c|c|c|c|}
\hline & & \multicolumn{14}{|c|}{ Size (Staff) } \\
\hline & & $<500$ & $\begin{array}{l}501- \\
1000\end{array}$ & $\begin{array}{c}1001- \\
1500\end{array}$ & $\begin{array}{c}1501- \\
2000\end{array}$ & $\begin{array}{c}2001- \\
2500\end{array}$ & $\begin{array}{c}2501- \\
3000\end{array}$ & $\begin{array}{c}3001- \\
3500\end{array}$ & $\begin{array}{c}3501- \\
4000\end{array}$ & $\begin{array}{c}4001- \\
4500\end{array}$ & $\begin{array}{c}4501- \\
5000\end{array}$ & $\begin{array}{c}5001- \\
5500\end{array}$ & $\begin{array}{c}5501- \\
6000\end{array}$ & $\begin{array}{l}7501- \\
8000\end{array}$ & Total \\
\hline \multirow{3}{*}{$\begin{array}{l}\text { Mode of Entry } \\
\text { (Categorical) }\end{array}$} & Non-equity & 27 & 55 & 46 & 42 & 21 & 37 & 10 & 20 & 1 & 8 & 2 & 1 & 2 & 272 \\
\hline & $\begin{array}{l}\text { Joint venture } \\
\text { (minority equity) }\end{array}$ & 1 & 0 & 0 & 0 & 1 & 0 & 0 & 0 & 0 & 0 & 0 & 1 & 0 & 3 \\
\hline & $\begin{array}{l}\text { Joint venture } \\
\text { (50/50 equity) }\end{array}$ & 0 & 0 & 1 & 1 & 1 & 1 & 0 & 0 & 0 & 0 & 0 & 0 & 0 & 4 \\
\hline
\end{tabular}




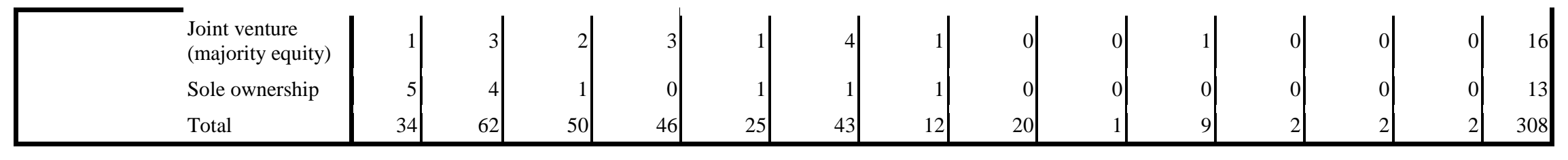

Mode of Entry (Categorical Measure) and Age

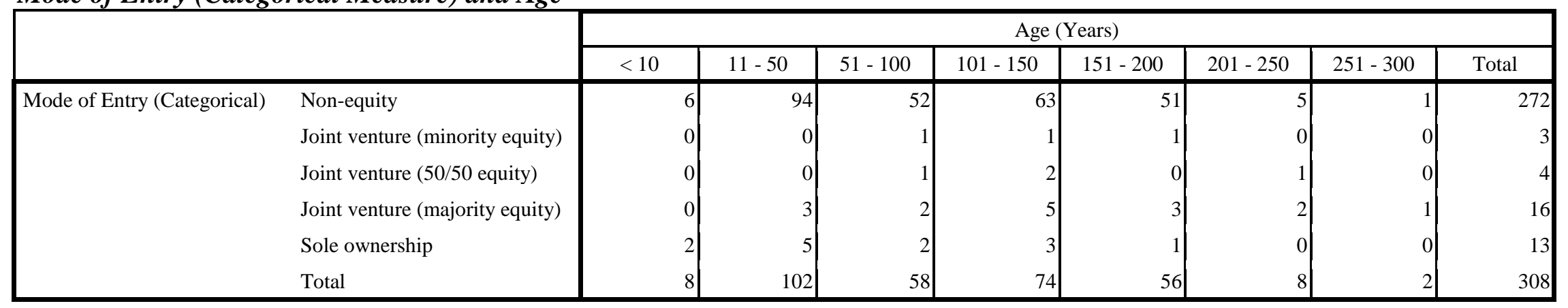

\section{Mode of Entry (Categorical Measure) and Worldwide Rankings}

\begin{tabular}{|c|c|c|c|c|c|c|c|c|c|c|}
\hline & & \multicolumn{9}{|c|}{ World Ranking } \\
\hline & & $\begin{array}{c}90^{\text {th }} \\
\text { percentile }\end{array}$ & $\begin{array}{c}80^{\text {th }} \\
\text { percentile }\end{array}$ & $\begin{array}{c}70^{\text {th }} \\
\text { percentile }\end{array}$ & $\begin{array}{c}60^{\text {th }} \\
\text { percentile }\end{array}$ & $\begin{array}{c}50^{\text {th }} \\
\text { percentile }\end{array}$ & $\begin{array}{c}40^{\text {th }} \\
\text { percentile }\end{array}$ & $\begin{array}{c}30^{\text {th }} \\
\text { percentile }\end{array}$ & $\begin{array}{c}20^{\text {th }} \\
\text { percentile }\end{array}$ & Total \\
\hline \multirow{4}{*}{$\begin{array}{l}\text { Mode of Entry } \\
\text { (Categorical) }\end{array}$} & Non-equity & 7 & 23 & 27 & 43 & 76 & 55 & 39 & 2 & 272 \\
\hline & Joint venture (minority & & & & & & & & & \\
\hline & equity) & 1 & 0 & 1 & 0 & 0 & 0 & 1 & 0 & 3 \\
\hline & Joint venture (50/50 & 0 & 1 & 2 & 0 & 1 & 0 & 0 & 0 & \\
\hline
\end{tabular}




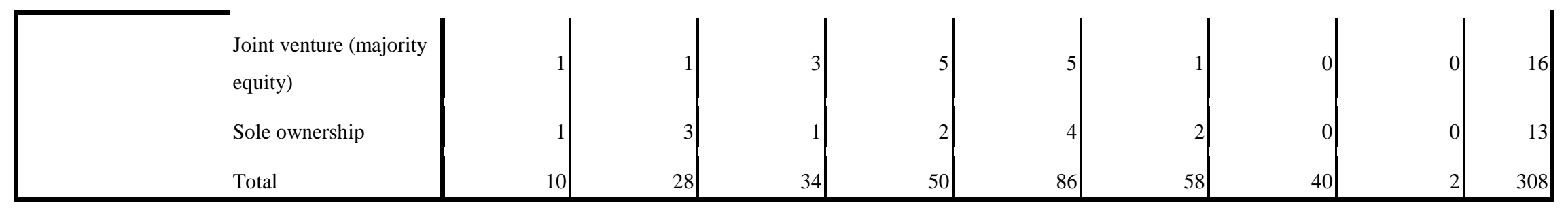

\section{Mode of Entry (Categorical Measure) and Domestic Rankings}

\begin{tabular}{|c|c|c|c|c|c|c|c|c|c|c|c|}
\hline & \multicolumn{10}{|c|}{ Domestic Ranking } \\
\hline & & $\begin{array}{c}90^{\text {th }} \\
\text { percentile }\end{array}$ & $\begin{array}{c}80^{\text {th }} \\
\text { percentile }\end{array}$ & $\begin{array}{c}70^{\text {th }} \\
\text { percentile }\end{array}$ & $\begin{array}{c}60^{\text {th }} \\
\text { percentile }\end{array}$ & $\begin{array}{c}50^{\text {th }} \\
\text { percentile }\end{array}$ & $\begin{array}{c}40^{\text {th }} \\
\text { percentile }\end{array}$ & $\begin{array}{c}30^{\text {th }} \\
\text { percentile }\end{array}$ & $\begin{array}{c}20^{\text {th }} \\
\text { percentile }\end{array}$ & $\begin{array}{c}90^{\text {th }} \\
\text { percentile }\end{array}$ & Total \\
\hline \multirow{6}{*}{$\begin{array}{l}\text { Mode of Entry } \\
\text { (Categorical) }\end{array}$} & Non-equity & 23 & 36 & 33 & 47 & 87 & 36 & 7 & 1 & 2 & 272 \\
\hline & $\begin{array}{l}\text { Joint venture } \\
\text { (minority equity) }\end{array}$ & 1 & 1 & 0 & 0 & 0 & 0 & 1 & 0 & 0 & 3 \\
\hline & $\begin{array}{l}\text { Joint venture (50/50 } \\
\text { equity) }\end{array}$ & 0 & 2 & 1 & 1 & 0 & 0 & 0 & 0 & 0 & 4 \\
\hline & $\begin{array}{l}\text { Joint venture } \\
\text { (majority equity) }\end{array}$ & 1 & 4 & 2 & 1 & 6 & 2 & 0 & 0 & 0 & 16 \\
\hline & Sole ownership & 3 & 1 & 2 & 1 & 5 & 0 & 0 & 1 & 0 & 13 \\
\hline & Total & 28 & 44 & 38 & 50 & 98 & 38 & 8 & 2 & 2 & 308 \\
\hline
\end{tabular}




\section{Appendix Q}

\section{UNIVARIATE STATISTICS}

\begin{tabular}{|c|c|c|c|c|c|c|}
\hline & $\mathrm{N}$ & Minimum & Maximum & Mean & Median & Std. Deviation \\
\hline QC - Continuous Dependent & 308 & 1 & 22 & 7.65 & 7.00 & 4.071 \\
\hline QDi - Categorical Dependent & 308 & 1 & 5 & 1.36 & 1.00 & 1.038 \\
\hline QDii - Learning Intent (i) & 23 & 3 & 5 & 4.00 & 4.00 & .674 \\
\hline QDii - Learning Intent (ii) & 23 & 1 & 4 & 2.43 & 2.00 & .843 \\
\hline QDii - Learning Intent (iii) & 23 & 1 & 4 & 2.26 & 2.00 & .752 \\
\hline QDii - Learning Intent (iv) & 23 & 6 & 9 & 7.61 & 8.00 & .656 \\
\hline QDii - Learning Intent (v) & 23 & 6 & 9 & 7.61 & 8.00 & .783 \\
\hline QDii - Learning Intent (vi) & 23 & 6 & 9 & 7.39 & 7.00 & .722 \\
\hline QDii - Learning Intent (vii) & 23 & 6 & 9 & 7.70 & 8.00 & .876 \\
\hline Qdiii - Regulative Distance (i) & 308 & 4 & 9 & 7.24 & 7.00 & 1.033 \\
\hline Qdiii - Regulative Distance (ii) & 308 & 4 & 9 & 7.31 & 8.00 & 1.244 \\
\hline Qdiii - Regulative Distance (iii) & 308 & 4 & 9 & 6.56 & 7.00 & 1.319 \\
\hline QF - Geo Experience (i) & 308 & 3 & 9 & 6.89 & 7.00 & 1.456 \\
\hline QF - Geo Experience (ii) & 308 & 2 & 9 & 6.96 & 7.00 & 1.513 \\
\hline QF - Geo Experience (iii) & 308 & 4 & 9 & 7.13 & 7.00 & 1.257 \\
\hline QF - Geo Experience (iv) & 308 & 3 & 9 & 7.07 & 7.00 & 1.347 \\
\hline
\end{tabular}




QF - Geo Experience (v)
QG - Ind Experience (i)
QG - Ind Experience (ii)
QG - Ind Experience (iii)
QG - Ind Experience (iv)
QG - Ind Experience (v)
QG - Ind Experience (vi)
QI - Know how (i)
QI - Know how (ii)
QI - Know how (iii)
QI - Know how (iv)
QI - Know how (v)
QJ - Org Culture (i)
QJ - Org Culture (ii)
QJ - Org Culture (iii)
QJ - Org Culture (iv)
QJ - Org Culture (v)
QK - Financial Resources
QM - Reputation (i)
QM - Reputation (ii)
QM - Reputation (iii)
QM - Reputation (iv)

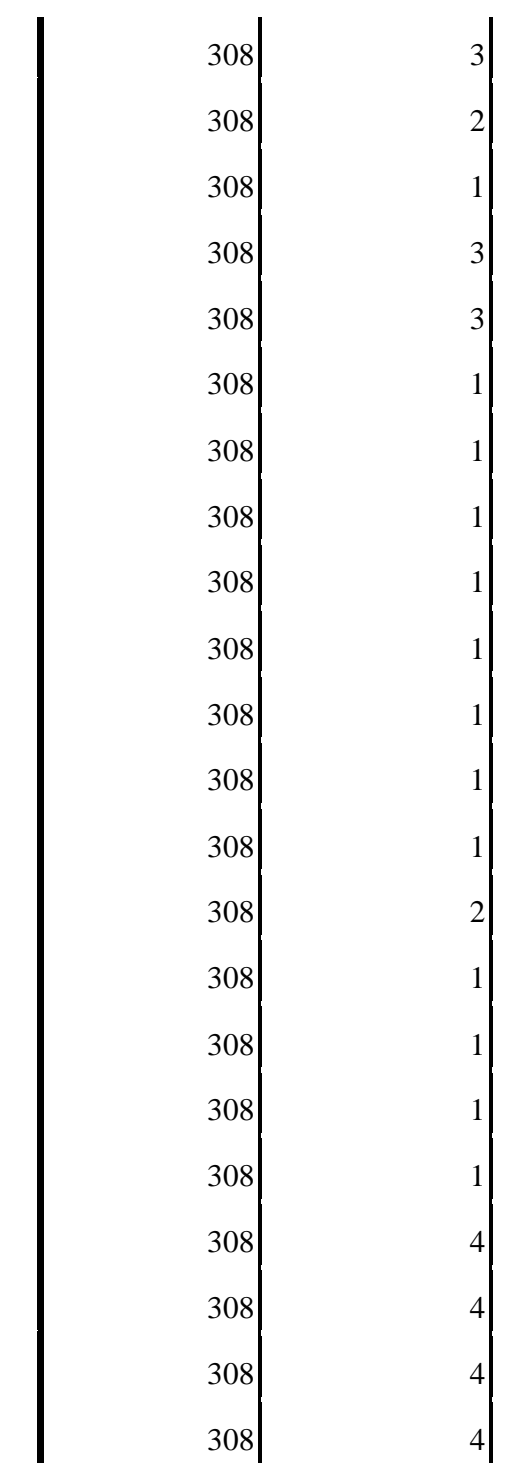

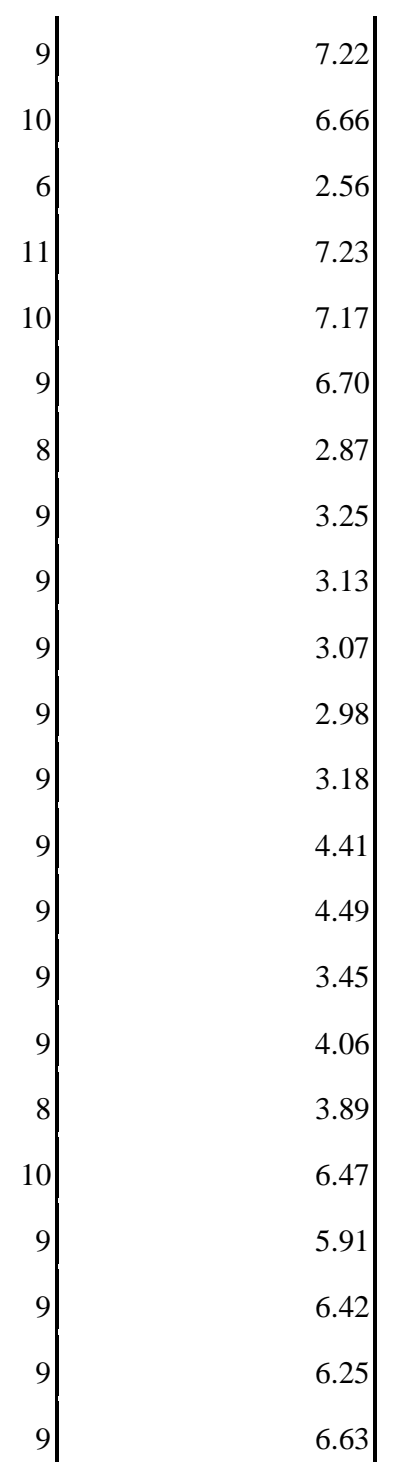

7.00
7.00
2.00
8.00
7.00
7.00
3.00
3.00
3.00
2.00
2.00
3.00
4.00
4.00
3.00
4.00
4.00
6.00
6.00
6.00
6.00
7.00 


\begin{tabular}{|c|c|c|c|c|c|c|}
\hline QN - Size (UG) & 308 & 0 & 46000 & 17241.92 & 16000.00 & 9392.900 \\
\hline QN - Size (PG) & 308 & 0 & 16037 & 5118.82 & 4122.00 & 3279.169 \\
\hline QN - Size (Staff) & 308 & 94 & 8000 & 1973.71 & 1800.00 & 1341.793 \\
\hline Age (Years) & 308 & 4 & 254 & 92.15 & 97.00 & 58.082 \\
\hline QQ (i) - World Ranking & 308 & 1 & 8 & 4.68 & 5.00 & 1.617 \\
\hline QQ(ii) - Domestic Ranking & 308 & 1 & 9 & 4.02 & 4.00 & 1.683 \\
\hline QR - Reasons for Offshore egmnt (i) & 308 & 5 & 9 & 7.05 & 7.00 & .961 \\
\hline QR - Reasons for Offshore egmnt (ii) & 308 & 3 & 8 & 5.15 & 5.00 & 1.002 \\
\hline QR - Reasons for Offshore egmnt (iii) & 308 & 5 & 9 & 7.04 & 7.00 & .999 \\
\hline QR - Reasons for Offshore egmnt (iv) & 308 & 3 & 7 & 5.15 & 5.00 & 1.006 \\
\hline QR - Reasons for Offshore egmnt (v) & 308 & 5 & 9 & 7.12 & 7.00 & .961 \\
\hline QR - Reasons for Offshore egmnt (vi) & 308 & 5 & 9 & 7.15 & 7.00 & 1.062 \\
\hline QR - Reasons for Offshore egmnt (vii) & 308 & 1 & 7 & 3.31 & 3.00 & 1.185 \\
\hline QT - Extent of influence of QS & 293 & 1 & 8 & 2.97 & 3.00 & 1.385 \\
\hline QU - Future engmnt intent & 308 & 1 & 9 & 5.70 & 6.00 & 1.754 \\
\hline IMD Normative ID (Yr of entry) & 307 & .1287 & 6.6255 & 1.6245 & 1.2163 & 1.2670 \\
\hline IMD Regulative ID (Yr of entry) & 307 & .03373 & 9.7633 & 1.5245 & 1.2123 & 1.0604 \\
\hline EFI Regulative ID (Yr of entry) & 308 & .0000 & 9.5079 & 3.4333 & 4.8000 & 2.3484 \\
\hline Hostede Cultural distance & 307 & .0198 & 4.7864 & 2.7065 & 2.7066 & .8467 \\
\hline Control - Edu demand (yr of entry) & 308 & .006 & .332 & .09317 & .2175 & .0992 \\
\hline
\end{tabular}

Note: means and standard deviations for categorical variables should be interpreted with caution. Medians are more applicable for those variables. 


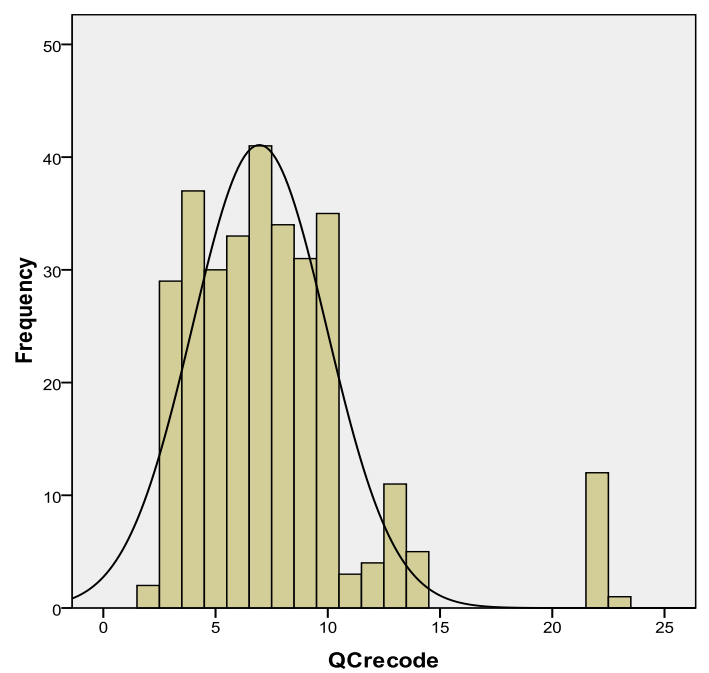

Mean $=7.65$
Stdd. Dev
$N=4.071$
$N=308$

QDii - Learning Intent (i)

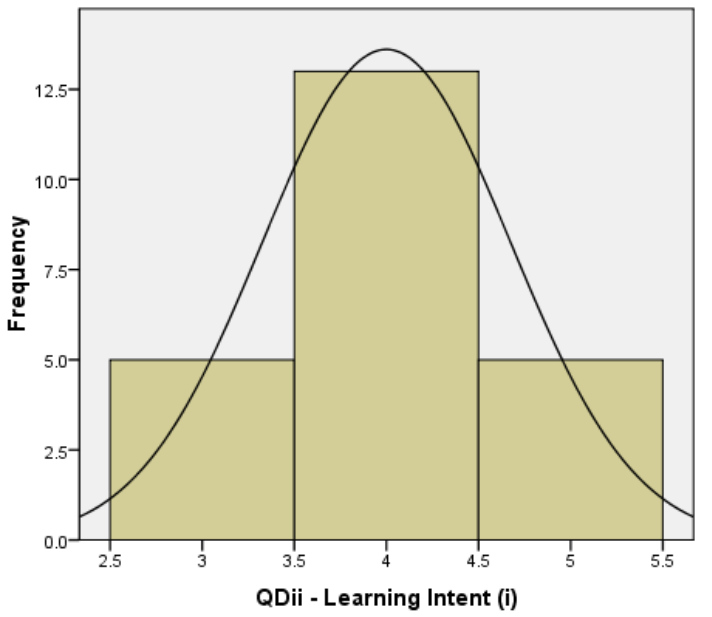

QDi - Categorical Dependent

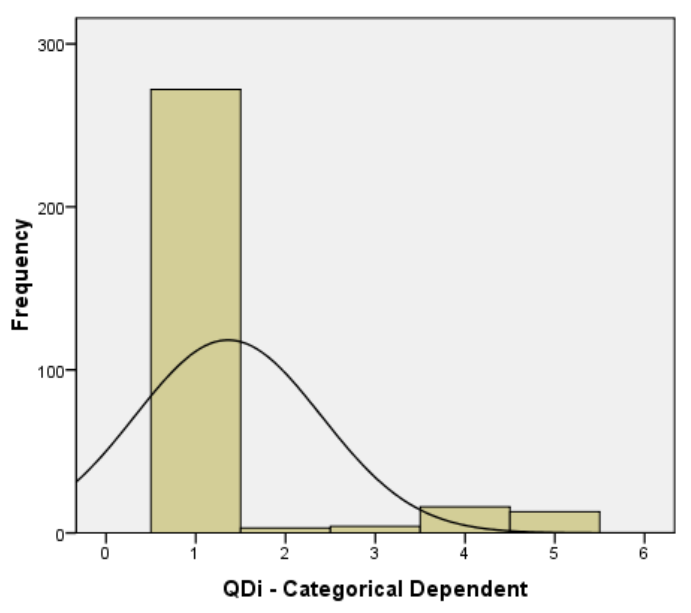

Mean $=1.36$
Std. Dev. $=1.038$
$N=308$

QDii - Learning Intent (ii)

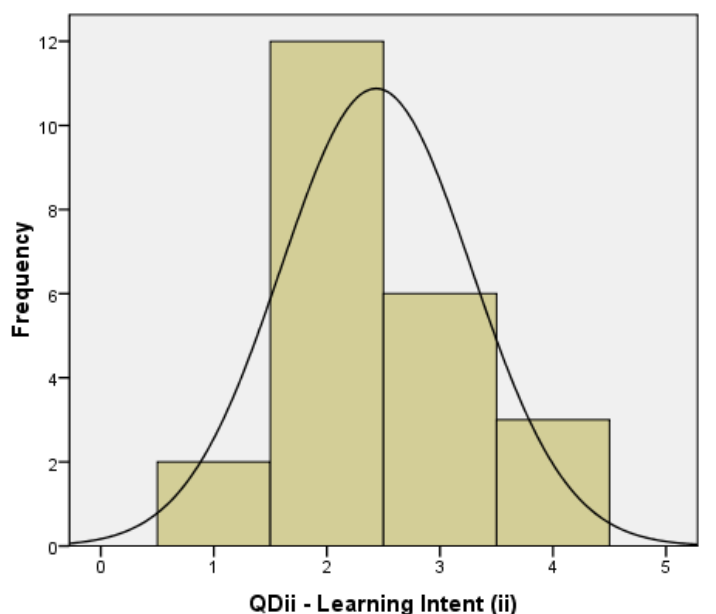

Mean $=2.43$
Std. DeV $=0.843$
$N=23$

Mean $=4$
Std. Dev. $=0.674$
$N=23$

QDii - Learning Intent (ii) 
QDii - Learning Intent (iii)

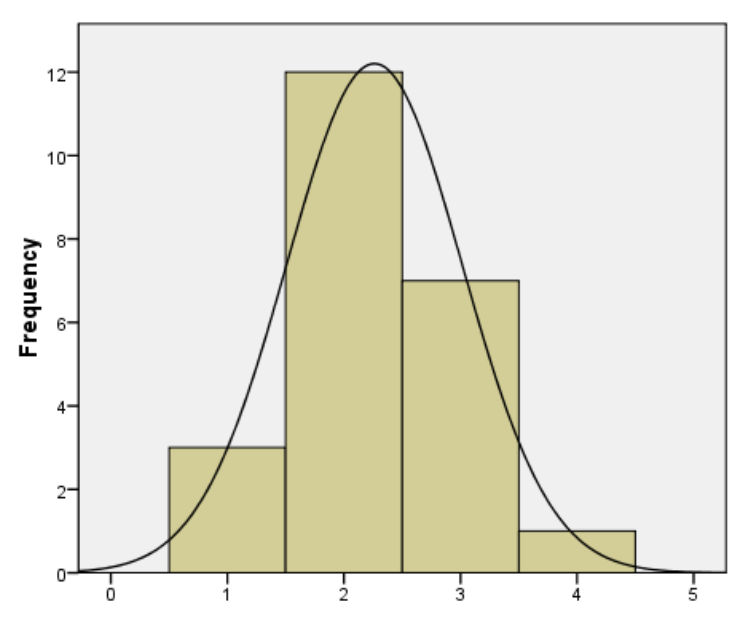

QDii - Learning Intent (iii)

QDii - Learning Intent (v)

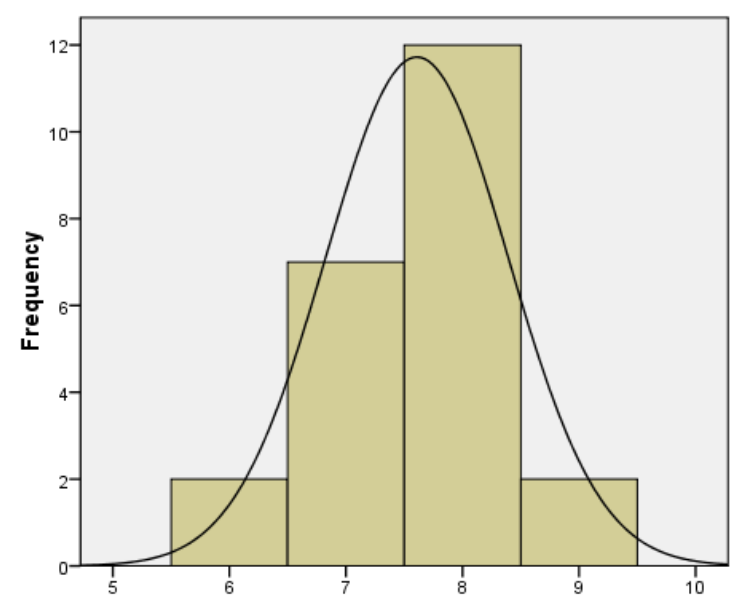

QDii - Learning Intent (v)
QDii - Learning Intent (iv)

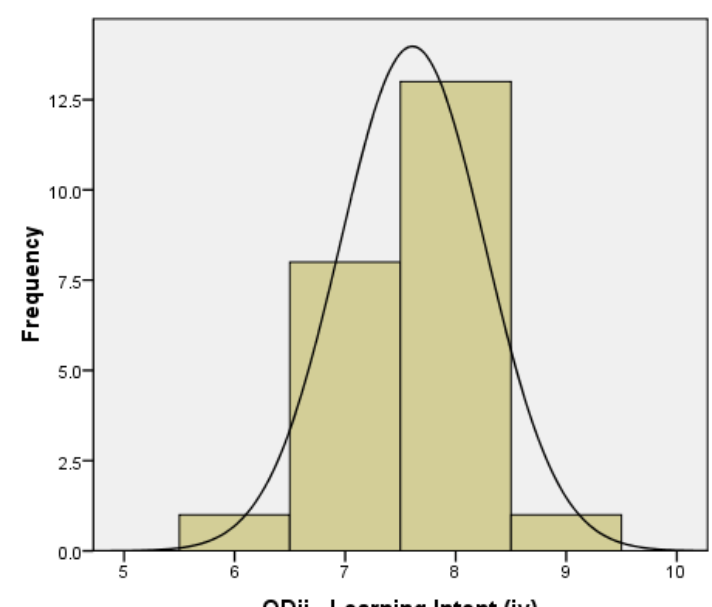

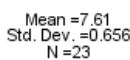

Mean $=2.26$
Std. Dev. $=0.752$
$N=23$

QDii - Learning Intent (iv)

QDii - Learning Intent (vi)

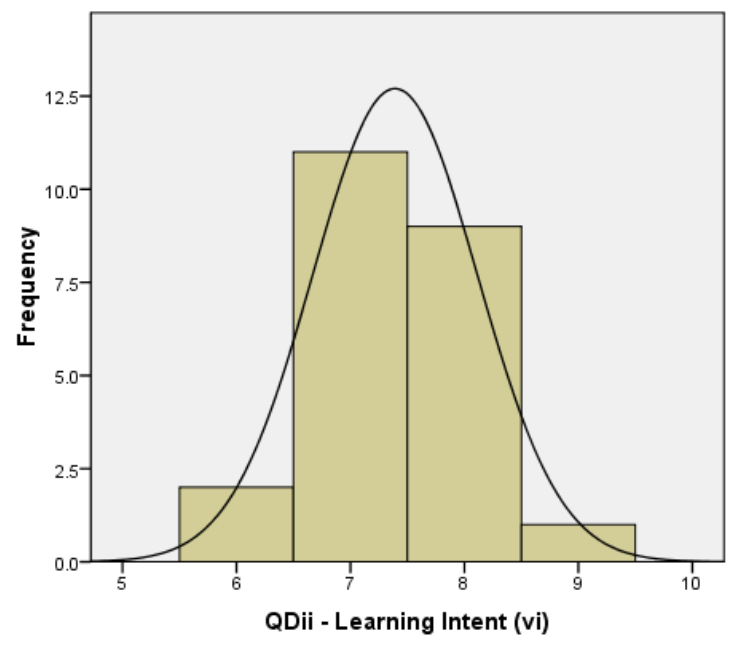

Mean $=7.39$
Std. Deve. $=0.722$
$N=23$ 
QDii - Learning Intent (vii)

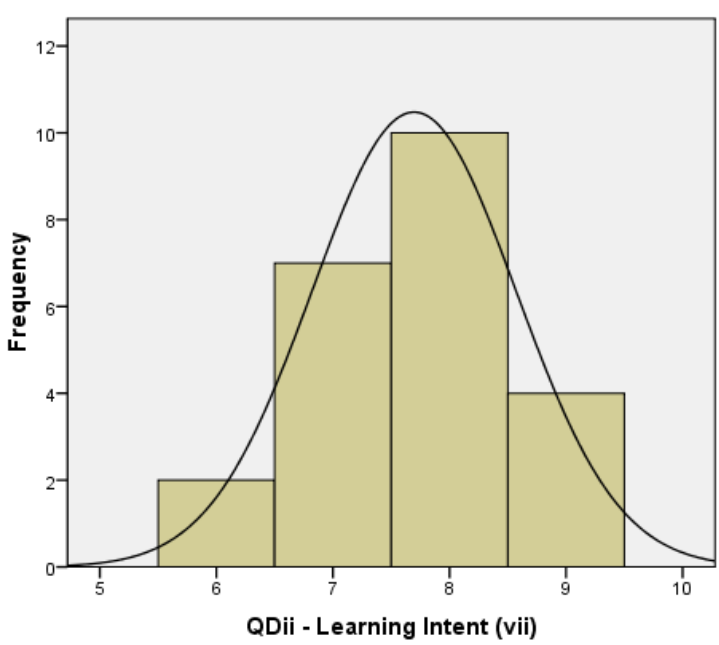

Qdiii - Regulative Distance (ii)

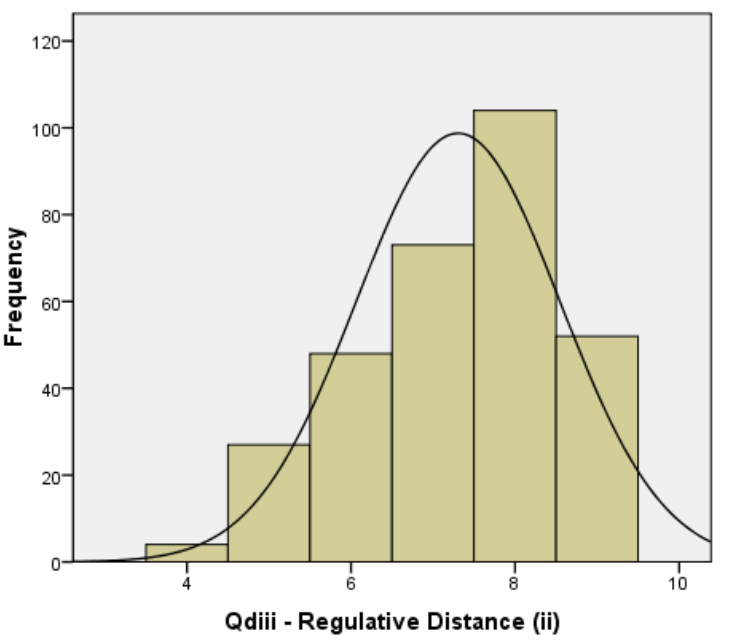

Qdiii - Regulative Distance (i)

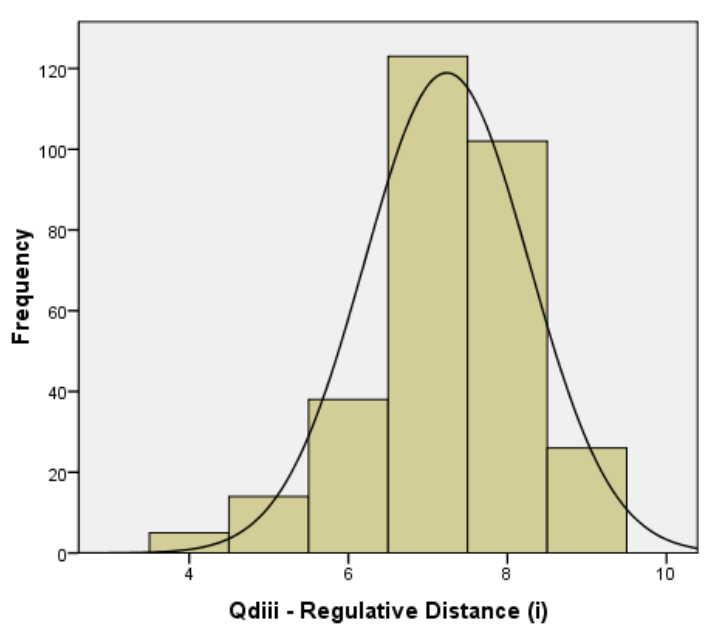

Mean $=7.24$
Std. Dev. $=1.033$
$N=308$

Mean $=7.7$
Std. Dev. $=0.876$
$N=23$

Qdiii - Regulative Distance (i)

Qdiii - Regulative Distance (iii)

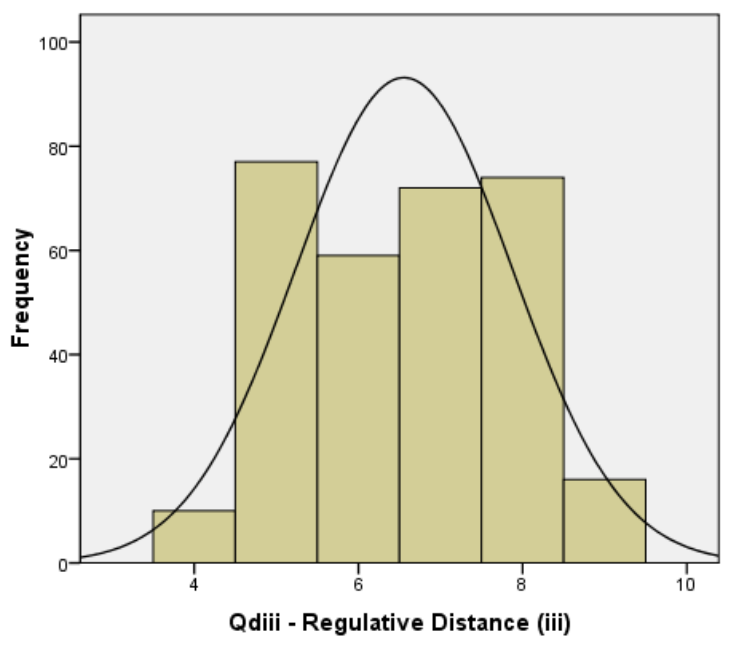

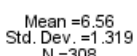

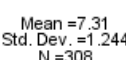


QF - Geo Experience (i)

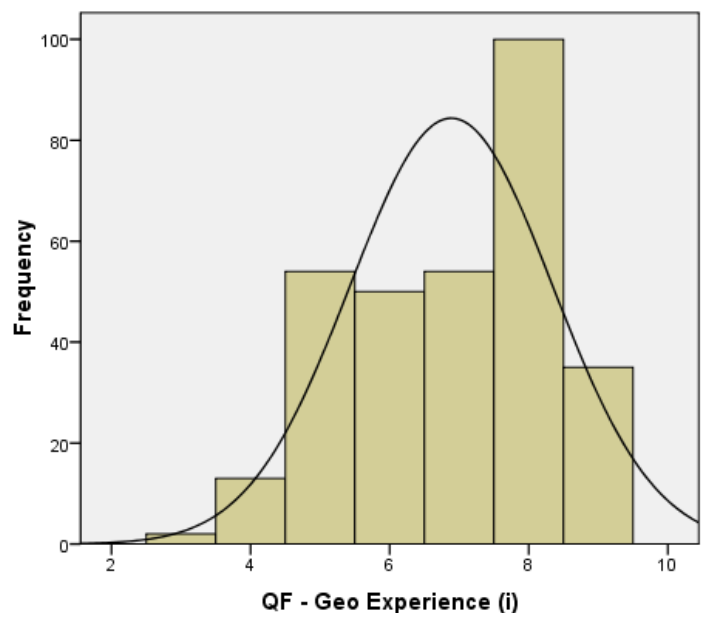

QF - Geo Experience (iii)

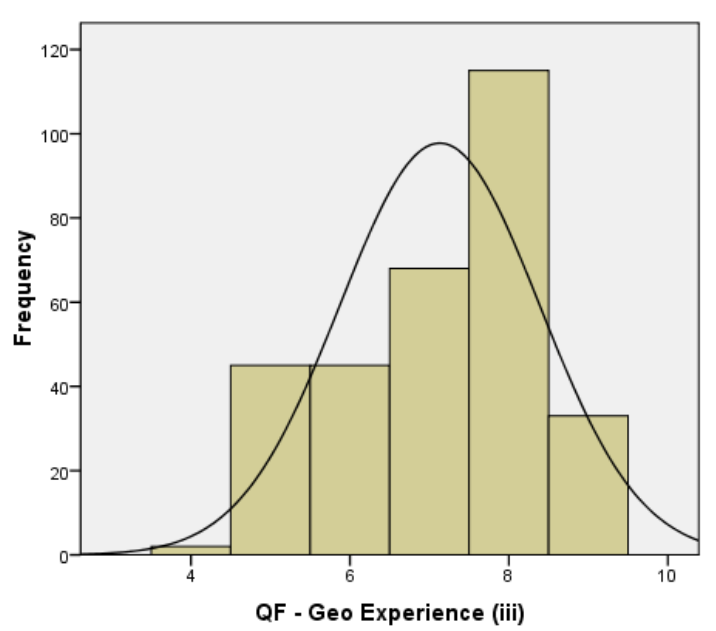

QF - Geo Experience (ii)

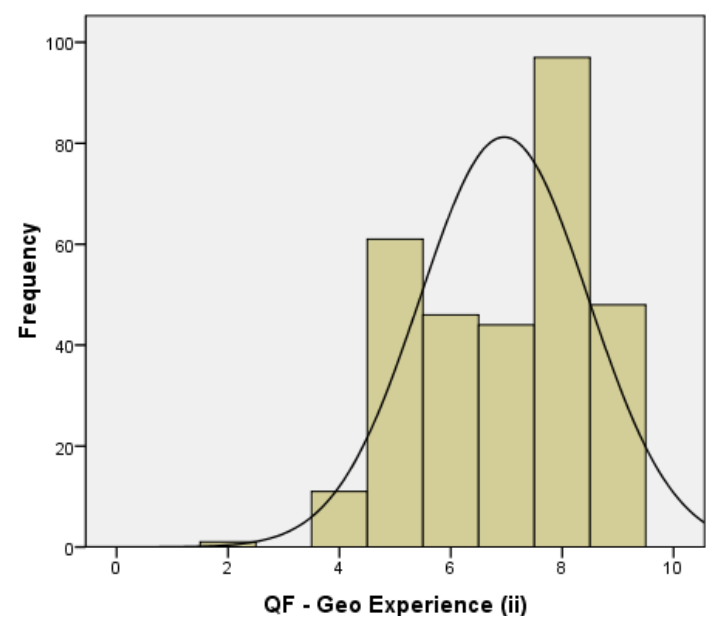

Mean $=6.96$
Std. Dev. $=1.513$
$N=308$

$\begin{aligned} \text { Mean } & =6.89 \\ \text { Std. Dev } & =1.456\end{aligned}$

QF - Geo Experience (ii)

QF - Geo Experience (iv)

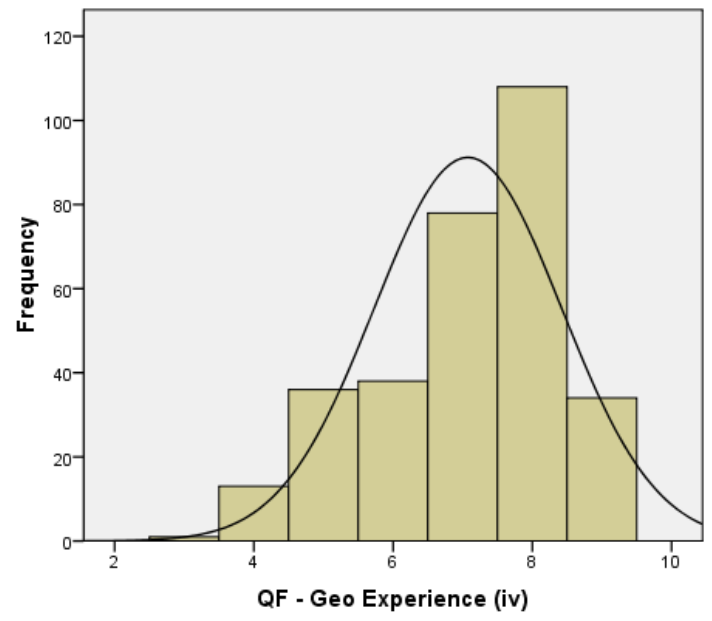

Mean $=7.07$
Std. Dev. $=1.347$
$N=308$

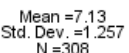


QF - Geo Experience (v)

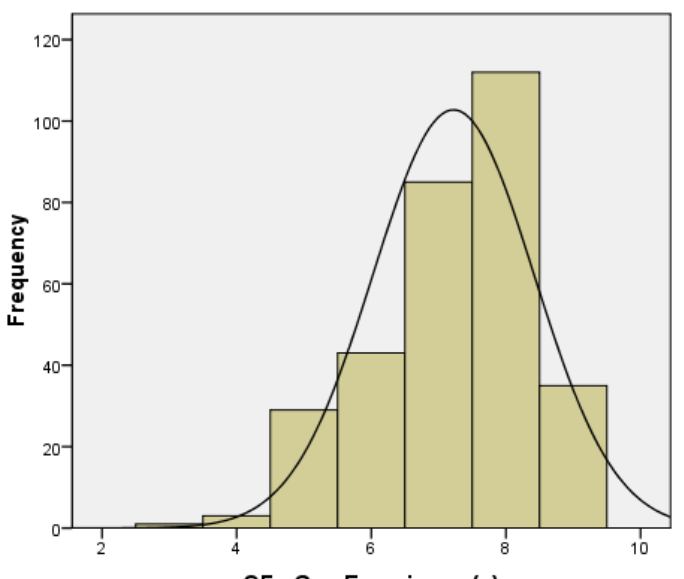

QF - Geo Experience (v)

QG - Ind Experience (ii)

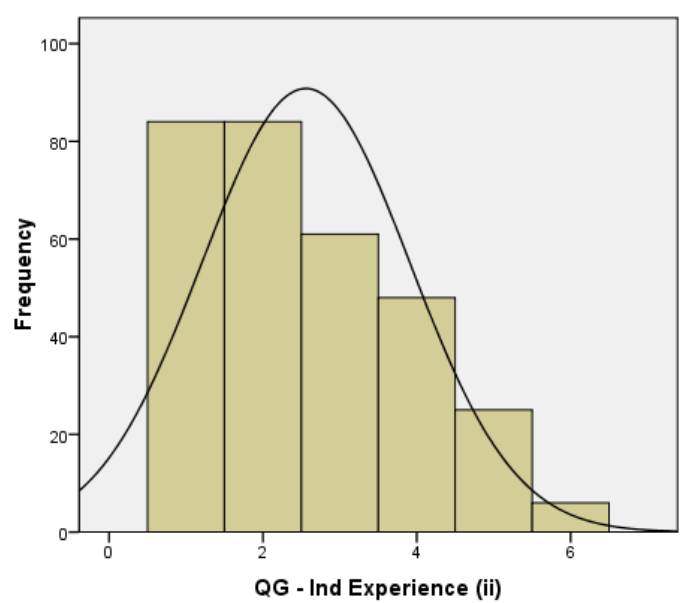

QG - Ind Experience (i)

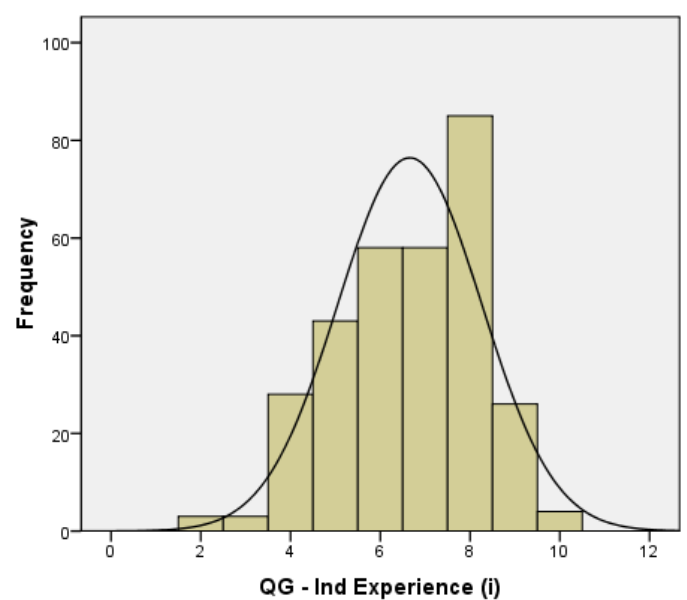

Mean $=6.66$
Std. Dev. $=.6 .608$
$N=308$

Mean $=7.22$
Std. Dev. $=1.196$
$N=308$

QG - Ind Experience (i)

QG - Ind Experience (iii)

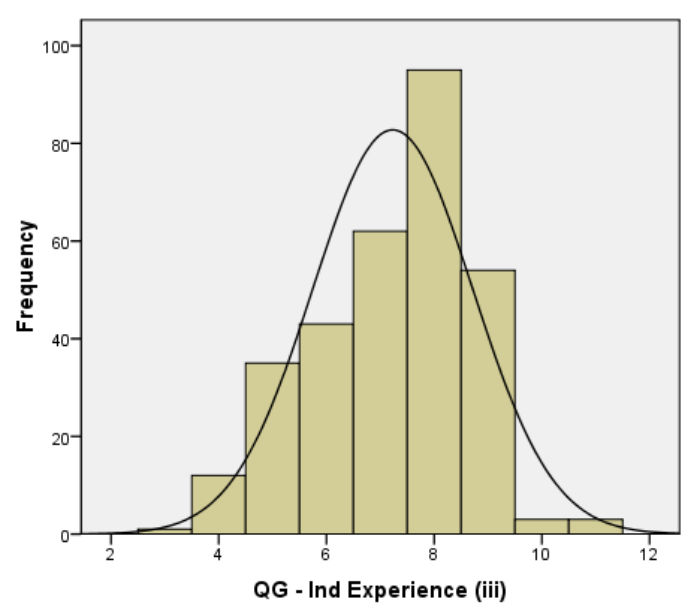


QG - Ind Experience (iv)

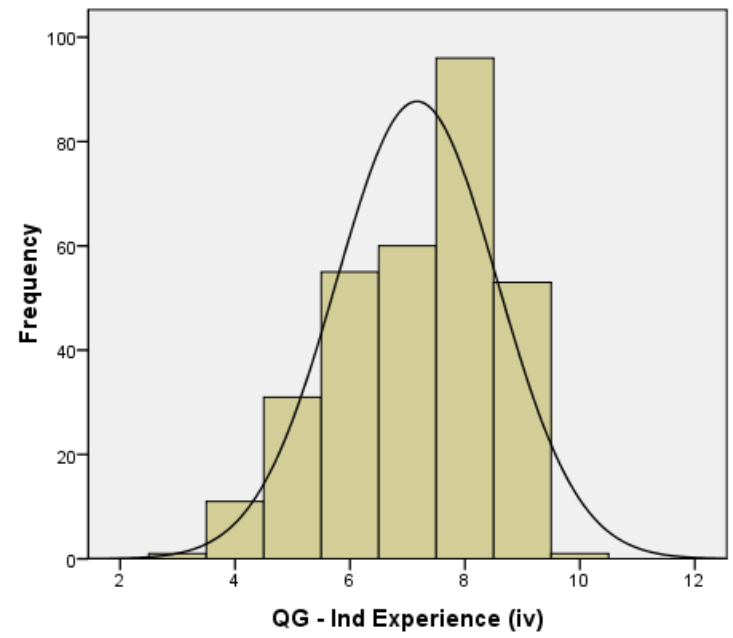

QG - Ind Experience (vi)

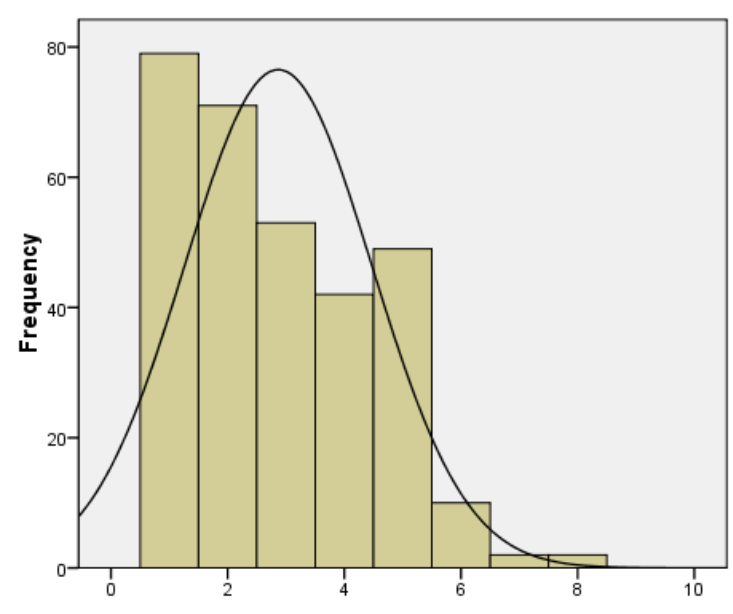

QG - Ind Experience (vi)

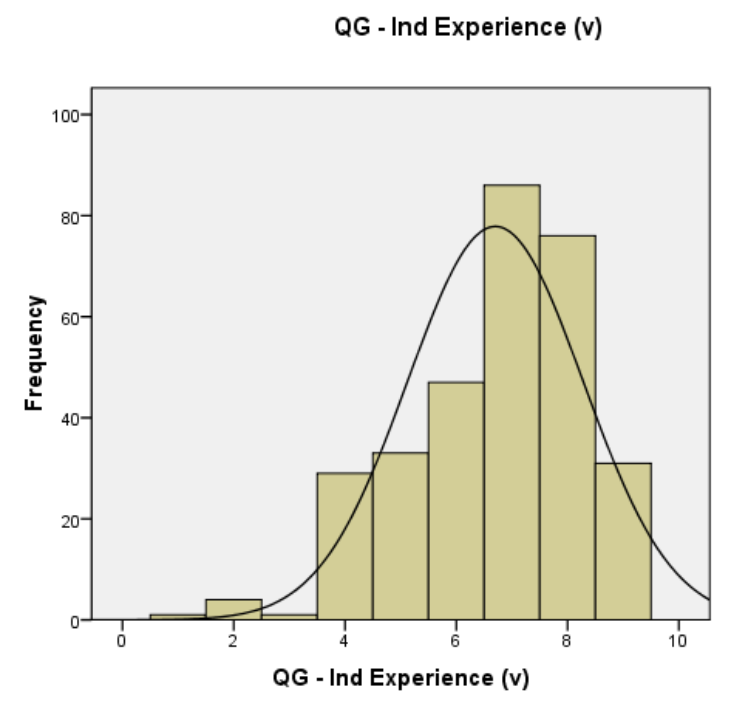

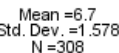

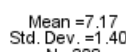

QI - Know how (i)

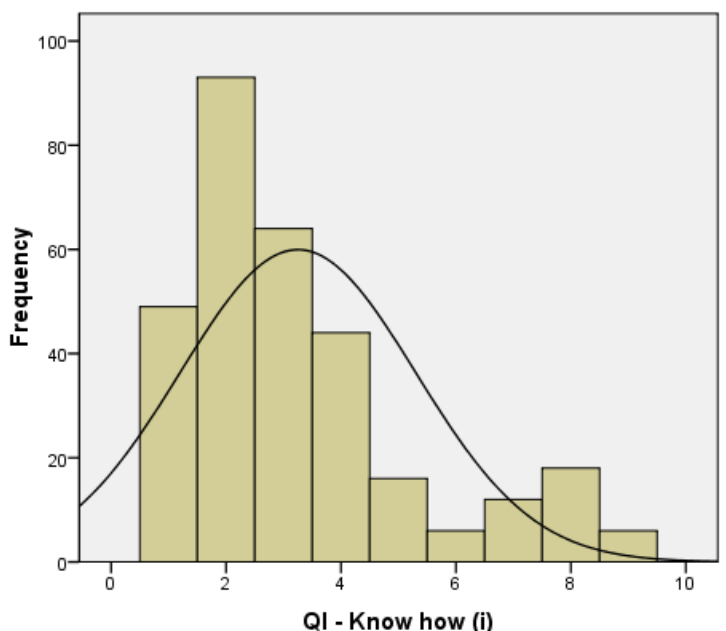

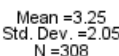

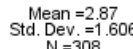

QI - Know how (i) 


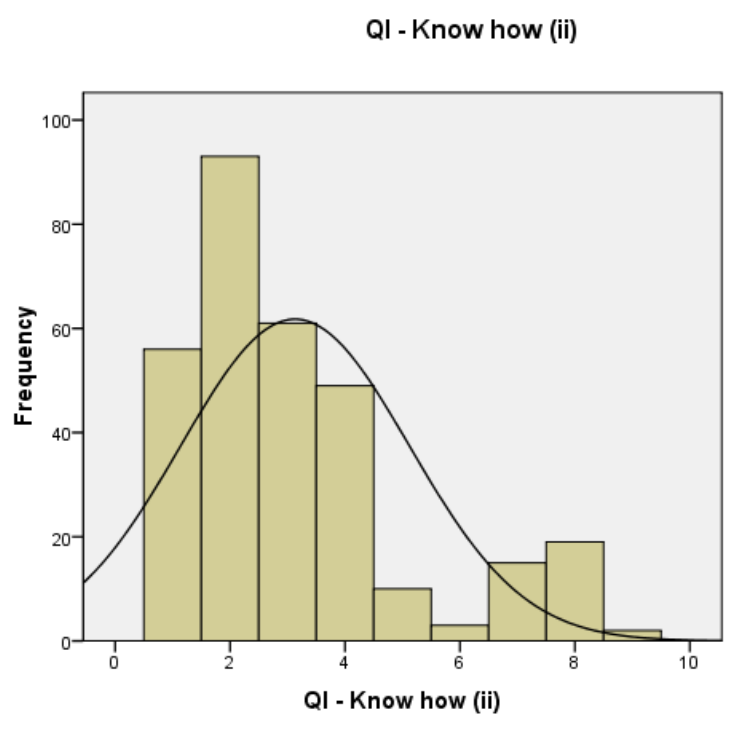

QI - Know how (iv)

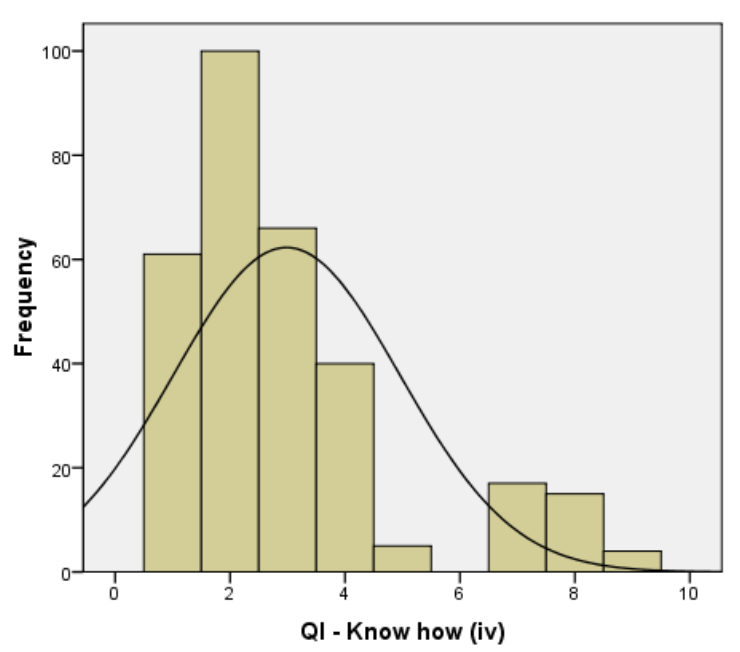

QI - Know how (iii)

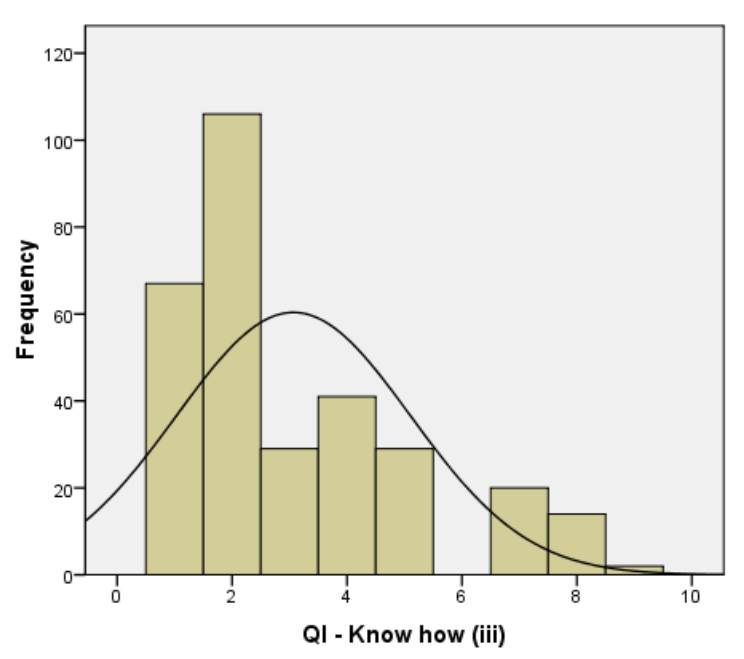

Mean $=3.07$
Std. Devev $=2.035$
$N=308$

Mean $=3.13$
Std. DeV $=1.989$
$N=308$

QI - Know how (v)

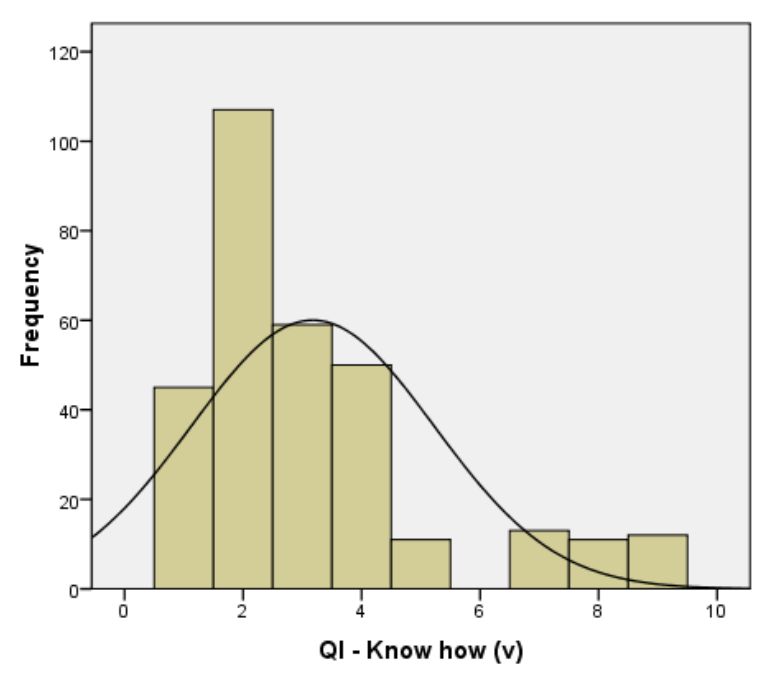


QJ - Org Culture (i)

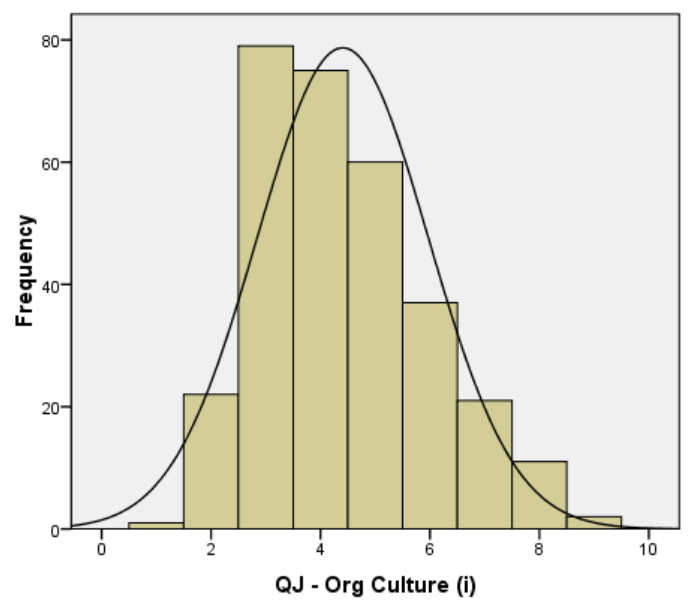

QJ - Org Culture (iii)

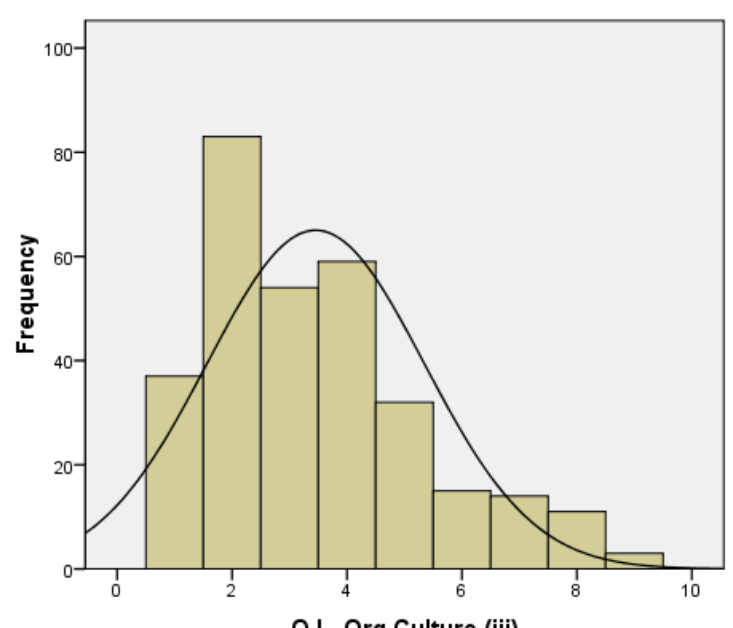

QJ - Org Culture (iii)
QJ - Org Culture (ii)

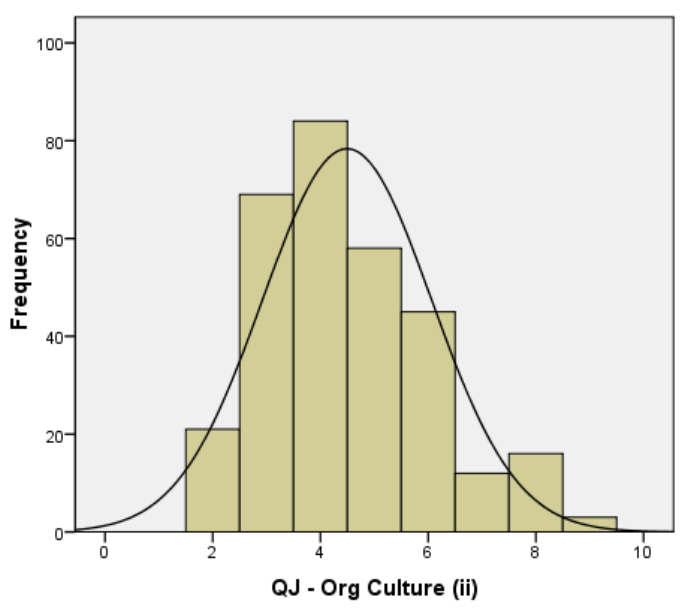

Mean $=4.49$
Std. DeV $\begin{gathered}\text {. } \\ N=308 \\ N=368\end{gathered}$

Mean $=4.41$
Std. Dev. $=.51$
$N=308$

- Org Culture (iv)

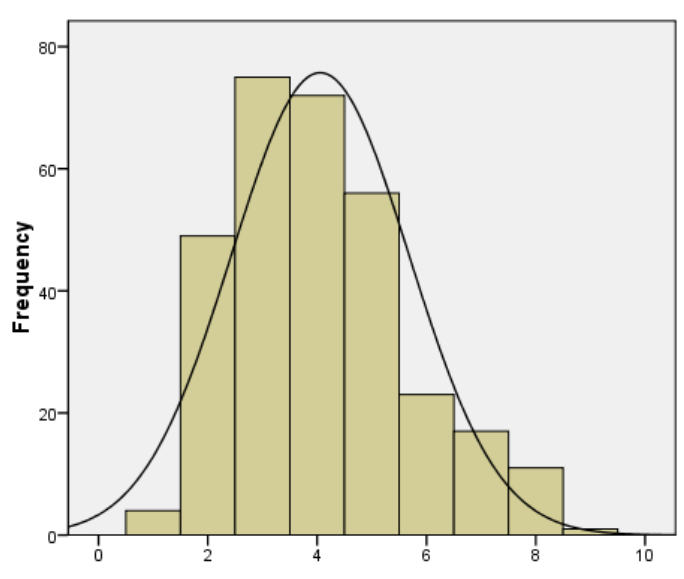

QJ - Org Culture (iv)

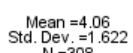


QJ - Org Culture (v)

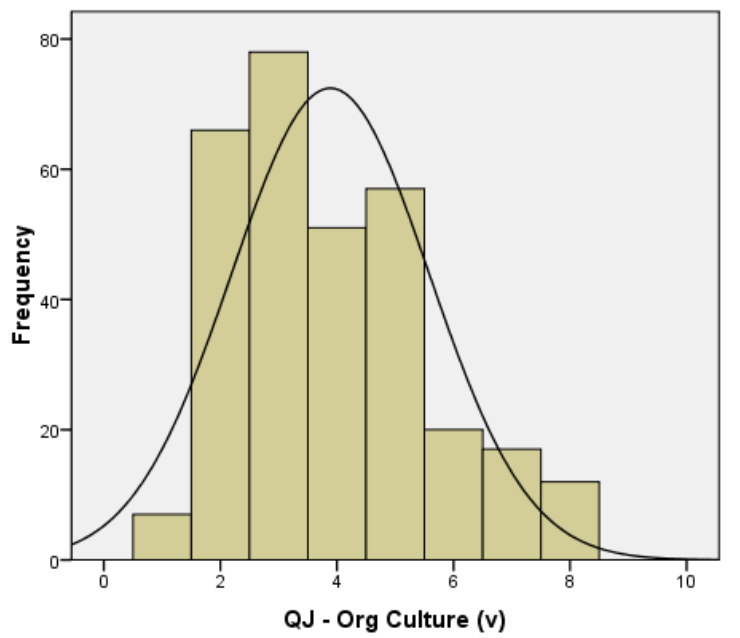

QM - Reputation (i)

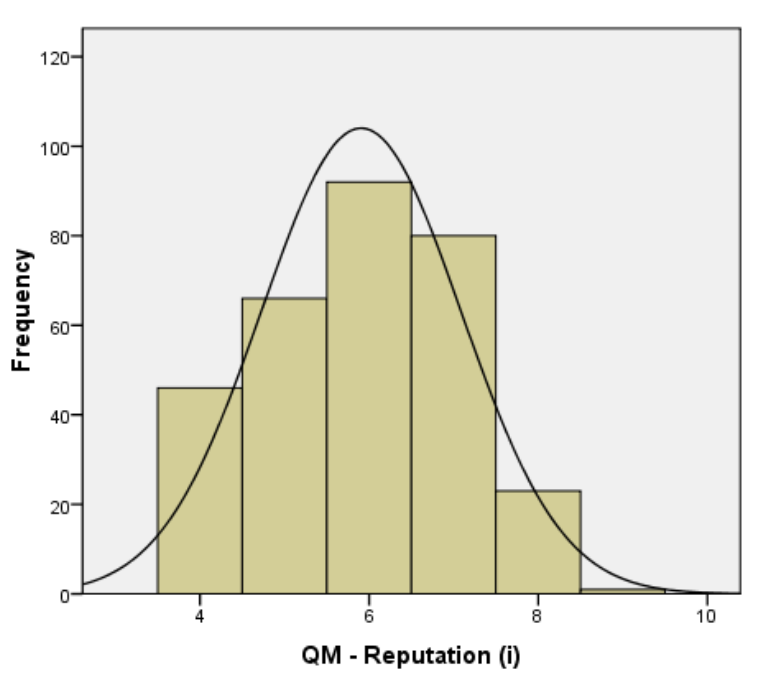

QK - Financial Resources

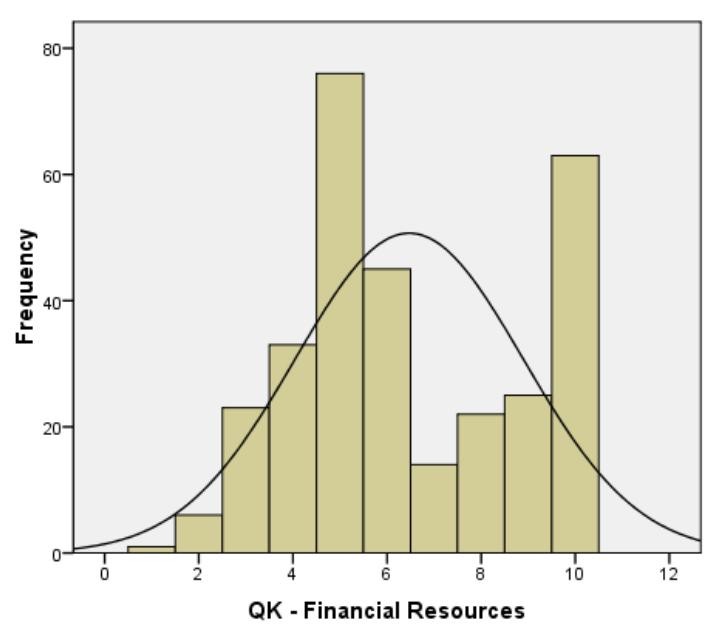

Mean $=6.47$
Std. Devev. $=2.423$
$N=308$

Mean $=3.89$
Std. Dev. $=1.696$
$N=308$

QM - Reputation (ii)

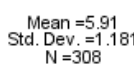

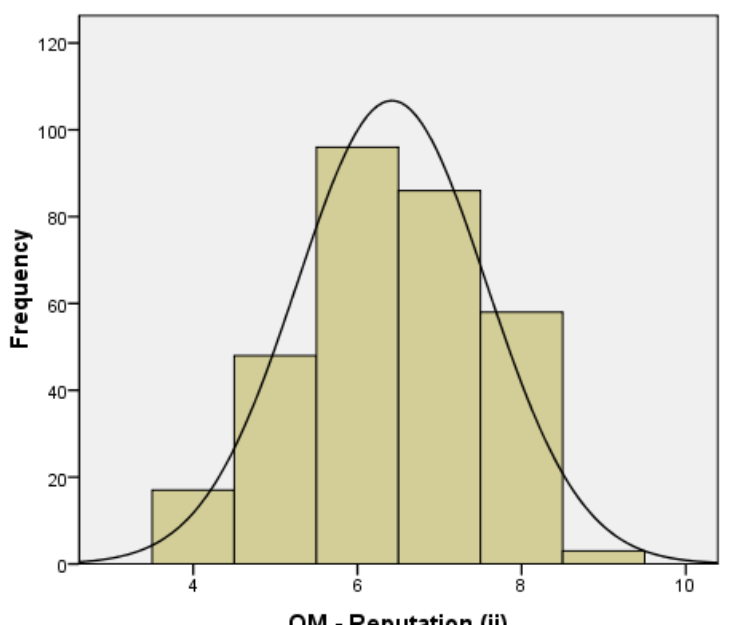


QM - Reputation (iii)

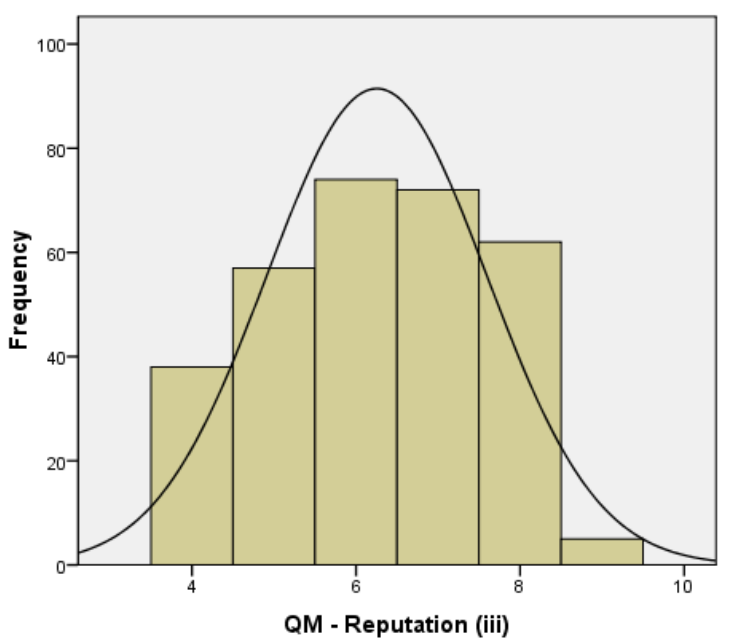

QN - Size (UG)

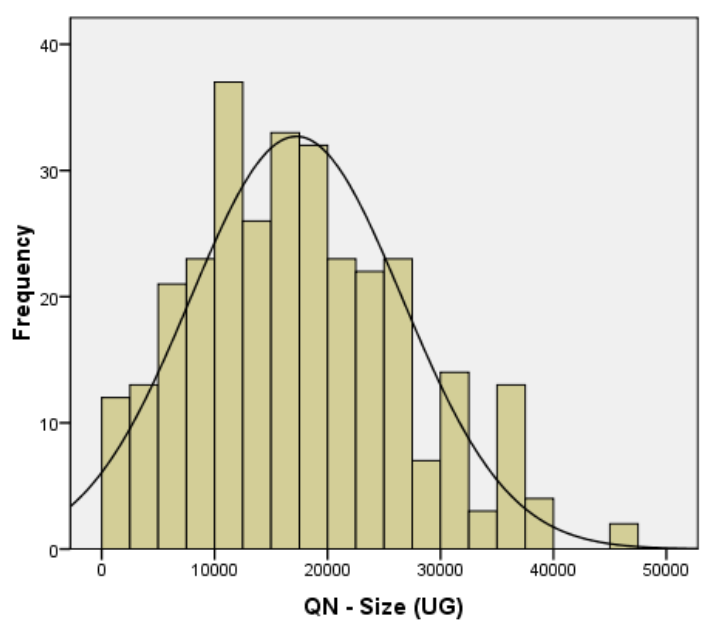

QM - Reputation (iv)

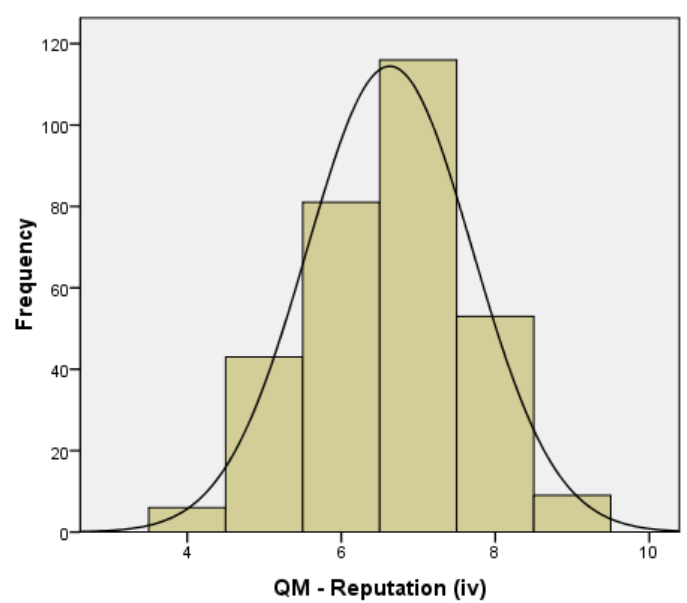

Mean $=6.63$
Std. Dev $=1.074$
$N=308$

QN - Size (PG)

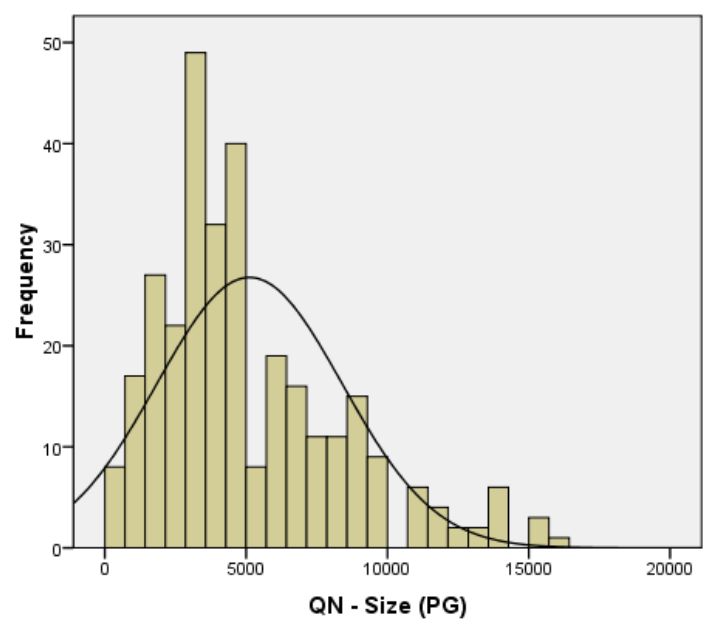


QN - Size (Staff)

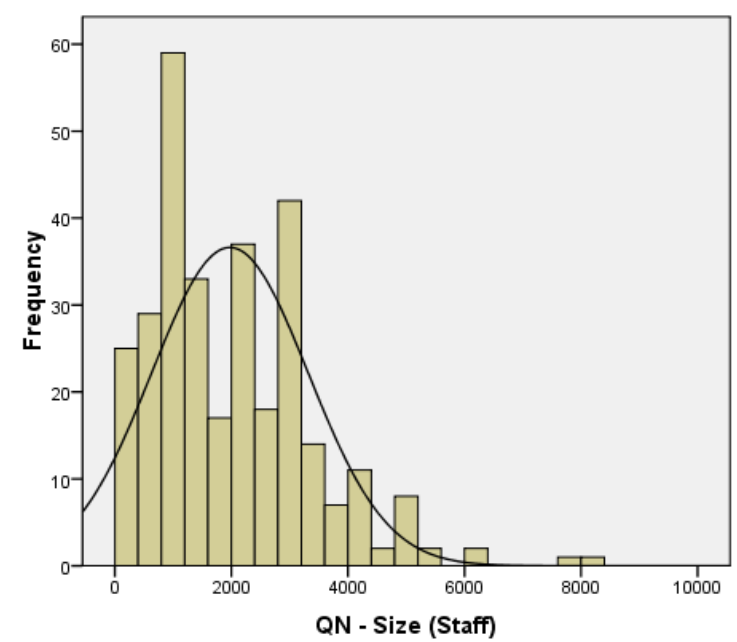

QQ (i) - World Ranking

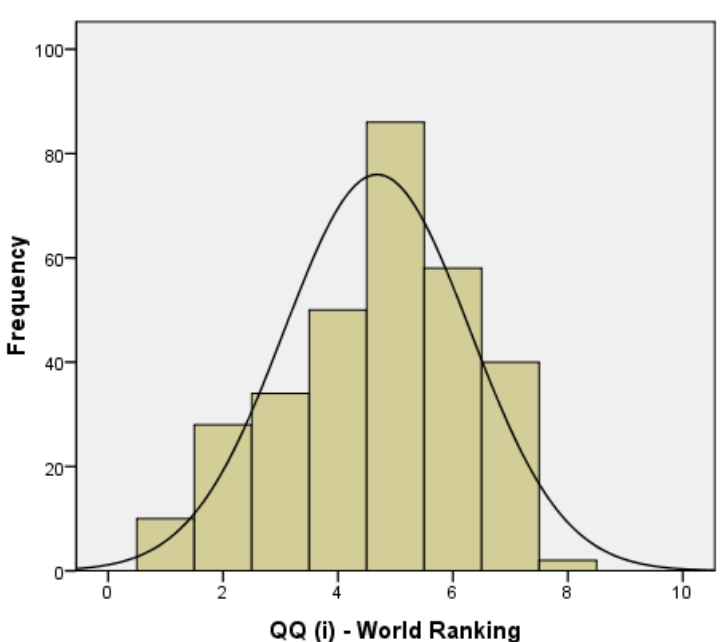

Histogram

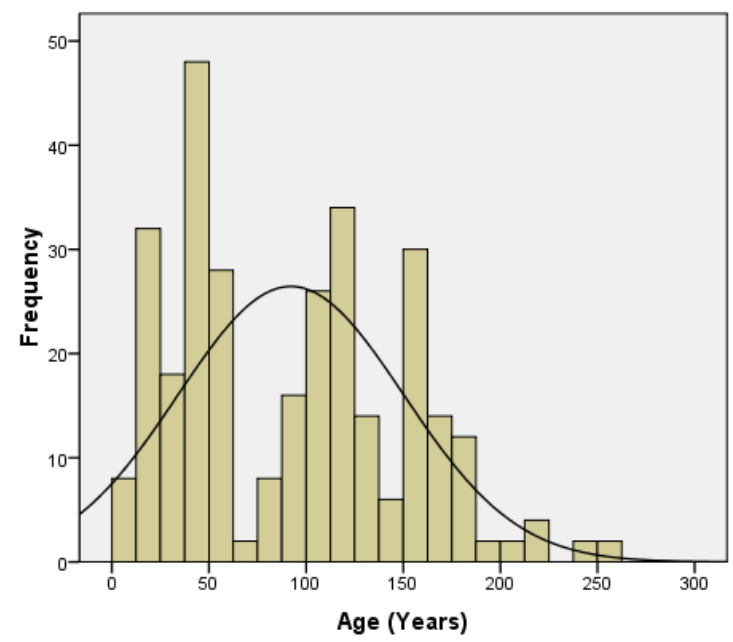

Mean $=92.15$
Std. Dev. .58 .082
$N=30.082$

Mean $=1973.71$
Std. Dev $=1934.793$
$N=303$

QQ(ii) - Domestic Ranking

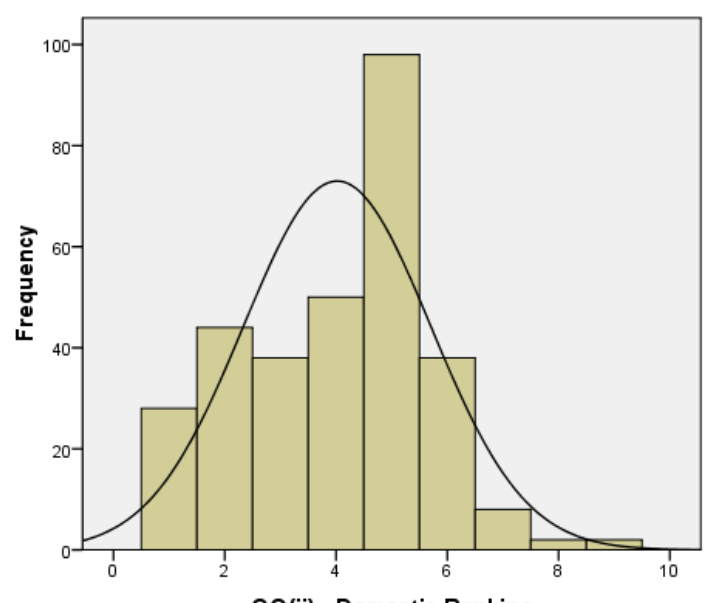

QQ(ii) - Domestic Ranking 
QR - Reasons for Offshore egmnt (i)

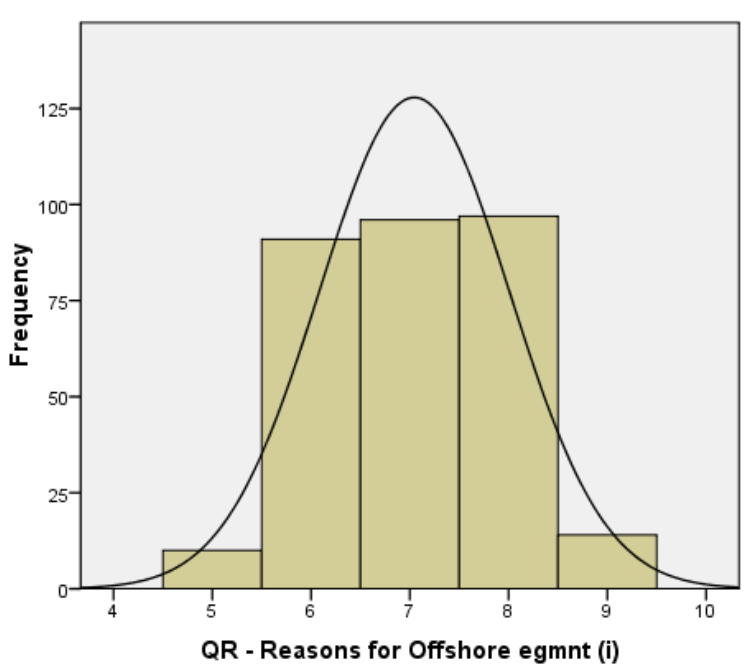

QR - Reasons for Offshore egmnt (iii)

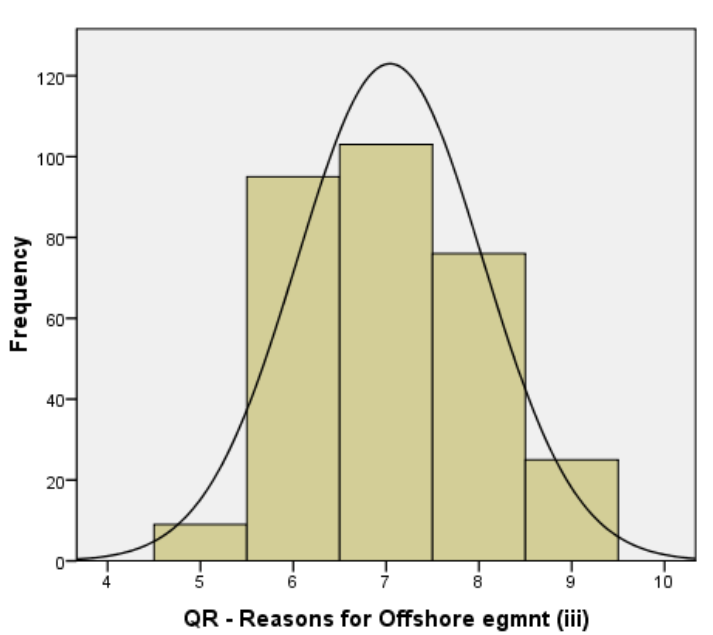

QR - Reasons for Offshore egmnt (ii)

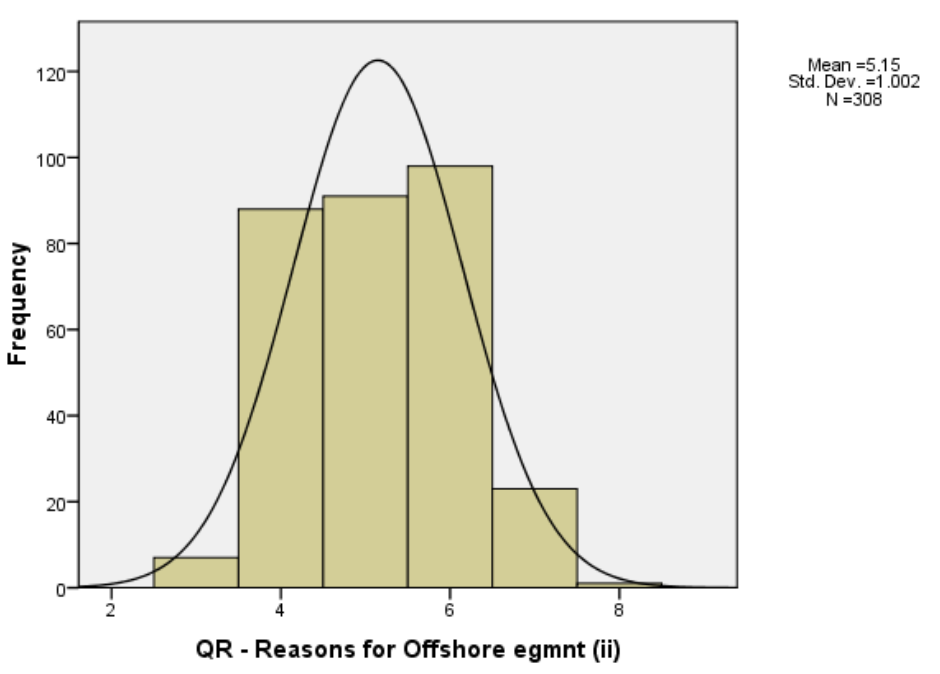

QR - Reasons for Offshore egmnt (iv)

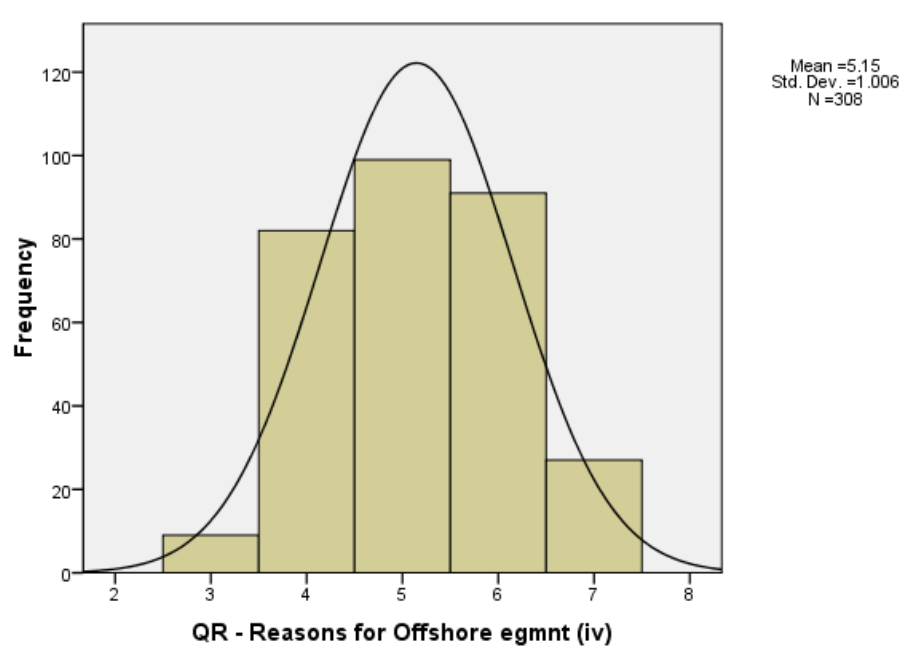


QR - Reasons for Offshore egmnt (v)

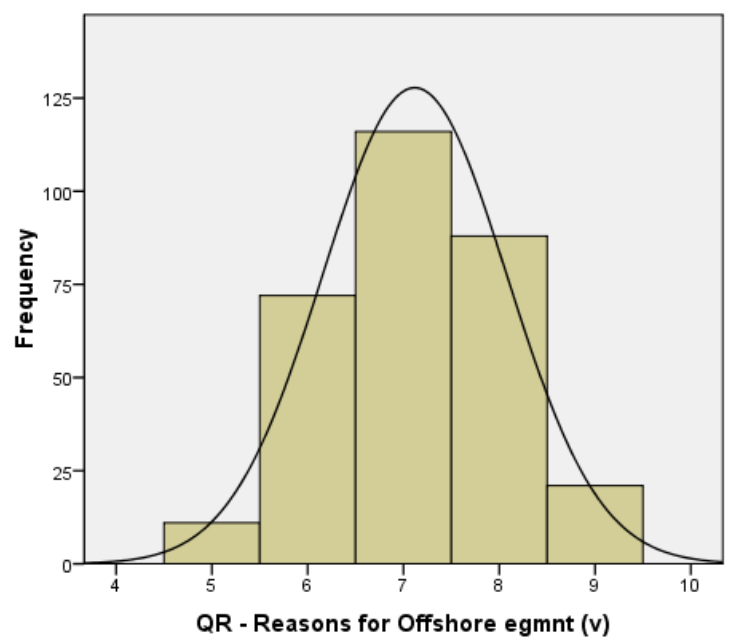

QR - Reasons for Offshore egmnt (vii)

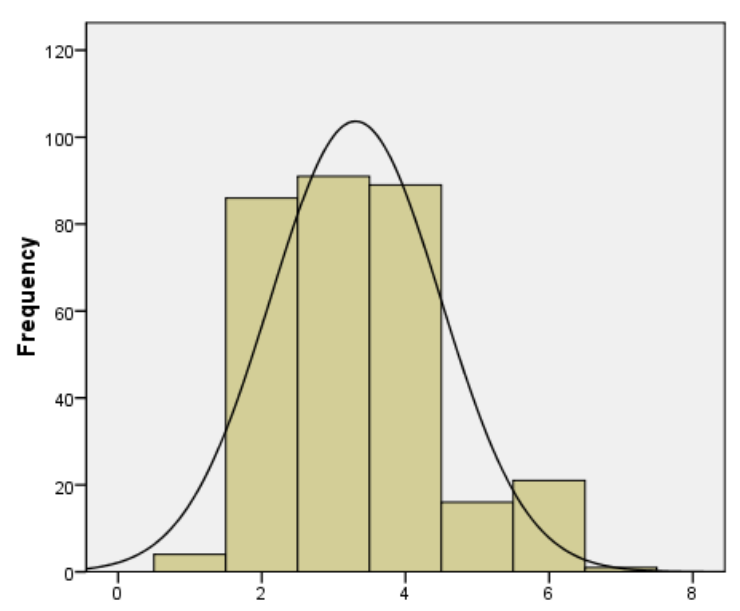

QR - Reasons for Offshore egmnt (vii)
QR - Reasons for Offshore egmnt (vi)

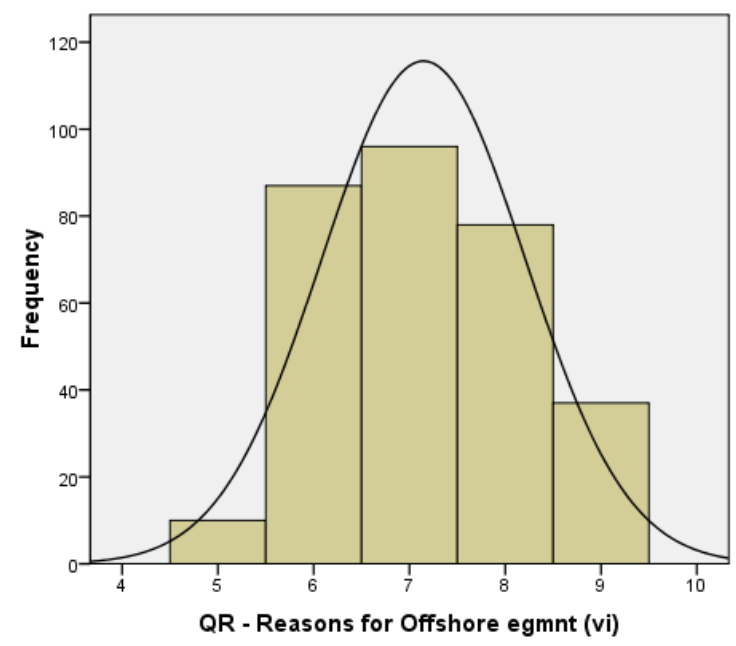

Mean $=7.15$
Std. Dev $=1.062$
$N=308$
Mean $=7.12$
Std. Dev $=0.961$
$N=308$

Mean $=3.31$
Std. Dev $=1.1 .185$
$N=308$

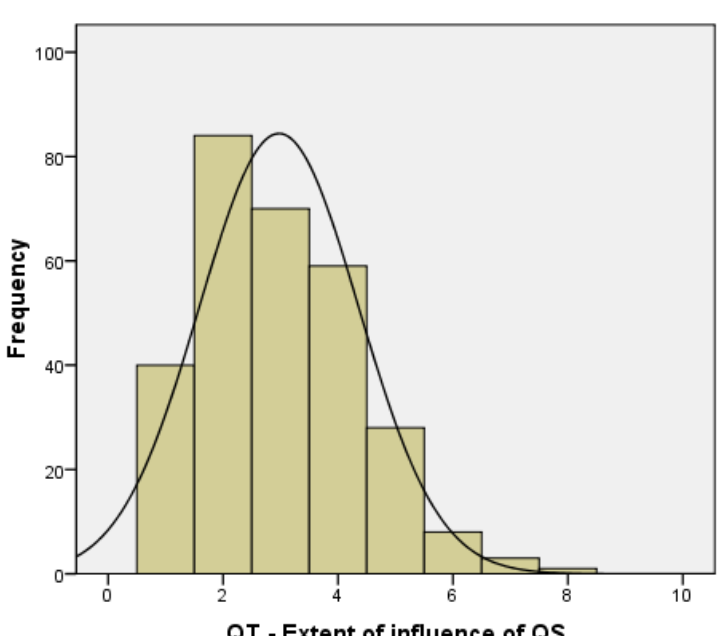

Mean $=2.97$
Std. Dev. $=1.385$
$N=293$ 
QU - Future engmnt intent

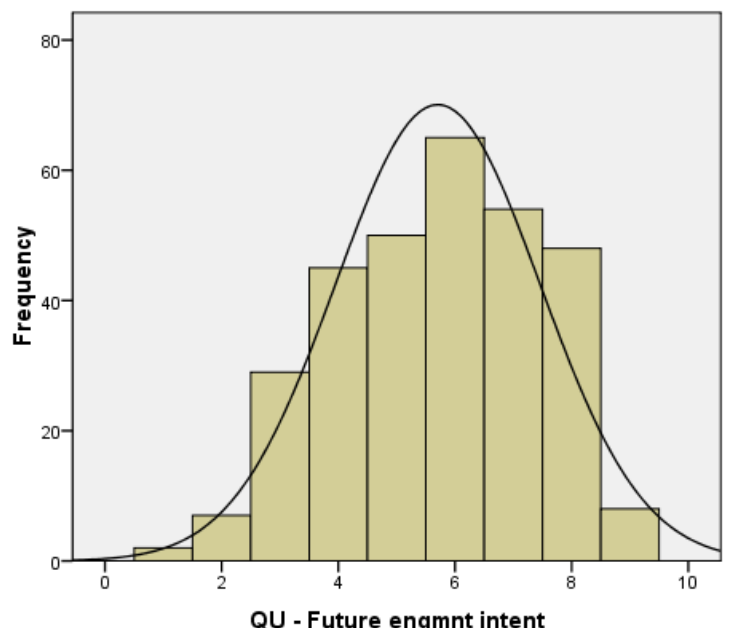

IMD Regulative ID (Yr of entry)

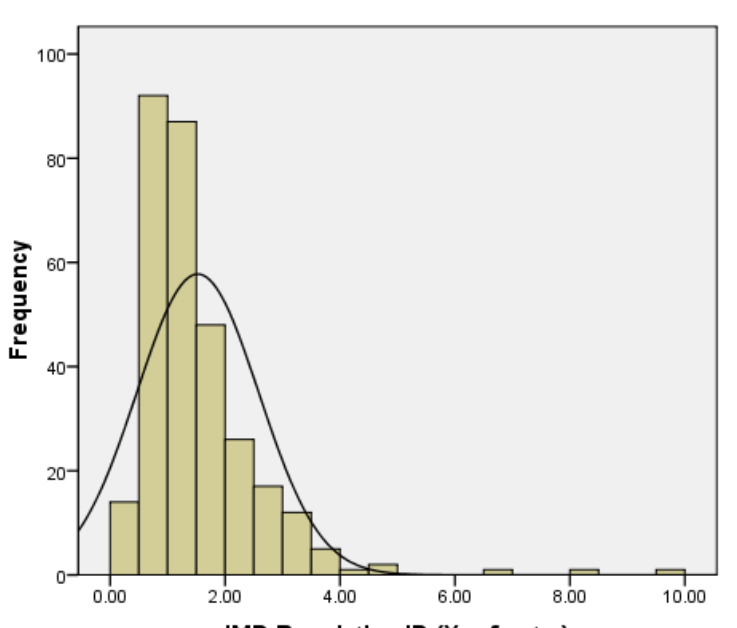

Mean $=5.7$
Std. Dev. $=1.754$
$N=308$

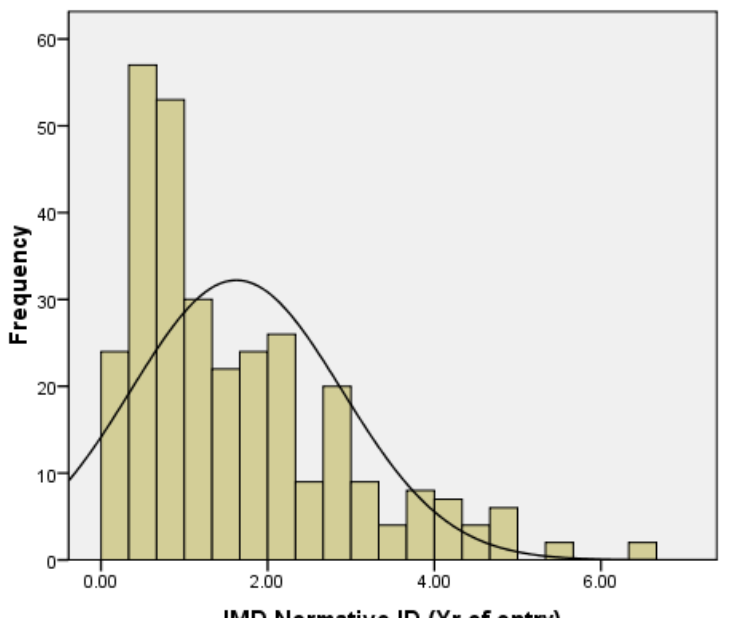

IMD Normative ID (Yr of entry)

EFI Regulative ID (Yr of entry)

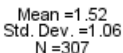

Mean $=1.52$
Std. Dev. $=1.0$
$N=307$

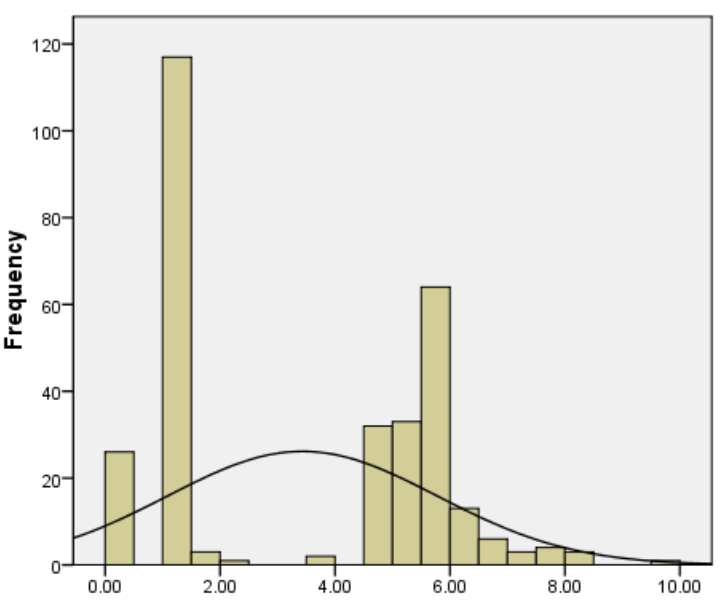

Mean $=1.62$
Std. Dev. $=1.267$
$N=307$

Mean $=3.43$
Std. Dev $=2.348$
$N=308$ 
Hostede Cultural distance

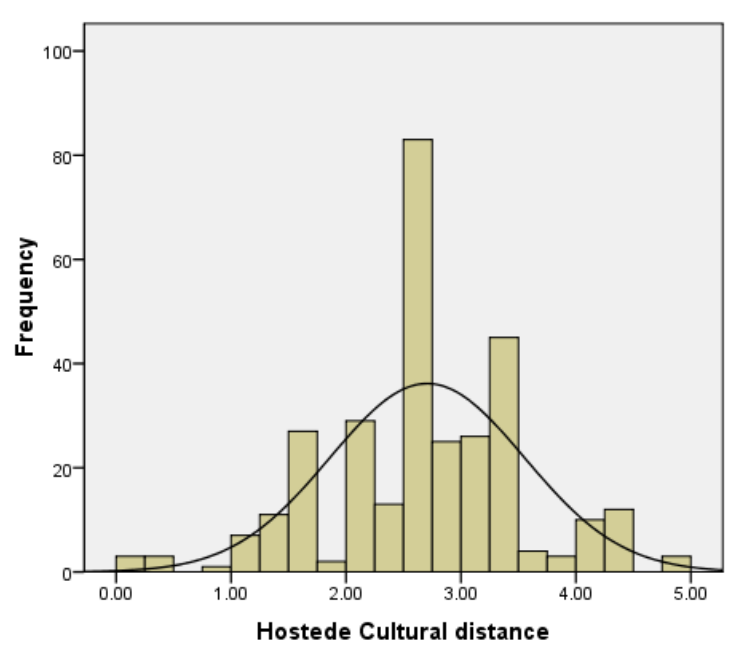

Control - Edu demand (yr of entry)

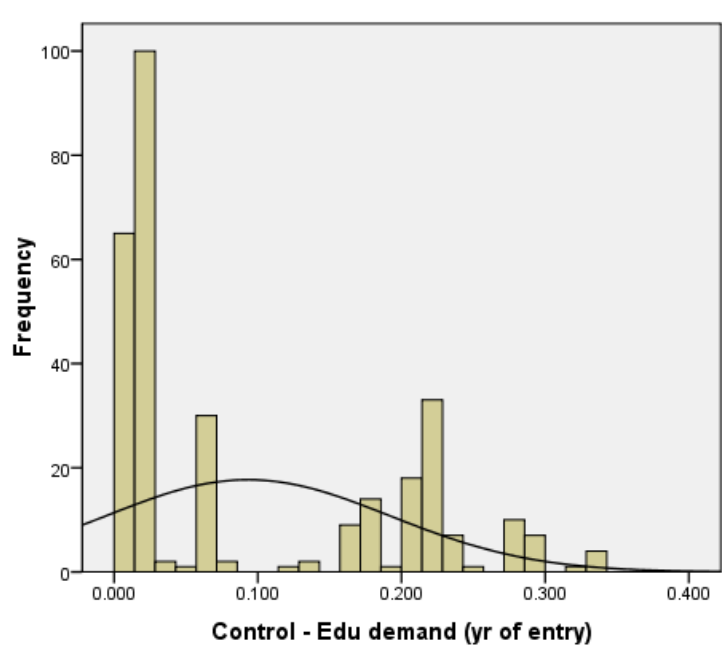

Control - Edu demand (yr of entry)
Mean $=0.09$
Std. Dev. $=0.099$
$N=308$
Mean $=2.71$
Std. Dev. $=0.847$
$N=307$ 


\section{Appendix R}

\section{LEVENE'S TEST OF HOMOGENEITY OF VARIANCE}

\begin{tabular}{|c|c|c|c|c|c|c|c|}
\hline \multicolumn{8}{|c|}{ Case Processing Summary } \\
\hline & \multirow{3}{*}{$\begin{array}{l}\text { Into } \\
\text { numerical } \\
\text { values }\end{array}$} & \multicolumn{6}{|c|}{ Cases } \\
\hline & & \multicolumn{2}{|c|}{ Valid } & \multicolumn{2}{|c|}{ Missing } & \multicolumn{2}{|c|}{ Total } \\
\hline & & $\mathrm{N}$ & Percent & $\mathrm{N}$ & Percent & $\mathrm{N}$ & Percent \\
\hline \multirow[t]{6}{*}{ QC - Continuous Dependent } & AU & 70 & $100.0 \%$ & 0 & $.0 \%$ & 70 & $100.0 \%$ \\
\hline & CA & 30 & $100.0 \%$ & 0 & $.0 \%$ & 30 & $100.0 \%$ \\
\hline & IR & 6 & $100.0 \%$ & 0 & $.0 \%$ & 6 & $100.0 \%$ \\
\hline & $\mathrm{NZ}$ & 10 & $100.0 \%$ & 0 & $.0 \%$ & 10 & $100.0 \%$ \\
\hline & US & 94 & $100.0 \%$ & 0 & $.0 \%$ & 94 & $100.0 \%$ \\
\hline & UK & 98 & $100.0 \%$ & 0 & $.0 \%$ & 98 & $100.0 \%$ \\
\hline
\end{tabular}

Test of Homogeneity of Variance

\begin{tabular}{|c|c|c|c|c|c|}
\hline & & Levene Statistic & df1 & $\mathrm{df} 2$ & Sig. \\
\hline \multirow[t]{4}{*}{ QC - Continuous Dependent } & Based on Mean & 5.701 & 5 & 302 & .000 \\
\hline & Based on Median & 4.161 & 5 & 302 & .001 \\
\hline & Based on Median and with adjusted df & 4.161 & 5 & 189.939 & .001 \\
\hline & Based on trimmed mean & 5.108 & 5 & 302 & .000 \\
\hline
\end{tabular}




\section{Appendix S \\ FACTOR ANALYSIS}

Industry experience (AU and NZ combined data set)

Cumulative variance explained by retained factor: $51 \%$

KMO and Bartlett's Test

\begin{tabular}{|ll|r|}
\hline Kaiser-Meyer-Olkin Measure of Sampling Adequacy. & & .570 \\
Bartlett's Test of Sphericity & Approx. Chi-Square & df \\
& Sig. & 15.000 \\
& .000 \\
\hline
\end{tabular}

Rotated Component Matrix ${ }^{\mathrm{a}}$

\begin{tabular}{|c|c|c|c|}
\hline & \multicolumn{3}{|c|}{ Component } \\
\hline & 1 & 2 & 3 \\
\hline QG - Ind Experience (i) & -.162 & .500 & .537 \\
\hline QG - Ind Experience (ii) & .101 & -.067 & .906 \\
\hline QG - Ind Experience (iii) & .842 & .116 & .100 \\
\hline QG - Ind Experience (iv) & .855 & -.028 & -.055 \\
\hline QG - Ind Experience (v) & .402 & .639 & -.144 \\
\hline QG - Ind Experience (vi) & .030 & -.753 & -.078 \\
\hline
\end{tabular}

Extraction Method: Principal Component Analysis.

Rotation Method: Varimax with Kaiser Normalization.

a. Rotation converged in 5 iterations. 
Industry experience (UK and IR combined data set)

Cumulative variance explained by retained factor: $64 \%$

KMO and Bartlett's Test

\begin{tabular}{|ll|r|}
\hline Kaiser-Meyer-Olkin Measure of Sampling Adequacy. & & .519 \\
Bartlett's Test of Sphericity & Approx. Chi-Square & \\
& df & 42.386 \\
15.000 & Sig. \\
\hline
\end{tabular}

Rotated Component Matrix

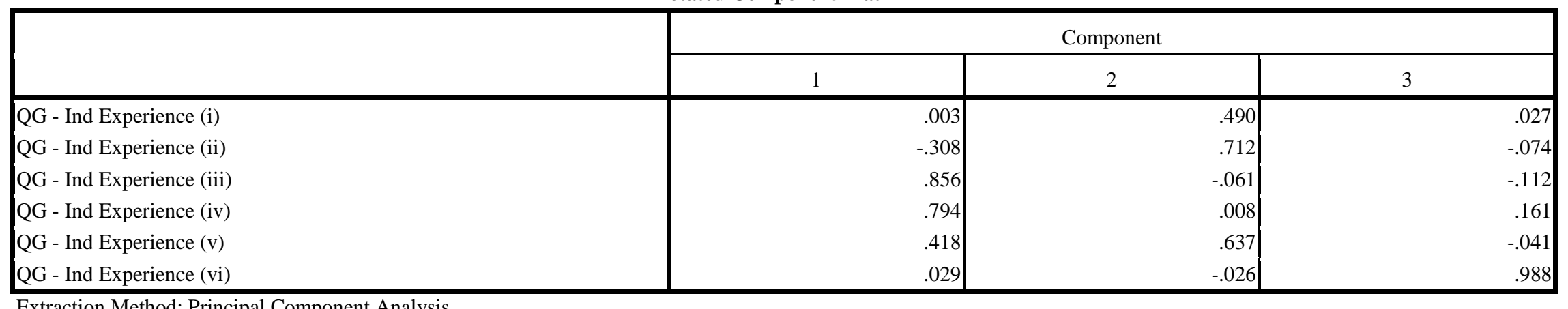

Extraction Method: Principal Component Analysis.

Rotation Method: Varimax with Kaiser Normalization.

a. Rotation converged in 4 iterations.

Industry experience (US and CA combined data set)

Cumulative variance explained by retained factor: $63 \%$

KMO and Bartlett's Test

Kaiser-Meyer-Olkin Measure of Sampling Adequacy.

Bartlett's Test of Sphericity 


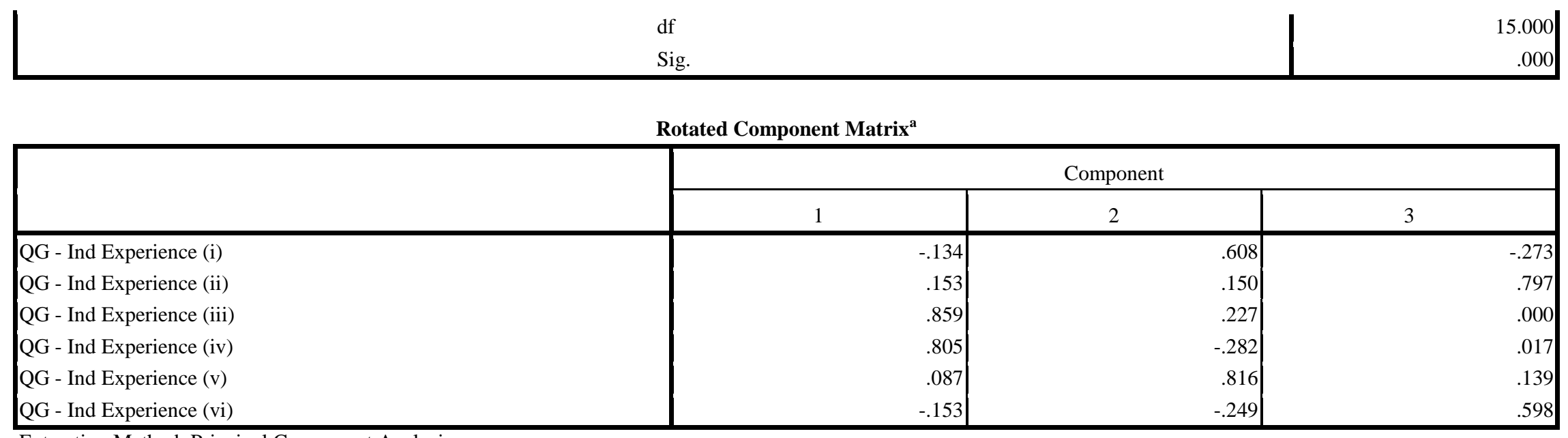

Extraction Method: Principal Component Analysis.

Rotation Method: Varimax with Kaiser Normalization.

a. Rotation converged in 4 iterations.

\section{Industry experience (Complete data set)}

Cumulative variance explained by retained factor: $62 \%$

KMO and Bartlett's Test

\begin{tabular}{|ll|r|}
\hline Kaiser-Meyer-Olkin Measure of Sampling Adequacy. & & .495 \\
Bartlett's Test of Sphericity & Approx. Chi-Square & df \\
& Sig. & 106.899 .000 \\
.000 & \\
\hline
\end{tabular}


Rotated Component Matrix

\begin{tabular}{|c|c|c|c|}
\hline & \multicolumn{3}{|c|}{ Component } \\
\hline & 1 & 2 & 3 \\
\hline QG - Ind Experience (i) & -.112 & .669 & .060 \\
\hline QG - Ind Experience (ii) & -.025 & .002 & .972 \\
\hline QG - Ind Experience (iii) & .848 & .146 & .001 \\
\hline QG - Ind Experience (iv) & .832 & -.179 & -.030 \\
\hline QG - Ind Experience (v) & .327 & .558 & .207 \\
\hline QG - Ind Experience (vi) & .028 & -.611 & .138 \\
\hline
\end{tabular}

Extraction Method: Principal Component Analysis.

Rotation Method: Varimax with Kaiser Normalization.

a. Rotation converged in 4 iterations.

\section{Know-how (AU and NZ combined data set)}

Cumulative variance explained by retained factor: $72 \%$

KMO and Bartlett's Test

\begin{tabular}{|ll|r|}
\hline Kaiser-Meyer-Olkin Measure of Sampling Adequacy. & & .869 \\
Bartlett's Test of Sphericity & Approx. Chi-Square & 231.796 \\
& $\mathrm{df}$ & Sig. \\
\hline
\end{tabular}

Component Matrix ${ }^{a}$

\begin{tabular}{|l|r|r|}
\hline & Component \\
\cline { 2 - 3 } & \\
\hline QI - Know how (i) & .860 \\
QI - Know how (ii) & .847 \\
QI - Know how (iii) & .846 \\
\hline
\end{tabular}


QI - Know how (iv)

QI - Know how (v)

Extraction Method: Principal Component Analysis.

a. 1 components extracted.

\section{Know-how (UK and IR combined data set)}

Cumulative variance explained by retained factor: $60 \%$

KMO and Bartlett's Test

\begin{tabular}{|ll|r|}
\hline Kaiser-Meyer-Olkin Measure of Sampling Adequacy. & Approx. Chi-Square & .815 \\
Bartlett's Test of Sphericity & df & 183.472 \\
& Sig. & 10.000 \\
& .000 \\
\hline
\end{tabular}

Component Matrix

\begin{tabular}{|c|c|}
\hline & Component \\
\hline & \\
\hline QI - Know how (i) & .793 \\
\hline QI - Know how (ii) & .830 \\
\hline QI - Know how (iii) & .651 \\
\hline QI - Know how (iv) & .768 \\
\hline QI - Know how (v) & .812 \\
\hline
\end{tabular}

Extraction Method: Principal Component Analysis.

a. 1 components extracted. 


\section{Know-how (US and CA combined data set)}

Cumulative variance explained by retained factor: $82 \%$

KMO and Bartlett's Test

\begin{tabular}{|c|c|c|}
\hline & .909 \\
\hline \multirow{3}{*}{$\begin{array}{l}\text { Kaiser-Meyer-Olkin Measure of Sampling Adequacy. } \\
\text { Bartlett's Test of Sphericity }\end{array}$} & Approx. Chi-Square & 546.622 \\
\hline & $\mathrm{df}$ & 10.000 \\
\hline & Sig. & .000 \\
\hline
\end{tabular}

Component Matrix ${ }^{\mathrm{a}}$

\begin{tabular}{|l|r|r|}
\hline & & \\
\cline { 2 - 3 } & & \\
\hline QI - Know how (i) & .881 \\
QI - Know how (ii) & .899 \\
QI - Know how (iii) & .909 \\
QI - Know how (iv) & .912 \\
QI - Know how (v) & .916 \\
\hline
\end{tabular}

Extraction Method: Principal Component Analysis.

a. 1 components extracted.

\section{Know-how (Complete data set)}

Cumulative variance explained by retained factor: $75 \%$

KMO and Bartlett's Test

\begin{tabular}{|ll|r|}
\hline Kaiser-Meyer-Olkin Measure of Sampling Adequacy. & & .901 \\
Bartlett's Test of Sphericity & Approx. Chi-Square & \\
& df & Sig. \\
& 1039.239 \\
10.000 & .000 \\
\hline
\end{tabular}


Component Matrix ${ }^{\mathrm{a}}$

\begin{tabular}{|l|r|}
\hline & \\
\cline { 2 - 3 } & \\
\hline QI - Know how (i) & .863 \\
QI - Know how (ii) & .878 \\
QI - Know how (iii) & .843 \\
QI - Know how (iv) & .874 \\
QI - Know how (v) & \\
\hline
\end{tabular}

Extraction Method: Principal Component Analysis.

a. 1 components extracted.

\section{Organisational culture (AU and NZ combined data set)}

Cumulative variance explained by retained factor: $60 \%$

KMO and Bartlett's Test

\begin{tabular}{|ll|r|}
\hline Kaiser-Meyer-Olkin Measure of Sampling Adequacy. & & .838 \\
Bartlett's Test of Sphericity & Approx. Chi-Square & 131.650 \\
& $\mathrm{df}$ & Sig. \\
10.000 & .000 \\
\hline
\end{tabular}

Component Matrix

\begin{tabular}{|l|r|r|}
\hline & Component Matrix $^{\mathrm{a}}$ & \\
\cline { 2 - 3 } & & \\
\hline QJ - Org Culture (i) & .725 \\
QJ - Org Culture (ii) & .753 \\
QJ - Org Culture (iii) & .766 & \\
\hline
\end{tabular}


QJ - Org Culture (iv)

QJ - Org Culture (v)

Extraction Method: Principal Component Analysis.

a. 1 components extracted.

\section{Organisational culture (UK and IR combined data set)}

Cumulative variance explained by retained factor: 57\%

KMO and Bartlett's Test

\begin{tabular}{|ll|r|}
\hline Kaiser-Meyer-Olkin Measure of Sampling Adequacy. & & .696 \\
Bartlett's Test of Sphericity & Approx. Chi-Square & \\
& df & Sig. \\
42.023 \\
10.000 \\
.000 \\
\hline
\end{tabular}

Rotated Component Matrix ${ }^{\mathrm{a}}$

\begin{tabular}{|l|r|r|}
\hline & \multicolumn{2}{|c|}{ Component } \\
\cline { 2 - 3 } & \multicolumn{2}{|c|}{1} \\
\hline QJ - Org Culture (i) & .924 \\
QJ - Org Culture (ii) & .164 \\
QJ - Org Culture (iii) & .006 \\
QJ - Org Culture (iv) & .657 \\
QJ - Org Culture (v) & .631 \\
\hline
\end{tabular}

Extraction Method: Principal Component Analysis.

Rotation Method: Varimax with Kaiser Normalization.

a. Rotation converged in 3 iterations. 


\section{Organisational culture (US and CA combined data set)}

Cumulative variance explained by retained factor: 69\%

\begin{tabular}{|ll|r|}
\hline Kaiser-Meyer-Olkin Measure of Sampling Adequacy. & KMO and Bartlett's Test & \\
Bartlett's Test of Sphericity & Approx. Chi-Square & .877 \\
& df & 310.574 \\
& 10.000 \\
& Sig. & .000 \\
\hline
\end{tabular}

Component Matrix ${ }^{\mathrm{a}}$

\begin{tabular}{|l|r|}
\hline & \multicolumn{1}{|c|}{ Component } \\
\cline { 2 - 2 } & 1 \\
\hline QJ - Org Culture (i) & 1 \\
QJ - Org Culture (ii) & .827 \\
QJ - Org Culture (iii) & .806 \\
QJ - Org Culture (iv) & .849 \\
QJ - Org Culture (v) & .856 \\
\hline
\end{tabular}

Extraction Method: Principal Component Analysis.

a. 1 components extracted.

\section{Organisational culture (Complete data set)}

Cumulative variance explained by retained factor: $60 \%$

KMO and Bartlett's Test

\begin{tabular}{|ll|r|}
\hline Kaiser-Meyer-Olkin Measure of Sampling Adequacy. & & .855 \\
Bartlett's Test of Sphericity & Approx. Chi-Square \\
& df & Sig. \\
\hline
\end{tabular}


Component Matrix

\begin{tabular}{|l|r|}
\hline & Component \\
\cline { 2 - 2 } & 1 \\
\hline QJ - Org Culture (i) & 1 \\
QJ - Org Culture (ii) & .744 \\
QJ - Org Culture (iii) & .756 \\
QJ - Org Culture (iv) & .784 \\
QJ - Org Culture (v) & .800 \\
\hline
\end{tabular}

Extraction Method: Principal Component Analysis.

a. 1 components extracted.

\section{Reputation (AU and NZ combined data set)}

Cumulative variance explained by retained factor: $69 \%$

KMO and Bartlett's Test

\begin{tabular}{|ll|r|}
\hline Kaiser-Meyer-Olkin Measure of Sampling Adequacy. & & .772 \\
Bartlett's Test of Sphericity & Approx. Chi-Square & 133.498 \\
& df & Sig. \\
& 6.000 \\
\hline
\end{tabular}

Component Matrix

\begin{tabular}{|l|r|}
\hline & Component \\
\cline { 2 - 3 } & \\
\hline QM - Reputation (i) & .878 \\
QM - Reputation (ii) & .818 \\
QM - Reputation (iii) & .811 \\
QM - Reputation (iv) & .807 \\
\hline Extraction Method: Principal Component Analysis. & \\
\hline
\end{tabular}

Extraction Method: Principal Component Analysis. 
Component Matrix

\begin{tabular}{|l|r|}
\hline & Component \\
\cline { 2 - 2 } & 1 \\
\hline QM - Reputation (i) & 1 \\
QM - Reputation (ii) & .878 \\
QM - Reputation (iii) & .818 \\
QM - Reputation (iv) & .811 \\
\hline
\end{tabular}

Extraction Method: Principal Component Analysis.

a. 1 components extracted.

\section{Reputation (UK and IR combined data set)}

Cumulative variance explained by retained factor: $68 \%$

KMO and Bartlett's Test

\begin{tabular}{|c|c|c|}
\hline \multicolumn{2}{|l|}{ Kaiser-Meyer-Olkin Measure of Sampling Adequacy. } & .794 \\
\hline Bartlett's Test of Sphericity & Approx. Chi-Square & 163.311 \\
\hline & df & 6.000 \\
\hline & Sig. & .000 \\
\hline
\end{tabular}

Component Matrix

\begin{tabular}{|l|r|}
\hline & \\
\cline { 2 - 2 } & \\
\hline QM - Reputation (i) & .851 \\
QM - Reputation (ii) & .810 \\
QM - Reputation (iii) & .854 \\
QM - Reputation (iv) & .777 \\
\hline
\end{tabular}

Extraction Method: Principal Component Analysis.

a. 1 components extracted. 


\section{Reputation (US and CA combined data set)}

Cumulative variance explained by retained factor: $70 \%$

KMO and Bartlett's Test

\begin{tabular}{|ll|r|}
\hline Kaiser-Meyer-Olkin Measure of Sampling Adequacy. & & .822 \\
Bartlett's Test of Sphericity & Approx. Chi-Square & df \\
& Sig. & 6.000 \\
\hline
\end{tabular}

Component Matrix ${ }^{\mathrm{a}}$

\begin{tabular}{|l|r|r|}
\hline & Component \\
\cline { 2 - 2 } & & \\
\hline QM - Reputation (i) & .838 \\
QM - Reputation (ii) & .858 \\
QM - Reputation (iii) & .877 \\
QM - Reputation (iv) & .783 \\
\hline
\end{tabular}

Extraction Method: Principal Component Analysis.

a. 1 components extracted.

\section{Reputation (Complete data set)}

Cumulative variance explained by retained factor: 69\%

KMO and Bartlett's Test

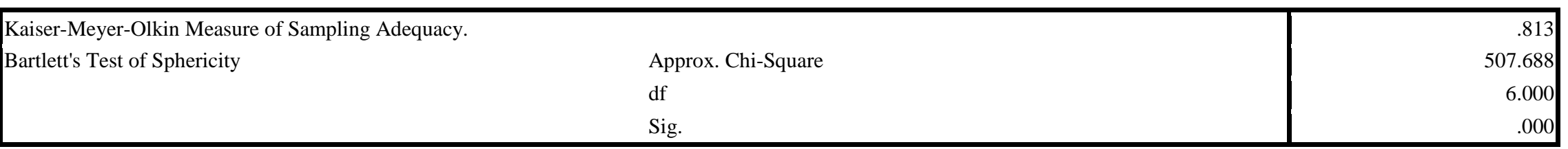


Component Matrix ${ }^{\mathrm{a}}$

\begin{tabular}{|l|r|}
\hline & Component \\
\cline { 2 - 3 } & 1 \\
\hline QM - Reputation (i) & 1 \\
QM - Reputation (ii) & .852 \\
QM - Reputation (iii) & .833 \\
QM - Reputation (iv) & .849 \\
Extraction Method: Principal Component Analysis. & .783 \\
\hline
\end{tabular}

a. 1 components extracted. 


\section{Appendix T}

\section{RELIABILITY ANALYSIS FOR FACTOR VARIABLES}

\begin{tabular}{|c|c|c|}
\hline Variable & Cronbach's Alpha & Number of Items \\
\hline Industry Experience (AU/NZ) & 6.682 & 2 \\
\hline Industry Experience (UK/IR) & .652 & 2 \\
\hline Industry Experience (US/CA) & .600 & 2 \\
\hline Industry Experience (Complete data set) & .640 & 2 \\
\hline Know-how (AU/NZ) & .902 & 5 \\
\hline Know-how (UK/IR) & .827 & 5 \\
\hline Know-how (US/CA) & .943 & 5 \\
\hline Know-how (Complete data set) & .918 & 5 \\
\hline Organisational Culture (AU/NZ) & .827 & 5 \\
\hline Organisational Culture (UK/IR) & .491 & 3 \\
\hline Organisational Culture (US/CA) & .885 & 5 \\
\hline Organisational Culture (Complete data set) & .830 & 5 \\
\hline Reputation (AU/NZ) & .844 & 4 \\
\hline Reputation (UK/IR) & .839 & 4 \\
\hline Reputation (US/CA) & .859 & 4 \\
\hline Reputation (Complete data set) & .847 & 4 \\
\hline
\end{tabular}




\section{Appendix U}

\section{CORRELATION MATRIX ${ }^{44}$}

\begin{tabular}{|c|c|c|c|c|c|c|c|c|c|c|}
\hline AU/NZ data set & 1 & 2 & 3 & 4 & 5 & 6 & 7 & 8 & 9 & 10 \\
\hline 1.Continuous Dependent & 1 & & & & & & & & & \\
\hline 2.Geo Experience (i) & & 1 & & & & & & & & \\
\hline 3.Geo Experience (ii) & & & 1 & & & & & & & \\
\hline 4.Geo Experience (iii) & & & & 1 & & & & & & \\
\hline 5.Geo Experience (iv) & & & & & 1 & & & & & \\
\hline 6.Geo Experience (v) & & & & & $.240 *$ & 1 & & & & \\
\hline 7.Ind Experience (i) & & & & & & & 1 & & & \\
\hline 8.Ind Experience (ii) & & $.223 *$ & $-.229 *$ & & & & & 1 & & \\
\hline $\begin{array}{l}\text { 9.Industry Experience } \\
\text { Factor }\end{array}$ & & & & & & & & & 1 & \\
\hline 10.Ind Experience (v) & & & & & & & & & $.290 * *$ & 1 \\
\hline 11.Ind Experience (vi) & & & & & & & & & & \\
\hline 12. Transfer Experience & $-.726 * *$ & & & & & & & & & \\
\hline 13. Know-how factor & $-.686 * *$ & & & & & & & & & \\
\hline $\begin{array}{l}\text { 14. Organisational culture } \\
\text { factor }\end{array}$ & $-.657 * *$ & & & & & & & & $-.226 *$ & \\
\hline 15. Financial resources & & & & & & & & & & \\
\hline 16. Importance of protection & & & & & & & & & & \\
\hline
\end{tabular}

${ }^{44}$ Only Significant correlations are highlighted. The correlations of learning intent and the dummy variables are not shown because of small $\mathrm{n}$.

** Correlation is significant at the 0.01 level (2-tailed). ${ }^{*}$ Correlation is significant at the 0.05 level (2-tailed). 


\begin{tabular}{|c|c|c|c|c|c|c|c|}
\hline \multicolumn{8}{|l|}{ of reputation } \\
\hline 17. Reputation factor & $-.328 * *$ & & & & $.223 *$ & & \\
\hline \multicolumn{8}{|l|}{ 18. Size (UG) } \\
\hline \multicolumn{8}{|l|}{ 19. Size (PG) } \\
\hline \multicolumn{8}{|l|}{ 20. Size (Staff) } \\
\hline 21. Age & & $.264 *$ & & & & & \\
\hline \multicolumn{8}{|l|}{ 22. World ranking } \\
\hline 23. Domestic ranking & & & & & & $.243 *$ & \\
\hline \multicolumn{8}{|l|}{$\begin{array}{l}\text { 24. Reasons for offshore } \\
\text { engagement } 1\end{array}$} \\
\hline $\begin{array}{l}\text { 25. Reasons for offshore } \\
\text { engagement } 2\end{array}$ & & & $-.250 *$ & & & & $.252^{*}$ \\
\hline \multicolumn{8}{|l|}{$\begin{array}{l}\text { 26. Reasons for offshore } \\
\text { engagement } 3\end{array}$} \\
\hline \multicolumn{8}{|l|}{$\begin{array}{l}\text { 27. Reasons for offshore } \\
\text { engagement } 4\end{array}$} \\
\hline \multicolumn{8}{|l|}{$\begin{array}{l}\text { 28. Reasons for offshore } \\
\text { engagement } 5\end{array}$} \\
\hline \multicolumn{8}{|l|}{$\begin{array}{l}\text { 29. Reasons for offshore } \\
\text { engagement } 6\end{array}$} \\
\hline $\begin{array}{l}\text { 30. Reasons for offshore } \\
\text { engagement } 7\end{array}$ & & & & & & & $-.236^{*}$ \\
\hline \multicolumn{8}{|l|}{ 31. IMD normative ID } \\
\hline 32. IMD regulative ID & & & $.237 *$ & & & & \\
\hline 33. EFI regulative ID & $.320 * *$ & & & & & & \\
\hline \multicolumn{8}{|l|}{$\begin{array}{l}\text { 34. Hofstede cultural } \\
\text { distance }\end{array}$} \\
\hline \multicolumn{8}{|l|}{ 35. Education demand } \\
\hline 36. Host country regulations & & & & $.263 *$ & & & \\
\hline $\begin{array}{l}\text { 37. Home country } \\
\text { regulations }\end{array}$ & & & & & & & \\
\hline
\end{tabular}




\begin{tabular}{|c|c|c|c|c|c|c|c|c|c|c|}
\hline AU/NZ data set & 11 & 12 & 13 & 14 & 15 & 16 & 17 & 18 & 19 & 20 \\
\hline 11.Ind Experience (vi) & 1 & & & & & & & & & \\
\hline 12. Transfer Experience & & 1 & & & & & & & & \\
\hline 13. Know-how factor & & $.883 * *$ & 1 & & & & & & & \\
\hline $\begin{array}{l}\text { 14. Organisational culture } \\
\text { factor }\end{array}$ & & $.839 * *$ & $.838 * *$ & 1 & & & & & & \\
\hline 15. Financial resources & & & & & 1 & & & & & \\
\hline $\begin{array}{l}\text { 16. Importance of protection } \\
\text { of reputation }\end{array}$ & & $.398 * *$ & $.383^{* *}$ & $.353^{* *}$ & & 1 & & & & \\
\hline 17. Reputation factor & & $.461 * *$ & $.432 *$ & $.347 * *$ & & & 1 & & & \\
\hline 18. Size (UG) & & & & & $.407 * *$ & & $.274 *$ & 1 & & \\
\hline 19. Size (PG) & & $.363 * *$ & $.263 *$ & $.240 *$ & $.559 * *$ & & $.310 * *$ & $.673 * *$ & 1 & \\
\hline 20. Size (Staff) & & $.335 * *$ & $.272 *$ & $.222 *$ & $.516 * *$ & & & $.576 * *$ & $.603 * *$ & 1 \\
\hline 21. Age & & & & & $.537 * *$ & & & $.282 *$ & $.249 *$ & \\
\hline 22. World ranking & & & & & $-.720 * *$ & & & $-.412 * *$ & $-.578 * *$ & $-.503^{* *}$ \\
\hline 23. Domestic ranking & & & & & $-.723 * *$ & & & $-.394 * *$ & $-.572 * *$ & $-.478 * *$ \\
\hline $\begin{array}{l}\text { 24. Reasons for offshore } \\
\text { engagement } 1\end{array}$ & & & & & & & & & & \\
\hline $\begin{array}{l}\text { 25. Reasons for offshore } \\
\text { engagement } 2\end{array}$ & & & & & & & $.271 *$ & & & \\
\hline $\begin{array}{l}\text { 26. Reasons for offshore } \\
\text { engagement } 3\end{array}$ & & & & & & & & & & \\
\hline $\begin{array}{l}\text { 27. Reasons for offshore } \\
\text { engagement } 4\end{array}$ & & & & & & & & & & \\
\hline $\begin{array}{l}\text { 28. Reasons for offshore } \\
\text { engagement } 5\end{array}$ & & & & & & & & & & \\
\hline $\begin{array}{l}\text { 29. Reasons for offshore } \\
\text { engagement } 6\end{array}$ & $-.359 * *$ & & & & & & & & & \\
\hline $\begin{array}{l}\text { 30. Reasons for offshore } \\
\text { engagement } 7\end{array}$ & & & & & & & & & & \\
\hline 31. IMD normative ID & & & $-.250 *$ & & & & $-.249 *$ & & & \\
\hline 32. IMD regulative ID & & & $-.242 *$ & & & & $-.307 * *$ & & & \\
\hline 33. EFI regulative ID & & $-.411 * *$ & $-.363 * *$ & $-.308^{* *}$ & & & & & & \\
\hline
\end{tabular}




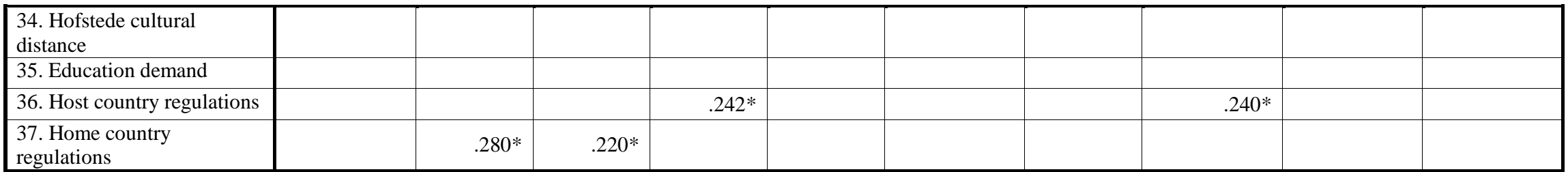

\begin{tabular}{|c|c|c|c|c|c|c|c|c|c|c|}
\hline AU/NZ data set & 21 & 22 & 23 & 24 & 25 & 26 & 27 & 28 & 29 & 30 \\
\hline 21. Age & 1 & & & & & & & & & \\
\hline 22. World ranking & $-.537 * *$ & 1 & & & & & & & & \\
\hline 23. Domestic ranking & $-.609 * *$ & $.908 *$ & 1 & & & & & & & \\
\hline $\begin{array}{l}\text { 24. Reasons for offshore } \\
\text { engagement } 1\end{array}$ & $.225^{*}$ & & & 1 & & & & & & \\
\hline $\begin{array}{l}\text { 25. Reasons for offshore } \\
\text { engagement } 2\end{array}$ & & & & & 1 & & & & & \\
\hline $\begin{array}{l}\text { 26. Reasons for offshore } \\
\text { engagement } 3\end{array}$ & & & & & & 1 & & & & \\
\hline $\begin{array}{l}\text { 27. Reasons for offshore } \\
\text { engagement } 4\end{array}$ & & & & & & & 1 & & & \\
\hline $\begin{array}{l}\text { 28. Reasons for offshore } \\
\text { engagement } 5\end{array}$ & & & & & & & & 1 & & \\
\hline $\begin{array}{l}\text { 29. Reasons for offshore } \\
\text { engagement } 6\end{array}$ & & & & & & & & & 1 & \\
\hline $\begin{array}{l}\text { 30. Reasons for offshore } \\
\text { engagement } 7\end{array}$ & & & & & & & & & & 1 \\
\hline 31. IMD normative ID & & & & & & & & & & \\
\hline 32. IMD regulative ID & & & & & & & & & & \\
\hline 33. EFI regulative ID & & & & & & & & & & \\
\hline $\begin{array}{l}\text { 34. Hofstede cultural } \\
\text { distance }\end{array}$ & & & & & & & & & & \\
\hline 35. Education demand & & & & & & & & & & \\
\hline 36. Host country regulations & & & & & & & & & & \\
\hline
\end{tabular}




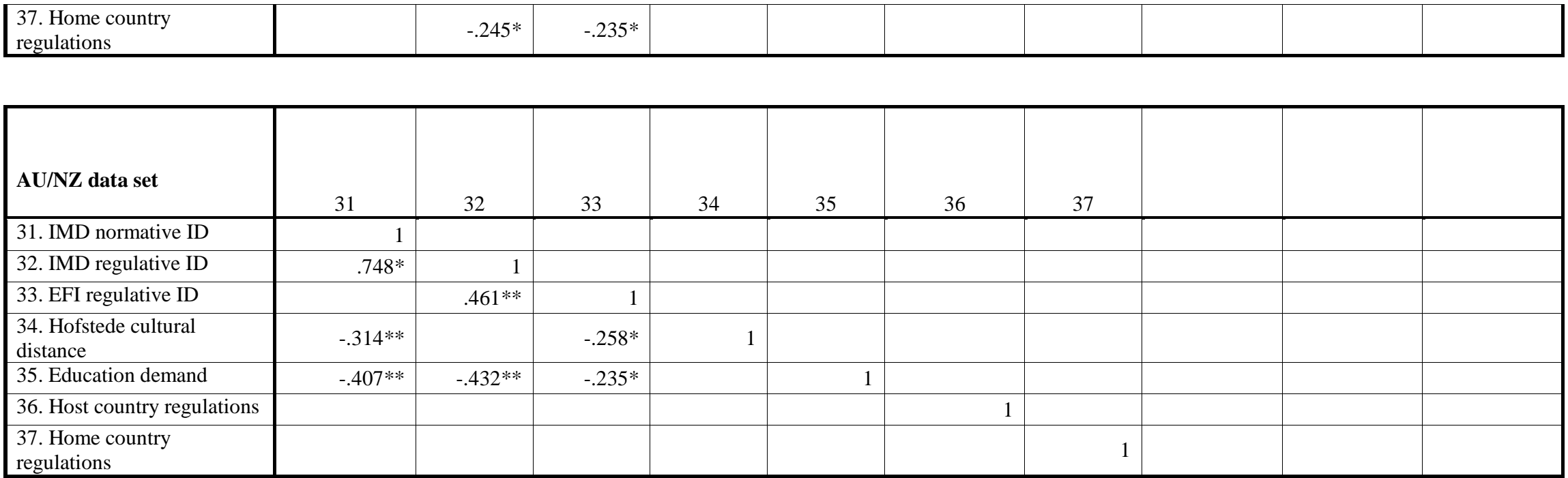

\begin{tabular}{|c|c|c|c|c|c|c|c|c|c|c|}
\hline UK/IR data set & 1 & 2 & 3 & 4 & 5 & 6 & 7 & 8 & 9 & 10 \\
\hline 1.Continuous Dependent & 1 & & & & & & & & & \\
\hline 2.Geo Experience (i) & & 1 & & & & & & & 1 & \\
\hline 3.Geo Experience (ii) & & & 1 & & & & & & & 1 \\
\hline 4.Geo Experience (iii) & & & & 1 & & & & & & \\
\hline 5.Geo Experience (iv) & & & & & 1 & & & & & \\
\hline 6.Geo Experience (v) & & & & & & 1 & & & & \\
\hline 7.Ind Experience (i) & & & & $.206 *$ & $-.242 *$ & & 1 & & & \\
\hline 8.Ind Experience (ii) & & & & & & & & 1 & & \\
\hline $\begin{array}{l}\text { 9.Industry Experience } \\
\text { Factor }\end{array}$ & & & & & & & & & 1 & \\
\hline 10.Ind Experience (v) & & & & & & & & & $.213 *$ & 1 \\
\hline 11.Ind Experience (vi) & & & & & & & & & & \\
\hline
\end{tabular}




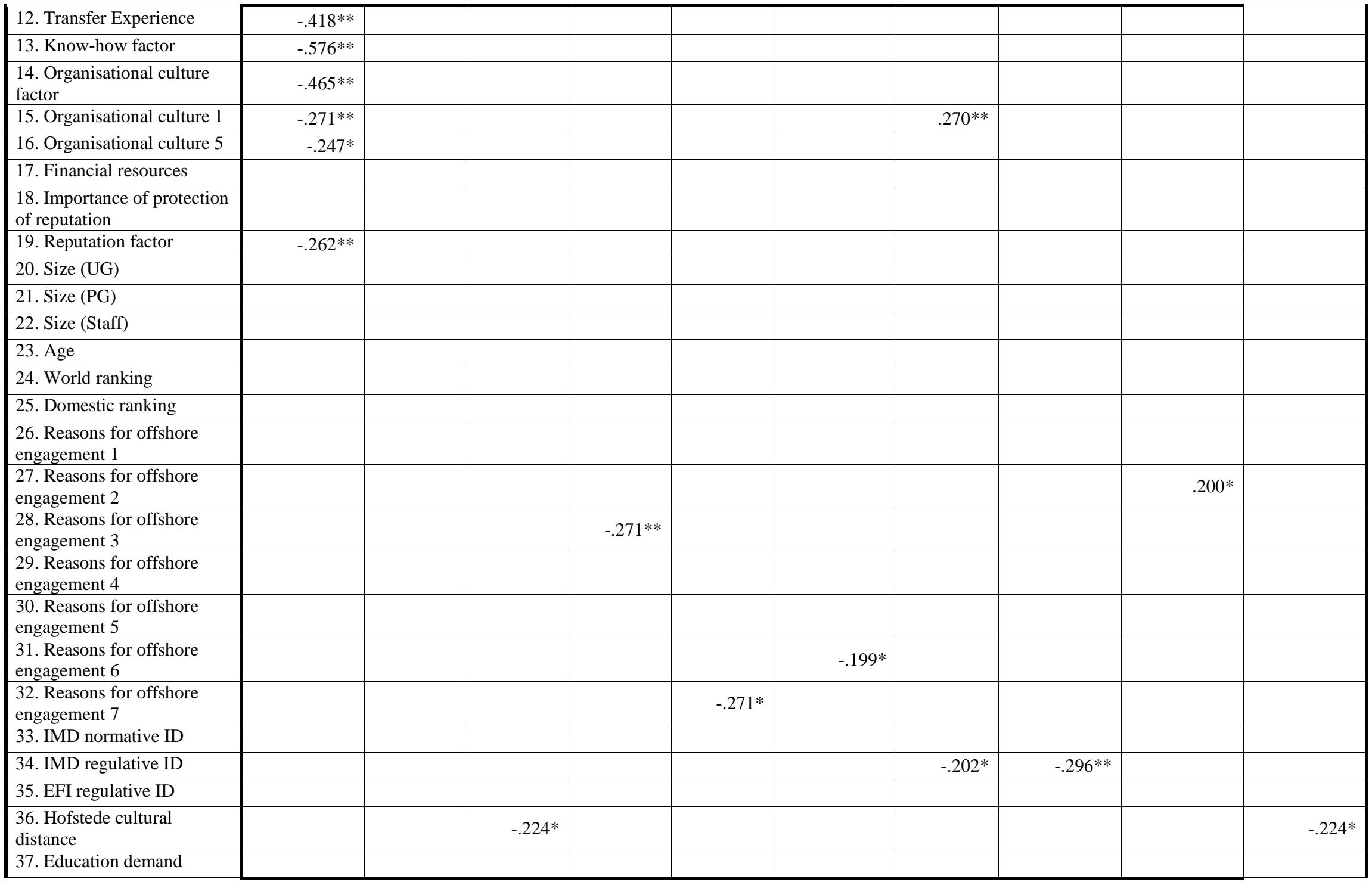


38. Host country regulation

39. Home country

regulations

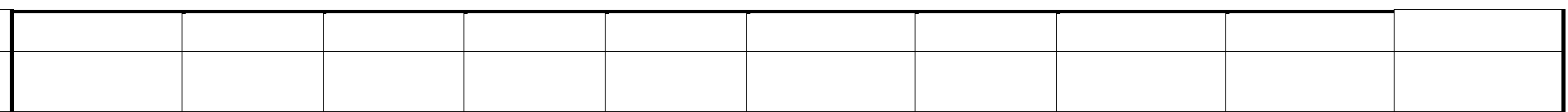

\begin{tabular}{|c|c|c|c|c|c|c|c|c|c|c|}
\hline UK/IR data set & 11 & 12 & 13 & 14 & 15 & 16 & 17 & 18 & 19 & 20 \\
\hline 11.Ind Experience (vi) & 1 & & & & & & & & & \\
\hline 12. Transfer Experience & & 1 & & & & & & & & \\
\hline 13. Know-how factor & & $.566 * *$ & 1 & & & & & & & \\
\hline $\begin{array}{l}\text { 14. Organisational culture } \\
\text { factor }\end{array}$ & & $.540 * *$ & $.507 * *$ & 1 & & & & & & \\
\hline 15. Organisational culture 1 & & $.362 * *$ & $.347 * *$ & & 1 & & & & & \\
\hline 16. Organisational culture 5 & $-.193 *$ & $.374 * *$ & $.471^{* *}$ & $.379 * *$ & $.263 * *$ & 1 & & & & \\
\hline 17. Financial resources & & & & & & & 1 & & & \\
\hline $\begin{array}{l}\text { 18. Importance of protection } \\
\text { of reputation }\end{array}$ & & & $.265^{* *}$ & & & $.285^{* *}$ & & 1 & & \\
\hline 19. Reputation factor & & $.304 * *$ & $.314 * *$ & $.278 * *$ & & & & & 1 & \\
\hline 20. Size (UG) & & $-.215 *$ & & & & & & & & 1 \\
\hline 21. Size (PG) & & & & & & & $.390 * *$ & & & $.504 * *$ \\
\hline 22. Size (Staff) & & & & & & & $.396 * *$ & & & $.537 * *$ \\
\hline 23. Age & & & & & & & & & & $.249 *$ \\
\hline 24. World ranking & & & & & & & $-.594 * *$ & & & \\
\hline 25. Domestic ranking & & & $-.224 *$ & $-.293 * *$ & & & $-.542 * *$ & & & \\
\hline $\begin{array}{l}\text { 26. Reasons for offshore } \\
\text { engagement } 1\end{array}$ & & & & & & & & & & \\
\hline $\begin{array}{l}\text { 27. Reasons for offshore } \\
\text { engagement } 2\end{array}$ & & & & & & & & & & \\
\hline $\begin{array}{l}\text { 28. Reasons for offshore } \\
\text { engagement } 3\end{array}$ & & & & & & & & & & \\
\hline $\begin{array}{l}\text { 29. Reasons for offshore } \\
\text { engagement } 4\end{array}$ & & & & & & & & $-.245^{*}$ & & \\
\hline $\begin{array}{l}\text { 30. Reasons for offshore } \\
\text { engagement } 5\end{array}$ & & & & & & & & & & \\
\hline
\end{tabular}




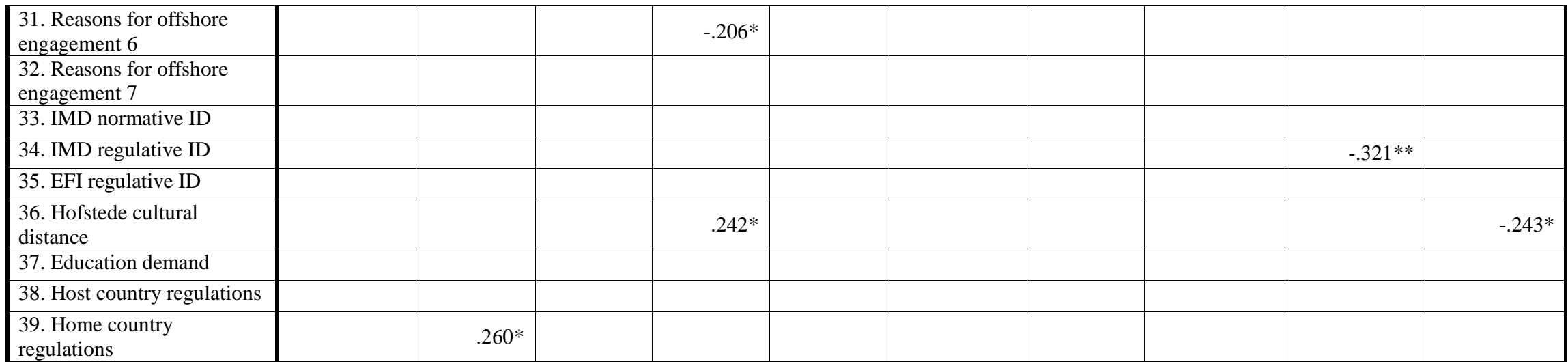

\begin{tabular}{|c|c|c|c|c|c|c|c|c|c|c|}
\hline UK/IR data set & 21 & 22 & 23 & 24 & 25 & 26 & 27 & 28 & 29 & 30 \\
\hline 21. Size (PG) & 1 & & & & & & & & & \\
\hline 22. Size (Staff) & $.709 * *$ & 1 & & & & & & & & \\
\hline 23. Age & $.346 * *$ & & 1 & & & & & & & \\
\hline 24. World ranking & $-.356 * *$ & $-.385 * *$ & & 1 & & & & & & \\
\hline 25. Domestic ranking & $-.336 * *$ & $-.297 * *$ & & $.883 * *$ & 1 & & & & & \\
\hline $\begin{array}{l}\text { 26. Reasons for offshore } \\
\text { engagement } 1\end{array}$ & & & $-.219 *$ & & & 1 & & & & \\
\hline $\begin{array}{l}\text { 27. Reasons for offshore } \\
\text { engagement } 2\end{array}$ & & & $-.229 *$ & & & & 1 & & & \\
\hline $\begin{array}{l}\text { 28. Reasons for offshore } \\
\text { engagement } 3\end{array}$ & & & & & & & & 1 & & \\
\hline $\begin{array}{l}\text { 29. Reasons for offshore } \\
\text { engagement } 4\end{array}$ & & & & & & & & & 1 & \\
\hline $\begin{array}{l}\text { 30. Reasons for offshore } \\
\text { engagement } 5\end{array}$ & & & & & & $-.243 *$ & & & & 1 \\
\hline $\begin{array}{l}\text { 31. Reasons for offshore } \\
\text { engagement } 6\end{array}$ & & & & & & $.228 *$ & & & & \\
\hline 32. Reasons for offshore & & & & & & & & & & \\
\hline
\end{tabular}




\begin{tabular}{|l|l|l|l|l|l|l|l|l|}
\hline engagement 7 & & & & & & & & \\
\hline 33. IMD normative ID & & & & & & & \\
\hline 34. IMD regulative ID & & & & & & & \\
\hline $\begin{array}{l}\text { 35. EFI regulative ID } \\
\text { 36. Hofstede cultural } \\
\text { distance }\end{array}$ & $-.208 *$ & & & & & & & \\
\hline $\begin{array}{l}37 . \text { Education demand } \\
\text { 38. Host country regulations }\end{array}$ & & & & & & & & \\
\hline $\begin{array}{l}\text { 39. Home country } \\
\text { regulations }\end{array}$ & & & & & & & & \\
\hline
\end{tabular}

\begin{tabular}{|c|c|c|c|c|c|c|c|c|c|}
\hline UK/IR data set & 31 & 32 & 33 & 34 & 35 & 36 & 37 & 38 & 39 \\
\hline $\begin{array}{l}\text { 31. Reasons for offshore } \\
\text { engagement } 6\end{array}$ & 1 & & & & & & & & \\
\hline $\begin{array}{l}\text { 32. Reasons for offshore } \\
\text { engagement } 7\end{array}$ & & 1 & & & & & & & \\
\hline 33. IMD normative ID & & & 1 & & & & & & \\
\hline 34. IMD regulative ID & $-.220 *$ & & $.242 *$ & 1 & & & & & \\
\hline 35. EFI regulative ID & & & $-.582 * *$ & & 1 & & & & \\
\hline $\begin{array}{l}\text { 36. Hofstede cultural } \\
\text { distance }\end{array}$ & & & $.314 * *$ & & $-.471 * *$ & 1 & & & \\
\hline 37. Education demand & & & $.641 * *$ & & $-.758 * *$ & & 1 & & \\
\hline 38. Host country regulations & & & & & & & & 1 & \\
\hline $\begin{array}{l}\text { 39. Home country } \\
\text { regulations }\end{array}$ & & & $-.222 *$ & & & & & & 1 \\
\hline
\end{tabular}

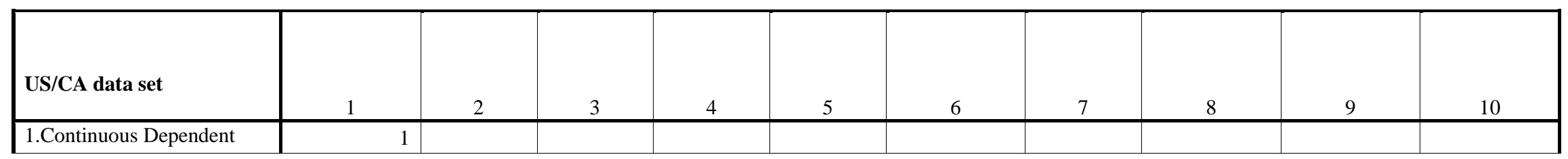




\begin{tabular}{|c|c|c|c|c|c|c|c|c|c|c|}
\hline 2.Geo Experience (i) & & 1 & & & & & & & & \\
\hline 3.Geo Experience (ii) & & & 1 & & & & & & & \\
\hline 4.Geo Experience (iii) & & & & 1 & & & & & & \\
\hline 5.Geo Experience (iv) & & & & & 1 & & & & & \\
\hline 6.Geo Experience (v) & & & & & & 1 & & & & \\
\hline 7.Ind Experience (i) & & $.272 *$ & & & & $.179 *$ & 1 & & & \\
\hline 8.Ind Experience (ii) & & & & & & & & 1 & & \\
\hline $\begin{array}{l}\text { 9.Industry Experience } \\
\text { Factor }\end{array}$ & & & & & & & & & 1 & \\
\hline 10.Ind Experience (v) & & & & $-.179 *$ & & & $.180^{*}$ & & & 1 \\
\hline \multicolumn{11}{|l|}{ 11. Ind Experience (vi) } \\
\hline 12. Transfer Experience & $-.789 * *$ & & $-.235^{*}$ & & & & & & & \\
\hline 13. Know-how factor & $-.782 * *$ & & $-.185^{*}$ & & & & & & & \\
\hline $\begin{array}{l}\text { 14. Organisational culture } \\
\text { factor }\end{array}$ & $-.707 * *$ & & & & & & & & & \\
\hline \multicolumn{11}{|l|}{ 15. Financial resources } \\
\hline $\begin{array}{l}\text { 16. Importance of protection } \\
\text { of reputation }\end{array}$ & $-.562 * *$ & $.179 *$ & & & & & & & & $.207^{*}$ \\
\hline 17. Reputation factor & $-.521 * *$ & & $-.193 *$ & & & & & & & \\
\hline \multicolumn{11}{|l|}{ 18. Size (UG) } \\
\hline \multicolumn{11}{|l|}{ 19. Size (PG) } \\
\hline \multicolumn{11}{|l|}{ 20. Size (Staff) } \\
\hline \multicolumn{11}{|l|}{ 21. Age } \\
\hline \multicolumn{11}{|l|}{ 22. World ranking } \\
\hline \multicolumn{11}{|l|}{ 23. Domestic ranking } \\
\hline \multicolumn{11}{|l|}{$\begin{array}{l}\text { 24. Reasons for offshore } \\
\text { engagement } 1\end{array}$} \\
\hline $\begin{array}{l}\text { 25. Reasons for offshore } \\
\text { engagement } 2\end{array}$ & $.221 *$ & & $.181^{*}$ & & & & & & & \\
\hline \multicolumn{11}{|l|}{$\begin{array}{l}\text { 26. Reasons for offshore } \\
\text { engagement } 3\end{array}$} \\
\hline $\begin{array}{l}\text { 27. Reasons for offshore } \\
\text { engagement } 4\end{array}$ & & & & & $-.193^{*}$ & & & & & \\
\hline $\begin{array}{l}\text { 28. Reasons for offshore } \\
\text { engagement } 5\end{array}$ & & & & $-.178 *$ & & & & & $-.191 *$ & \\
\hline
\end{tabular}




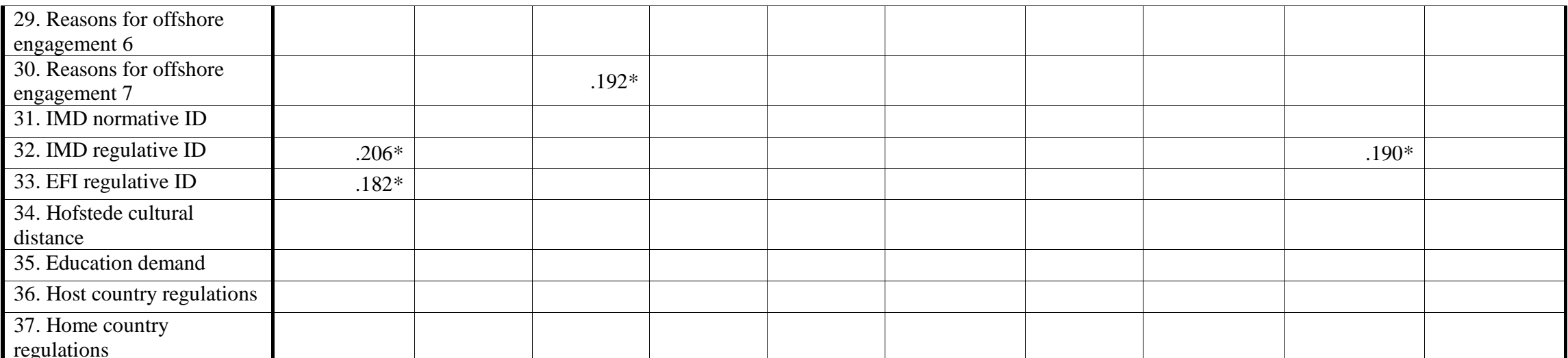

\begin{tabular}{|c|c|c|c|c|c|c|c|c|c|c|}
\hline US/CA data set & 11 & 12 & 13 & 14 & 15 & 16 & 17 & 18 & 19 & 20 \\
\hline 11. Ind Experience (vi) & 1 & & & & & & & & & \\
\hline 12. Transfer Experience & & 1 & & & & & & & & \\
\hline 13. Know-how factor & & $.931 *$ & 1 & & & & & & & \\
\hline $\begin{array}{l}\text { 14. Organisational culture } \\
\text { factor }\end{array}$ & & $.878^{* *}$ & $.908^{* *}$ & 1 & & & & & & \\
\hline 15. Financial resources & & & & & 1 & & & & & \\
\hline $\begin{array}{l}\text { 16. Importance of protection } \\
\text { of reputation }\end{array}$ & & $.544^{* *}$ & $.545^{* *}$ & $.519 * *$ & & 1 & & & & \\
\hline 17. Reputation factor & $-.182 *$ & $.664 * *$ & $.633 * *$ & $.638 * *$ & & $.370 * *$ & 1 & & & \\
\hline 18. Size (UG) & & & & & $.268 * *$ & & & 1 & & \\
\hline 19. Size (PG) & & & & & $.424 * *$ & & & $.501 * *$ & 1 & \\
\hline 20. Size (Staff) & & & & & $.424 * *$ & & & $.720 * *$ & $.671^{* *}$ & 1 \\
\hline 21. Age & & & & & $.402 * *$ & & & & $.460 * *$ & $.246 * *$ \\
\hline 22. World ranking & & & & $-.187 *$ & $-.658 * *$ & & & $-.292 * *$ & $-.587 * *$ & $-.519 * *$ \\
\hline 23. Domestic ranking & & & & & $-.549 * *$ & & & $-.281^{* *}$ & $-.425^{* *}$ & $-.474 * *$ \\
\hline 24. Reasons for offshore & & & & & & & & & & \\
\hline
\end{tabular}




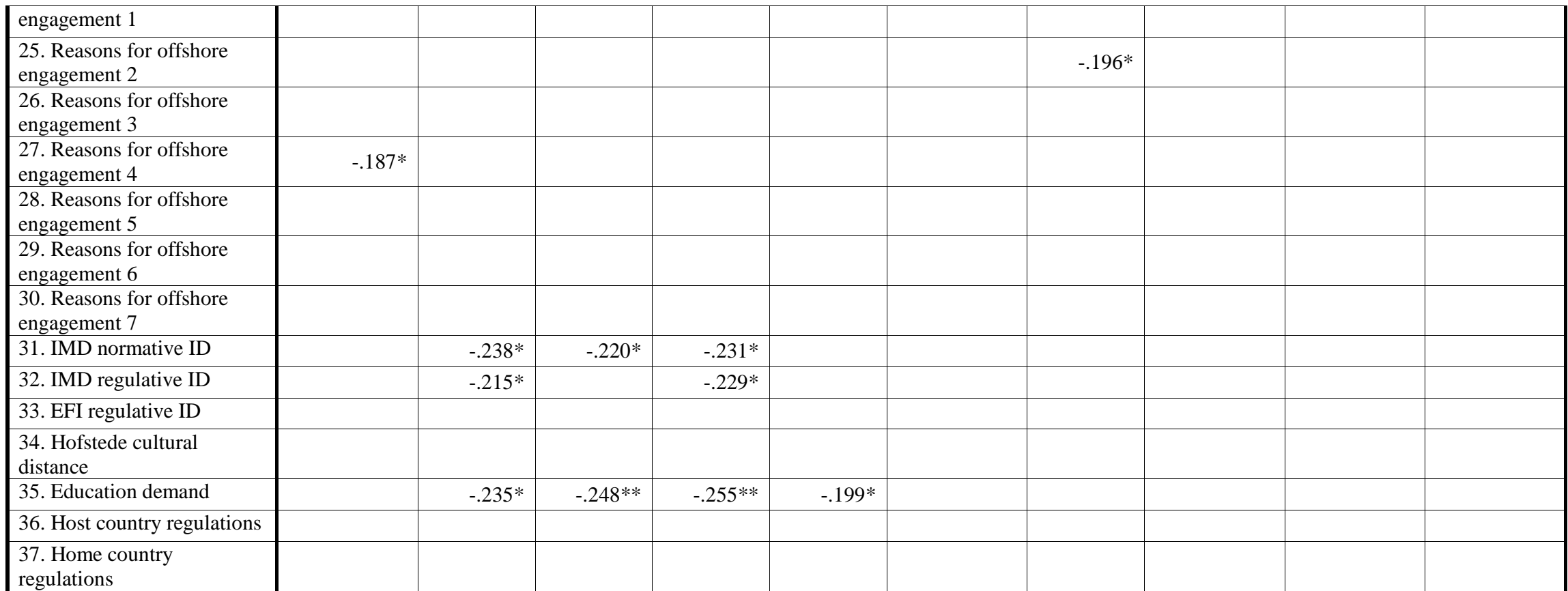

\begin{tabular}{|c|c|c|c|c|c|c|c|c|c|c|}
\hline US/CA data set & 21 & 22 & 23 & 24 & 25 & 26 & 27 & 28 & 29 & 30 \\
\hline 21. Age & 1 & & & & & & & & & \\
\hline 22. World ranking & $-.347 * *$ & 1 & & & & & & & & \\
\hline 23. Domestic ranking & $-.411 * *$ & $.840 * *$ & 1 & & & & & & & \\
\hline $\begin{array}{l}\text { 24. Reasons for offshore } \\
\text { engagement } 1\end{array}$ & & & & 1 & & & & & & \\
\hline $\begin{array}{l}\text { 25. Reasons for offshore } \\
\text { engagement } 2\end{array}$ & & & & & 1 & & & & & \\
\hline $\begin{array}{l}\text { 26. Reasons for offshore } \\
\text { engagement } 3\end{array}$ & & & & & & 1 & & & & \\
\hline
\end{tabular}




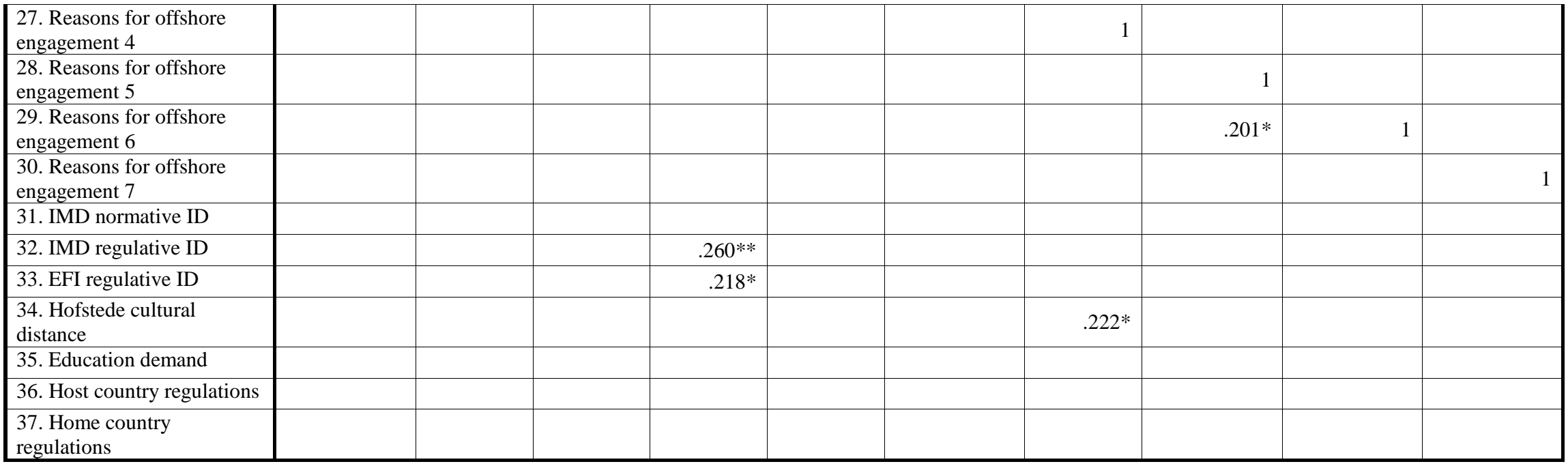

\begin{tabular}{|l|r|r|r|r|r|r|r|}
\hline US/CA data set & & & & & & & \\
\end{tabular}




\begin{tabular}{|c|c|c|c|c|c|c|c|c|c|c|}
\hline Complete data set & 1 & 2 & 3 & 4 & 5 & 6 & 7 & 8 & 9 & 10 \\
\hline 1.Continuous Dependent & 1 & & & & & & & & & \\
\hline 2.Geo Experience (i) & & 1 & & & & & & & & \\
\hline 3.Geo Experience (ii) & & & 1 & & & & & & & \\
\hline 4.Geo Experience (iii) & & & & 1 & & & & & & \\
\hline 5.Geo Experience (iv) & & & & & 1 & & & & & \\
\hline 6.Geo Experience (v) & & & & & & 1 & & & & \\
\hline 7.Ind Experience (i) & & & & $.151^{* *}$ & & & 1 & & & \\
\hline 8.Ind Experience (ii) & & & & & & & & 1 & & \\
\hline $\begin{array}{l}\text { 9.Industry Experience } \\
\text { Factor }\end{array}$ & $.144^{*}$ & & & & & & & & 1 & \\
\hline 10.Ind Experience (v) & & & & & & & $.126 *$ & & $.162 * *$ & 1 \\
\hline \multicolumn{11}{|l|}{ 11.Ind Experience (vi) } \\
\hline 12. Transfer Experience & $-.723 * *$ & & & & & & & & & \\
\hline 13. Know-how factor & $-.732 * *$ & & & & & & & & & \\
\hline $\begin{array}{l}\text { 14. Organisational culture } \\
\text { factor }\end{array}$ & $-.668 * *$ & & & & & & & & $-.116^{*}$ & \\
\hline \multicolumn{11}{|l|}{ 15. Financial resources } \\
\hline $\begin{array}{l}\text { 16. Importance of protection } \\
\text { of reputation }\end{array}$ & $-.331 * *$ & & & $-.114^{*}$ & & & & & & $.125^{*}$ \\
\hline 17. Reputation factor & $-.407 * *$ & & & & & & $.145^{*}$ & & & \\
\hline \multicolumn{11}{|l|}{ 18. Size (UG) } \\
\hline \multicolumn{11}{|l|}{ 19. Size (PG) } \\
\hline \multicolumn{11}{|l|}{ 20. Size (Staff) } \\
\hline \multicolumn{11}{|l|}{ 21. Age } \\
\hline 22. World ranking & $.134 *$ & & & & & & & & & \\
\hline \multicolumn{11}{|l|}{ 23. Domestic ranking } \\
\hline \multicolumn{11}{|l|}{$\begin{array}{l}\text { 24. Reasons for offshore } \\
\text { engagement } 1\end{array}$} \\
\hline $\begin{array}{l}\text { 25. Reasons for offshore } \\
\text { engagement } 2\end{array}$ & & & & & & & & & $.139 *$ & \\
\hline
\end{tabular}




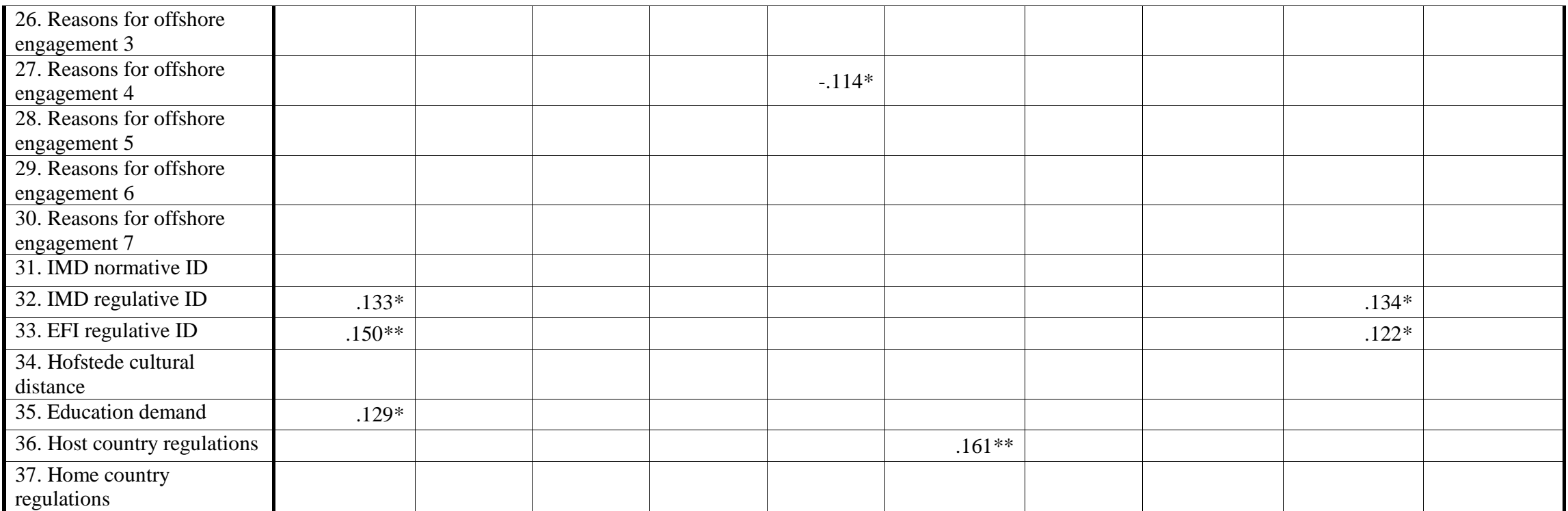

\begin{tabular}{|c|c|c|c|c|c|c|c|c|c|c|}
\hline Complete data set & 11 & 12 & 13 & 14 & 15 & 16 & 17 & 18 & 19 & 20 \\
\hline 11.Ind Experience (vi) & 1 & & & & & & & & & \\
\hline 12. Transfer Experience & & 1 & & & & & & & & \\
\hline 13. Know-how factor & & $.868 * *$ & 1 & & & & & & & \\
\hline $\begin{array}{l}\text { 14. Organisational culture } \\
\text { factor }\end{array}$ & $-.118 *$ & $.833 * *$ & $.851^{* *}$ & 1 & & & & & & \\
\hline 15. Financial resources & & $.184 * *$ & $.170 * *$ & $.220 * *$ & 1 & & & & & \\
\hline $\begin{array}{l}\text { 16. Importance of protection } \\
\text { of reputation }\end{array}$ & & $.416 * *$ & $.432 * *$ & $.90 * *$ & & 1 & & & & \\
\hline 17. Reputation factor & $-.152 * *$ & $.526 * *$ & $.500 * *$ & $.474 * *$ & & $.213^{* *}$ & 1 & & & \\
\hline 18. Size (UG) & & & & & $.201 * *$ & & & 1 & & \\
\hline 19. Size (PG) & & & & & $.372 * *$ & & $.168 * *$ & $.554 * *$ & 1 & \\
\hline
\end{tabular}




\begin{tabular}{|c|c|c|c|c|c|c|c|c|c|}
\hline 20. Size (Staff) & & & & $.272^{* *}$ & & & $.605^{* *}$ & $.637^{* *}$ & 1 \\
\hline 21. Age & $-.181 * *$ & $-.169 * *$ & & $.404 * *$ & & & & $.309 * *$ & \\
\hline 22. World ranking & & & $-.200 * *$ & $-.638 * *$ & & $-.119 *$ & $-.272 * *$ & $-.529 * *$ & $-.422 * *$ \\
\hline 23. Domestic ranking & & & & $-.470 * *$ & & & $-.254 * *$ & $-.453 * *$ & $-.424 * *$ \\
\hline $\begin{array}{l}\text { 24. Reasons for offshore } \\
\text { engagement } 1\end{array}$ & & & & & & & & & \\
\hline $\begin{array}{l}\text { 25. Reasons for offshore } \\
\text { engagement } 2\end{array}$ & & & & & & & & & \\
\hline $\begin{array}{l}\text { 26. Reasons for offshore } \\
\text { engagement } 3\end{array}$ & & & & & & & & & \\
\hline $\begin{array}{l}\text { 27. Reasons for offshore } \\
\text { engagement } 4\end{array}$ & & & & & & & & & \\
\hline $\begin{array}{l}\text { 28. Reasons for offshore } \\
\text { engagement } 5\end{array}$ & & & & & & & & & \\
\hline $\begin{array}{l}\text { 29. Reasons for offshore } \\
\text { engagement } 6\end{array}$ & & & & & & & & & \\
\hline $\begin{array}{l}\text { 30. Reasons for offshore } \\
\text { engagement } 7\end{array}$ & & & & & $.128 *$ & & & & \\
\hline 31. IMD normative ID & $-.187 * *$ & $-.154^{* *}$ & $-.146 *$ & & & $-.154 * *$ & & & \\
\hline 32. IMD regulative ID & $-.155 *$ & $-.139 *$ & $-.118 *$ & & & $-.160 * *$ & & & \\
\hline 33. EFI regulative ID & $-.138 *$ & $-.130 *$ & & & & & & & \\
\hline $\begin{array}{l}\text { 34. Hofstede cultural } \\
\text { distance }\end{array}$ & & & & & & & & & \\
\hline 35. Education demand & $-.145 *$ & $-.115 *$ & $-.146 *$ & & & & & & \\
\hline 36. Host country regulations & & & & & & & & & \\
\hline $\begin{array}{l}\text { 37. Home country } \\
\text { regulations }\end{array}$ & & & & & & & & & \\
\hline
\end{tabular}

\begin{tabular}{|c|c|c|c|c|c|c|c|c|c|c|}
\hline Complete data set & 21 & 22 & 23 & 24 & 25 & 26 & 27 & 28 & 29 & 30 \\
\hline 21. Age & 1 & & & & & & & & & \\
\hline 22. World ranking & $-.297 * *$ & 1 & & & & & & & & \\
\hline 23. Domestic ranking & $-.291 * *$ & $.833 * *$ & 1 & & & & & & & \\
\hline
\end{tabular}




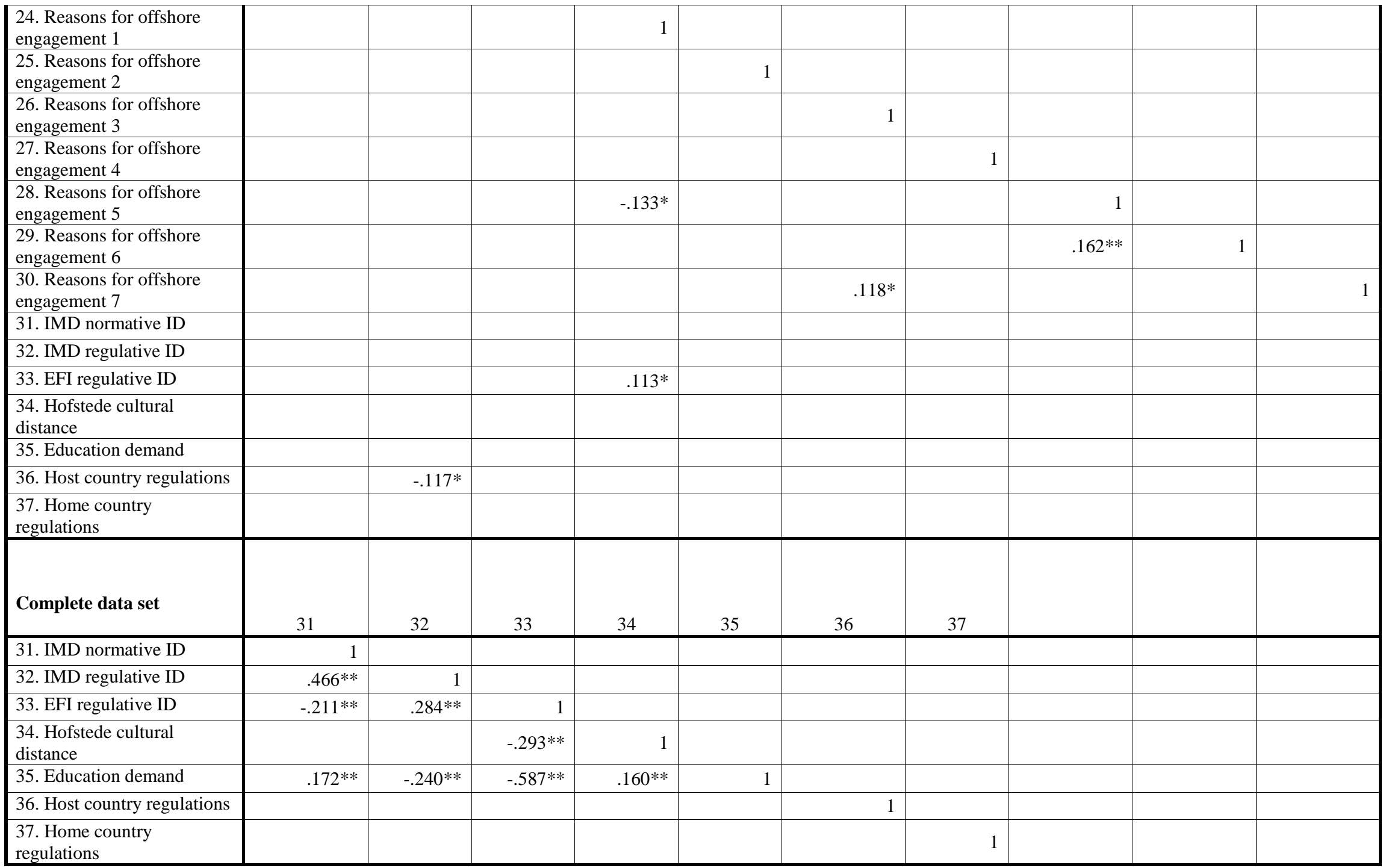


